NIST GCR 17-012

\title{
Proceedings of the International Workshop on Modeling of Physical, Economic, and Social Systems for Resilience Assessment
}

Lauren A. Mudd

Francis M. Lavelle

This publication is available free of charge from:

https://doi.org/10.6028/NIST.GCR.17-012

National Institute of 
NIST GCR 17-012

\section{Proceedings of the International Workshop on Modeling of Physical, Economic, and Social Systems for Resilience Assessment}

Prepared for

National Institute of Standards and Technology

Gaithersburg, MD

This publication is available free of charge from:

https://doi.org/10.6028/NIST.GCR.17-012

May 2017

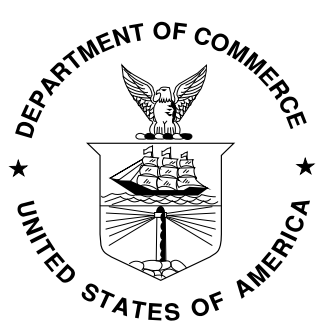

U.S. Department of Commerce Wilbur L. Ross Jr., Secretary

National Institute of Standards and Technology Kent Rochford, Acting NIST Director and Under Secretary of Commerce for Standards and Technology 
This publication was produced as part of contract SB1341-12-CQ-0014/15-370 with the National Institute of Standards and Technology. The contents of this publication do not necessarily reflect the views or policies of the National Institute of Standards and Technology or the US Government. 
International Workshop on Modeling of Physical, Economic, and Social Systems for Resilience Assessment

This page intentionally left blank. 


\section{Acknowledgements}

The International Workshop on Modeling of Physical, Economic, and Social Systems for Resilience Assessment was held at the Washington Dulles Airport Marriott in Dulles, VA on 1921 October 2016. This workshop was organized by the National Institute of Standards and Technology (NIST) and Colorado State University (CSU) to collect information on the state-ofthe-art methods to support the development of community resilience models from international researchers and subject matter experts in systems modeling of physical, social, and economic systems.

The workshop co-chairs who led the planning, organization, and execution of a successful workshop were:

- Dr. Terri McAllister, National Institute of Standards and Technology

- Dr. Bruce Ellingwood, Colorado State University

- Dr. John van de Lindt, Colorado State University

The workshop benefitted from the expertise, insight, and experience of the following speakers:

- Dr. Dennis Mileti, University of Colorado Boulder, CO

- Dr. Michael Faber, Technical University of Denmark, Denmark

- Dr. Adam Rose, University of Southern California, CA

- Dr. Rae Zimmerman, New York University, NY

- Dr. Georgious Giannopoulos, EU Joint Research Centre, Italy

- Dr. Nick Horspool, GNS Science, New Zealand

- Dr. Dongping Fang, Tsinghua University, China

- Dr. David Mendonça, National Science Foundation, VA

- Mr. Matthew Coats, US Department of Homeland Security, Washington DC

- Dr. Royce Francis, The George Washington University, VA

- Ms. Katherine Klise, Sandia National Laboratory, NM

- Dr. Charles Macal, Argonne National Laboratory, IL

- Dr. Matteo Broggi, Leibniz University, Hannover, Germany

- Dr. Auroop Ganguly, Northeastern University, MA

- Dr. Judith Mitrani-Reiser, National Institute of Standards and Technology, Johns Hopkins University, MD

- Dr. Terje Haukass, The University of British Columbia Vancouver, British Columbia

- Dr. Himmet Karaman, Istanbul Technical University, Turkey

- Dr. Quanwang Li, Tsinghua University, China

- Dr. Joshua Behr, Old Dominion University, VA

- Dr. Carolyn Kousky, Resources for the Future, Washington DC

- Dr. Nathanael Rosenheim, Texas A\&M University, TX

- Dr. Cindy Bruyère, National Center for Atmospheric Research, CO

- Dr. Keith Porter, University of Colorado Boulder, CO

- Dr. John van de Lindt, Colorado State University, CO 
Session moderators led facilitated discussions which were recorded by scribes:

- Session 1: Moderators: Dr. Georgios Giannopoulos, EU Joint Research Centre, Italy Dr. John van de Lindt, Colorado State University

Scribes: $\quad$ Ms. Hana Chmielewski, National Institute of Standards and Technology

Dr. Eun Cha, University of Illinois Urbana-Champaign

- Session 2: Moderators: Dr. Adam Rose, University of Southern California

Dr. Bruce Ellingwood, Colorado State University

Scribes: $\quad$ Dr. Ken Harrison, National Institute of Standards and

Technology

Dr. Andre Barbosa, Oregon State University

- Session 3: Moderators: Dr. Hussam Mahmoud, Colorado State University

Dr. Terje Haukaas, The University of British Columbia, Vancouver

Scribes: $\quad$ Dr. Juan Fung, National Institute of Standards and Technology

Dr. Ken Harrison, National Institute of Standards and

Technology

- Session 4: Moderators: Dr. Harvey Cutler, Colorado State University

Dr. Keith Porter, University of Colorado Boulder

Scribes: Dr. Jennifer Helgeson, National Institute of Standards and

Technology

Dr. Suren Chen, Colorado State University

- Session 5: Moderators: Dr. Elise Miller-Hooks, George Mason University

Dr. Judith Mitrani-Reiser, National Institute of Standards and

Technology, Johns Hopkins University

Scribes: $\quad$ Dr. Maria Dillard, National Institute of Standards and

Technology

Dr. Steve Cauffman, National Institute of Standards and

Technology

The following participants also provided valuable input based on their expertise, knowledge, and experience:

\begin{tabular}{|l|l|l|}
\multicolumn{1}{|c|}{ Last Name } & \multicolumn{1}{c|}{ First Name } & \multicolumn{1}{c|}{ Organization } \\
\hline Averill & Jason & National Institute for Standards and Technology \\
\hline Barbosa & Andre & Oregon State University \\
\hline Behr & Joshua & Old Dominion University \\
\hline Broggi & Matteo & Leibniz University Hannover \\
\hline Bruyere & Cindy & National Center for Atmospheric Research \\
\hline Butry & David & National Institute for Standards and Technology \\
\hline Cauffman & Stephen & National Institute for Standards and Technology \\
\hline Cha & Eun Jeong & University of Illinois at Urbana-Champaign \\
\hline Chen & Cynthia & University of Washington \\
\hline Chen & Suren & Colorado State University \\
\hline Chmielewski & Hana & National Institute for Standards and Technology \\
\hline
\end{tabular}




\begin{tabular}{|c|c|c|}
\hline Last Name & First Name & Organization \\
\hline Cimellaro & Gian Paolo & Politecnico di Torino \\
\hline Clifford & Megan & Argonne National Laboratory \\
\hline Coats & Matthew & Department of Homeland Security \\
\hline Cutler & Harvey & Colorado State University \\
\hline Dillard & Maria & National Institute for Standards and Technology \\
\hline Ellingwood & Bruce & Colorado State University \\
\hline Faber & Michael & Technical University of Denmark \\
\hline Fang & Dongping & Tsinghua University \\
\hline Forbes & Nathaniel & Forbes Calamity Prevention \\
\hline Francis & Royce & The George Washington University \\
\hline Frye & Emily & The MITRE Corporation \\
\hline Fung & Juan & National Institute for Standards and Technology \\
\hline Ganguly & Auroop & Northeastern University \\
\hline Giannopoulos & Georgios & EU Joint Research Centre (JRC) \\
\hline Graeden & Ellie & Talus Analytics \\
\hline Graettinger & Andrew & The University of Alabama \\
\hline Harrison & Ken & National Institute for Standards and Technology \\
\hline Haukaas & Terje & University of British Columbia Vancouver \\
\hline Hong & Liu & Huazhong University of Science and Technology \\
\hline Horspool & Nick & GNS Science \\
\hline Hummel & John & Argonne National Laboratory \\
\hline Karaman & Himmet & Istanbul Technical University \\
\hline Kerr & Justin & Talus Analytics \\
\hline Klise & Katherine & Sandia National Laboratory \\
\hline Kousky & Carolyn & Resources for the Future \\
\hline Lavelle & Francis & Applied Research Associates \\
\hline $\mathrm{Li}$ & Nan & Tsinghua University \\
\hline $\mathrm{Li}$ & Quanwang & Tsinghua University \\
\hline Macal & Charles & Argonne National Laboratory \\
\hline Mahmoud & Hussam & Colorado State University \\
\hline McAllister & Therese & National Institute for Standards and Technology \\
\hline Mendonça & David & National Science Foundation \\
\hline Mileti & Dennis S. & Retired \\
\hline Miller-Hooks & Elise & George Mason University \\
\hline Mitrani-Reiser & Judith & National Institute for Standards and Technology \\
\hline Mudd & Lauren & Applied Research Associates \\
\hline Mujumdar & Vilas & Self Employed \\
\hline Ouyang & Min & Huazhong University of Science and Technology \\
\hline Porter & Keith & University of Colorado Boulder \\
\hline Pozdnoukhov & Alexei & University of California Berkeley \\
\hline Qiu & Feng & Argonne National Laboratory \\
\hline Rajczak & William & The MITRE Corporation \\
\hline Ranjithan & Ranji & North Carolina State University \\
\hline Rose & Adam & University of Southern California \\
\hline
\end{tabular}


International Workshop on Modeling of Physical, Economic, and Social Systems for Resilience Assessment

Last Name

\section{First Name}

\section{Organization}

\begin{tabular}{|l|l|l|}
\hline Rosenheim & Nathanael & Texas A\&M University \\
\hline Slater & David & The MITRE Corporation \\
\hline Sutley & Elaina & University of Kansas \\
\hline Tien & Iris & Georgia Institute of Technology \\
\hline van de Lindt & John & Colorado State University \\
\hline van Maele & Michael & Talus Analytics \\
\hline Walton & Eli & Environmental Protection Agency \\
\hline Wang & Naiyu & University of Oklahoma \\
\hline Wang & Zhenghua & American Bureau of Shipping \\
\hline Zimmerman & Rae & New York University \\
\hline
\end{tabular}




\section{Table of Contents}

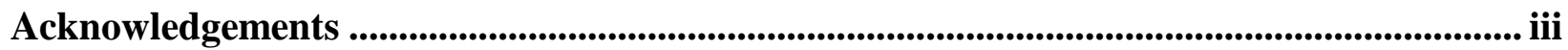

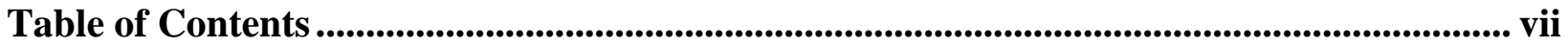

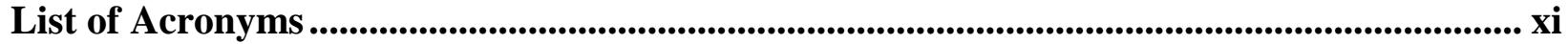

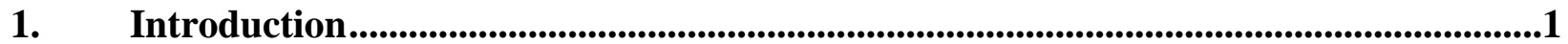

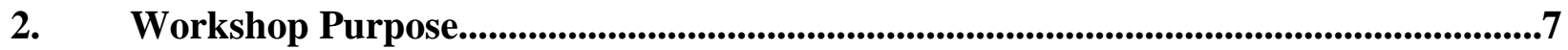

3. Session 1: Decision-Making and Evaluation of Proposed Alternatives ..........................9

3.1. Session Presentations ..................................................................................

3.1.1. Public Warning Timing: Issuance, Diffusion, Protective Action Initiation for Dam Breaches, Controlled Dam Releases, and Levee Breaches or Overtopping

3.1.2. Resilience of Societal Infrastructure Systems - Modeling and Decision Analysis Framework

3.1.3. Total Economic Consequences of Disasters ......................................................11

3.1.4. Infrastructure System Interconnectivity Effects on Resilience............................12

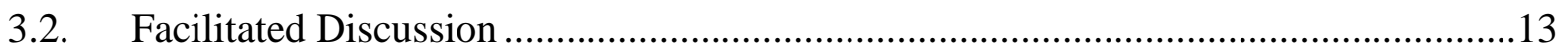

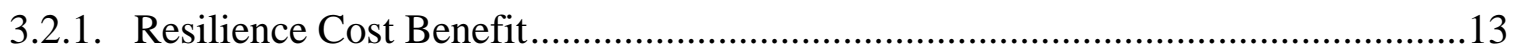

3.2.2. Effects of Human Behavior on System Design ...............................................14

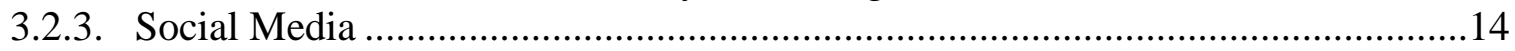

3.2.4. Model Library to Address Resilience Issues ...................................................14

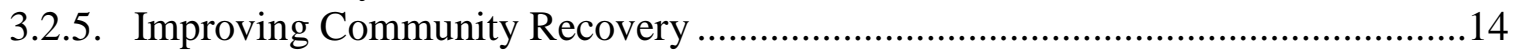

3.2.6. Resilient Failure and Management of Recovery..............................................15

3.2.7. Evolution of Thinking Regarding Tools and Methods ......................................15

3.3. Common Themes and Key Points (Session 1)........................................................16

4. Session 2: Resilience Modeling Research Around the World.........................................17

4.1. Session Presentations ........................................................................................17

4.1.1. Interdependencies, Cascading Effects and Resilience Optimization of

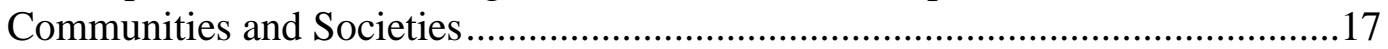

4.1.2. Resilience Modeling Research in New Zealand ................................................17

4.1.3. Urban Resilience: Asian Research Initiatives ....................................................18

4.1.4. NSF Investments and Opportunities in Interdisciplinary Research on Critical Infrastructure Systems .........................................................................19

4.1.5. DHS Sponsored Research: DHS Approaches to Supporting Infrastructure

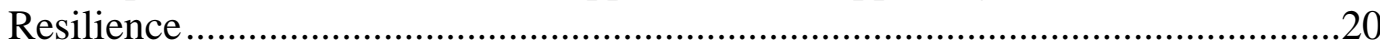

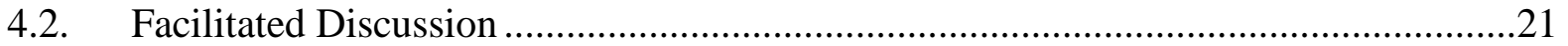

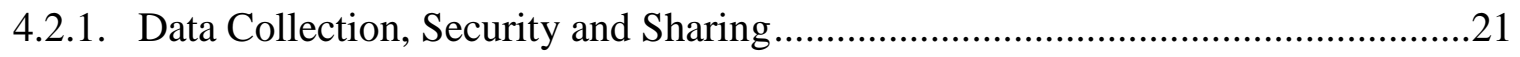

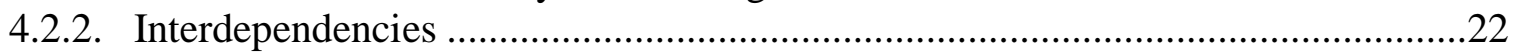

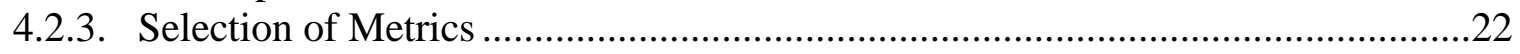

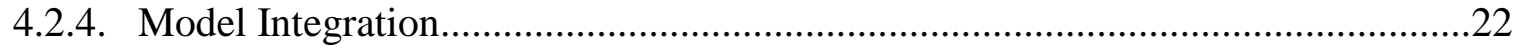

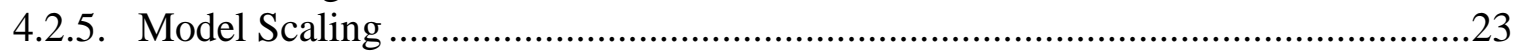

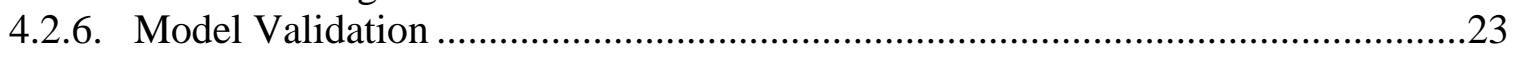


4.3. Common Themes and Key Points (Session 2).................................................23

5. Session 3: Modeling of Systems and Dependencies.......................................................25

5.1. Session Presentations .........................................................................................25

5.1.1. Evolution of Hazard Assessment Modeling in Drinking Water Systems ..............25

5.1.2. Modeling Water Distribution System Resilience ..............................................25

5.1.3. Power Distribution Systems: Modeling of System Dependencies and

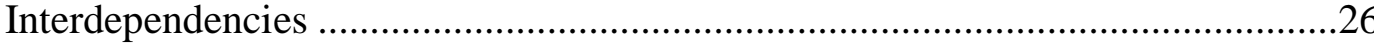

5.1.4. Reliability Assessment of Interdependent Networks Based on Survival

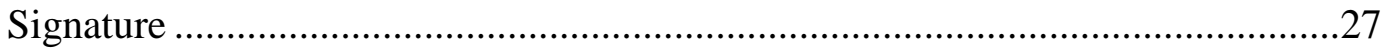

5.1.5. Climate \& Complexity: The Resilience of Natural-Built-Human Systems ...........27

5.1.6. Natural Disaster Impacts on Infrastructure Systems and Society .........................28

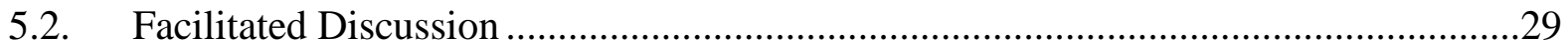

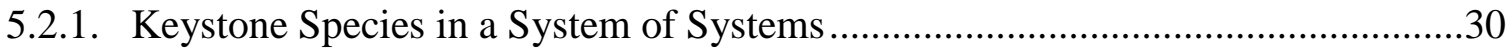

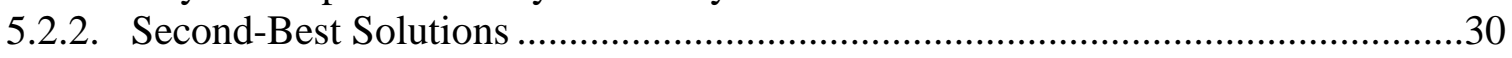

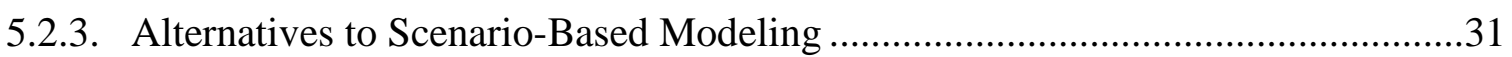

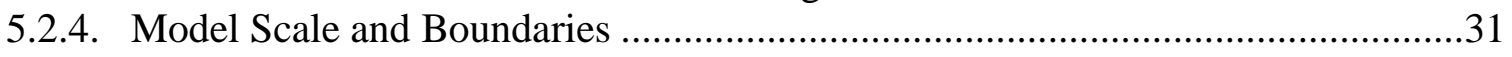

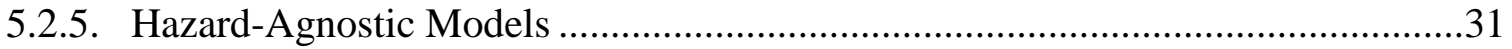

5.3. Common Themes and Key Points (Session 3).....................................................32

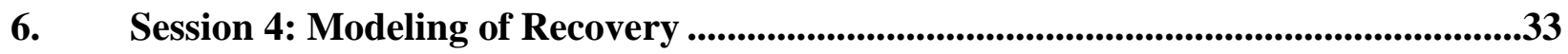

6.1. Session Presentations ...........................................................................................33

6.1.1. Physical and Economic Impacts of Earthquakes and Other Hazards ....................33

6.1.2. Road Network Functionality of Istanbul Following an Earthquake ......................34

6.1.3. Measuring and Improving the Resilience of the Built Environment in a

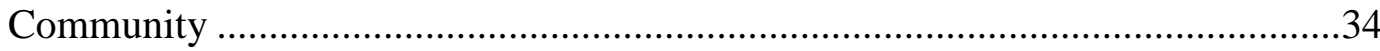

6.1.4. Housing Demand \& Capacity for Medically Fragile \& Vulnerable

Populations: Modeling Transition through Temporary, Interim, and

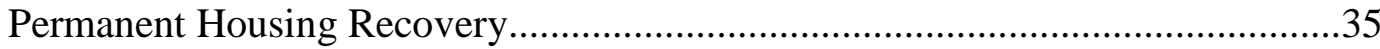

6.1.5. Modeling Recovery: The Role of Disaster Financing .........................................36

6.1.6. An Integration of Social Vulnerability Data and Mapping Tools for Community Vulnerability Assessments and Recovery Modeling ........................37

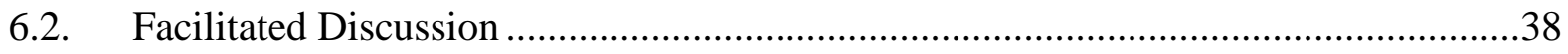

6.2.1. Defining, Communicating, and Modeling Metrics .........................................38

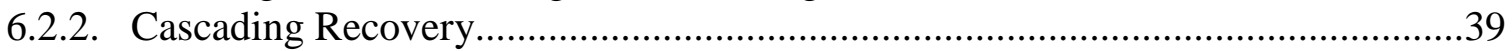

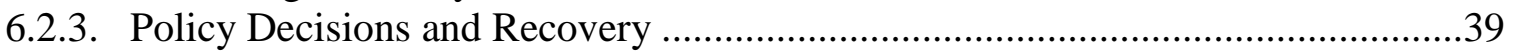

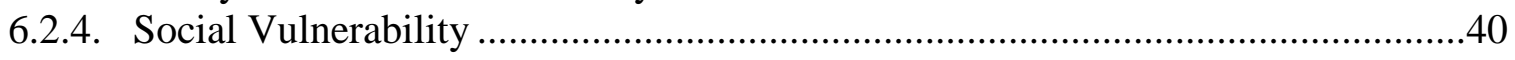

6.3. Common Themes and Key Points (Session 4) .....................................................40

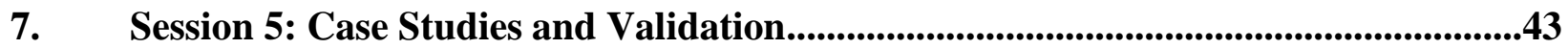

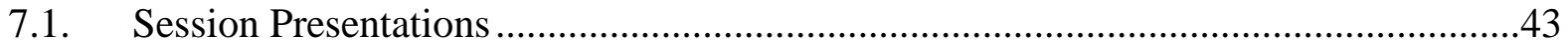

7.1.1. GRRIT ${ }^{\circledR}$ - a Sustainable Tool for Reducing Weather and Climate Impacts..........43

7.1.2. Resilience Modeling Innovations of the HayWired Planning Scenario ................44

7.1.3. Model Validation: The Joplin Tornado Hindcast .............................................45

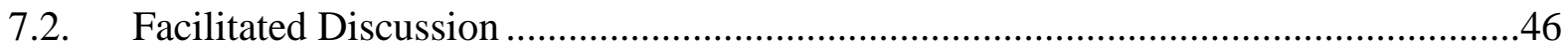




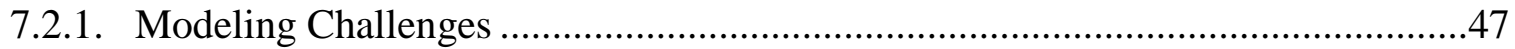

7.2.2. Increased Resilience and Hardened Design .......................................................47

7.2.3. Comparison of Modeling Approaches ..............................................................48

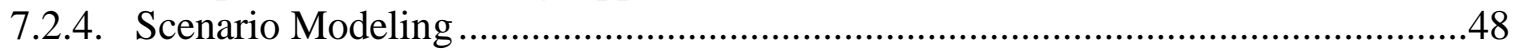

7.2.5. Model Complexity and Calibration ............................................................49

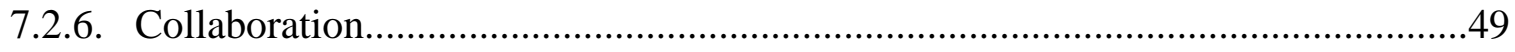

7.3. Common Themes and Key Points (Session 5).....................................................50

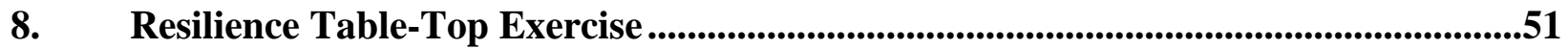

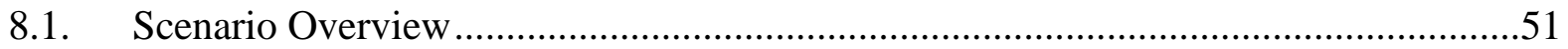

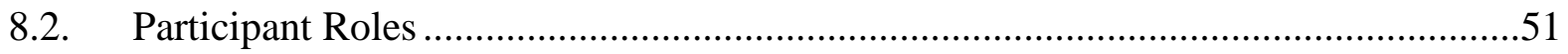

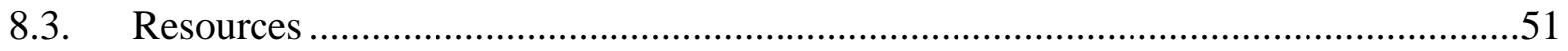

8.4. Post-Disaster Time Periods.............................................................................51

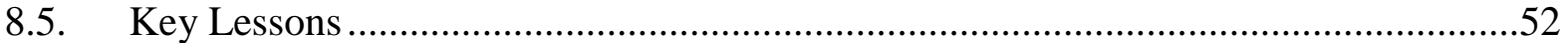

9. Summary of Systems Modeling Needs and Challenges for Resilience

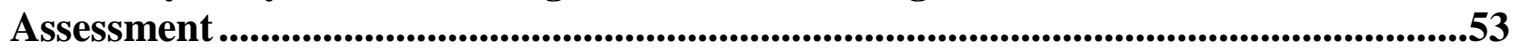

9.1. Overview of Systems Modeling for Community Resilience ....................................53

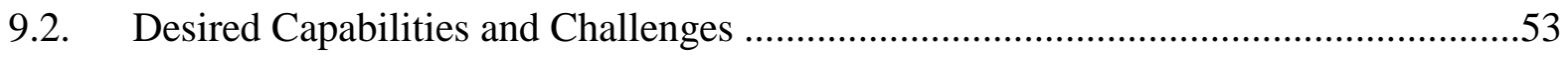

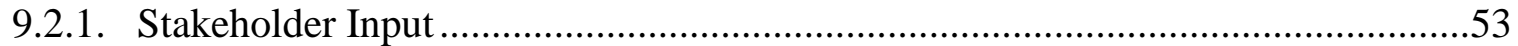

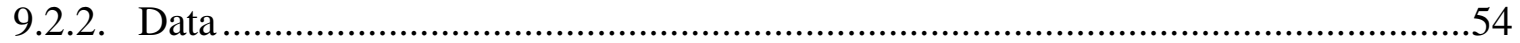

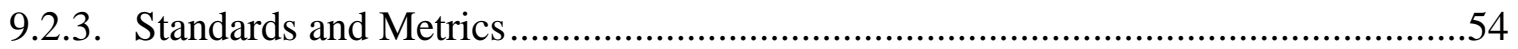

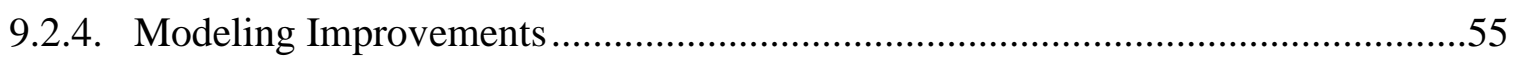

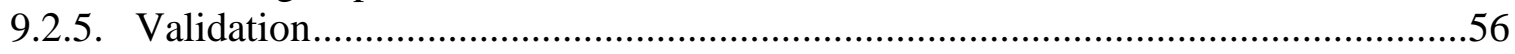

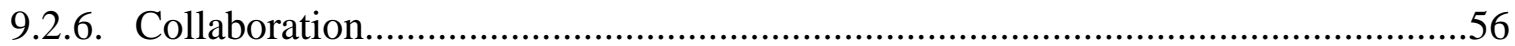

Appendix A: Decision-Making and Evaluation of Proposed Alternatives (Session 1)...... A-1

Appendix B: Resilience Modeling Research Around the World (Session 2)...................... B-1

Appendix C: Modeling of Systems and Dependencies (Session 3) ..................................... C-1

Appendix D: Modeling of Recovery (Session 4) .................................................................... D-1

Appendix E: Case Studies and Validation (Session 5) ................................................... 
International Workshop on Modeling of Physical, Economic, and Social Systems for Resilience Assessment

This page intentionally left blank. 
List of Acronyms

\begin{tabular}{|c|c|}
\hline Acronym & Definition \\
\hline AWWA & American Water Works Association \\
\hline BCA & Benefit Cost Analysis \\
\hline BI & Business Interruption \\
\hline BIM & Building Information Model \\
\hline C3WE & Capacity Center for Climate and Weather Extremes \\
\hline CDBG-DR & Community Development Block Grant Disaster Recovery Program \\
\hline CGE & Computable General Equilibrium \\
\hline CIbSS & Critical Infrastructure-based Societal Systems \\
\hline CIRI & Critical Infrastructure Research Institute \\
\hline CoE & Community Resilience Center of Excellence \\
\hline CoPE-WELL & Composite of Post-Event Well Being \\
\hline CREATE & Center for Risk and Economic Analysis \\
\hline CRISP & Critical Resilient Interdependent Infrastructure Systems and Processes \\
\hline CSU & Colorado State University \\
\hline DHS & Department of Homeland Security \\
\hline ECA & Economic Consequence Analysis \\
\hline E-CAT & Economic Consequences Analysis Tool \\
\hline EPA & Environmental Protection Agency \\
\hline FEM & Finite Element Model \\
\hline GIS & Geographic Information Systems \\
\hline GPS & Global Positioning System \\
\hline GRRIT & Global Risk Resilience and Impacts Toolbox \\
\hline IBC & International Building Code \\
\hline IFCI & Institute for Future Cities and Infrastructures (Tsinghua University) \\
\hline MAEViz & Multi-hazard Approach to Engineering Visualization \\
\hline NCAR & National Center for Atmospheric Research \\
\hline NIST & National Institute of Standards and Technology \\
\hline NSF & National Science Foundation \\
\hline PAI & Protective Action Initiation \\
\hline PBEE & Performance-based Earthquake Engineering \\
\hline PEER & Pacific Earthquake Engineering Research \\
\hline PEPPER & Program for Evaluating Payment Patterns Electronic Report \\
\hline RAMCAP & Risk and Resilience Management of Water and Wastewater Systems \\
\hline SBC & Structural Building Code \\
\hline USACE & U.S. Army Corps of Engineers \\
\hline VSAT & Vulnerability Self-Assessment Tool \\
\hline WNTR & Water Network Tool for Resilience \\
\hline WHEAT & Water, Health, and Economic Analysis Tool \\
\hline
\end{tabular}


International Workshop on Modeling of Physical, Economic, and Social Systems for Resilience Assessment

This page intentionally left blank. 


\section{Introduction}

The International Workshop on Modeling of Physical, Economic, and Social Systems for Resilience Assessment was held on 19-21 October 2016 at the Washington-Dulles Airport Marriott in Dulles, VA. The purpose of the workshop was to solicit input from researchers and subject matter experts in areas related to the modeling of physical, economic, and social systems to inform systems modeling research being conducted by NIST and Colorado State University.

Dr. Terri McAllister, Community Resilience Group Leader and Program Manager at the National Institute of Standards and Technology (NIST), welcomed attendees and presenters to the workshop and introduced the session topics:

1. Decision-Making and Evaluation of Proposed Alternatives

2. Resilience Modeling Research Around the World

3. Modeling of Systems and Dependences

4. Modeling of Recovery

5. Case Studies and Validation

Sessions were held consecutively as shown in Tables 1 to 3 . At the end of the workshop, a discussion regarding next steps, future activities, and challenges of community resilience systems modeling brought the session to a close.

This document summarizes key points and discussions from the workshop presentations and facilitated discussions. 
Table 1. Workshop Agenda - Day 1

\begin{tabular}{|c|c|c|c|}
\hline \multicolumn{3}{|c|}{ Topic } & \multirow[t]{2}{*}{ Speakers / Moderators } \\
\hline \multicolumn{3}{|r|}{ Wednesday, October 19, 2016} & \\
\hline 8:00-8:30 & \multicolumn{2}{|c|}{$\begin{array}{l}\text { Welcome and Introductions } \\
\text { Breakfast Buffet }\end{array}$} & $\begin{array}{l}\text { Terri McAllister } \\
\text { Community Resilience Group } \\
\text { Leader and Program Manager } \\
\text { National Institute of Standards } \\
\text { and Technology }\end{array}$ \\
\hline \multirow[t]{5}{*}{ 8:30-10:30 } & Session 1 & \multicolumn{2}{|c|}{ Decision-Making and Evaluation of Proposed Alternatives } \\
\hline & $8: 30-9: 00$ & $\begin{array}{l}\text { Public Warning Timing: Issuance, Diffusion, } \\
\text { Protective Action Initiation for Dam } \\
\text { Breaches, Controlled Dam Releases, and } \\
\text { Levee Breaches or Overtopping }\end{array}$ & $\begin{array}{l}\text { Dennis Mileti } \\
\text { Professor Emeritus of Behavior } \\
\text { Science } \\
\text { University of Colorado Boulder }\end{array}$ \\
\hline & 9:00-9:30 & $\begin{array}{l}\text { Resilience of Societal Infrastructure Systems } \\
\text { - Modeling and Decision Analysis } \\
\text { Framework }\end{array}$ & $\begin{array}{l}\text { Michael Faber } \\
\text { Professor of Civil Engineering } \\
\text { Technical University of Denmark }\end{array}$ \\
\hline & 9:30-10:00 & Total Economic Consequences of Disasters & $\begin{array}{l}\text { Adam Rose } \\
\text { Research Professor of Public } \\
\text { Policy } \\
\text { University of Southern California }\end{array}$ \\
\hline & 10:00-10:30 & $\begin{array}{l}\text { Infrastructure System Interconnectivity } \\
\text { Effects of Resilience }\end{array}$ & $\begin{array}{l}\text { Rae Zimmerman } \\
\text { Professor of Planning and Public } \\
\text { Administration } \\
\text { New York University } \\
\end{array}$ \\
\hline 10:30-11:00 & \multicolumn{3}{|l|}{ Break } \\
\hline \multirow[t]{2}{*}{ 11:00-12:00 } & Session 1 & \multicolumn{2}{|c|}{ Decision-Making and Evaluation of Proposed Alternatives (continued) } \\
\hline & 11:00-12:00 & Facilitated Discussion & $\begin{array}{l}\text { Georgios Giannopoulos } \\
\text { Scientific Officer } \\
\text { EU Joint Research Centre } \\
\text { John van de Lindt } \\
\text { Distinguished Professor in } \\
\text { Infrastructure, Co-Director of } \\
\text { Center for Risk-Based } \\
\text { Community Resilience Planning } \\
\text { Colorado State University }\end{array}$ \\
\hline 12:00-1:00 & Lunch & & \\
\hline \multirow[t]{3}{*}{ 1:00-2:30 } & Session 2 & \multicolumn{2}{|c|}{ Resilience Modeling Research Around the World } \\
\hline & $1: 00-1: 30$ & $\begin{array}{l}\text { Interdependences, Cascading Effects and } \\
\text { Resilience Optimization of Communities and } \\
\text { Societies }\end{array}$ & $\begin{array}{l}\text { Georgios Giannopoulos } \\
\text { Scientific Officer } \\
\text { EU Joint Research Centre }\end{array}$ \\
\hline & $1: 30-2: 00$ & $\begin{array}{l}\text { Resilience Modeling Research in New } \\
\text { Zealand }\end{array}$ & $\begin{array}{l}\text { Nick Horspool } \\
\text { Natural Hazard Risk Scientist } \\
\text { GNS Science }\end{array}$ \\
\hline
\end{tabular}


International Workshop on Modeling of Physical, Economic, and Social Systems for Resilience Assessment

\begin{tabular}{|c|c|c|c|}
\hline Time & \multicolumn{2}{|l|}{ Topic } & \multirow{2}{*}{$\begin{array}{l}\text { Speakers / Moderators } \\
\text { Dongping Fang } \\
\text { Department Head and Professor in } \\
\text { Construction Management } \\
\text { Tsinghua University }\end{array}$} \\
\hline & $2: 00-2: 30$ & Urban Resilience: Asia Research Initiatives & \\
\hline 2:30-3:00 & Break & & \\
\hline \multirow[t]{4}{*}{ 3:00-5:00 } & Session 2 & \multicolumn{2}{|c|}{ Resilience Modeling Research Around the World (continued) } \\
\hline & 3:00-3:30 & $\begin{array}{l}\text { NSF Investments and Opportunities in } \\
\text { Interdisciplinary Research on Critical } \\
\text { Infrastructure Systems }\end{array}$ & $\begin{array}{l}\text { David Mendonça } \\
\text { Director of Infrastructure } \\
\text { Management and Extreme Events } \\
\text { Program } \\
\text { National Science Foundation }\end{array}$ \\
\hline & $3: 30-4: 00$ & $\begin{array}{l}\text { DHS Sponsored Research: DHS Approaches } \\
\text { to Supporting Infrastructure Resilience }\end{array}$ & $\begin{array}{l}\text { Matthew Coats } \\
\text { Program Manager Science and } \\
\text { Technology Directorate } \\
\text { Department of Homeland Security }\end{array}$ \\
\hline & $4: 00-5: 00$ & Facilitated Discussion & $\begin{array}{l}\text { Adam Rose } \\
\text { Research Professor of Public } \\
\text { Policy } \\
\text { University of Southern California } \\
\text { Bruce Ellingwood } \\
\text { Professor of Civil and } \\
\text { Environmental Engineering, Co- } \\
\text { Director of Center for Risk-Based } \\
\text { Community Resilience Planning } \\
\text { Colorado State University }\end{array}$ \\
\hline
\end{tabular}


Table 2. Workshop Agenda - Day 2

\begin{tabular}{|c|c|c|c|}
\hline Time & Topic & & Speakers / Moderators \\
\hline \multicolumn{4}{|c|}{ Thursday, October 20, 2016} \\
\hline \multirow[t]{5}{*}{ 8:30-9:50 } & Session 3 & Modeling of Systems and Dependencies & \\
\hline & 8:30-8:50 & $\begin{array}{l}\text { Evolution of Hazard Assessment Modeling in } \\
\text { Drinking Water Systems }\end{array}$ & $\begin{array}{l}\text { Royce Francis } \\
\text { Assistant Professor of } \\
\text { Engineering Management and } \\
\text { Systems Engineering } \\
\text { The George Washington } \\
\text { University }\end{array}$ \\
\hline & 8:50-9:10 & $\begin{array}{l}\text { Modeling Water Distribution System } \\
\text { Resilience }\end{array}$ & $\begin{array}{l}\text { Katherine Klise } \\
\text { Senior Member of the Technical } \\
\text { Staff } \\
\text { Sandia National Laboratory }\end{array}$ \\
\hline & 9:10-9:30 & $\begin{array}{l}\text { Power Distribution Systems: Modeling of } \\
\text { System Dependencies and Interdependencies }\end{array}$ & $\begin{array}{l}\text { Charles Macal } \\
\text { Senior Systems Engineer } \\
\text { Argonne National Laboratory }\end{array}$ \\
\hline & 9:30-9:50 & $\begin{array}{l}\text { Reliability Assessment of Interdependent } \\
\text { Networks Based on Survival Signature }\end{array}$ & $\begin{array}{l}\text { Matteo Broggi } \\
\text { Senior Research Associate } \\
\text { Leibniz University Hannover }\end{array}$ \\
\hline 9:50-10:20 & Break & & \\
\hline \multirow[t]{5}{*}{ 10:20-12:00 } & Session 3 & \multicolumn{2}{|c|}{ Modeling of Systems and Dependencies (continued) } \\
\hline & $10: 20-10: 40$ & $\begin{array}{l}\text { Climate \& Complexity: The Resilience of } \\
\text { Natural-Built-Human Systems }\end{array}$ & $\begin{array}{l}\text { Auroop Ganguly } \\
\text { Associate Professor of Civil and } \\
\text { Environmental Engineering } \\
\text { Northeastern University } \\
\end{array}$ \\
\hline & 10:40-11:00 & $\begin{array}{l}\text { Natural Disaster Impacts on Infrastructure } \\
\text { Systems and Society }\end{array}$ & $\begin{array}{l}\text { Judith Mitrani-Reiser } \\
\text { Assistant Professor of Civil } \\
\text { Engineering and Co-Director of } \\
\text { Center for Systems Science and } \\
\text { Engineering } \\
\text { Johns Hopkins University } \\
\text { Director of Disaster and Failure } \\
\text { Studies } \\
\text { National Institute of Standards } \\
\text { and Technology }\end{array}$ \\
\hline & 11:00-12:00 & Facilitated Discussion & $\begin{array}{l}\text { Hussam Mahmoud } \\
\text { Assistant Professor and Director, } \\
\text { Structural Laboratory } \\
\text { Colorado State University }\end{array}$ \\
\hline & & & $\begin{array}{l}\text { Terje Haukaas } \\
\text { Professor of Civil Engineering } \\
\text { University of British Columbia }\end{array}$ \\
\hline 12:00-1:30 & Lunch & & \\
\hline
\end{tabular}




\begin{tabular}{|c|c|c|c|}
\hline Time & \multicolumn{2}{|l|}{ Topic } & \multirow[t]{2}{*}{ Speakers / Moderators } \\
\hline \multirow[t]{7}{*}{ 1:30-3:30 } & Session 4 & Modeling of Recovery & \\
\hline & $1: 30-1: 50$ & $\begin{array}{l}\text { Physical and Economic Impacts of } \\
\text { Earthquakes and Other Hazards }\end{array}$ & $\begin{array}{l}\text { Terje Haukaas } \\
\text { Professor of Civil Engineering } \\
\text { University of British Columbia }\end{array}$ \\
\hline & $1: 50-2: 10$ & $\begin{array}{l}\text { Road Network Functionality of Istanbul } \\
\text { Following an Earthquake }\end{array}$ & $\begin{array}{l}\text { Himmet Karaman } \\
\text { Associate Professor of Civil } \\
\text { Engineering } \\
\text { Istanbul Technical University }\end{array}$ \\
\hline & $2: 10-2: 30$ & $\begin{array}{l}\text { Measuring and Improving the Resilience of } \\
\text { the Built Environment in a Community }\end{array}$ & $\begin{array}{l}\text { Quanwang Li } \\
\text { Associate Professor of Civil } \\
\text { Engineering } \\
\text { Tsinghua University }\end{array}$ \\
\hline & $2: 30-2: 50$ & $\begin{array}{l}\text { Housing Demand \& Capacity for Medically } \\
\text { Fragile \& Vulnerable Populations: Modeling } \\
\text { Transition through Temporary, Interim, and } \\
\text { Permanent Housing Recovery }\end{array}$ & $\begin{array}{l}\text { Joshua Behr } \\
\text { Research Associate Professor of } \\
\text { Analysis and Social Sciences } \\
\text { Old Dominion University }\end{array}$ \\
\hline & $2: 50-3: 10$ & $\begin{array}{l}\text { Modeling Recovery: The Role of Disaster } \\
\text { Financing }\end{array}$ & $\begin{array}{l}\text { Carolyn Kousky } \\
\text { Fellow } \\
\text { Resources for the Future } \\
\end{array}$ \\
\hline & 3:10-3:30 & $\begin{array}{l}\text { An Integration of Social Vulnerability Data } \\
\text { and Mapping Tools for Community } \\
\text { Vulnerability Assessments and Recovery } \\
\text { Modeling }\end{array}$ & $\begin{array}{l}\text { Nathanael Rosenheim } \\
\text { Assistant Research Scientist in } \\
\text { Architecture } \\
\text { Texas A\&M University }\end{array}$ \\
\hline $3: 30-4: 00$ & Break & & \\
\hline \multirow[t]{2}{*}{ 4:00-5:00 } & Session 4 & Modeling of Recovery (continued) & \\
\hline & $4: 00-5: 00$ & Facilitated Discussion & $\begin{array}{l}\text { Harvey Cutler } \\
\text { Professor of Economics } \\
\text { Colorado State University } \\
\text { Keith Porter } \\
\text { Research Professor of Civil, } \\
\text { Environmental, and Architectural } \\
\text { Engineering } \\
\text { University of Colorado Boulder }\end{array}$ \\
\hline $5: 00-5: 30$ & Break & & \\
\hline \multirow[t]{2}{*}{$5: 30-7: 30$} & \multicolumn{3}{|c|}{ Resilience Table Top Exercise and Reception } \\
\hline & \multicolumn{2}{|c|}{ Hot appetizers and cash bar available. } & $\begin{array}{l}\text { Nathaniel Forbes } \\
\text { Director } \\
\text { Forbes Calamity Prevention }\end{array}$ \\
\hline
\end{tabular}


Table 3. Workshop Agenda - Day 3

\begin{tabular}{|c|c|c|c|}
\hline \multirow[t]{2}{*}{ Time } & \multicolumn{2}{|l|}{ Topic } & \multirow[t]{2}{*}{ Speakers / Moderators } \\
\hline & & Friday, October 21, 2016 & \\
\hline \multirow[t]{4}{*}{ 9:00-10:30 } & Session 5 & Case Studies and Validation & \\
\hline & 9:00-9:30 & $\begin{array}{l}\text { GRRIT }^{\circledR} \text { - a Sustainable Tool for Reducing } \\
\text { Weather and Climate Impacts }\end{array}$ & $\begin{array}{l}\text { Cindy Bruyere } \\
\text { Project Scientist II } \\
\text { National Center for Atmospheric } \\
\text { Research }\end{array}$ \\
\hline & 9:30-10:00 & $\begin{array}{l}\text { Resilience Modeling Innovations of the } \\
\text { HayWired Planning Scenario }\end{array}$ & $\begin{array}{l}\text { Keith Porter } \\
\text { Research Professor of Civil, } \\
\text { Environmental, and Architectural } \\
\text { Engineering } \\
\text { University of Colorado Boulder }\end{array}$ \\
\hline & 10:00-10:30 & $\begin{array}{l}\text { Model Validation: The Joplin Tornado } \\
\text { Hindcast }\end{array}$ & $\begin{array}{l}\text { John van de Lindt } \\
\text { Distinguished Professor in } \\
\text { Infrastructure, Co-Director of } \\
\text { Center for Risk-Based } \\
\text { Community Resilience Planning } \\
\text { Colorado State University } \\
\end{array}$ \\
\hline 10:30-10:45 & Break & & \\
\hline \multirow[t]{2}{*}{ 10:00-11:45 } & Session 5 & Case Studies and Validation (continued) & \\
\hline & $10: 45-11: 45$ & Facilitated Discussion & $\begin{array}{l}\text { Elise Miller-Hooks } \\
\text { Professor and Chair in } \\
\text { Infrastructure Engineering } \\
\text { George Mason University } \\
\text { Judith Mitrani-Reiser } \\
\text { Assistant Professor of Civil } \\
\text { Engineering and Co-Director of } \\
\text { Center for Systems Science and } \\
\text { Engineering } \\
\text { Johns Hopkins University } \\
\text { Director of Disaster and Failure } \\
\text { Studies } \\
\text { National Institute of Standards } \\
\text { and Technology }\end{array}$ \\
\hline 11:45-12:00 & \multicolumn{2}{|c|}{ Closure, Next Steps, and Future Activities } & $\begin{array}{l}\text { Terri McAllister } \\
\text { Community Resilience Group } \\
\text { Leader and Program Manager } \\
\text { National Institute of Standards } \\
\text { and Technology }\end{array}$ \\
\hline
\end{tabular}




\section{Workshop Purpose}

The Community Resilience Group at NIST is conducting research to promote resilient communities, with a focus on buildings and infrastructure systems. A key research activity is the development of integrated systems models for simulating physical, social, and economic systems in a community, including their performance, recovery, functionality, and dependencies. Systems modeling research is being conducted in collaboration with the NIST-funded Community Resilience Center of Excellence (CoE), which is led by Colorado State University (CSU).

To support these activities, NIST and CSU co-sponsored a systems modeling workshop to identify and benefit from international research on modeling methods, characterizing systems, and data needs and sources being developed and used by other researchers.

The workshop brought together researchers and subject matter experts on state-of-the-art methods for topics that support community resilience modeling to identify gaps and research needs to further strengthen systems modeling research plans.

Areas of expertise of workshop speakers and participants include: physical and functional modeling of buildings and infrastructure systems (e.g., energy, communication, water, wastewater, and transportation), economic systems, and social systems (e.g., government, health, education). 
International Workshop on Modeling of Physical, Economic, and Social Systems for Resilience Assessment

This page intentionally left blank. 
3. Session 1: Decision-Making and Evaluation of Proposed Alternatives

\subsection{Session Presentations}

\subsubsection{Public Warning Timing: Issuance, Diffusion, Protective Action Initiation for Dam Breaches, Controlled Dam Releases, and Levee Breaches or Overtopping}

Presenter: Dr. Dennis Mileti, Professor Emeritus of Behavioral Science, University of Colorado Boulder

The work presented by Dr. Mileti was funded by the U.S. Army Corps of Engineers (USACE) Risk Management Center in Davis, California. Dr. Mileti began his presentation with a discussion of the behavioral aspects of issuing emergency warnings. He stated issuing public alerts and warnings involve human behavior, such as physical scientists who detect a threat, emergency managers who manage the threat, message authors who communicate the threat, and finally the public who must take heed of the warning. The presentation had four parts: Part I Introduction, Part II Warning issuance delay, Part III Warning diffusion delay, and Part IV Protective action initiation (PAI) delay.

The objectives of the work presented were to provide USACE with estimates of human behavior for life loss estimation, to develop methods to measure behavior time estimates, and to prepare a community guidebook that could inform emergency managers about social science evidencebased warning practices. To achieve these objectives, quantitative curves for warning issuance delay, warning diffusion delay, and mobilization delay were developed. To develop the curves, quantitative research findings on time/delay were reviewed for all three stages of the warning process, data from empirical studies were identified, and model equations were developed and fitted to historical events.

Four categories of research-based factors affect warning issuance delay: plans and procedures, performance and inter-personal relations, system performance, and situational factors. Each of the four categories contained several factors (e.g., day vs. night is an example situational factor), which were assigned weights dependent upon their respective significance in affecting delay time. Case studies of rapid warning issuance (Boulder, Colorado Flood 2013) and slow warning issuance (Graniteville, South Carolina Train Derailment 2005) were discussed and compared.

Research-based categories of factors that influence warning diffusion times are: timing and methods for sending first warning, and situational aspects of receiving the first warning. Examples of factors include types of technologies available to send the warning message, and access to technology to receive the warning message. Each of the factors in the two categories was assigned a weight based on empirical data and expert opinion, and modeling of warning diffusion time was discussed. Examples of rapid vs. slow warning diffusion were presented.

Research-based factors that affect PAI time are: message characteristics, receiver characteristics, and context characteristics. Examples of factors belonging to each category are message length, personal preparedness, and environmental cues, respectively. The factors were assigned weights in the modeling of PAI time. Examples of rapid and slow PAI times, during a historical hazardous materials train derailment event near Pittsburgh, PA, were presented and compared.

To combine the analysis of delay times, an interview schedule was created to measure significant factors specific to local communities to enable the USACE to estimate likely future warning delays and resulting life loss and injury consequences. The interview contains a total of 58 
interview questions, which will guide USACE in assigning issuance, diffusion, and PAI curves to communities.

Finally, the guidebook ${ }^{1}$ was discussed which was developed based on synthesized empirical social science quantitative evidence to provide emergency managers with practical ways to enhance future public alert and warning practices. Tools provided in the guidebook include the ability to: catalogue and define protective actions, identify protective actions based on time and location, identify the threat versus actions and warnings, rank community planning steps for upgrading issuance/diffusion/PAI, analyze the anatomy of warning messages/examples/templates, and catalogue and evaluate available warning dissemination channels.

${ }^{1}$ Mileti, Dennis. S., and John H. Sorensen (2015). A Guide to Public Alerts and Warnings for Dam and Levee Emergencies. Davis CA: U.S. Army Corps of Engineers Risk Management Center.

\subsection{2. $\quad$ Resilience of Societal Infrastructure Systems - Modeling and Decision Analysis Framework}

Presenter: Dr. Michael Faber, Professor of Civil Engineering, Technical University of Denmark Dr. Faber outlined the context for his presentation by stating societal developments are approaching the carrying capacities of Earth's ecosystems. Dr. Faber acknowledged that couplings between civilizations and living conditions are observable and can be projected into the future using different hypotheses for future societal development. In order to do so, however, there is a need for improved knowledge such as: representations of dynamic interactions in integral systems models of the coupling between society and nature; determination of vulnerability and resilience in nature/society systems for particular ecosystems and livelihoods; and utilization of decision support systems.

The presentation covered: frameworks for systems decision analysis; probabilistic systems representation; a principal example of an infrastructure/social/hazard/ ecological system; followed by conclusions and a future outlook.

Regarding frameworks for systems decision analysis, hierarchical representation of social and natural systems were presented with respect to their exposure to hazards, shared environmental and societal responses, and decision-making processes. Several questions still to be answered regarding frameworks include: how to prioritize investments on design and management of interlinked systems; how to select target reliabilities and performances of individual systems and constituents; and how to plan and budget for the future economy/environment/society/health. Bayesian decision analysis was presented as a consistent "book-keeping" method for calculating the expected value of the utility associated with different decision alternatives.

Probabilistic system representation was next discussed including system robustness, resilience, and sustainability. A framework for decision analysis and probabilistic modeling of systems for optimal design and life-cycle management was proposed. The overall probabilistic system representation model was comprised of a graph model, constituents model, probabilistic model, and decision alternatives. Robustness modeling was presented as a function of all relevant scenarios and consequences. Consequences include impacts to health and safety, to the environment, and to the economy. Social preparedness systems modeling was presented as a benefit versus time analysis accounting for pre-disturbance performance, time of disturbance, period of reorganization, interim operations, and the period of renewal/rehabilitation. A system is 
deemed not resilient if at any given time one or more of its capacities are exceeded. Resilience failure modeling was presented as a time variant reliability (or vector out-crossing) problem. An example resilience failure model might consider ecological stress versus financial stress versus time.

A principal example of an interlinked infrastructure/social/hazard/ecological system model was next presented. Examples of resilience management of the system were presented as: increasing redundancy through increasing the number of system constituents; improvements of social preparedness; design of individual system constituents with respect to natural and anthropological hazards; and saving sufficient benefits to recover from disturbances. Social systems with low and high preparedness were presented and compared. The conditional resilience failure of both systems was presented in terms of the joint probability distribution function of recovery time and the magnitude of lost benefit for a given disturbance event.

Concluding, several areas of systems modeling were identified that are lacking, including: best practices and optimized techniques for modeling and analysis of individual systems; best practices and optimized techniques for identification and representation of connections between systems; and robust and generic representations and analysis methods of relevance for the complexity of real life coupled systems.

\subsubsection{Total Economic Consequences of Disasters}

Presenter: Dr. Adam Rose, Research Professor of Public Policy, University of Southern California

Dr. Rose delivered a presentation on the evolution of Economic Consequence Analysis (ECA) of natural, man-made and technological disasters and introduced a broad ECA framework developed at the Center for Risk and Economic Analysis (CREATE) to estimate the total impacts of a disasters on an economy. Dr. Rose's presentation covered the CREATE ECA framework, three example applications, implications for decision-making, and the Economic Consequence Analysis Tool (E-CAT).

The ECA framework developed at CREATE has been expanded to include resilience, behavior responses, supply-chain linkages, remediation, mitigation, and spillovers. The disaster event scenario feeds into a computable general equilibrium model (CGE), with added aspects such as resilience adjustments, behavior linkages, and mitigation costs. These extensions can have positive or negative impacts, depending on the economy.

Two definitions of economic resilience were presented. A static definition was presented as the efficient use of remaining resources at a given point in time to produce as much as possible; a dynamic definition was presented as the efficient use of resources over time for investment in repair and reconstruction. One operational metric of economic resilience was defined as averted losses as a percentage of potential losses. This metric was used to assess the economic resilience of the US and NYC economies in the aftermath of the 2001 World Trade Center terrorist attacks.

Regarding economic resilience, two major perspectives were discussed. The first perspective was outlined to include pre- and post-disaster actions to reduce property damage; while the second perspective is limited to post-disaster actions to improve resilience in the future. Business interruption (BI) was identified as the new paradigm in economic loss estimation, including behavioral and policy considerations. An overview of computable general equilibrium (CGE) 
modeling was next presented. CGE represents the economy as a set of interdependent supply chains with individual responses to price signals.

The state-of-the-art ECA framework proposed by CREATE was then compared to the standard approach from two port closure studies. The standard approach for estimating port impact was identified as direct economic activity times some multiplier. This approach misses the value of the cargo and its contribution to the rest of the economy and thus has been shown to underestimate the economic impact of disruption. The state-of-the art approach without resilience overestimates losses, while including resilience accounts for adaptations.

Behavior linkages translate into direct and indirect BI losses. BI losses can be two to three orders of magnitude higher than direct losses. Methods to measure extreme behavioral responses to disasters, and to translate them into direct economic costs so that they can be integrated into CGE models, were presented.

Several general policy implications for economic consequence analysis were outlined including: measuring both property damage and business interruption in risk management; considering nonstructural causes of economic loss; and considering the demand side.

Finally, an overview of E-CAT was presented. The objectives of E-CAT were to develop a standardized capability to estimate economic consequences of over 30 hazards and to transition the research into a user-friendly, fast software tool for high-level decision makers. The tool starts from direct impacts and a number of user-specified scenario parameters; it then performs randomized draws of 100 variable combinations converted to CGE inputs to estimate GDP and employment impacts. Uncertainty distributions are generated using reduced-form results and displayed in the user interface.

\subsubsection{Infrastructure System Interconnectivity Effects on Resilience}

Presenter: Dr. Rae Zimmerman, Professor of Planning and Public Policy, New York University

Dr. Zimmerman gave a presentation highlighting resilience and interconnectivity as related to infrastructure and its services. She expressed the fact that environmental threats are increasing in some locations at the same time as the vulnerability of infrastructure and populations in those locations; and that condition, performance and investment analyses typically do not include interconnectivity. The interconnectivity between environmental conditions and the design of infrastructure were identified as key inputs to resilience modeling.

The presentation began with an overview of selected natural and human hazards affecting infrastructure, infrastructure condition, siting, materials, and resources. Infrastructure interconnections, consequences of interdependences, failure modes and resilience, and future research needs were discussed in Parts II, III, IV, and V, respectively.

Attributes of infrastructure connections were identified as connections between sectors; flows of goods and services between infrastructures; the scale of components and interactions; types of dependencies (temporal, physical, cyber, spatial, and logical), and implications and likelihood of cascading failures from interconnections. Examples of interconnections potentially vulnerable to cascading disruptions were presented as electric power and rail transport, and energy and water sectors. Concentration of infrastructure was identified as an important contributor to cascading failures. For example, approximately half of the power plants in the U.S. are in just a dozen states. 
The consequences of interdependencies relating to general failure modes were next discussed. Interconnectivity consequence scenarios include direct and indirect failures, infrastructure vulnerability, and the targeting of certain sectors and their indirect impact on various other sectors. An electrical power outage was presented as an example consequence scenario assessing the interdependency of energy connections, transportation, and water systems.

Conventional and distributed or alternative infrastructure systems were compared, with the latter being more resilient by enabling more flexible and simple interconnections. Diversifying the dependency of networks through methods such as alternative infrastructure layout or increased redundancy was highlighted as a means of promoting resilience of interconnected infrastructure systems. Promoting infrastructure investment, as opposed to relying solely on emergency funding, is one approach to increasing infrastructure system resilience. Several specific techniques were highlighted for improving system specific resilience, such as the use of sensor technologies to detect contamination in water systems (interconnectivity between IT and water). Finally, the interconnectivity of environmental, social, and infrastructure systems was discussed.

Inputs to modeling were identified as a key first step to future research needs and lessons for policy. These included providing a greater (more precise) specification and quantification of system linkages; understanding overall conditions which strengthen or weaken interconnections; identifying and reducing means of cascading effects; and better understanding the role of how to shape human behavior to support more resilient interconnected systems.

\subsection{Facilitated Discussion}

Moderators: Dr. Georgios Giannopoulos, Scientific Officer, EU Joint Research Centre

Dr. John van de Lindt, Distinguished Professor in Infrastructure, Co-Director of Center for Risk-Based Community Resilience Planning, Colorado State University

Scribes: Ms. Hana Chmielewski, Pathways Intern, National Institute of Standards and Technology

Dr. Eun Cha, Assistant Professor of Civil and Environmental Engineering, University of Illinois Urbana-Champaign

\subsubsection{Resilience Cost Benefit}

In quantifying the total economic impact, how receptive are resilience investors to quantifying the resilience benefit versus the total cost of investment?

Past experience in working with water and power utilities has shown them to be receptive to resilience indicators. Economic consequence analysis (ECA) informs the benefits of avoided losses, an analysis result in which investors and government agencies are increasingly interested. The consequence analysis is just another way of getting to the benefits. The government has been performing benefit-cost analysis for resilience decisions for the past ten years. Interest and funding to do a similar analysis for the private sector has recently been on the rise. Such an assessment for the private sector will require an additional focus on the business interruption aspect of the problem. 


\subsubsection{Effects of Human Behavior on System Design}

Systems must be seen as applied technology. They have to be directly designed, maintained, operated, and regulated in an adaptive manner to the organization, environment, and society. To what extent are people trained to use systems in ways that take into account human behavior?

There was a consensus among the panel that this was one of the big questions currently facing community resilience researchers. It is often difficult to determine when people will recognize the importance of resilience initiatives and, in turn, how to get them to actually implement such initiatives. There are many different kinds of people who would or wouldn't use new systems. There is not only the question of how to design systems, but how to present, package, and market them to improve different audiences' reception.

\subsubsection{Social Media}

Social media, and the impact it has on the public, is an extremely hot issue in social sciences. Data mining is being used to analyze social media data and how it influences public behavior. The panel was asked how the role of social media compares to traditional warning and recovery.

Often, information communicated over social media is helpful news (e.g., intersection closures). The FCC has recently approved warning messages on cell phones up to 360 characters, which will open the door regarding how social media technologies can inform and warn people. Compared to traditional methods (e.g., radio, television, warning siren), messages can be distributed on at the individual level. This increases the effectiveness over traditional distribution of messages at the county level, particularly in places such as the western U.S., where county sizes are very large. There was a consensus among the panel that it will likely be years before the role and effect of social media on public behavior are understood and optimized.

\subsubsection{Model Library to Address Resilience Issues}

The issue of sharing of data and tools was raised several times during the workshop. A question was posed to the panel as to the possibility of developing a model library to address resilience issues.

There are resilience centers around the world (e.g., Tsinghua University, Singapore, Shanghai, Taipei, Europe, U.S.) that can contribute to the development of toolboxes. An open platform for the community to develop and use such tools for decision problems is the goal. The biggest challenge to this task is how the systems are linked. Prior to creating model inventories, the linkage between systems needs to be planned and coordinated. There was a consensus among the panel that there is currently no one responsible for this critical role.

\subsubsection{Improving Community Recovery}

Since many infrastructure systems are public, they are built and operated for the long-term. When there is damage to the system there is often pressure from the public to build back to the same state. A question was raised regarding plans for recovery where capacity is incrementally added. Is there any learning or improving following an event?

This phenomenon has been well documented in social science research since the 1950s. It has been shown that if a community does not have plans in place for rebuilding better, it will not happen after a disaster event. There is societal pressure to restore services to the same state as opposed to building back better when critical infrastructure services (e.g., power, water, housing, etc.) are missing. Instead of waiting until after a disaster occurs, it should be asked if there are 
subsystems that can be cost effectively improved over the long-term to improve overall system resilience before the next disaster happens. The concept of staging was also presented as a means to assess needs at various time scales (e.g., one day, month, year, 5 years, lifetime) following an event. Climate change was identified as a major factor that will influence rebuilding decisions into the future. In some cases, relocation might be a better alternative for resilience than rebuilding.

A related question was asked on the management of emergency/short term response plans following an event. It was put forward by an audience member that systems with the highest probability of failure are the ones which rarely rehearse or re-evaluate their response plans. How should communities be managing their response plans to reduce negative impacts of a major event?

It was suggested that the problem is not that communities don't read or rehearse their response plans, but that they do not have plans in place at all. Whether a response plan is in place or not, more research is needed regarding general methods of rapidly disseminating information into the community as strategy to improve post-event recovery.

\subsubsection{Resilient Failure and Management of Recovery}

Most resilience analysis assumes the community can recover and tends to measure the speed of recovery. The panel was asked not about resilient recovery, but about resilient failure and what it might look like.

There is a need to focus on capacities to support community activities and to then determine events which will exceed those capacities. In reality, there are no isolated communities. In the cases where a community cannot handle an event, the next level of society will contribute resources. Resource allocation should be optimized across communities by evaluating the risk associated with bankruptcy of some critical resources. This will provide communities with an idea of the amount of resources that they should be sharing.

\subsubsection{Evolution of Thinking Regarding Tools and Methods}

There is discussion within the research community regarding risk and resilience and how tools can be modified. What has changed as we think about tools and methods for community resilience? Is the change in the objective function? How much of climate change should we look at for specific signatures of risk?

Several paradigm shifts were identified, such as going beyond property damage and emphasizing business interruption, assessing post-disaster recovery, and focusing on the customer side. Regarding climate change, a position was presented that the market will take care of part of the issue. Examples include the possibility of decreasing coastal property values and the recent declaration of bankruptcy by coal companies in the U.S. It was ventured by one panel member that market adjustments will help address climate change in an orderly pace, but acknowledged market failures (e.g., infrastructure with no single entity as owner) exist. There was skepticism among other panel members to the free-market approach. Insurance policies which encourage or allow owners to rebuild in increasingly vulnerable locations were cited as a reason for such skepticism. It was also speculated that relying on the free market will not be as efficient from the perspective of society and the allocation of tax money. Communities should be careful regarding sustainability (e.g., consider discount rate and present value) in order to make decisions now that continue to use societal resources efficiently in the future. 


\subsection{Common Themes and Key Points (Session 1)}

The following themes and issues emerged from the presentations and discussion on decisionmaking and evaluation of proposed alternatives:

- There is a need for best practices regarding the modeling of individual systems and connections between systems. Best practices should address the levels of generality and complexity needed/desired by end-users.

- The evolution of the infrastructure, economic, social, and ecological capacities or reserves of a community under a broad range of disruptive scenarios is a key modeling need for assessing resilience.

- Interruptions to the flows of goods and services can produce stresses and losses that greatly exceed the direct costs of repairing or replacing physical damage to buildings and infrastructure.

- There is a need for increased understanding of cascading failure effects between and within infrastructure and societal systems.

- There are important questions to be addressed on how to shape human behavior to support resilient systems and how to design and present resilient systems to improve reception and implementation.

- Resilience initiatives should be community specific. Resources and capacity should be assessed to determine if a community has the ability to recover. Community objective functions should be taken into account during decision-making to ensure policy is aligned with resilience and recovery goals (e.g., building back to pre-event performance vs. building beyond).

- If a community does not have plans in place for rebuilding better, it will not happen after a disaster event because of the pressure to restore services quickly.

- There are no isolated communities. In cases where a community cannot cope with an event or a threat, the next levels of society (e.g., regional, state, or national) will contribute resources. Thus, the problem of defining the spatial boundaries of the systems to be modeled can be very complex. 


\section{4. $\quad$ Session 2: Resilience Modeling Research Around the World}

\subsection{Session Presentations}

\subsubsection{Interdependencies, Cascading Effects and Resilience Optimization of Communities and Societies}

Presenter: Dr. Georgios Giannopoulos, Scientific Officer, EU Joint Research Centre

Dr. Giannopoulos began his talk on resilience research performed at the European Commission's Joint Research Centre (JRC) by introducing three tiers of resilience modeling. He then presented the Geospatial Risk and Resilience Assessment Platform (GR ${ }^{2} \mathrm{ASP}$ ), a web oriented architecture which brings together geospatial technologies and computational tools for the analysis and simulation of critical infrastructures. Several case studies were provided to support the discussion of the $\mathrm{GR}^{2} \mathrm{ASP}$ methodology and its application to selected disruptive scenarios.

Tier 1 of resilience modeling was related to the network analytics. Understanding network components and interactions enables quick analysis of intrinsic network properties, resilience analysis of directed/random disruptions, and resilience investment prioritization.

Dynamical functional modeling of vulnerability and independencies of critical infrastructure (DMCI) is handled in Tier 2 of resilience modeling. Modular, cross-sectoral models are adaptable at different levels of granularity (e.g., local, regional, country, international). Generic variables that were identified for critical infrastructure representation include, but are not limited to: inoperability, service capacity, recovery time, and buffer time. Boolean representations of physical and organizational aspects are used in the DMCI, which helps to reduce analysis complexity. The objective of resilience optimization during Tier 2 is to minimize cascading effects and to maintain functionality for prioritized nodes.

Tier 3 consists of the dynamic interoperability inventory optimization model. The work presented is based on W. Leontief's inventory optimization approach. In Tier 3, service loss propagation in economic sectors can be assessed as a consequence of critical events using public economic databases. The model is applicable at different levels of granularity.

$\mathrm{GR}^{2} \mathrm{ASP}$ is a server-client architecture that employs WebGIS technologies in conjunction with analysis tools for critical infrastructures and economic sectors. The platform is based on opensource software and supports multi-user collaboration and data sharing.

Concluding the presentation, a scenario builder was identified as a key next step to link all three categories of models, from asset disruption to socio-economic impact. He stated that we need more involvement from the community; models should facilitate collaboration and not intimidate the end users. It was stated that we do not necessarily need an increased quantity of models, but closer links between existing models are needed.

\subsubsection{Resilience Modeling Research in New Zealand}

Presenter: Dr. Nick Horspool, Natural Hazard Risk Scientist, GNS Science

Dr. Horspool delivered an overview of resilience modeling research in New Zealand. He began his talk by setting the stage for social and infrastructure systems in New Zealand, where two thirds of the population live in three major cities exposed to significant and diverse natural hazard risk (e.g., earthquake, tsunami, volcanic eruption, flood). Recent disasters in New Zealand have dramatically highlighted the impact shocks can have on society and its ability to 
function and grow. The Canterbury earthquakes affecting Christchurch in 2010-2011 caused catastrophic damages, but also provided a platform for a dialogue on resilience across various sectors of New Zealand. An overview was provided of New Zealand-based resilience modeling research programs and demonstrated through case-studies how these are leading to a more resilient New Zealand.

The first resilience program discussed by was QuakeCoRE, a centre for earthquake resilience. Flagship programs of QuakeCoRE include: 1) ground motion simulation and validation; 2) liquefaction impacts on infrastructure; 3) earthquake-prone buildings; 4) next-generation infrastructure; 5) pathways to resilience; and 6) distributed infrastructure.

An outline of the National Science Challenge's "Resilience to Nature's Challenges” program was presented. The mission of this program is to partner with stakeholders to build a transformative pathway towards natural hazard resilience. This goal is achieved through prioritydriven co-creation laboratories and targeted research, suited to the diverse and rapidly changing social, economic, built and natural environments.

On-going risk-based resilience modeling in New Zealand has been largely adapted from a NIST framework for community resilience. The objective of this research is to translate the damage of the built environment into social and economic disruption experienced by each significant sector of the community.

The Modeling the Economic Resilience of Infrastructure Tool (MERIT) was discussed next. MERIT is a fully dynamic model that is able to show status under various post-impact scenarios. MERIT has approximately 50 economic sectors/commodities which can be differentiated spatially, and it covers all geographic regions in New Zealand. MERIT is composed of a nested suite of models that can be used for different size and scale outages, and for different purposes.

A case study of a magnitude 8.1 earthquake associated with a $400 \mathrm{~km}$ long rupture between Milford Sound and the Ahaura River was discussed. The case study covered direct losses, damages, casualties; road network impacts; rail network impacts; hydroelectric power and water outages; and restoration timelines under various scenarios.

\subsubsection{Urban Resilience: Asian Research Initiatives}

Presenter: Dr. Dongping Fang, Department Head and Professor in Construction Management, Tsinghua University

Dr. Fang presented an overview of Asian research initiatives in the context of urban resilience. Dr. Fang's presentation starts with an overview of world urbanization trend and the impact of disasters. Next, a framework and a vision of a city simulator for modeling urban resilience were presented. Current initiatives in urban resilience research at Tsinghua University were presented, and finally current researches in Asia were summarized.

Rapid urbanization is being experienced around the world and the global urban population is expected to increase to $69 \%$ of the total population by 2050. In order to achieve a better quality of life for all, future cities should be resilient, efficient, and sustainable. Challenges to urbanization include natural disasters (e.g. earthquake, mudslide, typhoon); accidents (e.g., explosion, fire); social safety (e.g., stampede, violence, group events); public health (e.g., SARS, H1N1, melamine); and coupled disasters (e.g., snow and ice, fire caused by earthquake). 
Modeling urban resilience through the development of the city simulator concept, which is a system of systems view of the urban system, was presented. Societal, cyber and physical spaces can all be captured in the resilience modeling of the City Simulator. It was emphasized that crossing academic boundaries is essential for making new breakthroughs, and could possibly lead to new disciplines.

Initiatives at the Institute for Future Cities and Infrastructures (IFCI) at Tsinghua University were next discussed. The IFCI is an interdisciplinary and transdisciplinary research program, conducting research on themes such as: data sensing and big data analytics in urban and infrastructure systems; sustainable life-cycle management of urban and infrastructure systems; and social and economic theories and models for future cities and infrastructures.

Research initiatives throughout Asia were discussed. First discussed was the CREATE program at the Singapore ETH Centre, a part of the Future Resilient Systems program. The mission of the CREATE program is to address the challenges with critical infrastructure systems that provide essential services to modern societies. The Resilience Engineering Research Center (RERC) at the University of Tokyo was also highlighted. The RERC consists of three divisions to promote research into the principles and methodologies for realizing resilient systems. Finally, several specific research projects were highlighted such as technology management strategy for future social needs driven innovation at the global level; an assessment of massive integration of renewable energy by developing a multi-regional optimal power generation mix model; and a study on the strategies and scenarios required to achieve the reformation of power generation mix in light of the Great East Japan Earthquake.

\subsubsection{NSF Investments and Opportunities in Interdisciplinary Research on Critical Infrastructure Systems}

Presenter: Dr. David Mendonça, Director of Infrastructure Management and Extreme Events Program, National Science Foundation

Dr. Mendonça's presentation highlighted NSF funded research in the broad area of critical infrastructure systems. His presentation focused first on the critical resilient interdependent infrastructure systems and processes (CRISP) program and the current projects under this program. Next, the discussion shifted to methods and data, and finally an overview of the current CRISP solicitation was presented.

NSF has made an investment of \$59 million, over 37 projects, 71 project teams, and 180 individuals. The composition of awardees can be broken down across 3 divisions: engineering, computer and information sciences and engineering (CISE), and social behavior and economic sciences (SBES). Most projects tend to be led by engineering, with CISE at a distant second. Members of SBES play an important role as co-project investigators on many projects.

The various methodological approaches being used in the CRISP program were categorized as: optimization, statistical, simulation-based, physics-based, system dynamics, game theory, data extraction, surveys, and others. Regarding the data being used and generated under the CRISP program, several types were highlighted, such as: social media data, surveys, and activity diaries to better understand how humans operate with respect to critical infrastructure; metered data on different kinds of use, inventory, and maintenance; archival and public data; and synthetic or simulated data. The need for growth of archival and simulated data and the relationship between those was stressed. 
Next the need for integrative methods to support or explain cognition and adaptive behavior within infrastructures and the role of human agency beyond the demand for service was presented. Concerns beyond demand include how interdependent critical infrastructure systems are designed to behave during restoration and recovery and how they are controlled. Regarding data, the talk was framed with the question "what are the requirements of a common data block to support the CRISP research enterprise?” A workshop is being hosted by NSF on shared public data for critical infrastructure and materials sciences and the intersection between the two.

Finally, the current CRISP solicitation for fiscal year 2017 was introduced. The recent announcement has four goals: community building enterprise / interdisciplinary research, design of interdependent critical infrastructures, creating knowledge for innovation in interdependent critical infrastructures, and improving infrastructure performance.

\subsection{5. $\quad$ DHS Sponsored Research: DHS Approaches to Supporting Infrastructure Resilience}

Presenter: Mr. Matthew Coats, Program Manager Science and Technology Directorate, Department of Homeland Security

Mr. Coats delivered a presentation on Department of Homeland Security (DHS) sponsored research. He began his talk with a discussion of emerging trends and technologies, then explained the role of resiliency within DHS, gave an overview of DHS Office of University Programs funded modeling projects, and finally discussed the DHS Critical Infrastructure Resilience Institute.

Threats to cyber infrastructure were identified as a constant risk underlying all sectors. Key emerging trends and technologies related to information technology highlighted were: a focus on challenges posed by cyber-attacks on the critical infrastructure supply chain, evolution towards smart manufacturing, and developing digital threads to make manufacturing infrastructure more resilient.

The 2013 National Infrastructure Protection Plan was presented as an outline for how government and private sector participants in the critical infrastructure community work together to manage risks and achieve security and resilience outcomes. The three critical infrastructure sectors of focus for DHS are: the critical manufacturing sector, the energy sector, and the transportation systems sector.

Several modeling projects funded by the DHS Science \& Technology Directorate's Office of University Programs were outlined. The first initiative discussed was the AgConnect software products developed at the Institute for Infectious Animal Diseases. This suite of software supports real-time situational awareness and decision-making regarding animal diseases. The Criticality Spatial Analysis Program, developed at the Food Protection and Defense Institute, assists with identifying and understanding the vulnerability of supply chains. The Economic Consequences Analysis Tool (E-CAT) provides quick estimates of the economic impact of various threats. The advanced circulation storm surge model (ADCIRC), developed at the Coastal Resilience Center, is used to predict when, where and to what extent flooding will inundate a community.

Finally, an overview was provided of the Critical Infrastructure Resilience Institute (CIRI), led by the University of Illinois at Urbana-Champaign, and how it supports the DHS mission. Projects at CIRI include: analyzing and supporting the development of the cyber-insurance 
market; analyzing regulatory options for managing systemic risks; assessing supply-chain cybersecurity assurance for critical infrastructure; and measuring business and economic resilience in disasters.

\subsection{Facilitated Discussion}

Moderators: Dr. Adam Rose, Research Professor of Public Policy, University of Southern California

Dr. Bruce Ellingwood, Professor of Civil and Environmental Engineering, CoDirector of Center for Risk-Based Community Resilience Planning, Colorado State University

Scribes: $\quad$ Dr. Ken Harrison, Operations Research Analyst, National Institute of Standards and Technology

Dr. Andre Barbosa, Assistant Professor of Civil \& Construction Engineering, Oregon State University

\subsubsection{Data Collection, Security and Sharing}

Data is an integral part of creating and validating systems models for community resilience. As such, several questions related to data were raised regarding issues such as collection of data, security of data, and methods to increase sharing of data. First, the panel was asked to comment on the temporal scale of data collection. Historically, data has not been collected for many sectors. Where data does exist, it is often at an inadequate resolution.

There was a consensus among the panel that the quantity and quality of data needs to be improved. Many operators know they have interdependencies with others, but not the extent of the interdependency. Incentives could motivate utilities to undertake such data collection and analysis initiatives. The quantity of data collected on restoration was agreed to be particularly poor. There are vast dependencies (e.g., resource allocation, effects of human behavior, etc.) on the restoration side are largely unknown.

Next, issues of data security were raised.

Several of the panelists discussed the need for high resolution data of the infrastructure in their presentations, though (in the U.S. specifically) these data are frequently not publicly available. Similar issues exist with healthcare data. The panel was asked if sharing metadata characteristics of the data (not the detailed data itself) could be a solution to possible security concerns. One of the drivers of solutions to the secure data problem in New Zealand was the fact that as the quality of the data improves, the quality of the asset management does as well. Lifeline operators who are able to see the benefit to sharing the data are much more likely to participate.

Another approach to the secure data issue is the requirement of data sharing. The panel was asked to comment on the notion that there is some data that simply have to be shared. One argument was that tax money is invested to develop strategic plans to confront risk and therefore the data must be made available.

It is important to understand what data is relevant for what effort. Sometimes disseminating excess information can leave a data owner vulnerable (e.g., to physical threats, competitiveness, ideological criticism, etc.). If the benefit does not outweigh the vulnerability, data owners will be 
less likely to share their data. Compliance with data availability requirements will not guarantee the quality of the data will be acceptable. There needs to be an incentive for the data owners.

\subsubsection{Interdependencies}

Several questions arose regarding interdependencies between systems. As a start, the panel was asked how they would define interdependency and what they would like to see in research and in practice.

The panel was careful not to overly-narrow the definition of an interdependency and its feedback processes. Interactions between systems are extremely rich and need to be two-way at every step possible. The panel agreed that in general the research community is data-limited on the dependency front. There is a need for organizations to communicate and understand the importance of the value added in defining, quantifying, and sharing data on their own interdependencies.

Though redundancies and interdependencies exist between utilities, each utility is operated independently. The panel was asked how this aspect of resiliency is addressed from a modeling standpoint.

One parameter that is used to assess the overall dependency of a community or system is the time that a group needs to get back to operability. This parameter is highly scenario dependent but is inherently able to capture effects of cascading failure and recovery.

\subsubsection{Selection of Metrics}

All of the presentations given in this session discussed research and models to address some aspect of community resilience. The panel was asked what types of metrics are used to determine if a solution will improve a situation and what types of questions the models are trying to answer.

Typically, the metrics that are chosen to evaluate model performance or decision alternatives are driven by the end user of the information or application. Given an interest of the end-user, the modeler should look at causes and indicators of that interest to develop metrics and objectives. Metrics should be determined over the full time-scale from pre- to post-event to assess the needs of the community at various stages of recovery. As more aspects (e.g., physical, social, economy, cyber, etc.) are considered, the metrics will become more complicated, but this is a fundamental need to assess the overall performance of the system.

\subsubsection{Model Integration}

Resiliency deals with modeling of complex systems. A question arose as to how components of resilience models are integrated in the context of interconnected systems of systems.

Creating more resilient systems is challenging due to the complex nature of the interconnections (such as interdependencies between the social and technical systems). Currently it is all piecemeal with no ability to model the systems altogether. The panel was in agreement that integrating and combining current models is a major challenge and future need. A modular framework within a computational platform could potentially present an opportunity for the research community to plug and play different with models and couplings of models. The concept of a framework to drive the definition and collection of data needed for quantifying resiliency rather than looking at step-by-step modeling processes was also highlighted. Ideally, 
such a framework would account for various hazards, including any social hazards, and serve as a means to tie the research and models together.

\subsubsection{Model Scaling}

There has been much discussion on the temporal aspects of modeling and data collection. What would be challenges to creating models which are physically scalable?

The panel was in agreement that model scaling must be assessed on a case by case basis. Some models are scalable, but limits should be provided as to where the models are accurate. Economic models have been shown to scale well between the county and state levels but may not perform well at lower granularities. At the community scale, local knowledge of dependencies is required. State and national scale models can utilize coarser data. Beyond that, data would need to originate from physical dynamics or actual regulators in the community.

\subsubsection{Model Validation}

The first question that is often asked of modelers is how the models were validated. In cases for which we do not have data, such as looking at future states or when trying to answer what-if questions, how are models being validated?

Stress tests, routinely done in banking and nuclear testing, are an option for validation. In this case, model components are failed individually or in combination with other model components to determine the impact on the overall system. The amount of theoretical validation is strikingly low compared to empirical validations. The panel was in agreement that the area of model validation needs significant improvement if the models are to be used to support decisionmaking and evaluation of resilience alternatives.

\subsection{Common Themes and Key Points (Session 2)}

The following themes and issues emerged from the presentations and discussion on resilience modeling research around the world:

- The current availability and quality of empirical data on physical, economic and social dependencies and restoration times for disruptive events is poor. To a large degree, the research community is data limited. An increase in quantity, quality, and access to data is needed, as it is essential to creating and validating models. Historically, data has often not been collected at spatial and temporal scales useful for modeling.

- Security concerns and constraints have made it difficult to obtain data necessary for systems modeling. Sharing of metadata (i.e., information about the data, as opposed to the data itself) may be an important first step. Access to metadata may allow researchers to show utility owners and other potential data providers the potential benefits of sharing data.

- Integrating and combining existing system models is a greater priority than developing new system models. The concept of a model library or framework could facilitate creating an interconnected system of systems.

- There are many efforts underway around the world to develop and improve resilience modeling. Collaboration across academic and geo-political boundaries could foster new breakthroughs in systems modeling. 
International Workshop on Modeling of Physical, Economic, and Social Systems for Resilience Assessment

- Increased input from the community and stakeholders is desired. There is a need to better understand human cognition and adaptive behavior within infrastructures. 


\section{Session 3: Modeling of Systems and Dependencies}

\subsection{Session Presentations}

\subsubsection{Evolution of Hazard Assessment Modeling in Drinking Water Systems}

Presenter: Dr. Royce Francis, Assistant Professor of Engineering Management and Systems Engineering, The George Washington University

Dr. Francis delivered a presentation on the evolution of hazard assessment modeling in drinking water systems, detailing the shift from primarily intentional events (e.g., terrorism) towards an all-hazards risk management approach. The talk covered the rationale for vulnerability assessment from the Bioterrorism Act of 2002, the transition to all-hazards analysis in Environmental Protection Agency (EPA) and American Water Works Association (AWWA) frameworks, and concluded with two example assessment tools representing the expanding scope of the knowledge bases required for all-hazards assessment.

The Bioterrorism Act of 2002, Title IV provided the initial impetus for securing drinking water systems following the 2001 World Trade Center attacks. Key innovations of the bioterrorism act were presented, such as the requirement for utilities to conduct vulnerability assessments, to prepare an emergency response plan, and to prepare for various system disruptions (e.g., supply disruptions or the intentional introduction of chemical or other agents to the system). The act resulted in significant advances in distribution system monitoring research.

Transitioning to an all-hazards analysis, how and why it has become apparent that natural hazards are more of a threat to drinking water systems than man-made hazards was discussed. Hardening a system for all-hazards may also be effective against man-made attacks or hazards, while hardening a system to protect against man-made attacks may be less effective against natural hazards. Tools developed to support all-hazards analyses for drinking water utilities updating their bioterrorism act vulnerability assessments include the EPA Vulnerability SelfAssessment Tool (VSAT), the EPA Water, Health, and Economic Analysis Tool (WHEAT), and the AWWA J100-10 Risk and Resilience Management of Water and Wastewater Systems (RAMCAP). The EPA tools, VSAT and WHEAT, are intended to be used in combination. The objective of VSAT is to identify critical assets, threats, effective countermeasures and costs; and the objective of WHEAT is to support the assessment of economic and health consequences. The AWWA J100-10 RAMCAP requires a ranking of critical assets for prioritization of resilience activities.

Two examples used to highlight the expanding area of all-hazards assessment within the water systems framework include the development of an Adaptation Toolbox for Southeast Florida Water Supplies and the Water Resources Dashboard. The former includes a set of adaptation tools and fundamental empirical and physical data; and the later aims to be a one-stop location for water-relevant climate data sets.

\subsubsection{Modeling Water Distribution System Resilience}

Presenter: Ms. Katherine Klise, Senior Member of Technical Staff, Sandia National Laboratory

Ms. Klise's presentation outlined the modeling components of the Water Network Tool for Resilience (WNTR), demonstrated the use of the tool, and showed how WNTR can help water utilities estimate potential damage, evaluate preparedness, prioritize repair strategies, and identify worse case scenarios. 
The motivation and background for the development of WNTR was the fact that water utilities are vulnerable to a wide-variety of human-caused and natural disasters. Additionally, influences on the resilience of drinking water systems were identified as design, maintenance, operations, and interdependence with other infrastructure. Several important questions were laid out that must be considered in drinking water infrastructure resilience: What kind of infrastructure damage would be expected? How long would the system continue to provide water to customers? Which customers are impacted the most? What kind of restoration actions would be helpful and how should they be prioritized? What can utilities do to prepare?

Software capabilities of WNTR include: simulation of disaster scenarios; measurement of quantitative resilience indicators; benefit evaluation of utility response; and evaluation of improvement in resilience. The WNTR modeling framework was discussed in detail. The general flow of the framework was presented as generating a network model, defining disruptive events and restoration actions, simulating hydraulics and water quality, computing resilience, and finally analyzing and visualizing the results.

WNTR employs tools such as EPANET and NetworkX to define and analyze network structure, components, leaks, and interconnectivity. Disruptive events are defined using fragility and survival curves to capture the probability of damage. The controls, demand, components, and attributes of the network can also be modified to match a particular scenario. Restoration actions (e.g., type of repair, number of crews, time to repair, firefighting capacity) are similarly defined. Hydraulic simulation and water quality simulation are performed using EPANET. WNTR includes a leak model to explicitly model water lost between the time when the leak starts and the time when crews can isolate and repair the leak. Metrics that are used to quantify the resilience include topography (e.g., shortest path lengths, bridges, articulation points, centrality), hydraulics (e.g., availability, pressure, Todini index, entropy, population impacted), water quality/security (e.g., water age, mass consumed, extent of contamination, population impacted), and economic (e.g., network cost, greenhouse gas emissions). Finally, results are stored in a time indexed database in order to analyze and visualize the results.

\subsubsection{Power Distribution Systems: Modeling of System Dependencies and Interdependencies}

Presenter: Dr. Charles Macal, Senior Systems Engineer, Argonne National Laboratory

The presentation by Dr. Macal discussed modeling of system dependencies and interdependencies as related to power distribution systems. Part 1 of the presentation discussed the background motivation for the modeling; in Part 2 modeling methods were presented; in Part 3 modeling results were discussed; and in Part 4 future challenges to modeling of power distribution systems were covered.

First, the issue of increasing interdependencies between infrastructure systems was discussed. For example, the growing reliance of the electric power system on cheap and clean natural gas for power generation is creating a significant and growing dependency of the electric power system on the natural gas system, while, at the same time, the natural gas system heavily relies on electricity for its operation. These interdependencies create the possibility that both systems are potentially more vulnerable. Eight top questions, determined from literature review and interview, to best assist with understanding stakeholder requirements were presented. 
Next, the link between infrastructure modeling and infrastructure resilience was explored. Two specific power distribution infrastructure models were presented: EPfast and NGfast (for electric power and natural gas systems, respectively). These models are linked by modeling the critical interdependencies between these systems. To demonstrate the model interdependencies, a hypothetical power disruption scenario set in North Dakota was presented. Local effects (e.g., downed power substations, local blackouts, disruption to natural gas processing plant, and reduced flow of natural gas), downstream effects (e.g., reduced gas delivery, reduced/halted production at gas-fired electric power plants, widespread blackouts), and restoration of the system were all discussed.

Concluding, several challenges for advancing infrastructure and interdependency modeling were identified. Challenges include: better defining end-user requirements; identifying data needs for modeling; exploring development of integrated modeling approaches; delivering useful and useable models to users; and fostering collaboration among the R\&D community.

\subsubsection{Reliability Assessment of Interdependent Networks Based on Survival Signature}

Presenter: Dr. Matteo Broggi, Senior Research Associate, Leibniz University Hannover

The subject of Dr. Broggi's presentation was reliability assessment of interdependent networks based on survival signature. Due to the complexity of the interdependencies, dependent failures are very difficult to predict and hence dangerous, in particular, when the initiating event is seemingly unimportant. Dr. Broggi proposed a solution based on the concept of survival signature which provides a novel basis for complex network analysis, outperforming traditional analysis techniques in numerical efficiency when estimating the reliability of networks.

An introduction to the current analysis of complex systems began the presentation. Traditional analysis techniques were identified as fault tree analysis and reliability block diagrams. Limitations of traditional analysis techniques were identified as dependencies, common-cause failures, time-dependent behavior, lack of information, and complex network structure.

Next, an overview of survival signature was presented. The key advantage of the survival signature approach is the complete separation of the structure of the network from its probabilistic characteristics. Once the signature of the network has been analyzed, the reliability analysis can be carried out by evaluating only the probabilistic structure, which makes the analysis particularly efficient.

A numerical example of modeling a hydro-electric power plant system was presented. Results from such an analysis allow the modeler to: assess sensitivities of the system reliability with respect to the imprecision of the model; control for the modeling accuracy; and control for detection precision and modeling refinement in critical parts of the system.

\subsubsection{Climate \& Complexity: The Resilience of Natural-Built-Human Systems}

Presenter: Dr. Auroop Ganguly, Associate Professor of Civil and Environmental Engineering, Northeastern University

Dr. Ganguly's presentation focused on transferring climate science to impacts with uncertainties and what this could mean for the resilience of systems in general. He began his presentation with a discussion of climate risks, presented an example of a cascading failure in India, and introduced a framework for assessing climate impacts on infrastructure systems and evaluating 
post-disruption recovery options. Finally, the generalization and application of this framework towards ecological systems is discussed.

The presentation began with Hurricanes Sandy and Katrina as examples of climate risks to natural-built-human systems. The disastrous effects witnessed after these hurricanes were the result of the climate (e.g., hurricane), the state of the built environment (e.g., an ASCE infrastructure rating of $\mathrm{D}+$ ), and society (e.g., increasing population in vulnerable urban-coastal areas). Modeling challenges surrounding climate change were identified as uncertainty and nonstationarity that are often difficult to quantify. Additionally, in climate there are certain things that are known at large dynamical scales and translating what that means to an infrastructure scale is not always an easy task. In the context of natural/climate hazards, it is important to not just understand at a high level but to also understand what the signatures of the impacts on infrastructures are. As example, he discussed heat waves (projected to be longer, more intense) and hurricanes (projected to be fewer, more intense). He stressed that those kinds of changes have important implications for urban coastal areas.

An example of a cascading failure in India during the delayed monsoon season of 2012 was presented. Much of the agricultural sector in India is dependent upon the monsoon season, hence the delay caused an increase in energy demand to pump additional groundwater. The delayed monsoons were also accompanied by an extreme heat wave, leading to increase air conditioning usage and further increasing the energy demand. This combined energy demand increased the stress on the electric power grid, leading to failures which quickly spread across the system, resulting in widespread blackouts across India. The cascading failures ultimately led to impacts on the Indian Railway Network - which is the supply lifeline of the nation.

Lifeline infrastructure networks in the framework of national resilience were next presented. By analyzing hazards in conjunction with sophisticated network representations, system resilience can be enhanced. A framework for assessing climate impacts on infrastructure systems was introduced. The framework follows a general risk-centric approach, considering the threat, vulnerability, and consequence of a hazard in conjunction with network sciences. This framework was used to assess the Indian Railway Network. Using ticketing information, an entire network of the railway system was developed. Metrics of the system include strength, degree, and betweenness. This network then allows for the ability to begin quantifying the response and recovery conceptual curve.

Finally, generalizing this framework and its application to ecosystems was presented. In ecology there is a concept called a keystone species. A keystone species was defined as one which will disproportionately help sustain the ecosystem. If the ecosystem is damaged, the keystone species are those which should be introduced back into the society to achieve near pre-event performance of the ecosystem. This is similar to an infrastructure system where a centralized node might be more critical to the overall system performance than an outlying node with fewer interdependencies.

\subsubsection{Natural Disaster Impacts on Infrastructure Systems and Society}

Presenter: Dr. Judith Mitrani-Reiser, Assistant Professor of Civil Engineering and Co-Director of Center for Systems Science and Engineering, Johns Hopkins University; Director of Disaster and Failure Studies, National Institute of Standards and Technology 
Dr. Mitrani-Reiser delivered a presentation on natural disaster impacts on infrastructure systems and society. She began her talk with a review of performance-based design and resilience-based design. She then presented an NSF-funded Resilient Interdependent Infrastructure Processes and Systems project titled Resilience of Critical Infrastructure-based Societal Systems (CIbSS), as well as a CDC funded project titled Composite Post-Event Well Being (CoPE-WELL).

Using the Pacific Earthquake Engineering Research (PEER) Center's Performance-Based Earthquake Engineering (PBEE) methodology, a conceptual overview of performance-based design as related to buildings was delivered. This general methodology was outlined to include hazard, structural, damage, and loss analyses. Performance-based design must also take into account downtime in buildings including repair time (e.g., the length of time necessary to procure items and conduct repairs) and mobilization time (e.g., the delay before construction begins including: time for damage inspection, consulting with professionals, bidding process, clean-up). For some building occupancies (such as hospitals) performance-based design procedures will not suffice in capturing the loss of important services.

The example of a hospital was used to convey the process of resilience-based design, which is able to take into account various services within the structure. Using this methodology, resilience curves (e.g., functionality versus recovery time) can be created for various hazard scenarios. This methodology can be seen as a continuous feed process, including: design, hazard analysis, structural analysis, damage analysis, repair and operability analysis, and resilience analysis. It was summarized that while helpful for individual buildings, it will not suffice in capturing disaster impacts on important community institutions.

CIbSS and their interdependencies with other infrastructure systems such as water, wastewater, power, natural gas, communication/cyber, and transportation were next discussed. The resilience of the entire CIbSS is assessed by employing discrete event simulation, care-paths that span critical units, resource tracking, and metamodeling with interactions. The concept can be applied to population displacement, food security, economic security, and healthcare delivery. Key needs to advance modeling of resilience in CIbSS were identified as: a holistic approach to capture community functioning over time, models that span multiple scales, effective use of data that is collected over a wide range of time scales, and models that capture the complex interactions of many community institutions.

Finally, the CoPE-WELL model was presented. CoPE-WELL is a linked conceptual and computational model with the eventual goal of serving as a decision support tool for local, state, and federal stakeholders seeking to enhance community functioning and resilience. This model takes into account various community functioning domains such as communication, economy, education, food and water, government, housing, healthcare, public safety, and transportation. Given an event, its effects on natural and engineered systems, countermeasures, and information on population vulnerability, inequality, and deprivation are used to inform the community functioning over time (including social cohesion, preparedness \& response, and external resources).

\subsection{Facilitated Discussion}

Moderators: Dr. Hussam Mahmoud, Assistant Professor and Director, Structural Laboratory, Colorado State University

Dr. Terje Haukaas, Professor of Civil Engineering, University of British Columbia 
Scribes: $\quad$ Dr. Juan Fung, National Institute of Standards and Technology

Dr. Ken Harrison, Operations Research Analyst, National Institute of Standards and Technology

\subsubsection{Keystone Species in a System of Systems}

In his talk, Dr. Ganguly mentioned keystone species in ecological systems as being those that are essential to a functioning ecosystem. This raised the question of whether the concept of keystone species is useful in thinking about built systems. In other words, can and should we identify critical components in a system of systems?

Extending this concept to the built environment is not straightforward. For a particular component, for instance, an electric power network, keystone species might be analogous to critical nodes on the network. Within a more complex system of systems, however, interactions are more complex, and identifying keystone species is not obvious. Each component is itself a piece of critical infrastructure and so arguably critical to the larger system. While the concept is intriguing, care should be taken in defining exactly what a "keystone" infrastructure is and why this concept would be necessary. For instance, does it add something to the analysis of networks that a measure of centrality cannot capture?

\subsubsection{Second-Best Solutions}

In the context of restoring post-event functionality, is it possible to accept suboptimal performance? The question raised concern over a possible disconnect between optimal functionality in a model and practical functionality for a community facing limited resources. To some extent, this depends on the community's objective function. What does a community prioritize in terms of functionality?

An example of patient throughput was presented by the panel. While optimizing such functionality, it may be more effective to strengthen long-term care facilities, which may go down following a major event and surge a hospital, as opposed to increasing hospital throughput capacity that will rarely be utilized. Giving a community the option to think through its priorities can help pin down the objective.

A similar concern arose over reaching a socially acceptable level of recovery. For instance, treating patients on sidewalks may work in the immediate aftermath of the event and having a dedicated space (such as a school gymnasium) may be a "socially acceptable" short-term intermediate step to recovering hospital functionality. How do we know when we are there and how do we model such a scenario?

Again, what is socially acceptable is up to a community to define. The models can account for how long it takes to reach a socially acceptable level, but broadly defining what that means is beyond the scope of existing models. Addressing this question in the planning stage, before an event occurs, can ensure socially acceptable recovery is appropriately defined. For example, in post-Sandy New York City took advantage of excess office space for medical use. The panel cautioned that recovery metrics and time scales will vary between communities and so should be customized to best use the available resources and alternative spaces. Within the model framework, metrics should be weighted with time to reflect the transition from suboptimal to optimal decisions as recovery progresses. 


\subsubsection{Alternatives to Scenario-Based Modeling}

Existing models seem predominantly focused on particular scenarios, which can appear ad hoc when compared to a probabilistic estimate of the damage under a given event. The panel was asked how difficult it would be to perform this type of analysis and what the barriers might be.

Scenario-based modeling aligns naturally with the way decision-makers (e.g., a utility) think about the problem, and so eases the issue of communicating the potential risk. The consensus among the panel was that such an analysis is possible but must ultimately rely on simulating thousands and thousands of scenarios, either through Monte Carlo or parameterized simulations, in order to infer damage distributions. Analytical probabilities are often intractable. In some cases (e.g., EPANET), such extensions are not possible in the existing software. In others (e.g., WNTR), the software can be extended. There is interest among researchers in developing more rigorous, statistically-based models, but this often conflicts with end-users' goals.

\subsubsection{Model Scale and Boundaries}

Dr. Macal presented a model of an electric power distribution network (EPN) and pointed out that such networks are regional. Several questions arose as to how models scale across different boundaries (e.g., a region versus a community) when defining resilience.

Distribution networks are far more complex than transmission networks, though electrical transmission networks often extend over large regions and sometimes cannot be directly scaled down to a community level. Electrical transmission systems must be solved as whole, from which part of the grid can be extracted. In other words, the infrastructure often defines the scale.

When scaling models or extracting a subset of a critical infrastructure system corresponding to a community, how should boundaries be determined for each system?

An example of hospitals in California wanting to plan for a possible influx of Mexican patients was presented by the panel. In this case, modeling outside of the typical network area is important to the critical infrastructure of interest to the community. A second example of Boston and Cambridge, neighbors who face similar issues but often have conflicting policy across jurisdictions, was presented by the panel. An audience member remarked that ethical and legal boundaries raise similar issues, so perhaps the problem itself needs a boundary.

\subsubsection{Hazard-Agnostic Models}

Dr. Francis presented the evolution of resilience thinking in water distribution, which has shifted focus from man-made threats to the broader area of natural hazards. This presentation sparked the question of whether it is possible to map man-made hazards onto natural hazards, so that we can focus on the latter while confidently addressing the former in the process.

The consensus was that this approach may be undesirable. Water supply systems do not behave like electric power systems. Water supply systems can take advantage of demand and supply imbalances and ample storage to create slack in their systems. Electric power systems, on the other hand, must continuously balance supply and demand. More generally, there is skepticism regarding the value of developing hazard-agnostic models. Epidemics and bio-threats evolve differently on a temporal scale than natural hazards. Additionally, while translating fragility curves across hazards (e.g., from earthquakes to floods) may be possible in some cases, it may not actually make the system more resilient. As in the discussion regarding scenario-based modeling, there is a tension between analytical generality and practicality. 


\subsection{Common Themes and Key Points (Session 3)}

The following themes and issues emerged from the presentations and discussion on modeling of systems and dependencies:

- Response strategies should be prioritized and modeled based on community objectives and available resources. Metrics, time scales, and desired level of recovery will vary between communities. Models must be flexible to address a wide range of questions, objectives, alternatives, and time scales.

- Improvements are needed in scaling of processes, data, and models. Some systems operate at a national or regional level and therefore cannot be modeled solely at the community level. Data has historically been collected at non-standard spatial and temporal scales.

- Interdependencies between and within systems are often complex and can lead to unpredictable cascading failures. Interdependencies between infrastructure systems have been increasing and are likely to continue to increase. This trend will further increase the need for integrated modeling approaches and interdisciplinary collaboration within the research community.

- Attempting to build generalized, hazard-agnostic models to account for the wide array hazards to a system is undesirable. This approach can neglect spatial and temporal scales of the hazard as well as capacities available to a system.

- There is a tension in the research community regarding the generality vs. practicality of models. Probabilistic models provide a greater generality and rigor than scenario-based models but can be more difficult to communicate to the end-user. 


\section{Session 4: Modeling of Recovery}

\subsection{Session Presentations}

\subsubsection{Physical and Economic Impacts of Earthquakes and Other Hazards}

Presenter: Dr. Terje Haukaas, Professor of Civil Engineering, University of British Columbia

Dr. Haukaas began his presentation with a discussion on model granularity, general model organization, the computer program Rts (developed by his research group) which coordinates the analyses of many probabilistic models, and an optimization model that can be used in the Rts program. The second portion of Dr. Haukaas' talk covered interdependent network systems and assessing the resiliency of a community. In the third portion of his talk, Dr. Haukaas discussed the integration of building information models (BIM) with traditional finite element modeling (FEM), and what role such an integration may have in building optimization.

A high-level overview of model organization was presented. Broadly speaking, at the regional, building, and component levels, there are occurrence models, intensity models based on location and magnitude, damage models and loss models. A model framework, Rts, was developed which is implemented in a C++ environment and allows the modeler to plug-and-play with different model components. Response objects are used to carry information from one model to the other to handle model interdependencies. From such a model, one of the main outputs is loss curves. Several types of loss curves were identified, including: the probability of exceeding a given loss in a certain period of time considering a given hazard (here earthquakes), structural and nonstructural drift sensitivities, and acceleration sensitivities. An optimization module which takes some sort of risk measure has also been developed for use in the Rts framework. The optimization is performed by carrying response object sensitivity through each model and utilizing gradient-based observations.

Looking at networked systems, Dr. Haukaas presented efforts to assess the resiliency of coastal communities of Vancouver. In the event of an earthquake, getting resources (e.g., food, fuel, medical supplies) to these communities was identified as a significant challenge. In the Rts framework, ports, refineries, trucks and ships are modeled as objects that can transport resources. As the recovery takes place, some of the ports come back online and deliveries of resources are able to continue. A case study was presented of the Powell River community (approximately $120 \mathrm{~km}$ northwest of Vancouver) experiencing an earthquake scenario. The community has 10 days of reserve fuel storage and receives deliveries every 3 days. Typical issues that can be analyzed in the model include how keeping the reserve fuel tanks full affects the vulnerability to earthquakes and how the demand might change following an earthquake.

Finally, the ability to communicate across BIM and FEM to establish information rich, structural models was discussed. Doing so allows for the consideration of impacts from issues that structural engineers typically do not consider (e.g., environmental impacts, down time, etc.) throughout the life of a building (e.g., manufacturing, construction, operation, structural analysis, demolition). Multivariate fragility curves were identified as a potential improvement to currently available damage models. An example of a building with a central core and gravity bearing columns experiencing an increasing level of damage was explored. In this case, his research group's approach is to predict damage as a surrogate for the repair action. Once the repair action is known, an analysis of recovery time and cost can begin. 


\subsubsection{Road Network Functionality of Istanbul Following an Earthquake}

Presenter: Dr. Himmet Karaman, Associate Professor of Civil Engineering, Istanbul Technical University

Dr. Karaman presented a study on road network functionality of Istanbul following an earthquake. The outline of his presentation was as follows: aim of the study, data, analyses (including building damage, debris area and radius, debris direction, and bridge damage), results, and conclusion.

Many scientific studies have concluded that Istanbul will experience a major earthquake resulting in extensive structural damage to the built environment. There have been many studies performed to assess building damages, but the study presented is the first to focus on the transportation functionality following such an event.

The effects of road blockage due to building collapse and due to bridge damage were both considered. Building and bridge damages in Istanbul were estimated in HAZTURK for a 7.5 magnitude hypothetical earthquake occurring along the main Marmara fault line. It was found that buildings near the coast have a probability of collapse or heavy damage exceeding $40 \%$.

The general modeling methodology for road blockages due to building collapse was outlined based on a database of damage experienced in Gölcük, Turkey during the 1999 Kocaeli Earthquake. The database is then used to define the building collapse direction and the debris spreading distance. Building damage analysis was performed for Istanbul in order to identify buildings that have a mean damage of more than 30\%. Trends determined using the Gölcük database regarding debris spreading were then applied to Istanbul. The results were overlaid onto the Istanbul road network to define post-event road functionality. Using the Gölcük database, the average distance of the debris spreading of collapsed buildings was 17.45 meters. Using this measure as a buffer applied to Istanbul, it was estimated that 3,000 kilometers of road would be blocked following a 7.5 magnitude earthquake.

In modeling bridge damages, considerations were made regarding: bridge classes/types, distribution of bridges, and bridge functionality over time (includes anticipated restoration time). Immediately following the earthquake, 194 bridges were expected to have a complete loss of function. Three and seven days following the event 32 bridges and 1 bridge, respectively, were still inoperable. Finally, combining the bridge and building damage assessments, a total of 3,500 kilometers of road were estimated to be blocked following the hypothetical scenario.

\subsubsection{Measuring and Improving the Resilience of the Built Environment in a Community}

Presenter: Dr. Quanwang Li, Associate Professor of Civil Engineering, Tsinghua University

Dr. Li's presentation covered measuring and improving the resilience of the built environment in a community. The study considered four building sectors that provide essential functions to a community: housing, education, business, and public services.

The presentation began with a discussion of natural hazard threats to the built environment, such as hurricanes, earthquakes, landslides, floods, and storm surge. Regarding resilience of the built environment, he pointed out the need for a pre-event measure of functionality to be defined. In this study, the population outmigration (or the percentage of people in a community who dislocate following a disaster as a result of physical damages to buildings) is used as a resilience 
metric. This metric was chosen as its occurrence would adversely impact a community's ability to function normally.

Community functionality is defined as the percentage of population remaining in the community. The functionality of the community is based upon the performance of building sectors that support essential community activities. Measuring the community functionality is a probabilistic function that takes into account the probability of loss of a single community sector (e.g., building sector) and the probability of population outmigration conditioned on the loss of one or more community sectors.

A framework for linking community resilience goals to specific performance targets for the built environment was developed. Given various probabilities of loss for each of the building sectors, a risk matrix is developed. It was assumed that out of four risk categories, if two or more sectors are in the most extreme risk category, or if three or more sectors are in the second highest risk category, significant outmigration may occur.

A second framework was presented for determining optimum retrofit strategies based upon the community functionality framework. This framework takes into account building type, quantity of buildings, cost of retrofit, and time to retrofit. The optimization largely focuses the retrofitting in building sectors which have relatively fewer buildings as well as towards buildings with lower retrofitting costs, ultimately resulting in higher cost efficiencies.

A third framework for evaluating the performance of the traffic system to support the community recovery process was next presented. This framework accounts for the capacity of the traffic system (e.g., ratio of resources shipped daily to resources needed daily for recovery) and the probability of damage to, or collapse of, critical bridges. Considerations of the traffic system can be incorporated with the community functionality framework and the optimum retrofitting framework to determine the recovery time of a community following a given event.

The frameworks presented can be used to compare and evaluate various pre-event measures and what effect they might have on the community recovery time. As an example, a hypothetical case was presented where all bridges in Beijing were hypothetically retrofitted for a magnitude 9 earthquake. Under the given earthquake event, the recovery time of the community with retrofitted bridges is significantly reduced compared to the current state.

\subsubsection{Housing Demand \& Capacity for Medically Fragile \& Vulnerable Populations: Modeling Transition through Temporary, Interim, and Permanent Housing Recovery}

Presenter: Dr. Joshua Behr, Research Associate Professor, Virginia Modeling, Analysis, and Simulation Center, Old Dominion University

Dr. Behr presented a study to develop a set of simulation models that allow for the forecast of the demand and transition times for temporary, interim, and permanent housing of displaced households stemming from weather events. Dr. Behr highlighted the utility of the model in its ability to evaluate 'what if' testing scenarios for local pre-disaster housing recovery planning practices and policies. The definition of resilience used in this study is the ability of the system of systems to temper the impact, to hasten the rebound, and to transcend the pre-event normal.

A high-level overview of the model was presented as starting with housing and neighborhood vulnerabilities, regenerative capacities for housing, and storm surge and wind speed data projections. This data is fed into a demand-capacity model to obtain information on housing 
damage by type of structure, displaced populations, and housing production capacity. The demand-capacity model then informs the dynamic modeling and production chain models. The output from the model is the forecasted housing recovery including supply-demand gap in interim, temporary, and permanent housing.

Next, the storm scenario selection and modeling process was discussed. Three categories of storms were selected. The first was a historic tropical cyclone that had previously struck Hampton Roads, Virginia causing significant flooding and high winds along its track. The second was identified as a quasi-historic storm, where the track of a historical tropical cyclone that made landfall outside of Hampton Roads was modified to pass directly over the community. The third was called a black swan event, which was mostly user defined and intended to generate a truly catastrophic event. Selected storm scenarios included: 1933 Chesapeake-Potomac storm (historic), 2012 Sandy (quasi-historic), 1996 Fran (quasi-historic), 1989 Hugo (black swan).

In estimating the vulnerability of Hampton Roads to each of the selected storm scenarios, many dimensions of vulnerability were assessed. For example, financial, mobility, healthcare, insurance, social-familial, aged, dependent, and theft vulnerability all fed into the overall vulnerability of the localities comprising Hampton Roads. Combining the scenario modeling with the spatial vulnerability modeling yields displaced population versus vulnerability and owner/renter status.

In estimating the housing stock reconstruction and repair, time, labor, equipment, and materials were all taken into account. Factors such as the type of trade, materials required, cost of labor, and time of repair all factor into a construction schedule that can inform overall recovery time. Finally, a conceptual supply chain model can also be incorporated to inform the overall recovery time, demand and transition times for temporary, interim, and permanent housing, and likelihood of persons relocating outside of the community.

\subsubsection{Modeling Recovery: The Role of Disaster Financing}

Presenter: Dr. Carolyn Kousky, Fellow, Resources for the Future

In her presentation, Dr. Kousky discussed the role of financing systems in recovery, risk perceptions and preferences in recovery, and factors missed by aggregate impacts.

First two types of disaster financing, ex-ante and ex-post, were discussed. Types of ex-ante (preevent) financing include insurance or other forms of securitization; types of ex-post (post-event) financing include disaster aid and access to credit. Insurance can provide larger and timelier payments to individual households than can disaster relief funding, ultimately leading to: a greater likelihood of rebuilding, limited negative economic shock to households; limited negative multiplier effects throughout communities.

Despite the benefits of disaster insurance, take-up rates remain low, and households are unlikely to voluntarily insure against disasters. Only about 50\% of households in the 100-year floodplain have flood insurance and only about $10-12 \%$ of households in California have earthquake insurance, despite the high risk associated with each of these communities and events. Reasons for the low take-up rate of disaster insurance were presented as: high cost, heuristics and biases, inaccurate or incomplete information, bounded rationality, transaction/search costs, and myopia.

The disaster declaration process begins with the disaster itself. The governor must request funds from the federal government. A rapid assessment will be performed by FEMA who sends a recommendation to the president. The president then decides to issue a declaration that can be for 
individual assistance and/or public assistance. FEMA then disburses the funds from the disaster relief fund, and finally supplemental legislation may be needed. Historically, the number of presidential disaster declarations shows a positive increasing trend since about 1950. For households, FEMA offers individual assistance grants, though many applications are denied. The assistance is often limited in amount and purpose, and can take months or years before it actually makes it to the household.

A new trend in disaster aid is the community development block grant disaster recovery program (CDBG-DR). Local governments have a greater flexibility in how the funds are used and the funds can be used for recovery and for mitigation (disaster declaration funds can only be used for recovery).

Between 2002-2014, 89\% of FEMA spending on hazard mitigation occurred after a disaster had occurred, with only $11 \%$ of funding occurring before the disaster. A significant portion of possible mitigation is not performed for reasons such as: high upfront costs, high transaction costs, no compensation for disruption or loss of use during mitigation, risk perceptions, and inaccurate/unavailable information.

Aggregate measures of community wide economic activity may be misleading as there is often significant variation in disaster financing at the household level. Some aggregate measures (e.g., gross domestic product) can mask critical distributional impacts of disasters or do not equate with overall welfare. Impacts from disasters are often localized and distributional consequences can be large even when the overall aggregate consequences are low.

\subsubsection{An Integration of Social Vulnerability Data and Mapping Tools for Community Vulnerability Assessments and Recovery Modeling}

Presenter: Dr. Nathanael Rosenheim, Assistant Research Scientist in Architecture, Texas A\&M University

Dr. Rosenheim began his talk by highlighting the biggest challenges of social science recovery modeling as: integrating qualitative and quasi-experimental research with engineering fragility models; model resolutions that show spatial variance in impacts across demographic subgroups; modeling short-, medium- and long-term recovery when available data is annual or quarterly; modeling variations in infrastructure at the neighborhood level to capture investment, divestment, and equity issues.

Pre-existing community characteristics (e.g., hazard exposure, physical vulnerability, and social vulnerability) shape and determine the specific impacts of hazards. When these three community characteristics increasingly overlap, the community becomes more vulnerable, and the recovery becomes slower. In order to integrate social science into hazard assessments and recovery modeling, community characteristics must first be defined in order to identify spatial clusters where vulnerabilities overlap.

Areas with higher vulnerabilities experience disproportionate losses and greater displacement. To support this claim, housing recovery indices were presented for Hurricane Andrew in MiamiDade Florida and Hurricane Ike in Galveston, Texas. In Miami-Dade owners, who are often the less vulnerable population, recovered past the pre-existing level within three years, while renters only achieved a $70 \%$ recovery in four years. In Galveston, Texas, in four years' time owners had achieved a $67 \%$ recovery level while renters had achieved only a $36 \%$ recovery level. 
As an example of social science integration, an analysis was presented of Cameron and Willacy Counties in Texas under a hypothetical hurricane causing significant storm surge. Metrics of the hazard included pre-defined hurricane surge zones and hurricane evacuation zones. The physical vulnerability was based upon several factors such as geographic location, critical facilities, interdependencies, and age, and was measured using vulnerability assessment and risk analysis. Several metrics of social vulnerability were identified such as: household structure, socioeconomic status, gender, race, age, tenure, urban/rural, special needs populations, and employment status. Combining these metrics, the entire set can be used in mapping tools to capture areas of hyper-vulnerability in context of the hazard exposure.

Several model dimensions and the fact basis supporting them were presented (e.g., fiscal model impacts are supported by tax base and sales data). Several challenging issues that were highlighted in regards to community recovery modeling include: time scale, spatial scale, uneven and inconsistent data across sectors, analysis expense and time (increasingly so at smaller spatial scales), public versus private proprietary data, and compiling all impacts into a single picture.

\subsection{Facilitated Discussion}

Moderators: Dr. Harvey Cutler, Professor of Economics, Colorado State University

Dr. Keith Porter, Research Professor of Civil, Environmental, and Architectural Engineering, University of Colorado Boulder

Scribes: Ms. Jennifer Helgeson, Office of Applied Economics, National Institute of Standards and Technology

Dr. Suren Chen, Associate Professor of Civil and Environmental Engineering, Colorado State University

\subsubsection{Defining, Communicating, and Modeling Metrics}

The needs of a community may be different at different times following a disaster. Metrics should be defined that are dependent on a temporal period following a major event. For example, critical services could be identified for the recovery process. Similarly, metrics of human agency as a function of time following a disaster are needed. For instance, the loss of water service for one week might not be a significant problem, but no water service for several months might result in population outmigration.

The panel cautioned that metrics and time scales will differ from society to society. Following the 2008 earthquake in China, people stayed in temporary housing provided by the government for several years, trusting that the government would fix everything.

It was proposed to the panel that metric definitions and capabilities to define metrics are satisfactory, and instead that the real issue is that modelers are not very good at communicating the metrics. It is not always clear which metrics should be highlighted and important metrics (such as system capacity) are often omitted. Which are the pre-disturbance metrics that are important to communicate to communities?

Valuable pre-disturbance metrics for vulnerability should be defined in three areas: social, economic, and building stock. Populations that are vulnerable to all three should be identified and communicated prior to an event. Tools can be used to ameliorate issues before a disaster occurs. Metrics in the response phase may be similar to the pre-event phase. Ideally the city 
manager will be able to give meaningful estimates based on implementing a resilience plan that was put together before the event.

What are the key metrics and how close are modelers to being able to model them in advance of a hazard event being realized? How easy is it to estimate and control these metrics ahead of time?

One key metric that was suggested is the time to repair. Repair time (e.g., to homes, businesses, schools, hospitals, infrastructure) is indicative of the time it takes for community functioning to resume. One panel member suggested key measures are relatively easy to determine ahead of time, but the question is how to appropriately combine them. Appropriate network analysis of how these things fit together is missing. However, there was disagreement among the panel with the notion that the metrics are easily defined or stable, noting that there are still challenges particularly with metrics related to social issues. For example population recovery might not be a positive indicator if there are some people that are better off (long-term) relocating than coming back to the community.

\subsubsection{Cascading Recovery}

Dependencies and cascading failure effects have been discussed in throughout this session. The question was raised as to whether recovery can be viewed as cascading in the same way as is on the risk side?

The panel was in agreement that a community is a system of systems and repair of one system will affect the recovery in another system. As such, methodologies could be developed to find optimum strategies to effectively and efficiently improve the systems. Several key links in communities that could be formulations of such cascading effects in recovery were identified by the panel, such as the links between: business recovery and housing recovery; retaining key societal roles (e.g., teachers, fire fighters, etc.) and community functioning; the establishment of a safety-net health system and societal well-being; and satisfying Maslow's hierarchy of individual needs during recovery and community recovery.

\subsubsection{Policy Decisions and Recovery}

How do policy or governance decisions affect recovery? Is it positive, negative, or both?

When governments issue travel bans or mandatory curfews or closures during hazard events, this can have a positive impact on safety but a negative impact on business. In terms of recovery, there are requirements when federal dollars are involved. In the U.S., FEMA provides tools and guidance on how to conduct a benefit-cost analysis, but this is usually problematic for communities that want to build back better. There are a number of blanket policies without appropriate flexibilities. An example was provided from Hampton Roads where different localities have different views and plans for resilience. Some are interested in building back stronger while others are interested in a speedier recovery. Communities that use build back better approaches may be more resilient, but they will pay up front for delaying the rebuilding process. This is also an additional burden on the poor.

The possibility of community-wide insurance policies was presented to the panel for discussion. For example, a hypothetical city could buy an insurance policy, on behalf of those who can't afford the cost of insuring, in order to guarantee how soon the expected recovery status may actually return. 
Situations similar to the hypothetical presented to the panel are currently being explored and enacted with mixed results. A study was discussed where taxes from property level assessment were found to be a viable funding source for a community flood insurance policy for places with low income populations located in vulnerable areas. This solution could act to increase the community resilience and decrease recovery time. An example was provided following an earthquake affecting Istanbul, where the government placed the most disadvantaged population in new housing with a guarantee of housing for 15 years. At the end of the 15 years, the population was forced to leave the housing. This solution essentially delayed the original consequences of the disaster.

Regarding storm surge hazard, the main discount for insurance is on elevating your house. The panel was asked from the policy perspective what other options, which are more easily incorporated, are good for resilience?

Affordability is a key issue of flood insurance. The riskiest properties are the most expensive to insure. Places that have a discount on insurance premiums have issues with insurance companies, as the companies don't think that the discounts are connected to their rates and reduced risk. Additionally, discounts are typically so low that they don't spark new mitigation.

\subsubsection{Social Vulnerability}

Depending on economical and societal conditions, communities have been found to behave differently and have differing priorities following a major disruptive event. Should the social vulnerability matrix consider the intention of the community?

The panel was in agreement that it would be better for overall resilience and recovery to have a community create a set of indices or metrics unique to its own priorities and preferences. Regarding increased social cohesion, in recognizing the importance of a process and bringing people together, it is important to figure out preferences and values ahead of a disaster event. The capacities of groups in communities should be recognized and made visible in the modeling.

Social cohesion and community bonds can be positive but so far social vulnerability has been discussed in a negative light. Can it be considered on the positive side? There may be community characteristics that result in needing different services but that may ultimately help in recovery.

Dissatisfaction with the term social vulnerability was expressed. It is negative and does not capture social abilities. Thinking about vulnerability as increased uncertainty may be one solution to overcome this negative casting.

\subsection{Common Themes and Key Points (Session 4)}

The following themes and issues emerged from the presentations and discussion on modeling of recovery:

- Social vulnerabilities and interdependencies have a significant effect on the impact of hazards and should be taken into account in modeling recovery. Pre-existing community characteristics (e.g., physical and social vulnerabilities) will shape and determine the path and speed of recovery.

- Similar to failure, recovery can also be viewed as a cascading process. Recovery of one system is likely have a positive impact on other connected systems. This information should be used to optimize resilience and recovery measures. One example is the linkage between housing recovery and business recovery. 
- Pre-event community metrics should be quantified regarding social, economic and infrastructure systems. This information can be used to inform resilience policies prior to an event and to evaluate the performance of such policies in the days, months, and years following an event. Consideration should be given to both social vulnerabilities and social abilities (e.g., social cohesion and community belonging).

- Key challenges to modeling recovery include temporal and spatial scales of data, inconsistent data across sectors, and high analysis time and expense.

- Aggregate measure of social and economic recovery at the community level can mask more severe impacts and slower recovery within specific neighborhoods or population groups.

- Households are unlikely to voluntarily insure against rare events. As a result, recovery is often dependent upon access to disaster aid and credit.

- Modeling of recovery must be flexible. Different communities will have different resilience and recovery goals. For example, some communities may focus on building back faster while others may focus on building back better. There may also be different preferences in terms of resources allocated to pre-event mitigation vs. post-event recovery. 
International Workshop on Modeling of Physical, Economic, and Social Systems for Resilience Assessment

This page intentionally left blank. 
7. Session 5: Case Studies and Validation

7.1. Session Presentations

\subsubsection{GRRIT ${ }^{\circledR}$ - a Sustainable Tool for Reducing Weather and Climate Impacts}

Presenter: Dr. Cindy Bruyère, Deputy Director, Capacity Center for Climate and Weather Extremes, Mesoscale and Microscale Meteorology Laboratory, National Center for Atmospheric Research

Dr. Bruyère gave a presentation on the management of data and models for resilience assessment. She began with an overview of the National Center for Atmospheric Research (NCAR) and its Capacity Center for Climate and Weather Extremes (C3WE). She next discussed the motivation for the initiatives underway at C3WE and the importance of effective communication in resilience analysis and planning. She then presented the Global Risk Resilience and Impacts Toolbox (GRRIT). Finally, two tools in GRRIT were presented as examples of its capabilities.

The number and cost of natural hazards are increasing. Potential causes for the increasing cost of natural disasters include: climate change and variability; increased development and relocation to vulnerable areas; and lack of planning. The largest contributor to the increased cost of natural disasters was identified as the communication gap, with over $60 \%$ of engineering failures directly attributed to lack of knowledge and communication of information as their primary causes of failure. One example given was in communicating climate change effects on hurricane activity to a manager. Instead of communicating in terms friendly to scientists and engineers (e.g., change of power distribution of storms in the North Atlantic), it would be more effective to communicate in terms friendly to the manager (e.g., the cost of hurricanes due to climate change is projected to increase by $\mathrm{X}$ dollars in $\mathrm{Y}$ years).

Next, GRRIT was presented. This toolbox is an entire framework that is supported at its foundation by data of various types hosted on a large number of computers not necessarily owned or operated by NCAR. NCAR does not want to be the custodian of others' data, but wants to provide links between databases and serve as a resource to point the community in the right direction. Once captured, relevant data are fed to decision-making tools to provide insights to critical questions related to extreme weather and climate risk and resilience.

Two tools of GRRIT were next presented as examples of its capability. The first tool was specifically for the off-shore oil industry to assess their vulnerability to hurricane hazards and estimate the amount of damage that is likely to occur. There exists a weak relationship between the Saffir-Simpson hurricane scale and expected damages (10\% correlation) and the need for a more reliable index. Using wind speed, translational velocity, and storm size, a cyclone damage potential index was developed, which yielded an $80 \%$ correlation to the expected damages. This tool also has an extension for a future scenario, so a manager can use possible future trends to determine what level of damage they might expect into the future and evaluate alternative actions they might want to take.

The second tool discussed was one developed for the construction industry. When the construction industry bids for a contract, they look at the historical period and estimate contingencies for budgeting. This method could be vastly improved if the construction industry had advance knowledge of weather they might expect. For example, instead of projecting an average of 5 days contingency for rain in Houston, what if it was known to be an El Niño (or La 
Niña) year and Houston was projected to be much wetter (or drier)? The three major weather sensitivities of the construction industry are temperature, rainfall, and winds. From this foundation, a tool was created that can produce maps which provide more useful information than standard above, at, or below normal projection estimates for a geographic area. The tool can yield information such as the number of days when temperatures will be above $40^{\circ} \mathrm{F}$ and when in the season a particular extreme (e.g., cold/hot, wet, windy) trend might occur.

\subsection{2. $\quad$ Resilience Modeling Innovations of the HayWired Planning Scenario}

Presenter: Dr. Keith Porter, Research Professor of Civil, Environmental, and Architectural Engineering, University of Colorado Boulder

Dr. Porter's presentation highlighted several resilience modeling capabilities of the USGS's HayWired earthquake planning scenario project. He first provided an overview of the HayWired project and its origins. Dr. Porter then discussed the societal impacts of building code performance objectives and the fact that life-safety design can lead to unacceptable building impairment in a large urban earthquake. Public preference, expectation, and willingness to pay for seismic resilience of new buildings were also discussed. Next, Dr. Porter presented a new water supply model with lifeline restoration, interaction, and resilience measures. Concluding the talk, several open questions were posed to the audience and broader resilience modeling community.

Building codes, particularly the IBC and ASCE7, aim to protect life safety and in earthquakes they do that job very well. The audience was led through an example of two cases where the entire building stock of the San Francisco Bay Area was assumed to be: 1) compliant with current design codes, and 2) 50\% stronger (e.g., can survive 50\% stronger shaking) than current design code requirements. The second case reduced the probability of collapse due to shaking by about one third. For each collapsed structure there are approximately 60 impaired (red or yellow tagged) structures. Results presented from the HayWired experiment show roughly 8,000 structures collapse at the code compliant level compared to 2,000 with the $50 \%$ increase in strength. For an entirely code compliant building stock, there are 100,000 and 400,000 red and yellow tags, respectively, affecting 1.5 million people, 150,000 businesses, and $24 \%$ of the buildings in the 9 counties surrounding the San Francisco Bay area. There isn't enough vacancy in the San Francisco Bay area to accommodate all of the displaced people, which will result in out-migration. In the HayWired experiment with the $50 \%$ stronger buildings, results show only $6 \%$ of the building stock is impaired, which almost meets an urban planning objective of $95 \%$ of people being able to shelter in place after a large EQ.

An overview of a first-ever large survey conducted to determine what the public would prefer if they knew realistic cost and performance options was presented. Results from the survey show the largest number of people expected a building to at least be occupiable after a once in a lifetime earthquake event and nearly $20 \%$ of them expected or wanted a building to be functional. Only about $22 \%$ of survey respondents expected or wanted the performance that the building code actually provides (life safety). Next, the survey sought to determine if and how much the public would be willing to pay for the desired increase in structural performance. Putting the cost in familiar terms, the increase in performance would cost approximately \$3/sf. The majority of survey respondents said they would be willing to pay at least that much. He also reported, however, that this finding is in direct contradiction with interactions with most builders and owners. The issue is not in the cost itself but with cost competitiveness. Builders would 
prefer a mandatory design strengthening applicable to all in lieu of an optional code plus design alternative that may increase their bid over their competitors.

The second topic presented covered a new water supply model that includes lifeline restoration, interaction, and resilience. In a shakeout scenario for southern California, business interruption losses due to loss of water service accounted for about a quarter of the overall loss. Damage from shaking, liquefaction, landslide, post-seismic slip, and after-slips are included in the damage analysis. He stated that a simple approach (that of HAZUS) is used to estimate the level of service after the earthquake. A linearized approach was presented to including lifeline interaction without performing a full system of systems analysis, which can be overly demanding in terms of data and analytical requirements. In the linearized model, up-stream lifelines are characterized and impairment is only propagated downstream. The overall loss to society caused by a loss of water service is estimated at $\$ 770$ per customer per day of no water service. From the HayWired scenario, comparing the initial level of service on two sides of the Oakland/Berkeley Hills, 85\% of customers east of the hills have water supply and $10 \%$ of customers west of the hills have water supply after the main shock. Having a fuel plan in place results in a resilience benefit of 200,000 service days less loss or restoration of water service 1 day sooner for the average customer, or $\$ 150$ million in savings to society.

Concluding his presentation, two issues were posed to the audience. The first is the issue of human agency. For example, how long will people tolerate dry taps before they will relocate? The second issue is a lack of understanding in what motivates and restrains utilities from making resilience investments and what influences the decision-making process.

\subsubsection{Model Validation: The Joplin Tornado Hindcast}

Presenter: Dr. John van de Lindt, Distinguished Professor in Infrastructure, Co-Director of Center for Risk-Based Community Resilience Planning, Colorado State University

Dr. van de Lindt's presentation focused on model validation through hindcasting of the Joplin tornado. The general outline of Dr. van de Lindt's presentation was: an overview of the 2011 Joplin, Missouri tornado event, field study data collection and mapping, single sector validation focused on the electric power network and on the building sector individually, and multi-sector validation considering the electric power network and the building sector simultaneously. The presentation was concluded with a discussion of next steps and future solutions.

First, the demographics of Joplin including: geographic size, population, total retail sales per capita, median household income, travel time to work, gender, race/ethnicity, and age were covered. The tornado that struck Joplin on May 22, 2011 was an EF5 on the Enhanced Fujita scale and had multiple vortices. There were 161 fatalities, 1150 injuries, and monetary losses of $\$ 2.8$ billion as a result of the event. Damage that occurred to critical infrastructure such as the water network, the communications network, and the electric power network, was presented.

Next, the field study, data collection, and mapping efforts were discussed. The high level mapping procedure involved syncing camera and GPS equipment, conducting a field study with GPS running, geo-locating photos, and creating a web map. Data collection consisted of 2,600 photographs, interviews with residents and builders, and $360^{\circ}$ videos along streets in affected areas. Damage contours were derived from the geo-located photographs. Information on the recovery of buildings, housing, water, and electric power networks was also developed. Key impacts of the 2011 tornado include: more than 17,000 people were affected and 9,200 were 
displaced; five of 19 schools were totally destroyed; four additional schools were damaged or partially destroyed; and 4,200 of the 7,500 students in Joplin were displaced. Several mental health statistics were highlighted, including: a $40 \%$ increase in domestic violence, $10 \%$ increase in suicide, and an increase in the population experiencing mental health issues from 13,000 to 14,500 .

Key elements of the electric power network that are captured in the model include: transmission and distribution lines, substations, and transmission and distribution poles. To perform a damage analysis, fragility functions were used for each network component. The distribution of damage along a tornado path is based on statistics of all tornadoes on record in the U.S., with the probability of damage in each of the EF scales as a function of distance from the storm track. Using a simplified straight line path simulation technique, an average of 70 transmission towers and 3,446 distribution poles were modeled as damaged in the EF2 damage region. Using the actual track and damage path, an average of 113 transmission towers and 3,744 distribution poles were modeled as damaged in the EF2 damage region. This was compared to 135 transmission towers and 3,850 distribution poles actually damaged in the same area.

Eighteen new fragility functions were developed for various structure types (e.g., single story residential with hip roof, strip mall, etc.). For each structure type, fragilities for each of four damage categories (e.g., slight, moderate, extensive, complete) are defined. To estimate the damage in the buildings, each building is converted to a point that maps back to various characteristics for the sake of data compression. Preliminary results were presented, where all buildings less than 5,000 square feet were randomly assumed to be one of five residential building types. Results from this scenario show 2,780 buildings in the EF3 region were modeled as heavily damaged or demolished, compared to 3,181 buildings that were actually heavily damaged or demolished in the same region.

For multi-sector model validation using the electric power network and the building stock, the two systems can be overlaid within the model. This enables the model to determine which buildings have lost power and how long it will take for recovery. Within the model, resource units can be limited to replicate a post-event supply chain scenario. No results were presented for multi-sector validation, which was still in progress at the time of the workshop.

Concluding the presentation, several next steps for the Joplin Tornado Hindcast were identified, including: accurate fragility assignments to all buildings; coupling of buildings and the electric power network; inclusion of other physical infrastructure sectors and costs; one-way dependencies; and coupling of buildings with social demographics.

\subsection{Facilitated Discussion}

Moderators: Dr. Elise Miller-Hooks, Professor and Chair in Infrastructure Engineering, George Mason University

Dr. Judith Mitrani-Reiser, Assistant Professor of Civil Engineering and CoDirector of Center for Systems Science and Engineering, Johns Hopkins University; Director of Disaster and Failure Studies, National Institute of Standards and Technology

Scribes: $\quad$ Dr. Maria Dillard, Research Social Scientist, National Institute of Standards and Technology 
Dr. Steve Cauffman, Research Engineer, National Institute of Standards and Technology

\subsubsection{Modeling Challenges}

The first two talks discussed the importance of performing exercises. Dr. Bruyère and Dr. Porter were asked to comment on the challenges they each overcame to get theoretical models into the hands of practitioners in a way that would be useful during these types of exercises.

Dr. Bruyère's top modeling challenge was related to developing a practical, useful, accurate, and simple tool that the off-shore oil industry could use to relate hurricane damages to insurance claim payouts. Dr. Porter's top modeling challenge was focused on the water side of the model and obtaining the utility data necessary to create it. Utilities are concerned about data security, researchers issuing information that might cause unintended public response, unfavorable information being published, use of time (i.e., doing work that falls outside of normal job duties), and working with unfamiliar people or outsiders. Solutions to the challenges were identified as: involving the utilities in the peer review process and allowing the utilities to have approval rights of the model, non-disclosure agreements formed between the private consulting company and utility companies, incentives (tech transfer and ownership of research, free research on a topic of value to their industry, partnership with USGS), and developing on-going relationships with utilities.

\subsubsection{Increased Resilience and Hardened Design}

In his presentation, Dr. Porter presented a 1\% increase in construction costs associated with a higher level of design. He was asked to comment on the feasibility of the $1 \%$ cost increase, how it may be generalized to different sized projects, and whether it is inclusive of fabrication and the uncertainty of performance.

As further support for the figure, a previous NIST study was cited that looked at change from 1999 SBC to 2000 IBC and the resulting cost for 7 building types, where similar cost increases were seen. The $1 \%$ figure is the overall average increase in construction costs for society, and cannot be assumed to be uniform over all projects or buildings. An increase in strength may lead to other costs which are not included in the figure.

The hardened design concept was further explored in a similar question. It is easy to pick a scenario and say spending 1\% more to strengthen our infrastructure will result in less damage. The question was raised as to whether this is resilient design or not. Could it be that making something harder is less resilient because it takes longer to recover from an event? It was postulated that if the load is ignored and it is assumed that the system is going to fail, assessing how long recovery takes may allow different design choices to emerge other than hardening.

There were mixed opinions among the panel members regarding this topic. One point of view was that an ounce of prevention really is worth a pound of cure. From this standpoint, the definition of resilience as faster recovery is seen as a false premise and strengthening is viewed as one of many pragmatic approaches for reduction of loss. On the other hand, it was acknowledged that a community is not always able or willing to design to the ultimate level. It should be evaluated what is absolutely necessary to harden and where something can be allowed to fail. Some current concepts do allow for damage and replacement of components after damage. 
Dr. Porter closed his presentation with the question as to what drives investment in resilience and Dr. Bruyère mentioned a communication gap. Is it enough to provide more information (such as the resilience benefit for utilities or the 1\% figure for developers) in order ensure more resilience actions and investments or are incentives a necessity?

The panel was in agreement that incentives are a better driving force than only providing information or data showing the cost benefit of resilience. However, there are many more factors that affect resilience decisions than incentives. For example, organizations do not typically like to be the first to implement such measures. By following others, it helps them to understand practical decisions that had to be made in order to make the investment work. The National Institute of Building Sciences has issued a white paper on how to provide incentives to the private industry.

\subsubsection{Comparison of Modeling Approaches}

Dr. Porter was asked if the linear supply chain approach of lifeline interaction discussed in his presentation had been compared to or evaluated against a system of systems model.

No system of systems analysis had been performed and therefore the differences that might exist between the two models could not be defined. Some interaction loops do exist in real processes, but they aren't significant enough that it has caused significant pushback. The simplified approach was the only way that partners would participate, as the security concerns of the utility associated with providing detailed data for use with MaeViz were too overwhelming. Furthermore, HayWired's program objectives would not allow for the scholarly investigation of benefits of each model type. This is the reason that makes the Centerville model such a great value. We can gain valuable insights without getting the detailed proprietary data. The work for HayWired was intended for decision makers and for providing value to stakeholders.

This research community deals with uncertainty regarding hazards and responses but it seems most of the quantification of uncertainty originates from simulation. The panel was asked to talk about the role of analytical approaches in dealing with uncertainty.

Appropriate analytical approaches were identified, including: first order second moment treatment of uncertainty and moment matching. The IN-CORE modeling environment currently in development was also discussed. Analytical models play into this broader model through fragility development. The panel was in agreement that harder or more complex approaches to modeling are not necessarily better. It was argued that Monte Carlo simulation can provide better measures of uncertainty than moment matching, is relatively simple to implement, and is easily understood by end-users. Additionally, in Dr. Bruyère's presentation, it was shown through comparisons of modeling approaches that the complex model did not yield better results than the simple model.

\subsubsection{Scenario Modeling}

It was mentioned that stakeholders prefer a single scenario to design for and prepare for. How is uncertainty with a single scenario to be handled when making design decisions?

The panel was in agreement that a scenario needs to be realistic so that users can wrap their heads around it, even if it differs dramatically from an actual event. A scenario is one realistic outcome that is worth planning for. End-users want a solid, tangible, well-accepted story and uncertainty often undermines this desire and ultimately leads to paralysis in decision-making. As a result, HayWired provides a realistic outcome, but no measures of uncertainty. Actual 
outcomes of the event may differ and this is acknowledged and communicated to the community of users. There are also on-going efforts of the NIST CoE in addressing scenarios by creating and providing an array of scenarios instead of a single scenario.

A follow-up question was raised regarding scenario development. Given that there is so much uncertainty around the impacts of a hazard, what is the decision-making process in designing a perfect scenario for a community? What are the key components of a table top exercise or scenario event given that there will be just one event and one impact scenario?

The value of stakeholder input in scenario development was acknowledged by all panelists. It is important to have discussions initially help to determine the scenario design and to find out what people really want to know. A one in one million event is not usually a scenario that people want to plan for. Simple scenarios are used to create the uncertainty bounds and to present a final result.

A final comment on the use of scenario modeling was presented to the panel for discussion. There are design criteria that communities are meant to be designing to. From the discussion, it sounds as if scenarios are being offered that might not be consistent with design scenarios. This has been a source of confusion in the past because communities didn't really understand what they should be designing for and what they should be expecting.

The panel responded that there are people in the community who have the expertise to understand these differences. There is a mismatch between scenario and design criteria, and the building code illustrates this tension. Scenarios help to explain the distinction. Some people see design maps and shake maps and don't understand the distinction.

\subsubsection{Model Complexity and Calibration}

For the end-user driven projects, how much do they care about the complexity, accuracy, and precision of the models and how much would that change their decisions on the results of the models? Do the end-users care that the trend is towards more complex models?

End-users seem to be less interested in complex models and more interested in high resolution data, fast running models, and solid measures of uncertainty. With increasing complexity, the opportunity for technology transfer decreases due to the inability of end-users to review and evaluate the model. The CoE is currently evaluating the level of complexity, aggregation, how uncertainty propagates through a system, and whether uncertainty is so high that decision support cannot be provided.

As models increase in complexity, are they being calibrated? If so, how is the calibration being performed?

No panelists were aware of current calibration capabilities. Future calibration exercises are planned for the Joplin Tornado Hindcast, to be performed after version 1 . The calibration is intended to be systematically documented to reduce bias. NCAR has a wealth of expertise and knowledge to develop their theoretical models but has historically lacked data, in appropriate quality and quantity, needed for model calibration.

\subsubsection{Collaboration}

Regarding the possibility of NCAR as a hub for software and data, Dr. Bruyère was asked if others would contribute to such a library? Would the library include individual pieces of 
software code or would it serve as a link to different code hosted elsewhere? How do you envision this software library?

The preference by NCAR is for partners to share more than just a link to another website or tool, though there is awareness that not all partners may be willing to share all source code for tools. The software doesn't necessarily have to reside at NCAR but could link back to another institution. The aim is for a seamless framework or environment that looks and behaves the same with all tools or models.

\subsection{Common Themes and Key Points (Session 5)}

The following themes and issues emerged from the presentations and discussion on case studies and validation:

- Data availability is a major challenge for realistic case studies and model validation. Solutions to obtaining utility system data include developing ongoing relationship with utilities, involving utilities in peer reviews, executing non-disclosure agreements, and incentives such as providing research or advice to utilities in exchange for data. Other solutions include the development of synthetic communities such as the Centerville model from which valuable insights can be gained without the need for detailed proprietary data.

- Model calibration and validation have significant room for growth. Calibration and validation are crucial if the models are to be used in the decision-making process. Comparisons are needed across models to understand the effects of model granularity and complexity. Simplifying assumptions and sensitivity studies, such as one-way coupling between systems and deterministic scenarios, can be used to develop simplified models and test the components of more rigorous and complex models.

- There is a potential disconnect between scenario-based modeling and probabilistic modeling and in the level of complexity desired by the research community and end-users. This can be addressed by developing an integrated set of rigorous probabilistic models for use by the research community and a more practical, scenario-based tool for community planners that provides realistic measures of uncertainty that have been derived from the research models.

- Communication and collaboration with stakeholders, the public, and amongst the research community should be improved and built upon. Better communication with stakeholders and the public can increase reception and implementation of resilience measures.

- A common library to access data and models could facilitate collaboration and shed new light on critical questions of resilience. 


\section{8. $\quad$ Resilience Table-Top Exercise}

Presenter: Nathaniel Forbes, Director, Forbes Calamity Prevention

\subsection{Scenario Overview}

Mr. Forbes ran a table-top exercise under the fictitious scenario of Hurricane Nat. The main objectives of this exercise were to demonstrate the importance of cooperation between public and private sectors in disaster preparation, response, and recovery, and to provide workshop participants with a small glimpse of the decision-making process.

The Hurricane Nat scenario was loosely based upon Hurricane Matthew, which made landfall in South Carolina approximately two weeks prior to the conference. The setting of the scenario was on an island called the Republic of Resilia, which was said to be inaccessible by land, two hours by air from the nearest airport, and with a port big enough for only two ships.

\subsection{Participant Roles}

Participants were assigned to one of five sectors common to any disaster: the private sector, the government, non-governmental organizations (NGO), survivors, and witnesses. The private sector was in control of many basic resources such as bottled water, portable electric generators, portable toilets, building supplies, fuel, and groceries. The private sector also included entities such as the news media, telecom providers, and the insurance industry. The government consisted of the roles of mayor, police, fire \& rescue, hospitals, schools, public utilities, and a national disaster management agency. All government roles were seated at one table to facilitate inter-agency communication. NGO's were able to address needs such as medical treatment, logistics, food distribution, shelter, disease management, and religious faith support. This table had the smallest number of people to simulate the relative proportion of relief workers to survivors and other responders. Each survivor exhibited one of eight common disaster consequences: broken leg, parents missing, spouse died, home damaged, home destroyed, restaurant damaged, lost pet, and undocumented immigrant with limited language capability. Finally, witnesses included anyone local or remote that was not directly affected by the event but was looking for information on it. Witnesses were to remain at their tables and had to get information delivered to them, as you would observing on a television or computer screen. No information was provided as to the roles of each table or other participants.

\subsection{Resources}

Immediately following a disaster event, there often are not enough resources to meet the demand of the community. To simulate resource constraints, in the role descriptions, the quantities of supplies, maximum capacity of shelters and hospitals, etc. were limited or restricted in time. These restrictions ultimately forced those in NGO and government roles to turn away people in need, and further led to the private sector increasing prices as a response to increased demand and limited resources.

\subsection{Post-Disaster Time Periods}

Two post-event time periods were presented to participants. On Day 1 following the disaster consequences included no electricity, water, communications, sanitation infrastructure, or ground transportation. There were many injuries and deaths reported. Destroyed and damaged buildings led to many business closures. Both the airport and port were inoperable, further complicating relief and recovery. On Day 3, NGO's were provided with enough supplies (tents, portable 
toilets, bottled water) for ten people, communications systems are back up, and the public water utility confirms Resilia's water reservoir was contaminated. The community members are not satisfied with the level of relief and recovery and have begun to protest. Many community members are still injured, without electricity or water, and are missing pets. Each participant's role description included expectations of their role corresponding to the amount of time that had passed after the disaster. For example, on the first day following the disaster, the Red Cross equivalent was instructed that they had only enough local, trained volunteer doctors and nurses to treat two survivors.

Following each post-event time period, a group debriefing was performed where each person could report on their experiences in the various roles. Questions posed to the groups following the Day 1 Time Period included: What did survivors need and was it obtained? How many people came to NGO's for help and was help able to be provided? Who came to the government and what did they want? Did the private sector have enough of what people wanted, how were resources allocated? It was following the Day 1 Time Period that participants were notified what sector each table represented and what resources or needs they had. Following the Day 3 Time Period, participants were able to recognize how far planning, knowledge, and practicing can go in a disaster scenario.

\subsection{Key Lessons}

One key lesson participants were able to take away was that responses would have been more effective if communication had been better, faster, and clearer both before and during the event. Having no pre-disaster planning in place, no awareness of what societal needs and resources were or where they were concentrated, and no inclination as to what services would be available led to chaos on Day 1 following the disaster. After communication began, another key lesson regarding the importance of cooperation between public and private sectors was realized. The government realized the importance of private sector resources. The private sector was able to find a balance between commercial advantage and effective distribution of resources and assistance to those in need. 


\section{Summary of Systems Modeling Needs and Challenges for Resilience Assessment}

\subsection{Overview of Systems Modeling for Community Resilience}

The functioning, performance and well-being of communities relies upon increasingly interdependent infrastructure systems. Such interdependencies create the possibility for cascading failures which can have widespread negative impacts upon a community, such as loss of housing or utilities, outmigration, and business interruption. The development, improvement, and validation of integrated systems models for simulating physical, social, and economic systems in a community is critical in achieving the goal of improving community resilience for all hazards. Improved modeling methodologies assessing the performance, recovery, functionality, and dependencies of systems will better inform the planning, design, investment, and decision-making processes.

Throughout this workshop, common themes that emerged as critical areas for discussion and ongoing work included: stakeholder input, data, standards and metrics, modeling improvements, validation, and collaboration. These areas, which attendees felt would significantly advance the area of systems modeling for resilience, are highlighted in the following section.

\subsection{Desired Capabilities and Challenges}

\subsubsection{Stakeholder Input}

Input from stakeholders (e.g., planners, managers, members of the community, owners/operators, builders, etc.) is critical in developing guidance and tools that will be needed for model development and identifying key criteria for the decision-making process.

When developing a model, the needs and desires of stakeholders should be taken into account. This is a difficult task in itself, as stakeholders are often not in a position to define realistic needs and expectations prior to an event. One approach to this issue is engaging the stakeholders in an iterative process of communication following their initial responses and input, where stakeholders are presented with the results and consequences of various options to determine their preferences.

Due to the size, scale, and interdependencies of the physical, social, and economic systems being considered, many systems models can be complex. Determining what stakeholders need for decision-making might yield different needs regarding the complexity, scientific basis (e.g., empirical or physics-based), and granularity of the systems models. While a complex, high granularity, empirical or physics-based model may not readily support decision-making; such models do provide the foundation for higher-level models that do support decision-making.

Further, the need for quantitative assessment of factors that influence the decision-making process was identified. It is not clear to many systems modelers what steps, formats, or processes are the best way to socialize community resilience issues with stakeholders and the general public. Identifying how to inform decision-making before, during, and after a hazard event, and determining how to quantitatively incorporate decision factors into systems models could improve evaluation of alternative community resilience measures.

Another key issue regarding stakeholder input is how to communicate and incorporate uncertainty and risk. One current approach is to present stakeholders with a single (or ensemble of) real-world scenario(s) and associated consequences. This single scenario method does not communicate hazard uncertainty to stakeholders, but it does present an opportunity to simulate a 
variety of possible outcomes for a given hazard. Often though, stakeholders want to know how certain modelers are about the model results and projections. However, current methods of communicating uncertainty and risk (e.g., annual probability of hazard occurrence or failure) can lead to confusion or even dismissal of model projections. More effective ways to communicate uncertainty and risk to the stakeholders and to the public is an important issue that should involve collaboration between critical infrastructure modelers and social scientists.

\subsubsection{Data}

Accurate systems modeling techniques cannot be developed and validated without data. The first data challenge identified is defining what data is needed and whether specific data types, formats, and sources are available. For example, from a modeler’s perspective, a virtual city with all infrastructure system information and interdependencies known is an ideal case. For an actual city or community, such availability of data may not be feasible or even necessary for the decision-making process. It may be that common patterns (e.g., when do the patterns emerge, what patterns exist when systems are coupled, etc.) are more important to understand and model. The cleaning and sharing of metadata was posed as a possible solution to defining overarching patterns relevant to community resilience.

Another important data issue is the temporal aspects of data collection and data sharing for system performance. Historically, temporal data has not been collected on the performance of many systems, or it is often at varying time scales or an inadequate resolution. Where data has been collected, it is often at an inadequate resolution. Furthermore, data is often not collected through the recovery and restoration phases. Evaluation of community resilience requires data on a time scale at a useful frequency both prior to and following hazards events up to 5 or 10 years. Improving the amount and quality of data is vital, as the lowest quality of data drives the overall quality of the analysis.

In the U.S., high resolution data (e.g., electric power, healthcare, etc.) are restricted in their availability, which poses significant challenges to system modeling. Utilities have concerns about sharing their proprietary data that include security and competition issues. Proposed solutions to the challenges of accessing and utilizing secure data include involving the utilities in the peer review process, allowing the utilities to have approval rights of the model, nondisclosure agreements, and incentives. If the value of the data use for the community and their own systems can be presented, the stakeholders may see benefits for sharing their data.

There was also an on-going discussion at the workshop regarding data availability and whether some data must be shared. Tax money is often invested to develop strategic plans to confront risk and therefore data regarding community functioning are required. It was pointed out however, that compliance with data availability requirements does not necessarily guarantee the quality of the data will be acceptable. Some incentive for the data owners should be presented.

\subsubsection{Standards and Metrics}

Standards and metrics would enhance the development and evaluation of systems modeling capabilities for community resilience. Current motivations for the selection of metrics include end-user objectives and data availability. Many indicators of community resilience were discussed during the workshop, including: population outmigration, time for repair, employment levels, household income, and tax revenue per person. These metrics are heavily dependent upon the community though, and may not always indicate recovery. For example, while outmigration 
might have a negative impact on a community functioning as a whole, individuals may be much better off in the long-term relocating than coming back to their community. Several examples from Hurricane Katrina were provided where a positive or negative metric actually implied the opposite. For example, income per person increased because many dislocated persons were from disadvantaged neighborhoods (e.g., income metric increased but populations did not return). A standardization of metrics that could be used across models and different communities would be valuable in comparing modeling capabilities and decision alternatives.

In defining metrics of community resilience and recovery, the importance of a temporal definition was highlighted. Choices that are optimal on the first day following a disaster will likely become suboptimal over time. For example, it would be acceptable in the short-term to utilize a school gymnasium as a medical clinic immediately following a disaster if hospital facilities are overwhelmed or cannot be accessed. A proposed solution was to develop a weighting scheme over time for various recovery metrics. The metrics themselves as well as the time scales at which they might vary will likely be unique to each community.

The importance of pre-event metrics in evaluating performance and recovery following a disaster event was discussed in the workshop. If no pre-event performance metrics are defined, it will be impossible to evaluate the post-event recovery. Advocacy groups often have the best idea of preevent levels of performance for vulnerable populations, but it is difficult to obtain quantitative information. Ideally, the city manager will be able to give meaningful estimates based on implementing a resilience plan that was put together prior to the event. Metrics in the response phase may be similar to the pre-event phase.

Many metrics were discussed at the workshop, but the question still exists of how to appropriately combine the various measures to evaluate a system on multiple levels. Additional research is needed regarding the network analysis of how these metrics fit together. Communicating metrics to stakeholders and the public was also identified as an area for improvement. There is no consensus as to which metrics are the best to communicate to communities. Additionally, important metrics regarding capacity building are often neglected.

From a modeling perspective, a key standardization that is needed is in the definition of resilience. There are several definitions of resilience and each will dramatically influence how models are created and what is put together. This workshop brings together international researchers and modelers of community resilience, yet many languages have no word for resilience.

\subsubsection{Modeling Improvements}

Throughout the workshop several presentations highlighted current capabilities of modeling individual systems or utilities (e.g., housing, water utility, electric, transportation, supply chain, education, etc.). A major challenge was identified as combining the various systems into a complete model. Handling the interdependencies is complicated by the intricate nature of the connections (e.g., between the social and technical systems) and also by the varying temporal and spatial scales of models. There is a need for a modular framework within a computational platform. This could potentially create an opportunity for the community to explore different modules and couplings of modules, which may ultimately provide guidance for modeler collaboration. Inherently, linking individual systems models to provide an overall picture of a community will result in an increase in the complexity of the model. Characterizing the model complexity, precision accuracy, and how the two affect one another, is necessary. 
Appropriately defining model boundaries was also highlighted as an area for improvement during the workshop. When modeling critical infrastructure in a community, it is not always clear how to determine the boundary for each system. The simplest method would be to base the boundaries on the geographical community boundary area, but this method still requires dealing with boundary conditions. If a model framework is being developed for decision-making, it could be customized to the local or even building level. This presented further boundary options such as school districts, jurisdiction boundaries, and ethical model boundaries.

Several challenges to creating scalable models were identified. At the community scale, models require local knowledge of dependencies, while models at the state and national level can be useful with coarser data. It was pointed out that some models are scalable, but limits should be better defined as to where the scaling is applicable. Furthermore, the nature of some systems requires modeling at a specific levels. For example, the electrical transmission system must be solved as a whole at the regional level. The electrical distribution system, however, is more important for community level resilience.

The temporal scale of resilience modeling and analysis in the scope of decision-making support was also stressed as an area for concentration and improvement. If the temporal scale of the event is ignored or misconstrued, an essential point of resilience (capacity building) will be absent from the models. If dynamic effects are not explicitly modeled, the capability to assess transient behavior and failure scenarios through other, more simplified, methods is necessary

\subsubsection{Validation}

A critical area for improvement of systems modeling is in validation. Very little was discussed in the presentations or facilitated discussions regarding validation, indicating there is significant room for growth. Data that is used to validate models is often the same data that was used to create the model. Furthermore, the models that are being created are often extremely complex. If they are to be used for decision-making, it is crucial that the models are validated and the uncertainties are quantified. Once the models are deemed to be accurate and their potential errors are quantified, then the models can be refined and the uncertainties can be reduced. Proposed options for validating the models included stress tests (which are more common in the fields of banking and nuclear power generation), analytical approaches, and theoretical analyses.

\subsubsection{Collaboration}

This workshop brought together researchers and modelers from around the world, highlighting an opportunity for further collaboration in the systems modeling community. Often, multiple research groups are unknowingly working towards similar goals, with little or no exchange of information or resources. This workshop is a step towards increased international collaboration, and several initiatives (e.g., at NCAR and Tsinghua University) are already underway to create reference toolboxes where users can contribute to and benefit from an exchange of knowledge and abilities. 
International Workshop on Modeling of Physical, Economic, and Social Systems for Resilience Assessment

This page intentionally left blank. 


\section{Appendix A: Decision-Making and Evaluation of Proposed Alternatives (Session 1)}

Public Warning Models: Issuance, Diffusion and Protective Initiation [Dennis Mileti]

Abstract A-2

Slides A-3

References A-43

Biography..... A-44

Resilience of Societal Infrastructure Systems - Modeling and Decision Analysis Framework [Michael Faber].

Abstract A-45

Slides A-46

References A-61

Biography..... A-62

Total Economic Consequences of Disasters [Adam Rose] A-63

Abstract A-63

Slides A-64

References A-78

Biography. A-80

Infrastructure System of Interconnectivity Effects on Resilience [Rae Zimmerman] ..... A-81

Abstract A-81

Slides A-82

References A-104

Biography..... A-107 


\author{
ABSTRACT \\ Dennis S. Mileti, PhD \\ Public Warning Models: Issuance, Diffusion and Protective Action Initiation \\ For Dam and Levee Emergencies
}

October 19, 2016

\begin{abstract}
Quantitative curves for use in life loss estimation models for dam and levee emergencies based on public warning delay for warning issuance delay, warning diffusion delay in the population at risk, and protective action initiation delay by people at risk are presented. The curves were based on a synthesis of available social science research and data on these topics. The factors that contribute to these delay types were summarized, weighted and linked to life loss estimation. An interview schedule was constructed to measure the factors in local communities to enable the U.S. Army Corps to estimate likely future warning delays and life loss in future events. A local community guidebook was developed to assist local communities in public warning planning to reduce delays and minimize life and injury consequences.
\end{abstract}




\section{PUBLIC WARNING TIMING:}

Issuance, Diffusion and Protective Action Initiation For

Dam Breaches, Controlled Dam Releases, and Levee

Breaches or Overtopping

Dennis S. Mileti, Ph.D. Professor Emeritus

Univ. of Colorado, Boulder

John H. Sorensen, Ph.D. Distinguished Researcher

Emeritus, ORNL

Presented to

USACE Risk Management Center, Davis, California

27 July 2016

\section{ACKNOWLEDGEMENTS}

USACE, Risk Management Center, Davis

Jason Needham \& William Lehman

RAC Engineers \& Economists

David S. Bowles

Academic Reviewers

Susan Cutter, Univ. of So. Carolina, Columbia

Michael Lindell, Univ. of Washington, Seattle

Emergency Manager Reviewers

Sacramento \& Yollo Counties

HNTB Corporation

David W. Renetzky 


\section{PART 1 INTRODUCTION}

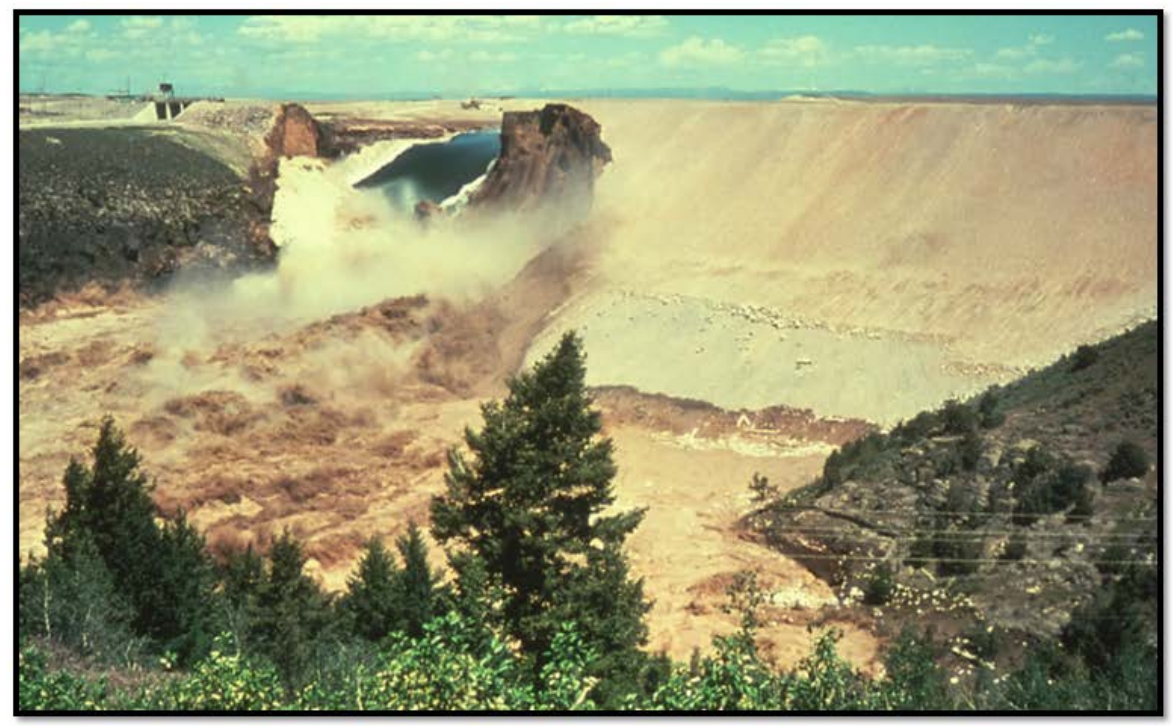

\section{WARNING BEHAVIOR}

\section{Public alerts and warnings involve the behavior of people who}

1. Detect threat (physical scientists)

2. Manage threat (emergency managers)

3. Communicate threat (message authors)

4. Take protective action (public)

Social scientists study all of them 


\section{TIME SEQUENCE CHART}
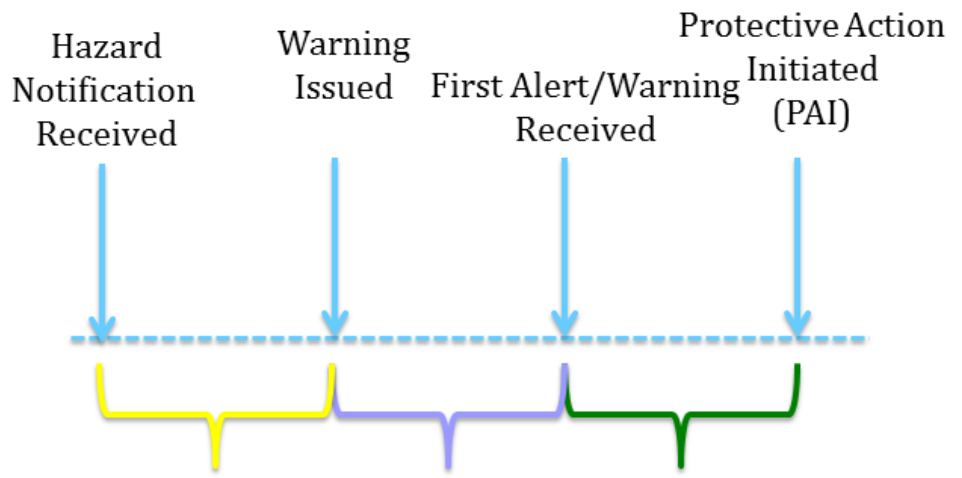

Warning Issuance Warning Diffusion Mobilization Time

Delay Time

Time

or PAI Delay

TIME

\section{OBJECTIVES}

\section{Provide USACE with}

Estimates of human behavior for life loss estimation

\section{Develop methods \& procedures}

To measure/classify local community behavior time estimates for warning issuance, diffusion \& PAI for

Dam breaches \& controlled releases

Levee breaches or overtopping

\section{Prepare a local community guidebook}

To inform emergency managers about social scientific evidence-based best warning practices 


\section{APPROACH}

Synthesis of hazards research literature

Behavior \& timing of actions - officials and public

Prepare influence factors catalogues

For issuance, diffusion \& PAI

Include factor's relative influence weights

Interview questions for community measurement

Pre-tested, revised, pre-tested again

Measure synthesis for classifying communities

Likely future issuance, diffusion \& PAI delay

External review \& revision as needed

\section{ALL HAZARDS OBSERVATION}

\section{Hazard types differ regarding}

Physical properties

Alert \& warning lead times

Appropriate public protective action(s)

\section{Human warning behavior predictors}

Are the same across hazard types

Because people stay people

Apply to dam \& levee warning events 


\section{CURVE DEVELOPMENT FOR ISSUANCE, DIFFISUION \& PAI}

Reviewed quantitative research findings on warning timing/delay for all 3 stages

Identified data from empirical studies

Point observations

Distributions

Developed model equations

Fitted equations to historical events

Developed parameters for planning curves

\section{PART 2 \\ WARNING ISSUANCE DELAY}

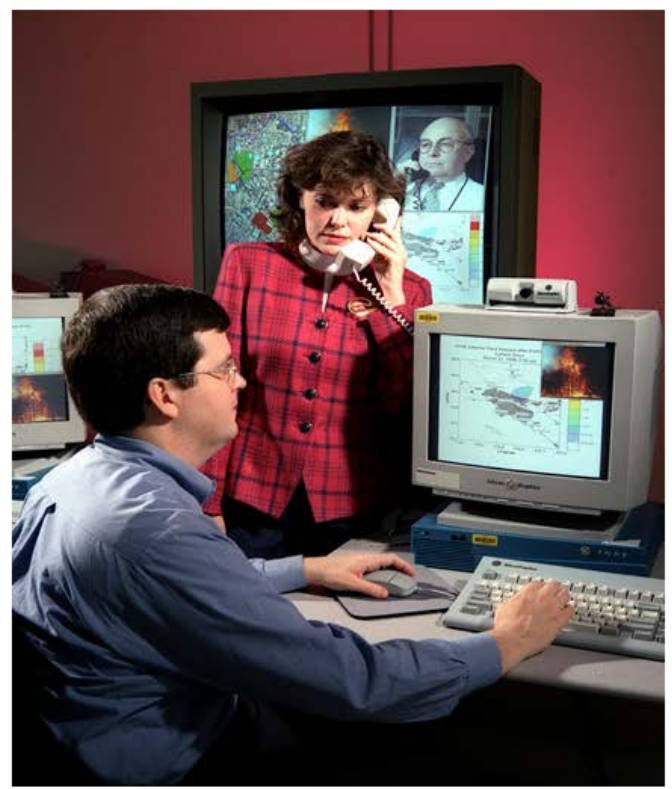




\section{PROCESS \& DEFINITIONS}

\section{Warning issuance is both}

Formal: from the official system

Informal: from friends \& relatives

Formal warnings across communities

Warning point is diverse

Decision process to issue warning varies

Definition of issuance "time" differs

\section{RESEARCH BASED FACTORS THAT REDUCE WARNING ISSUANCE DELAY}




\section{PLANS \& PROCEDURES}

Written Warning Plan

Written Standard Operating Procedures (SOPs)

Warning Thresholds In Place

Succession Planning Detailed in SOPs

Warning Responsibilities Identified

Clearly Defined Authority To Issue Warnings

Interagency Communication Rules/Procedures

Two-Way Communication Among Organizations

Threat Verification Procedures Defined
RANK WEIGHT

HIGH $\quad .20-.25$

HIGH $\quad .25-.30$

HIGH $\quad .15-.20$

LOW $\quad .01-.05$

MEDIUM $.10-.15$

MEDIUM $\quad .10-.15$

HIGH $\quad .11-.17$

LOW . $01-.05$

LOW . .01-.03

\section{PERFORMANCE \& INTER- PERSONAL RELATIONS}

$\begin{array}{lll} & \text { RANK } & \text { WEIGHT } \\ \text { SOP Practice Is Conducted (Drills or Exercises) } & \text { MEDIUM } & .13-.16 \\ \text { Knowledge Of Communicating Personnel } & \text { LOW } & .01-.03 \\ \text { Frequency Of Interaction } & \text { LOW } & .01-.03 \\ \text { Ability To Improvise } & \text { LOW } & .02-.04\end{array}$

\section{SYSTEM PERFORMANCE FACTORS}

Failsafe Communication Mechanisms In Place LOW .06-.07

Redundancies In Communications In Place LOW .06-.07 


\section{SITUATIONAL FACTORS}

Day Or Night

Power Availability (Electricity)

Damage To Infrastructure

Environmental Cues

Time To Impact

Number Of People Involved

Experience of Community

$\begin{array}{ll}\text { RANK } & \text { WEIGHT } \\ \text { MEDIUM } & .10-.13 \\ \text { LOW } & .01-.03 \\ \text { LOW } & .01-.03 \\ \text { LOW } & .01-.03 \\ \text { MEDIUM } & .05-.09 \\ \text { MEDIUM } & .12-.16 \\ \text { LOW } & .02-.04\end{array}$

\section{LINKS TO INTERVIEW SCHEDULE 15 ISSUANCE QUESTIONS (Example: Responsibilities Identified)}

Q 8. Is a particular person or position responsible for getting a first alert or warning out to the public? YES/NO

(IF YES) What is their name and title?

Do they have legal authority to do so? YES/NO

(IF NO) What is the name and title of who does?

Is the responsible person or position written down? YES/NO

(IF YES) Where? 


\section{COMMUNITY CLASSIFICATION (warning issuance delay)}

Formalization Of Planning And Implementation Procedures

Standard Warning Plan Is Written Down

Standard Operating Procedures (SOP) Are Written Down

Warning Thresholds Are In Place (Matrix)

Succession Planning Is Detailed Within SOPS

Responsibilities Are Identified

Clearly Defined Authority To Issue Warnings

Interagency Communication Follows Rules And Procedures

There Is Two-Way Communication Among Organizations

Threat Verification Procedures Are Defined

Performance And Interpersonal Relations

SOP Practice Is Conducted (Drills or Exercises)

Personal Knowledge of Communicating Personnel

Frequency of Interaction

Ability To Improvise

System Performance Factors

Redundancies In Communications Are In Ploce

Situational Factors

Day Or Night

Power Availability (Electricity)

Damage To Infrastructure

Time To Impact

Number Of People Involved

Experience of Community

\author{
OUESTION/RULE \\ 4,6 (if either is yes) \\ 5,7 (if either is yes) \\ 11 (if yes) \\ 9 (if yes) \\ 8 (if yes) \\ $8-2^{\text {nd }}$ sub (if yes) \\ $14-3^{\text {rd }}$ sub (if written down) \\ 15 (if yes) \\ 13-sub (if written down) \\ 21 (if yes) \\ 17 (if yes) \\ 17 (if 4 times/year+) \\ 18 (if yes) \\ 16 (if yes) \\ 10 (if yes) \\ $16-2^{\text {nd }}$ sub (if yes) \\ $16-3^{\text {rd }}$ sub (if yes) \\ 19 (if yes) \\ 20 (if 3 or less) \\ 48 (if yes)
}

\section{WHAT DOES RAPID VS. SLOW ISSUANCE LOOK LIKE?}




\section{RAPID ISSUANCE Boulder, CO (9/2013) Flood}

\section{9:46 p.m. NWS issues life threatening flash flood warning}

9:55 p.m. Boulder OEM sounds outdoor sirens in selected areas stating: "Warning. Flash flood of Boulder Creek is imminent. Leave immediately. Proceed to higher ground. Do not cross Boulder Creek"

10:01 p.m. Warning notice posted on OEM website stating: "Flood sirens activated near Boulder Creek. The City of Boulder has activated flood sirens near Boulder Creek urging anyone near the waterway to seek higher ground immediately. Do not cross or attempt to flee the area in a vehicle. The safest routs is on foot, away from low lying areas. If you are on the south side of the creek, head south along Broadway to the university. If you are on the north side of the creek, head north to Spruce Street of higher"

Note: Multiple additional warning channels/times added to these

\section{SLOW ISSUANCE Graniteville Train Derailment}

\begin{tabular}{|c|c|}
\hline Time & Event \\
\hline 2:39 a.m. & Norfolk Southern Railway train \#192 derailed. \\
\hline 2:42 a.m. & County sheriff and fire personnel on scene at accident \\
\hline $2: 40-2: 50$ a.m. & 12 calls received by Sheriff's office 911 center regarding smoke and bleach smells \\
\hline 2:49 a.m. & $\begin{array}{l}\text { Warrenville Volunteer Fire Department Chief requests dispatch to initiate the Aiken County Reverse } \\
911 \text { Emergency Notification System, with instructions for residents to shelter indoors. }\end{array}$ \\
\hline 3:45 a.m. & South Carolina Emergency Management Division (SCEMD) was notified. \\
\hline 5:00 a.m. & Media, hearing of the wreck on police scanners, began broadcasting news coverage. \\
\hline 5:09 a.m. & $\begin{array}{l}\text { The Emergency Alert System used by the SCEMD to alert media was activated and the message told } \\
\text { residents to evacuate. }\end{array}$ \\
\hline 6:01 a.m. & $\begin{array}{l}\text { The National Weather Service weather band radio system was activated; radios emit a loud noise } \\
\text { that could wake sleeping residents. }\end{array}$ \\
\hline 6:27 a.m. & $\begin{array}{l}\text { Reverse } 911 \text { was used to notify } 3,600 \text { homes with a shelter-in-place message. The shelter-in-place } \\
\text { order was also given by local officials. Residents were told to turn off their air conditioning and } \\
\text { heating systems. }\end{array}$ \\
\hline 2:30 p.m. & $\begin{array}{l}\text { All residents within one mile of the crash site were asked to evacuate by } 6: 00 \text { p.m. A two-mile radius } \\
\text { curfew was in effect from 6:00 p.m. to 7:00 a.m. }\end{array}$ \\
\hline 4:00 - 4:30 p.m. & An evacuation message was delivered via reverse 911. \\
\hline
\end{tabular}




\title{
HISTORICAL ISSUANCE TIMES
}

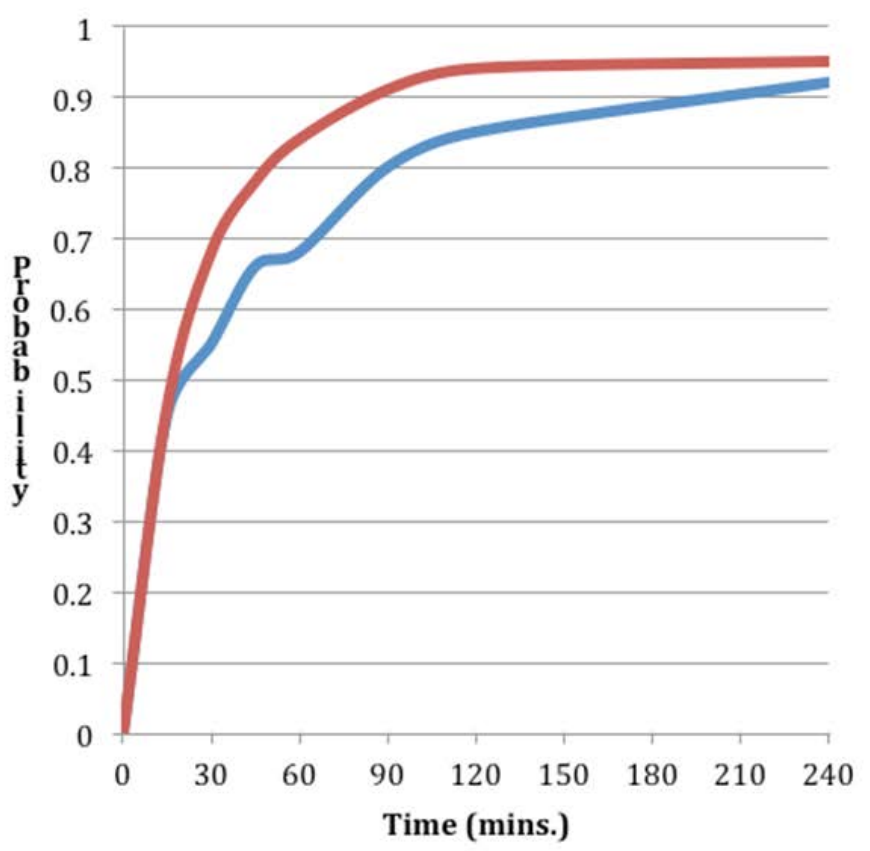

Probability of Warning Issuance 1994

Probability of Warning 1990

MODELING WARNING ISSUANCE

\author{
$P_{t}=1-\exp \left(-a t^{b}\right)$ \\ $\mathrm{t}=$ time \\ $\mathrm{a}$ and $\mathrm{b}$ are constants
}

fit simulated curves to empirically derived curves 


\section{COMPARISON}

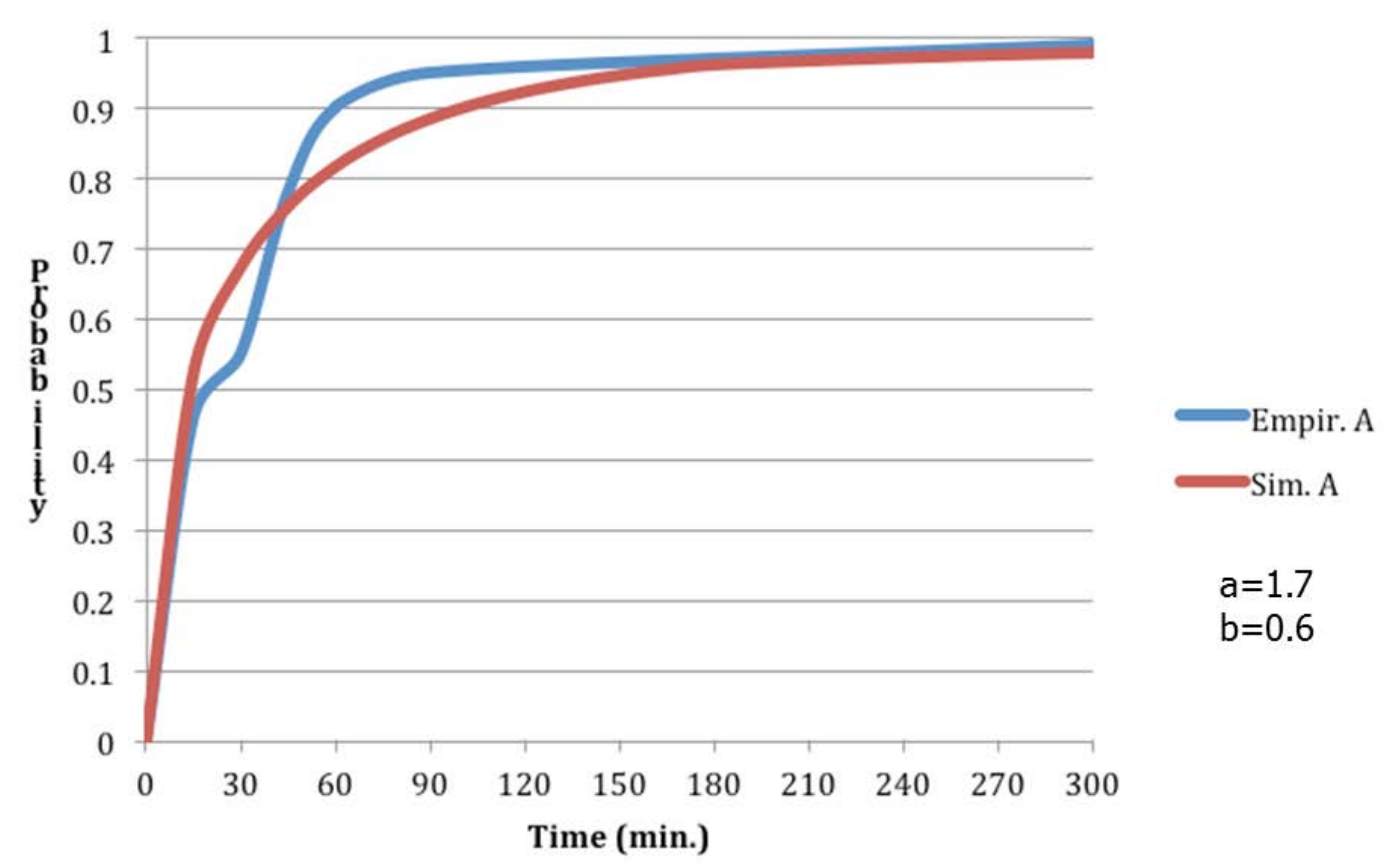

\section{ISSUANCE PLANNING CURVES}

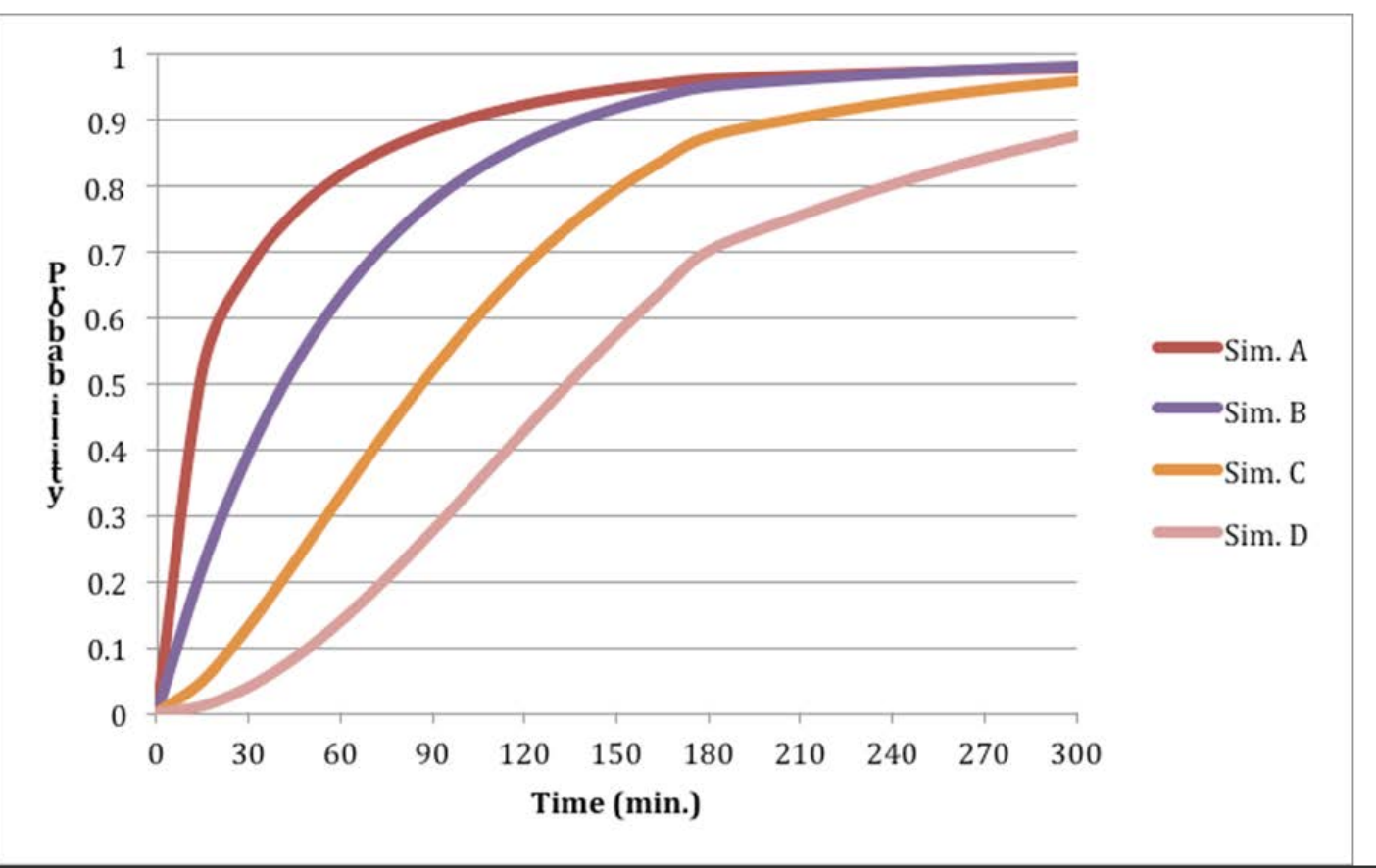




\section{MORE DETAILS IN}

Sorensen, J., and D. Mileti (2014a). First Alert and/or Warning Issuance Time Estimation for Dam Breaches, Controlled Dam Releases, and Levee Breaches of Overtopping. Davis, CA: U.S. Army Corps of Engineers, Institute for Water Resources, Risk Management Center.

\section{PART 3 \\ WARNING DIFFUSION DELAY}
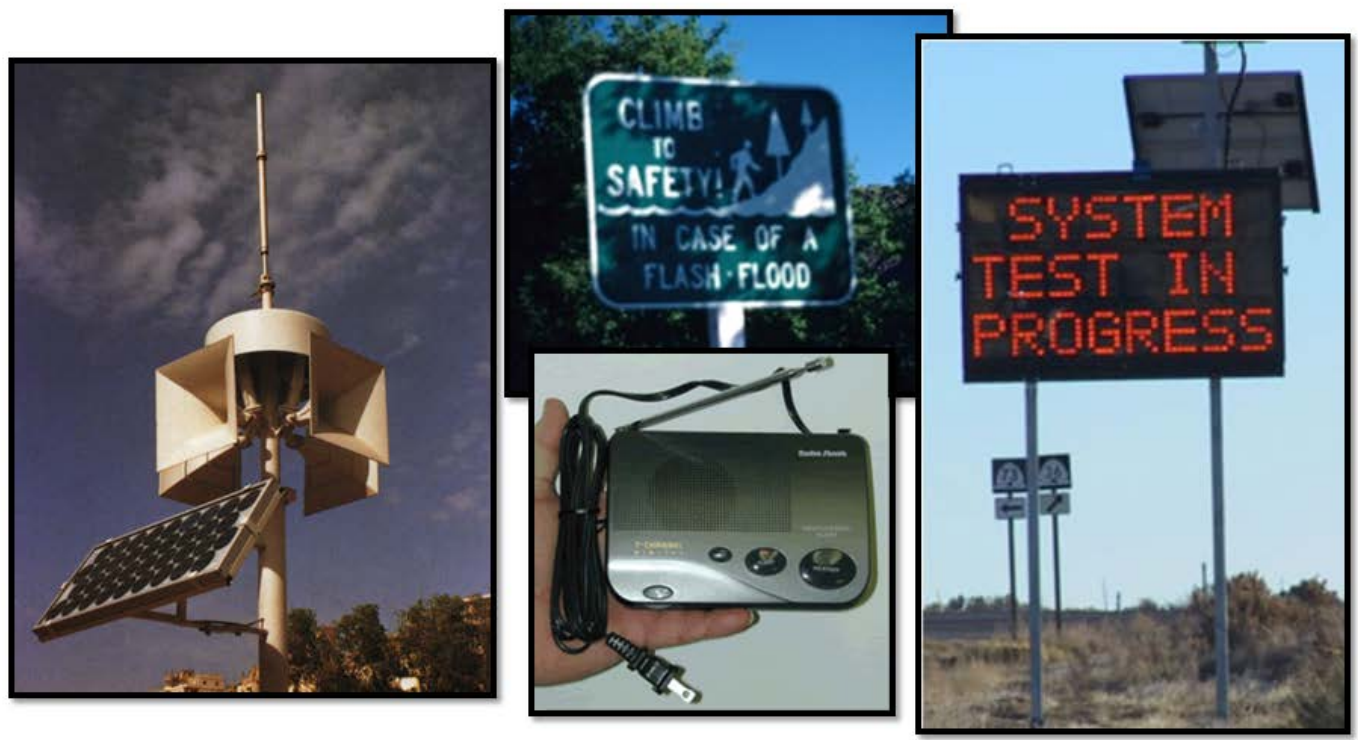


\section{PROCESS \& DEFINITIONS}

Direct warning (broadcast)

Informal warning (contagion)

Alert vs. notification

First vs. multiple warnings

Targeted vs. non-targeted warnings

\section{RESEARCH BASED FACTORS THAT INFLUENCE DIFFUSION TIME}




\section{SENDING THE $1^{\text {st }}$ ALERT/WARNING}

Channels: Types Of Technologies

Channels: Disruption To Infrastructure

Channels: Number \& Mix Of Channels

Frequency Of Distribution

Informal Notification

Environmental and Social Cues
RANK WEIGHT

HIGH $\quad .17-.20$

LOW . .00 - .05

HIGH $\quad .25-.30$

HIGH . .21 - .26

MOD. $\quad .10-.13$

LOW . .01-.05

\section{RECEIVING THE $1^{\text {st }}$ ALERT/WARNING}

Activity-Task

Activity - Location And Proximity To The Hazard

Activity - Time Of Day

Impediments - Sensory (Hearing, Visual)

Impediments - Cognitive

Impediments - Linguistic And Cultural

Resources - Access To Technology

Social Media Participation

Socio-Economic Status
RANK WEIGHT

MODERATE $.10-.16$

MODERATE $.12-.18$

HIGH .05 - .25*

MODERATE $.08-.13$

LOW $\quad .01-.02$

LOW $\quad .03-.06$

MODERATE $\quad .05-.12$

LOW .01-.02

MODERATE $\quad .07-.12$

*NOTE: Weights should not to be used in assigning curves since separate curves exist. 


\section{LINKS TO INTERVIEW SCHEDULE 14 DIFFUSION QUESTIONS (Example: Message Consistency)}

Q 28. Do you have ways to monitor what others might be telling the public to find rumors and incorrect information? YES/NO

(IF YES) How would that be done?

If rumors or incorrect information were detected, would you issue subsequent messages to correct for misinformation? YES/NO

(IF YES) How would it be done?

\section{COMMUNITY CLASSIFICATION (Warning Diffusion Delay)}

\section{Sending The First Alert/Warning}

Channels - Types Of Technologies

Channels - Disruption To Infrastructure

Channels - Number And Mix Of Channels

Frequency Of Distribution

Informal Notification

\section{Receiving The First Alert/Warning}

Activity -Task

Activity - Location \& Proximity To The Hazard

Activity - Time Of Day

Impediments - Sensory (Hearing, Visual)

Age

Impediments - Linguistic And Cultural

Resources - Access To Technology

Social Media Participation

Socio-Economic Status

\section{QUESTION/RULE}

2 (if 2 or more of a certain type)

35 (if yes)

22 (if 5 or more)

25 (if yes)

27 (if yes)

29 (if yes - any special warning)

30 (if yes)

31 (if yes)*

32,33 (if yes to either)

52 (if yes)

34 (if yes)

24 (if any of special type)

24 (if $50 \%$ or more)

$53,54,55,57$ (if none are yes)

*NOTE: Weights should not to be used in assigning curves since separate curves exist. 


\section{WHAT DOES RAPID VS. SLOW DIFFUSION LOOK LIKE?}

\section{RAPID DIFFUSION Boulder (9/2013) CO Flood}

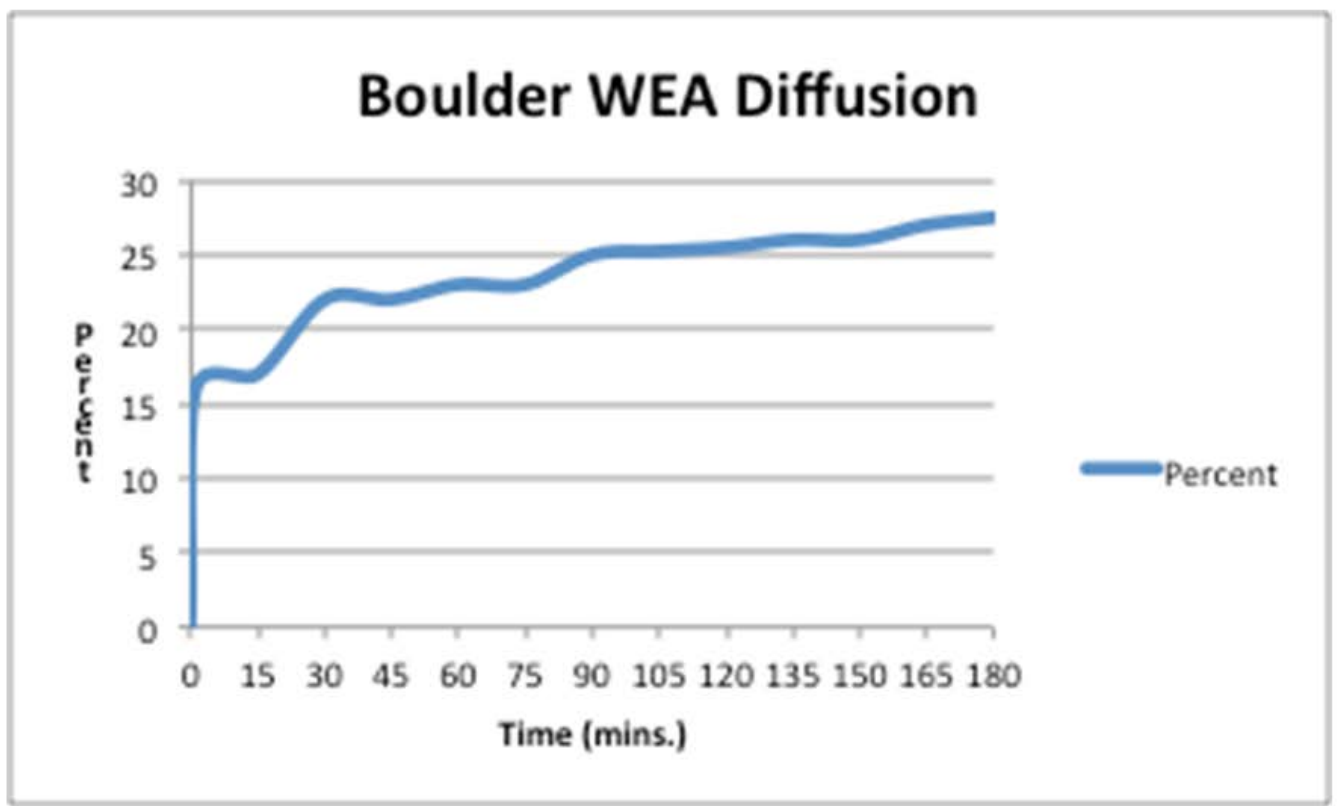




\section{SLOW DIFFUSION: Nanticoke, PA}

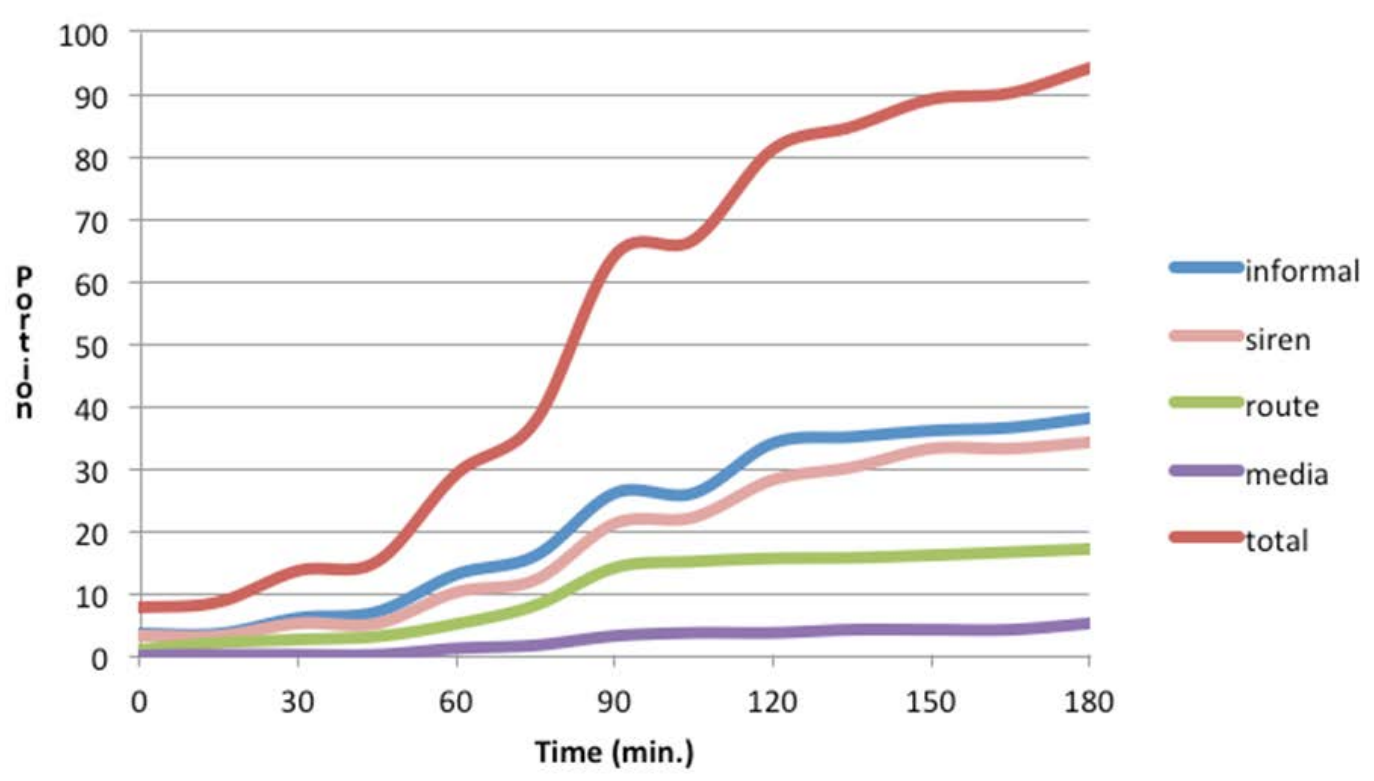

\section{HISTORIC DIFFUSION DATA}

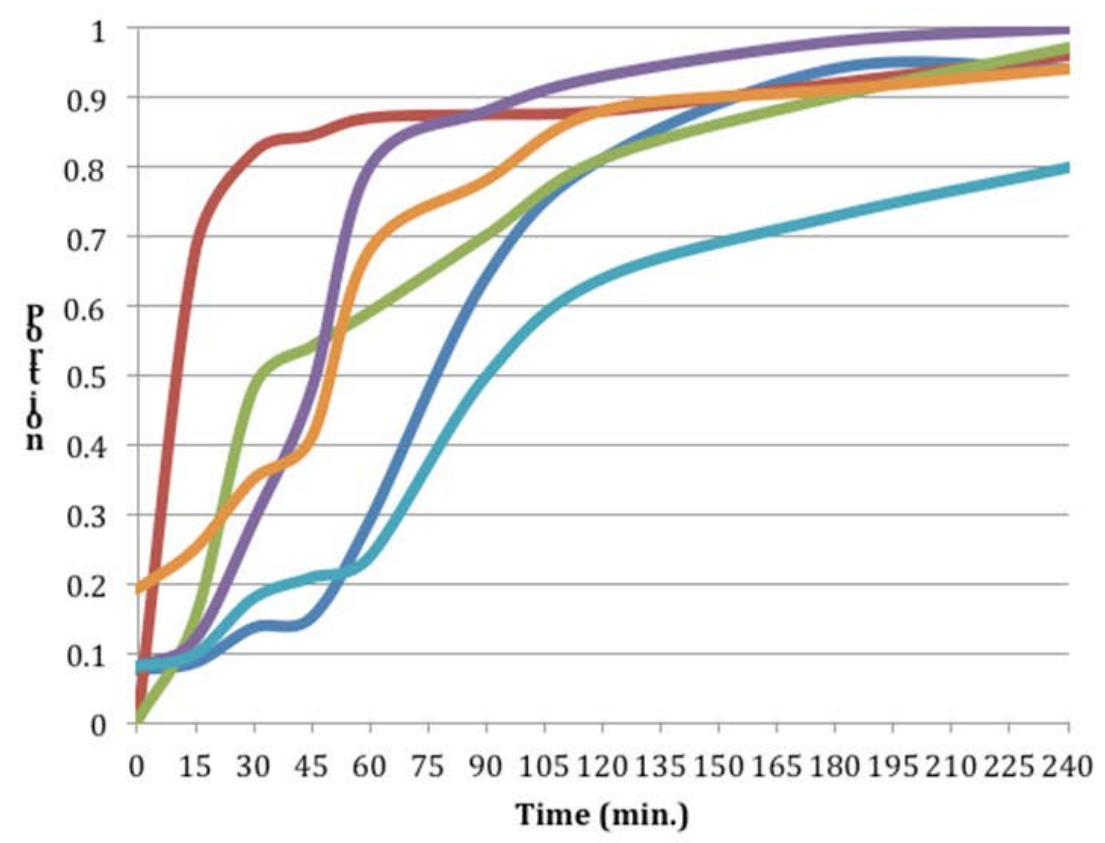

Nanticoke

Toutle

Woodland

Helena

Pittsburg

Confluence 


\section{MODELING WARNING DIFFUSION}

\section{$\Delta W / \Delta t=P U t^{*}\left(B t+C t-B t^{*} C t\right)$}

Where:

$\mathrm{W}=$ Alerted population

$\mathrm{t}=$ time

$\Delta \mathrm{W} \Delta t=$ the rate of population being alerted per time step $P U t=$ The population unwarned for time step $\mathrm{t}$

$B t=$ the effectiveness of the broadcast systems in time step $t$ $C t=$ the effectiveness of the indirect warning in time step $t$

\section{COMPARING SIMULATED AND EMPIRICAL}

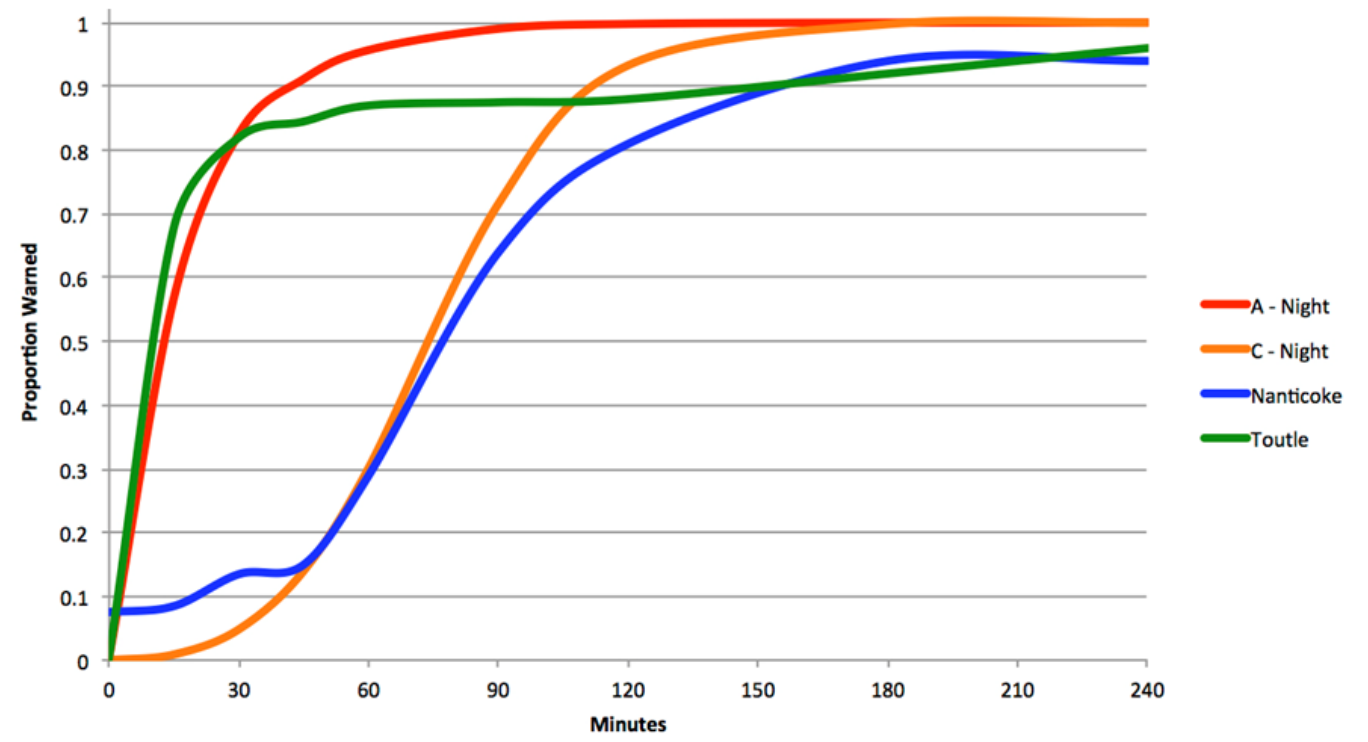




\section{DIFFUSION CURVES}
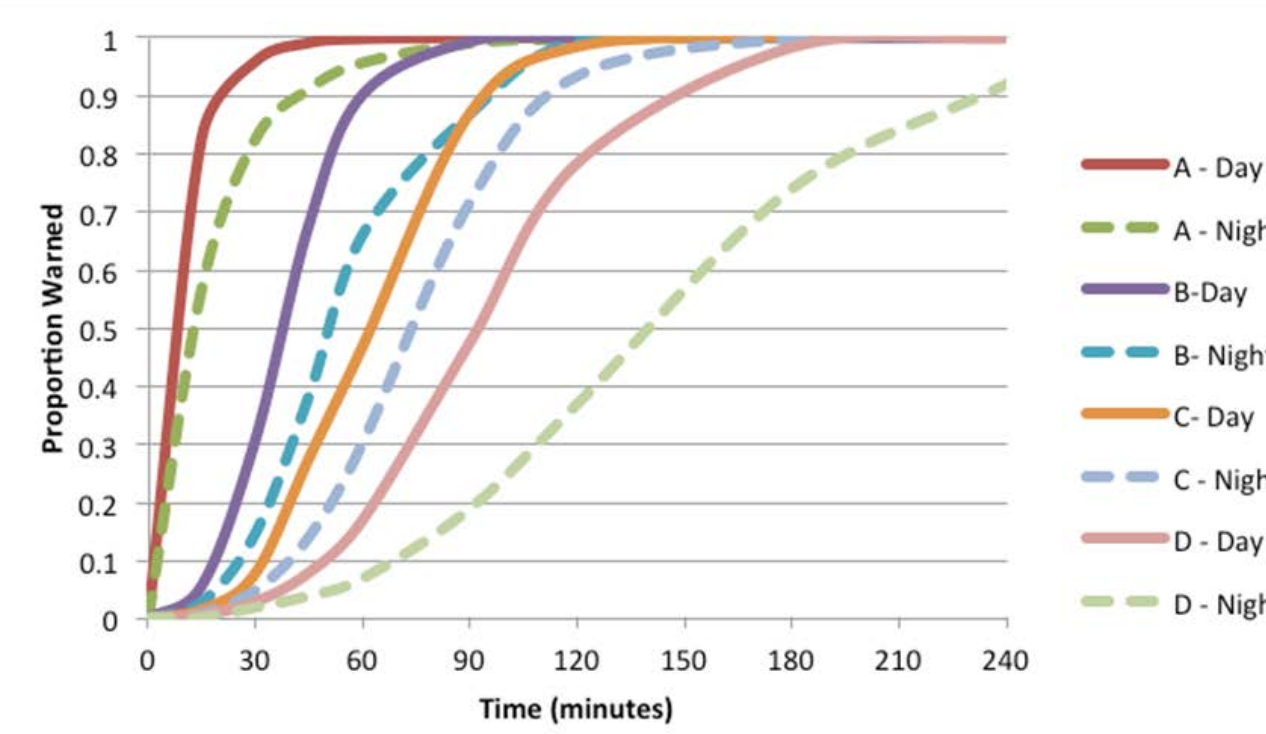

- A - Night

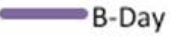

- B- Night

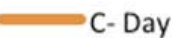

- C - Night

D - Day

- - D - Night

\section{SUGGESTED Bt VALUES FOR WARNING TECHNOLOGIES}

\begin{tabular}{|l|l|}
\hline Route alerting & $60-120$ \\
\hline $\begin{array}{l}\text { Loudspeakers and } \\
\text { PA systems }\end{array}$ & $5-10$ \\
\hline $\begin{array}{l}\text { Wireless Emergency } \\
\text { Alerts (WEA) }\end{array}$ & $5-15$ \\
\hline $\begin{array}{l}\text { Wireless } \\
\text { communications }\end{array}$ & $3-20$ \\
\hline Radio & $40-90$ \\
\hline Television & $40-90$ \\
\hline Newspaper & $90-180$ \\
\hline $\begin{array}{l}\text { Dedicated tone alert } \\
\text { radios }\end{array}$ & $5-10$ \\
\hline $\begin{array}{l}\text { Telephone } \\
\text { automatic dialers }\end{array}$ & $10-20$ \\
\hline
\end{tabular}

\begin{tabular}{|l|l|}
\hline $\begin{array}{l}\text { NOAA Weather } \\
\text { Radio }\end{array}$ & $5-15$ \\
\hline Audio sirens/alarms & $5-10$ \\
\hline Broadcast sirens & $5-10$ \\
\hline Message signs & $2-5$ \\
\hline Aircraft & Unknown \\
\hline Visual alerting & $5-10$ \\
\hline $\begin{array}{l}\text { Internet protocol } \\
\text { (IP) based } \\
\text { technology }\end{array}$ & $10-30$ \\
\hline Social media & Unknown \\
\hline & \\
\hline
\end{tabular}




\section{DIFFUSION FOR TWO SPECIFIC TECHNOLOGIES (Bt=10; 40)}

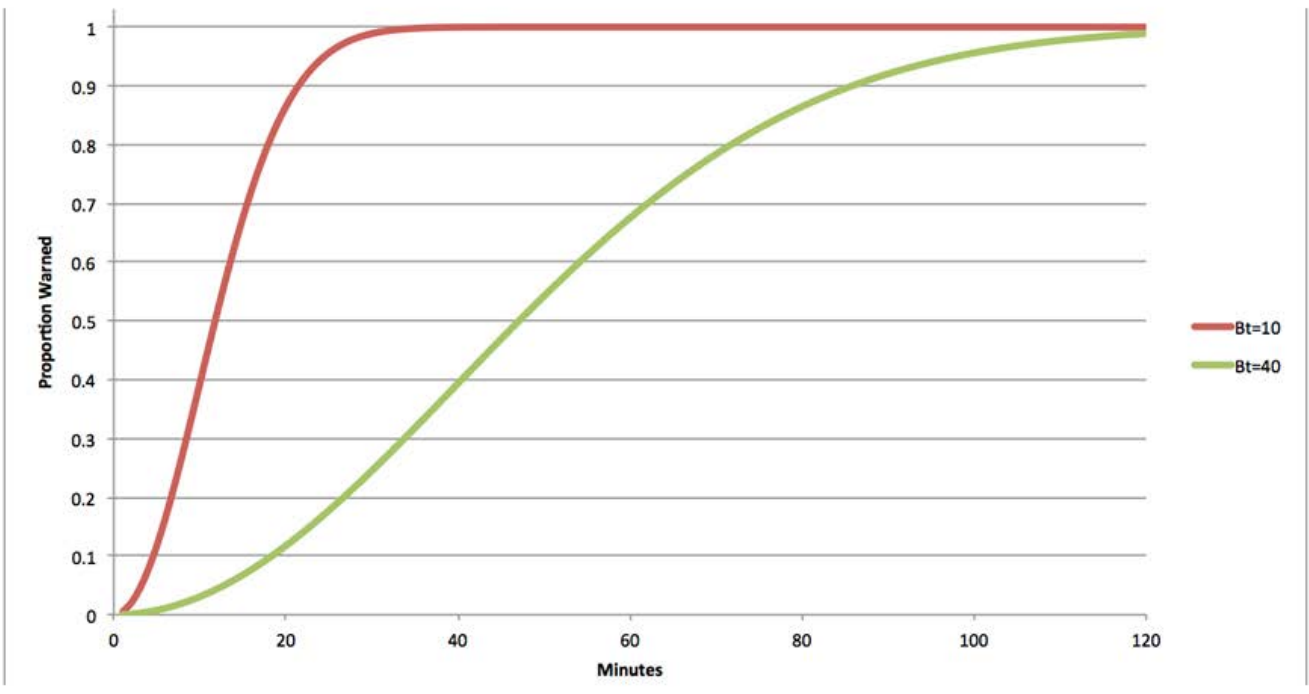

\section{MORE DETAILS IN}

Sorensen, J., and D. Mileti (2014b). First Alert and Warning Diffusion Time Estimation for Dam Breaches, Controlled Dam Releases, and Levee Breaches of Overtopping. Davis, CA: U.S. Army Corps of Engineers, Institute for Water Resources, Risk Management Center. 


\section{PART 4 PAI DELAY}

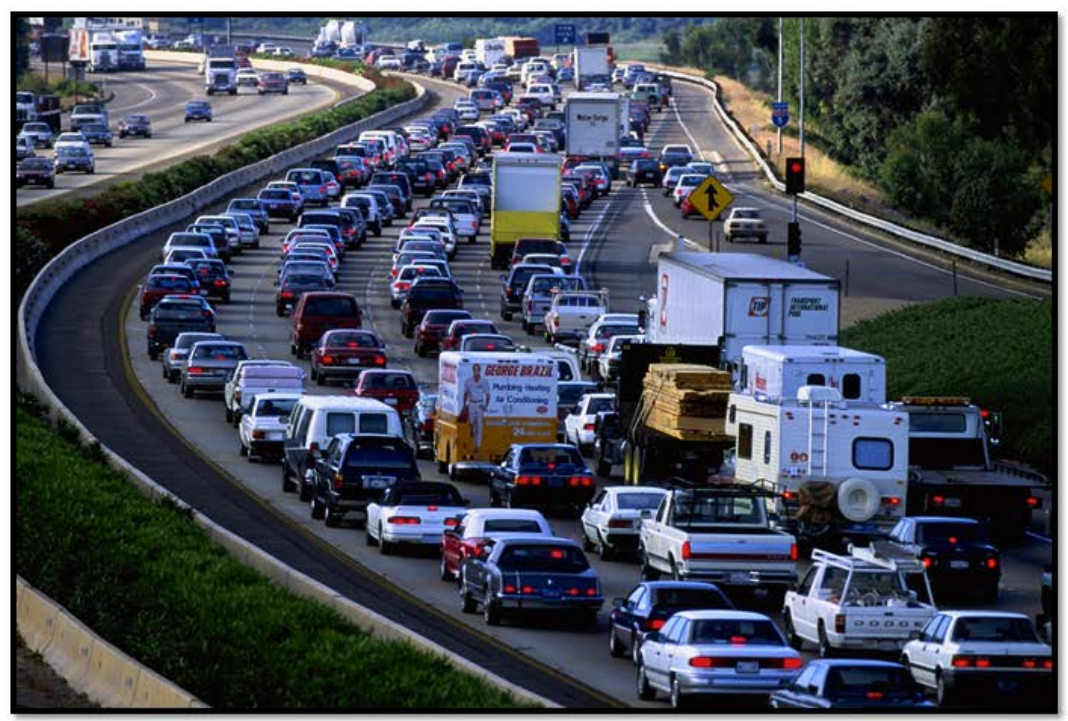

\section{PROCESS \& DEFINITIONS}

\section{Choice of actions}

When does initiation begin

Area warned versus at risk

What is compliance? 


\section{RESEARCH BASED FACTORS \\ THAT \\ INFLUENCE PAI \\ TIME}

\section{MESSAGE \\ CHARACTERISTICS}

\section{Appropriate Content}

Style

Message Length Adequacy

Personal Channel

Delivery (Frequency)

Protective Action Type
RANK

HIGH

HIGH

MODERATE

HIGH

HIGH

MODERATE
WEIGHT

$.25-.30$

$.17-.22$

$.12-.16$

$.13-.18$

$.12-.15$

$.05-.10 *$

* NOTE: Not to be used in estimating vertical evacuation since separate curves exist 


\section{RECEIVER \\ CHARACTERISTICS}

Status Attributes

Role Characteristics

Personal Preparedness

Pre Event Knowledge

Experience

Member Isolated Group
RANK

MODERATE

HIGH

LOW

LOW

MODERATE

MODERATE
WEIGHT

$.01-.10$

$.10-.17$

$.10-.02$

$.01-.02$

$.01-.14$

$.01-.11$

\section{CONTEXT \\ CHARACTERISTICS}

Environmental Cues

Social Cues

Location/Activity

Day Versus Night

Time To Impact

Impact Intensity
RANK

HIGH

MODERATE

MODERATE

LOW

HIGH

HIGH
WEIGHT

$.05-.28$

$.05-.13$

$.05-.15$

$.01-.05$

$.10-.17$

$.10-.17$ 


\section{LINKS TO INTERVIEW SCHEDULE 11 PAI QUESTIONS (Example: Guidance \& Consequence Reduction)}

Q 39. Would you recommend in alerts, warnings and press releases that the public take specific protective actions, for example, evacuate, move vertically, or check local media? YES/NO

Q 40. Would the alerts, warnings and emergency press releases you issue tell the public about any of the following?

The consequences of the flood and why taking the recommended protective action(s) would reduce them? YES/NO

(IF YES) What would alerts and warnings say?

What would press releases say?

\section{COMMUNITY CLASSIFICATION}

\section{(Protective Action Initiation Delay)}

\section{Message Characteristics}

Appropriate Content

Style

Message Length Adequacy

Personal Channel

\section{Receiver Characteristics}

Status Attributes

Role Characteristics

Personal Preparedness/Planning

Pre Event Knowledge

Experience

Membership In A Socially Isolated Group

\section{Context Characteristics}

Environmental Cues

Location/Activity

Day Versus Night

Time To Impact

\section{QUESTION/RULE}

$38,39,40 a, 40 b, 40 c$ (if yes to $39 \& 2$ others)

$41 a, 41 b, 44,28$ (if yes to 2 or more)

$38,39,40 \mathrm{a}, 40 \mathrm{~b}, 40 \mathrm{c}, 40 \mathrm{~d}, 40 \mathrm{e}, 49 \mathrm{f}, 40 \mathrm{~g}$ (if yes to $5+$ )

22 (if yes to any of a special type)

$53,54,55,57$ (if none is yes)

$40 \mathrm{e}, 40 \mathrm{f}$ (if yes to either)

46 (if yes)

46 (if yes)

48 (if yes)

58 (if yes)

$40 \mathrm{~g}$ (if yes)

29 (if yes to 1 or more)

31 (if yes)

43 (if yes) 


\section{RAPID (Confluence, PA) AND SLOW (Pittsburgh, PA) PAI}

(Hazardous Materials Train Derailment Events)
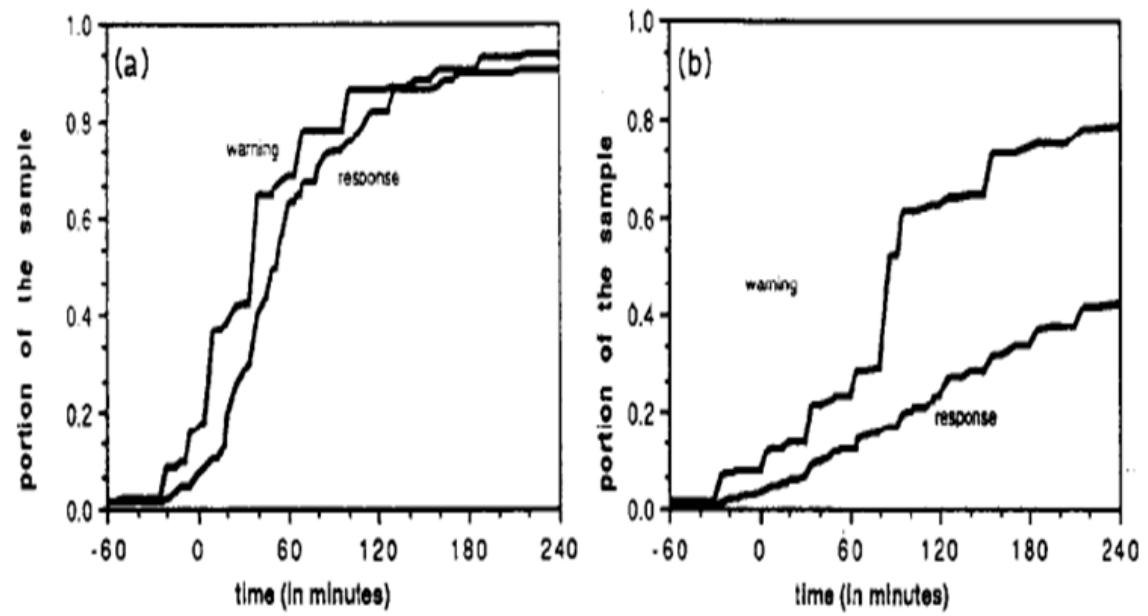

Fig. 3. The timing and warning response for (a) the Confluence and (b) the Pittsburgh event.

\section{DIFFERENCE}

\section{Definition of area of risk}

\section{Time of day}

Day of the week

Method of warning receipt

Urgency of message 


\section{HISTORIC PAI DATA}

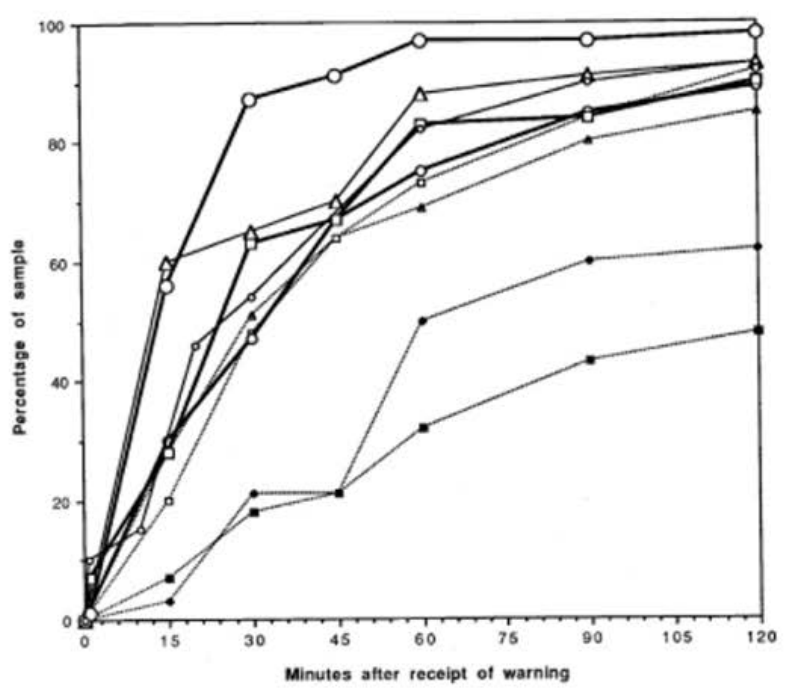

ORNL-DWG 90M-5852

$\triangle-$ Mississauga-15t

- Valley Flood

- Fillmore Flood

- Thequimio Flood

- Missisgauga-Al

$\rightarrow-$ Pittsburgh

$-\infty$ Confluence

$\longrightarrow$ Nanticoke

Fig, 5.2 Mobilization time in selected events. "First wave of evacuation for Mississauga accident designated (1st), all Mississauga evacuations designated (all).

\section{EVACUATION COMPLIANCE (\% warned)}

Table 6. Estimated compliance rates for dam breaches and levee flooding. (Based on Lindell and Prater, 2007 and Cutter et. al., 2011)

\begin{tabular}{|l|c|c|c|}
\hline \multirow{2}{*}{ Zone } & \multicolumn{3}{|c|}{ Event Intensity } \\
\cline { 2 - 4 } & Minor & Moderate & Major \\
\hline $\begin{array}{l}\text { Flood Area: Short } \\
\text { Response Time }\end{array}$ & $51-59$ & $81-89$ & $94-100$ \\
\hline $\begin{array}{l}\text { Flood Area: Long } \\
\text { Response Time }\end{array}$ & $28-34$ & $70-76$ & $83-89$ \\
\hline Adjacent Zones & $10-16$ & $32-38$ & $65-71$ \\
\hline
\end{tabular}




\section{MODELING PROTECTIVE ACTION INITIATION}

$P_{t}=1-e^{\left.\left[-\left(t^{\wedge} 2\right) / a b^{\wedge} 2\right)\right]}$

$\mathrm{t}$ is time

$\mathrm{a}$ and $\mathrm{b}$ constants

a: acceleration

b: overall timing (midpoint)

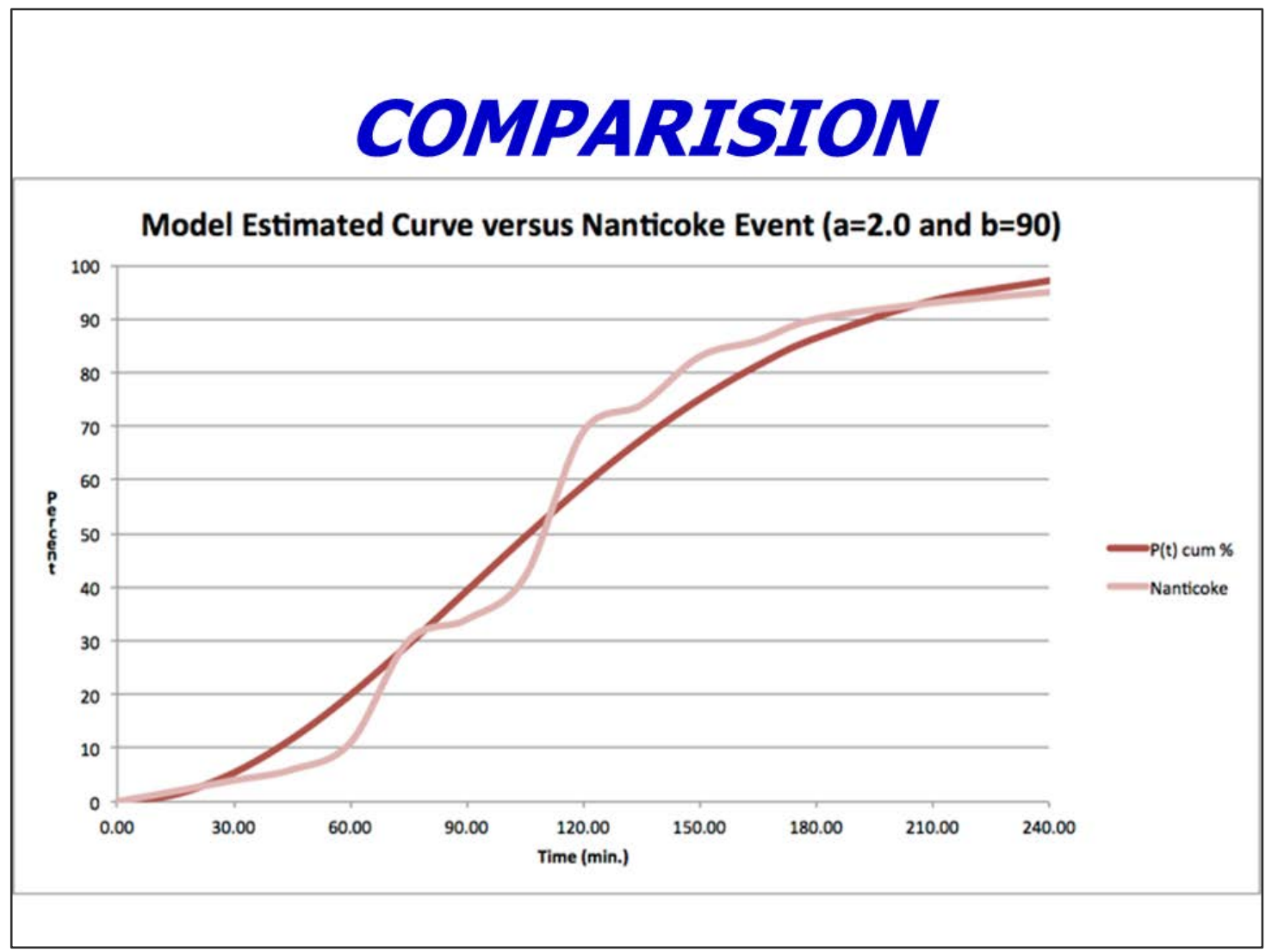




\section{PAI PLANNING CURVES}

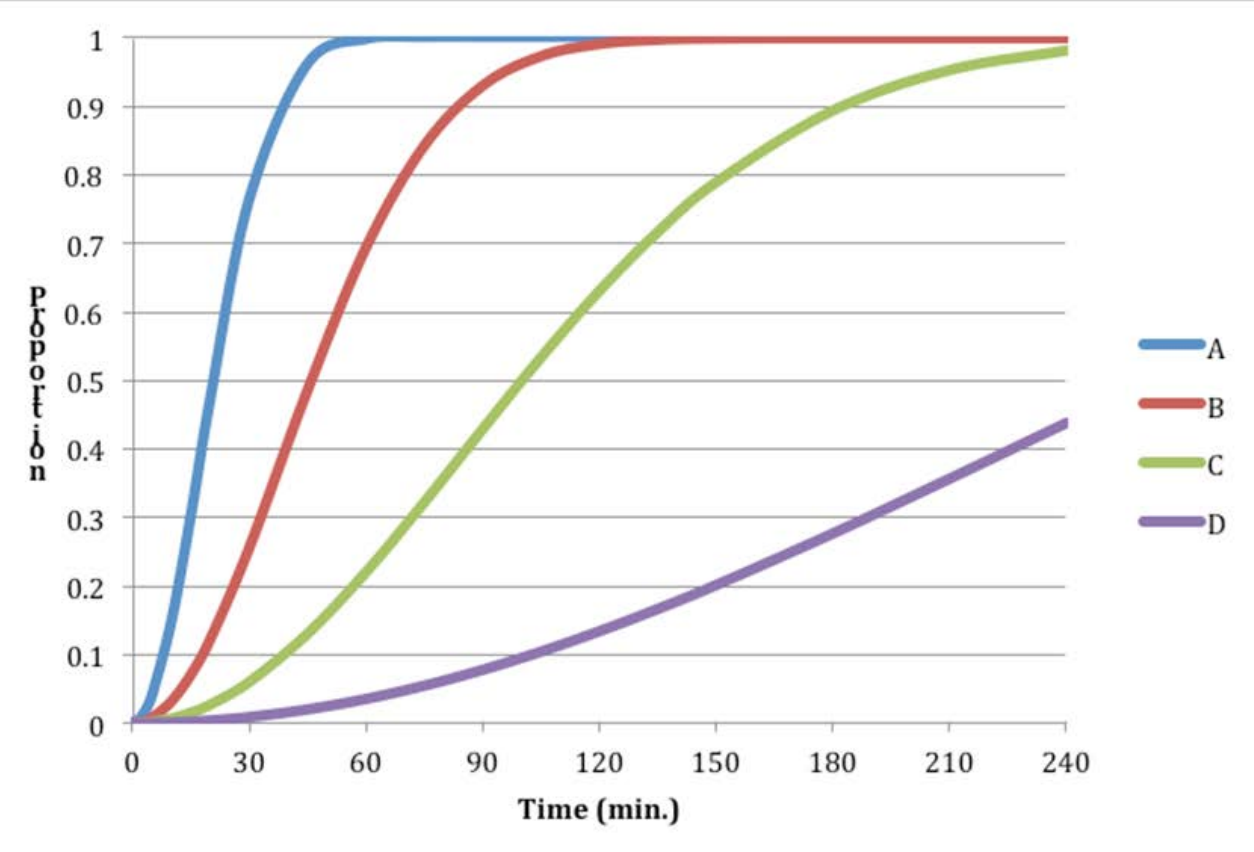

\section{PAI PLANNING CURVES}

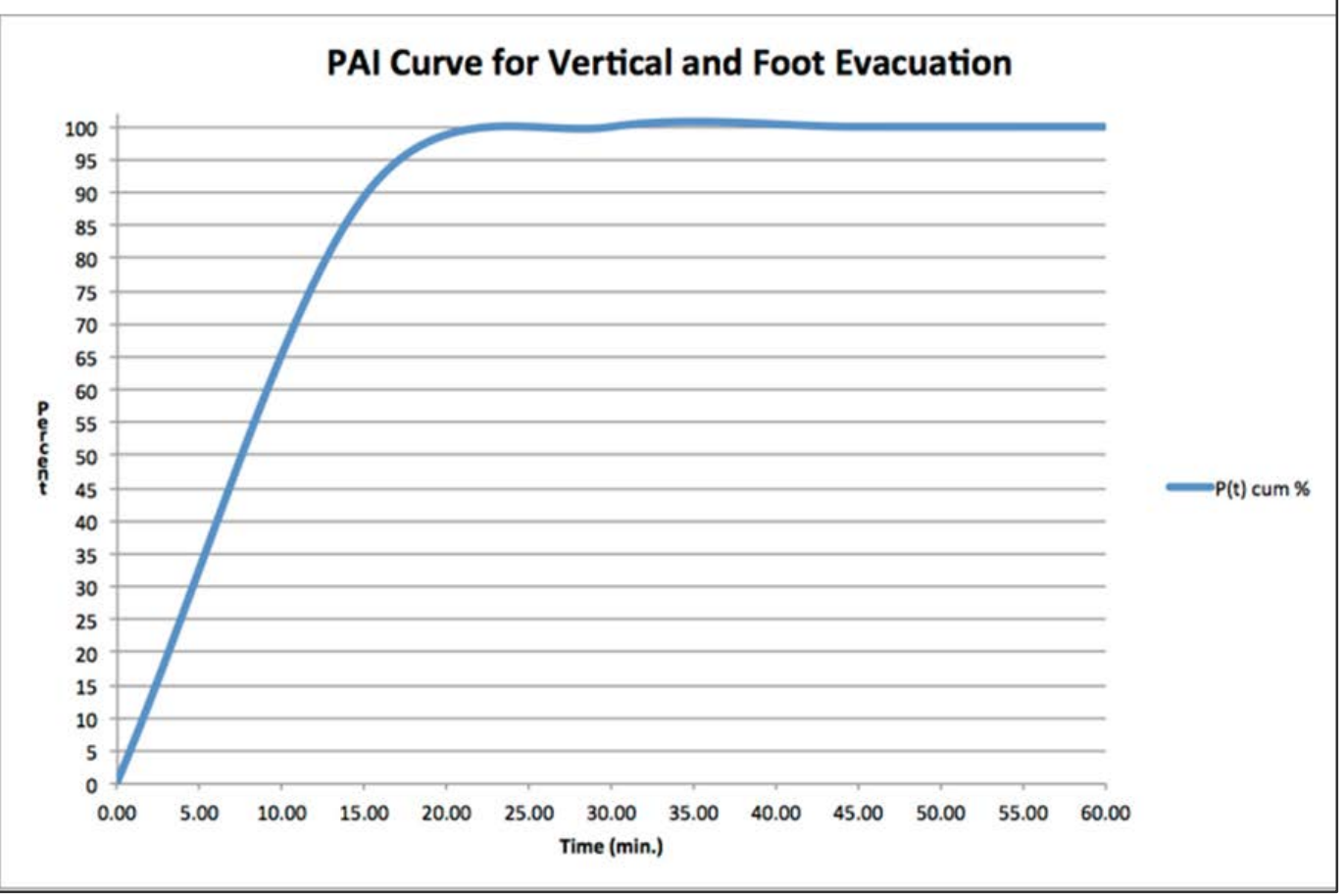




\section{PAI PLANNING CURVES}

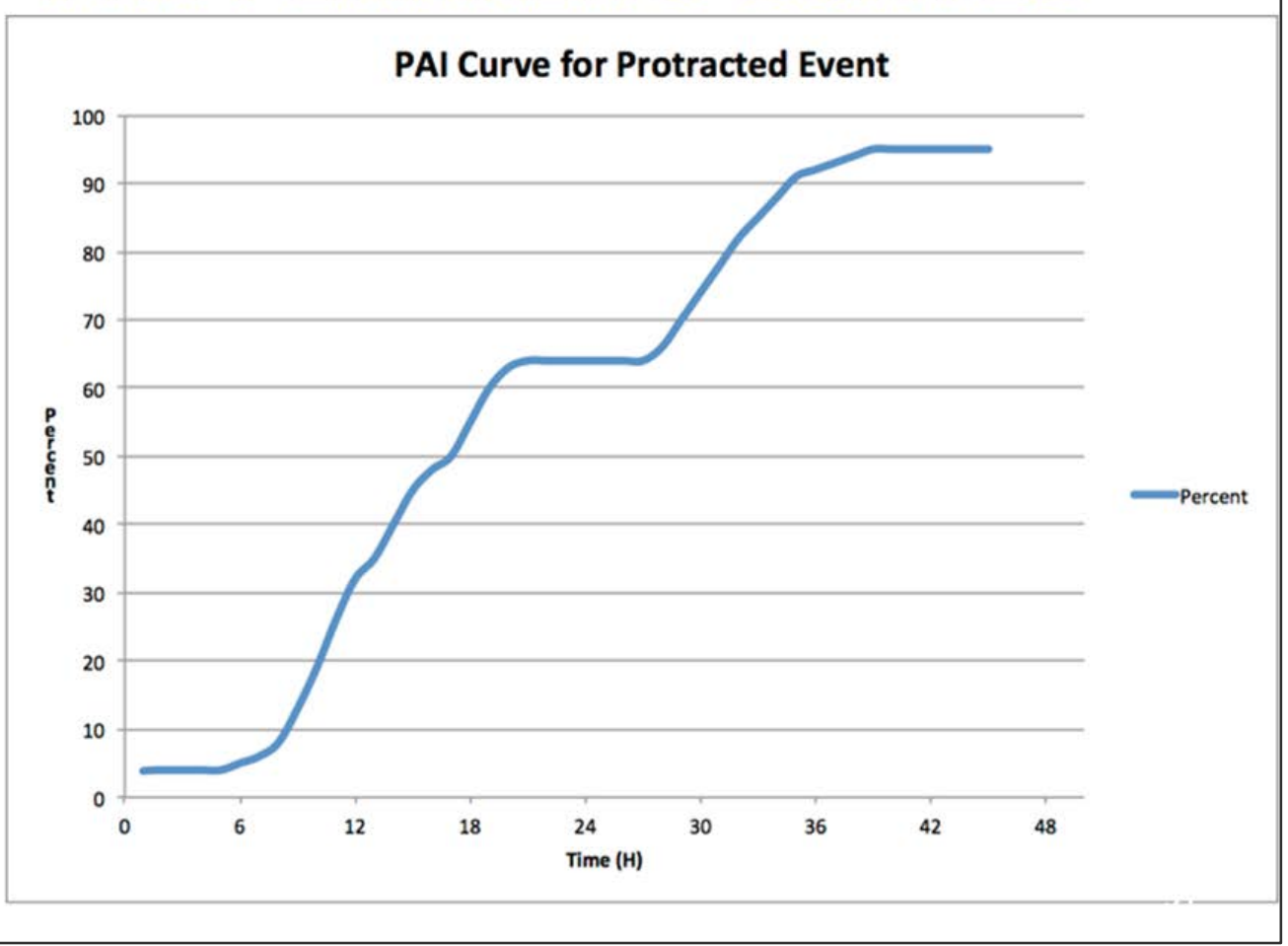

\section{MORE DETAILS IN}

Sorensen, J., and D. Mileti (2014c). Protective Action Initiation Time Estimation for Dam Breaches, Controlled Dam Releases, and Levee Breaches of Overtopping. Davis, CA: U.S. Army Corps of Engineers, Institute for Water Resources, Risk Management Center. 


\section{BRINGING IT TOGETHER FOR LIFE LOSS ESTIMATION}

\section{RESEARCH FINDINGS, CURVES INTERVIEWS, LIFE LOSS ESTIMATES}

58 interview questions

15 Issuance questions

14 Diffusion question

11 Protective action initiation questions

18 Other questions (local threat type \& more)

USACE will use the rules we developed to combine question answers to assign issuance, diffusion and PAI curves to communities to estimate future life loss 


\section{MORE DETIALS IN}

Mileti, D., and J. Sorensen. (2015d). Interview Schedule: Community Warning Issuance, Diffusion, and Protective Action Initiation Estimation. Davis, CA: U.S. Army Corps of Engineers, Institute for Water Resources, Risk Management Center.

Sorensen, J., and D. Mileti (2015e). Influence Weights and Measures for the Factors Shaping First Alert/Warning Delay, Diffusion and Protective Action Initiation Curves for Dam Breaches, Controlled Dam Releases, and Levee Breaches or Overtopping. Davis, CA: U.S. Army Corps of Engineers, Institute for Water Resources, Risk Management Center.

\section{PART 5 COMMUNITY PLANNING GUIDEBOOK}

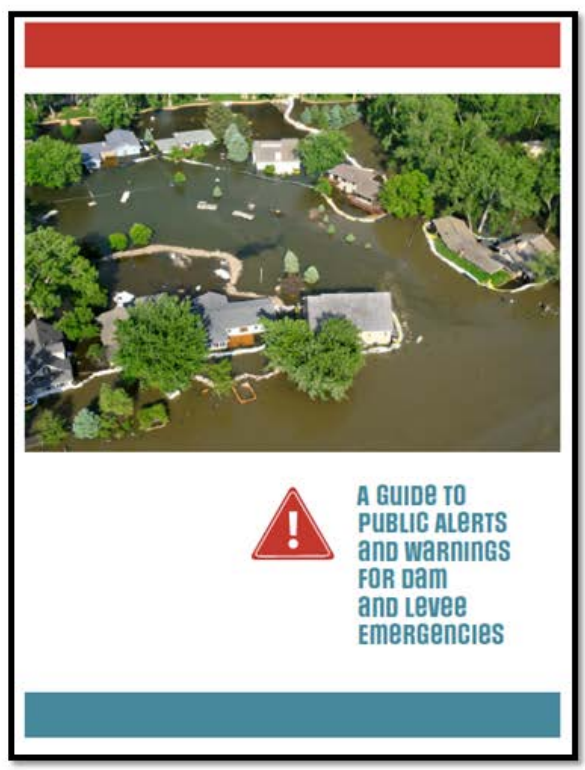




\section{GUIDEBOOK PURPOSE}

Prepare a guidebook based on synthesized empirical social science quantitative evidence

Provide emergency managers with practical ways to enhance future public alert \& warning practices that could

Reduce issuance time delay

Minimize diffusion time

Accelerate protective action initiation

\section{GUIDEBOOK TOOLS}

Catalogue and define protective actions

Protective actions based on time \& area

Threat vs. actions and warning content planning matrices

Ranked community planning steps for upgrading issuance, diffusion, and PAI

Anatomy of warning messages, examples, and templates

Catalogue of available warning dissemination channels \& their merits and shortcomings 


\section{TABLE 1 - PROTECTIVE ACTIONS LIST}

\section{PUBLIC PROTECTIVE ACTION DEFINITION}

\begin{tabular}{|l|l|}
\hline Evacuate - Vehicle & Driving away from area at risk \\
\hline Evacuate - Pedestrian & $\begin{array}{l}\text { Moving away from the threat by walking, running or } \\
\text { dimbing to higher ground }\end{array}$ \\
\hline Evacuate - Vertical & Moving to a higher floor in a structure \\
\hline Evacuate - Safer Structure & Moving to a nearby structure offering more protection \\
\hline Avoid Area & Not entering an area of potential threat \\
\hline Expedient* Protection of People & Grabbing hold of a sturdy or floating item \\
\hline Expedient* Protection of Structures & $\begin{array}{l}\text { Improving water resistance or strength of structure (short } \\
\text { term - sandbag, seal door frame, etc.) }\end{array}$ \\
\hline Expedient* Protection of Possessions & Moving valuables and pets to a safer location \\
\hline Seek or Monitor Information & $\begin{array}{l}\text { Seeking more information about the event and recom- } \\
\text { mended protective actions }\end{array}$ \\
\hline Prepare To Evacuate & $\begin{array}{l}\text { Packing evacuation kit and supplies, reviewing household } \\
\text { plan, securing valuables, contacting others }\end{array}$ \\
\hline Continue Normal Activities & Not changing daily behavior \\
\hline
\end{tabular}

"Expedient refers to actions that require no planning or preparation and are implemented with those resources at hand. Note: The protective actions in this table are not necessarily mutually exclusive.

\section{TABLE 2 - PROTECTIVE ACTION BASED ON THREAT AREA AND RESPONSE TIME}

\begin{tabular}{|c|c|c|c|}
\hline \multirow{2}{*}{ PROTECTIVE ACTION } & \multicolumn{2}{|c|}{ FLOOD AREA } & \multirow{2}{*}{ ADJACENT AREA } \\
\hline & SHopt & LoNG & \\
\hline Evacuate -Vehicle & 0 & 口 & \\
\hline Evacuate - Pedestrian & ] & & \\
\hline Evacuate-Vertical & 0 & 0 & \\
\hline Evacuate - Safer Structure & 0 & प & \\
\hline Avold Area & 0 & प & 0 \\
\hline Expedient Protection of People & 0 & & \\
\hline Expedient Protection of Structures & & 0 & \\
\hline Expedient Protection of Possessions & & 0 & \\
\hline Seek or Monitor Information & & 0 & 口 \\
\hline Prepare To Evacuate & & 0 & \\
\hline Continue Normal Activities & & & 口 \\
\hline
\end{tabular}




\section{TABLE 3 - THREAT VS. PUBLIC ACTIONS FOR DAM BREACHES}

\begin{tabular}{|c|c|c|c|}
\hline $\begin{array}{l}\text { PHYSICAL } \\
\text { OBSERVATIONS* }\end{array}$ & $\begin{array}{l}\text { THREAT LEVEL } \\
\text { DESIGNATION }\end{array}$ & $\begin{array}{l}\text { FLOOD } \\
\text { THREAT }\end{array}$ & $\begin{array}{l}\text { PROTECTIVE } \\
\text { ACTION } \\
\text { OPTIONS }\end{array}$ \\
\hline $\begin{array}{l}\text { Water flowing } \\
\text { through breach in } \\
\text { embankment }\end{array}$ & $\begin{array}{l}\text { LEVEL IV } \\
\text { Dam breaching or } \\
\text { breached }\end{array}$ & $\begin{array}{l}\text { Imminent or in } \\
\text { progress }\end{array}$ & $\begin{array}{l}\text { Evacuate - vehicle } \\
\text { Evacuate - pedestrian } \\
\text { Evacuate - vertical } \\
\text { Evacuate - safer structure } \\
\text { Expedient protection } \\
\text { of people } \\
\text { Avoid area }\end{array}$ \\
\hline $\begin{array}{l}\text { Rapidly enlarging } \\
\text { sinkhole }\end{array}$ & $\begin{array}{l}\text { LEVEL III } \\
\text { Dam breach very } \\
\text { likely }\end{array}$ & Very likely & $\begin{array}{l}\text { Evacuate - vehicle } \\
\text { Expedient protection } \\
\text { of possessions } \\
\text { Avoid area }\end{array}$ \\
\hline $\begin{array}{l}\text { New seepage } \\
\text { areas with doudy } \\
\text { discharge or } \\
\text { increasing flow rate }\end{array}$ & $\begin{array}{l}\text { LEVEL II } \\
\text { Conditions at dam } \\
\text { may or may not lead } \\
\text { to breach }\end{array}$ & $\begin{array}{l}\text { Possible but not } \\
\text { certain }\end{array}$ & $\begin{array}{l}\text { Expedient protection } \\
\text { of possessions } \\
\text { Seek or monitor } \\
\text { information } \\
\text { Prepare to evacuate }\end{array}$ \\
\hline $\begin{array}{l}\text { New seepage areas } \\
\text { in or near the dam }\end{array}$ & $\begin{array}{l}\text { LEVEL I } \\
\text { Safety issues being } \\
\text { investigated }\end{array}$ & $\begin{array}{l}\text { Potential being } \\
\text { determined }\end{array}$ & $\begin{array}{l}\text { Seek or monitor } \\
\text { information }\end{array}$ \\
\hline
\end{tabular}

-This column contains examples of physical observations; these observations should be tailored to fit individual projects

FIGURE 2 - FIRST ALERT/WARNING ISSUANCE CHECKLIST

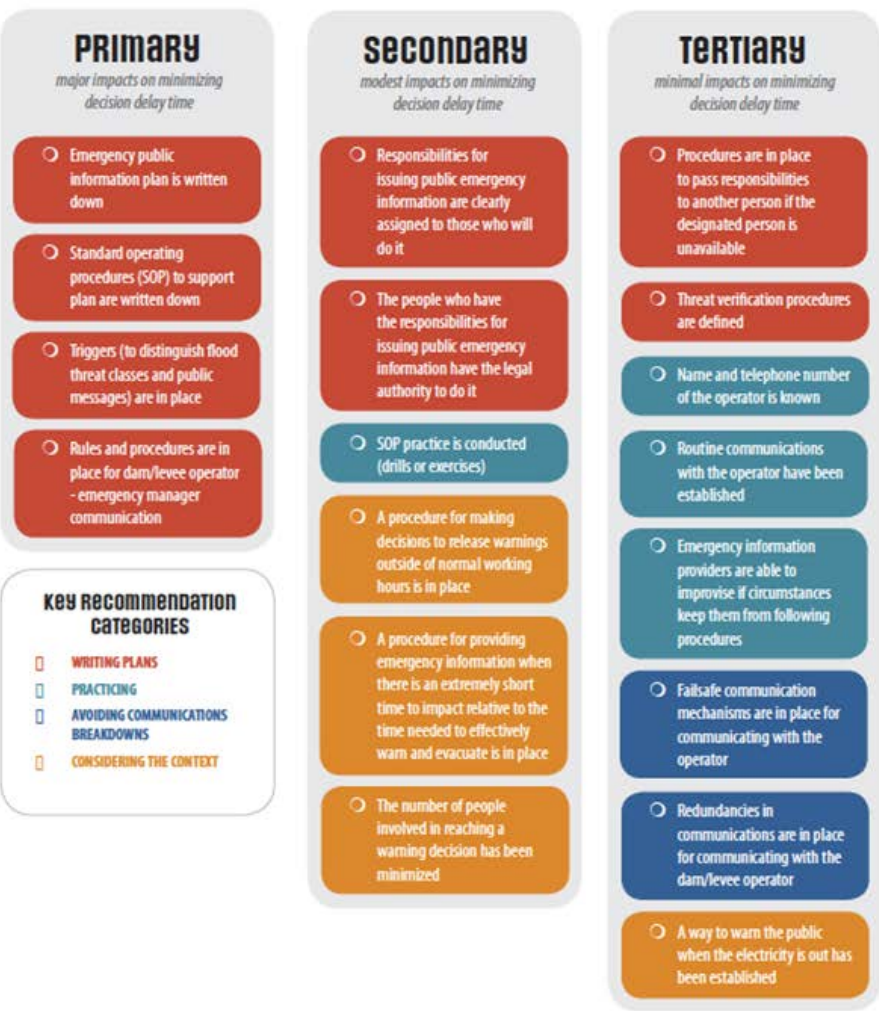




\section{FIGURE 3 - FIRST ALERT/WARNING DIFFUSION CHECKLIST}

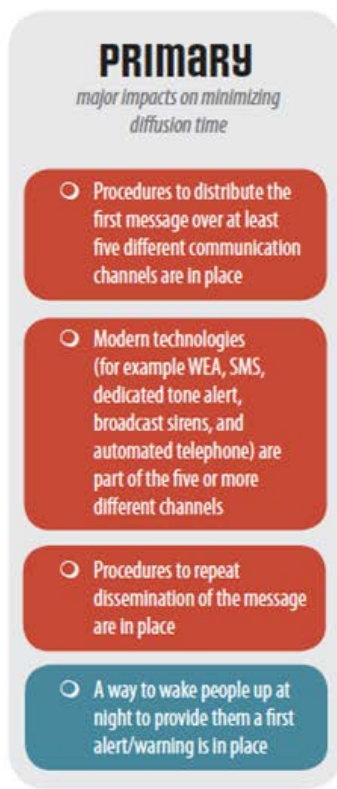

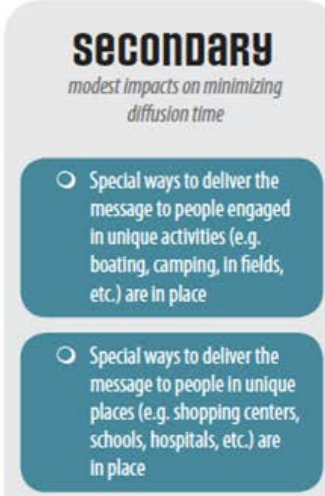

Special ways to dellwer the message to people very close to the dam or levee are in place
Speclal ways to dellwer the message to people who are hearing or visually impalred are in place
The percentage of the population that cannot be reached by the primary channels is known and have a way to reach them

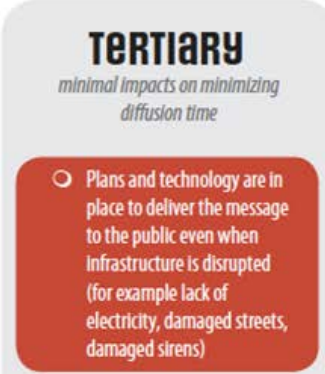

O Ablity to dellver the message to people who only speaka forelign language is in place

\section{Key Recommennation} CATEGORIES

[) SENDING THE FIRST

ALERT/WARNING

D. CONSIDERING YOUR
AUDIENCE

FIGURE 4 - PROTECTIVE ACTION INITIATION CHECKLIST

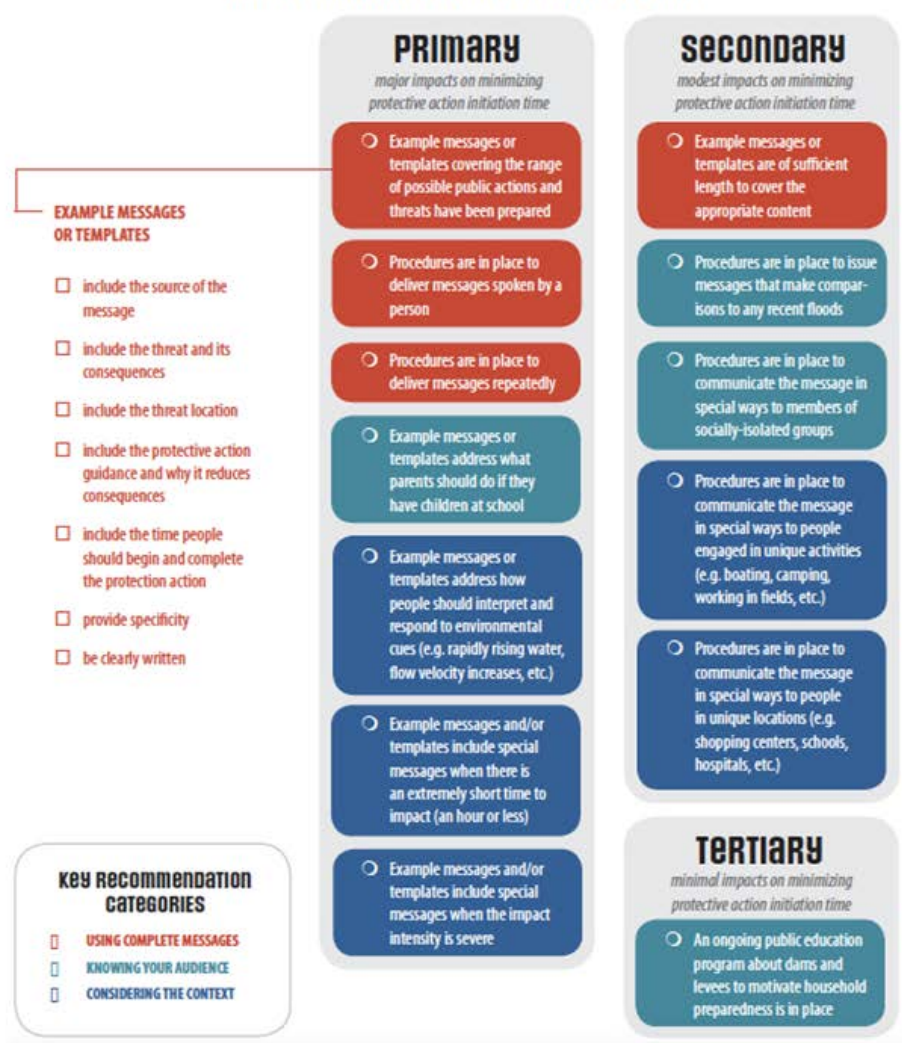




\begin{tabular}{l}
$\begin{array}{l}\text { SOURCE: say who the message } \\
\text { is from }\end{array}$ \\
THREAT: describe the flooding \\
event and its impacts \\
LOCATION: state the impact area \\
boundaries in a way that can be \\
easily understood (for example use \\
street names, landmarks, natural \\
features, and political boundaries) \\
GUIDANCE/TIME: tell people \\
what protective action to take, \\
the time when to do it, how to \\
accomplish it, and how doing it \\
reduces impacts \\
\hline EXPIRATION TIME: tell people \\
when the alert/warning expires \\
and/or new information will be \\
received
\end{tabular}

EXAMPLE:

LCPD Check and monitor local media now Explosion at Superior dam Potential damage and flooding in Sherman Heights Message expires 8:00 AM PDT

\section{TEMPLATE:}

[insert title and organization of a local, familiar, authoritative message source] Check and monitor local media now [insert description of event, dam name, and threat here] in [insert location of threat here] Message expires [insert time here] 


\section{EXAMPLE:}

Los Cerritos Police Department. An explosion occurred at 5:00 AM PDT today at Superior Dam. The explosion may have damaged the dam. Engineers are examining the dam to determine if there are any safety and downstream flood issues. People in Sherman Heights who are downstream from the dam should check and monitor local media now and continue to do so frequently. There is no reason to do anything more at this time. This message will be updated when new information becomes available.

\section{TEMPLATE:}

[insert title and organization of a local, familiar, authoritative message source]. A [insert a brief description of the event] occurred at [insert the time the event occurred] at [insert the name of the dam]. The [insert the same brief description of the event] may have damaged the dam. Engineers are examining the dam to determine if there are any safety and downstream flood issues. People in [insert a brief description of the boundaries of the area at risk] who are downstream from the dam should check and monitor local media now and continue to do so frequently. There is no reason to do anything more at this time. This message will be updated when new information becomes available.

\begin{tabular}{|c|c|c|c|c|}
\hline $\begin{array}{l}\text { DISSEMINATION } \\
\text { CHANNELS }\end{array}$ & SPEED 1 & COVERAGE ${ }^{2}$ & CONCENTRATION 3 & $\begin{array}{c}\text { MESSAGE } \\
\text { COMPREHENSIVENESS }\end{array}$ \\
\hline Route alerting & Slow & Limited & Concentrated & High \\
\hline $\begin{array}{l}\text { Loudspeakers and public } \\
\text { address (PA) systems }\end{array}$ & Fast & Limited & Concentrated & Medium \\
\hline $\begin{array}{l}\text { Wireless Emergency Alerts } \\
\text { (WEA) }\end{array}$ & Very Fast & Widespread & Dispersed & Very Low \\
\hline $\begin{array}{l}\text { Wireless } \\
\text { communications (SMS) }\end{array}$ & Very Fast & Widespread & Dispersed & Very Low \\
\hline Radio & Moderately Fast & Widespread & Dispersed & High to Low \\
\hline Television broadcast & Moderately Fast & Widespread & Dispersed & Very High to Medium \\
\hline Television message scrolls & Moderately Fast & Widespread & Dispersed & Low \\
\hline Newspaper & Very Slow & Widespread & Dispersed & Very High \\
\hline Dedicated tone alert radios & Very Fast & Limited & Concentrated & High \\
\hline $\begin{array}{l}\text { Tone alert and NOAA } \\
\text { Weather Radio }\end{array}$ & Fast & Widespread & Dispersed & High \\
\hline $\begin{array}{l}\text { Text Telephone } \\
\text { (TDD/TTY) }\end{array}$ & Fast & Widespread & Dispersed & Low \\
\hline $\begin{array}{l}\text { Reverse telephone distribu- } \\
\text { tion systems }\end{array}$ & Fast & Limited & Dispersed & High \\
\hline Audio sirens and alarms & Fast & Limited & Concentrated & Very Low \\
\hline Broadcast sirens & Fast & Limited & Concentrated & Medium \\
\hline Message boards & Fast & Limited & Concentrated & Low \\
\hline Aircraft & Slow & Limited & Concentrated & Low \\
\hline Visual alerting & Fast & Limited & Concentrated & Low \\
\hline $\begin{array}{l}\text { Internet protocol (IP) based } \\
\text { technology }\end{array}$ & Fast & Widespread & Dispersed & Very High to Medium \\
\hline Social media & Fast & Widespread & Dispersed & Low \\
\hline
\end{tabular}




\section{GUIDEBOOK SYNTHESIS}

Minimize issuance delay

Written plan beforehand \& what should be in it

Accelerate diffusion

Disseminate over mix of channels/technology

Reduce protective action delay

Warning messages based on repetitive social behavioral science empirical evidence

\section{MORE DETAILS IN}

Mileti, Dennis. S., and John H. Sorensen (2015f). $\underline{A}$ Guide to Public Alerts and Warnings for Dam and Levee Emergencies. Davis CA: U.S. Army Corps of Engineers Risk Management Center.

Download at:

http://silverjackets.nfrmp.us/Portals/0/doc/WarningGuidebo ok USACE.pdf?ver $=$ 2015-08-10-213008-520 


\section{QUESTIONS?}




\section{REFERENCES}

Dennis S. Mileti, PhD

Sorensen, J., and D. Mileti (2014a). First Alert and/or Warning Issuance Time Estimation for Dam Breaches, Controlled Dam Releases, and Levee Breaches of Overtopping. Davis, CA: U.S. Army Corps of Engineers, Institute for Water Resources, Risk Management Center.

Sorensen, J., and D. Mileti (2014b). First Alert and Warning Diffusion Time Estimation for Dam Breaches, Controlled Dam Releases, and Levee Breaches of Overtopping. Davis, CA: U.S. Army Corps of Engineers, Institute for Water Resources, Risk Management Center.

Sorensen, J., and D. Mileti (2014c). Protective Action Initiation Time Estimation for Dam Breaches, Controlled Dam Releases, and Levee Breaches of Overtopping. Davis, CA: U.S. Army Corps of Engineers, Institute for Water Resources, Risk Management Center.

Mileti, D., and J. Sorensen. (2015d). Interview Schedule: Community Warning Issuance, Diffusion, and Protective Action Initiation Estimation. Davis, CA: U.S. Army Corps of Engineers, Institute for Water Resources, Risk Management Center.

Sorensen, J., and D. Mileti (2015e). Influence Weights and Measures for the Factors Shaping First Alert/Warning Delay, Diffusion and Protective Action Initiation Curves for Dam Breaches, Controlled Dam Releases, and Levee Breaches or Overtopping. Davis, CA: U.S. Army Corps of Engineers, Institute for Water Resources, Risk Management Center.

Mileti, Dennis. S., and John H. Sorensen (2015f). A Guide to Public Alerts and Warnings for Dam and Levee Emergencies. Davis CA: U.S. Army Corps of Engineers Risk Management Center. 


\section{BIOGRAPHICAL SKETCH}

Dennis S. Mileti, Ph.D.

Dennis S. Mileti is professor emeritus at the University of Colorado, Boulder where he served as Director of the Natural Hazards Center from 1994-2003. He is the author of over 100 publications most of which discuss societal aspects of hazards and disasters. His book Disasters by Design summarized knowledge in all fields of science and engineering regarding natural hazards and disasters, and made recommendations for shifts in national policies and programs. Dennis has had the opportunity to work on a variety of exciting hazards-related projects over the years including providing oversight to the U.S. Army Corps' investigation of why the levees failed during Hurricane Katrina and ensuing societal impacts for which he was awarded the U.S. Army's Civilian Medal of Honor; and he designed and oversaw NIST's congressional study of evacuation of the World Trade Center towers on 9/11. He has chaired the Committee on Disasters in the National Research Council of the National Academies, and the Board of Visitors to FEMA’s Emergency Management Institute. Dennis also served as a California Seismic Safety Commissioner, and he has worked as a consultant in the private, public, and legal sectors in matters related to emergency management and public risk communication. Dennis has recently completed a Department of Homeland Security project testing Wireless Emergency Alert public warning messages for communication over mobile devices, and another for the U.S. Army Corps of Engineers resulting in a public warning guidebook for emergency managers. 


\title{
Resilience of Societal Infrastructure Systems - Modeling and Decision Analysis Framework
}

\author{
M. H. Faber \\ Department of Management Engineering, Technical University of Denmark, Kgs. Lyngby DK-2800, Denmark. \\ Correspondence e-mail address: mihf@dtu.dk
}

\begin{abstract}
:
Starting point is taken in a motivation for the consideration of the intertwined topics of resilience and sustainability and different interpretations of systems resilience are presented and discussed. A hierarchical presentation of societal systems in their context of exposures to hazards, shared environmental and societal resources and decision-making processes is presented through which the requirements to resilience modeling and assessments are highlighted. On this basis a framework for decision analysis and probabilistic modeling of systems for optimal design and life-cycle management is proposed. The framework allows for the joint consideration of life cycle benefits, vulnerability, robustness and resilience of systems comprised by interlinked spatially distributed sub-systems evolving over time; including governance, regulatory, social, infrastructure, environmental, geo-hazard and anthropological hazard subsystems. The framework allows for assessing the tradeoffs between efficiency, robustness and resilience and facilitates decision optimization in dependency of considered temporal and spatial scales and assumptions regarding disturbance scenarios. A principal example covering a range of phenomena and parameters normally associated with systems complexity (including number of constituents, causal and stochastic dependencies, multi-hazard exposures, cascading failure events, direct and indirect consequences, etc.) is presented to illustrate the use of the framework, highlight needs for further research and facilitate discussions.
\end{abstract}




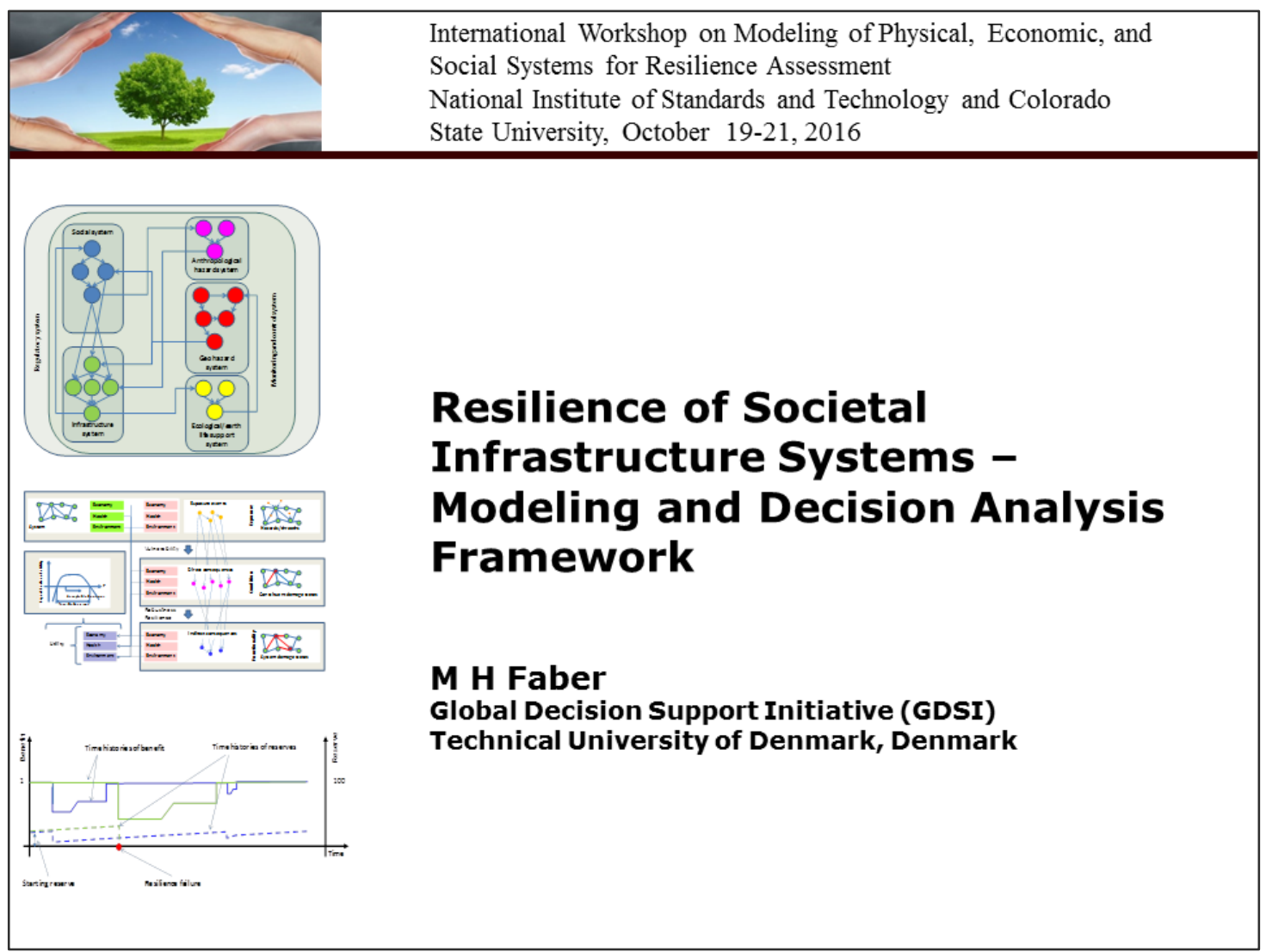

\section{Preamble}

\section{Acknowledgements}

Thanks for the support from:

- The CoWIfonden, Denmark

-Research Team of Professor Mark Stewart University of Newcastle, NSW, Australia

- Research Team of Professor Jie Li, Tongji University, China 


\section{Context}

\section{Pressing boundaries for societal developments:}

At local and global scales it is increasingly appreciated that societal developments are approaching the limits of the capacities of the ecological systems and the Earth life support system

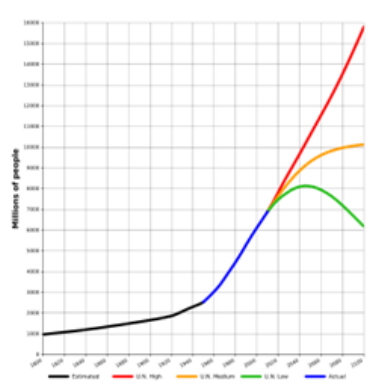

Population growth, Wikepedia, UN

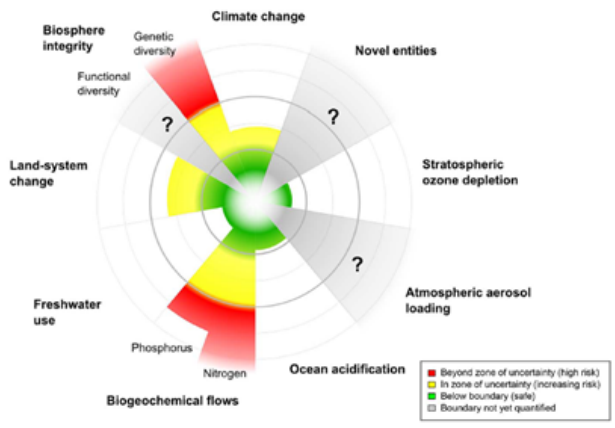

Planetary boundaries, Steffen et al. 2015 3/30 M. H. Faber NIST \& CSU Resilience Workshop October 19-21, 2016

\section{Context}

\section{Pressing boundaries for societal developments:}

Significant signs of the back-coupling between civilizations and living conditions for civilization are observable

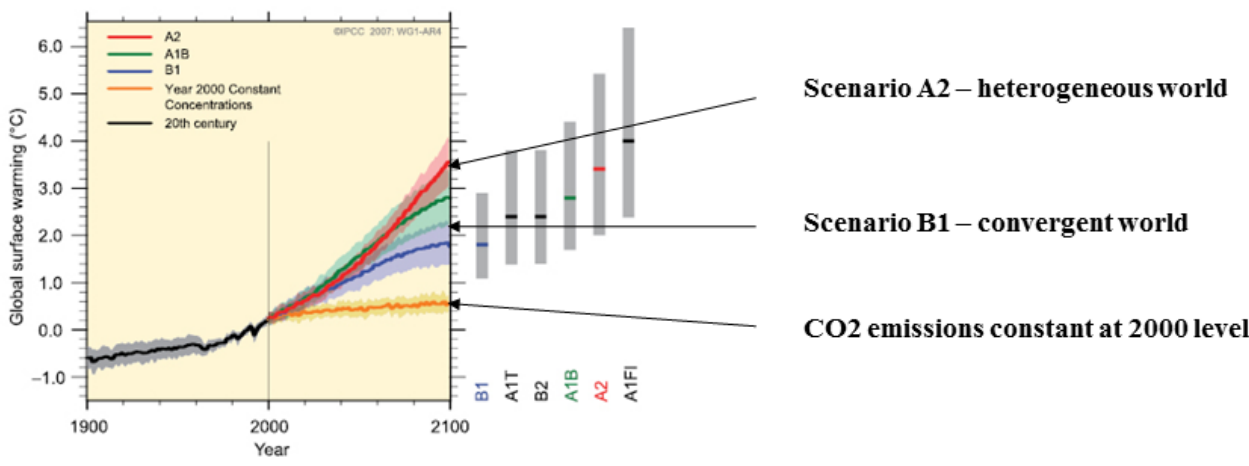

IPCC homepage

4/30 M. H. Faber NIST \& CSU Resilience Workshop October 19-21, 2016




\section{Context}

\section{Pressing boundaries for societal developments:}

Significant signs of the back coupling between civilizations and living conditions for civilization are observable

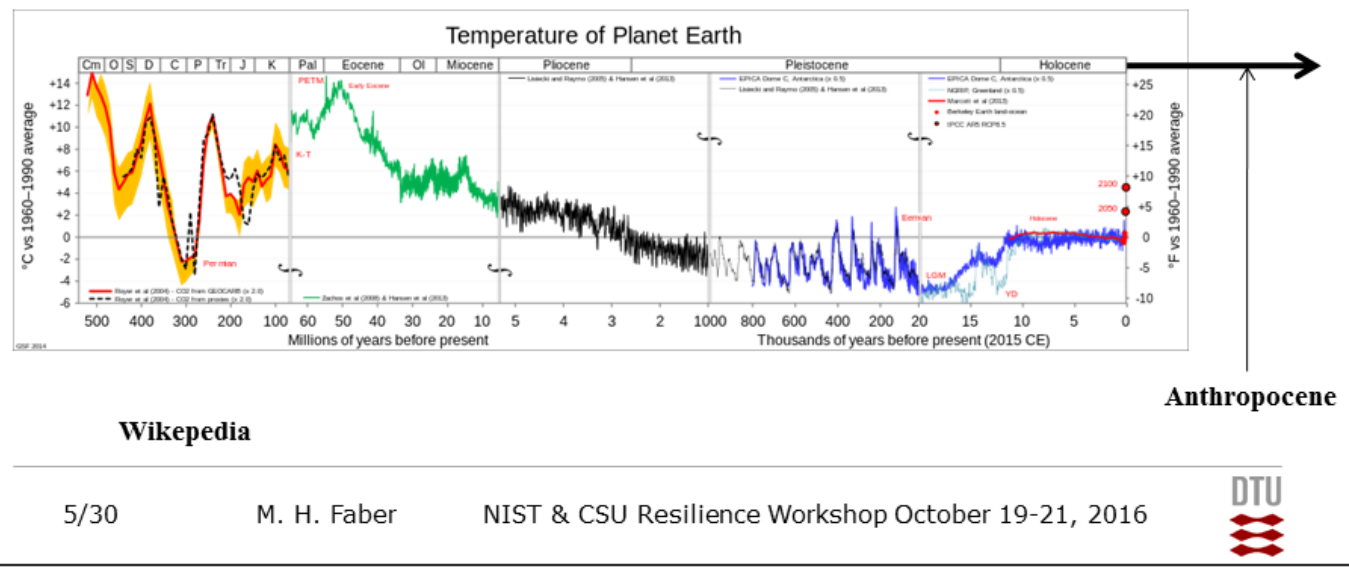

\section{Context}

\section{Improved knowledge is required}

Kates et al. (Science, 2001) calls for

i) representations of the dynamic interactions in integral systems models of society and nature

ii) understanding how trends in society and environment affect sustainability

iii) what determines vulnerability and resilience of nature society systems for particular ecosystems/livelihoods

iv) criteria for early warning of developments or conditions which seriously changes nature society systems

v) incentive structures, markets, regulatory frameworks which leads society on a more sustainable track

vi) how monitoring and reporting might be utilized and improved for enhanced sustainable developments

vii) utilization of decision support systems, systems modeling 


\section{Context}

\section{Resilience definitions}

Pimm (1984) - Resilience....the time it takes till a system which has been subjected to a disturbance returns to its original mode and level of functionality

Holling (1996) - Resilience....the measure of disturbance which can be sustained by a system before it shifts from one equilibrium to another

Cutter (2010) - Resilience.... capacity of a community to recover from disturbances by their own means

Bruneau (2009) - Resilience.... a quality inherent in the infrastructure and built environment; by means of redundancy, robustness, resourcefulness and rapidity

National Academy of Science (NAS, USA) - Resilience.... a systems ability to plan for, recover from and adapt to adverse events over time

\section{Contents of Presentation}

- Context

- Framework for systems decision analysis

- Questions to be answered

- Probabilistic systems representation

- Robustness of systems

- Resilience of systems

- Sustainability of systems

- Principal example -infrastructure-social-hazard-ecological system

- Conclusions and outlook 


\section{Framework for Systems Decision Analysis}

\section{A hierarchy of systems}

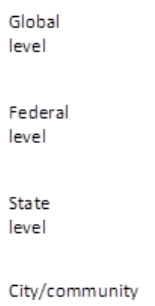

level

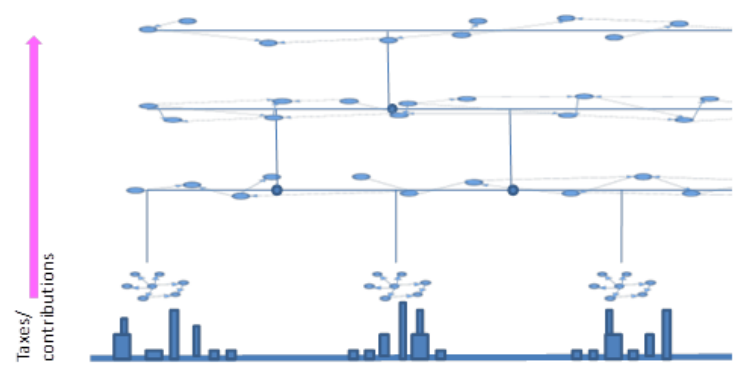

Environment

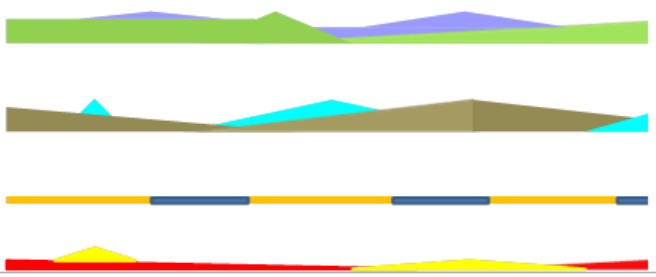

\section{Framework for Systems Decision Analysis}

\section{Questions to be answered}

How to:

- prioritize investments on design and management of interlinked systems (economy, environment, health)

- select target reliabilities and performances of individual systems and constituents

- plan and budget for the future (economy, qualities of the environment, social capacity, health)

How resilient is resilient enough?

at all levels in the hierarchy of societal systems utilizing communication and democratic decision making processes to decide on the allocation and sharing of resources 


\section{Framework for Systems Decision Analysis}

\section{A generic framework}

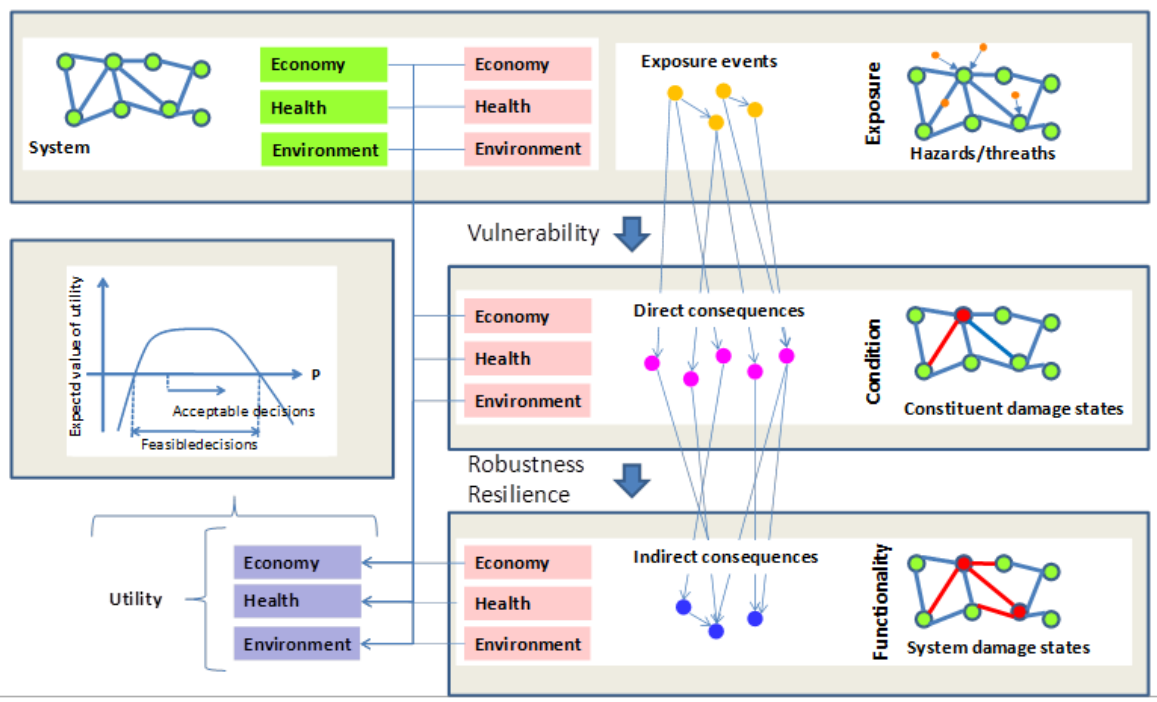

$11 / 30$

M. H. Faber

NIST \& CSU Resilience Workshop October 19-21, 2016

\section{Framework for Systems Decision Analysis}

\section{Bayesian decision analysis}

Consistent "book keeping" of the expected value of the utility associated with different decision alternatives -(Raiffa and Schlaifer (1961), von Neumann and Morgenstern (1947))

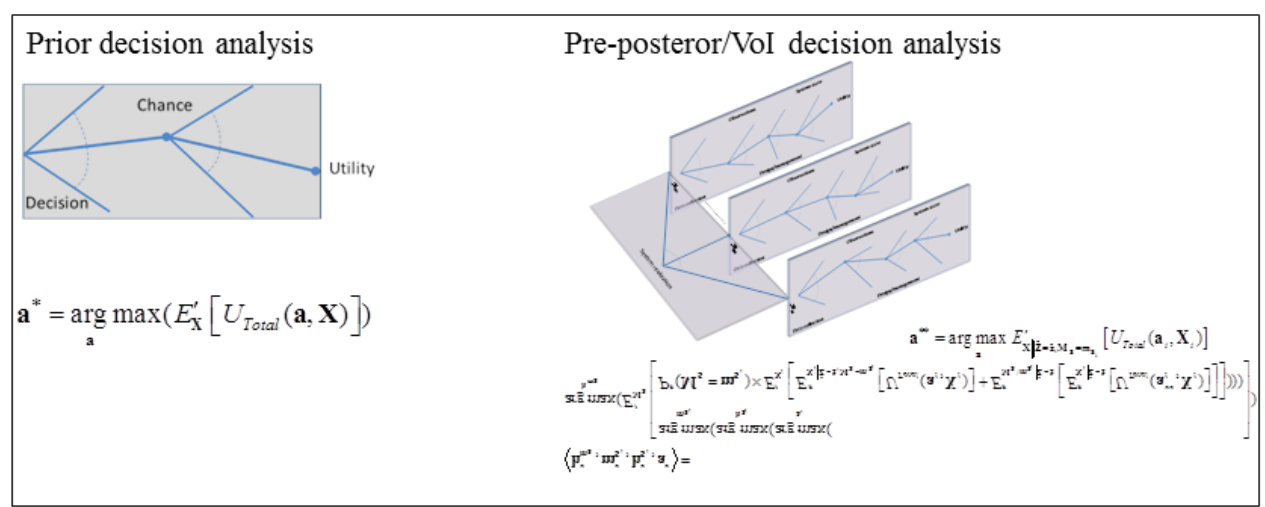

Epistemic Uncertainty... System Choice - Faber, M.H. and Maes, M.A. (ICOSSAR2005)

12/30 M. H. Faber NIST \& CSU Resilience Workshop October 19-21, 2016




\section{Probabilistic System Representation}

\section{Infrastructure as part of an interlinked system}
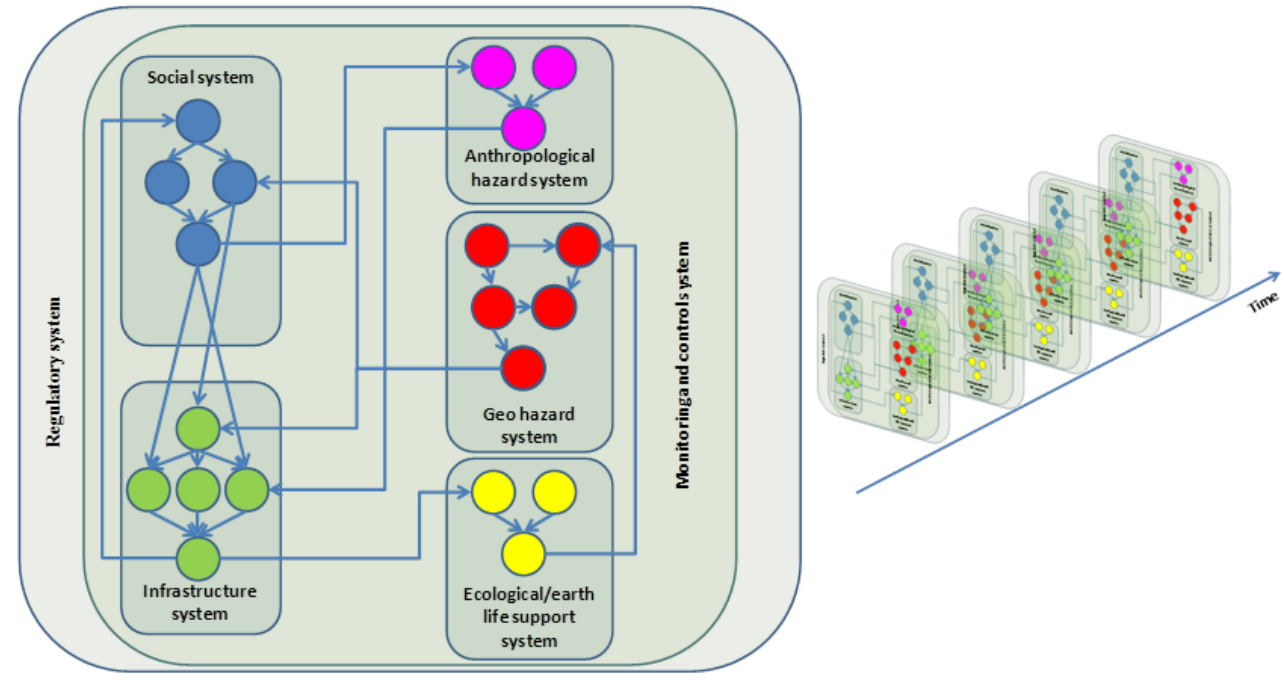

\section{Probabilistic System Representation}

\section{System representation}

System model

Graph model

Constituents model

Probabilistic model

Decision alternatives
$\mathbf{m}_{\mathbf{S}}(\mathbf{a})=\left(\mathbf{m}_{\Sigma}(\mathbf{a}), \mathbf{m}_{\mathbf{c}}(\mathbf{a}, \mathbf{X}), \mathbf{X}(\mathbf{a})\right)^{T}$

$$
\mathbf{m}_{\Sigma}(\mathbf{a})
$$

$\mathbf{m}_{\mathrm{c}}(\mathbf{a}, \mathbf{X})$

$\mathbf{X}(\mathbf{a})$

a

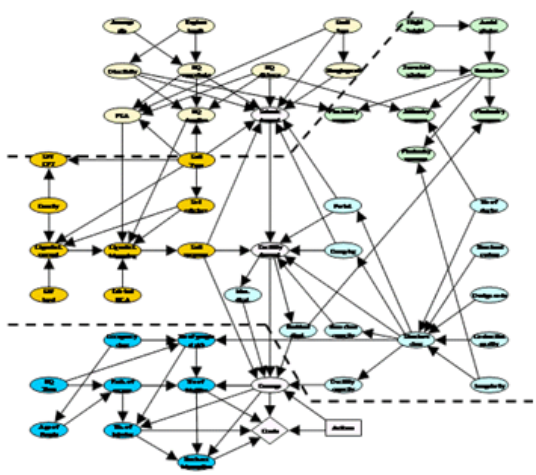




\section{Probabilistic System Representation}

\section{Cascading failure scenarios and evolution of consequences}

Inîtiation phase Propagation phase

Disturbanceeffects

Redistribution effects

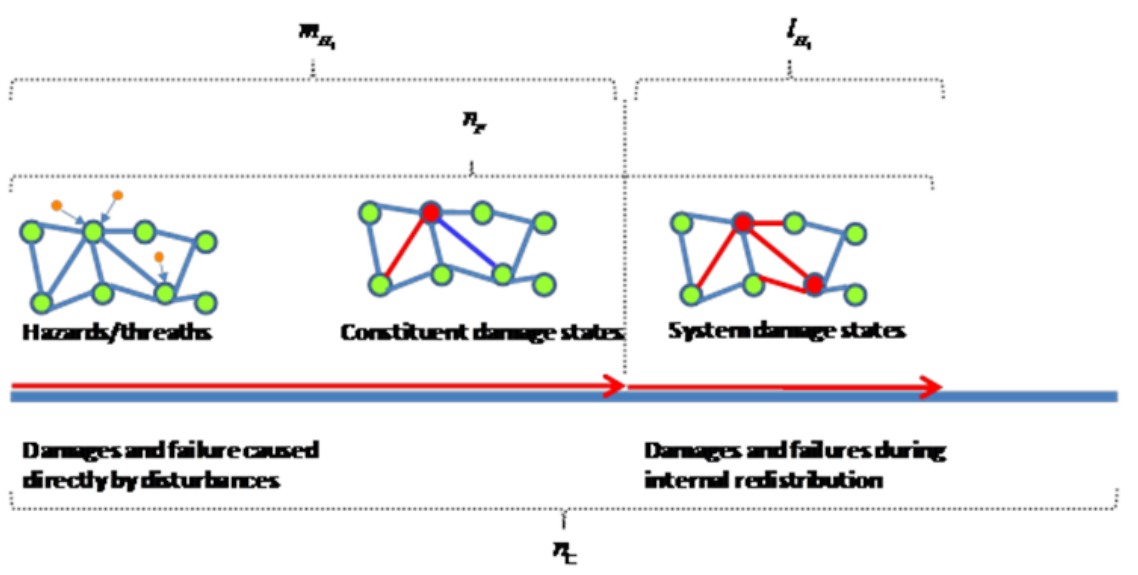

\section{Probabilistic System Representation}

\section{Robustness modeling}

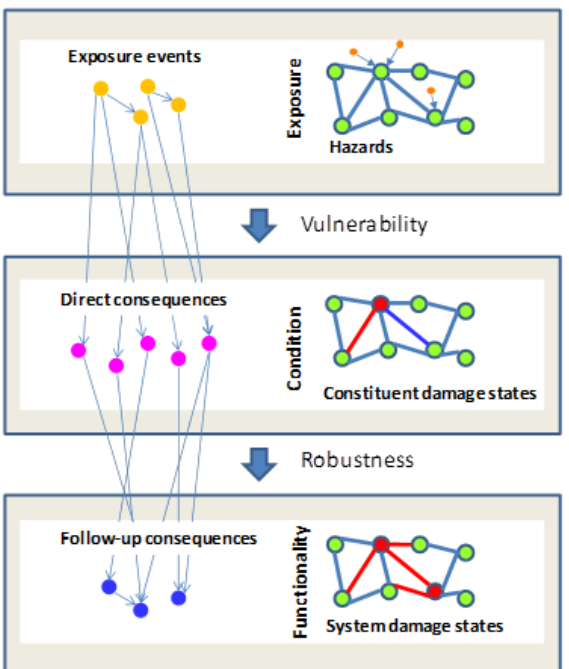

It is assumed that all relevant scenarios have been identified

$$
\begin{aligned}
& \left.\mathbf{S}=\left(i, p(i), c_{D, I}(i), c_{D, P}(i), c_{I D}(i)\right)\right) \\
& i=1,2, \ldots, n_{s} \\
& I_{R}(i)=\frac{c_{D}(i)}{c_{T}(i)} \\
& I_{R}(i)=\frac{c_{D, I}(i)}{c_{D, I}(i)+c_{D, P}(i)} \\
& I_{R}(i)=\frac{c_{D, I}(i)+c_{D, P}(i)}{c_{D, I}(i)+c_{D, P}(i)+c_{I D}(i)}
\end{aligned}
$$




\section{Probabilistic System Representation}

\section{Consequences to health, environment and economy}

Impacts to health and safety are addressed through the relative utility function comprised by the Life Quality Index (LQI)

(Nathwani et al, 1997)

Impacts to the environment are addressed through:

- Quantitative Life Cycle Analysis (substances/energy)

(Hauschild, 2015)

- Eco-system services (space/landscape/capacities)

(Constanza et al, 1997)

Impacts to the economy are addressed through:

- Monetary benefits (input-output functions)

- Monetary losses (input-output functions)

\section{Probabilistic System Representation}

\section{"Matter" book-keeping - Life Cycle Analysis (LCS)}

\begin{tabular}{l|l|l|l|l|l|l}
\hline System states & LCA quantifications & Consequences
\end{tabular}

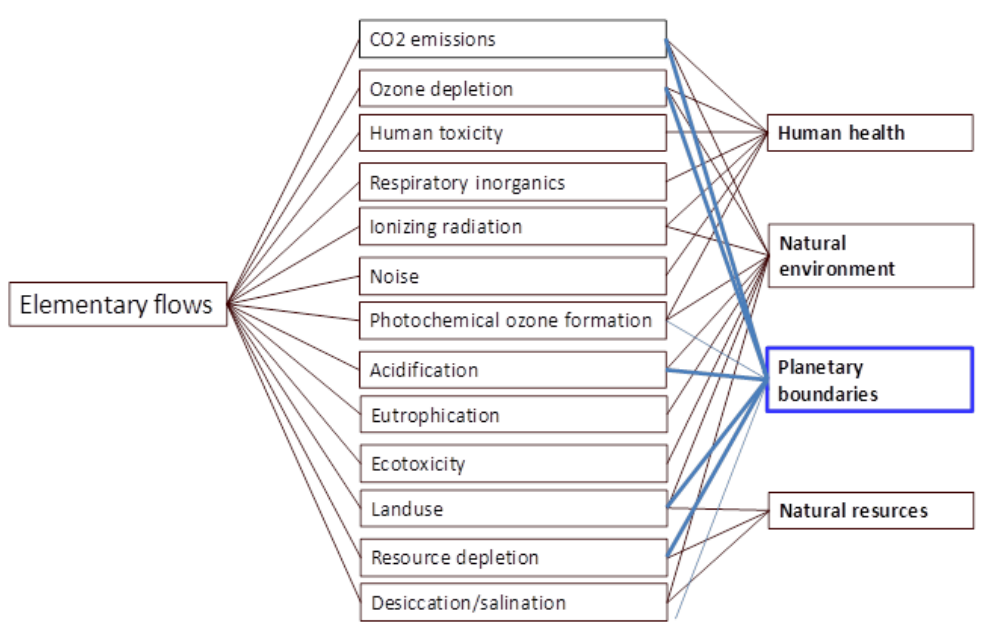




\section{Probabilistic System Representation}

\section{Social preparedness modeling}

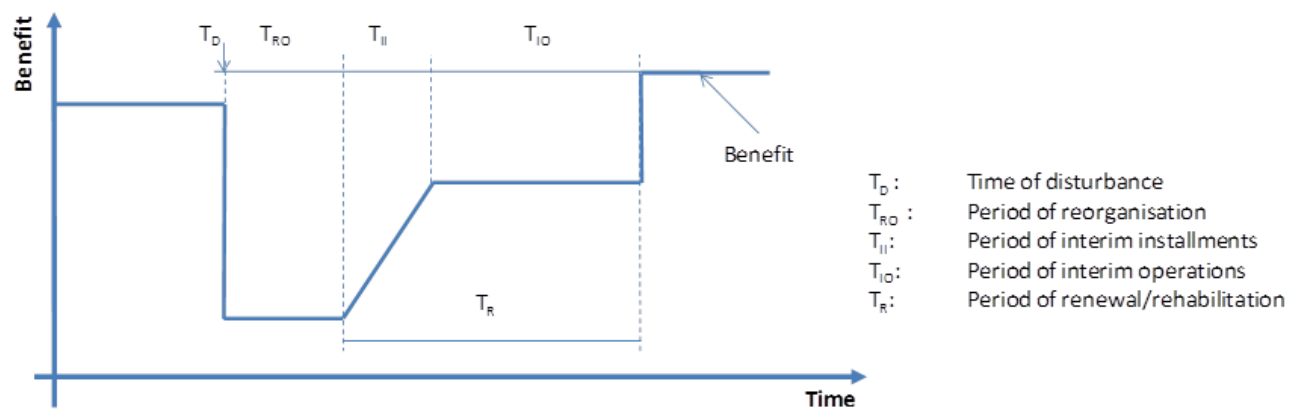

\section{Probabilistic System Representation}

\section{Resilience interpretation}

The system is not resilient if within a given timeframe one or more of its capacities/reserves are exceeded

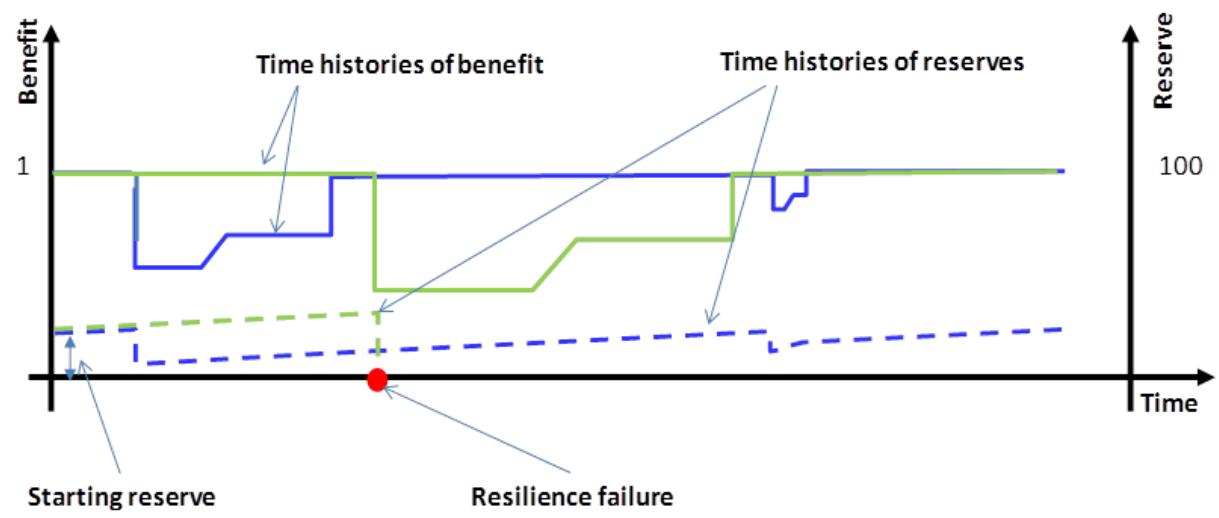

$20 / 30$

M. H. Faber

NIST \& CSU Resilience Workshop October 19-21, 2016 


\section{Probabilistic System Representation}

\section{Resilience failure modeling}

Resilience as a time variant reliability problem vector out-crossing problem

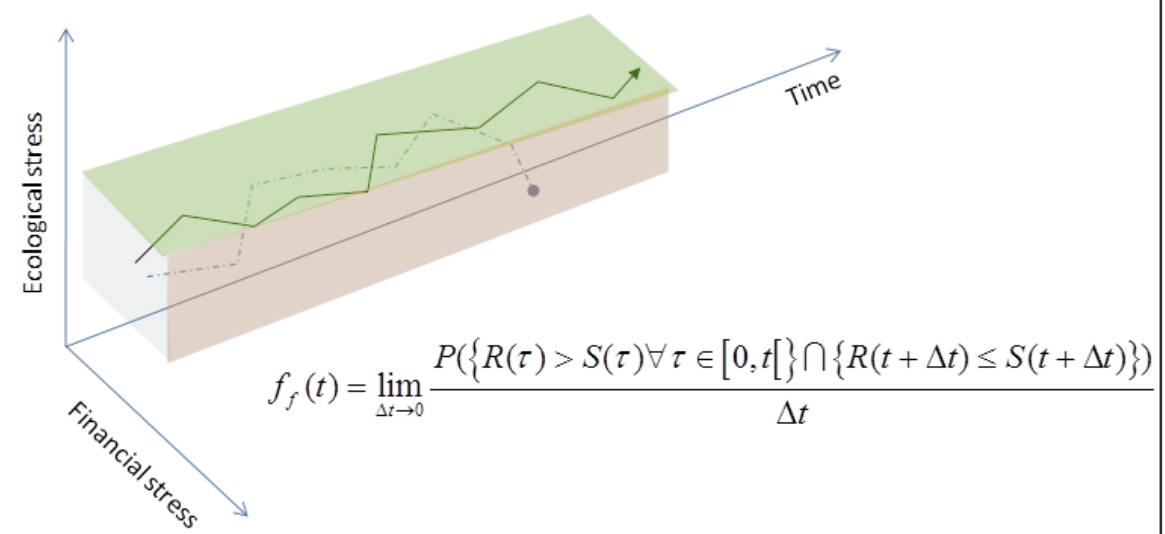

\section{Principal Example}

Interlinked infrastructure system model

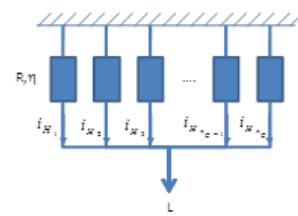

Return period $=100 \mathrm{y}$ Annual $\mathrm{Pf}=10^{-4}$
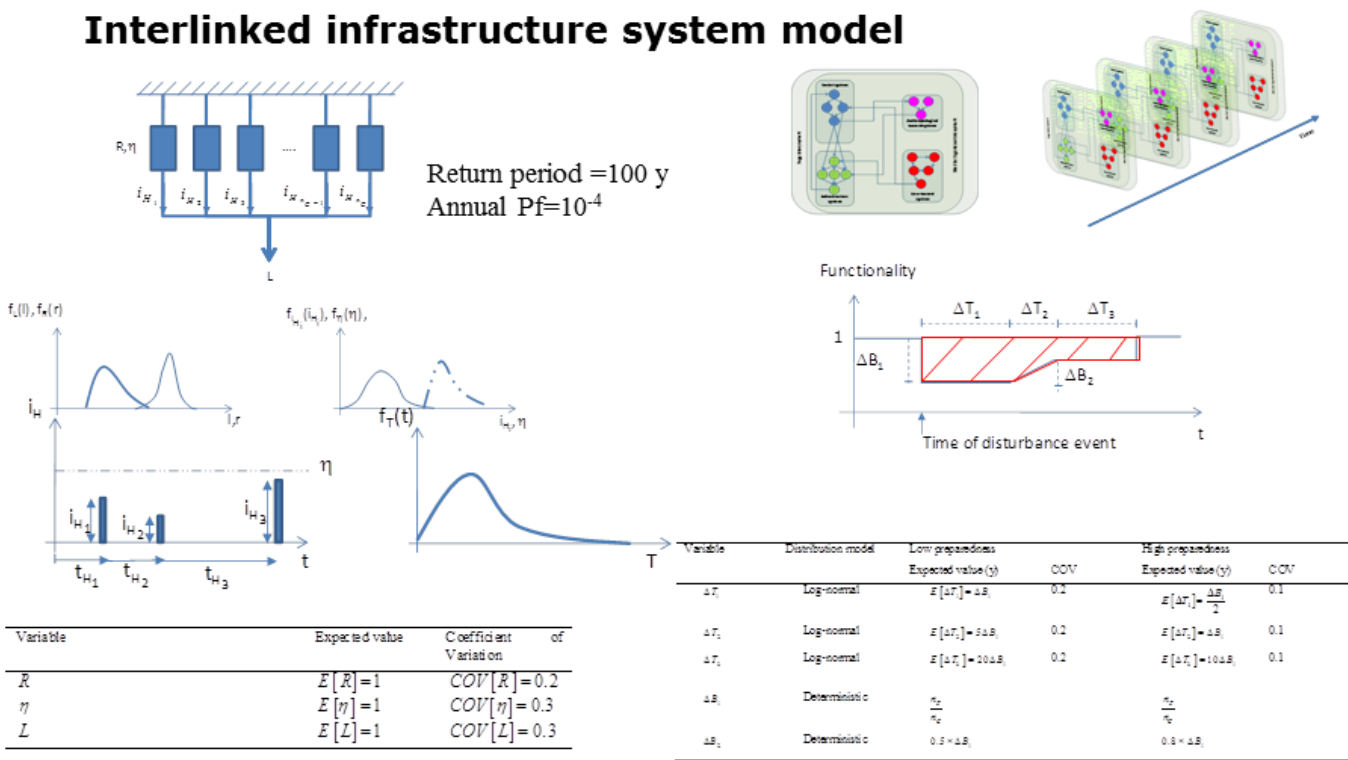

$22 / 30$

M. H. Faber

NIST \& CSU Resilience Workshop October 19-21, 2016 


\section{Principal Example}

\section{Resilience management}

Resilience may be managed by:

- increasing redundancy (number of constituents)

- improvements of (social) preparedness

- design of individual constituents wrt nat. and ant. hazards

- saving sufficient benefits to recover from disturbances

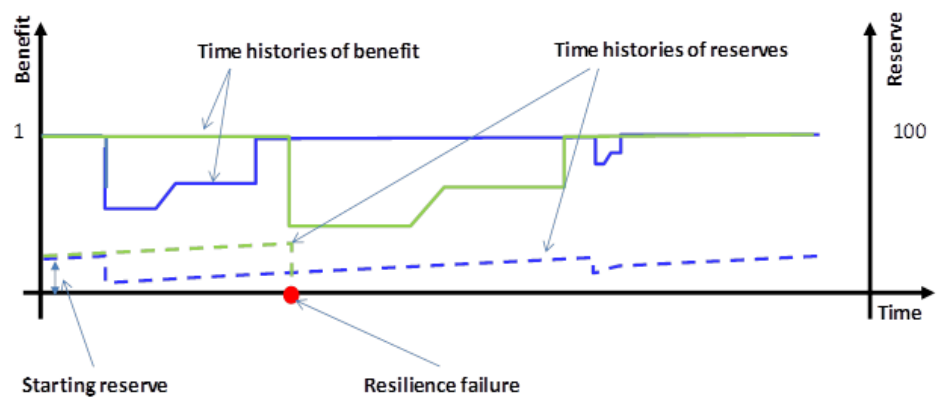

\section{Principal Example}

\section{Robustness wrt operational and natural hazards}
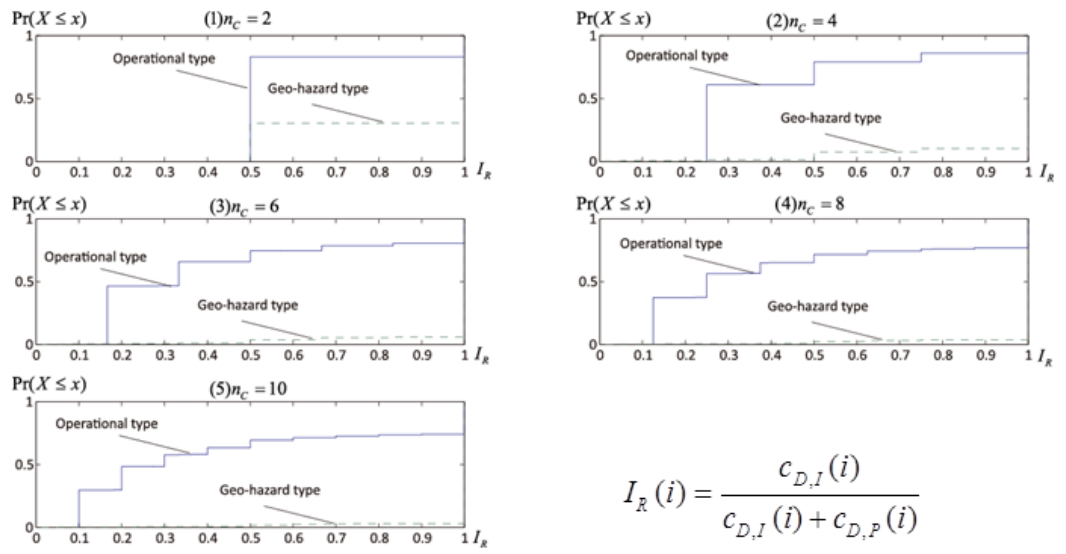

$$
I_{R}(i)=\frac{c_{D, I}(i)}{c_{D, I}(i)+c_{D, P}(i)}
$$




\section{Principal Example}

\section{Resilience failure (social system with low preparedness)}

Resilience is a probabilistic characteristic of a system - quantified e.g. in terms of annual probability of resilience failure

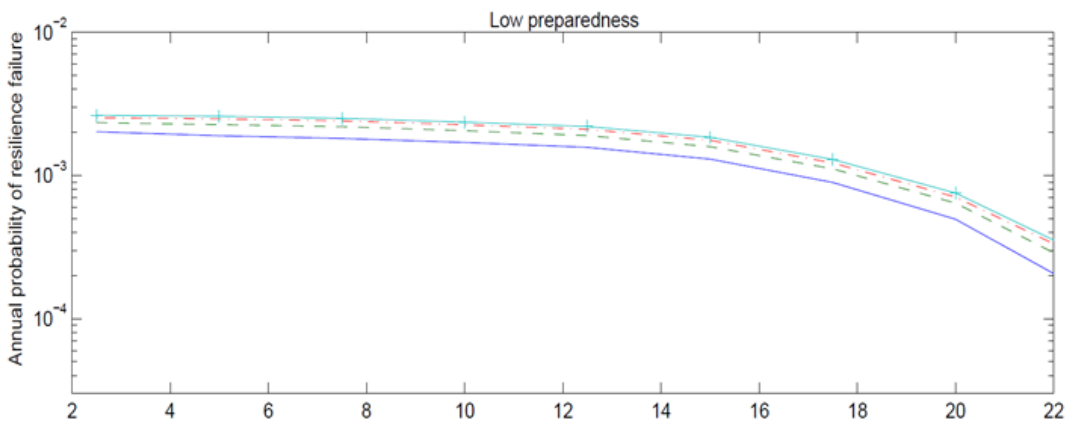

Probability of resilience failure as function of the percentage of annual benefits saved as reserve for Daniels systems with different number of components

\section{Principal Example}

\section{Resilience failure (social system with high preparedness)}

Resilience is a probabilistic characteristic of a system - quantified e.g. in terms of annual probability of resilience failure

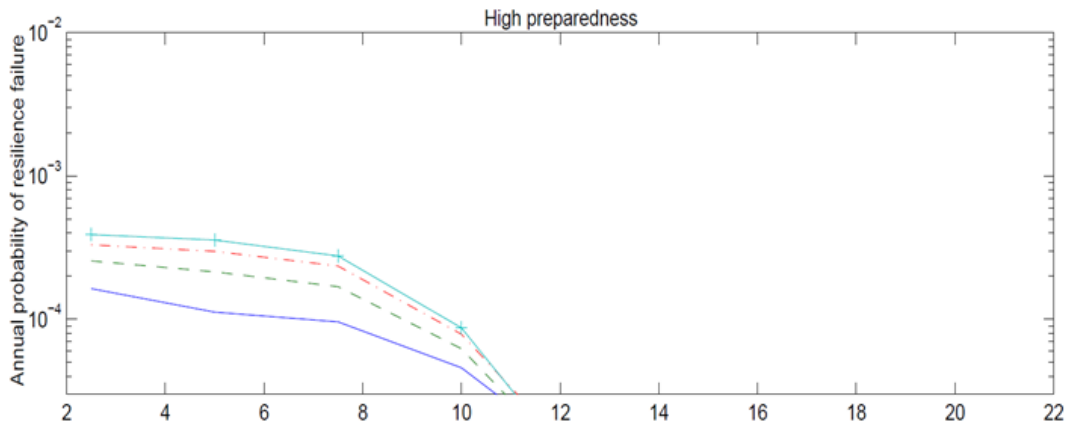

Probability of resilience failure as function of the percentage of annual benefits saved as reserve for Daniels systems with different number of components 


\section{Principal Example}

\section{Conditional resilience failure (given disturbance damage)}

Indicators of resilience can be assessed in terms of the joint probability distribution function of the recovery time and the magnitude of lost benefit for a given disturbance event $\left(n_{f} / n_{c}=0.5\right)$
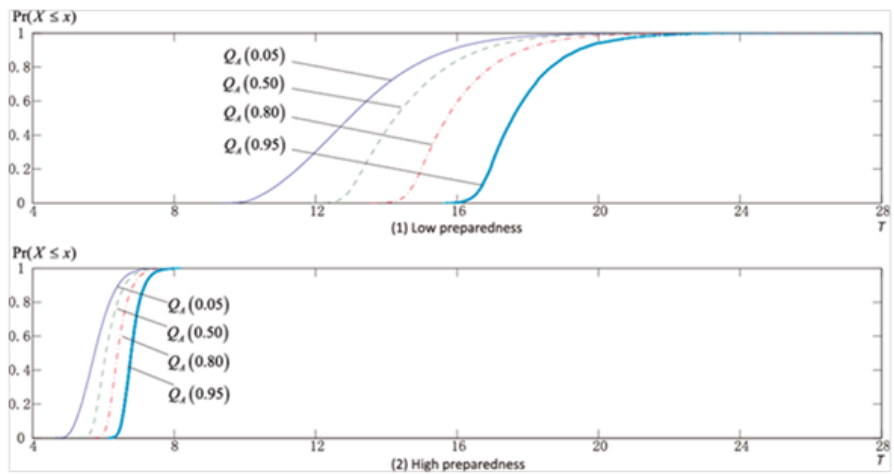

\section{Conclusions and outlook}

\section{What have we?}

Framework for decision analysis for coupled systems

Useful formulations for the representation and quantification of robustness and resilience - and sustainability...

\section{What have we not?}

Best practices and optimized techniques for:

- modeling and analysis of individual systems

- identification and representation of connections between systems

Robust and generic representations and analysis methods of relevance for the complexity of real life coupled systems 


\section{Thanks for your attention!}

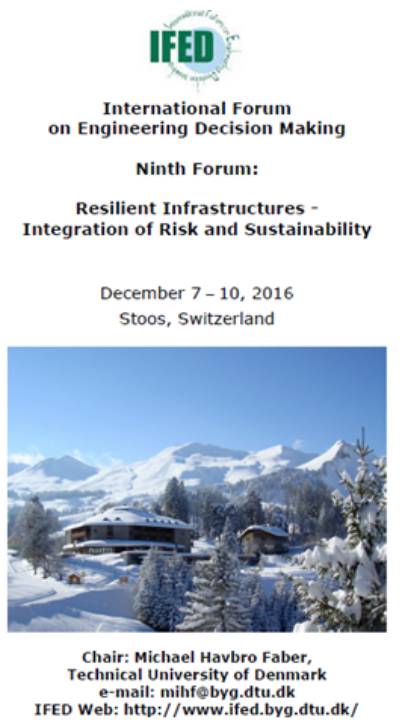

$29 / 30$

M. H. Faber

NIST \& CSU Resilience Workshop October 19-21, 2016

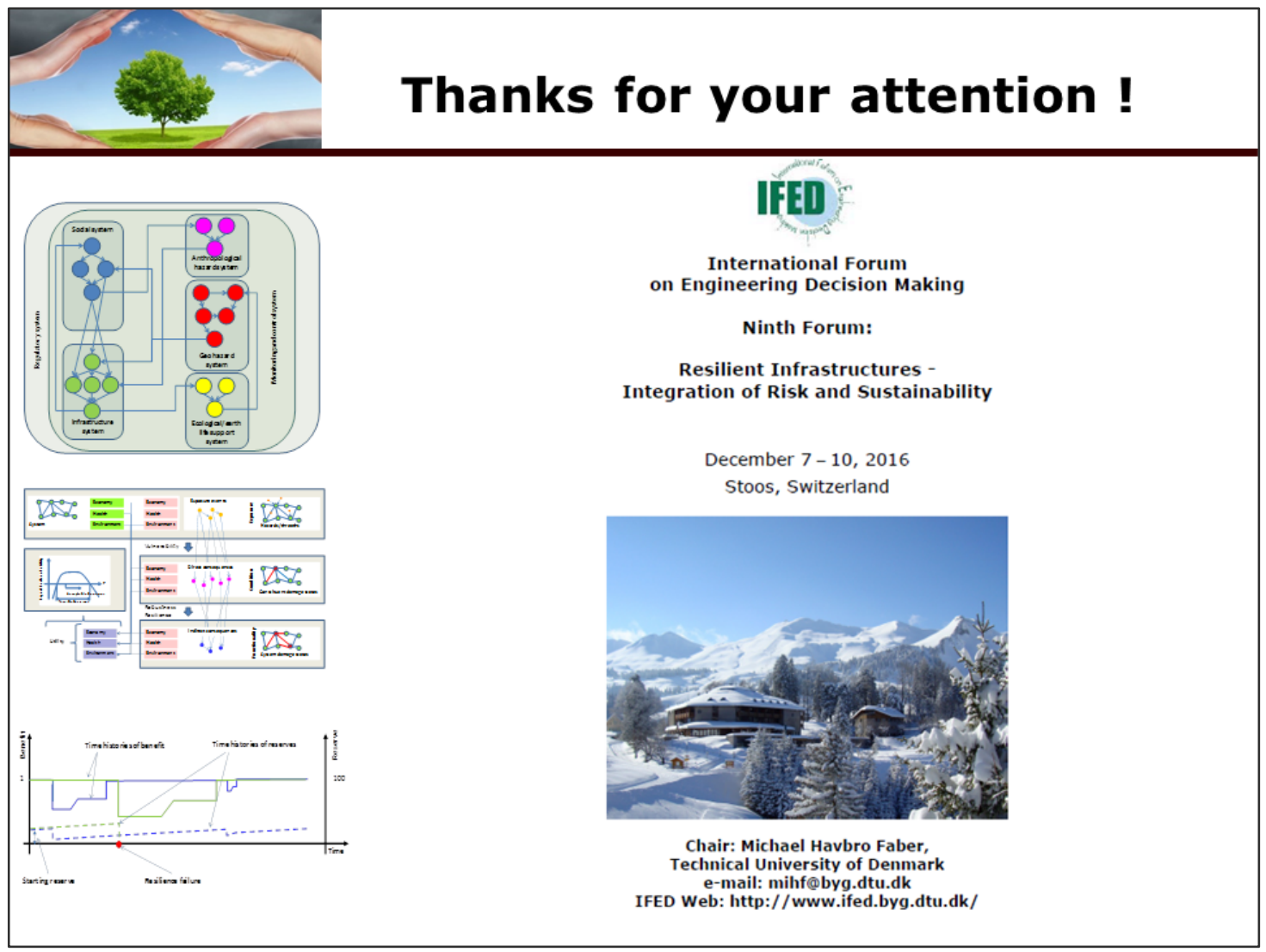




\section{Resilience of Societal Infrastructure Systems - Modeling and Decision Analysis Framework M. H. Faber}

Konakli K, Sudret B and Faber MH (2015) Numerical Investigations into the Value of Information in Lifecycle Analysis of Structural Systems, ASCE-ASME J. Risk Uncertainty Eng. Syst., Part A: Civ. Eng., 2015, pp 1-13.

Narasimhan, Ferlisi, Cascini, De Chiara, Faber, M H (2016). A cost-benefit analysis of mitigation options for optimal management of risks posed by flow-like phenomena, Natural Hazards, Volume 81, Supplement 1, Springe, 2016, pp 117-144.

Faber, M H, Giuliani, L, Revez, A, Jayasena, S; Sparf, J; Mendez, JM (2014). Interdisciplinary approach to disaster resilience education and research, Procedia Economics and Finance, 18, 2014, pp 601-609.

Fischer, K.; Virguez, E.; Sánchez-Silva, M.; Faber, M.H. (2013) On the assessment of marginal life saving costs for risk acceptance criteria, Structural Safety, No. 44, Elsevier Ltd., 2013, p. 3746.

Schubert, M. and M. H. Faber (2012). Common cause effects in portfolio loss estimation. Structure and Infrastructure Engineering 8(5): pp 497-506.

Faber, M.H.(2011). On the governance of global and catastrophic risks. International Journal of Risk Assessment and Management 15(5), 2011, pp 400-416.

Bayraktarli, Y. Y., J. W. Baker and M. H. Faber. (2011). Uncertainty treatment in earthquake modelling using Bayesian probabilistic networks. Georisk 5(1), 2011, pp 44-58.

Bayraktarli, Y. Y. and Faber, M. H.(2011) Bayesian probabilistic network approach for managing earthquake risks of cities, Georisk: Assessment and Management of Risk for Engineered Systems and Geohazards, 5, (1), 2011, pp 2 - 24.

Faber MH, (2015) Codified Risk Informed Decision-making for Structures: Keynote Lecture at the International Symposium on Reliability of Engineering Systems (SRES2015), Hangzhou, China 15-17 October, 2015.

Faber, M H, Vrouwenvelder T A C W M, Lind, N C (2015) Protocols for Communication and Governance of Risks. In proc. to the 12th International Conference on Applications of Statistics and Probability in Civil Engineering, ICASP12, Vancouver, Canada, July 12-15, 2015.

Faber, M H, Sørensen, J D and Vrouwenvelder T A C W M (2015) On the Regulation of Life Safety Risk. In proc.to the 12th International Conference on Applications of Statistics and Probability in Civil Engineering, ICASP12, Vancouver, Canada, July 12-15, 2015.

Virguez-Rodriguez, E. and Faber, M. H. (2011): Supporting decisions on global health and life safety investments. In proc. to 11th International Conference on Applications of Statistics and Probability in Civil Engineering, ICASP11 August 1-4, 2011, Zurich, Switzerland.

Faber M.H., Maes M.A., Baker J.W., Vrouwenvelder T., and Takada T. (2007). "Principles of risk assessment of engineered systems," in 10th International Conference on Application of Statistic and Probability in Civil Engineering (ICASP10), Tokyo, Japan, 8 p 


\section{Resilience of Societal Infrastructure Systems - Modeling and Decision Analysis Framework M. H. Faber}

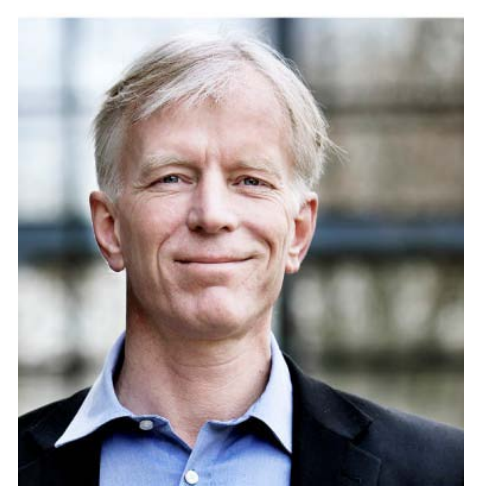

Michael Havbro Faber Since November 1, 2015 Michael Havbro Faber is fully engaged in research and teaching in the field of reliability, risk and safety in engineering in the newly established Global Decision Support Initiative (GDSI) at the Technical University of Denmark. Prior to this position he was head of the department of civil engineering at DTU in Denmark from 2011-2015 and professor and head of the group on risk and safety at ETH, Zurich, Switzerland in the period 2000-2011.

His research interests are directed on decision theory, life safety investments, risk assessment, resilience, global catastrophic risks, uncertainty modeling, Bayesian probability theory and applied statistics. Application areas include risk and reliability informed decision-making for design, assessment and maintenance optimization of bridges, tunnels, buildings, offshore installations, ship structures, roadway traffic systems and space structures as well as for management of natural hazards including earthquakes, hurricanes, floods, rock-fall and avalanches. His industrial experience mostly originates from COWI, Denmark, Det Norske Veritas, Norway and on-going consultancy work through the specialist consulting company Matrisk GmbH of which he is a founding partner since 2001.

He has been actively involved and taken leadership in several international committees, including: The Joint Committee on Structural Safety (JCSS);past president, the International Forum on Engineering Decision-making (IFED); founding and acting president, the ISO 2394 Principles of Reliability of Structures; convener, the international Civil Engineering Reliability and Risk Association (CERRA); president, The World Economic Forum, member of Global Expert Network on Risk and Resilience, the OECD High Level Risk Forum and the Danish Academy of Technical Sciences. 


\title{
Total Economic Consequences of Disasters
}

\author{
Adam Rose
}

University of Southern California

\begin{abstract}
Economic Consequence Analysis (ECA) of natural, man-made and technological disasters continues to evolve. This presentation will introduce the broad ECA framework developed at the Center for Risk and Economic Analysis (CREATE) to estimate the total impacts of a disasters on an economy. The presentation will illustrate the workings of the framework in relation to three aspects of ECA that can have major influences on the bottom-line. First, we have specified the multiple dimensions of economic resilience and developed an operational metric that has been applied to several major case studies, including the resilience of the US and New York Metropolitan Area economies in the aftermath of the 2001 World Trade Center terrorist attacks. Second, we have refined computable general equilibrium (CGE) analysis as the over-arching modeling framework to estimate total economic consequences of actual and simulated disasters. We illustrate its workings in relation to the extensive supply-chain impacts of a simulated seaport disruption. Third, we have developed ways to measure extreme behavioral responses to disasters, and to translate them into direct economic costs so that they can be integrated into CGE models. We illustrate this advance with a simulation of a "dirty-bomb" attack. Public and private sector risk management implications of these examples are then offered. The presentation will conclude with a brief discussion of the CREATE Economic Consequence Analysis Tool (E-CAT), and illustrate its applicability to earthquake scenarios.
\end{abstract}




\title{
USC $\overline{\mathbb{I I}}$ CREATE
}

\section{Total Economic Consequences of Disasters}

\author{
Adam Rose
}

Center for Risk and Economic Analysis of Terrorism Events CREATE

October 19, 2016

\section{(4) USC $\overline{\text { III CREATE }}$}

\section{Overview}

- CREATE has broadened the ECA Framework

- resilience

- extreme behavioral responses

- complex supply-chain linkages

- remediation

- mitigation and spillovers

- Overview

- CREATE ECA Framework

- example applications: resilience, behavioral, supply-chain

- implications for decision-making

- E-CAT Tool 

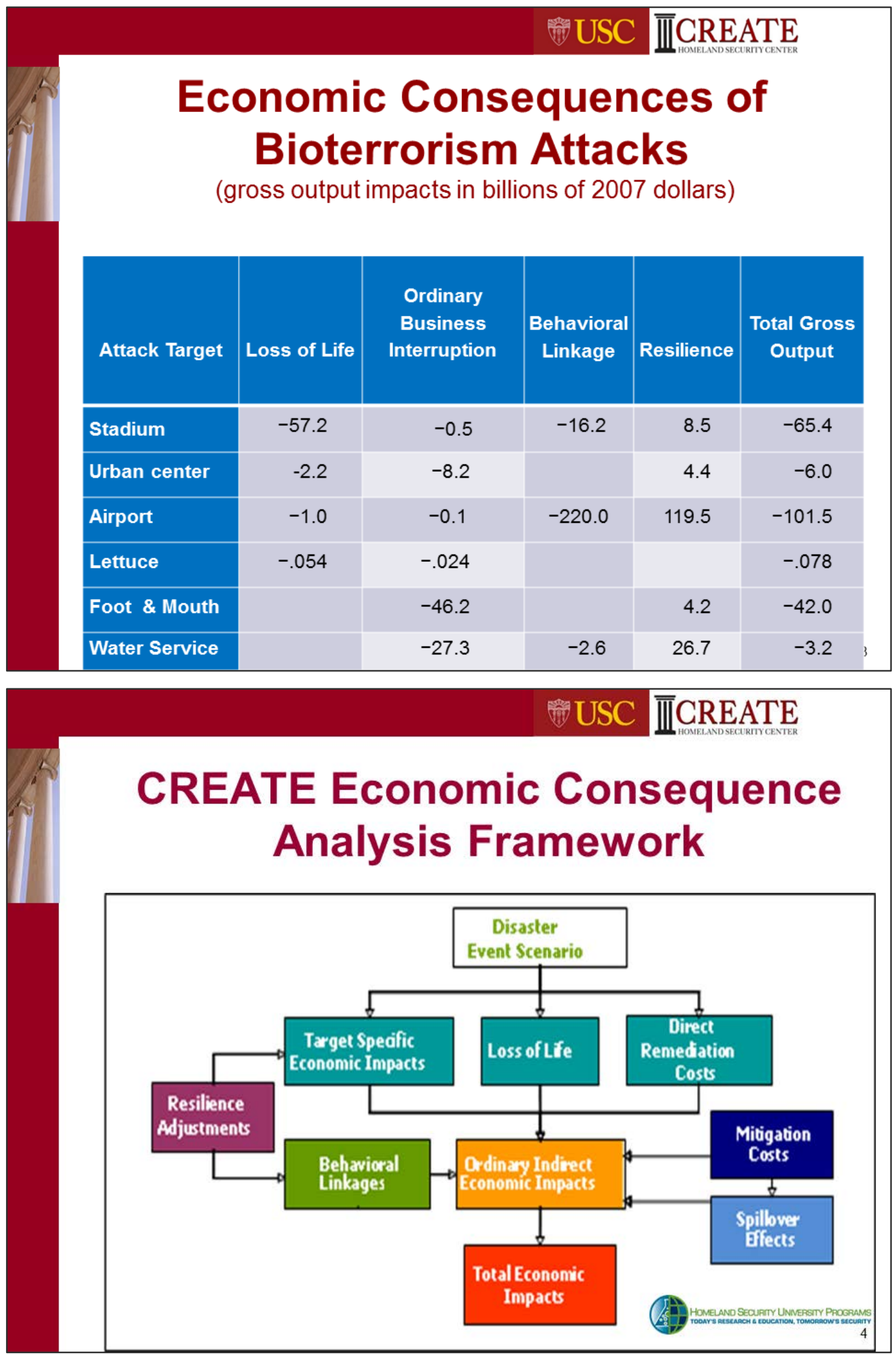


\section{USC $\overline{\mathbb{I}}$ CREATE Economic Resilience}

- Static:

- General Definition: Ability of a system to maintain function when shocked.

- Econ Definition: Efficient use of remaining resources at a given point in time to produce as much as possible.

- Dynamic

- General: Ability \& speed of a system to recover.

- Economic: Efficient use of resources over time for investment in repair and reconstruction, including expediting the process \& adapting to change.

- Metric: averted losses as \% of potential losses

\section{USC $\overline{\mathbb{I}}$ CREATE}

\section{Background on Economic Resilience}

- Two major perspectives:

1. Include everything done to reduce losses, pre- and post-disaster (focus is mitigation of property damage)

2. Limit to actions implemented after the disaster hits (acknowledging that resilience is a process; things can be done to advance to build resilience capacity)

- e.g., emergency drills, back-up generators, alternative suppliers - however, these are not implemented until after the disaster hits

- The latter perspective may strike some as odd: How can you reduce property damage post-disaster? 


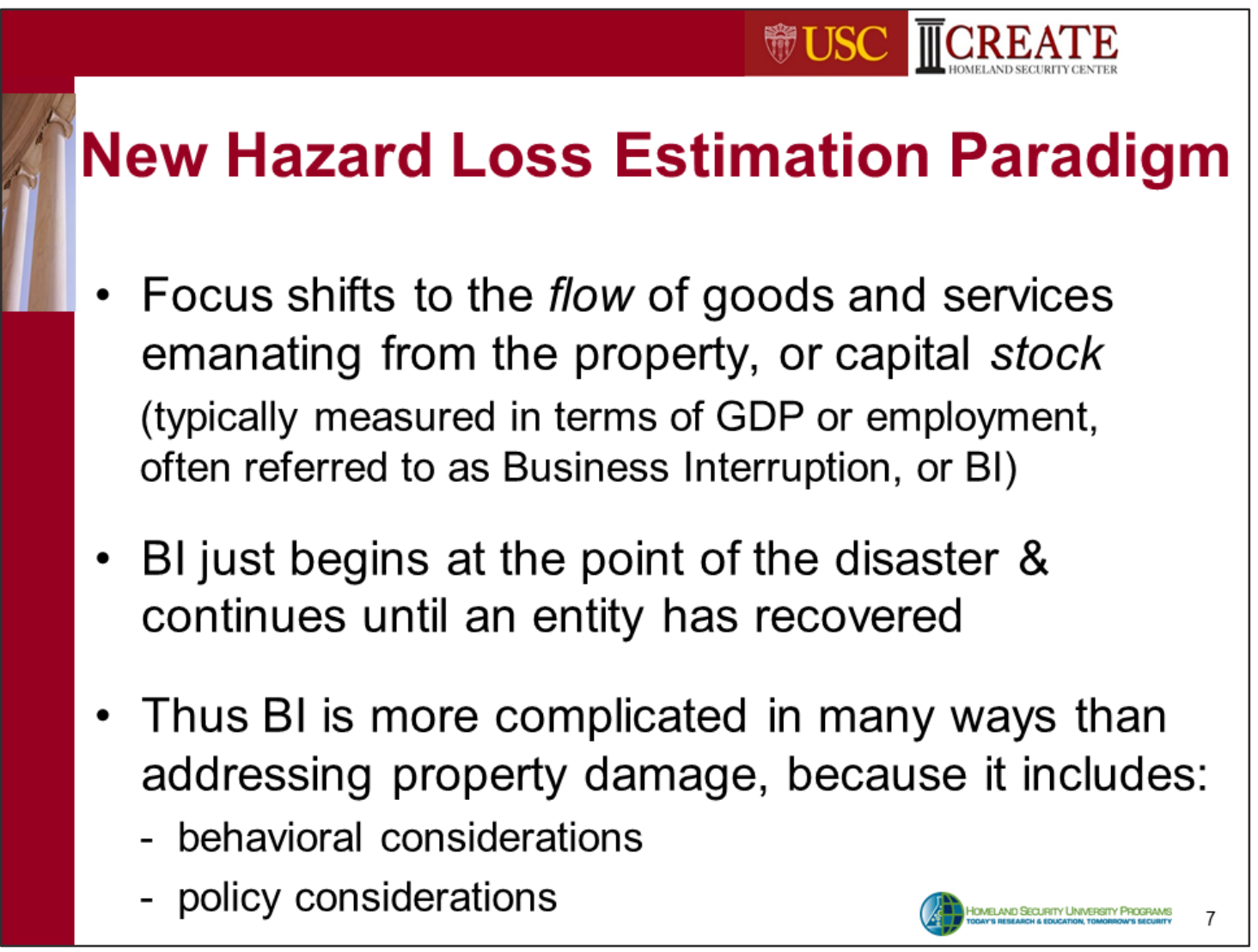

\section{USC $\overline{\mathbb{I}}$ CREATE}

\section{Measuring Econ Resilience of 9/11}

- $95 \%$ of over 1,100 WTC area firms relocated after $9 / 11$

- If all of firms in the WTC area went out of business, direct business interruption $(\mathrm{BI})$ loss would $=\$ 58.4 \mathrm{~B}$

- If all relocation were immediate, then $\mathrm{BI}=\$ 0$

- Businesses relocated 2 to 4 months, $\mathrm{BI}=\$ 16.1 \mathrm{~B}$

- Resilience Metric: Avoided Loss $\div$ Max Potential Loss

$$
\$ 42.3 B \div \$ 58.4 B=72 \%
$$




\section{USC $\overline{\mathbb{I}}$ CREEATE}

\section{Illustration: Port Valuation}

- Recent CREATE studies

- 90-day closure of Port Arthur/Port Beaumont [USCG]

- 2-day tsunami closure of Ports of LA/LB [USGS]

- Standard approach for estimating economic impact of a port: direct econ activity $X$ multiplier

- Direct economic activity $=$ Port revenue

- PA/PB: $\$ 220$ million X $5.9=\$ 1.3$ billion

- But the standard approach misses the value of the cargo \& its contribution to rest of the economy

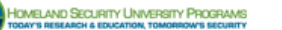

\section{USC $\overline{\mathbb{I}}$ CREATE}

Estimating Total Economic Impacts of a Port Disruption, Cargo Damages \& Terminal Downtime

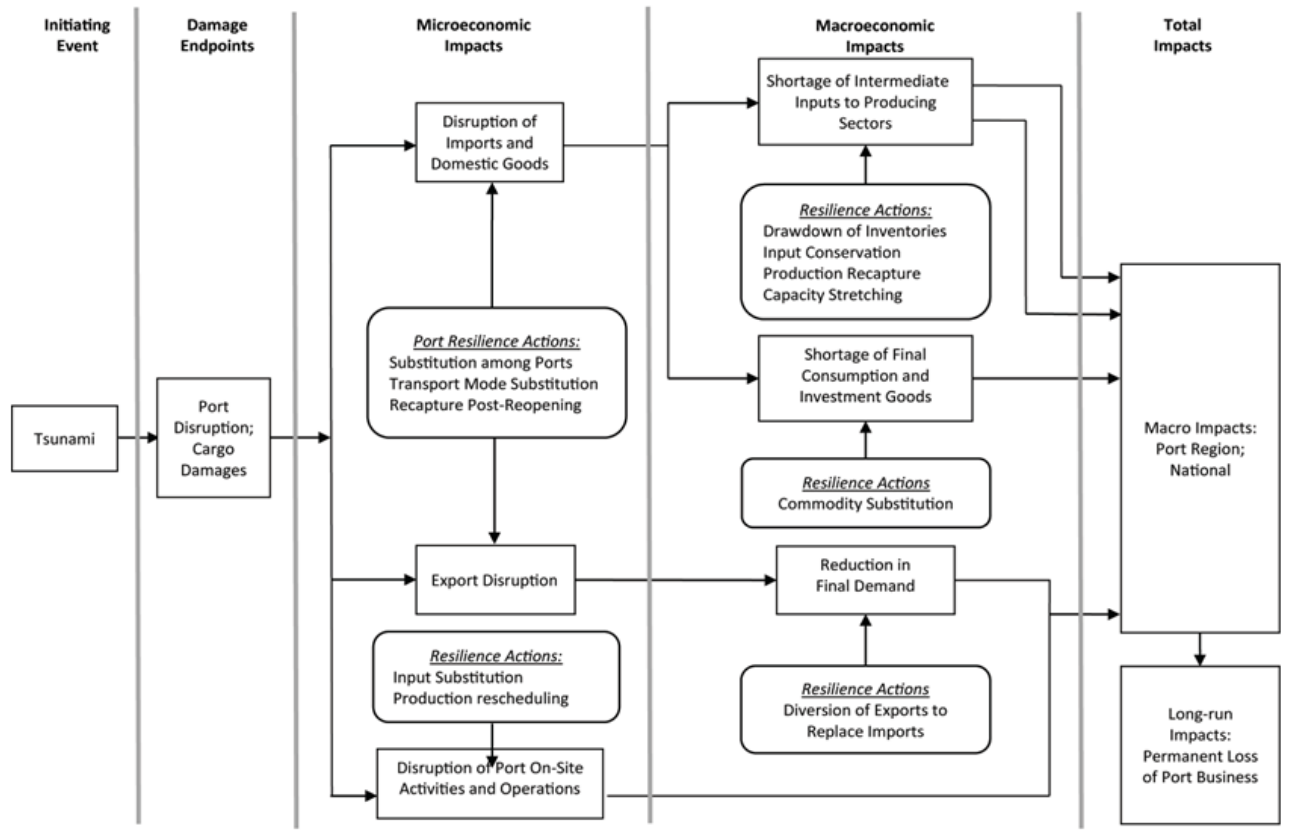




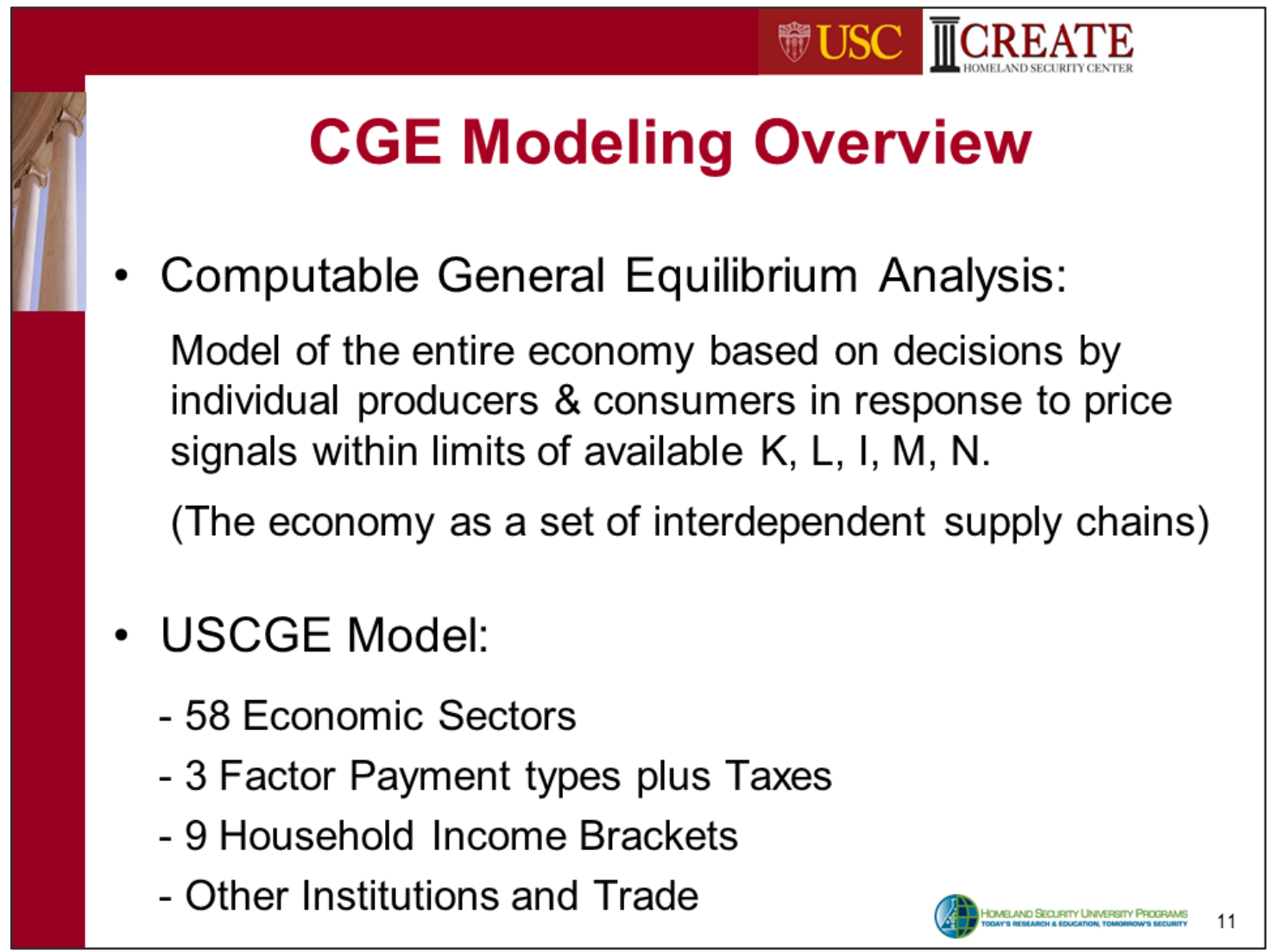

$\begin{aligned} & \text { Resilience to Port Disruptions } \\ & \text { (90-day Port Arthur Disruption; Avoided BI Losses) }\end{aligned}$
Strategic Petroleum Reserve
Ordinary Inventories of All Goods
Conservation by Customers
Import Ship Rerouting
Export Diversion (Replace Imports)
Production Rescheduling
Total Resilience (not additive)




\section{USC $\overline{\mathbb{I}}$ CREATE}

Results for Port Arthur/Beaumont (90-day Disruption; Business Interruption Loss)

- Standard estimate:

$\$ 1.3$ billion

- SOA approach (w/o resilience): $\$ 14.8$ billion

- SOA approach (w/ resilience): $\$ 4.8$ billion

- Moreover, resilience is relatively low-cost

Behavioral Linkages
- Off-site responses associated with behavioral
changes (business, household, investor, worker)
- Emanates from social amplification of risk \&
stigma effects (media coverage, rumor)
- Fear feeds on itself and spreads
- Translates into direct and indirect BI losses
- Can be 2 to 3 orders of magnitude higher




\section{USC $\overline{\mathbb{I}}$ CREATE}

\section{Behavioral Linkage Examples}

- 9/11 led to a 2-year reduction in air travel

- Workers fear of riding the subway/bus

- Business fear of staying open after dark

- Investor fear of taking high risk

- General avoidance behavior

- Gov't premature shutdown or evacuation

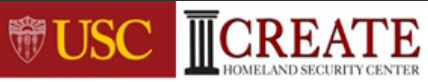

\section{RDD Attack: Direct Economic Losses}

- Casualties: \$26.2 million for labor input (modeled as decrease in $L$ productivity)

- Capital Damage: relatively minor (capital simply off limits for 30 days)

- Business interruption: \$1.4 billion of lost output (recalibration of technical efficiency parameter) 


\section{USC $\overline{\mathbb{I}}$ CREATE \\ Direct Behavioral Effects (Burns and Slovic Experiment)}

- Consumer/tourist risk perceptions

- 15 to 23\% price discount (subset of goods)

- mid-range of stigma-related WTP literature

- Employee risk perceptions

- $>25 \%$ risk premium in affected area

- much higher than WTP literature

- Investor risk perceptions (non-survey)

- $20 \%$ rate of return premium

- mid-range of property value studies, factoring in Tobin's $Q$

\section{\% USC $\overline{\mathbb{I}}$ CREEATE}

\section{Behavioral Results \& Conclusions}

- Psychological impacts of disasters are measurable (fear \& uncertainty from SAR and Stigma)

- Can translate them into changes in econ behavior

- For terrorist attacks may be especially high in L-R

- 19.4 times as high as ordinary direct effects

- 14.9 times as high as total ordinary effects

- Policy Implications

- gov't needs to provide assurances of safety

- media has a responsibility in reporting 

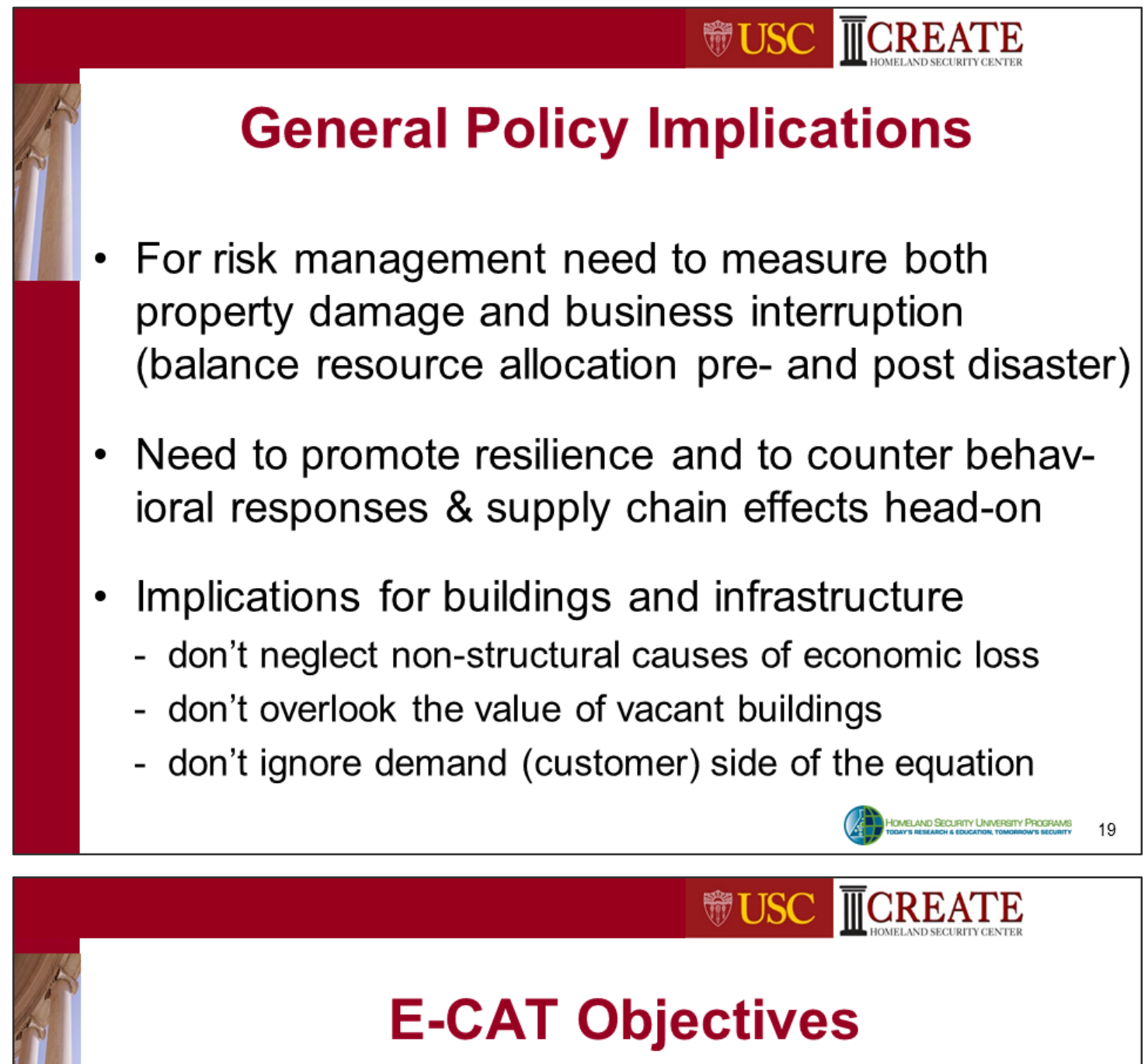

- Develop a standardized capability to estimate economic consequences of 30+ types of threats

- includes a comprehensive set of impact categories

- standardization facilitates comparisons

- Transition the research into a user-friendly, fast software tool for high-level decision-makers

- risk mgt: resource allocation across multiple threats

- rapid response: estimates for remediation/aid/recovery 

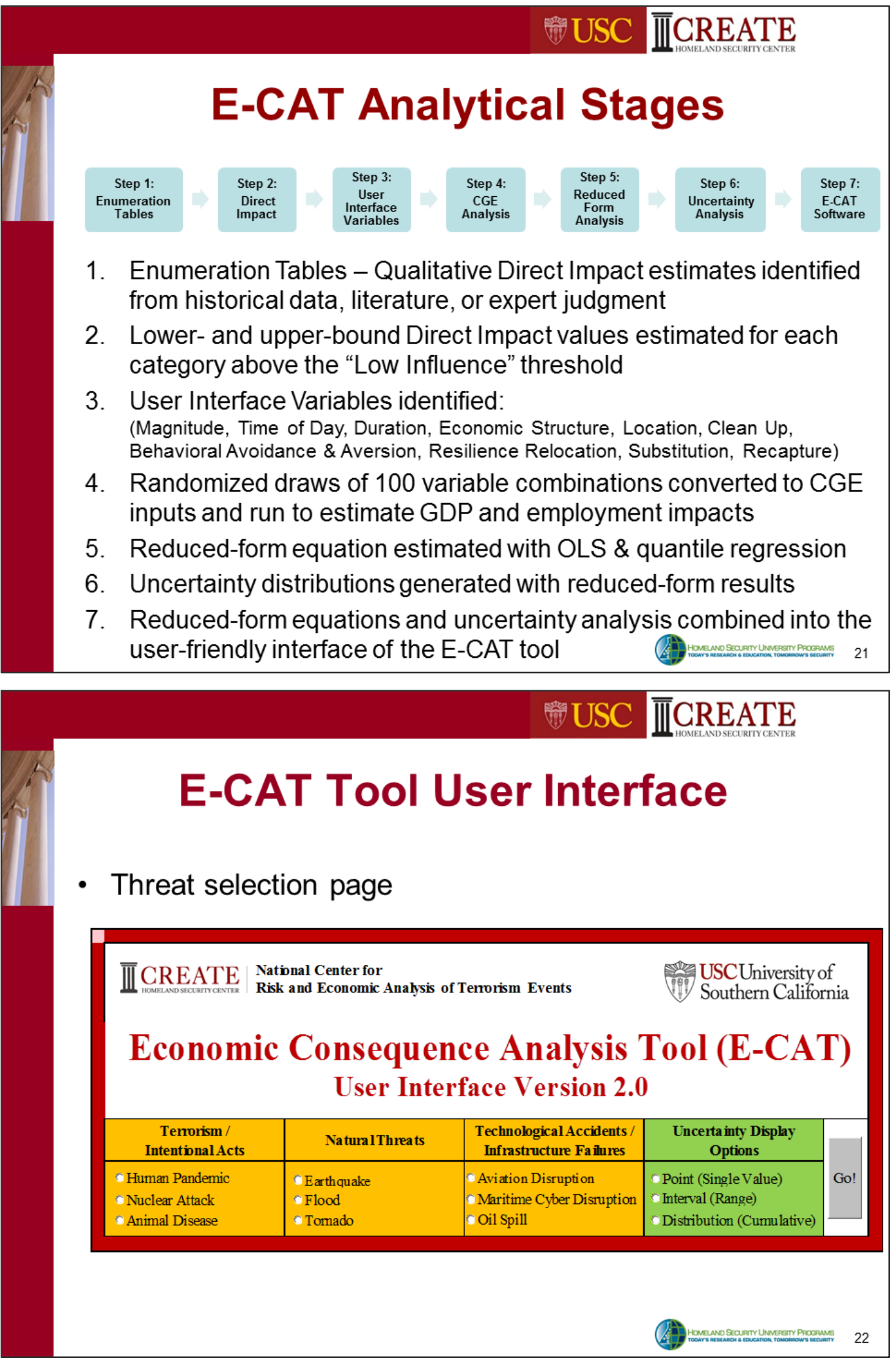


\section{WSC $\overline{\mathbb{I}}$ CREATE}

\section{E-CAT Tool Results}

- Earthquake - Default Options (Step 1)
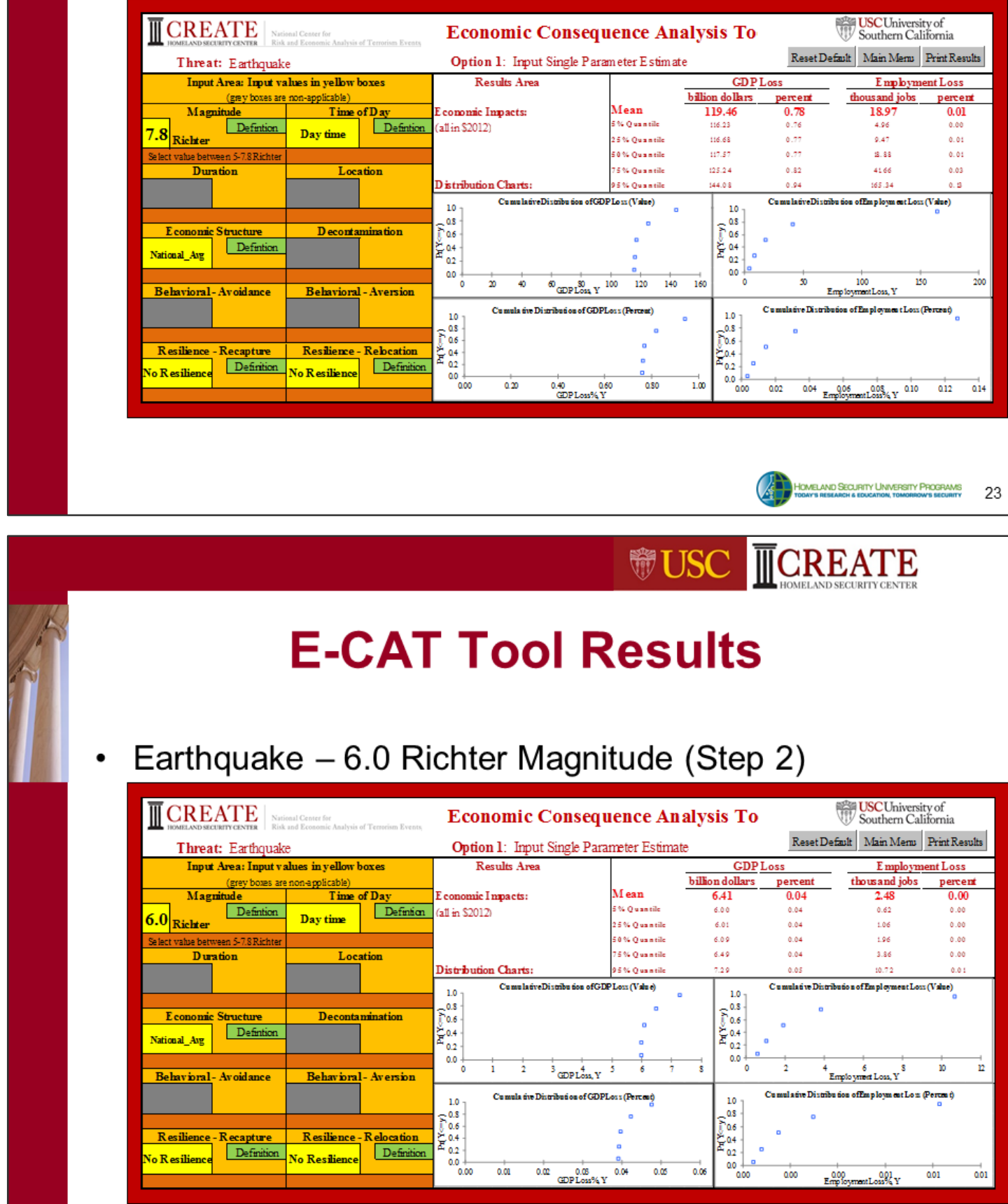


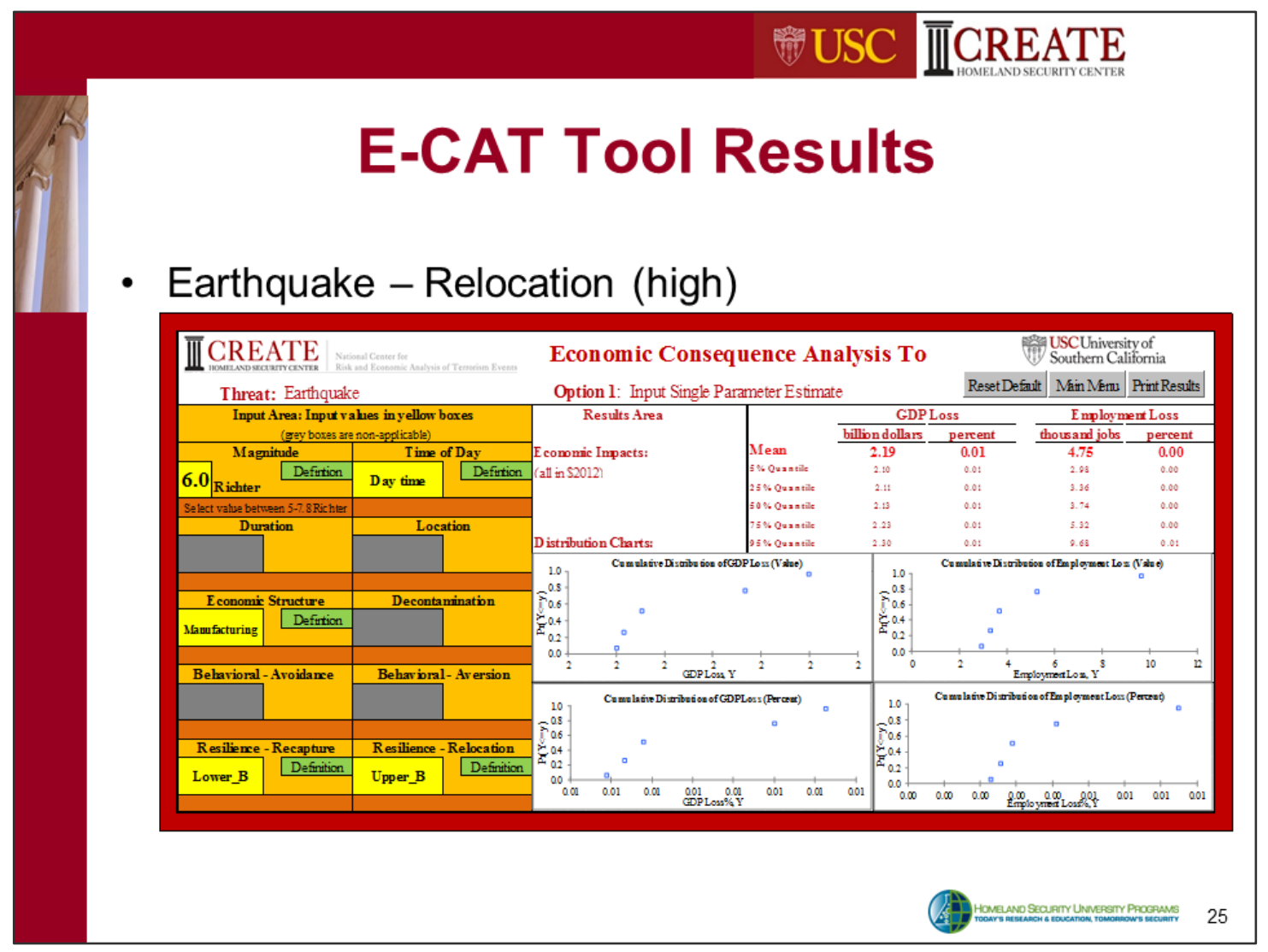

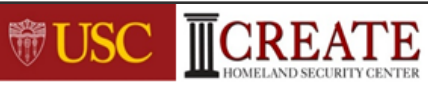

\section{E-CAT Summary}

- Comprehensive and standardized estimates of economic consequences for $30+$ HSNRC threats

- Solid foundation

- based on 10 years of research on ECA

- use of computable general equilibrium analysis

- vetted in case studies \& peer reviewed literature

- Incorporates major types of uncertainty

- User friendly

- reduced form format (single equation, major drivers)

- programmed in Excel VBA (Visual Basic Applications) 


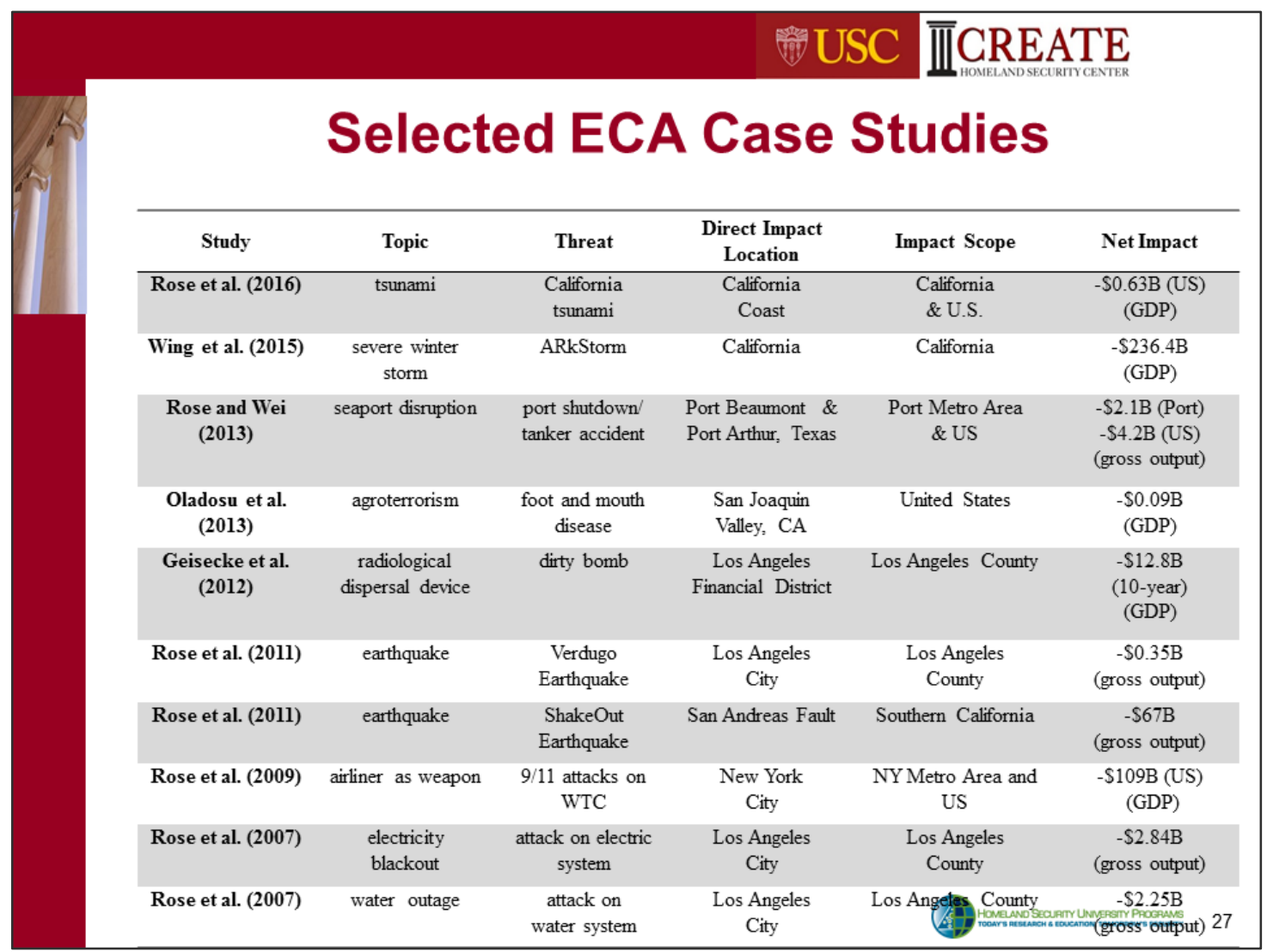

\section{NSC $\overline{\mathbb{I}}$ CREATE}

\section{Economic Consequence Publications}

- Rose, A., M. Avetisyan, W. Burns, H. Rosoff, and P. Slovic. 2016. "The Role of Behavioral Responses in the Total Economic Consequences of Terrorist Attacks on U.S. Air Travel Targets," Risk Analysis, forthcoming

- Chen, Z, A. Rose, F. Prager, and S. Chatterjee. 2016. Economic Consequences of Aviation System Disruptions: A Reduced-Form Computable General Equilibrium Analysis, Risk Analysis, forthcoming

- Rose, I. Sue Wing, D. Wei and A. Wein. 2016. "Economic Impacts of a California Tsunami," Natural Hazards Review, published on-line thus far

- Prager F., D. Wei, and Rose. 2016. Total Economic Consequences of an Influenza Outbreak in the United States," Risk Analysis, published on-line thus far

- $\quad$ Rose, A. and C. Huyck. 2016. "Improving Catastrophe Modeling for Business Interruption Insurance Needs," Risk Analysis, published on-line thus far.

- Rose, A. 2015. "Macroeconomic Consequences of Terrorist Attacks: Estimation for the Analysis of Policies and Rules," in C. Mansfield and V.K. Smith (eds.), Benefit Transfer for the Analysis of DHS Policies and Rules, Cheltenham, UK: Edward Elgar

- Rose, A. and E. Krausmann. 2013 "An Economic Framework for the Development of a Resilience Index for Business Recovery," International Journal of Disaster Risk Reduction 5(October): 73-83

- Rose, A. and D. Wei. 2013. "Estimating the Economic Consequences of a Port Shutdown: The Special Role of Resilience," Economic Systems Research 25(2): 212-32.

- Geisecke, J., A. Rose, P. Slovic et al. 2012. "Assessment of the Regional Economic Impacts of Catastrophic Events: A CGE Analysis of Resource Loss and Behavioral Effects of a Radiological Dispersion Device Attack Scenario," Risk Analysis 32: 583-600

- $\quad$ Rose, A., S. Liao and A. Bonneau. 2011. "Regional Economic Impacts of a Verdugo Earthquake Disruption of Los Angeles Water Supplies: A Computable General Equilibrium Analysis," Earthquake Spectra 27(3): 881-906.

- Rose, A. 2009. "A Framework for Analyzing and Estimating the Total Economic Impacts of a Terrorist Attack and Natural Disaster," Journal of Homeland Security and Emergency Management6: Article 4.

- Rose, A., G. Oladosu, B. Lee and G. Beeler Asay. 2009. "The Economic Impacts of the 2001 Terrorist Attacks on the World Trade Center: A Computable General Equilibrium Analysis," Peace EconomicseReace Science, and Public Policy 15: Article 6. 


\title{
Total Economic Consequences of Disasters
}

\author{
Adam Rose \\ University of Southern California
}

\begin{abstract}
References
Bruneau, M., S. Chang, R. Eguchi, G. Lee, T. O'Rourke, A. Reinhorn, M. Shinozuka, K. Tierney, W. Wallace, and D. von Winterfeldt. 2003. "A Framework to Quantitatively Assess and Enhance Seismic Resilience of Communities," Earthquake Spectra 19: 733-52.

Chen, Z, A. Rose, F. Prager, and S. Chatterjee. 2016. Economic Consequences of Aviation System Disruptions: A Reduced-Form Computable General Equilibrium Analysis, Risk Analysis, forthcoming.

Cho, S., P. Gordon, J. Moore II, H. Richardson, M. Shinozuka, and S. Chang. 2001. Integrating Transportation Network and Regional Economic Models to Estimate the Costs of a Large Urban Earthquake," Journal of Regional Science 41(1): 39-65.

Coffman, M. and I. Noy. 2011. Hurricane Iniki: Measuring the Long-Term Economic Impact of a Natural Disaster Using Synthetic Control," Environment and Development Economics 17(2): 187-205.

Cutler, H., M. Shields, D. Tavani, and S. Zahran. 2016. "Integrating Engineering Outputs from Natural Disaster Models into a Dynamic Spatial Computable General Equilibrium Model of Centerville," Sustainable and Resilient Infrastructure, forthcoming.
\end{abstract}

Cutter, S. 2016. "The Landscape of Disaster Resilience Indicators in the USA," Natural Hazards 80: 74158.

Dixon, P. and M. Rimmer. 2013. "Validation in Computable General Equilibrium Modeling," In P. Dixon and D. Jorgenson (eds.) Handbook of Computable General Equilibrium Modeling. Amsterdam: NorthHolland.

Geisecke, J., W. Burns, A. Barrett, E. Bayrak, A. Rose, P. Slovic, and M. Suher. 2012. "Assessment of the Regional Economic Impacts of Catastrophic Events: A CGE Analysis of Resource Loss and Behavioral Effects of a Radiological Dispersion Device Attack Scenario," Risk Analysis 32: 583-600.

Hallegate, S. 2014. Natural Disasters and Climate Change: An Economic Perspective. Heidelberg: Springer.

Kajitani, Y., and H. Tatano. 2009. "Estimation of Lifeline Resilience Factors based on Empirical Surveys of Japanese Industries," Earthquake Spectra 25(4): 755-76.

Okuyama, Y. 2007. "Economic Modeling for Disaster Impact Analysis: Past, Present, and Future.," Economic Systems Research 19(2): 115 - 24. 
Prager F., D. Wei, and Rose. 2016. Total Economic Consequences of an Influenza Outbreak in the United States," Risk Analysis, published on-line thus far.

Rose, A. 2009. "A Framework for Analyzing and Estimating the Total Economic Impacts of a Terrorist Attack and Natural Disaster," Journal of Homeland Security and Emergency Management 6: Article 4.

Rose, A. 2015. "Macroeconomic Consequences of Terrorist Attacks: Estimation for the Analysis of Policies and Rules," in C. Mansfield and V.K. Smith (eds.), Benefit Transfer for the Analysis of DHS Policies and Rules, Cheltenham, UK: Edward Elgar.

Rose, A. and C. Huyck. 2016. "Improving Catastrophe Modeling for Business Interruption Insurance Needs," Risk Analysis, published on-line thus far.

Rose, A. and E. Krausmann. 2013. "An Economic Framework for the Development of a Resilience Index for Business Recovery," International Journal of Disaster Risk Reduction 5(October): 73-83

Rose, A. and D. Wei. 2013. "Estimating the Economic Consequences of a Port Shutdown: The Special Role of Resilience," Economic Systems Research 25(2): 212-32.

Rose, A., S. Liao and A. Bonneau. 2011. "Regional Economic Impacts of a Verdugo Earthquake Disruption of Los Angeles Water Supplies: A Computable General Equilibrium Analysis," Earthquake Spectra 27(3): 881-906.

Rose, A., G. Oladosu, B. Lee and G. Beeler Asay. 2009. "The Economic Impacts of the 2001 Terrorist Attacks on the World Trade Center: A Computable General Equilibrium Analysis," Peace Economics, Peace Science, and Public Policy 15: Article 6.

Rose, I. Sue Wing, D. Wei and A. Wein. 2016. "Economic Impacts of a California Tsunami," Natural Hazards Review, published on-line thus far.

Rose, A., M. Avetisyan, W. Burns, H. Rosoff, and P. Slovic. 2016. "The Role of Behavioral Responses in the Total Economic Consequences of Terrorist Attacks on U.S. Air Travel Targets," Risk Analysis, forthcoming.

Santos, J. 2006. "Inoperability Input-Output Modeling of Disruptions to Interdependent Economic Systems," Systems Engineering 9(1): 20-34.

Tierney, K. 2007. "Businesses and Disasters: Vulnerability, Impacts, and Recovery," Handbook of Disasters. Heidelberg: Springer.

West, C. and D. Lenze. 1994. Modeling the Regional Impact of Natural Disaster and Recovery: A general Framework and an Application to Hurricane Andrew. International Regional Science Review 17(2): 12150. 


\section{Adam Rose}

Ralph and Goldy Lewis Hall 230

Los Angeles, CA 90089-0626

Email: Adam.Rose@usc.edu

Phone: (213) 740-8022

Fax: (213) 740-0001

Ph.D. in Economics, Cornell University

\section{Expertise}

Energy and environmental economics, public policy, natural hazards and terrorism, regional science, applied general equilibrium modeling

\section{Biographical sketch}

Adam Rose is a Research Professor in the USC Sol Price School of Public Policy.

Much of Professor Rose's research is on the economics of natural and man-made hazards. He currently serves as an advisor on disaster resilience to the United Nations Development Progamme. He is currently working on DHS-sponsored studies on economic consequence analysis of radiological and biological threats, and on the economic impacts of U.S Customs and Border Protection institutions and policies. He recently completed studies sponsored by NSF and DHS analyzing the economic consequences of behavioral reactions to terrorism. He has served on a National Research Council panel on Earthquake Resilience, was the lead researcher on the Multi-Hazard Mitigation Council report to the U.S. Congress on the net benefits of FEMA hazard mitigation grants, and coordinated 8 studies to arrive at a definitive estimate of the economic consequences of 9/11. A major focus of his research has been on resilience to natural disasters and terrorism at the levels of the individual business, market, and regional economy.

Professor Rose's other research area is the economics of energy and climate change policy. . He has served as a member of an EPA Scientific Advisory Board Panel and as a coordinating team member for the federal interagency State of the Carbon Cycle Review. As a consultant to the United Nations, he played a major role in the development of the first proposal for a system of globally tradable emission allowances. More recently, he has advised government agencies in several U.S. states on the development of cap \& trade programs and on the job impacts of climate action plans. He is currently helping governments in China and Mexico develop climate action planning capabilities.

Rose is the author of several books and 200 professional papers. He has been appointed to the editorial boards of the Energy Journal, Resource and Energy Economics, Energy Policy, Pacific and Asian Journal of Energy, Resource Policy, Journal of Regional Science, International Journal of Disaster Risk Science, and Journal of Integrated Disaster Risk Management.

Rose has served as the American Economic Association Representative to the American Association for the Advancement of Science and as a member of the Board of Directors of the Center for National Policy Resilience Forum, the National Institute of Building Sciences Multi-Hazard Mitigation Council, and the American Association of Geographers Energy and Environment Specialty Group. He is the recipient of a Woodrow Wilson Fellowship, East-West Center Fellowship, American Planning Association's Outstanding Program Planning Honor Award, Applied Technology Council Outstanding Achievement Award, and Regional Economic Models, Inc., George Treyz Award for Excellence in Economic Analysis. 
Zimmerman Abstract for the NIST Resilience Workshop

Rae Zimmerman

Professor of Planning and Public Administration

Director, Institute for Civil Infrastructure Systems (www.nyu.edu/icis)

Robert F. Wagner Graduate School of Public Service

New York University

295 Lafayette Street - 2nd floor

New York, NY 10012

Phone: 212 998-7432

FAX: 212 995-4162

Email: rae.zimmerman@nyu.edu

Infrastructure system resilience reflects interconnectivity that involves many scales, ranging from global systems to facility components, and has human and physical dimensions.

Zimmerman's research primarily emphasizes infrastructures, their interconnections, and the effect of these characteristics on the services they provide for society. This work is primarily used as a foundation for modeling of infrastructure vulnerability and recovery capacity relating to accidents and weather related extremes. She identifies and evaluates vulnerability and recovery rates for infrastructure characteristics and services using event-based information or scenarios to develop and analyze indicators, metrics, and rates of actual and anticipated consequences and recovery. Her research includes the role of risk communication on outcome severity following accidents, applied primarily to transportation and energy pipelines. Her interests cover policy responses to disasters and their effect on infrastructure service performance. Funding is primarily through National Science Foundation (NSF) and U.S. Department of Transportation (UTRC Region 2). As part of the research Zimmerman has produced numerous publications, participated as Principal Investigator on about three dozen grants, and held other roles in a dozen other projects. Education: B.A. in Chemistry, University of California (Berkeley); Master of City Planning, University of Pennsylvania; and a Ph.D. in planning, Columbia University. URL: http://wagner.nyu.edu/zimmerman 


\title{
Infrastructure System Interconnectivity Effects on Resilience
}

\author{
Rae Zimmerman \\ Professor of Planning and Public Administration \\ New York University - Wagner School \\ International Workshop on Modeling of Physical, Economic, and \\ Social Systems for Resilience Assessment \\ National Institute of Standards and Technology \\ October 19-21, 2016 \\ Washington Dulles Airport Marriott, Dulles, VA
}

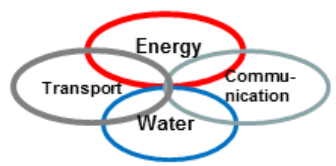

Cite material used from this presentation as: R. Zimmerman (2016) Infrastructure System Interconnectivity Effects on Resilience, presented at the National Institute of Standards and Technology International Workshop on Modeling of Physical, Economic, and Social Systems for Resilience Assessment, Dulles, VA; include other references cited within this presentation.

\section{Introduction}

\section{Concepts and Scope}

- Resilience and interconnectivity are addressed for infrastructure and its services including relationships to society and the environment.

- Resilience is used in numerous contexts. A generic concept for infrastructure resilience used here is: "bounce back," "bounce forward" or don't bounce at all.[1]

- Interconnectivity connotes multiple-directional or one-directional dependencies or interdependencies among infrastructures. It can influence vulnerability and resilience.[2]

- Many types of infrastructure resilience and interconnectivity exist and at many scales.

\section{The Backdrop}

- Environmental threats are increasing in some areas, but more importantly the consequences are increasing regardless of the extent of the threats.

- Condition, performance and investment typically do not include interconnectivity, which can exacerbate weaknesses in single infrastructures.

- Siting, material, structural, design, and resource (financial and institutional) factors contribute to the resilience of interconnectivity and resilience relationships to society.

\section{Going Forward}

- Interconnectivity, its contribution to infrastructure vulnerability, and its value in promoting resilience are key inputs to infrastructure resilience modeling.

Sources:

[1]L. Vale (2014) The politics of resilient cities: Whose resilience and whose city? Building Research \& Information, 42(2), 191-201; summarized in R Zimmerman (2016) Resilient Urban Infrastructure for Adapting to Environmental Disruptions, in Handbook on Urbanization and Global Environmental Change, K. C. Seto, W. D. Solecki, and C. A. Griffith, eds., London, UK: Routledge, 2016, pp. 488-512; 492.

[2]S.M. Rinaldi, J.P. Peerenboom and T.K. Kelly (December 2001) Identifying, understanding and analyzing critical infrastructure interdependencies, IEEE Control Systems Magazine, 11-25 


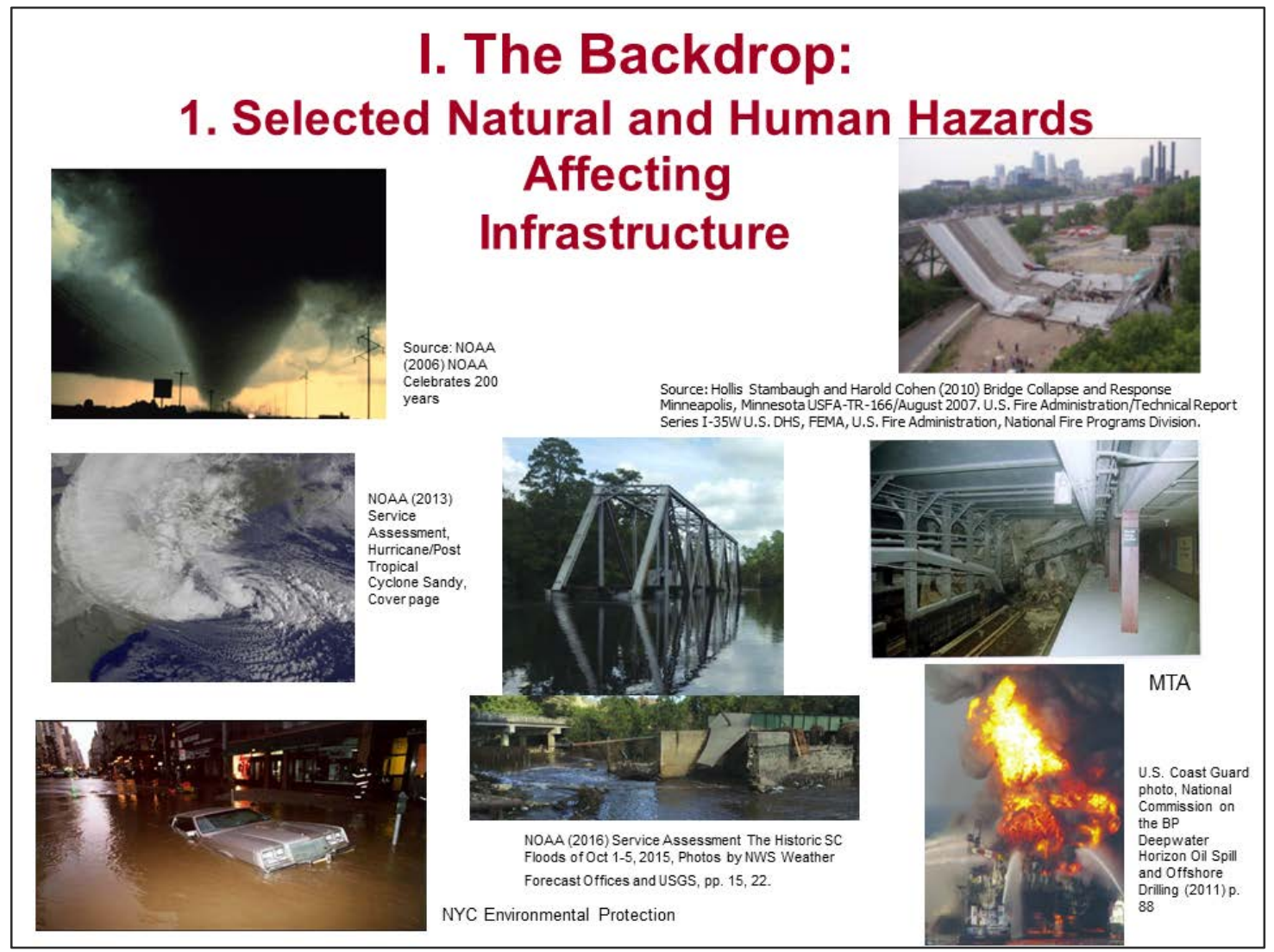

\section{Selected Trends in Natural Hazards}

- NOAA's National Climate Data Center (2016) reported the continued prominence of severe storms and flooding among other weather or climate related events whose losses exceed a billion dollars.[1]

- NOAA's National Hurricane Center reported that the recent couple of decades accounted for the most severe storms in dollar losses and other factors.[2]

- The National Climate Assessment trends and projections reported increases in most climate change-related extreme phenomena: temperature, sea level rise, heavy precipitation, hurricanes.[3]

- Swiss Re reported generally increasing trends in catastrophic losses (according to their threshold definitions based on "insured losses (claims), economic losses, and casualties"): "353 catastrophe events across the world in 2015, up from 339 in 2014. Of those, 198 were natural catastrophes, the highest ever recorded in one year," most of which are weather-related.[4]

- NOAA reported that records are being exceeded or almost being exceeded for temperature (NOAA's State of the Climate), hurricane extremes, and ice loss (NOAA National Snow and Ice Data Center).[5]

Sources

[1]NOAA National Climate Data Center (2016)

[2]Blake, E.S., C.W. Landsea and E.J. Gibney (August 2011) The Deadliest, Costliest, and Most Intense United States Tropical Cyclones from 1851 to 2010 NWS NHC-6, available at http://www.nhc.noaa.gov/pdf/nws-nhc-6.pdf

[3]Walsh, J., et al. (2014) Ch. 2: Our Changing Climate. Climate Change Impacts in the United States: The Third National Climate Assessment, J. M. Melillo, Terese (T.C.) Richmond, and G. W. Yohe, Eds., U.S. Global Change Research Program, 19-67. doi:10.7930/J0KW5CXT. pp. 19-67.Page 20-

21. http://nca2014.globalchange.gov/re port/our-chan ging-climate/introduction

[4]Swiss Re (2016) Sigma Report No. 1/2016, pages 2 and 5

[5]NOAA (2016) State of the Climate; National Snow and Ice Data Center

Note: These findings can vary by location. 


\section{Infrastructure Condition and Selected Factors Influencing Condition}

Condition 2013: U.S. average is "D", from D- (water) to C+ (bridges).[1] Age: NYC infrastructure age ranges, according to CUF[2], are: sewer mains and subway facilities (about $80-90$ years old) to airport support facilities (40-50 years old); water mains and bridges are in the middle.

Design and environmental issues: However, age may not be the whole story, since many bridge collapses have occurred in newer bridges.[3]

Usage and capacity:

- According to EIA, energy production and use steadily increased nationwide.[4]

- Vehicle Miles of Travel steadily increases (U.S. DOT[5]) and transit ridership also (APTA)

Vehicle Registrations, Fuel Consumption, and

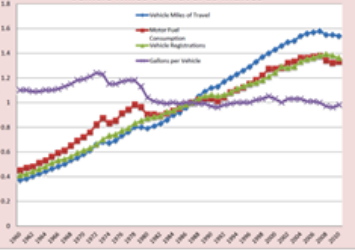

- CTIA indicates the exponential growth in cellular technologies.[6]

- The Pew Center [7] indicates dramatic increases in information technology usage, i.e., for the internet, computers and cell phones. Investment: ASCE estimates a \$3.6 trillion need to 2020.[1]

Sources: [1] ASCE (2013) Cumulative Infrastructure Needs by System Based on Current Trends Extended to 2020 (Dollars in $\$ 2010$ billions) http://www.infrastructurereportcard.org/a/\#p/grade-sheet/americas-infrastructure-investment-needs [2]Center for Urban Future (2015) Caution Ahead, New York, NY: CUF, p. 11; [3]R. Zimmerman, Transport the Environment and Security, 2012; [4] http://www.eia.gov/totalenergy/data/monthly/pdf/mer.pdf, [5] U.S. DOT, FHWA Highway Statistics (April 2013) [6] CTIA (2014) Annual Wireless Industry Survey; [7] Pew Research Center, February 2014, "The Web at 25," summarized from pp. 4,11 and 13 Available at: http://www. pewinternet.org/2014/02/27/the-web-at-25-in-the-u-s.

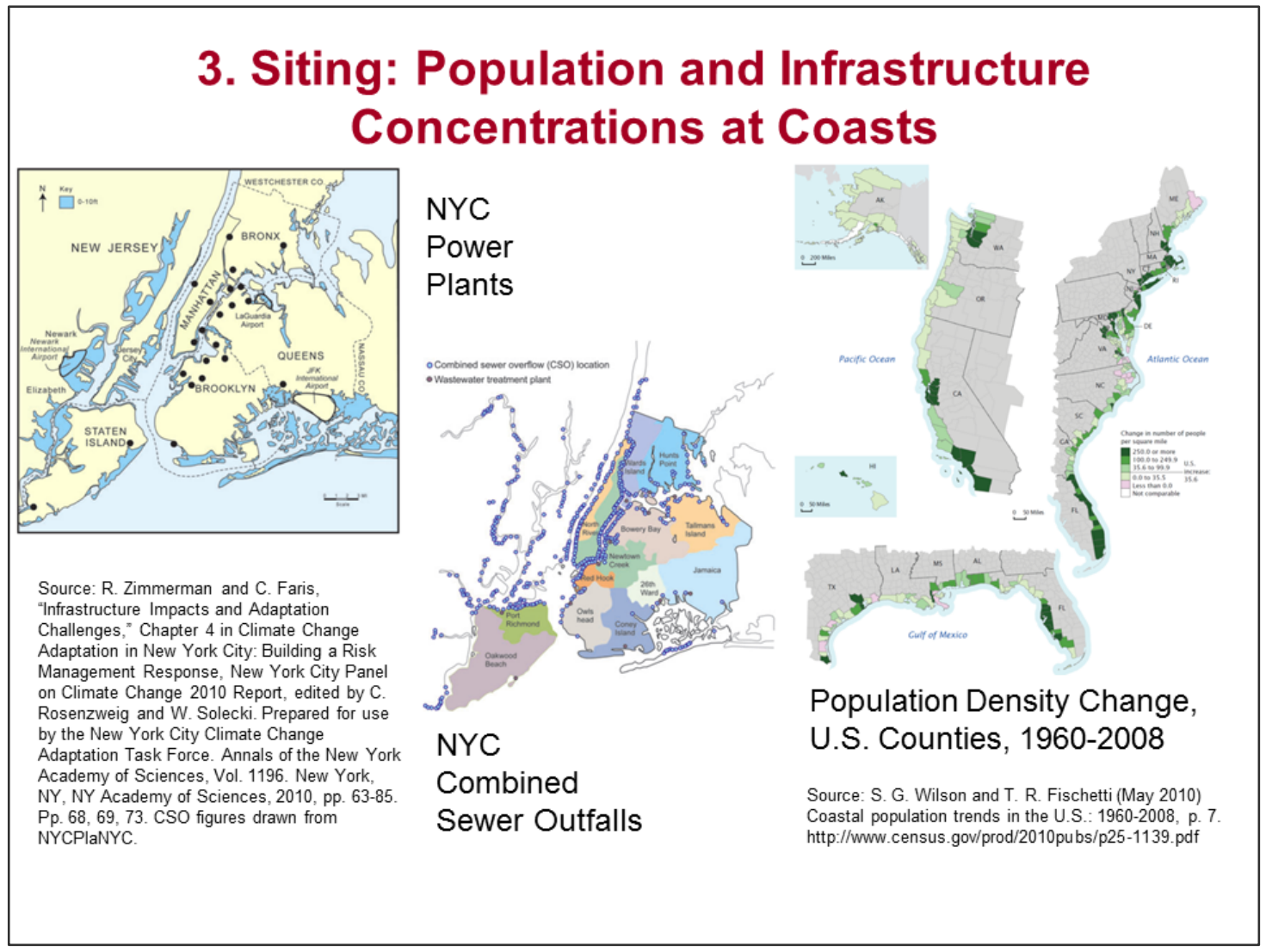




\section{Siting Consequences: NYC Subway Stations Flooded in the August 8, 2007 Storm}

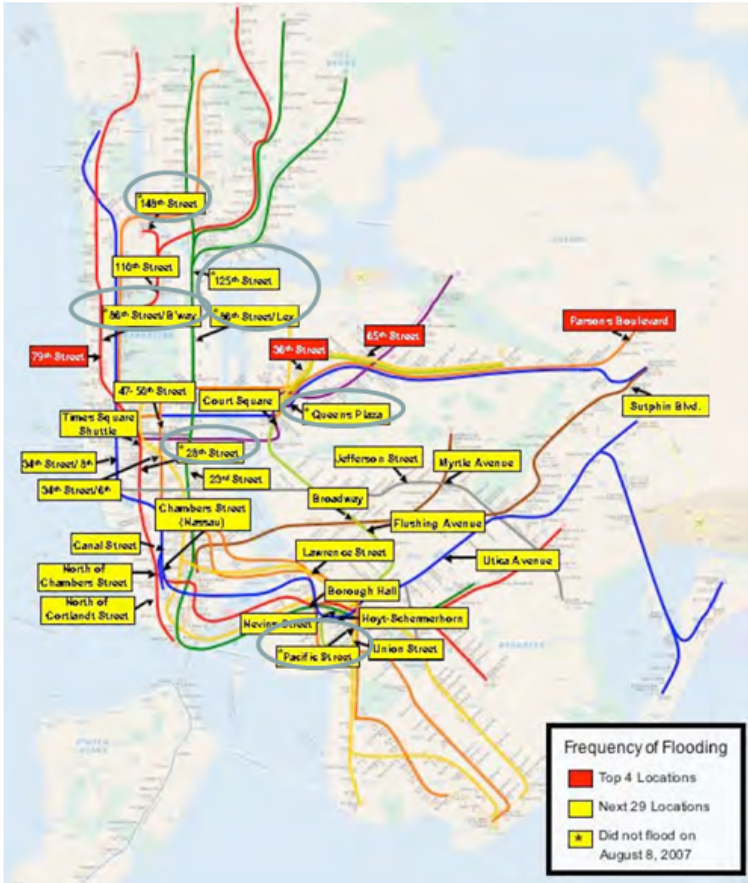

September 8, 2004 storm 7 lines were disrupted

April 15, 2007, 12 were disrupted, July 18,

2007, 9 were disrupted,

- August 8, 2007, 19 lines had reduced or no service

Most of the lines were back within 12 hours.

In 2007, some outer areas where poorer populations live were spared flooding, but others were flooded (Brooklyn and Queens)

- 31 of 468 stations; 15 of 25 lines vulnerable

Source: Metropolitan Transportation Authority (2007), August 8, 2007 Storm Report, New York, NY: MTA, p. 49 (Ovals added signifying affected stations in 2007).

\section{Materials: Example - Non-Absorbent, Impervious Surfaces Selected U.S. Cities}

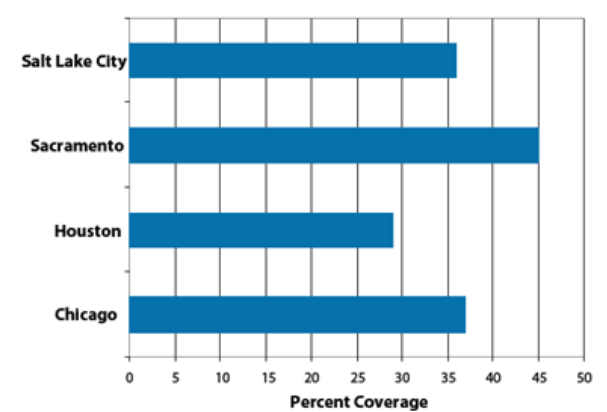

Extent of pavement coverage

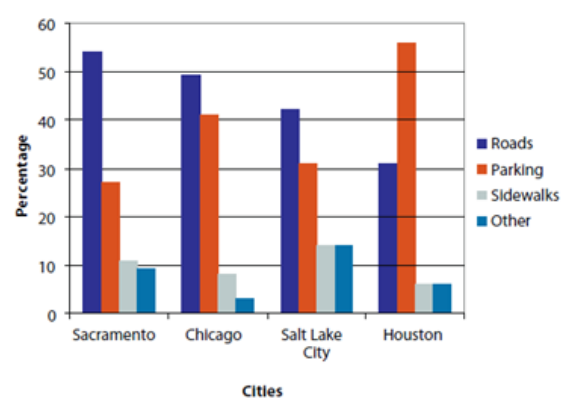

Extent of pavement coverage by land use

Source: U.S. EPA (October 2008) Reducing Urban Heat Islands: Compendium of Strategies, Chapter 5, ${ }^{a} \mathrm{Cool}$

Pavements," p. 1 and p. 12, http://www.epa.gov/heatisld/resources/compendium.htmLawrence Berkeley National Labs.

From Litman (2011):

- Roadway area is the largest contributor to impervious surfaces for housing lots (2000 sq ft)

- Roadway area is greatest for single-family large lot homes and least for high-rise apartments

- Other impervious surface contributors are the housing area itself and parking

Source: T. Litman (2011) Why and how to reduce the amount of land paved for roads and parking facilities, Environmental Practice 13 (1), p. 40. 


\section{Resources: Example of Cities with Increasingly Suburban Poor Populations and Availability of Rail Transit}

- About a dozen cities were identified by the Brookings Institution where the ratio of 2010 to 2000 share of poor populations exceeded $20 \%$.[1]

- These cities were aligned with heavy rail, commuter rail and light rail, and the percent share of these types of rail transit were computed for each city.[2]

- Results showed that only one city, Chicago, exceeded a ten percent share for any rail type, and most of the cities, except for Chicago accounted for less than $5 \%$ of the share.[2]

Sources:

[1]E. Kneebone, The Brookings Institution.

[2] R. Zimmerman (2012) Transport, the Environment and Security. Making the Connection, Cheltenham, UK and Northampton, MA: Edward

Elgar Publishing, Ltd., p. 16. http://www.e-elgar.com/bookentry_mainUS.lasso?id=13884; Information on trips was compiled from U.S. DOT

National Transit Database.

- Generic infrastructure interconnections:

\section{Infrastructure Interconnections: \\ Attribute Summary}

- Electric Power - with Transportation, Water, and IT;

- Transportation - with Water, and IT;

- Water - IT

- Specification of the direction and magnitude of flows of goods, services, and/or information among infrastructures

- Scale: Component-level connections (ranging from small parts to large multiple interrelated systems ${ }^{*}$ )

- Types:

- Temporal Interconnections

- Physical ${ }^{*}$

- Cyber*

- Spatial Interconnections (geographic) ${ }^{*}$

- Logical*

- Implications: Impact and Likelihood of Cascading Failures from Interconnections 


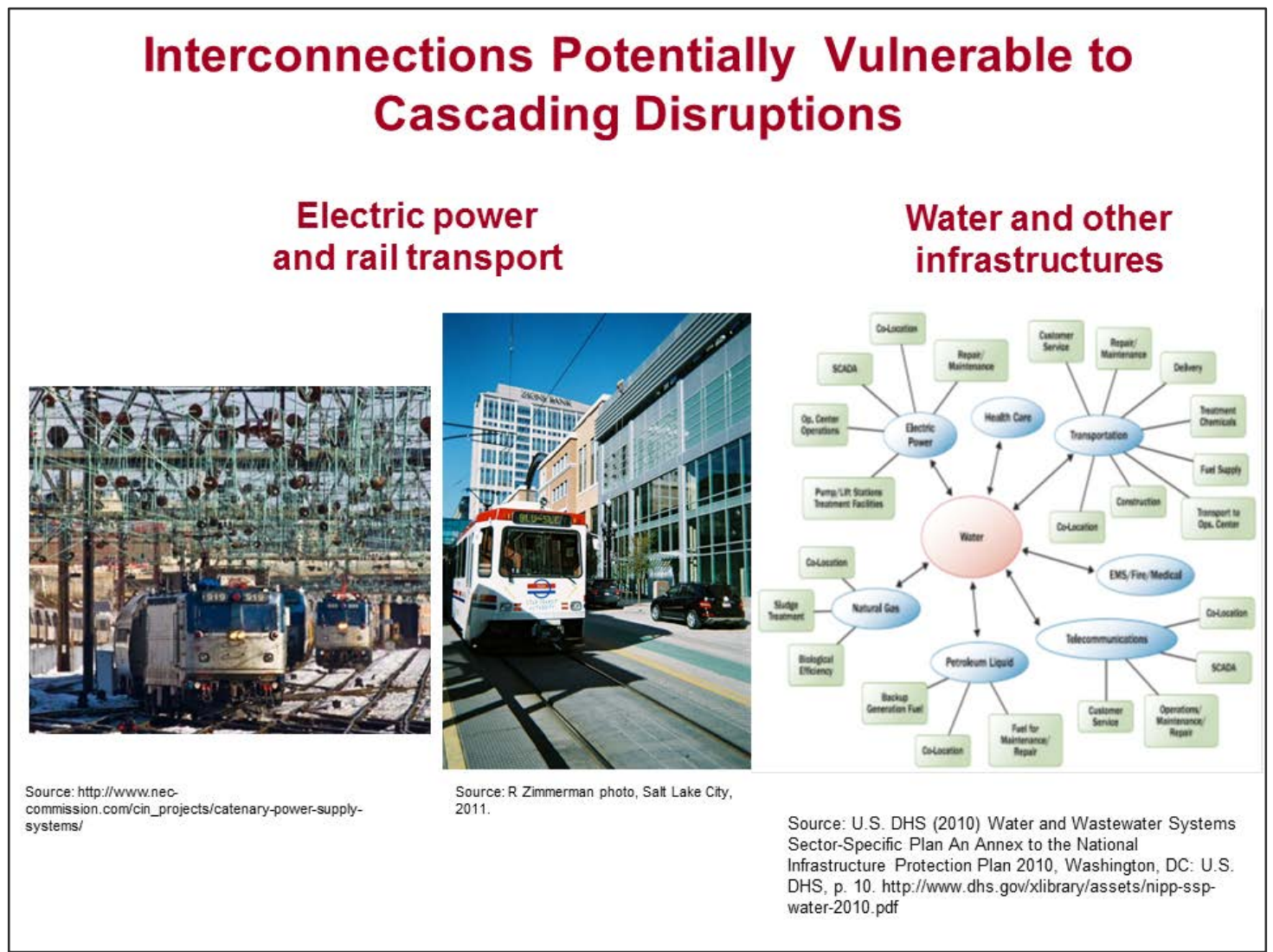

\section{Energy-Water Connections: Quantified Sector Level Flows Nationwide, U.S., 2011}

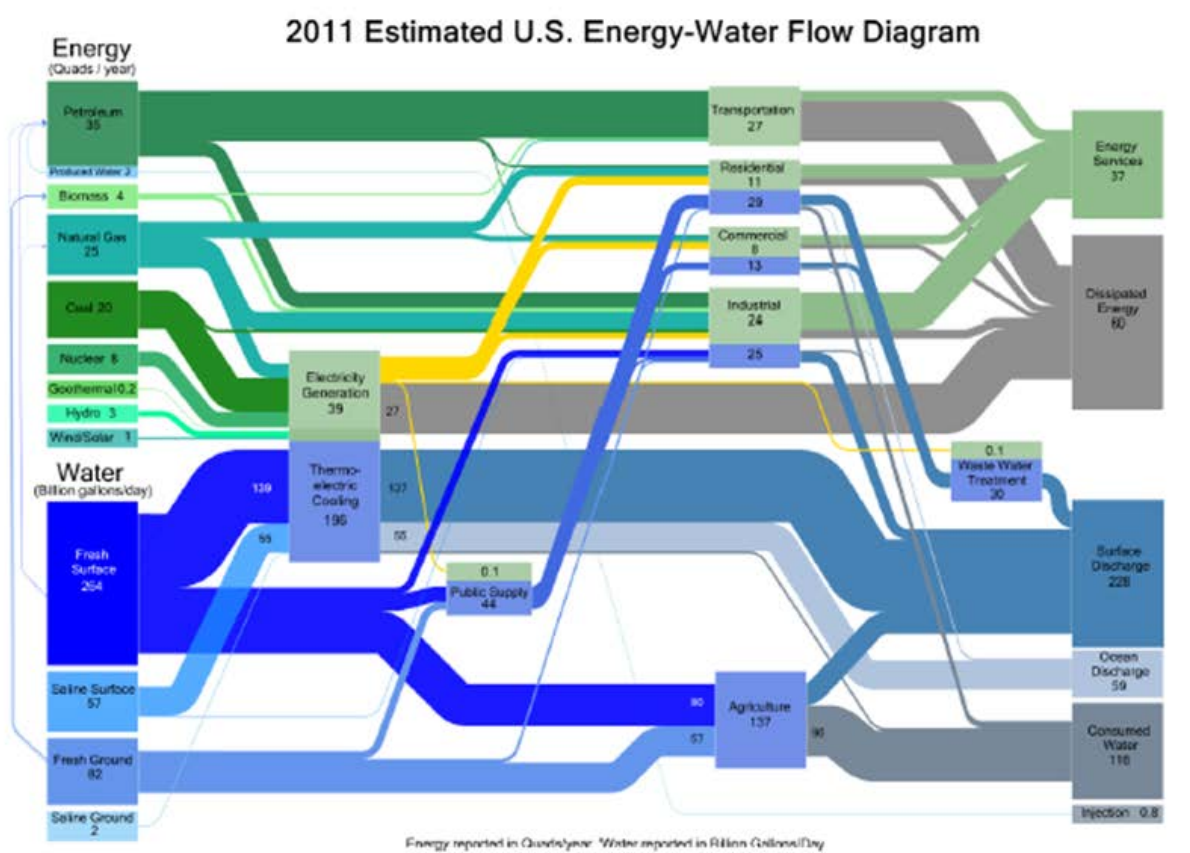

Source: U.S. Department of Energy (June 2014) The Water-Energy Nexus: Challenges and Opportunities. Washington, D.C.: U.S. DOE, p. 210. 


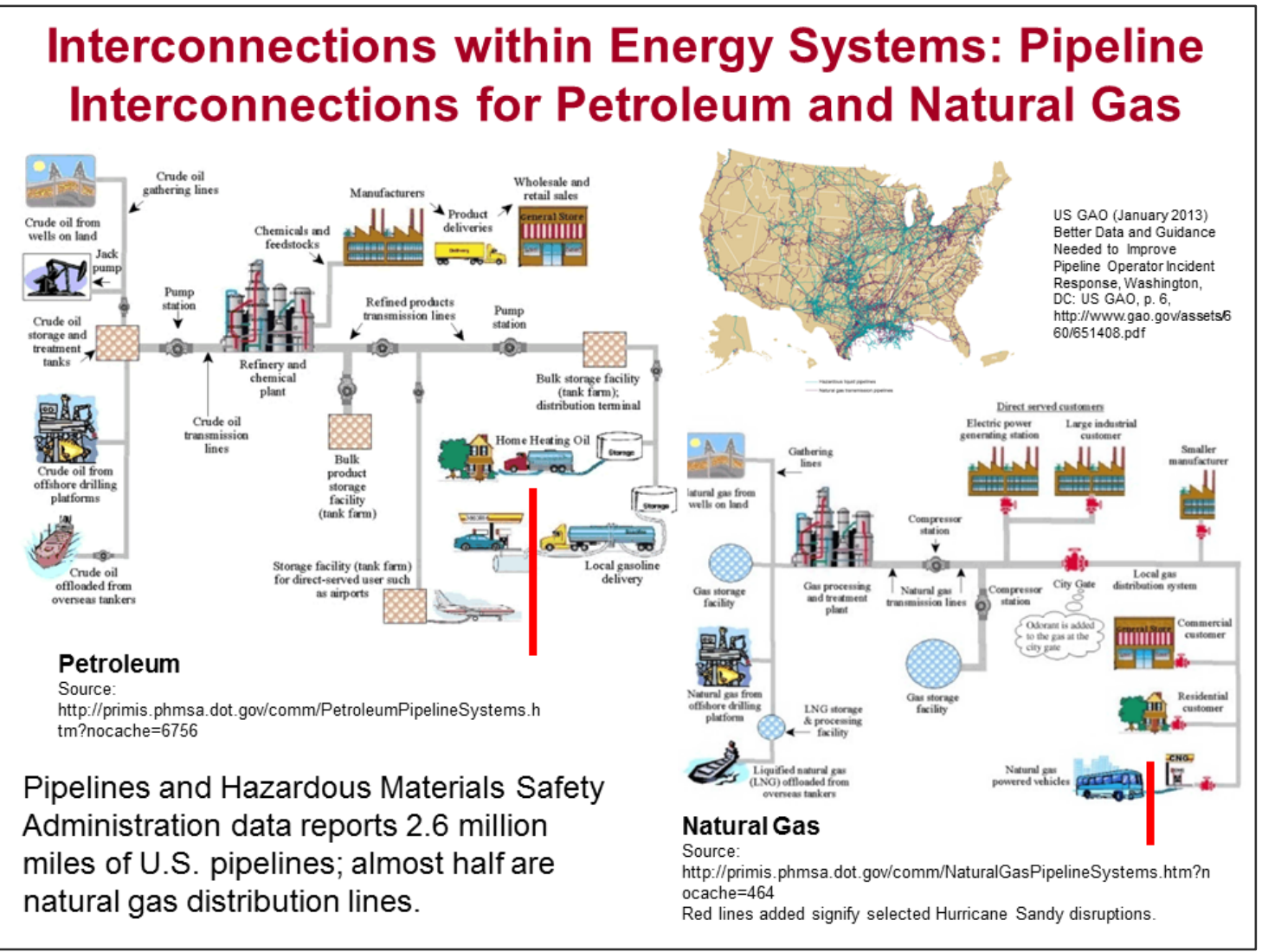

\section{Concentration as a Characteristic of Interconnections: Transportation Example}

- Transportation ridership and its infrastructure are highly concentrated in a few states

- About half of U.S. transit ridership, transit user populations, and transportation infrastructure is concentrated in just a handful of states.[1,2]

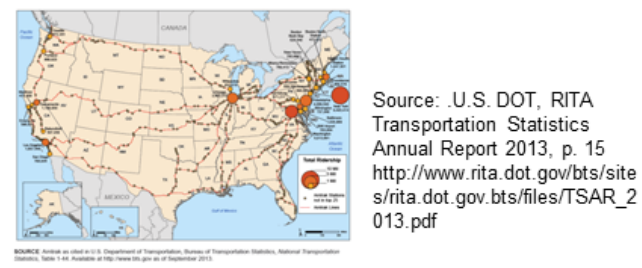

- About half of automobile ridership (annual vehicle miles of travel) is concentrated in 9 states.[1]

- About half of roadway mileage is within 14 states.[1]

- Half of enplanements occur at under five \% of U.S. major airports.[1]

- Transportation infrastructure and use is similarly concentrated within just a few urban areas.[3,4]

Sources:

[1] Calculated from U.S. Department of Transportation, Bureau of Transportation Statistics databases

[2] R. Zimmerman, "Critical Infrastructure and Interdependency," Chapter 35 in The McGraw-Hill Homeland Security Handbook, edited by D. G. Kamien. New York, NY: The McGraw-Hill Companies, Inc., 2006, pp. 523-545; p. 532

[3] R. Zimmerman, "Critical Infrastructure and Interdependency Revisited," Chapter 20 in The McGraw-Hill Homeland Security Handbook - 2nd edition, edited by D. G. Kamien. New York, NY: The McGraw-Hill Companies, Inc., 2012, pp. 437-460. Page 446-447.

[4] R. Zimmerman (2012) Transport, the Environment and Security: Making the Connection, Cheltenham, UK and Northampton, MA: Edward Elgar 


\section{Concentration: Energy, Water and Telecom}

\section{Energy}

- About half of the petroleum refineries in the U.S. are in just a few states.[1]

- About half of the power plants in the U.S. are in about a dozen states.[1]

- Henry's Hub is an area in the U.S. where major oil distribution lines converge.[2]

- Energy transmission lines tend to enter urban areas at only a few locations.

Water

- About $7 \%$ of community water supply systems serve half of the population. $[3,4]$

- A similar disproportionality exists in the area of wastewater treatment systems.

- Distribution and storage facilities for urban areas tend to be highly concentrated.

Telecom [5]

"The growth in cellular technology has been very dramatic. According to the 2010 Cellular Telecommunications and Internet Association (CTIA) semi-annual survey of the wireless industry, between 1985 and 2010, the number of estimated connections for all uses increased almost 1000-fold (889 times); Interestingly, this reflects a growing centralization and hence vulnerability, since ... from 1985 to 2010, the connections per cell site grew from 373 to 1,197."

Sources:

[1] R. Zimmerman, "Critical Infrastructure and Interdependency Revisited," Chapter 20 in The McGraw-Hill Homeland Security Handbook - 2nd edition, edited by D. G. Kamien. New York, NY: The McGraw-Hill Companies, Inc., 2012, pp. 437-460. Page 446.

[2] R. Zimmerman (2012) Transport, the Environment and Security: Making the Connection, Cheltenham, UK and Northampton, MA: Edward Elgar Publishing.

[3] R. Zimmerman, "Critical Infrastructure and Interdependency Revisited," 2012, Page 446

[4] R. Zimmerman, "Water," Chapter 5 in Digital Infrastructures: Enabling Civil and Environmental Systems through Information Technology, edited by R Zimmerman and T. Horan. London, UK: Routledge, 2004, pp. 75-95; p. 81.

5] R. Zimmerman (2012), citing CTIA

\section{Consequences of Interdependencies: General Failure Modes (all infrastructures)}

- Obliteration/inundation, e.g., submersion or debris entrainment

- Undermining

- Disintegration

- Physical impingement / structural collapse

- Corrosion; other material failures and distortions

- Facility inoperability through functional impairment

Service disruption

- Social, economic and environmental consequences 

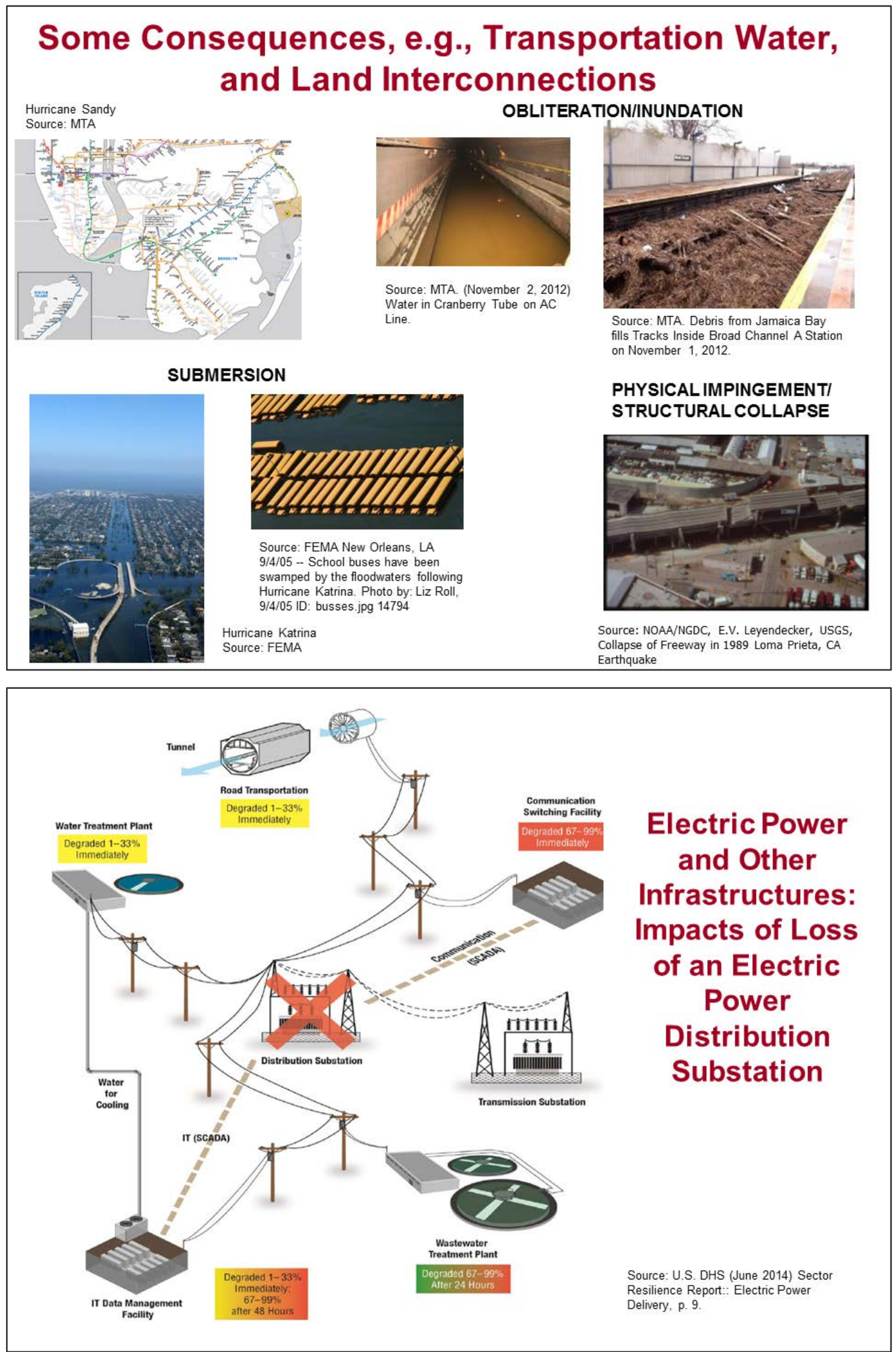


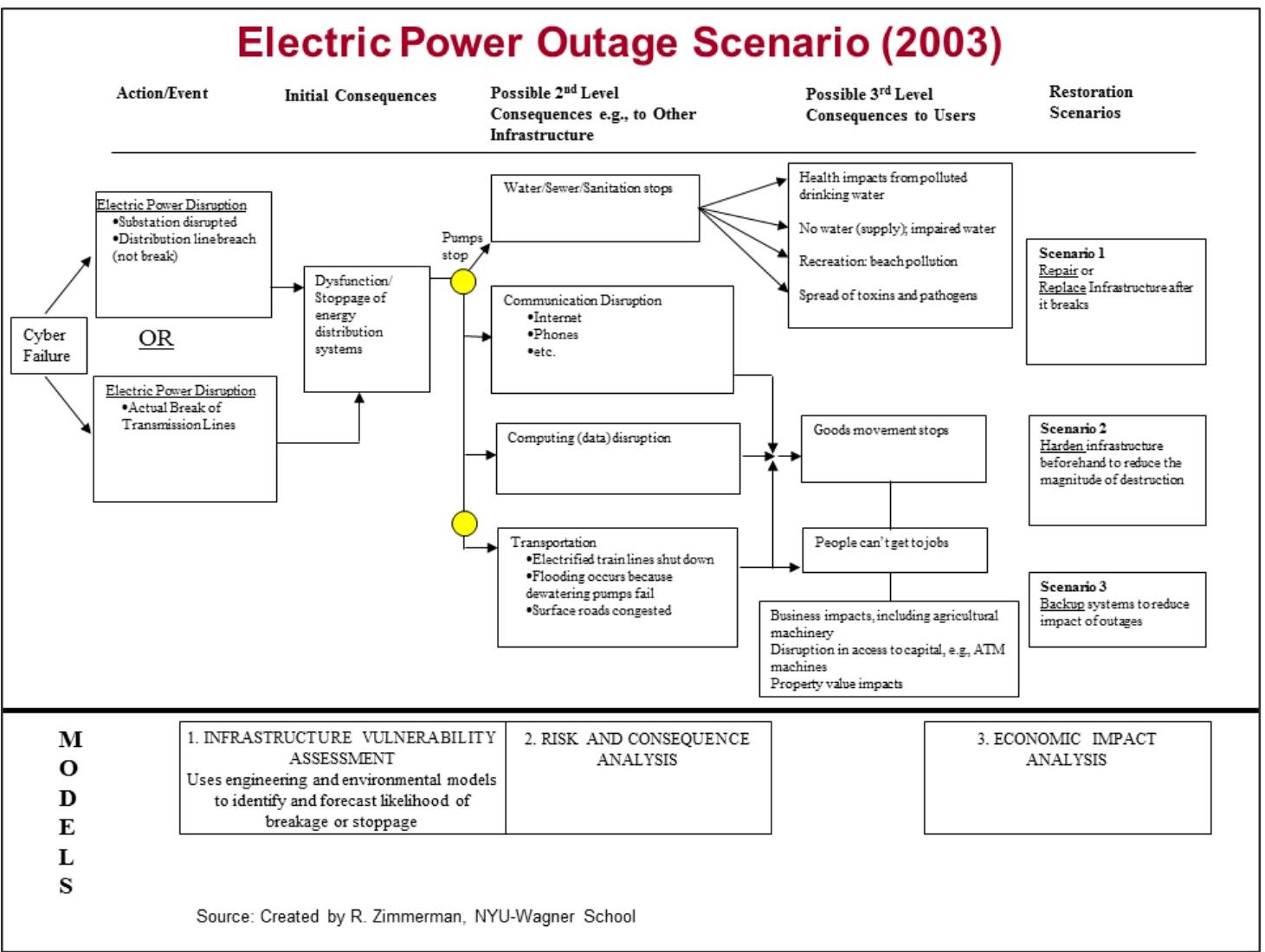

\title{
Using Energy Connections with Transportation and Water to Estimate Recovery from Electricity Outages, 2003 Blackout
}

\author{
Outage Durations, August 2003 Blackout (Total Duration = approx. 42-72 hrs)$$
\mathrm{T}(\mathrm{i}) / \mathrm{T}(\mathrm{e})^{*}
$$ \\ Infrastructure Outage/Electricity Outage \\ Transit-electrified rail (NYC) \\ Traffic Signals (NYC) \\ 2.6 \\ Cleveland Water Supply System \\ 2.0 \\ Detroit Water Supply System \\ 3.0
}

Source:* R. Zimmerman and C. Restrepo, "The Next Step: Quantifying Infrastructure Interdependencies to Improve Security," International Journal of Critical Infrastructures, Vol. 2, Nos. 2/3,2006, pp. 215-230. Summarized from Table 3 


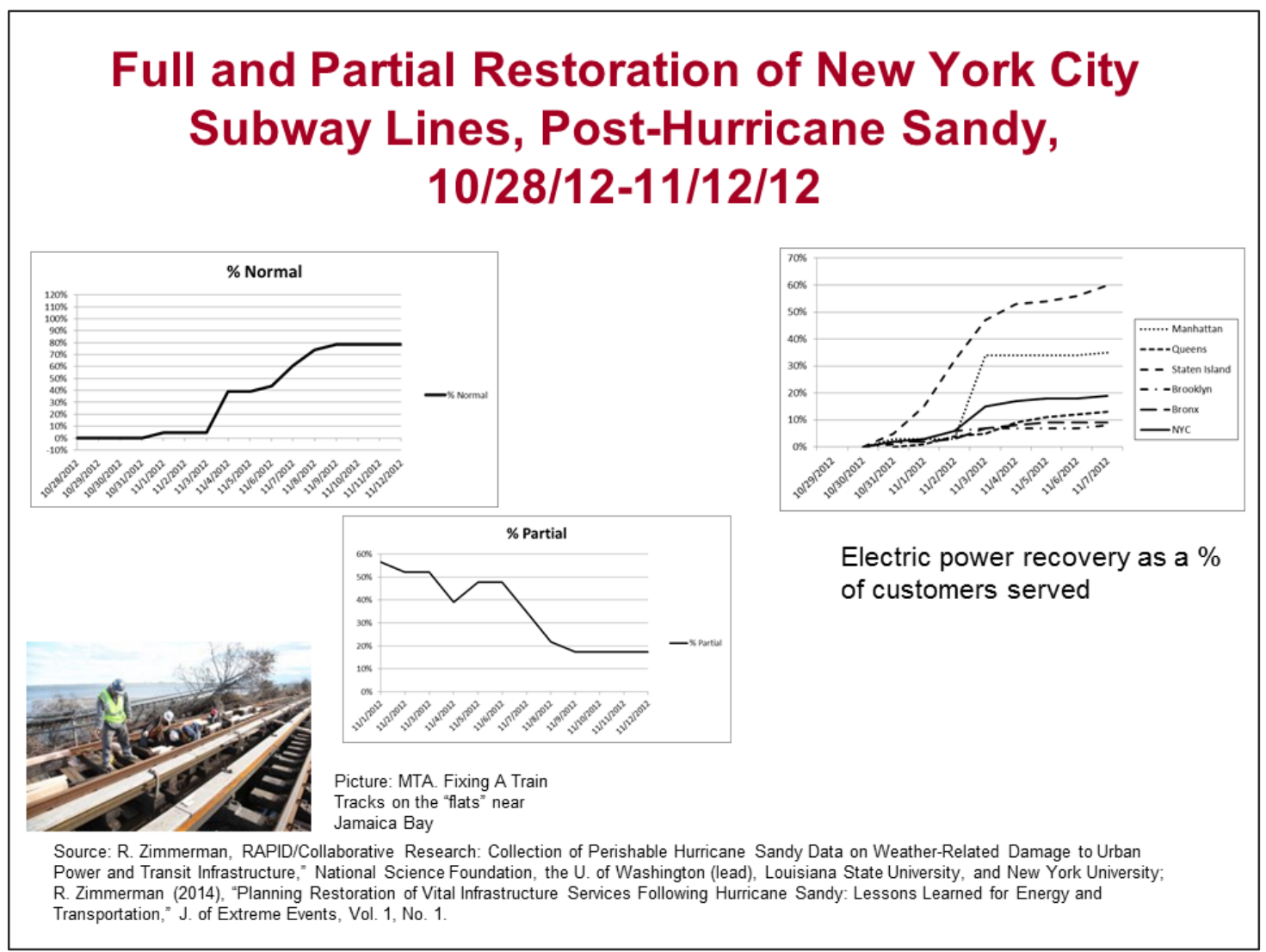

\section{Failure Mode Examples for Infrastructure Interconnections: Electric Power, Transportation, Water, IT}

- IT and all infrastructures: [1]

- In 2012, ICF-CERT reported the following based on 198 attacks: 41\% (82) in energy; $3 \%(6)$ in nuclear; $15 \%(29)$ in water, and 3\% (5) in transportation;

- By 2013, based on 256 attacks they reported: $59 \%$ (151) in energy; $3 \%$ (8) in nuclear; $5 \%$ (13) in water, and $5 \%(12)$ in the transportation sector

- Electric Power and Transportation:

- Hurricane Sandy resulted in extensive flooding of electric power infrastructure that was a key factor in transit vehicle, signal, and switch outages and lighting and signal outages for road transportation[2]

- Transportation and Water:

- For water management, unavailable or blocked drainage infrastructure can cause road, rail and vehicle flooding in the short-run and material disruptions in the long run [3]

Note: Only examples of two-way infrastructure interconnections are given, but in reality, many examples exist of more complex interconnections Sources:

[1] Industrial Control Systems Cyber Emergency Response Team (October/November/December 2012) ICS-CERT Monitor http://ics-cert.uscert.gov/sites/default/files/ICS-CERT_Monthly_Monitor_Oct-Dec2012_2.pdf summarize from p. 5. Industrial Control Systems Cyber Emergency Response Team (October-December 2013) ICS-CERT Monitor, p. 1, https://ics-cert.us-cert.gov/sites/default/files/Monitors/lCS-CERT_Monitor_Oct-Dec2013.pdf [2] NYS 2100 Commission (2013) 2100 Commission Report. Recommendations to Improve the Strength and Resilience of the Empire State's Infrastructure, Albany, NY: The Commission.

[3] A contributing factor to the Mianus Bridge Collapse in CT was the paving over of drainage facilities which created water damage to steel components on the bridge. NTSB (1984), Highway Accident Report: Collapse of a Suspended Span of Interstate Rte 95 Highway Bridge over the Mianus R. Greenwich, CT. 6/28/83, Washington, DC, USA, NTSB. 


\section{From Failure Modes to Resilience: A Basis for Modeling}

Note: Only two-way, simple infrastructure interconnections are shown, but more complex interconnections occur involving more infrastructures. Created by R. Zimmerman, NYU-Wagner School.

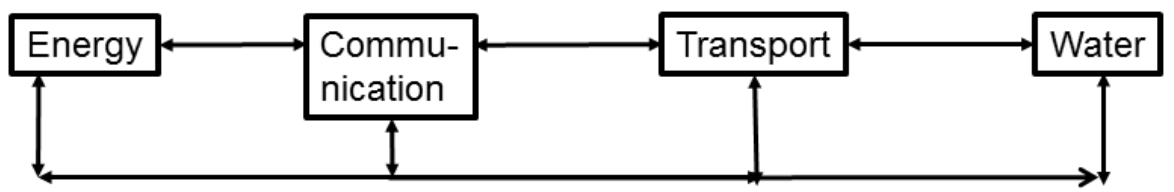

Conventional infrastructure interdependencies are potentially vulnerable to breaks in single links that can cause cascading damages across multiple infrastructure systems.

wind

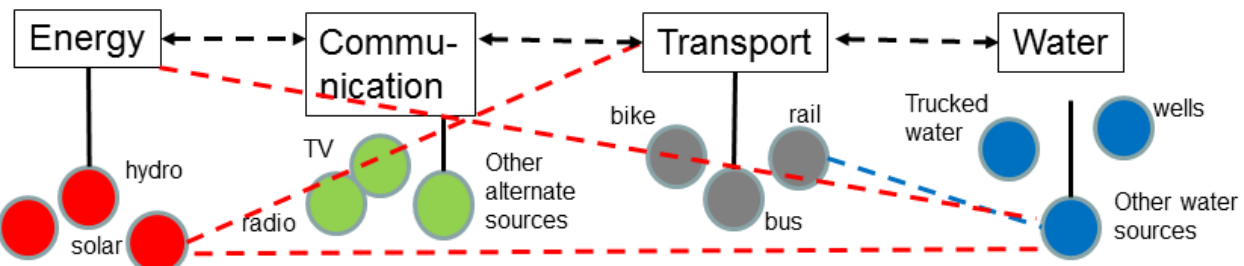

Distributed or alternative infrastructure systems enable more flexible, relatively simpler interconnections by adding additional resources that can perform and connect independently (dashed lines) or through traditional interdependent system linkages (dashed double arrows). Lines exemplify linkages.

\section{Using Interconnectivity to Promote Resilience}

- Selected Measures

- Intersection of hazards and infrastructure nodes and links

- Recovery time

- Illustrative Methods

- Multiple routes, modes, and resources (decentralized, redundant, shared, and "slack resources")

- Redundant structures and functions

- Effective communication

- Hazard mitigation and emergency planning

- Components

- Resources

- Physical Systems

- Environmental Systems

- Social Systems

From Spoke Structure to Interconnected Structure

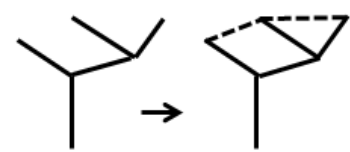

Note: Illustrates redundancy and flexibility from interconnectivity; best applied to dense systems

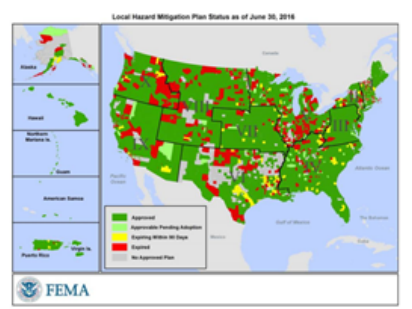

Source:FEMA (2016) Local Hazard Mitigation Plan Status RiskMap http://www.fema.gov/hazard-mitigation-plan-status 


\section{Resources}

Valuing Infrastructure

- Contributions to the economy:

- Infrastructure capital as a contributor to the Gross Domestic Product (GDP) (World Bank)

- High value of infrastructure assets (in the trillions of dollars) (U.S. Bureau of the Census)

- How do we capture that value to support future infrastructure investment?

- The cost of not investing

- Reliance on disaster funding in the shortterm

\section{Financing via Emergency Funding: Disaster Relief Appropriations Act of 2013 (DRAA)}

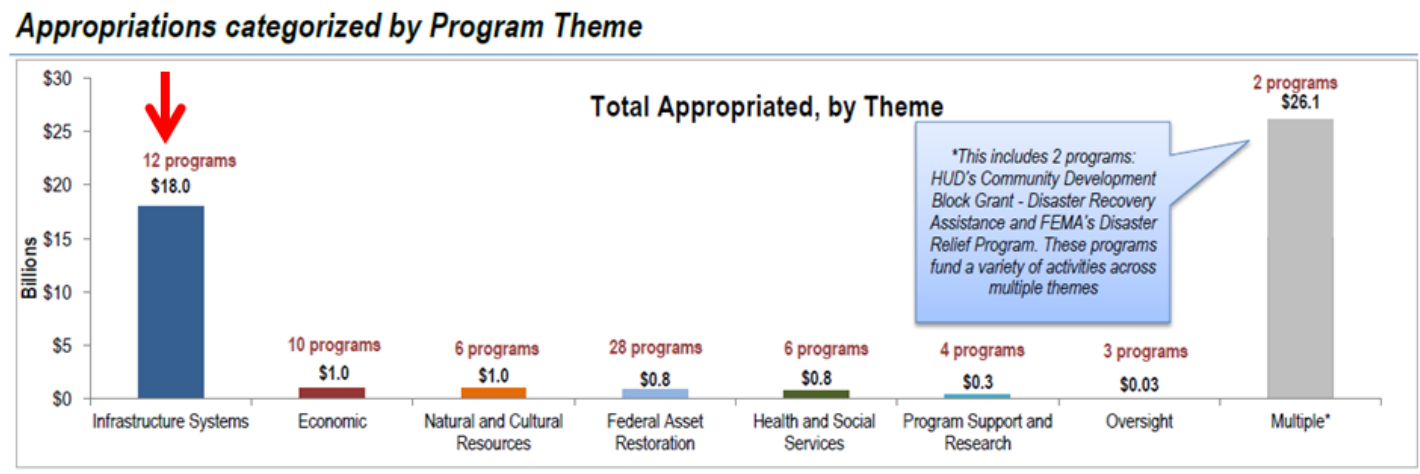

Source: U.S. Department of Housing and Urban Development (HUD), Sandy Program Management Office (PMO) (January 23, 2014) Monthly Public Financial Update, Washington, DC: US HUD, p. 4 available at http://portal.hud.gov/hudportal/documents/huddoc?id=HSTFSupp_Rpt123113.pdf 


\section{Financing via Capital Budgeting \\ Selected Permanent Restorations After Hurricane Sandy - Making Old Things New, 2013 and beyond}

- April 4, 2013. Number 1-train service to South Ferry substituted by service through the old South Ferry station[1]

- May 31, 2013. A-train service to the Rockaways restored[1]

- September 3, 2014. Greenpoint Tube repaired - G Line [2] September 15, 2014. Montague Tunnel repaired - R Line [3]

- Other tunnels, stations, and transit facilities under construction to 2016 and beyond[4]

Source: Based on data compiled by R. Zimmerman from MTA public information sources:

[1] $]^{*}$ ttp://web. mta.info/sandy/timeline.htm

[2]MTA (September 2, 2014) Full G Service Resumes Between Long Island City and Greenpoint Fix\&Fortify Work Improves Resiliency \& Reliability of G Line http://www.mta.info/press-release/nyc-transit/full-g-service-resumes-between-long-island-city-and-greenpoint

[3]MTA (September 15, 2014) Services Resumes Through Montague Tubes (13 months) http://www.mta.info/news/2014/09/15/services-resumesthrough-montague-tubes; MTA (September 15, 2014) Governor Cuomo Announces Early Completion of Superstorm Sandy Recovery Work In

Montague Subway Tunnel R Train Service between Brooklyn and Manhattan Restored Ahead of Schedule and Under Budget

http://www.mta.info/press-release/nyc-transit/governor-cuomo-announces-early-completion-superstorm-sandy-recovery-work

[4]MTA CPOIC April 2014 presentations

\section{Physical Systems: Electric Power, Water, Transportation}

- Electric Power and Transportation:

- Back up power (short-term)

- Decentralized power and alternative transportation modes with different energy inputs (long term)

- Alter Transportation to Avoid Disabled or Vulnerable Electric Power Systems: Modes (demand responses, surface vs. rail), Routes, Timing, Usage, e.g., telecommuting

- Technology - robotics

- Facility design: Elevate, Relocate

- Electric Power and Water

- Alternative/backup power

- Alternative water sources

- Alternative supplies and supply routes

- Electric Power distribution and production with all other Infrastructure: Strengthen, Relocate, Underground, Seal ${ }^{\star}$ 


\section{Alternatives for Avoiding Adverse Effects of Interconnections between Water and Energy}

- Energy Usage for Water Reduction

- Water recycling in energy systems

- Fluids other than water for energy cooling systems

- Water Usage for Energy Reduction

- Energy recovery

- Alternative (renewable) energy sources

- Less energy and/or water intensive products or methods of production, including those in other infrastructure sectors, e.g., transportation

Source: Summarized from U.S. Department of Energy (June 2014) The Water-Energy Nexus: Challenges and Opportunities. Washington, D.C.: U.S DOE, p. 109, 97

\section{Adapting Interconnectivity for Resilience: IT}

- IT and all infrastructure

- Capacity expansion

- Alternative communication modes

- Alternative structures

- Alternative procedures to improve communication management

- IT and Transportation

- Capacity expansion

- Redundancy

- Improved operational performance and usage training

- IT and Water

- Capacity expansion

- Sensor technologies to detect contamination, adhere to water quality standards, detect intrusions, and other IT security

- IT and Electric Power (both fossil fuels and renewables):

- IT has considerable value for energy systems in detecting anomalies, managing production, routing, accident avoidance through detection, distribution under normal conditions (bringing dispersed resources from extraction sites to concentrated production points and concentrated production resources to dispersed consumption points), and emergency conditions, e.g., detecting conditions that warrant preemptive shutdowns 


\section{Alternative Vehicles (Fueling Stations)}

Connecting Transportation (Vehicles) to Energy

Fueling Station Location for Alternative Fuels, miles from interstate highways, U.S.*:

- Only about $10 \%$ were under one quarter mile

- Almost half were between one quarter mile and 5 miles

- About a third were greater than 10 miles

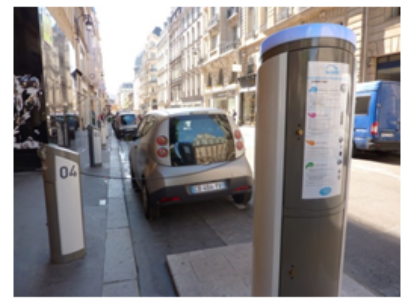

Autolib, Paris, France ${ }^{\star \star}$

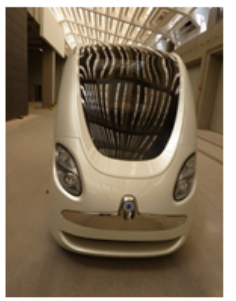

Automated vehicle, Masdar, UAR ${ }^{\star \star}$

Source: *R. Zimmerman (2012) Transport, the Environment and Security. Making the Connection, Cheltenham, UK and Northampton, MA: Edward Elgar Publishing, Ltd., p. 142,143. http://www.e-elgar.com/bookentry_mainUS.lasso?id=13884. **Pictures from R. Zimmerman

\section{Flexibility Through Multi-Modal Interconnections: \\ Bus Connections at Subway Stations, NYC}

- Connections between buses and subways do and can continue to provide alternative transportation modes in emergencies

- Nationally, bus connectivity is highest with rail transit

- The New York City subway stations vary in numbers of buses stopping at stations from a couple of dozen to none

- Bus connectivity is in part related to the number of train tracks located at each

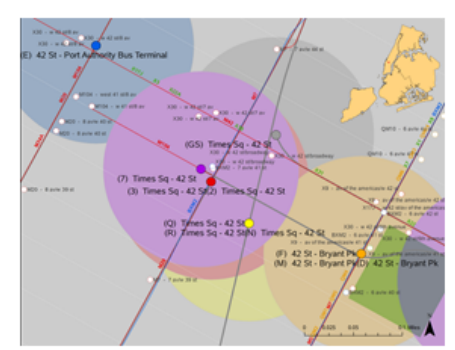
station

Source: Zimmerman, R. et al. (2014) Promoting Transportation Flexibility in Extreme Events through Multi-Modal Connectivity, New York, NY: NYU-Wagner. Funded by the U.S. DOT Region 2 University Transportation Research Center (UTRC). Photos from R. Zimmerman. 


\section{Adaptation for Flood Protection by Integrating Innovative Pedestrian Transportation Corridors, St. Jean de Luz}

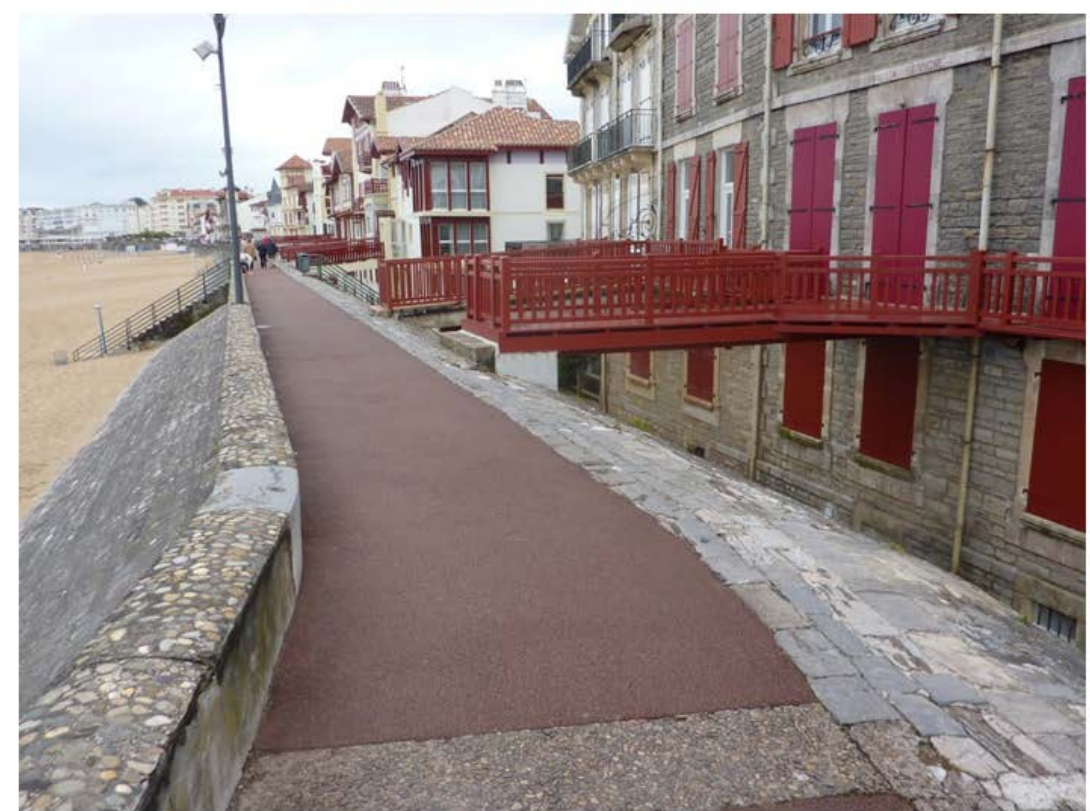

Photograph by R. Zimmerman

\section{Interconnecting Wastewater Treatment, Electric Power, and Water Management Infrastructure, NYC Post-Hurricane Sandy}

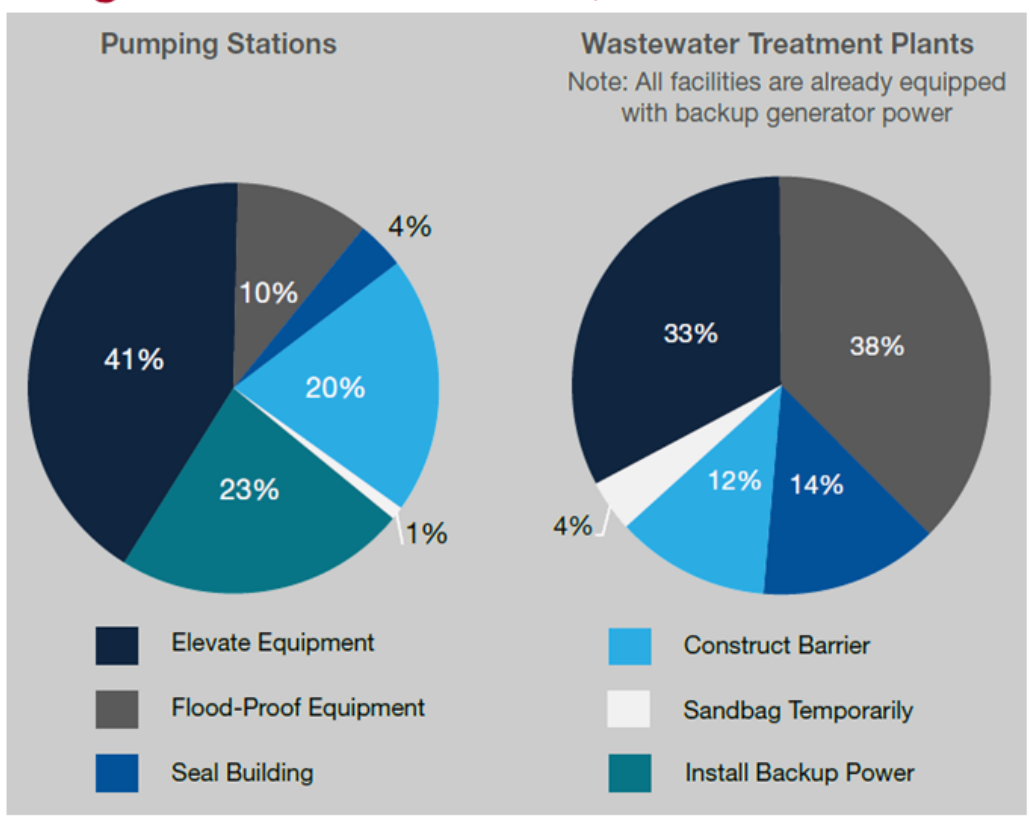




\section{Changing Materials to Balance Heat Exchange Changes in Cooling over Time for Concrete and Asphalt}

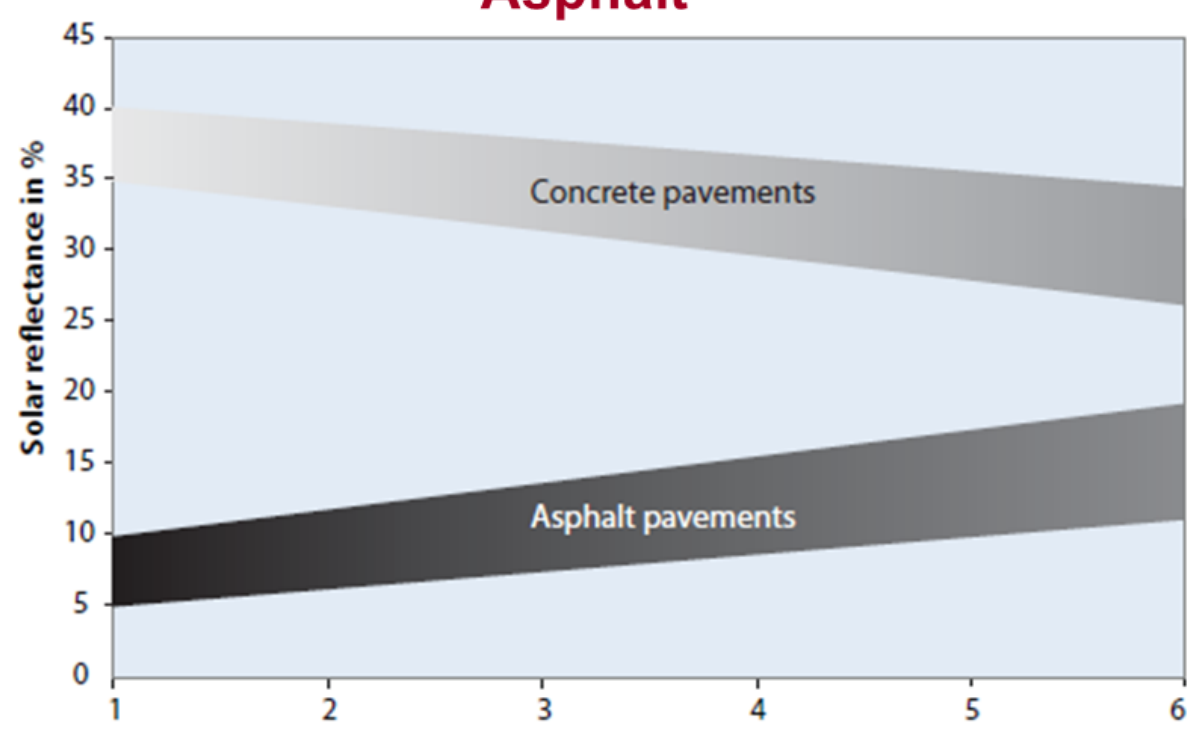

Source: U.S. EPA (October 2008) Reducing Urban Heat Islands: Compendium of Strategies, Chapter 5, "Cool Pavements," p. 5 ,

http://www.epa.gov/heatisld/resources/compendium.htmLawrence Berkeley National Labs. Asphalt lightens over time thus increasing reflectivity and concrete darkens over time thus decreasing reflectivity.

\section{Innovative Streets: Controlling Water, Heat and Other Factors}

- Strategies

- Spatial and temporal adaptations

- Reducing Roadways: Decentralizing streets; Deconstructing large roadways

- Specialized Approaches

- Cooling pavement surfaces for heat absorption

- Green corridors for ecological protection

- Green corridors for pedestrian thoroughfares

- Streets for stormwater control (Kuala Lumpur, Malaysia)

- Streets for waste recycling (glasphalt, plastic bags)

- Streets for electric power generation

- Streets as utility corridors

Source: R. Zimmerman (2012) Transport, the Environment and Security. Making the Connection, Cheltenham, UK and Northampton, MA: Edward Elgar Publishing, Ltd., p. 140-150. http://www.e-elgar.com/bookentry_mainUS.lasso?id=13884 


\section{Environmental Systems and Infrastructure: Wildlife Corridors at Roads and Bridges}
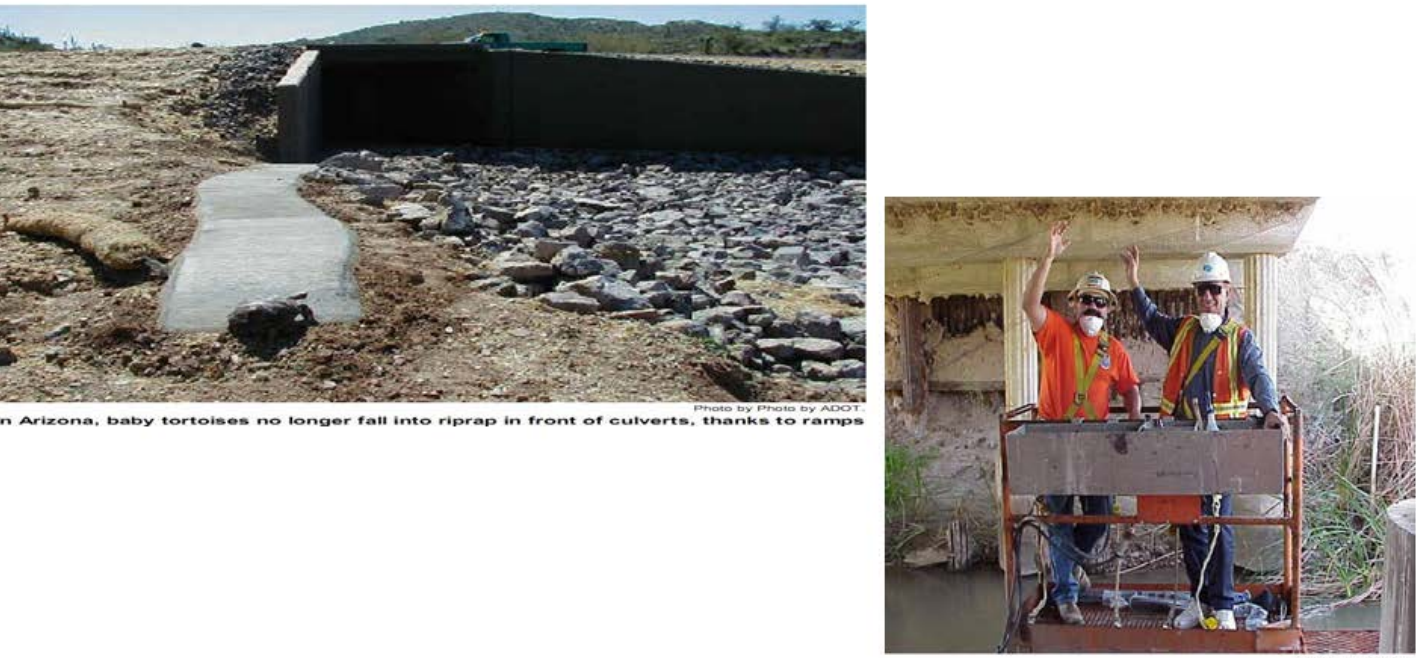

Source: U.S. Department of Transportation, Federal Highway Administration (2006), 'Along roads', available at http://www. fhwa.dot.gov/environment/wildlifeprotection/ind ex.cfm? fuseaction=home.viewTopic\&topicID=1



Roadside Swale, Salt Lake City, Utah
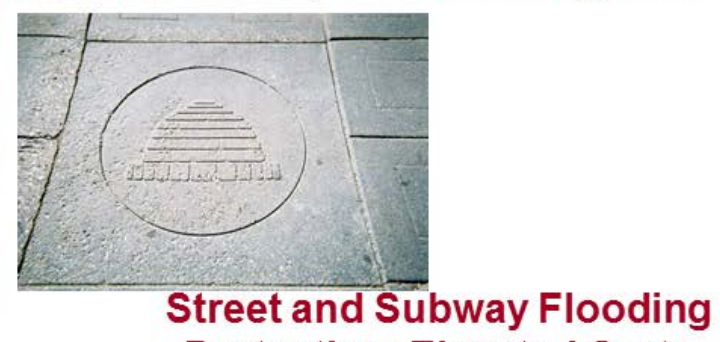
Protection: Elevated Grate Barriers, NYC

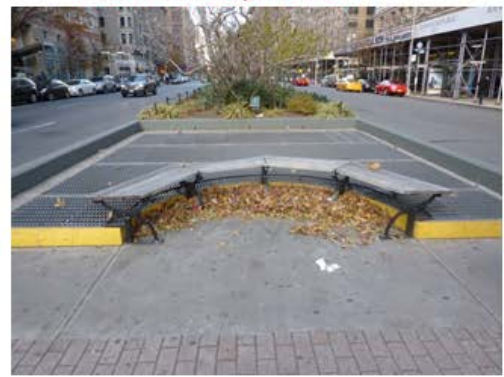

Source: Photos by R. Zimmerman 2012 


\section{Connecting CSO Basins (Gray Infrastructure) with Green Infrastructure Technologies}

Figure 16: Green Roof at DEP's Paerdegat Basin CSO Detention Facility

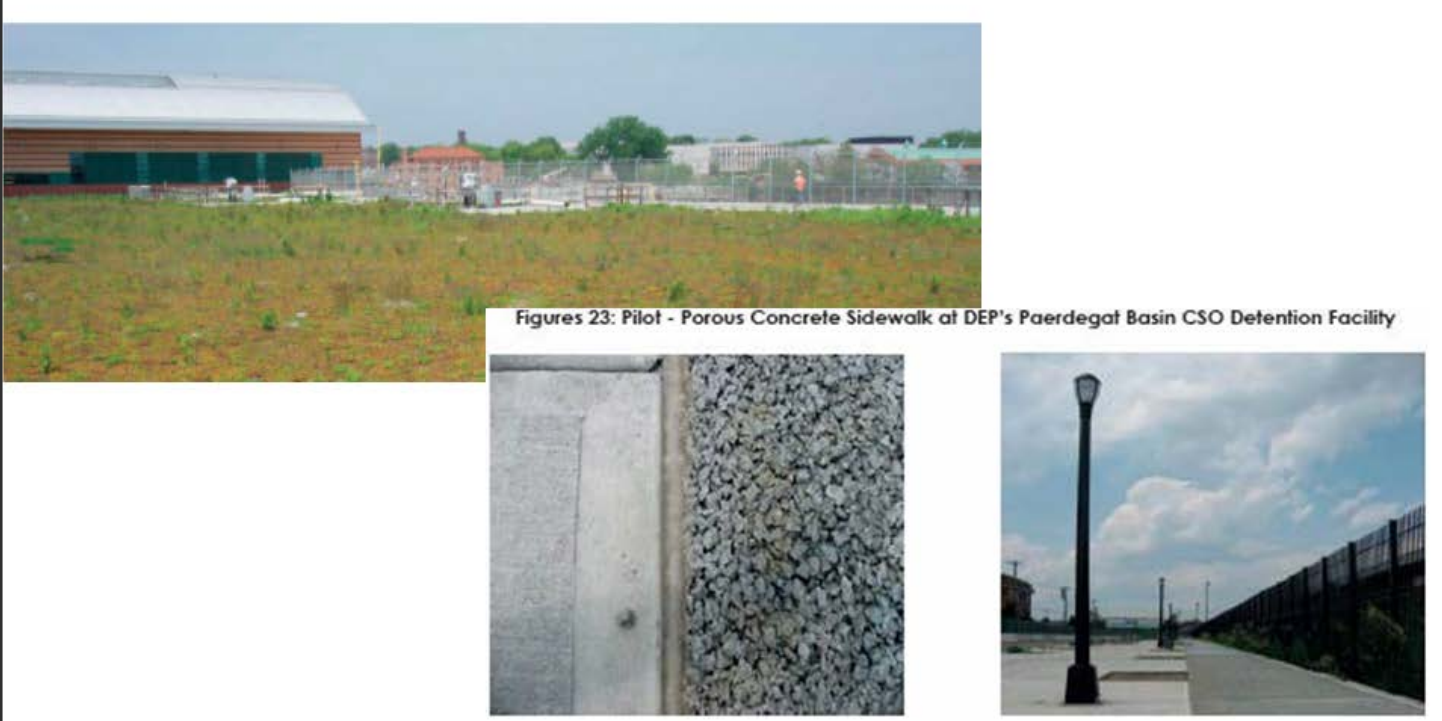

Source: NYC DEP (2009) NYC Green Infrastructure Plan 2009, p. 54, p. 62

\section{Social Systems \\ Role of Human Behavior: Will People Adapt}

Transportation Example: Psychological and Social Determinants of Route Choice Potential Factors Influencing Individual Choice of Transportation Routes

- Crowding, congestion

- Reliability

- Predictability / certainty

- Number of transfers required

- Time (transfer among modes and waiting time)

- Cost

- Safety

- Number of alternatives

- Aesthetics (including cleanliness)

- Access to other non-transit related resources (stops for shopping, etc.)

Potential Factors Contributing to Variations in Individual Choice Decisions

- Income

- Trip purpose

- Trip length (scale)

- Health status

- Miscellaneous travel preferences and customs

Source: Z. Guo and N. H.M. Wilson (2011) Assessing the cost of transfer inconvenience in public transport systems: A case study of the London Underground, Transportation Research Part A 45 (2011) 91-104; Z. Guo (2011) Mind the map! The impact of transit maps on path choice in public transit, Transportation Research Part A: Policy and Practice, 45 (7), 625-639. 


\section{How We Use Land}

Population Density, Household Characteristics and Transportation Per Household $\mathrm{CO}_{2}$ Emissions, U.S.

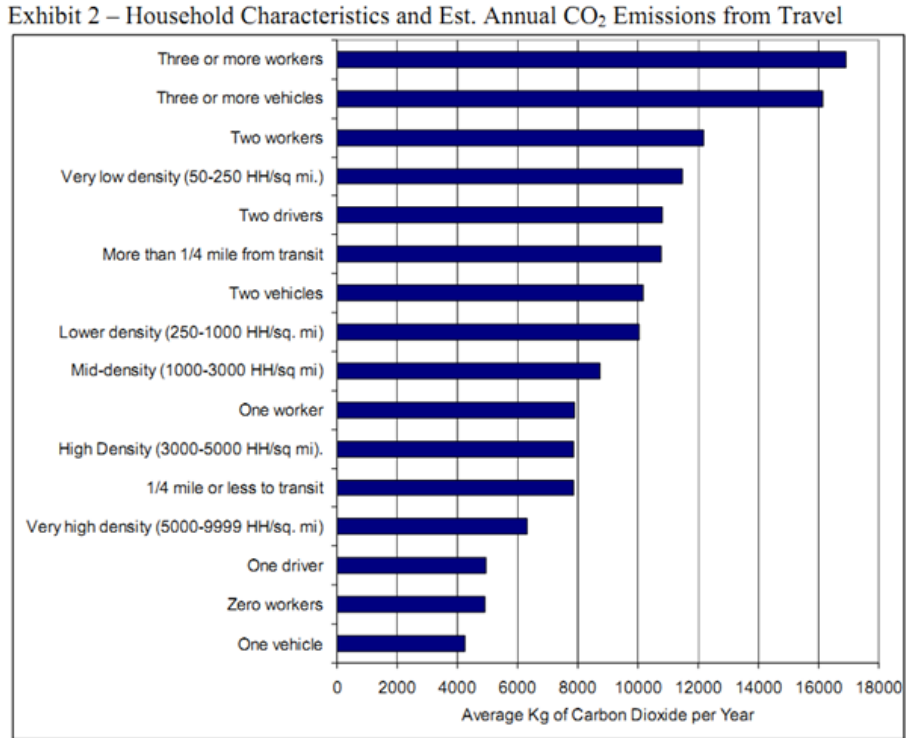

Source: U.S.DOT, FHWA (March 2009) NHTS Brief. The Carbon Footprint of Travel, p. 2 http.//nhts. ornl.gov/briefs/Carbon $\% 20$ Footprint $\% 20$ of $\% 20$ Travel.pdf

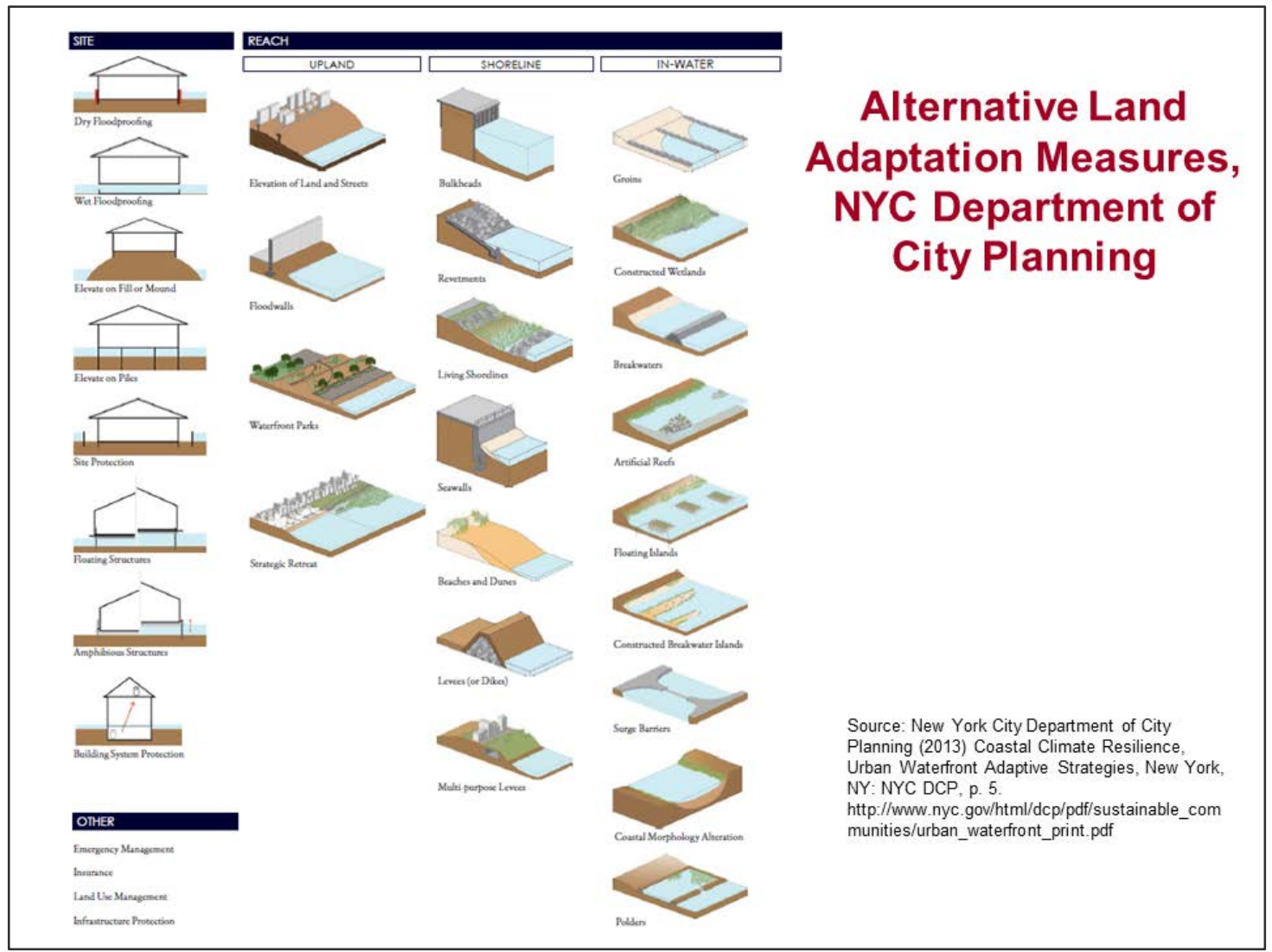




\section{Future Research Needs and Lessons for Policy as Inputs to Modeling}

- Provide greater specification and quantification of linkages, e.g., in terms of flows, inputs and outputs, acknowledging the uncertainty and variability

- Understand overall conditions (internal/external to systems) under which interconnections are strengthened or weakened (network theory; concentration effects)

- Identify interventions that change adverse cascading effects: Address concentration effects by reducing them through alternative designs

- Understand the role of and how to shape human behavior to support interconnections that reinforce resilience

\section{Acknowledgements}

The author acknowledges support from the following grants for portions of this presentation:

"Resilient Interdependent Infrastructure Processes and Systems (RIPS) Type 1: A Meta-Network System Framework for Resilient Analysis and Design of Modern Interdependent Critical Infrastructures," funded by the National Science Foundation (NSF), 2014-2016(1441140).

Critical Resilient Interdependent Infrastructure Systems and Processes (CRISP) Type 1Reductionist and integrative approaches to improve the resiliency of multi-scale interdependent critical infrastructure," funded by the NSF (1541164)

"Urban Resilience to Extreme Weather Related Events Sustainability Research Network (UREx SRN)," funded by the NSF (1444755).

"RAPID/Collaborative Research: Collection of Perishable Hurricane Sandy Data on Weather-Related Damage to Urban Power and Transit Infrastructure," funded by the NSF, with the U. of Washington (lead) and Louisiana State University (1316335).

"Promoting Transportation Flexibility in Extreme Events through Multi-Modal Connectivity" Faculty research grant from the University Transportation Research Center, Region 2.

Portions of this presentation were presented at other venues.

Disclaimer: The views expressed in this presentation are the opinions of the author and not necessarily those of the NSF or the U.S. DOT. 
Illustrative Bibliography for "Infrastructure System Interconnectivity Effects on Resilience" Professor Rae Zimmerman

NYU-Wagner School

For the NIST International Workshop on Modeling of Physical, Economic, and Social Systems for Resilience Assessment

\section{Infrastructure Interdependencies}

Chang, S.E., McDaniels, T. L., Mikawoz, J. and Peterson, K. (2007) Infrastructure failure interdependencies in extreme events: power outage consequences in the 1998 Ice Storm, Natural Hazards, 41(2), pp 337-358.

Grubesic, T.H. and Murray, A.T. (2008) Vital Nodes, Interconnected Infrastructures, and the Geographies of Network Survivability, Annals of the Association of American Geographers, pp. 64-83

Little, R.G. (2004) A socio-technical systems approach to understanding and enhancing the reliability of interdependent infrastructure systems, International Journal of Emergency Management, 2 (1-2).

Perrow, C. (1984) Normal Accidents: Living with High-Risk Technologies. New York: Basic Books.

Petit, F., Verner, D., Brannegan, D., Buehring, W., Dickinson, D., Guziel, K., Haffenden, R., Phillips, J. and Peerenboom, J. (June 2015) Analysis of Critical Infrastructure Dependencies and Interdependencies, Argonne National Laboratory.

Rinaldi, S.M., Peerenboom, J.P., and Kelly, T.K. (2001) Complex Networks, Identifying, Understanding, and Analyzing Critical Infrastructure Interdependencies, IEEE Control Systems Magazine, December 2001, pp. 11-25.

U.S. Department of Energy (June 2014) The Water-Energy Nexus: Challenges and Opportunities. Washington, D.C.: U.S. DOE

U.S. DHS (June 2014) Sector Resilience Report: Electric Power Delivery. Washington, DC: U.S. DHS

Xu, N., Nozick, L.K., Turnquist, M.A. and Jones, D.A. (2007) Optimizing Investment for Recovery in Interdependent Infrastructure. In: HICSS 2007 - 40th Hawaii International International Conference on Systems Science 3-6 January, 2007, Waikoloa, Big Island, HI, USA.

Zimmerman, R. (2012) Critical Infrastructure and Interdependency Revisited, Chapter 20 in The McGraw-Hill Homeland Security Handbook - 2nd edition, edited by D. G. Kamien. New York, NY: The McGraw-Hill Companies, Inc., pp. 437-460. 
Zimmerman, R. (2012) Transport, the Environment and Security. Making the Connection. Cheltenham, UK and Northampton, MA: Edward Elgar Publishing, Ltd.

Zimmerman, R. and Restrepo, C. (2006) The Next Step: Quantifying Infrastructure Interdependencies to Improve Security, International Journal of Critical Infrastructures, 2 (2/3), pp. 215-230.

Zimmerman, R., Restrepo, C.E., Sellers, J., Amirapu, A., Pearson, T. R. and Kates, H. B. (2015) MultiModal Transit Connectivity for Flexibility in Extreme Events, Transportation Research Record: Journal of the Transportation Research Board, No. 2532, pp. 64-73.

Zimmerman, R., Zhu, Q. and Dimitri, C. (2016) Promoting Resilience for Food, Energy and Water Interdependencies, Journal of Environmental Studies and Sciences, 6 (1), pp. 50-61.

\section{Resilience (infrastructure focus)}

(Note: Many of these references are listed in Zimmerman (2016) cited below, and discussed in the text of that reference).

Beatley, T. (2012) Green cities of Europe: Global lessons on green urbanism. Washington, DC: Island Press.

Chang, S. E., McDaniels, T., Fox, J., Dhariwal, R., and Longstaff, H. (2014) Toward disasterresilient cities: Characterizing resilience of infrastructure systems with expert judgments. Risk Analysis, 34(3), 416-434.

National Academies, Committee on Increasing National Resilience to Hazards and Disasters, Committee on Science, Engineering, and Public Policy (2012) Disaster resilience: A national imperative. Washington, DC: The National Academies Press.

New York State 2100 Commission (2013) Recommendations to improve the strength and resilience of the empire state's infrastructure. Available at http://www.rockefellerfoundation.org/uploads/files/7c012997-176f-4e80-bf9cb473ae9bbbf3.pdf

O'Rourke, T. D. (2007) Critical infrastructure, interdependencies, and resilience, The Bridge 37 (1), pp. 22-29.

Perrow, C. (2007) The Next Catastrophe. Princeton, NJ, USA: Princeton University Press.

Reed, D., Kapur, K., and Christie, R. (2009), Methodology for assessing the resilience of networked infrastructure. IEEE Systems Journal, 3(2), 174-180. 
Roe, E., and Schulman, P. R. (2012) Toward a comparative framework for measuring resilience in critical infrastructure systems, Journal of Comparative Policy Analysis: Research and Practice, 14(2), 114-125.

U.S. Department of Homeland Security (2013) Partnering for Critical Infrastructure Security and Resilience, U.S. DHS, Washington, DC.

Zimmerman, R. (2016) Resilient Urban Infrastructure for Adapting to Environmental Disruptions, Chapter 32 in Handbook on Urbanization and Global Environmental Change, edited by K. C. Seto, W. D. Solecki, and C. A. Griffith, London, UK: Routledge, pp. 488-512. 
Infrastructure System Interconnectivity Effects on Resilience Professor Rae Zimmerman, NYU-Wagner

October 19, 2016

\section{Biosketch}

Rae Zimmerman is Professor of Planning and Public Administration and the Institute for Civil Infrastructure Systems Director at NYU's Wagner Graduate School of Public Service, and directed NYU-Wagner's Urban Planning Program five times. She is an elected Fellow of AAAS and Fellow, past president and outstanding service award recipient of the Society for Risk Analysis. Her teaching and research are interdisciplinary encompassing infrastructure and its interdependencies, climate change, environment, natural hazards, and equity as they influence the quality of life in cities. Current and past research funding sources include the National Science Foundation (NSF), U. S. DOT, and U.S. EPA. Of the approximately four dozen grants she has received, she served as principal Investigator on about three dozen. In 2015, she completed NSF-funded research on electric power and transit recovery following Hurricane Sandy and a U.S. DOT Region 2 University Transportation Research Center (UTRC) funded project on multimodal transportation use in emergencies and its relationship to poverty in New York City. Current NSF and UTRC funded research emphasizes infrastructure interdependencies from physical, social and natural environmental perspectives and how these relationships influence consequences from extreme events. Her current professional committee appointments include the third NYC Panel on Climate Change, the National Academies Committee on Pathways to Urban Sustainability, and the Transportation Research Board's Critical Transportation Infrastructure Protection committee. She authored and co-authored numerous publications and in 2012 authored Transport, the Environment and Security: Making the Connection (Edward Elgar). Education: B.A., Chemistry, University of California (Berkeley); MCP, University of Pennsylvania; Ph.D., Planning, Columbia University. URL: http://wagner.nyu.edu/zimmerman 
International Workshop on Modeling of Physical, Economic, and Social Systems for Resilience Assessment

This page intentionally left blank.

A-108 


\section{Appendix B: Resilience Modeling Research Around the World (Session 2)}

Interdependencies, Cascading Effects and Resilience Optimization of Communities

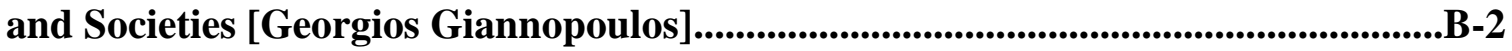

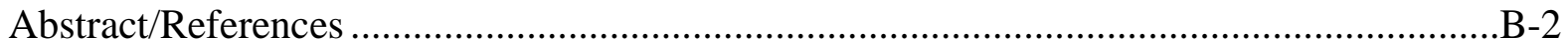

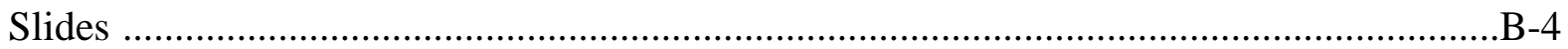

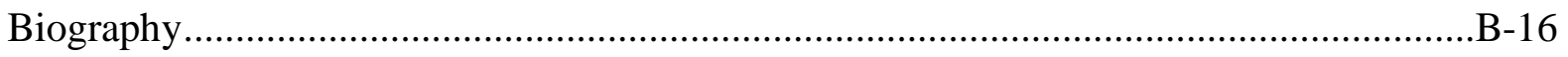

Resilience Modeling Research in New Zealand [Nick Horspool] ......................................B-17

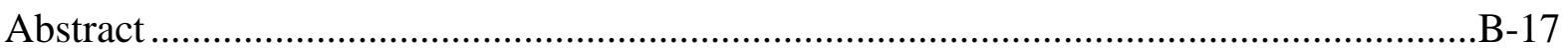

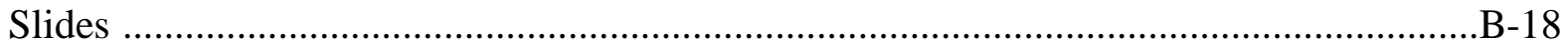

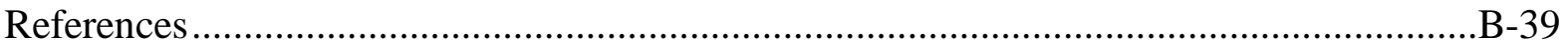

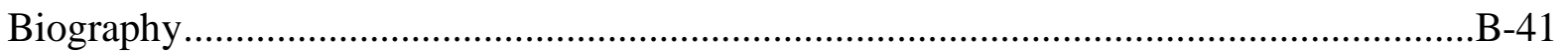

Urban Resilience: Asia Research Initiatives [Dongping Fang] .......................................42

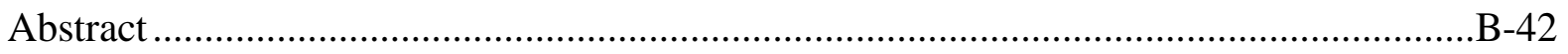

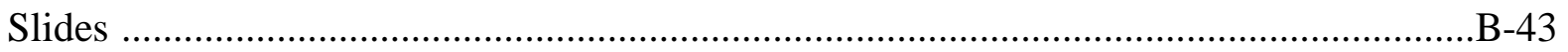

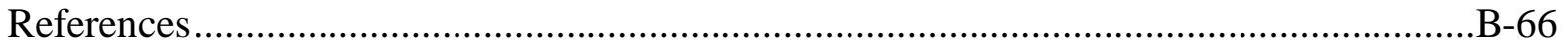

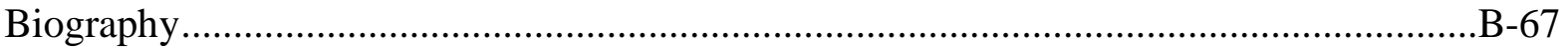

NSF Investments and Opportunities in Interdisciplinary Research on Critical

Infrastructure Systems [David Mendonça] ...............................................................B-68

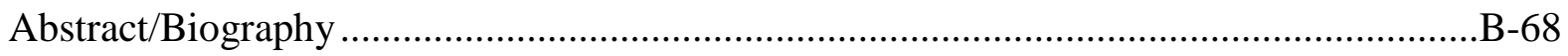

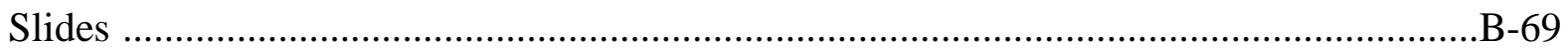

DHS Sponsored Research: DHS Approaches to Supporting Infrastructure

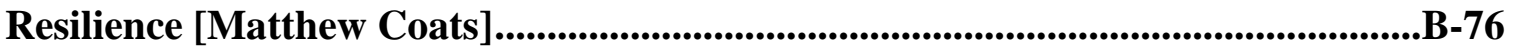

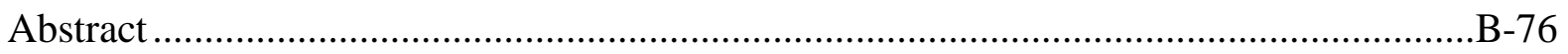

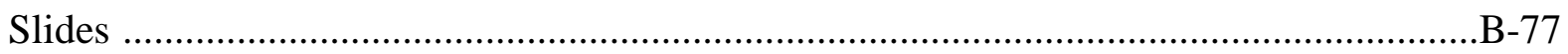

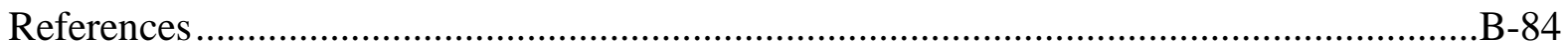

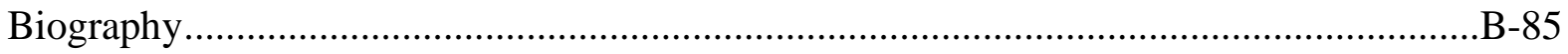




\title{
Interdependencies, cascading effects and resilience optimization of communities and societies
}

Georgios Giannopoulos, Luca Galbusera, Marianthi Theocharidou

\begin{abstract}
European Commission, Joint Research Centre, Directorate E - Space, Security and Migration, Technology Innovation in Security Unit, Via E. Fermi 2749, 21027 Ispra, Italy
\end{abstract}

Communities and societies rely on a network of infrastructures and technological systems that provide indispensable services for the citizens. These often display high degree of interconnectedness: functional, geographical, cyber and logical interdependencies play a role in enhancing the level of services provided to the citizens (e.g. ICT penetration in the electricity sector to develop smart grids), but they might also expose the system to harmful domino effects.

Accordingly, the Joint Research Centre (JRC) and partner research institutions are conducting a broad research initiative related to the analysis of critical infrastructures and their response to hazardous events of different origin (e.g. natural events, technical failures, and man-made attacks).

The research conducted at JRC in this realm obeys a principle of layering: resilience assessment of complex systems starts with static and dynamic interdependency analysis, incorporates service-oriented models, and involves economic impact assessment in relation to business discontinuities. This approach allows different levels of granularity (local, regional, national, international scales) and supports decision-making towards an optimization-based improvement of preparedness, recovery processes and other resilience factors.

Case studies are provided to support the discussion of the methodologies and their application to selected disruptive scenarios.

\section{References}

Barrat, Alain, Marc Barthelemy, and Alessandro Vespignani. Dynamical processes on complex networks. Cambridge University Press, 2008.

Liu, Yang-Yu, Jean-Jacques Slotine, and Albert-László Barabási. "Controllability of complex networks." Nature 473.7346 (2011): 167-173. 
Barker, Kash, and Joost R. Santos. "Measuring the efficacy of inventory with a dynamic inputoutput model." International Journal of Production Economics 126.1 (2010): 130-143.

Marseguerra, Marzio, Enrico Zio, Luca Podofillini, and David W. Coit. Optimal design of reliable network systems in presence of uncertainty. Reliability, IEEE Transactions on, 54(2) (2005): 243-253.

Galbusera, Luca, Georgios Theodoridis, and Georgios Giannopoulos. "Intelligent Energy Systems: Introducing Power-ICT Interdependency in Modeling and Control Design." IEEE Transactions on Industrial Electronics 62.4 (2015): 2468-2477.

Theodoridis, Georgios, Luca Galbusera, and Georgios Giannopoulos. "Controllability Assessment for Cascade Effects in ICT-enabled Power Grids." International Conference on Critical Information Infrastructures Security. Springer International Publishing, 2015.

Lian, Chenyang, and Yacov Y. Haimes. "Managing the risk of terrorism to interdependent infrastructure systems through the dynamic inoperability input-output model." Systems Engineering 9.3 (2006): 241-258.

Haimes, Yacov Y., et al. "Inoperability input-output model for interdependent infrastructure sectors. I: Theory and methodology." Journal of Infrastructure Systems 11.2 (2005): 67-79.

Haimes, Yacov Y., et al. "Inoperability input-output model for interdependent infrastructure sectors. II: Case studies." Journal of Infrastructure Systems 11.2 (2005): 80-92.

Barker, Kash, and Joost R. Santos. "Measuring the efficacy of inventory with a dynamic inputoutput model." International Journal of Production Economics 126.1 (2010): 130-143.

Galbusera, Luca, et al. "Inoperability Input-Output Modeling: Inventory Optimization and Resilience Estimation during Critical Events." ASCE-ASME Journal of Risk and Uncertainty in Engineering Systems, Part A: Civil Engineering (2016): B4016001. 


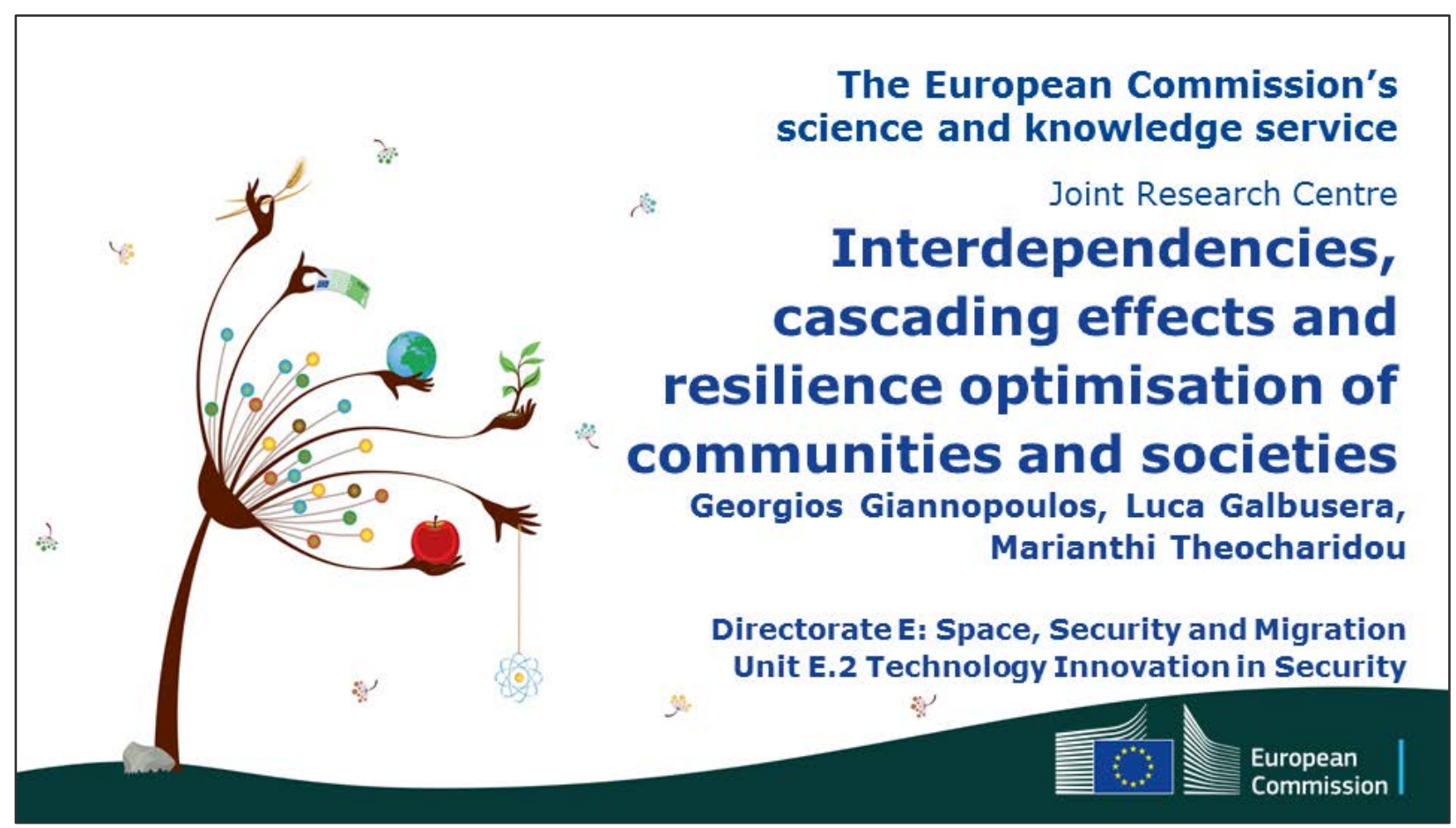

\section{DG JRC Role: facts \& figures}

- 6 locations in 5 Member States

- 1500 core research staff, out of around 3000 total staff

- Research fellows and visiting scientists

- 42 large scale research facilities, more than 110 online databases

- More than 100 economic, biophysical and nuclear models

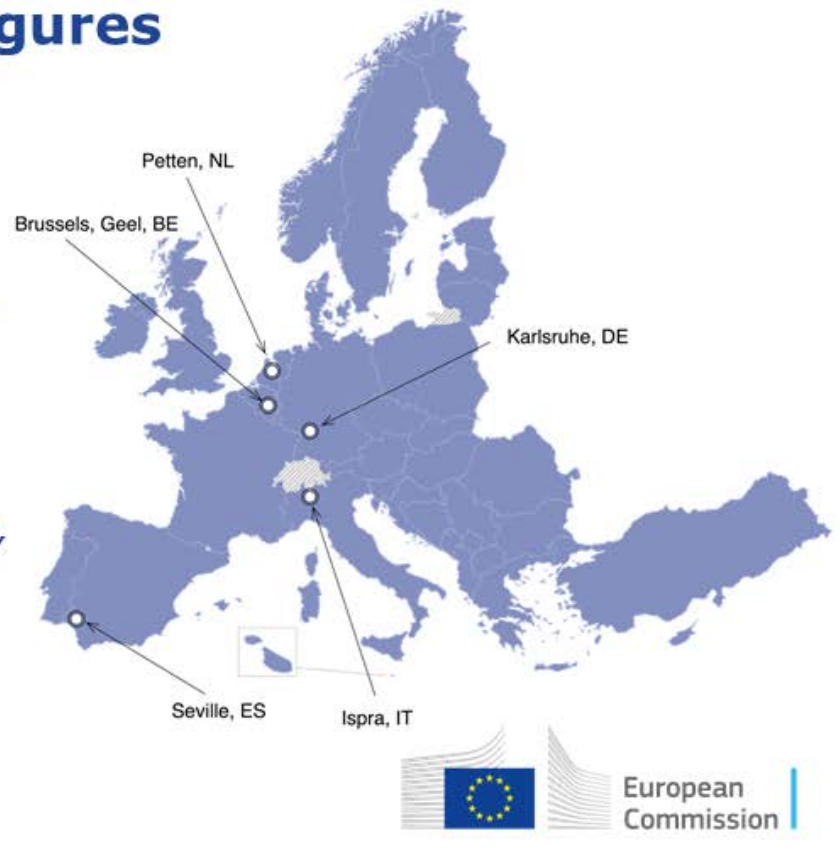




\section{Resilience modelling based on 3 Tiers}

\section{Tier 1: Sectoral/Asset analysis}

- Critical Infrastructure sector-specific models (power, ICT, gas, traffic,...)

- Network analytics

- ...

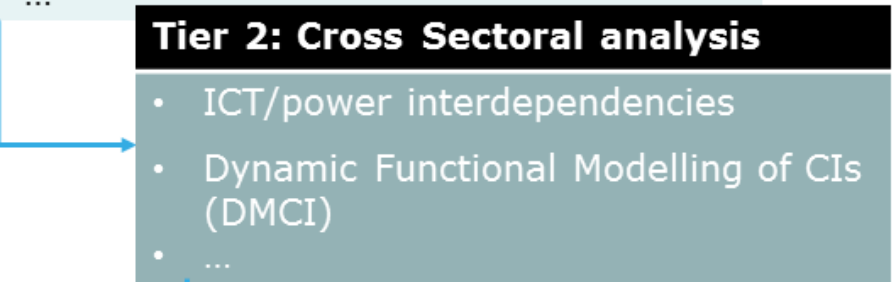

Tier 3: Societal/Economic impact

- Leontief I/O models

- CGE models

- ...

\section{Tier 1: Network analytics}

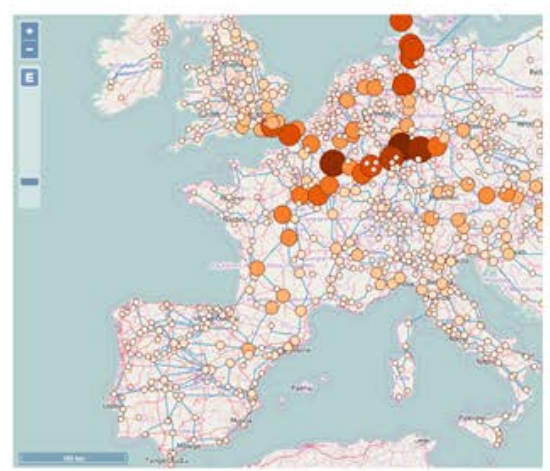

Importance of network analytics

1 Quick analysis of intrinsic network properties (critical

elements)

2 Resilience analysis due to directed/random disruptions

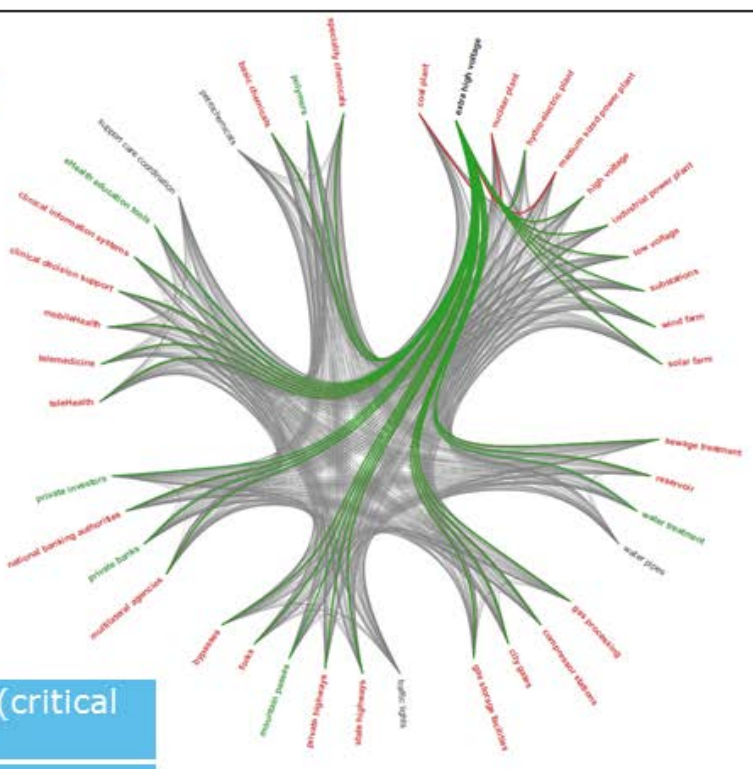




\section{Tier 2: DMCI}

Dynamical Functional Modelling of Vulnerability and Interdependencies in CIs

Jointly developed with Polytechnic School of Milan*: Leveraging expertise in the CIP community

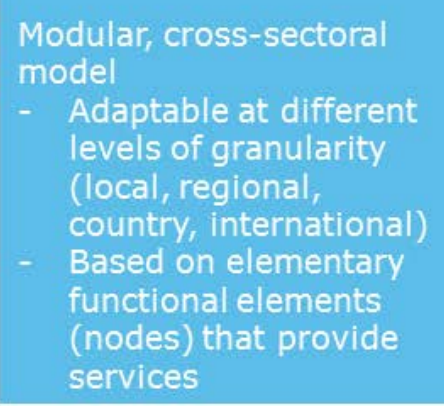

Modular, cross-sectoral mode

Adaptable at different levels of granularity

(local, regional

country, international)

on elementary

services

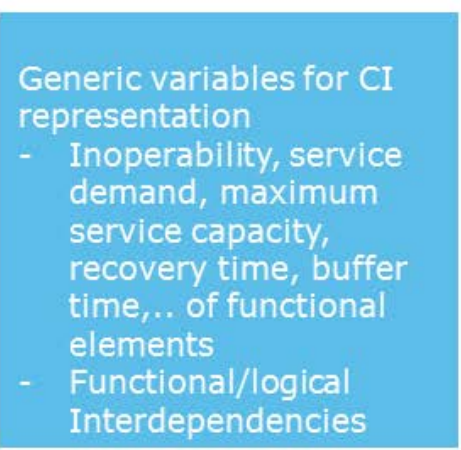

* Paolo Trucco, Enrico Cagno and Massimiliano De Ambroggi, Dynamic functional modelling of vulnerability and interoperability of Reliability Engineering \& System Safety, vol. 105 (2012): 51-63
representation
Inoperability, service
demand, maximum
service capacity,
recovery time, buffer
elements
Functional/logical
Interdependencies

Tier 2: DMCI

Dynamical Functional Modelling of Vulnerability and Interdependencies in CIs

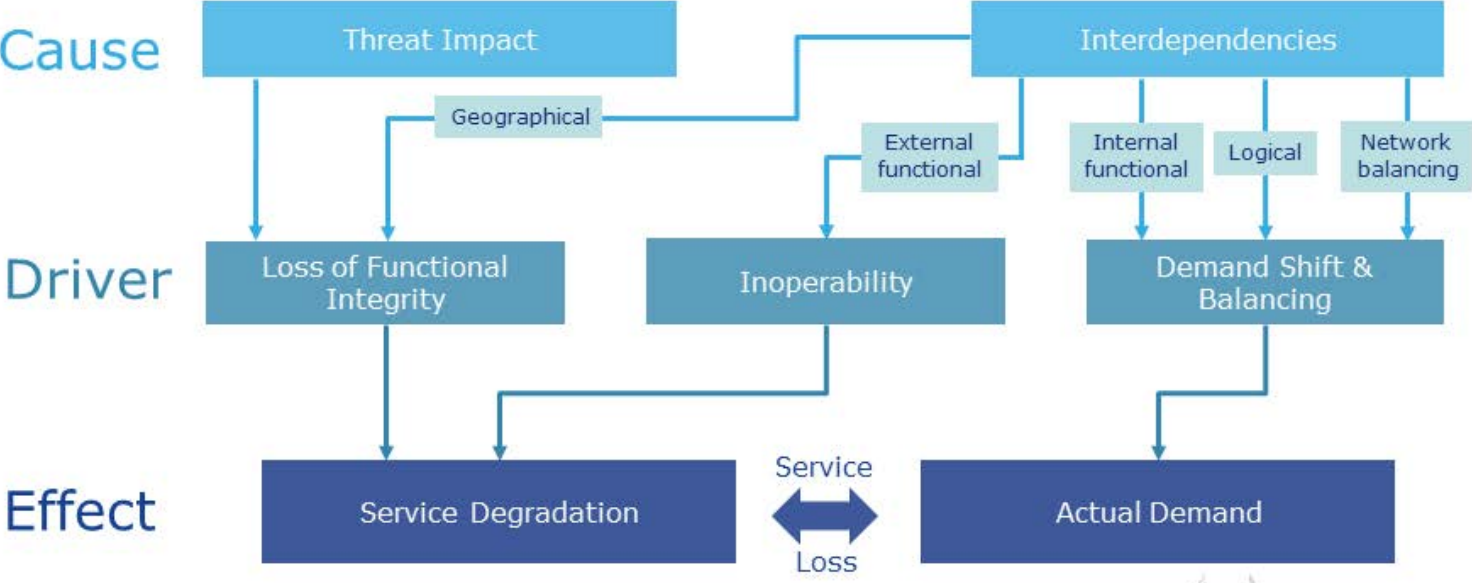




\section{Tier 2: Boolean dynamic networks}

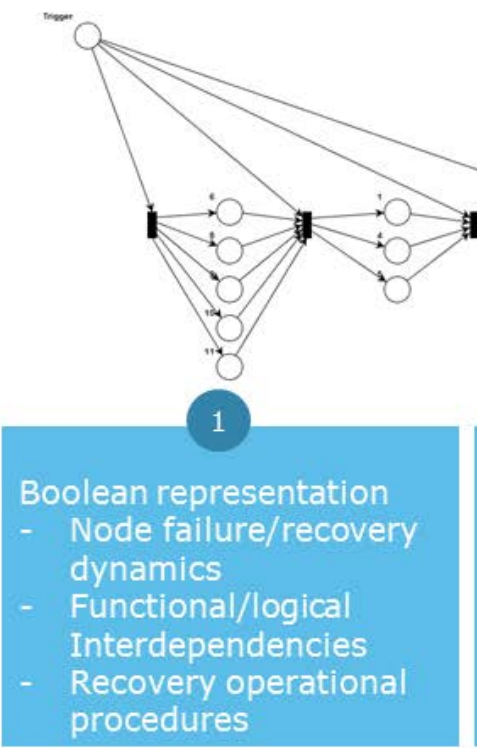

Physics and

organisational aspects

are expressed through

Boolean relationships -

helps to reduce

analysis complexity

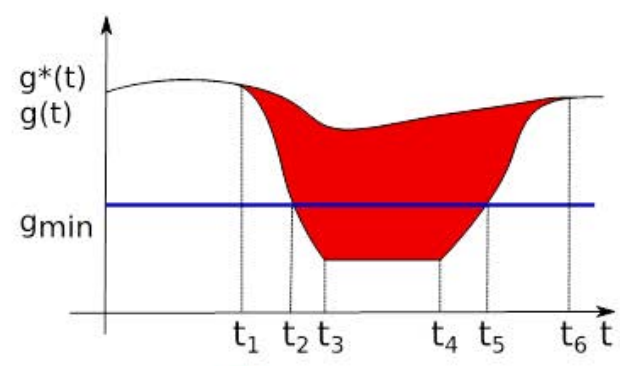

3

Recovery sequence is

prioritised based on

Component

Importance

Availability of

resources

\section{Tier 2: Resilience optimisation}
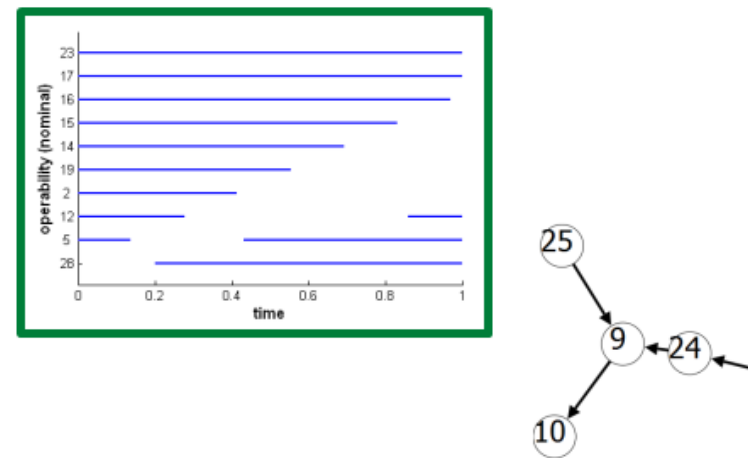

1 Objective: Minimise cascade effects and maintain functionality for prioritised nodes

2 Identify failure propagation path \& assign nodes' priority

3 Solution: Modify network parameters to minimise failure propagation

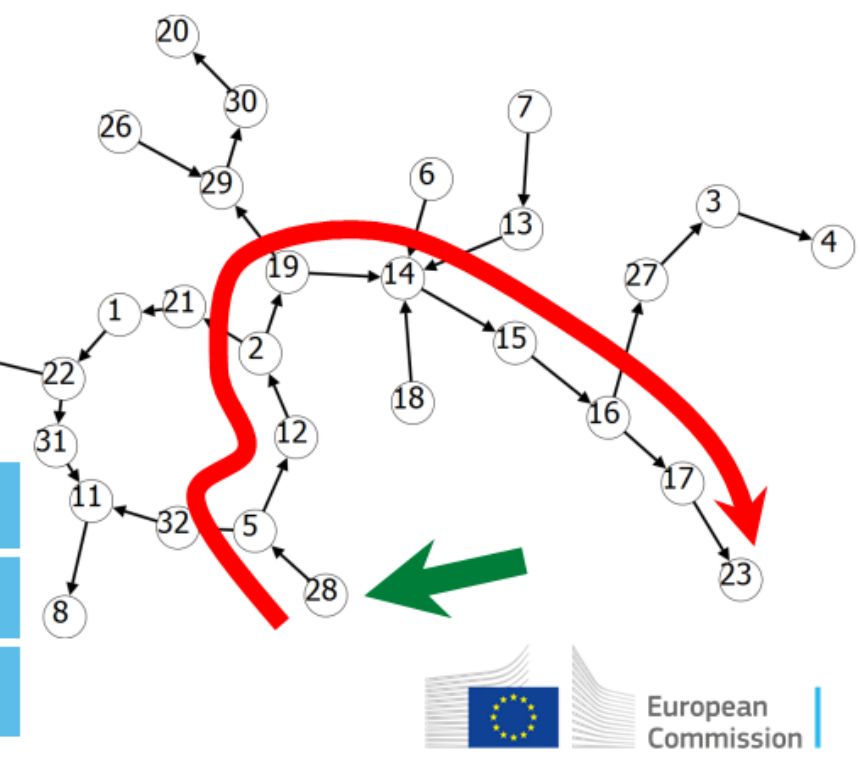




\section{Tier 2: Resilience optimisation}
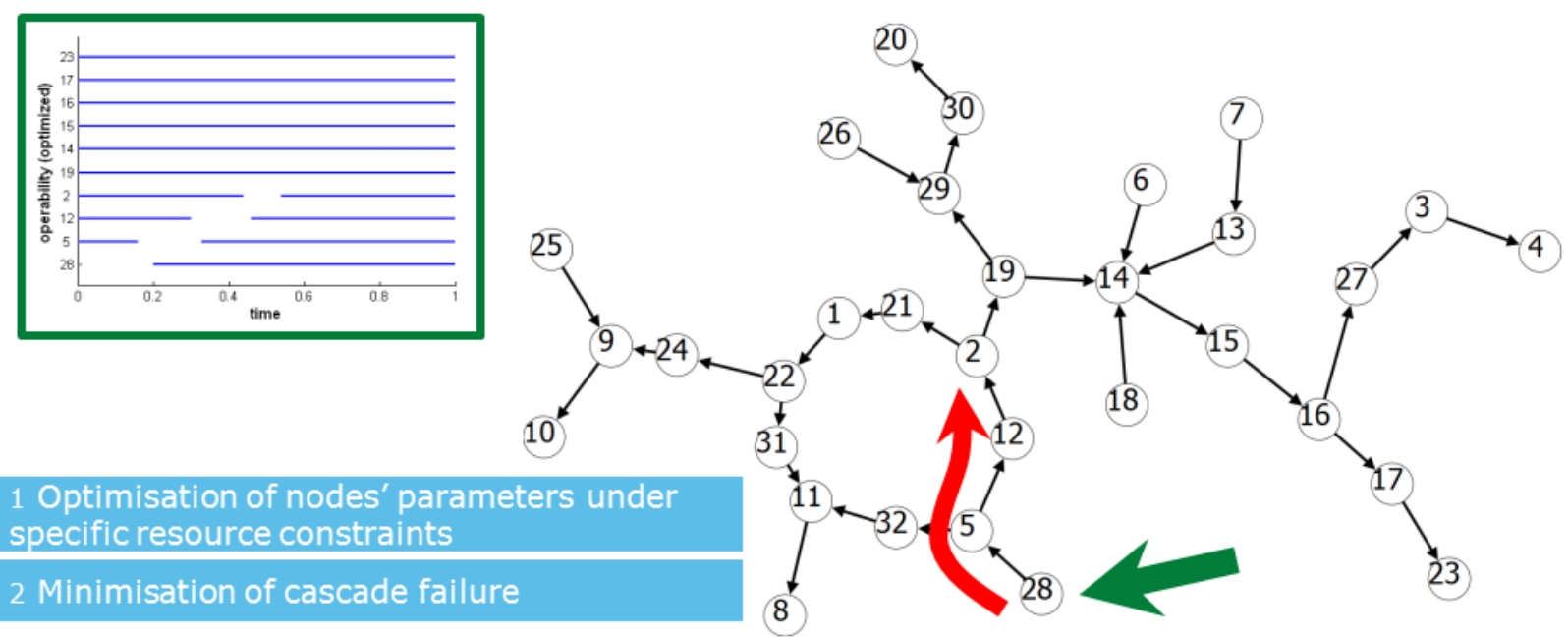

2 Minimisation of cascade failure

Tier 3: Dynamic inoperability I/O model

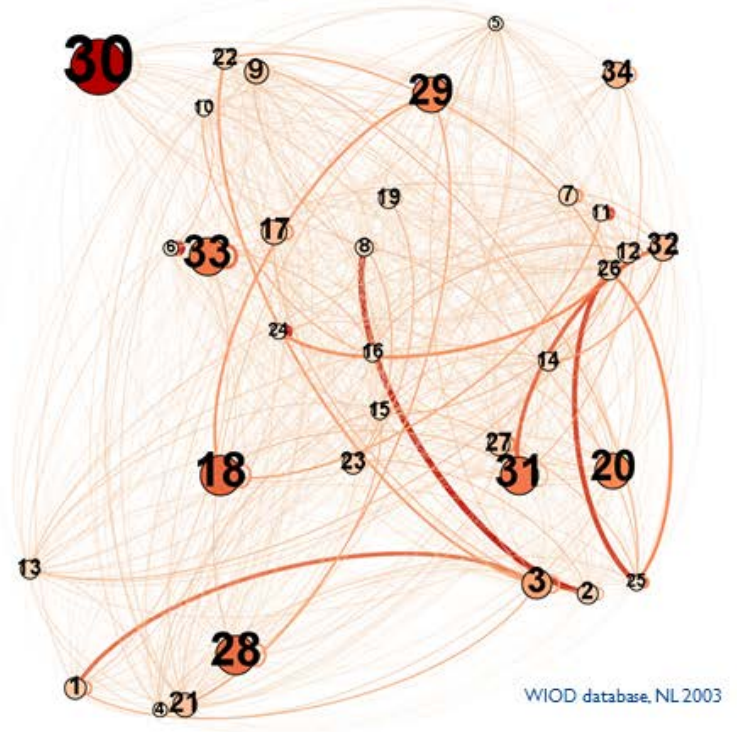

Based on W. Leontief's I/O approach

Assessing service loss

propagation in economic

sectors as a consequence

of critical events

Exploiting data from public economic DBs

Applicable at different

levels of granularity

(regional, country) 


\section{Tier 3: Inventory optimisation in economic sectors for resilience}

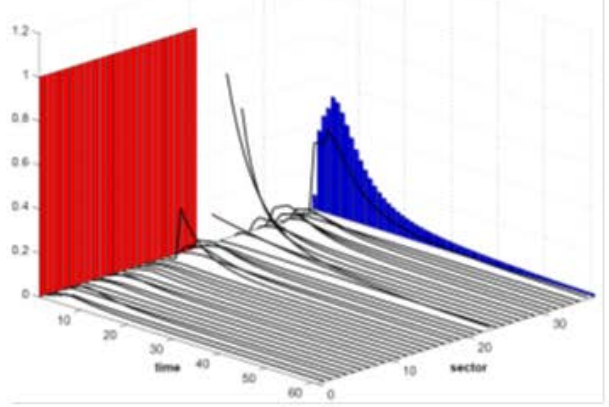

Nominal inventory levels

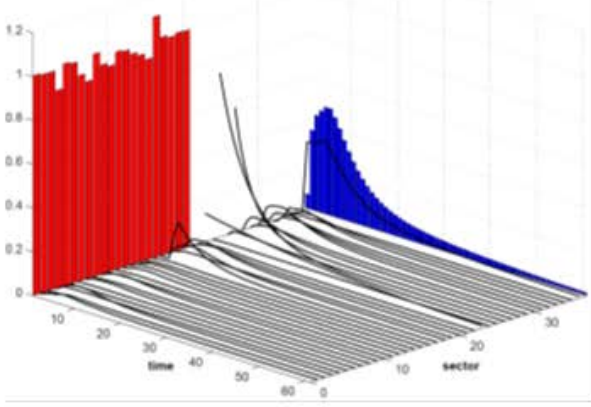

Optimised inventory levels

\section{Geospatial Risk and Resilience Assessment Platform - GR²ASP}

\section{Server client architecture}

WebGIS technologies +

analysis tools for CIs and

economic sectors

Based on open-source software

Supporting multi-user

collaboration and data

sharing ( $\mathrm{CI}$ analysis

community)

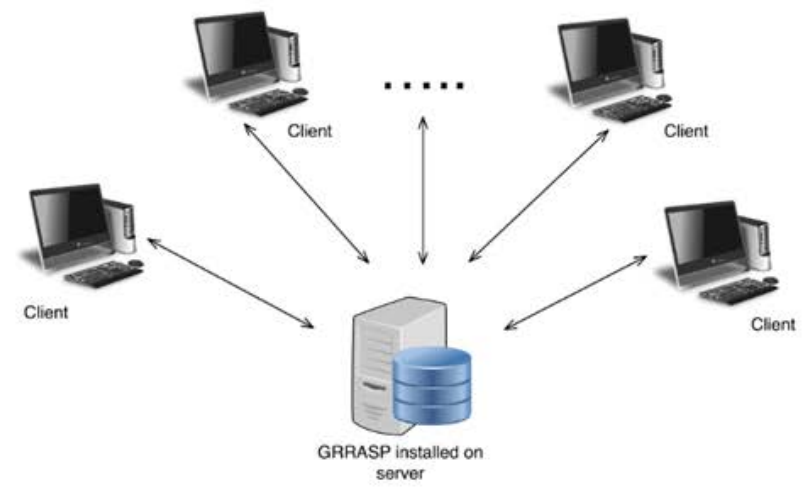

Available for download at: https://ec.europa.eu/jrc/en/grrasp 


\section{GR²ASP Architecture}

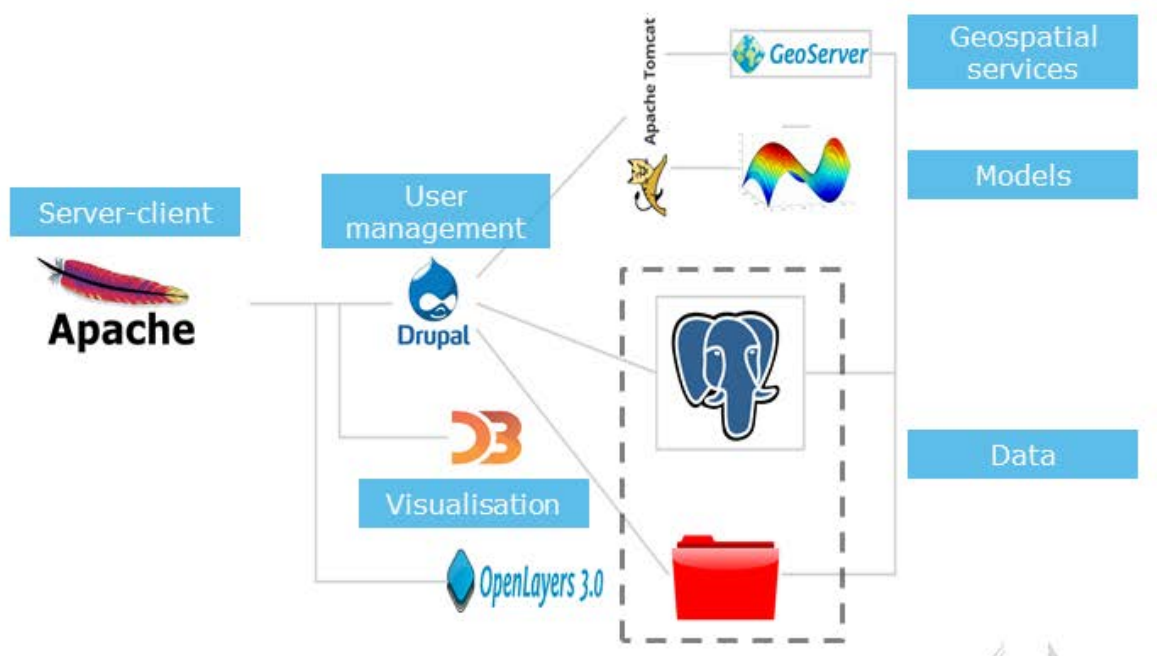

\section{Applying GR²ASP: Use case 1}

Applying Tier 1 and Tier 2 models to assess the resilience of an Urban area
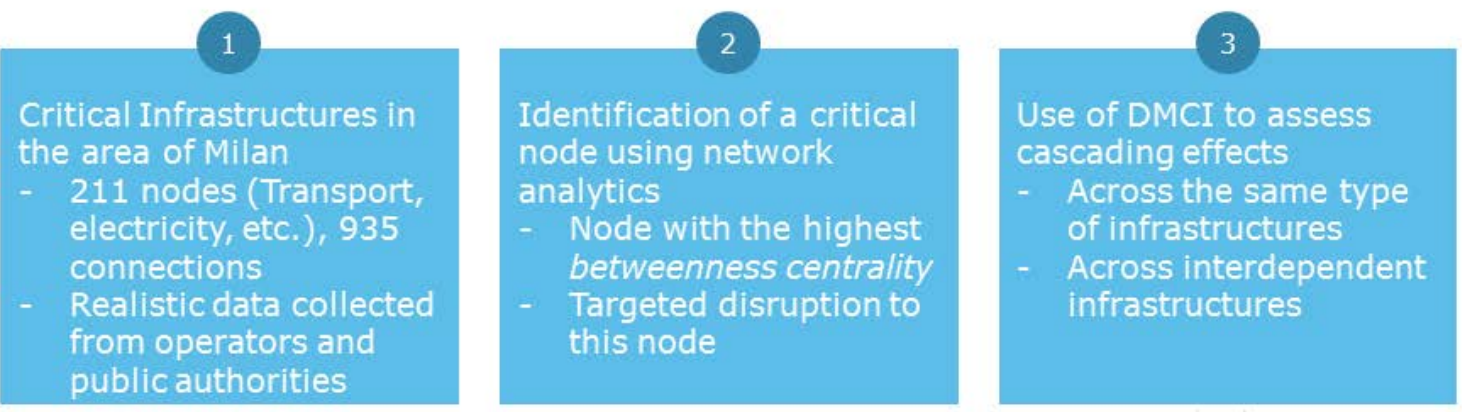


\section{Applying GR ${ }^{2}$ ASP: Use case 1}
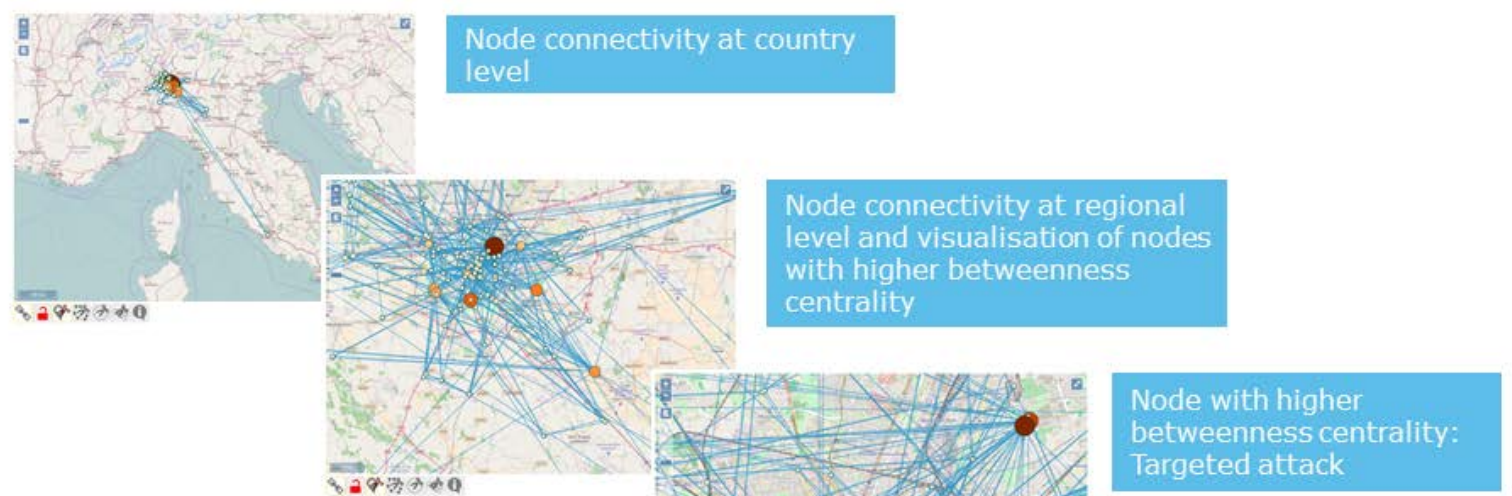

\section{Applying GR²ASP: Use case 1}
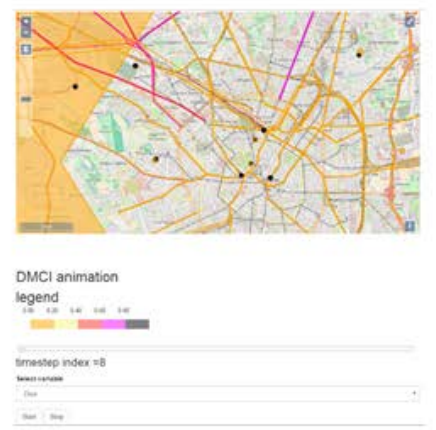

Dynamic visualization of results on maps

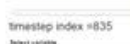

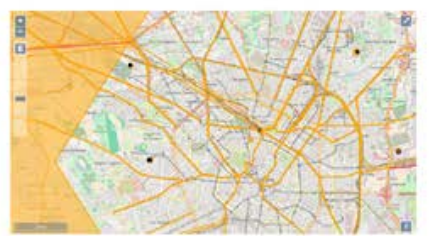

DMCI animation

logend .......

$=$

$\sim$

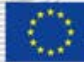

European Commission
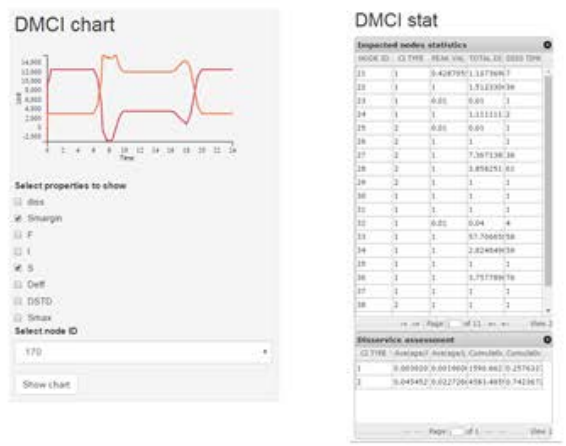

Node graphs (Service margin, disservice) and node statistics

Data input, interaction with the model and output visualization takes place through web-browser 


\section{Applying GR ${ }^{2}$ ASP: Use case 2}

Recovery optimization of a port infrastructure:

\section{Main assets are cranes and electrical substations}
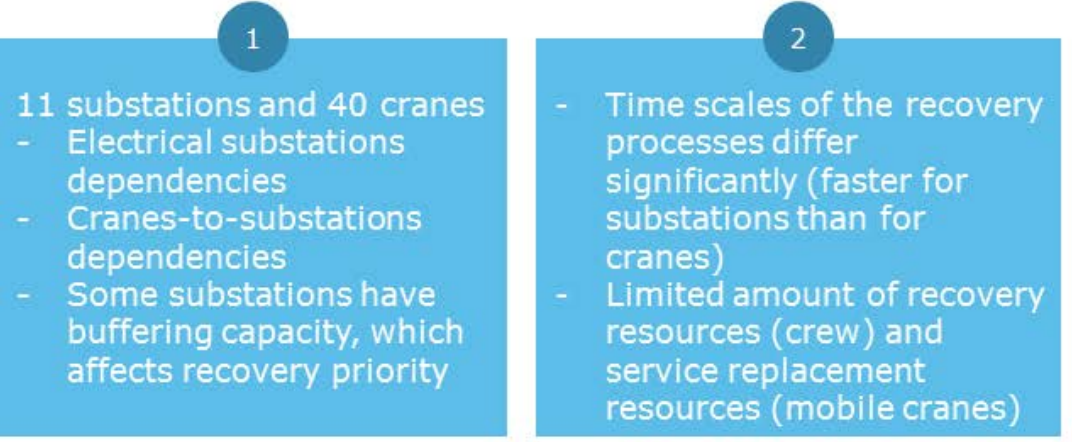

\section{Applying GR $\mathbf{R}^{\mathbf{2}}$ ASP: Use case $\mathbf{2}$}

Port topology in GRRASP and cranes' classification groups defining recovery priority

\begin{tabular}{ll}
$\begin{array}{l}\text { Classification } \\
\text { group }\end{array}$ & $\begin{array}{l}\text { Minimum } \\
\text { number of } \\
\text { required } \\
\text { functional } \\
\text { cranes }\end{array}$ \\
\hline 1 & 4 \\
2 & 4 \\
3 & 8 \\
4 & 10
\end{tabular}

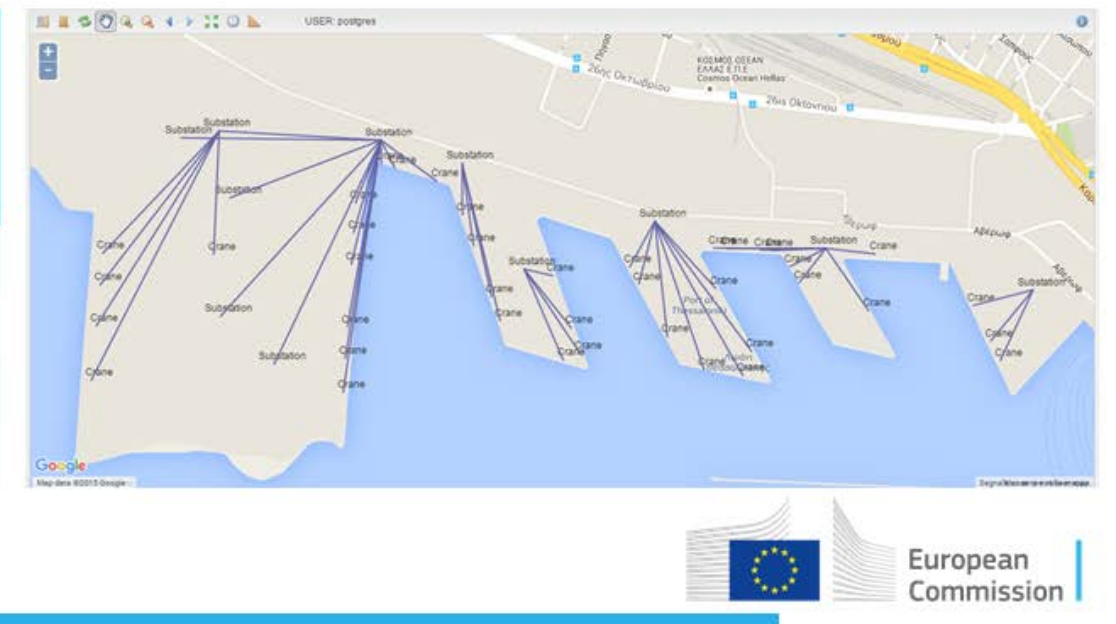




\section{Applying GR ${ }^{2}$ ASP: Use case 2}

Simulation of the recovery process ( $t=8$ days)

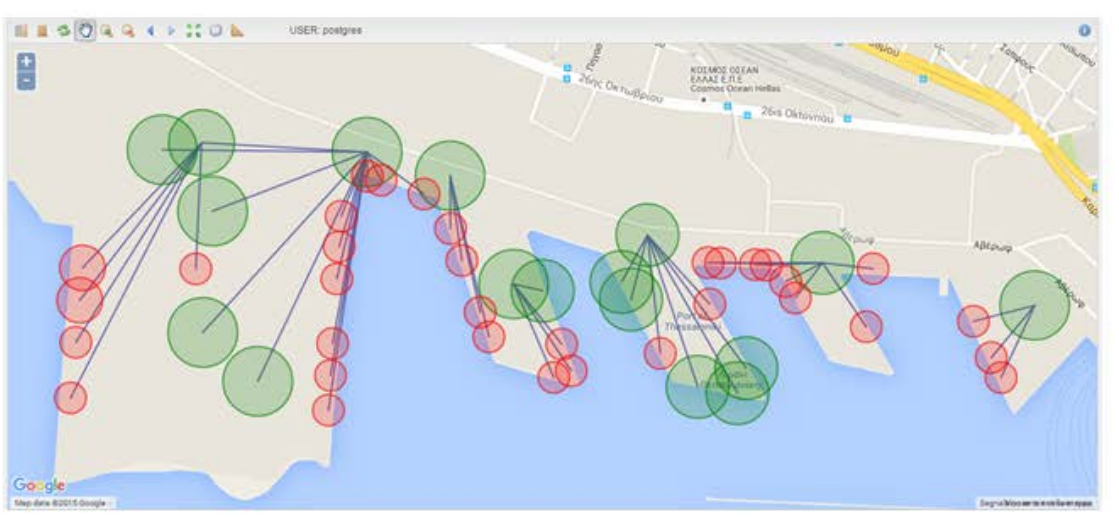

Recovered asset

Inoperable asset

\section{Applying GR ${ }^{2}$ ASP: Use case $\mathbf{2}$}

Simulation of the recovery process ( $t=102$ days)

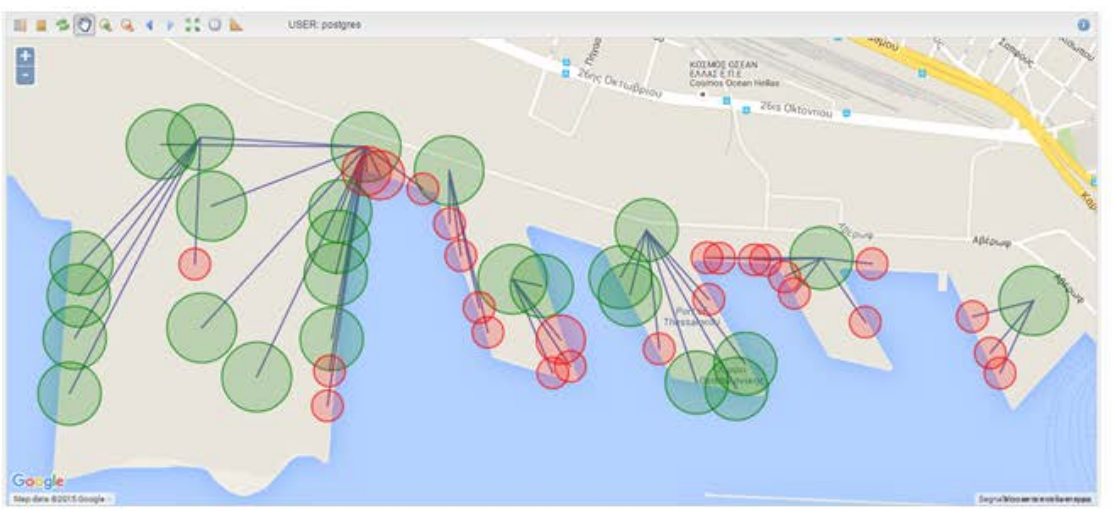

Recovered asset

Inoperable asset 


\title{
Conclusions \& Future work
}

\author{
We presented a framework for resilience analysis applicable \\ at various levels of granularity involving various models
}

Next steps: A scenario builder to link all three categories of

models - from asset disruption to socio-economic impact

We need more involvement from the community: Models

should facilitate collaboration and involvement and not

intimidate the end users

We do not necessarily need more models - but closer links

between our models: Interoperability is the key

We need to start thinking on training for the use of scientific

models: Bringing closer academia and end-users

\section{A closer collaboration at international level on modelling and training is needed!}

\section{References}

1. Alain Barrat, Marc Barthelemy, and Alessandro Vespignani, Dynamical processes on complex networks, Cambridge University Press, 2008.

2. Yang-Yu Liu, Jean-Jacques Slotine, and Albert-László Barabási, Controllability of complex networks, Nature 473.7346 (2011): 167-173.

3. Kash Barker, and Joost R. Santos, Measuring the efficacy of inventory with a dynamic input-output model, International Journal of Production Economics 126.1 (2010): 130-143.

4. Marzio Marseguerra, Enrico Zio, Luca Podofillini, and David W. Coit, Optimal design of reliable network systems in presence of uncertainty, Reliability, IEEE Transactions on, 54(2) (2005): 243-253.

5. Luca Galbusera, Georgios Theodoridis, and Georgios Giannopoulos. Intelligent Energy Systems: Introducing Power-ICT Interdependency in Modeling and Control Design, IEEE Transactions on Industrial Electronics 62.4 (2015): 2468-2477

6. Theodoridis, Georgios, Luca Galbusera, and Georgios Giannopoulos, Controllability Assessment for Cascade Effects in ICT-enabled Power Grids, International Conference on Critical Information Infrastructures Security. Springer International Publishing, 2015.

7. Chenyang Lian, and Yacov Y. Haimes, Managing the risk of terrorism to interdependent infrastructure systems through the dynamic inoperability input-output model, Systems Engineering 9.3 (2006): 241-258.

8. Yacov Y. Haimes et al., Inoperability input-output model for interdependent infrastructure sectors. I: Theory and methodology, Journal of Infrastructure Systems 11.2 (2005): 67-79.

9. Yacov Y. Haimes et al., Inoperability input-output model for interdependent infrastructure sectors. II: Case studies, Journal of Infrastructure Systems 11.2 (2005): 80-92.

10. Kash Barker, and Joost R. Santos, Measuring the efficacy of inventory with a dynamic input-output model, International Journal of Production Economics 126.1 (2010): 130-143.

11. Luca Galbusera et al., Inoperability Input-Output Modeling: Inventory Optimization and Resilience Estimation during Critical Events, ASCE-ASME Journal of Risk and Uncertainty in Engineering Systems, Part A: Civil Engineering (2016): B4016001

12. Paolo Trucco, Enrico Cagno and Massimiliano De Ambroggi, Dynamic functional modelling of vulnerability and interoperability of Critical Infrastructures, Reliability Engineering \& System Safety, 105 (2012): 51-63 
International Workshop on Modeling of Physical, Economic, and Social Systems for Resilience Assessment

\section{Stay in touch}

EU Science Hub: ec.europa.eu/jrc

๖ Twitter: @EU_ScienceHub

f Facebook: EU Science Hub - Joint Research Centre

in LinkedIn: Joint Research Centre

You YouTube: EU Science Hub 


\section{Georgios Giannopoulos short biography}

Dr Georgios Giannopoulos holds a degree in Mechanical and Aeronautical Engineering and a PhD in Engineering from Vrije Universiteit Brussel and a management degree from Solvay Brussels School in Economics and Management. He joined the Joint Research Centre in 2007. He is currently working in the domain of critical infrastructures risk and resilience modeling with focus on systems of systems perspective. In this framework he is carrying out research in the domain of GIS for risk assessment and resilience for critical infrastructures, interdependencies and economic impact of critical infrastructure disruption. In addition he has been actively involved in the review process of the Council Directive 2008/114/EC on the identification and designation of European Critical Infrastructures supporting DG HOME efforts, he is running the Thematic Network on Critical Energy Infrastructure Protection (TNCEIP) supporting DG ENERGY and he is the project leader of the European Reference Network for Critical Infrastructure Protection (ERNCIP). He has been the author of about 40 scientific publications in peer reviewed journals and scientific conferences. 
Resilience Modelling Research in New Zealand

Nick Horspool, GNS Science, Lower Hutt, New Zealand

Recent disasters in New Zealand have dramatically highlighted the impact shocks can have on society and its ability to function and grow. The Christchurch Earthquakes of 2010-2011 caused over NZ\$40B of economic losses, 185 fatalities and 10,000 injuries, but also of note is that the rebuild and recovery of the city is still ongoing, some 6 years following the first earthquake. Issues with the recovery of Christchurch have provided the platform for a dialogue on resilience across various sectors in New Zealand. In order to shift to a resilience-based way of thinking a step-change in attitudes is required to transform governance, business, public and science responsibilities. Underpinning the resilience movement are a number of resilience focussed research programs that span the physical, engineering and social sciences. These research programmes are advancing the science and implementation pathways of resilience through system-level science with highly integrated cross-disciplinary collaborations. One area of focus is the development of resilience modelling workflows that will be delivered through decision support tools to enable decision makers to better assess resilience-building strategies in their dayto-day decisions as well as actioning community led activities. This presentation will provide an overview of NZ based resilience modelling research programmes and demonstrate through casestudies how these are leading to a more resilient New Zealand. 


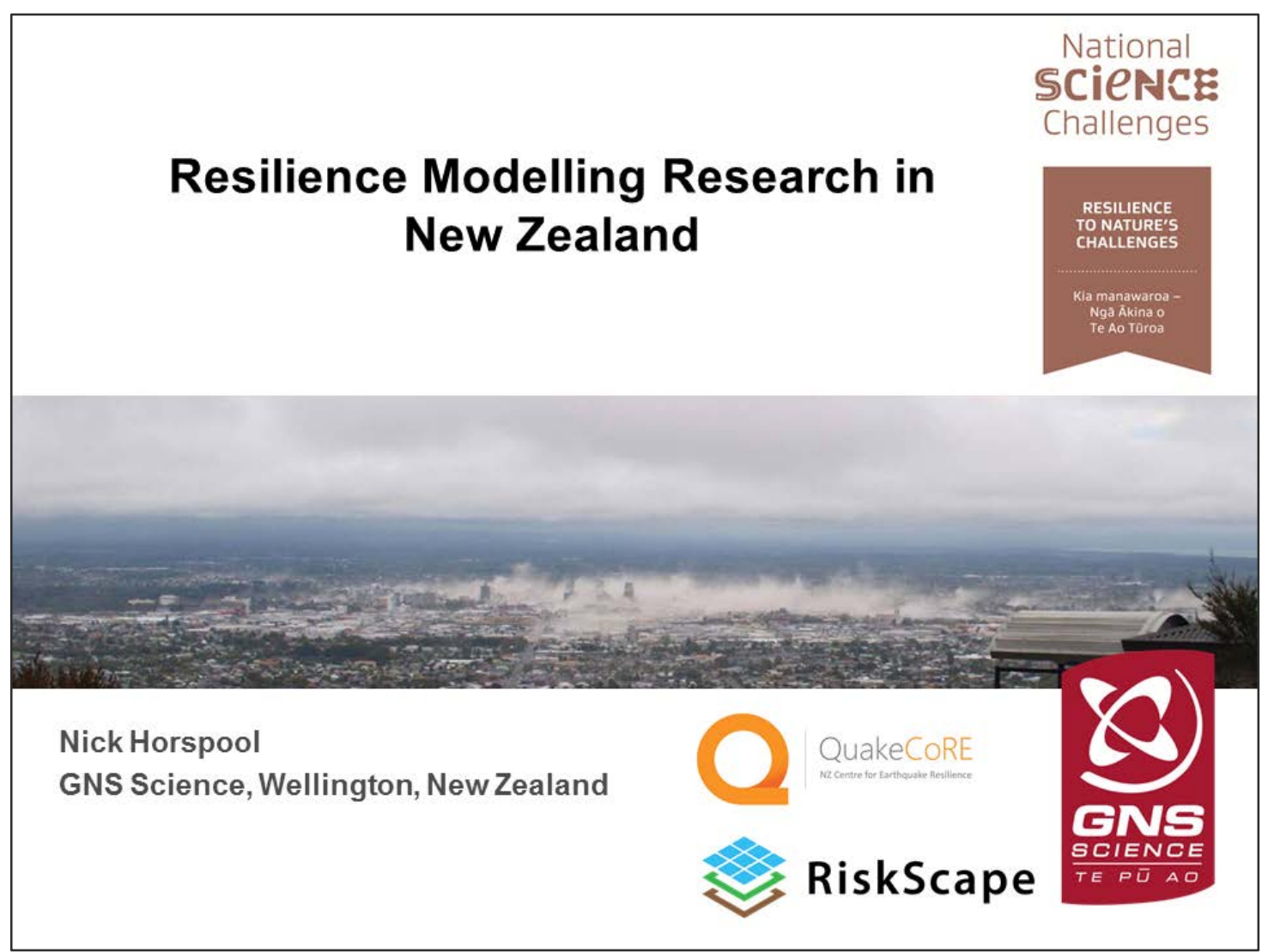

\section{Consortium of Universities, Government and Private Research Institutes}
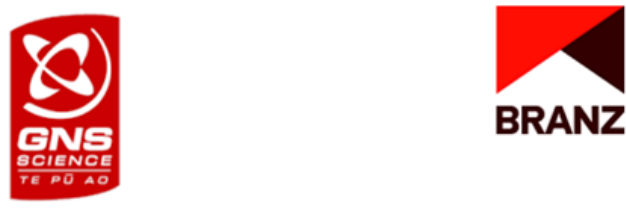

BRANZ

MASSEY UNIVERSITY TE KUNENGA KI PÖ́REHURO

UNIVERSITY OF NEW ZEALAND

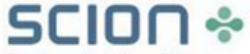

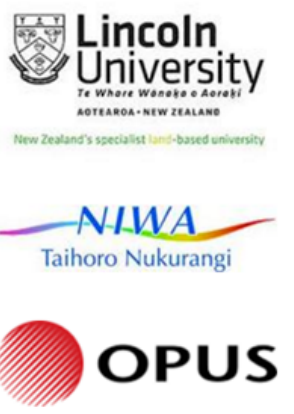

Taihoro Nukurangi

OPUS m.e market economics

Resilient

ORGANISATIONS

\section{UC항}

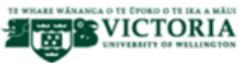

OT:Ẍtro

rimernagosous 


\section{New Zealand Snapshot}

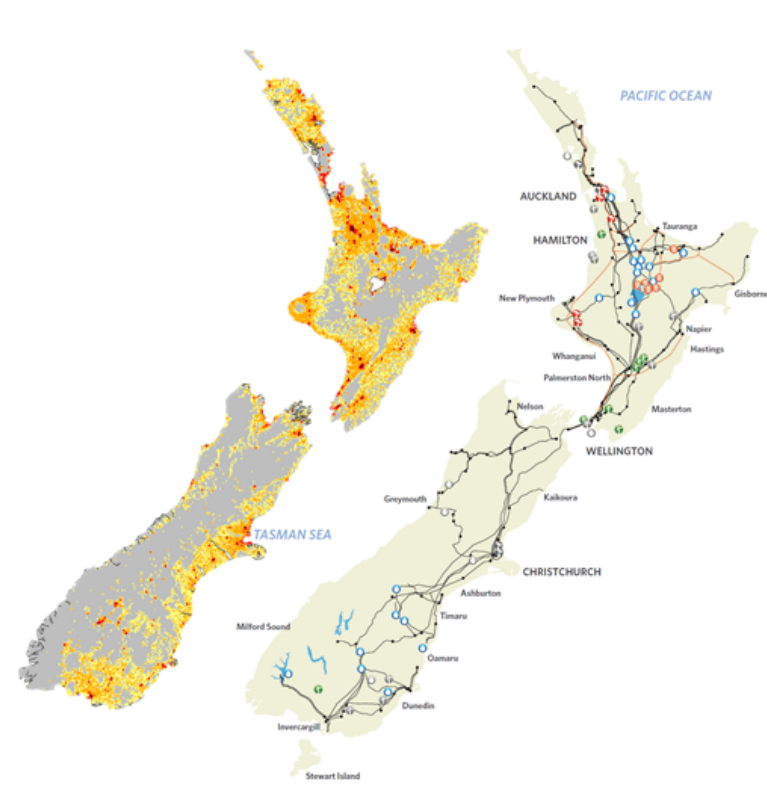

- Very high insurance penetration (>95\%)

- High risk awareness

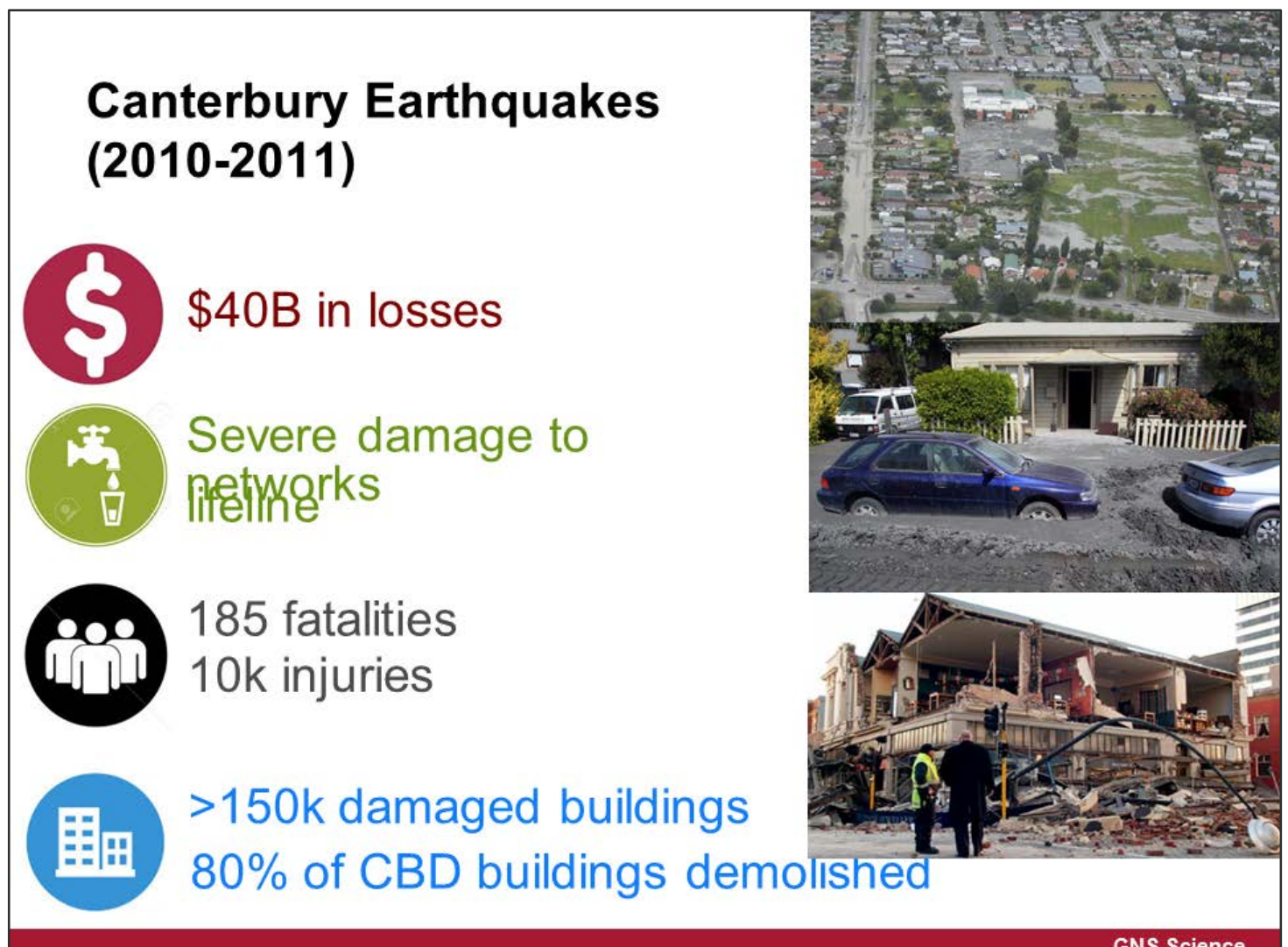




\section{Ground Motions Were Above Design Level}

- Response Spectra from CBD

- 2 buildings collapsed

- $80 \%$ CBD buildings demolished ( 1200)
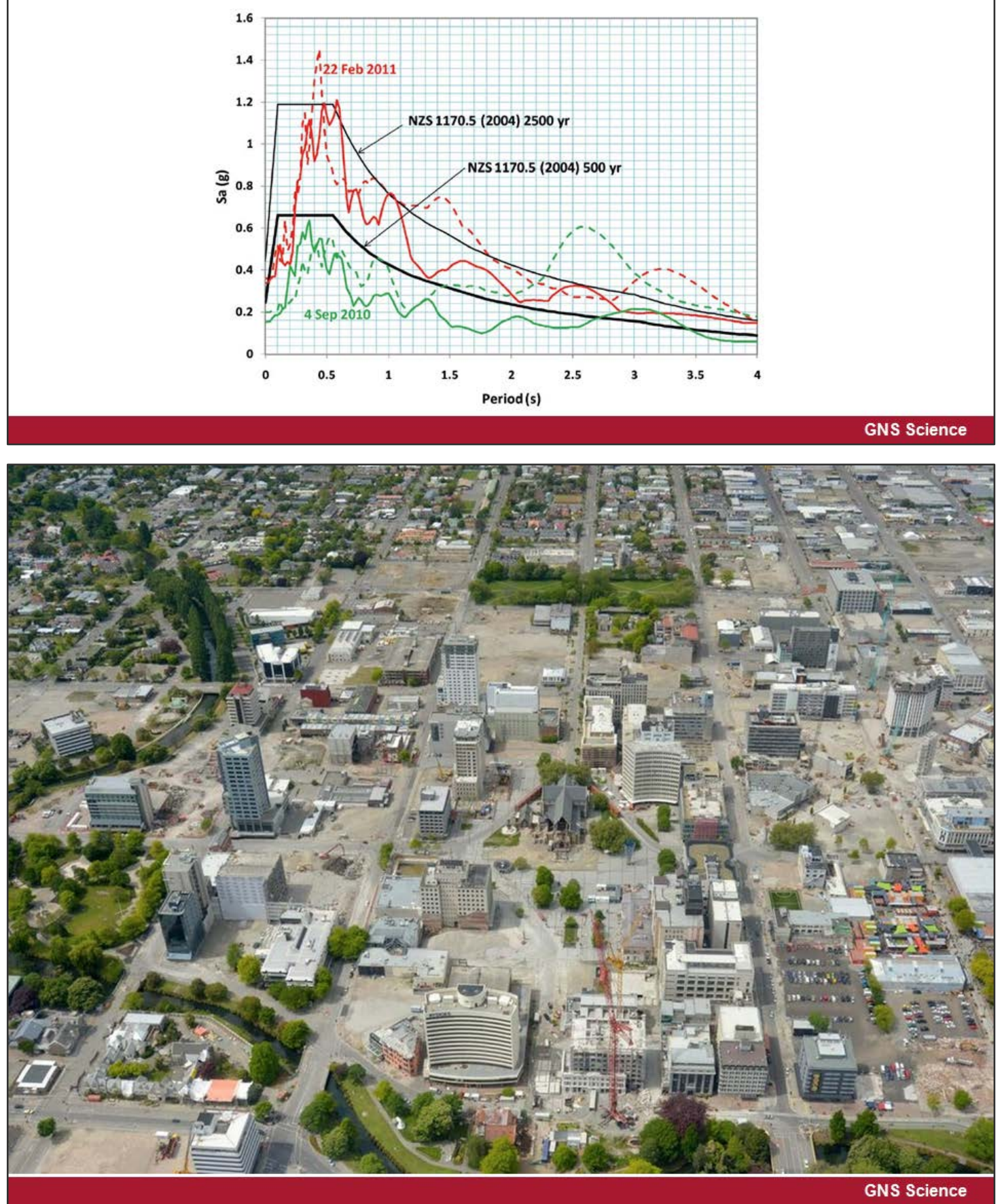


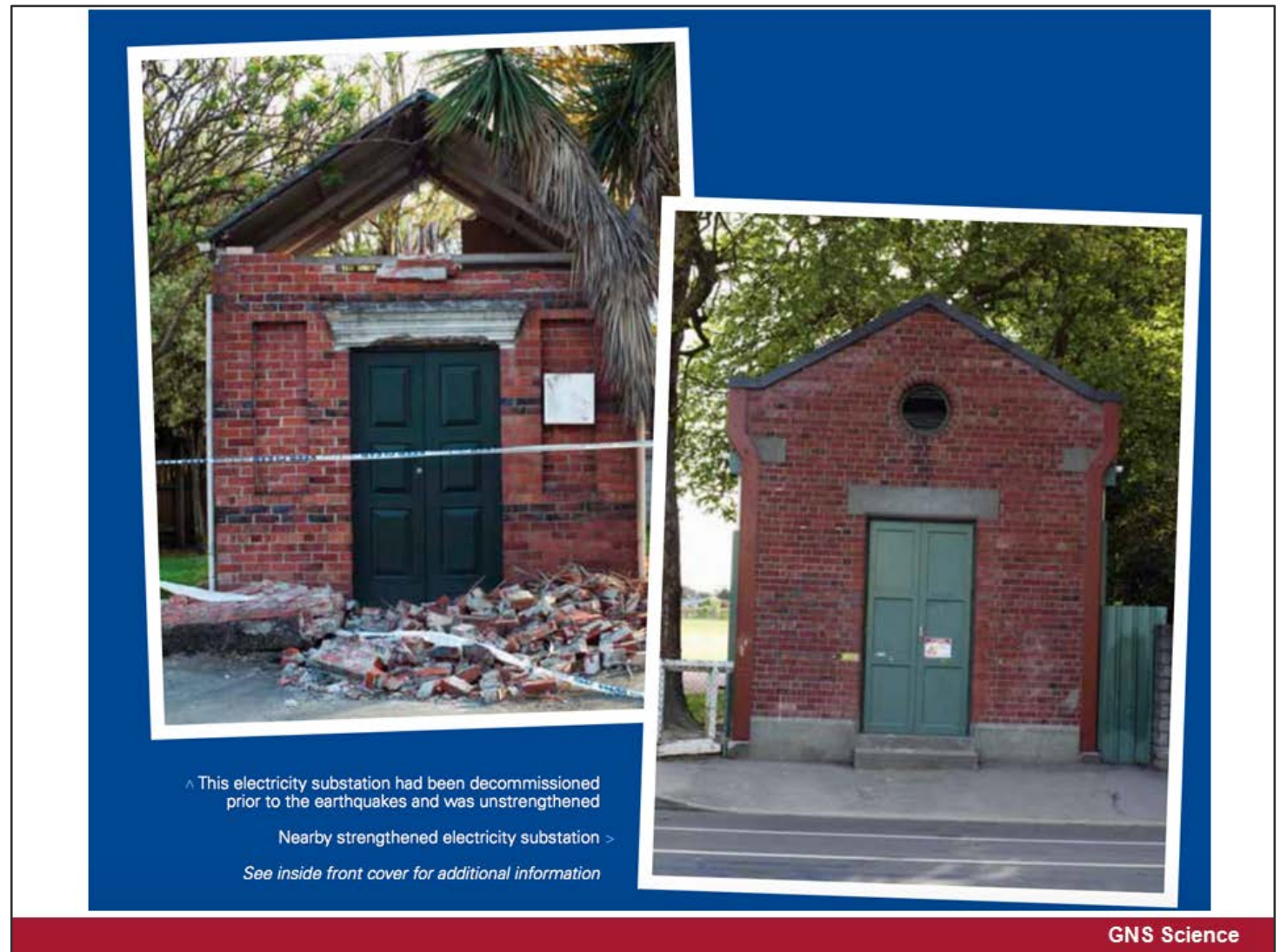

\section{Two New 10 year Resilience Programs (2016-2026)}
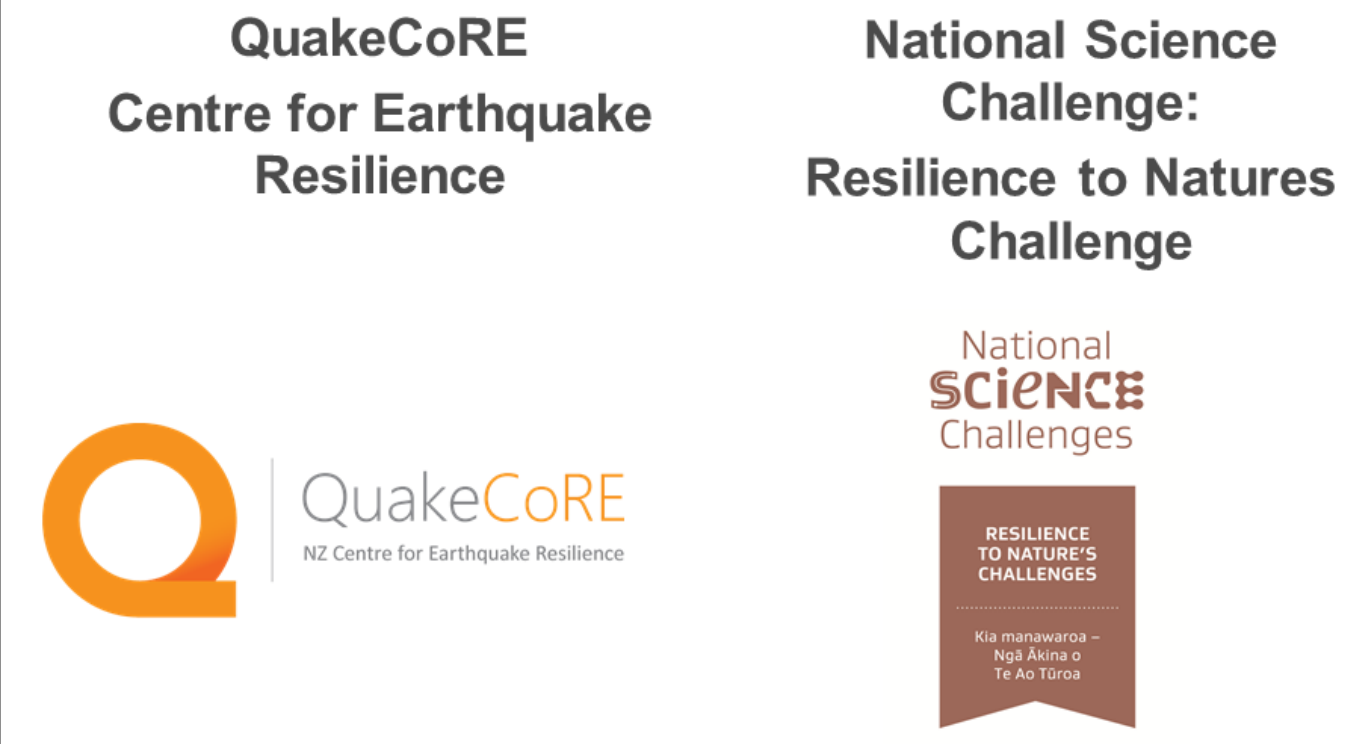


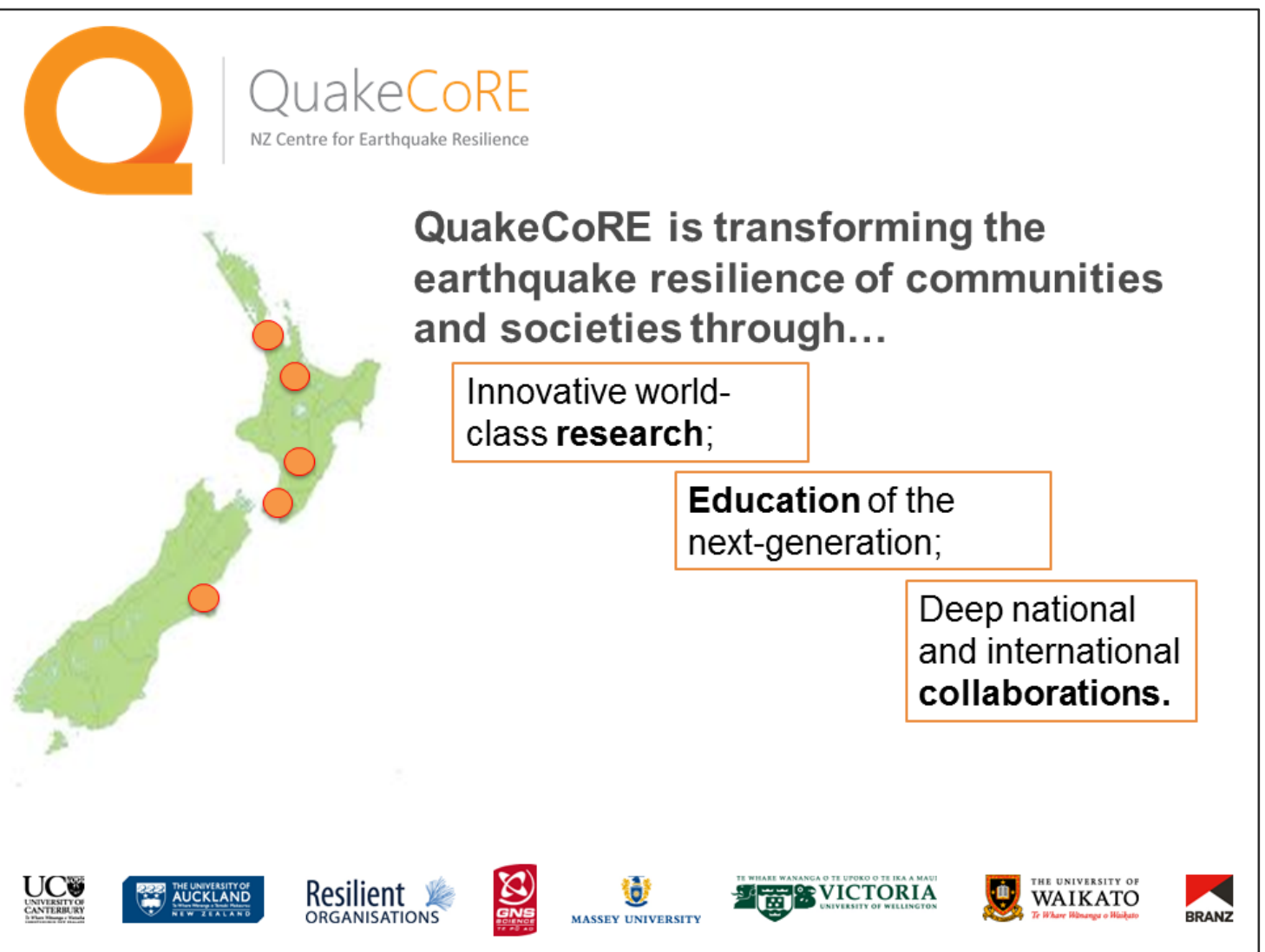

\section{QuakeCoRE Structure}

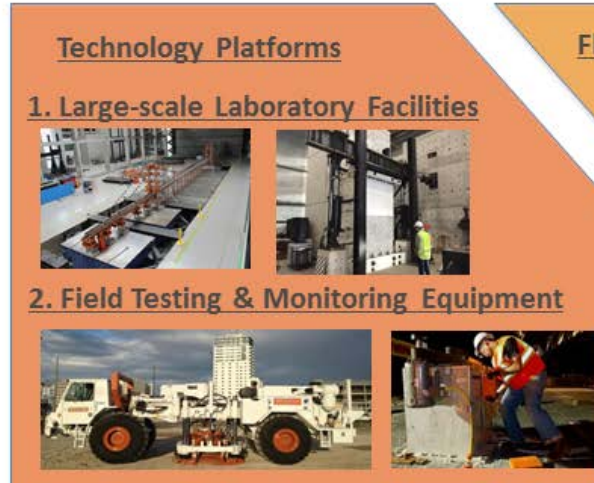

Flagship Programmes

1. Ground motion

simulation \& validation

3. Multi-disciplinary Community Datasets

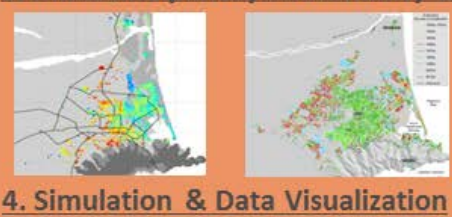

2. Liquefaction

impacts on

infrastructure
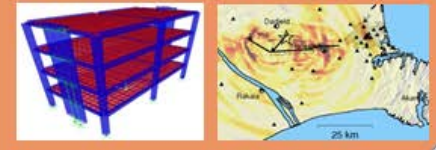

6. Distributed

infrastructure

(with RNC-NSC) 
Flagship 1:

Ground motion simulation \& validation Leader: B. Bradley

Developing a fundamental tool for engineering and earthquake resilience applications.

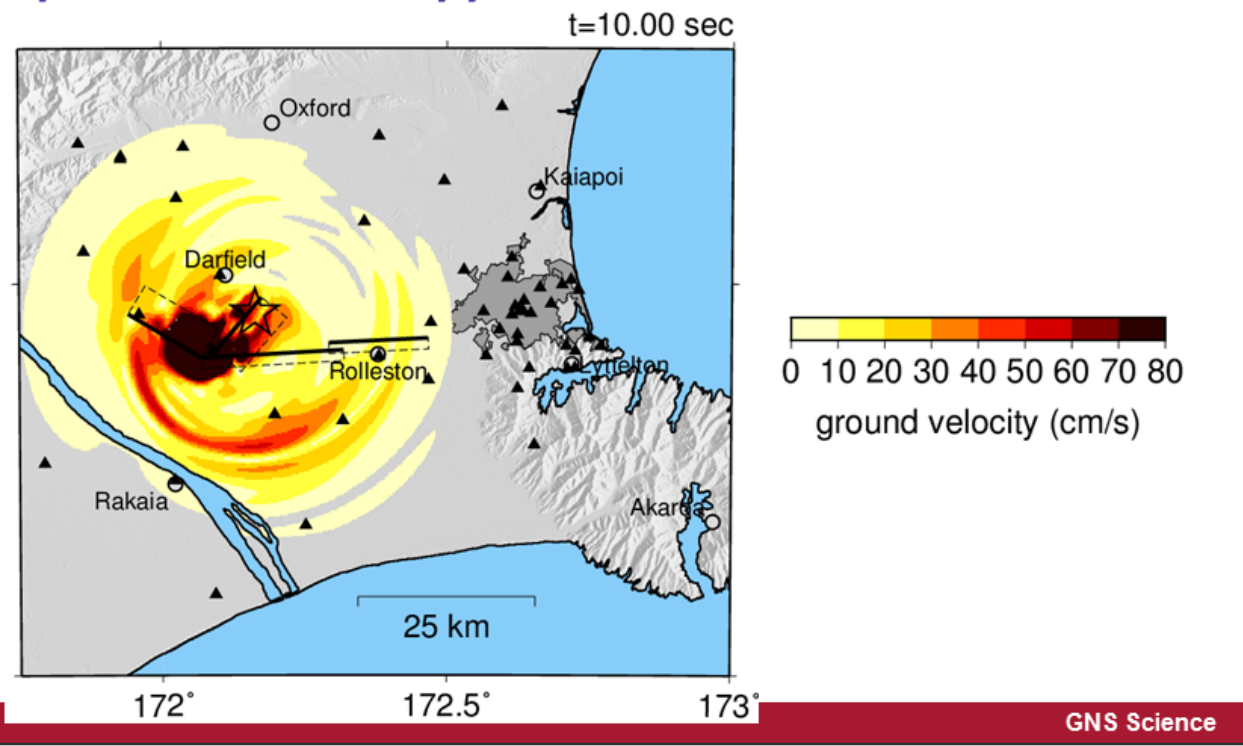

\section{Flagship 2:}

Liquefaction Impacts on Infrastructure Leader: M. Cubrinovsh

\section{Next-generation assessment methods and mitigation} strategies for liquefaction hazards.

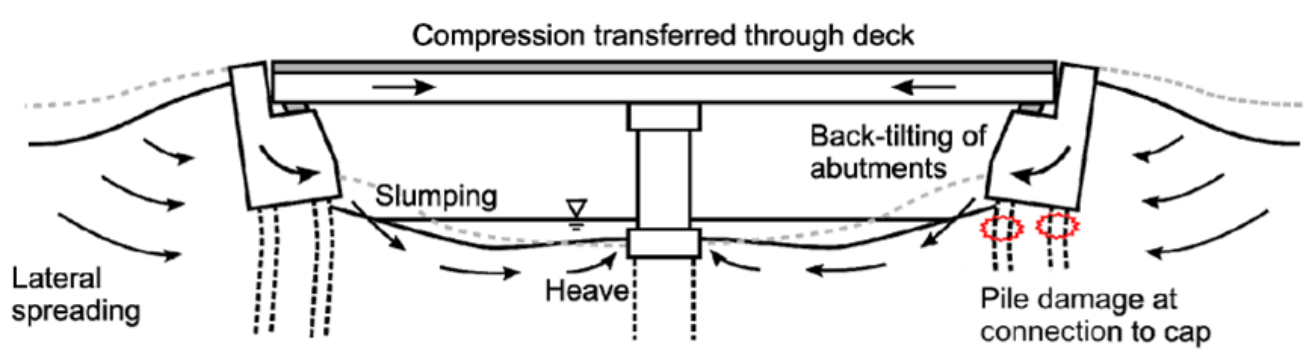




\section{Flagship 3: Earthquake-Prone Buildings Heritage, safety and economics}

Addressing the risk posed by earthquake-prone buildings through a multi-disciplinary lens.

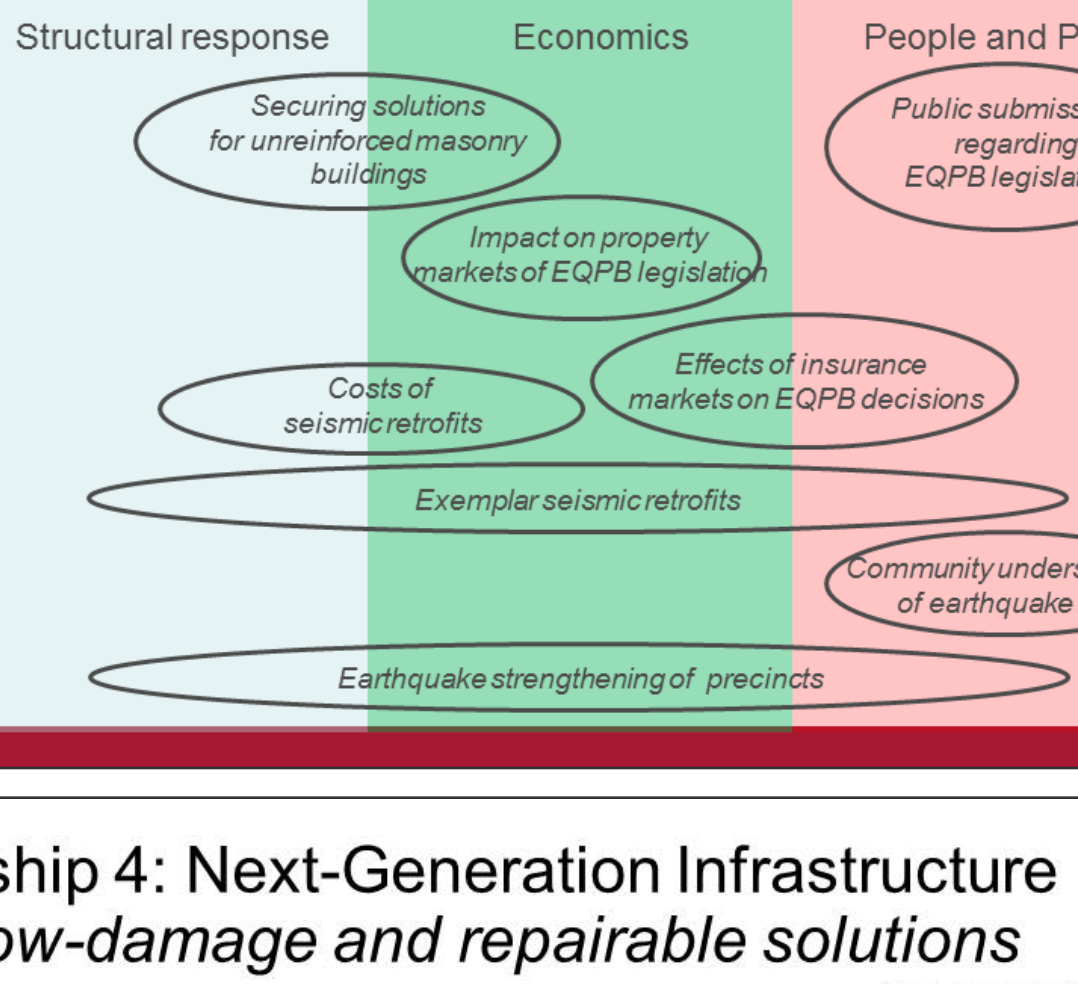

Co-Leaders: K. Elwood

S. Pampanin

A new design paradigm whereby reparability and damage-control is explicitly considered in the design process.

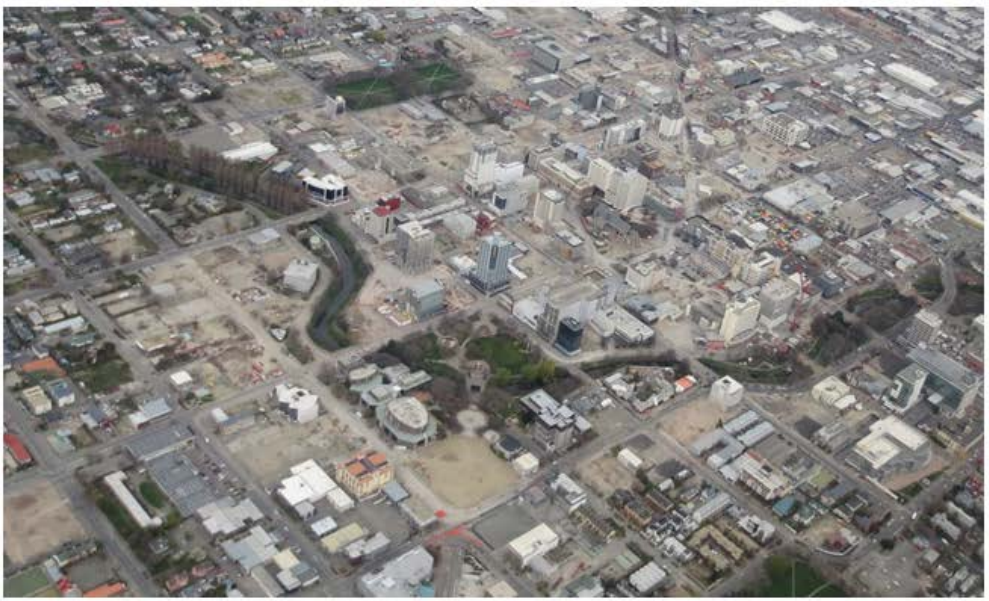




\section{Flagship 5: Pathways to Resilience}

\section{How best to invest to improve New}

Leader: E. Seville

\section{Zealand's resilience to earthquakes?}

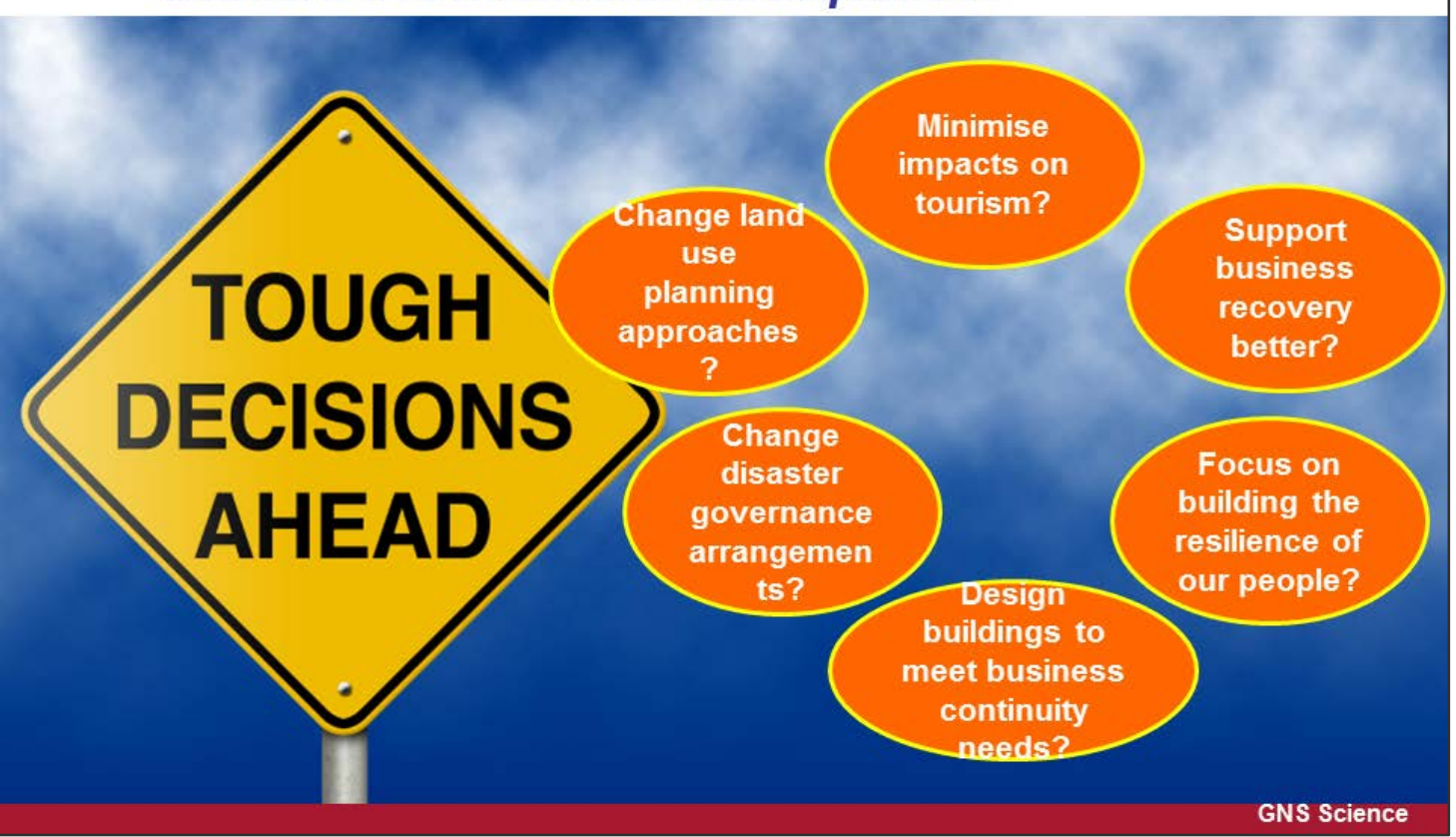

\section{Flagship 6: Distributed Infrastructure}

Quantifying the resilience of distributed infrastructure

Leader: L. Wotherspoor networks and the benefits of mitigation measures.
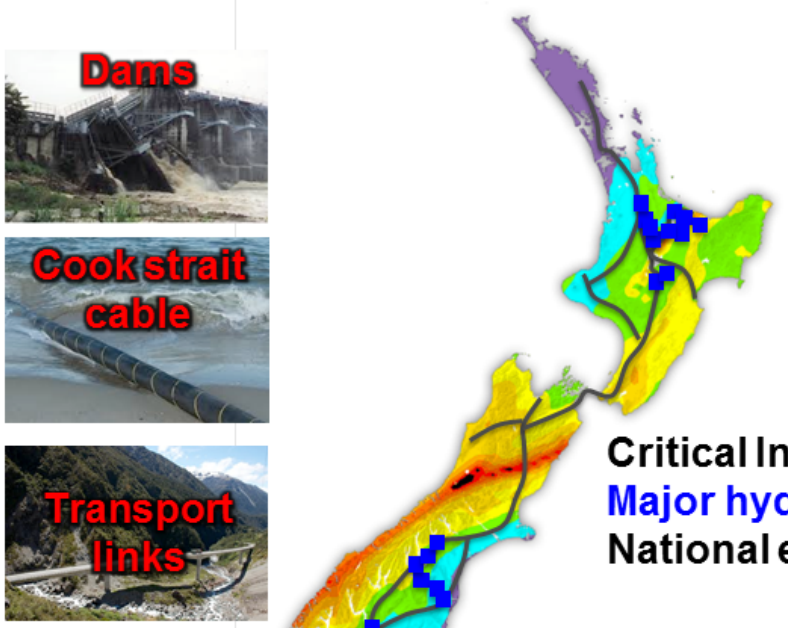

Critical Infrastructure - too big to fail? Major hydro/geothermal generators National electricity grid 


\section{Resilience Challenge - Mission}

Partner with stakeholders to build a transformative pathway towards natural hazard resilience.

Delivered by priority-driven co-creation laboratories and targeted underpinning research, suited to our diverse and rapidly changing cultural, economic, built and natural environments.

RESILIENCE

TO NATURE'S

CHALLENGES
Kia manawaroa

- Ngā Ākina o

Te Ao Tūroa
National SCIENCE: Challenges

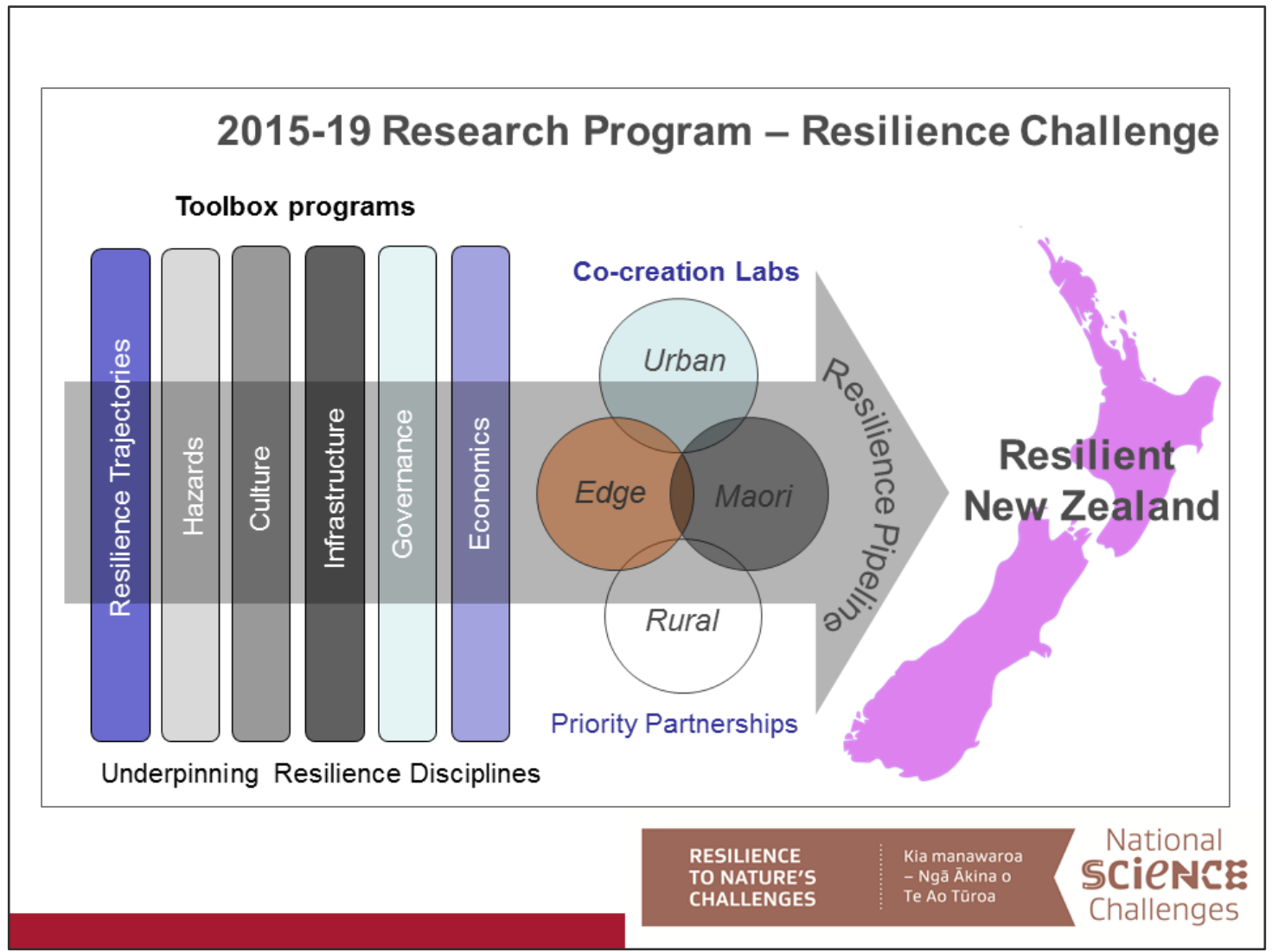


Case studies

\section{National} ScieNcE: challenges

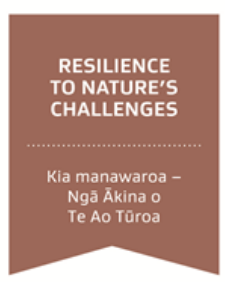

\section{AUCKLAND \\ Urban (Lead)}

Economics

Culture

Edge

Infrastructure

Trajectories






\section{Resilience Modelling in NZ}

- "Risk-based resilience modelling"

- Transition from risk assessment approach to risk informed resilience

- Adapted from NIST framework for community resilience for $\mathrm{NZ}$ context

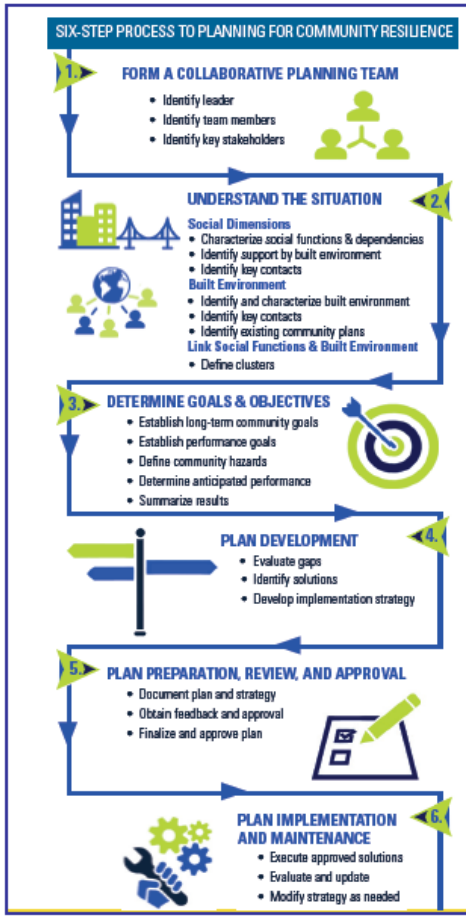

GNS Science

\section{Detailed framework for community impact}

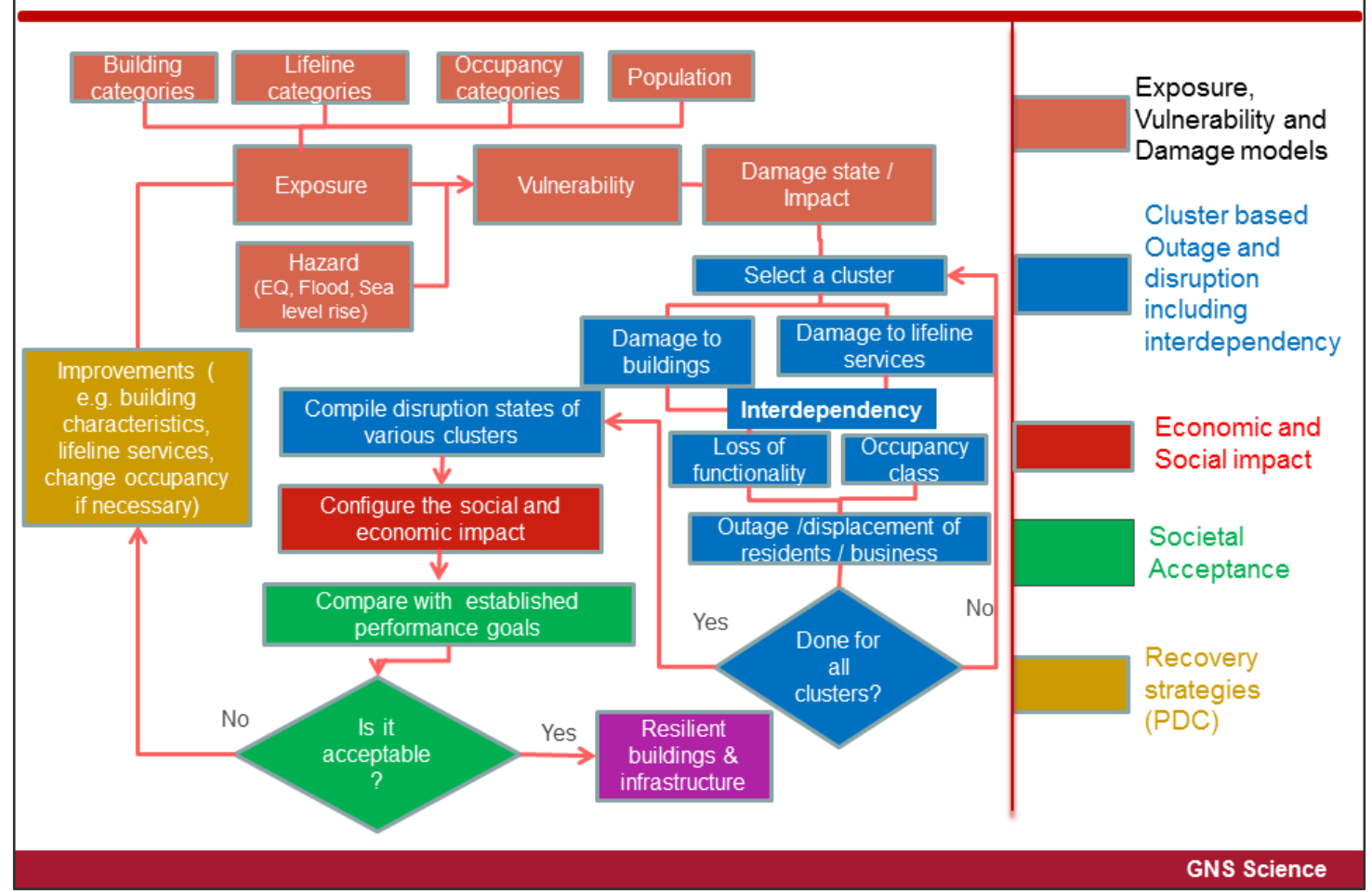




\section{Interface with Risk and Socio-Economic models}
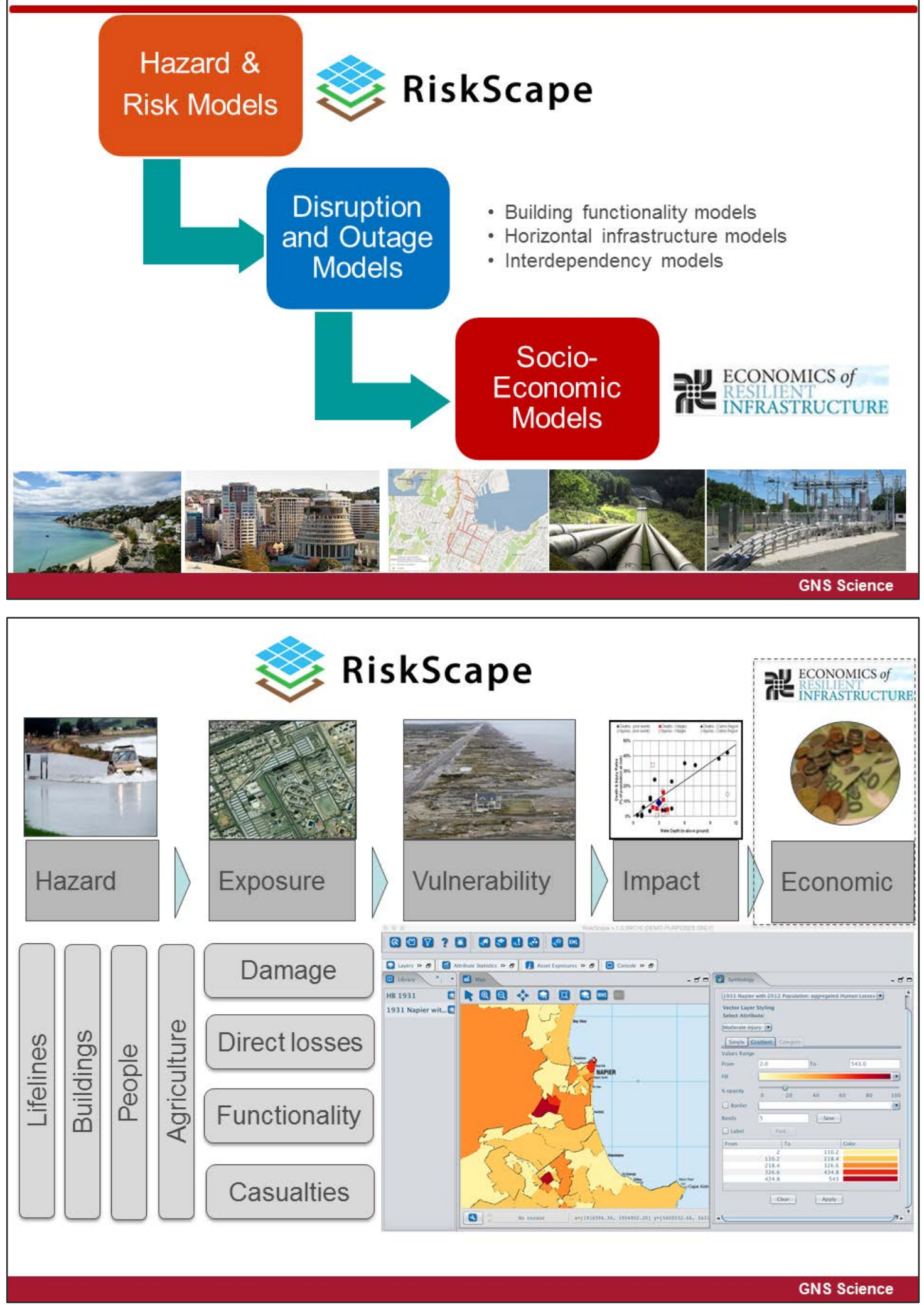


\section{Interdependency Modelling}

- National Sector Network Models

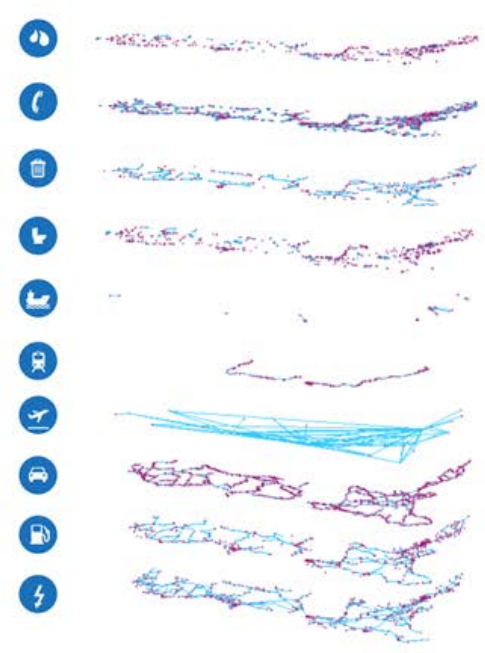

\section{Network Interdependency}

- Driven by expert elicitation

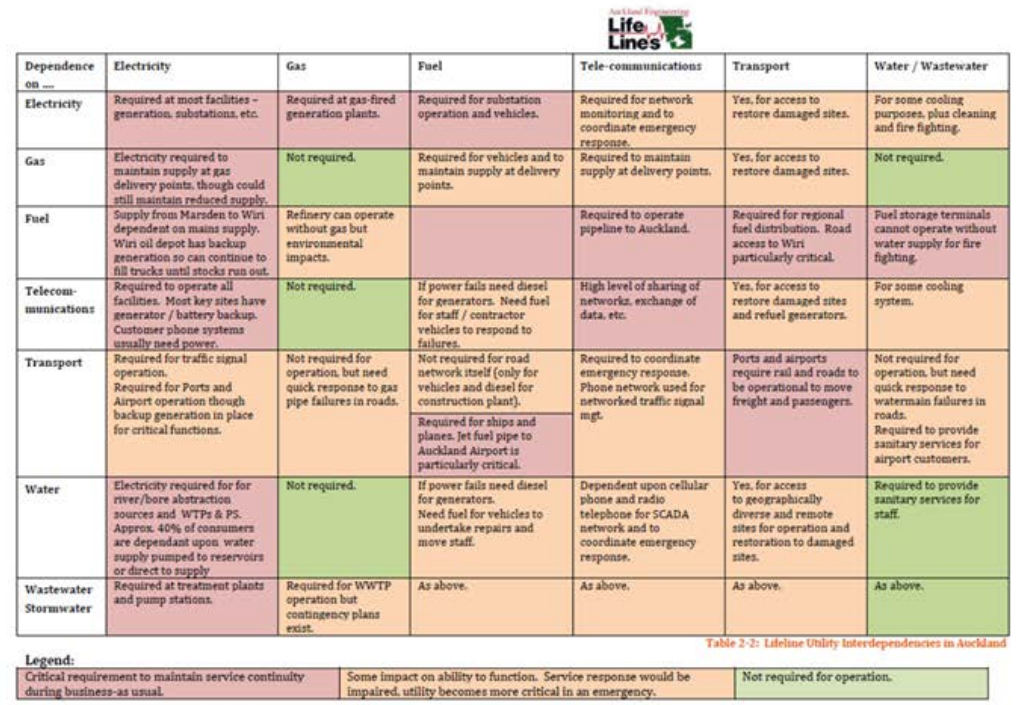




\section{Network Interdependency}

- E.g. Electricity substation removal

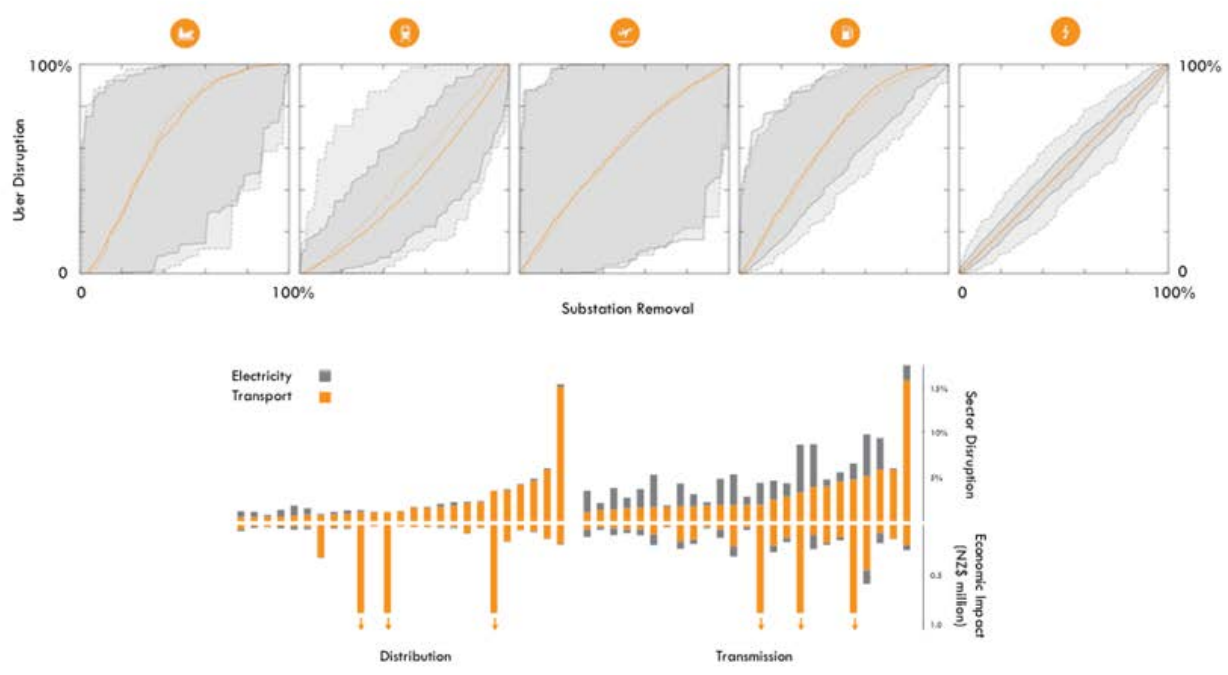

\section{MERIT = Modelling the Economic Resilience of Infrastructure Tool}

- Fully dynamic. The model is able to show transition pathways under various postimpact scenarios.

- Sectoral and commodity coverage. The model has between 40 and 60 aggregate economic sectors/ commodities included.

- Spatial. The model allows economic impacts to be differentiated spatially in an area or community.
- Multi-regional. Covers all regions in New Zealand. This allows cross-boundary economic impacts associated with infrastructure failure to be accounted for including impacts in labour markets, capital markets, housing markets and infrastructure provisioning.

- Based on General Equilibrium principles. The model uses prices to equilibrate supply and demand. 

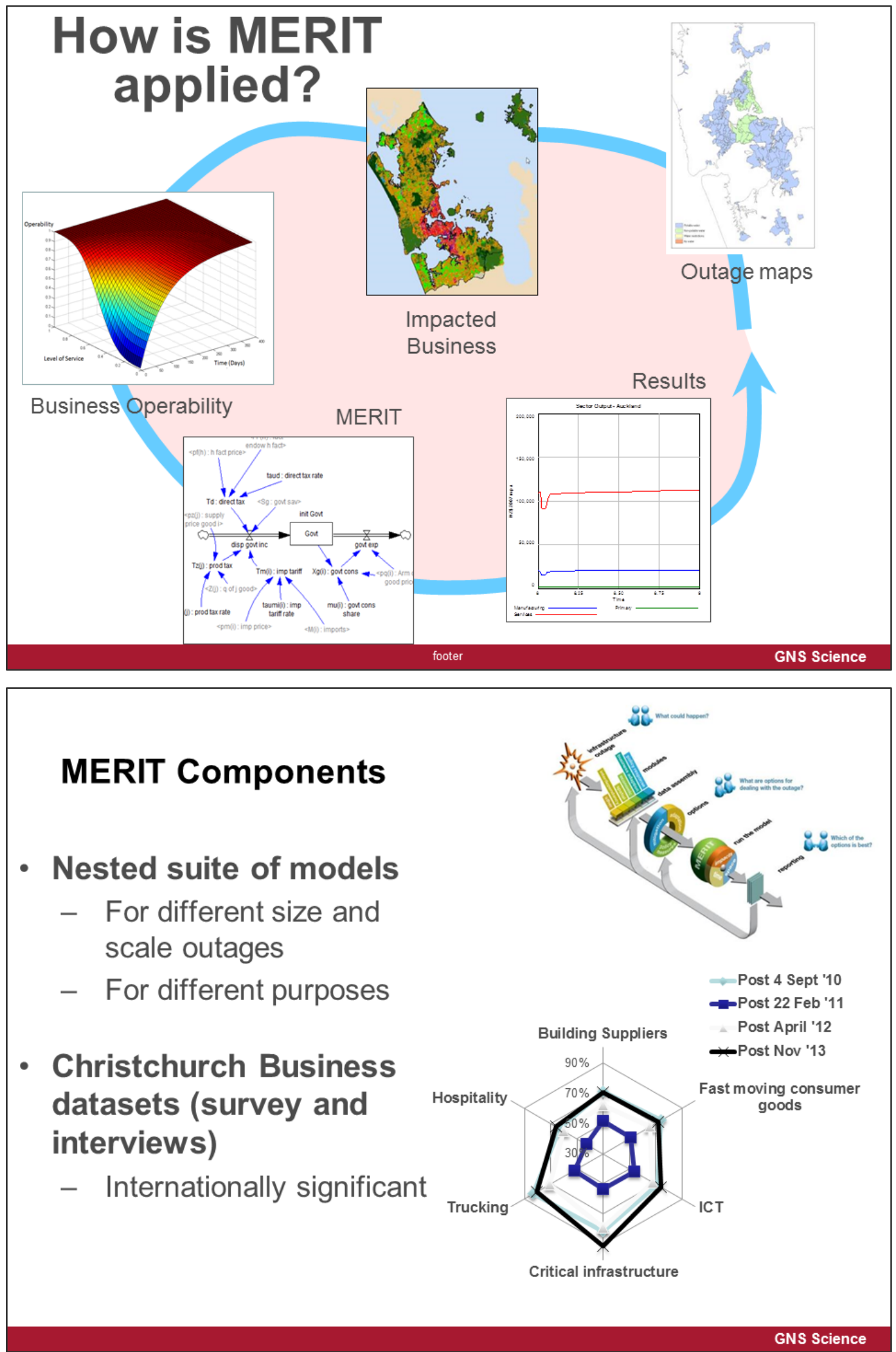


\section{MERIT Suite of Models}

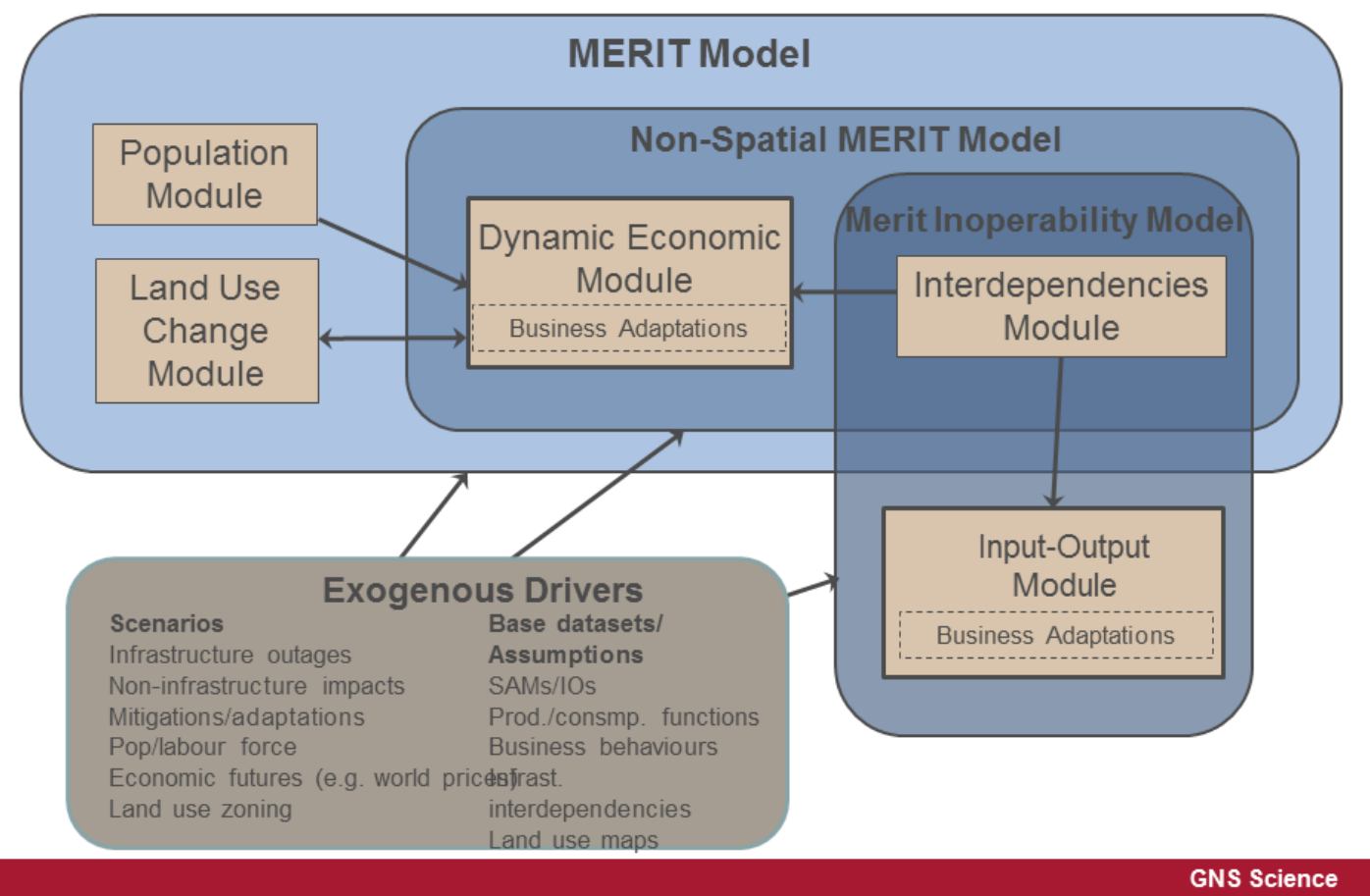

\section{Alpine Fault Case Study}




\section{Alpine Fault}

- The scenario earthquake included in the 2013/6 CDEM exercise and replicates a M8.1

earthquake involving $\sim 400$ $\mathrm{km}$ rupture between Milford Sound and the Ahaura River

- $50 \%$ probability in next 30 years

- Strong shaking intensities over large areas of S. Island

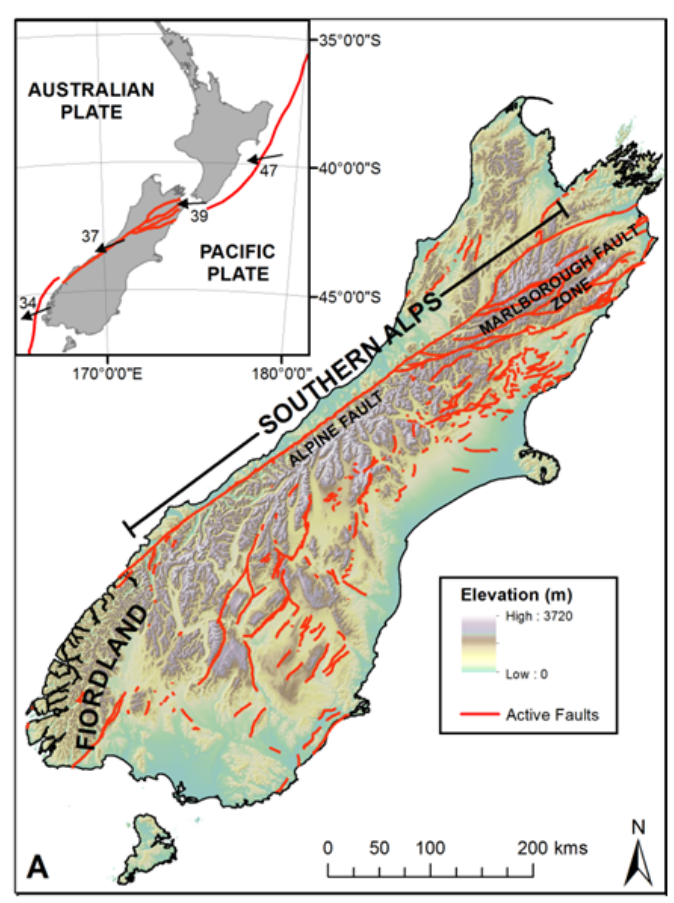

GNS Science

\section{Ground Motion Simulation}

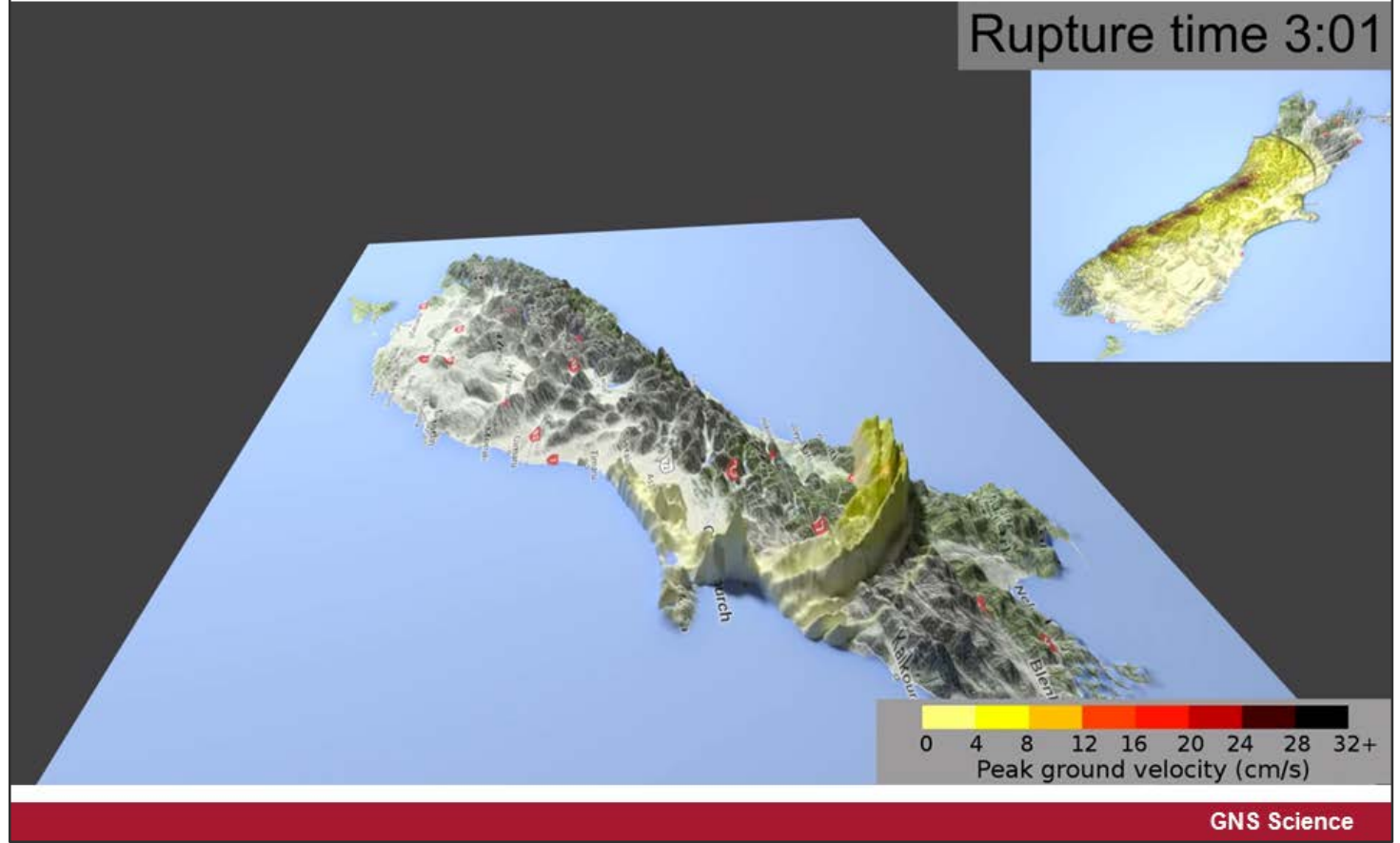




\section{The modelled impacts - direct losses, damage, casualties}
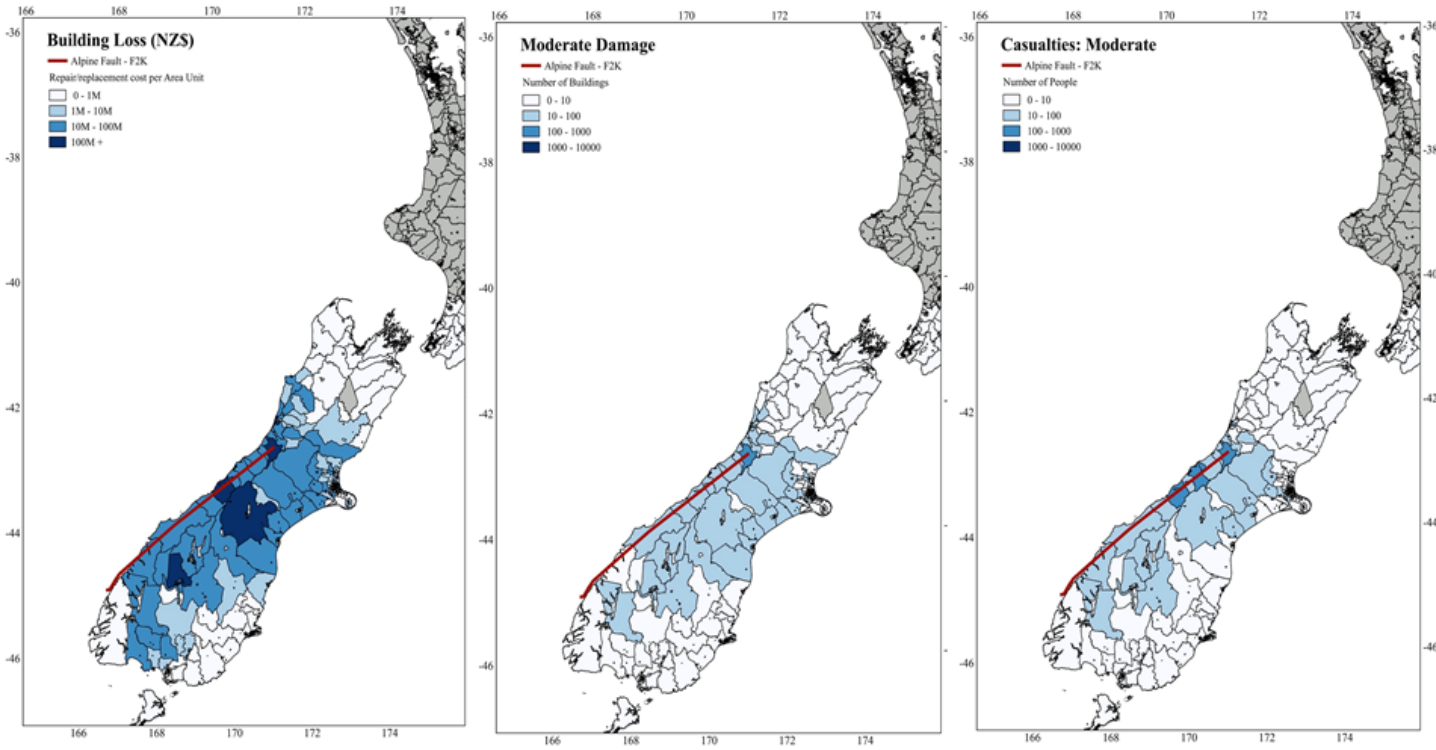

\section{The modelled road impacts}

- Surface rupture

- multiple sites SH6 between Hokitika and Haast

- Landsliding

- Alpine passes SH7 \& SH73

- SH6 \& SH94

- Damage to bridges

- 5 bridges total failure

- 13 bridges weight restrictions $(>3.5 t)$
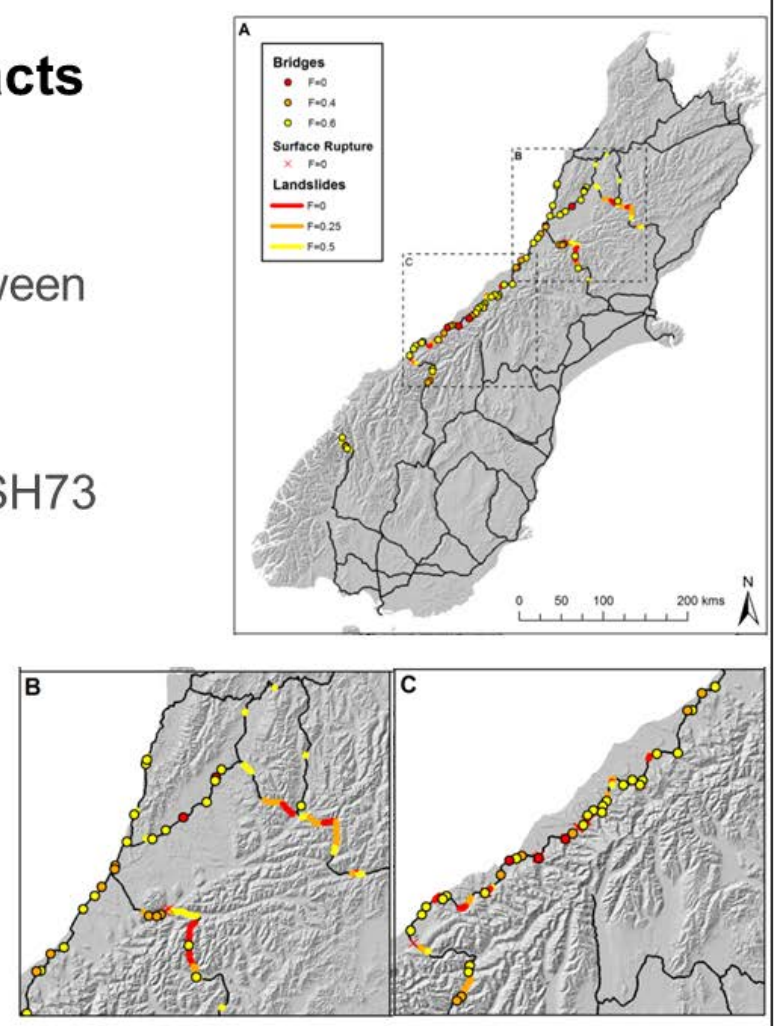


\section{The modelled rail impacts}

- The Midland Line and Stillwater-Westport Line are the most affected

- Surface rupture affects the railway at Lake Poerua

- Landsliding affects the railway at 10 locations

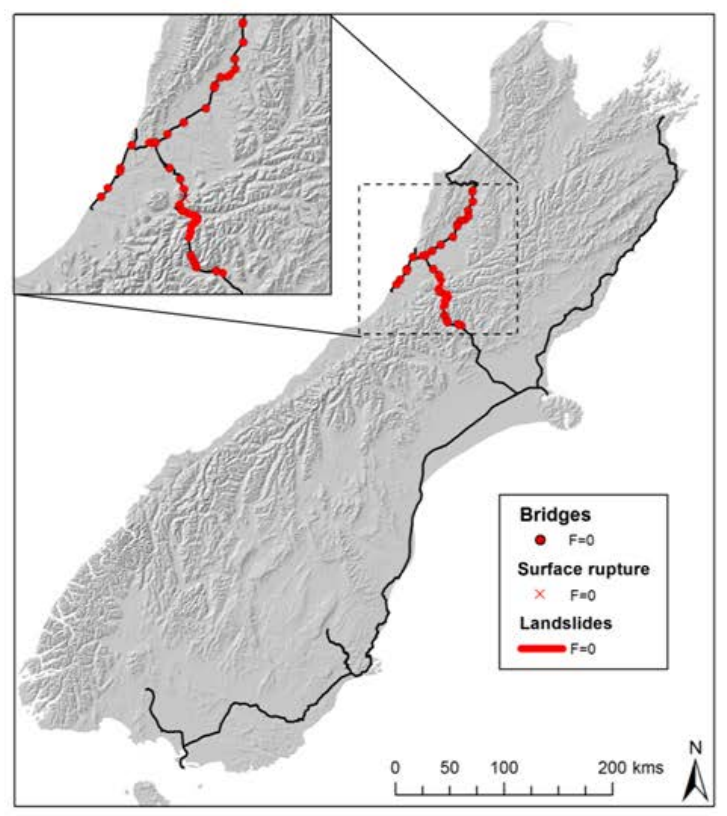

\section{Hydro-Electric Power and Water Outages}

- HEP outages at Franz Josef, Greymouth and Hokitika caused by pylon/powerpole damage

- Damage to water/sewers

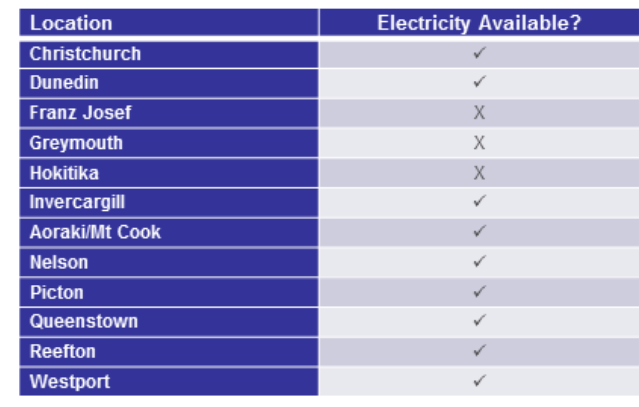
and stormwater systems in the listed townships is extreme in some cases

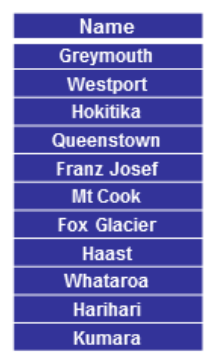




\section{Restoration Timelines}

Roads

- After 90 days Haast remains the only location inaccessible

Rail

- After 180 days final blockages on the Midland line cleared

Hydro Electric Power and 3-Waters

- After 60 days Greymouth has water supply restored, after 160 days Greymouth and Hokitika have sewers restored.

- After 80 days Franz Josef has electricity supply restored.

\section{Alpine Fault Scenario - Results}

Net Change in Gross Domestic Product after Event (\$2015mil)

\begin{tabular}{lcccc}
\hline & 1 month & 2 months & 6 months & 1 year \\
\hline Infrastructure Disruptions Only & -103 & -174 & -245 & -232 \\
$\begin{array}{l}\text { Infrastructure and Non- } \\
\text { Infrastructure Disruptions }\end{array}$ & -134 & -222 & -308 & -296 \\
\hline
\end{tabular}

Industry Share of GDP Loss at 1 Year after Event Infrastructure and Non Infrastructure Disruptions

\begin{tabular}{|lrrr}
\hline \multicolumn{1}{c}{ Industry } & West Coast & $\begin{array}{c}\text { Rest of New } \\
\text { Zealand }\end{array}$ & Total \\
\hline 1 Dairy cattle farming & $4 \%$ & $2 \%$ & $6 \%$ \\
\hline 2 Other agriculture and forestry & $2 \%$ & $1 \%$ & $3 \%$ \\
\hline 3 Mining and quarrying & $6 \%$ & $0 \%$ & $6 \%$ \\
\hline 4 Dairy product manufacturing & $52 \%$ & $-4 \%$ & $48 \%$ \\
5 Other food manufacturing & $1 \%$ & $0 \%$ & $1 \%$ \\
6 Other manufacturing & $0 \%$ & $2 \%$ & $2 \%$ \\
7 Utilities and construction & $5 \%$ & $2 \%$ & $7 \%$ \\
8 Retail and hospitality & $2 \%$ & $4 \%$ & $6 \%$ \\
9 Transportation services & $6 \%$ & $4 \%$ & $10 \%$ \\
10 Other services & $7 \%$ & $3 \%$ & $11 \%$ \\
\hline Total & $88 \%$ & $12 \%$ & $100 \%$ \\
\hline \hline
\end{tabular}

Note:

Figures represent economic losses from infrastructure disruption only i.e. they are not representative of a total economic loss. 


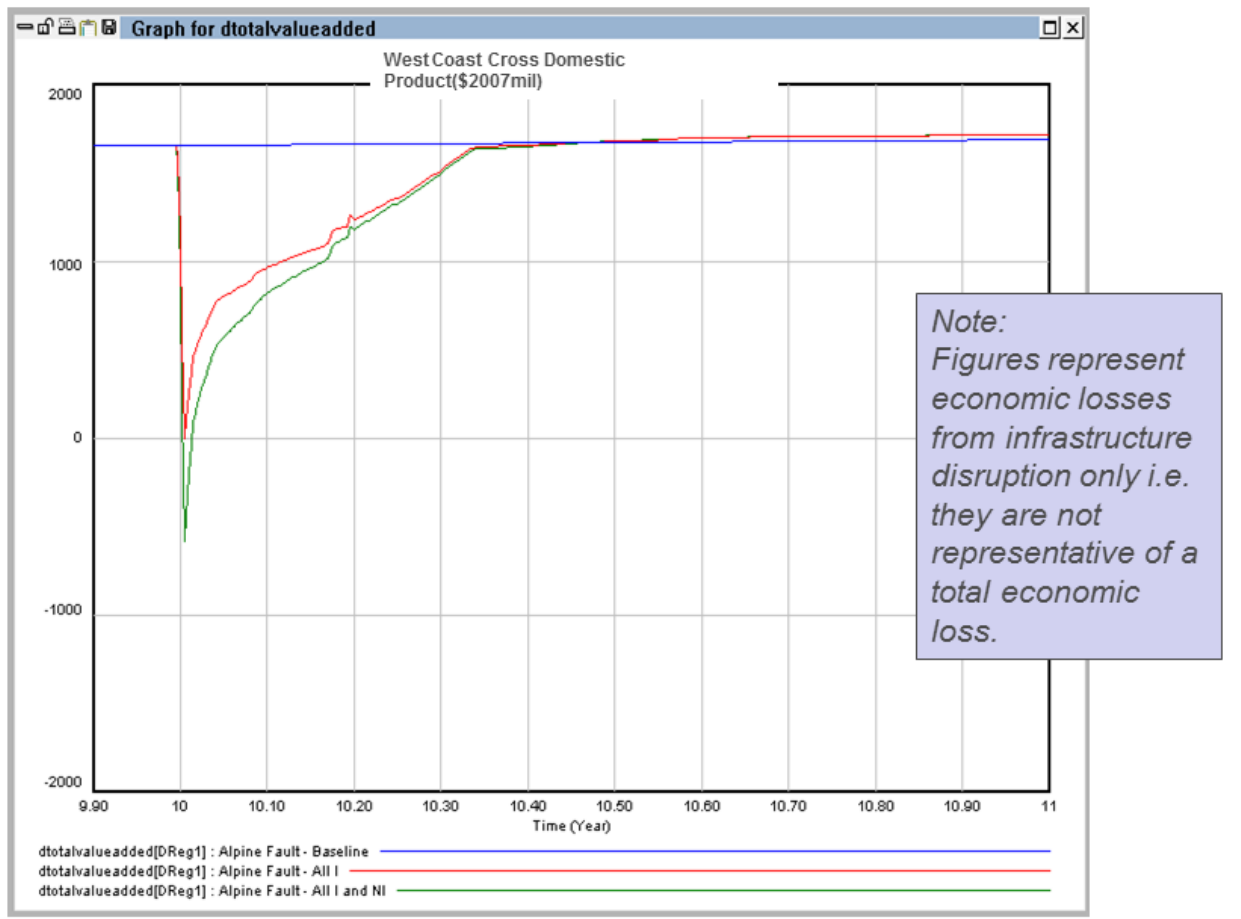

\section{Summary}

- New Zealand has recent experience in recovery from significant events

- Driving change in thinking towards resilience

- New research programs (2016-2026) are addressing the gaps in our understanding and modelling with direct links to decision makers

- QuakeCoRE

- Resilience To Nature's Challenges 


\section{NZ Resilience Programs Bibliography}

Bradley BA, Quigley MC, Van Dissen RJ, Litchfield NJ. Ground motion and seismc source aspects of the Canterbury earthquake sequence. Earthquake Spectra, 2014, Vol 30, No 1, pp 1-15.

Brown C, Seville E, Stevenson JR, Giovinazzi S, Vargo J (2015) Developing the Business Behaviours Module within MERIT. ERI Research Report 2015/02 August 2015/Addendum. http://www.naturalhazards.org.nz/NHRP/Hazard-themes/Societal-Resilience/EoRI/EoRI-OutputsPublications/EoRI-Research-Reports.

Buxton R, Wright KC, Daly MC, Timar L, Mieler D (2014) Single infrastructure failures: capturing outage information for MERIT Modelling the Economics of Resilient Infrastructure Tool. ERI Research Report 2014/01.56 p. http://www.naturalhazards.org.nz/NHRP/Hazard-themes/Societal-Resilience/EoRI/EoRIOutputs-Publications/EoRI-Research-Reports.

Horspool, N.A.; King, A.B. 2015 What drives seismic risk in New Zealand? : Insights from a next-generation loss model. paper no. 97 IN: Proceedings of the Tenth Pacific Conference on Earthquake Engineering : building an earthquake-resilient Pacific, 6-8 November 2015, Sydney, Australia. Melbourne, Vic.: Australian Earthquake Engineering Society. http://www.aees.org.au/wpcontent/uploads/2015/12/Paper_97.pdf

Horspool, N.A.; King, A.B.; Lin, S.-L.; Uma, S.R. 2016 Damage and losses to residential buildings during the Canterbury earthquake sequence. paper no. O-04 IN: Reducing risk raising resilience : New Zealand Society for Earthquake Engineering Technical Conference, 1-3 April 2016, Christchurch. New Zealand Society for Earthquake Engineering. http://www.nzsee.org.nz/db/2016/Papers/O-04\%20Horspool.pdf

Kwasinski, A., Trainor, J., Wolshon, B., Lavelle, F.M.2016. A Conceptual Framework for Assessing Resilience at the Community Scale NIST GCR 16-001.

https://www.nist.gov/sites/default/files/documents/el/resilience/NIST-GCR-16-001.pdf

National Science Challenges: Resilience to Nature's Challenges. http://resiliencechallenge.nz/

Post-disaster functioning of cities. GNS Science. http://www.gns.cri.nz/Home/Our-Science/NaturalHazards/Research-Programmes/Post-Disaster-Functioning-of-Cities

RiskScape Regional Loss Modelling Tool. http://riskscape.co.nz

Robinson, T. R; Buxton, R.; Wilson, T. M.; Cousins, W. J.; Christophersen, A. M. 2015. Multiple infrastructure failures and restoration estimates from an Alpine Fault earthquake: Capturing modelling information for MERIT, ERI Research Report 2015/04. 80 p.

http://www.naturalhazards.org.nz/NHRP/Hazard-themes/Societal-Resilience/EoRI/EoRI-OutputsPublications/EoRI-Research-Reports.

Sherson, A.K.; Nayyerloo, M.; Horspool, N.A. 2015 Seismic performance of underground pipes during the Canterbury Earthquake Sequence. paper no. 202 IN: Proceedings of the Tenth Pacific Conference on Earthquake Engineering : building an earthquake-resilient Pacific, 6-8 November 2015, Sydney, Australia. Melbourne, Vic.: Australian Earthquake Engineering Society. http://www.aees.org.au/wpcontent/uploads/2015/12/Paper_202.pdf

Smith, N.J., Zhang, Y., Cardwell, R.J., McDonald, G.W., Kim, J.H., Murray, C.F. (2015) Development of a Regional Social Accounting Framework for New Zealand. ERI Research Report 2015/01, 65 p. http://www.naturalhazards.org.nz/NHRP/Hazard-themes/Societal-Resilience/EoRI/EoRI-OutputsPublications/EoRI-Research-Reports. 
The 2010-2011 Canterbury Earthquake Sequence: Personal, Social, Governance and Environmental Consequences, Douglas Paton \& David Johnston (Eds.) IN International Journal of Disaster Risk Reduction, Volume 14, Part 1, Pages 1-104 (December 2015)

QuakeCoRE. New Zealand Centre for Earthquake Resilience. http://www.quakecore.nz/research/

Uma, S.R.; Dhakal, R.P.; MacRae, G.A. 2013 Implementation of low damage construction : what are the challenges?. paper no. 52 (10 p.) In: Same risks, new realities : New Zealand Society for Earthquake Engineering Technical Conference and AGM, April 26-28, 2013, Wellington

Zorn, C. R., \& Shamseldin, A. Y. (2015). Post-disaster infrastructure restoration: A comparison of events for future planning. International Journal of Disaster Risk Reduction, 13, 158-166.

Zorn, C.R., and Shamseldin, A. Y (2016) Quantifying Directional Dependencies from Infrastructure Restoration Data. Earthquake Spectra: August 2016, Vol. 32, No. 3, pp. 1363-1381. 
Nick Horspool is a Risk Scientist with GNS Science, a Crown Research Institute in New Zealand. He is the leader of the RiskScape research program which focuses on developing and applying risk and resilience models for a range of applications from disaster management, landuse planning to the (re)insurance industry. Nick is on the leadership team of QuakeCoRE New Zealand Centre for Earthquake Resilience and a management committee member for the New Zealand Society of Earthquake Engineering. Prior to joining GNS Science, Nick worked as a Risk Modeler for Geoscience Australia an Australian Government research agency. Nick has over 10 years experience developing and applying risk models in New Zealand, Australia, Indonesia, Philippines and Pacific Island Countries. 


\title{
URBAN RESILIENCE: ASIA RESEARCH INITIATIVES
}

\author{
Professor Dongping Fang \\ Institute for Future Cities and Infrastructures \\ Tsinghua University \\ Email: fangdp@mail.tsinghua.edu.cn
}

\begin{abstract}
The world is undergoing rapid urbanizing at an unprecedented speed. The global urban population increased from 29\% in 1950 to $50 \%$ in 2010, and is expected to further increase to $69 \%$ by 2050. This presentation starts with an overview of world urbanization trend and the impact of disasters. A framework and a vision of city simulator that we have proposed to model urban resilience will be illustrated. Current researches in Asia will be summarized and current initiatives in urban resilience research at Tsinghua University will be presented.
\end{abstract}




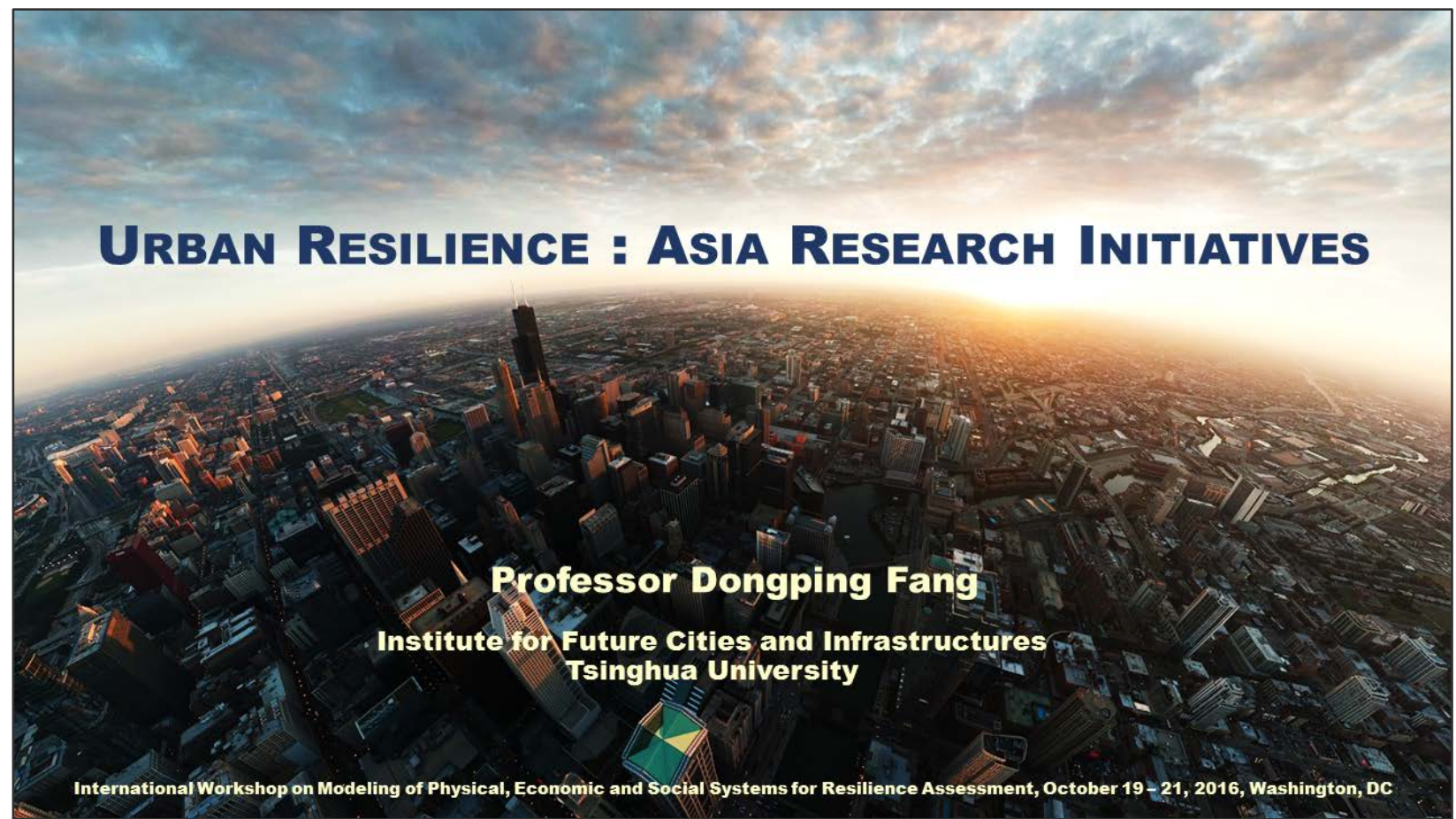

\section{OUTLINE}

I Urbanization and challenges

II Modelling urban resilience - City simulator

III Initiatives at IFCI@TSINGHUA

IV Asia research initiatives

V Concluding remarks 


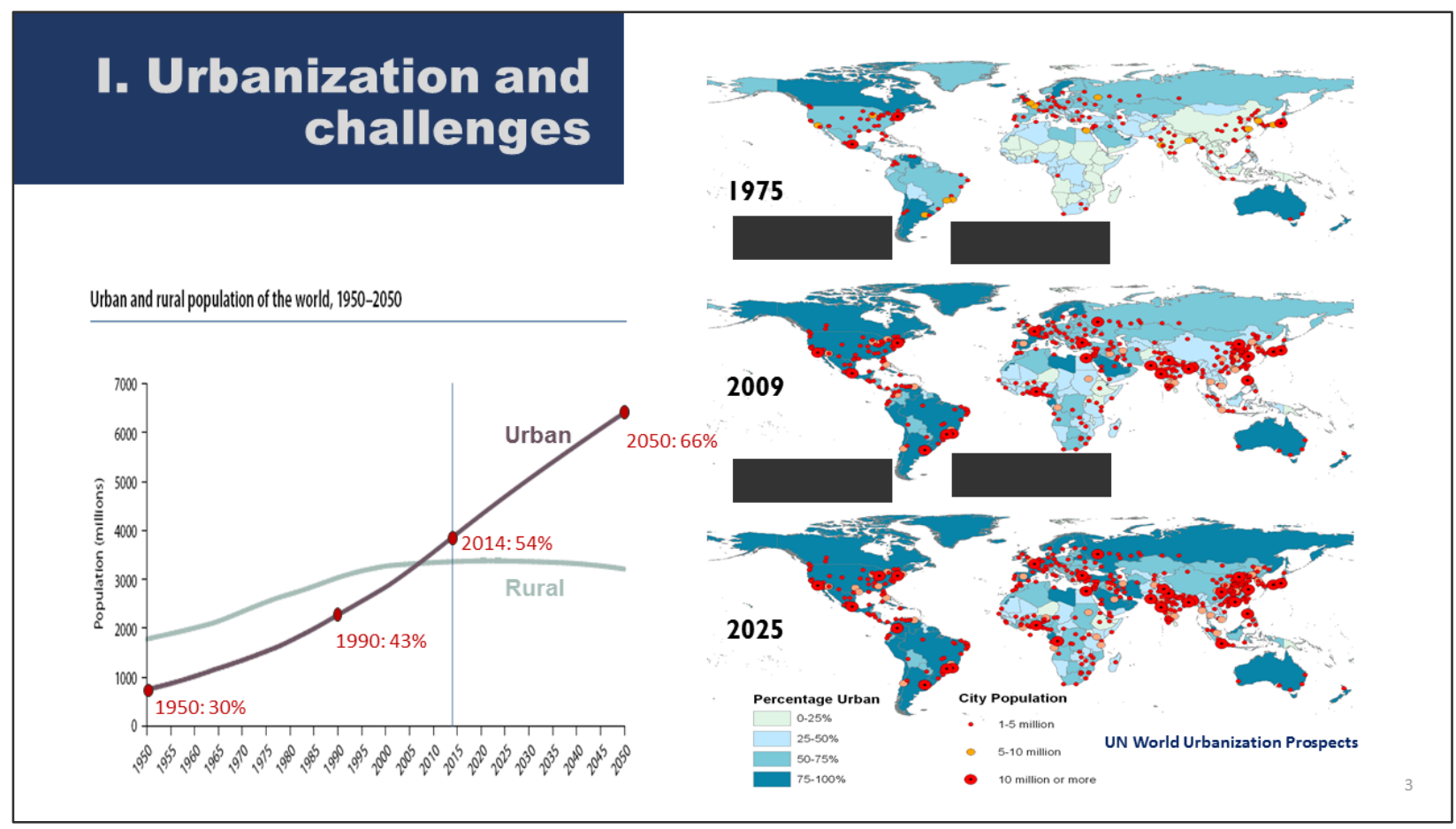

\section{Urbanization and challenges}

\begin{tabular}{|c|c|c|c|c|}
\hline & $\begin{array}{r}\text { 末来 } \\
\text { Future }\end{array}$ & $\begin{array}{l}\text { 成镇 } \\
\text { Cities }\end{array}$ & $\begin{array}{l}\text { 全体市民更 } \\
\text { Better qual }\end{array}$ & $\begin{array}{l}\text { 生活品质 } \\
\text { f life for all }\end{array}$ \\
\hline & & & & \\
\hline $\begin{array}{l}\text { 智慧 } \\
\text { 城市 }\end{array}$ & $\begin{array}{l}\text { 低碳 } \\
\text { 城市 }\end{array}$ & $\begin{array}{l}\text { 海绵 } \\
\text { 城市 }\end{array}$ & $\begin{array}{r}\begin{array}{r}\text { 可持 } \\
\text { ( 经济/ }\end{array} \\
\text { Sustainable }\end{array}$ & $\begin{array}{l}\text { 镇化 } \\
\text { (环境 ) } \\
\text { banization }\end{array}$ \\
\hline Smart & Low & Sponge & $\widehat{૫}$ & $\widehat{ن}$ \\
\hline Cities & $\begin{array}{c}\text { Carbon } \\
\text { Cities }\end{array}$ & Cities & $\begin{array}{c}\text { 韧性 } \\
\text { Resiliency }\end{array}$ & $\begin{array}{c}\text { 高效 } \\
\text { Efficiency }\end{array}$ \\
\hline
\end{tabular}




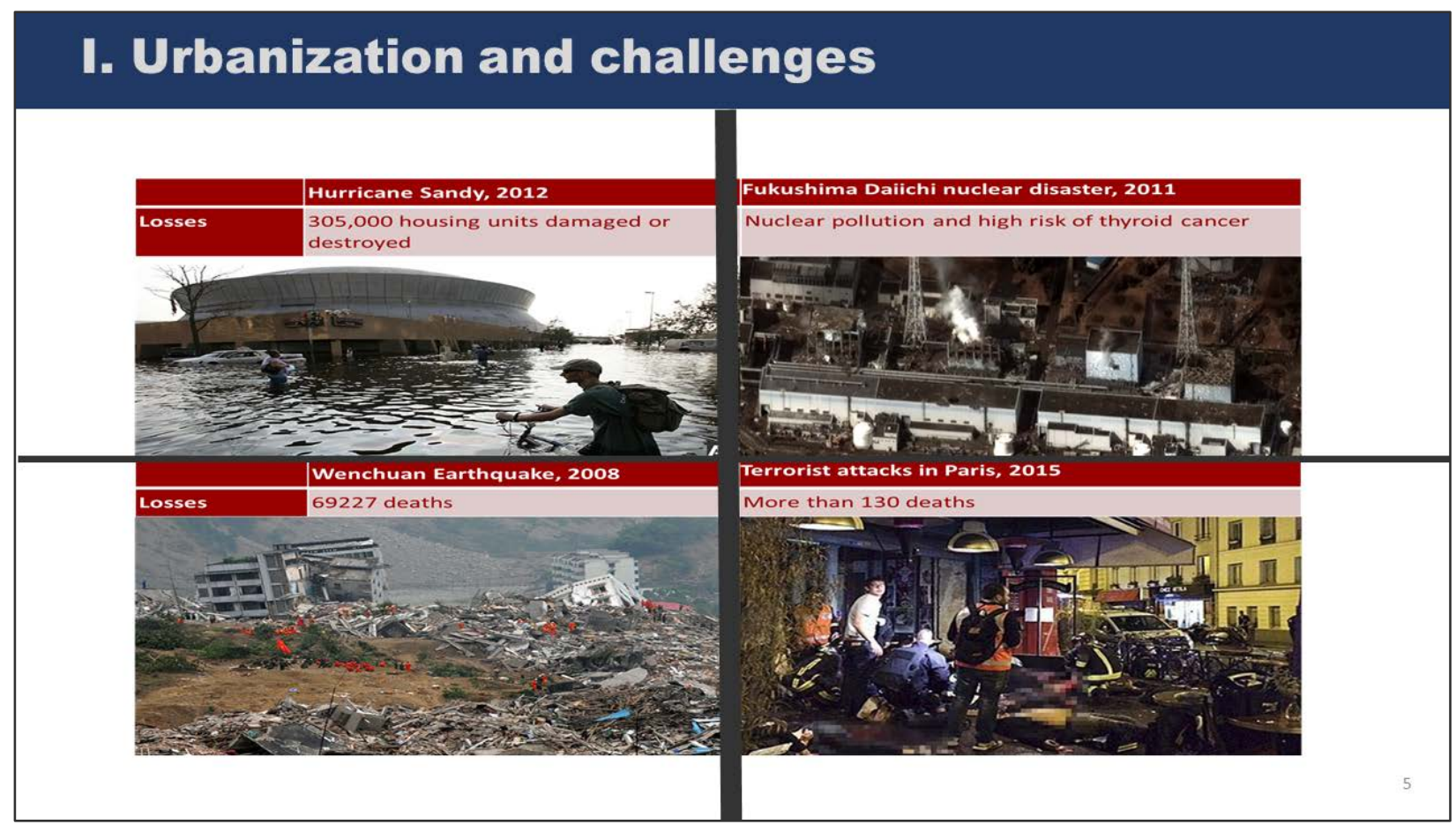

\section{Urbanization and challenges}

The magnitude of losses in cities due to natural disasters has been increasing disproportionately
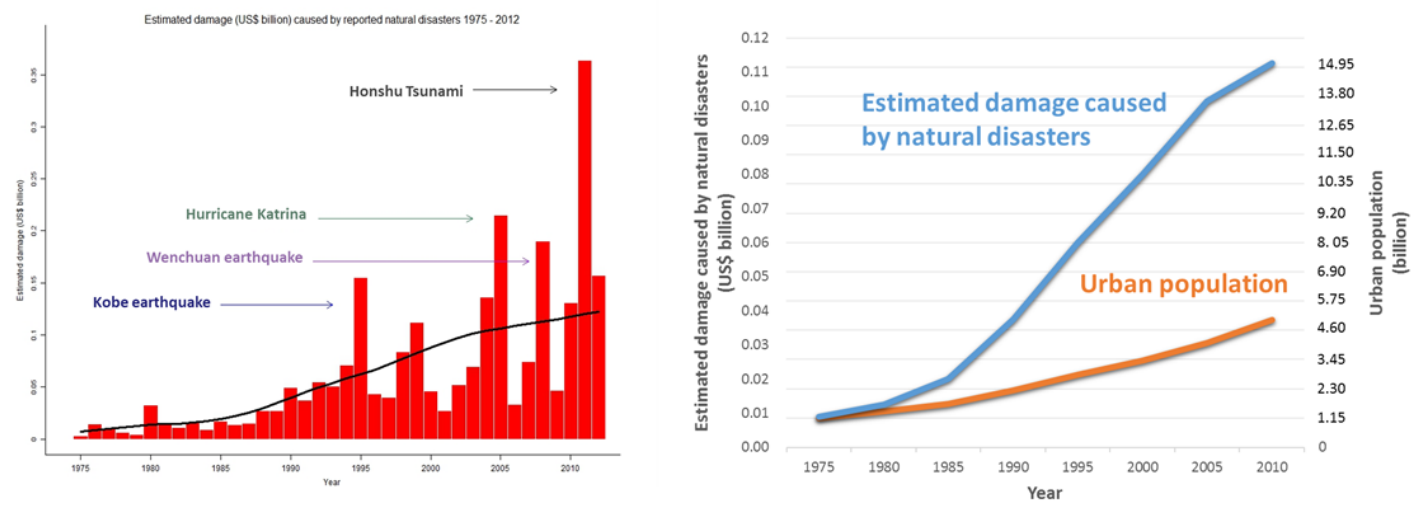


\section{Urbanization and challenges}

\section{China enjoyed fast urbanization, but with huge regional inequity}

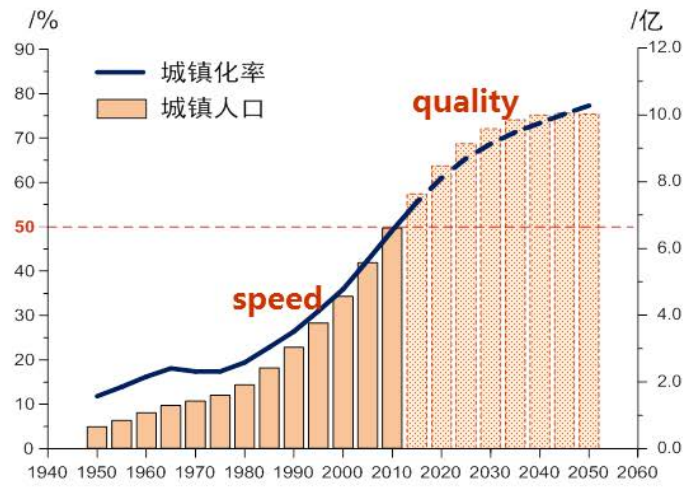

Rapid urbanization in China
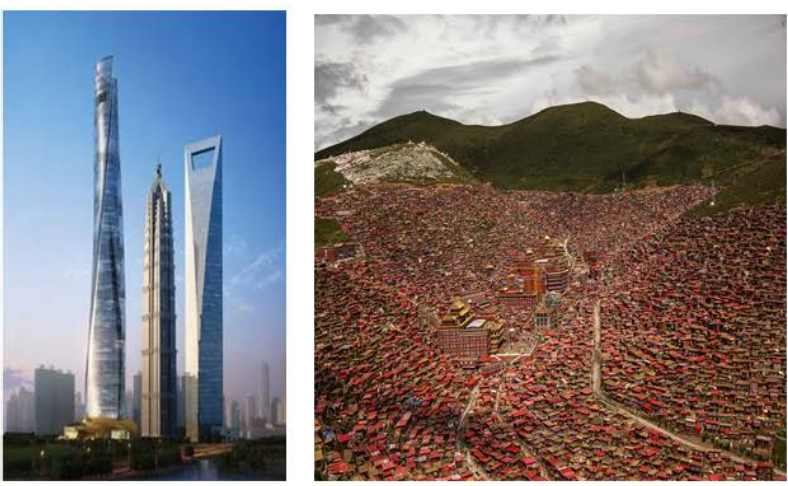

Regional Inequity in China

\section{Urbanization and challenges}
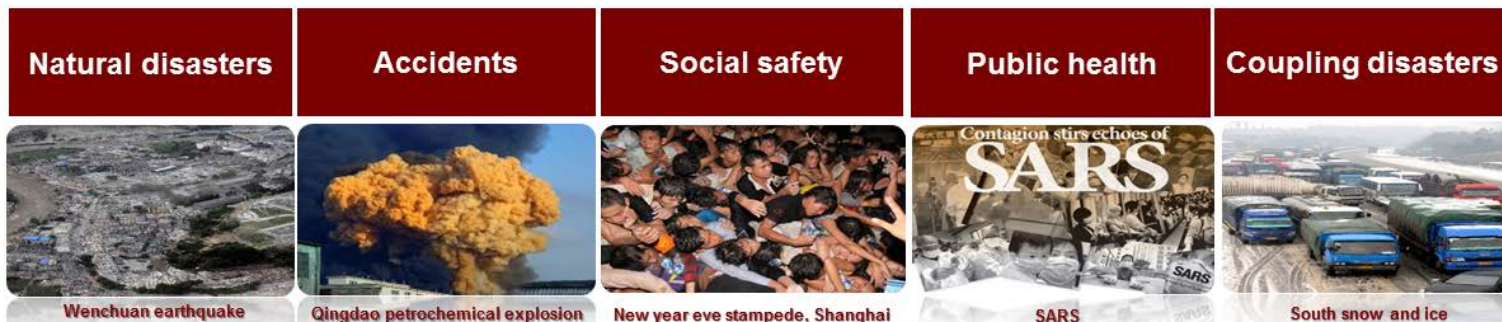

Wenchuan earthquake

Qingdao petrochemical explosio

New year eve stampede, Shanghai
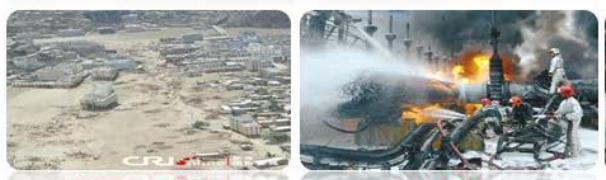

Zhouqu mudslides

Dalian716
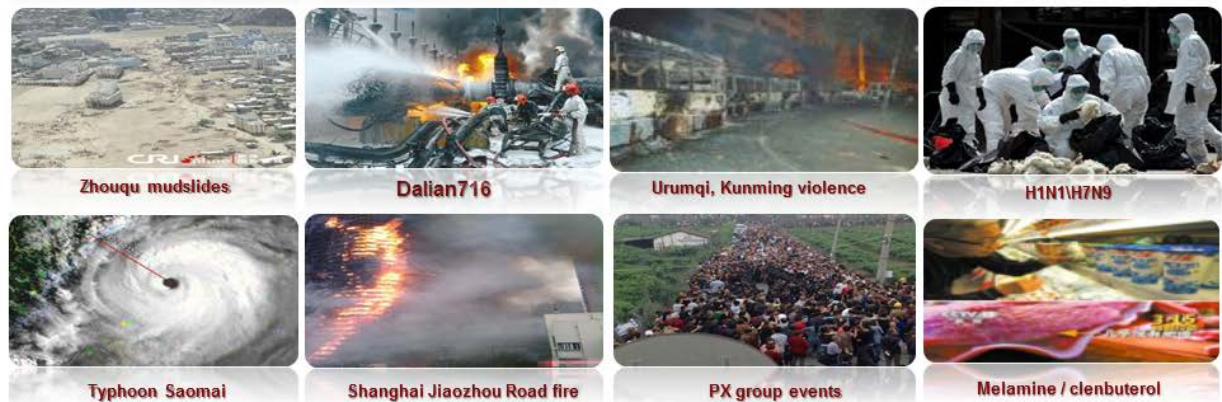

South snow and ice
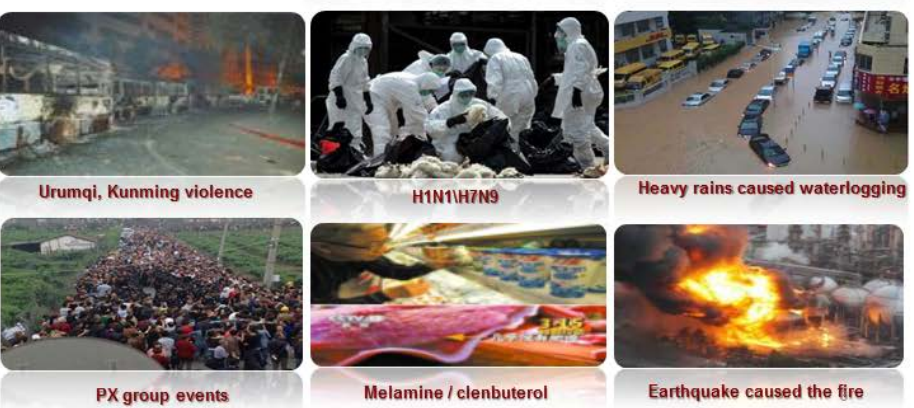

Heavy rains caused waterlogging

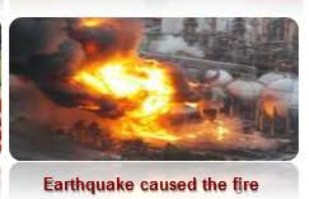




\section{Modelling urban resilience - City simulator}

\section{Are our cities prepared to respond to these disasters?}

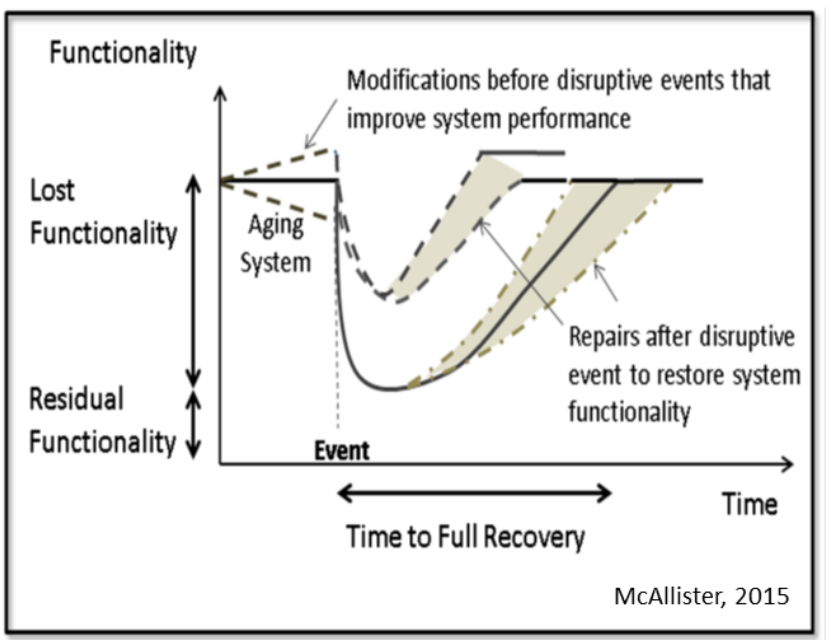

Resilience refers to the ability of an urban system to

- plan and prepare for,

- absorb,

- response to,

- recover from,

- and adapt to

adverse events.

Resilience measurement:

- robustness,

- rapidity,

- resourcefulness,

- redundancy

\section{Resilience}

Center for Risk-Based Community Resilience Planning

Colorado A NIST-funded Center of Excellence

\section{Traditional approaches to risk management of the built environment A critical appraisal}

- Standards-based, with a focus on individual hazards and facilities

- Measures of performance among interdependent systems are inconsistent

- Margins of safety and functionality are not commensurate with uncertainty

- Competing risks cannot be assessed

- Critical social institutions are not considered

- Investments in risk mitigation and management may be misdirected 


\section{Modelling urban resilience - City simulator}

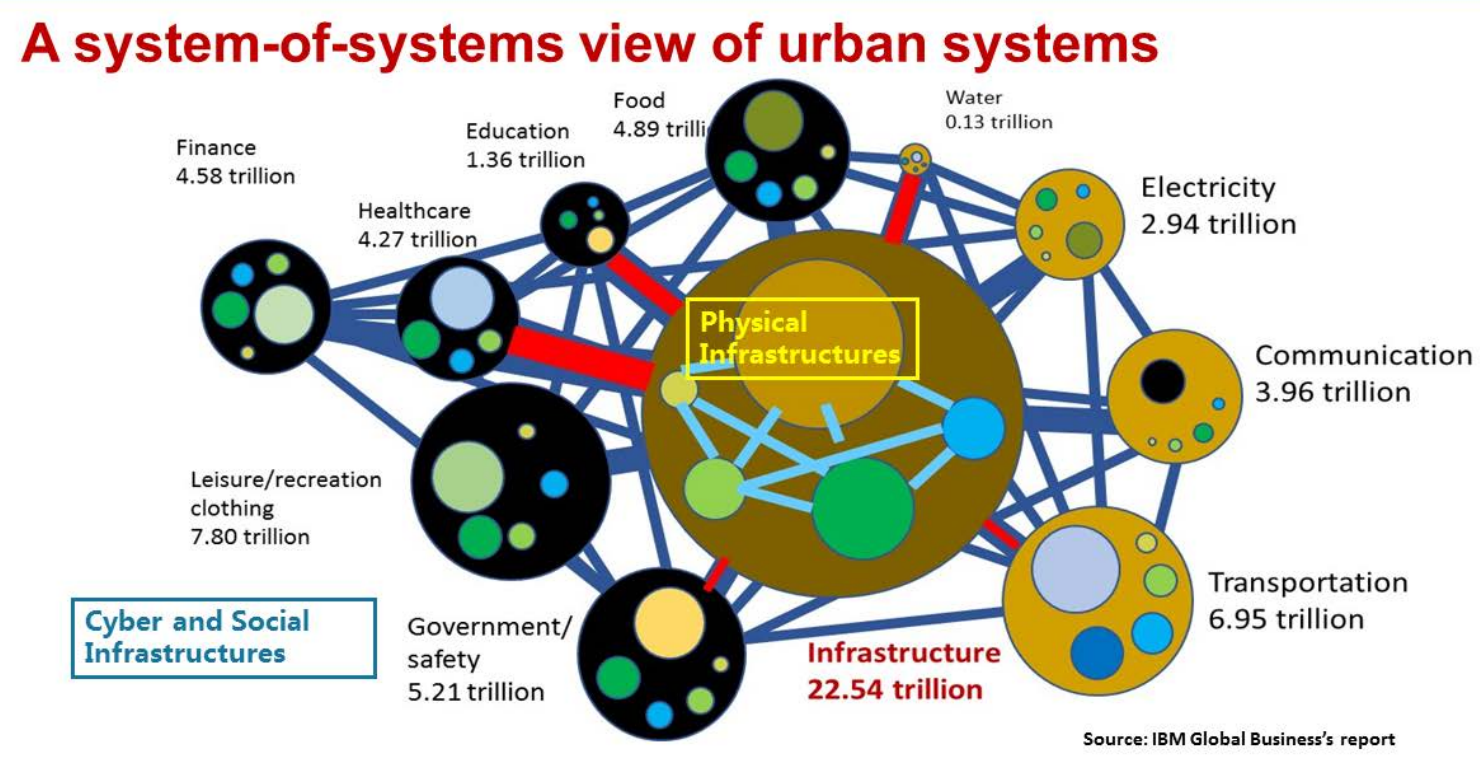

\section{Modelling urban resilience - City simulator}

\section{A trio-space perspective}

\section{Most challenges in urban system are caused by incongruity between the three spaces, which increases the complexity of the challenges exponentially.}

Domain experts of the three spaces don't always talk to each other, which hinders the solution to challenges in urban systems.

\section{Societal space}

- Social sciences,

- humanities,

- psychology,

- urban planning,

- public management, - etc.

\section{Physical space}

\section{Cyber space}

- Information technology,

- automation and control,

- data science,

- artificial intelligence,

- communication,

- etc.
- Civil engineering,

- architecture,

- construction management,

- mechanical engineering,

- building science,

- etc. 


\section{Modelling urban resilience - City simulator}

\section{Managing Resilience: shock cycle + trio-space + 4Rs}

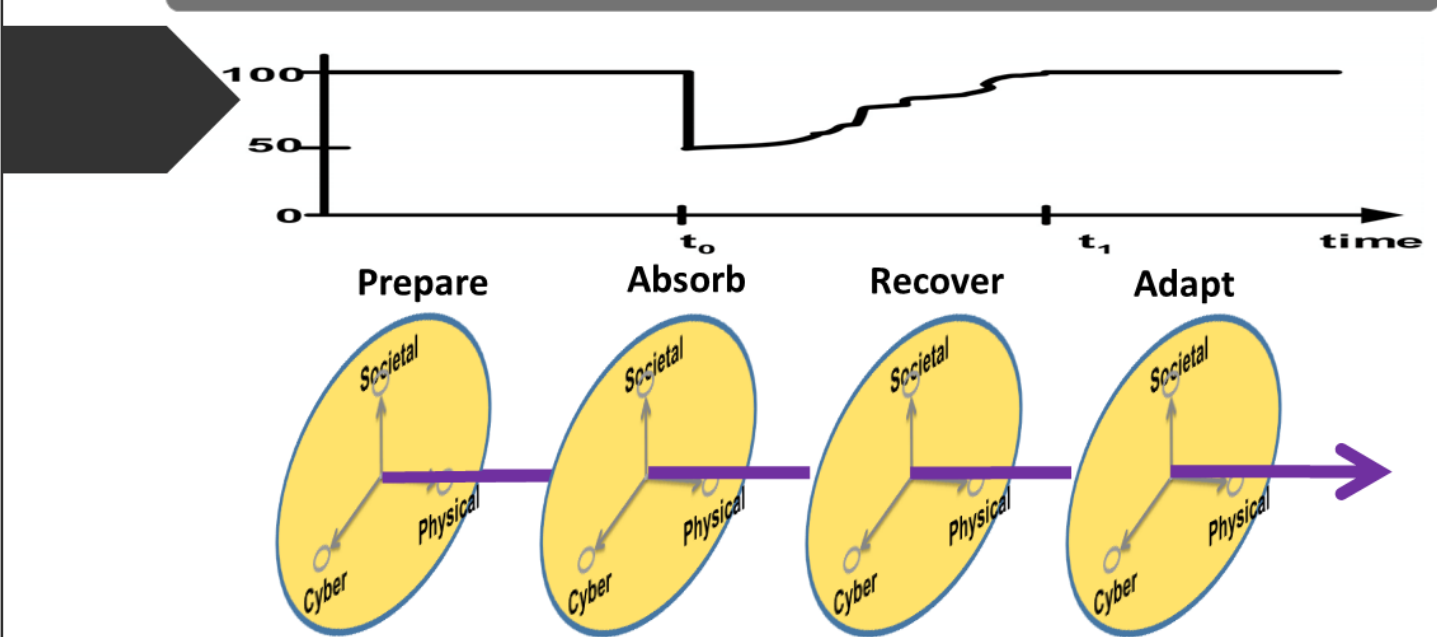

4Rs: robustness, rapidity, resourcefulness, and redundancy

\section{Modelling urban resilience - City simulator}

Crossing the boundary is essential for making new breakthroughs in respective academic disciplines, and possibly leading to emerging disciplines.

We advocate an interdisciplinary approach, which is also inherently determined by the nature of urban systems, and complexity of the impacts of disasters.

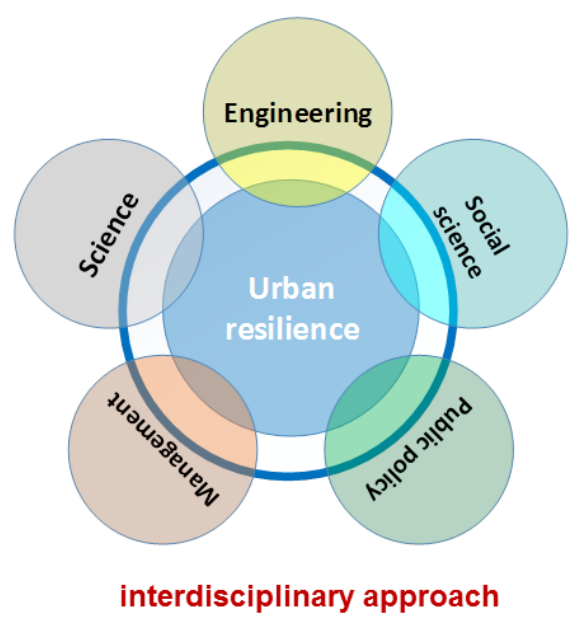




\section{Modelling urban resilience - City simulator}

Crossing the boundary is essential for making new breakthroughs in respective academic disciplines, and possibly leading to emerging disciplines.

We even advocate a transdisciplinary approach, where we move beyond discipline-specific approaches, and address urban challenges in the most integrated and creative way.
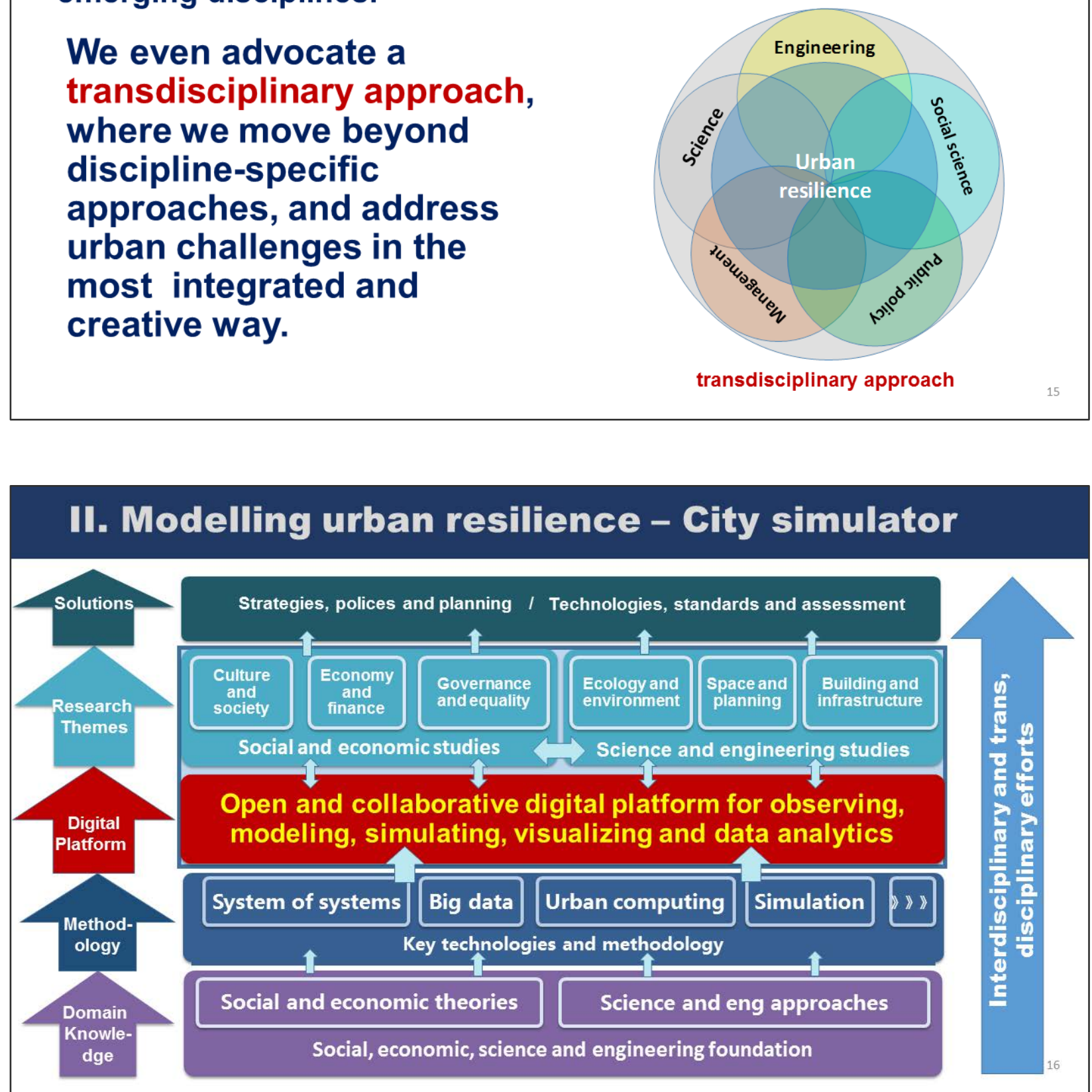


\section{Modelling urban resilience - City simulator}

\section{City Simulator}

\section{City Simulator}
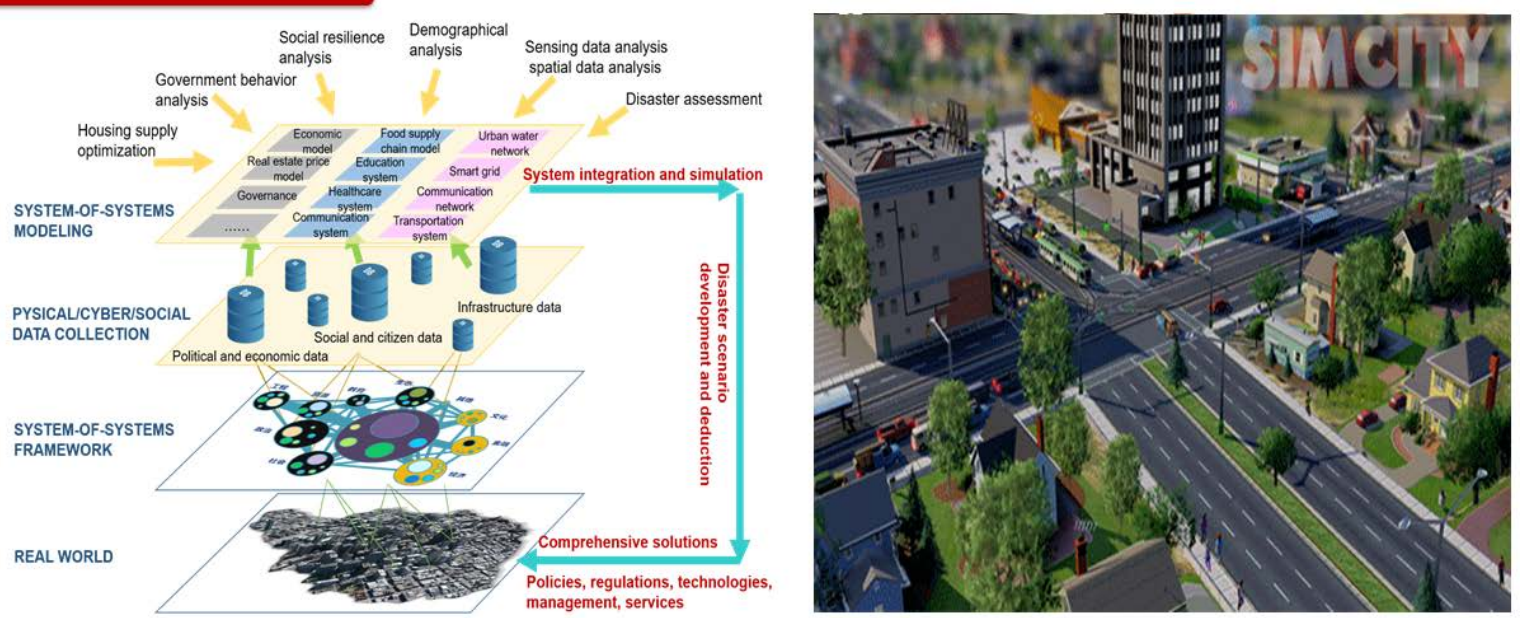

Open and collaborative digital platform for modeling, simulation, visualization and data analytics

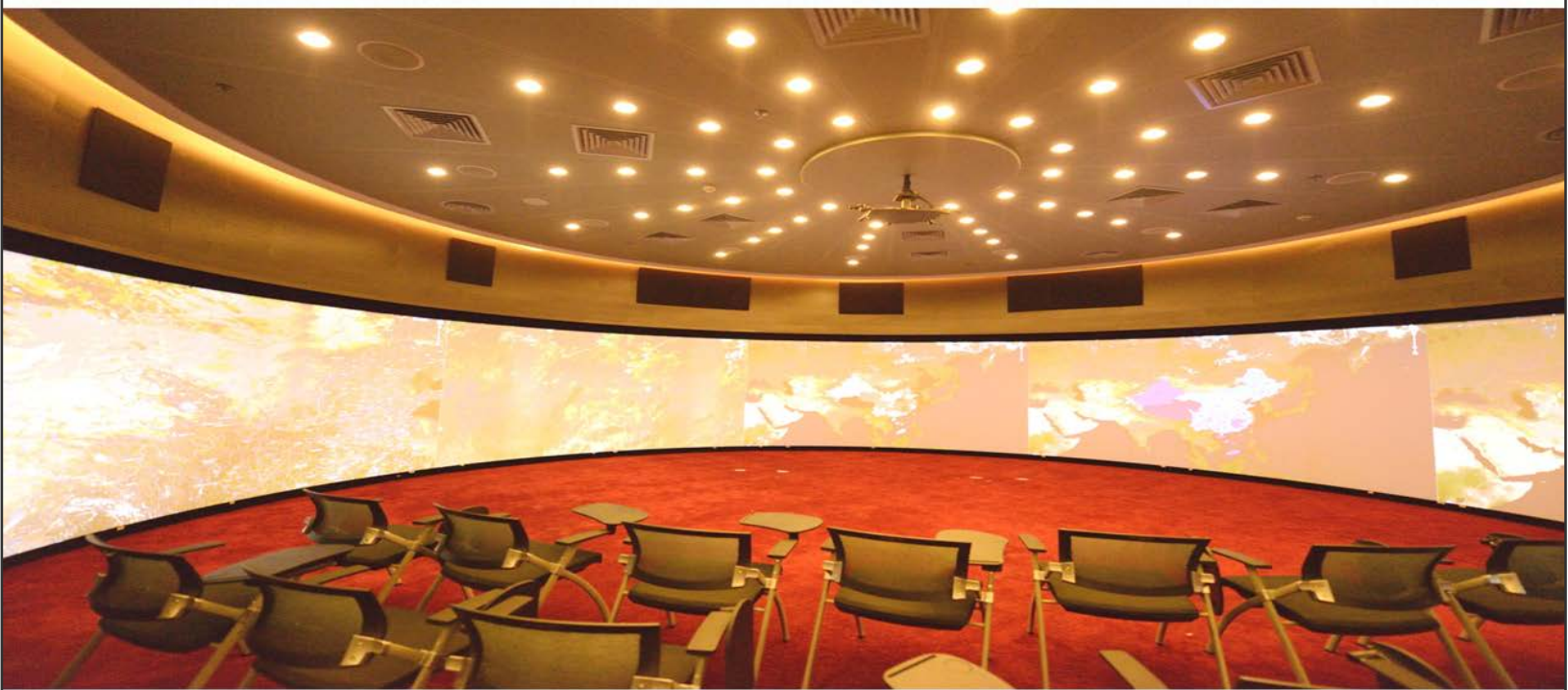




\section{City Simulator}

Model convergence, system integration, simulation, scenario development and deduction, comprehensive solutions Through visualizations to support policies, regulations, governance, standards, management and services.

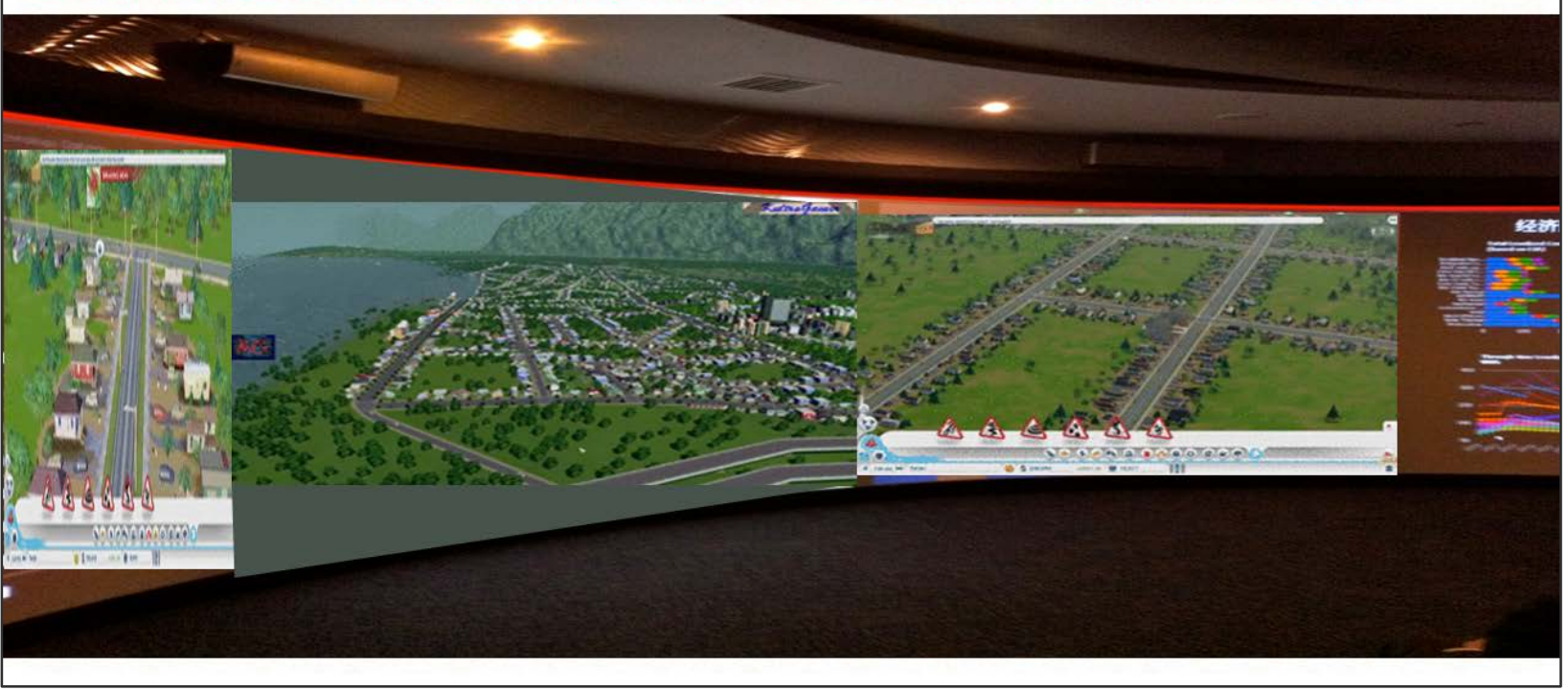

\section{City Simulator}

Model convergence, system integration, simulation, scenario development and deduction, comprehensive solutions Through visualizations to support policies, regulations, governance, standards, management and services.

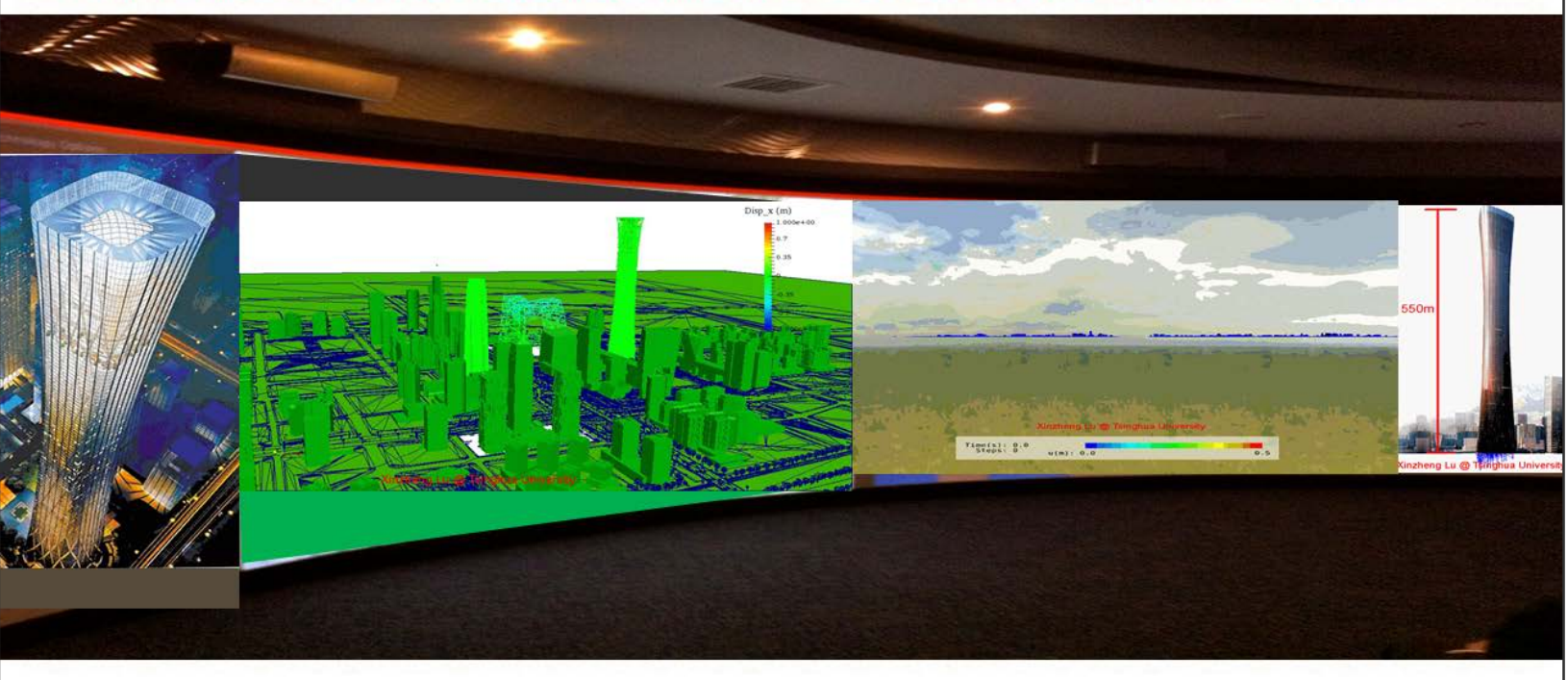




\section{City Simulator}

Model convergence, system integration, simulation, scenario development and deduction, comprehensive solutions Through visualizations to support policies, regulations, governance, standards, management and services.

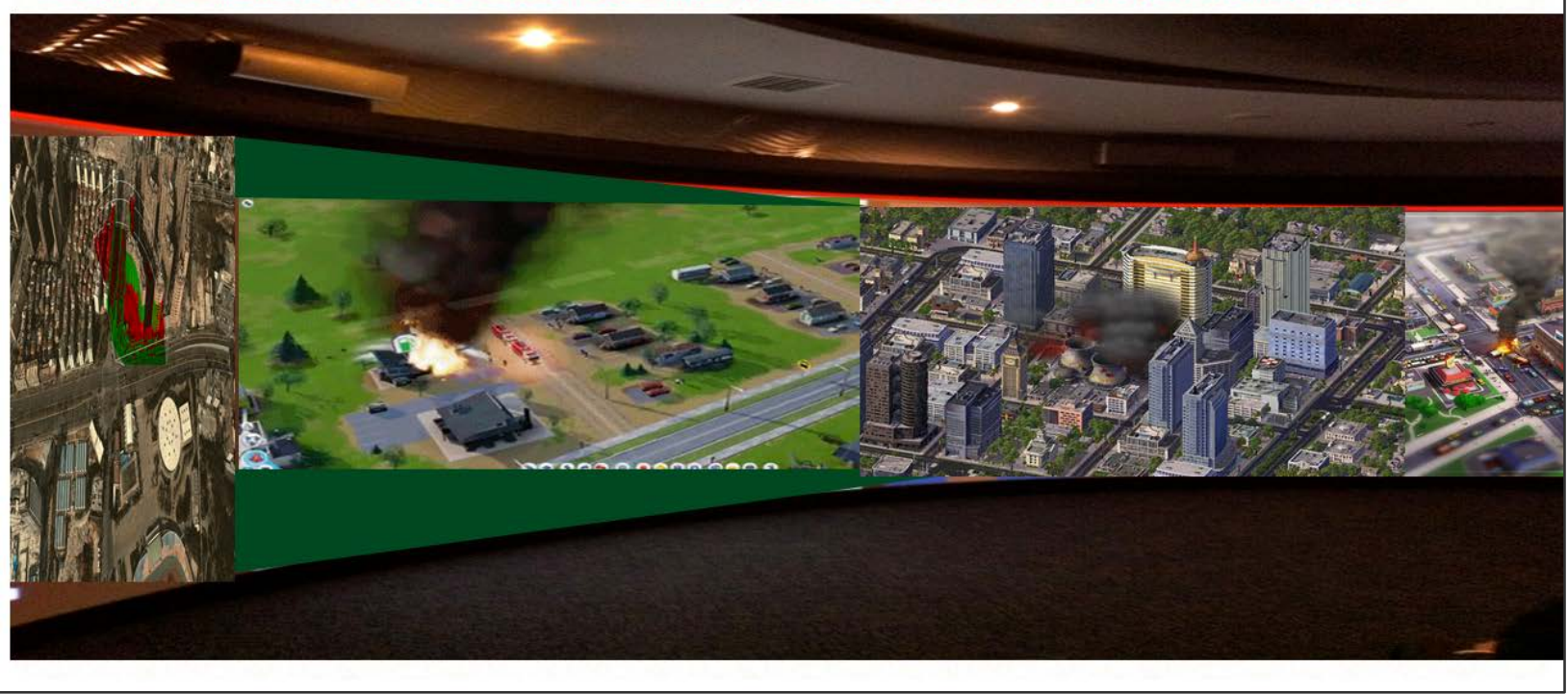

\section{City Simulator}

Model convergence, system integration, simulation, scenario development and deduction, comprehensive solutions Through visualizations to support policies, regulations, governance, standards, management and services.

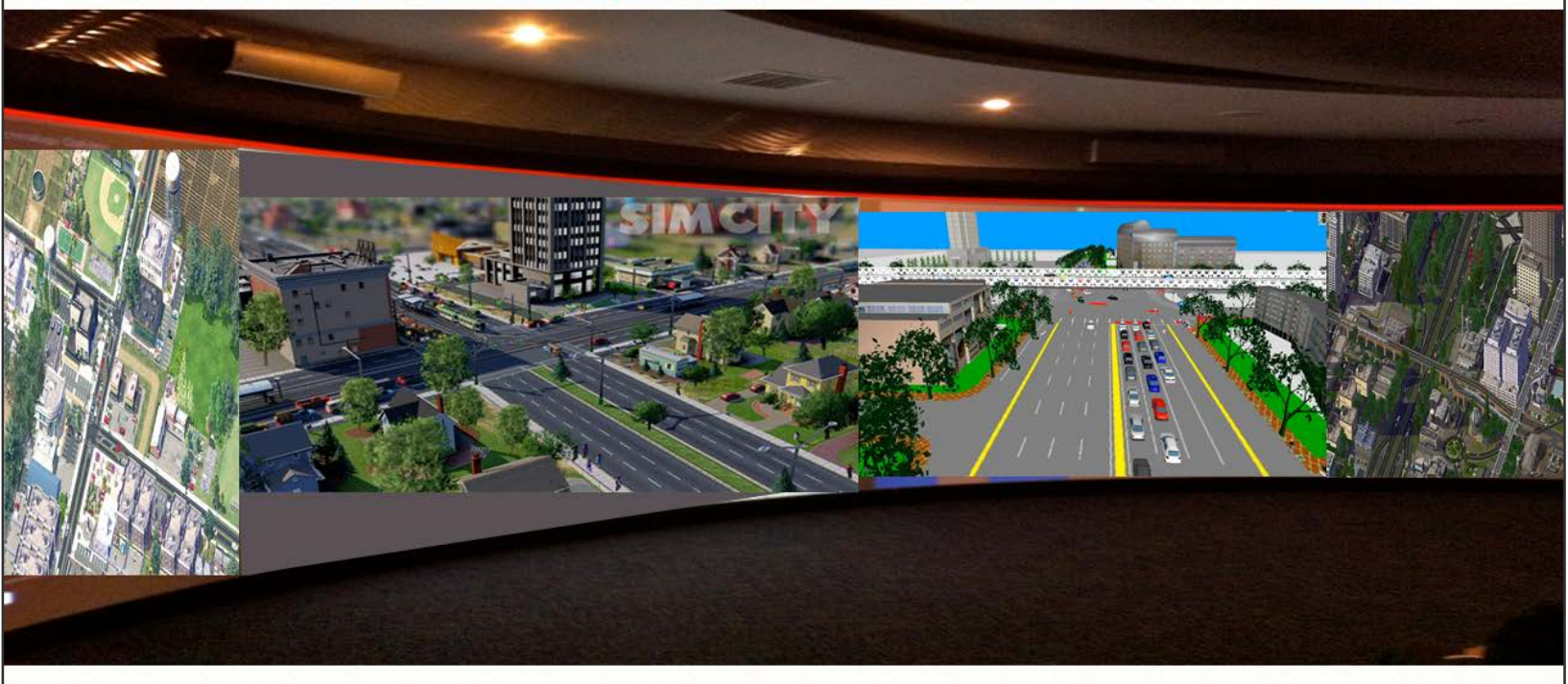




\section{Initiatives at IFCI@TSINGHUA}

\section{IFCI@TSINGHUA}

\section{Institute for future cities and infrastructures, Tsinghua University}

- Co-founded by the Schools of Civil Engineering, Architecture, Environmental Engineering, Public Management, and Social Sciences.

- The vision of this new institute is to foster interdisciplinary and transdisciplinary research in sustainable urbanization and lifecycle management of urban infrastructure systems.

- Resilience is one of the major research themes of IFCI@Tsinghua.

\section{Initiatives at IFCI@TSINGHUA}

\section{IFCI@TSINGHUA}

We encourage interdisciplinary \& transdisciplinary research.
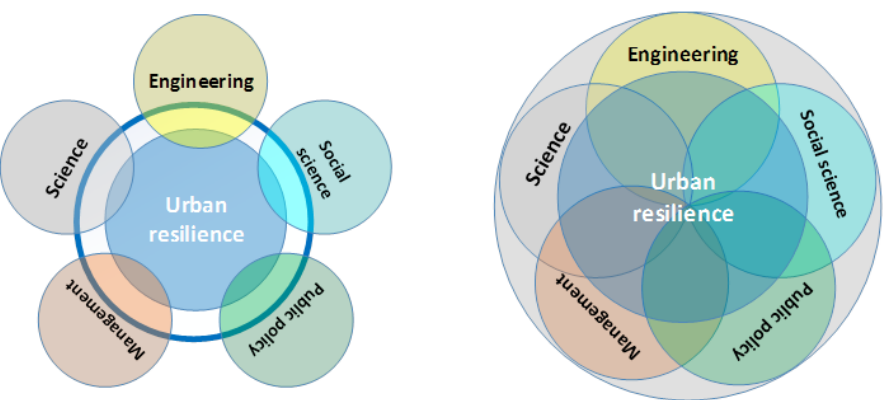

- Integrate intelligence and resources, in various academic disciplines, within and outside Tsinghua, for cutting-edge collaboration;

- Integrate and move beyond discipline-specific approaches and ultimate adopt an transdisciplinary, or even antidisciplinary approach;

- So as to create new conceptual, theoretical, methodological, and translational innovations, to address grand challenges in resilient urbanization. 


\section{Initiatives at IFCI@TSINGHUA}

\section{IFCI@TSINGHUA}

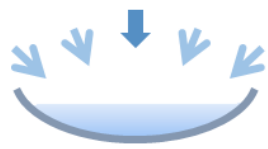

Collaboration model at IFCl: Attraction of resources and intelligence

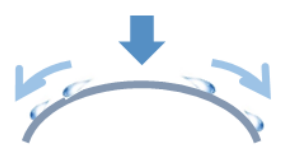

Traditional collaboration model: Distribution of resources

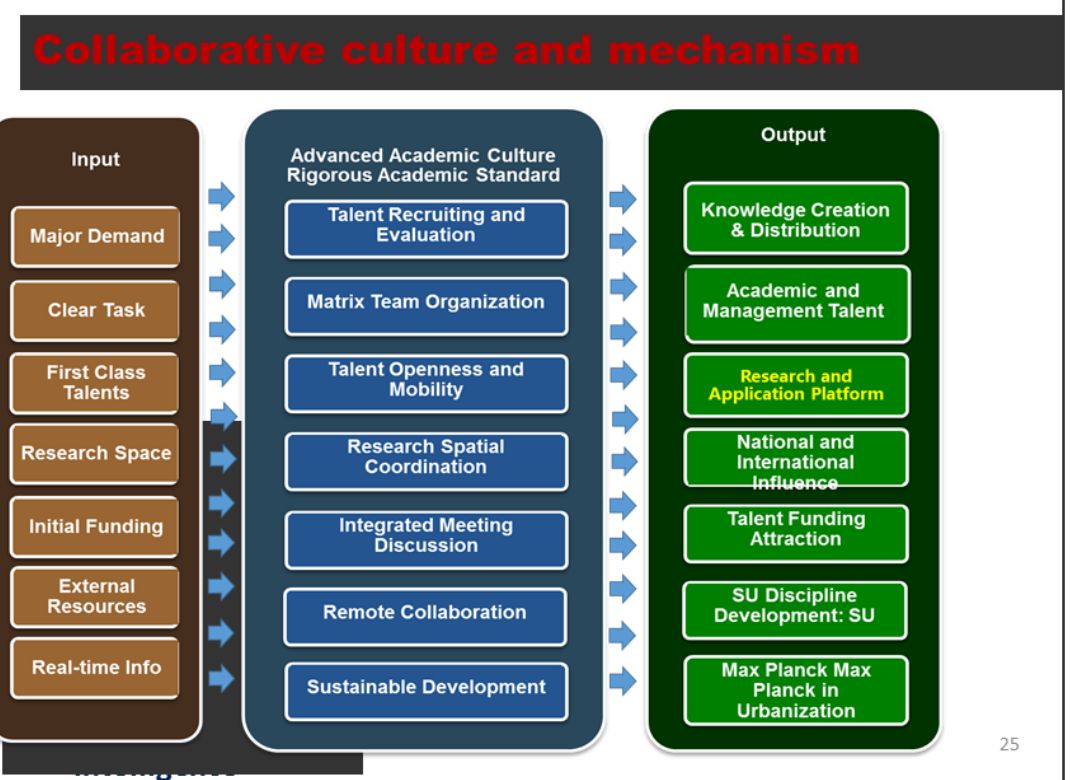

\section{Initiatives at IFCI@TSINGHUA}

\section{IFCI@TSINGHUA}

Data Sensing and Big Data Analytics in Urban and Infrastructure Systems

- Urban and infrastructure data sensing and warehousing

- Urban and infrastructure data mining and visualization

- Open and collaborative digital platiorm for urban and infrastructure systems studies

Sustainable Life-Cycle Management of Urban and Infrastructure Systems

-High-performance and resilience-based building materials and structural design

-Life-cycle design and operation technologies for infrastructure systems

-Disaster prevention and reduction and "scenario-response" management in urban systems

Social and Economic Theories and Models for Future Cities and Infrastructures

-Physical-social-economic interdependency mechanism of urban and infrastructure systems

- Evolution and behavior modeling of urban and infrastructure systems

-Complexity theory based urban and infrastructure modeling and computing 


\section{Initiatives at IFCI@TSINGHUA}

\section{I-SERCHES - Increasing SEismic Resilience of Chinese Healthcare Systems: physical, economic and social interdependencies}

- The project was developed in collaboration with University College London in response to a joint NSFC-NERC CFP that focused on earthquakeprone regions in China.

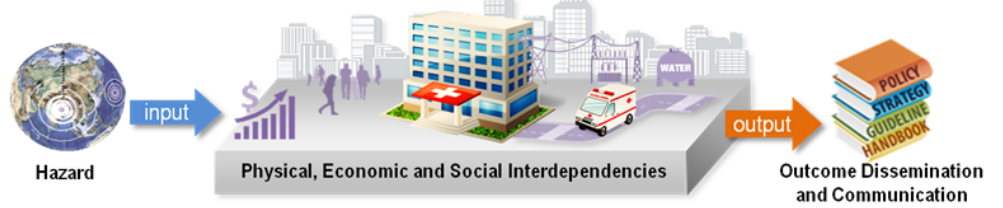

- We formed a highly interdisciplinary team including researchers in geomechanics, civil engineering, construction management, financial management, healthcare, sociology and economy.

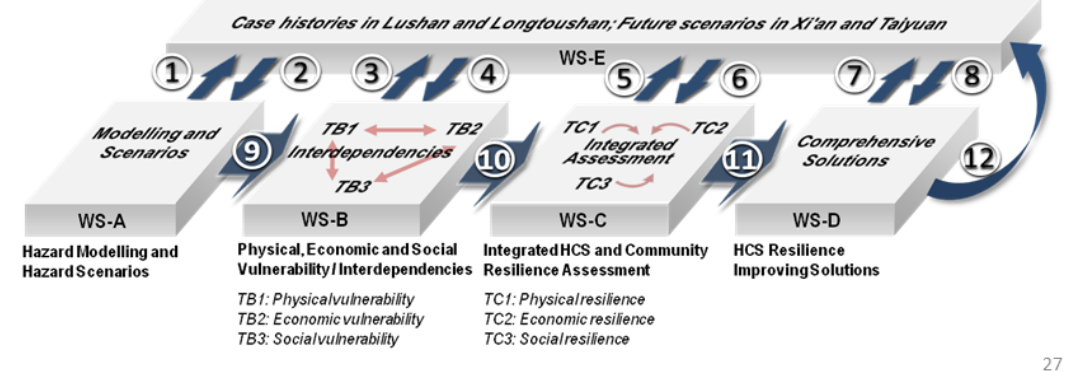

\section{Initiatives at IFCI@TSINGHUA}

\section{I-SERCHES - Increasing SEismic Resilience of Chinese Healthcare} Systems: physical, economic and social interdependencies

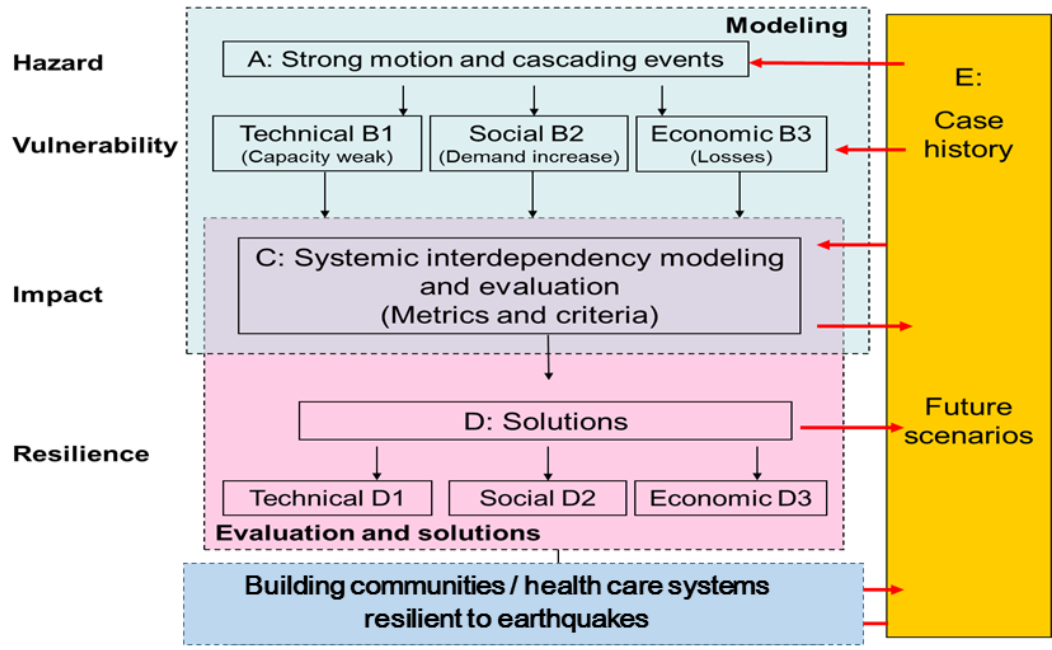

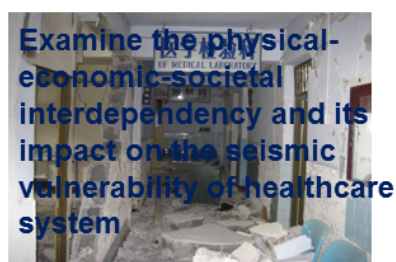

- Develop a framework for seismic resilience assessment that considers the interdependencies Propese seismicresilence improvement technologyes, fivise arrangements ind public policy sugg in 


\section{Initiatives at IFCI@TSINGHUA}

\section{Resilient cities to earthquake and beyond}
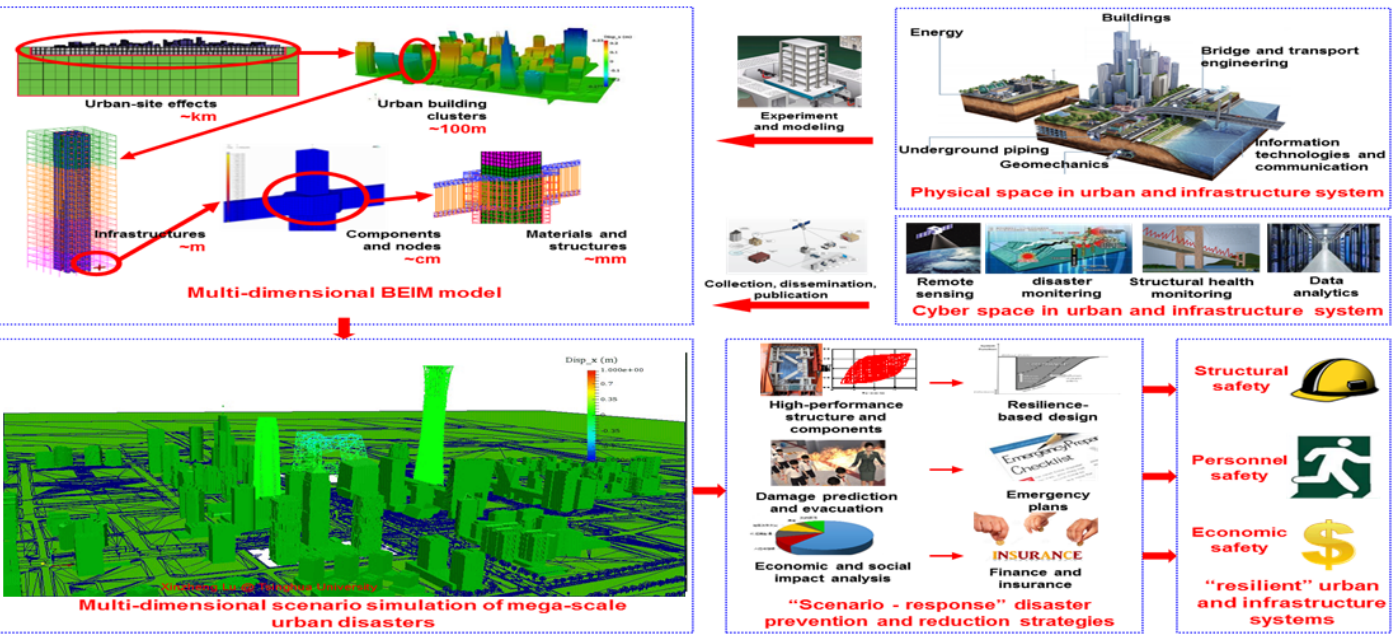

Simulate mage-disaster scenarios, and develop 'scenario-response' strategies for seismic damage prevention and reduction Courtesy to X. Lu

\section{Initiatives at IFCI@TSINGHUA}

\section{Resilient smart cities to earthquake and beyond}

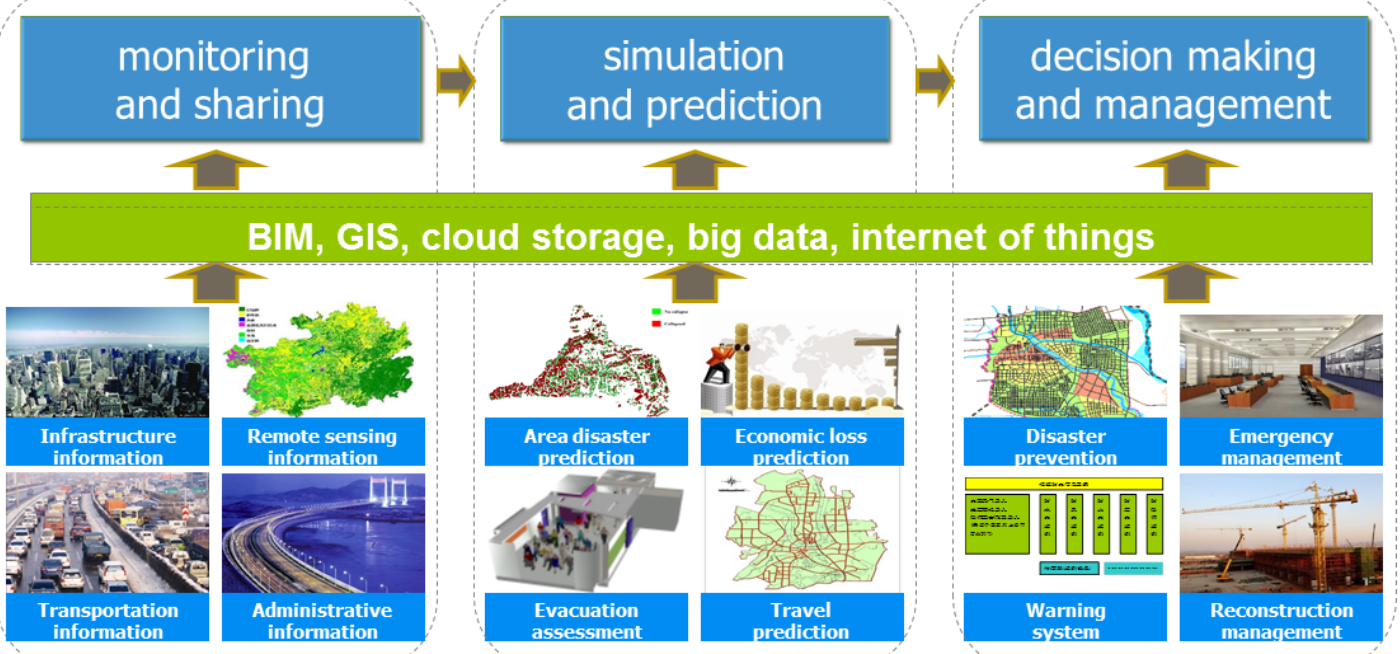

(prediction \& warning, risk analysis, simulation, assistant decision making) Courtesy to X. Lu 


\section{Initiatives at IFCI@TSINGHUA}

\section{Modeling interdependencies and cascading failures in networked infrastructure systems}
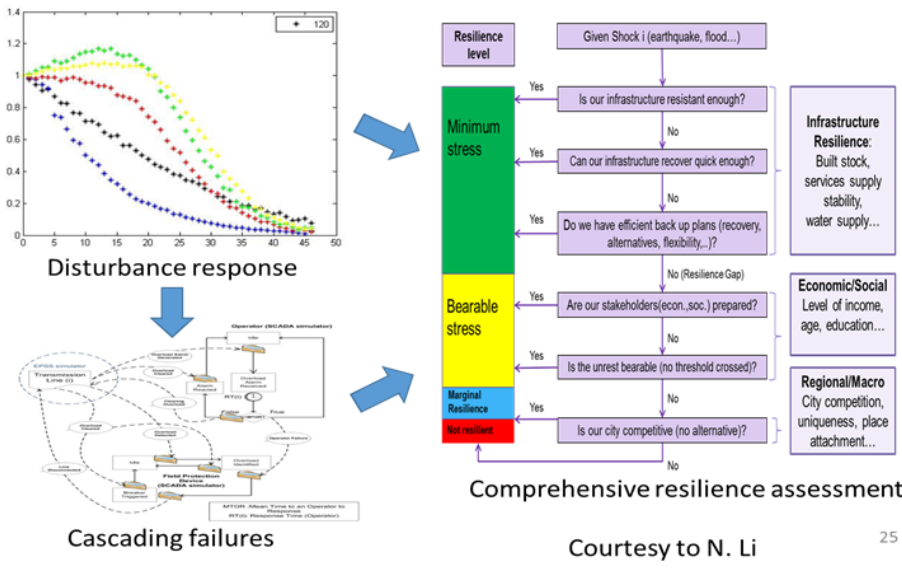

weighted directed complex network approach

Simulate cascading failure processes and assess ripple effects of disturbances

\section{Initiatives at IFCI@TSINGHUA}

4. Modeling the coupling between transportation system and healthcare system

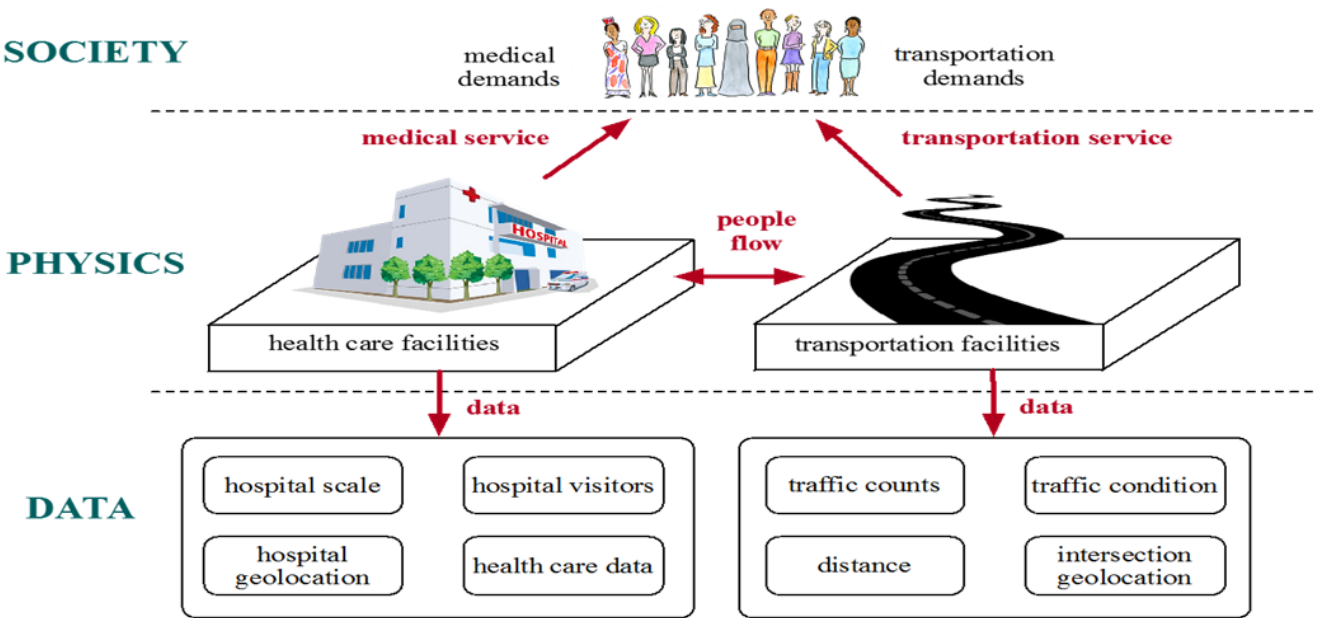




\section{Initiatives at IFCI@TSINGHUA}

\section{Measuring the functionality of built environment} considering the inter-dependency of community functions

Lost functionality:

$$
L_{C}=\left\{\begin{array}{llll}
1 & 1 & 1 & 1
\end{array}\right\}[\mathbf{D A M}]_{4 \times 4}\left\{l_{i}\right\}_{4 \times 1}
$$

[DAM]: Damage Augmentation Matrix

$\left\{l_{i}\right\}$ : disrupted capacity of community component

$$
[\mathbf{D A M}]=\left[\begin{array}{cccc}
0.128 & 0.297 l_{1} & 0.373 l_{1} & 0.268 l_{1} \\
0.297 l_{2} & 0.0436 & 0.279 l_{2} & 0.101 l_{2} \\
0.373 l_{3} & 0.279 l_{3} & 0.126 & 0.278 l_{3} \\
0.268 l_{4} & 0.101 l_{4} & 0.278 l_{4} & 0.051 l_{4}
\end{array}\right]
$$

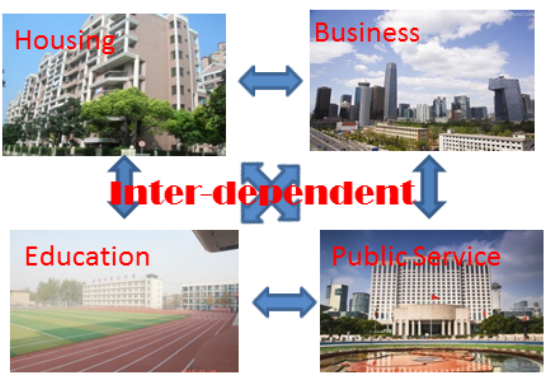

With the measurement,

- Identify ways to improve community resilience

- Evaluate the relative contributions of different loss-reduction measures to resilience

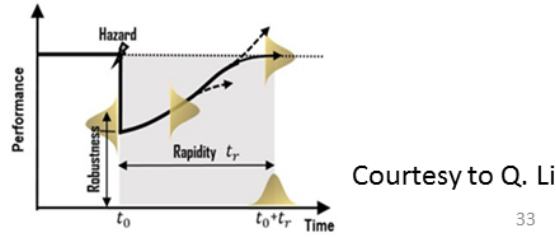

\section{Initiatives at IFCI@TSINGHUA}

\section{Modeling human behaviors and social resilience}
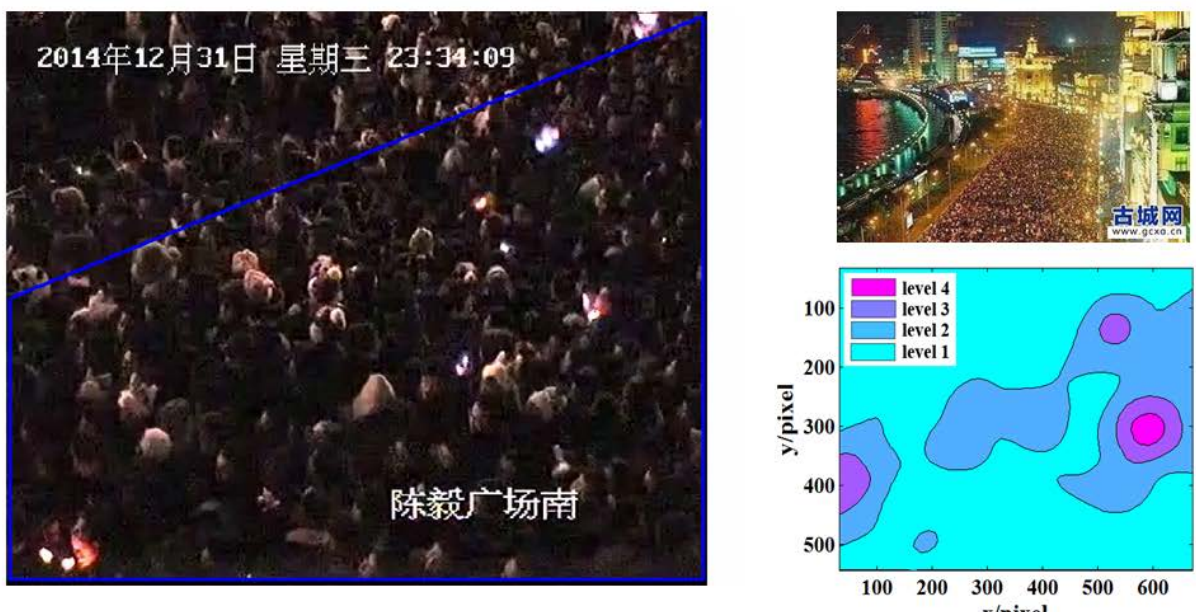

x/pixel Courtesy to W. Weng 


\section{Initiatives at IFCI@TSINGHUA}

\section{Resilience Index}

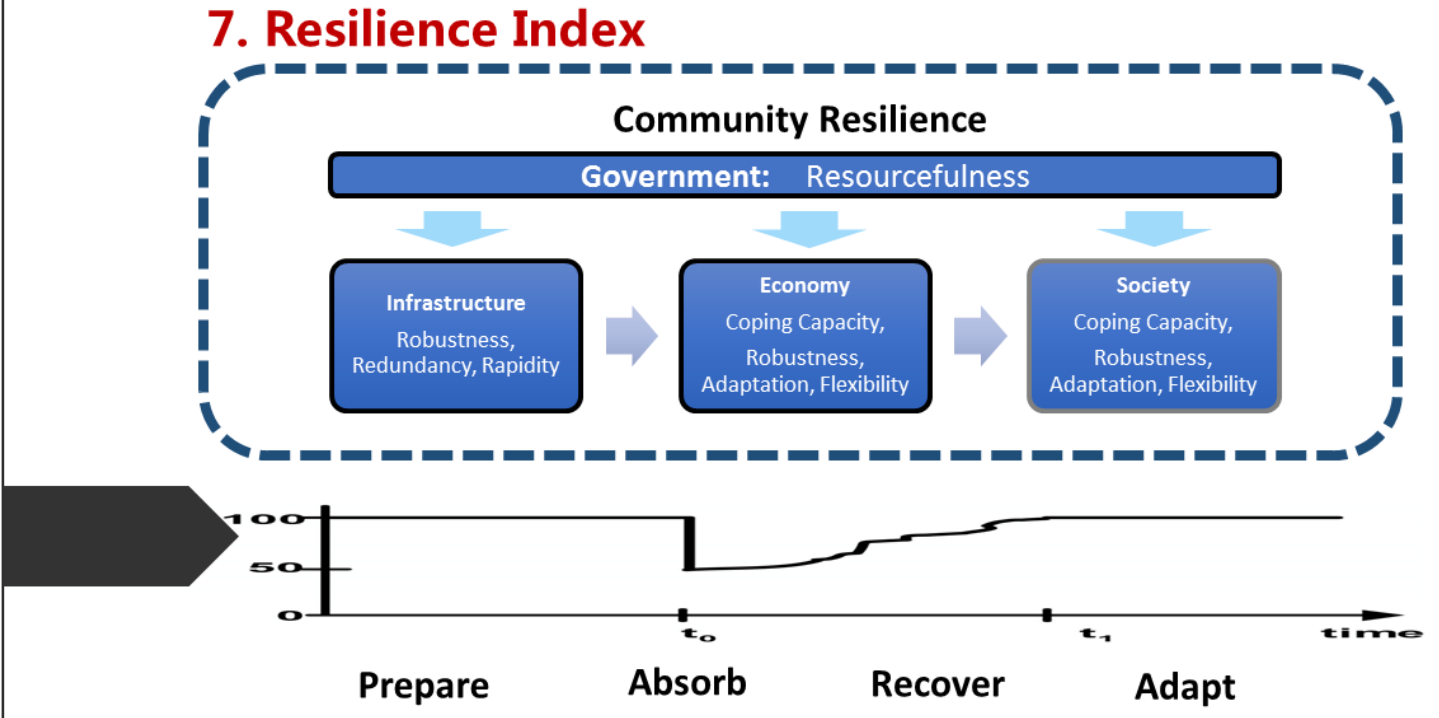

\section{Initiatives at IFCI@TSINGHUA}

Test-bed community for research, validation and application

- Dinglan Town, Hangzhou City (G20, 2016), Zhejiang Province

- Area $\approx 15.6 \mathrm{~km}^{2}$, population $\approx 72,000$

- The Dinglan community is highly committed to collaborate with IFCI to:

- Develop a resilient smart community master plan

- Improve resilience of its transportation, tourism and healthcare infrastructures

- Create an ecological system that enables continuous research and capital investment on community resilience

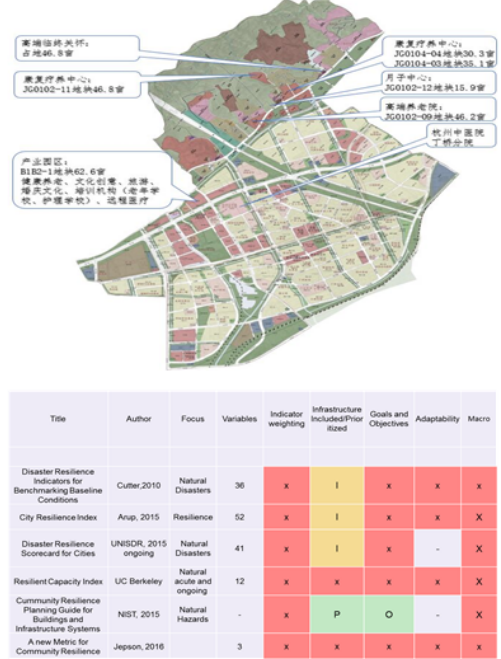

Metrics for Resilient Urbanization 


\section{Initiatives at IFCI@TSINGHUA}

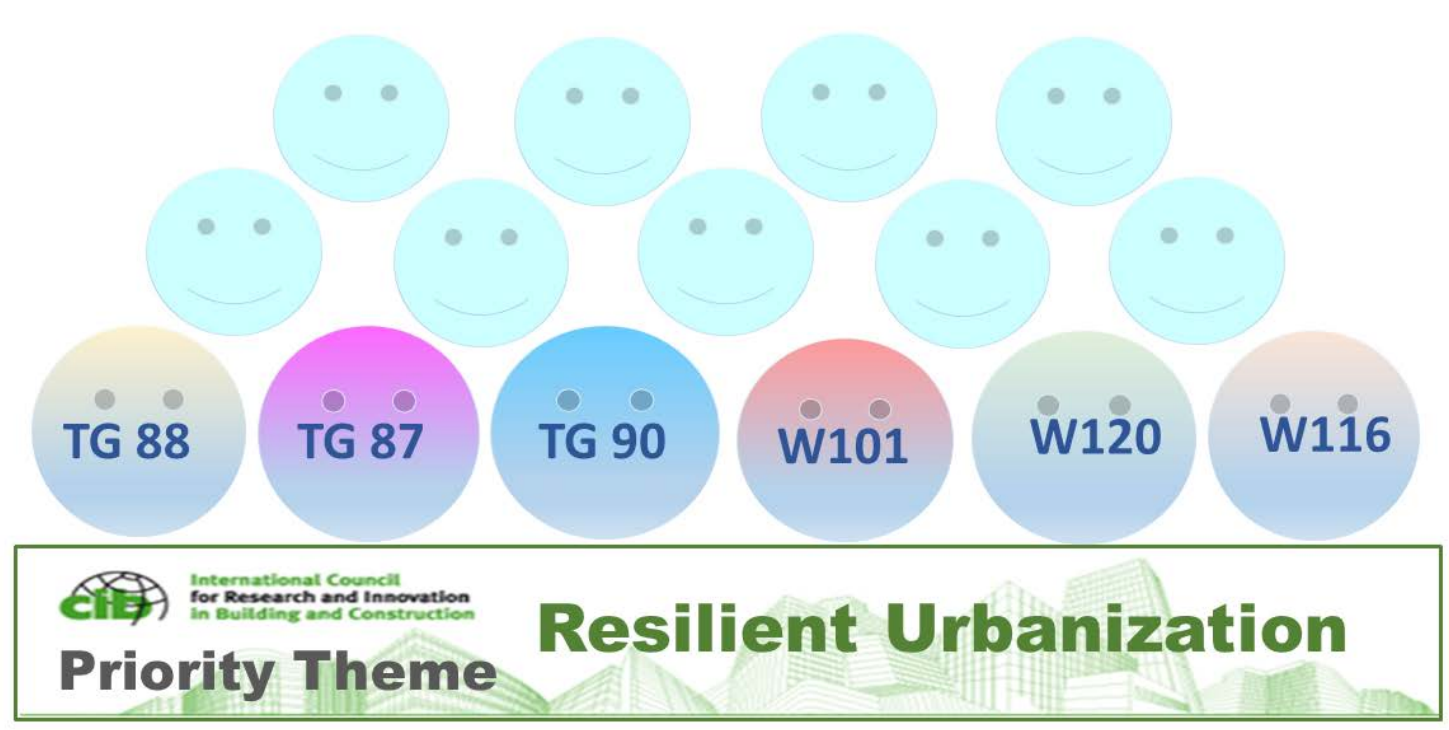

\section{Asia research initiatives}

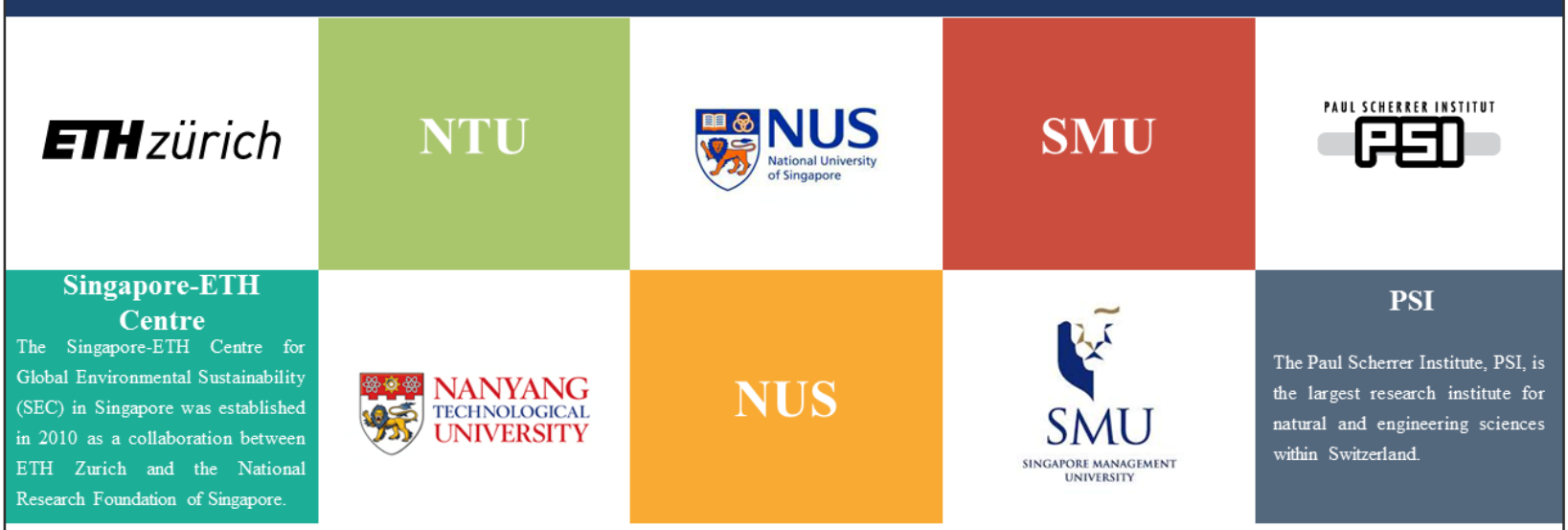

Background:

FUTURE RESILIENT SYSTEMS

Future Resilient Systems (FRS) is a research project under the Singapore-ETH Centre (SEC). Through the Singapore-ETH Centre (SEC), ETH partnering NTU, NUS and SMU has launched the CREATE program on Future Resilient Systems (FRS)

Mission:

To address the challenges with critical infrastructure systems that provide essential services to modern societies 


\section{Asia research initiatives}

\section{FUTURE RESILIENT SYSTEMS}

\section{Clusters:}

1. Interdependent Critical Infrastructure Systems

2. Energy Systems and Comparative System Assessment

3. Social and Behavioural Factors in Decision-Making

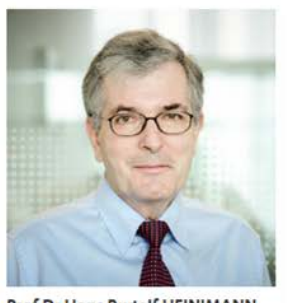

Prof Dr Hans Rudolf HEINIMANN FRS Programme Director and Centre

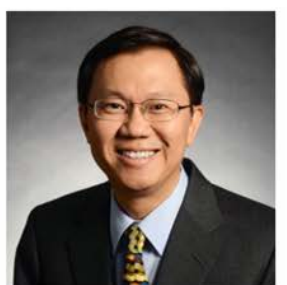

Prof Dr HO Teck Hua FRS Management Committee Member \& NUS Deputy President (Research \& Technology)

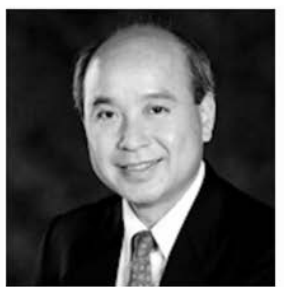

Prof Dr PAN Tso-Chien FRS Management Committe Member, Executive Director, Institute of Catastrophe Risk

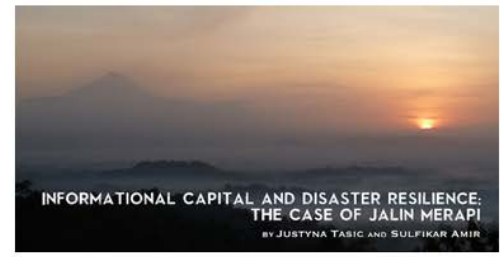

Reference:

http://www.frs.ethz.ch/about/frs/

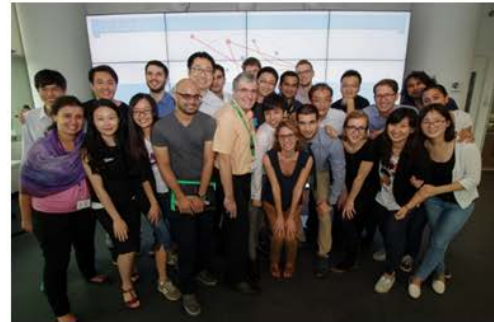

FRS Framing \& Collaboration Workshop http://www.frs.ethz.ch/department/principal_investigators/

\section{Asia research initiatives}

\section{The University of Tokyo \\ The Resilience Engineering Research Center (RERC)}

\section{Background:}

The Resilience Engineering Research Center (RERC), which consists of three divisions, was established in April 2013 to promote research into the principles and methodologies for realizing resilient systems. Mission:

The center intends to contribute to a safe and secure society by establishing a new risk management framework that exceeds the conventional and static approaches of risk management.

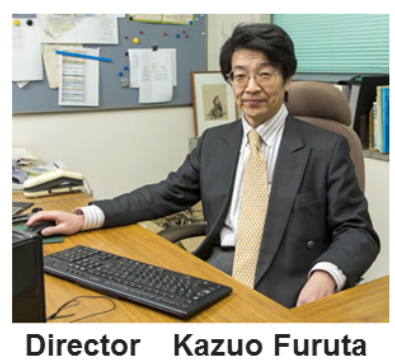

RESILIENCE ENGINECRING RESEARCIH CENTER

TIHE UNIUERSITY ()F T()KY() 


\section{Asia research initiatives}

\section{Featured projects:}

Industry

1. Development of numerical models for lithium ion battery fabrication processes;

2. Empirical Studies on Japanese-style Open Innovation;

3. Technology management strategy for future social needs driven innovation at global level.

Grant-in-Aid for Scientific Research (KAKENHI)

1. Assessment of massive integration of renewable energy by developing a multi-regional optimal power generation mix model;

2. Numerical study on rheological properties of highly viscous fluids including fine particles;

3. Construction of Innovation Database and International Comparison of Open Innovation.

\section{Others}

1. Collaborative researches into information technology for financial instrument market stability and efficiency;

2. Framework for Administration of Social Simulations on Massively Parallel Computers;

3. A study on the strategy and scenario to achieve the reformation of power generation mix in light of the Great East Japan Earthquake;

4. Resilience Analysis for Social Safety Policy;

\section{Professor Kazuo Furuta's research}

1.Interactive Method for Service Design

2.Incident management

3.Resilience of network service systems

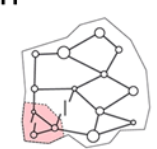

\section{Asia research initiatives}

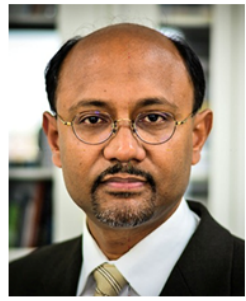

PROFESSOR RAJIB SHAW Management[AUEDM]

\section{京都大学}

KYOTO UNIVERSITY

Graduate School of Global Environmental Studies of Kyoto University, Japan President of Asian University Network of Environment and Disaster

Co-chair of UN ISDR Science Technology Academia Stakeholder Group

\section{BIOGRAPHY:}

Rajib Shaw is Professor in the Graduate School of Global Environmental Studies of Kyoto University, Japan. He worked closely with the local communities, NGOs, governments and international organization, including United Nations, especially in the Asian countries. Professor Shaw has extensive publications in different journals, books and edited volumes.

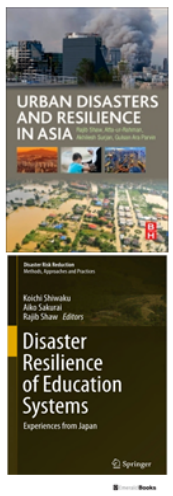

\section{RESEARCH INTERESTS}

1. community based disaster risk management;

2. climate change adaptation;

3. urban risk management;

4. disaster and environmental education.
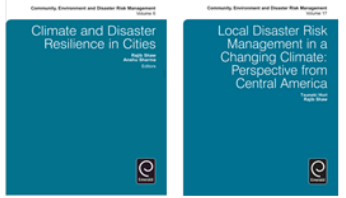


\title{
V. Concluding remarks
}
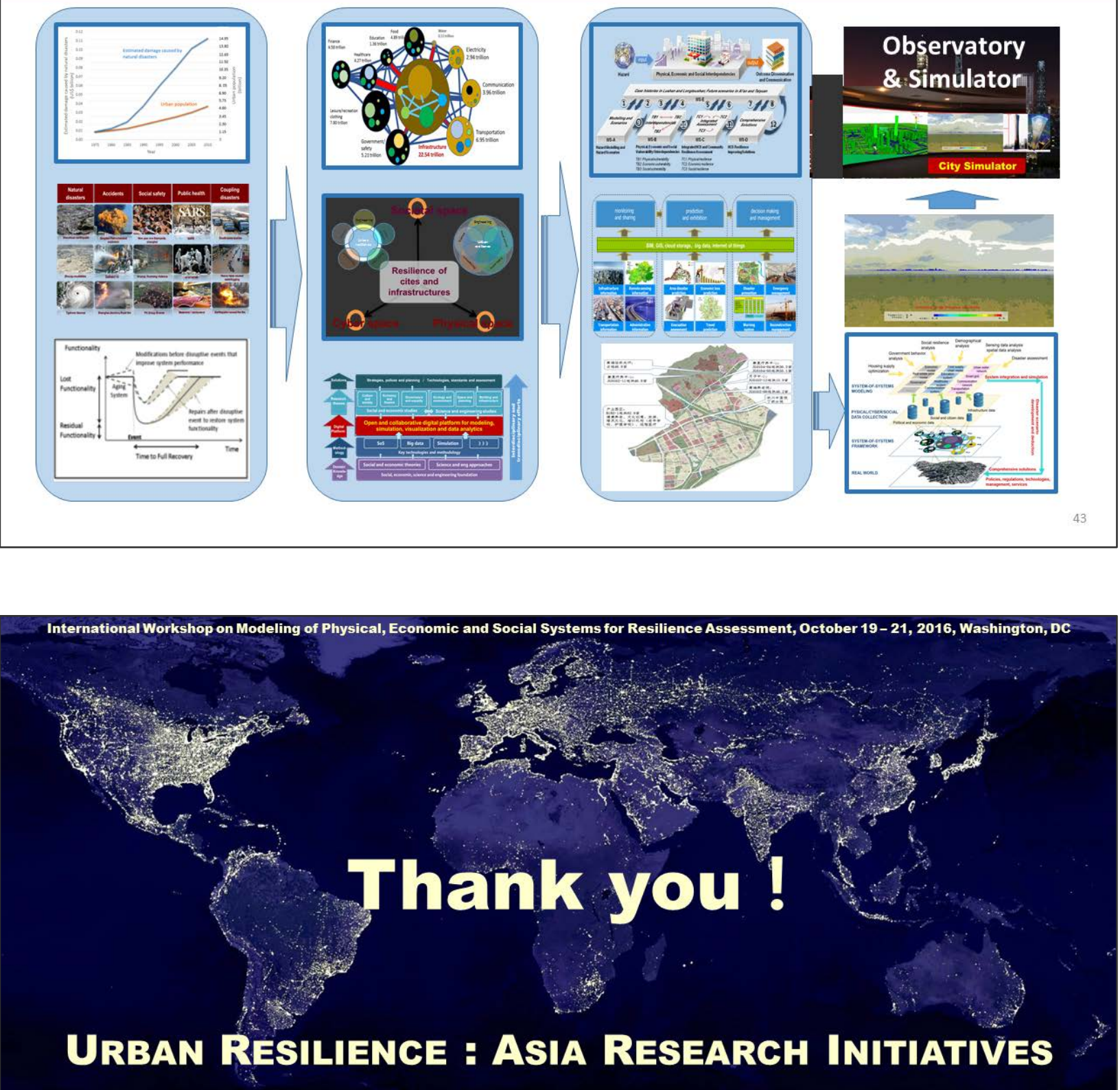

\author{
Professor Dongping Fang \\ Institute for Future Cities and Infrastructures \\ Tsinghua University
}

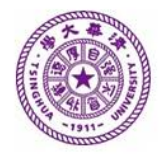




\section{Bibliography}

- United Nations, Department of Economic and Social Affairs, Population Division (2015). World Urbanization Prospects: The 2014 Revision, (ST/ESA/SER.A/366).

- EM-DAT, The OFDA/CRED International Disaster Database-www.emdat.be - Universite Catholique de Louvain, Brussels, Belgium.

- McAllister, T. (2015). Research Needs for Developing a Risk-Informed Methodology for Community Resilience. Joumal of Structural Engineering, 10.1061/(ASCE)ST.1943-541X.0001379, C4015008.

- Ellingwood, B. R. et al (2016). A fully integrated model of interacting physical and social infrastructure systems: introducing the center for risk-based community resilience planning. Urban Transitions Global Summit 2016, September 5-9, 2016, Shanghai, China, Elsevier.

- http://www.luxinzheng.net/commontop.html

- Zhao, C., Li, N., Fang, D. (2015). A Conceptual Framework for Modeling Critical Infrastructure Interdependency: Using a Multilayer Directed Network Model and Targeted Attack-Based Resilience Analysis. Proc., 2015 ASCE International Workshop on Computing in Civil Engineering (IWCCE 2015), June 21-23, 2015, Austin, TX, American Society of Civil Engineers (ASCE)

- Feng, K., Li, Q. (2016). Measuring and improving the functionality of built environment considering the inter-dependency of community components. Urban Transitions Global Summit 2016, September 5-9, 2016, Shanghai, China, Elsevier.

- Wang, J., Wang, W., Zhang, X. (2014). Empirical study of the characteristics of the pedestrians' velocity in crowded places based on crosscorrelation algorithm. Journal of Safety Science and Technology, 10(6), 5-11.

- Top Plan Report of Golden Dinglan (2015).

- Vallet, J., Liao, P., Li, N., Fang, D. (2016). An improved index for benchmarking urban resilience. Urban Transitions Global Summit 2016 , September 5-9, 2016, Shanghai, China, Elsevier.

- http://www.frs.ethz.ch/about/frs/

- http://rerc.t.u-tokyo.ac.jp/html_en/center.html

- http://www.frs.ethz.ch/about/frs/

- http://rerc.t.u-tokyo.ac.jp/html_en/project.html

- http://www.frs.ethz.ch/department/principal_investigators/

- https://www.researchgate.net/profile/Rajib_Shaw 


\section{Bibliography}

- United Nations, Department of Economic and Social Affairs, Population Division (2015). World Urbanization Prospects: The 2014 Revision, (ST/ESA/SER.A/366).

- EM-DAT, The OFDA/CRED International Disaster Database- www.emdat.be - Universite Catholique de Louvain, Brussels, Belgium.

- McAllister, T. (2015). Research Needs for Developing a Risk-Informed Methodology for Community Resilience. Journal of Structural Engineering, 10.1061/(ASCE)ST.1943541X.0001379, C4015008.

- Ellingwood, B. R. et al (2016). A fully integrated model of interacting physical and social infrastructure systems: introducing the center for risk-based community resilience planning. Urban Transitions Global Summit 2016, September 5-9, 2016, Shanghai, China, Elsevier.

- http://www.luxinzheng.net/commontop.html

- Zhao, C., Li, N., Fang, D. (2015). A Conceptual Framework for Modeling Critical Infrastructure Interdependency: Using a Multilayer Directed Network Model and Targeted Attack-Based Resilience Analysis. Proc., 2015 ASCE International Workshop on Computing in Civil Engineering (IWCCE 2015), June 21-23, 2015, Austin, TX, American Society of Civil Engineers (ASCE).

- Feng, K., Li, Q. (2016). Measuring and improving the functionality of built environment considering the inter-dependency of community components. Urban Transitions Global Summit 2016, September 5-9, 2016, Shanghai, China, Elsevier.

- Wang, J., Wang, W., Zhang, X. (2014). Empirical study of the characteristics of the pedestrians' velocity in crowded places based on cross-correlation algorithm. Journal of Safety Science and Technology, 10(6), 5-11.

- Top Plan Report of Golden Dinglan (2015).

- Vallet, J., Liao, P., Li, N., Fang, D. (2016). An improved index for benchmarking urban resilience. Urban Transitions Global Summit 2016, September 5-9, 2016, Shanghai, China, and Elsevier.

- http://www.frs.ethz.ch/about/frs/

- http://www.frs.ethz.ch/about/frs/

- http://www.frs.ethz.ch/department/principal_investigators/

- http://rerc.t.u-tokyo.ac.jp/html_en/center.html

- http://rerc.t.u-tokyo.ac.jp/html_en/project.html

- https://www.researchgate.net/profile/Rajib_Shaw 


\section{Prof. Dongping FANG}

\section{A Short CV}

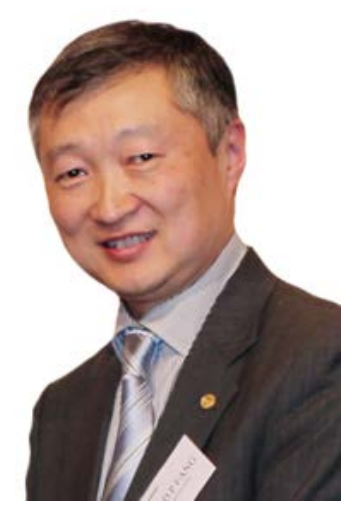

Dr. Dongping FANG is a Professor at Tsinghua University, China. He is the head of Department of Construction Management in the School of Civil Engineering and the executive director of Institute of Future Cities and Infrastructures.

Prof Fang is a former vice president of CIB ( International Council for Research and Innovation in Building and Construction ) and the current leader of CIB priority theme - Resilient Urbanization. He has been sitting on boards and committees of many international and national organizations of government, industry and academics. He has been honored as Visiting Professors in Australia, Sweden and the UK, and invited as keynote speakers for many international conferences such as CIB world building congress.

Prof Fang is an expert on construction safety and sustainable urbanization. He serves as members of editorial board of several renowned international journals and is the author/coauthor of more than 200 books, book chapters, journal papers, conference papers and reports. 
"NSF Investments and Opportunities in Interdisciplinary Research on Critical Infrastructure Systems"

David Mendonca

Program Director

Infrastructure Management and Extreme Events

National Science Foundation

The National Science Foundation (NSF) is in the midst of a series of major, multi-directorate, multi-year investments in the basic science and engineering underlying society's ability to prepare for--and respond to--hazards and disasters that pose risks to critical infrastructure systems. This talk reviews recent results, opportunities and challenges in this area, focusing on strongly interdisciplinary research on phenomena where humans are inextricably "in the loop." Examples are drawn primarily from recent research funded through two NSF programs: Infrastructure Management and Extreme Events (IMEE) and Critical Resilient Infrastructure Systems and Processes (CRISP). The talk will include opportunity for discussion.

About the speaker. David Mendonca currently directs the IMEE program and participates in the administration of a number of others. While at NSF, he is on leave from the Industrial and Systems Engineering Department at Rensselaer Polytechnic Institute, where he is an Associate Professor. His research focuses on the role of systems engineering in disaster response. 


\title{
NSF Investments and Opportunities in Interdisciplinary Research on Critical Infrastructure Systems
}

\author{
David Mendonça
}

Division of Civil, Mechanical and Manufacturing Innovation

National Science Foundation mendonca@nsf.gov

\section{Outline}

- Overview of CRISP Program

- Features of recent CRISP awards

- Infrastructures

- Topics

- Methods

- Data

- Observations and questions

- Funding opportunities 


\section{CRISP FY17 Overview}

- Critical Resilient Interdependent Infrastructure Systems and Processes program goals:

- Foster interdisciplinary research community

- Enhance understanding and design of interdependent critical infrastructure (ICI) systems and processes

- Create knowledge for innovation in ICls

- Improve ICI performance

- FY 2017: \$22.9M, 15-20 awards

- Type 1: Duration 2 years, max total budget $\$ 500 \mathrm{k}$.

- Type 2: Duration 3-4 years, budget range $\$ 1 \mathrm{M}-\$ 2.5 \mathrm{M}$.

\section{A Burgeoning, Interdisciplinary Community}

- Since 2014 (CRISP\&RIPS), an investment of $\$ 59 \mathrm{M}$ across 37 projects, 71 project teams and 180 distinct individuals

- Composition of research teams (CRISP+RIPS)

- Infrastructures (CRISP)

- For FY16 Type 2 CRISP awards ( $<=\$ 2.5 \mathrm{M} /$ award):

- Topics

- Methodological approaches

- Data 


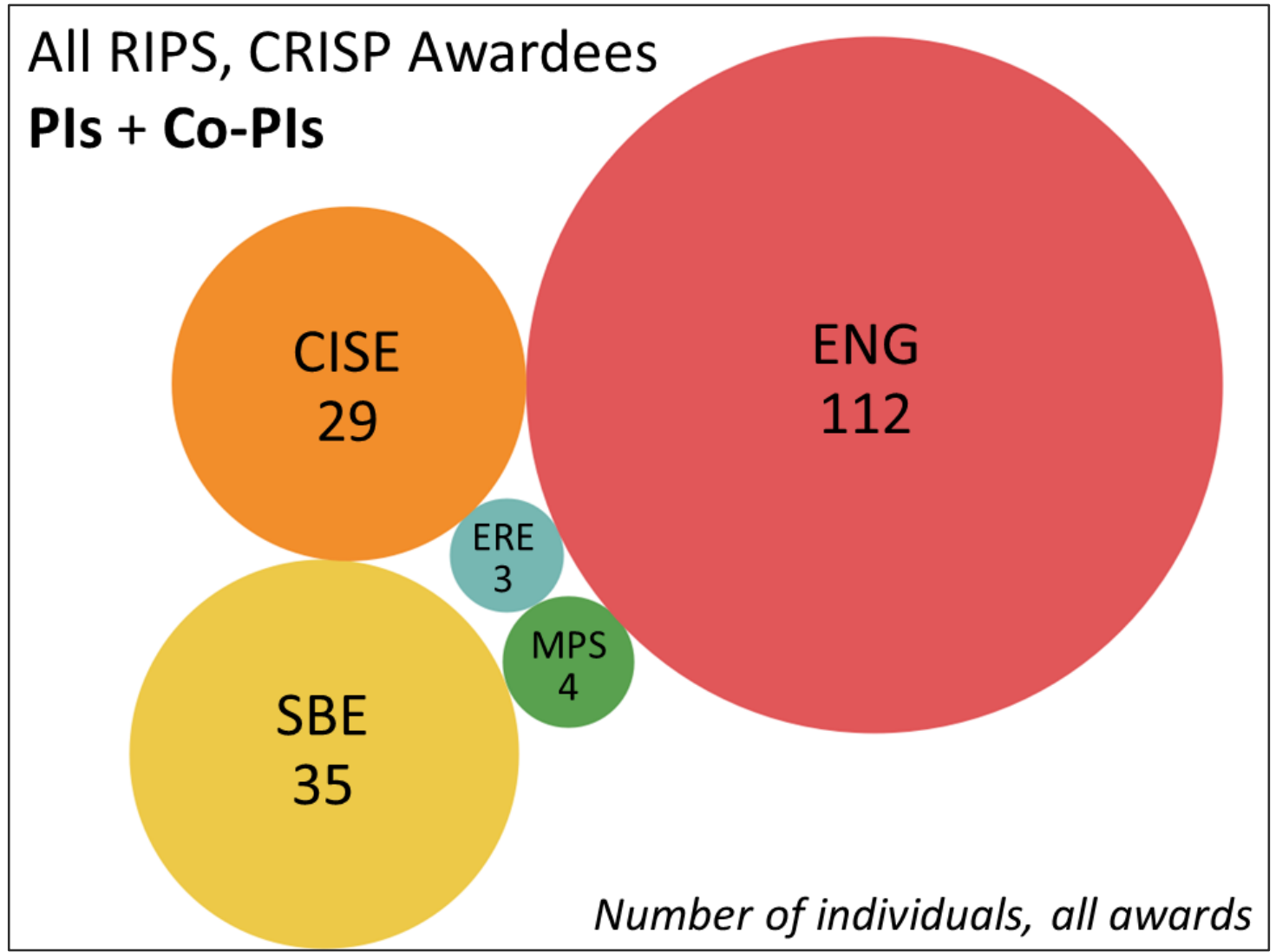

\section{All RIPS, CRISP Awardees}

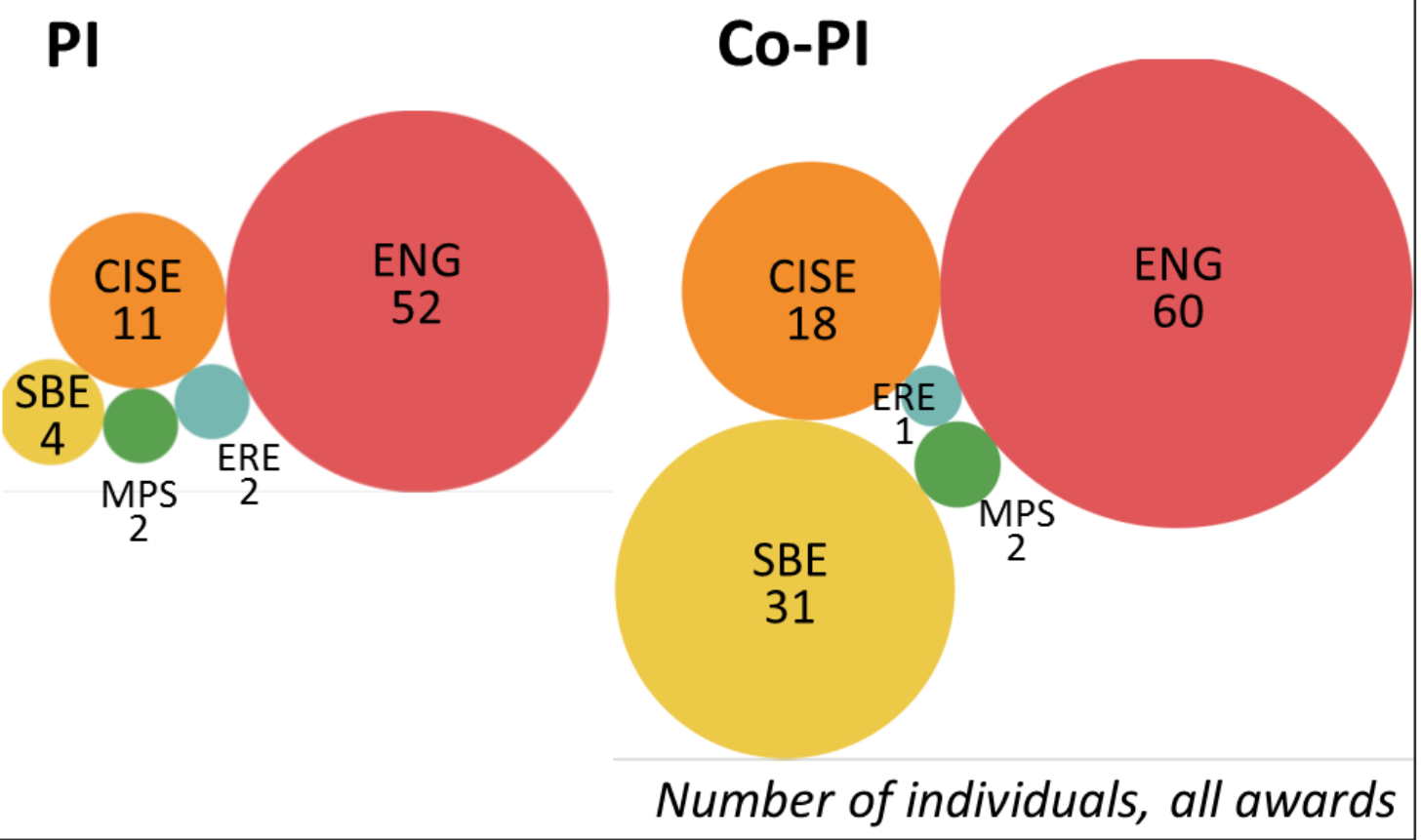




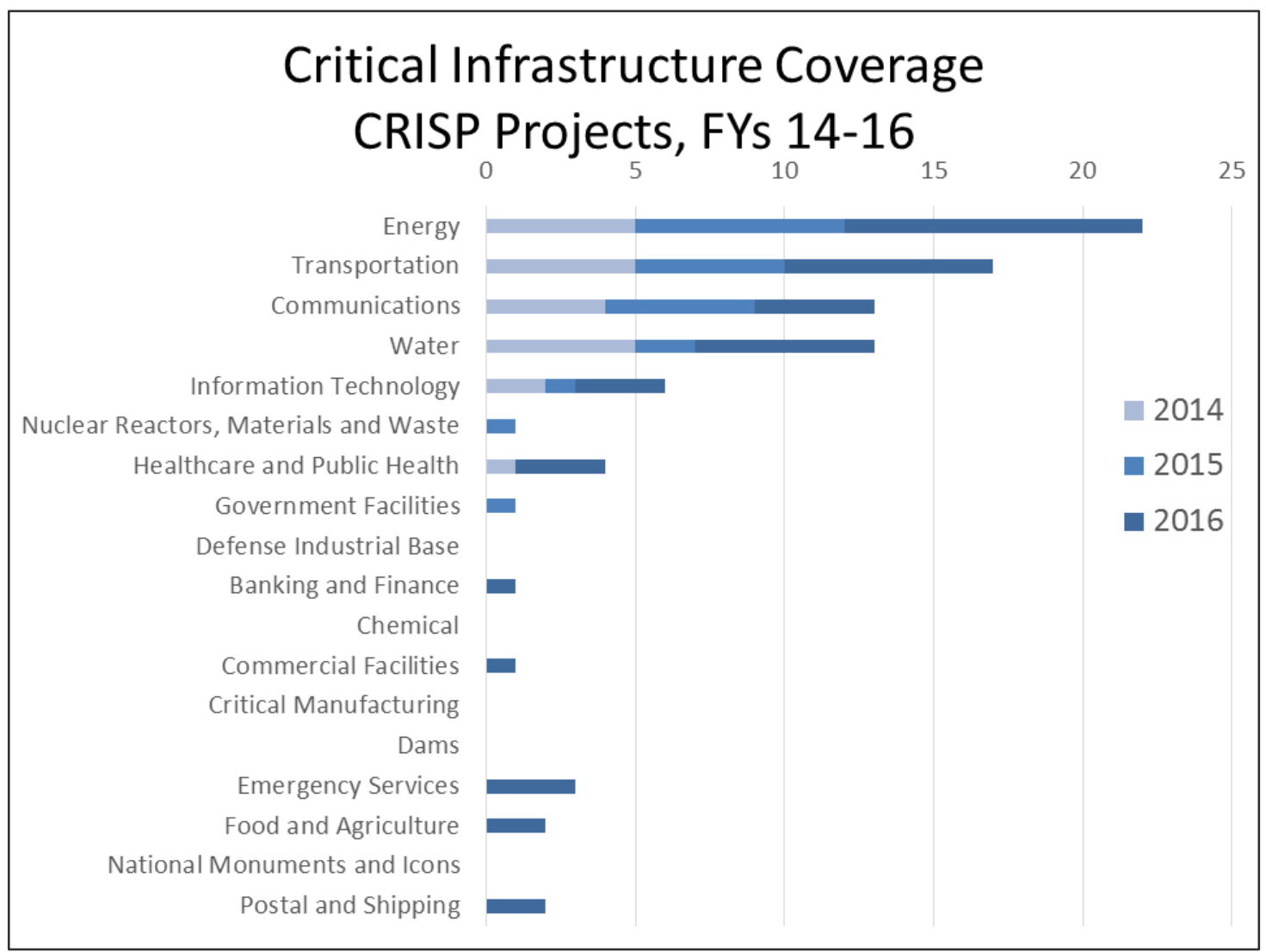

\section{FY16 CRISP Type 2 Awards}

- Interdependencies in Community Resilience: A Simulation Framework

- Coordinated, Behaviorally-Aware Recovery for Transportation and Power Disruptions

- Identification and Control of Uncertain, Highly Interdependent Processes Involving Humans with Applications to Resilient Emergency Health Response

- Natural Gas Production, Electricity, and Water Infrastructures- Economic, Environmental and Agricultural Impacts in the Texas-Mexico Border Region

- Scalable Decision Model to Achieve Local and Regional Resilience of Interdependent Critical Infrastructure Systems and Communities

- Harnessing Interdependency for Resilience: Creating an Energy Sponge with Cloud Electric Vehicle Sharing

- Integrative Decision Making Framework to Enhance the Resiliency of Interdependent Critical Infrastructures

- Critical Transitions in the Resilience and Recovery of Interdependent Social and Physical Networks

- Multi-Agent Sustainable Water Decision Theory: Nexus of Water, Road, and Hierarchic Social Contractual Systems 


\section{FY16 T2 Methodological Approaches}

1) Optimization: network, stochastic and nonlinear

2) Simulation: agent-based, Markov chain Monte Carlo, POMDP

3) Statistics: classical inference; machine learning; (social) network analysis, hierarchical Bayesian

4) Information Science. Software/data eng, viz

5) Explanatory sciences. Physics-based modeling, systems dynamics, graph theory, game theory 6) Other. Gaming experimentation, survey/interview

Illustrative, not comprehensive.

\section{FY16 T2 Data Targets}

- Human

- Social media

- Subj. judgment, interviews, surveys

- Activity diaries

- Technological

- System design, use, inventory, maintenance

- Mixed

- Archival/public (HIS, LODES, Census/PUMS, HUD, LBD, USDA, EPANET, SWMM, NGMD)

- Synthetic, Simulated

Illustrative, not comprehensive. 


\section{Preliminary Observations\&Questions}

- Infrastructures

- Other theoretically interesting possibilities?

- Driven by data availability?

- "Good" data on interdependencies?

- Methods

- Integrative methods to explain/support

- Cognition and adaptive behavior-including redesign?

- Human agency beyond demand for service-including $\mathrm{ICI}$ design and control?

\section{Preliminary Observations\&Questions}

- Data

- What are the requirements for a common data block to support the CRISP research enterprise?

- Related opportunities

- DIBBS (NSF 17-500), 03 January 2017

- IMEE (NSF 17-1638), 30 Dec 2016-15 January 2017 


\title{
FY17 CRISP Solicitation
}

NSF 16-618

- Due date: 8 February 2017 by 5p proposer's local time

- Webcast: 5 December 2016 at 1:30pm EST

- Questions/inquiries: Contact member of project team (see solicitation)

\section{NSF Investments and Opportunities in Interdisciplinary Research on Critical Infrastructure Systems*}

\author{
David Mendonça
}

Division of Civil, Mechanical and Manufacturing Innovation

National Science Foundation

mendonca@nsf.gov

\begin{abstract}
*Thanks to past and present members of the CRISP team, especially Elise Miller-Hooks for her organizing vision.
\end{abstract}




\title{
DHS Sponsored Research: DHS Approaches to Supporting Infrastructure Resilience
}

\section{Matthew Coats, Program Manager, U.S. Department of Homeland Security, Science and Technology Directorate}

\begin{abstract}
NIST Presentation
The Federal government has a compelling interest in getting industries and regional economies working again as soon as possible after a catastrophic event. This is always true, but particularly so in locales where there is an intersection of significant probabilities of catastrophes, infrastructure systems and industries that are critical to the functioning of the U.S. economy. DHS must work closely with infrastructure owners and operators to craft technically feasible and economically efficient partnerships that will make the Nation more resilient. Moreover, DHS must understand the interactions of the multiple public and private sector linkages that comprise an infrastructure system and community, and how these linkages respond to the severe stress brought on by catastrophic events. DHS is partnering with its Centers of Excellence to provide insights into promising areas of research and to address specific challenges faced by DHS operational components. The DHS Critical Infrastructure Resilience Institute will focus on improving understanding of the role cyber plays in infrastructure systems, how businesses can use this information to make risk informed decisions, and how to test those approaches in the real world. The Center will also address the cascading effects of a disruption across infrastructure sectors and the issues surrounding legal, legislative, and policy frameworks necessary to define responsibility and support recover.
\end{abstract}




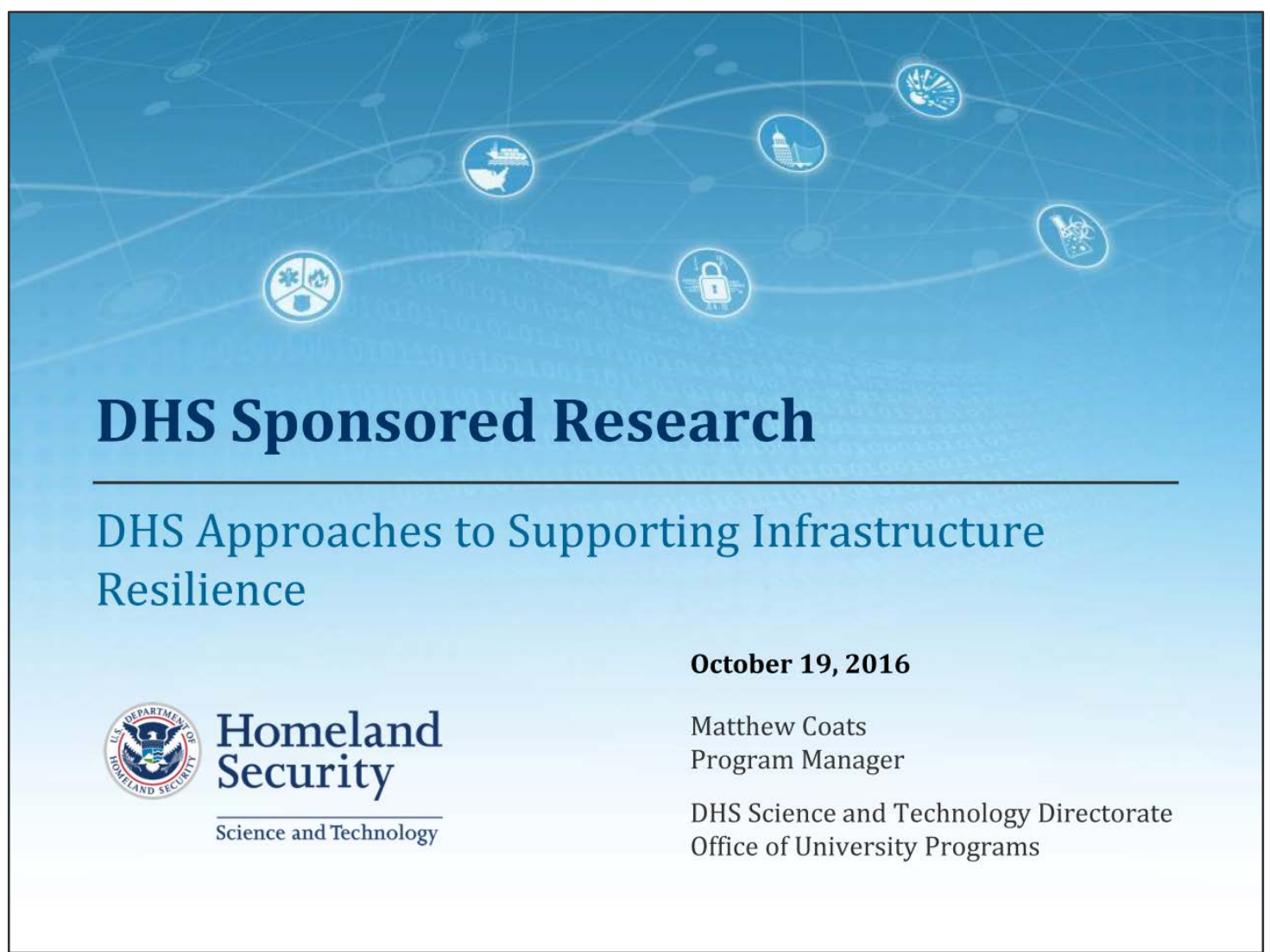

\section{Overview}

- Emerging Trends and Technologies

- DHS and Resilience

- DHS Office of University Programs Funded Modeling Projects

- DHS Critical Infrastructure Resilience Institute 


\section{Perspective of Critical Infrastructure Interdependencies}

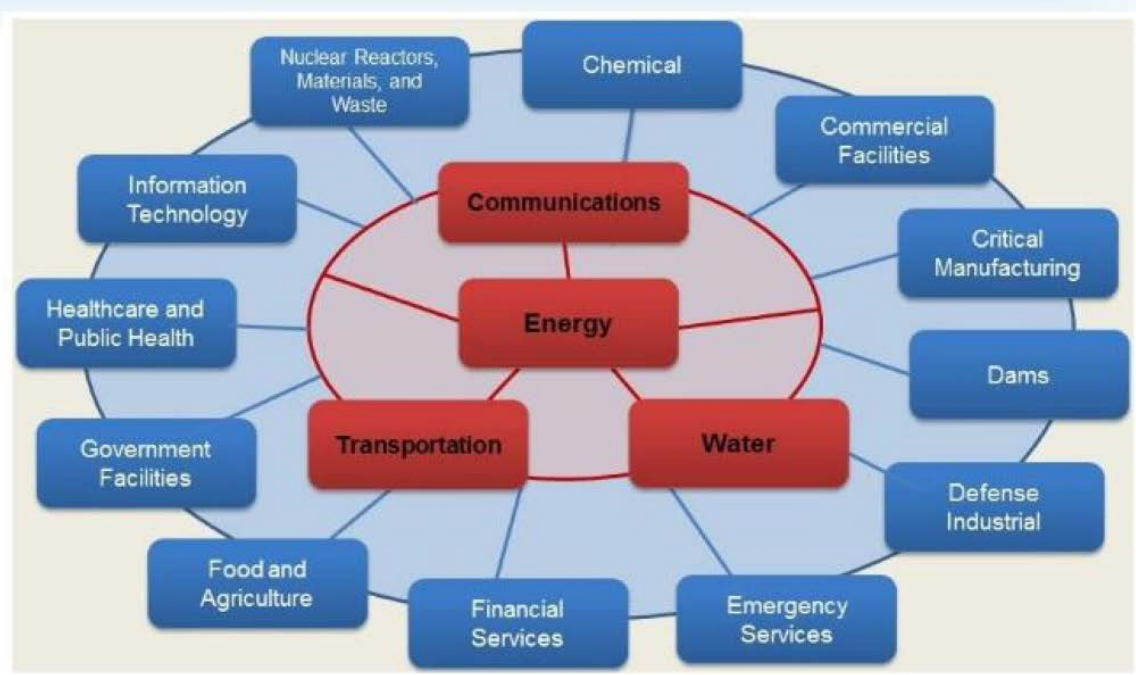

Source: Energy : 2015 Sector-Specific Plan - NIPP

Homeland Security

Science and Technology

\section{Threats to Cyber Infrastructure are a} constant risk underlying all sectors

\section{Emerging Trends and Technologies in Information Technology}

- In the News:

- 9/23/16 Trend Micro security researchers reported the Locky ransomware was responsible for an increase in certain methods of malware delivery ${ }^{1}$

- 9/22/16 Yahoo confirms massive data breach of 500 million accounts. ${ }^{1}$

- 9/19/16 Rockwell patches code execution flaw in RSLogix product. Rockwell Automation released patches for several of its RSLogix products used in the food and agriculture, critical manufacturing, water, and chemical sectors 1

- 9/16/16 loT Village uncovers 47 security vulnerabilities across 23 devices used in home security and municipal power facilities

\section{- Market Overview:}

- Cyber-insurance providers to play a pivotal role in combatting information insecurity on a global scale by providing price incentives to companies to adhere to strict data security standards.

- Typical limit capacity for stand-alone coverage is in the range of \$5 million to \$25 million per carrier, with total market per risk capacity estimated at $\$ 500$ million. $^{2}$

- Gross written premiums for US cyber insurance are about $\$ 2.75$ billion, up from roughly $\$ 2.0$ billion in $2014 .^{2}$

- Focus on challenges posed by cyberattacks on the critical infrastructure supply chain.

- Evolution toward smart manufacturing and developing digital threads to make the critical manufacturing infrastructure more resilient.

Homeland

Security

Science and Technology 


\section{NIPP Goals, Priorities, and Activities}

\section{NATIONAL STRATEGY}

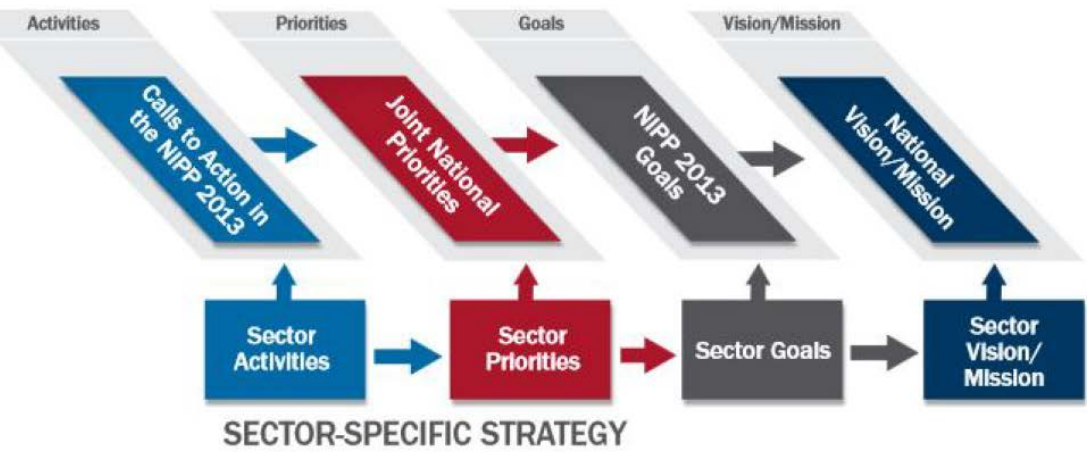

The 2013 National Infrastructure Protection Plan (NIPP) outlines how government and private sector participants in the critical infrastructure community work together to manage risks and achieve security and resilience outcomes.

Homeland

Security

\section{Critical Infrastructure Sectors of Focus}

\section{- Critical Manufacturing Sector}

- Sector Specific Agency: Department of Homeland Security

- Produces 12.5 percent of the U.S. gross domestic product (2012).

- 60 percent of U.S. exports are produced by the manufacturing industry

\section{- Energy Sector}

- Sector Specific Agency: Department of Energy

- More than 80 percent of the country's energy infrastructure is owned by the private sector.

\section{- Transportation Systems Sector}

- Sector Specific Agencies : Department of Homeland Security, and the Department of Transportation

- Maritime Transportation System consists of about 95,000 miles of coastline, 361 ports, more than 25,000 miles of waterways, and intermodal landside connections.

Homeland

Security

Science and Technology
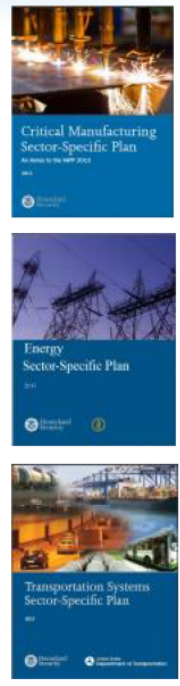


\section{DHS S\&T Efforts in Resilience}

\section{- Mission}

- The DHS Science and Technology Directorate's (S\&T) mission is to deliver effective and innovative insight, methods and solutions for the critical needs of the Homeland Security Enterprise.

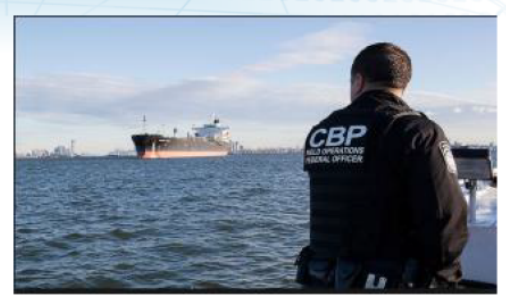

- Visionary Goals

- Screening at Speed: Security that Matches the Pace of Life

- Trusted Cyber Future: Protecting Privacy, Commerce, and Community

- Enable the Decision Maker: Actionable Information at the Speed of Thought

- Responder of the Future: Protected, Connected, and Fully Aware

- Resilient Communities: Disaster-Proofing Society

Homeland

Security

\section{Additional Resilience Efforts}

- 100 Resilient Cities - Pioneered by the Rockefeller Foundation, dedicated to helping cities around the world become more resilient to the physical, social and economic challenges. .

- NIST funded Disaster Resilience Center of Excellence led by Colorado State University - works with NIST researchers and partners from 10 universities to accelerate the development of system-level models and support tools to help local governments decide how to best invest resources.

- NIST Community Resilience Planning Guide - Provides a practical and flexible approach to help communities improve their resilience by setting priorities and allocating resources to manage risks for their prevailing hazards.

Homeland Security

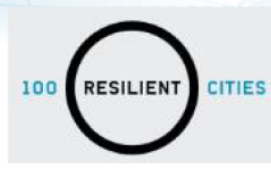

Science and Technology 


\section{Moving from Models to Action}

- What will drive business owners to change their practices?

- Many models are theoretical in nature and although these models can provide insights for decision makers, modelling of critical infrastructure systems should focus more on realistically identifying data flows, dependencies and interdependencies.

- Models provide answers to discrete questions. There is no one model that can be used to make a community resilient. Stakeholder needs are too diverse.

- Additional Challenges:

- Threat centric vs Resilience centric?

- Ontology coordination across disciplines for model integration - what questions are being asked?

- Securing necessary data

- Intellectual property rights

- Operations and maintenance

Homeland

Security

Executing research to support resilience:

- DHS S\&T Office of University Programs Funded Modeling Projects support elements of building resilience

- AgConnect - Institute for Infectious Animal Diseases (IIAD). A suite of customizable software products designed to enhance real-time situational awareness and support decision-making for emerging, zoonotic and/or transboundary animal diseases.

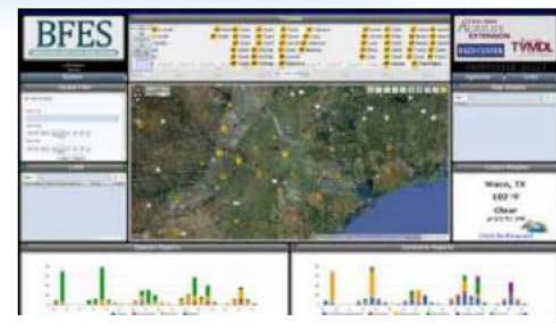

- Criticality Spatial Analysis (CRISTAL) Program Food Protection and Defense Institute (FPDI). CRISTAL develops new ways of identifying and understanding how and where supply chains are vulnerable to disruptions.

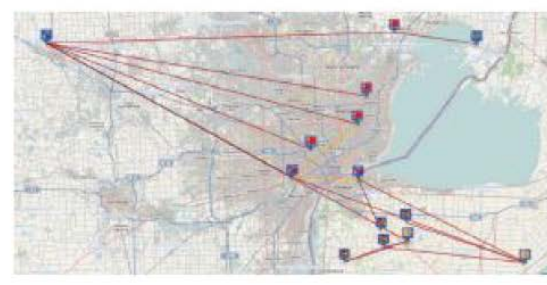

Homeland

Security 


\section{DHS S\&T Office of University Programs Funded Modeling Projects}

- Economic Consequences Analysis Tool (E-CAT) - National Center for Risk and Economic Analysis of Terrorism Events (CREATE). Provides quick estimates of the economic impact of threats listed in the Homeland Security National Risk Characterization Register.

- ADvanced CIRCulation (ADCIRC) storm surge model - Coastal Resilience Center $(C R C)$. Predicts when, where, and to what extent flooding will inundate a coastal community with greater precision than other available models.

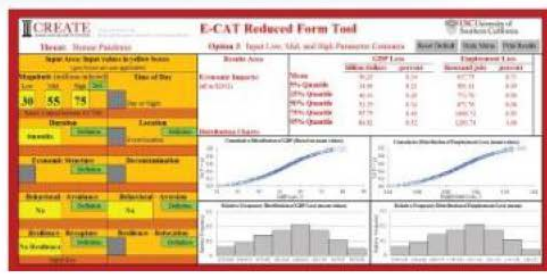

Homeland

Security

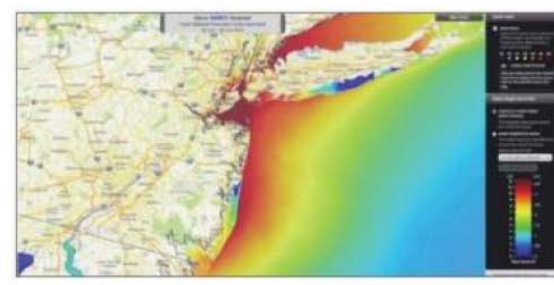

\section{How CIRI Supports the DHS Mission}

- Lead University: University of Illinois at Urbana-Champaign

- Key Customers: NPPD, FEMA, Department of Commerce, State and local governments

- Alignment:

- QHSR: Safeguard and Secure Cyberspace, Strengthen National Preparedness and Resilience

- S\&T Visionary Goals: Trusted Cyber Future, Resilient Communities, Enable the Decision Maker

- Capability Gaps:

- Understanding of the impacts of catastrophic disruptions to infrastructures and across infrastructures- using cyber physical systems as a focus

- Private Sector adoption of risk reduction measures

- Models for public-private partnerships

Homeland 


\section{Focus on CIRI Modeling Projects}

- Analyzing and Supporting the Development of the Cyberinsurance Market as a Market-Based Solution for Cyber

Resiliency - Will model specific threats affecting the cyber insurance market.

- Regulatory Options for Managing Systemic Risks - Will create a model that can evaluate both systemic risk in the electric power grid and the potential impact of regulatory options on systemic risk.

- Supply Chain Cybersecurity Assurance For Critical Infrastructure - Will develop assessment computer models that will be specifically designed to facilitate identification and mitigation of supply chain-induced cyber vulnerabilities in critical infrastructure

- Measuring Business and Economic Resilience in Disasters - Will develop a formal microeconomic model of business responses to disasters

Homeland

Security

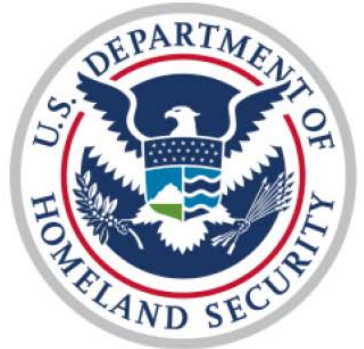

Homeland

Security

Science and Technology 


\section{DHS Sponsored Research: DHS Approaches to Supporting Infrastructure Resilience}

\section{Matthew Coats, Program Manager, U.S. Department of Homeland Security, Science and Technology Directorate}

\section{Bibliography}

The White House. March 30, 2011. Presidential Policy Directive/PPD 8 - National Preparedness. Available online at: http://www.dhs.gov/presidential-policy-directive-8-national-preparedness.

The White House. February 12, 2013. Presidential Policy Directive/PPD 21 - Critical Infrastructure Security and Resilience. Available online at: http://www.whitehouse.gov/the-pressoffice/2013/02/12/presidential-policy-directive-critical-infrastructure-security-and-resil

Executive Order No. 13636 of February 12, 2013. Improving Critical Infrastructure Cybersecurity. Available online at: http://www.whitehouse.gov/the-press-office/2013/02/12/executive-orderimproving-critical-infrastructure-cybersecurity.

United States Department of Homeland Security. February 2010. Quadrennial Homeland Security Review Report: A Strategic Framework for a Secure Homeland. Available online at: http://www.dhs.gov/xlibrary/assets/qhsr_report.pdf.

United States Department of Homeland Security, National Protection and Programs Directorate. August 2012. Office of Infrastructure Protection Strategic Plan: 2012-2016. Available online at: https://www.dhs.gov/sites/default/files/publications/IP\%20Strategic\%20Plan\%20FINAL.pdf.

United States Department of Homeland Security. 2013. National Infrastructure Protection Plan. Online at: http://www.dhs.gov/national-infrastructure-protection-plan. 


\section{DHS Sponsored Research: DHS Approaches to Supporting Infrastructure Resilience}

\section{Matthew Coats, Program Manager, U.S. Department of Homeland Security, Science and Technology Directorate}

Mr. Matt Coats is the Program Manager for three DHS Science and Technology Centers of Excellence that conduct research and development activities in zoonotic and animal disease, critical infrastructure resilience, and food protection and defense. He also serves as the Office of University Programs Transition lead responsible for overseeing technology transition. In 2012, he jointly served as the acting Deputy Director of the DHS International Cooperative Programs Office, overseeing the team's management of the Department's 12 International Agreements. He participates in the Department's integrated product teams, National Security Council working groups, and the White House Foreign Animal Disease Threat Subcommittee where he served as the co-chair for the Work Force Task Force.

Prior to his work at DHS, Mr. Coats was a Senior Consultant and Booz Allen Hamilton supporting diverse public sector clients responsible for developing technologies to protect the Nation. He has worked in the biotech private sector as an International Business Development Associate at KARD Scientific where he was responsible for managing brand development, account management, and assisting in the execution of new research and development partnerships globally.

He is a graduate of Georgetown University's Medical School with an M.S. in Biomedical Science Policy, Union College with a B.S. in biology, and Massachusetts Institute of Technology Sloan School of Business' Executive Program. Mr. Coats is a certified Program Manager from the Defense Acquisition University, a Contracting Officer Representative, and a member in the Phi Alpha Theta and Sigma Xi Honor Societies. 
International Workshop on Modeling of Physical, Economic, and Social Systems for Resilience Assessment

This page intentionally left blank. 


\section{Appendix C: Modeling of Systems and Dependencies (Session 3)}

Evolution of Hazard Assessment Modeling in Drinking Water Systems [Royce

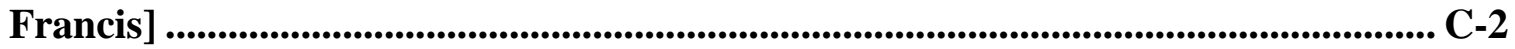

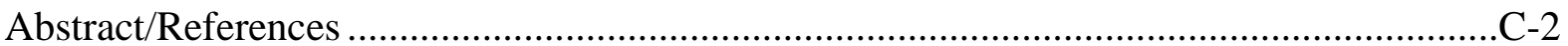

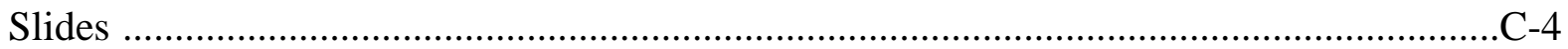

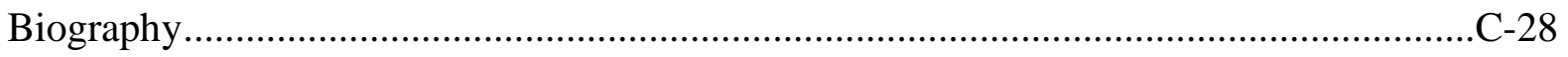

Modeling Water Distribution System Resilience [Katherine Klise]................................. C-30

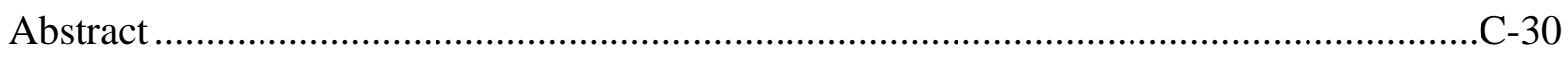

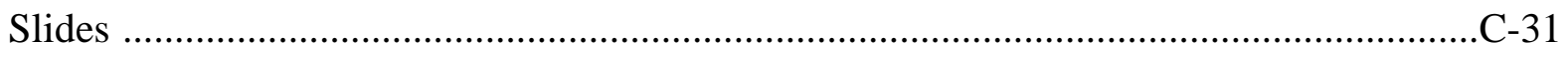

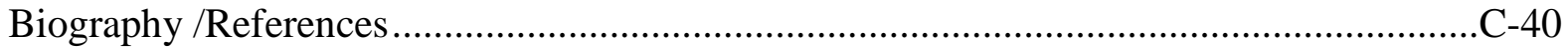

Power Distribution Systems: Modeling of System Dependencies and Interdependencies [Charles Macal] ........................................................................... C-41

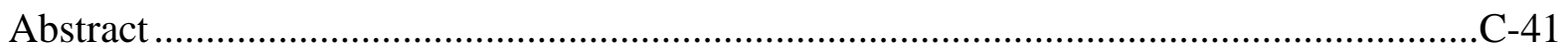

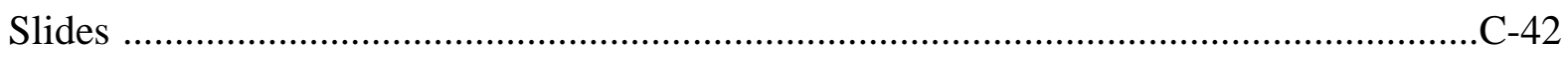

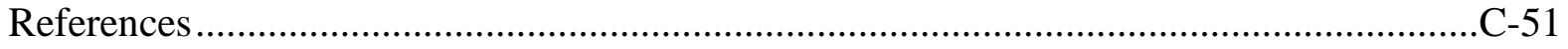

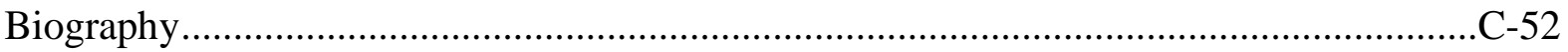

Reliability Assessment of Interdependent Networks Based on Survival Signature [Matteo Broggi] ............................................................................................................. C-53

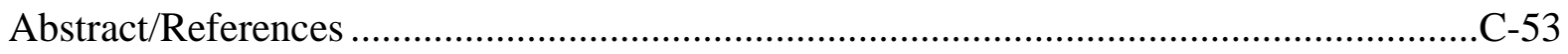

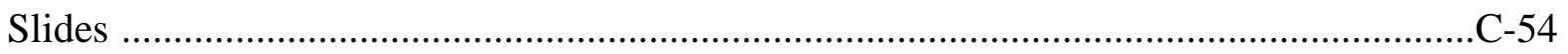

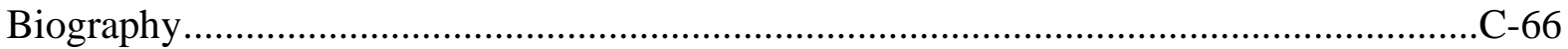

Climate \& Complexity: The Resilience of Natural-Built-Human Systems [Auroop Ganguly]......................................................................................................

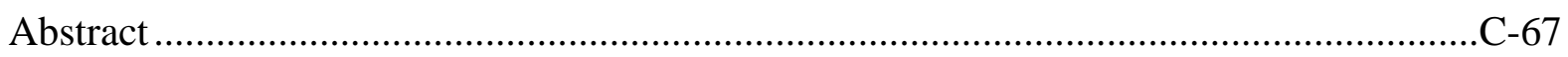

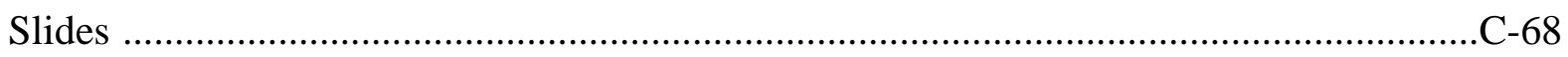

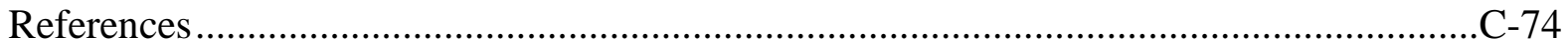

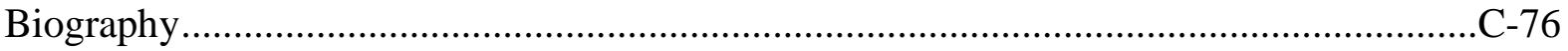

Natural Disaster Impacts on Infrastructure Systems and Society[Judith MitraniReiser] .................................................................................................................. C-77

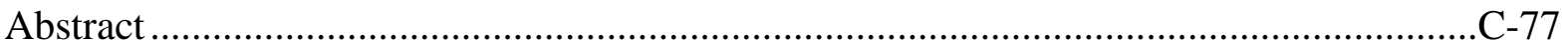

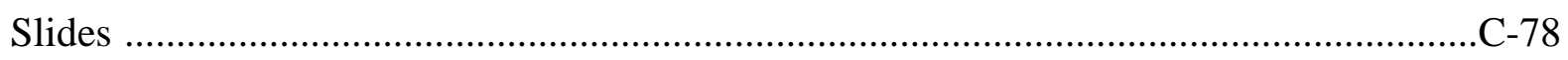

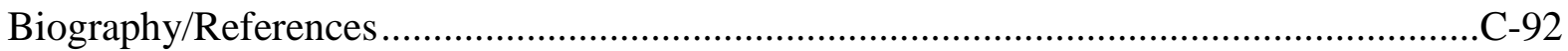




\section{Evolution of Hazard Assessment Modeling in Drinking Water Systems}

Royce Francis, George Washington University, seed@email.gwu.edu

The drinking water sector has focused intently during the last 15 years on hazard and risk assessment for potential service disruptions attributable to intentional and unintentional events. During the first 6-8 years of this period, the focus was primarily devoted to assessment risk attributable to intentional, i.e., terrorist, events. During the last 6-8 years, however, the sector has come to a consensus that an all-hazards risk management approach is more appropriate. The justification for the change from a focus on intentional events to all-hazards is based on the need to allocate limited resources to event preparations and response. The sector has come to the realization that preparing under an all-hazards approach would harden most systems to terrorist attacks, while preparing for terrorist attack scenarios would not harden most systems to the most important natural hazards. Consequently, most utilities in the industry assert that assessment procedures are more important than any particular modeling framework. In response to this consensus position, drinking water utilities have engaged in risk and vulnerability assessment using standardized tools such as EPA VSATMHEAT and SEMS/RAMCAP, EPA or AWWA table top exercises and incident checklists.

Notable References:

1. Ostfeld et al. (2008). "The Battle of the Water Sensor Networks (BWSN): A Design Challenge for Engineers and Algorithms." ASCE Journal of Water Resources Planning and Management, 134(6):556-568.

2. Krause, A., Leskovec, J., Guestrin, C., VanBriesen, J., \& Faloutsos, C. (2008). Efficient sensor placement optimization for securing large water distribution networks. Journal of Water Resources Planning and Management, 134(6), 516526.

3. Leskovec, J., Krause, A., Guestrin, C., Faloutsos, C., VanBriesen, J., \& Glance, N. (2007, August). Cost-effective outbreak detection in networks. In Proceedings of the 13th ACM SIGKDD international conference on Knowledge discovery and data mining (pp. 420-429). ACM.

4. Preis, A., \& Ostfeld, A. (2008). Multiobjective contaminant sensor network design for water distribution systems. Journal of Water Resources Planning and Management, 134(4), 366-377.

5. Hart, W. E., \& Murray, R. (2010). Review of sensor placement strategies for contamination warning systems in drinking water distribution systems.Journal of Water Resources Planning and Management, 136(6), 611-619.

6. Isovitsch, S. L., \& VanBriesen, J. M. (2008). Sensor placement and optimization criteria dependencies in a water distribution system. Journal of Water Resources Planning and Management, 134(2), 186-196.

7. US EPA Office of Water, US EPA Office of Ground Water, and US EPA Office of Drinking Water, "Conduct a Drinking Water or Wastewater Utility Risk Assessment," 2015. [Online]. https://www.epa.gov/waterriskassessment/conductdrinking-water-or-wastewaterutility-risk-assessment. [Accessed: 07-Jul-2016]. 
8. US EPA Office of Water, US EPA Office of Ground Water, and US EPA Office of Drinking Water, "Water Health and Economic Analysis Tool (WHEAT) 3.0," 2016. [Online]. Available: https://www.epa.gov/waterriskassessment/find-outabouthealth-and-economic-impacts-water-utility-emergencies. [Accessed: 07-Jul2016].

9. S. D. Spence and C. M. Tuozzoli, (2011). Gap Assessment for ASME-ITI/ AWWA J100-10 Standard and Leading Vulnerability Assessment Tools, Water Research Foundation Web Report No. 4358.

10. US EPA Office of Research and Development, National Homeland Security Research Center, (2015). Systems Measures of Water Distribution System Resilience. Report No. EPA 600/R-14/383.

11. Roberson and Morley (2006). "Shifting to an All-Hazards Resiliency Approach." Journal of the American Water Works Association, 95(5):46-47.

12. Bloetscher et al. (2011). "Development of an adaptation toolbox to protect southeast Florida water supplies from climate change." Eniron. Rev. 19:397-417.

13. The Water Resources Dashboard, <http://toolkit.climate.gov/topics/waterresources/water-resources-dashboard> 


\section{Evolution of Hazard Assessment Modeling in Drinking Water Systems}

Royce A. Francis

Dept. of Engineering Management and Systems

Engineering

The George Washington University, Washington, DC

Email: seed@gwu.edu, Phone: 412-780-6453

\section{Overview of the Drinking Water System}




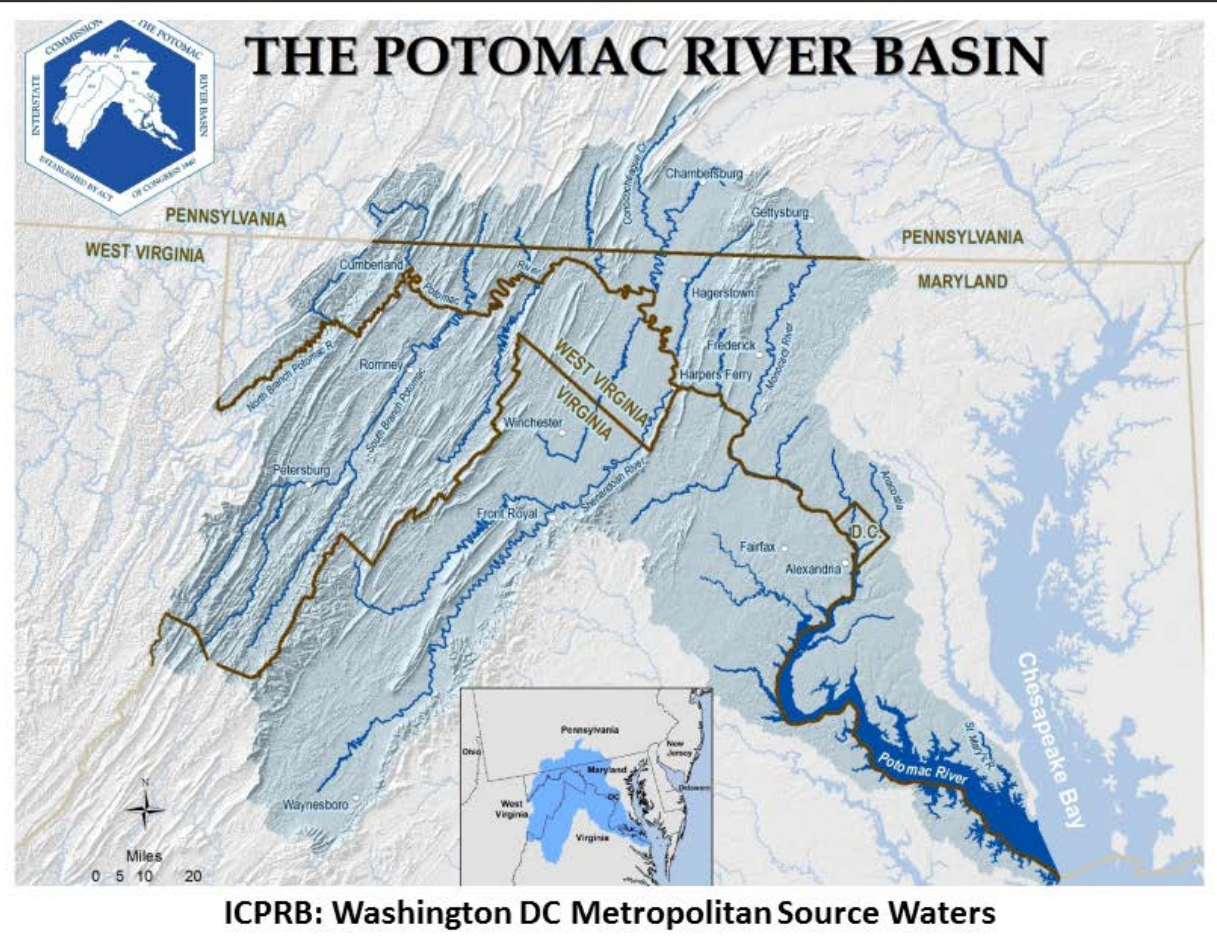

Authorized by an Act of Congress in 1940, the Interstate Commision on the Potomac River Basin is an advisory,
non-regulatory interstate compact agency of the Potomac basinstates of Maryland, Pennsylvania Virginia, West Virginia, and the District of Columbia The federalgovernment also is a member. The ICPRB was formed in response to extreme pollution levelsthat required aregional, cooperative response by all the jurisdictions. In
1970 , amendments to the compact empowered ICPRB to address not just pollution issues, but water resources and related land issues by two or more jurisdictions.

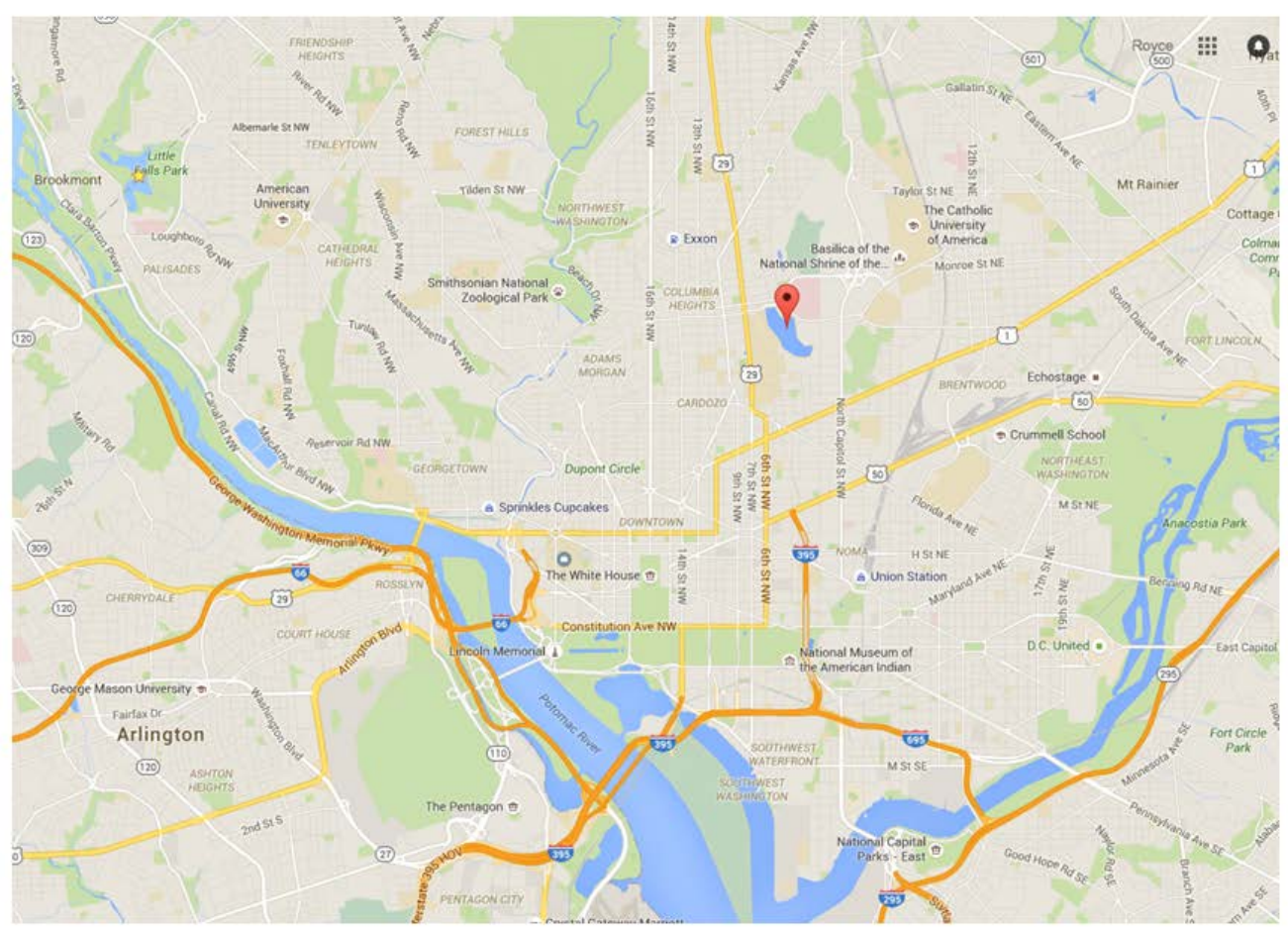

Location of Source Water Intake and Washington Aqueduct Reservoir and Treatment Facilities 


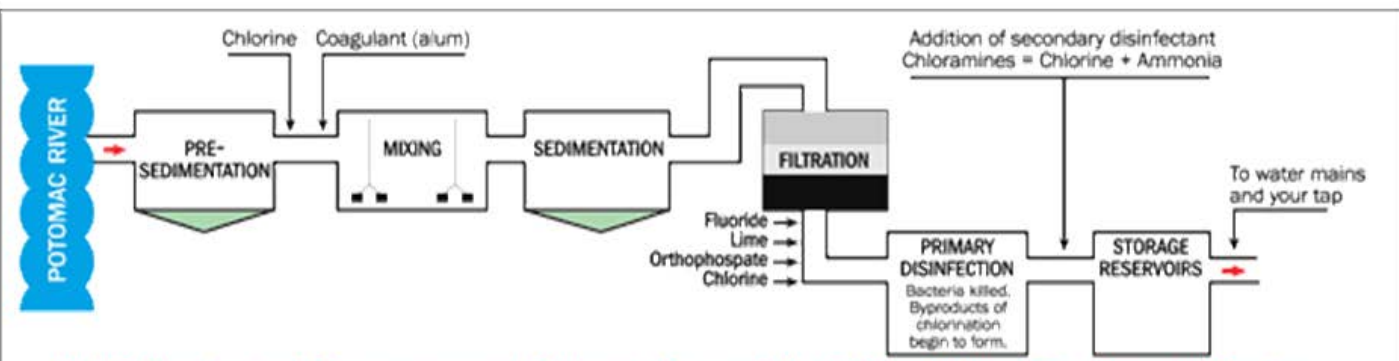

Main Treatment Processes at Dalecarlia and McMillan Water Treatment Plants

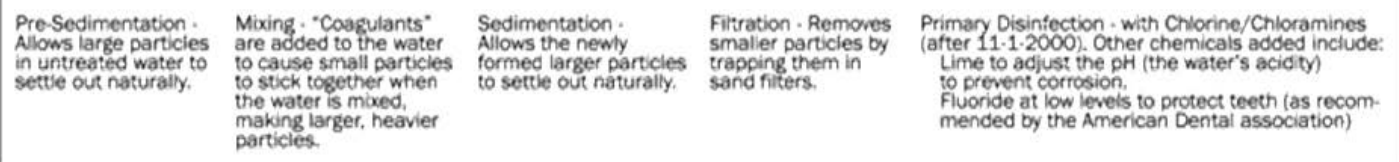

Water Treatment Example Process

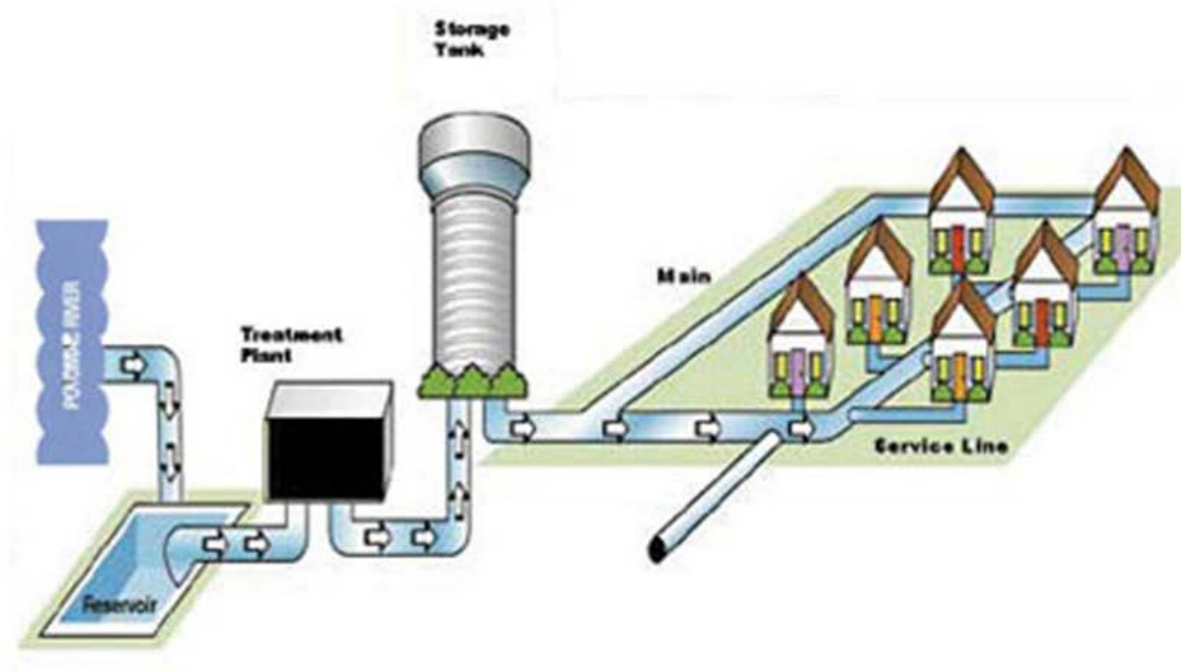

Water Distribution 


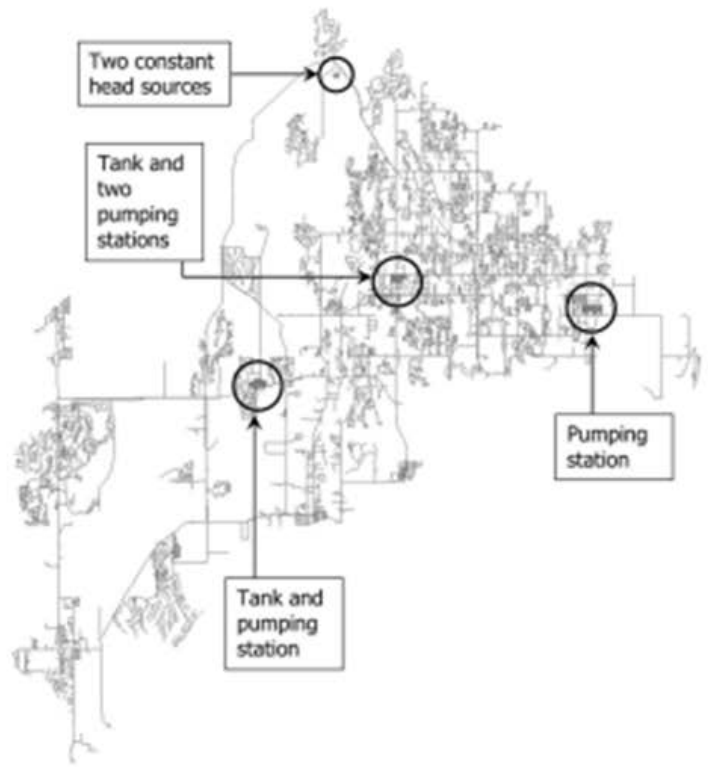

Fig. 2. Layout of Network 2 (12,523 nodes, 2 sources, 2 tanks, 14,822 pipes, 4 pumps, 5 valves)

Example Distribution System Illustrating Topology and Complexity of a Sample System

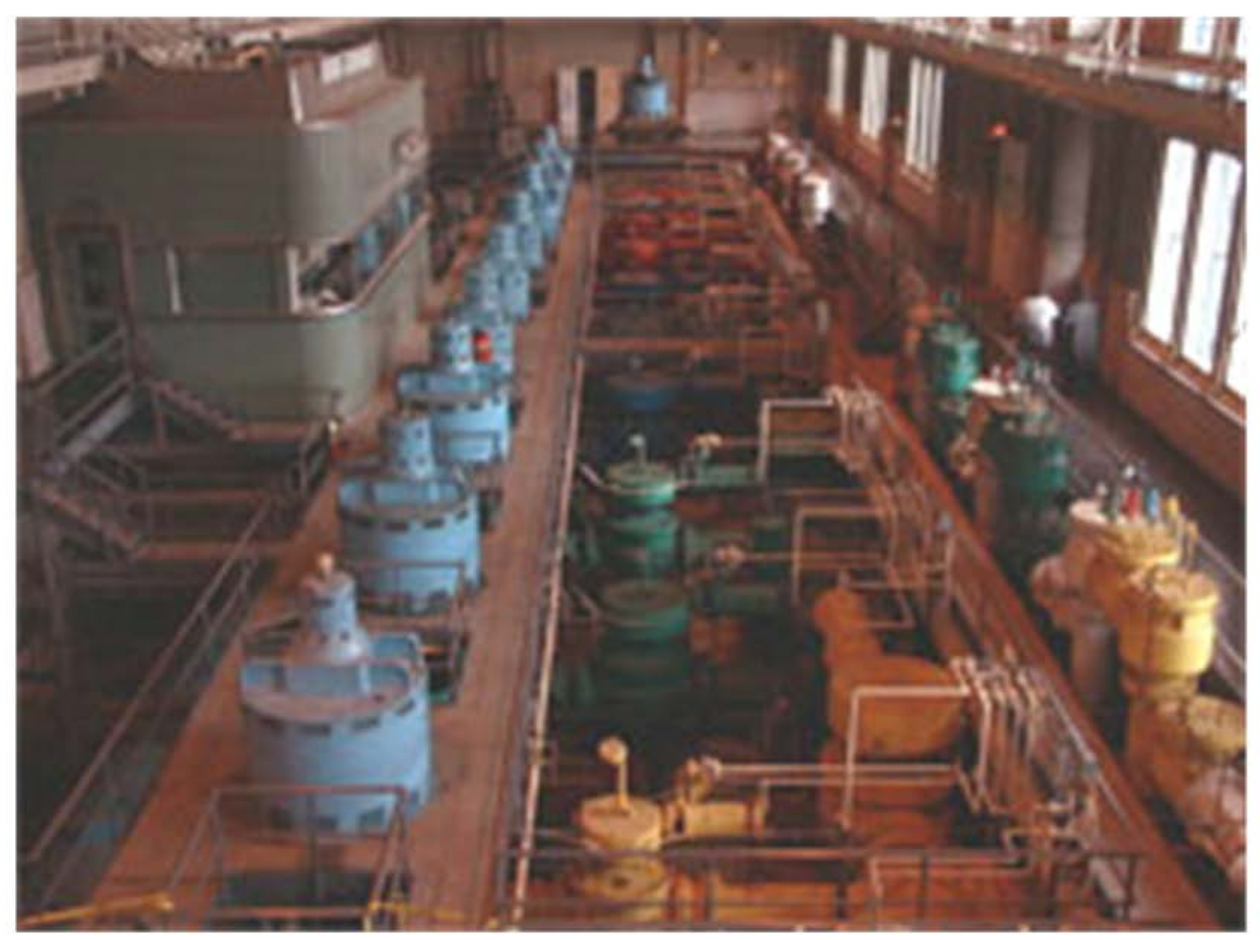

Pumping Stations 


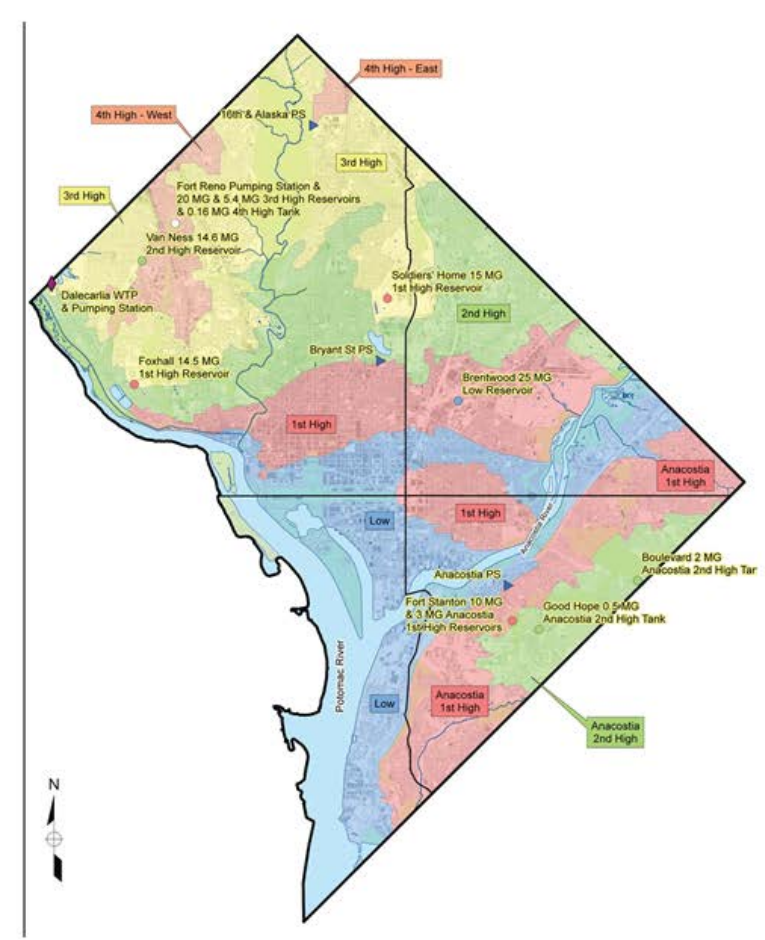

Washington DC Pressure Zones

DC is split into 7 pressure zones (yet, I can only count $5 . .$.

\section{Infrastructure Interdependence}

- There are 4 types of Interdependence:

- Physical: Water supply requires electric power for pumping

- Geographic: Water quality in the Potomac depends on land use and wastewater treatment practices in sub-watersheds

- Logical: EPA MS4 discharge permit requirements influences tech adopted in MD, VA, W. VA WWTPs

- Cyber: Water distribution and pumping is monitored via SCADA systems requiring information infrastructure

For more details, see: Rinaldi, S. M.; Peerenboom, J. P.; Kelly, T. K. Identifying, understanding, and analyzing critical infrastructure interdependencies. IEEE Control Systems Magazine 2001, 21, 11-25. 


\section{The Basis of Drinking Water Models is EPANET}

- EPANET ${ }^{1}$ is a software package used to model drinking water distribution system pipe networks written by Lew Rossman

- It is an extended-period hydraulic solver that can model:

- the flow of water in each pipe,

- the pressure at each node,

- the height of the water in each tank,

- the type of chemical concentration throughout the network during a simulation period,

- water age,

- source, and

- tracing

${ }^{1}$ Available at: https://www.epa.gov/water-research/epanet

\section{The Basis of Drinking Water Models is EPANET}

- EPANET is freely available as open-source ANSI compliant $\mathrm{C} / \mathrm{C}++$ code

- For this reason, EPANET is the engine behind many academic/research, commercial, and proprietary software used to study and design drinking water systems

- EPANET is extended by EPANET-MSX (multispecies extension) to model complex reactions (with more than one species) that may be important in distributed drinking water quality 
Hazard assessment in the

drinking water sector is

characterized by a transition in

its focus. Starting with a focus

on terrorism, the sector is now

embracing all-hazards risk and

vulnerability assessments.

\section{Objectives}

- After today's presentation, the participant will:

1. Observe key features of the rationale for vulnerability assessment from the Bioterrorism Act of 2002;

2. Consider the nature of models responsive to thse rationales;

3. Note the transition to all-hazards analysis using EPA and AWWA frameworks; and,

4. Consider two example assessment tools representing the expanding scope of the knowledge bases required for all-hazards assessment

NOTE: I do not review articles integrating qualitative or quantitative social science with hazard assessment models in the drinking water sector. 


\section{Risk and vulnerability analysis in the drinking water sector}

The drinking water sector focused intently during the period from ca. 2001-2010 on hazard and risk assessment for potential service disruptions attributable to intentional (i.e., terrorist) acts.

\section{Terrorism Provided Initial Impetus}

- The Bioterrorism Act of 2002, Title IV ${ }^{1}$ provided the initial impetus for securing drinking water systems post-9/11/2001 


\section{Bioterrorism Act Innovations}

- The Bioterrorism Act of 2002 required utilities to:

1. "... conduct an assessment of the vulnerability

of its system to a terrorist attack or other intentional acts intended to substantially disrupt the ability of the system to provide a safe and reliable supply of drinking water..."

\section{Bioterrorism Act Innovations}

- The Bioterrorism Act of 2002 required utilities to:

2. "... prepare or revise, where necessary, an emergency response plan that incorporates the results of vulnerability assessments that have been completed." 


\section{Bioterrorism Act Innovations}

- The Bioterrorism Act of 2002 required government partners to:

3. "... review (or enter into contracts or cooperative agreements to provide for a review of) current and future methods to prevent, detect and respond to the intentional introduction of chemical, biological or radiological contaminants community water systems and source water for community water systems..."

\section{Bioterrorism Act Innovations}

- The Bioterrorism Act of 2002 required government partners to:

4. "...shall review (or enter into contracts or cooperative agreements to provide for a review of) methods and means by which terrorists or other individuals or groups could disrupt the supply of safe drinking water or take other actions against water collection, pretreatment, treatment, storage and distribution facilities..." 


\section{Key Foci}

- A quick survey of key terms supporting these important advances include:

- Vulnerability assessment

- Terrorist attack

- Intentional acts

- Emergency response plan

- Prevent, detect, respond

- Intentional introduction of contaminants

- Supply disruption

- These focus on intentional human acts!

\section{Advances in Distribution System Monitoring Research}

- Distribution system modeling and monitoring research was responsive to these themes

- Drinking water distribution systems can have complex network topology.

- The complexity of the topology implies a very large number of potential attack scenarios

- The topological features of the network provide some clues for prioritizing critical attack scenarios 


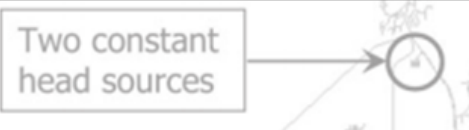

\section{Tank and \\ two \\ pumping \\ stations}

\section{Example: Battle of the} Water Sensor Networks ${ }^{2}$

The BWSN competition fused computer science, hydraulics, and environmental engineering to produce innovative approaches to characterizing the consequences of various attack scenarios.

${ }^{2}$ Consider: Ostfeld et al. (2008). "The Battle of the Water Sensor Networks (BWSN): A Design Challenge for Engineers and Algorithms." ASCE Journal of Water Resources Planning and Management, 134(6):556-568.

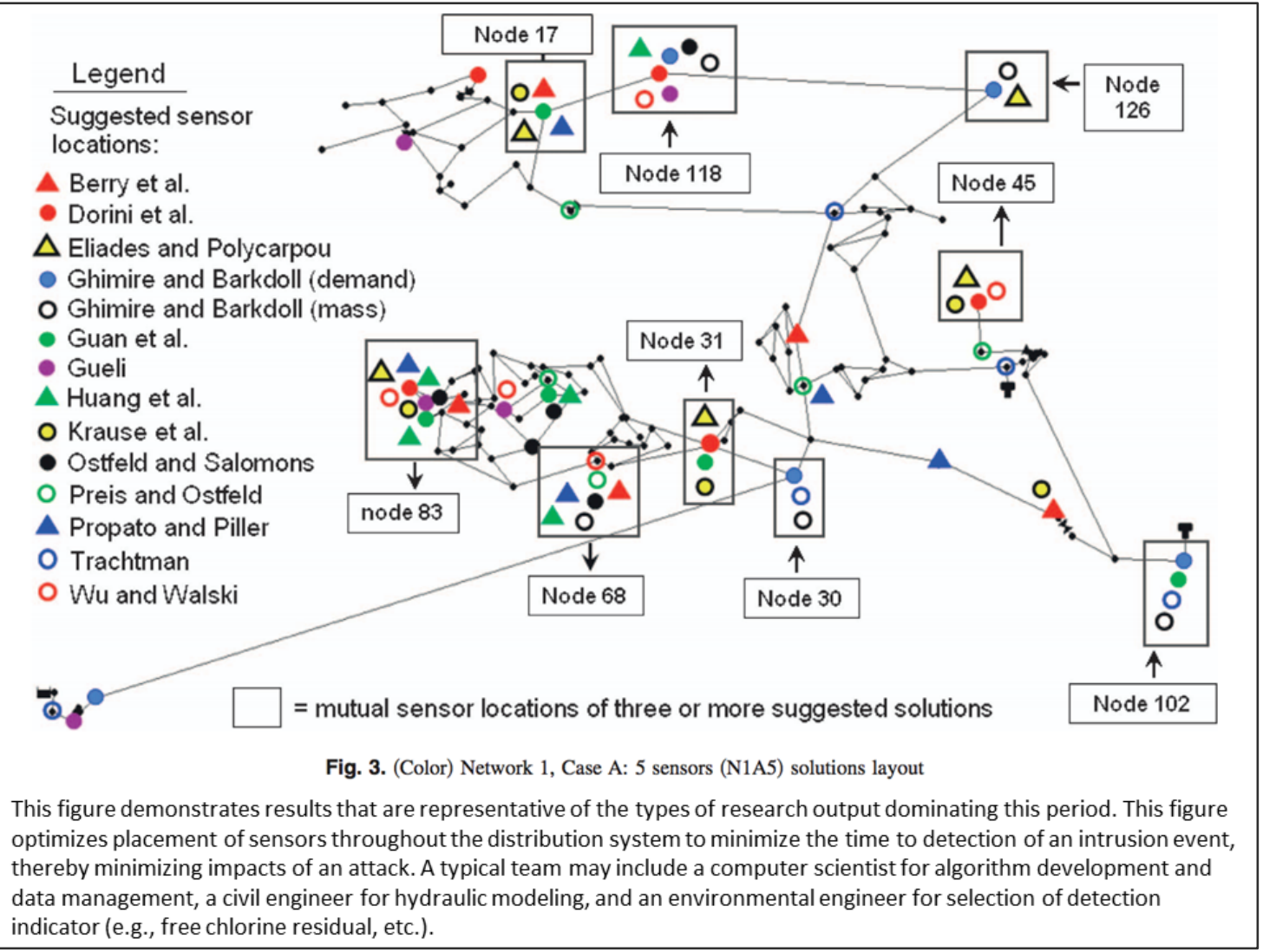




\section{Other Examples}

- Zechman [Berglund] and Ranjithan (2009). "Evolutionary Computation-Based Methods for Characterizing Contaminant Sources in a Water Distribution System," ASCE J. Water Resources Planning and Management, 135(5):334-343

- This paper uses evolutionary search algorithms to solve the inverse problem of identifying contaminant sources in a water distribution system. This is important in the need for algorithms used to process sensor data that could then aid in contaminant source characterization.

\section{Other Examples}

- J-P Watson, R. Murray, and W.E. Hart (2009). "Formulation and Optimization of Robust Sensor Placement Problems for Drinking Water Contamination Warning Systems." ASCE Journal of Infrastructure Systems, 15(4):330-339.

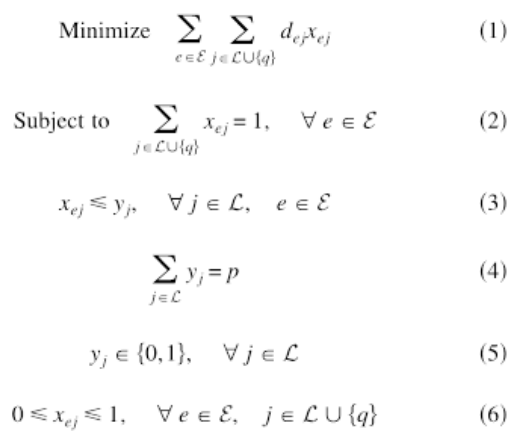

The objective of papers like this one is to optimize sensor placement so as to minimize the impact on the population served within the distribution system. 


\section{Representative Citations on Attack Detection Models}

- Krause, A., Leskovec, J., Guestrin, C., VanBriesen, J., \& Faloutsos, C. (2008). Efficient sensor placement optimization for securing large water distribution networks. Journal of Water Resources Planning and Management, 134(6), 516526.

- Leskovec, J., Krause, A., Guestrin, C., Faloutsos, C., VanBriesen, J., \& Glance, N. (2007, August). Cost-effective outbreak detection in networks. InProceedings of the 13th ACM SIGKDD international conference on Knowledge discovery and data mining (pp. 420-429). ACM.

- Preis, A., \& Ostfeld, A. (2008). Multiobjective contaminant sensor network design for water distribution systems. Journal of Water Resources Planning and Management, 134(4), 366-377.

- Hart, W. E., \& Murray, R. (2010). Review of sensor placement strategies for contamination warning systems in drinking water distribution systems.Journal of Water Resources Planning and Management, 136(6), 611-619.

- Isovitsch, S. L., \& VanBriesen, J. M. (2008). Sensor placement and optimization criteria dependencies in a water distribution system. Journal of Water Resources Planning and Management, 134(2), 186-196.

Morley (2013) reports that utilities with the need to update their vulnerability assessments did so in accordance with AWWA J100-10RAMCAP all-hazards guidance.

Transitioning to All-Hazards Analysis 


\section{All-Hazards vs. Terrorism Analysis}

- It has become apparent that natural hazards would be more of a threat than man-made hazards

- Hurricanes Katrina and Sandy demonstrate that the impacts of natural hazards may be more extensive and more severe

- Natural hazards can escalate impacts due to dependence of water systems on other systems

- Natural hazards activate considerable geographic interdependence among infrastructure systems

- Natural hazards can cause cascading failures via disruption in functionality of interdependent systems

\section{All-Hazards vs. Terrorism Analysis}

- The diversity inherent in all-hazards vulnerabilities will require system hardening that may also be effective against man-made attacks or hazards

- Hardening activities required to protect against man-made attacks may be less effective against natural hazards 


\section{VSAT, WHEAT, and RAMCAP}

- EPA and AWWA have developed tools to support all-hazards analyses for utilities updating their Bioterrorism Act vulnerability assessments

- EPA Vulnerability Self-Assessment Tool (VSAT)

- EPA Water, Health, and Economic Analysis Tool (WHEAT)

- AWWA J-100-10 RAMCAP

\section{VSAT, WHEAT, and RAMCAP}

- The AWWA J-100-10 standard requires a ranking of critical assets for prioritization of resilience activities.

- The main analytical steps are: asset characterization; threat characterization; consequence analysis; vulnerability analysis; threat analysis; risk/resilience analysis; and, risk/resilience management. 


\section{VSAT, WHEAT, and RAMCAP}

- EPA WHEAT and EPA VSAT are intended to be used in combination.

- The objective of WHEAT is to support the assessment of economic and health consequences of attacks, service disruptions, natural disasters, or otherwise adverse events by prioritizing assets; while,

- The objective of VSAT is to help utilities identify critical assets, threats, effective countermeasures, and costs in order to facilitate comprehensive vulnerability assessment.

\section{VSAT Main Steps}

1. Asset Identification

2. Countermeasure Evaluation

3. Threat Identification: Man-made or Natural

4. Baseline assessment: Utility information

5. Improvement assessment: Propose new countermeasures

6. Cost/Risk evaluations

7. Summaries and Reports 


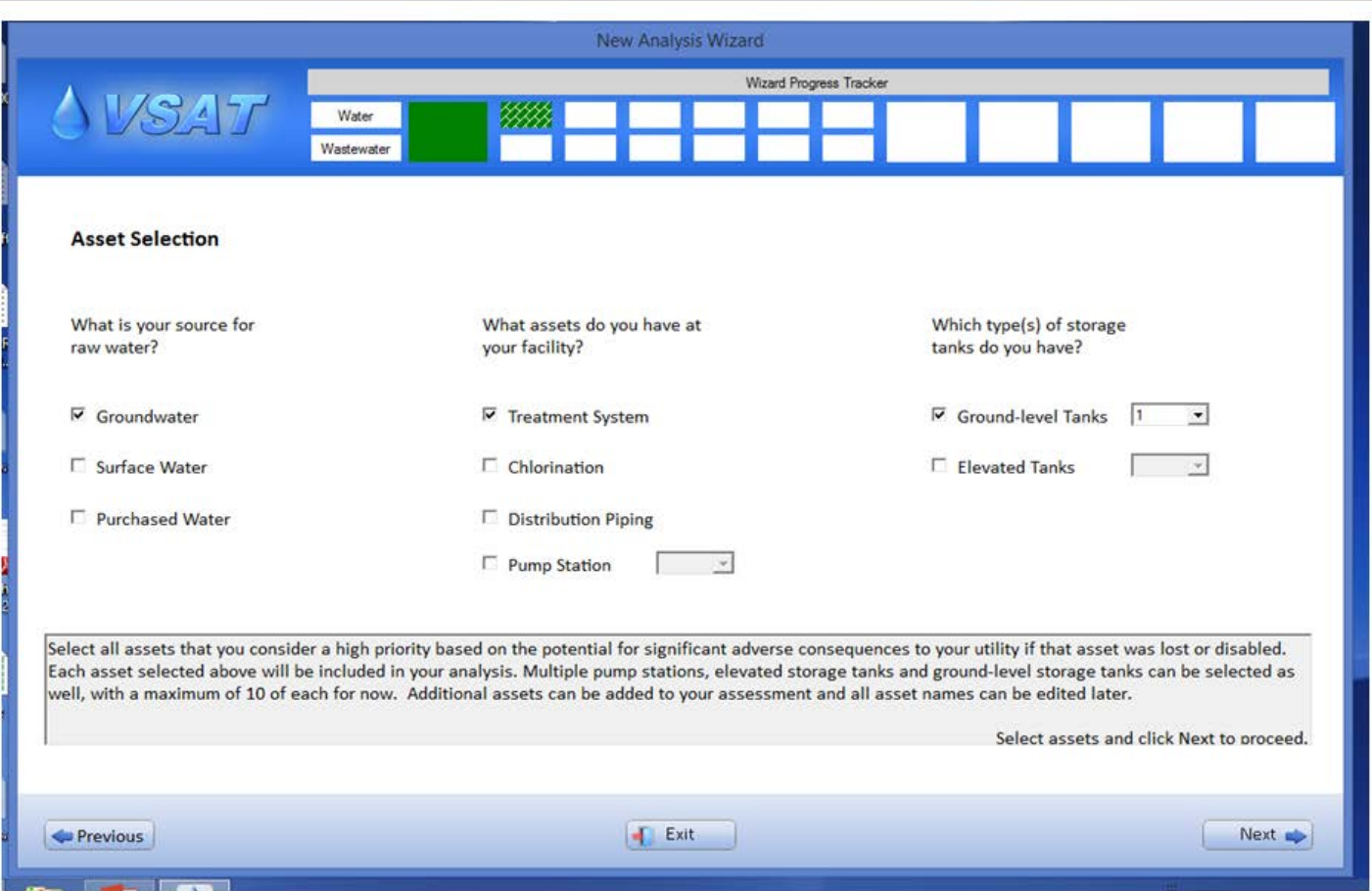

\section{WHEAT Main Steps}

1. System and Scenario Selection

2. Baseline Inputs: Asset condition

3. Scenario Inputs and Service Loss and Response

4. Contamination Event Consequences

5. Public Health Consequences

6. Utility-Level and Regional Economic Consequences

7. Downstream Impacts

8. Summary Reports 
System and Scenario Selection

The choice of system tpe and scenario tppe should be based on an assessment of how your chosen scenario is expected to impact your utility. If

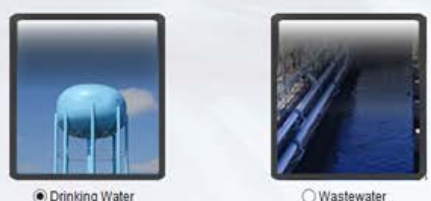

- Drinking Water

OWastewater

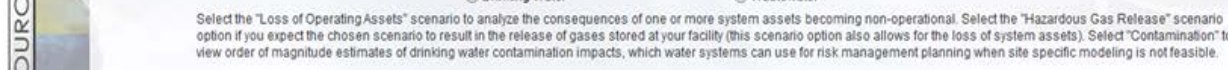

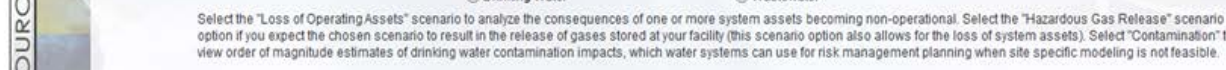

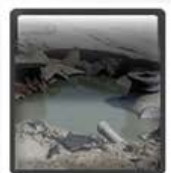

OLoss oftassets

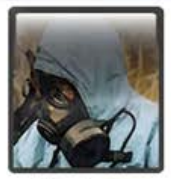

Hazardous Gas

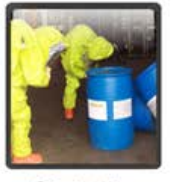

Ocontamination

Asset Name and Type

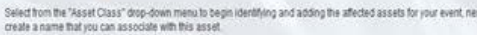

kssername

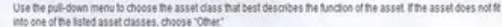

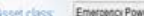

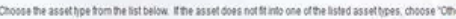

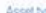

Geneabs:

Asset Condition

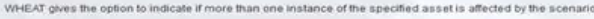

Enter how many of these assets are non-operationat.

Choose the fendivon of the acoer anter the ereren

- Nam-operationat but undamaged 


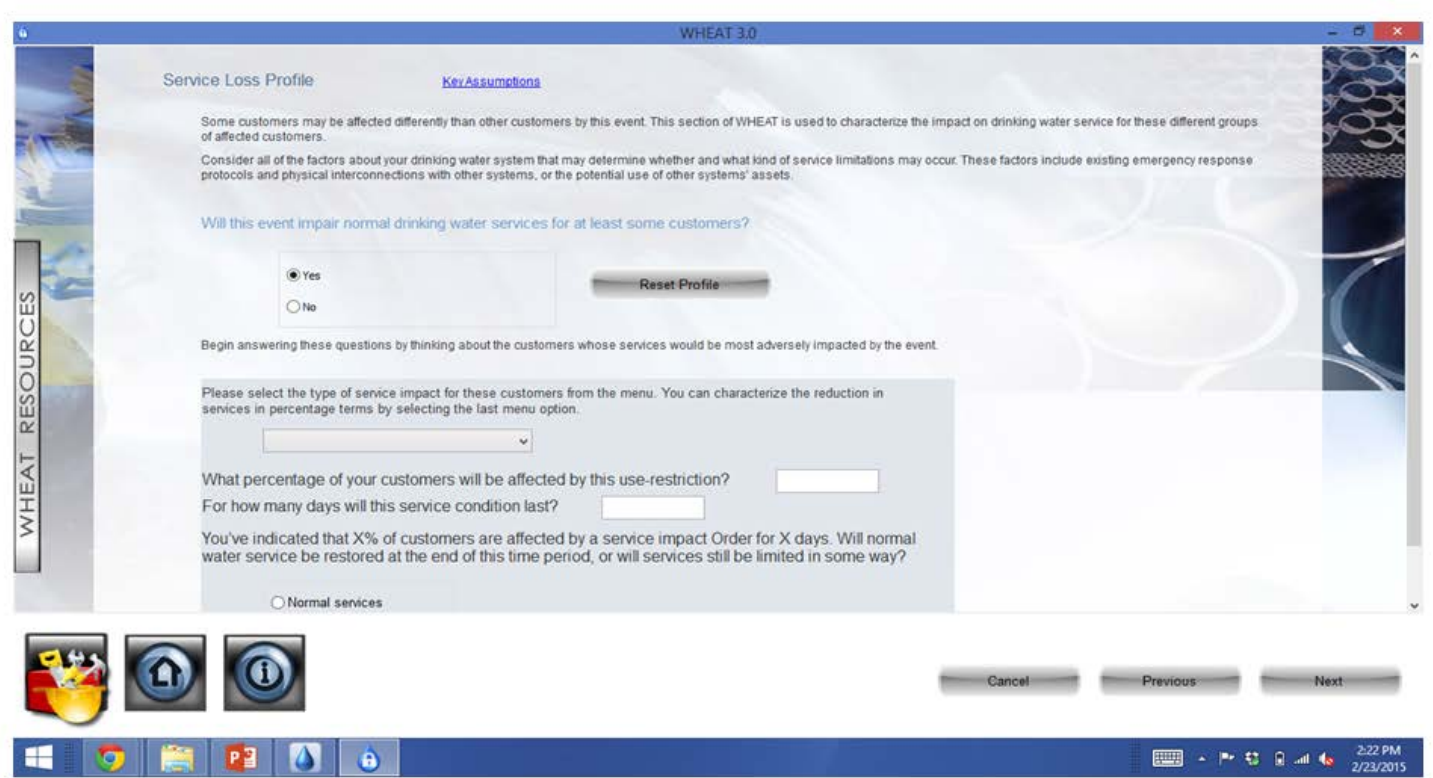

\section{VSAT, WHEAT, and RAMCAP}

VSAT is used to identify threatasset pairs to prioritize assets and vulnerabilities.

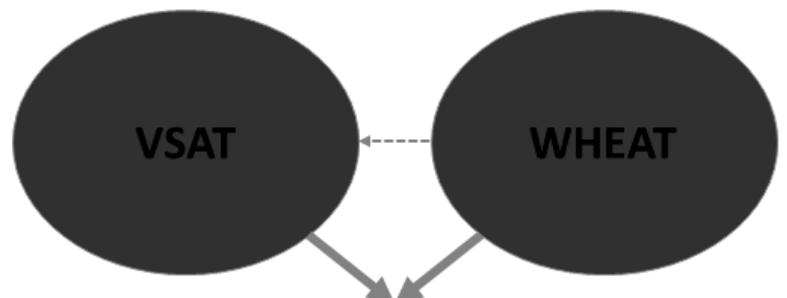

WHEAT is used to characterize consequences. WHEAT results may be provided as inputs to VSAT.

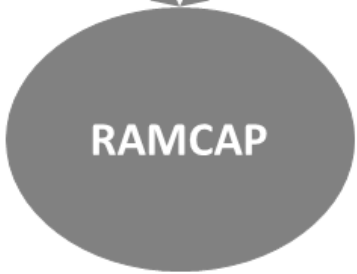

RAMCAP integrates WHEAT and VSAT under a standardized assessment framework. 


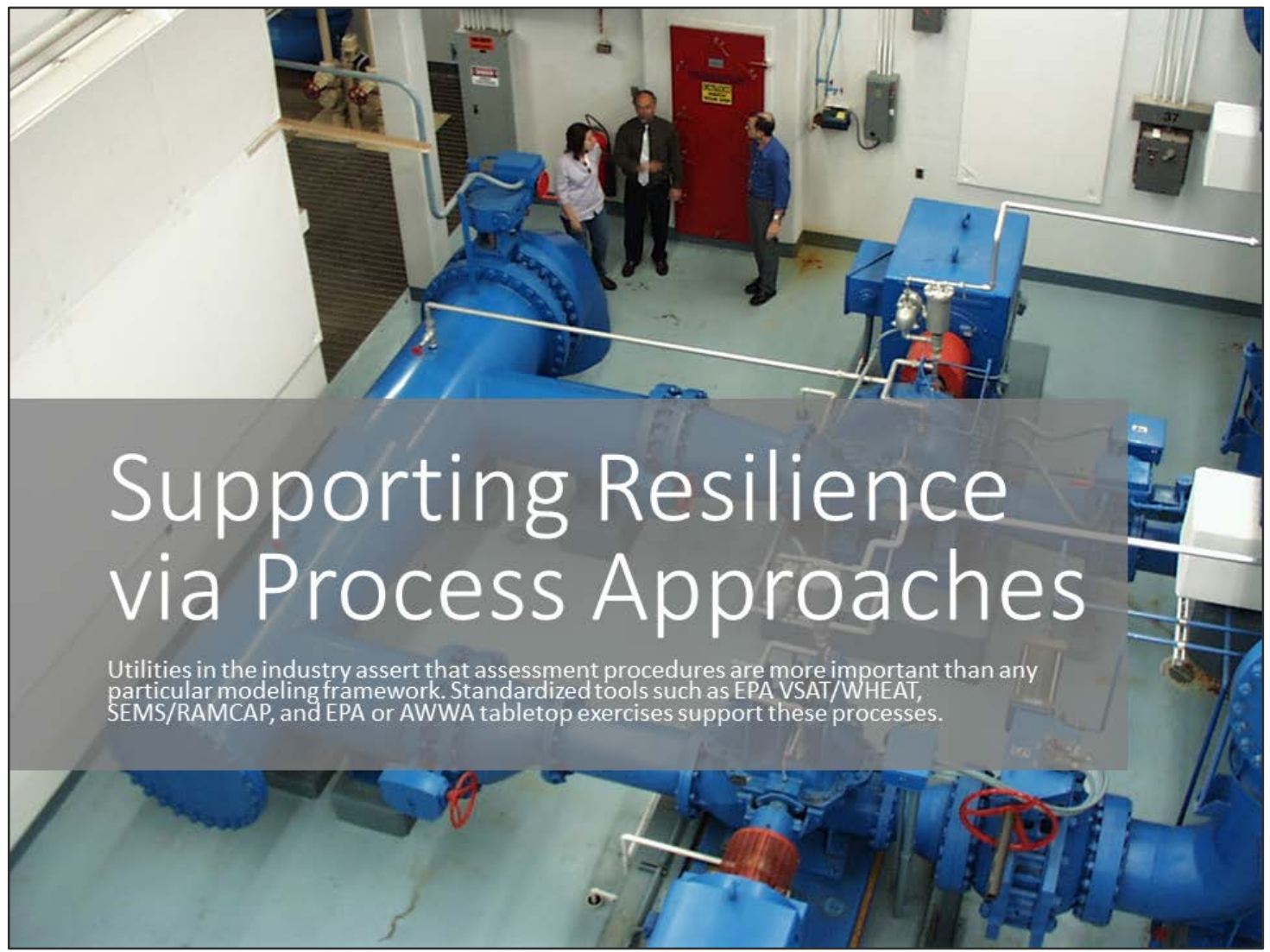

\section{The Value of Process-Based Approaches}

- At this stage, professionals in the water sector acknowledge the primacy of analytical process in resilience planning

- The assessment process may uncover important interdependence with other systems (e.g., emergency response becomes familiar with components of water systems)

- A process-oriented approach, rather than quantificationoriented approach, may give participants confidence that the analysis is responsive to the unique features of their system

- Drinking water systems may have sufficient storage and distribution system treatment capabilities that allow for slight departures from normal water quality. This means that collaboration with other infrastructure managers and longterm planning needs can be prioritized. 


\section{Morley (2011) Utility Resilience Indicator Reflects Process Focus}

- The URI, developed by Morley (2011), involves the development of a methodology that concisely and simply communicates a utility's readiness and resilience to disruptions attributable to all-hazards

- The URI operational indicators are:

1. emergency response plan readiness level;

2. national incident management system (NIMS) compliance;

3. mutual aid and assistance (e.g., interstate WARN agreement);

4. emergency power availability for critical operations;

5. ability to meet minimum daily demand (water) or treatment requirements (wastewater);

6. lead time for critical parts and equipment repair (shorter is better); and,

7. percentage of response-capable critical staff available for operations and maintenance with cross-trained staff backup.

\section{Morley (2011) Utility Resilience Indicator Reflects Process Focus}

- The URI, developed by Morley (2011), involves the development of a methodology that concisely and simply communicates a utility's readiness and resilience to disruptions attributable to all-hazards

- The URI financial indicators are:

1. business continuity planning exercised;

2. utility bond rating;

3. condition assessment coverage;

4. community unemployment (i.e., community unemployment is a proxy measure for utility financial stability); and

5. community median household income (i.e., another proxy for financial stability). 


\section{Representative Citations on All- Hazards Vulnerability Assessment}

- US EPA Office of Water, US EPA Office of Ground Water, and US EPA Office of Drinking Water, "Conduct a Drinking Water or Wastewater Utility Risk Assessment," 2015. [Online]. https://www.epa.gov/waterriskassessment/conduct-drinking-water-orwastewater-utility-risk-assessment. [Accessed: 07-Jul-2016].

- US EPA Office of Water, US EPA Office of Ground Water, and US EPA Office of Drinking Water, "Water Health and Economic Analysis Tool (WHEAT) 3.0," 2016. [Online]. Available: https://www.epa.gov/waterriskassessment/find-out-abouthealth-and-economic-impacts-water-utility-emergencies. [Accessed: 07-Jul2016].

- S. D. Spence and C. M. Tuozzoli, (2011). Gap Assessment for ASME-ITI/AWWA J100-10 Standard and Leading Vulnerability Assessment Tools, Water Research Foundation Web Report No. 4358.

- US EPA Office of Research and Development, National Homeland Security Research Center, (2015). Systems Measures of Water Distribution System Resilience. Report No. EPA 600/R-14/383.

- Roberson and Morley (2006). "Shifting to an All-Hazards Resiliency Approach." Journal of the American Water Works Association, 95(5):46-47.

\section{Conclusions}

- Hazard assessment modeling in drinking water systems will continue to evolve and become more inter-disciplinary.

- Drinking water systems will rely on a much broader knowledge base to remain resilient; and,

- Drinking water systems will continue to develop tools that have a wide scope of application to multiple decision contexts. 


\section{Example 1: Development of Adaptation Toolbox for SE Florida Water Supplies ${ }^{3}$}

South Florida Water Management District (SFWMD) evaluated plans to adapt to sea level rise (SLR), while at the same time considering options avallable to reverse adverse impacts of Everglades drainage on the native ecosystem and Biscayne aquifer. The toolbox includes a set of adaptation tools (e.g., pumping stations, water conservation, aquifer recharge, etc.) and fundamental empirical and physical data including a land-use change model, hydrological model of surface flows, temperature and SLR projections under climate change.

${ }^{3}$ Bloetscher et al. (2011). "Development of an adaptation toolbox to protect southeast Florida water supplies from climate change." Eniron. Rev. 19:397-417.

\section{Example 2: The Water Resources Dashboard ${ }^{4}$}

From NOAA: "Water resource managers, city planners, and the general public are witnessing changes in the climate, as well as associated impacts to our environment. To better plan for the future, the American Planning Association, American Water Works Association, Association of Metropolitan Water Agencies, National Oceanic and Atmospheric Administration, Water Environment Federation, Water Environment and Reuse Foundation, and the Water Resource Foundation collaborated to create a water resources dashboard - a one-stop location for water-relevant [climate] data sets."

${ }^{4}$ The Water Resources Dashboard, <http://toolkit.climate.gov/topics/water-resources/water-resourcesdashboard> 


\section{Royce Francis}

\section{Background}

Dr. Royce Francis leads the SEED research group-Strategic [urban] Ecologies, Engineering, and Decision-making. Currently, SEED is focusing on decision-analytic sustainability measurement in infrastructure systems, risk-based management of drinking water infrastructure rehabilitation and renewal, and integration of decision modeling with life cycle cost assessment methodologies for evaluating vulnerability of infrastructure systems to natural hazards. Dr. Francis is a member of the Association for Environmental Engineering and Science Professors, the American Society of Civil Engineers, and the Society for Risk Analysis where he serves as the 2012 Engineering Infrastructure Specialty Group (EISG) Chair.

\section{Current Research}

Dr. Royce Francis' research and professional interests are at the interface between environmental and sustainability engineering and policy, including:

- $\quad$ Risk and occurrence assessment of emerging contaminants;

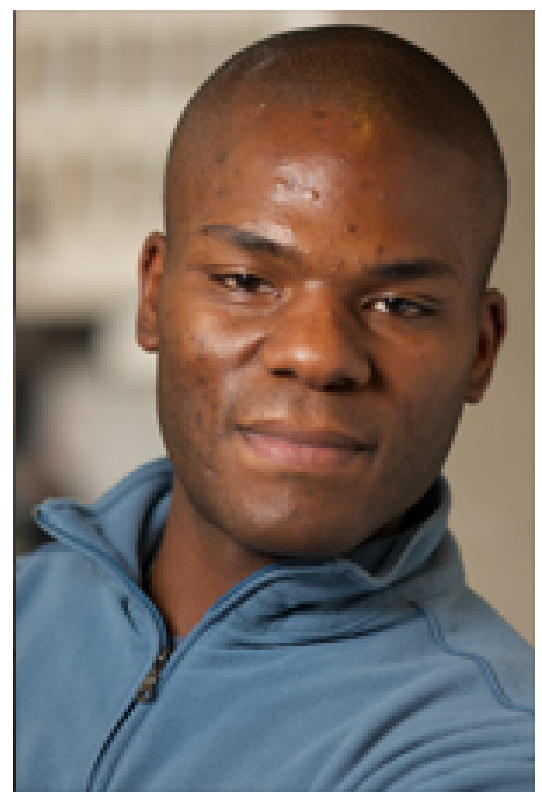

- $\quad$ Bayesian statistical modeling;

- Water infrastructure development, resilience, and sustainability

- Infrastructure systems analysis;

- Regulatory risk analysis

\section{Education}

- Postdoctoral Fellow: Department of Geography and Environmental Engineering, The Johns Hopkins University, 2009-2010

- $\quad$ Ph.D., Department of Engineering and Public Policy, Carnegie Mellon University, 2009 Department of Civil and Environmental Engineering, Carnegie Mellon University. 2009 Dissertation Title: Topics in Occurrence and Mixture Risk Assessment Supporting Formulation and Analysis of the Stage II Residual Disinfectants/Disinfection Byproducts (D/DBP) Rule.

- $\quad$ M.S., Civil and Environmental Engineering, Carnegie Mellon University, 2006

- $\quad$ B.S., Civil Engineering, Howard University, summa cum laude, 2004

\section{Publications}

\section{Journal Articles}

- $\quad$ Francis, R.A., Geedipally, S.R., Guikema, S.D., Lord, D., Dhavala, S.S., LaRocca, S. (2012). "Characterizing the performance of the Conway-Maxwell Generalized Linear Model." Risk Analysis. 32:167-183.

- $\quad$ Francis, R.A., Falconi, S., Nateghi, R., Guikema, S.D. (2011) "Probabilistic life cycle analysis model for evaluating electric power infrastructure risk mitigation investments." Climatic Change. 106:31-55.

- $\quad$ Francis, R.A., VanBriesen, J.M., Small, M.J. (2010). "Bayesian statistical modeling of disinfection byproduct (DBP) bromine incorporation in the Information Collection Rule (ICR) database." Environmental Science and Technology. 44:1232-1239. 
- $\quad$ Francis, R.A., Small, M.J., VanBriesen, J.M. (2009). "Multivariate Distributions of Disinfection Byproducts in Drinking Water." Water Research. 43:3453-3468.

Peer-Reviewed Conference Papers

- $\quad$ Pita, G., Francis, R., Liu, Z., Mitrani-Reiser, J., Guikema, S., Pinelli, J-P. "Statistical tools for populating/predicting input data of risk analysis models." ICVRAM 2011, College Park, MD.

- $\quad$ Francis, R., Guikema, S., and Henneman, L. "Bayesian belief networks for predicting drinking water distribution system pipe breaks." PSAM11/ESREL12, Helsinki, Finland.

\section{Distinctions}

- $\quad$ Assistant Professor: Department of Engineering Management and Systems Engineering, The George Washington University, September 2010-Present

- Visiting Scholar: Department of Geography and Environmental Engineering, The Johns Hopkins University, 20102011

- $\quad$ American Society of Civil Engineers (Assoc. M. ASCE)

- $\quad$ ASCE Environment and Water Resources Institute (EWRI)

- $\quad$ Society for Risk Analysis (SRA)

- $\quad$ Association for Environmental Engineering and Science Professors (AEESP) 


\section{Modeling Water Distribution System Resilience}

Water utilities are vulnerable to a wide variety of human-caused and natural disasters. These disruptive events can result in loss of water service, contaminated water, pipe breaks, and failed equipment.

Furthermore, long term changes in water supply and customer demand can have a large impact on the operating conditions of the network. The ability to maintain drinking water service during and following these types of events is critical. Simulation and analysis tools can help water utilities explore how their network will respond to disruptive events and plan effective mitigation strategies.

Sandia National Laboratories and the U.S. Environmental Protection Agency are developing new software tools to meet this need. The Water Network Tool for Resilience (WNTR) is a Python package designed to help water utilities investigate resilience of water distribution systems over a wide range of hazardous scenarios and to evaluate resilience-enhancing actions. This presentation will outline the modeling components in WNTR, demonstrate their use, and show how WNTR can help water utilities estimate potential damage, evaluate preparedness, prioritize repair strategies, and identify worse case scenarios. 


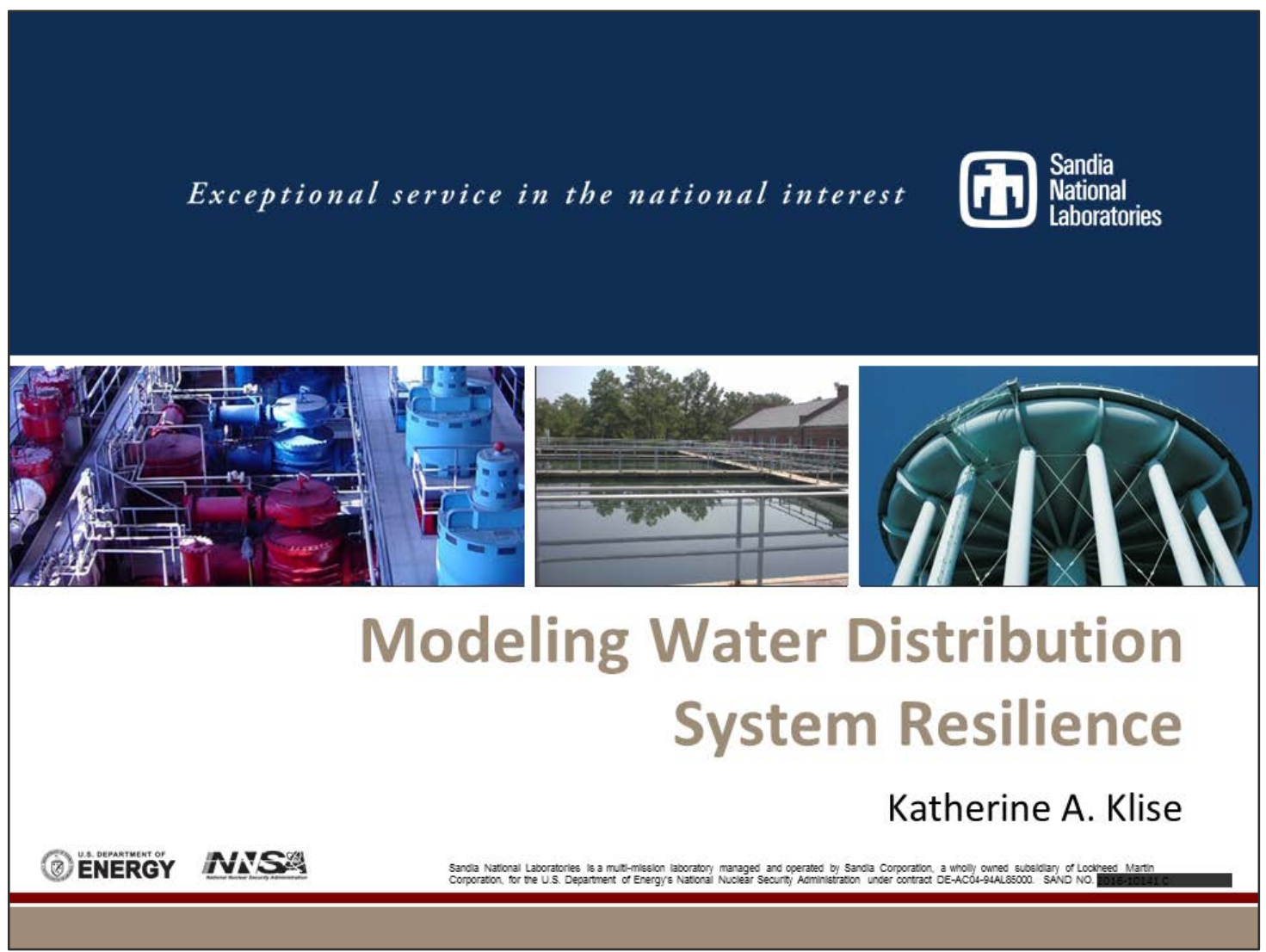

\section{Acknowledgements}

- This research was funded by the US Environmental Protection Agency

- US Environmental Protection Agency

- Regan Murray

- Terra Haxton

- Purdue University

- Michael Bynum

- Santiago Rodriguez

- Stephen Frechette

- Sandia National Laboratories

- Dylan Moriarty

- David Hart

- Carl Laird 


\section{Motivation and Background}

- Water distribution systems face multiple challenges

- The goal of a resilient system is to minimize the magnitude and duration of disruption

- Resilience of drinking water systems is influenced by

- Design

- Maintenance

- Operations

- Interdependence with other

\begin{tabular}{|l|}
\hline \multicolumn{1}{|c|}{ Potential Hazards } \\
Natural Disasters \\
- Drought \\
- Earthquakes \\
- Floods \\
- Turricanes \\
- Tornados \\
- Wunamis \\
- Wildfires \\
Terrorist Attacks \\
Cyber Attacks \\
Hazardous Materials Release \\
Climate Change \\
\end{tabular}

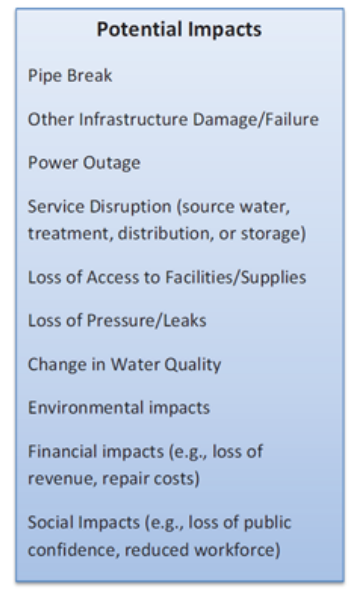
infrastructure

\section{Motivation and Background}

- Important questions to consider:

- What kind of infrastructure damage would be expected after a disruptive event?

- How long would the system continue to provide water to customers?

- Which customers are impacted the most?

- What kind of restoration actions would be helpful?

- How should restoration actions be prioritized?

- What can utilities do to prepare?

- Simulation and analysis tools can help water utilities explore how their network will respond to expected, and unexpected, events

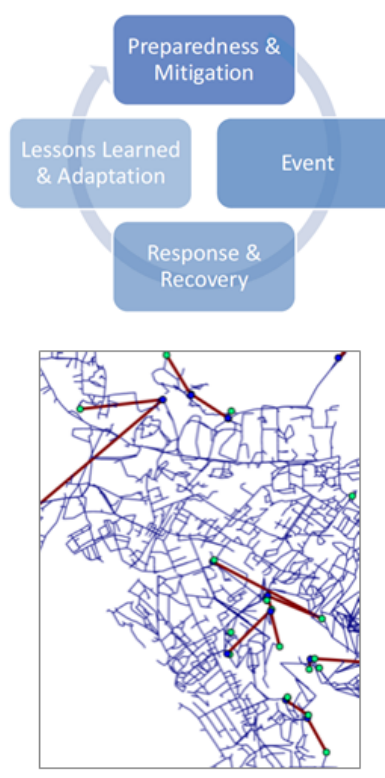




\section{Quantifying Resilience}

- Numerous metrics have been suggested to quantify reliability, robustness, redundancy, and security for water distribution networks

- Topographic metrics

- Hydraulic metrics

- Water quality metrics

- Economic metrics

- State transition plots graphically represent the meaning of resilience

- System performance function, event, and resilience action must be clearly defined

- Resilience is typically defined as a system measure, but could be measured for individual components of the network

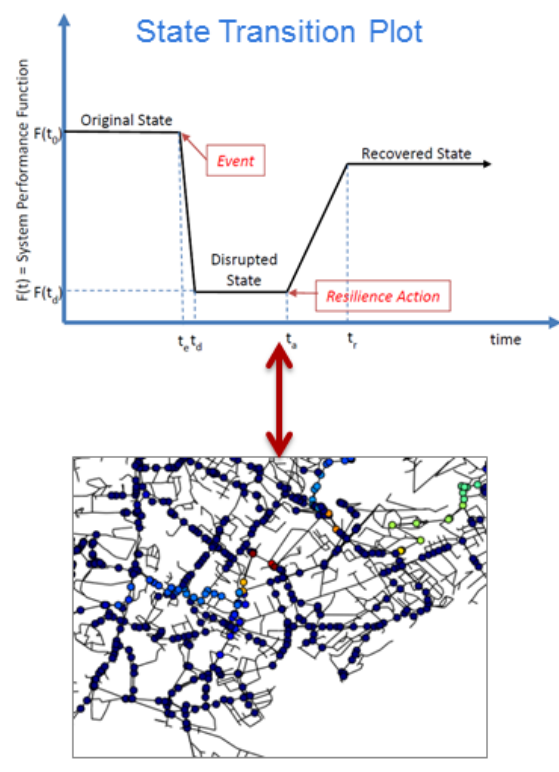

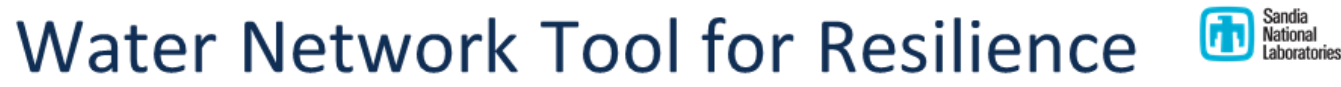

- Motivation

- Provide a single software framework for modeling disruptive incidents and repair strategies

- Intended users

- Researchers, consultants, water utilities

- Organizers of table top exercises

\section{- Software capabilities}

- Simulate disaster scenarios and damage to a specific water distribution system over time

- Measure quantitative resilience indicators

- Evaluate the benefit of utility response actions

- Evaluate improvements in resilience due to changes in system operation or design
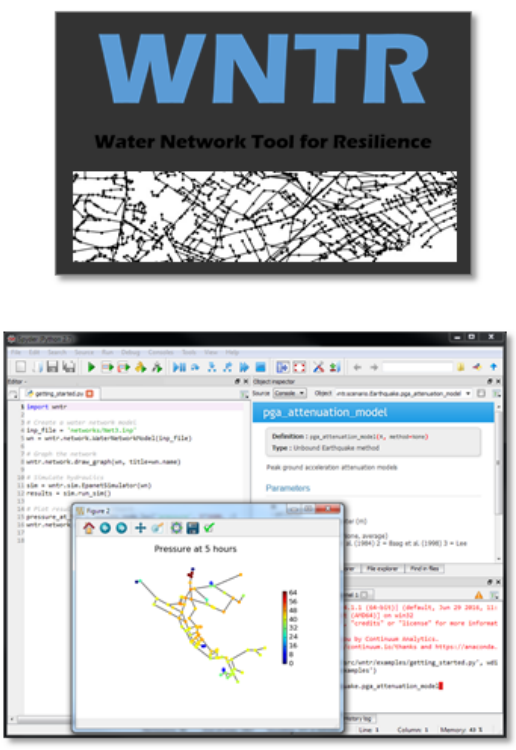


\section{WNTR Modeling Framework}

EPANET (DD) and PDD

hydraulic simulation

Water quality simulation

Pipe leak models

Stop/restart capabilities

Multiprocessing to run in parallel

Numpy and Scipy are used for efficient numerical computation

Pandas dataframes are used to store time-series simulation results

Topographic, hydraulic, water quality, and economic metrics

- Time-based and system wide metrics

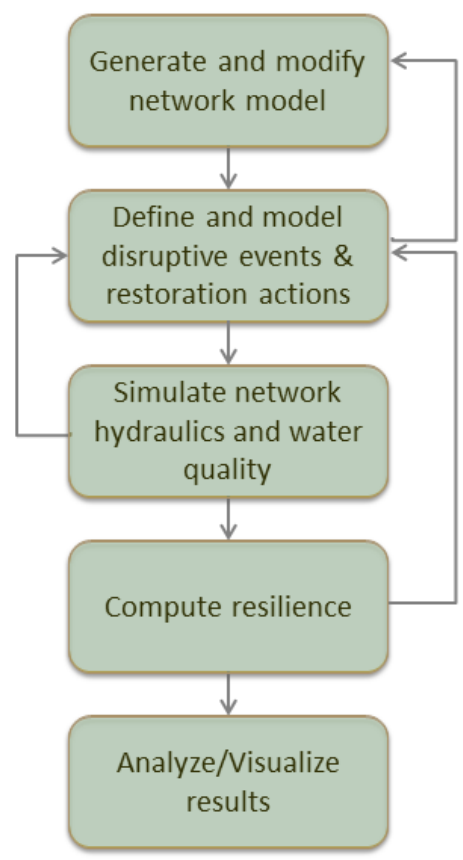

- Compatible with EPANET network model files

NetworkX graphs are used to analyze network structure

Deterministic or probabilistic events

- Fragility curves and damage models

Matplotlib is used to produce high-quality graphics, including network graphics and animation

\section{Network Models}

- Parse and write EPANET network model files

- Add/remove network components and controls

- Add/remove leaks

- Modify and query node/link attributes

- Plot node/link attributes on the network

- Analyze network structure using NetworkX

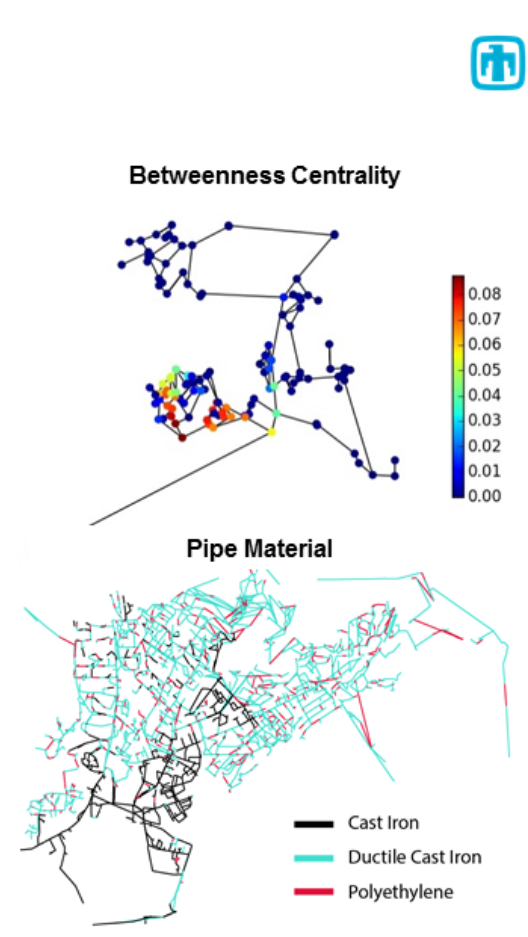




\section{Disruptive Incidents}

- Define disruptive incidents

- Type and consequence

- Informed by data or a model

- Define probability of damage

- Fragility and survival curves

- Modify the network

- Controls, demands, components, attributes to match each scenario
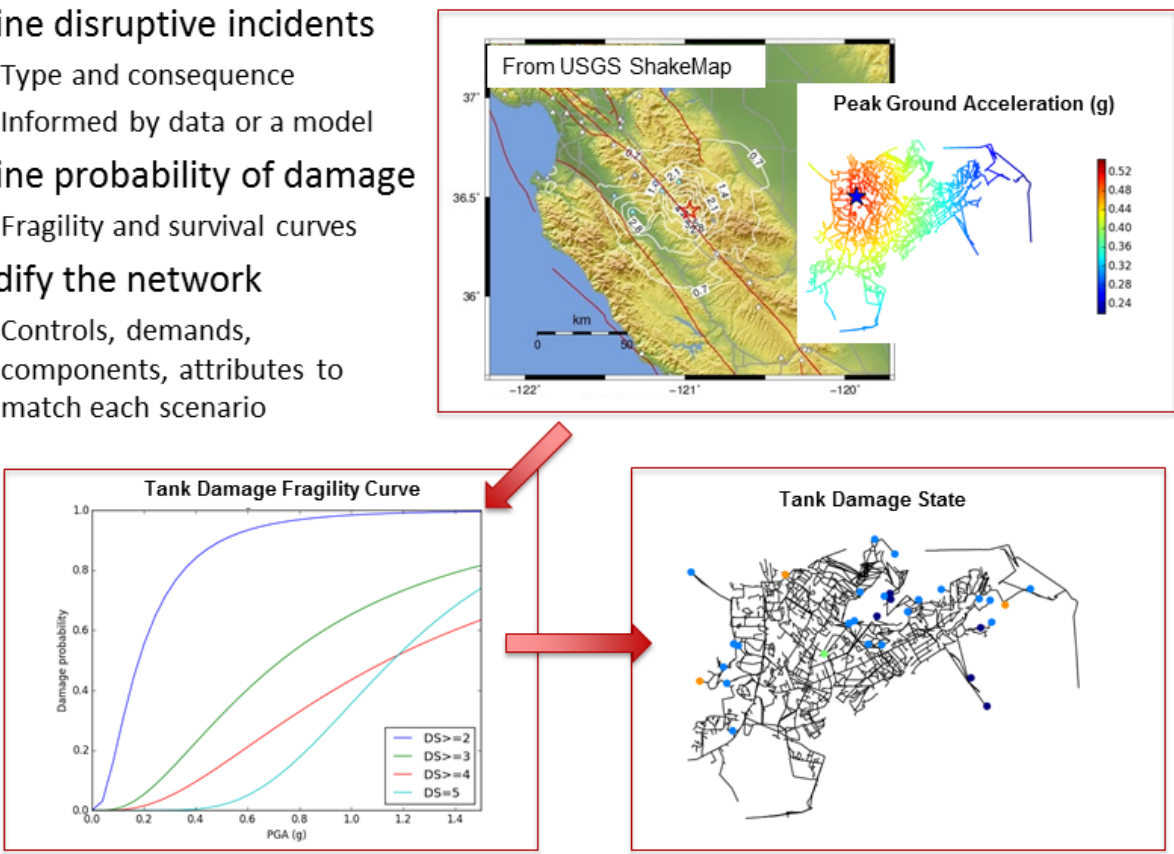

\section{Disruptive Incidents}

- Define disruptive incidents

- Type and consequence

- Informed by data or a model

- Define probability of damage

- Fragility and survival curves

- Modify the network

- Controls, demands, components, attributes to match each scenario
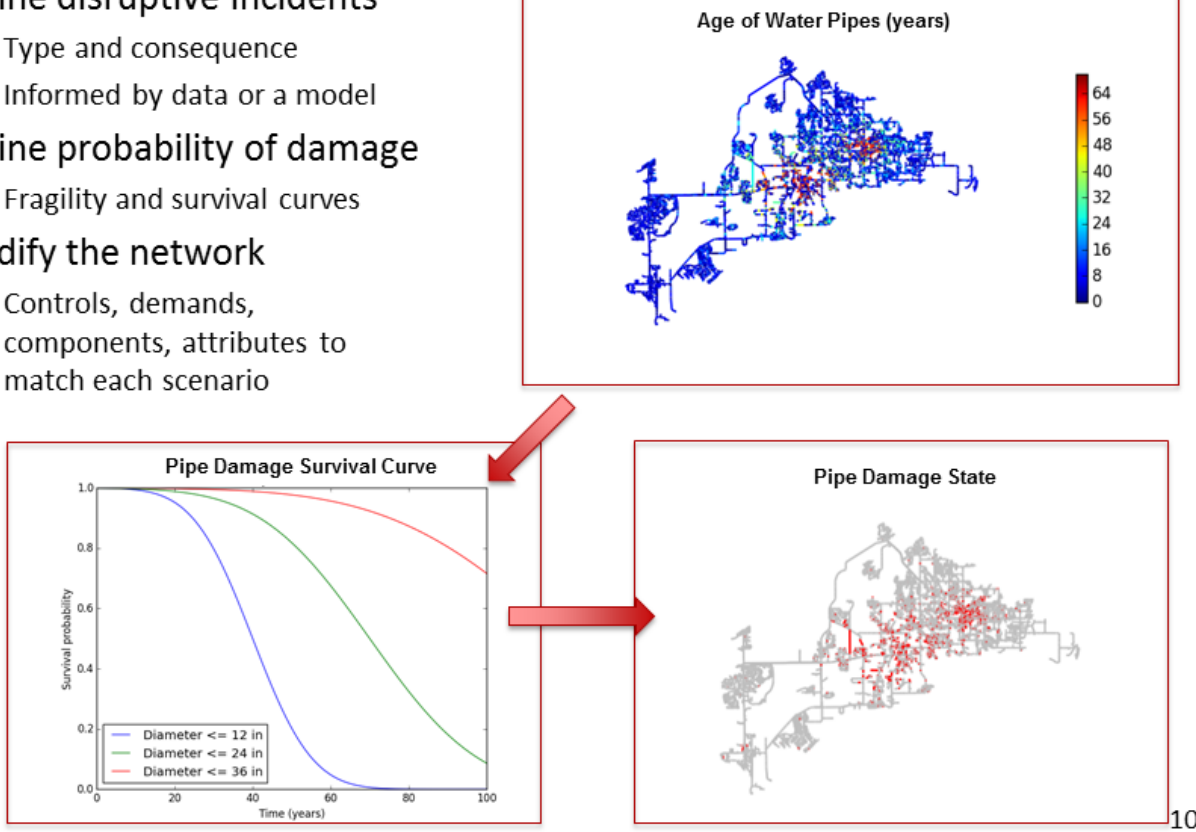


\section{Restoration Actions}

- Define the restoration action

- Type of repair actions

- Number of crews

- Time to repair

- Firefighting capacity

- Define priorities

- e.g., distance from source, magnitude of leak, number of people affected

- Modify the network

- Controls, demands, components, attributes to Repair Strategy Following Napa Valley Earthquake Number of repair crews -5

Repairs per day - 5 (120 breaksfixed in 5 days)

Repairs started 24 hours after earthquake

Separate team repaired tank

Prioritized repairs by proximity to limit travel time

Production maximized to feed leaks

Boil water order for affected regions match each scenario

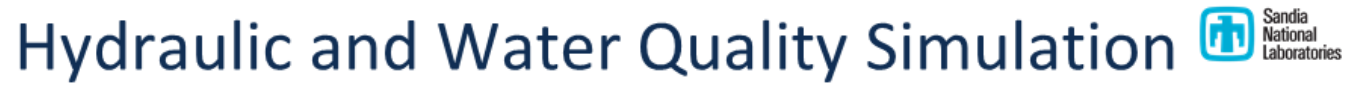

- Demand-driven hydraulic simulation and water quality simulation using EPANET

- Pressure driven-demand hydraulic simulation

- Demand at a node, $d$, depends on the pressure, $p$, available at the node

- Input parameters $=$ nominal pressure $\left(P_{f}\right)$ and minimum pressure $\left(P_{0}\right)$

$$
\begin{array}{ll}
d=D_{f} & \text { for } p \geq P_{f} \\
d=D_{f}\left(\frac{p-P_{o}}{P_{f}-P_{o}}\right)^{1 / e} & \text { for } P_{o}<p<P_{f} \\
d=0 & \text { for } p \leq P_{o}
\end{array}
$$

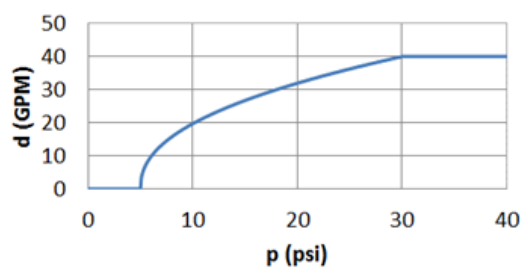

- Simulation start/stop capabilities

- Feedback loops

- Monte Carlo simulation

- Parallelization 


\section{Leak Model}

- Explicitly model water lost between the time when the leak starts and the time when crews can isolate/repair the leak

- New node added at location of leak

- Leak demand, $d_{\text {leak }}$ depends on pressure, $p$, at that node

- Input parameters: discharge coefficient $\left(C_{d}\right)$ and area $(A), \alpha$ set to 0.5

$$
d_{\text {leak }}=C_{d} A \sqrt{2 \rho} p^{\alpha}
$$

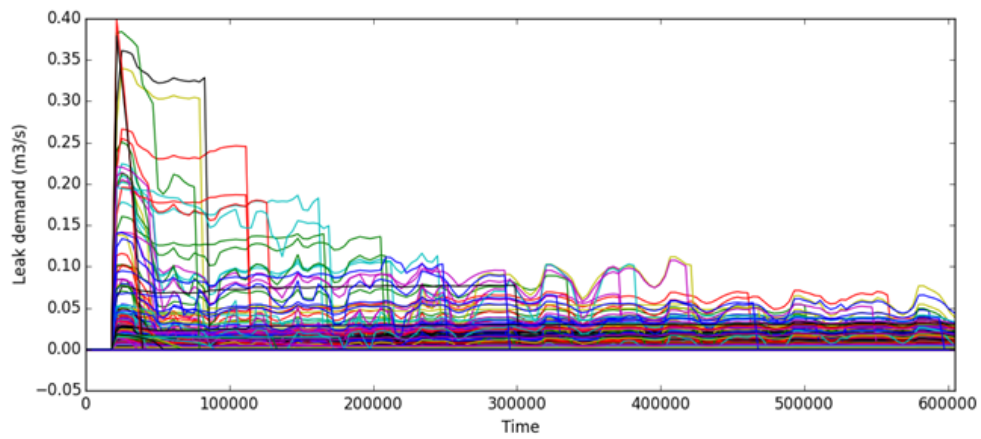

\section{Resilience Metrics}

\begin{tabular}{|l|l|}
\hline Topographic & Shortest path lengths, bridges, articulation points, betweenness centrality \\
\hline Hydraulic & $\begin{array}{l}\text { Water service availability, pressure within thresholds, Todini index, entropy, } \\
\text { population impacted }\end{array}$ \\
\hline $\begin{array}{l}\text { Water } \\
\text { quality/security }\end{array}$ & $\begin{array}{l}\text { Concentration above/below threshold, water age, mass consumed, extent } \\
\text { of contamination, population impacted }\end{array}$ \\
\hline Economic & Network cost, greenhouse gas emissions \\
\hline
\end{tabular}

Water service availability after a power outage

Population impacted after an earthquake
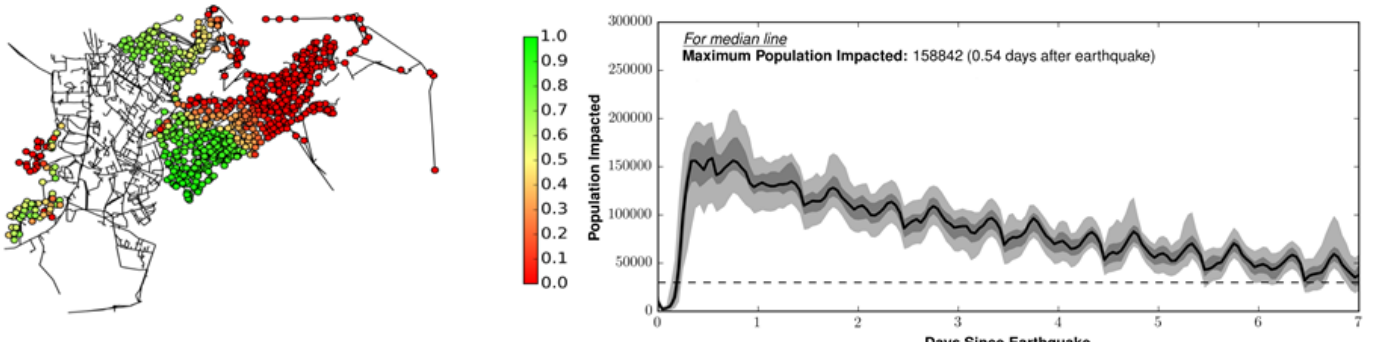


\section{Analyze/Visualize Results}

- Key concepts to extract:

- Critical network components

- Worse case scenarios

- Best restoration actions

- Results stored in a time indexed database using Pandas

- Easily saved to files (Excel, csv, hd5)

- Matplotlib and NetworkX graphing capabilities
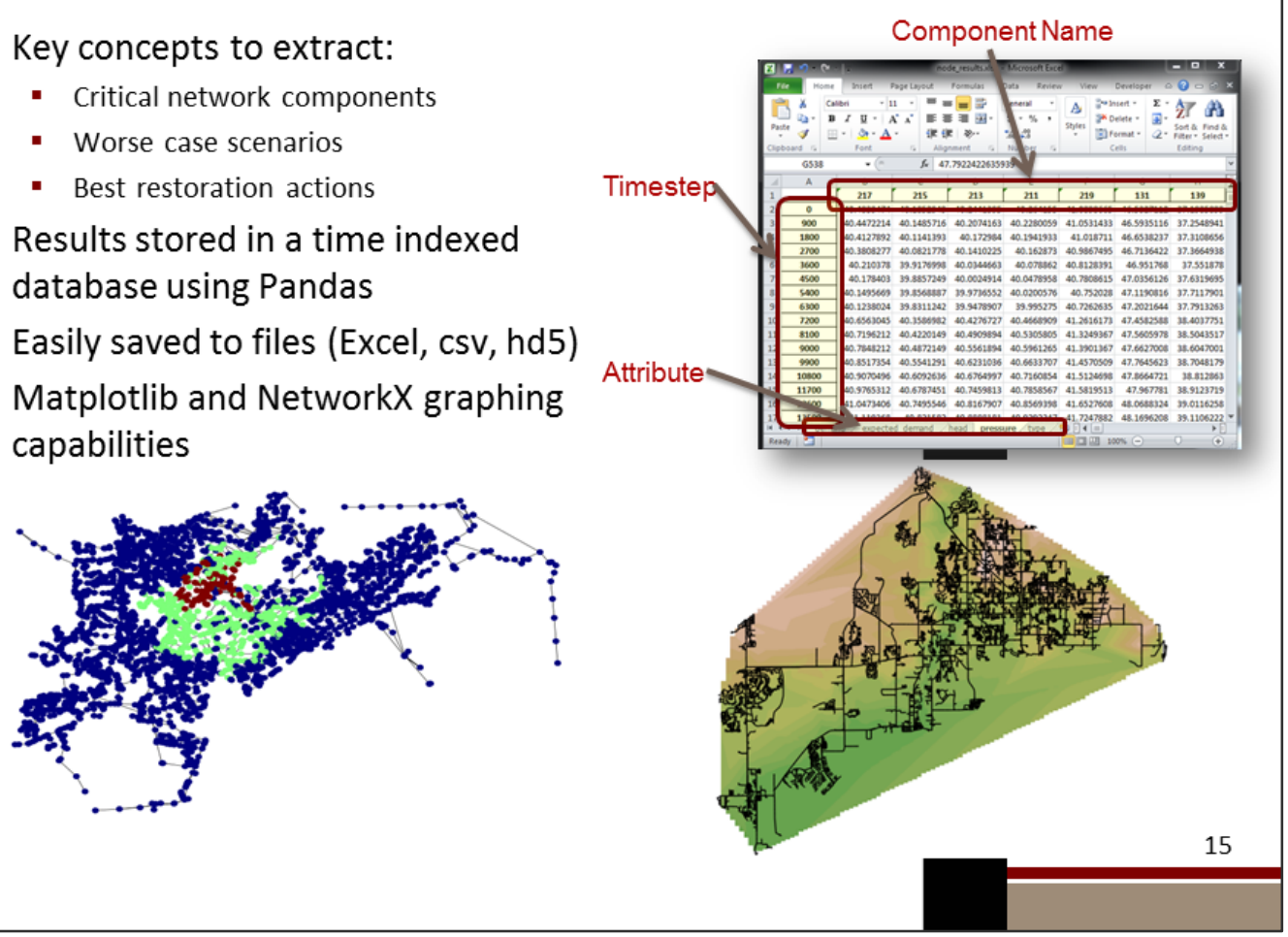

\section{WNTR Software}

- Open source and available on Github, https://github.com/usepa/wntr

- Examples and software tests

- 'Getting started guide' and API documentation coming soon

0

O

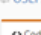
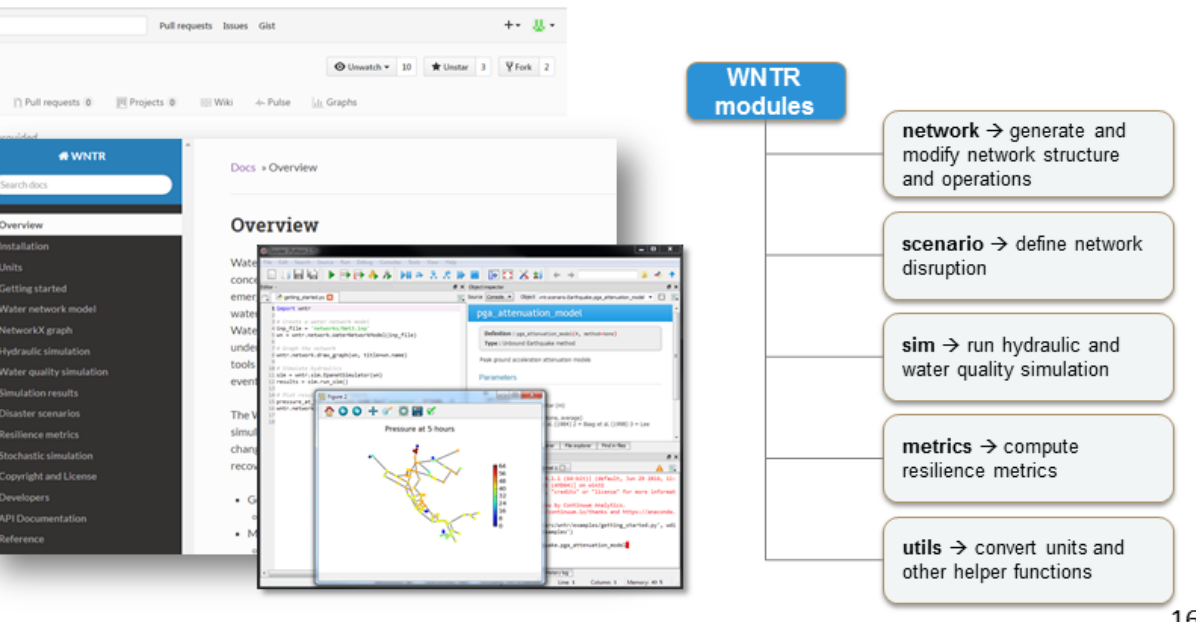


\section{Ongoing Research and Development}

- Develop additional disaster scenarios based on real events

- Improve code handling of rapid changes and failures

- Test and evaluate resilience functions/indicators

- Add optimization of response actions

- Perform case studies with utility partners

- Investigate network design for resilience 


\section{Modeling Water Distribution System Resilience}

\section{$\underline{B i o}$}

Katherine Klise joined Sandia National Laboratories in 2004 with a MS degree in Earth and Planetary Sciences. She is currently leading a collaborative research project with the EPA developing custom simulation and optimization software tools for water infrastructure security. Areas of specialization include network topography, image analysis, spatial heterogeneity, data mining, software development and quality assurance.

\section{Bibliography}

US Environmental Protection Agency (USEPA), "Systems Measures of Water Distribution System Resilience," US Environmental Protection Agency, Office of Research and Development, EPA 600/R14/383, Washington DC, 2015.

The software developers of WNTR (Water Network Tool for Resilience) wrote this report to review quantitative performance measures for water distribution systems with a focus on systems measures that can be used to quantify resilience to natural disasters, terrorist attacks, and other hazards. This report includes references for related work on modeling water distribution network resileince.

Henry D, Ramirez-Marquez JE. (2012). Generic metrics and quantitative approaches for system resilience as a function of time. Reliability Engineering and System Safety, 99, 114-122.

Barker K, Ramirez-Marquez JE, Rocco CM. (2013). Resilience-based network component importance measures. Reliability Engineering and System Safety, 117, 89-97.

These two publications describe methods to quantify resilience using state transitions. WNTR creates state transition plots to track water service availability and population impacted using simulations of disaster and recovery efforts.

Rossman LA. (2000). EPANET 2 Users Manual. [Technical report] Cincinnati, OH: U. S. Environmental Protection Agency. EPA/600/R-00/057

This report describes EPANET software used to model water distribution systems. The software is distributed by USEPA. WNTR uses EPANET for demand-driven hydraulic simulation and water quality simulation.

American Lifelines Alliance (2001), Seismic Fragility Formulations for Water Systems, Part 1 and 2, American Lifelines Alliance (ALA), April 2001

This report describes failure mechanisms in water systems after an earthquake. Fragility curves from this report can be included in WNTR. 
Title: Power Distribution Systems: Modeling of Systems Dependencies and Interdependencies

Presenter: Charles M. Macal, PhD, PE

Affiliation: Resilient Infrastructure Initiative, Global Security Sciences Division, Argonne National Laboratory

\begin{abstract}
:
Interdependencies between infrastructure systems are becoming more complex while our ability to understand these interdependencies continues to lag. The growing reliance of the electric power system on cheap and clean natural gas for power generation, for example, is creating a significant and growing dependency of the electric power system on the natural gas system, while, at the same time, the natural gas system heavily relies on electricity for its operation. These interdependencies create the possibility that both systems are potentially more vulnerable to disruptions cascading across multiple infrastructures and present challenges for recovery and restoration operations. This paper presents a framework for identifying and modeling critical interdependencies between infrastructure systems, focusing on electric power and natural gas. Proven infrastructure models (EPfast for electric power, NGfast for natural gas) are linked by modeling the critical interdependencies between these systems. Case studies are presented that illustrate the need for and value of such an approach. Technical challenges are identified, including data requirement for modeling interdependencies and the need for validation of linked infrastructure models beyond validation of individual infrastructure models. Research challenges for extensibility of the framework to modeling multiple infrastructure systems and their interdependencies are highlighted.
\end{abstract}




\section{POWER}

\section{DISTRIBUTION SYSTEMS}

Modeling of System Dependencies and Interdependencies

\section{CHARLES MACAL}

Chief Scientist, Argonne's Resilient Infrastructure Initiative

\section{MEGAN CLIFFORD}

Director, Argonne's Resilient Infrastructure Initiative $\Delta$

\section{OUTLINE}

- Background

- Methods

- Results

- Challenges

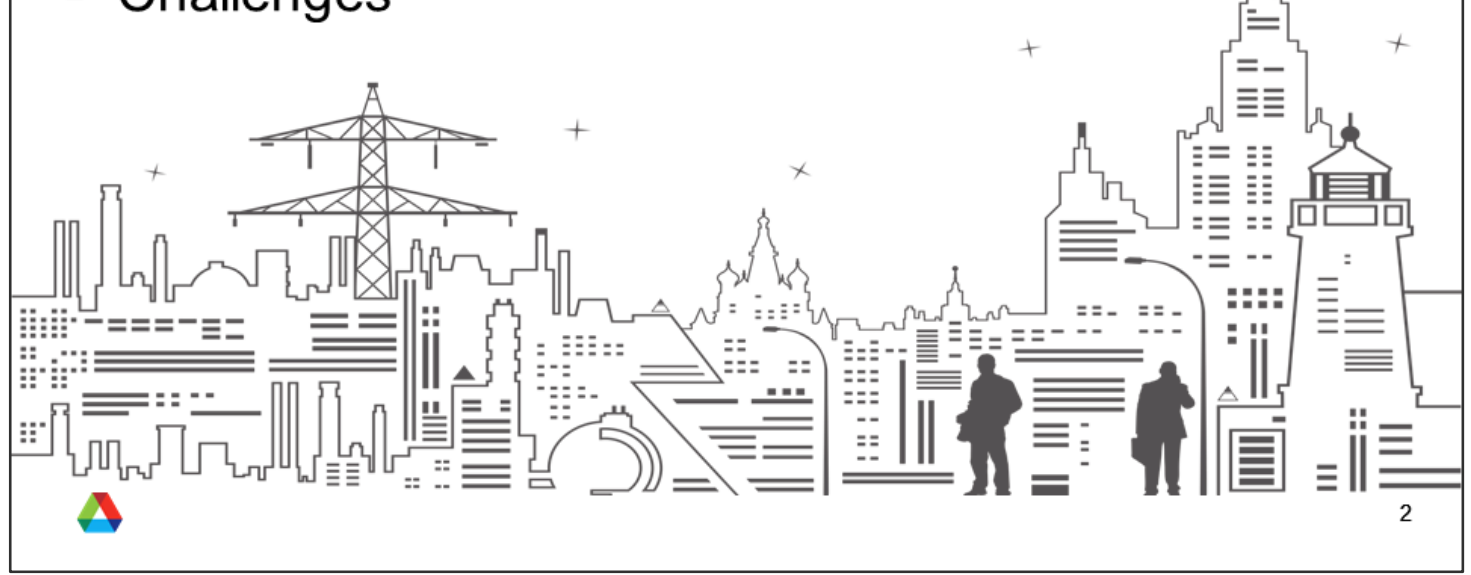




\section{INFRASTRUCTURES ARE BECOMING MORE INTERDEPENDENT}

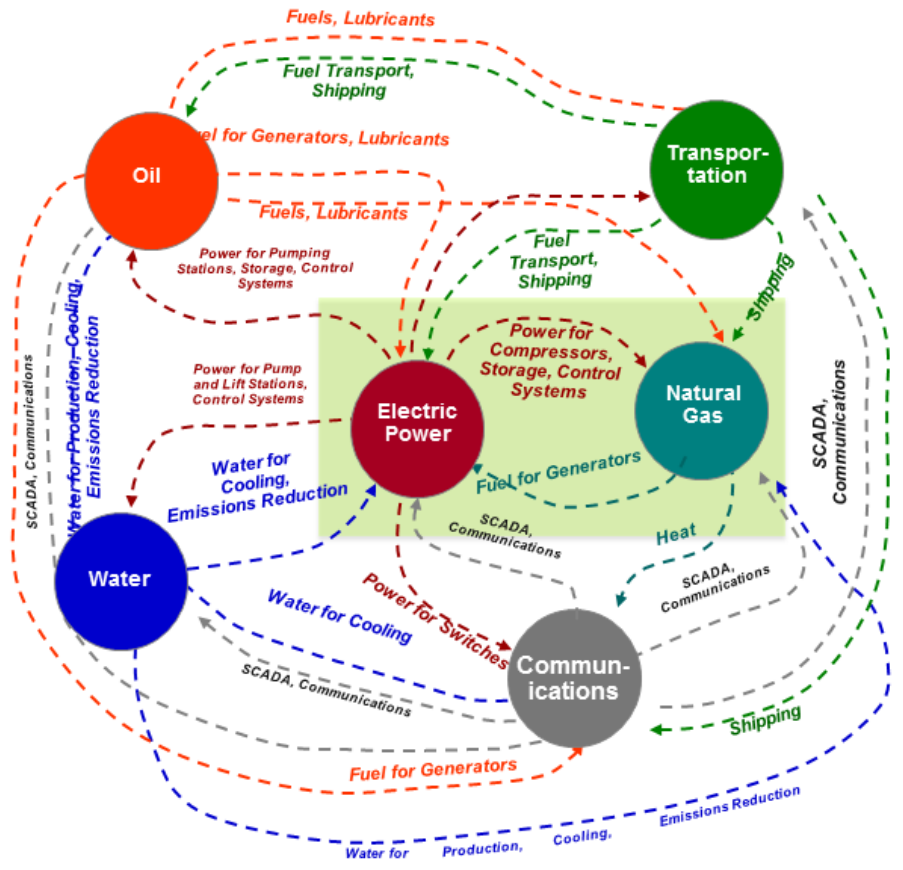

\section{INCREASING INTERDEPENDENCIES BETWEEN ELECTRIC POWER AND NATURAL GAS}

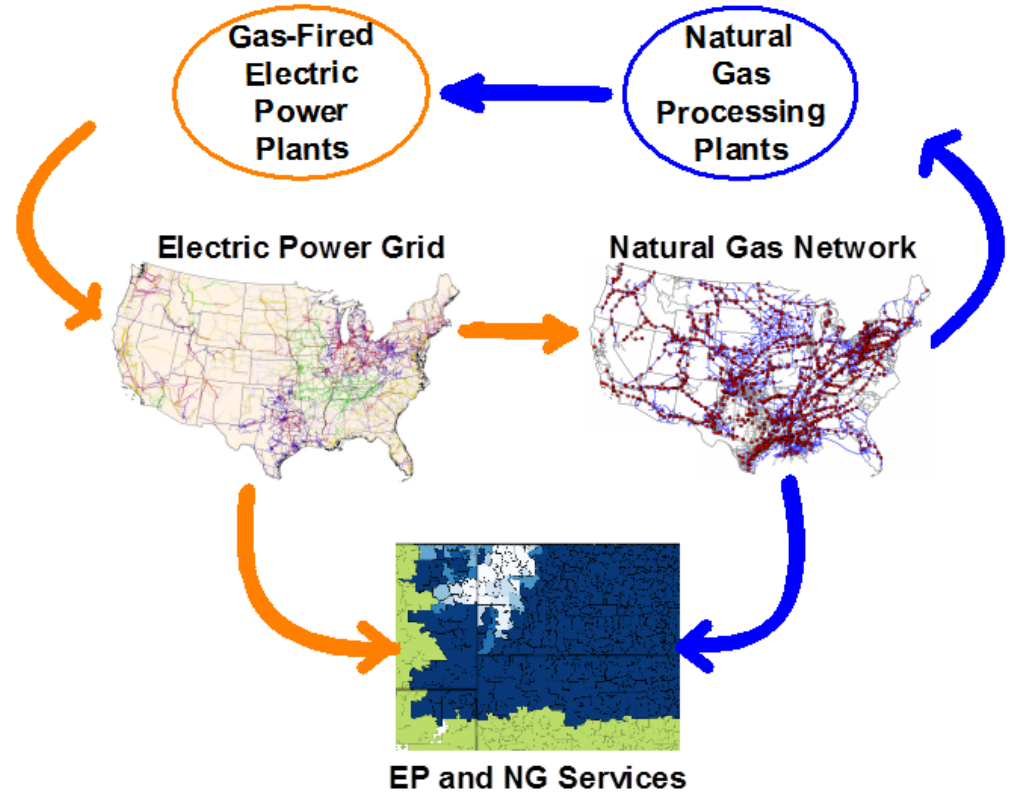




\section{DISRUPTIONS CASCADE BETWEEN INFRASTRUCTURE SYSTEMS}

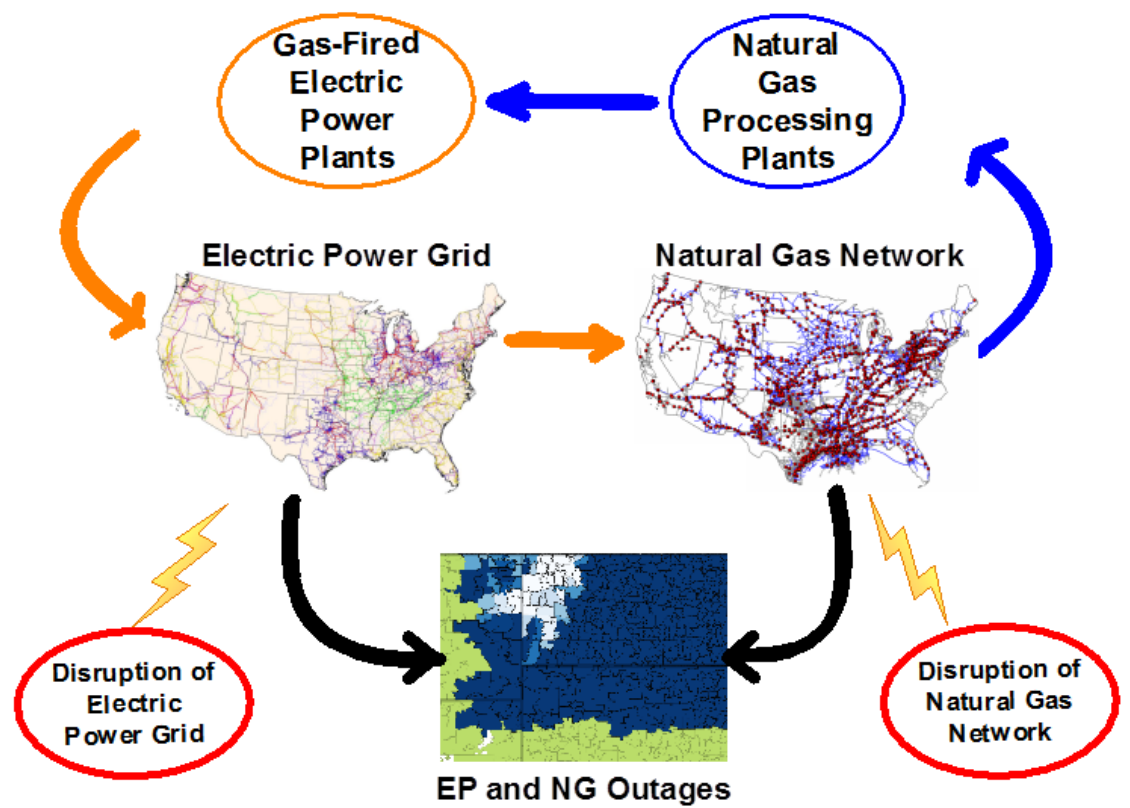

\section{UNDERSTANDING STAKEHOLDER REQUIREMENTS}

\section{Literature review (35 documents) and interviews (10) determined the top 8 questions:}

1. How are the different components of the DHS taxonomy (sector, subsector, asset) interconnected?

2. What infrastructures would be negatively impacted by a given event?

3. What are the interrelationships between the systems and potential for cross-system cascading disruptions?

4. What are the sequential and simultaneous impacts of a disruption?

5. What are the lifeline vulnerabilities to single points of failure?

6. How many people would be impacted?

7. What would be the consequences to the dysfunction of critical infrastructure?

8. What are the potential security and safety issues? 


\section{INFRASTRUCTURE MODELING AND RESILIENCE}

- Infrastructure Resilience: An infrastructure's ability to continue to provide critical services efficiently following a

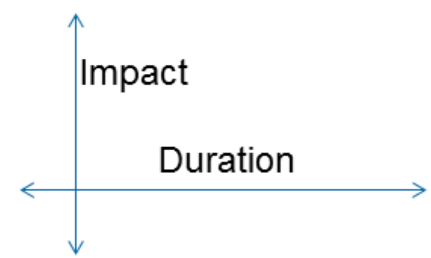
disruptive event

- Infrastructure Modeling

- Quantify the potential infrastructure impact and outage durations (people, business)

- Aid stakeholders by making recommending that increase critical infrastructure resilience

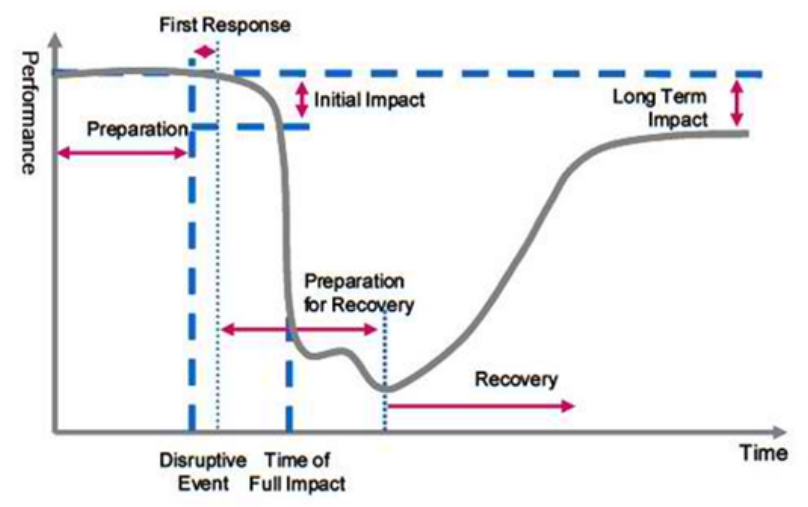

\section{EPFAST ELECTRIC GRID MODELING}

Direct Current, steady-state model provides quick estimates of disruptions

- Transmission line outages

- Substation outages

- Cascading line outages

- Uncontrolled islanding
Regional Scale

- Up to 60,000 nodes and 70,000 lines

- WECC, ERCOT, Eastern Interconnect

Results

- Load reduction per substation

- Generators shuttered

- Number and size of island grids formed

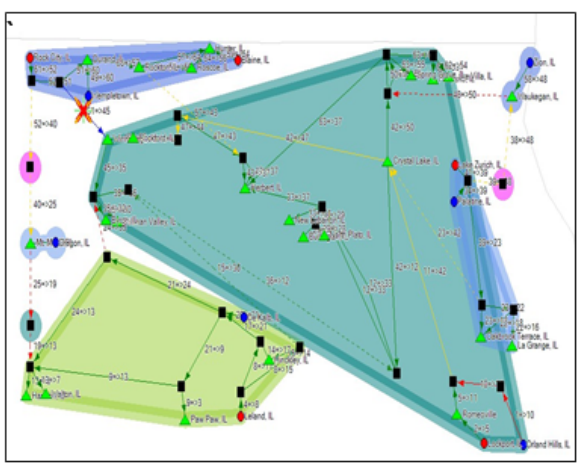




\section{NGFAST NATURAL GAS INTERSTATE PIPELINE MODELING}

- Linear, steady state model provides quick estimates of impacts

- Pipeline breaks

- Flow reduction due to production fields

- Accounts for effects of mitigating measures

- Underground storage (UGS)

- Liquefied Natural Gas (LNG)

- Production facilities

- Spare pipeline capacity

- National model that considers

- 80 interstate pipelines

- 1,800 Local Distribution Companies (LDCs)

$\Delta$ - 800 state border points

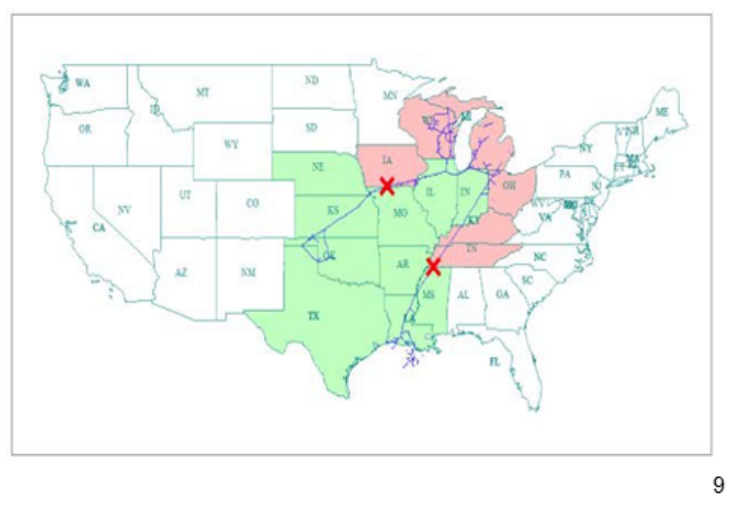

\section{MODELING THE PHYSICS OF INFRASTRUCTURE SYSTEMS}

\section{Example: Natural Gas Model Formulation}

Transport Equations for link $\ell \in \mathcal{L}:=\mathcal{L}_{p} \cup \mathcal{L}_{a}$

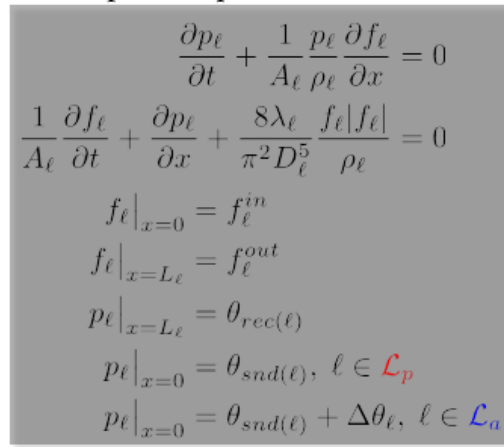

Conservation at node $n \in \mathcal{N}$
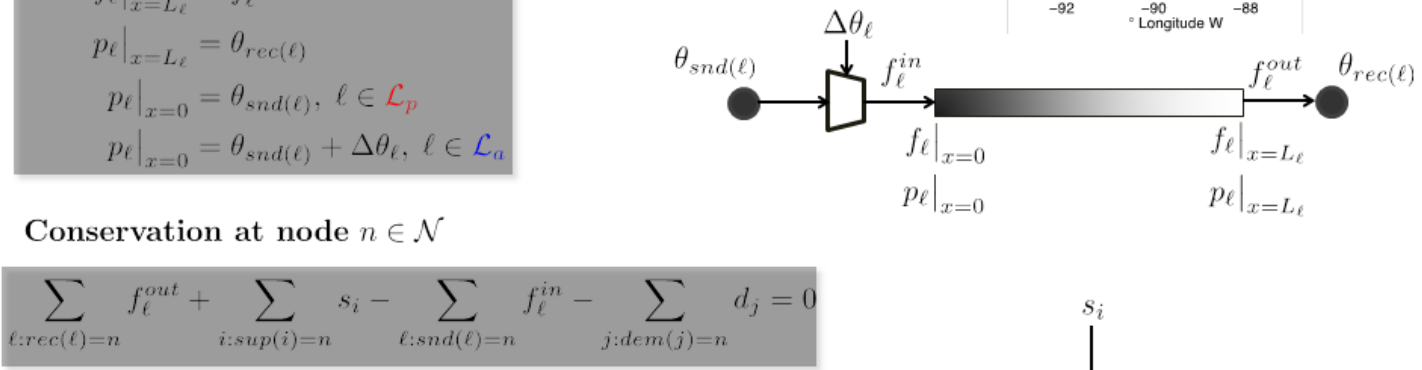

Compression Power for link $\ell \in \mathcal{L}_{A}$

$P_{\ell}=f_{\ell}^{i n} c_{p} T\left(\left(\frac{\theta_{\text {snd }(\ell)}+\Delta \theta_{\ell}}{\theta_{\text {snd }(\ell)}}\right)^{\frac{\gamma-1}{\gamma}}-1\right)$ 


\section{NORTH DAKOTA CASE: EP \& NG INTERDEPENDENCIES}

Nine EP substations disrupted

Outage area covers all NGPPs

NGPPs stop operating

Flow reduction along Northern Border PL

Flow reduced by $28 \%$

Impacts SD, IA, IL, and IN

Threatens 1,440 MW of gas-fired cap

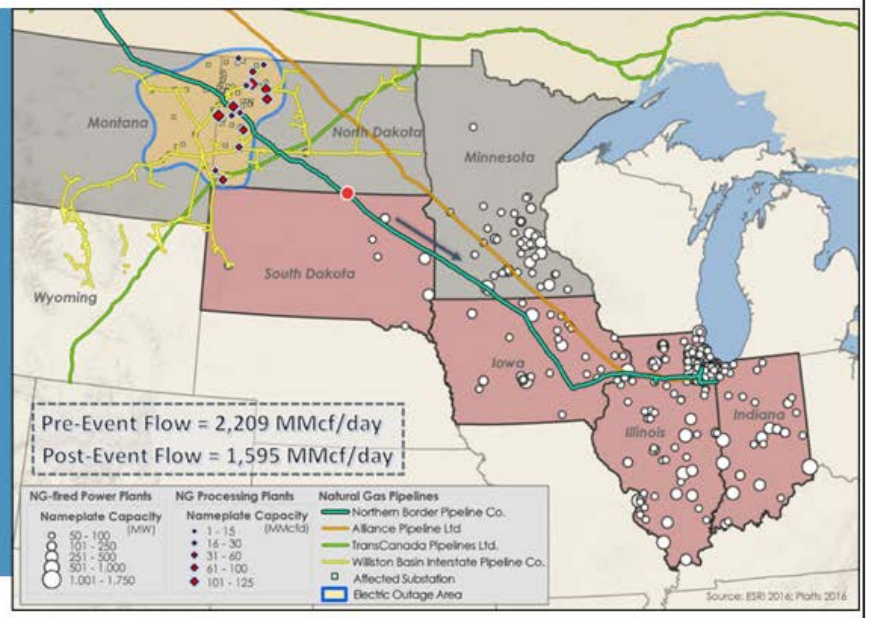

\section{TYPICAL DISRUPTION SCENARIO}

\section{Local Effects}

1. Disruption event brings down major electric power generating substations

2. Electric power network breaks into separate islands--blackouts occur in the immediate affected area

3. Loss of electricity disrupts natural gas processing plants

4. Cuts gas flow into interstate pipelines

Downstream Effects...

Restoration...

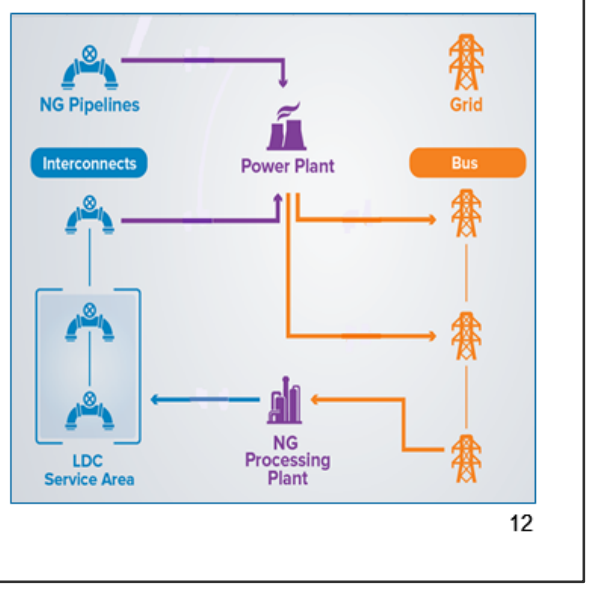




\section{TYPICAL DISRUPTION SCENARIO}

\section{Local Effects...}

\section{Downstream Effects}

1. Reduced gas delivery downstream results in curtailments to residential, commercial, industrial and electricity sectors across the region far removed from the initial event

2. Curtailed delivery to gas-fired electric power plants connected to affected pipelines

3. Electric power network breaks into separate islands-blackouts occur in the immediate affected area far from the point of the original disruption

Restoration...

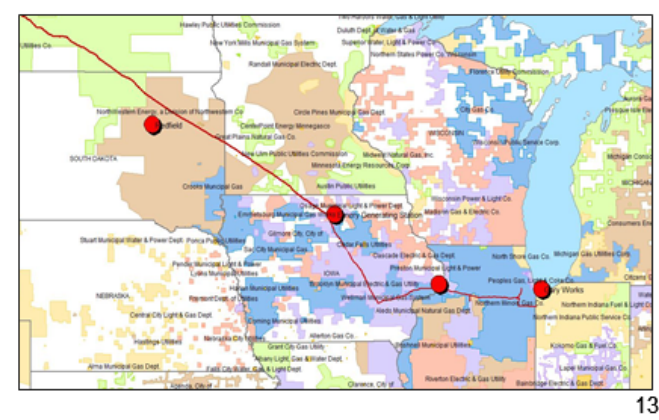

\section{TYPICAL DISRUPTION SCENARIO}

\section{Local Effects...}

\section{Downstream Effects...}

\section{Restoration}

1. Sequencing the start-up of the electricity generating units

2. Gas-fired plants may not accept unprocessed gas because of impurities and its heavy water contents

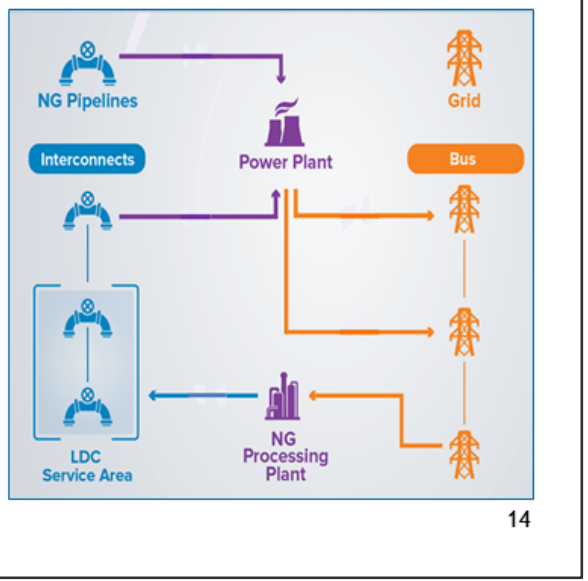




\section{CHALLENGES FOR ADVANCING} INFRASTRUCTURE DEPENDENCY AND INTERDEPENDENCY MODELING

- Better Define End-User Requirements

- Identify Data Needs for Modeling

- Explore Development of Integrated Modeling Approaches

- Deliver Useful and Usable Models to Users

- Foster Collaboration among the R\&D Community

Source: Clifford, Megan, and Charles Macal (2016) Advancing Infrastructure Dependency and Interdependency Modeling: A Summary Report from the Technical Exchange, Argonne $\Delta$ Report (August 2016).

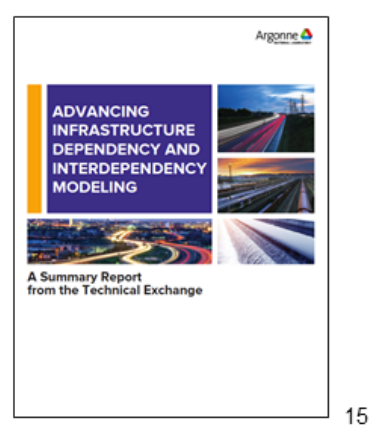

\section{BIBLIOGRAPHY}

- Clifford, Megan, and Charles Macal (2016) Advancing Infrastructure Dependency and Interdependency Modeling: A Summary Report from the Technical Exchange, Argonne Report (August 2016).

- Clifford, M. (2015) National Call to Action: The Resilient Infrastructure Initiative, Center for Infrastructure Protection and Homeland Security, George Mason University School of Law, Washington, DC, USA. <http://cip.gmu.edu/2015/12/02/national-call-to-action-the-resilient-infrastructure-initiative/> (April 25, 2016).

- Jalving, Jordan, Shrirang Abhyankar, Kibaek Kim, Mark Hereld, and Victor M. Zavala, A Graph-Based Computational Framework for Simulation and Optimization of Coupled Infrastructure Networks, Under review, 2016

- Portante, E.C., Craig, B.A., and Folga, S.F. (2007) NGfast: A Simulation Model for Rapid Assessment of Impacts of Natural Gas Pipeline Breaks and Flow Reductions at U.S. State Borders and Import Points Proc., 2007 Winter Simulation Conference, Henderson, S.G., Biller, B., Hsieh, M.-H., Shortle, J., Tew, J.D., and Barton, R.R., eds., IEEE, 1118-1126. <http://ieeexplore.ieee.org/xpls/abs_all.jsp?arnumber=4419711\&tag=1> (April 25, 2016)

- Portante, Edgar C., James A. Kavicky, Brian A. Craig, Leah E. Talaber, and Stephen M. Folga, Modeling Electric Power and Natural Gas Systems Interdependencies: Application to Natural Hazards, under Review, 2016.

- Portante, E.C., Craig, B.A., Talaber Malone, L.E., Kavicky, J.A., and Folga, S.F. (2011) EPFast: A Model for Simulating Uncontrolled Islanding in Large Power Systems Proc., 2011 Winter Simulation Conference, Jain, S., Creasey, R.R., Himmelspach, J., White, K.P., and Fu, M., eds., IEEE, 1758-1769. <http://ieeexplore.ieee.org/stamp/stamp.jsp?arnumber=06147891> (Oct. 9, 2013).

- Qi, J., K. Sun, and S. MEI (2015) An Interaction Model for Simulation and Mitigation of Cascading Failures, IEEE Trans. Power Systems, 30(2): 804-819, March.

- Zavala, V.M., and Chiang, N. (2016) Large-Scale Optimal Control of Interconnected Natural Gas and Electrical Transmission Systems, ANL/MCS-P5348-0515, Argonne National Laboratory, Argonne, IL, 


\section{ARGONNE'S RESILIENT INFRASTRUCTURE INITIATIVE}

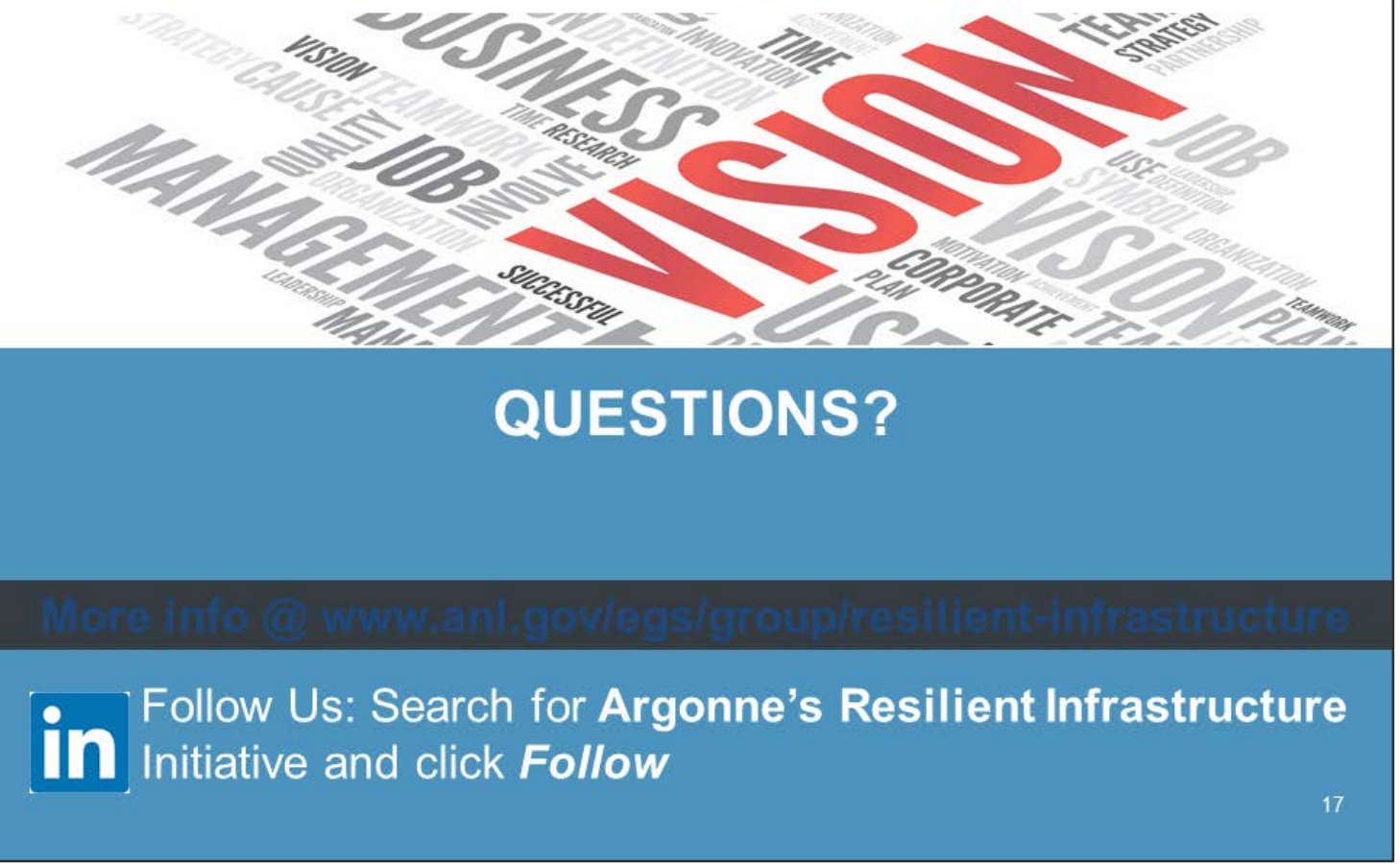


Title: Power Distribution Systems: Modeling of Systems Dependencies and Interdependencies

Presenter: Charles M. Macal, PhD, PE

Affiliation: Resilient Infrastructure Initiative, Global Security Sciences Division, Argonne National Laboratory

\section{BIBLIOGRAPHY}

Clifford, Megan, and Charles Macal (2016) Advancing Infrastructure Dependency and Interdependency Modeling: A Summary Report from the Technical Exchange, Argonne Report (August 2016).

Clifford, M. (2015) National Call to Action: The Resilient Infrastructure Initiative, Center for Infrastructure Protection and Homeland Security, George Mason University School of Law, Washington, DC, USA. <http://cip.gmu.edu/2015/12/02/national-call-to-action-theresilient-infrastructure-initiative/> (April 25, 2016).

Jalving, Jordan, Shrirang Abhyankar, Kibaek Kim, Mark Hereld, and Victor M. Zavala, “A Graph-Based Computational Framework for Simulation and Optimization of Coupled Infrastructure Networks,” Under review, 2016

Portante, E.C., Craig, B.A., and Folga, S.F. (2007) “NGfast: A Simulation Model for Rapid Assessment of Impacts of Natural Gas Pipeline Breaks and Flow Reductions at U.S. State Borders and Import Points.” Proc., 2007 Winter Simulation Conference, Henderson, S.G., Biller, B., Hsieh, M.-H., Shortle, J., Tew, J.D., and Barton, R.R., eds., IEEE, 1118-1126. $<$ http://ieeexplore.ieee.org/xpls/abs_all.jsp?arnumber=4419711\&tag=1> (April 25, 2016).

Portante, Edgar C., James A. Kavicky, Brian A. Craig, Leah E. Talaber, and Stephen M. Folga, "Modeling Electric Power and Natural Gas Systems Interdependencies: Application to Natural Hazards,” Under Review, 2016.

Portante, E.C., Craig, B.A., Talaber Malone, L.E., Kavicky, J.A., and Folga, S.F. (2011) "EPFast: A Model for Simulating Uncontrolled Islanding in Large Power Systems." Proc., 2011 Winter Simulation Conference, Jain, S., Creasey, R.R., Himmelspach, J., White, K.P., and Fu, M., eds., IEEE, 1758-1769. $<$ http://ieeexplore.ieee.org/stamp/stamp.jsp?arnumber=06147891> (Oct. 9, 2013).

Qi, J., K. Sun, and S. MEI (2015) “An Interaction Model for Simulation and Mitigation of Cascading Failures,” IEEE Trans. Power Systems, 30(2): 804-819, March.

Zavala, V.M., and Chiang, N. (2016) Large-Scale Optimal Control of Interconnected Natural Gas and Electrical Transmission Systems, ANL/MCS-P5348-0515, Argonne National Laboratory, Argonne, IL, 615April. 


\section{Argonne}

\section{Charles M. Macal}

\section{Chief Scientist, Resilient Infrastructure Initiative}

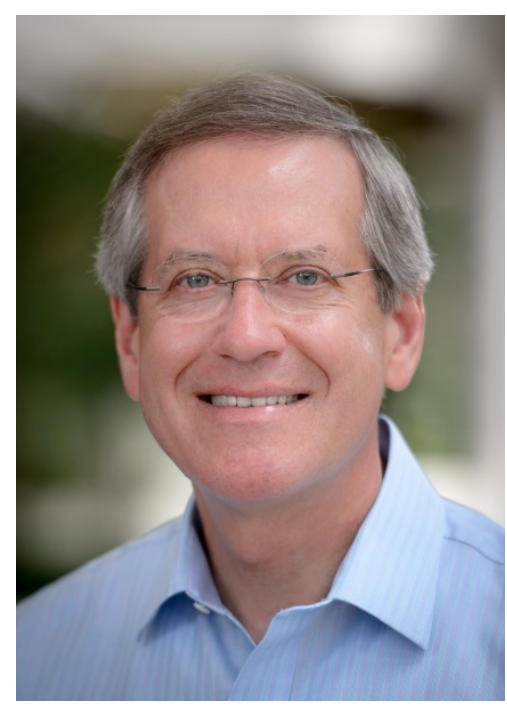

Charles Macal, PhD, PE, is the Chief Scientist of Argonne National Laboratory's Resilient Infrastructure Initiative. In this role, he leads a team of interdisciplinary researchers developing new computational models and simulations for infrastructure interdependency analysis, planning, and resilience.

Charles is recognized globally as a leader in the field of agent-based modeling and simulation, focusing on the modeling of social and behavioral components of sociotechnical systems. In addition to infrastructure, he has developed innovative computer simulation models in the areas of energy, critical materials, environmental sustainability, technology adoption, and infectious diseases. He holds Senior Fellow appointments at the Computation Institute of the University of Chicago and the Northwestern-Argonne Institute for Science and Engineering.

Charles received a $\mathrm{PhD}$ in Industrial Engineering \& Management Sciences from Northwestern University and holds an MS in Industrial Engineering and a BS in Engineering Sciences from Purdue University. He is a registered professional engineer in the State of Illinois and is a senior member of the Association for Computing Machinery (ACM), the Institute of Electrical and Electronics Engineers (IEEE), the Society for Computer Simulation International (SCSI), and the Institute for Operations Research and the Management Sciences (INFORMS). Charles serves on the editorial boards of several computer modeling and simulation publications. 


\title{
Reliability Assessment of Interdependent Networks Based on Survival Signature
}

\author{
M. Broggi, G. Feng, E. Patelli, F. Coolen, M. Beer
}

\begin{abstract}
The reliability analysis of complex networks is of key importance to the resilience of communities. However, a major obstacle for this analysis is the realistic consideration of interdependencies between different networks. Due to the complexity of the interdependencies dependent failures are very difficult to predict and hence dangerous, in particular, when the initiating event is seemingly unimportant.

We propose a solution based on the concept of survival signature, from systems theory, which provides a novel basis for complex network analysis, outperforming traditional analyses techniques in numerical efficiency when estimating the reliability of networks. The key advantage of the survival signature approach is the complete separation of the structure of the network from its probabilistic characteristics. Once the signature of the network has been analysed, the reliability analysis can be carried out by evaluating only the probabilistic structure, which makes the analysis particularly efficient. In addition, when only limited information is available for the specification of network parameters, imprecise probabilities can easily be implemented into the survival signature approach to capture the associated indeterminacy.
\end{abstract}

\section{References}

S.V. Buldyrev, R. Parshani, G. Paul, H. E. Stanley, S. Havlin, Catastrophic cascade of failures in interdependent networks, Nature 464, (2010) 1025-1028.

F. J. Samaniego. System signatures and their applications in engineering reliability. Springer Science \& Business Media (2007), vol. 110.

F. P. Coolen, T. Coolen-Maturi. Generalizing the signature to systems with multiple types

of components. Complex Systems and Dependability. (2012), pp. 115-130. 

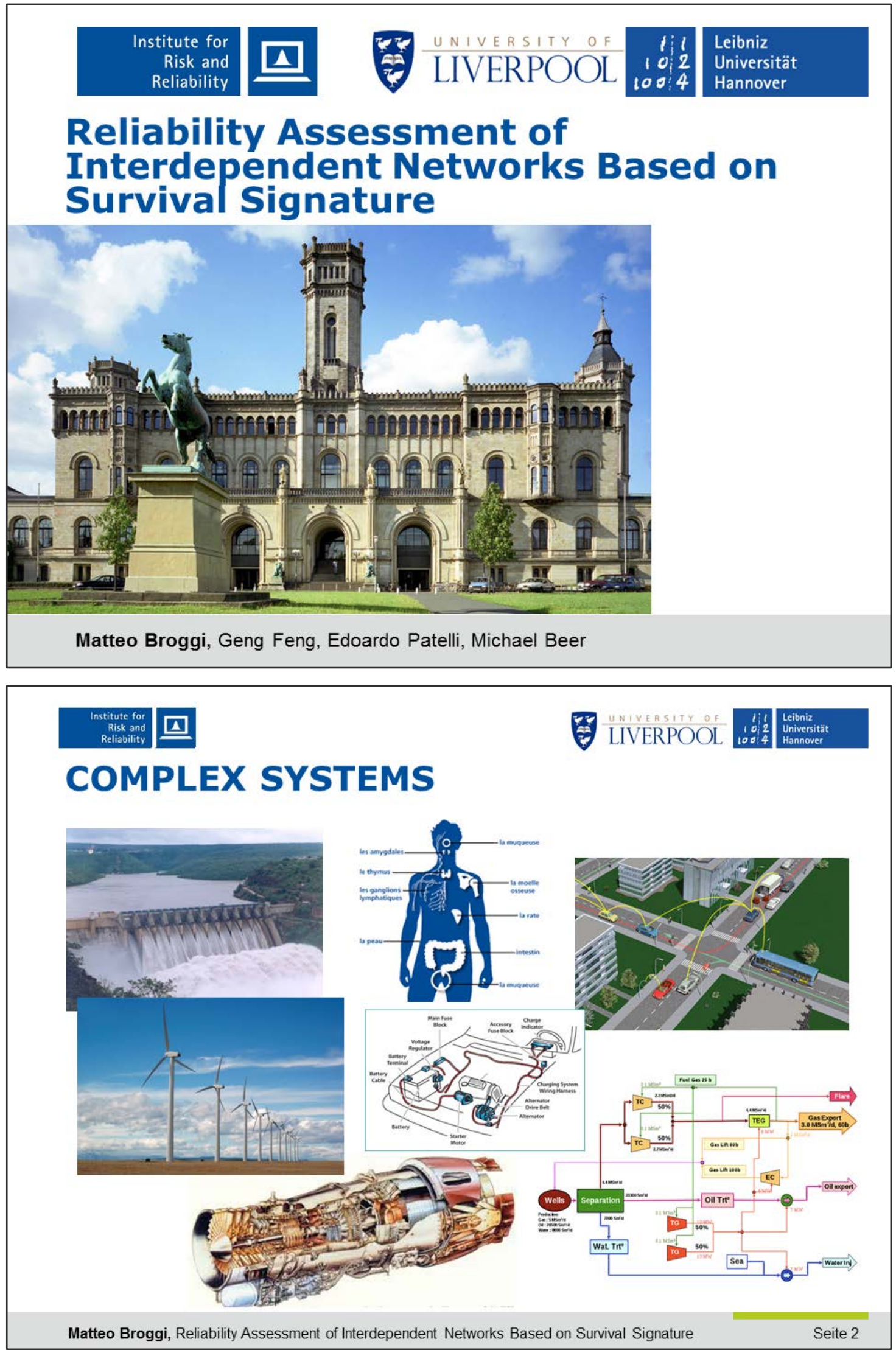

C-54 
- traditional approaches

- fault tree analysis

- reliability block diagrams

- ...

- limitations in modeling

- dependencies

- common-cause failures

- time-dependent behavior

- lack of information

- complex network structure

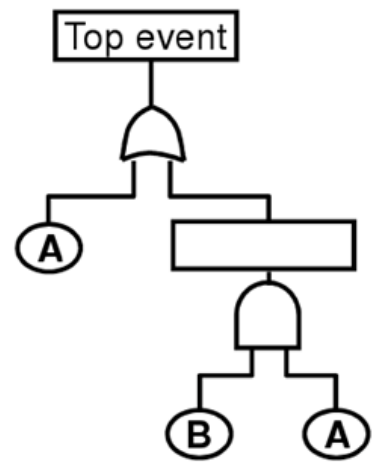

$\Rightarrow$ alternative modeling to address limitations

$\Rightarrow$ improved reliability and availability analysis of systems

» identify weak components

» define optimal maintenance strategies

\section{ANALYSIS OF COMPLEX SYSTEMS}

Limitations in classic modelling

- Common-cause failures

- Fault tree cannot capture dependencies between event

- Modify tree structure

- Additional events

- Problem: which probability to assign to rare dependent events?
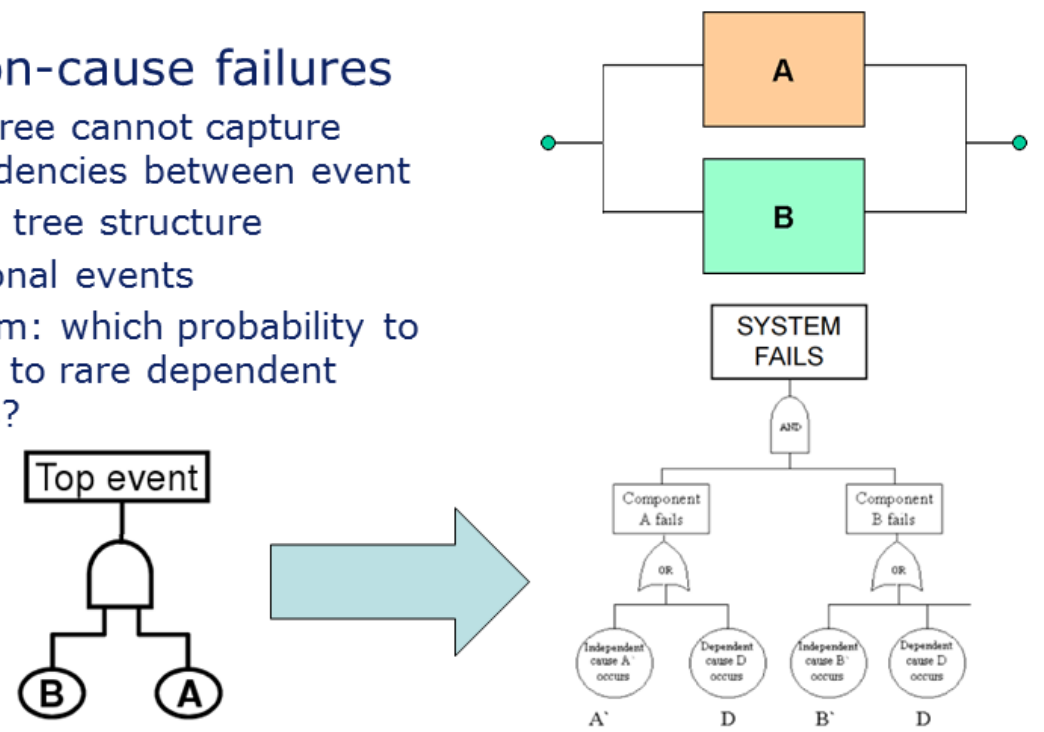

Picture source: "Zio E., Dependent Failures", http://www.lasar.polimi.it/wp-content/uploads/2016/05/Dependent-Failures.pdf 


\section{ANALYSIS OF COMPLEX SYSTEMS}

\section{Limitations in classic modelling}

- Dependent system failures

- Need to connect dependent systems structures in a common structure

- New structure (event tree + fault tree) needed for each top failure event analysed

- Which probability to assign to rare dependent events?
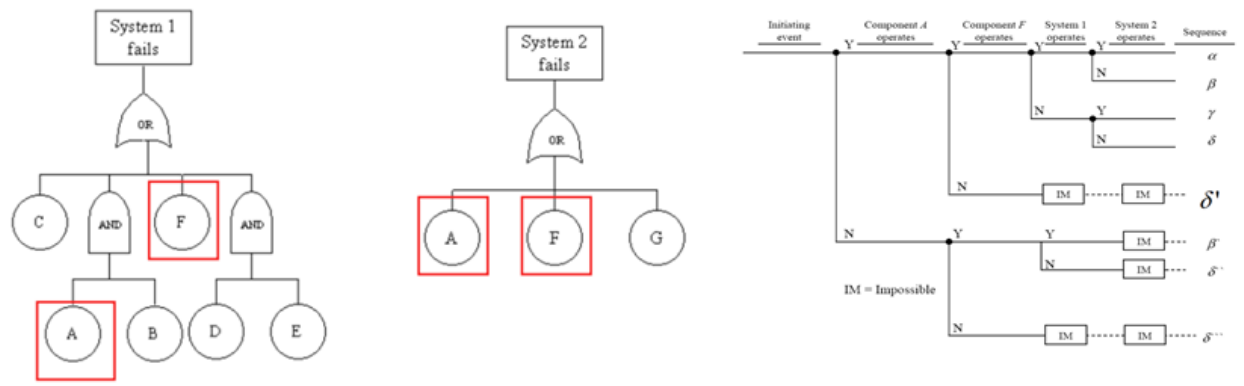

Picture source: "Zio E., DependentFailures", http://www.lasar.polimi.it/wp-content/uploads/2016/05/Dependent-Failures.pdf Matteo Broggi, Reliability Assessment of Interdependent Networks Based on Survival Signature Seite 5

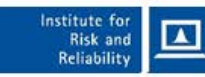

6

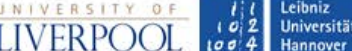

\section{VAGUE AND IMPRECISE INFORMATION}

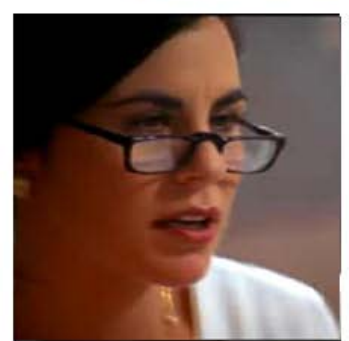

statistical analysis of imprecise and rare data

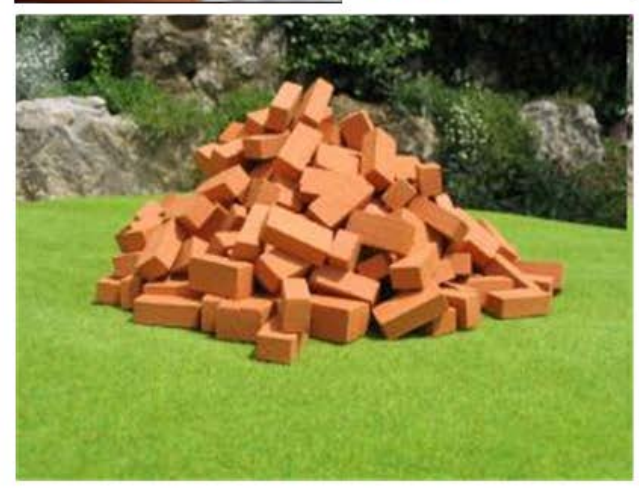

reliability analysis $\Rightarrow \widetilde{P}_{f}$

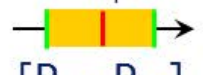

Is it safe?

$$
\left[P_{f, l}, P_{f, r}\right]
$$

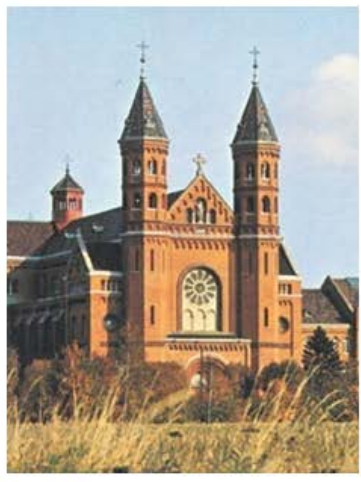

imprecision

Is the reflected in $\mathrm{P}_{f}$ still reliable?

$$
\text { Effects on } \mathrm{P}_{\mathrm{f}} \text { ? }
$$

Sensitivity of $P_{f}$ to imprecision ? 


\section{DEPENDENT NETWORK ANALYSIS WITH SURVIVAL SIGNATURE}

\section{SURVIVAL SIGNATURE}

» state vector

$\underline{x}=\left(x_{1}, \ldots, x_{m}\right) \quad x_{i}=1$ : component $i$ is working

$x_{i}=0$ : component $i$ is NOT working

$\gg$ structure function

$$
\begin{array}{ll}
\varphi(\underline{\mathbf{x}})=\varphi\left(\mathrm{x}_{1}, \ldots, \mathrm{x}_{\mathrm{m}}\right) \quad \varphi(\underline{\mathbf{x}})=1 \text { : system is working } \\
\varphi(\underline{\mathbf{x}})=0 \text { : system is NOT working }
\end{array}
$$

» example

$$
\varphi\left(\mathrm{x}_{1}=1, \mathrm{x}_{2}=0, \mathrm{x}_{3}=0\right)=\varphi(1,0,0)=0
$$

$\varphi\left(\mathrm{X}_{1}=1, \mathrm{X}_{2}=1, \mathrm{X}_{3}=0\right)=\varphi(1,1,0)=1$

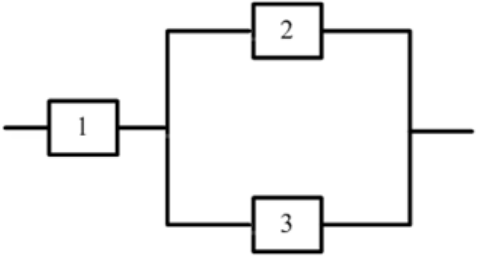




\section{Institute for
Risk and
Reliability}

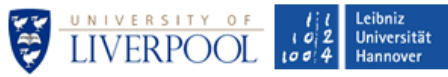

\section{SURVIVAL SIGNATURE}

- compressed representation of system availability

» system with $\mathrm{m}$ components of the same type

» I out of $\mathrm{m}$ components are working

$\Phi(\mathrm{l})=\left(\begin{array}{c}\mathrm{m} \\ \mathrm{l}\end{array}\right)^{-1} \cdot \sum_{\underline{x} \in \mathbf{S}} \varphi(\underline{\mathrm{x}}) \Longrightarrow \Phi(0)=0, \Phi(\mathrm{m})=1$

$\Rightarrow$ probability that system works when I out of $\mathrm{m}$ components are working

» example

$\mathrm{m}=3, \mathrm{I}=2$ :

$\mathrm{S}_{1}=\{(1,1,0),(1,0,1),(0,1,1)\}$

$\varphi(1,1,0)=1, \varphi(1,0,1)=1, \varphi(0,1,1)=0$

$\Phi(2)=\left(\begin{array}{l}3 \\ 2\end{array}\right)^{-1} \cdot \sum_{\underline{x} \in S_{1}} \varphi(\underline{x})=\frac{1}{3} \cdot(1+1+0)=\frac{2}{3}$

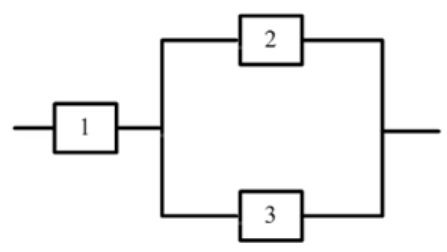

and further: $\Phi(0)=0, \Phi(1)=0, \Phi(3)=1$

\section{Institute for
Risk and
Reliability}

\section{SURVIVAL SIGNATURE}

- expansion to systems with several types of components » system with $\mathrm{K}$ component types, $\mathrm{m}_{\mathrm{k}}$ components of type $\mathrm{k}(\mathrm{k}=1, \ldots, \mathrm{K})$

$\gg I_{k}$ out of $m_{k}$ components are working $(k=1, \ldots, K)$

$$
\Phi\left(\mathrm{l}_{1}, \ldots, \mathrm{l}_{\mathrm{k}}\right)=\left[\prod_{\mathrm{k}=1}^{\mathrm{k}}\left(\begin{array}{c}
\mathrm{m}_{\mathrm{k}} \\
\mathrm{I}_{\mathrm{k}}
\end{array}\right)^{-1}\right] \cdot \sum_{\underline{\mathrm{x}} \in \mathrm{S}_{1, \ldots k}} \varphi(\underline{\mathbf{x}})
$$

》 example

$\mathrm{K}=2, \mathrm{~m}_{1}=3, \mathrm{~m}_{2}=3$ :

$$
\begin{aligned}
\Phi(1,2) & =\left(\begin{array}{l}
3 \\
1
\end{array}\right)^{-1} \cdot\left(\begin{array}{l}
3 \\
2
\end{array}\right)^{-1} \cdot \sum_{\underline{x} \in S_{1}} \varphi(\underline{x}) \\
& \left.=\frac{1}{3} \cdot \frac{1}{3} \cdot(0-1)+(0+0+0)+(0+0+0)\right)=\frac{1}{9} \\
\Phi(2,3) & =\left(\begin{array}{l}
3 \\
2
\end{array}\right)^{-1} \cdot\left(\begin{array}{l}
3 \\
3
\end{array}\right)^{-1} \cdot \sum_{\underline{x} \in S_{1}} \varphi(\underline{x})=\frac{1}{3} \cdot \frac{1}{1} \cdot(1+1+0)=\frac{2}{3}
\end{aligned}
$$




\section{Risk and
Reliability}

\section{SURVIVAL FUNCTION}

- time dependent system reliability $\mathrm{C}_{\mathrm{t}}^{\mathrm{k}}=$ number of components of type $\mathrm{k}$ still working at time $\mathrm{t}$

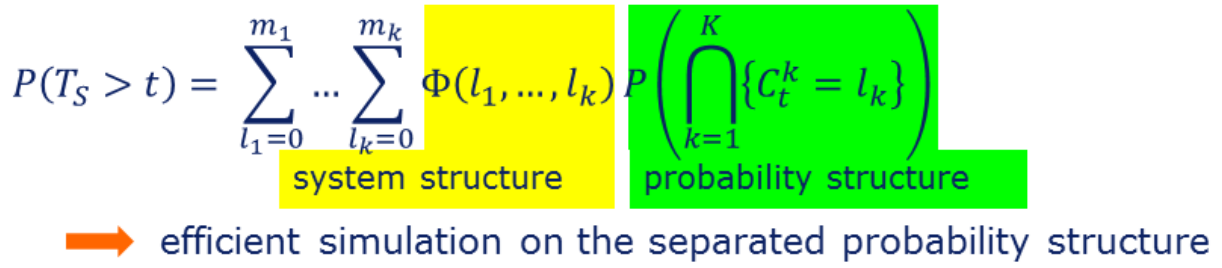

- superior features

» applicable to complex system structures

» can work with arbitrary dependency structures between failure events

» captures changing of component types (e.g., due to repair)

» can operate with imprecise probabilities (captures dependability issues)

\section{Ristitute for
Re and
Reliability}

\section{SURVIVAL FUNCTION} Probability Structures

- Probability structure captures dependencies between failures

- Example:

- Independent, crisp probabilities

$$
\mathbf{P}\left(\bigcap_{\mathrm{k}=1}^{\mathrm{K}}\left\{\mathbf{C}_{\mathrm{t}}^{\mathrm{k}}=\mathbf{l}_{\mathrm{k}}\right\}\right)=\prod_{\mathrm{k}=1}^{\mathrm{K}}\left(\begin{array}{c}
\mathbf{m}_{\mathrm{k}} \\
\mathbf{l}_{\mathrm{k}}
\end{array}\right)\left[\mathrm{F}_{\mathrm{k}}(\mathrm{t})\right]^{\mathrm{m}_{\mathbf{k}}-\mathbf{l}_{\mathrm{k}}}\left[\mathbf{1}-\mathbf{F}_{\mathrm{k}(\mathrm{t})}\right]^{\mathbf{l}_{\mathrm{k}}}
$$

- Independent imprecise distributions

$\Rightarrow$ bounds on survival function

$$
\begin{aligned}
& \underline{P}\left(T_{s}>t\right)=\sum_{l_{1}=0}^{m_{1}} \ldots \sum_{k=0}^{m_{k}} \Phi\left(l_{1}, \ldots, l_{k}\right) \cdot \prod_{k=1}^{K}\left(\begin{array}{c}
m_{k} \\
l_{k}
\end{array}\right)\left[\underline{F}_{k}(t)\right]^{m_{k}-l_{k}}\left[1-\underline{F}_{k}(t)\right]^{k_{k}} \\
& \bar{P}\left(T_{s}>t\right)=\sum_{l_{1}=0}^{m_{1}} \ldots \sum_{k=0}^{m_{k}} \Phi\left(l_{1}, \ldots, l_{k}\right) \cdot \prod_{k=1}^{k}\left(\begin{array}{c}
m_{k} \\
I_{k}
\end{array}\right)\left[\bar{F}_{k}(t)\right]^{m_{k}-l_{k}}\left[1-\bar{F}_{k}(t)\right]^{k}
\end{aligned}
$$

- Common cause of failure

- (Imprecise) Dependencies between random variables $\Rightarrow$ easily treated with MC 


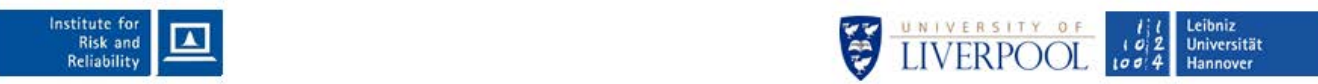

\section{HYDRO POWER PLANT SYSTEM}

- system model
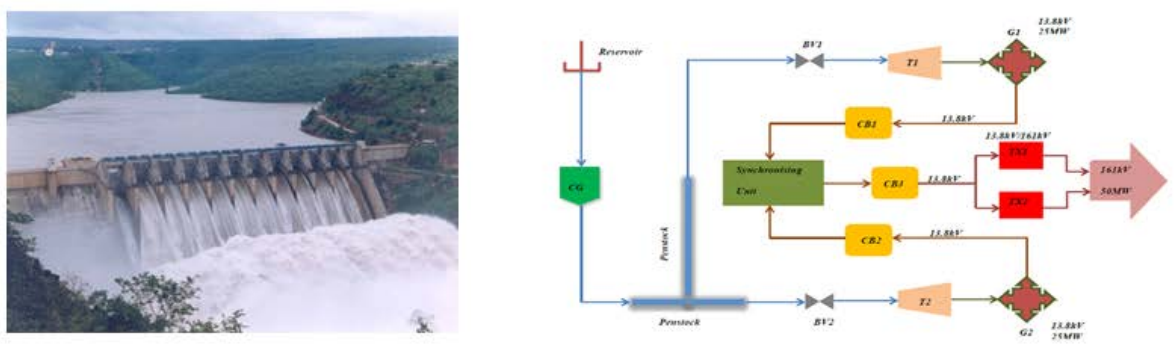

$\begin{array}{llr}\text { component type } & \text { distribution } & \text { parameters } \\ 1 & \text { Beta } & {[1.2,1.5][1.5,2.1]} \\ 2 & \text { Weibull } & {[1.0,1.6][2.1,2.5]} \\ 3 & \text { Exponential } & {[0.4,1.2]} \\ 4 & \text { Beta } & {[1.3,1.8][2.3,2.9]} \\ 5 & \text { Gamma } & {[1.2,1.4][2.8,3.3]} \\ 6 & \text { Gamma } & {[0.3,0.8][1.0,1.3]}\end{array}$




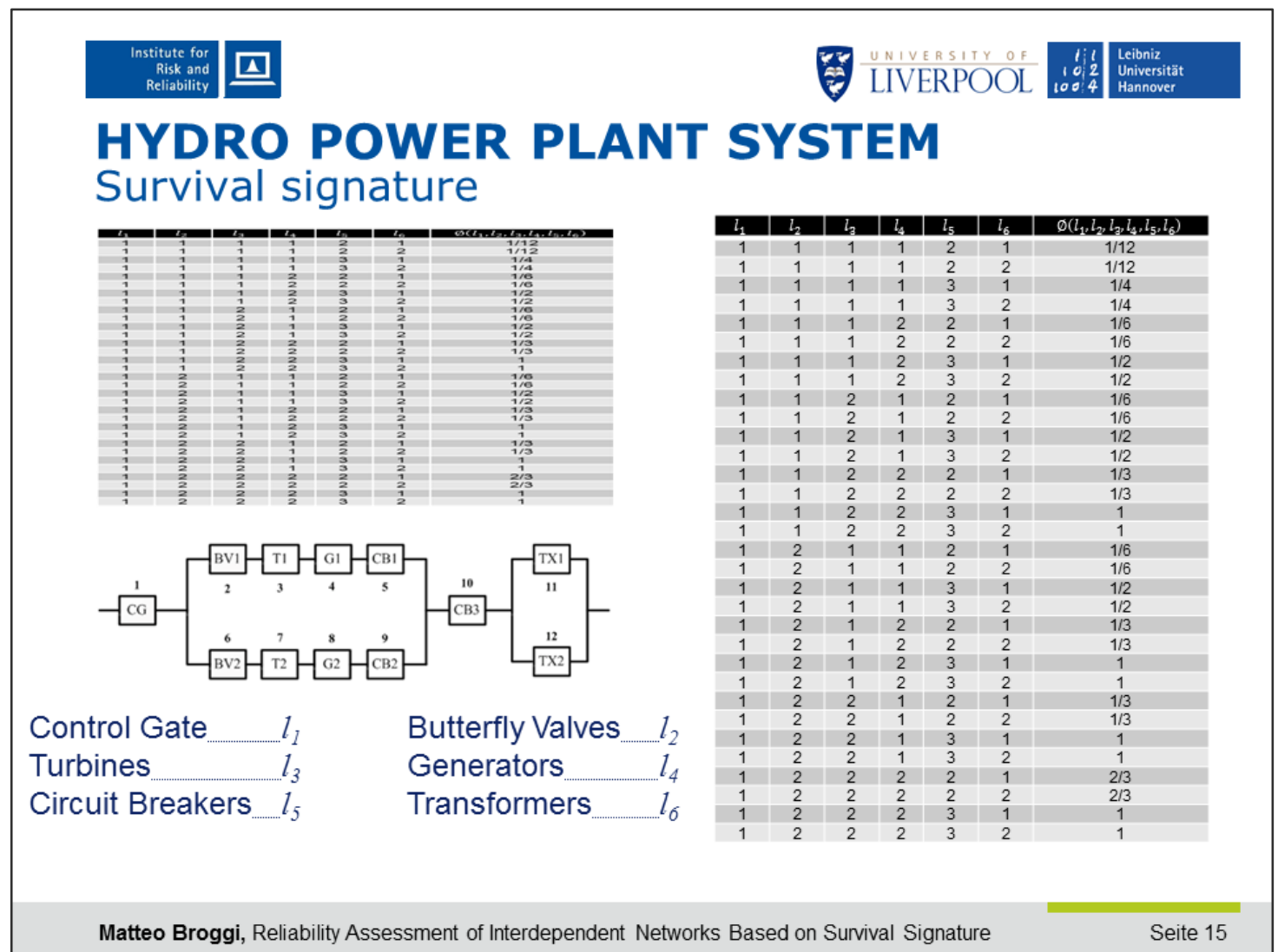

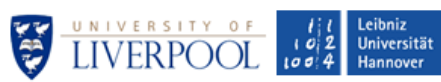

\section{HYDRO POWER PLANT SYSTEM} Results

- survival function

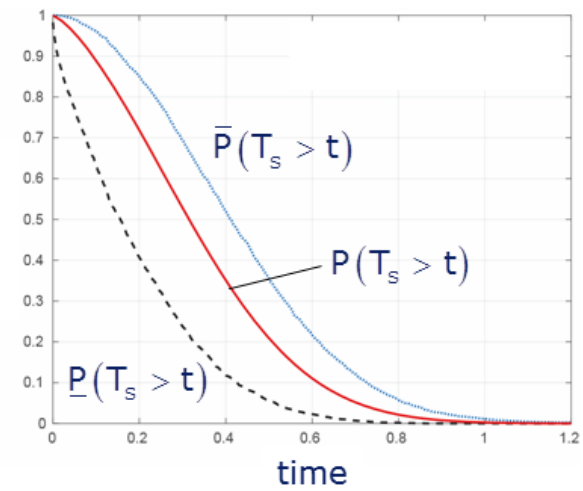

sensitivities of the system reliability wrt. the imprecision in the modeling

$\Rightarrow$ control of modeling accuracy
- relative importance index

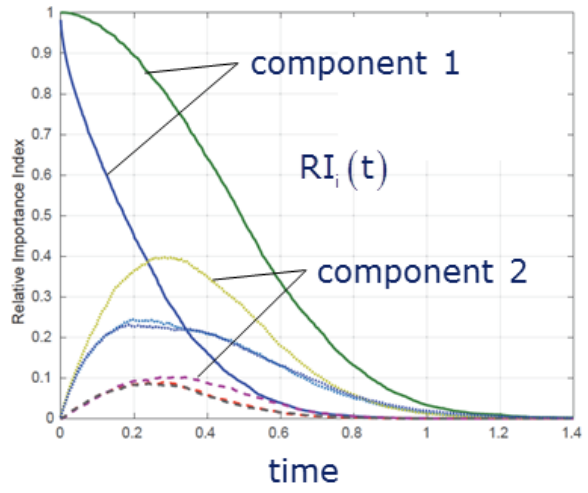

control of detection precision and modeling detailedness (model refinement in critical system parts) 
Institute for
Reliability

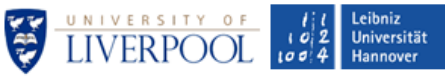

SYSTEM WITH COMMON CAUSE OF FAILURE

\begin{tabular}{llr} 
component & distribution & parameters \\
\hline A & Exponential & 1.5 \\
B & Exponential & 1.5
\end{tabular}

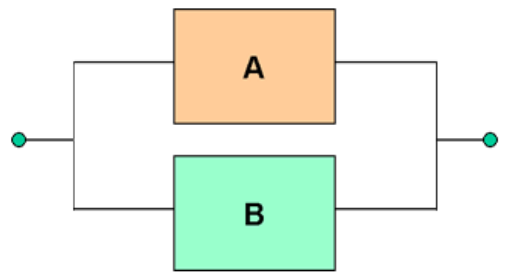

Common failure of components with a probability $\mathrm{p}=[.5, .9]$

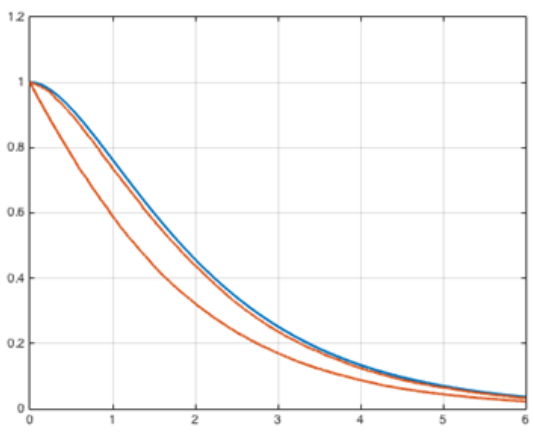

\section{SYSTEM WITH COMMON CAUSE OF FAILURE}

\begin{tabular}{llr} 
component & distribution & parameters \\
\hline A & Exponential & 1.5 \\
B & Exponential & 1.5
\end{tabular}

Common failure of components: largest extreme value distribution with parameters $\mu=[1.2,3]$ $\sigma=.1$
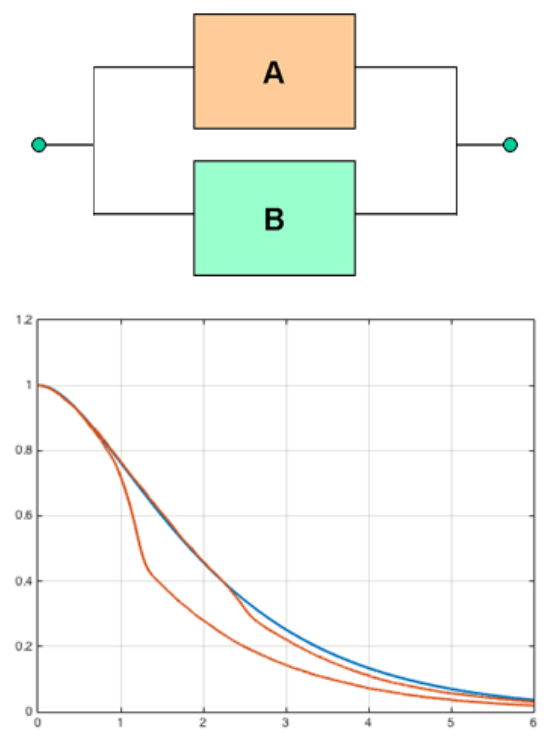
- Networks topology of IEEE RTS

- No other aspect taken

- Network 1

- Exponential components

- $\lambda$ chosen from uniform random in $[1,2]$

- Network 2

- Exponential components

- $\lambda$ chosen from uniform random in $[1,2]$

- Dependent failure as indicated

\section{TWO DEPENDENT SYSTEMS}

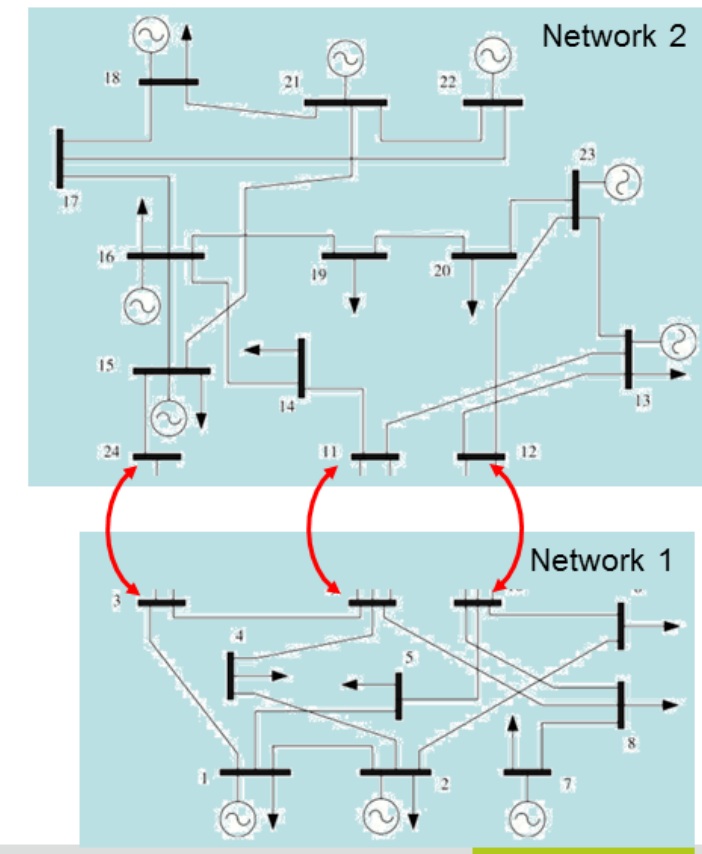

Matteo Broggi, Reliability Assessment of Interdependent Networks Based on Survival Signature

\section{Institute for
Reisk and
Reliability \\ TI UNIVERSITY OF

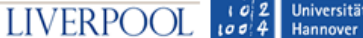

\section{TWO DEPENDENT SYSTEMS}

- Networks topology of IEEE RTS

- No other aspect taken

- Network 1

- Exponential components

- $\lambda$ chosen from uniform random in $[1,2]$

- Network 2

- Exponential components

- $\lambda$ chosen from uniform random in $[1,2]$

- Dependent failure as indicated
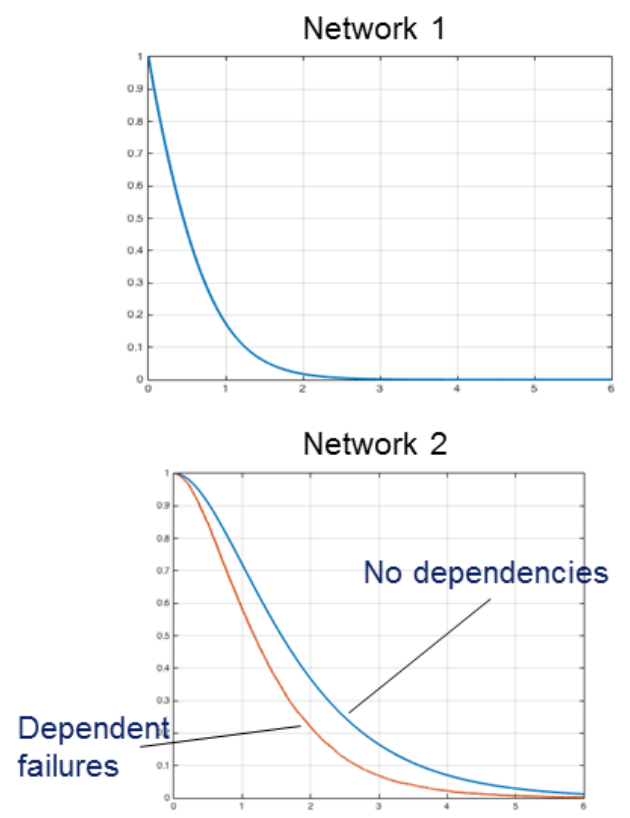


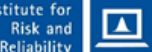

\section{CONCLUSIONS}

\section{LIVERPOOL $\log _{1002} \log _{4}$ Universitat}

Efficient and realistic reliability analysis of complex systems

Survival signature facilitates

- analysis of systems with multiple types of components

- stochastic sampling on a separated probability structure

- sensitivity analysis regarding importance of components

- model of dependent failures independent of network structure

Imprecise probabilities facilitate

- quantitative consideration of epistemic uncertainties

- identification of sensitivities wrt. model imprecision

- control of modeling accuracy

- identification of low-probability-high-consequence-events

\section{Institute for
Rels and
Reliability}

\section{BIBLIOGRAPHY}

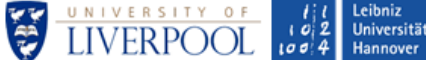

- F. J. Samaniego. System signatures and their applications in engineering reliability. Springer Science \& Business Media (2007), vol. 110

- S.V. Buldyrev, R. Parshani, G. Paul, H. E. Stanley, S. Havlin, Catastrophic cascade of failures in interdependent networks, Nature 464, (2010) 10251028

- F. P. Coolen, T. Coolen-Maturi, Generalizing the signature to systems with multiple types of components, in: Complex Systems and Dependability, 445 Springer, 2012, pp. 115-130.

- F. P. Coolen, T. Coolen-Maturi, A. H. Al-Nefaiee, Nonparametric predictive inference for system reliability using the survival signature, Proceedings of the Institution of Mechanical Engineers, Part O: Journal of Risk and Reliability 228 (5) (2014) 437-448.

- Geng Feng, Edoardo Patelli, Michael Beer, Frank P. Coolen, Imprecise System Reliability and Component Importance Based on Survival Signature, Reliability Engineering and System Safety, 150, 2016: 116-125 


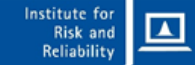

\section{Survival Function}

\section{T)}

- Reliability of the system based on survival signature

- The Survival Function is

- $C_{t}^{k}:=$ no. of components of type $k$ still working at time $t$

$$
P\left(T_{S}>t\right)=\sum_{l_{1}=0}^{m_{1}} \ldots \sum_{l_{k}=0}^{m_{k}} \Phi\left(l_{1}, \ldots, l_{k}\right) P\left(\bigcap_{k=1}^{K}\left\{C_{t}^{k}=l_{k}\right\}\right)
$$

- When probabilitipesof oromstoment are conditionally independent structure

$$
P\left(\bigcap_{k=1}^{K}\left\{C_{t}^{k}=l_{k}\right\}\right)=\prod_{k=1}^{K}\left(\begin{array}{c}
m_{k} \\
l_{k}
\end{array}\right)\left[F_{k}(t)\right]^{m_{k}-l_{k}\left[1-F_{k(t)}\right]^{l_{k}}}
$$

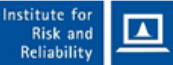 \\ G)}

\section{Relative Importance Index}

- The relative importance of a component $i$ is

$$
R I_{i}(t)=P\left(T_{S}>t \mid T_{i}>t\right)-P\left(T_{S}>t \mid T_{i} \leq t\right)
$$

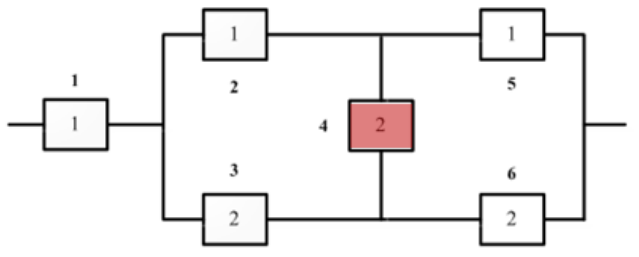

- $P\left(T_{S}>t \mid T_{1}>t\right)$ : $\mathrm{S}$ works given

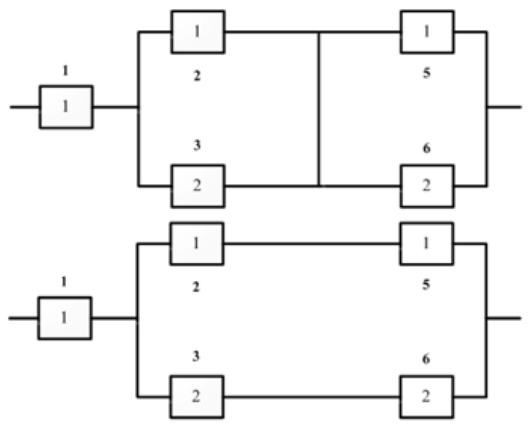




\section{Reliability Assessment of Interdependent Networks Based on Survival Signature}

Matteo Broggi graduated in Nuclear Engineering at the Polytechnic University of Milan in 2005. After working in AleniaAermacchi as a system safety engineer, in 2008 he joined the Institute of Engineerng Mechanics at the University of Innsbruck as a research assistant and post-graduate student, from where he received his Ph.D. in Civil Engineering at the end of 2011, specializing on numerical methods for structural reliability. In 2012, Dr. Broggi joined the Virtual Engineering Centre of the University of Liverpool as a post-doctoral research associate, where his main tasks were concentrated on the application of risk and reliability tools for problems of industrial interest. From 2014, Dr. Broggi held a dual position as project leader at the Virtual Engineering Centre and as a Lecturer at the Institute for Risk and Uncertainty, University of Liverpool. Since 2016, he joined the Institute for Risk and Reliability at the Leibniz University of Hannover as a senior research associate, where he is leader of the Uncertainty Quantification group at this institute. Dr. Broggi research interest is in numerical and computational tools for risk analysis, reliability and uncertainty quantification, in particular in the exploitation of high performance computing and artificial intelligence tools, with application in civil, mechanical and aerospace engineering. Recent research activities concentrated on model updating of finite element models and on development of algorithms for the reliability assessment of structural systems. 
Auroop Ganguly, PhD

Director, Sustainability \& Data Sciences Laboratory (SDS Lab)

Associate Professor, Civil \& Environmental Engineering

Northeastern University, Boston, MA 02115, USA

a.ganguly@neu.edu

(Prepared jointly with Udit Bhatia, PhD student, SDS Lab, with inputs from Dr. Evan Kodra, CEO, risQ)

SDS Lab: www.northeastern.edu/sds

risQ Corp: www.risq.io

Title:

Climate \& Complexity: The Resilience of Natural-Engineered-Human Systems

\begin{abstract}
:
The fundamental basis of design, operations and preservation of infrastructures and ecosystems have changed and are projected to alter significantly in the future owing to climate, hydrological, and socio-economic changes. The resilience of interdependent engineered lifeline networks, such as electric grids, water or waste water distribution systems, transportation and communication networks, and natural infrastructures such as open urban spaces and forests or marine or riverine ecosystems which protect against heat islands or storm surges and floods, to unprecedented climate induced extremes or changes in their statistical attributes, represent urgent societal priorities. Natural-built systems are intimately linked to human activity and depend on human intervention for strengthening and restoration. We describe novel network and data science methods and new methodological adaptations for climate-extremes resilient design, operations, and maintenance of critical infrastructures, which can in turn help us understand and develop actionable predictive insights on the stressors (climate hazards), as well as on the stressed (natural or built systems) and impacted (human or economic dimensions) systems. We discuss how computational and engineering tools can be brought to bear on these challenges, and how they can be aligned to provide useful and usable solutions for economic well-being, security, public health, governance and policy.
\end{abstract}




\title{
Climate \& Complexity: The Resilience of Natural-Built-Human Systems
}

\author{
Presenter: Auroop R. Ganguly \\ Preparers: Udit Bhatia and Auroop R. Ganguly* \\ Contributors: Current \& Former SDS Lab (and prior ORNL) Members \\ www.northeastern.edu/sds \\ (*Point of Contact: a.ganguly@neu.edu)
}

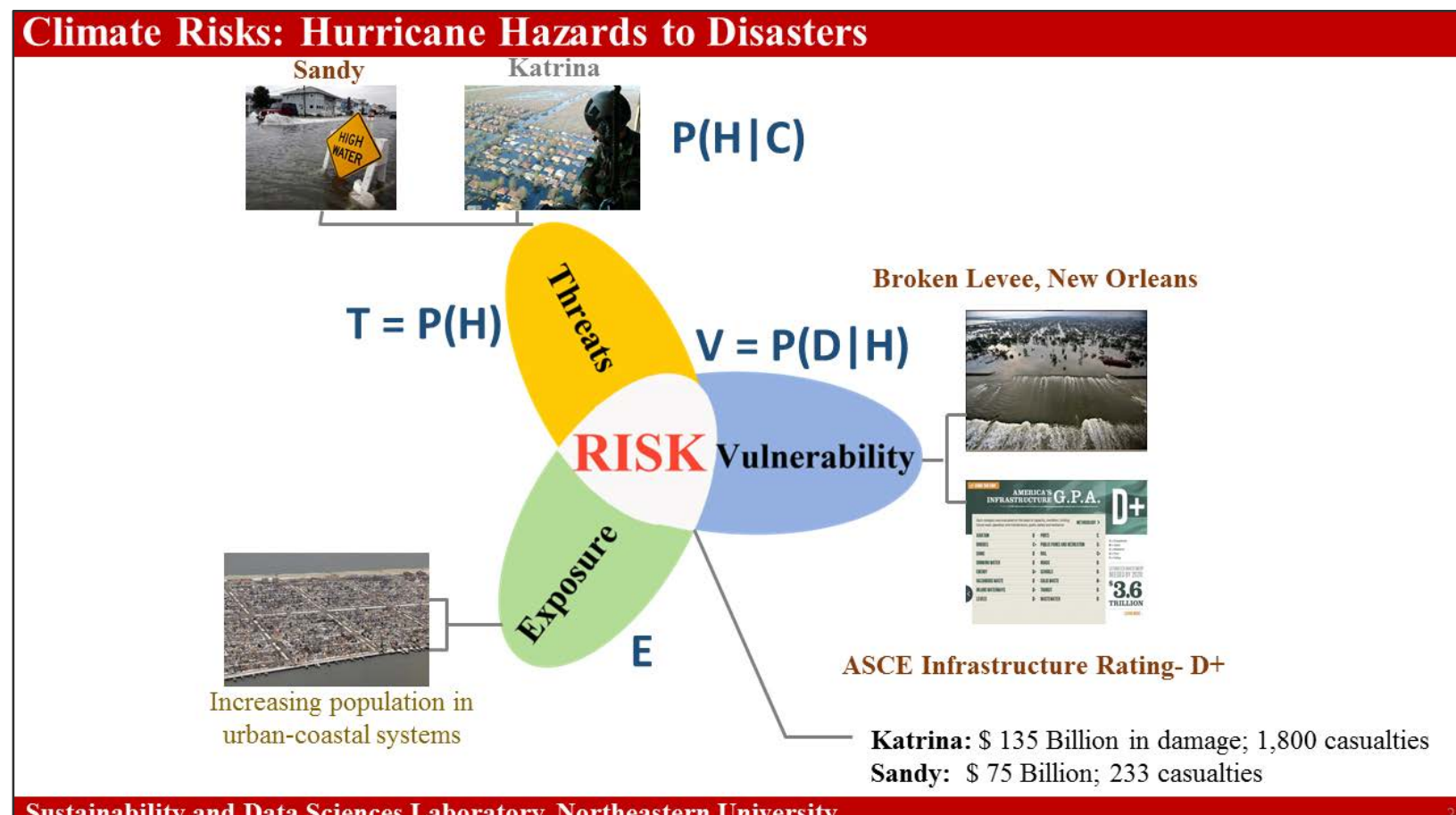




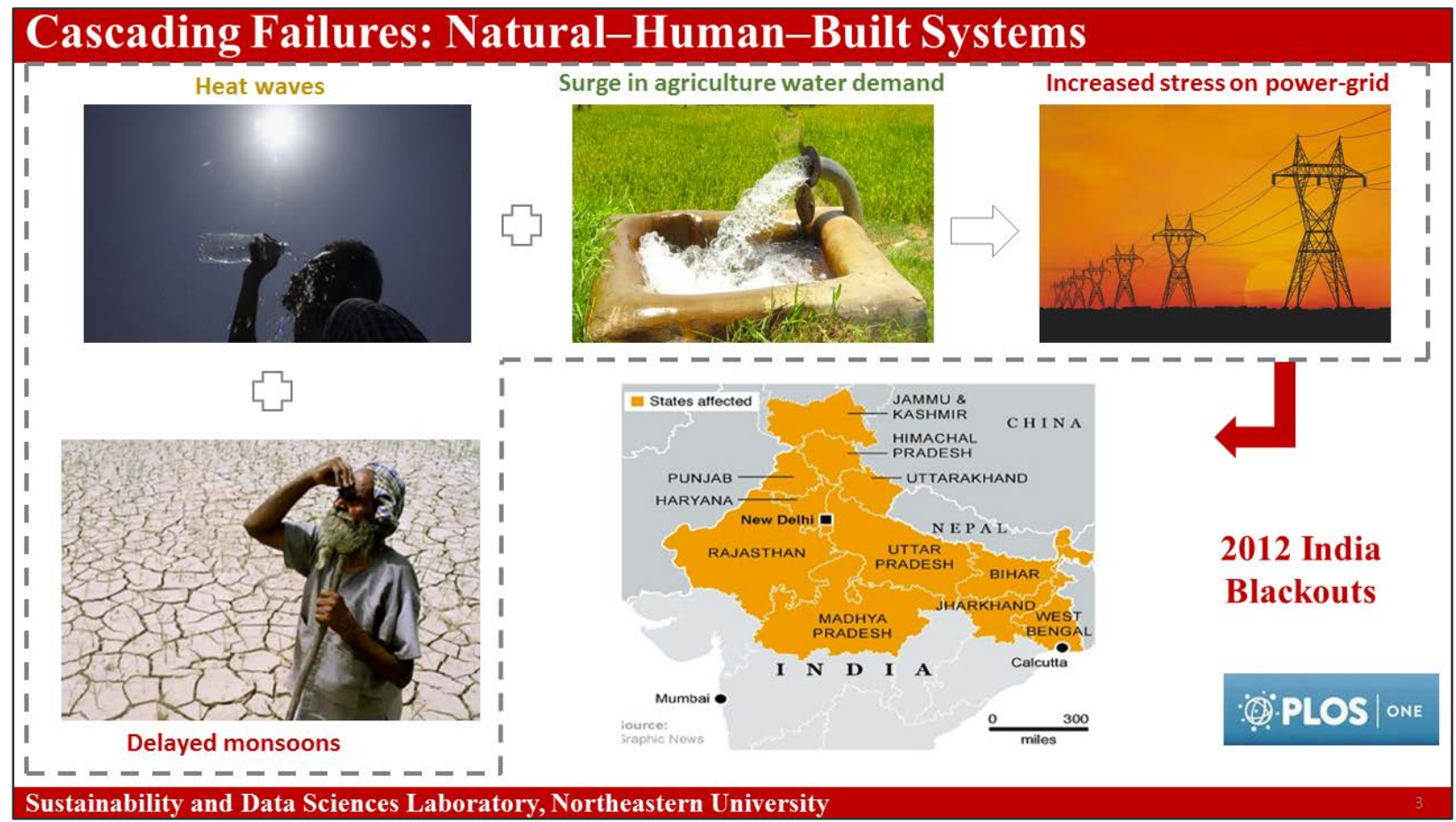

\section{Cascading Interdependencies: Network of Lifelines}

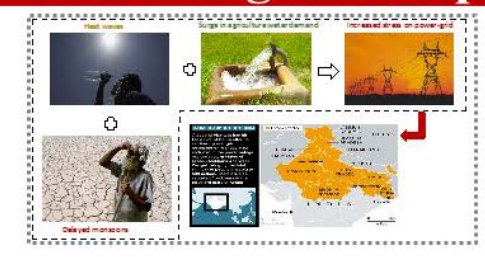

2012 Power Blackout Impacts on Indian Railways Network (IRN)
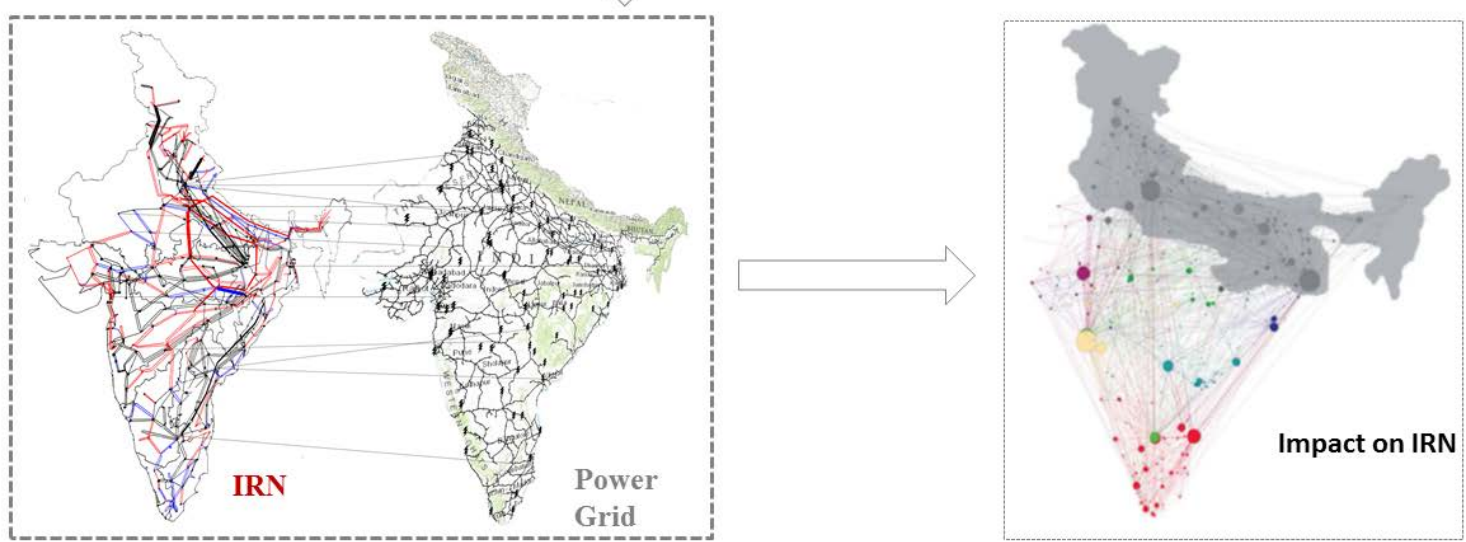

Sustainability and Data Sciences Laboratory, Northeastern University 


\section{Risk to Resilience: A Paradigm Shift}
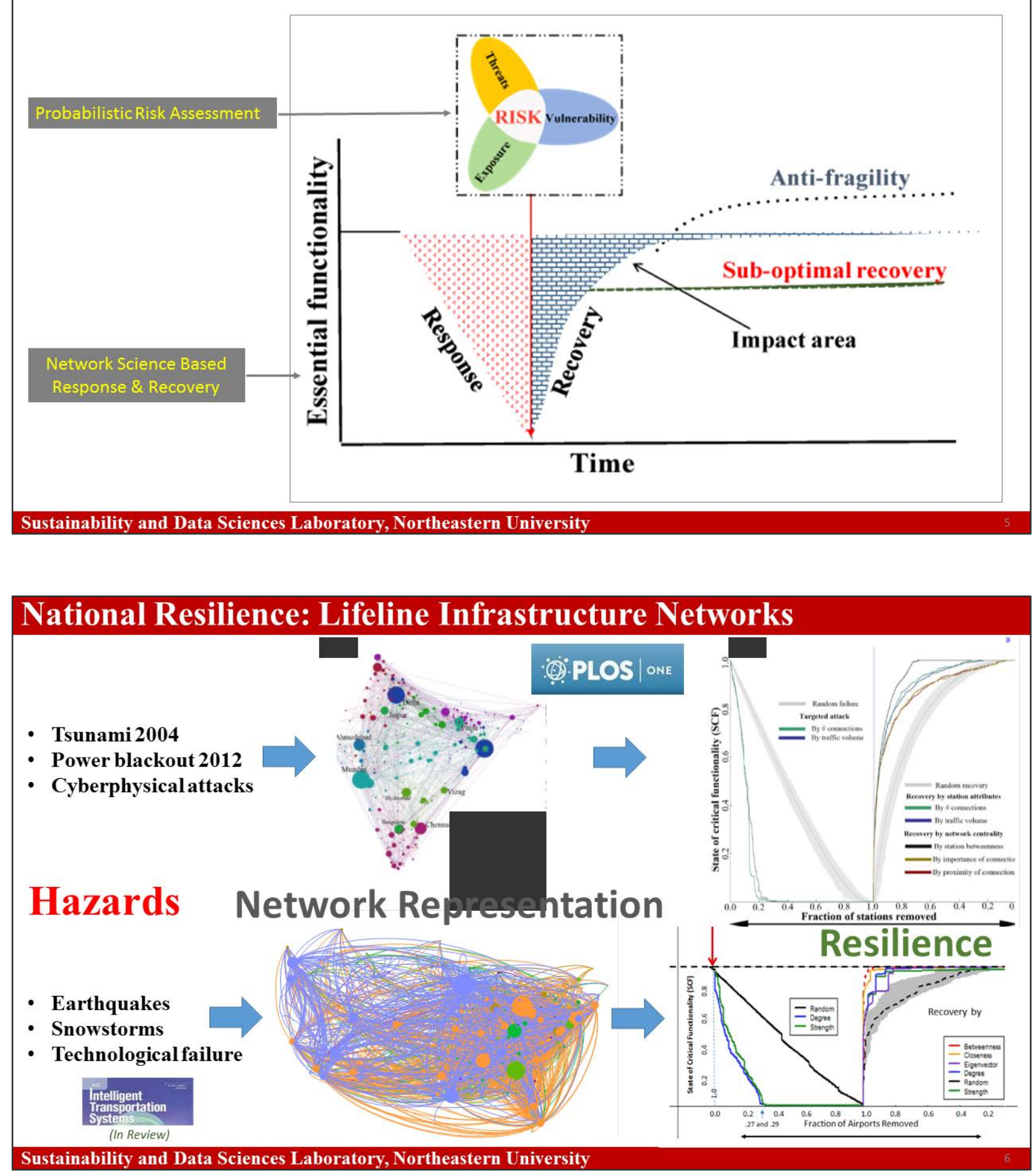

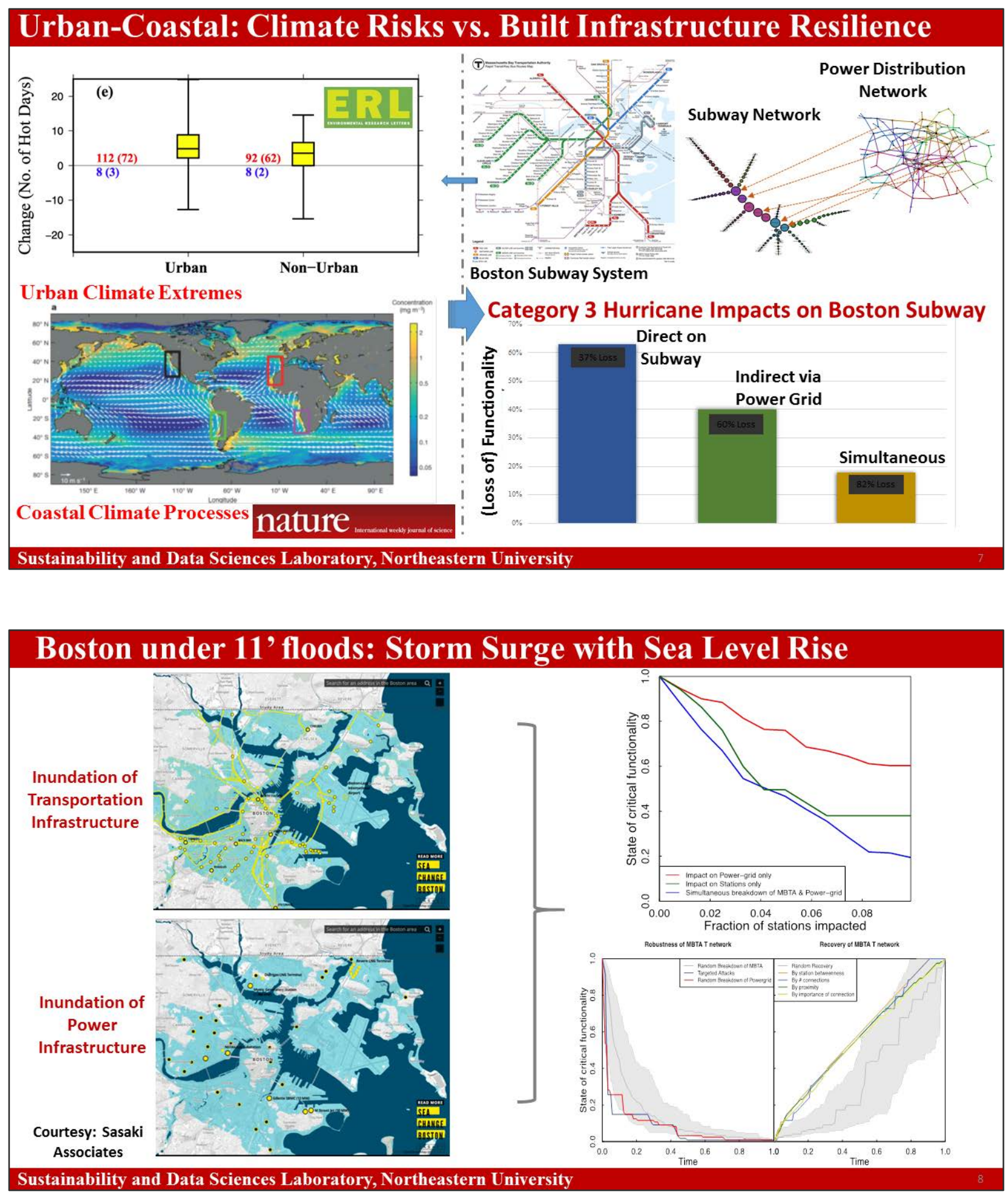


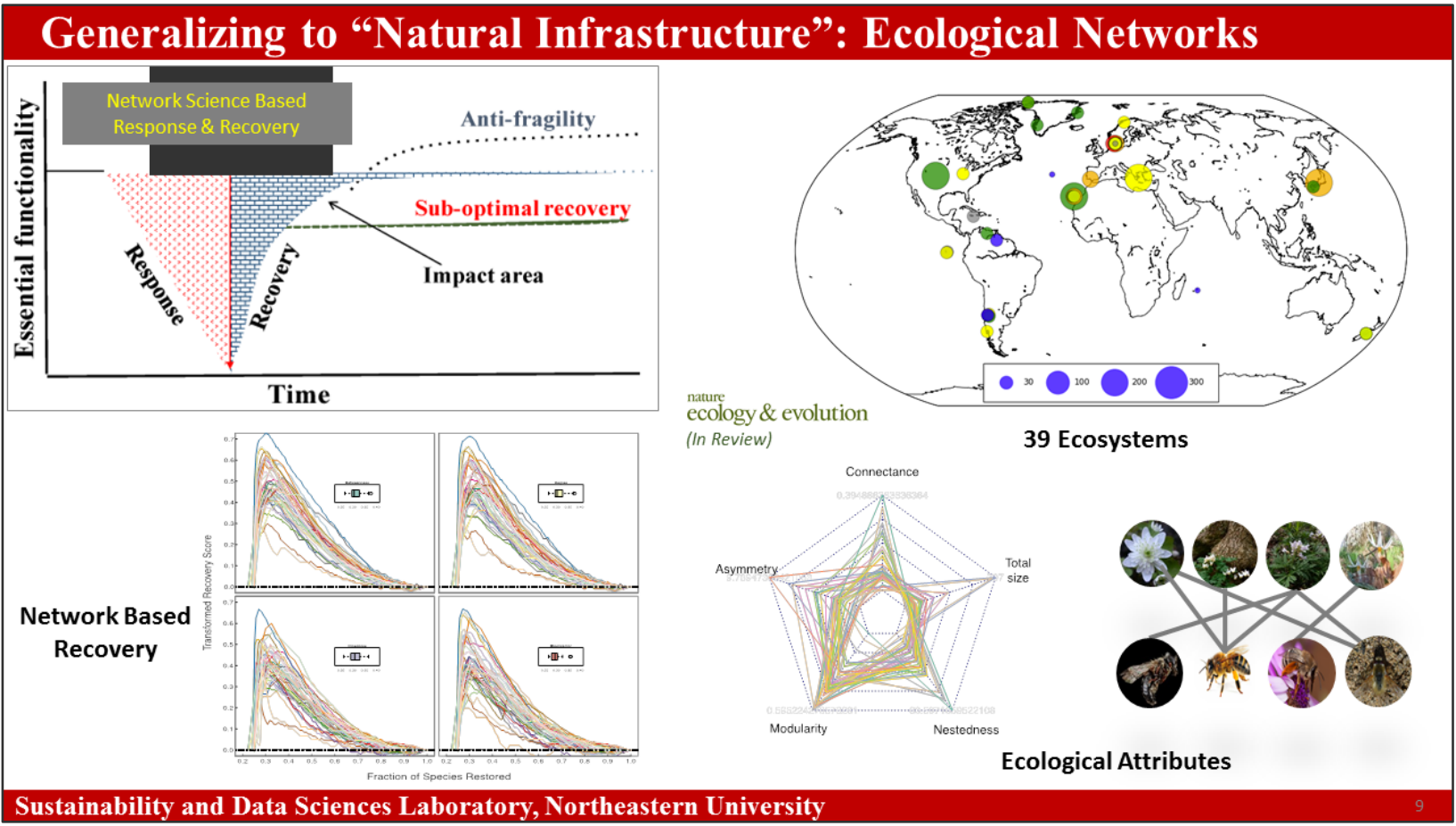

\section{Real-World Impacts: Resilience Best Practice}

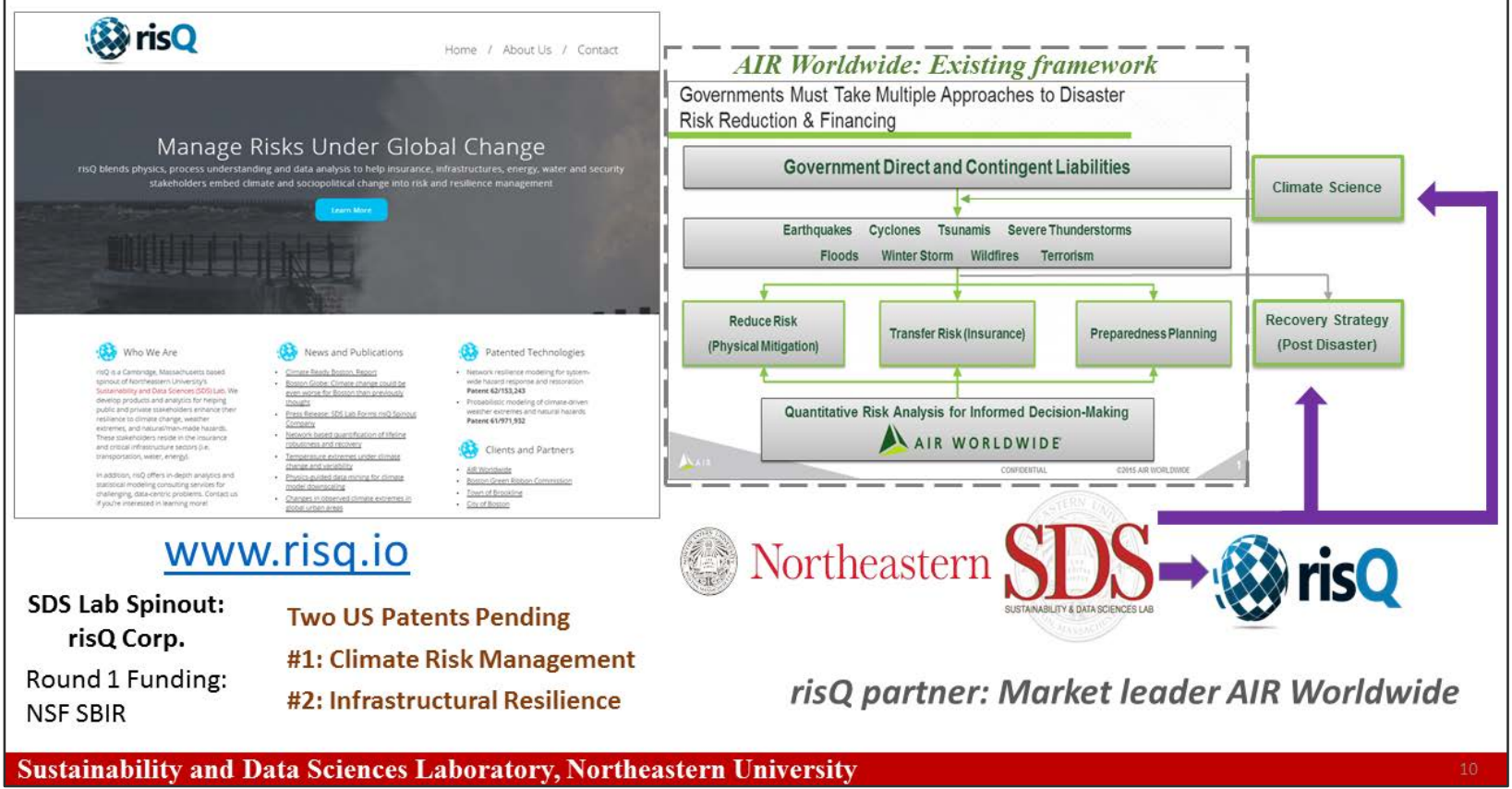




\title{
NIST \& Colorado State Resilience Workshop, October 19-21, 2016
}

\section{Climate \& Complexity: The Resilience of Natural-Built-Human Systems}

\author{
Presenter: Auroop R. Ganguly \\ Preparers: Udit Bhatia and Auroop R. Ganguly* \\ Contributors: Current \& Former SDS Lab (and prior ORNL) Members \\ www.northeastern.edu/sds \\ (*Point of Contact: a.ganguly@neu.edu)
}




\section{RELEVANT REFERENCES:}

\section{Our Publications}

1. Ganguli P, Ganguly AR. Robustness of Meteorological Droughts in Dynamically Downscaled Climate Simulations. JAWRA J Am Water Resour Assoc. 2016 Feb 1;52(1):138-67.

2. Ganguly AR, Steinhaeuser K, Erickson DJ, Branstetter M, Parish ES, Singh N, et al. Higher trends but larger uncertainty and geographic variability in 21st century temperature and heat waves. Proc Natl Acad Sci. 2009 Sep 15;106(37):15555-9.

3. Kodra E, Ganguly AR. Asymmetry of projected increases in extreme temperature distributions. Sci Rep [Internet]. 2014 Jul 30 [cited 2015 Apr 18];4. Available from: http://www.nature.com/srep/2014/140730/srep05884/full/srep05884.html

4. Kodra E, Steinhaeuser K, Ganguly AR. Persisting cold extremes under 21st-century warming scenarios. Geophys Res Lett. 2011 Apr 28;38(8):L08705.

5. Climate change: Cold spells in a warm world. Nature. 2011 Apr 14;472(7342):139-139.

6. Ghosh S, Das D, Kao S-C, Ganguly AR. Lack of uniform trends but increasing spatial variability in observed Indian rainfall extremes. Nat Clim Change. 2012 Feb;2(2):86-91.

7. Kao S-C, Ganguly AR. Intensity, duration, and frequency of precipitation extremes under 21stcentury warming scenarios. J Geophys Res Atmospheres. 2011;116(D16):D16119.

8. Bhatia U, Kumar D, Kodra E, Ganguly AR. Network Science Based Quantification of Resilience Demonstrated on the Indian Railways Network. PLoS ONE. 2015 Nov 4;10(11):e0141890.

9. Mishra V, Ganguly AR, Nijssen B, Lettenmaier DP. Changes in observed climate extremes in global urban areas. Environ Res Lett. 2015 Feb 1;10(2):24005.

10. Wang D, Gouhier TC, Menge BA, Ganguly AR. Intensification and spatial homogenization of coastal upwelling under climate change. Nature. 2015 Feb 19;518(7539):390-4.

11. Ganguli P, Ganguly AR. Space-time Trends in U.S. Meteorological Droughts. J Hydrol Reg Stud. 2016; In press.

12. Salvi K, Ghosh S, Ganguly AR. Credibility of statistical downscaling under nonstationary climate. Clim Dyn. 2015 Jun 10;1-33.

13. Ganguly AR, Kodra EA, Agrawal A, Banerjee A, Boriah S, Chatterjee S, et al. Toward enhanced understanding and projections of climate extremes using physics-guided data mining techniques. Nonlin Process Geophys. 2014 Jul 28;21(4):777-95.

14. Bhatia U, Gouhier TC, Ganguly AR. Universal and generalizable restoration strategies for degraded ecological networks. Rev. 2016.

15. Clark KL, Bhatia U, Kodra E, Ganguly AR. Resilience of the US National Airspace System Airport Network. Rev. 2015;

16. Bhatia U, Kumar D, Kodra E, Ganguly AR. Water Complexity and Physics Guided Data Mining. In: Water Diplomacy in Action: Contingent Approaches to Managing Complex Water Problems: An Edited Volume for the Anthem Water Diplomacy Series. Anthem press; 2016.

\section{Other publications}

1. IPCC. Climate Change 2014: Impacts, Adaptation, and Vulnerability. Part B: Regional Aspects. Contribution of Working Group II to the Fifth Assessment Report of the Intergovernmental Panel on Climate Change [Barros, V.R., C.B. Field, D.J. Dokken, M.D. Mastrandrea, K.J. Mach, T.E. Bilir, M. Chatterjee, K.L. Ebi, Y.O. Estrada, R.C. Genova, B. Girma, E.S. Kissel, A.N. Levy, S. 
MacCracken, P.R. Mastrandrea, and L.L. White (eds.)]. Cambridge, United Kingdom and New York, NY, USA: Cambridge University Press; 2014. 688 p.

2. Linkov I, Bridges T, Creutzig F, Decker J, Fox-Lent C, Kröger W, et al. Changing the resilience paradigm. Nat Clim Change. 2014 Jun;4(6):407-9.

3. Urban MC. Accelerating extinction risk from climate change. Science. 2015 May 1; 348(6234): 571-3.

4. Buldyrev SV, Parshani R, Paul G, Stanley HE, Havlin S. Catastrophic cascade of failures in interdependent networks. Nature. 2010 Apr 15;464(7291):1025-8.

5. Ouyang M, Wang Z. Resilience assessment of interdependent infrastructure systems: With a focus on joint restoration modeling and analysis. Reliab Eng Syst Saf. 2015 Sep;141:74-82.

6. Romero JJ. Blackouts illuminate India's power problems. IEEE Spectr. 2012 Oct;49(10):11-2.

7. Albert R, Jeong H, Barabási A-L. Error and attack tolerance of complex networks. Nature. 2000 Jul 27;406(6794):378-82.

8. Bhatia U, Kumar D, Kodra E, Ganguly AR. Network Science Based Quantification of Resilience Demonstrated on the Indian Railways Network. PLoS ONE. 2015 Nov 4;10(11):e0141890.

9. Mazdiyasni O, AghaKouchak A. Substantial increase in concurrent droughts and heatwaves in the United States. Proc Natl Acad Sci. 2015 Sep 15;112(37):11484-9.

\section{Acknowledgments}

We acknowledge the work by the current and former lab members of Sustainability and Data Sciences Laboratory (SDS Lab) at Northeastern University and former colleagues at US Department of Energy's Oak Ridge National Laboratory (ORNL). Specifically, we would like to thank Babak Fard, Kevin Clark, Hanieh Hassanzadeh, Poulomi Ganguli, and Stephen Flynn for helpful suggestions and contributions. Funding from the National Science Foundation's Expeditions in Computing, Big Data and Cyber SEES projects are gratefully acknowledged, as are contributions from the Department of Homeland Security and the Department of Energy. 


\section{Auroop Ganguly: Bio-sketch}

Auroop R. Ganguly works in climate and hydrological extremes and their impacts on water and infrastructure systems with data and computational methods. His research on climate change and weather extremes has led to novel and occasionally surprising insights for heat waves, cold snaps, heavy precipitation and droughts. New information for stakeholders and engineers have been generated for impacts on urban coastal and water-energy systems, leading to studies commissioned by large and small cities or federal agencies. Extending his work to man-made hazards, Ganguly has developed methods to extract potentially elusive indicators of threat from massive and heterogeneous data, for multiple federal agencies. Moving from hazards to risks and resilience, Ganguly has developed restoration and resilience methods for critical lifeline infrastructures, including for natural, technological and cyber-physical hazards, and perturbed ecological networks, at multiple scales. He has developed new "big data" science methods for rare or anomalous events and extremes, data mining and sparse learning from spatiotemporal data obtained from in-situ and remote sensors or dynamical model simulations, network science for dependence and teleconnections in dynamical systems and for timely and effective recovery in lifelines, uncertainty characterization in complex systems, nonlinear correlation with the mutual information for short and noisy data, nonlinear dimensionality reduction, and characterization of nonlinear behavior and dependence. Connection across domains such as climate or water versus infrastructures or sensors has been through impacts and system modeling, as well as via the underlying thread of computational and data methods with a focus on rare and extreme events in complex dynamical systems. He has also developed physics-guided data science approaches across domains.

Ganguly's research has been published in interdisciplinary journals such as Nature, PNAS, Nature Climate Change, Nature's Scientific Reports and PLOS One, climate or geophysics journals such as Journal of Climate, Journal of Geophysical Research, Geophysical Research Letters and Climate Dynamics, water journals such as Water Resources Research, Advances in Water Resources, Journal of Hydrology, Journal of Hydrometeorology, and JAWRA, nonlinear dynamics journals such as Physical Review E, and Nonlinear Processes in Geophysics, computer or data science journals such as Statistical Analysis and Data Mining and top peer-reviewed conferences such as SIAM Data Mining, and electrical engineering journals such as IEEE Transactions on Geoscience and Remote Sensing, IEEE Sensors, and IEEE Transactions on Intelligent Transportation Systems. He has published two edited books on Knowledge Discovery from Sensor Data and published multiple book chapters. His research has been highlighted in Nature news, NSF news, and the American Geophysical Union, and reported widely and over the last decade in the mainstream national and international media. He has won best paper awards in data and computer science conferences, and he has been interviewed by Live Science, NSF, and showcased under Faces of NSF. His research has been funded, among others, by NSF, DOE, ORNL and ARPA-E, DHS, DOD and DARPA, and other private or public sector organizations, influenced key investment and stakeholder decisions, and advanced best practices. He has two patents pending, which have led to new products in climate risk management and infrastructural resilience at a startup. He served as an associate editor of the AGU journal Water Resources Research and continues to be an associate editor for ASCE's Journal of Computing in Civil Engineering, besides currently serving on the editorial board of the journal Scientific Reports published by the Nature Publishing Group. He has received an outstanding reviewer award by ASCE, a faculty fellow award at Northeastern, an outstanding joint faculty award from the University of Tennessee, Knoxville, and scientific and mentorship awards from the Oak Ridge National Laboratory (ORNL) and US DOE.

Ganguly teaches climate change science and policy, as well as probability and engineering economy, to undergraduates, and critical infrastructures resilience, as well as applied time series and spatial analysis, to graduate students. A civil and environmental engineer with a PhD in hydrology from MIT and an undergraduate from the Indian Institute of Technology, he has five years of experience at Oracle Corporation in time series and demand forecasting, about a year at a semi-startup that got acquired by Oracle, and seven years at the Oak Ridge National Laboratory in both computational sciences and engineering as well as in climate change science. Over the last five years, he has been at Northeastern University in Boston, currently as a tenured associate professor of civil and environmental engineering, where he directs the Sustainability and Data Sciences Laboratory (SDS Lab). He is the Chief Scientific Advisor and co-founder, with his former PhD student, of risQ Corporation, a spinout from the SDS Lab. 


\title{
Natural Disaster Impacts on Infrastructure Systems and Society
}

\author{
Judith Mitrani-Reiser, Ph.D. \\ National Institute of Standards and Technology (NIST) \\ Johns Hopkins University (JHU)
}

\begin{abstract}
In recent years, many disasters have occurred which resulted in damage to critical community functions. The Christchurch, Bío-Bío, and Nepal earthquakes, Hurricanes Sandy and Katrina, and tornadoes in Joplin, MO and Moore, OK all resulted in severe damage to local hospitals, putting great strain on the healthcare systems of these regions. The continued functionality of critical infrastructure, such as healthcare facilities, is necessary following a major event. Healthcare delivery facilities are essential in disasters: they provide emergency medical care related to the event and regular health services required to maintain the health of the community they serve. In order to provide adequate services to patients, healthcare facilities rely on a wide range of internal and external functions, each of which are part of a complex network of interacting systems. The loss of a single function can severely disrupt the ability to provide care during the critical first hours.
\end{abstract}

In order to improve the resilience of facilities like these, decision makers first need a way to quantify their performance due to extreme loading from natural hazards, both predictively and retrospectively. This presentation will show a risk analysis framework for quantifying and predicting the loss, recovery, and resilience of critical facilities. The theoretical framework accounts for loss of service due to building and utility damage, as well as impacts to key personnel and resources/supplies needed to provide clinical and non-clinical services. The talk will introduce ongoing work focused on the resilience of critical infrastructure-based societal systems (CIbSS), and on the CoPE-WELL (Composite of Post-event Well-being) systems model of functioning and well-being of a community over time. 

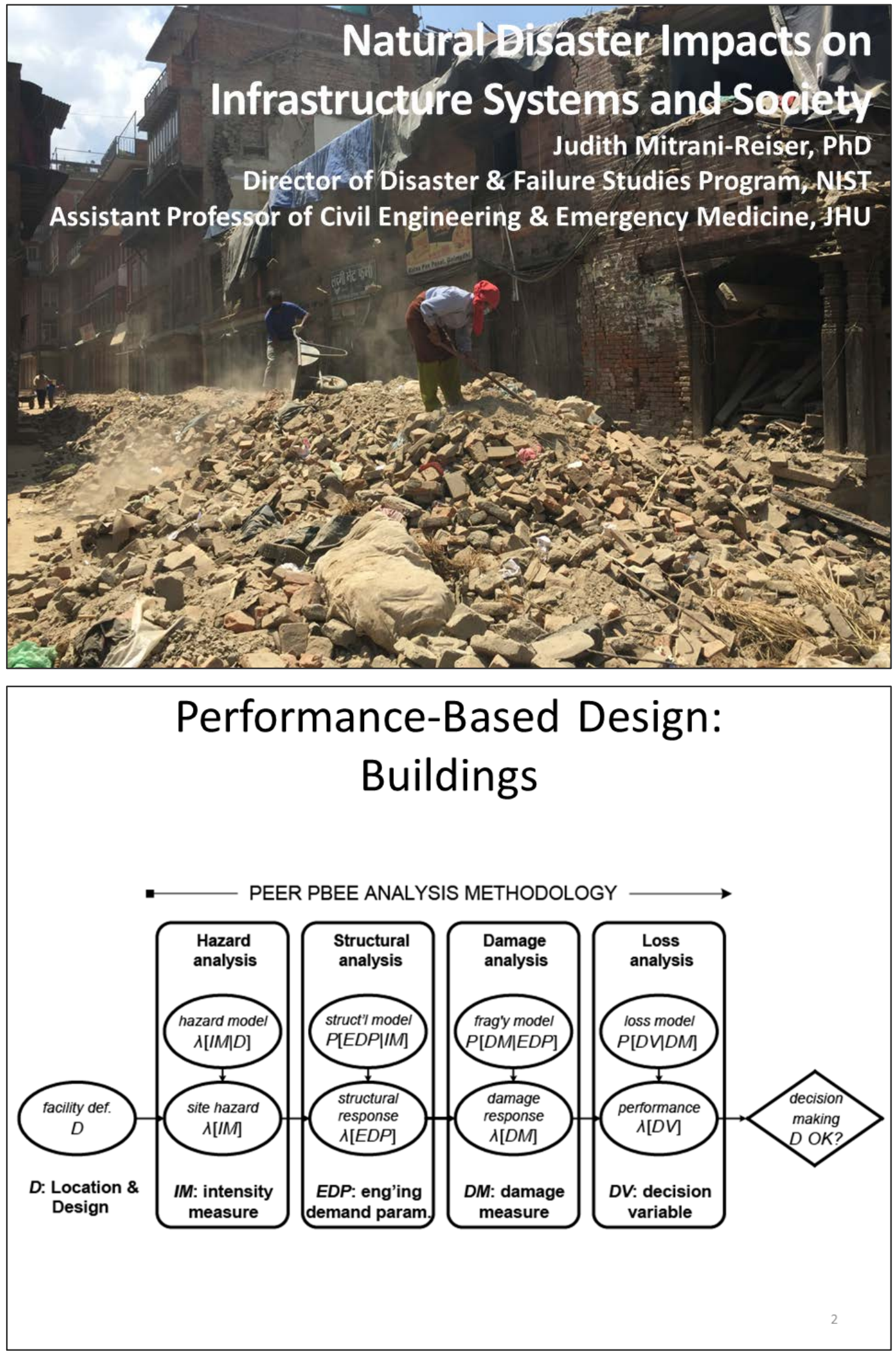

C-78 


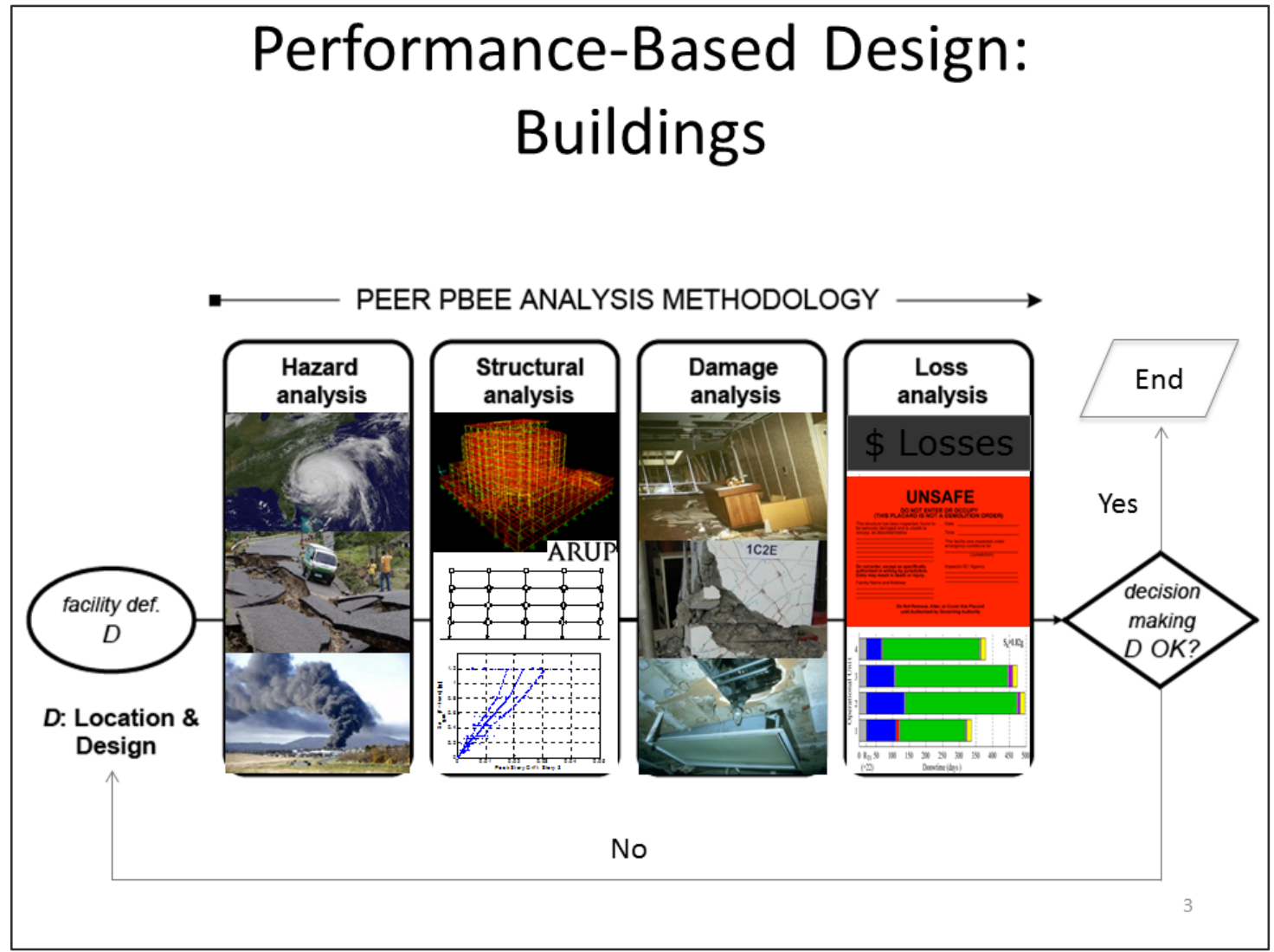

\section{Performance-Based Design: Downtime in Buildings}

ATC-58 procedures (Mitrani-Reiser) provide the following measures of occupancy interruption:

- The length of time necessary to conduct repairs,

- The need to procure items with long lead-times,

- The probability that the building will be placarded as unsafe for occupancy.

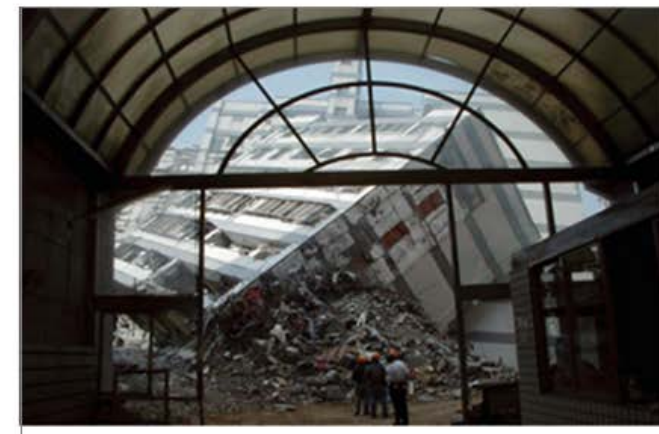

Seismic Performance Assessment of Buildings Volume 1 -Methodology

FEMA P-58-1 / September 2012 


\section{Performance-Based Design: Downtime in Buildings}

Repair time is the time needed to repair the earthquake damage and return the building to its pre-earthquake condition.

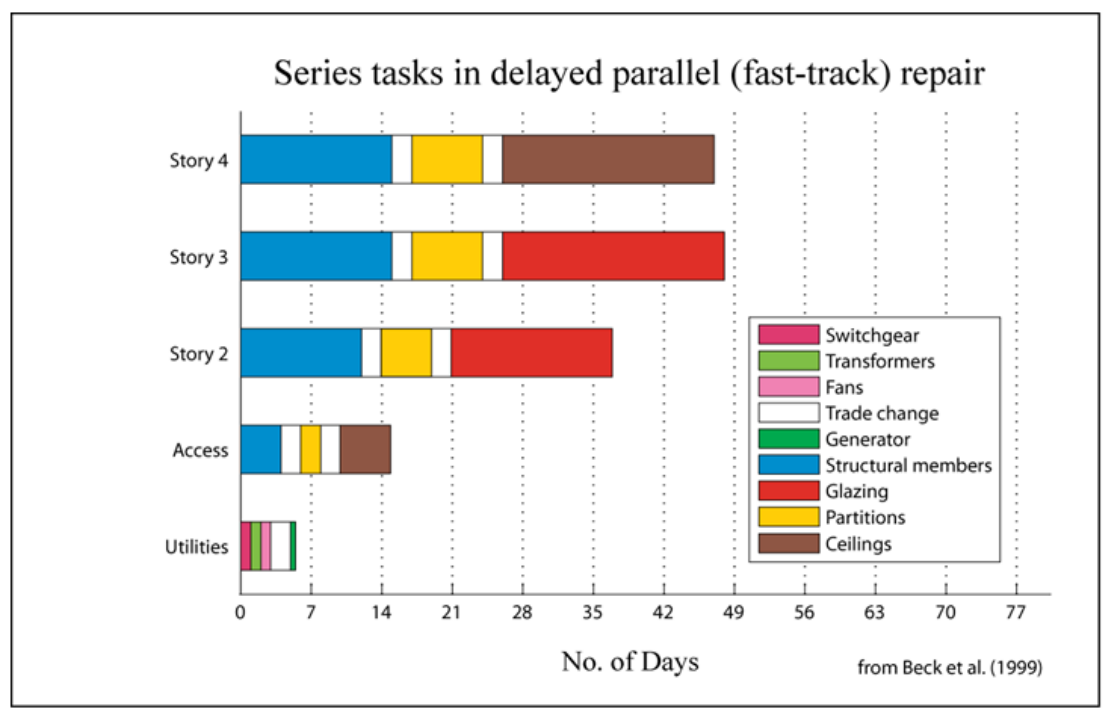

\section{Performance-Based Design: Downtime in Buildings}

Mobilization Time is the delay before construction begins needed to assess damage and inspect building, time to consult with professional engineers, time for bidding process, time for clean-up, time to acquire items with long lead times.

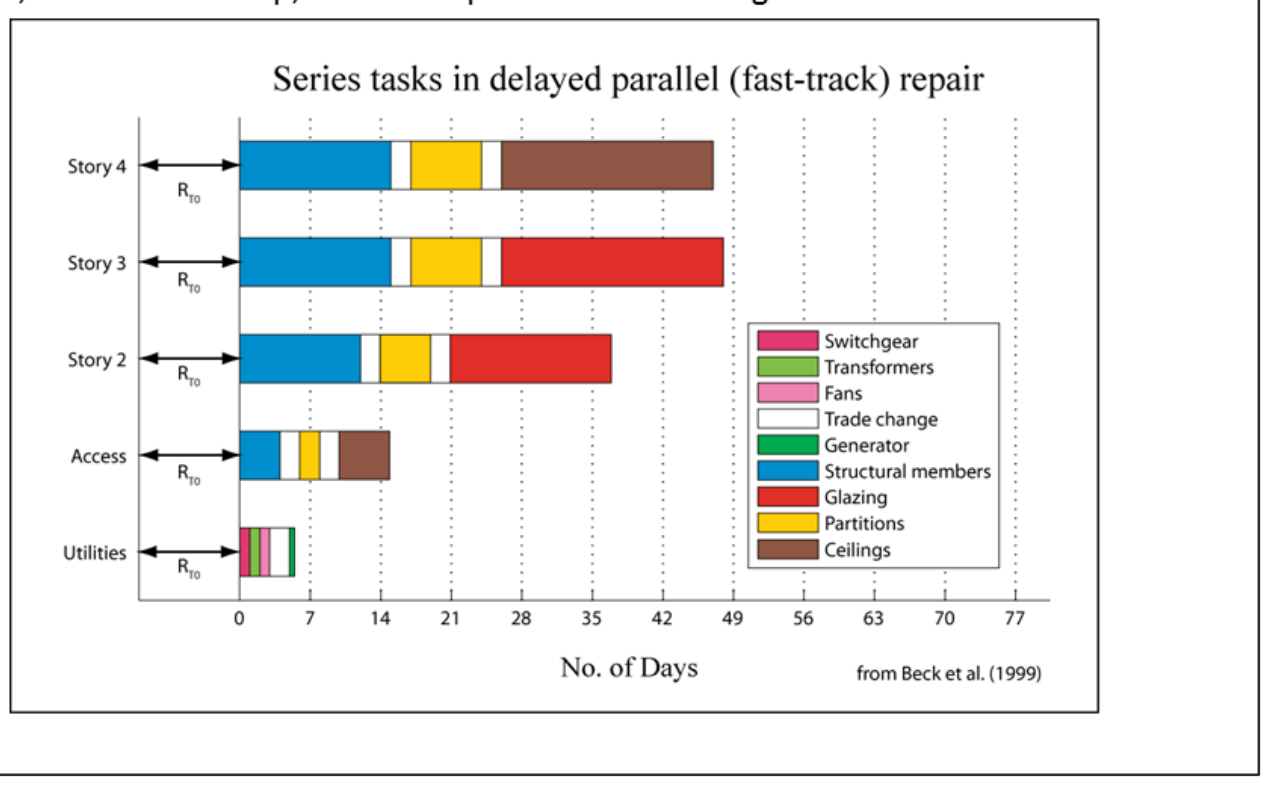




\section{Performance-Based Design: Downtime in Buildings}

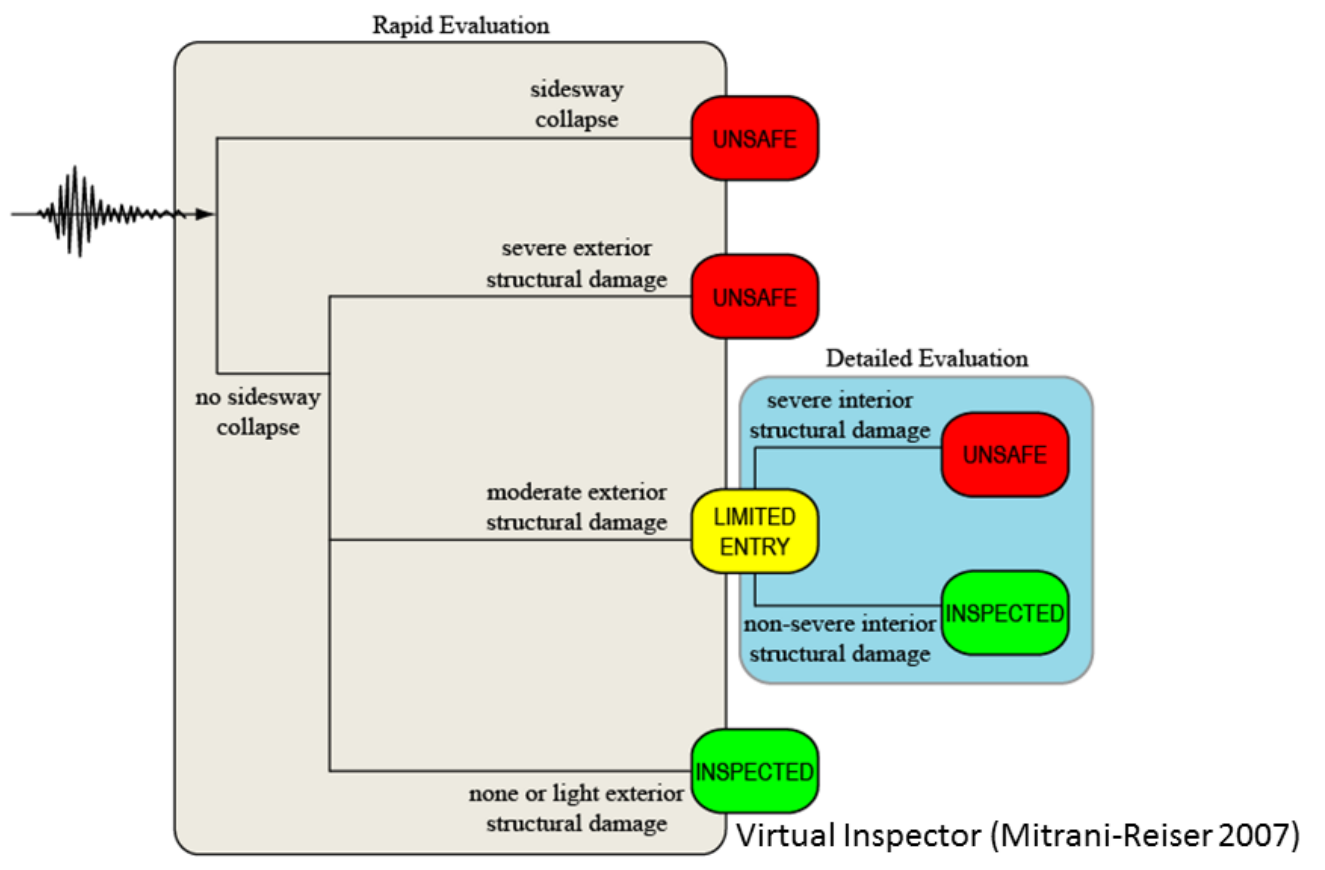

\section{Performance-Based Design: Downtime in Buildings}

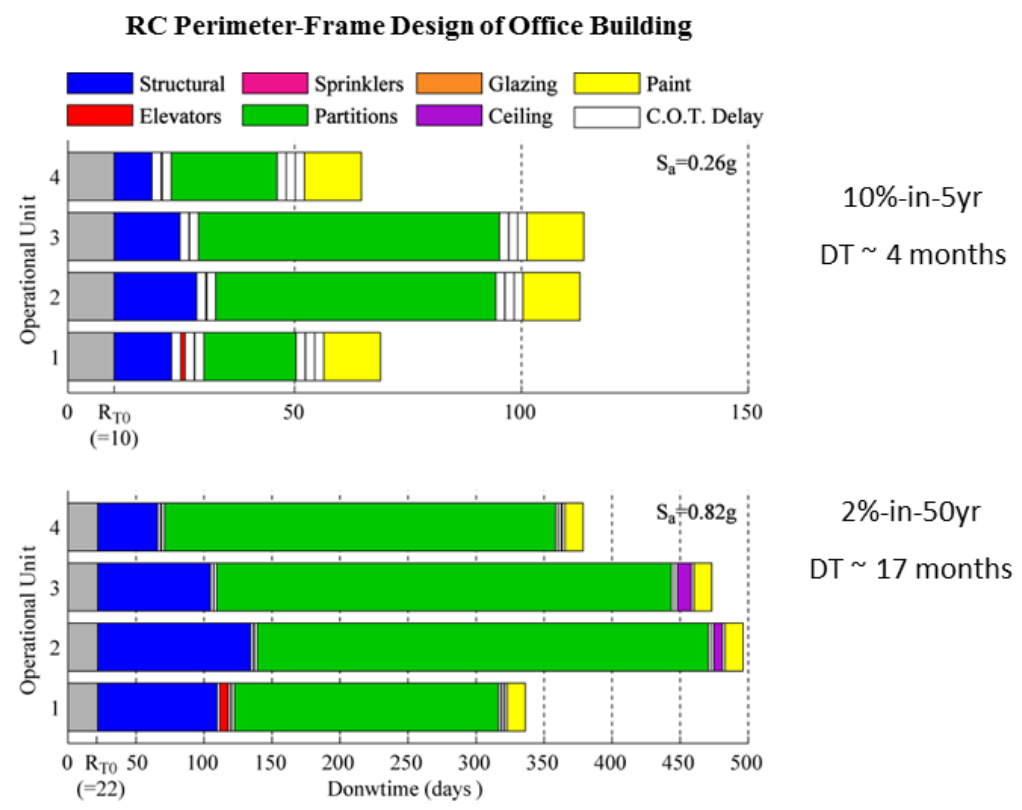




\section{Performance-Based Design: Summary}

For some building occupancies (i.e., hospitals), the above procedures will not suffice in capturing the loss of important services:

- Need models that include infrastructure failures outside the building.

- Need occupancy-specific models that incorporate human infrastructure.

- Need systematic procedures for capturing building damage and loss of function over time in the field (eq reconnaissance).

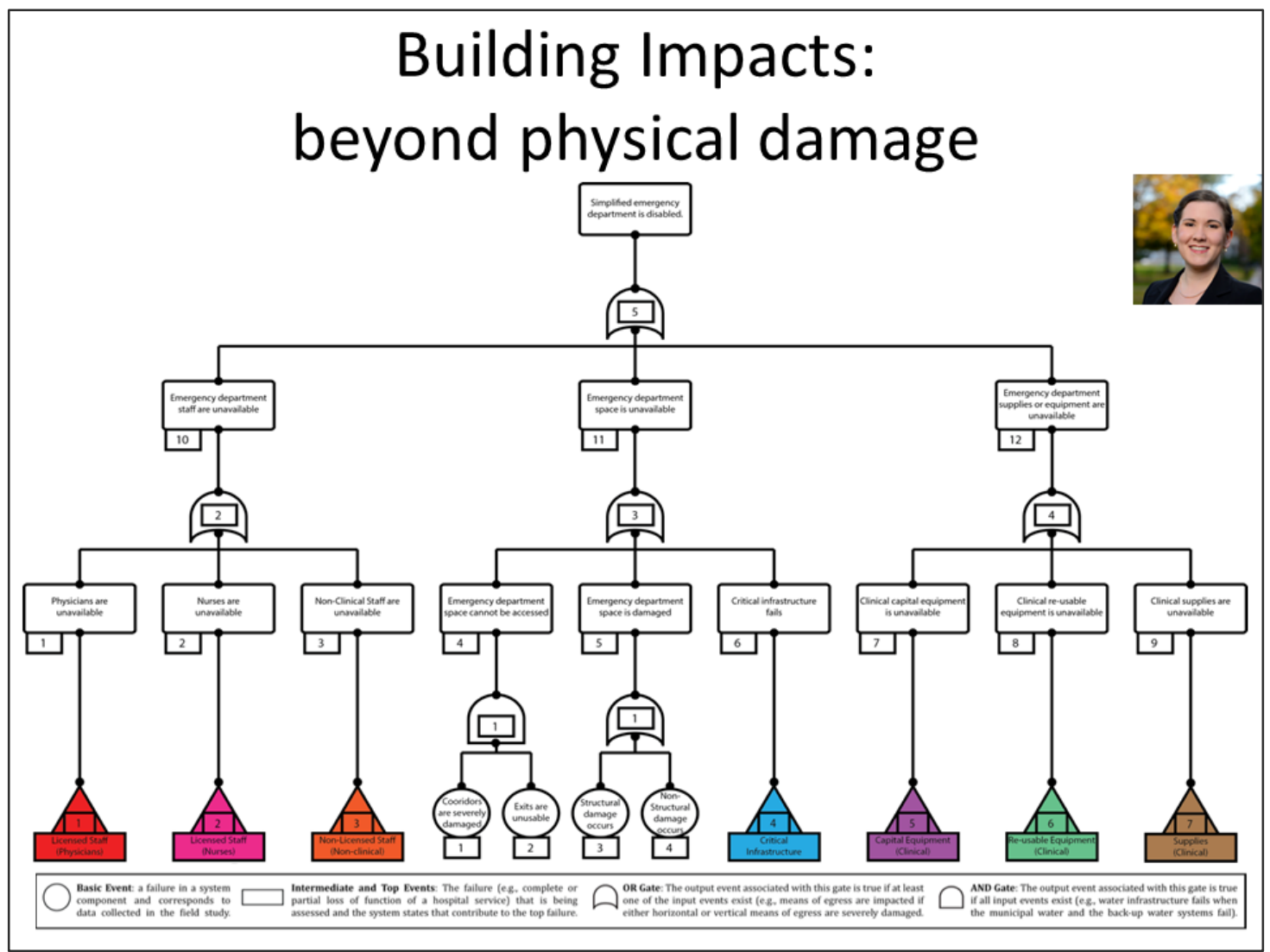




\section{Resilience-Based Design: Hospitals}

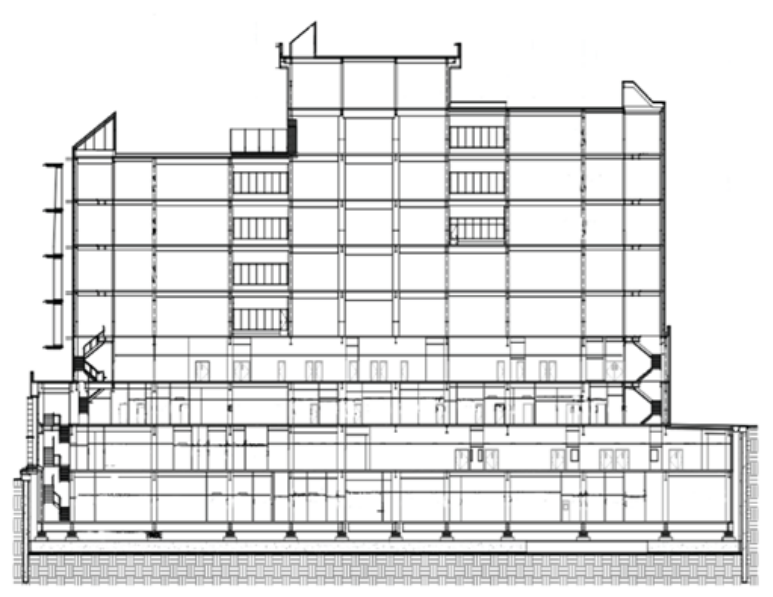

Mechanical Floor

Level 7: Medical/Surgical, Acute Care for Elderly Palliative Care, Roof Garden

Level 6: Medical/Surgical

Level 5: Medical/Surgical Unit, Forensic Unit

Leve1 4: Step Down Medical/Surgical, Step Down ICU, Dialysis

Level 3: Intensice Care Units (ICU)

Level 2: Labor and Delivery, Postpartum, Pediatrics, Neonatal Intensive Care

Level 1: Emergency Department and Trauma Center Basement 1: Operating Rooms, Pre-op, Post Op, Endoscopy, Blood Bank

Basement 2: Dietary. Phar macy, Cardiologloy, Pulmonary, Diagnostic Imaging (Xray), Sterile Processing

\section{Resilience-Based Design: Hospitals}

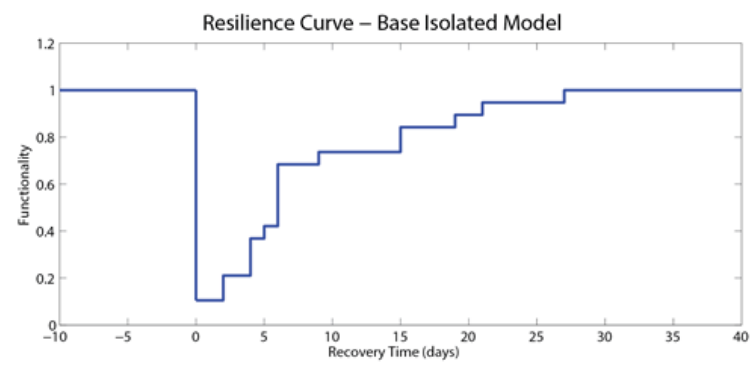

26 days until all hospital services are functional

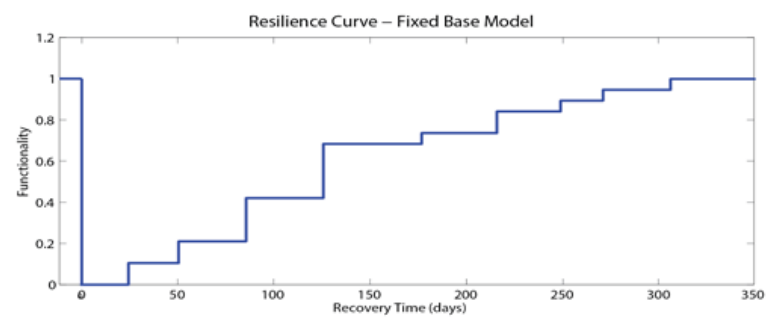

300 days untilall hospital services are functional 


\section{Resilience-Based Design: Hospitals}

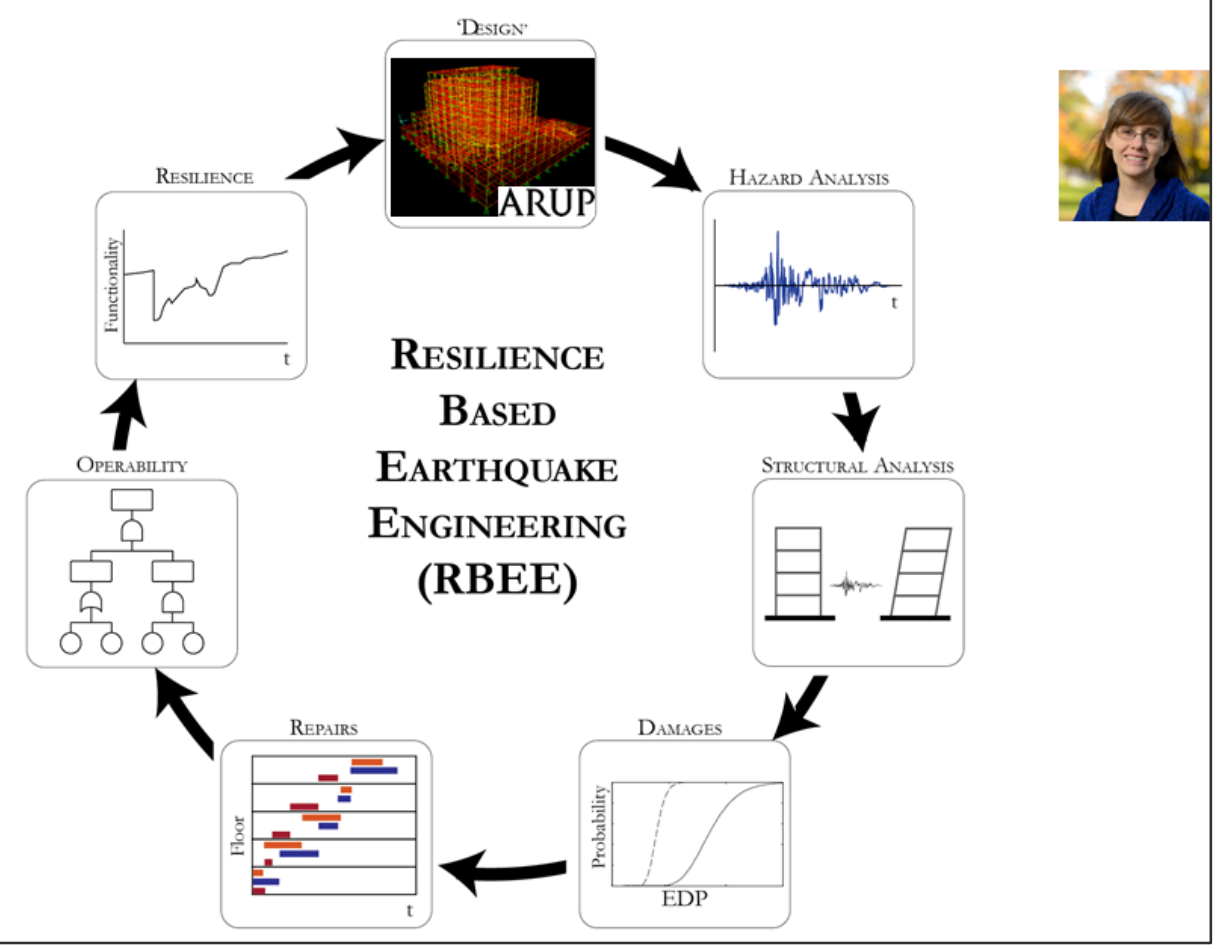

\section{Resilience-Based Design: Summary}

The above procedures, while helpful for individual buildings (nodes), will not suffice in capturing disaster impacts on important community institutions:

- Need models that include interdependent critical lifelines and supply chains.

- Need to capture the 'networked' system of buildings that provides specific community services.

- Need performance metrics that are relevant to the entire system and to the stakeholders managing these institutions. 


\section{NSF RIPS: \\ Resilience of Critical Infrastructure- based Societal Systems (ClbSS)}

U N I VERS I T Y OF

MARYLAND

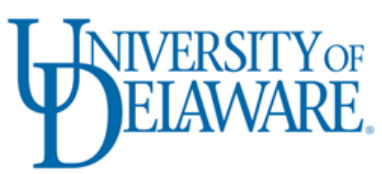

Critical infrastructure-Based Societal Systems (ClbSS) 


\section{Critical infrastructure-Based Societal Systems} (ClbSS)

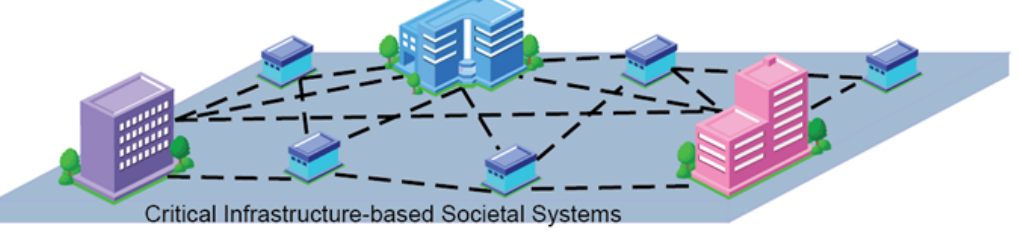

\section{Critical infrastructure-Based Societal Systems (ClbSS)}
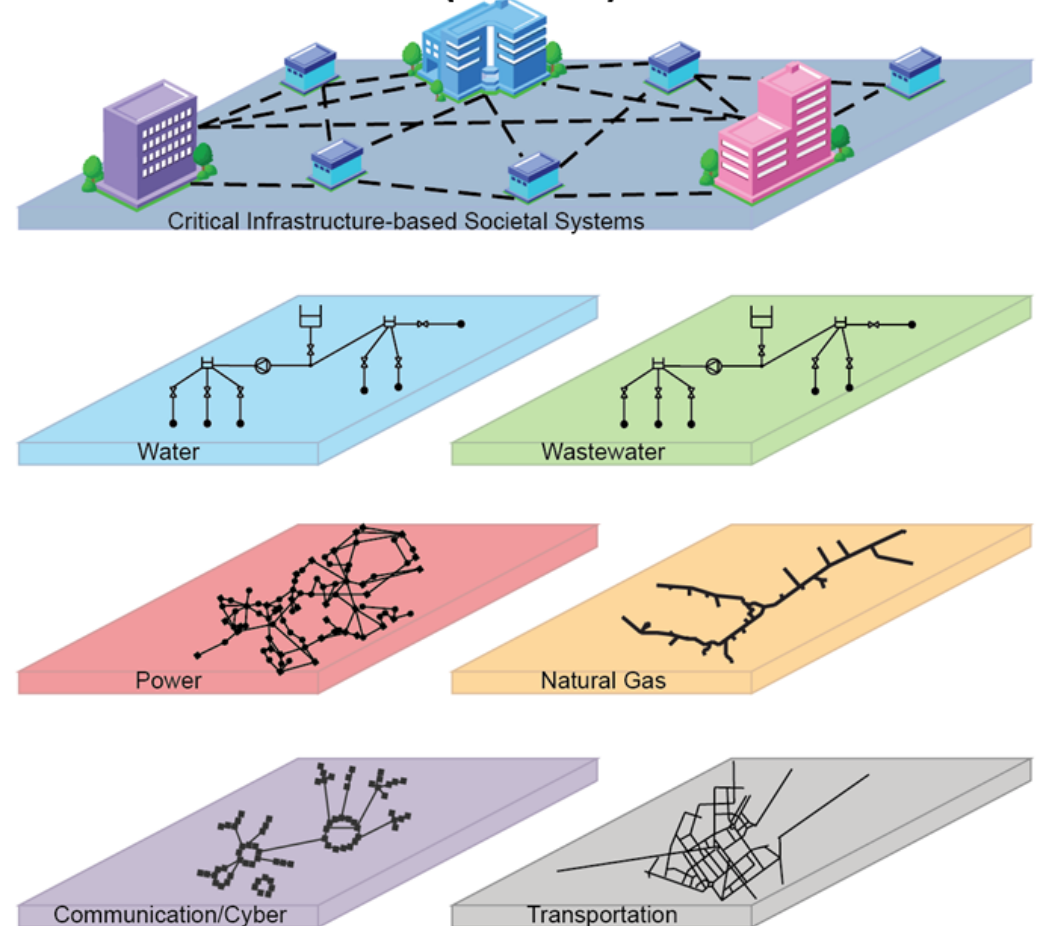

Natural Gas 


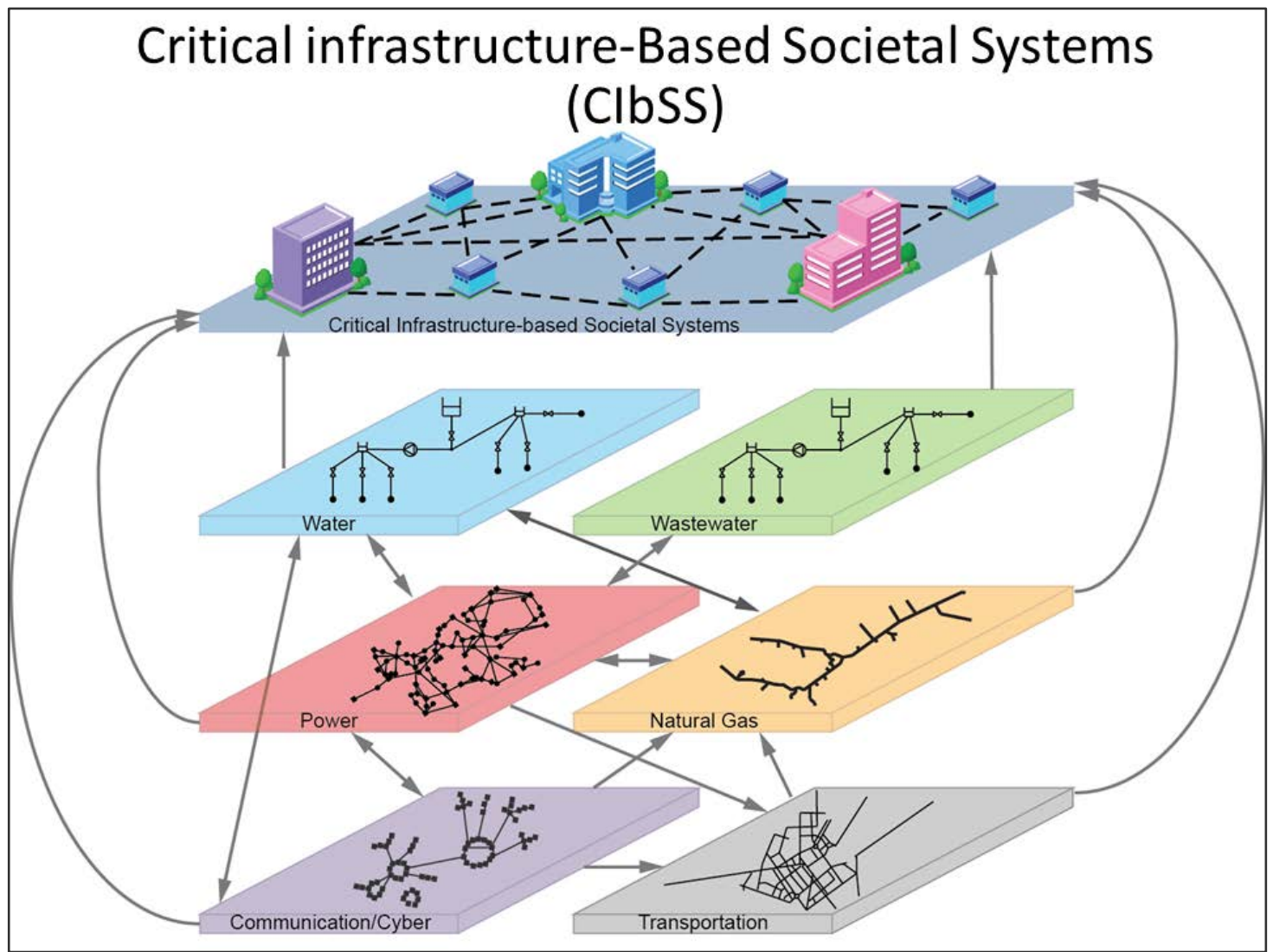

\section{Resilience of the entire ClbSS}
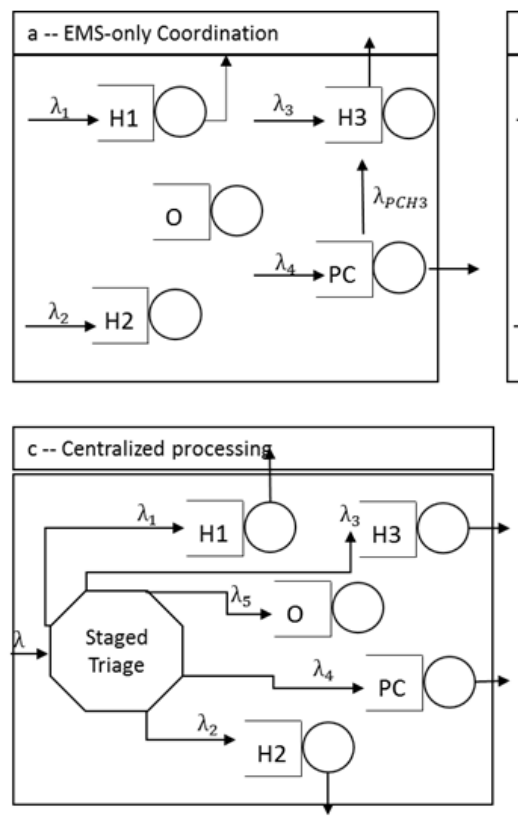

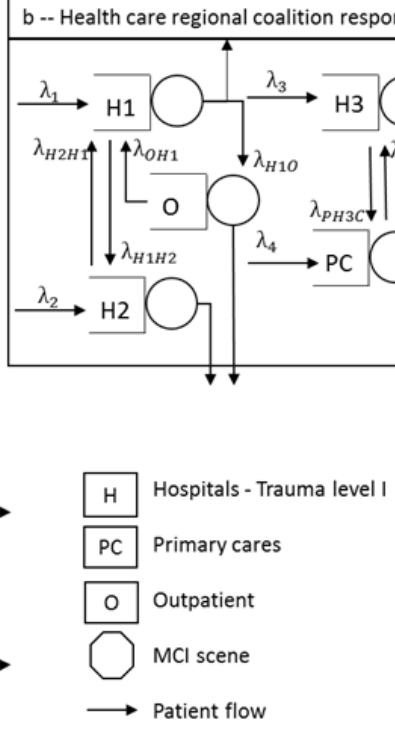

- Discrete event simulation

- Care-paths spanning critical hospital units

- Resource tracking

- Metamodeling with interactions

- Whole health care system coordinated patient flow management

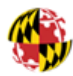

MNIVERSITY OF 


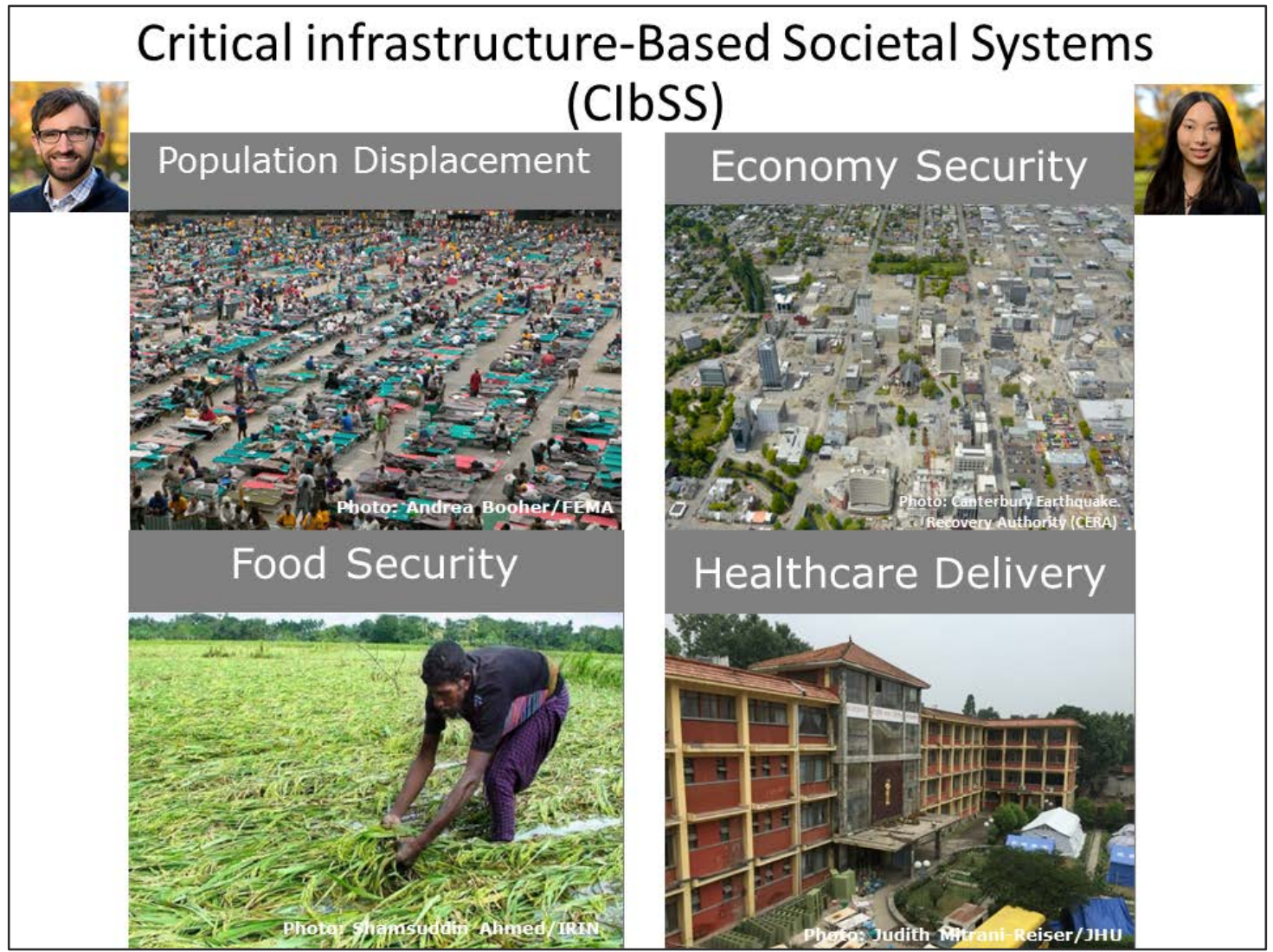

\section{Community Functioning Summary}

We're starting to scratch the surface of modeling the resilience of one CIbSS, but:

- Need holistic approach to capture community functioning over time.

- Need models that interface multiple scales (building institution - community).

- Need to effectively use data that is collected over a wide range of time scales (e.g., census, tax assessors, reconnaissance, etc.).

- Need models that capture the complex interactions of many community institutions. 


\section{CDC: \\ Composite of Post-Event Well Being (COPE-WELL)}

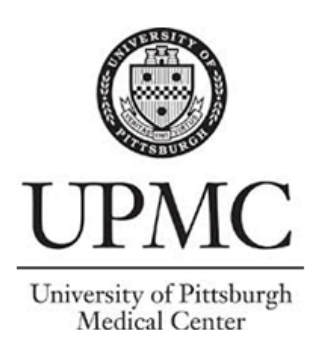

U N I V E R S I T Y

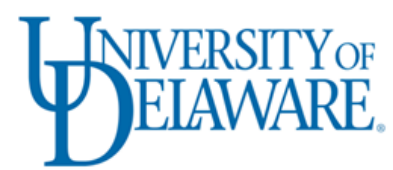
Medical Center

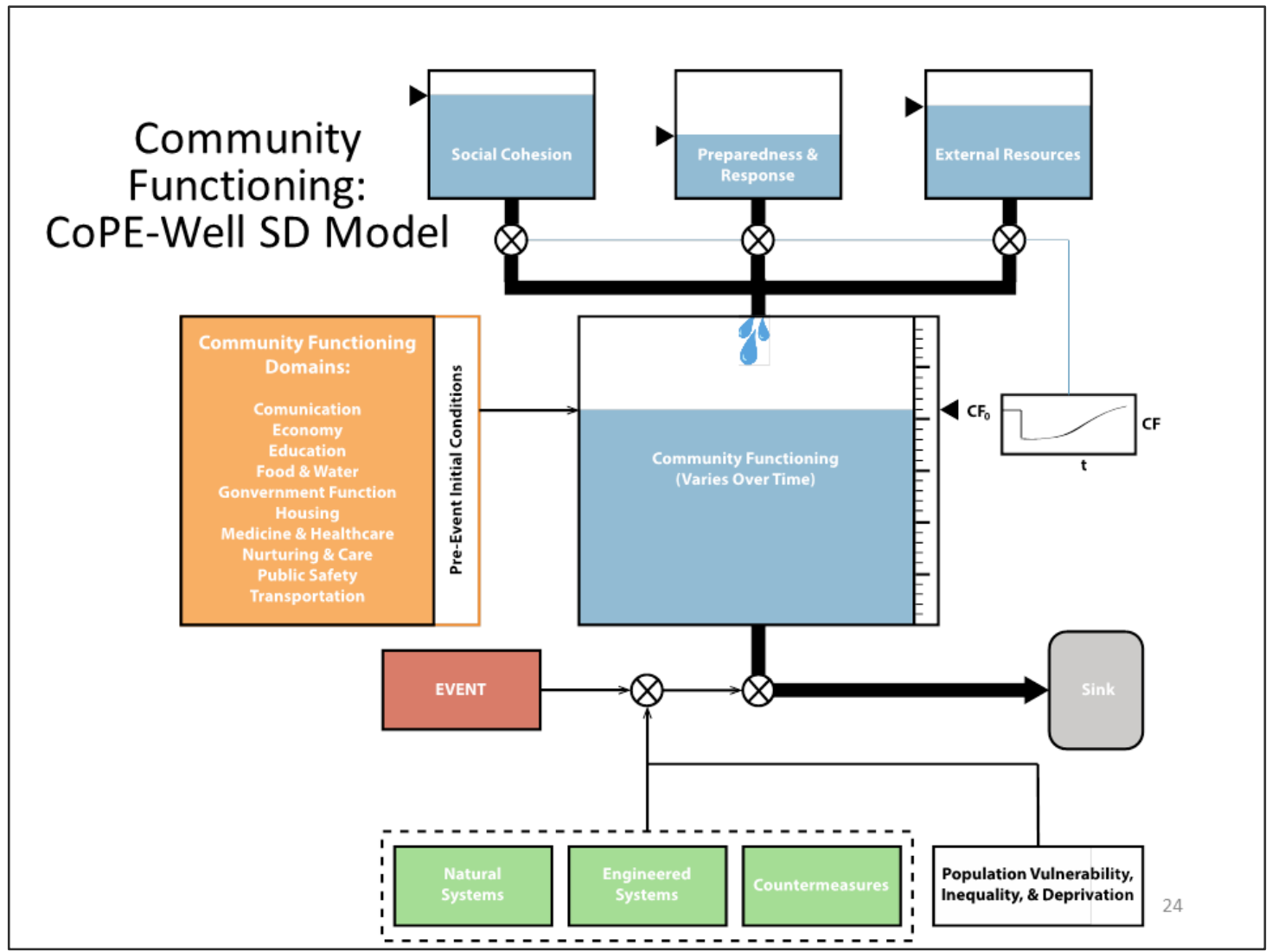




\section{Community Functioning: CoPE-Well SD Model}

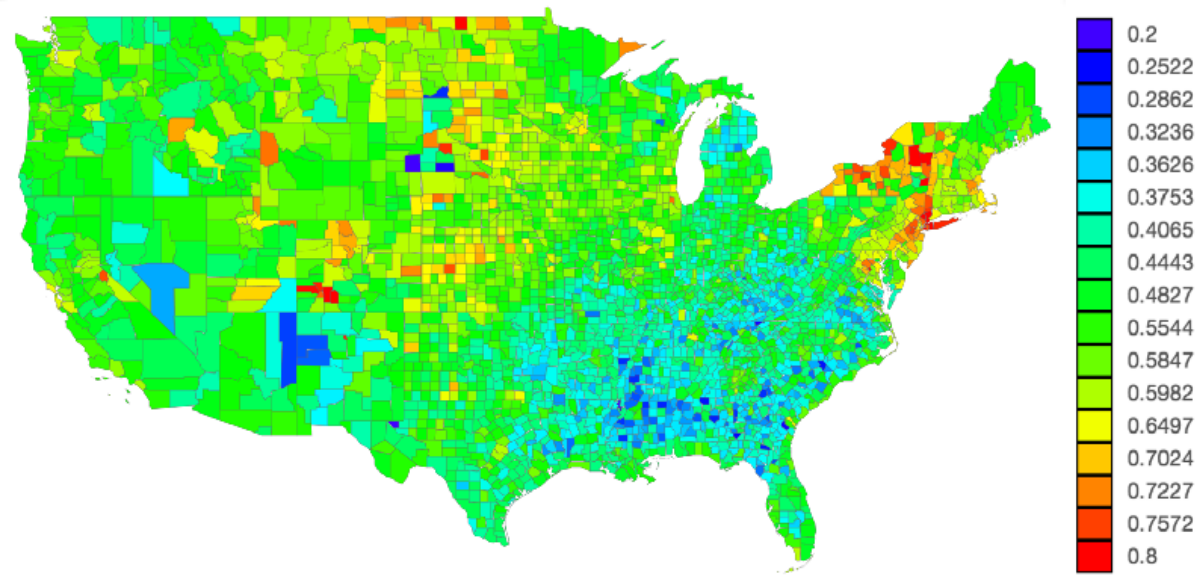

\section{STIRM Research Summary}

My JHU research is focused on using engineering tools to answer important questions at the interface of physical and societal systems:

n Adapting PBEE methods to other hazards (e.g., FPHLPM)

- Designing RBEE tools to assess functionality of infrastructure that's critical to communities

- Modeling human interaction with compromised infrastructure (building evacuations; patient transfers)

- Disaster field studies (acute and longitudinal)

n Creating tools that are useful to practitioners (e.g., States of Oregon, Utah, and California; Ministries/Departments of Health; USGS; Arup; CIGIDEN) 


\section{Acknowledgements}
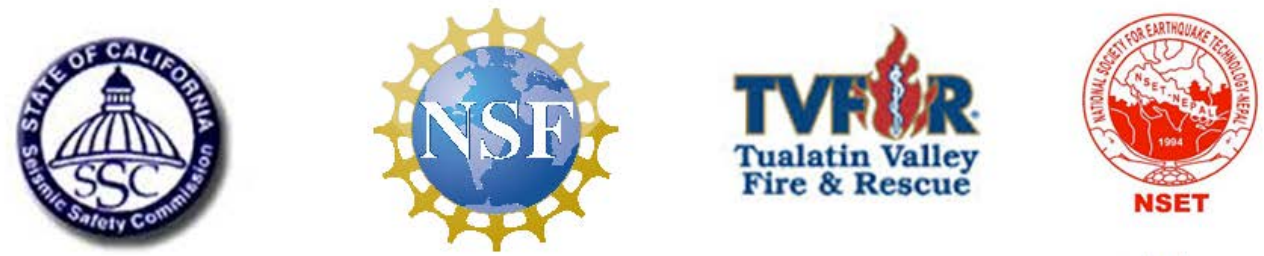

Canterbury

District Health Board

Te Poari Hauora o Waitaha

JOHNS HOPKINS

26

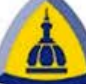

M D I C I N E

HEALTH

Baltimore Office

of Sustainability
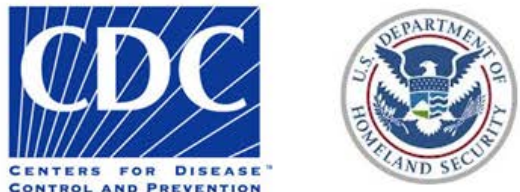

FEMA

\section{Thank you}

The Johns Hopkins Systems Institute

As an engineer I think you have a responsibility.

We have to not just care about erecting a structure.

We have to think about its lifetime and what kind

of impact it's going to have on society. 
Judith Mitrani-Reiser, Ph.D.

National Institute of Standards and Technology

(NIST)

Johns Hopkins University (JHU)

\author{
Education \\ California Institute of Technology, Ph.D. in Applied \\ Mechanics (2007) \\ University of California, Berkeley, M.S. in Civil and \\ Environmental Engineering (2001) \\ University of Florida, B.S. in Civil and Coastal \\ Engineering (2000)
}

\title{
Short Bio
}

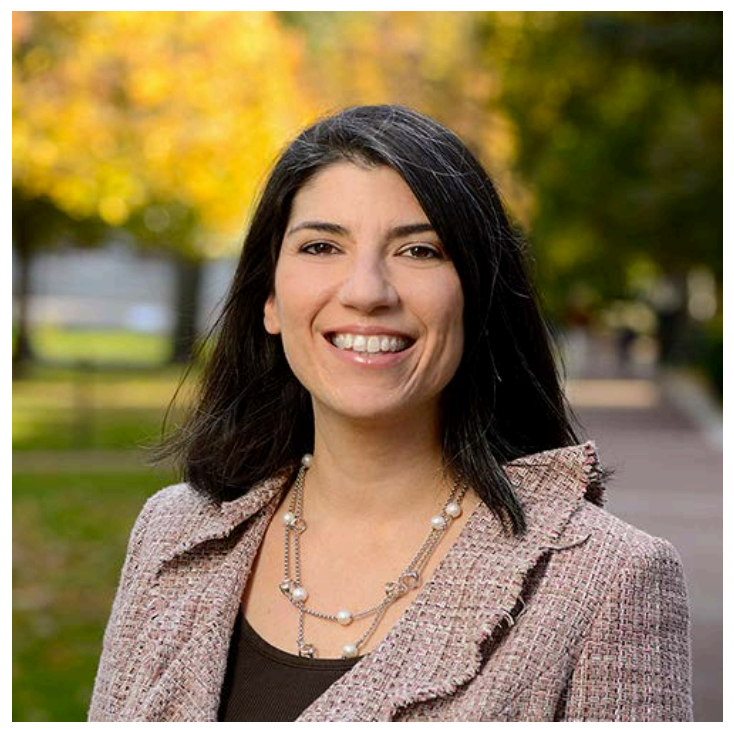

Dr. Mitrani-Reiser the Director of the Disaster and Failure Studies Program at the National Institute of Standards and Technology (NIST) and an Assistant Professor of Civil Engineering and Emergency Medicine at Johns Hopkins University (JHU). Her research is focused on the performance assessment of critical infrastructure, the safety and economic impact of hazards on the built environment, the effective communication of these risks to the public, informed decision-making for use in emergency management and policy making, and the interaction of humans with the built environment. Her multidisciplinary research program at JHU includes collaborations with the PEER (UC Berkeley), the Disaster Research Center (University of Delaware), the University of Maryland, the National Research Center for Integrated Natural Disaster Management (Pontificia Universidad Católica de Chile), and QuakeCORE (University of Canterbury in New Zealand). Her research program at NIST is focused on the metrology of disasters and failures, to improve building and fire codes, standards, and practices and to fill gaps in knowledge about buildings and infrastructure performance, emergency response, and human behavior in hazard events.

Dr. Mitrani-Reiser is an Associate of the Center for Refugee and Disaster Response (CRDR), a member of the American Society of Civil Engineers (ASCE), the Earthquake Engineering Research Institute (EERI), the Seismological Society of America (SSA), and the World Association for Disaster and Emergency Medicine (WADEM). She is the Vice Chair for ASCE's Subcommittee on Multi-Hazard Mitigation, and is a member of ASCE's Committee on Disaster Resilience of Structures and of the Committee of Critical Facilities in ASCE's Infrastructure Resilience Division, and a member of EERI's Learning From Earthquakes Committee. She is the founder of the Postdoctoral Association at Johns Hopkins University.

\section{Bibliography}

De la Llera, J. C., Rivera, F., Mitrani-Reiser, J., Jünemann, R., and Ríos, M. 2016. "Data Collection after the 2010 Maule earthquake in Chile." Bulletin of Earthquake Engineering (published online 11 May 2016).

Mieler, M.W., Uma, S.R., and Mitrani-Reiser, J. "Using Failure Analysis to Establish Seismic Resilience Objectives for Building Components and Systems." Bulletin of the New Zealand Society of Earthquake Engineering (in press).

Mitrani-Reiser, J., Wu, S., and Beck, J.L. 2016. "Virtual Inspector and its application to immediate pre-event and post-event earthquake loss and safety assessment of buildings." Natural Hazards, 81(3): pp. 18611878. 
Jacques, C., McIntosh, J.K., Giovinazzi, S., Kirsch, T.D., Wilson, T.M., and Mitrani-Reiser, J., 2014. "Resilience of the Canterbury Health Care System to the 2011 Christchurch $(\mathrm{Mw}=6.2)$ Earthquake, NZ", Earthquake Spectra, 30(1): pp. 533-554.

Mitrani-Reiser, J., Mahoney, M., Holmes, W.T., de le Llera, J.C., Bissell, R., and Kirsch, T.D., 2012. "A Functional Loss Assessment of a Hospital System in the Biobío Province." Earthquake Spectra, 28(S1): pp. S473S502.

Ramirez, M., Liel, A., Mitrani-Reiser, J., Haselton, C., Spear, A., Steiner, J., Miranda, E., and G.G. Deierlein, 2012. "Seismic Damage and Monetary Loss Performance of Modern Reinforced Concrete Frame Buildings", Earthquake Engineering and Structural Dynamics, 14(11): pp. 1455-1475.

Kirsch, T.D., Mitrani-Reiser, J., Bissell, R., Sauer, L.M., Mahoney, M., Holmes, W.T., Santa Cruz, N., de la Maza, F., 2010. "Impact on Hospital Functions Following the 2010 Chilean Earthquake." Journal of Disaster Medicine and Public Health Preparedness, 4(2): pp. 122-128.

Goulet, C., Haselton, C., Mitrani-Reiser, J., Beck, J.L., Deierlein, G., Porter, K.A., Stewart, J.P.. and Taciroglu, E., 2007. "Evaluation of the Seismic Performance of a Code-Conforming Reinforced-Concrete Building From Seismic Hazard to Loss Results," Earthquake Engineering and Structural Dynamics, 36(13): pp. 1973-1997. 
International Workshop on Modeling of Physical, Economic, and Social Systems for Resilience Assessment

This page intentionally left blank. 


\section{Appendix D: Modeling of Recovery (Session 4)}

Physical and Economic Impacts of Earthquakes and Other Hazards [Terje

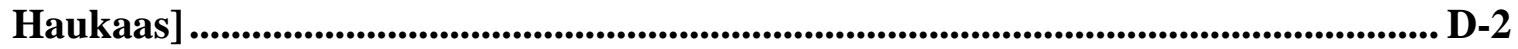

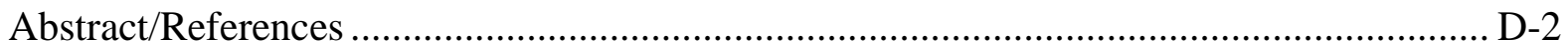

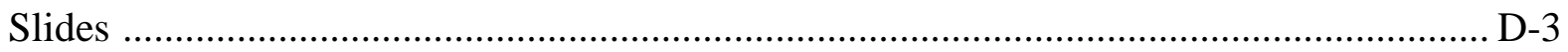

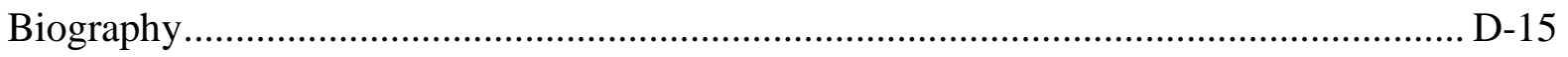

Road Network Functionality of Istanbul Following an Earthquake [Himmet

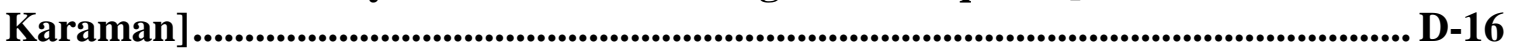

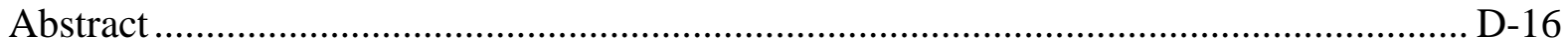

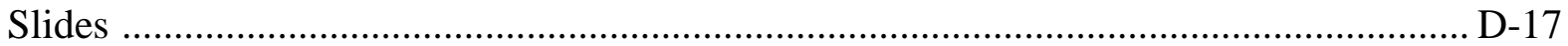

Biography....................................................................................................... D-36

Measuring and Improving the Resilience of the Built Environment in a Community

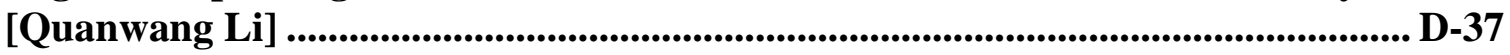

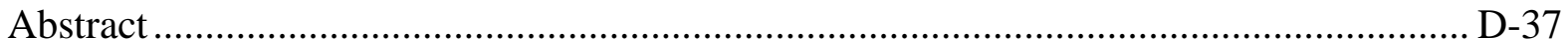

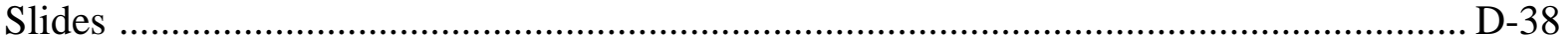

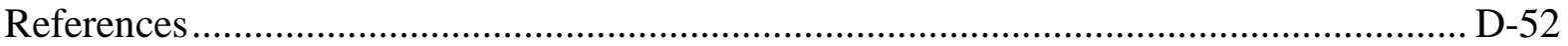

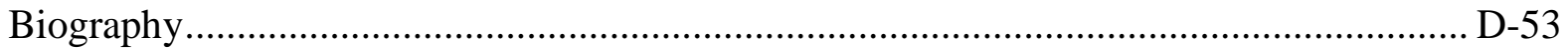

Housing Demaind \& Capacity for Medically Fragile \& Vulnerable Populations:

Modeling Transition through Temporary, Interim, and Permanent Housing

Recovery [Joshua Behr] ...................................................................................................... D-54

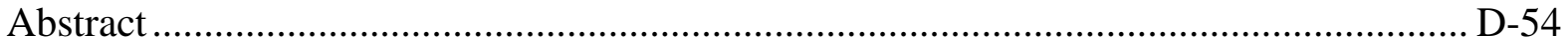

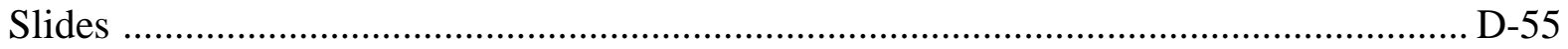

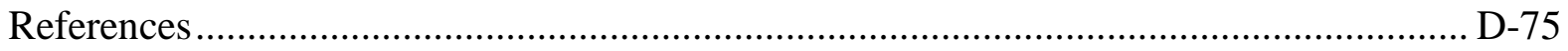

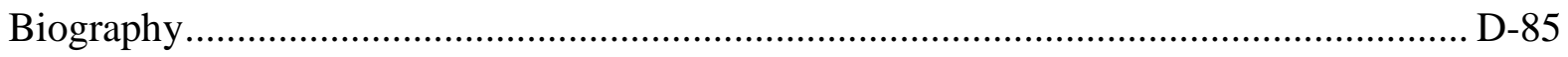

Modeling recovery: The Role of Disaster Financing [Carolyn Kousky] ............................. D-86

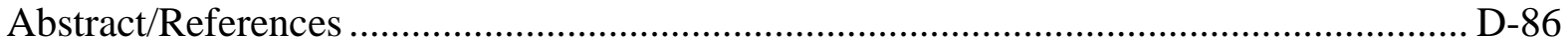

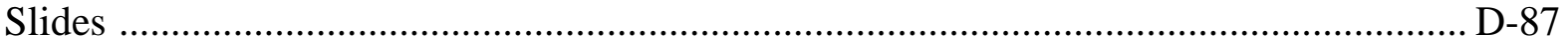

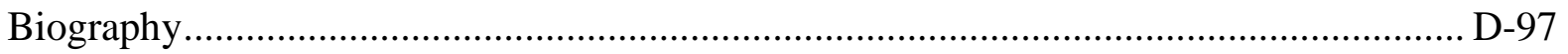

An Integration of Social Vulnerability Data and Mapping Tools for Community Vulnerability Assessments and Recovery Modeling [Nathanael Rosenheim]....... D-98

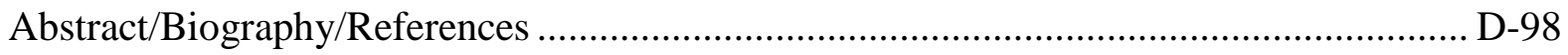

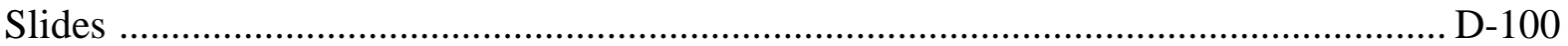




\section{Physical and Economic Impacts of Earthquakes and Other Hazards Professor Terje Haukaas, University of British Columbia, Vancouver}

The cornerstone of our work to improve the resilience of communities and individual structures is computer simulation models. The objective is to predict, in a probabilistic manner, the physical and economic impacts of earthquakes and other hazards. One result is the loss curve, which displays the probability distribution for economic losses in a specific time period. Based on such results we employ optimization algorithms to determine the design, or resource allocation, that minimizes the total expected cost. Our work is continually extending the computer program Rts, a program created in our research group to coordinate analyses with many probabilistic models. Today it contains a robust library of models for structural components, buildings, ports, bridges, hazards, costs, etc. The program and the approach grew out of efforts in performance-based earthquake engineering for buildings, but we now address networks and other hazards as well. A recent application is the interdisciplinary study of the resilience of the coastal communities in British Columbia. Rts is employed here to model the maritime transportation of food, fuel, and other supplies to remote communities that could become severely affected by transportation disruptions. Computer simulations provide the probability of disruption, and the effect of different mitigation options, allowing us to recommend mitigation decisions based on cost-benefit optimization.

\section{Bibliography}

Chang, Brown, Costa, Dobson, Dowlatabadi, Haukaas (2017) “A community resilience approach to assessing transportation risk in disasters.” 16th World Conference on Earthquake Engineering, January 9-13, Santiago Chile

Haukaas, T., Gavrilovic, S. (2016) "Feasibility and accuracy of reliability methods in gradient-based minimization of expected building cost.” Proceedings of IFIP WG-7.4 Reliability and Optimization of Structural Systems, May 18-20, Carnegie Mellon University, Pittsburgh, PA

Javaherian Yazdi, A., Haukaas, T., Yang, T., Gardoni, P. (2016) “Multivariate fragility models for earthquake engineering.” Earthquake Spectra, 32(1), pp. 441-461.

Mahsuli, M., Haukaas, T. (2013) “Sensitivity measures for optimal mitigation of risk and reduction of model uncertainty.” Reliability Engineering \& System Safety, 117, pp. 9-20.

Mahsuli, M., Haukaas, T. (2013) “Seismic risk analysis with reliability methods, Part II: Analysis.” Structural Safety, 42(1), pp. 63-74.

Mahsuli, M., Haukaas, T. (2013) “Seismic risk analysis with reliability methods, Part I: Models.” Structural Safety, 42(1), pp. 54-62.

Mahsuli, M., Haukaas, T. (2013) “Computer program for multi-model reliability and optimization analysis.” ASCE Journal of Computing in Civil Engineering. 27(1), pp. 87-98.

Haukaas, T. (2013) “Probabilistic models, methods, and decisions in earthquake engineering.” 11th International Conference on Structural Safety and Reliability, June 16-20, New York

Haukaas, T. (2008) “Unified reliability and design optimization for earthquake engineering.” Probabilistic Engineering Mechanics, 23, pp. 471-481. 


\title{
Physical and Economic Impacts of Earthquakes and Other Hazards
}

\author{
Professor Terje Haukaas \\ International Workshop on Modeling of Physical, Economic, \\ and Social Systems for Resilience Assessment \\ Washington, DC, October 19-21, 2016
}

Model Granularity
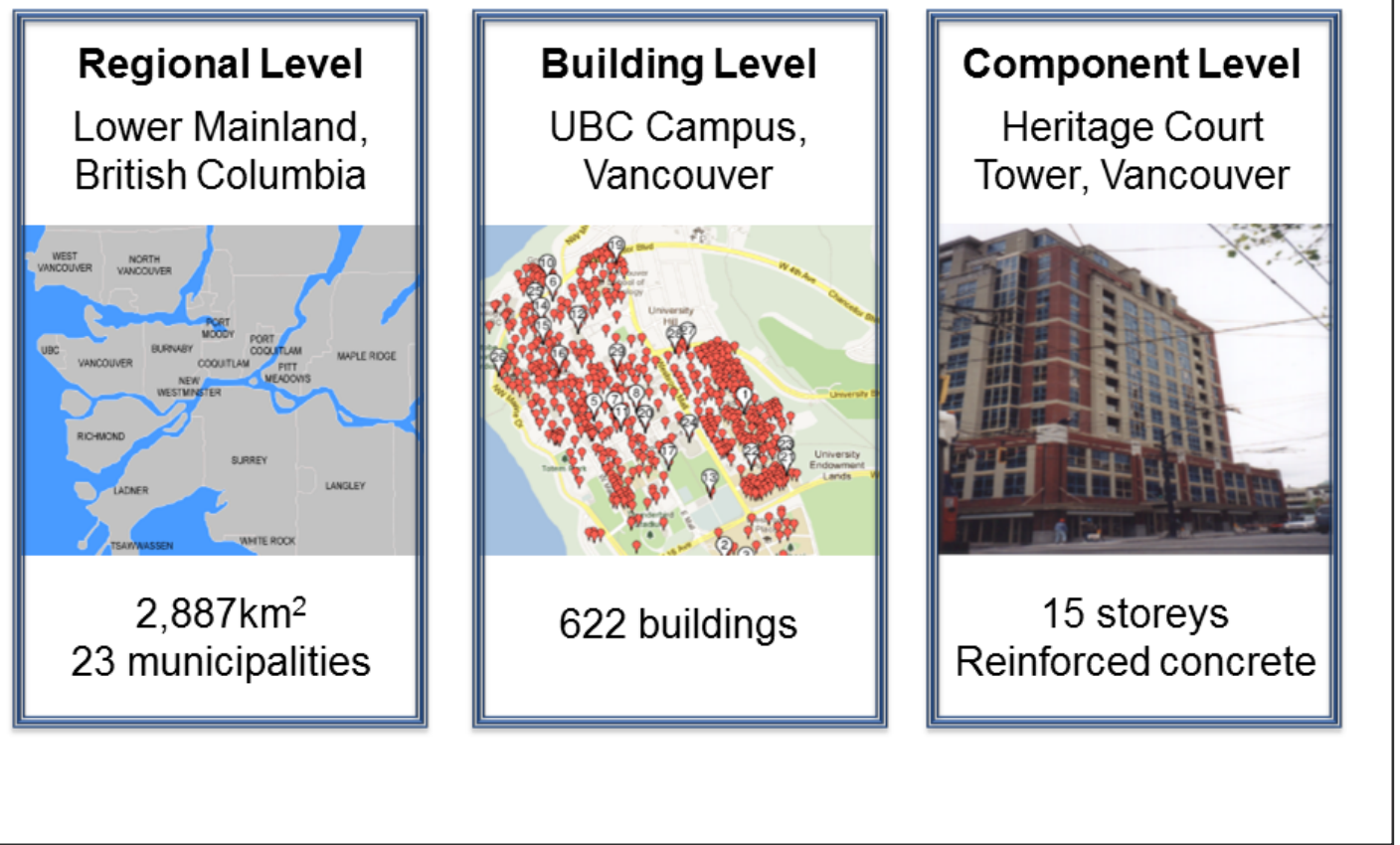
Model Matrix

\begin{tabular}{|c|c|c|c|}
\hline & & & \\
\hline & $\begin{array}{l}\text { Example: } \\
\text { Lower Mainland, } \\
\text { British Columbia }\end{array}$ & $\begin{array}{l}\text { Example: } \\
\text { UBC Campus, } \\
\text { Vancouver }\end{array}$ & $\begin{array}{c}\text { Example: } \\
\text { Heritage Court } \\
\text { Tower, Vancouver }\end{array}$ \\
\hline Loss & $\begin{array}{l}\text { Regional } \\
\text { Loss Model }\end{array}$ & $\begin{array}{l}\text { Building } \\
\text { Loss Model }\end{array}$ & $\begin{array}{l}\text { Component } \\
\text { Loss Model }\end{array}$ \\
\hline Damage & Regional & $\begin{array}{c}\text { Building } \\
\text { Damage Model }\end{array}$ & $\begin{array}{c}\text { Component } \\
\text { Damage Model }\end{array}$ \\
\hline Response & Model & $\begin{array}{c}\text { Building } \\
\text { Response Model }\end{array}$ & $\begin{array}{c}\text { Finite Element } \\
\text { Model }\end{array}$ \\
\hline $\begin{array}{l}\text { Ground } \\
\text { Motion }\end{array}$ & Intens & Model & $\begin{array}{c}\text { Ground Motion } \\
\text { Model }\end{array}$ \\
\hline Earthquake & $\begin{array}{c}\text { Occurrence } \\
\text { Model }\end{array}$ & $\begin{array}{l}\text { Location } \\
\text { Model }\end{array}$ & $\begin{array}{l}\text { Magnitude } \\
\text { Model }\end{array}$ \\
\hline
\end{tabular}

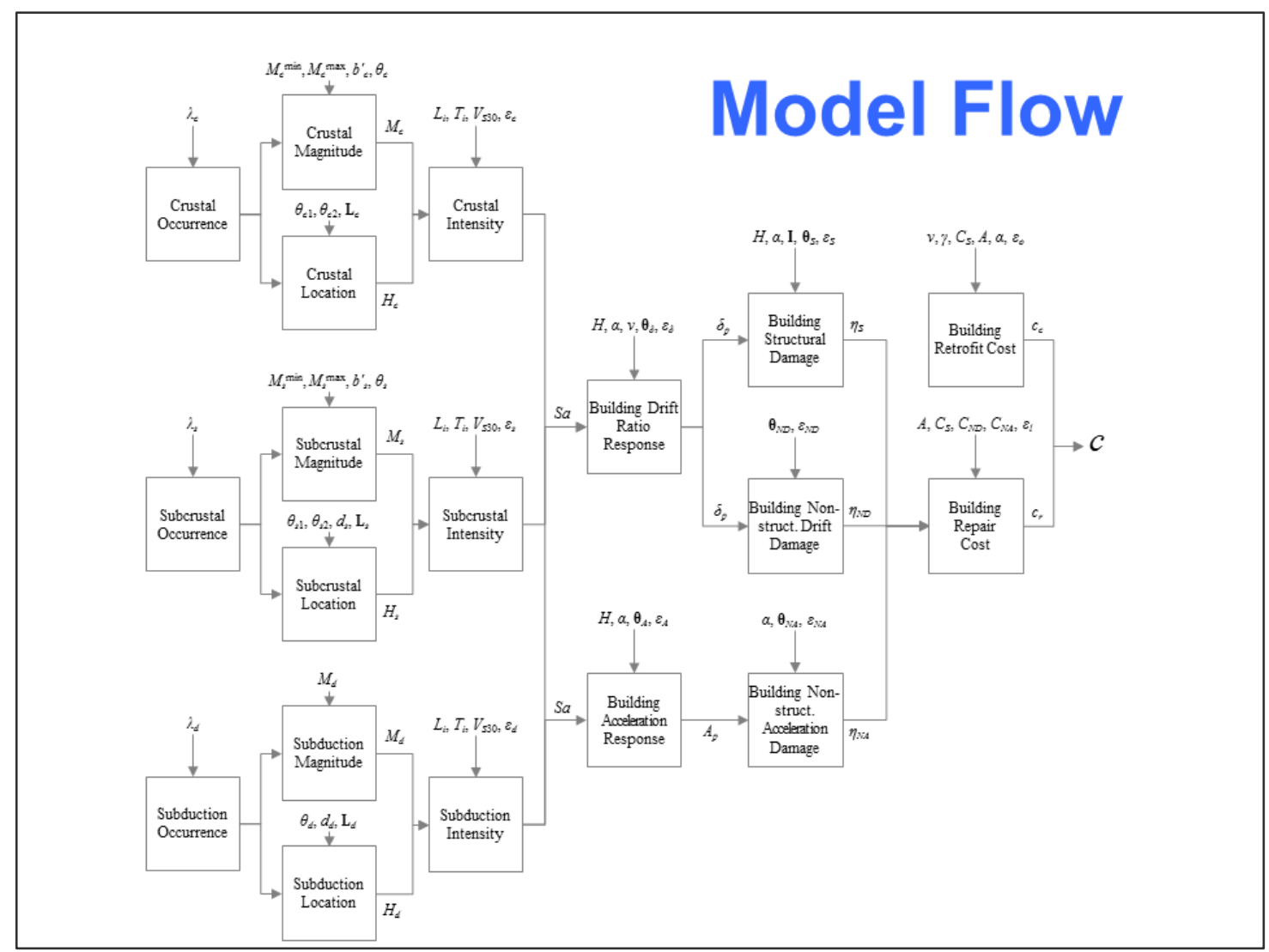




\section{Model Implementation}

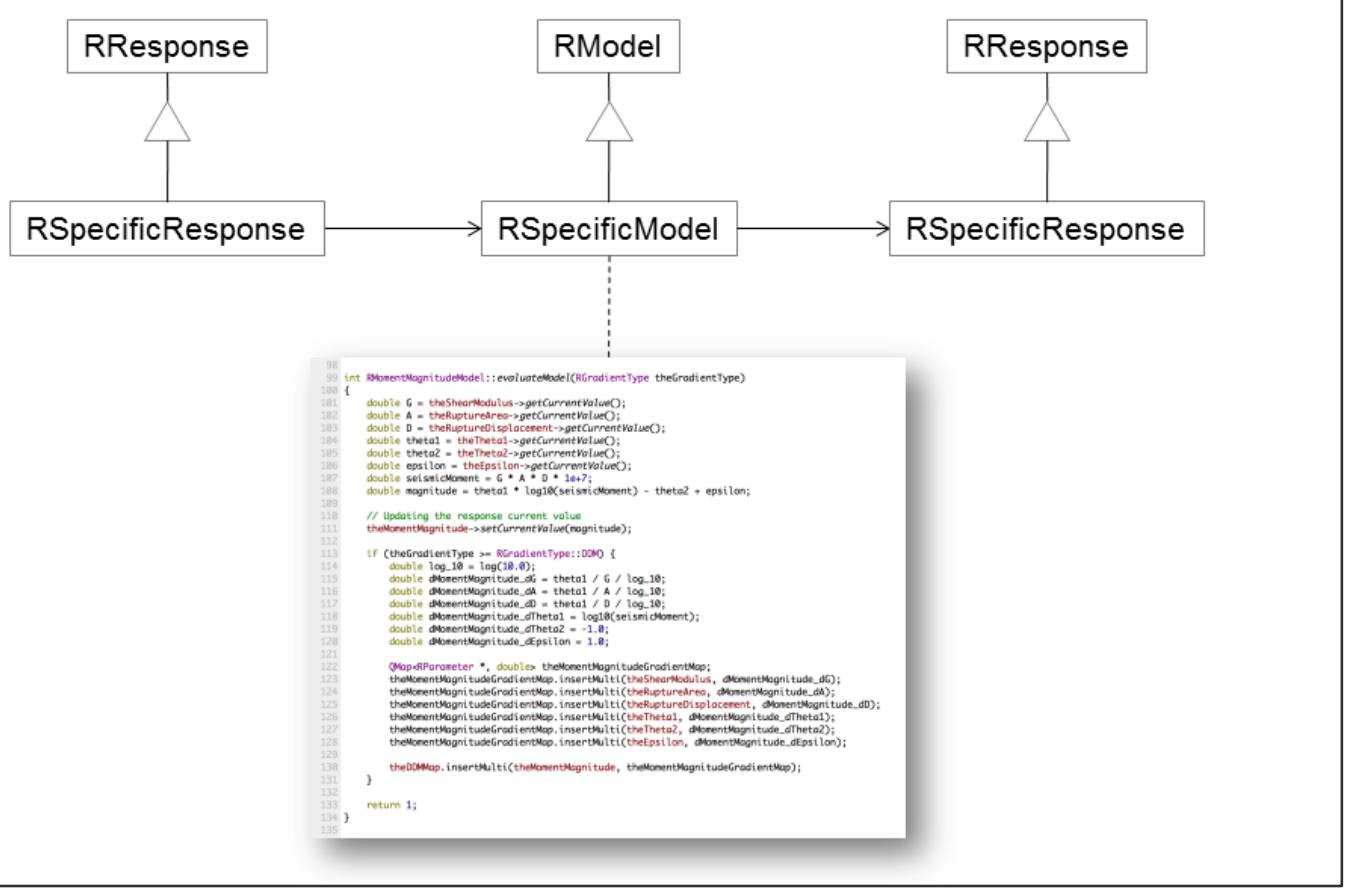

\section{Rts}

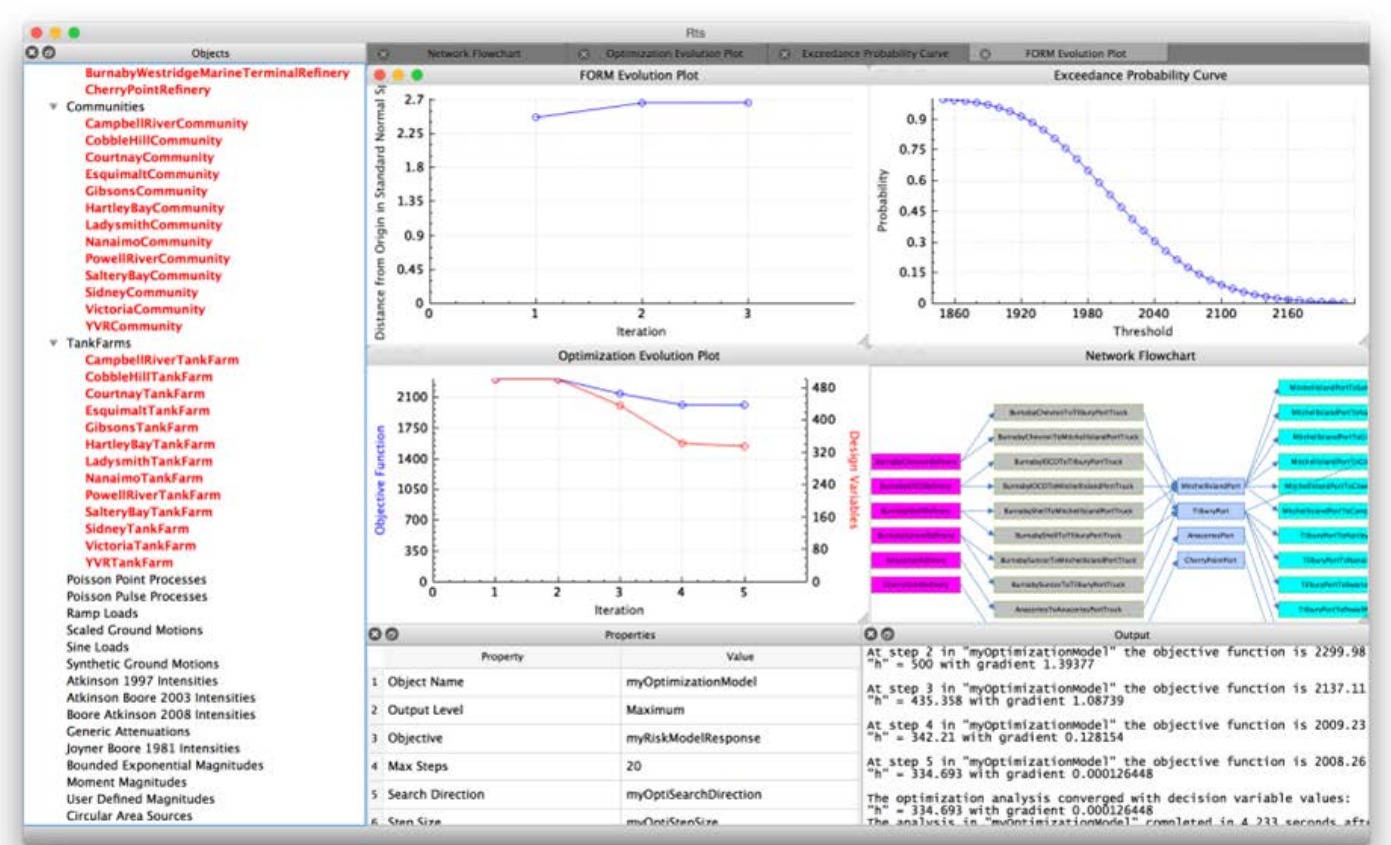




\section{Loss Curves}

Lower Mainland

British Columbia

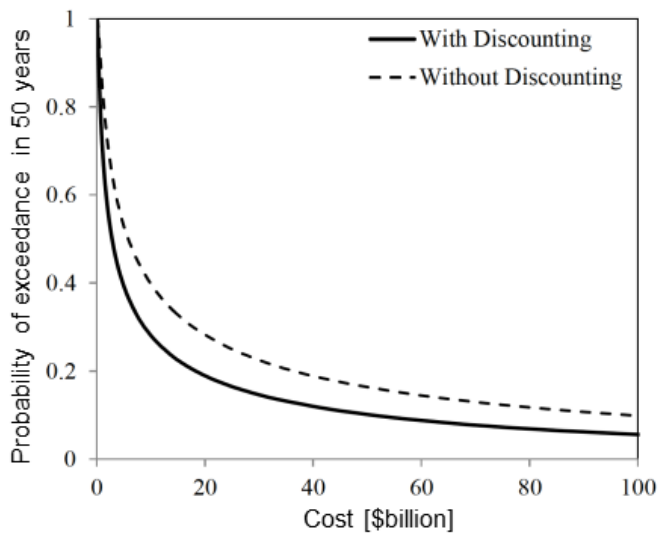

UBC Campus Vancouver

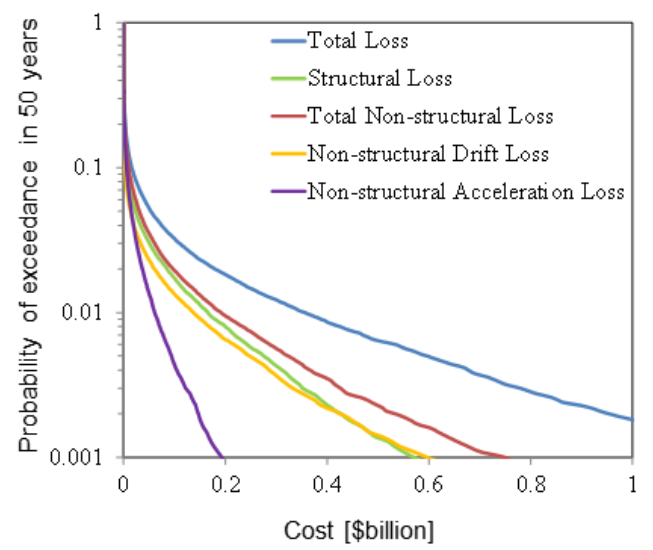

\section{Analysis}

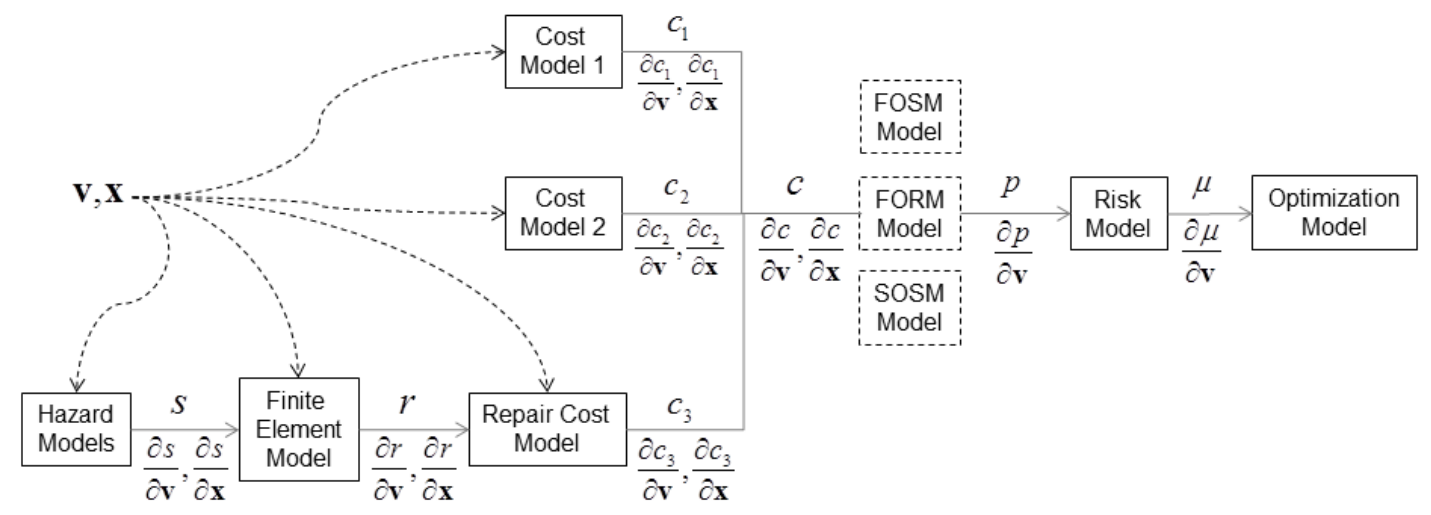




\section{Retrofit Prioritization}

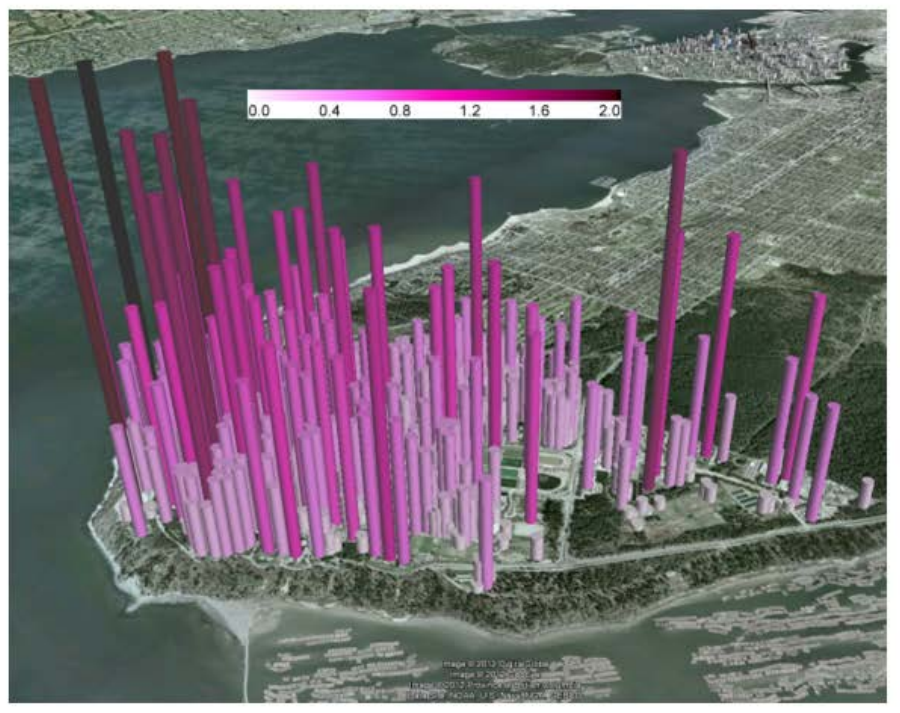

\section{Results}

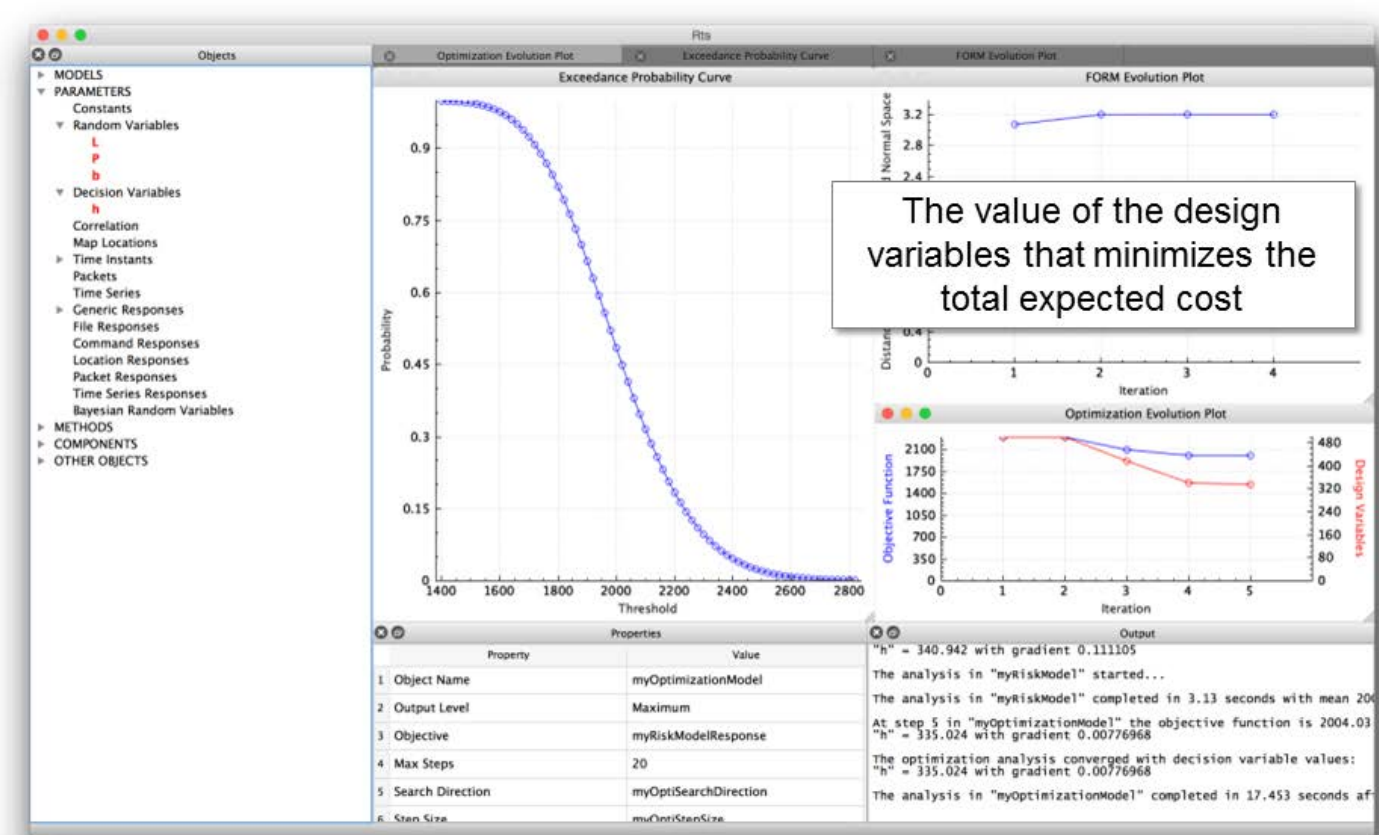




\section{Networks}

Objects
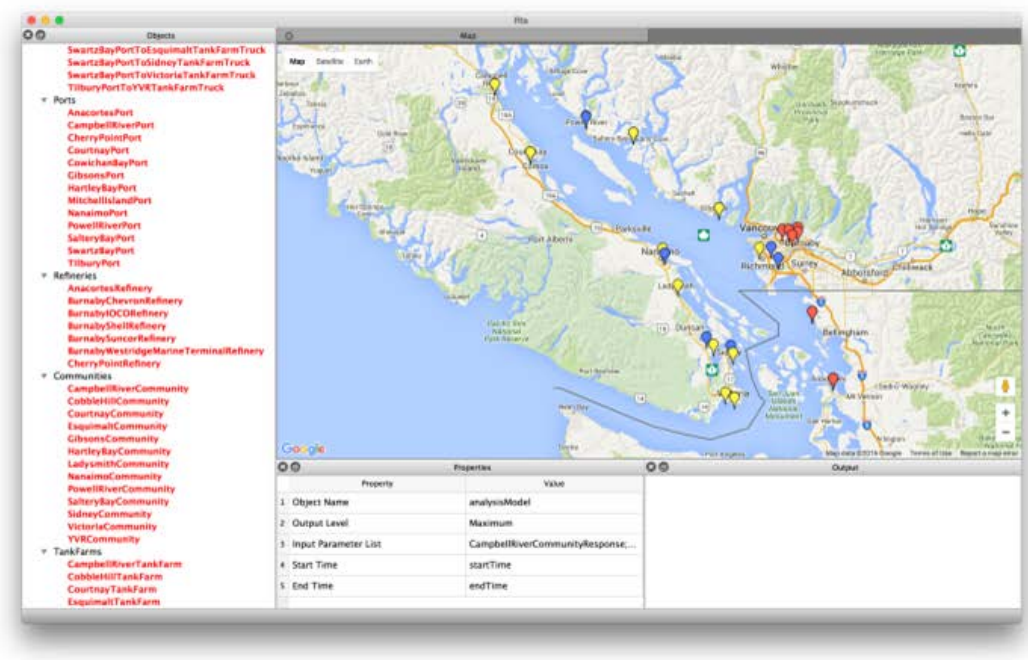

Ports

Tank farms

Refineries

Bridges

Communities

Trucks

Ships

\section{Flow of Packets}

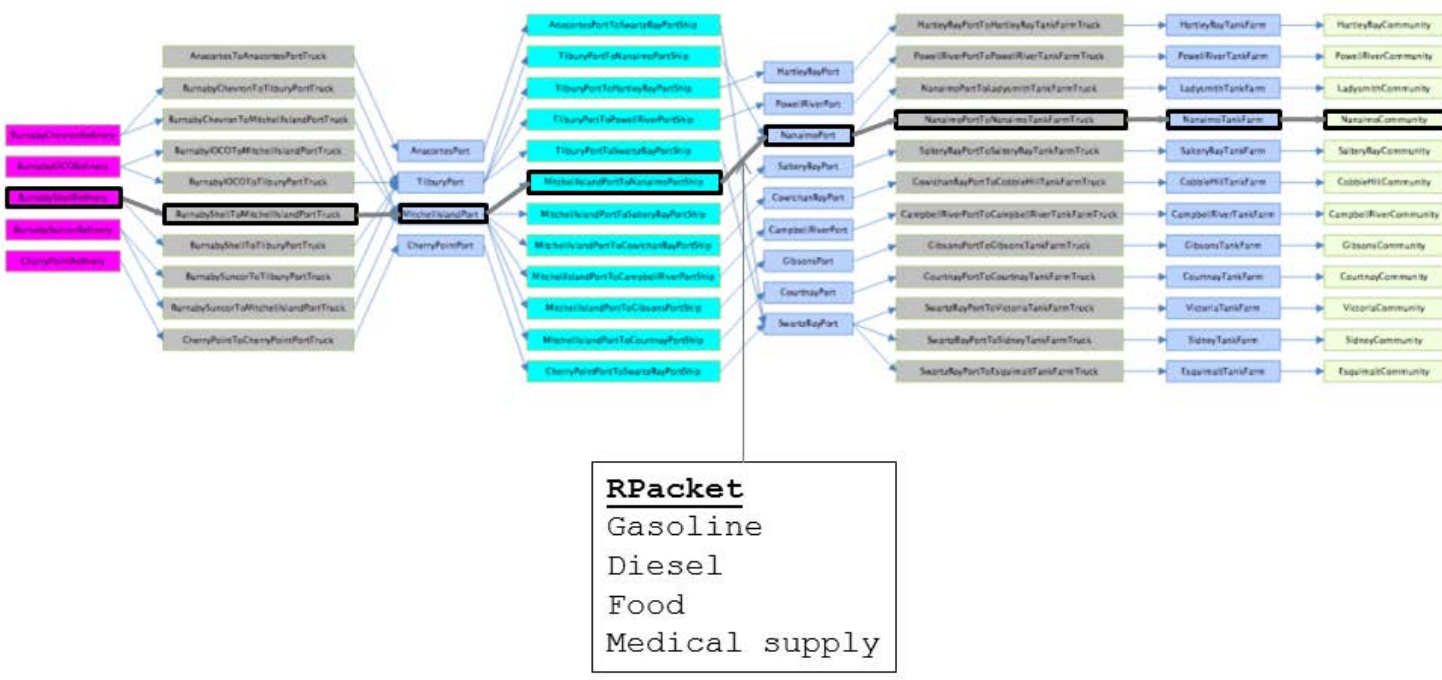




\section{Earthquakes}

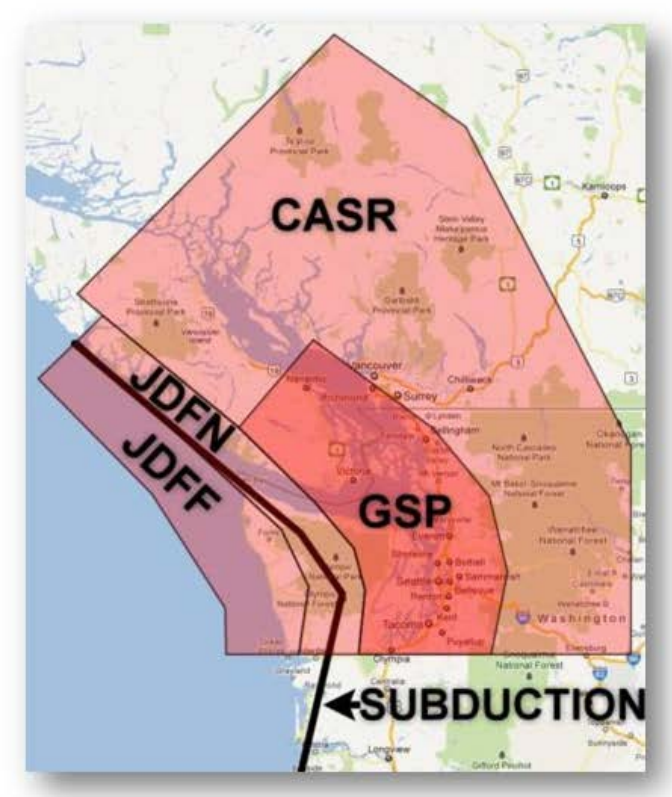

\section{Powell River}

- Population: 20,000

- Tank farm with 2 tanks filled to capacity

- Deliveries every 3 days

- Reserves would last 10 days

- Are the full tanks more vulnerable?

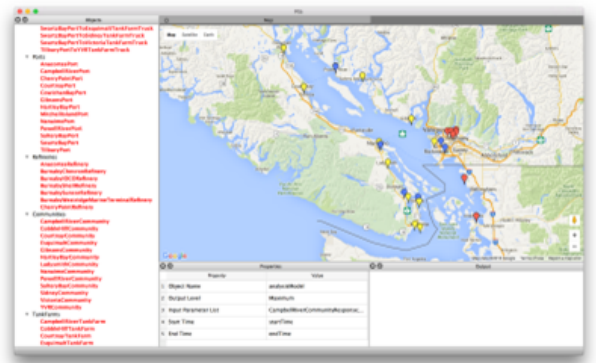

- Will the demand change after an earthquake? 


\section{Results}
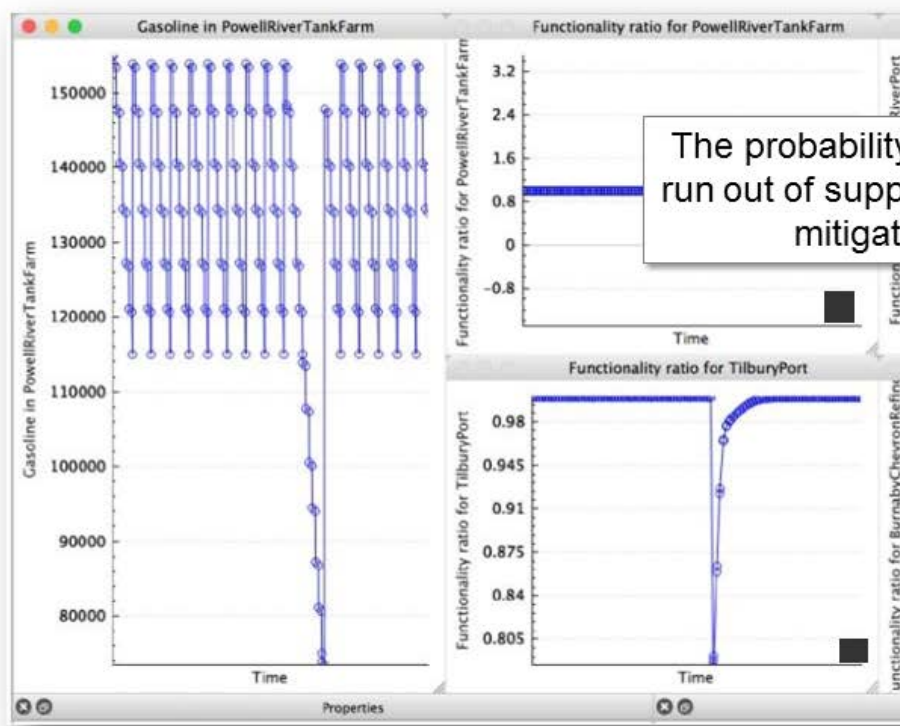

Functionality ratio for PowellikiverPort

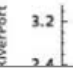

3.2

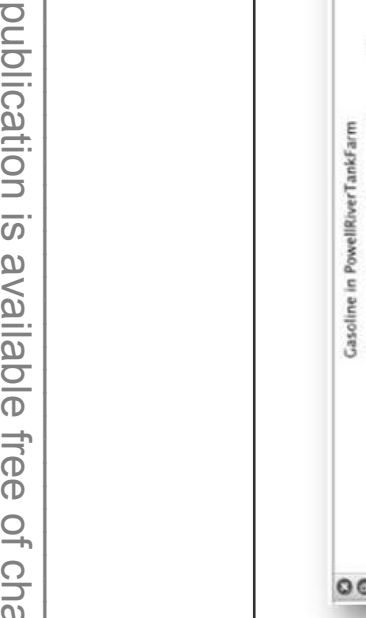

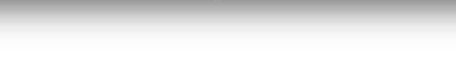

$y$ that communities

\section{New Model Matrix}

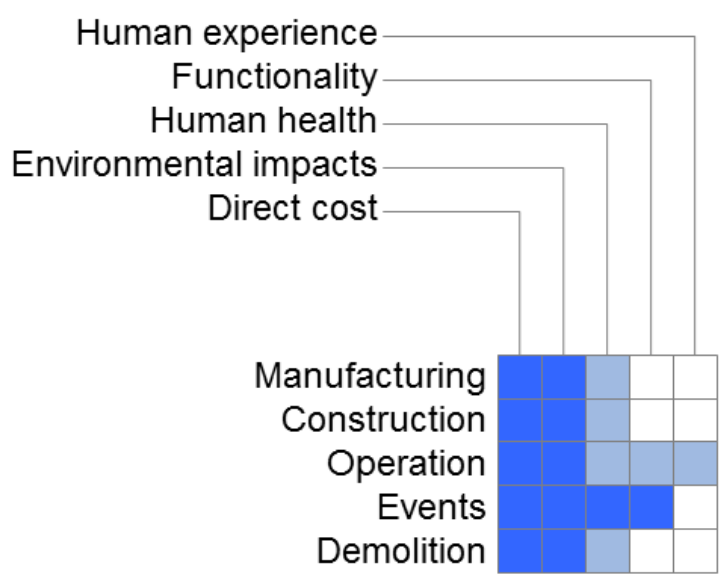




\section{$\mathrm{BIM} \rightarrow \mathrm{IFC} \rightarrow$ FEM}

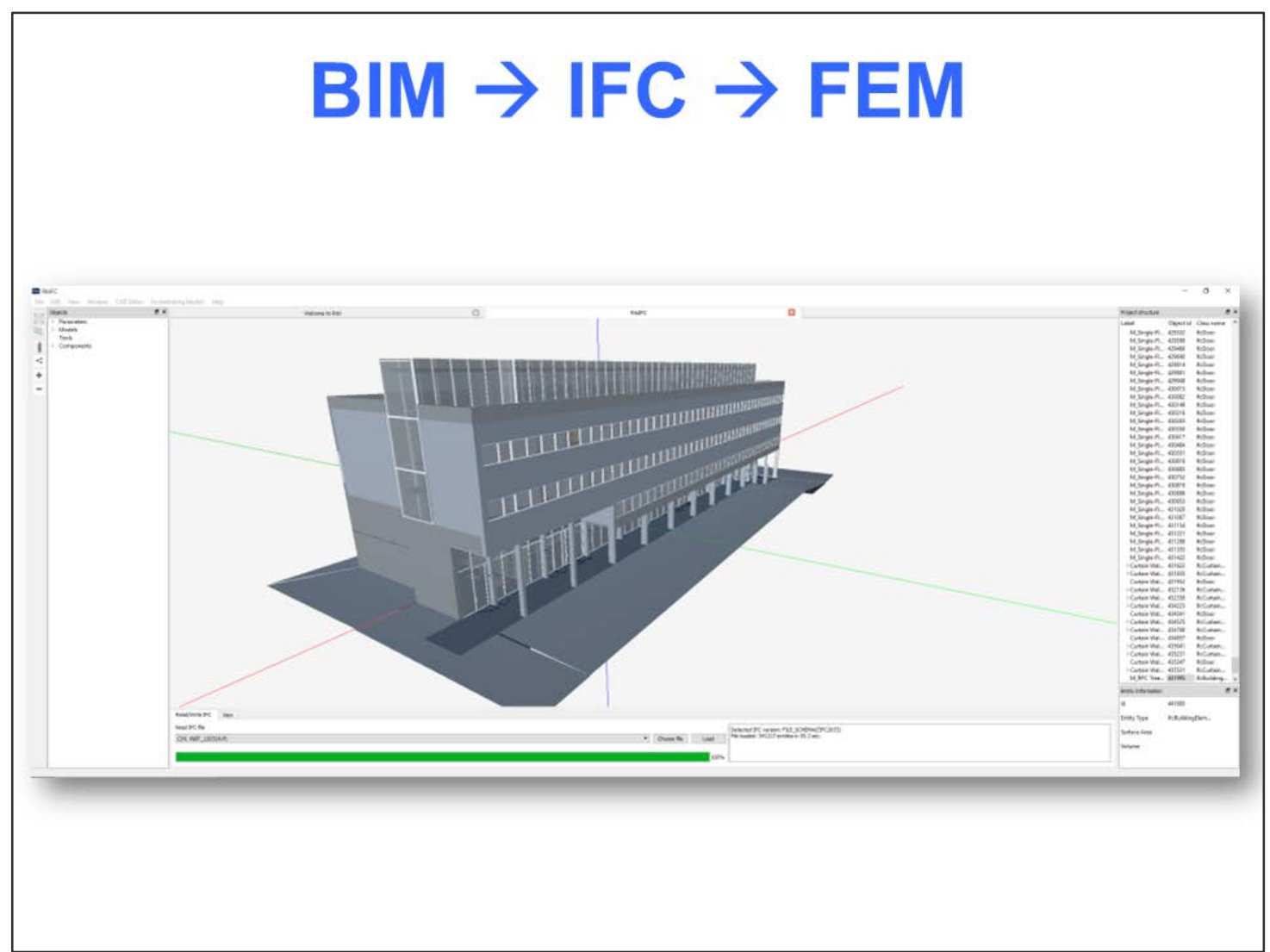

\section{Classic Damage Models}
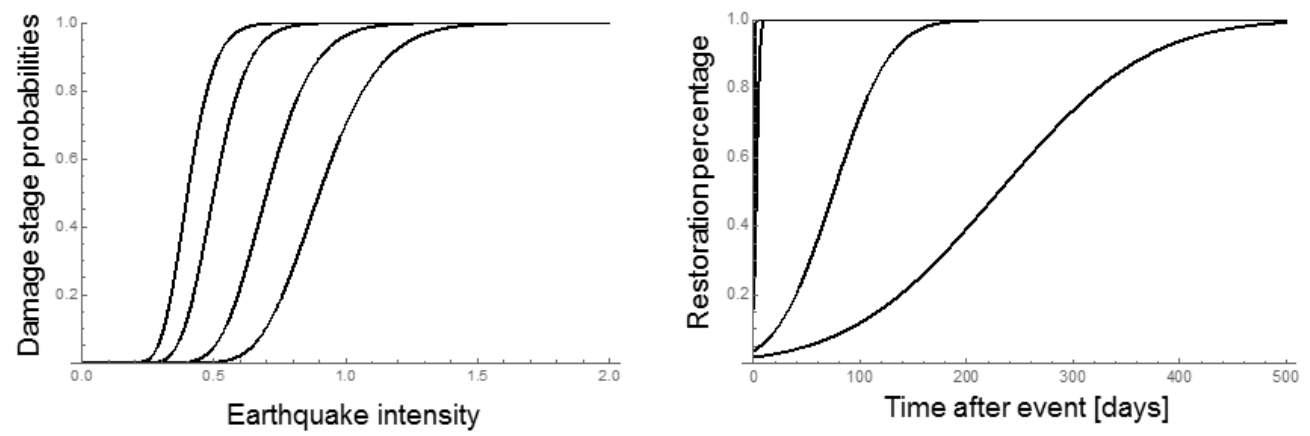


\section{New Models}

$$
\ln \left(\frac{p_{j}}{1-p_{j}}\right)=\theta_{d s_{j}}+\theta_{2} \cdot h_{2}(\mathbf{x})+\theta_{3} \cdot h_{3}(\mathbf{x})+\cdots+\theta_{m} \cdot h_{m}(\mathbf{x})
$$
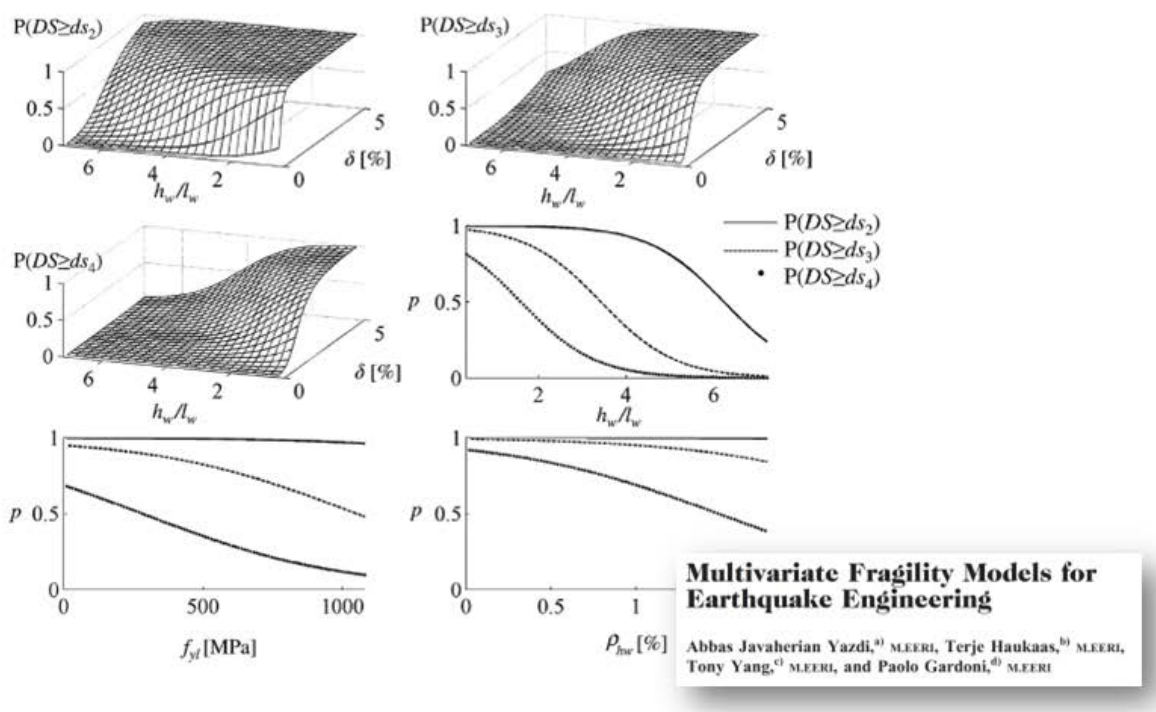

\section{Better Damage Models}

RComponent
getMaterialVolumes ( )
getResponse ()
getConstructionCost ()
getVisualDamage ()
getRepairActions ()
getCo2Emissions ()
...

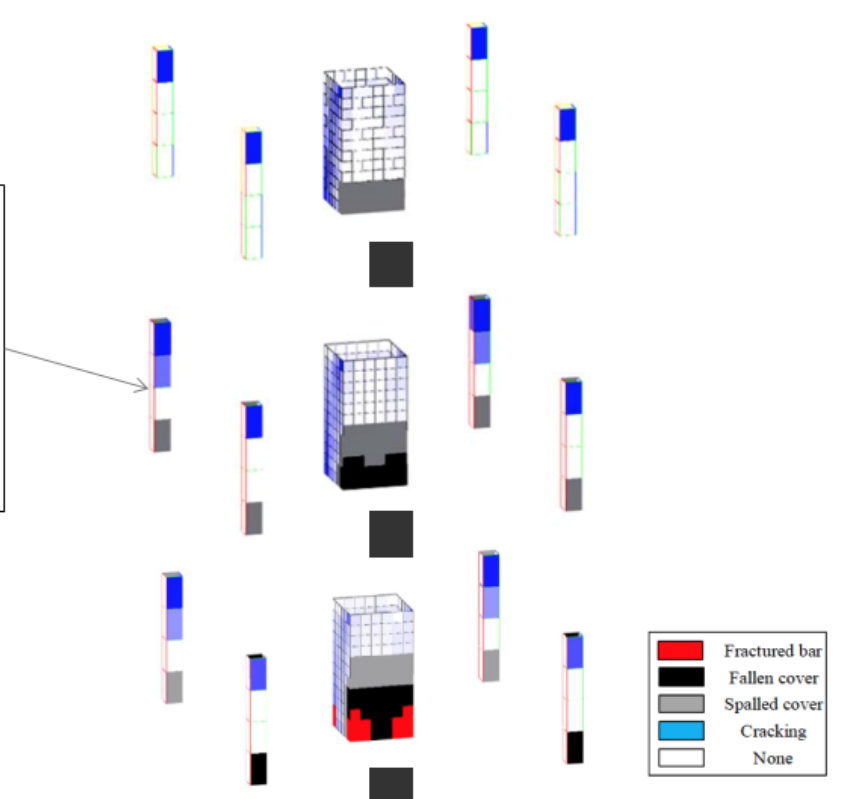

D-12 


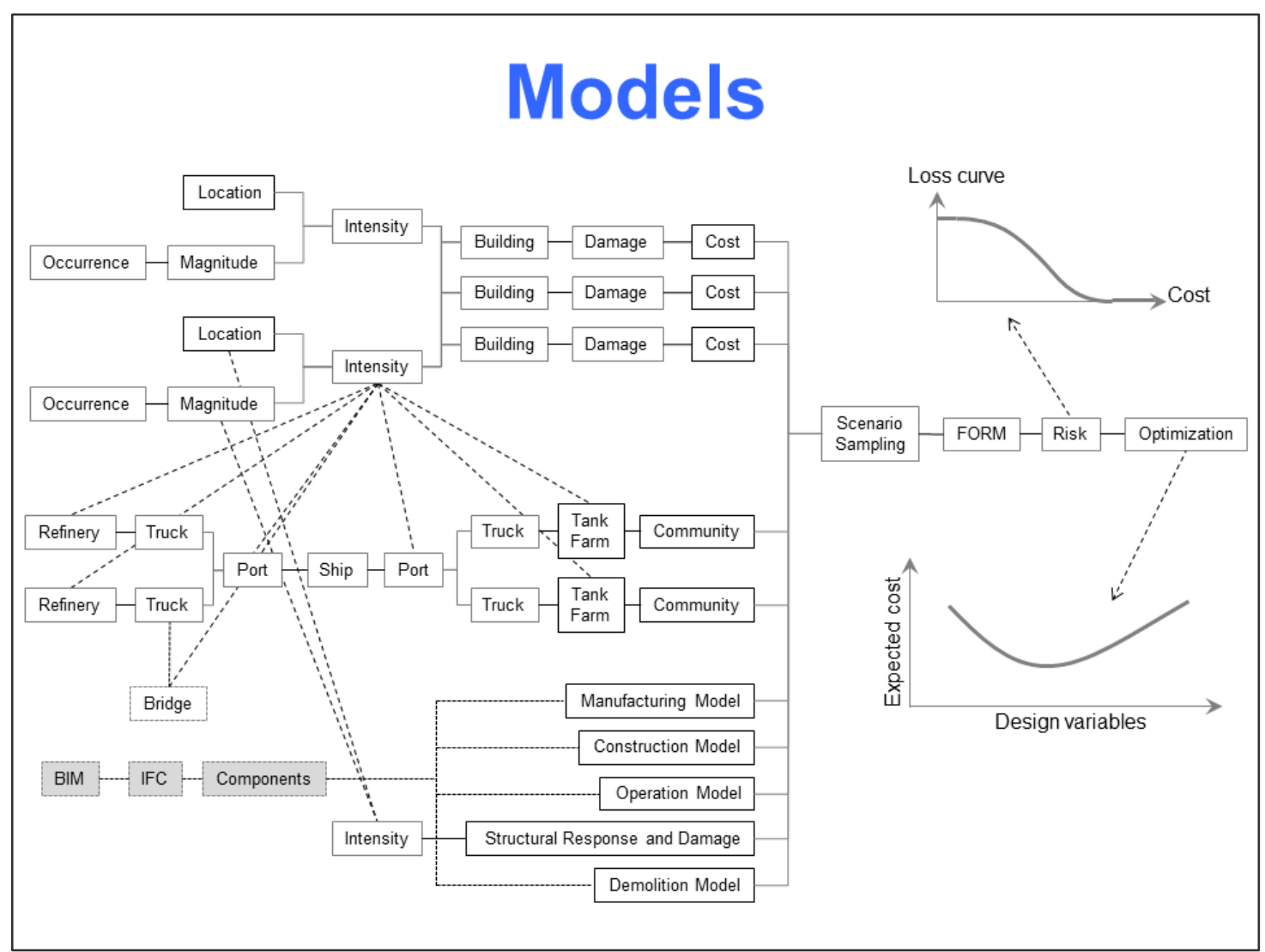

\section{Conclusions}

- Keywords

- Computer simulation models

- Cost-benefit optimization

- Holistic view

- Multi-hazard

- Multi-cost

- Model framework

- OpenSees introduced "plug \& play" with methods

- Rts introduces "plug \& play" with models

\section{- Current focus}

- Models rather than methods

- First go wide, then go deep 


\title{
Acknowledgements
}

\author{
NSERC Funding \\ Dr. Mojtaba Mahsuli \\ Mr. Stevan Gavrilovic, PhD student \\ Mr. Rodrigo Costa, PhD student \\ Dr. Abbas Javaherian Yazdi
}

\section{Thank You for Your Attention}

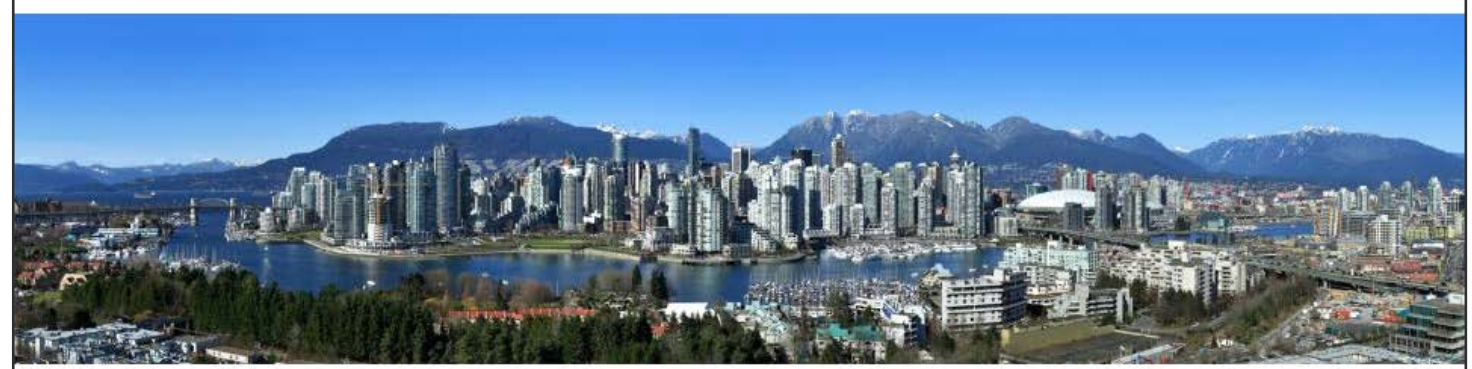




\section{Dr. Terje Haukaas}

Terje Haukaas is a Professor in the Department of Civil Engineering at UBC Vancouver, where he has been a member of the Structural Engineering group since 2003. He received his MS and $\mathrm{PhD}$ degrees from the University of California at Berkeley in 1999 and 2003. Originally from Norway, he obtained his undergraduate degree from the Norwegian University of Science and Technology in 1996 after obtaining an engineering degree from the Stavanger University College in 1994 and a technician degree from the Stavanger Technical College in 1992. He worked as an engineer in Norway from 1997 to 1998. Prior to entering the field of engineering, Dr. Haukaas had become a Journeyman and Master Builder of Carpentry. Dr. Haukaas conducts research on probabilistic modelling of a wide range of hazards, structures, and impacts, with particular emphasis on numerical simulation models. He has authored or coauthored more than thirty journal papers on reliability, sensitivity, and optimization analysis applied to civil engineering problems. Software development is an integral part of Dr. Haukaas' research. He developed the first version of the Matlab toolbox FERUM and he implemented the first reliability and sensitivity options in OpenSees. He later spearheaded the development of Rt, a program for multi-hazard, multi-model reliability and optimization analysis. Dr. Haukaas has received several teaching awards, and he is the recipient of a best-paper award from the ASCE Journal of Computing in Civil Engineering. He is a registered Professional Engineer in the province of British Columbia and he is a member of numerous scholarly committees. 


\section{Road Network Functionality of Istanbul Following an Earthquake}

\section{Himmet Karaman, Betül Ergün Konukcu}

(Istanbul Technical University)

Istanbul is one the most crowded megacities in the world with a population approaching 15 million, besides the city is located on one of the most active seismic zones in the world. Many scientific studies estimate that Istanbul is expecting a major earthquake and the possible damage to the structures will be extensive. Due to the earthquake risk, there have been many studies concerning on the building damages but, none of them focused on the transportation availability following an earthquake. As it is well known, disaster management consist of four phases as preparedness, mitigation, response and recovery. As it is also known, disaster management phases rely on reaching the locations on time. However, without estimating the functionality of the transportation networks, none of the disaster management phases can be planned correctly. This study aims to focus on the transportation functionality of Istanbul. This study firstly, estimated the building damages in Istanbul by using HAZTURK, then extracted buildings with high possibility of heavy damage and collapse. Then, the possible debris area and the road blockages due to those debris were estimated. Next step was to estimate the bridge damages of Istanbul by using HAZTURK. At the last step, all the blocked roads and non-functional bridges were merged and the available transportation network of Istanbul revealed.

Keywords: Road Blockage, Bridge Functionality, Transportation Functionality, Earthquake Damage, Hazturk 


\section{İT̈̈}

\section{Road Network Functionality of Istanbul Following an Earthquake}

Himmet Karaman, Ph.D.

Betül Ergün Konukcu, Ph.D.

ISTANBUL TEKNIK ONIVERSITEST-DEPARIMENT OF GBOMATICS ENANERENC

\section{Contents}

$\checkmark$ Aim of the study

$\circ$ Data

$\checkmark$ Analyses

- Building Damage

- Debris Area \& Radius

- Debris Direction

- Bridge Damage

$\checkmark$ Results

○ Conlusion 


\section{Study Area ${ }^{1}$}

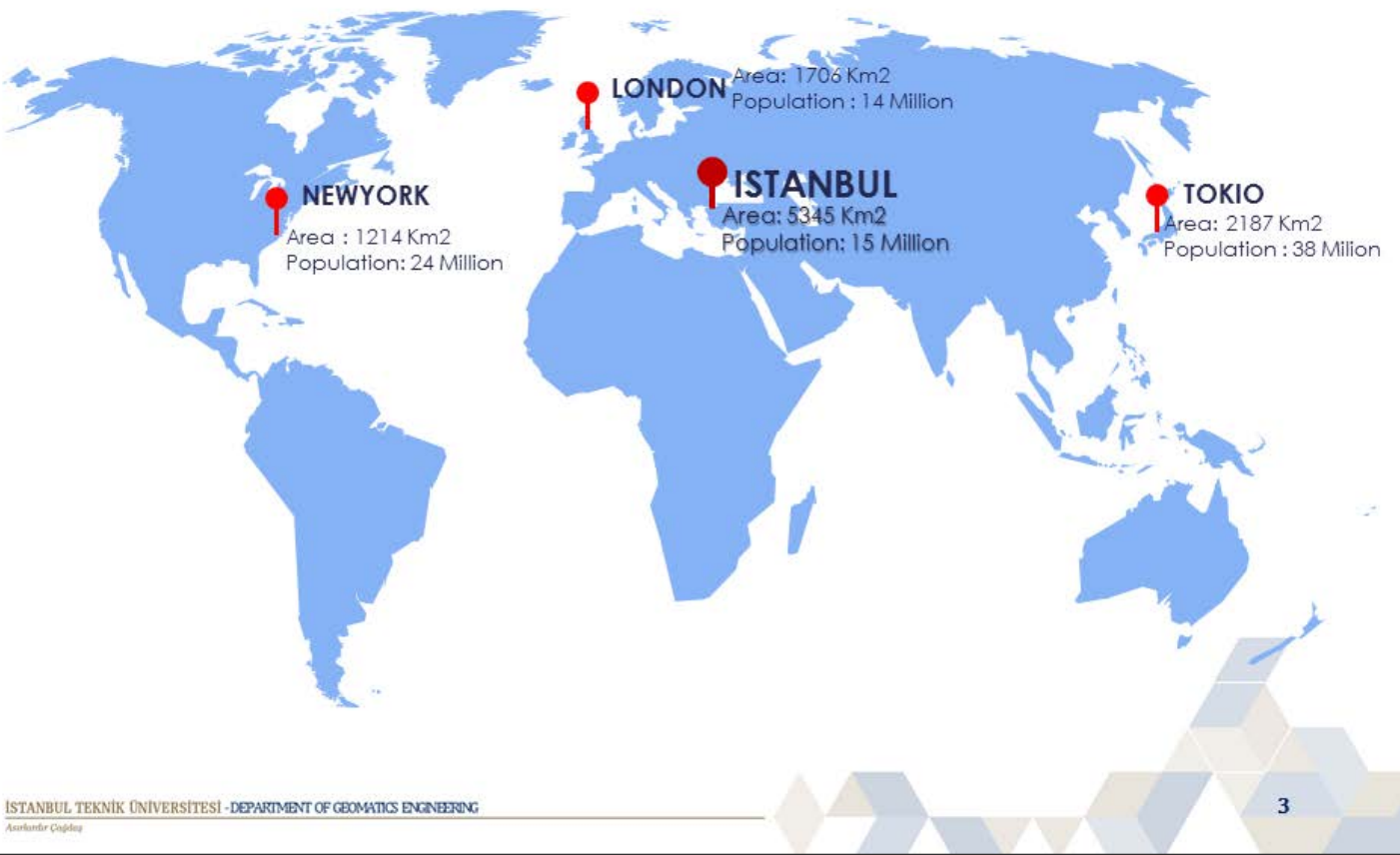

\section{Study Area ${ }^{2}$}

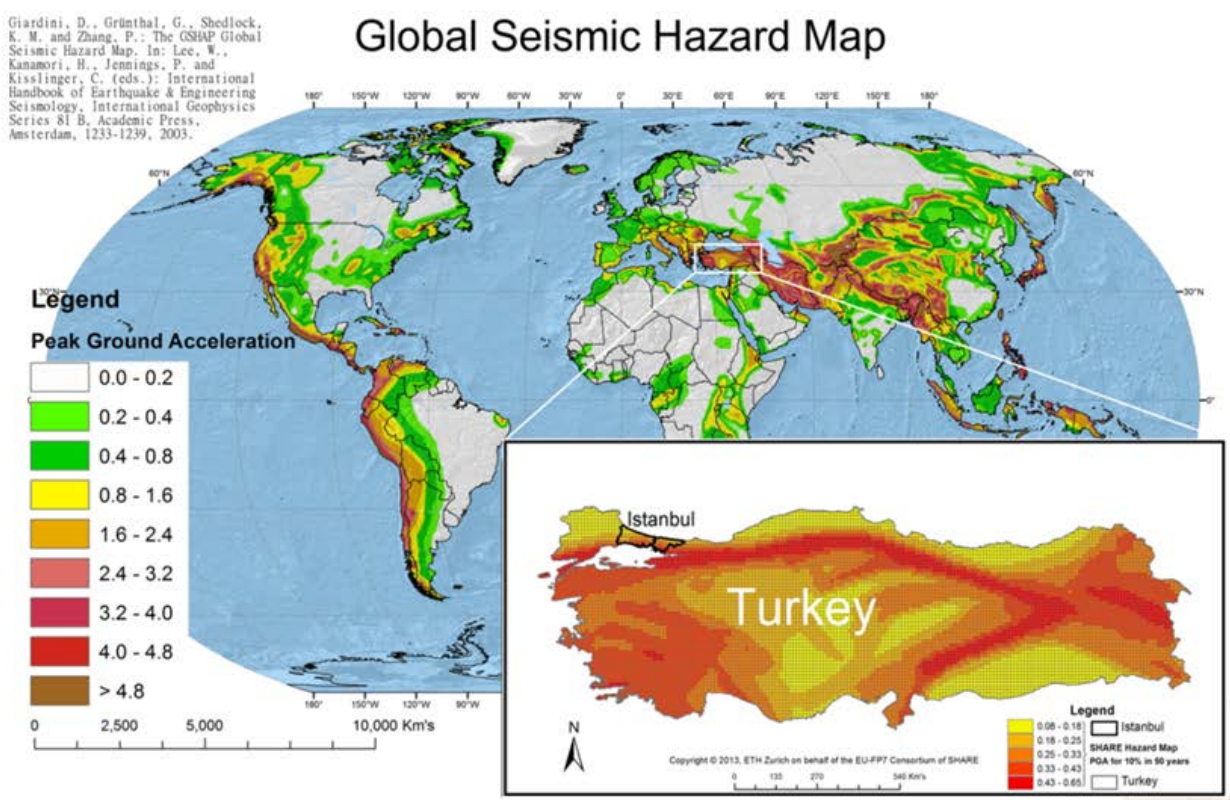




\section{Aim of the Study ${ }^{1}$}

$\checkmark$ Many scientific studies estimate that Istanbul is expecting a major earthquake and the possible damage to the structures will be extensive.

$\checkmark$ Due to earthquake risk, there have been many studies concerning on the building damages but, none of them focused on the transportation availability following an earthquake.

$\square$ This study aims to focus on the transportation functionality of Istanbul.

\section{Aim of the Study ${ }^{2}$}




\section{Building Distribution in Istanbul İ̈̈̈}
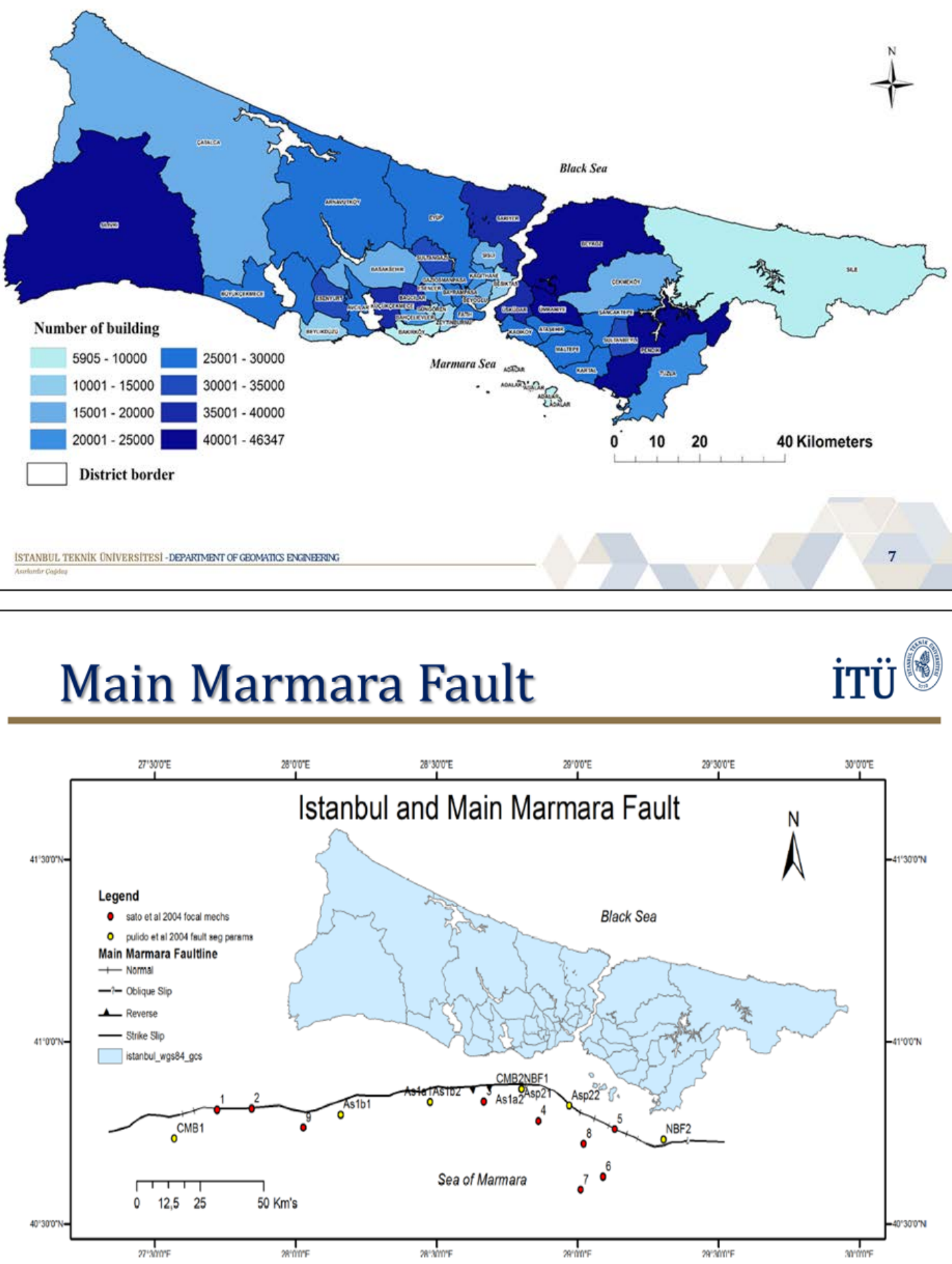


\subsection{Mw Earthquake Hazard Map İ̈̈ (8)}

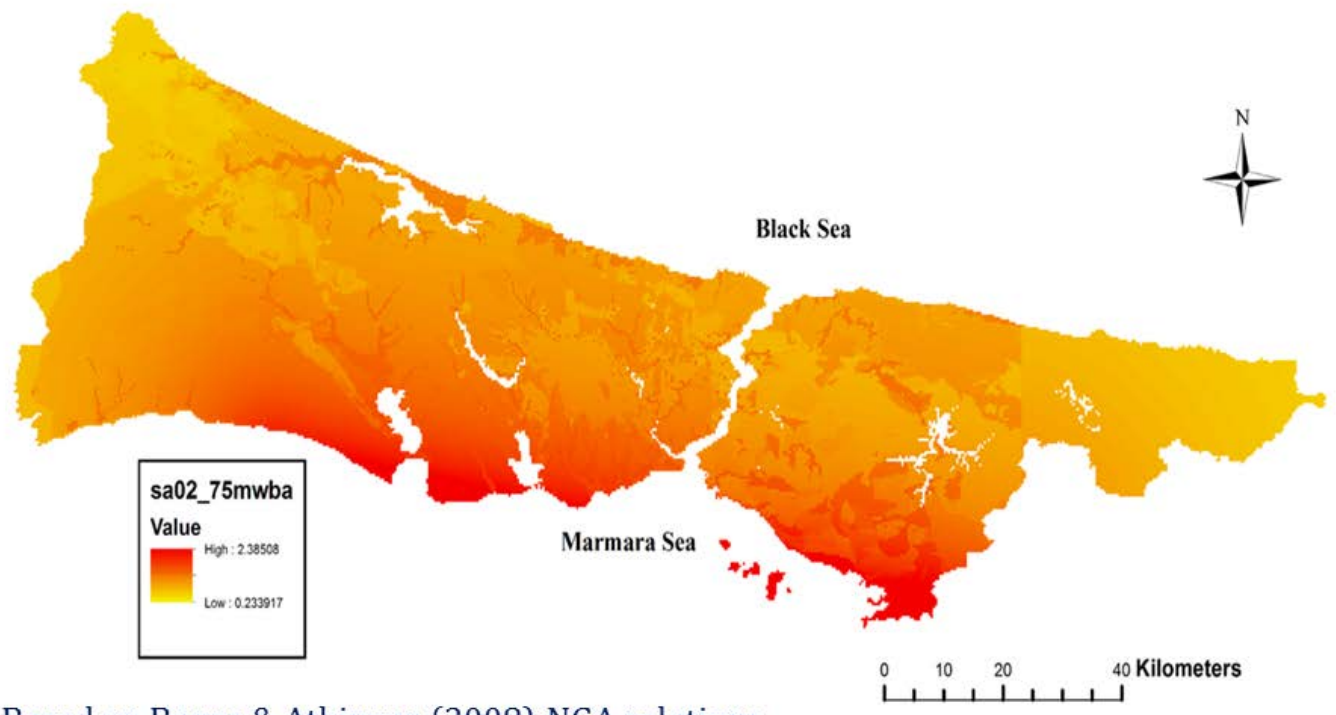

Based on Boore \& Atkinson (2008) NGA relations And JICA (2002) Earthquake Scenario Model A

\section{Building Damage Analyses Results İ̈̈}

Based on structure type

\begin{tabular}{cccccc}
\hline STR_TYP2 & $\begin{array}{c}\text { Insignificant } \\
(\%)\end{array}$ & $\begin{array}{c}\text { Moderate } \\
(\%)\end{array}$ & $\begin{array}{c}\text { Heavy } \\
(\%)\end{array}$ & $\begin{array}{c}\text { Complete } \\
(\%)\end{array}$ & $\begin{array}{c}\text { Mean } \\
\text { Damage } \\
(\%)\end{array}$ \\
\hline ClH & 22.7 & 48.1 & 21.7 & 7.4 & 26.2 \\
C1L & 22.8 & 37.2 & 27.4 & 12.4 & 32.1 \\
C1M & 22.0 & 49.0 & 21.3 & 7.5 & 26.2 \\
C2H & 26.7 & 50.4 & 17.9 & 4.8 & 22.1 \\
C2L & 35.6 & 35.7 & 22.3 & 6.2 & 23.6 \\
C2M & 32.5 & 43.8 & 17.2 & 6.4 & 22.2 \\
C3H & 15.1 & 40.3 & 28.4 & 16.0 & 36.3 \\
C3L & 19.0 & 28.8 & 31.5 & 20.6 & 40.4 \\
C3M & 17.2 & 37.8 & 27.8 & 17.1 & 36.6 \\
PC1 & 18.9 & 35.1 & 31.0 & 14.8 & 35.9 \\
PC2H & 21.5 & 46.1 & 25.5 & 6.7 & 27.3 \\
PC2L & 21.4 & 31.9 & 32.4 & 14.1 & 35.6 \\
PC2M & 16.3 & 37.8 & 31.5 & 14.2 & 36.1 \\
RM1L & 23.6 & 31.8 & 32.6 & 11.8 & 33.6 \\
RM2H & 11.5 & 39.8 & 34.5 & 14.0 & 37.8 \\
RM2M & 21.3 & 59.7 & 17.8 & 1.0 & 20.1 \\
S1H & 34.3 & 41.0 & 19.4 & 5.1 & 21.8 \\
SIM & 20.0 & 37.4 & 28.8 & 13.6 & 34.0 \\
S3 & 9.8 & 28.6 & 37.8 & 23.6 & 46.5 \\
URML & 12.7 & 23.1 & 27.6 & 36.4 & 51.6 \\
URMM & 11.5 & 26.2 & 33.4 & 28.7 & 48.4 \\
W1 & 25.9 & 39.6 & 23.1 & 11.1 & 29.0 \\
W2 & 32.8 & 44.1 & 17.2 & 6.2 & 22.1 \\
\hline
\end{tabular}

General distribution

$$
\begin{aligned}
& \begin{array}{cccccc}
\text { HAZTURK in } & \begin{array}{c}
\text { Insignificant } \\
2015
\end{array} & \begin{array}{c}
\text { Moderate } \\
\text { (\%) }
\end{array} & \begin{array}{c}
\text { Heavy } \\
(\%)
\end{array} & \begin{array}{c}
\text { Complete } \\
(\%)
\end{array} & \begin{array}{c}
\text { Mean } \\
\text { Damage }
\end{array}
\end{array} \\
& 2015
\end{aligned}
$$

\begin{tabular}{|c|c|c|c|c|c|}
\hline YEAR BUILT & $\begin{array}{l}\text { Insignificant } \\
(\%)\end{array}$ & $\begin{array}{c}\text { Moderate } \\
\text { (\%) }\end{array}$ & $\begin{array}{c}\text { Heavy } \\
\text { (\%) }\end{array}$ & $\begin{array}{c}\text { Complete } \\
(\%)\end{array}$ & $\begin{array}{c}\text { Mean } \\
\text { Damage } \\
(\%)\end{array}$ \\
\hline Before 1968 & 14.1 & 31.8 & 31.7 & 22.2 & 42.4 \\
\hline Between $1969-1982$ & 19.1 & 34.4 & 28.9 & 17.4 & 37.0 \\
\hline Between 1983- 1996 & 18.9 & 33.4 & 29.3 & 18.2 & 37.8 \\
\hline Between 1997-2004 & 27.0 & 39.9 & 23.7 & 9.8 & 27.7 \\
\hline Between 2005-2007 & 36.4 & 45.0 & 15.3 & 3.2 & 18.4 \\
\hline Between 2008- 2013 & 30.1 & 51.30 & 15.7 & 2.8 & 19.2 \\
\hline
\end{tabular}




\section{Building Damage Distribution İт̈ं}

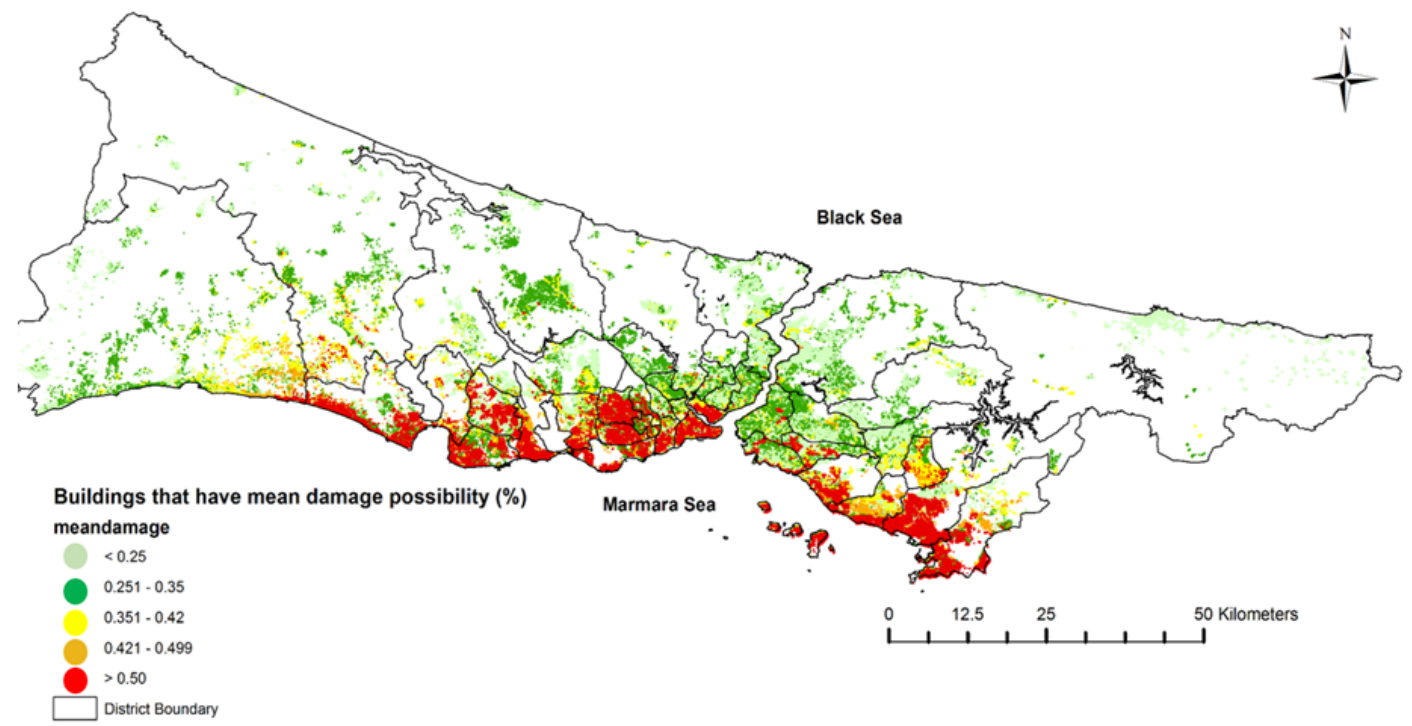

\section{Buildings with Complete \& Heavy Damage Possibilities more then $40 \%$

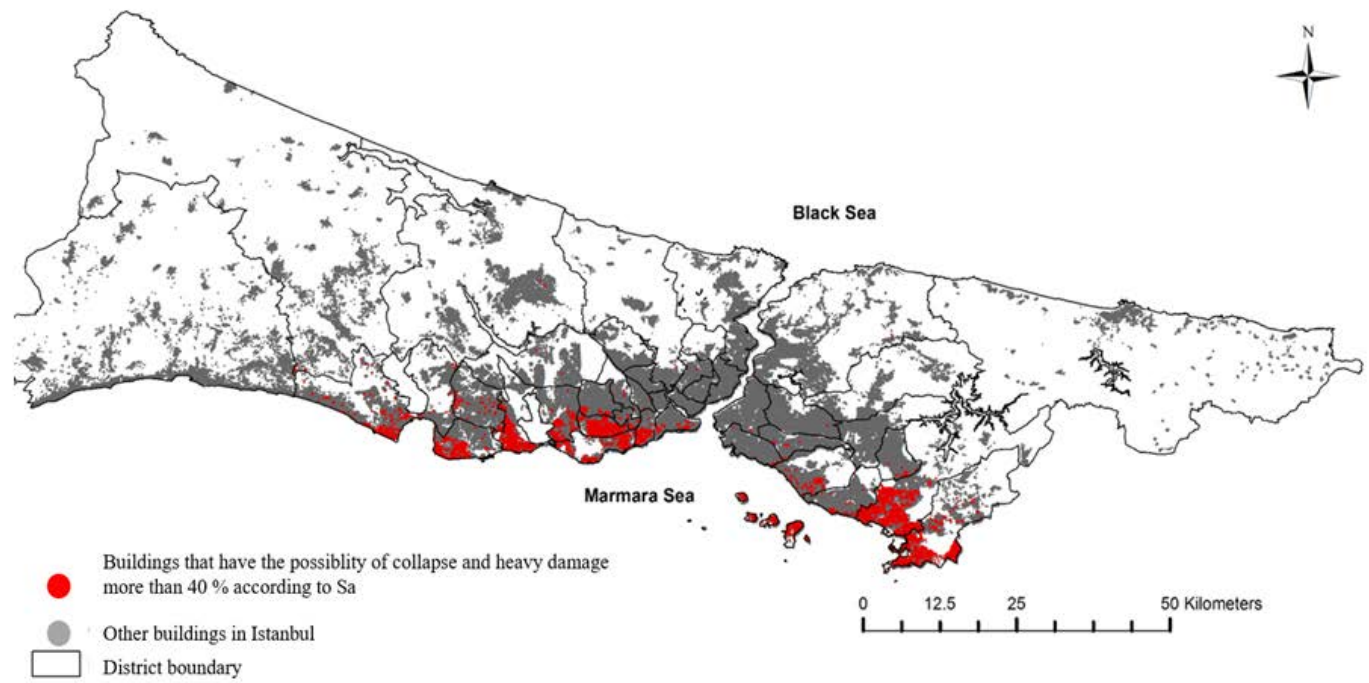




\section{Building Collapse Directions

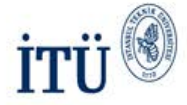

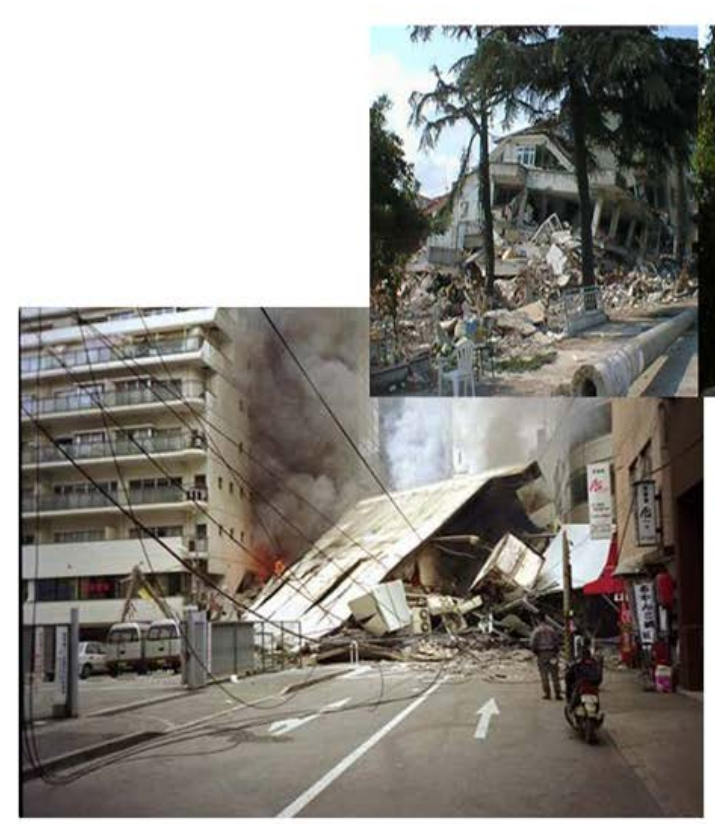

ISTANBUL TEKNIK ONIVERSITESI -DEPARIMENT OF CBOMATIS ENCNEETNC

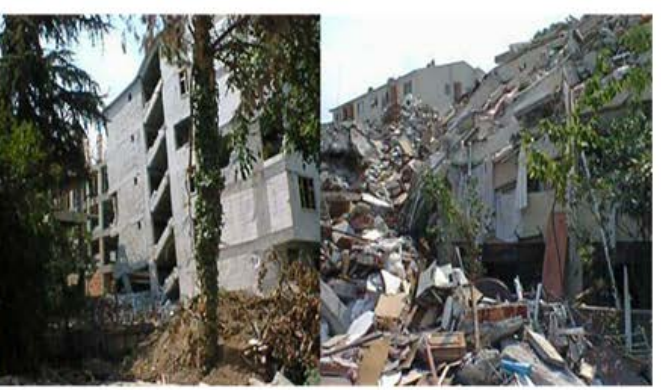

The scattered parts of collapsed buildings could create debris around the buildings and this debris could cause road blockages in the vicinity of the damaged buildings, especially on the narrow roads.

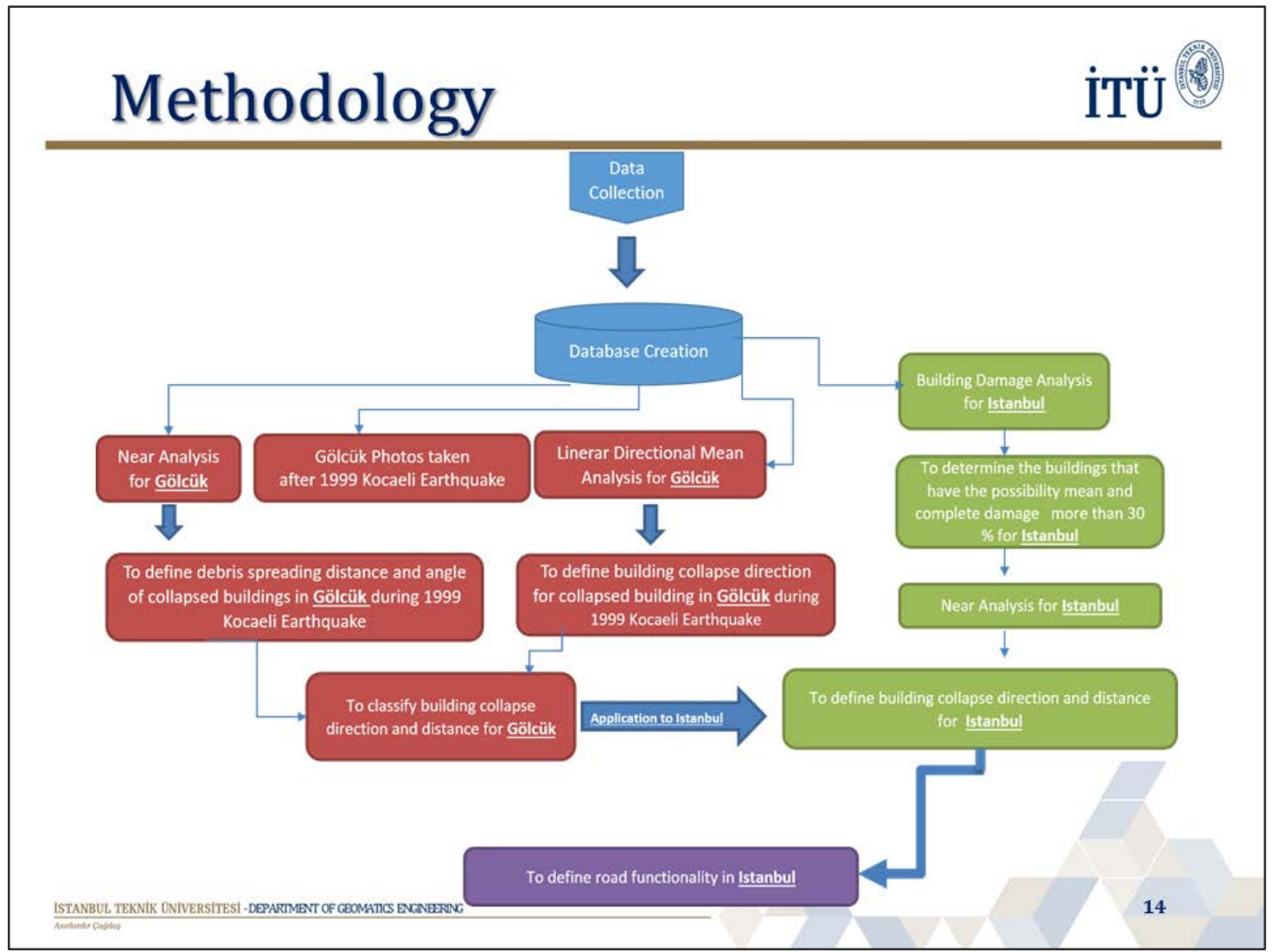




\section{Study Area ${ }^{1}$}

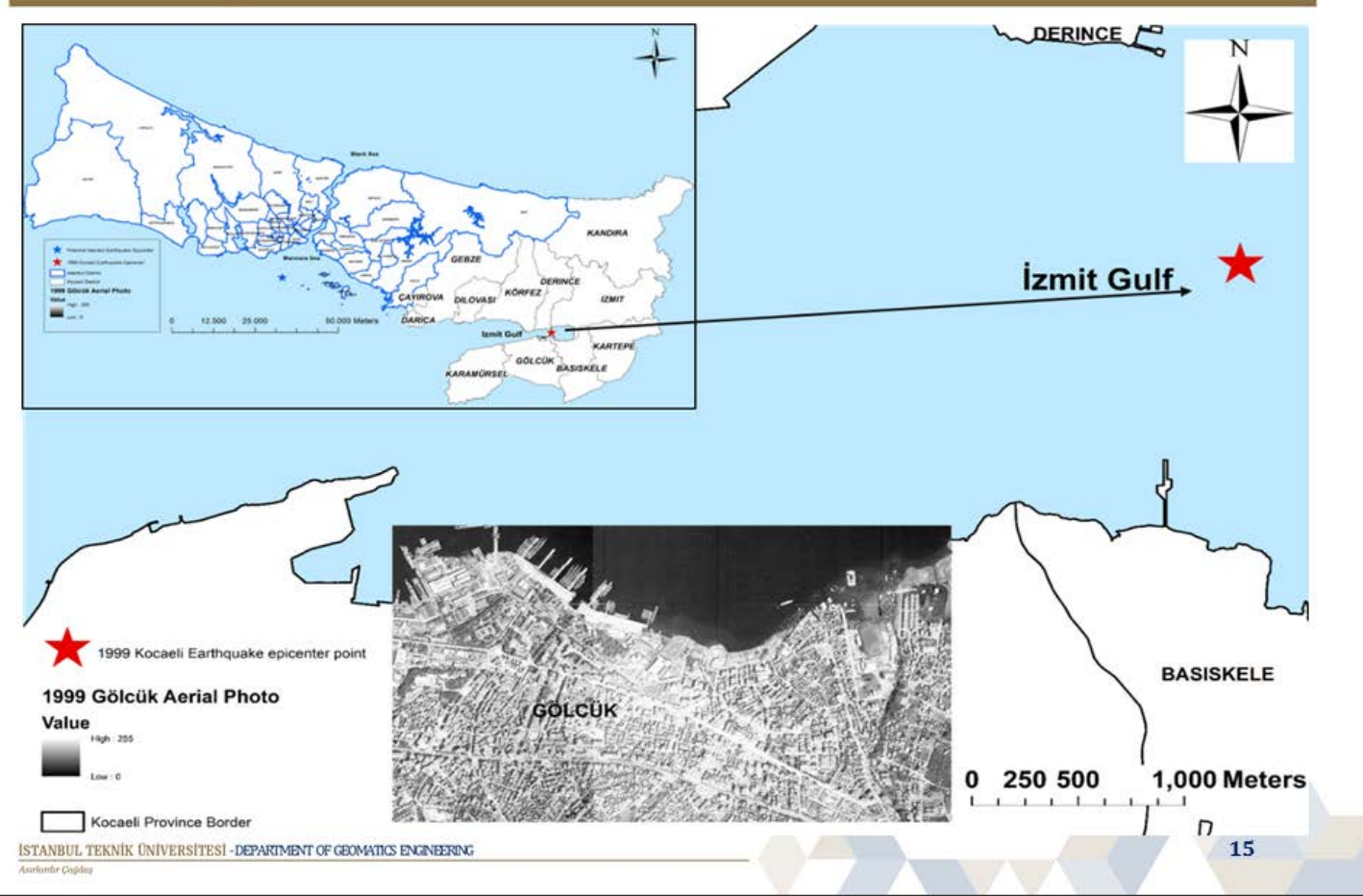

\section{Study Area ${ }^{2}$}
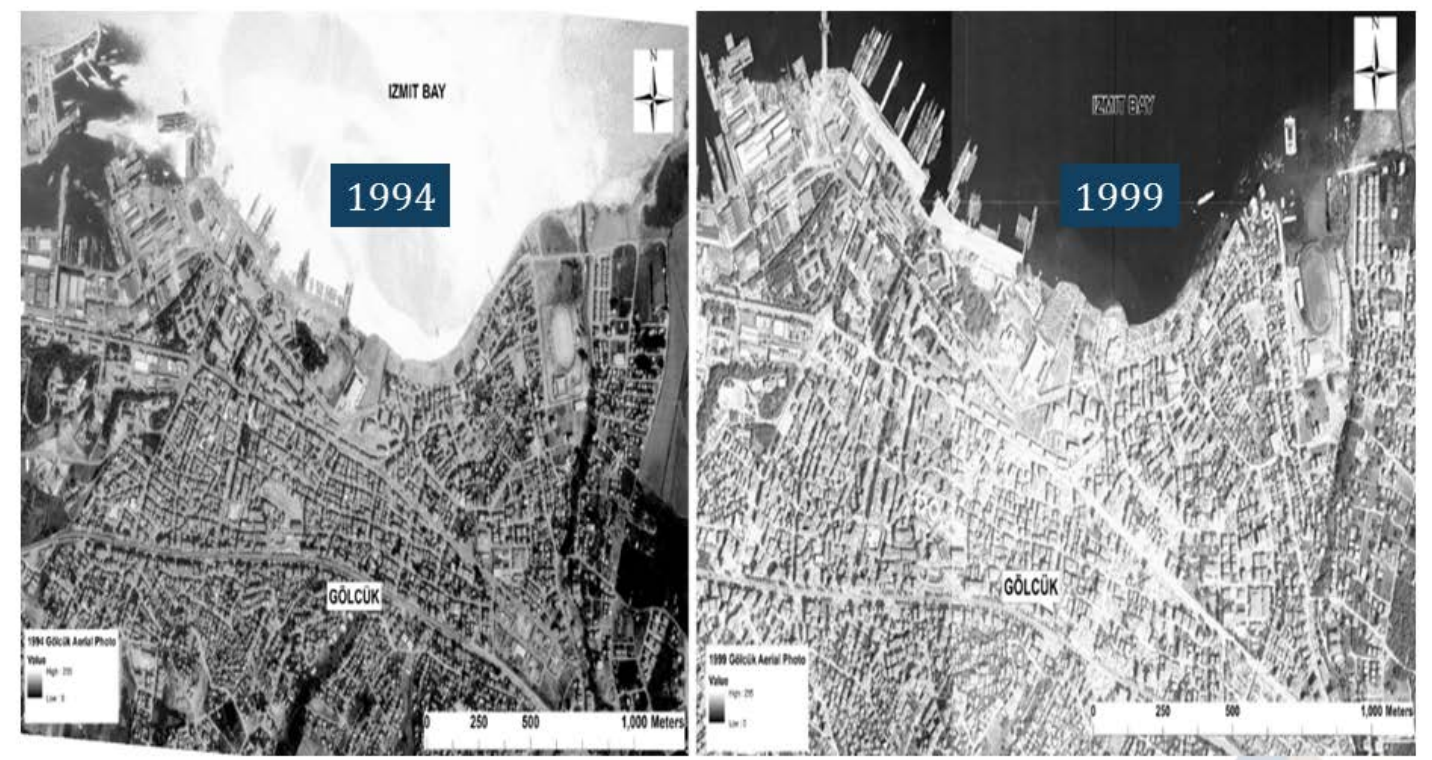

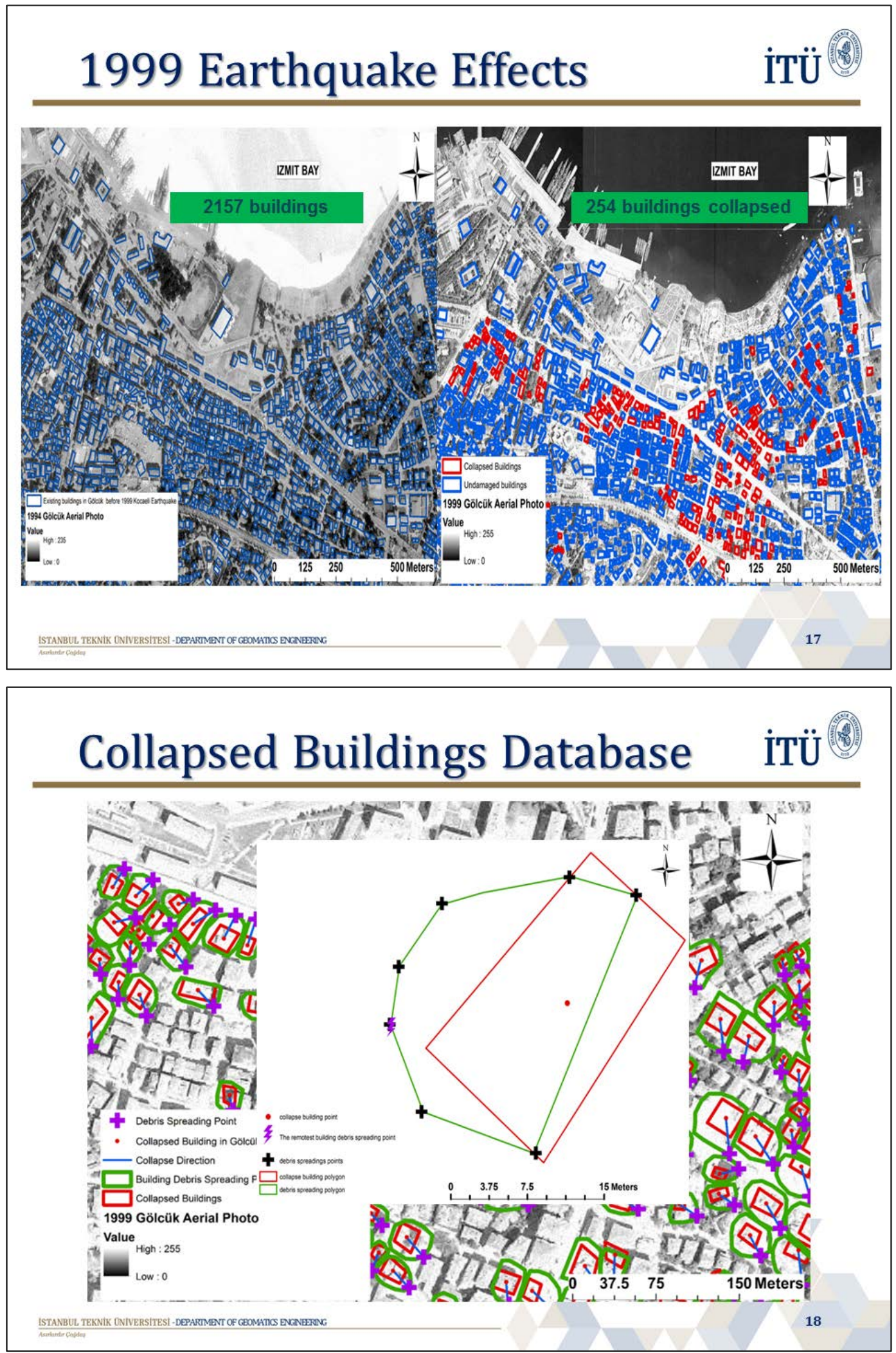

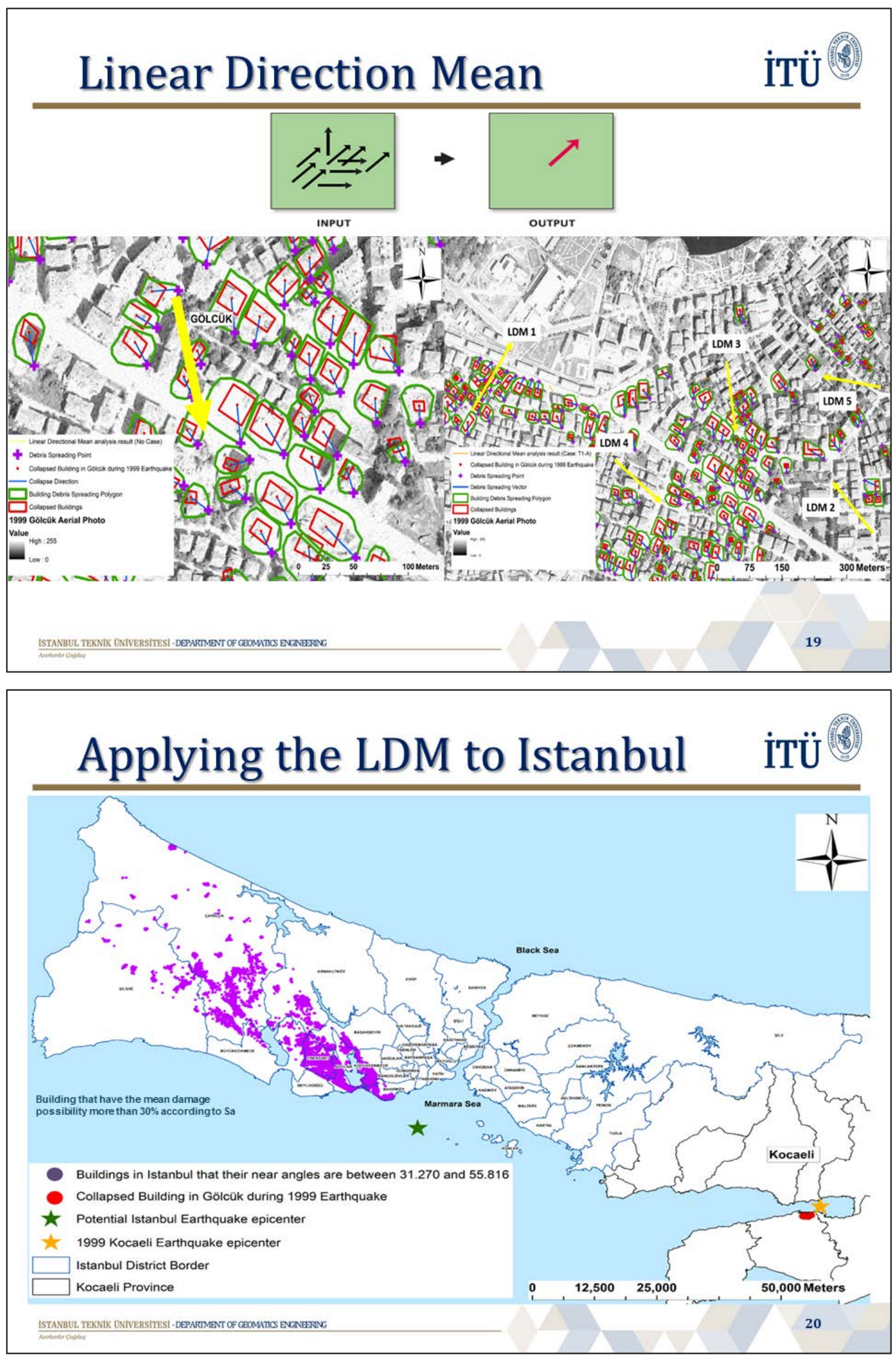

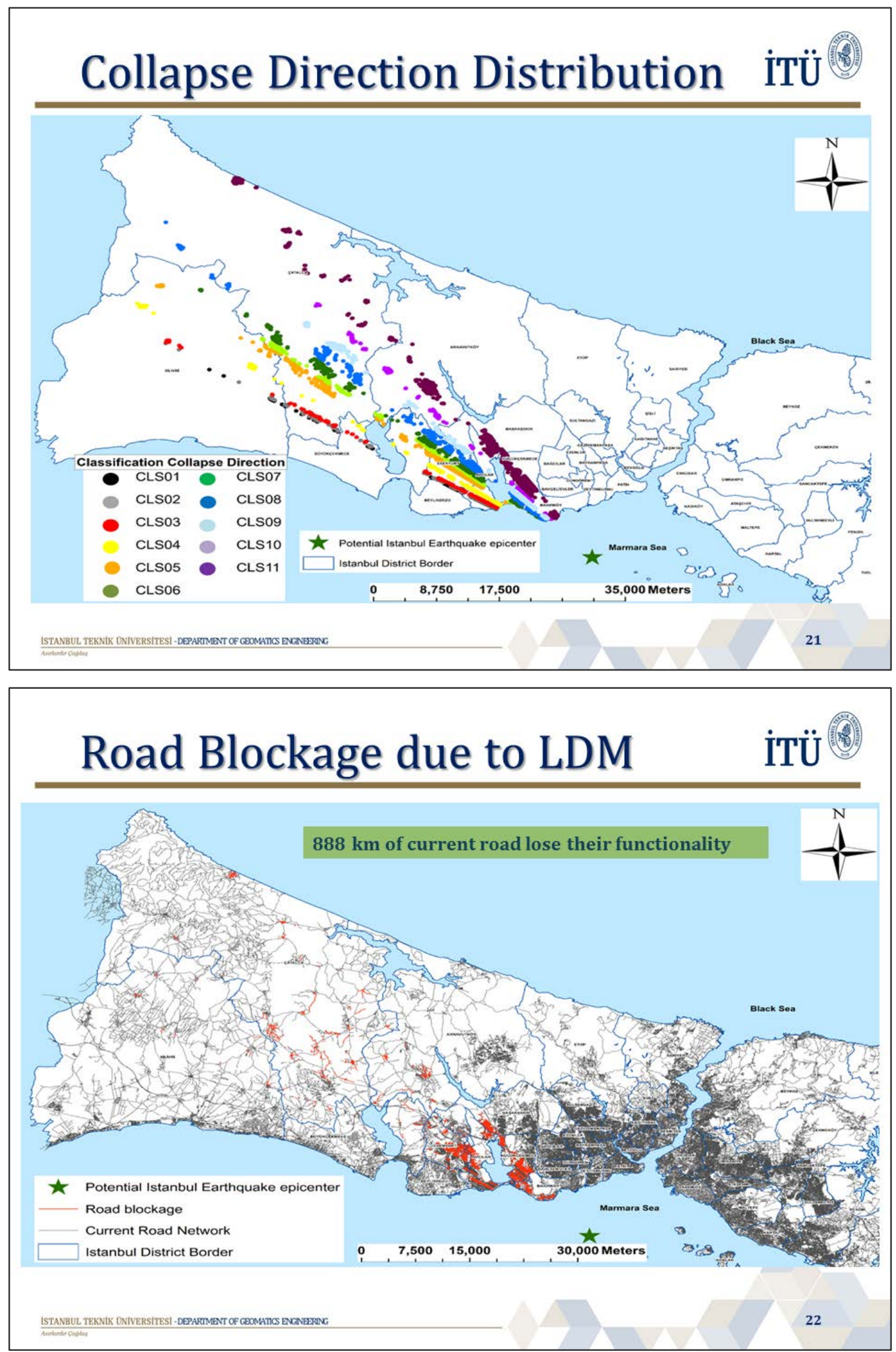


\section{Average Debris Distance}

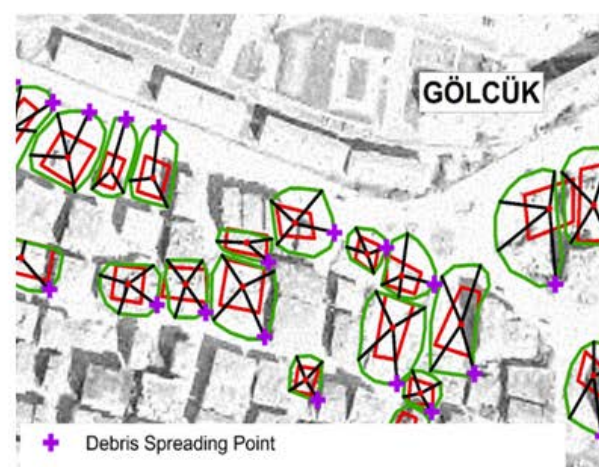

+ Debris Spreading Poin

- Collapsed Building in Gölcük during 1999 Earthquake

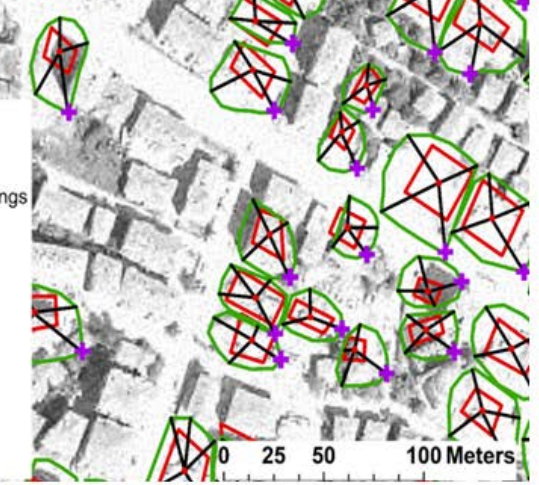

The

average distance of the debris of the collapsed buildings in Gölcük is

Debris Spreading Vectors Around The Collapsed Buildings 1999 Gölcük Aerial Photo Value

High : 255

Low : 0

\section{$17.45 \mathrm{~m}$}

\section{Road Blockage due to Buffer}
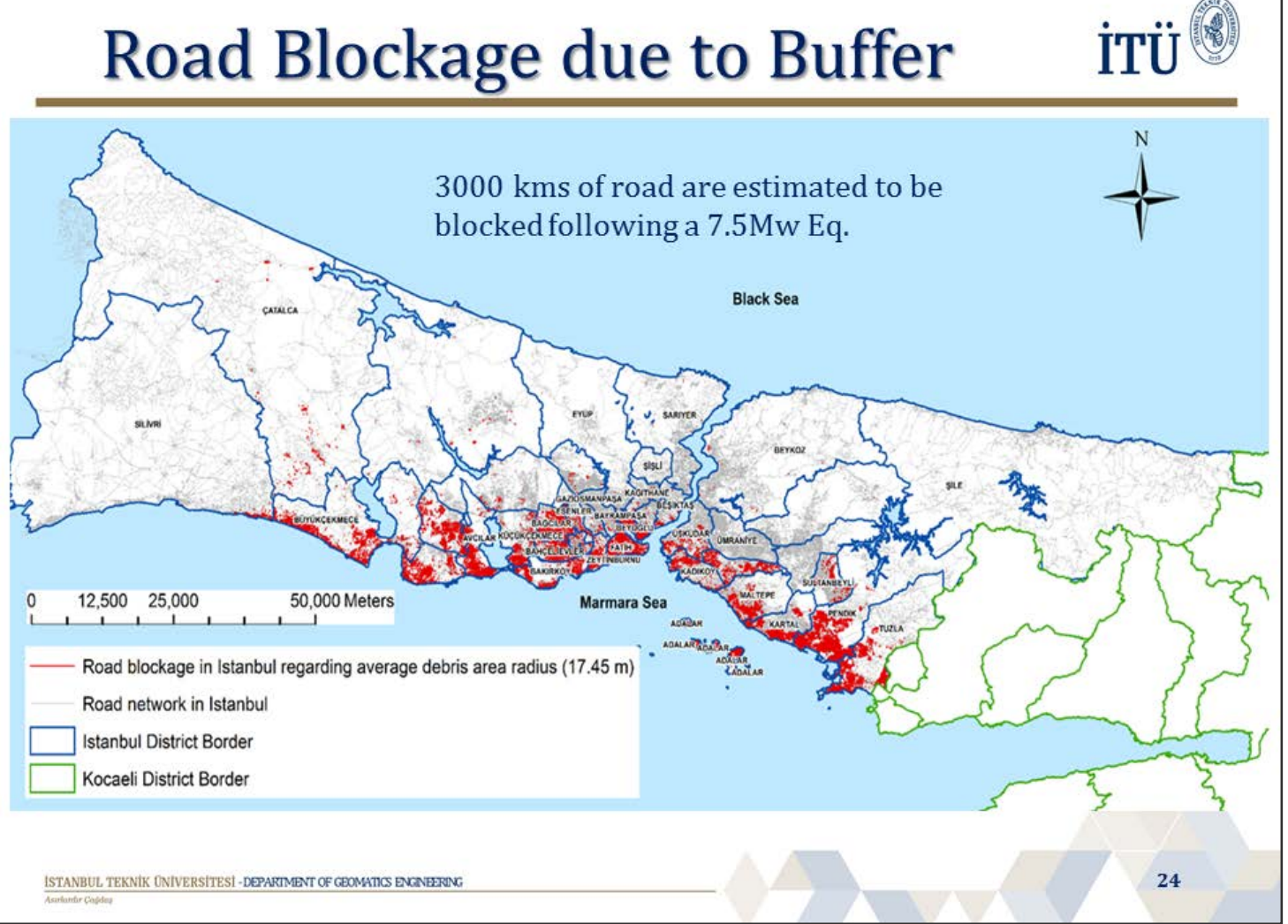


\section{A Case Study}
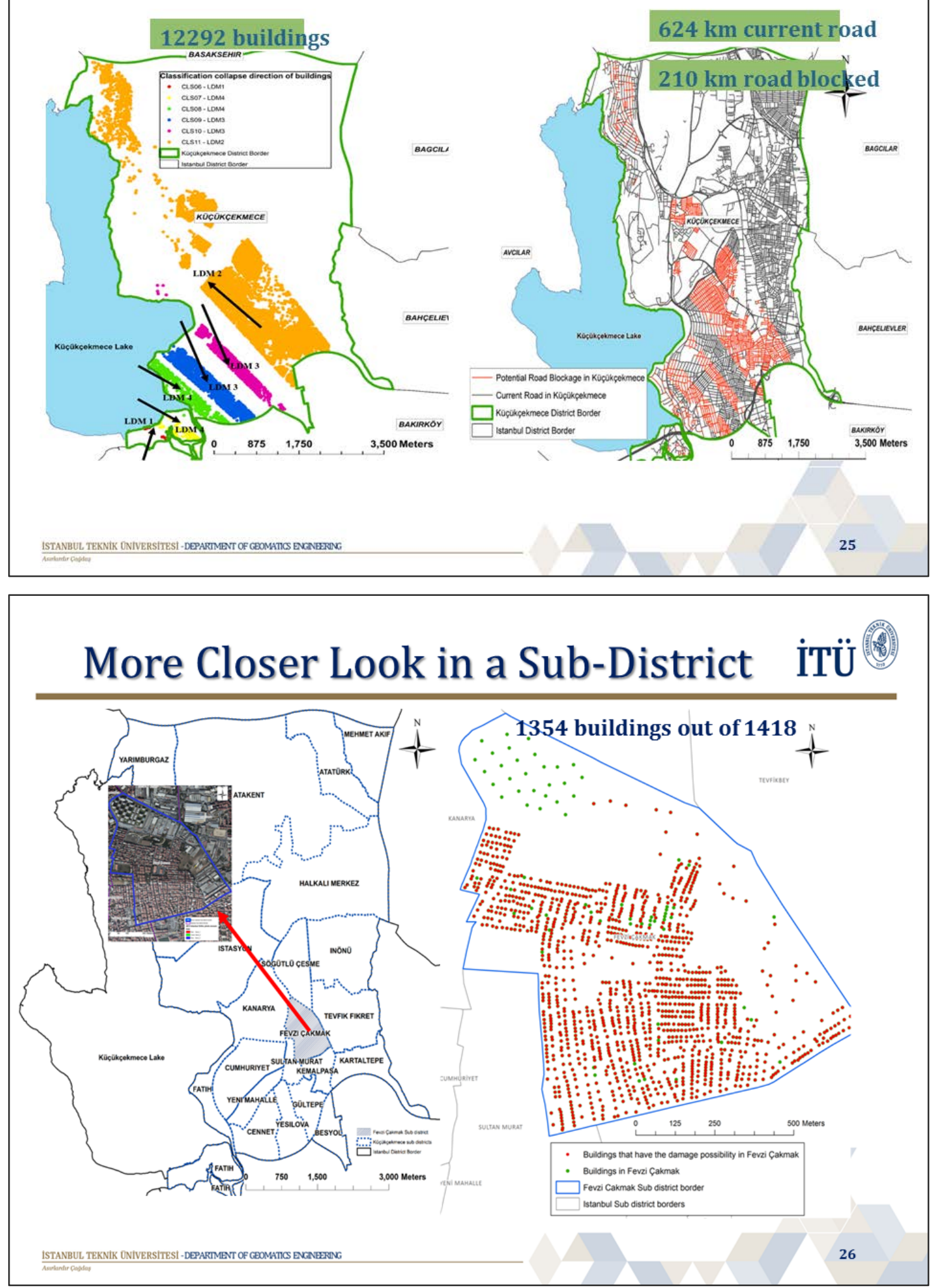


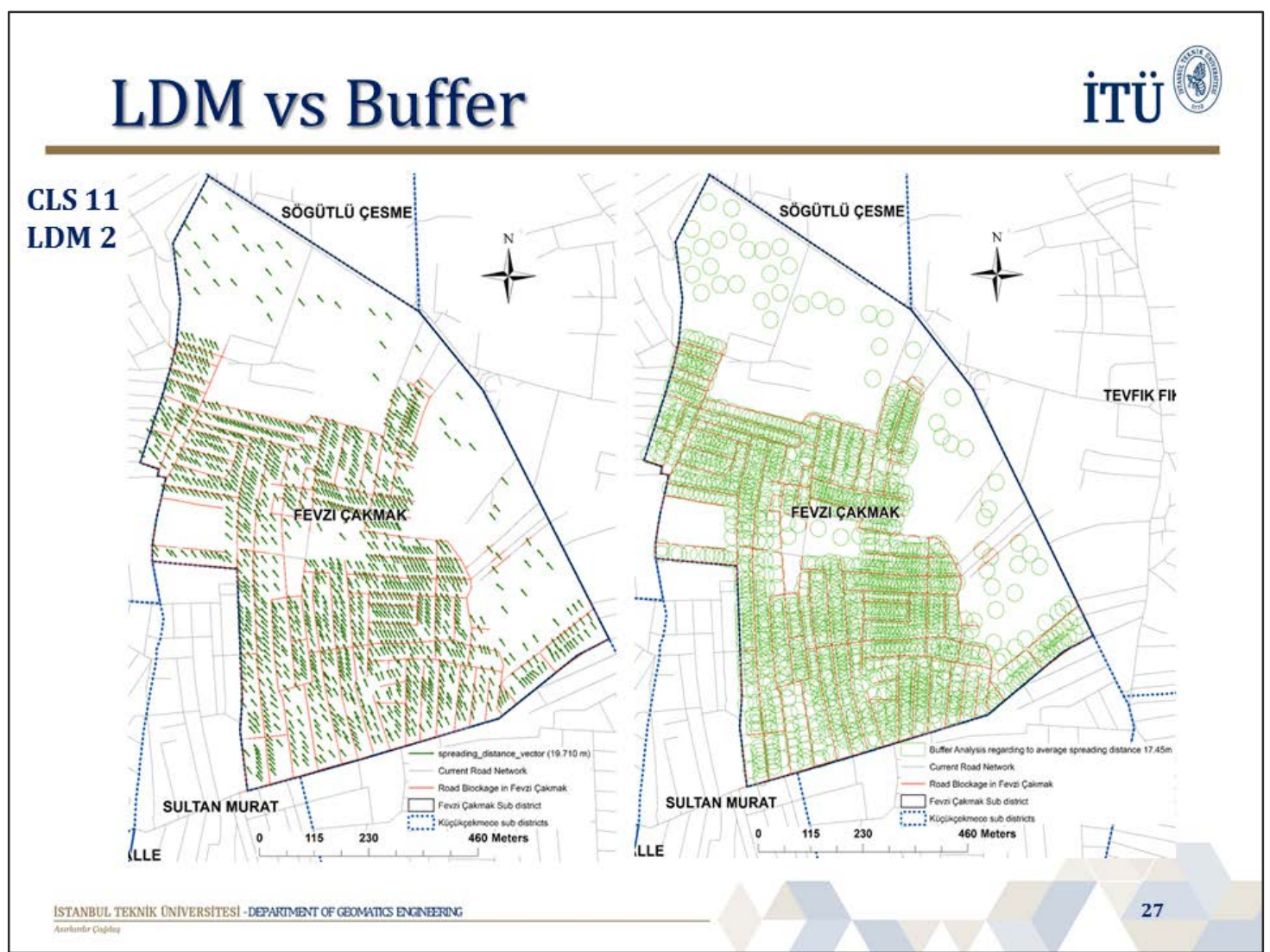

\section{Road Blockage}

їт̈ं

\section{$11741 \mathrm{~m}$ of road blockage out of $18745 \mathrm{~m}$}

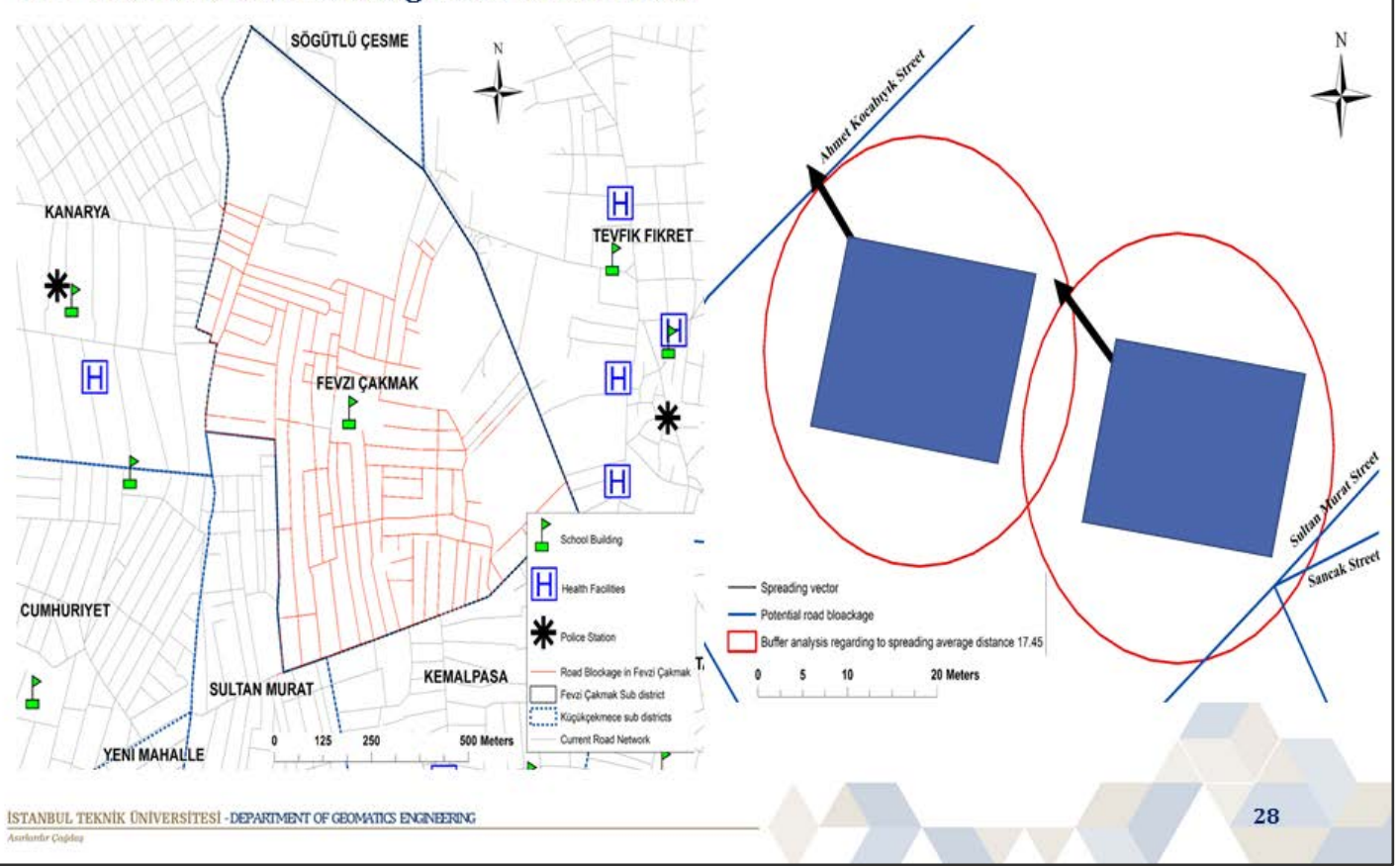




\section{Bridge Damages}
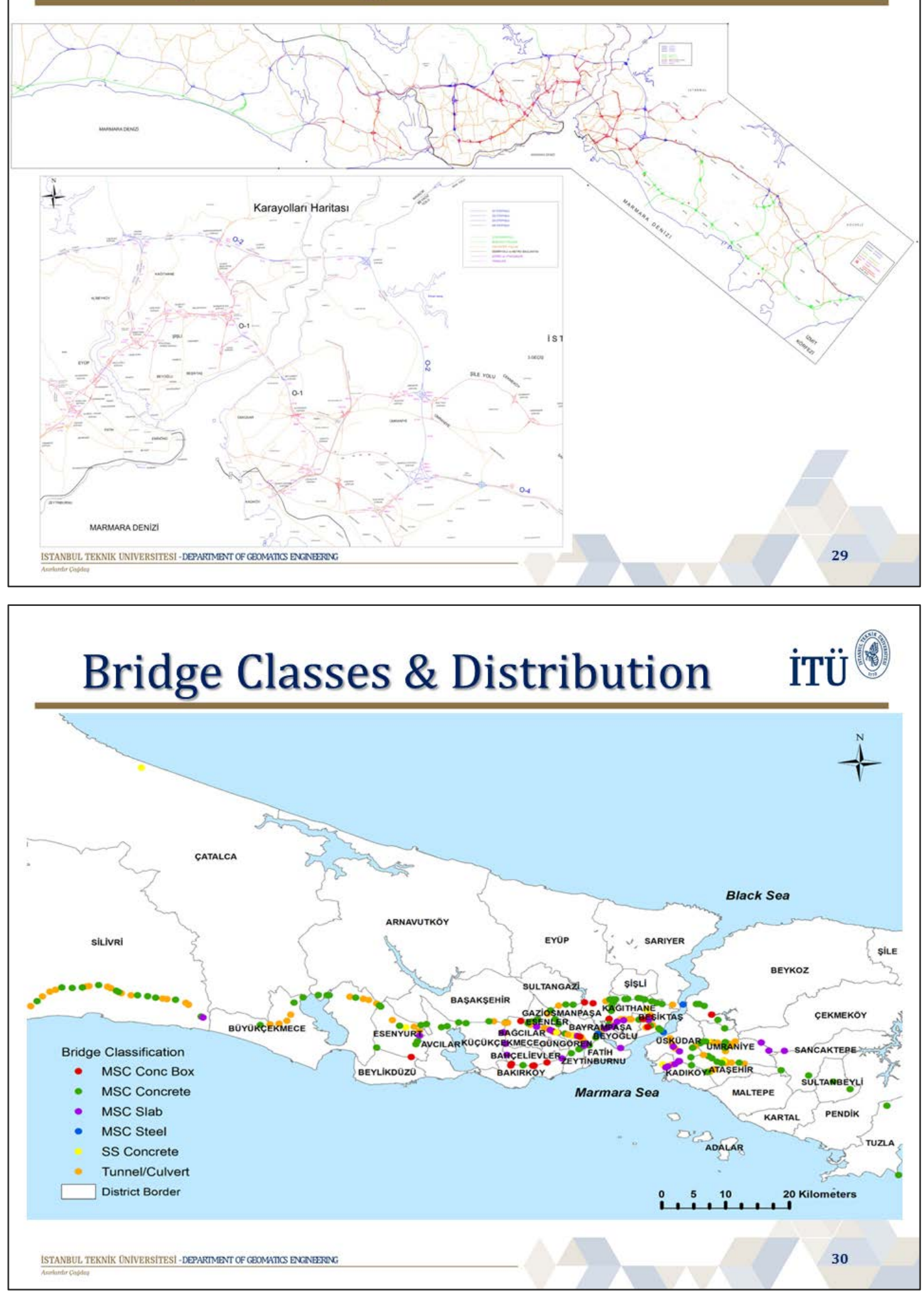


\section{Mean Damage Possibilities of Bridges in Istanbul}
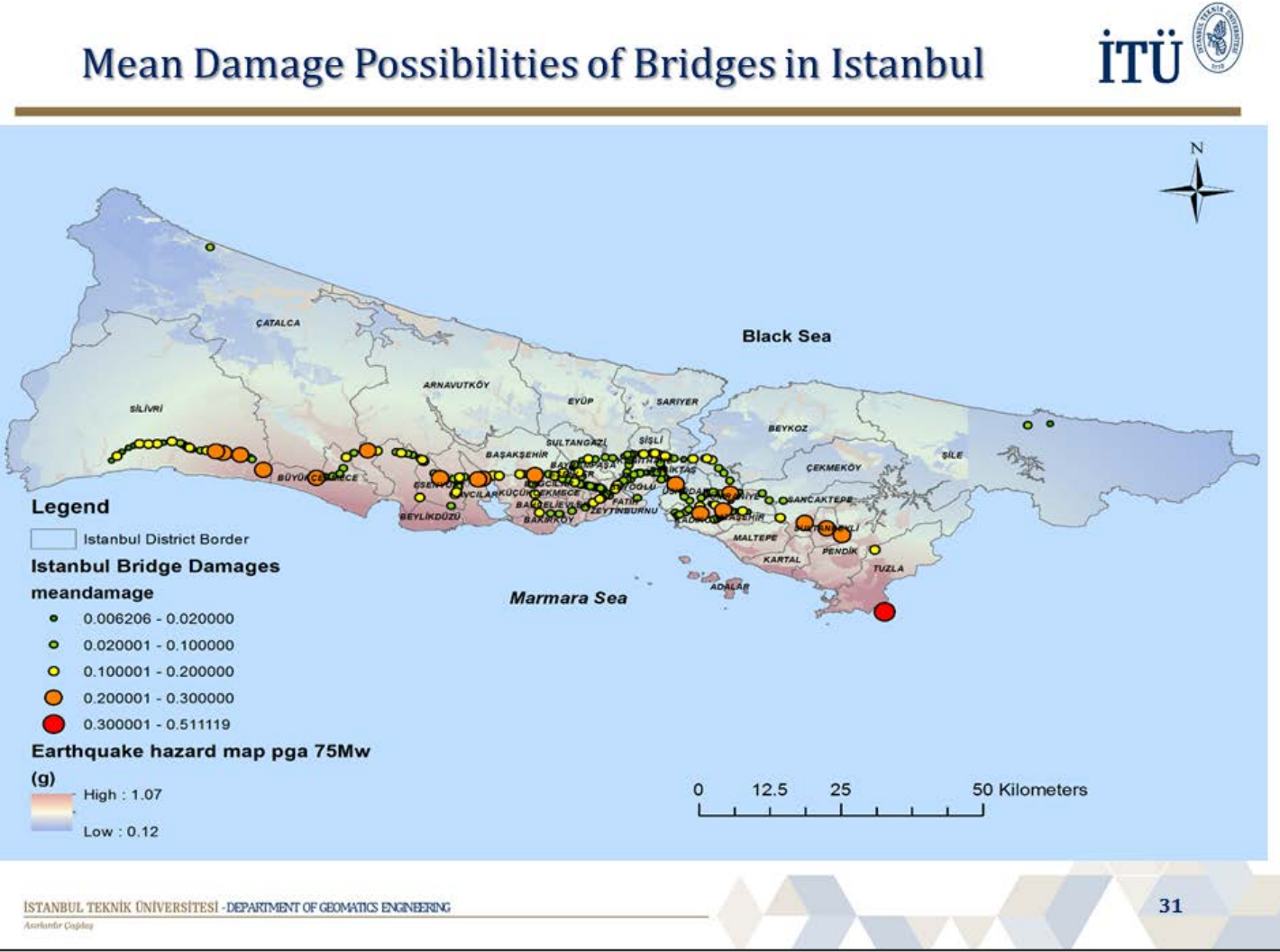

\section{Bridge Functionality}

\section{$\checkmark$ Analysis provides functionality of all of the transportation structures in the road network and reveals the anticipated restoration overtime}

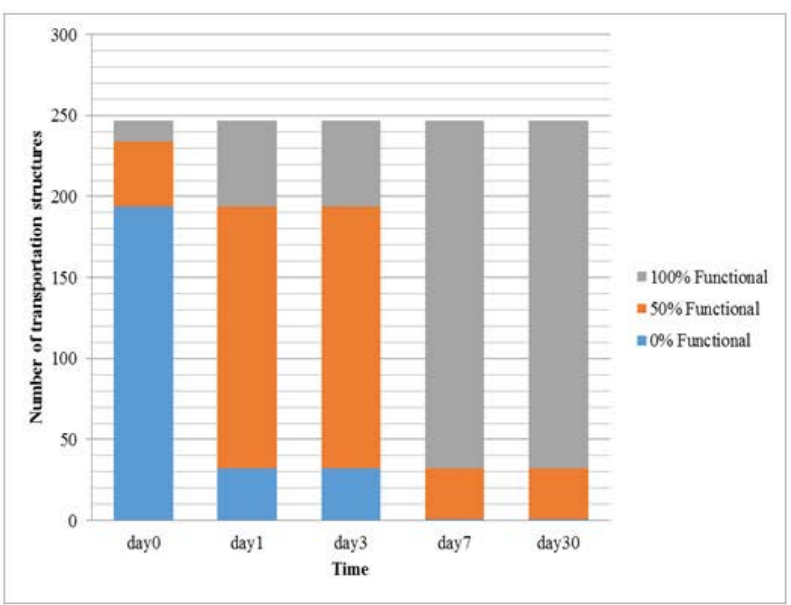

Step Functionality 


\section{Step Functionality Results}

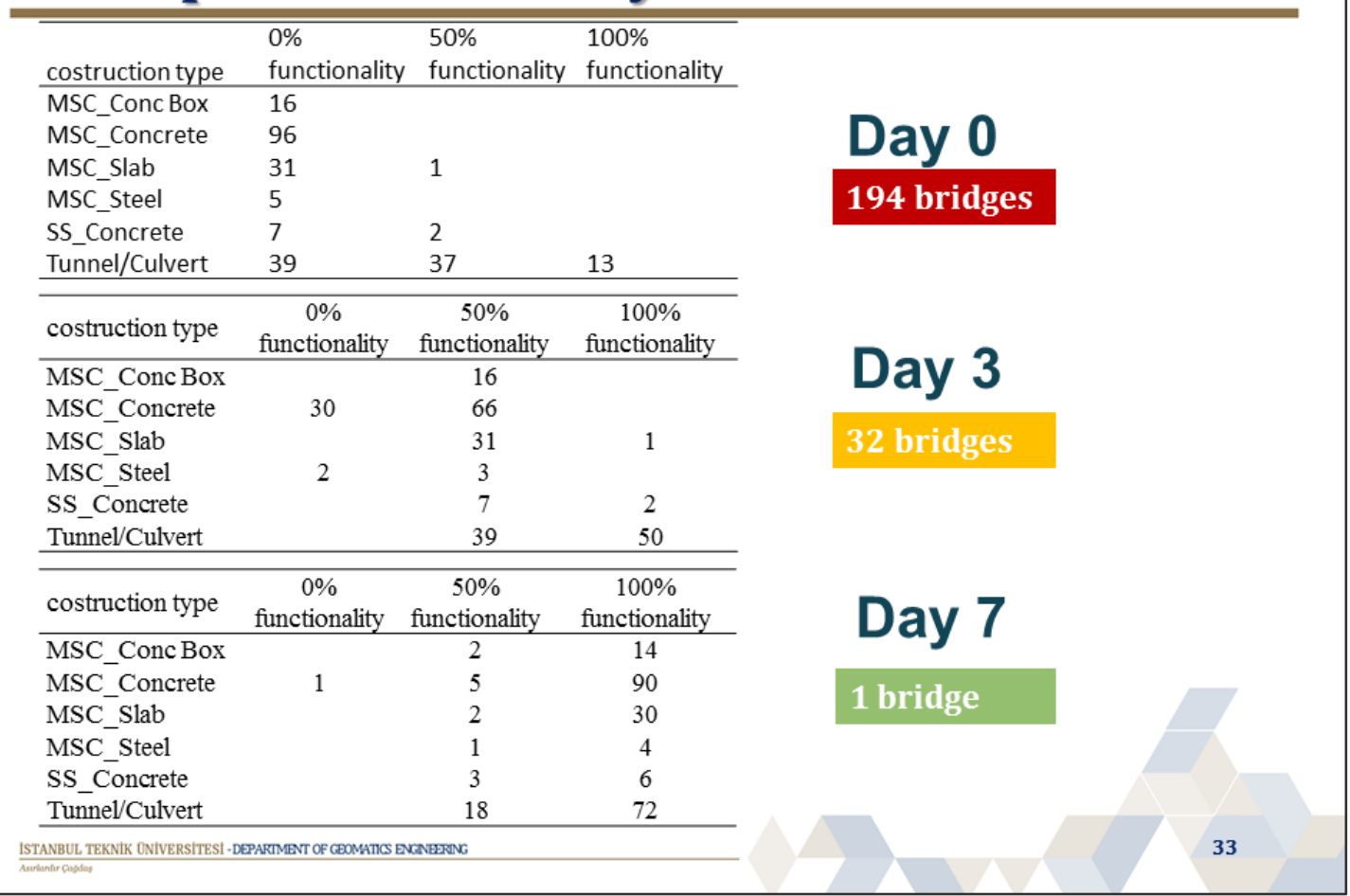

\section{Results of Retrofitted Bridge Damage Analysis İT̈̈}

$\checkmark 33$ bridges were retrofitted in Istanbul

- However the retrofitting types of retrofitted bridges can not be acquired anywhere

Retrofit (elastrometric)

\begin{tabular}{ccccccc}
\hline class & none & slight_mod & mod_extens & ext_comple & complete & meandamage \\
\hline MSC_Conc Box & 0.73 & 0.17 & 0.07 & 0.03 & 0.00 & 0.02 \\
MSC_Concrete & 0.60 & 0.26 & 0.03 & 0.01 & 0.10 & 0.06 \\
MSC_Slab & 0.92 & 0.32 & 0.20 & 0.12 & 0.01 & 0.06 \\
\hline
\end{tabular}

\section{Retrofit (steel jacket)}

\begin{tabular}{ccccccc} 
class & none & slight_mod & mod_extens & ext_comple & complete & meandamage \\
\hline MSC_Conc Box & 0.22 & 0.62 & 0.12 & 0.03 & 0.00 & 0.04 \\
MSC_Concrete & 0.15 & 0.60 & 0.13 & 0.06 & 0.05 & 0.07 \\
MSC_Slab & 0.08 & 0.61 & 0.21 & 0.09 & 0.00 & 0.06 \\
\hline
\end{tabular}



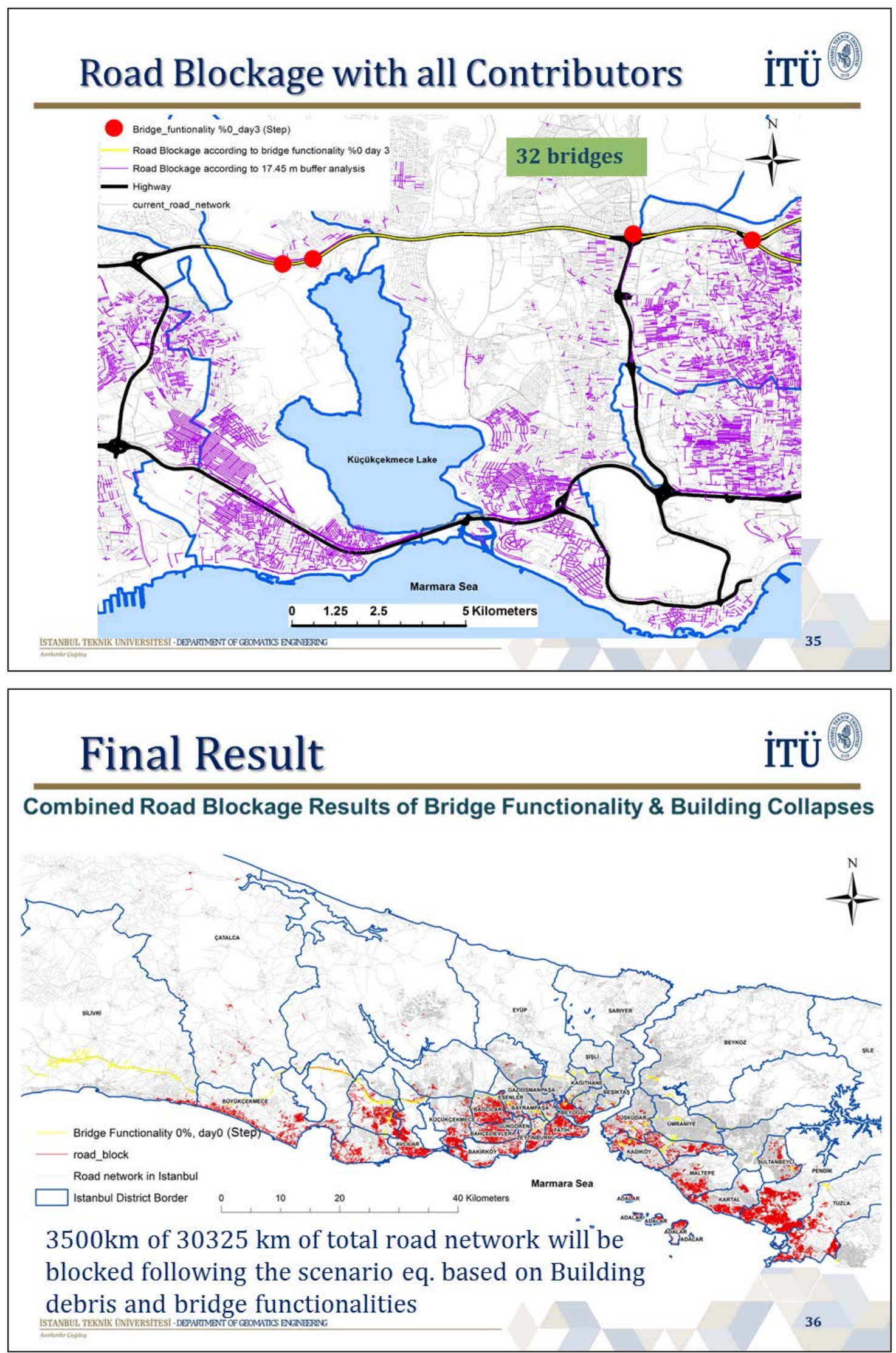


\section{One Last Analyses}

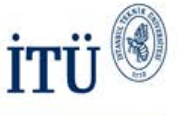

28 of the Fire brigades can be accessible following the eq. $37.8 \%$

11 of the Fire brigades can only be accessible after 3 days following the eq. 14.9\%

35 of the Fire brigades can not be accessible following the eq. $47.3 \%$

None of the Fire brigades can be accessible at the prince island district of Istanbul

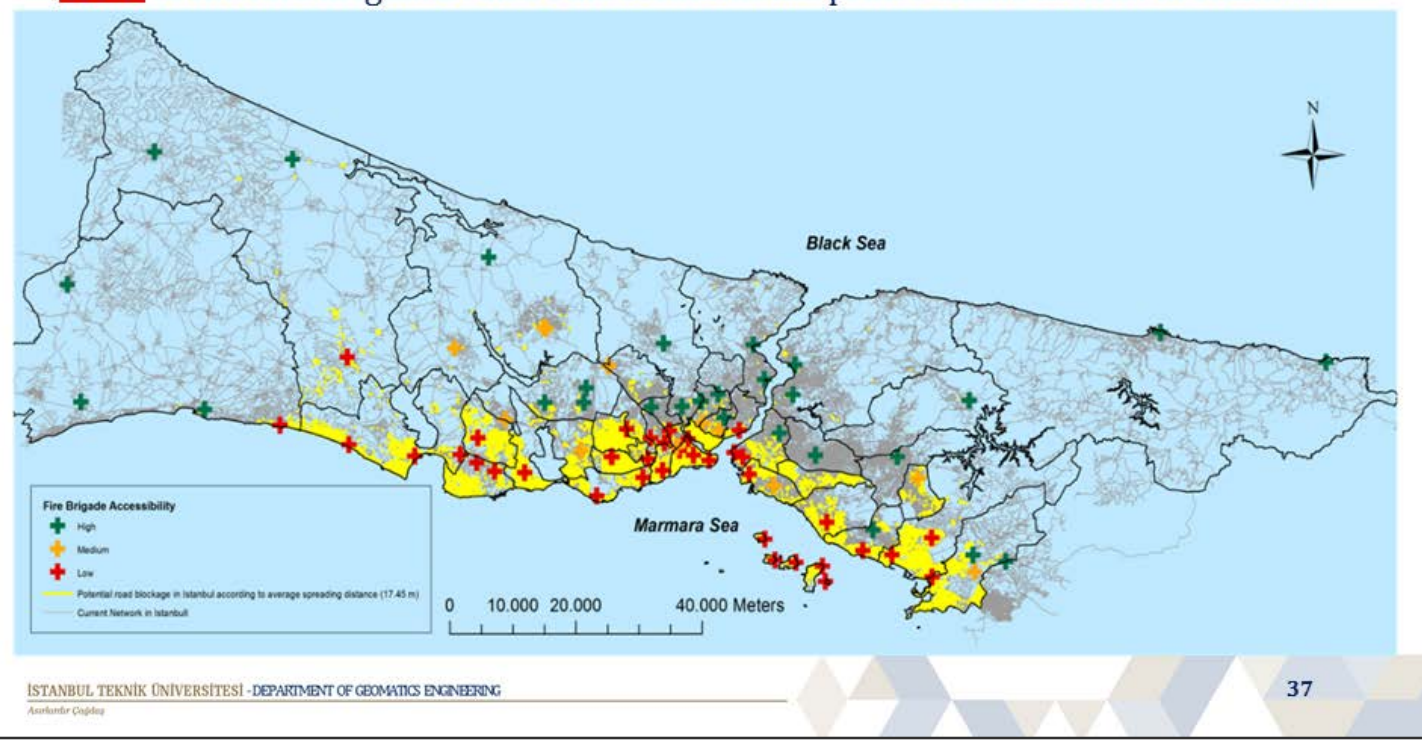

ітї (8)

\section{Thank you for your attention!}

Himmet Karaman, Ph.D.

Assoc. Prof. of Geomatics Engineering

Istanbul Technical University 


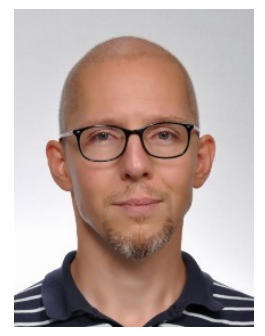

Himmet Karaman was born in Kutahya, Turkey, in 1979. He received the B.E. degree in geodesy and photogrammetry engineering from the Istanbul Technical University, Turkey, in 2001, and the M.Sc. and Ph.D. degrees in geomatics engineering from the Istanbul Technical University, Institute of Science and Technology, Turkey, in 2003 and 2008, respectively. He was a research scholar in University of Illinois at UrbanaChampaign between 2006 - 2007 at the Mid America Earthquake Center at the development of MAE-viz software. In 2009, he joined the Department of Geomatics Engineering, Faculty of Civil Engineering in Istanbul Technical University as an Assistant Professor, and in 2015 became an Associate Professor. Since December 2015, he has been the director of GIS Lab of the Civil Engineering Faculty and since 2016 he is serving as a member of Civil Engineering Faculty Board of Directors. His current research interests include disaster management, geographic information science, decision-making, earthquake engineering, surveying, and precise positioning. Dr. Karaman is a Fellow of the Board of Scientific Dictionary of Turkish Academy of Science; and the member of Disaster and Emergency Management Authority's Earthquake Advisory Board since 2009. 


\section{Measuring and Improving the Resilience of the Built Environment in a Community}

Kairui Feng, Guanjie Hou and Quanwang $\mathrm{Li}^{*}$

(Department of Civil Engineering, Tsinghua University)

*Corresponding author, Email: li_quanwang@tsinghua.edu.cn

Resilience is an attribute of communities, which is supported by community building sectors (occupancy types) with different functionalities. Evaluating community resilience and functionality requires quantification of metrics. This study, first, introduces a methodology to consider the functionality interdependencies between different building sectors in assessing community resilience metrics. Four building sectors that provide essential functions to a community, i.e. housing, education, business and public services, are considered. The percentage of people in a community who dislocate following a disaster as a result of the physical damages to buildings is selected as resilience metric in this conceptual study. Second, based on the proposed method to consider functionality interdependencies between building sectors, a framework is further developed to determine the optimum retrofit strategies for community building portfolios as a whole in order to achieve an overall community resilience objective expressed in terms of a threshold value of an interested community resilience metric (e.g. population dislocation). Third, recognizing the significant impact of the performance of traffic system has on the recovery rapidity of built environments, the paper proposes a measurement for evaluating the performance of traffic system to support the recovery process, which relates the capacity of traffic system to the recovery speed of built environment. Finally, the methodologies to quantify community functionality and traffic system performance are illustrated using a simplified Beijing City model exposed to potentially severe earthquakes, and the optimum retrofit strategies are discussed. 


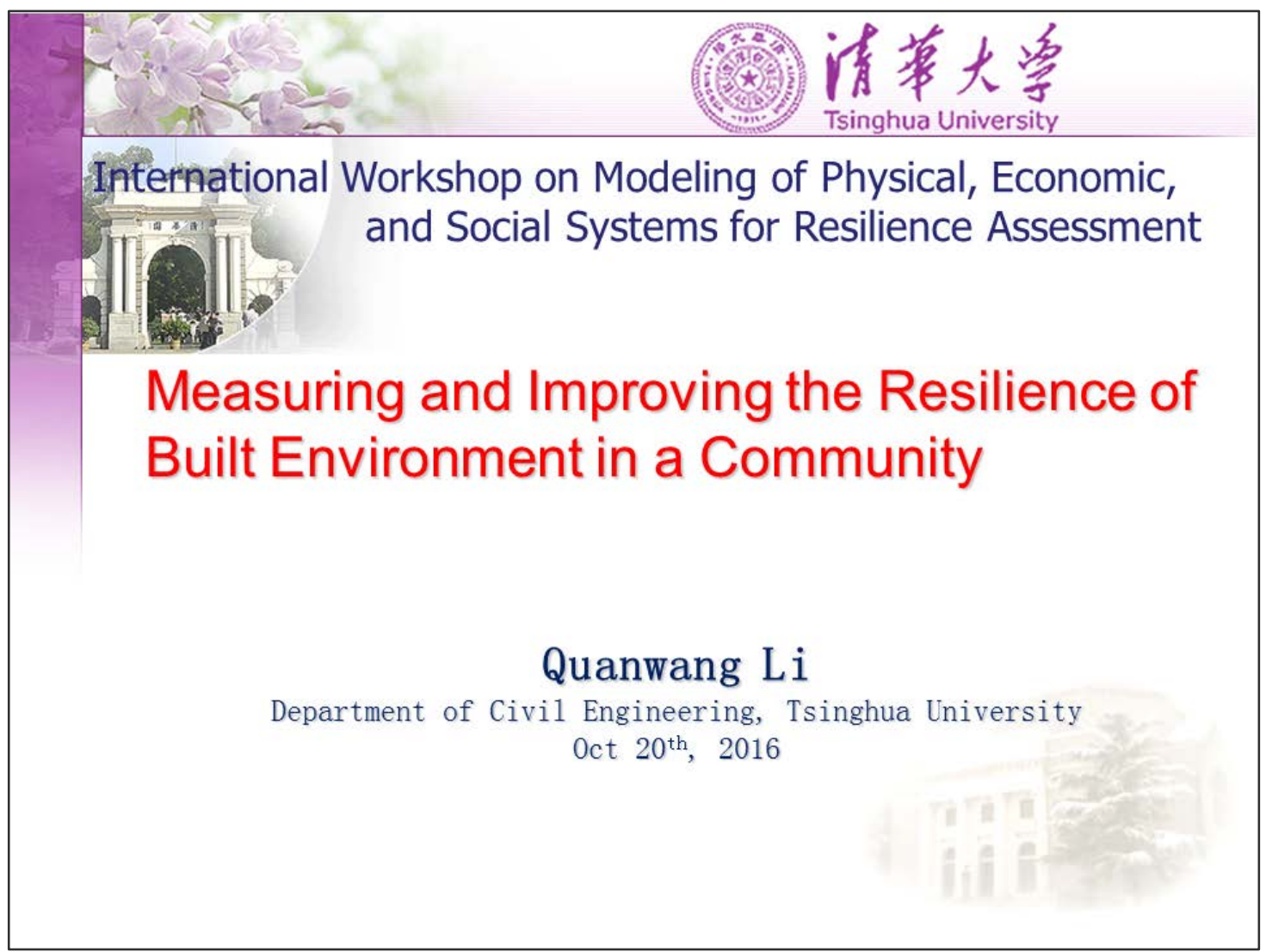

\section{Outline}

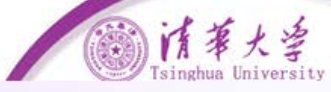

- Interdependency among building sectors of different functions

- Measurement for community functionality of built environment

- Community supply rate to evaluate the performance of traffic system 


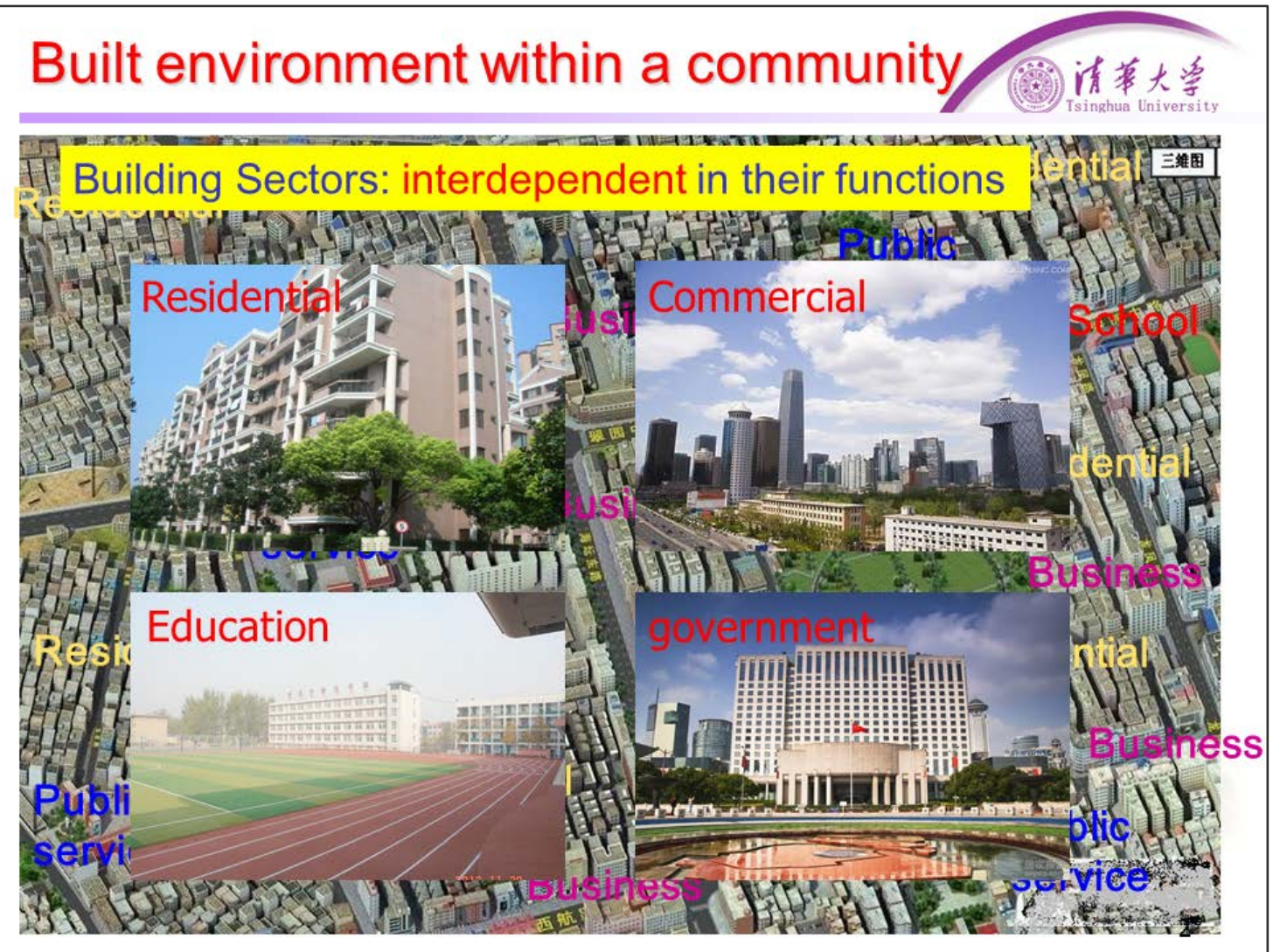

\section{Interdependency among building sectors}
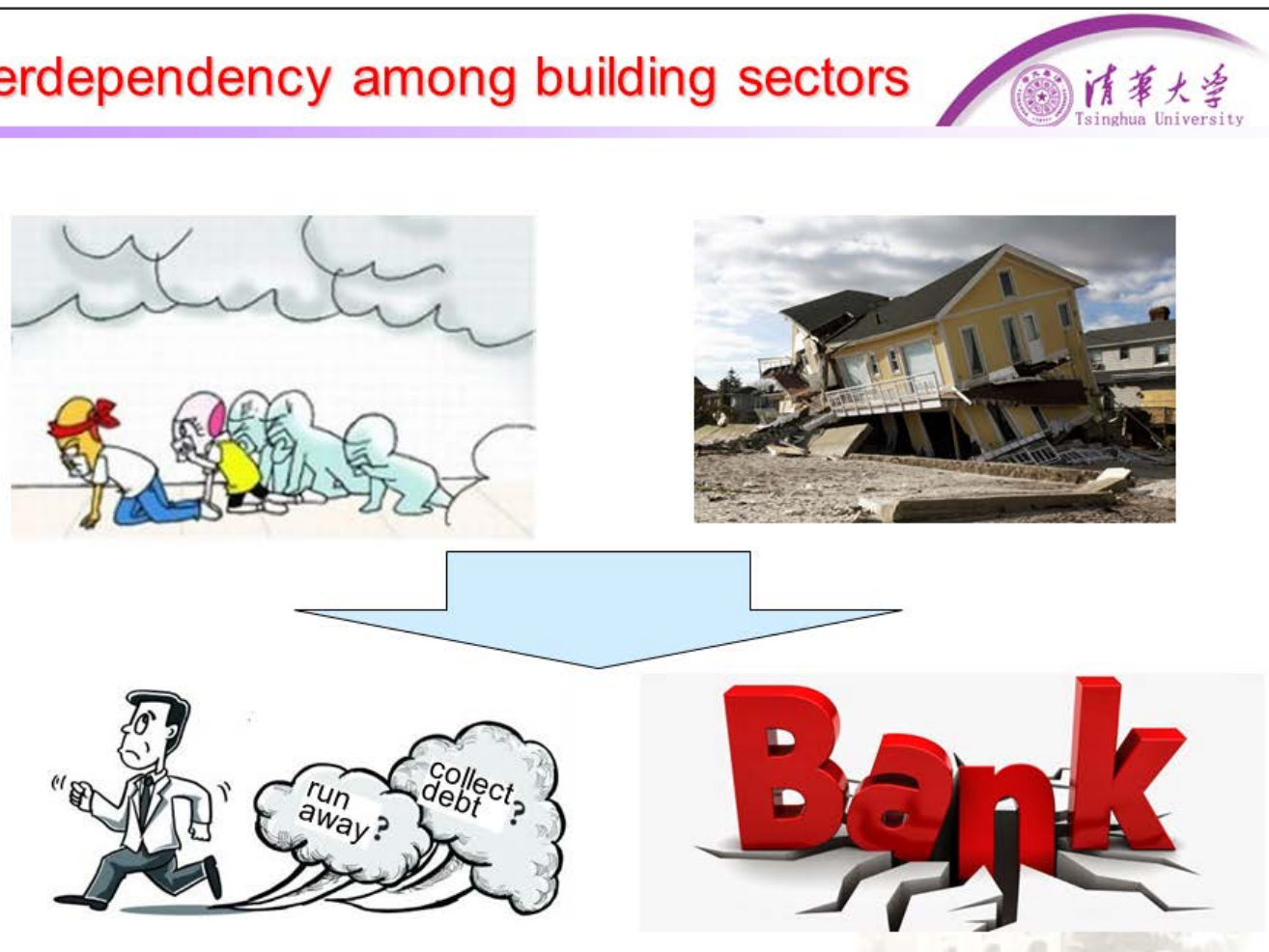


\section{Natural Hazards}

- Hurricanes

- Earthquakes

- Landslides

- Floods and storm surge

- 。。
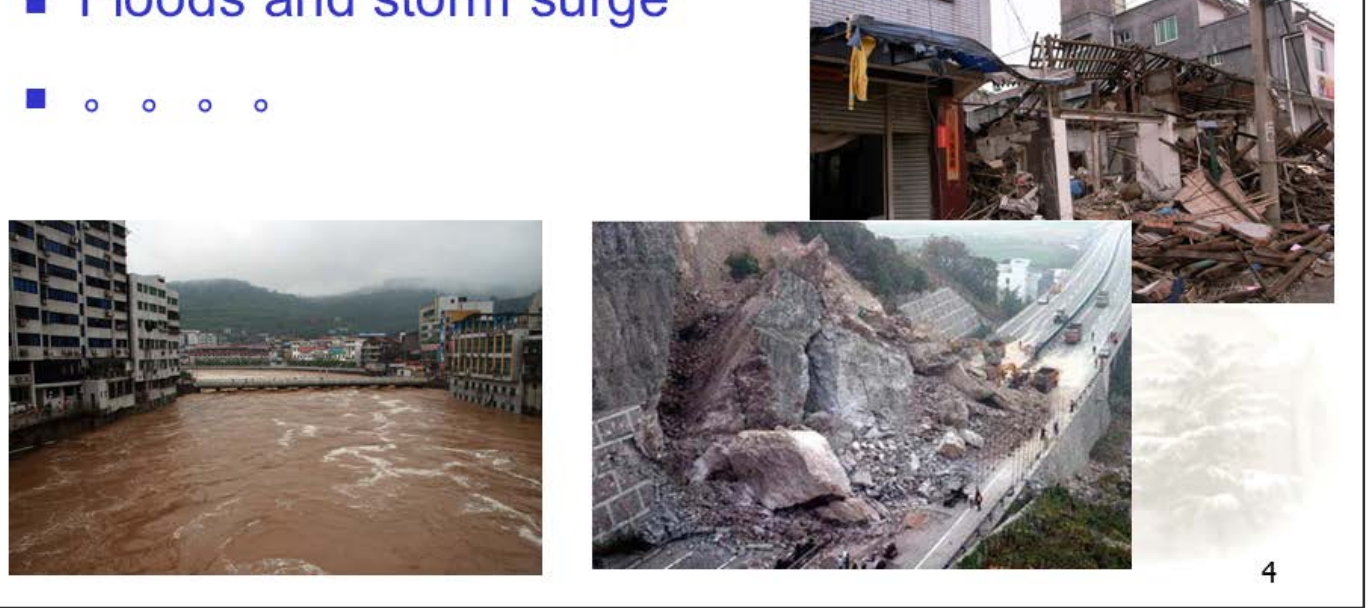

\section{Resilience of built environment}
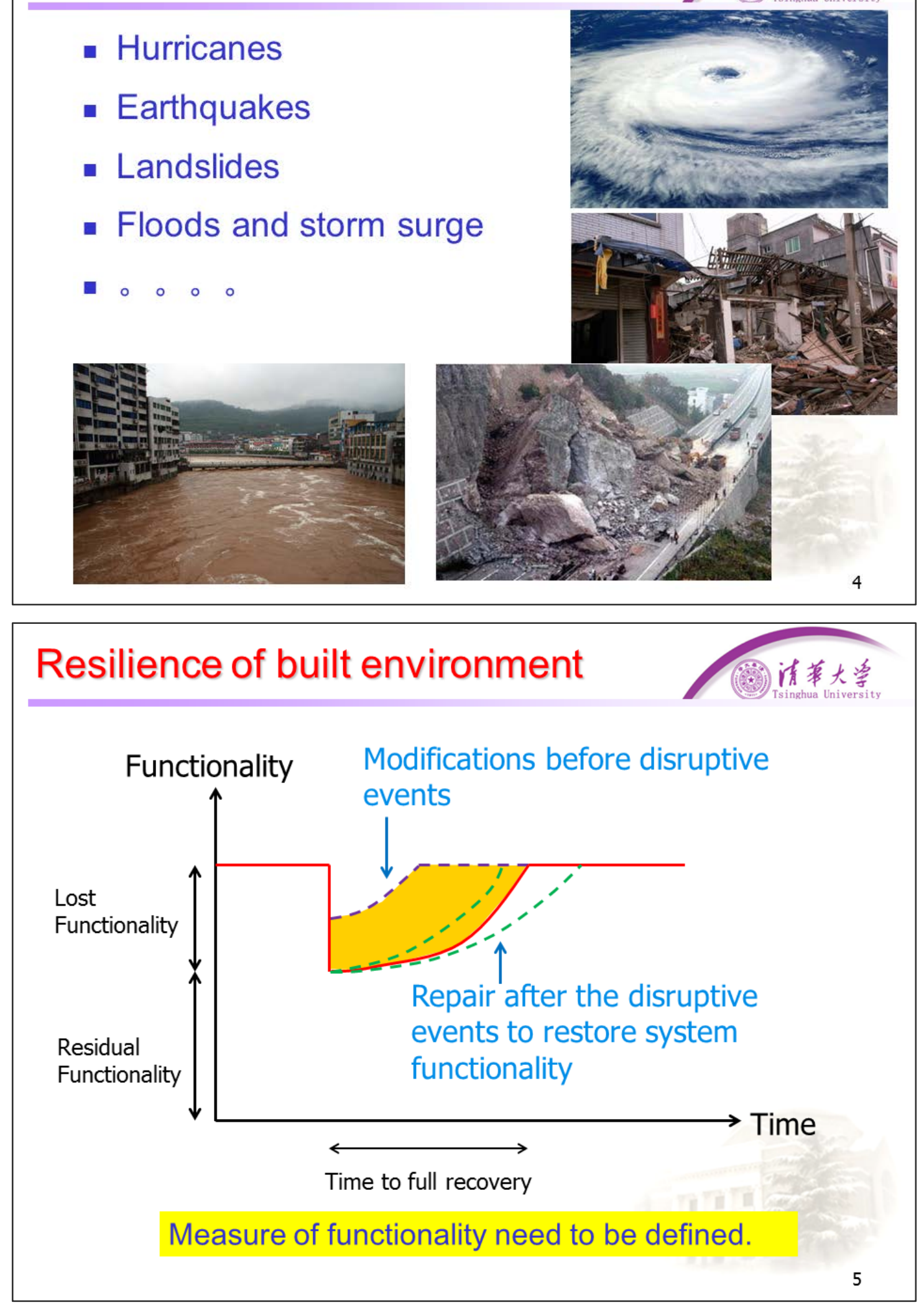


\section{Undesired outcome}
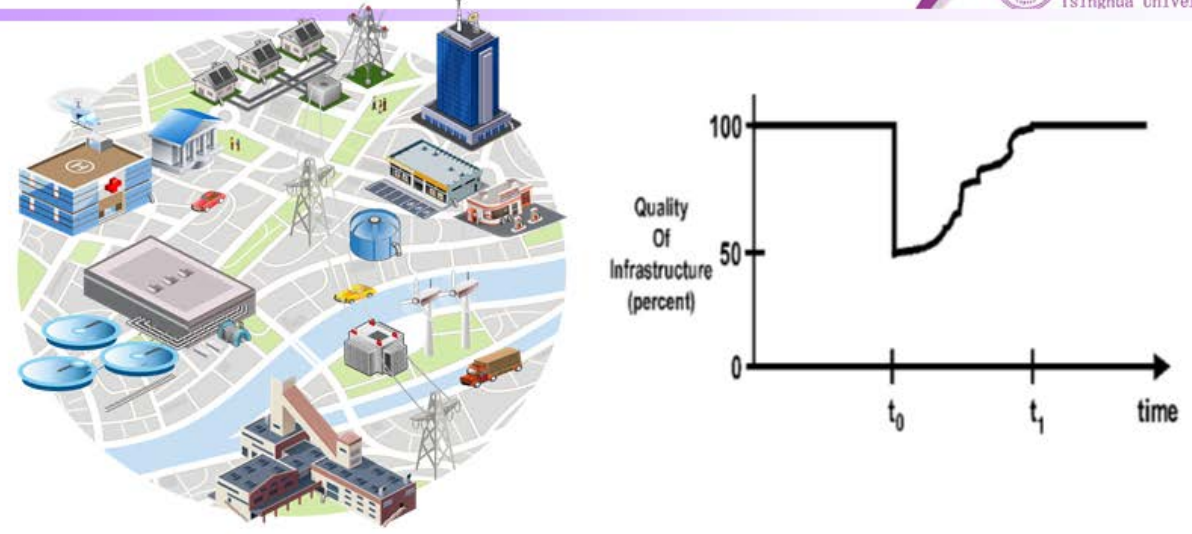

- An Undesired Outcome is an event whose occurrence would adversely impact a community's ability to function normally.

- Population Outmigration (PO) is chosen as a community resilience metric.

- Community Functionality is defined as the percentage of population remaining in the community.

\section{Essential community functions}

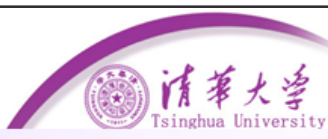

- 4 essential community functions

Housing (1); Employment(2); Education(3); Public service (4)

The functions are supported by building sectors

\section{- Measuring community functionality}

$\boldsymbol{P}_{i}$ : Probability of function $i$ being lost (independent)

$p_{o, i}$ : Probability of PO conditioned on the loss of function $i$ only

$p_{o, i j}$ : Probability of PO conditioned on the loss of both functions $i \& j$

$p_{o, j i k}$ or $p_{o, i j k h}$ : Probability of $\mathrm{PO}$ conditioned on the loss of more functions

$$
P_{P O}=\sum_{i=1}^{4} p_{o, i} P_{i} \prod_{\substack{j=1 \\ j \neq i}}^{4}\left(1-P_{j}\right)+\sum_{\substack{i, j=1 \\ i \neq j}}^{4} p_{o, j} P_{i} P_{j} \prod_{\substack{k=1 \\ k \neq i, j}}^{4}\left(1-P_{k}\right)+\sum_{\substack{i=1 \\ j, k, h \neq i}}^{4}\left(1-P_{i}\right) p_{o, j k h} P_{j} P_{k} P_{h}+p_{0,1234} P_{1} P_{2} P_{3} P_{4}
$$




\section{Measuring community functionality}

$$
\begin{aligned}
& P_{P O}=\sum_{i=1}^{4} p_{o, i} P_{i} \prod_{\substack{j=1 \\
j \neq i}}^{4}\left(1-P_{j}\right)+\sum_{\substack{i, j=1 \\
i \neq j}}^{4} p_{o, i j} P_{i} P_{j} \prod_{\substack{k=1 \\
k \neq i, j}}^{4}\left(1-P_{k}\right) \\
& 1-F_{C} \\
& p_{o, 1} \quad \frac{p_{o, 12}-p_{o, 1}-p_{o, 1}}{2} P_{1} \quad \frac{p_{o, 13}-p_{o, 1}-p_{o, 3}}{2} P_{1} \quad \frac{p_{o, 14}-p_{o, 1}-p_{o, 4}}{2} P_{1}
\end{aligned}
$$

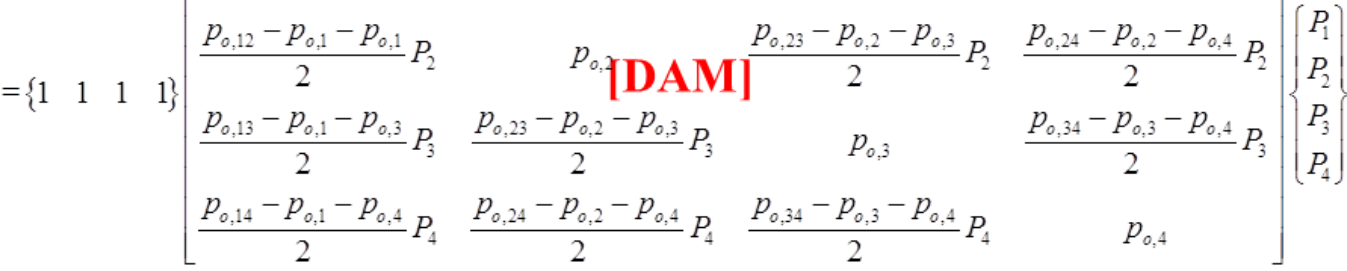

- Community Functionality

$$
F_{C}=1-\left\{\begin{array}{llll}
1 & 1 & 1 & 1
\end{array}\right\}[\mathbf{D A M}]_{4 \times 4}\left\{l_{i}\right\}_{4 \times 1}
$$

[DAM] can be determined by analyses of data from previous hazard events, or by computational models for physical damage and socioeconomic impact assessments

\section{Calibration according to Mieler's work : 请草大掌}

Mieler, M., Stojadinovic, B., Budnitz, R., Comerio, M., \& Mahin, S. (2015). "A framework for linking community-resilience goals to specific performance targets for the built environment". Earthquake Spectra, 31(3), 1267-1283.

\begin{tabular}{l|c|c|c|c|}
\hline $\begin{array}{c}\text { Outcomes } \\
\text { Function }\end{array}$ & Green & Yellow & Orange & Red \\
\hline Residents & $\mathrm{P}_{1} \leq 2 \%$ & $\mathrm{P}_{1} \leq 10 \%$ & $\mathrm{P}_{1} \leq 20 \%$ & $\mathrm{P}_{1}>20 \%$ \\
\hline Business & $\mathrm{P}_{2} \leq 5 \%$ & $\mathrm{P}_{2} \leq 20 \%$ & $\mathrm{P}_{2} \leq 50 \%$ & $\mathrm{P}_{2}>50 \%$ \\
\hline Education & $\mathrm{P}_{3} \leq 5 \%$ & $\mathrm{P}_{3} \leq 10 \%$ & $\mathrm{P}_{3} \leq 20 \%$ & $\mathrm{P}_{3}>20 \%$ \\
\hline Public Service & $\mathrm{P}_{4} \leq 5 \%$ & $\mathrm{P}_{4} \leq 20 \%$ & $\mathrm{P}_{4} \leq 50 \%$ & $\mathrm{P}_{4}>50 \%$
\end{tabular}

If two or more functions are in read, significant outmigration may occur

$$
[\mathbf{D A M}]=\left[\begin{array}{cccc}
0.128 & 0.297 l_{1} & 0.373 l_{1} & 0.268 l_{1} \\
0.297 l_{2} & 0.0436 & 0.279 l_{2} & 0.101 l_{2} \\
0.373 l_{3} & 0.279 l_{3} & 0.126 & 0.278 l_{3} \\
0.268 l_{4} & 0.101 l_{4} & 0.278 l_{4} & 0.051 l_{4}
\end{array}\right] \quad F_{C, c r i}=0.87
$$




\section{Beijing urban area}

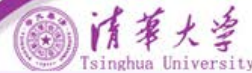

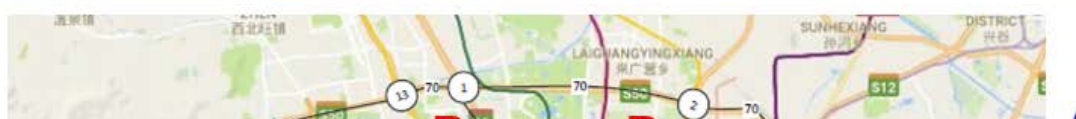

Area:

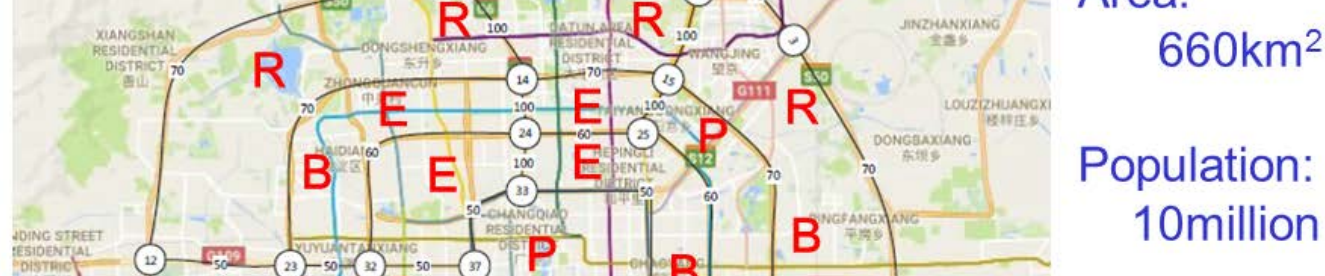

10

\section{Buildings}

\begin{tabular}{|c|c|c|c|}
\hline $\begin{array}{c}\text { Building } \\
\text { Sector }\end{array}$ & $\begin{array}{c}\text { Building } \\
\text { Category }\end{array}$ & Number of Buildings & $\begin{array}{c}\text { Design Seismic } \\
\text { Intensity Level (SIL) }\end{array}$ \\
\hline $\begin{array}{c}\text { Residential } \\
\text { buildings }\end{array}$ & $\mathrm{R} 6$ & 1621 & $6(0.07 \mathrm{~g})$ \\
\hline Rusiness & $\mathrm{R} 7$ & 2033 & $7(0.13 \mathrm{~g})$ \\
\hline buildings & $\mathrm{B} 6$ & 420 & $8(0.25 \mathrm{~g})$ \\
\hline Education & $\mathrm{B} 7$ & 445 & $6(0.07 \mathrm{~g})$ \\
\hline buildings & $\mathrm{E} 6$ & 307 & $7(0.13 \mathrm{~g})$ \\
\hline Public service & $\mathrm{E} 7$ & 66 & $8(0.25 \mathrm{~g})$ \\
\hline buildings & $\mathrm{P} 6$ & 231 & $6(0.07 \mathrm{~g})$ \\
\hline
\end{tabular}




\section{Seismic behavior}

Seismic fragility $\quad F_{R}(I M)=P\left(\theta>\theta_{c r} \mid I M=i m\right)=1-\Phi\left(\frac{\ln \theta_{c r}-\lambda_{\theta}}{\xi_{\theta}}\right)$

$\theta=a \cdot P G A^{b} \cdot \varepsilon$

A power-law relationship between structural inter-story drift ratio, $\theta$, and seismic intensity measure, PGA.

It is assumed the building becomes unoccupiable as $\theta$ exceeds $1 \%$.
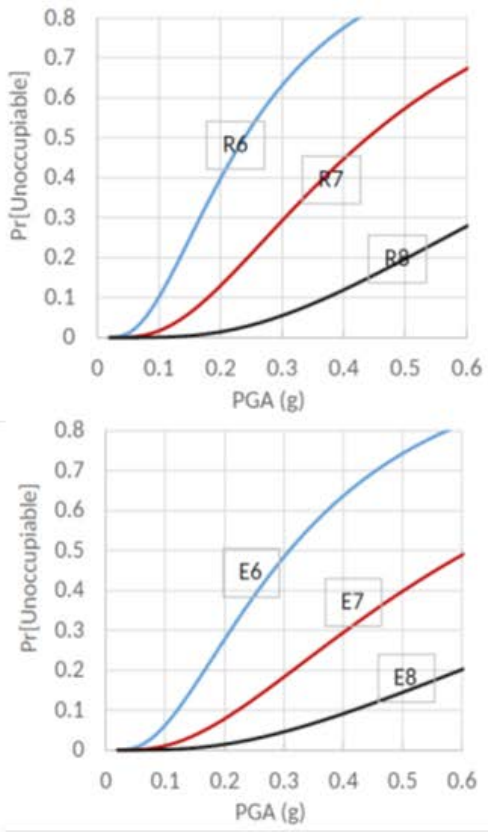
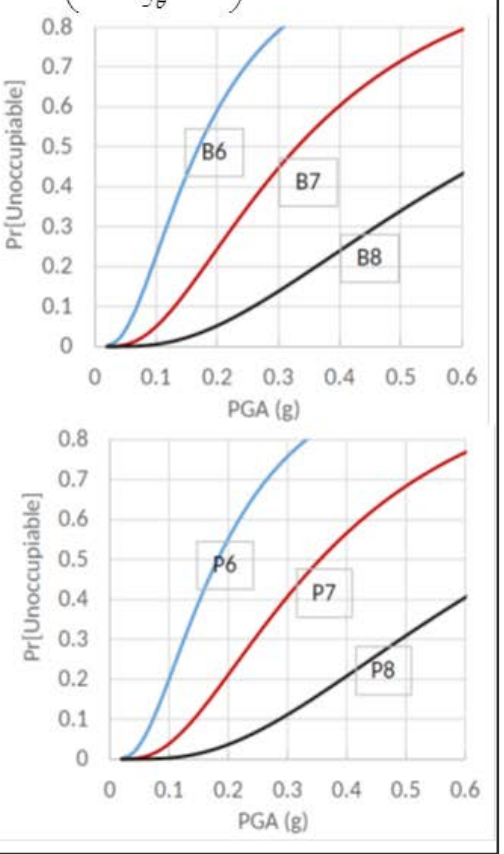

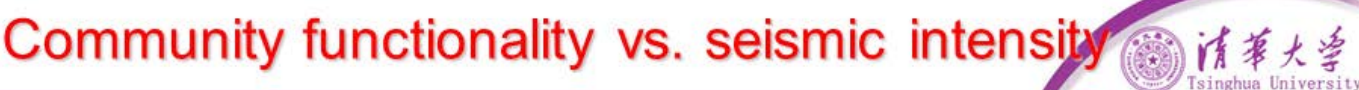

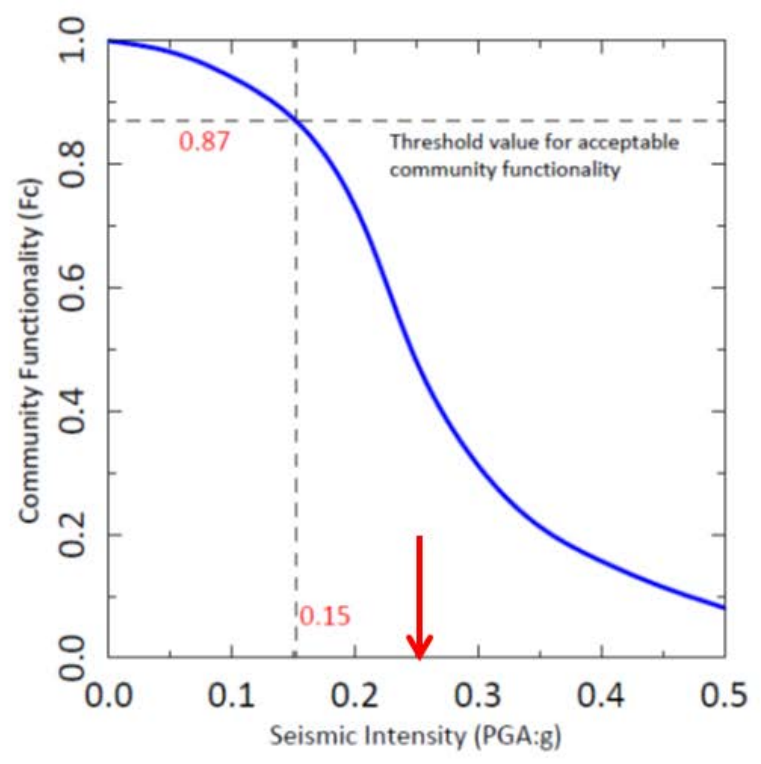

Community performance needs to be improved 


\section{Retrofit Cost}

- Retrofit cost of building cluster $i$ is give by:

$$
C_{i}=C_{0, i} \cdot n_{i} \cdot k_{i} \cdot\left(\frac{a_{i}^{*}}{a_{i}}-1\right)
$$

- Optimum retrofit strategy:

Cost efficiency of retrofitting sector

$$
z_{i}=\frac{\partial F_{C}}{\partial f_{i}} \cdot \frac{d f_{i}}{d C_{i}}
$$

$$
\longleftarrow \frac{\partial F_{C}}{\partial f_{i}}=a_{i i}+\sum_{j=1, j \neq i}^{m}\left(a_{i j}+a_{j i}\right) l_{j}
$$

$$
\frac{\mathbf{d} f_{i}}{\mathbf{d} C_{i}}=\frac{\mathbf{d} f_{i}}{\mathbf{d} \lambda_{\theta, i}} \cdot \frac{\mathbf{d} \lambda_{\theta, i}}{\mathbf{d} \mu_{\theta, i}} \frac{\mathbf{d} \mu_{\theta, i}}{\mathbf{d} C_{i}}
$$

\section{Optimum retrofit strategy}

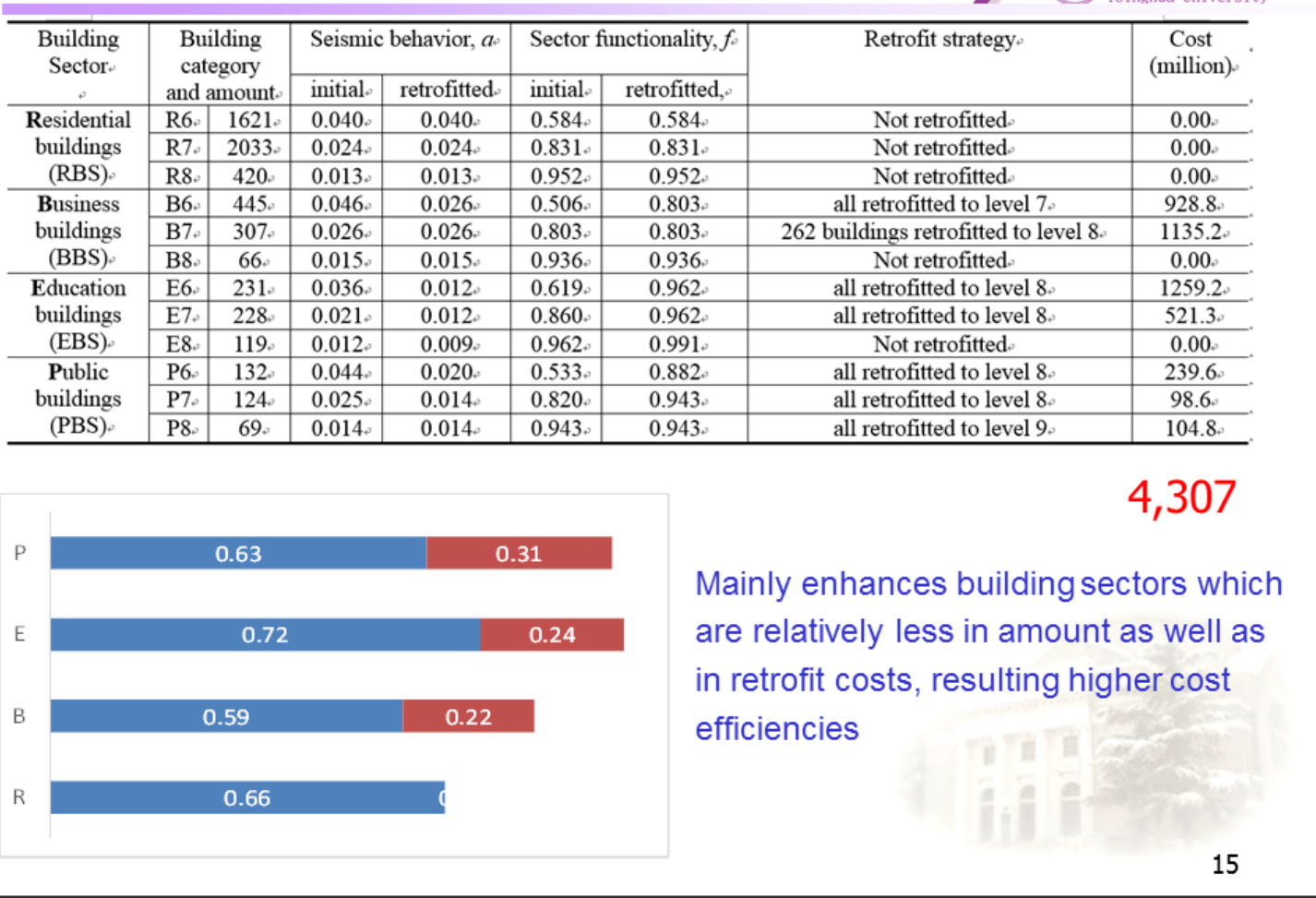




\section{Support of traffic system to recovery

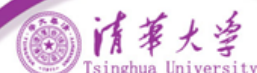

- Traffic system provides support to the recovery process of damaged buildings

- recourse supply rate of an individual building

$$
r=\frac{\Pi_{s}}{\Pi_{n}} \quad \text { resources shipped daily for recovery through traffic system }
$$

- Weighted supply rate of a community

$$
r=\sum_{j=1}^{4} \sum_{i} w_{j i} \frac{\Pi_{\mathrm{s}, j i}}{\Pi_{n, j i}} / \sum_{j=1}^{4} \sum_{i} w_{j i}=\frac{\partial F_{C}}{\partial f_{j}} \cdot \frac{\partial f_{j}}{\partial \Pi_{i}}
$$

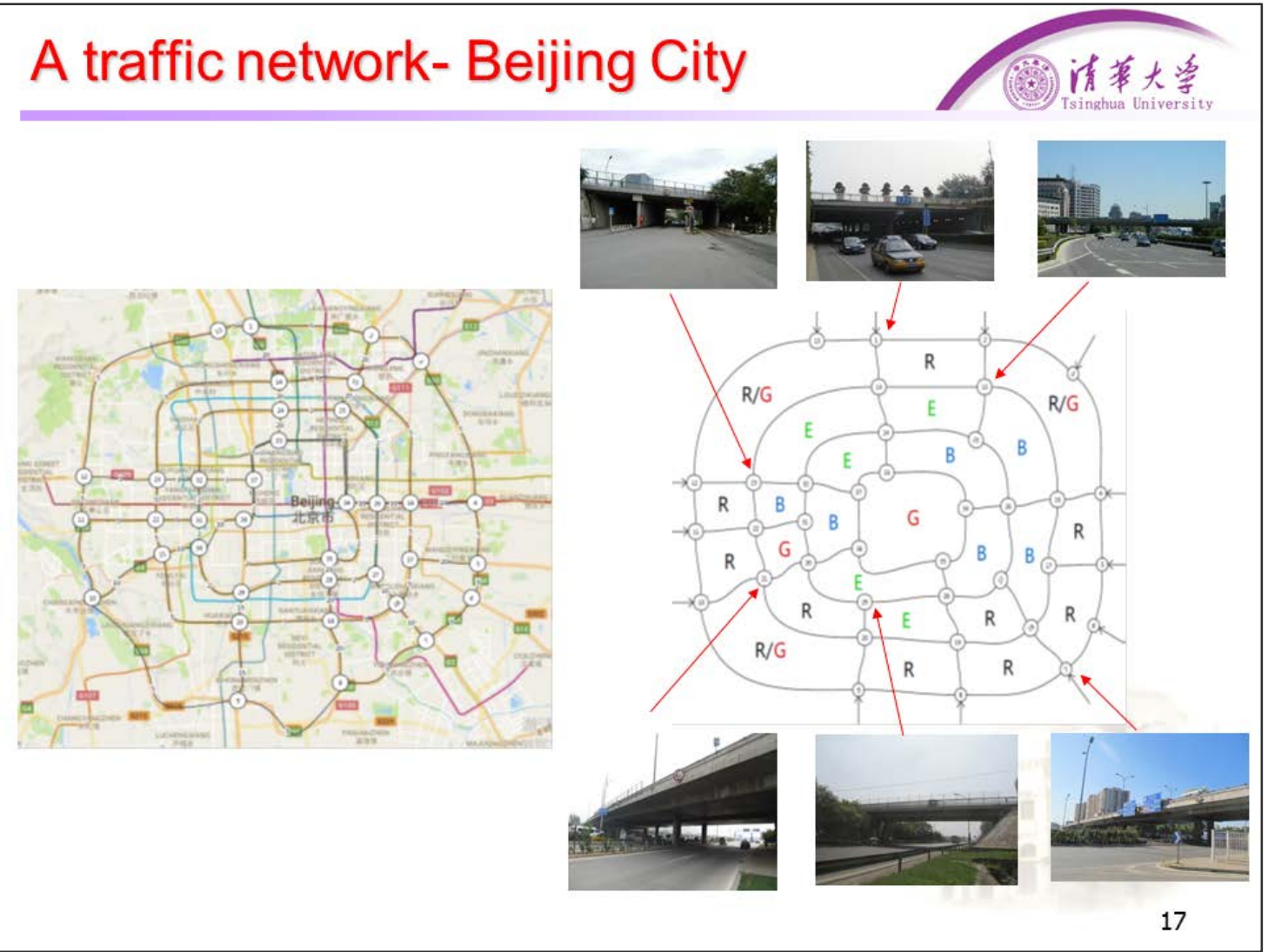




\section{Bridge Fragility}
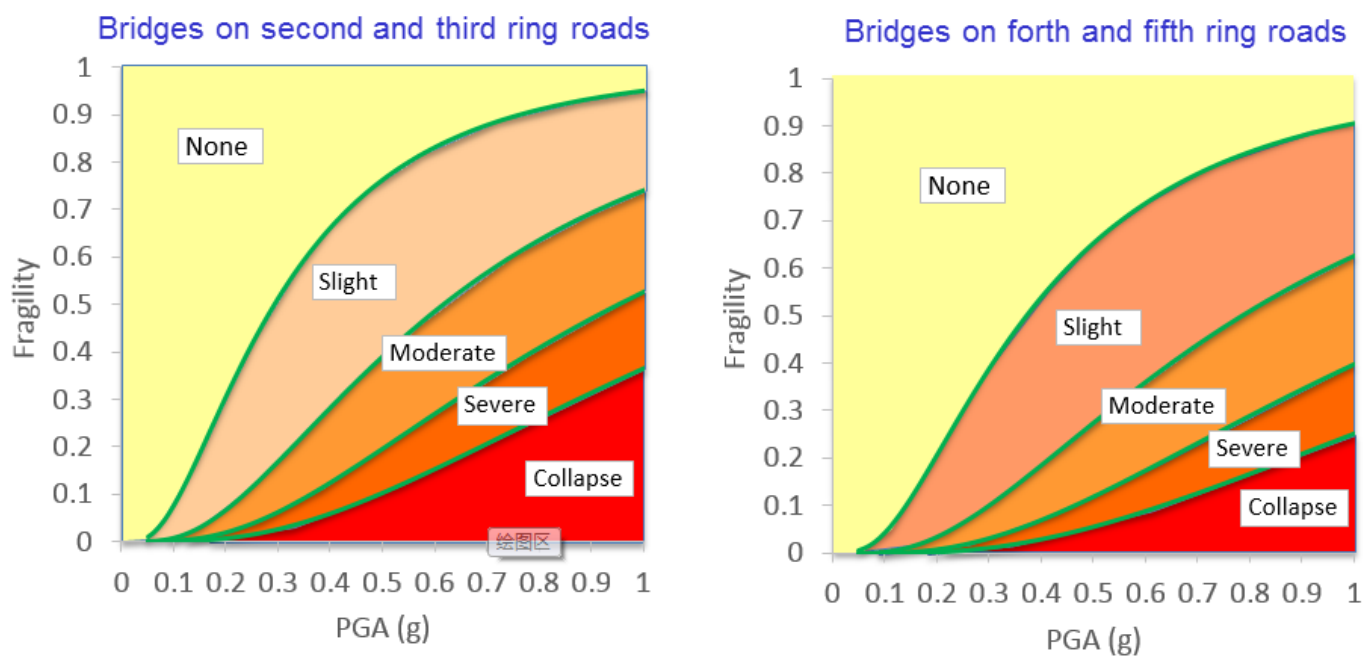

（焦池宇，北京市城市桥梁抗震性能的现状，《工程抗震与加固改造》2012)

\begin{tabular}{|c|c|c|c|c|}
\hline Damage State & Slight & Moderate & Severe & Collapse \\
\hline Remained Capacity & 1.0 & 0.75 & 0.5 & 0.0 \\
\hline
\end{tabular}

18

\section{Assumptions}

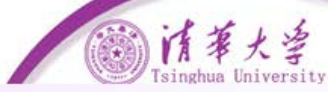

- The damages of individual bridge is assumed to be independent.

- For each zone, the needed resource

$$
\Pi_{n, \text { zone }}=\sum_{i=1}^{4} \sum_{j=1}^{5} n_{i j} \Pi_{i j}
$$

- An optimization problem

Target: maximizing the community supply rate using Simplex algorithm 


\section{Probability distribution of supply rate}

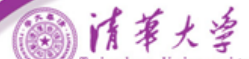

Subjected to an earthquake with seismic intensity of 8

Immediately after the disruptive event

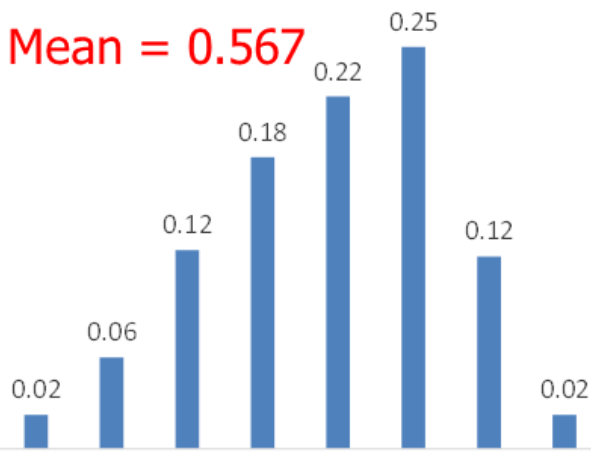

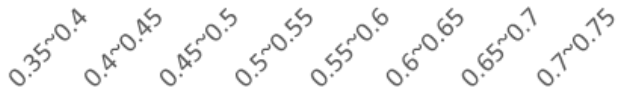

One month after the disruptive event

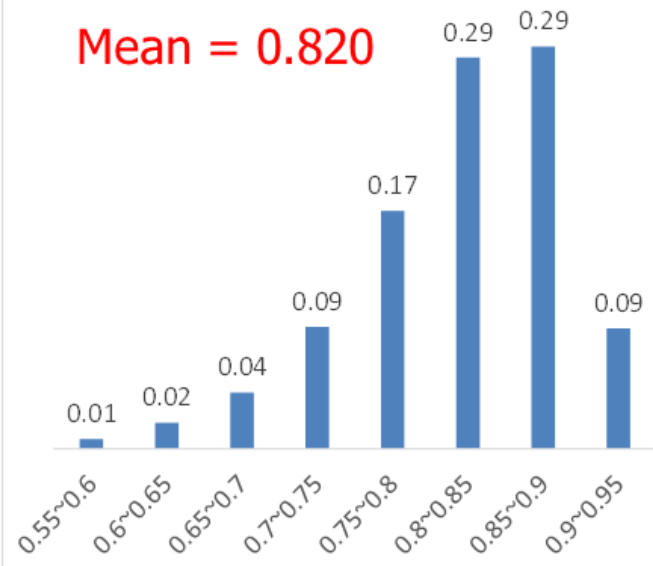

As some damaged buildings are repaired, the needed resources decrease.

20

\section{Recovery trajectory}

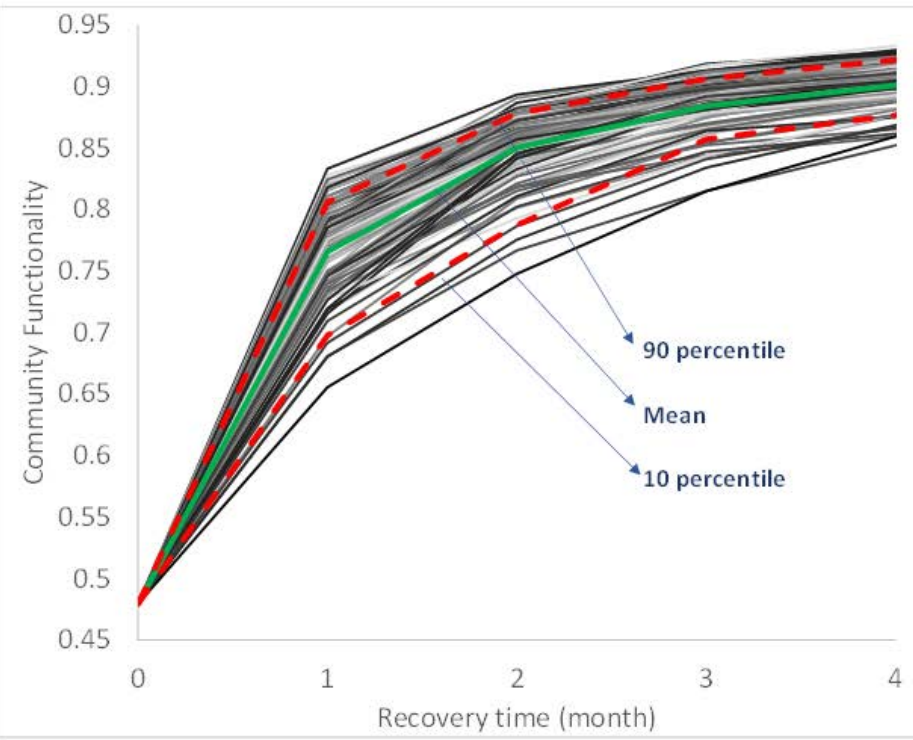

Averagely, the community is back to normal after 3 months 


\section{Seismic retrofitting to the bridges}

All bridges are retrofitted to the seismic level of 9

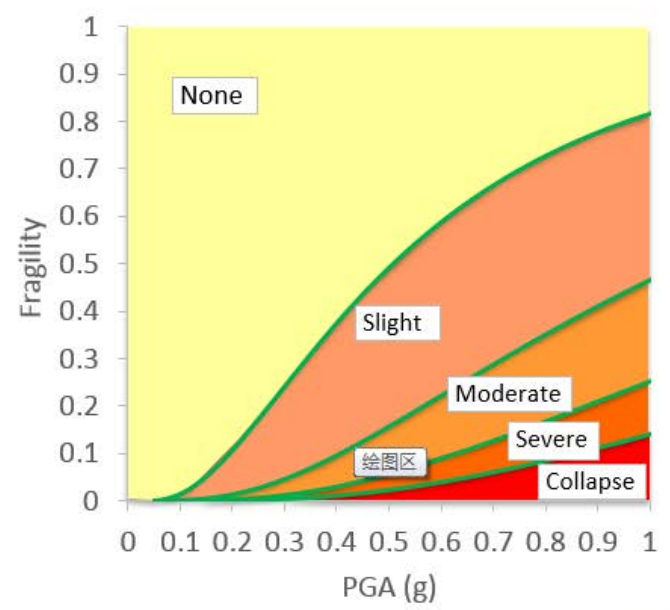

\section{Retrofitting effect - Comparison of supply rate: 请歎大密}

Immediately after the disruptive event

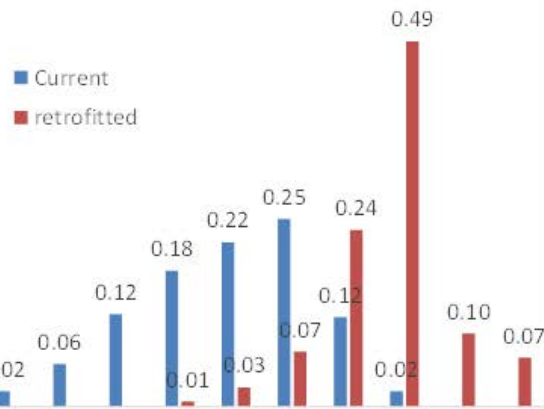

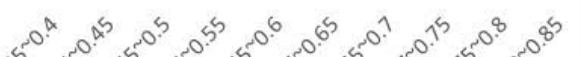

Mean: 0.567 vs. 0.703
One month after the disruptive event

current

- retrofitted

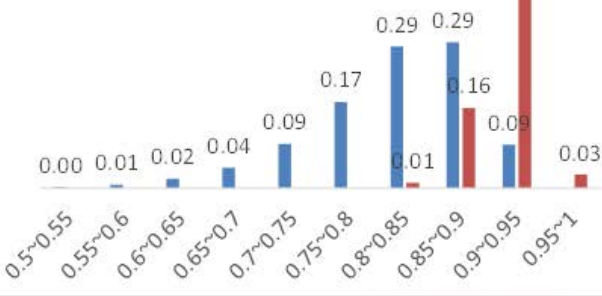

Mean: 0.820 vs. 0.922 


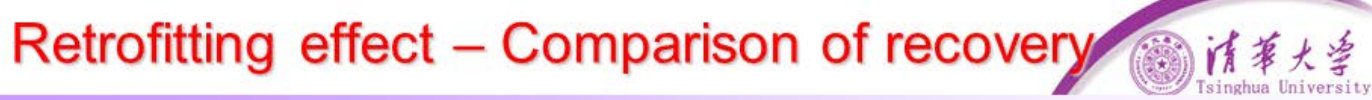

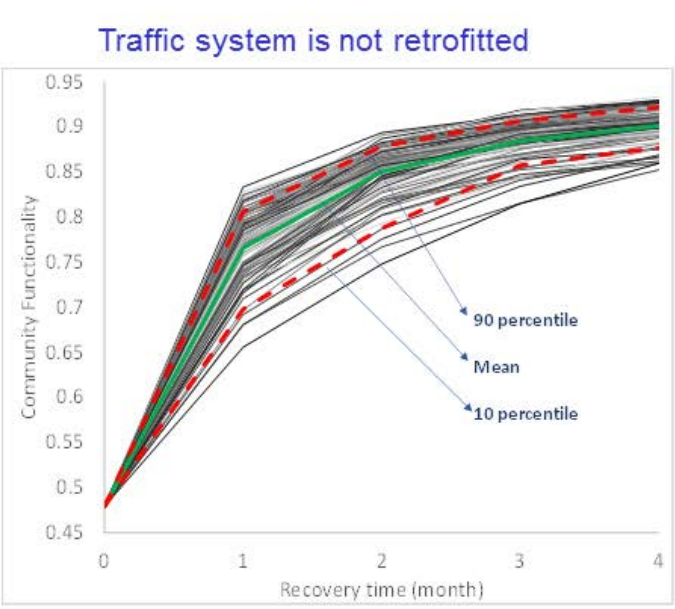

The average time to be back to normal: 3 months
Traffic system is retrofitted

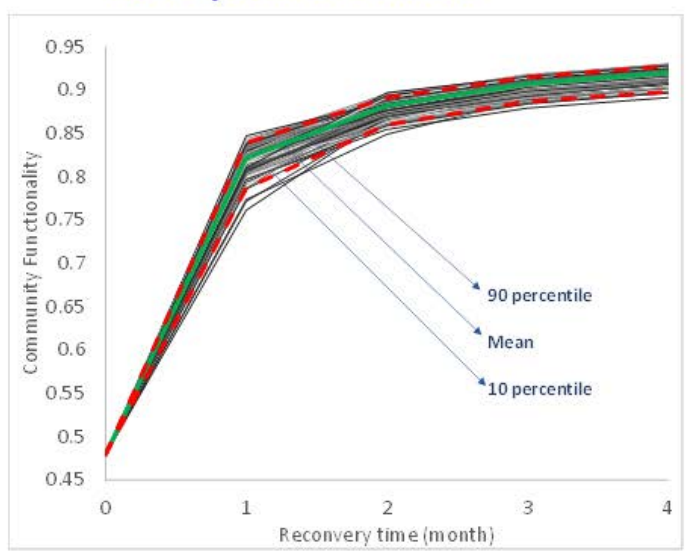

The average time to be back to normal: 2 months

\section{Summary}

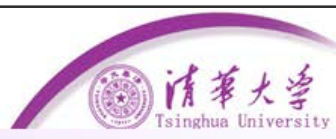

- A measurement of community functionality is proposed, and its application is demonstrated

- The community supply rate is proposed to evaluate the performance of traffic system to support the recovery of built environment, and its application is demonstrated 


\section{Translate Resilience into Chinese}

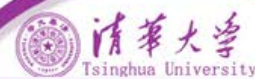

- 弹性

- 韧性

- 可恢复性

- 容灾性

\section{Thank you for your attention!}




\section{Measuring and Improving the Resilience of the Built Environment in a Community}

Dueñas-Osorio, L., Craig, J. I., Goodno, B. J., \& Bostrom, A. (2007). Interdependent response of networked systems. Journal of Infrastructure Systems, 13(3), 185-194.

Bocchini, P., and Frangopol, D. M. (2012). Restoration of bridge networks after an earthquake: multicriteria intervention optimization. Earthquake Spectra, 28(2), 426-455.

Bruneau, M., Chang, S., Eguchi, R., Lee, G., O’Rourke, T., Reinhorn, A.M., Shinozuka, M., Tierney, K., Wallace, W., and Winterfelt, D.V. (2003). "A framework to quantitatively assess and enhance the seismic resilience of communities.” Earthquake Spectra, 19 (4), 733-752.

Chang S E, Shinozuka M. (2004). "Measuring improvements in the disaster resilience of communities”. Earthquake Spectra, 20(3): 739-755.

Cutter, S.L. Burton, C.G., and Emrich, C.T. (2010). "Disaster resilience indictor for benchmarking baseline conditions.” J. Homeland Security and Emergency Management, 7(1): Article 51.

Francis, R., and Behailu, B. (2014). "A metric and frameworks for resilience analysis of engineered and infrastructure systems." Reliability Engineering \& System Safety 121, 90-103.

Goldberg A.V.(1998). "Recent Developments in Maximum Flow Algorithms”, Springer Berlin Heidelberg.

Lin P., Wang N. and Ellingwood BR. (2016). “A Risk De-aggregation Framework that Relates Community Resilience Goals to Building Performance Objectives”. Sustainable and Resilient Infrastructure add DOI

Lin, Y. (2009). "Development of algorithms to estimate post-disaster population dislocation: A research-based approach”. Ph.D. thesis, Texas A \& M University.

Lounis, Z., \& McAllister, T. P. (2016). Risk-based decision-making for sustainable and resilient infrastructure systems. Journal of Structural Engineering, F4016005.

McAllister, T. (2013). "Developing Guidelines and Standards for Disaster Resilience of the Built Environment: A Research Needs Assessment” National Institute of Standards and Technology (NIST) Technical Note 1795, March 2013.

Mieler, M., Stojadinovic, B., Budnitz, R., Comerio, M., \& Mahin, S. (2015). “A framework for linking community-resilience goals to specific performance targets for the built environment”. Earthquake Spectra, 31(3), 1267-1283.

Miles, S.B. and Chang, S.E. (2006) “Modeling community recovery from earthquakes,” Earthquake Spectra, 22(2): 439-458.

NIST. (2015). Community resilience planning guide for buildings and infrastructure systems. Volume 1 and Volume 2. National Institute of Standards and Technology, Gaithersburg, MD 20899 USA.

Xiao, Y., and Van Zandt, S. (2012). Building community resiliency: Spatial links between household and business post-disaster return. Urban Studies,49(11), 2523-2542.

D-52 


\section{Quanwang Li}

Department Civil Engineering, Tsinghua University

Beijing, China, 100084

Email: li_quanwang@tsinghua.edu.cn

Phone:86-10-62797673

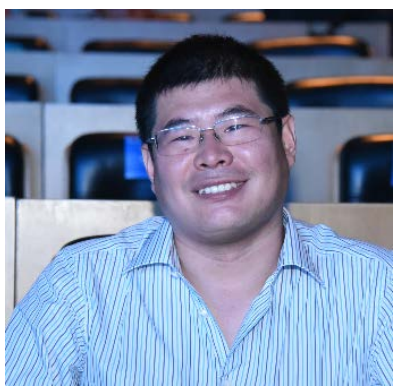

Quanwang $\boldsymbol{L i}$ is an associate professor in the Department of Civil Engineering at Tsinghua University, Beijing, China. His work focuses on applications of statistics and probability in civil engineering, structural safety and reliability assessment, and the time-dependent performance and reliability of deteriorating structures exposed to natural hazards. Currently, he is especially interested in the resilience of civil infrastructure systems, the performance and reliability of prefabricated reinforced concrete structures in high seismic intensity zones, and the durability reliability of reinforced concrete structures in marine environment. 


\title{
Title:
}

Modeling Temporary, Interim, and Permanent Housing Demand \& Capacity within Norfolk following a Catastrophic Event (special emphasis on medically fragile and vulnerable populations)

\section{Presenter:}

Joshua G. Behr

Research Associate Professor

Virginia Modeling, Analysis \& Simulation Center

Old Dominion University

\begin{abstract}
:
The central aim of this HUD-sponsored effort is the development of a set of simulation models that allow for the forecast of the demand and transition times for temporary, interim, and permanent housing of displaced households stemming from severe weather events. In particular, these efforts aim to identify the dynamics of displaced populations with social, medical, and financial vulnerabilities in the housing recovery process.

The models are intended to allow localities the ability to visualize how pre-disaster planning, zoning, and permitting practices may potentially impact the pace of recovery. The models should facilitate 'what if' scenario testing of current and theorized pre-disaster housing recovery planning, zoning, and permitting practices and policies to identify those factors that may enhance housing production capacity, speed the approach back to normalcy, and promote a further strengthening of resilience.
\end{abstract}



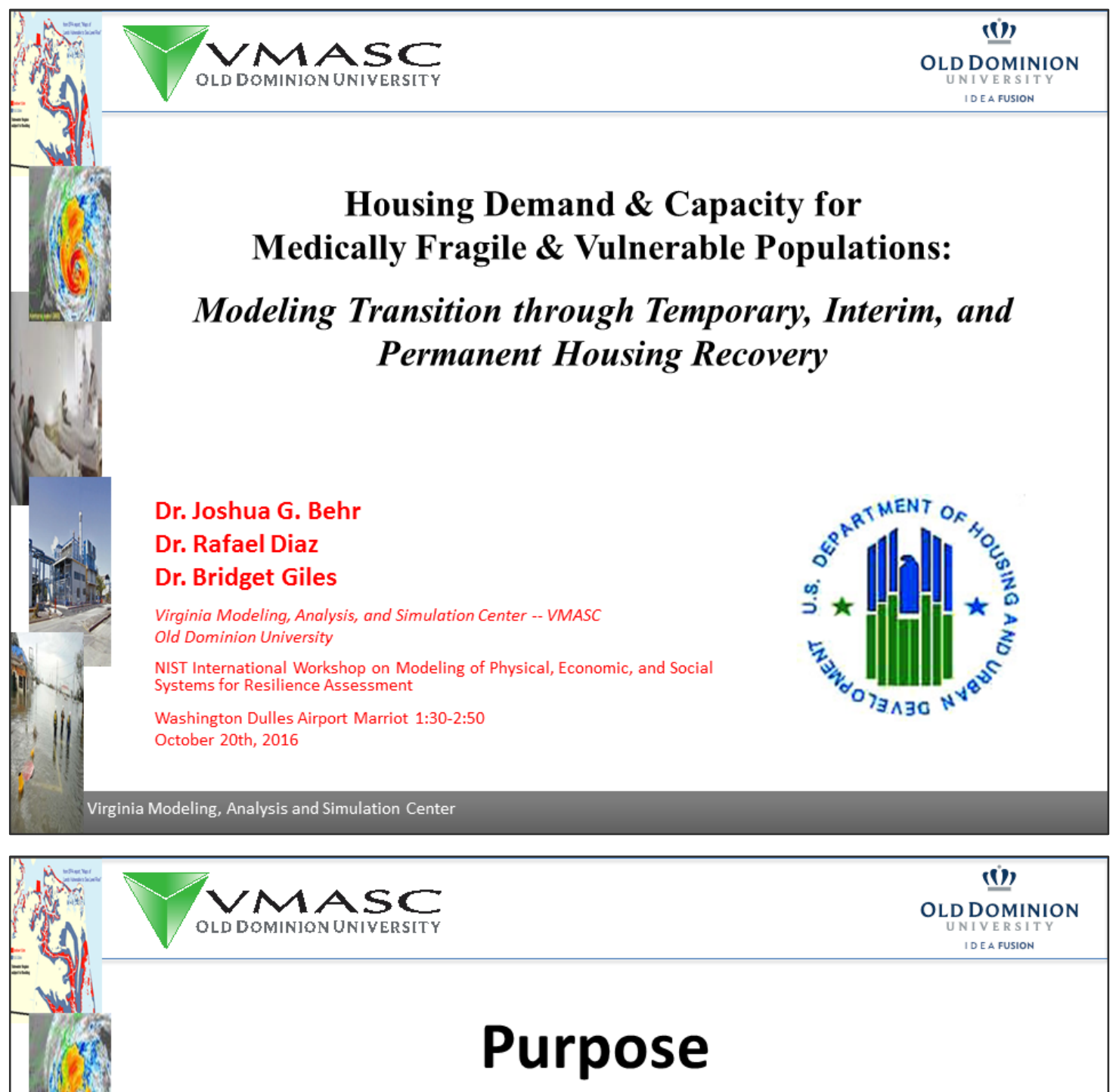

Through a series of Group Model Building events, guide the test bed cities in the development of models that forecast the housing transition times for displaced vulnerable populations. 

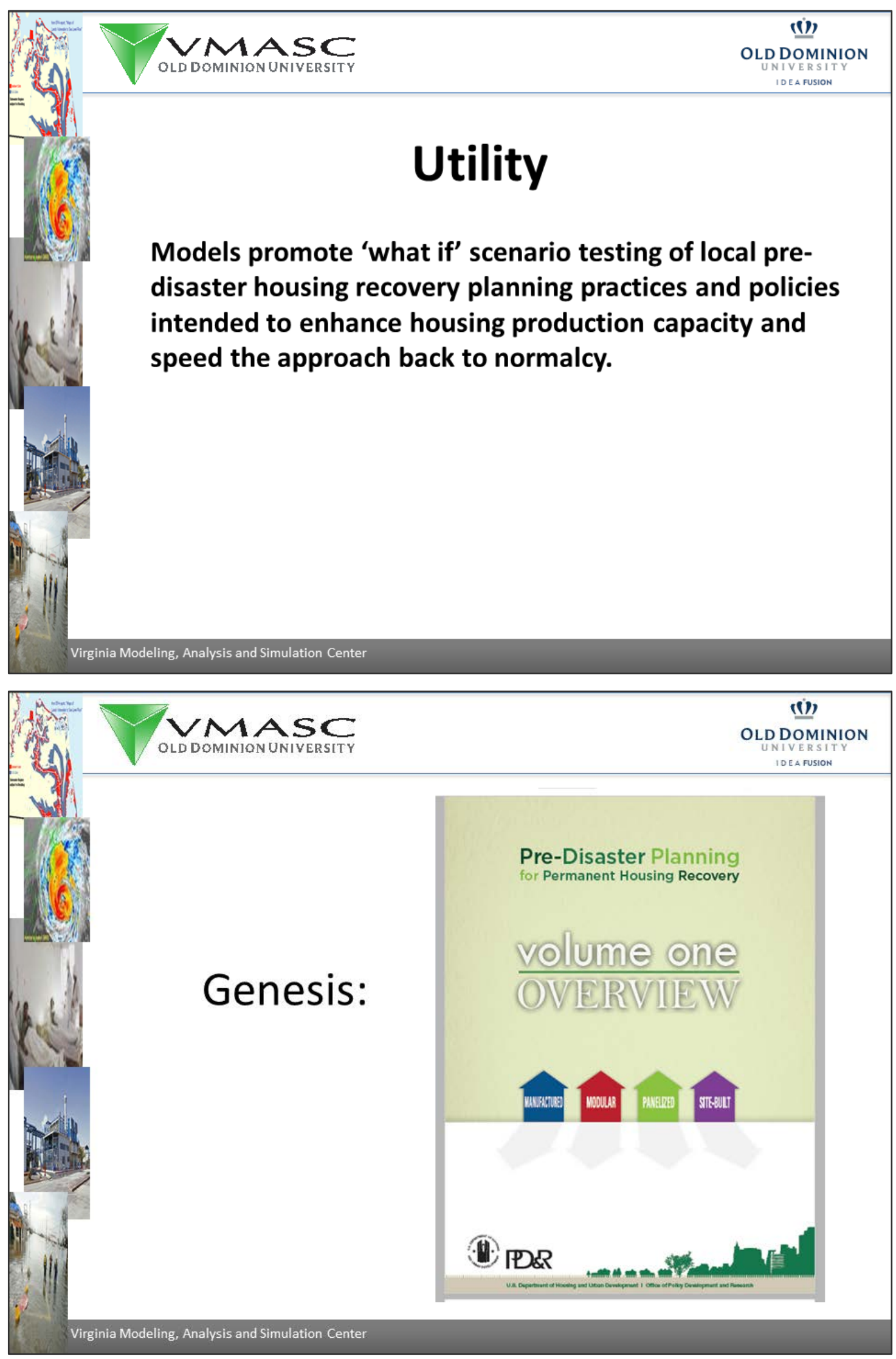

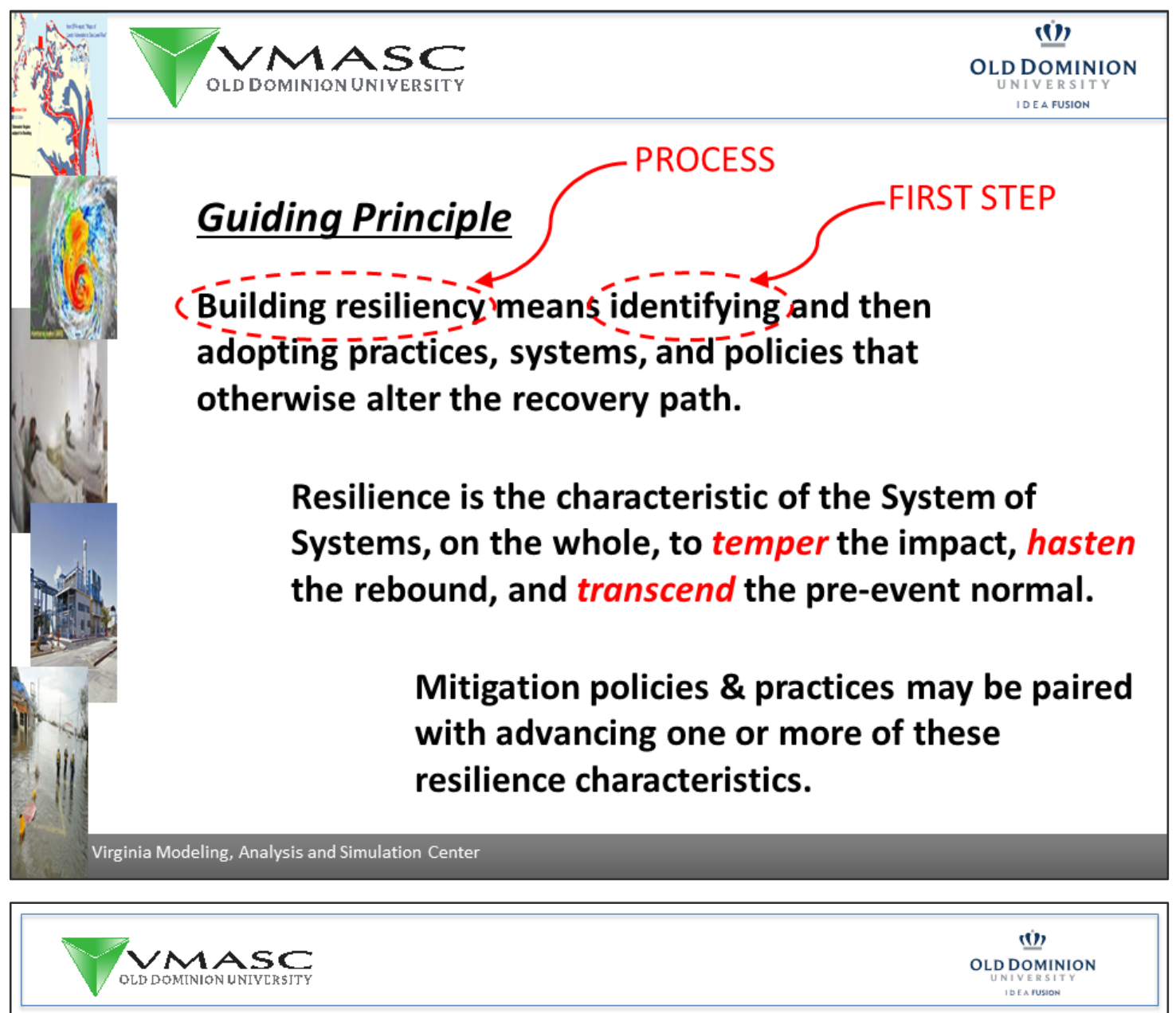

\section{Approaches to Building Resiliency}

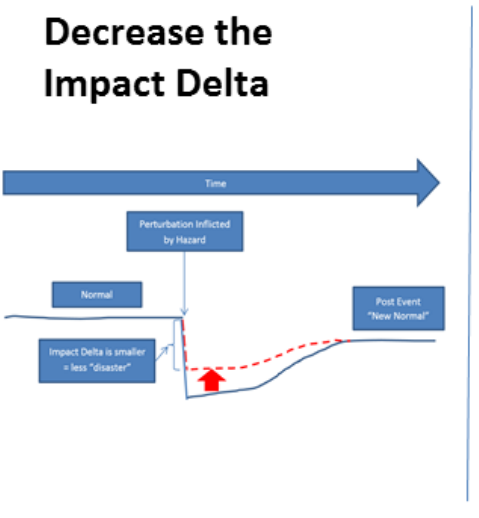

Temper

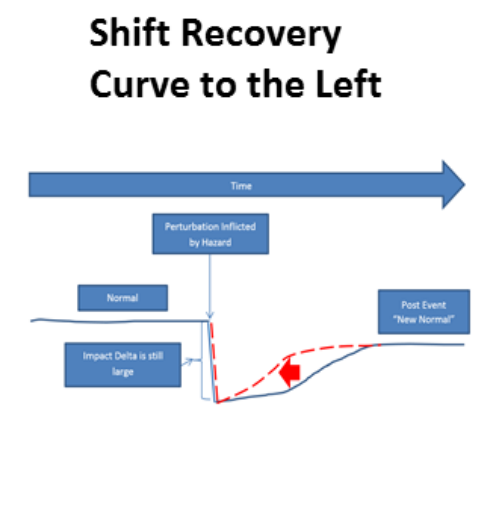

Hasten

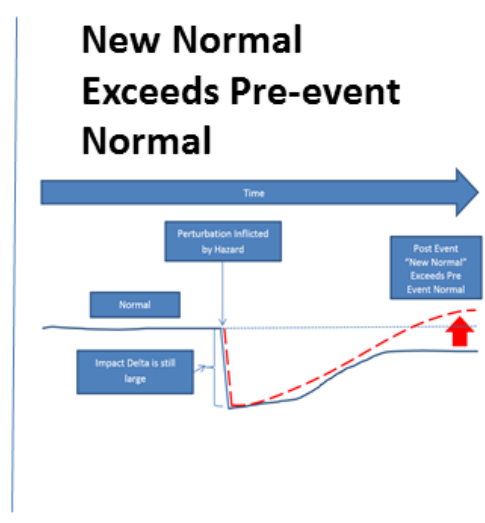

Transcend 

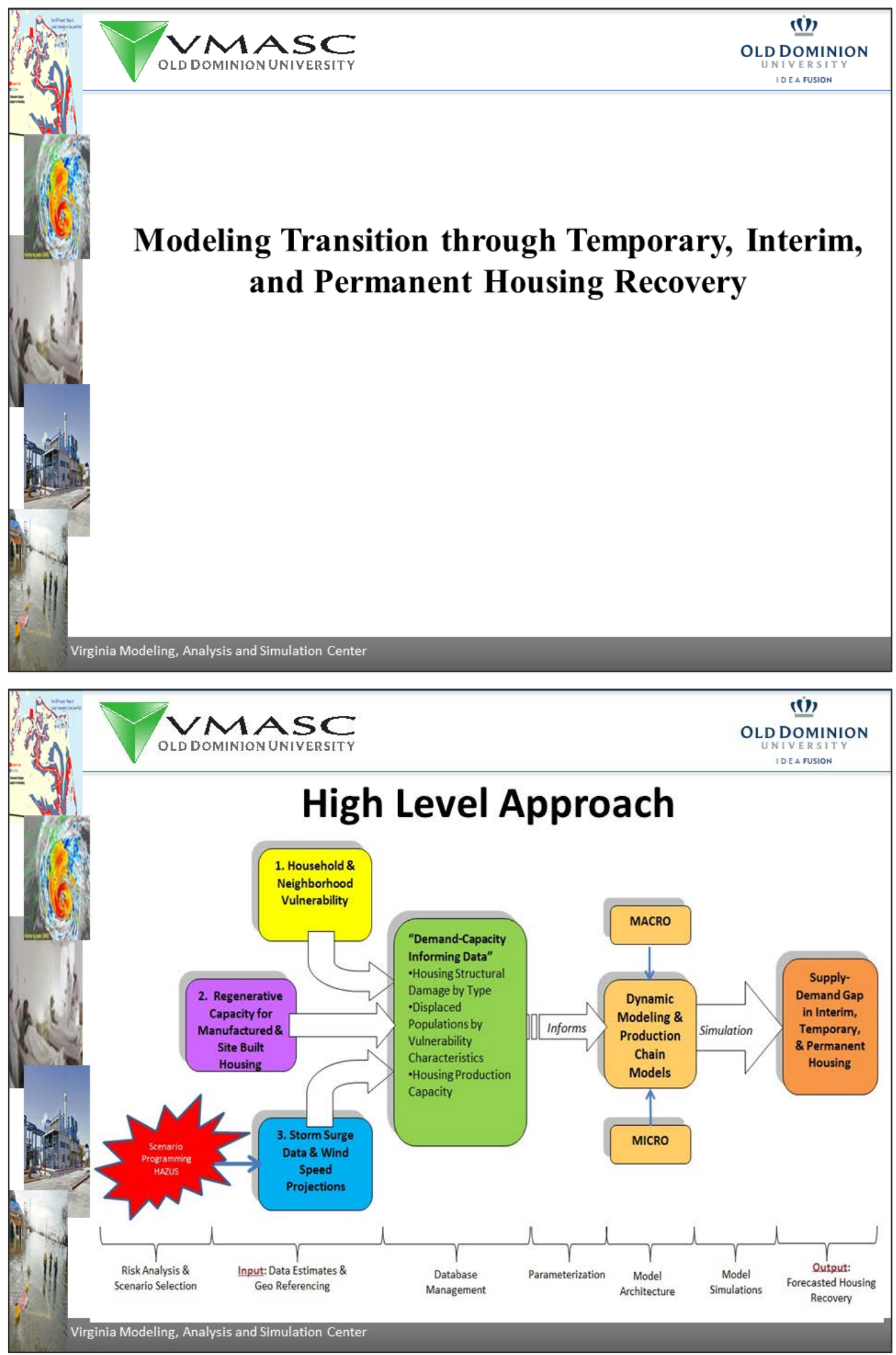

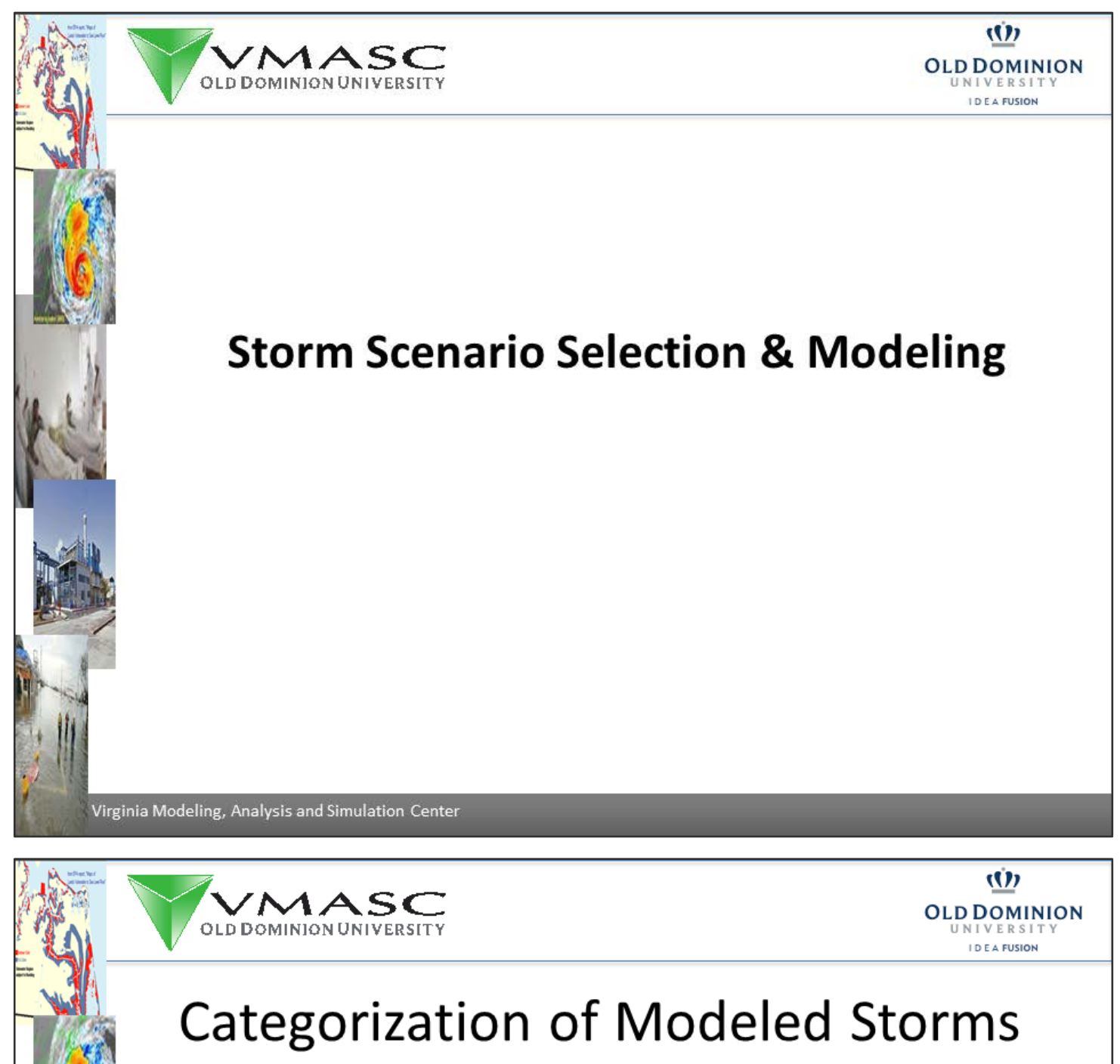

\section{Historic Hampton Roads Storm}

- Historic track, wind speeds, and flooding

Quasi-Historic Storm

- Adjusted track over Hampton Roads of historic storm making landfall outside Hampton Roads.

\section{Black Swan Event}

- Mostly user defined and intended to generate a truly catastrophic event; "worst possible case" 



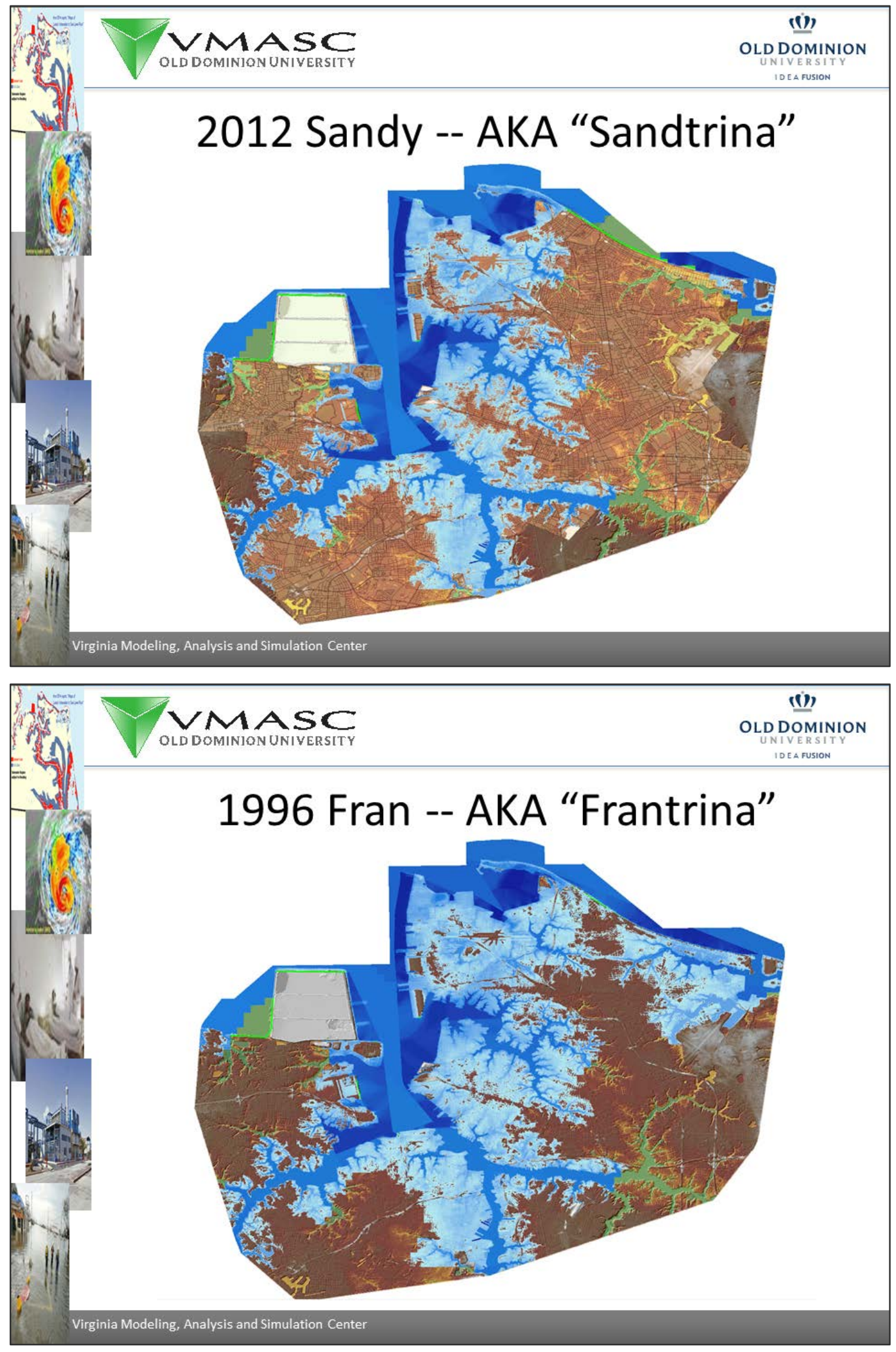

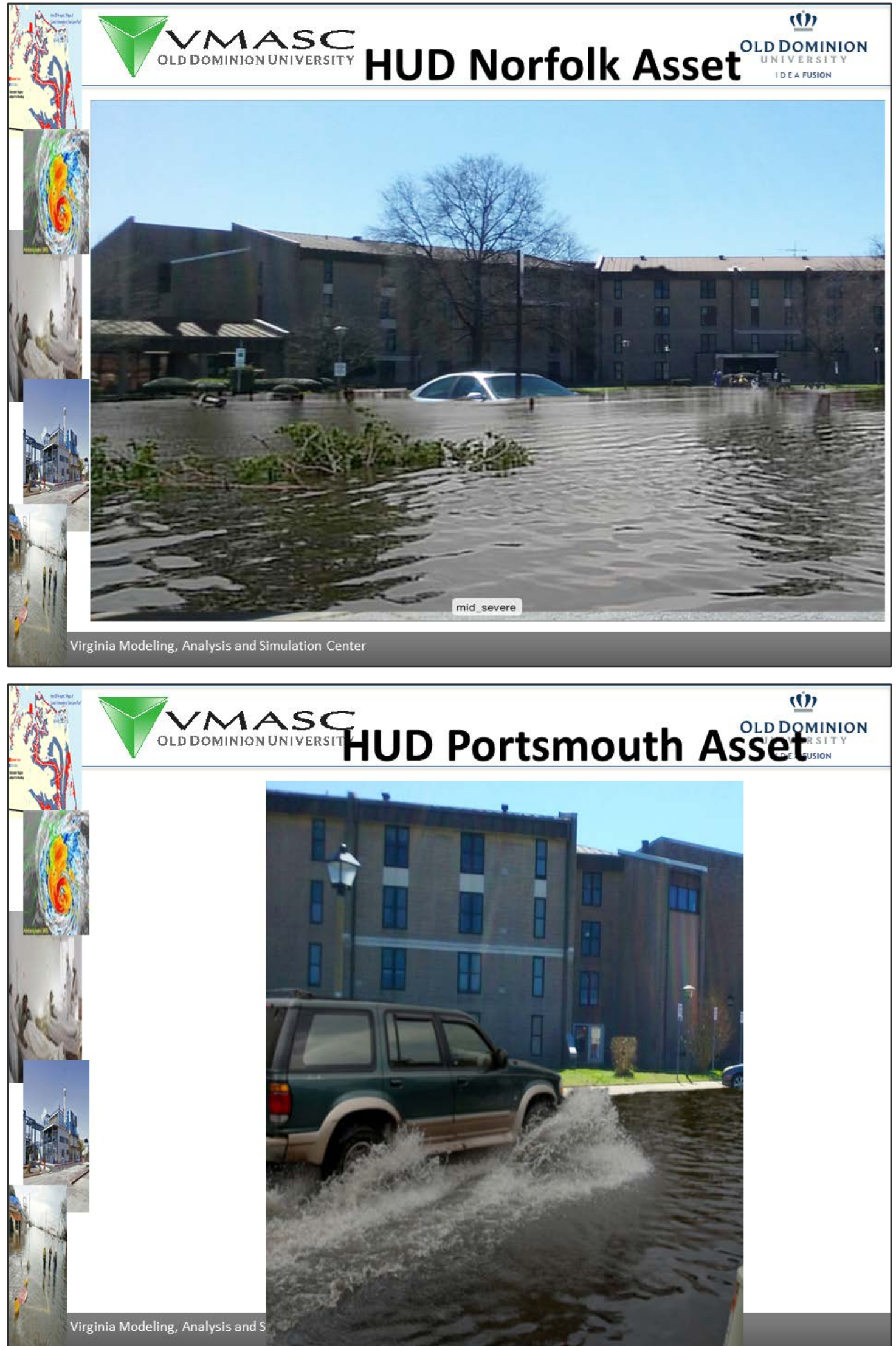

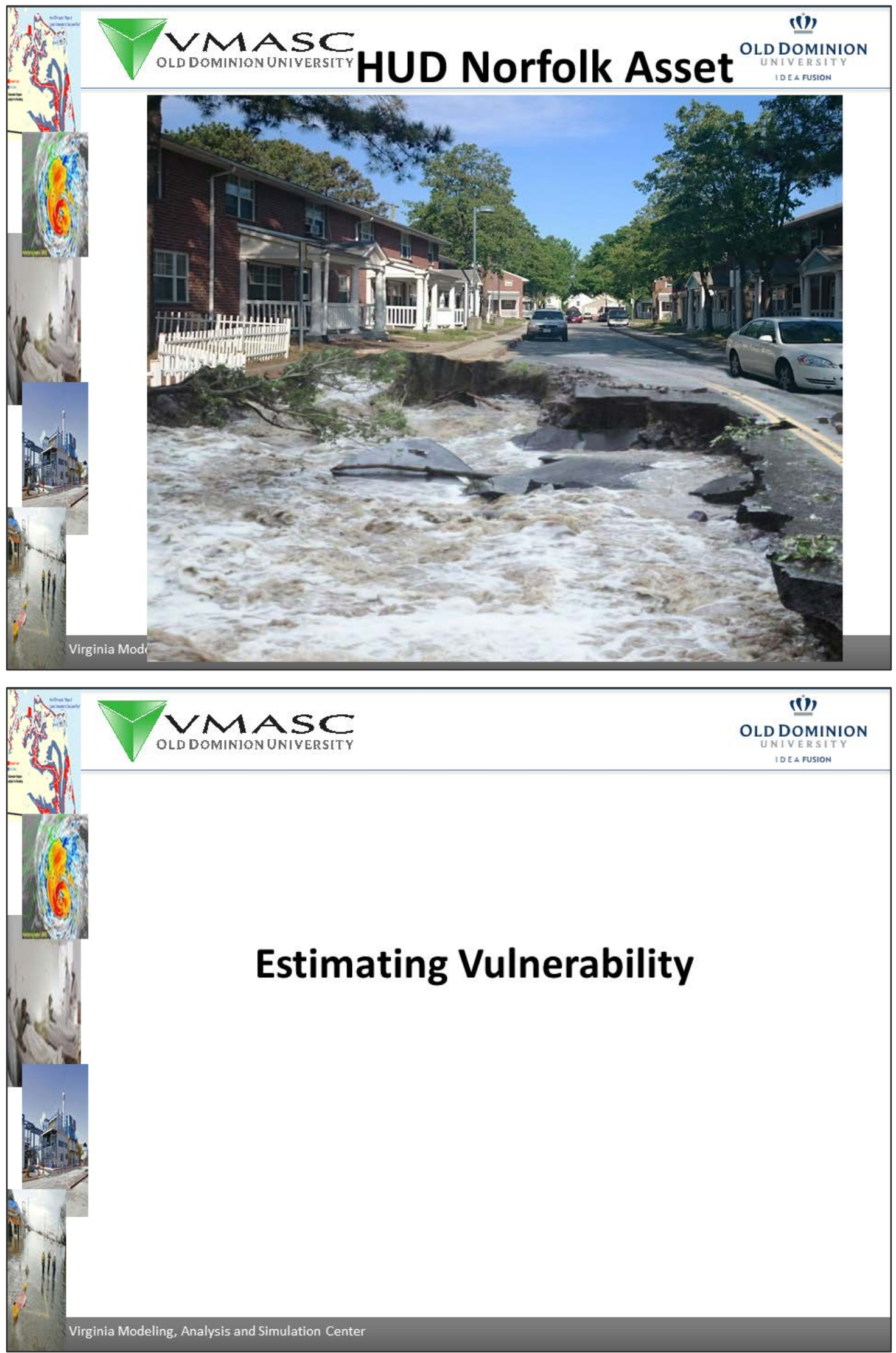

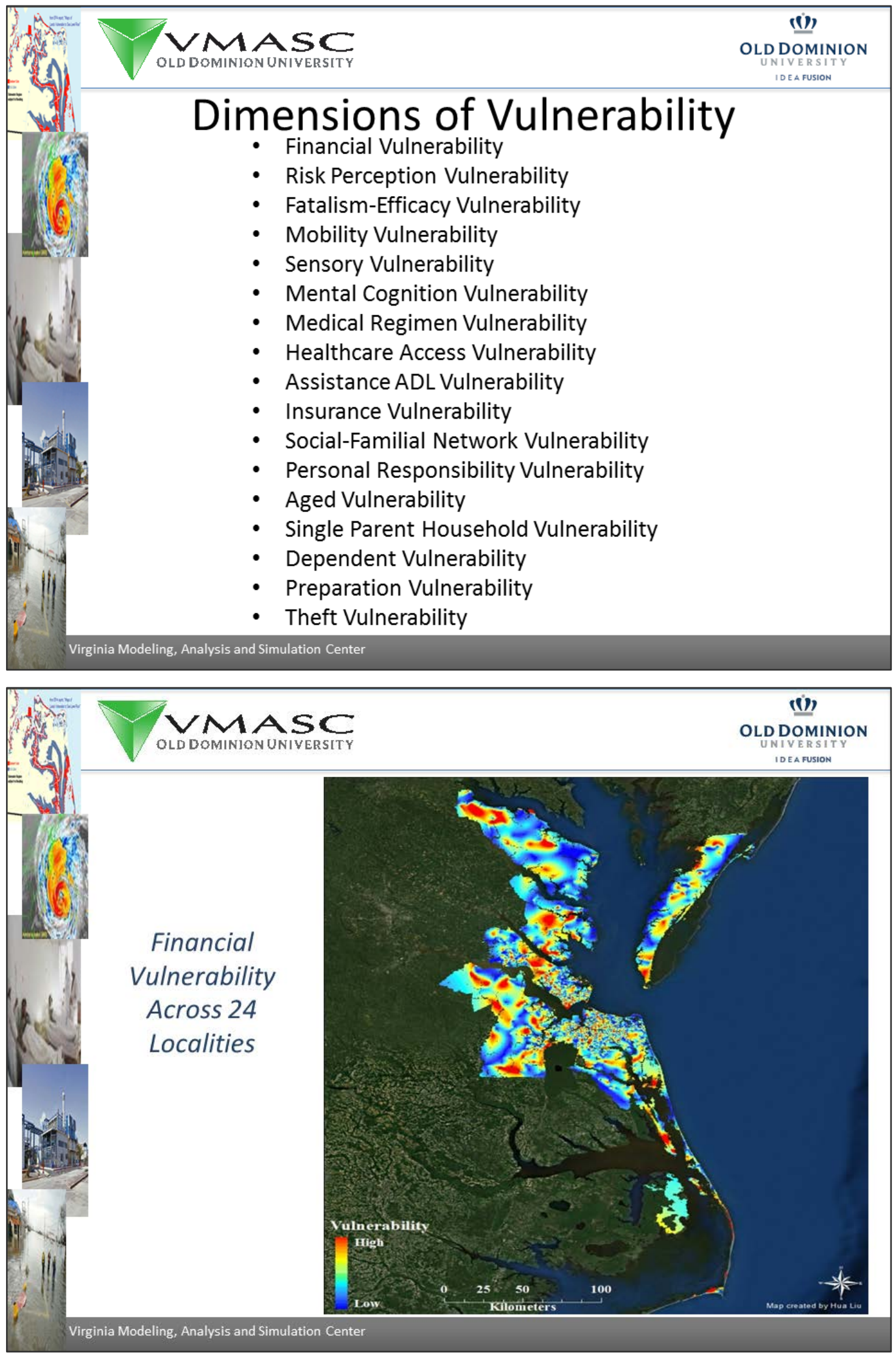

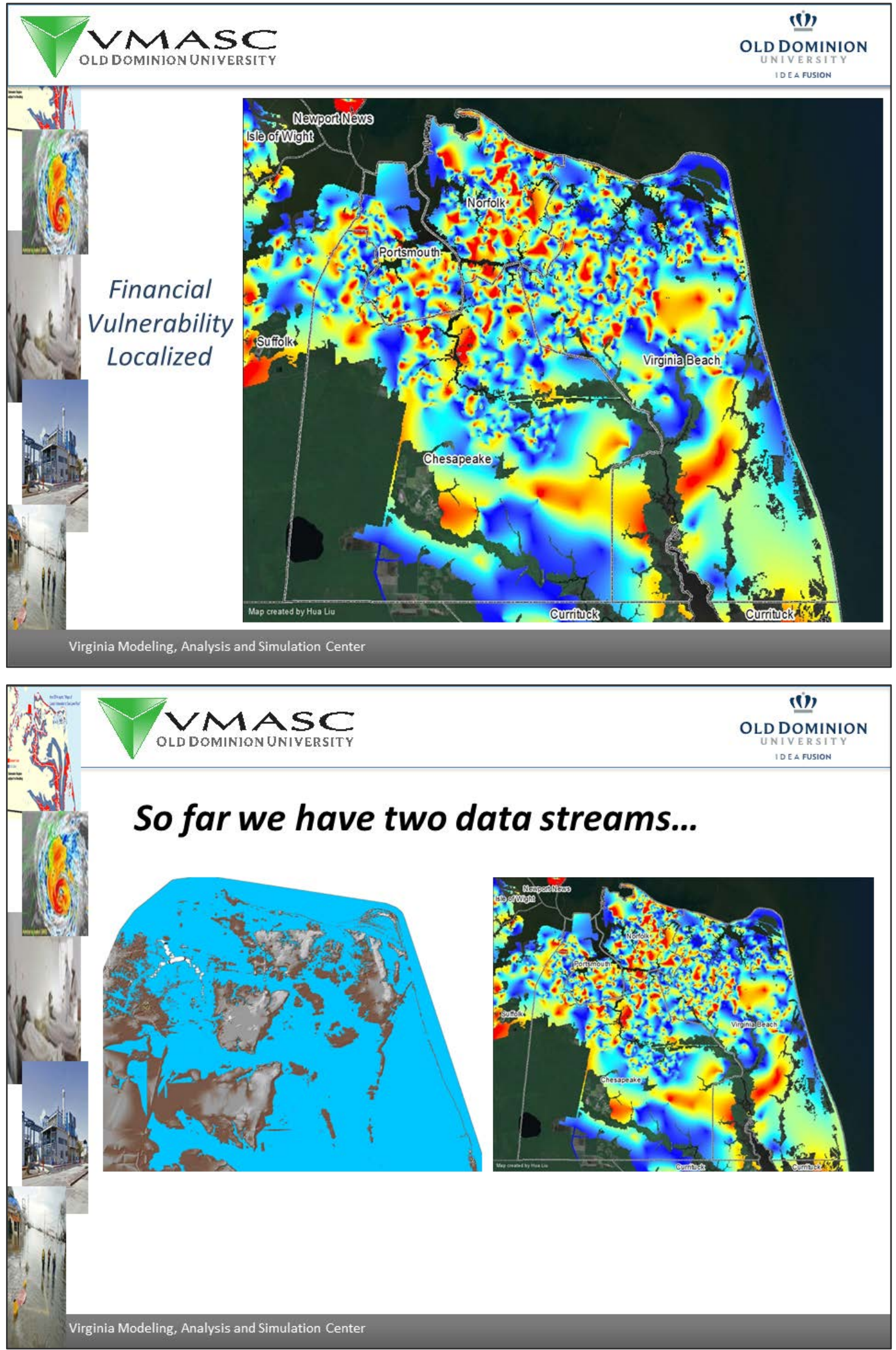


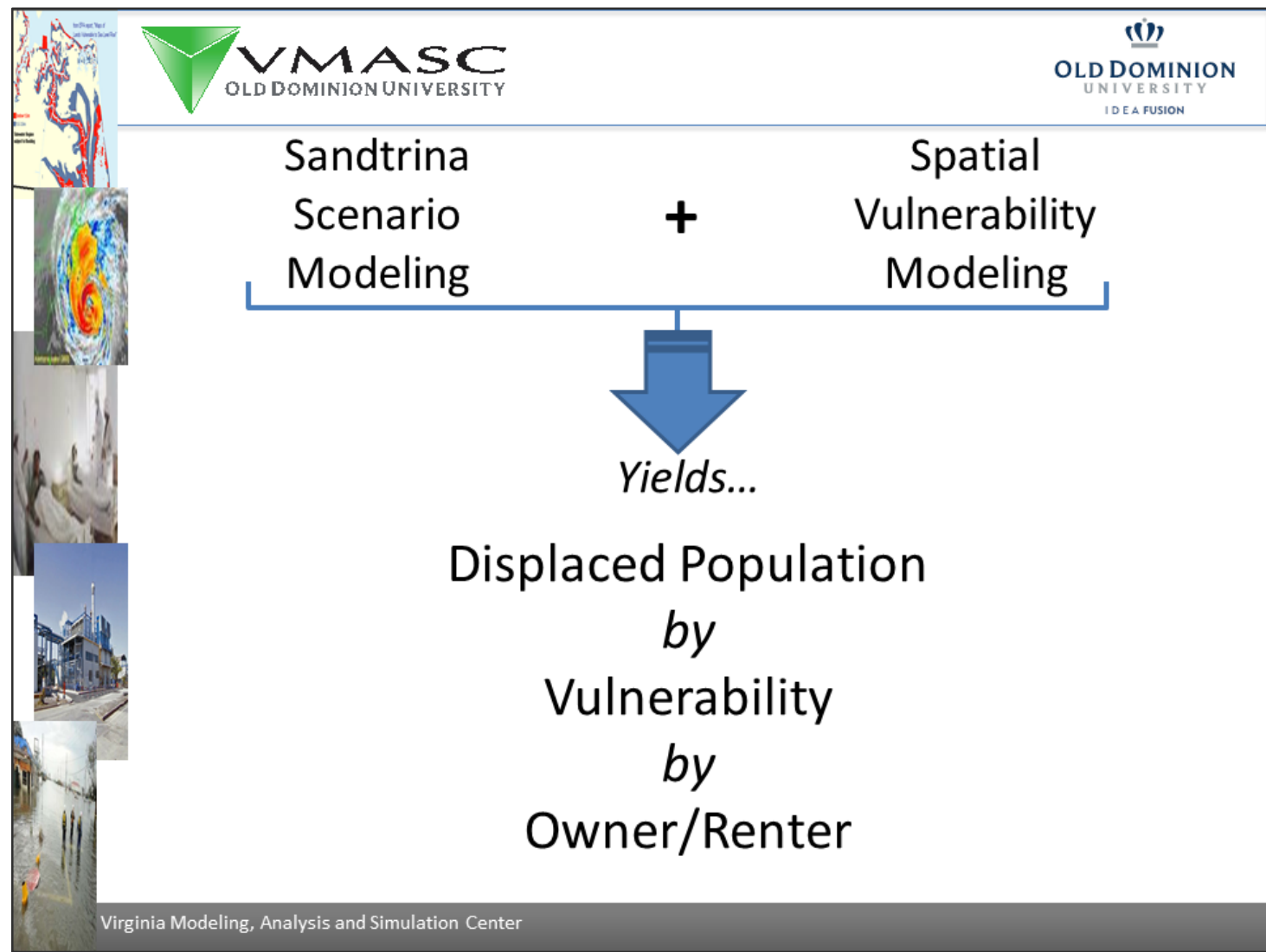

\begin{tabular}{|c|c|c|c|c|c|}
\hline & & LD DOMINION UNIVERS & & & $\underset{\substack{\text { ONIVERSITY } \\
\text { IDEAFUSION }}}{\text { OLD DOMINION }}$ \\
\hline & 1 & & $\begin{array}{l}\text { SEVA NENC P } \\
\end{array}$ & opulation Hazards & \\
\hline & 2 & Locality/Vulnerability & Sum of Displaced Population & Displaced Population (Owner) & Displaced Population (Renter) \\
\hline & 3 & Accomack County & 13,027 & 9416 & 3611 \\
\hline & 4 & 1- Low Vulnerability & 9,238 & 6783 & 2455 \\
\hline & 5 & 2-Medium Vulnerability & 3,326 & 2293 & 1033 \\
\hline & 6 & 3-High Vulnerability & 463 & 342 & 121 \\
\hline & 7 & Chesapeake city & 75,087 & 57746 & 17341 \\
\hline & 8 & 1- Low Vulnerability & 32,259 & 26113 & 6146 \\
\hline & 9 & 2-Medium Vulnerability & 37,307 & 27857 & 9450 \\
\hline & 10 & 3- High Vulnerability & 5,521 & 3377 & 2144 \\
\hline & 11 & Currituck County & 16,399 & 13322 & 3077 \\
\hline & 12 & 1-Low Vulnerability & 4,418 & 3631 & 787 \\
\hline & 13 & 2-Medium Vulnerability & 9,511 & 7803 & 1708 \\
\hline & 14 & 3-High Vulnerability & 2,470 & 1884 & 586 \\
\hline & 15 & Dare County & 26,117 & 19675 & 6442 \\
\hline & 16 & 1- Low Vulnerability & 6,727 & 5477 & 1250 \\
\hline & 17 & 2-Medium Vulnerability & 13,684 & 9968 & 3716 \\
\hline & 18 & 3- High Vulnerability & 5,706 & 4174 & 1532 \\
\hline & 19 & Gloucester County & 8,810 & 7272 & 1538 \\
\hline & 20 & 1- Low Vulnerability & 6,497 & 5424 & 1073 \\
\hline & 21 & 2-Medium Vulnerability & 1,969 & 1552 & 417 \\
\hline & 22 & 3-High Vulnerability & 344 & 305 & 39 \\
\hline & 23 & Hampton city & 105,525 & 63356 & 42169 \\
\hline & 24 & 1-Low Vulnerability & 32,098 & 22561 & 9537 \\
\hline & 25 & 2-Medium Vulnerability & 63,993 & 36651 & 27342 \\
\hline & 26 & 3- High Vulnerability & 9,434 & 3948 & 5486 \\
\hline & 27 & Isle of Wight County & 2,868 & 2351 & 517 \\
\hline Theoretical/ & 28 & 1- Low Vulnerability & 1,329 & 1088 & 241 \\
\hline Histrative & 29 & 2-Medium Vulnerability & 1,385 & 1124 & 261 \\
\hline plicitue & 30 & 3-High Vulnerability & 154 & 137 & 17 \\
\hline figures & 31 & James City County & 3,482 & 2785 & 697 \\
\hline & 32 & 1- Low Vulnerability & 2,511 & 2173 & 338 \\
\hline
\end{tabular}



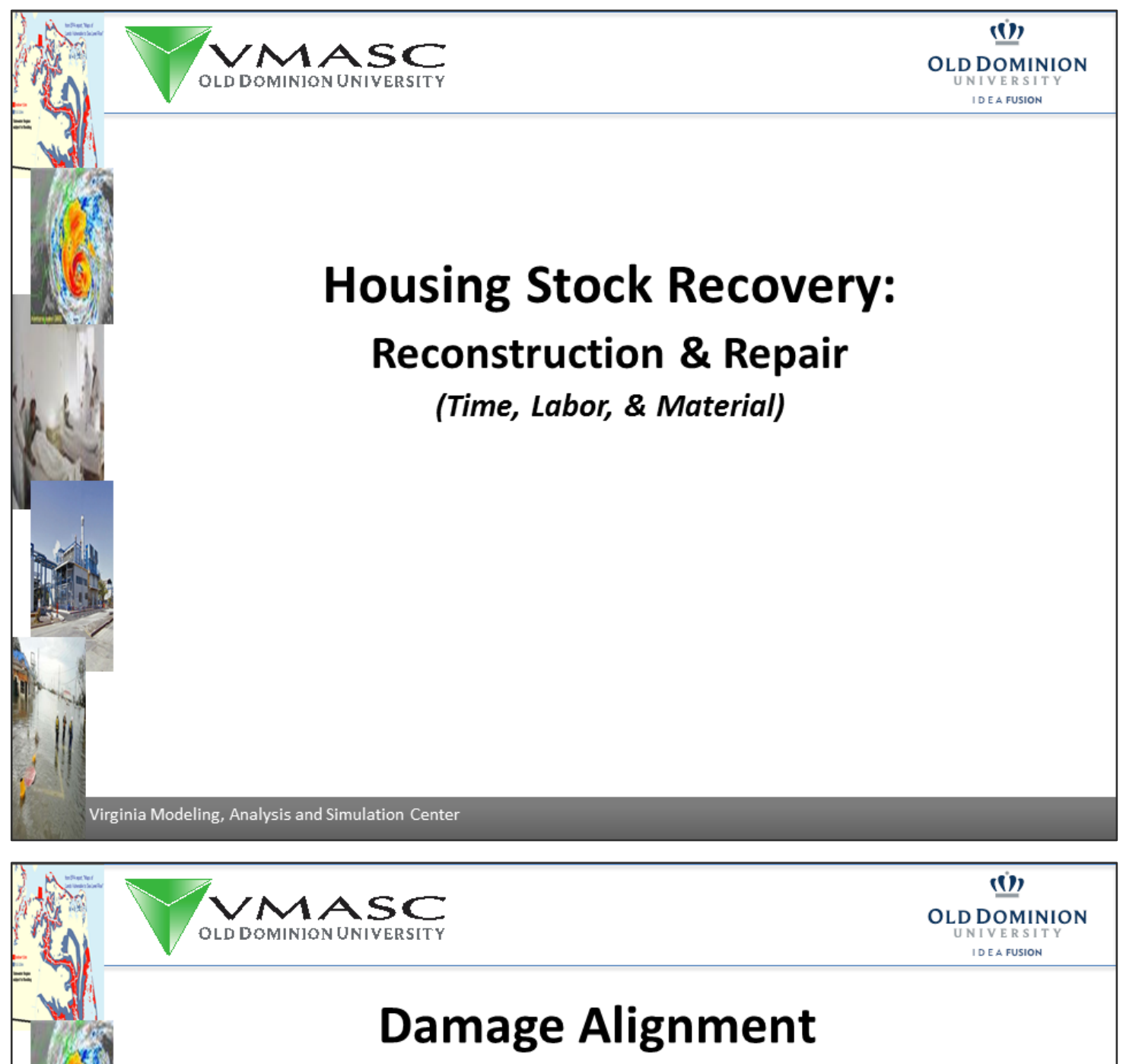

HazUS Damage States:

- Very Minor Damage \& Minor Damage

- Moderate

- Severe

- Destruction
FEMA Human

Services Preliminary Damage Assessment (PDA):

- Affected

- Minor

- Major

- Destroyed 


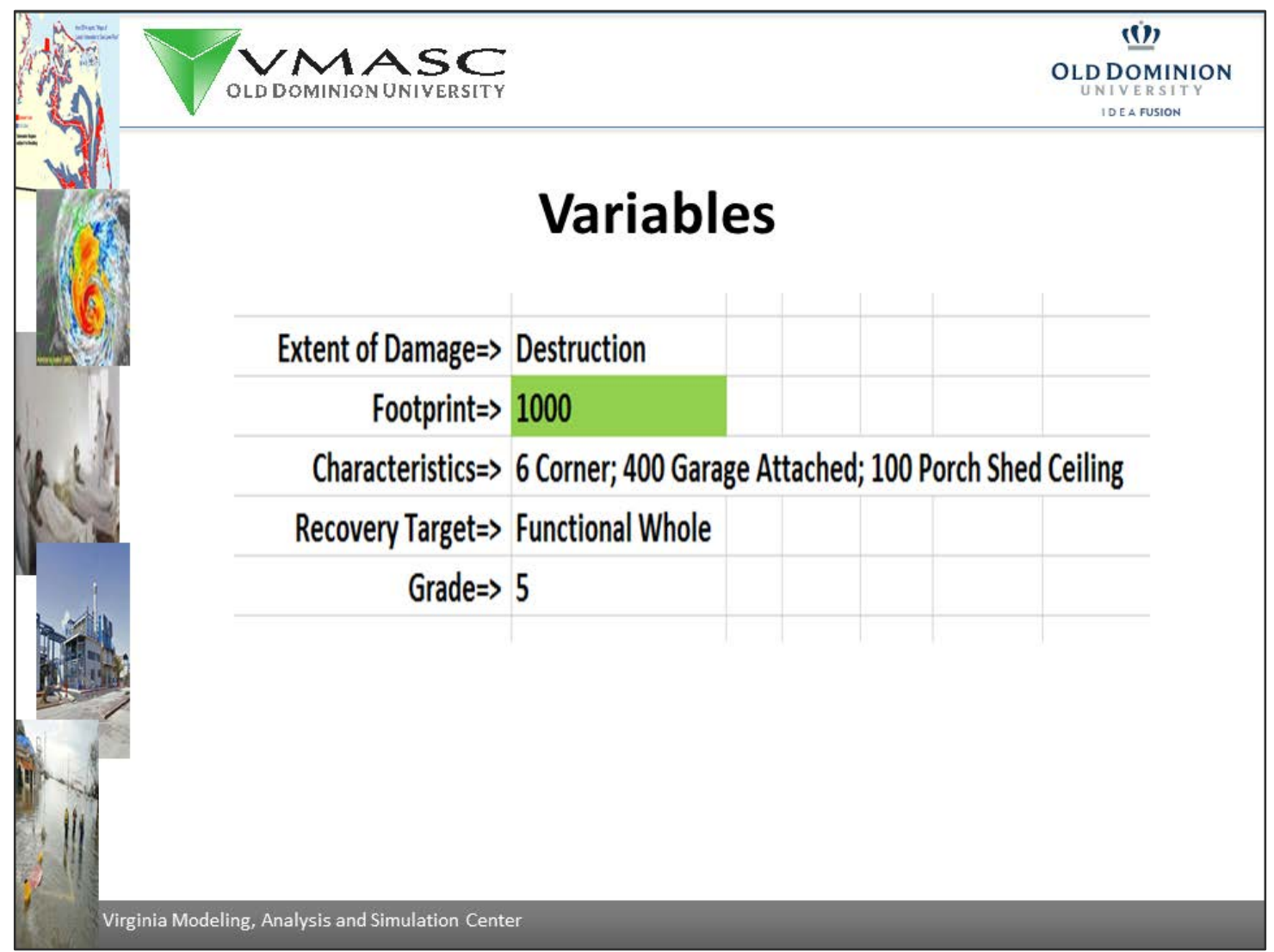

\begin{tabular}{|c|c|c|c|c|c|c|c|c|c|}
\hline \multirow{30}{*}{ 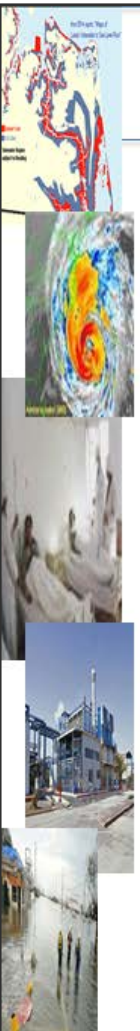 } & OLDDOMINIONU & & SITY & & & & & \multicolumn{2}{|c|}{$\stackrel{(i)}{\text { OLD DOMINION }}$} \\
\hline & & & & \multicolumn{2}{|r|}{ Material } & & & \multicolumn{2}{|r|}{ Contractor Labor } \\
\hline & Trade & & Material & $\%$ Materialcost & \%ofrotalcost1 & \%Totalcost2 & CLabor & c \%Laborcost & c\% \%otalcost1 \\
\hline & 1 Excavation & - & & & & & $1,257.00$ & $2.32 \%$ & $1.05 \%$ \\
\hline & 2 Foundation, Piers, Flatwork & s & $3,550.00$ & $5.66 \%$ & $2.98 \%$ & $2.91 \% s$ & $4,260.00$ & $7.85 \%$ & $3.57 \%$ \\
\hline & 3 Rough Hardware & s & 347.00 & $0.55 \%$ & $0.29 \%$ & $0.28 \% s$ & 417.00 & $0.77 \%$ & $0.35 \%$ \\
\hline & 4 Rough Carpentry & s & $11,478.00$ & $18.29 \%$ & $9.62 \%$ & $9.42 \% s$ & $12,928.00$ & $23.81 \%$ & $10.83 \%$ \\
\hline & 5 Insulation & $\$$ & $2,149.00$ & $3.42 \%$ & $1.80 \%$ & $1.76 \% \$$ & $1,121.00$ & $2.06 \%$ & $0.94 \%$ \\
\hline & 6 Exterior Finish & s & $6,110.00$ & $9.73 \%$ & $5.12 \%$ & $5.02 \% \$$ & $2,784.00$ & $5.13 \%$ & $2.33 \%$ \\
\hline & 7 Exterior Trim & s & 414.00 & $0.66 \%$ & $0.35 \%$ & $0.34 \% s$ & 497.00 & $0.92 \%$ & $0.42 \%$ \\
\hline & 8 Doors & s & $1,049.00$ & $1.67 \%$ & $0.88 \%$ & $0.86 \% s$ & 672.00 & $1.24 \%$ & $0.56 \%$ \\
\hline & 9 Windows & s & $1,807.00$ & $2.88 \%$ & $1.51 \%$ & $1.48 \% \mathrm{~s}$ & 937.00 & $1.73 \%$ & $0.79 \%$ \\
\hline & 10 Finish Hardware & s & 175.00 & $0.28 \%$ & $0.15 \%$ & $0.14 \% \$$ & 112.00 & $0.21 \%$ & $0.09 \%$ \\
\hline & 11 Garage Door & s & 495.00 & $0.79 \%$ & $0.41 \%$ & $0.41 \% s$ & 154.00 & $0.28 \%$ & $0.13 \%$ \\
\hline & 12 Roofing, Flashing, Fascia & s & $4,799.00$ & $7.65 \%$ & $4.02 \%$ & $3.94 \% \mathrm{~s}$ & $3,071.00$ & $5.66 \%$ & $2.57 \%$ \\
\hline & 13 Finish Carpentry & $\$$ & 637.00 & $1.01 \%$ & $0.53 \%$ & $0.52 \% s$ & $2,448.00$ & $4.51 \%$ & $2.05 \%$ \\
\hline & 14 Interior Wall Finish & s & $3,057.00$ & $4.87 \%$ & $2.56 \%$ & $2.51 \% \mathrm{~s}$ & $3,603.00$ & $6.64 \%$ & $3.02 \%$ \\
\hline & 15 Painting & $\$$ & $1,827.00$ & $2.91 \%$ & $1.53 \%$ & $1.50 \% \mathrm{~s}$ & $3,254.00$ & $5.99 \%$ & $2.73 \%$ \\
\hline & 16 Wiring & s & $1,855.00$ & $2.96 \%$ & $1.55 \%$ & $1.52 \% \mathrm{~s}$ & $2,672.00$ & $4.92 \%$ & $2.24 \%$ \\
\hline & 17 Lighting Fixtures & s & $1,390.00$ & $2.21 \%$ & $1.16 \%$ & $1.14 \% \mathrm{~s}$ & 334.00 & $0.62 \%$ & $0.28 \%$ \\
\hline & 18 Flooring & s & $1,366.00$ & $2.18 \%$ & $1.14 \%$ & $1.12 \% \mathrm{~s}$ & $1,479.00$ & $2.72 \%$ & $1.24 \%$ \\
\hline & 19 Carpeting & s & $2,718.00$ & $4.33 \%$ & $2.28 \%$ & $2.23 \% \mathrm{~s}$ & 740.00 & $1.36 \%$ & $0.62 \%$ \\
\hline & 20 Bath Accessories & s & 673.00 & $1.07 \%$ & $0.56 \%$ & $0.55 \% \mathrm{~s}$ & 316.00 & $0.58 \%$ & $0.26 \%$ \\
\hline & 21 Shower \& Tub Enclosure & s & 430.00 & $0.69 \%$ & $0.36 \%$ & $0.35 \% \mathrm{~s}$ & 275.00 & $0.51 \%$ & $0.23 \%$ \\
\hline & 22 Countertops & s & $1,300.00$ & $2.07 \%$ & $1.09 \%$ & $1.07 \% \mathrm{~s}$ & 831.00 & $1.53 \%$ & $0.70 \%$ \\
\hline & 23 Cabinets & s & $4,274.00$ & $6.81 \%$ & $3.58 \%$ & $3.51 \% \mathrm{~s}$ & $1,026.00$ & $1.89 \%$ & $0.86 \%$ \\
\hline & 24 Built In Appliances & \$ & $2,080.00$ & $3.31 \%$ & $1.74 \%$ & $1.71 \% \mathrm{~s}$ & 222.00 & $0.41 \%$ & $0.19 \%$ \\
\hline & 25 Plumbing Rough-in and Connec & s & $1,952.00$ & $3.11 \%$ & $1.64 \%$ & $1.60 \% \mathrm{~s}$ & $3,617.00$ & $6.66 \%$ & $3.03 \%$ \\
\hline & 26 Plumbing Fixtures & s & $3,965.00$ & $6.32 \%$ & $3.32 \%$ & $3.26 \% \$$ & 958.00 & $1.76 \%$ & $0.80 \%$ \\
\hline & 27 Heating and Cooling Systems & s & $2,872.00$ & $4.58 \%$ & $2.41 \%$ & $2.36 \% \mathrm{~s}$ & $4,308.00$ & $7.93 \%$ & $3.61 \%$ \\
\hline
\end{tabular}



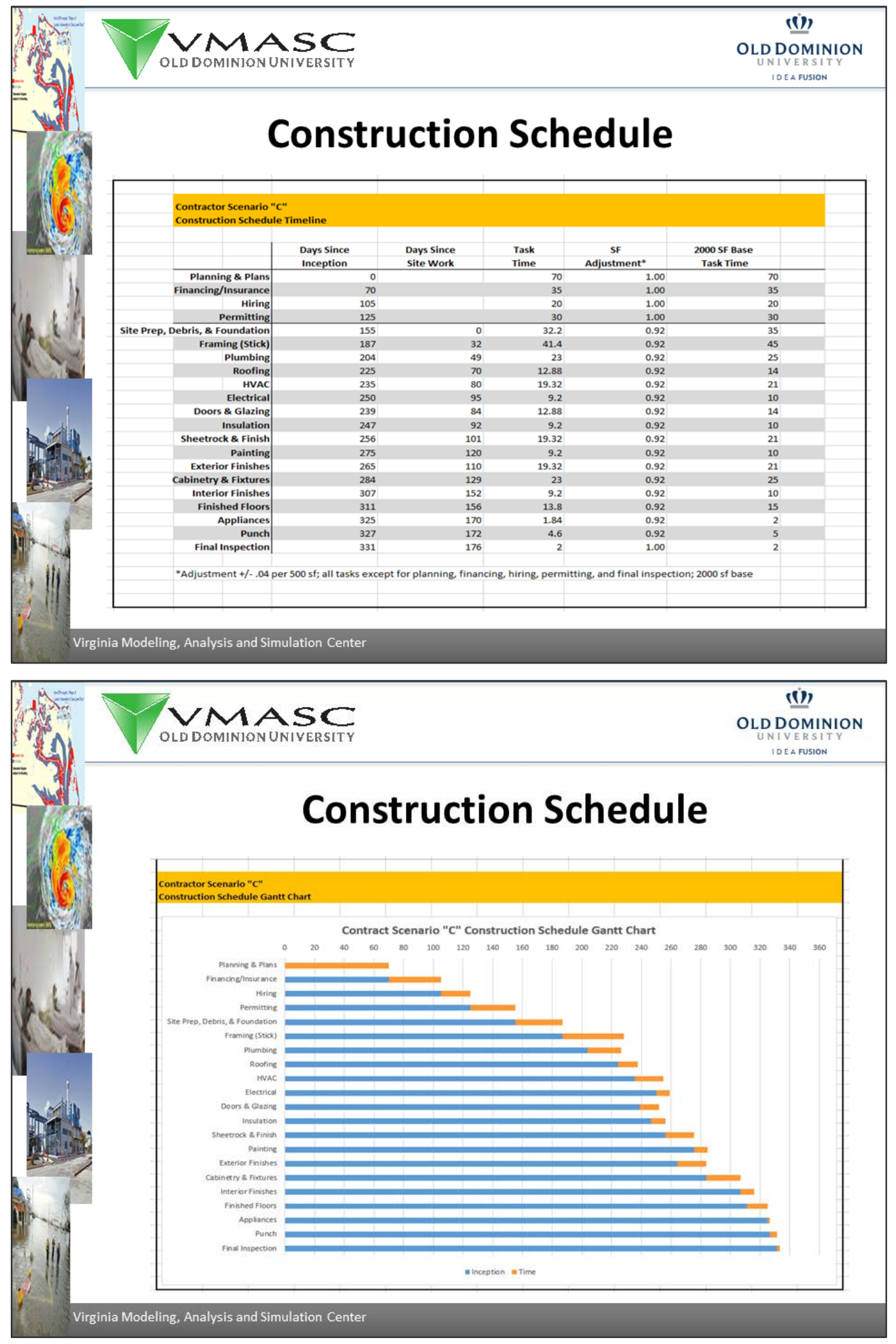

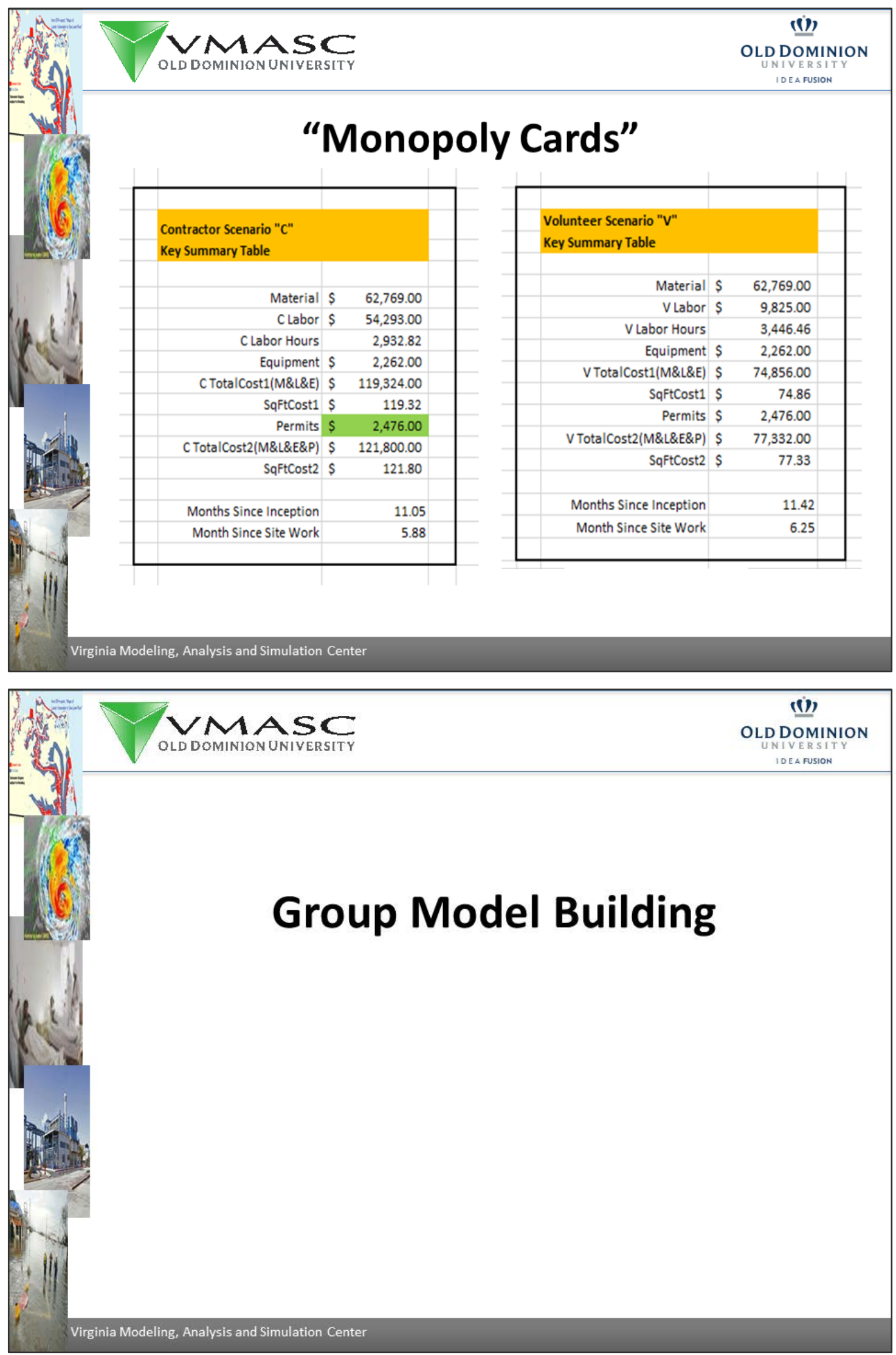

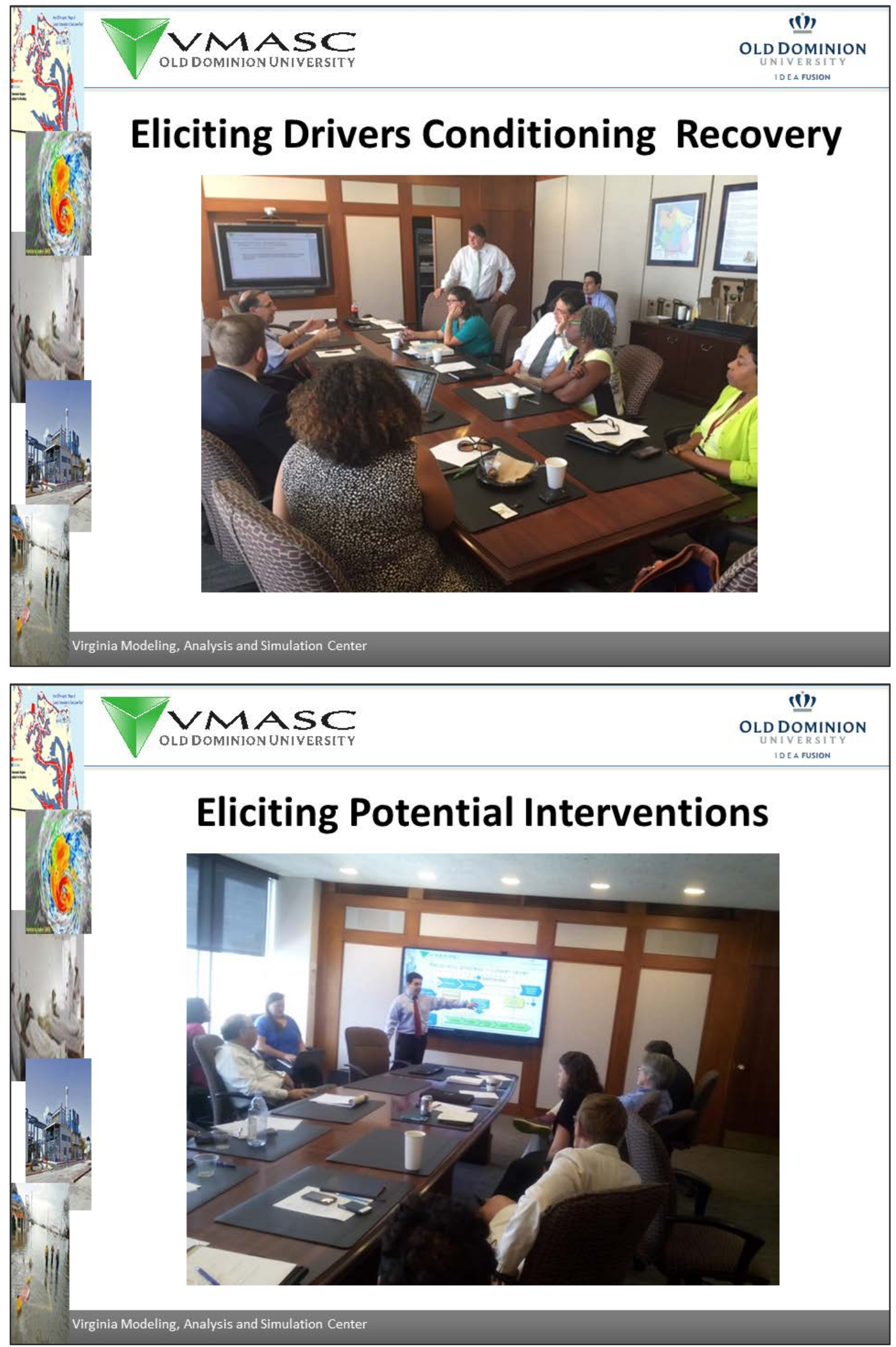

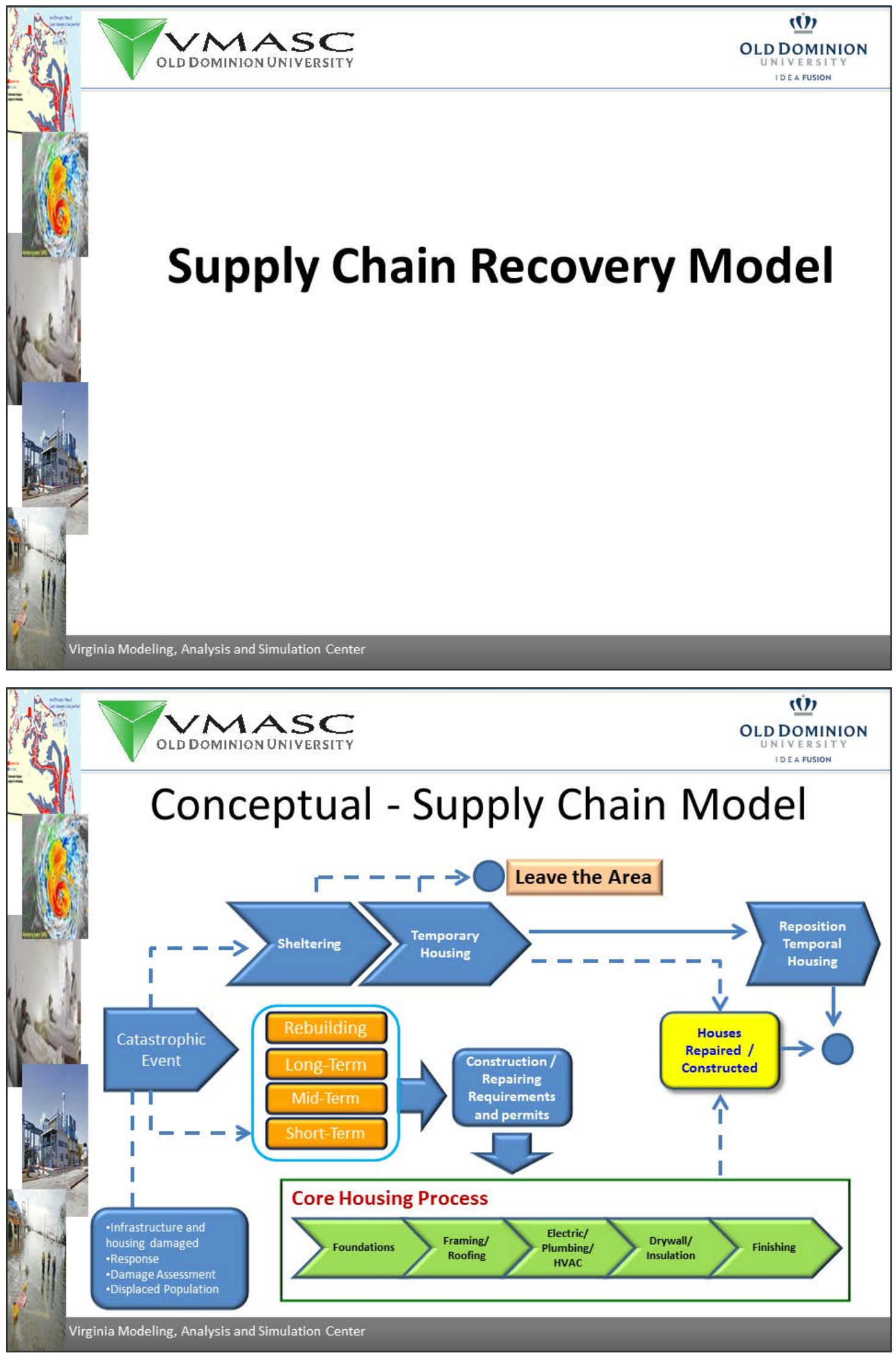

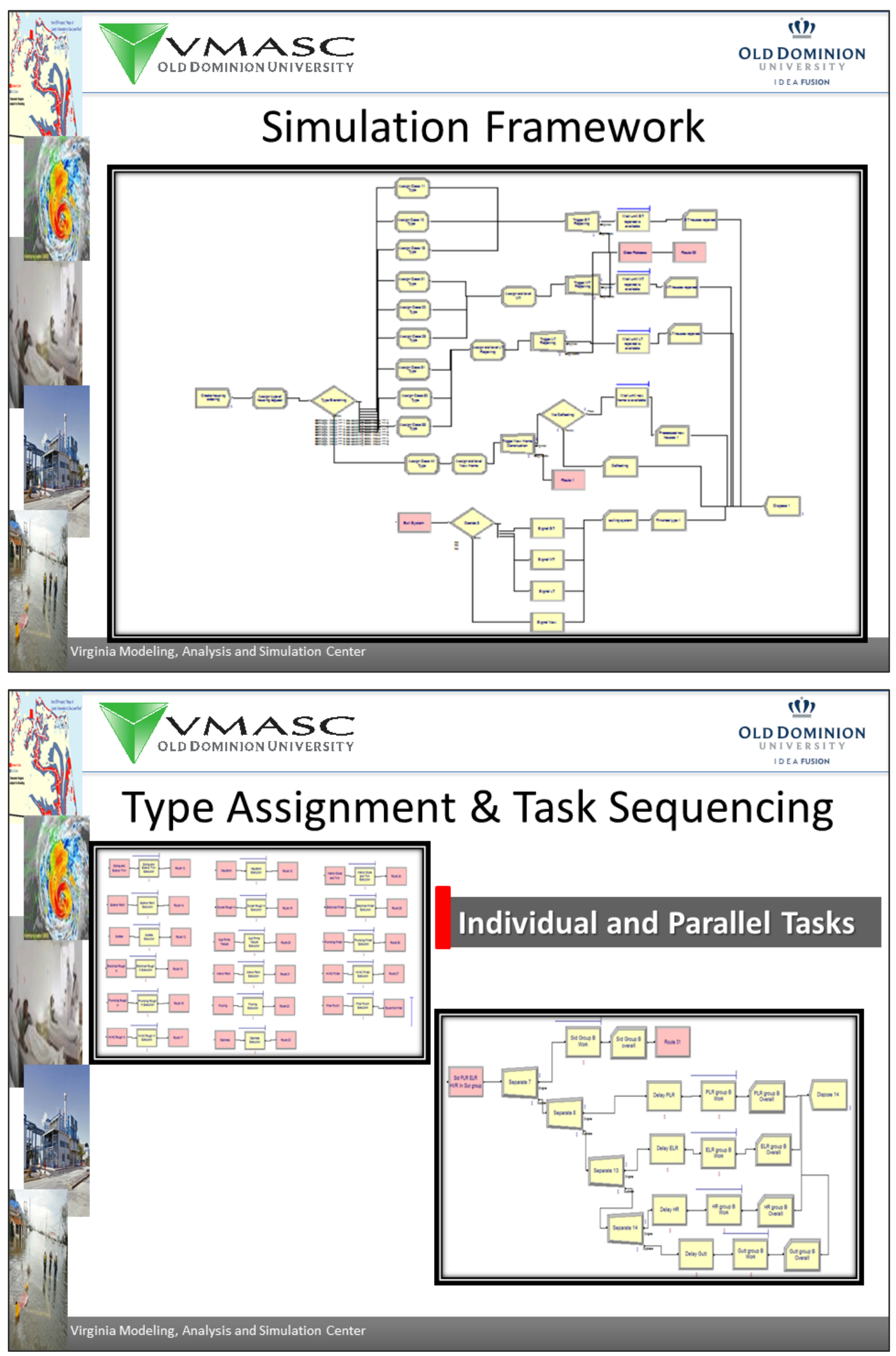

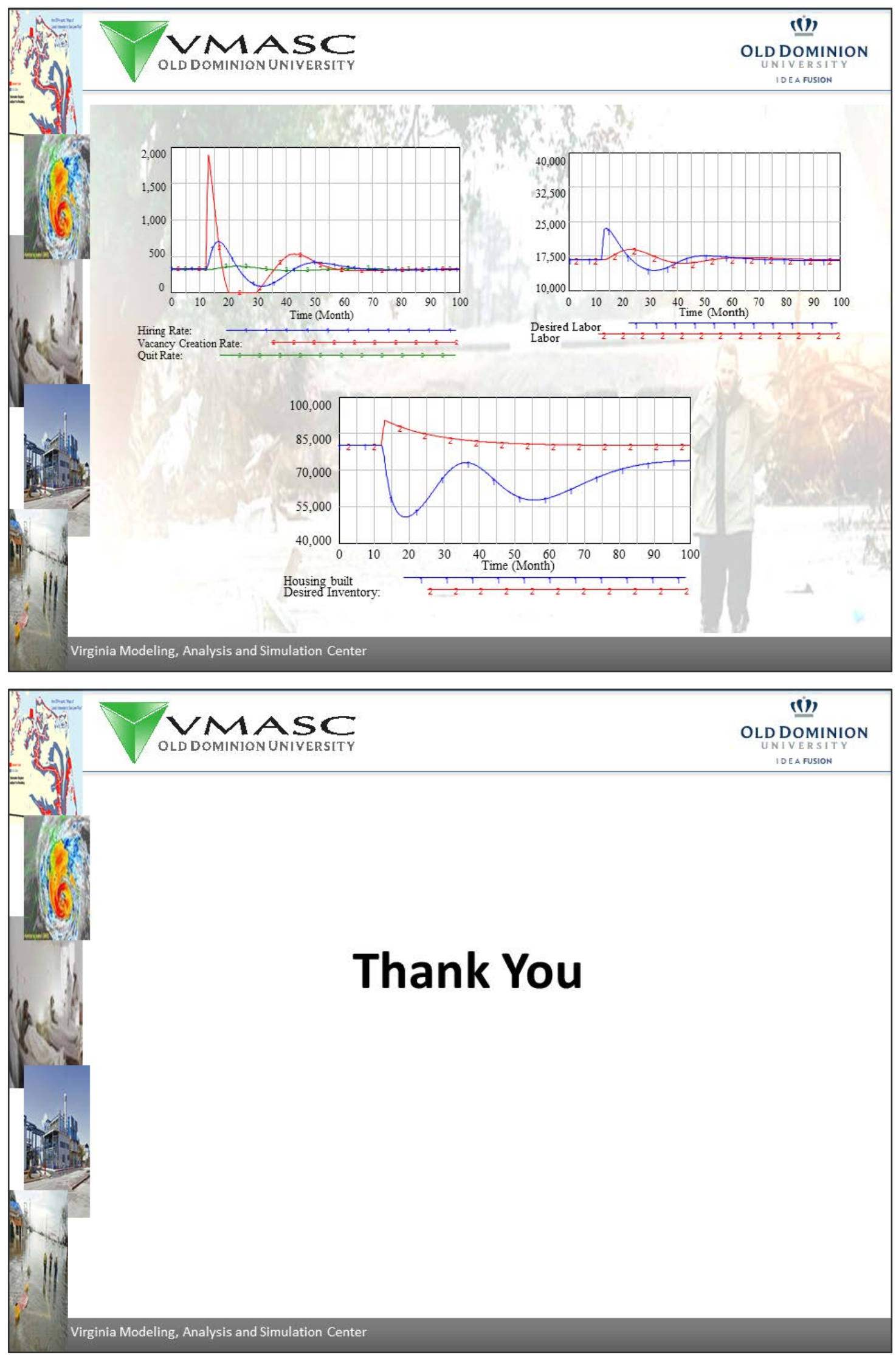
Bibliography for Joshua G. Behr's Presentation:

Housing Demand \& Capacity for Medically Fragile \&* Vulnerable Populations: Modeling Transition Times through temporary, Interim, and Permanent Housing stock Recovery

\section{BIBLIOGRAPHY}

Flanagan et al. 2011 "A Social Vulnerability Index for Disaster Management" Journal of Homeland Security and Emergency Management, Vol. 8, No. 1.

Phifer, James F. 1990. Psychological distress and somatic symptoms after natural disaster: Differential vulnerability among older adults. Psychology and Aging, Vol 5(3)

National Council on Disabilities, 2006 report, The impact of Hurricanes Katrina and Rita on People with Disabilities: A Look Back and Remaining Challenges

Bascetta C. Disaster Preparedness: Preliminary Observations on the Evacuation of Vulnerable Populations due to Hurricanes and Other Disasters. May 18, 2006, US Government Accountability Office, GAO-06-790T

National Council on Disabilities, 2006 report, "The Needs of People with Psychiatric Disabilities During and After Hurricanes Katrina and Rita: Position Paper and Recommendations."

National Council on Disabilities, 2009, Effective Emergency management: Making Improvements for Communities and People with Disabilities

National Council on Disabilities, 2005, Saving Lives: Including People with Disabilities in Emergency Planning

King, D., and C. MacGregor. 2000 Using Social Indicators to Measure Community Vulnerability to Natural Hazards, Australian Journal of Emergency Management, Spring.

Cova, T.J., et al. 2009. Protective Actions in Wildfires: Evacuate or Shelter-in-Place? Natural Hazards Review, November, 151-162.

Burton, C.J. 2010. Social Vulnerability and Hurricane Impact Modeling. Natural Hazards Review. May, 5968.

Cutter, S.L., et al. 2008. A Place-based Model for Understanding Community Resilience to Natural Disasters. Global Environment Change. Vol. 18, 598-606. 
French, S.P. and D. Lee 2010. Estimating the social and Economic Impact Consequences of natural hazards: Fiscal Impact Example, Natural Hazards Review, May, 49-57.

Baade et al. Baade, R.A., et al. 2007. Estimating the impact of Natural and Social Disasters, Urban Studies, Vol. 44, No.11, 2061-2076

Probvenzo, E.F. and A.B. Provenzo. 2002. In the Eye of Hurricane Andrew. University Press of Florida.

Devereaux, A., S. Burnes, and R. Gougelet. Delivering Acute Care to Chronically III Shelters. Chapter 11.

Brodie, M. et al. 2006. Experiences of Hurricane Katrina Evacuees in Houston Shelters: Implications for Future Planning. American Journal of Public Health, Vol. 96, No. 5.

Flint, C.G. and J. Stevenson. 2010. Building Community Disaster Preparedness with Volunteers. Natural Hazards Review.

Marrow, B.H. 1999. Identifying and mapping Community Vulnerability. Disasters Vol. 23, No. 1

Bloom, E. et al. 2009. Molds and Mycotoxins in Dust from Water-damaged Homes in New Orleans after Hurricane Katrina. Indoor Air, Vol. 19.

Flaskerud, J.H. and B.J. Winslow. 1998. Conceptualizing Vulnerable Populations Health-related Research. Nursing Research. Vol. 47.

Chen, A.C. et al. 2007. Hurricane Katrina: Prior Trauma, Poverty, and Health Among VietnameseAmerican Survivors. International Nursing Review, Vol. 54.

Finch, C., C.T. Emrich, and S.L. Cutter. 2010. Disaster Disparities and Differential Recovery in New Orleans. Population Environment, Vol. 31.

Grothmann, T. and F. Reusswig. 2006. People at Risk of Flooding: Why some Residents Rake Precautionary Action while Other Do Not. Natural Hazards, Vol. 38.

Kiefer, J.J. et al. 2008. Providing Access to Resilience-Enhancing Technologies for Disadvantaged communities and Vulnerable Populations. PARET report, Institute for Advanced Biometrics and Social Systems Studies.

Fox, M. et al. 2007. Disaster Preparedness and Response for Persons with Mobility Impairments. Journal of Disability Policy Studies, Vol. 17, No. 4.

West, D.and m. Orr. 2007. Race, Gender, and Communications in natural Disasters. The Policy Studies Journal, Vol. 35, No. 4

D-76 
Raid, J. et al. 2001. The Psychology of Evacuation and the Design of Policy. In Handbook of Crisis and Emergency Management, edited by A. Farazmand.

Fernandez, Lauren et al. 2002. Frail elderly as disaster Victims: Emergency Management Strategies. Prehospital and Disaster Medicine, Vol. 17 No. 2

Lawton, M.P. and E.M. Brody. 1969. Assessment of Older People: Self-maintaining and Instrumental Activities of Daily Living. Gerontologist, Vol . 9.

Hamerman, D. Toward an Understanding of Frailty. The Annals of Internal Medicine, Vol. 130, No. 11

Furner, S.E. et al. 1995. Medical conditions differentially Affect the Development of IADL Disability: Implications for Medical Care and Research. Gerontologist, Vol . 35, No. 4.

Gobbens, R.J. et al. 2010. Toward a Conceptual Definition of Frail Community Dwelling Older People. Nursing Outlook, Vol . 58, No. 2.

Nourhashemi, F. et al. 2001. Instrumental Activities of Daily Living as a Potential marker of Frailty. Journal of Gerontology, Vol. 56, No. 7.

Eisenman, D.P. 2007. Disaster Planning and Risk Communication with Vulnerable Communities: Lessons from Hurricane Katrina. American Journal of Public Health Vol. 97, No. 1.

Raid, J.K., et al. 1999. Predicting Evacuation in Major disasters: Risk Perception, Social Influence, and Access to Resources. Journal of Applied Social Psychology, Vol. 29, No. 5.

Neuman, B. and J. Fawcett. 2010. The Neuman Systems Model, $5^{\text {th }}$ edition. Prentice Hall.

Stergachis, A. et al. 2011. Health Care Workers' ability and Willingness to Report to Work During Public Health Emergencies. Disaster Medicine and Public Health Preparedness, Vol. 5, No. 4.

Cutter, S., B.J. Boruff, and W.L. Shirley. 2003. Social Vulnerability to Environmental Hazards. Social Science Quarterly, Vol. 84, No. 2.

Dow, K. and S. Cutter. 1998. Crying wolf: Repeat Response to Hurricane Evacuation Orders. Coastal Management, Vol. 26.

Baker, E.J. 1995. Public Response to Hurricane Probability Forecasts. Professional Geographer. Vol. 47, No. 2. 
Driscoll, P. and M.B. Salwen. 1996. Riding Out the Storm: Public Evaluations of News Coverage of Hurricane Andrew. International Journal of Mass Emergencies and Disasters. Vol. 14, No. 3.

U.S. Department of Transportation, Federal Highway Administration, Primer Series on Routes to Effective Evacuation Planning, Evacuating Populations with Special Needs

Kailes, J.I., and A. Enders. 2007. Moving Beyond "Special Needs": A Function-Based Framework for Emergency management and Planning. Journal of Disability Policy Studies, Vol. 17, No. 4.

Reis, J.P. et al. 2004. It takes more than ramps to solve the crises of healthcare for people with disabilities. Report issued by the Rehabilitation Institute of Chicago.

Pelling, M. 2003. The Vulnerability of Cities: Natural Disaster and Social Resilience. London Earthscan Publications

Norgaard, R.B. 1994. Development Betrayed: The end of progress and a Coevolutionary Revisioning of the Future. London: Routledge.

Brenanm V.M. 2009. Hurricanes Katrin, Rita, and Wilma and the Medically Underserved. In V.M. Brennan, Natural Disasters and Public Health: Hurricanes Katrina, Rita, and Wilma. Baltimore: The John Hopkins University Press.

Peacock, W.G. and C. Girard. 1997. Ethnic and Racial Inequalities in Hurricane Damage and Insurance Settlements. In Hurricane Andrew: Ethnicity, Gender and the Sociology of Disaster, W.G. Peacock, B.H. Maoorw, and H. Gladwin, eds. London: Routledge

Walker, B and R. Warren. 2009. Katrina Perspectives on Environment and Public Health. In V.M. Brennan, Natural Disasters and Public Health: Hurricanes Katrina, Rita, and Wilma. Baltimore: The John Hopkins University Press.

Mosca, N.G. et al. 2009. Dental Care as a Vital Service Response for Disaster Victims. In V.M. Brennan, Natural Disasters and Public Health: Hurricanes Katrina, Rita, and Wilma. Baltimore: The John Hopkins University Press.

Gray, B.H. and K. Hebert. 2009. Hospitals in Hurricane Katrina: Challenges Facing Custodial Institutions in Disaster. In V.M. Brennan, Natural Disasters and Public Health: Hurricanes Katrina, Rita, and Wilma. Baltimore: The John Hopkins University Press.

Curtis, A. et al. 2009. Katrina and Vulnerability: The Geography of Stress. In V.M. Brennan, Natural Disasters and Public Health: Hurricanes Katrina, Rita, and Wilma. Baltimore: The John Hopkins University Press. 
Krol, D.M., et al. 2009. A Mobile Medical Care Approach Targeting Underserved Populations in PostHurricane Katrina Misssissippi. In V.M. Brennan, Natural Disasters and Public Health: Hurricanes Katrina, Rita, and Wilma. Baltimore: The John Hopkins University Press.

Harvey, R. et al. 2009. The Hurricane Choir: remote Mental Health Monitoring of Participant in a Community-based Intervention in the Post-Katrina Period. In V.M. Brennan, Natural Disasters and Public Health: Hurricanes Katrina, Rita, and Wilma. Baltimore: The John Hopkins University Press.

Helsloot, I. and A. Ruitenberg. 2004. Citizen Response to Disasters: a Survey of Literature and Some Practical Implications Journal of Contingencies \& Crisis Management, Vol. 12 No. 3

Rath, B. et al. 2009. Adverse Health Outcomes after Hurricane Katrina among Children and Adolescents with Chronic Conditions. In V.M. Brennan, Natural Disasters and Public Health: Hurricanes Katrina, Rita, and Wilma. Baltimore: The John Hopkins University Press.

Seidenberg, J. 2009. Cultural Competency in Disaster Recovery: The Lessons of Hurricane Katrina for Serving Marginalized Communities. In V.M. Brennan, Natural Disasters and Public Health: Hurricanes Katrina, Rita, and Wilma. Baltimore: The John Hopkins University Press.

Powers, M.P. 2006. A Matter of Choice: Historical lessons for Disaster Recovery. In: There is No Such Thing as a Natural Disaster: Race, Class, and Hurricane Katrina., eds. C. Hartman and G.D. Squires. Taylor and Francis Publishing

Jones-Deweever, A.A. and H. Hartman. 2006. Abandoned Before the Storms: The Glaring Disaster of Gender, Race, and Class disparities in the Gulf. In: There is No Such Thing as a Natural Disaster: Race, Class, and Hurricane Katrina., eds. C. Hartman and G.D. Squires. Taylor and Francis Publishing

Gullette, M.M. 2006. Katrina and the Politics of Late Life. In: There is No Such Thing as a Natural Disaster: Race, Class, and Hurricane Katrina., eds. C. Hartman and G.D. Squires. Taylor and Francis Publishing

Franklin, E. 2006. A New Kind of Medical Disaster in the United States. In: There is No Such Thing as a Natural Disaster: Race, Class, and Hurricane Katrina., eds. C. Hartman and G.D. Squires. Taylor and Francis Publishing

Tobin, G.A. and B.E. Montz. 1997. Natural Hazards: Explanation and Integration. New York: The Guilford Press.

Brinkmann, J. 2006. Measuring Vulnerability to Promote Disaster-resilient Societies: Conceptual Frameworks and Definitions. In: Measuring Vulnerability to Natural Hazards: Towards Disaster Resilient Societies, Jorn Birkmann, editor. New York: United Nations University Press 
Mechler, R., S. Hochrainer, J. Linnerooth-Bayer, and G. Pflug. 2006. Public Sector Financial Vulnerability to Disasters: The IIASA CARSIM Model. In: Measuring Vulnerability to Natural Hazards: Towards Disaster Resilient Societies, Jorn Birkmann, editor. New York: United Nations University Press

Gladwin, H. and W.G. Peacock. 1997. Warning and Evacuation: A Night for Hard Houses. In Hurricane Andrew: Ethnicity, Gender and the Sociology of Disaster, W.G. Peacock, B.H. Maoorw, and H. Gladwin, eds. London: Routledge

Cutter, Susan L. American Hazardscapes: The Regionalization of Hazards and Disasters. Washington, D.C.: Joseph Henry, 2001.

Bonvicini, S., S. Ganapini, G. Spadoni, and V. Cozzani. 2012. The Description of Population Vulnerability in Quantitative Risk Analysis. Journal of Risk Analysis. DOI: 10.1111/j.1539-6924.2011.01766.

Blakie, Pliers M. et al. 1994. At Risk: Natural Hazards, People's Vulnerability,...

Benson, C. 2004. "Macro-economic Concepts of Vulnerability: Dynamics, Complexity and Public Policy" in Mapping Vulnerability: Disasters, Development and People, G. Bankoff, G. Frerks, and D. Hilhorst, Eds. Earth Science Press.

Fordham, Maureen. 2004. "Gendering Vulnerability Analysis: Towards a More Nuanced Approach" in Mapping Vulnerability: Disasters, Development and People, G. Bankoff, G. Frerks, and D. Hilhorst, Eds. Earth Science Press.

Alesch, D.J. 2009. Managing for Long-term Community Recovery in the Aftermath of Disaster. Published by The Public Entity Risk Institute.

Ronan, K.R. and D.M. Johnston. 2005. Promoting Community Resilience in Disasters: The Role for Schools, Youth, and Families. New York, Springer.

Norris, F.H, M.J. Friedman, P.J. Watson, C.M. Byrne, E. Diaz, C. North, and K. Kaniasty. 2002. 60,000 Disaster Victims Speak: Part I. An Empirical Review of the Empirical Literature, 1981-2001. Psychiatry, 65

Pfefferbaum, B. and D.E.E. Doughty. 2001. Increased Alcohol Use in a Treatment Sample of Oklahoma City Bombing Victims. Psychiatry: Interpersonal and Biological Processes. Vol. 64, No. 4.

Perrine, M.W., K.E.E. Schroder, R. Forester, P. McGonagle-Moulton, and F. Huessy. 2004. The Impact of the September 11, 2001, Terrorist Attacks on Alcohol Consumption and Distress: Reactions to a National Trauma 300 Miles from Ground Zero Journal of Studies on Alcohol and Drugs. Vol. 65., No. 1.

Stewart, S.H., T.L. Mitchell, K.D. Wright, and P. Loba. 2004. The relations of PTSD symptoms to alcohol use and coping drinking in volunteers who responded to the Swissair Flight 111 airline disaster. Journal of Anxiety Disorders. Vol. 18, No. 1. 
Beggs, J., V. Haines, and J. Hurlbert. 1996. The Effects of Personal Network and Local Community Contexts on the Receipt of Formal Aid during Disaster Recovery. International Journal of Mass Emergencies and Disasters. Vol. 14, No. 1.

Cook, J.D., L. Bickman. 1990. Social Support and Psychological Symptomology Following a Natural Disaster. Journal of Traumatic Stress. Vol. 3, No. 4

McClure, J., M.W. Allen, and F. Walkey. 2001. Countering Fatalism: Causal Information in News Reports Affects Judgments About Earthquake Damage._Basic \& Applied Social Psychology. Vol. 23, No. 2.

Charles E. Faupel, C.E. and S.P. Styles. 1993. Disaster Education, Household Preparedness, and Stress Responses Following Hurricane Hugo. Environment and Behavior March Vol. 25

Patton, D. 2003. Disaster preparedness: A social-cognitive perspective. Disaster Prevention and Management. Vol. 12, No. 3

Abramovitz, J.N. 2001. Unnatural Disasters. World Watch paper \#158, World Watch Institute, Danvers, MA.

Jasanoff, S. 2010. Beyond Calculation: A Democratic Response to Risk. In Disasters and the Politics of Intervention. Ed. A. Lackoff. The Columbia University Press.

Tiedemann, H. 1992. Earthquakes and Volcanic Eruptions: A Handbook on Risk Assessment. Geneva: Swiss Re.

Roberts, P. 2010. Private Choices, Public Harm: The Evolution of National Disaster Organizations in the United States. In Disasters and the Politics of Intervention. Ed. A. Lackoff. The Columbia University Press.

Gregory, R. and R. Mendelsohn. 1993. Perceived Risk, Dread, and Benefits. Risk Analysis. Vol 13, No.3 259-264.

Slovic, P. \& Weber, E.U. (2002). Perception of Risk Posed by Extreme Events. The Conference on Risk Management Strategies in an Uncertain World Held In April 12-13, 2002, Palisades, NY, 1-21.

Keeney, R.I., and D. von Winterfeldt. 1986. Improving Risk Communication. Risk Analysis, Vol. 6, No 4.

Palm, R.I. 1990. Natural Hazards: An Integrative Framework for Research and Planning. John Hopkins University Press.

Steinberg, T. 2000. Acts of God: The Unnatural History of Natural Disaster in America. Oxford University Press.

Alexander, D. 2000. Confronting Catastrophe: New Perspectives on Natural Disasters. Oxford university Press.

Platt, R.H, D. Scherf, and K.B. O'Donnell. 1999. Fire Island: The Politics of Coastal Erosion. In Disaster and Democracy: The Politics of Extreme Natural Events, Ed. R.H. Platt. Washington, D.C., Island Press. 
Bates, F.L. and C. Pelanda. 1994. An Ecological Approach to Disasters. In Disasters, Collective Behavior, and Social Organization, Eds. R.R. Dynes and K.J. Tierney. University of Delaware Press.

Fitzpatric, C. and D. Mileti. 1994. Public Risk Communication. In Disasters, Collective Behavior, and Social Organization, Eds. R.R. Dynes and K.J. Tierney. University of Delaware Press.

Thywissen, K. 2006. Core Terminology in Disaster Reduction. In: Measuring Vulnerability to Natural Hazards: Towards Disaster Resilient Societies, Jorn Birkmann, editor. New York: United Nations University Press.

Blaikie, P., T. Cannon, I. Davis, and B. Wisner. 1994. At Risk: Natural Hazards, People's Vulnerability, and Disasters. London: Routledge Press.

Mileti, D.S. 1999. Disasters by Design: A Reassessment of Natural Hazards in the United States. Washington, D.C: John Henry Press.

Whelan, R.K. and D. Strong. 2009. Rebuilding Lives Post Katrina: Choices and Challenges in New Orleans's Economic Development. In Race, Place, and Environmental Justice After Hurricane Katrina: Struggles to Reclaim, Rebuild, and Revitalize New Orleans and the Gulf Coast. Eds. R.D. Bullard and B. Wright. Westview Press.

Perry, R.W. and M.K. Lindell. 1991. The Effects of Ethnicity on Evacuation Decision-making. International Journal of Mass Emergencies and Disasters. Vol. 9, No. 1.

Dwyer, A., C. Zoppou, O, Neielson, S. Day, and S. Roberts. 2004. Quantifying Social Vulnerability: A Methodology for Identifying those at Risk to Natural Disasters. Geoscience Australia, Canberra.

Greenfield, K. 2011. The Myth of Choice: Personal Responsibility in a World of Limits. Yale New Haven: University Press.

Bentley, A., M. Earls, and M.J. O'Brien. 2007. I'Il Have What She's Having: Mapping Social Behavior. Cambridge: The MIT Press.

Wilkinson, I. 2010. Risk, Vulnerability and Everyday Life. London: Routledge.

Pidgeon, N., P. Simmons, and K. Henwood. 2006. Risk, Environment, and Technology. In Risk in Social Science. Eds. P. Taylor-Goodby and J. Zinn. New York: Oxford University Press.

Tibbetts, J.H. 2012. No Worries? The New Science of Risk and Choice. Coastal Heritage. Vol. 26, No. 4.

Zinn, J.O., and P. Taylor-Gooby. 2006. The Challenge of (Managing) New Risks. In Risk in Social Science. Eds. P. Taylor-Goodby and J. Zinn. New York: Oxford University Press. 
West, C.T. and D.G. Lenze. 1994. Modeling the Regional Impact of Natural Disaster and Recovery: A General Framework and an Application to Hurricane Andrew. International Regional Science Review. Vol. 17, No. 2.

Wemple, C. 2010. Challenges of Economic Recovery Following Natural Disasters: Insights Gleaned from Hurricane Ike. PERI Symposium: Community Recovery from Disaster.

Wilkinson, I. 2001. Anxiety in a Risk Society. London: Routledge.

Sunstein, C.R. 2007. Worst-case Scenarios. Cambridge: Harvard University Press.

Whitehead, J.C., B. Edwards, M. Van Willigen, .R. Maiolo, K. Wilson, and K.T. Smith. 2000. Heading for Higher Ground: Factors Affecting Real and Hypothetical Hurricane Evacuation Behavior. Environmental Hazards. Vol. 2.

Kesling, G. 2010. Hurricane Fatigue Syndrome. Annals of the American Psychtherapy s...STOP HERE

Kahneman, D. 2011. Thinking, Fast and Slow. New York: Farrar, Straus and Giroux.

Riad, J.K., F.H. Norris, and R.B. Ruback. 1999. Predicting Evacuation in Two Major Disasters: Risk Perception, Social Influence, and Access to Resources. Journal of Applied Social Psychology, 1999, Vol. 29. No. 5.

Taylor, R. 1989. Human territorial functioning: An empirical, evolutionary perspective on individual and small group territorial cognitions, behaviors, and consequences. New York: Cambridge University Press.

Some citizens are motivated to shelter in place for fear of looting, which has been characterized as territorial functioning (see Taylor 1989).

Baker, E.J. 1991. Evacuation Behavior in Hurricanes. International Journal of Mass Emergencies and Disasters. Vol. 9. No. 2.

Kleinpeter, Myra A. 2007. Shifts in Dialysis Patients from Natural Disasters in 2005. International Journal of Hemodialysis. Vol. 11

Fothergill, Alice and Lori A Peek. 2004. Poverty and Disasters in the United States: A Review of Recent Sociological Findings. Natural Hazards. Vol. 32.

Enarson, E. and B.H. Marrow (eds,). 1998. The Gendered Terrain of Disaster: Through Women's Eyes. Preager, Westport, CT.

Peacock, W.G., B.H. Morrow, H. Gladwin. 1997. Hurricane Andrew: Ethnicity, Gender, and the Sociology of Disasters. Routledge, London. 
Bergfren, R.E., and T.J. Curiel. 2006. After the Storm - Healthcare Infrastructure in Post-Katrina New Olereans. New England Journal of Medicine. Vol 354

Baker, E.J. 1979. Predicting Response to Hurricane Warnings: A Reanalysis of Data from Four Studies. Mass Emergencies. Vol. 4.

In 1985 Baker conducted a hurricane Gloria survey of residents from Virginia to Massachusetts, focusing on residents of four cities within Virginia (Newport News, Eastern Shore, Norfolk, Virginia Beach).

Baker, E.J. 1988. Hurricane Evacuation Behavior in the Middle Atlantic and Northeast States.

Tallahassee, FL: Hazards Management Group, Inc.

Childers, C.D. 1999. Elderly Female-headed Households in the Disaster Loan Process. International Journal of Mass Emergencies and Disasters. Vol. 17. No. 1.

Rendall, Michael S. 2011. Breakup of New Orleans Households After Hurricane Katrina. Journal of Marriage and Family. Vol. 73.

Kesling, Gary. 2010. Hurricane Fatigue Syndrome. Annals of the American Psychotheraphy Association. Spring.

Aldrich, Nancy, and William F. Benson. 2008. Disaster Preparedness and the Chronic Disease Needs of Vulnerable Older Adults. Centers for Disease Control and Prevention, Preventing Chronic Disease: Public Health Research, Practice, and Policy. Vol. 5, No. 1. 


\section{Joshua G. Behr}

Joshua G. Behr PhD is Research Associate Professor at the Virginia Modeling, Analysis and Simulation Center (VMASC) at Old Dominion University. Dr. Behr received his training at the University of New Orleans specializing in urban and minority politics. He is the governor's appointee to the Science and Technology Advisory Committee for the Chesapeake Bay Program. He has conducted studies and published insights related to evacuation behavior, recurrent flooding, community resilience, and the disposition of medically fragile and vulnerable populations in the post-event recovery process. 


\title{
Carolyn Kousky
}

Modeling of Recovery: The Role of Disaster Financing Systems

\begin{abstract}
The nature of disaster financing plays a critical role in recovery of households and communities. For many smaller disasters, no federal aid will be provided to those impacted. For larger events that merit a presidential disaster declaration, federal disaster aid will be offered. For households, however, this assistance is limited in amount, what it can be used for, and it can take months or even years before it gets into the pockets of households. Disaster insurance, on the other hand, can provide the necessary funds for rebuilding and replacing damaged property and previous work has shown that those with insurance are more likely to rebuild than households without insurance. Despite this, take-up rates for disaster insurance in the U.S. remain low. Disaster declarations may also authorize funds for investments in hazard mitigation during the rebuilding process. Households cannot rely on receiving such funds as there is substantial local government discretion. And even when large sums have been appropriated to improve post-disaster reconstruction, implementation has been plagued with delays, mismanagement, and other criticisms. Individual risk perceptions and preferences will also play a role in the rebuilding decision. The variation in disaster financing at a household level can lead to substantial heterogeneity in recovery. The distributional impacts of disasters may be masked in aggregate measures of economic activity.
\end{abstract}

\section{References}

**Note: a more complete list of references will be provided with my slides** Kousky, C. and L. Shabman (2012). The Realities of Federal Disaster Aid: The Case of Floods. Washington, DC, Resources for the Future.

Kousky, C. (2014). "Informing climate adaptation: A review of the economic costs of natural disasters." Energy Economics 46: 576-592.

Turnham, J., K. Burnett, C. Martin, T. McCall, R. Juras and J. Spader (2011). Housing Recovery on the Gulf Coast, Phase II: Results of Property Owner Survey in Louisiana, Mississippi, and Texas. Washington, DC, U.S. Department of Housing and Urban Development, Office of Policy Development and Research, August. 


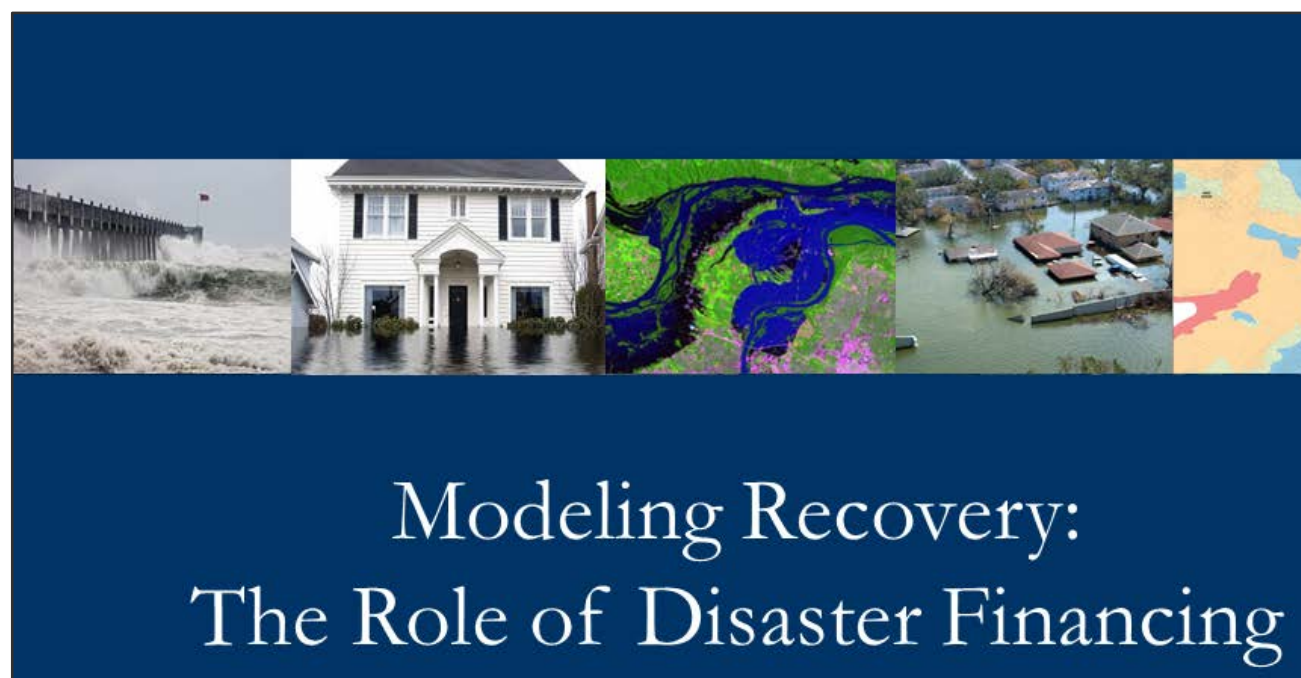

Carolyn Kousky

Resources for the Future

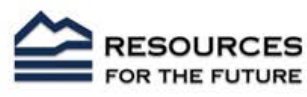

October 19, 2016

kousky@rff.org

\section{Outline}

1. Role of financing systems in recovery

i. Disaster insurance

ii. Federal disaster aid

2. Risk perceptions and preferences in recovery

3. What do aggregate impacts miss? 


\section{Financing Disaster Costs and Risk Reduction}

Ex-ante Financing

- Insurance

- Other forms of securitization

Ex-Post Financing

- Disaster aid

- Access to credit

Hazard Mitigation / Climate Adaptation

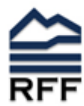

\section{Insurance for resilience}

- Insurance provides larger and timelier payments to households

Leading to:

- Greater likelihood of rebuilding

- Limited negative economic shock to households

- Limited negative multiplier effects in communities 


\section{Disaster insurance take-up rates are low}

- Very few households voluntarily insure against disasters

- Roughly $50 \%$ of those in 100 -year floodplains have flood insurance

- $10-12 \%$ of households in California have earthquake insurance

- Wind coverage is widespread since in homeowners policies, but may have high hurricane deductible

\section{Reasons for low take-up}

- Disaster insurance is expensive: there may not be a price at which disaster insurance can be profitably offered and homeowners are willing/able to pay.

- Heuristics and biases

- Inaccurate or incomplete information

- Bounded rationality; transaction/search costs

- Myopia

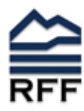




\section{The Disaster Declaration Process}

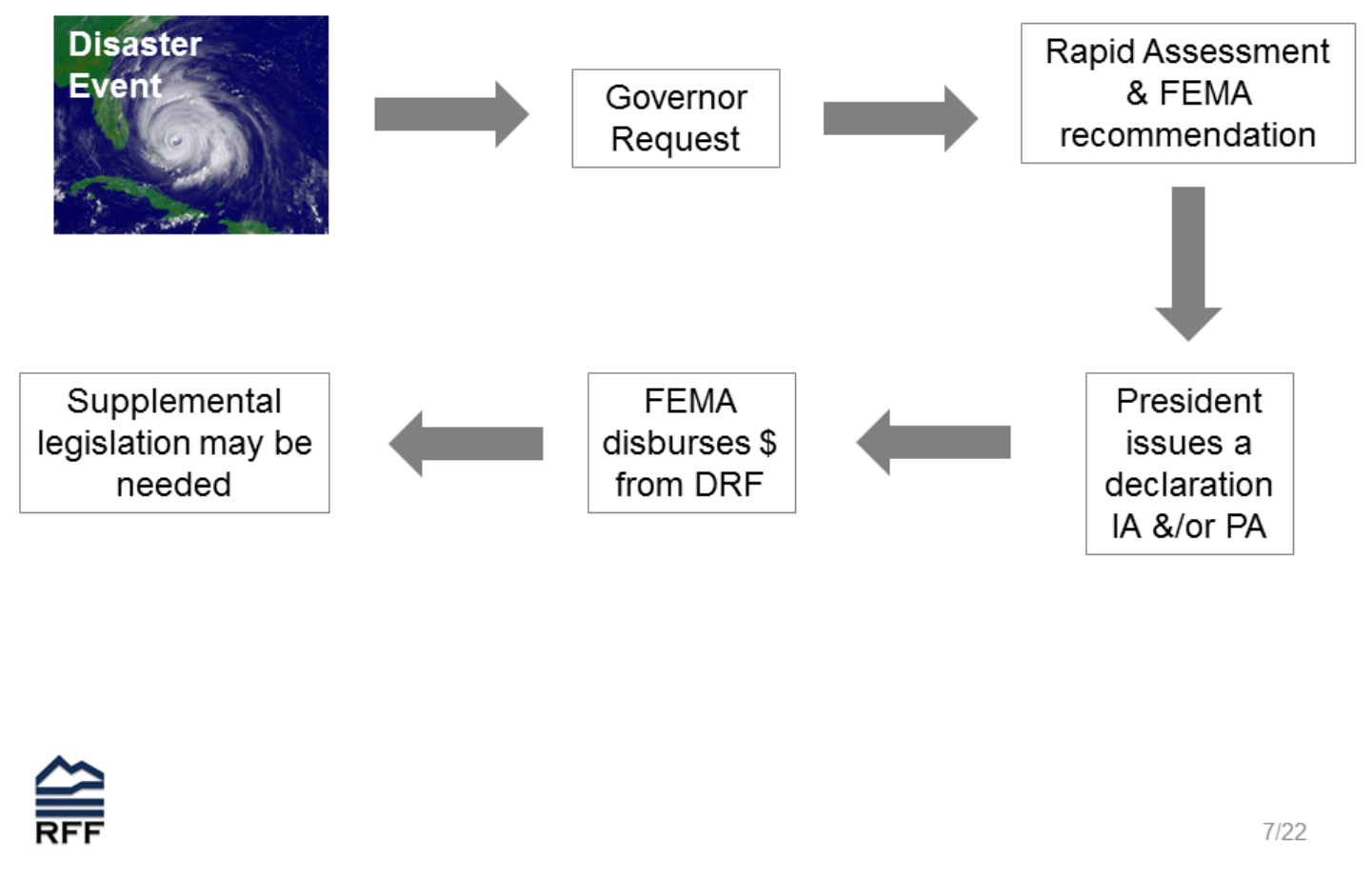

\section{Presidential Disaster Declarations, 1953-2015}

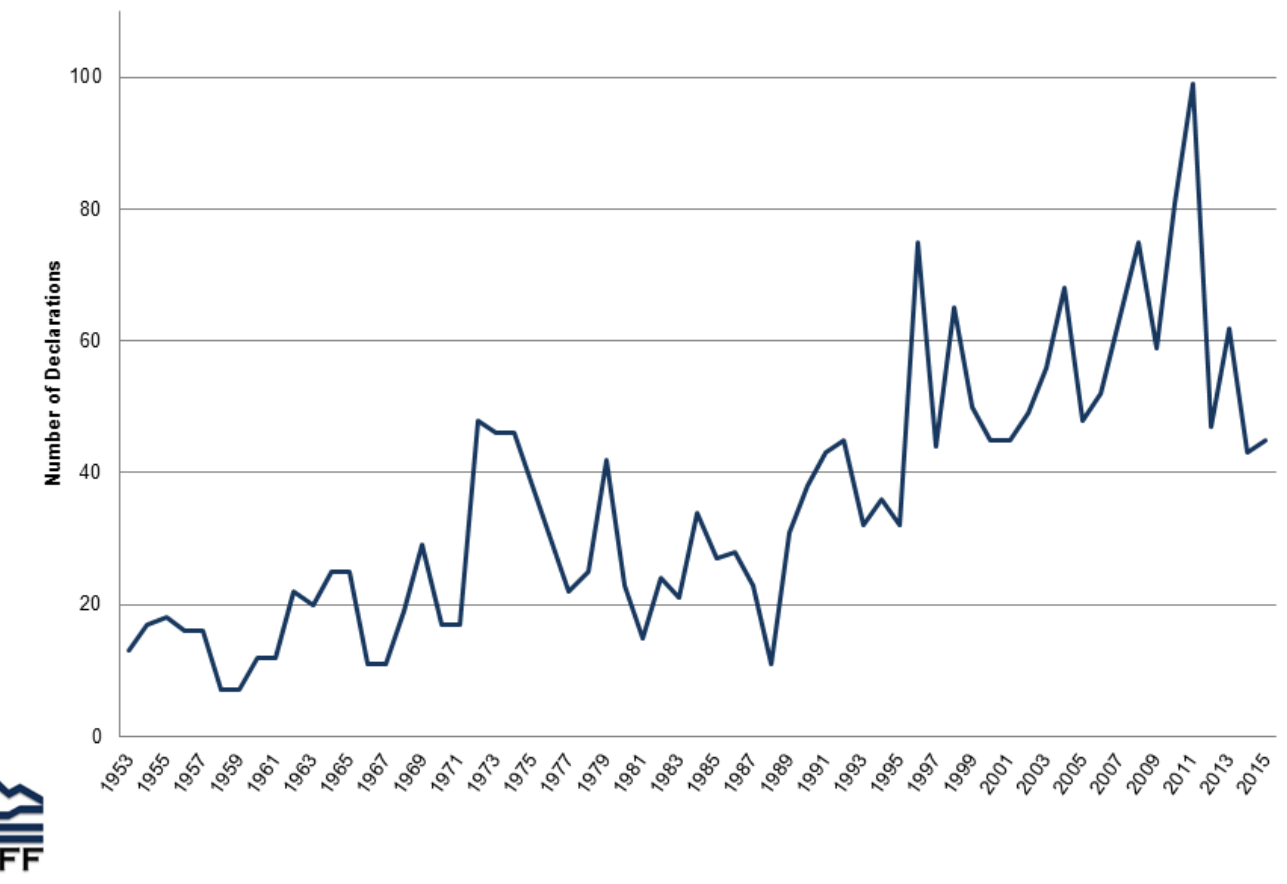




\section{OMB estimates of federal relief spending}
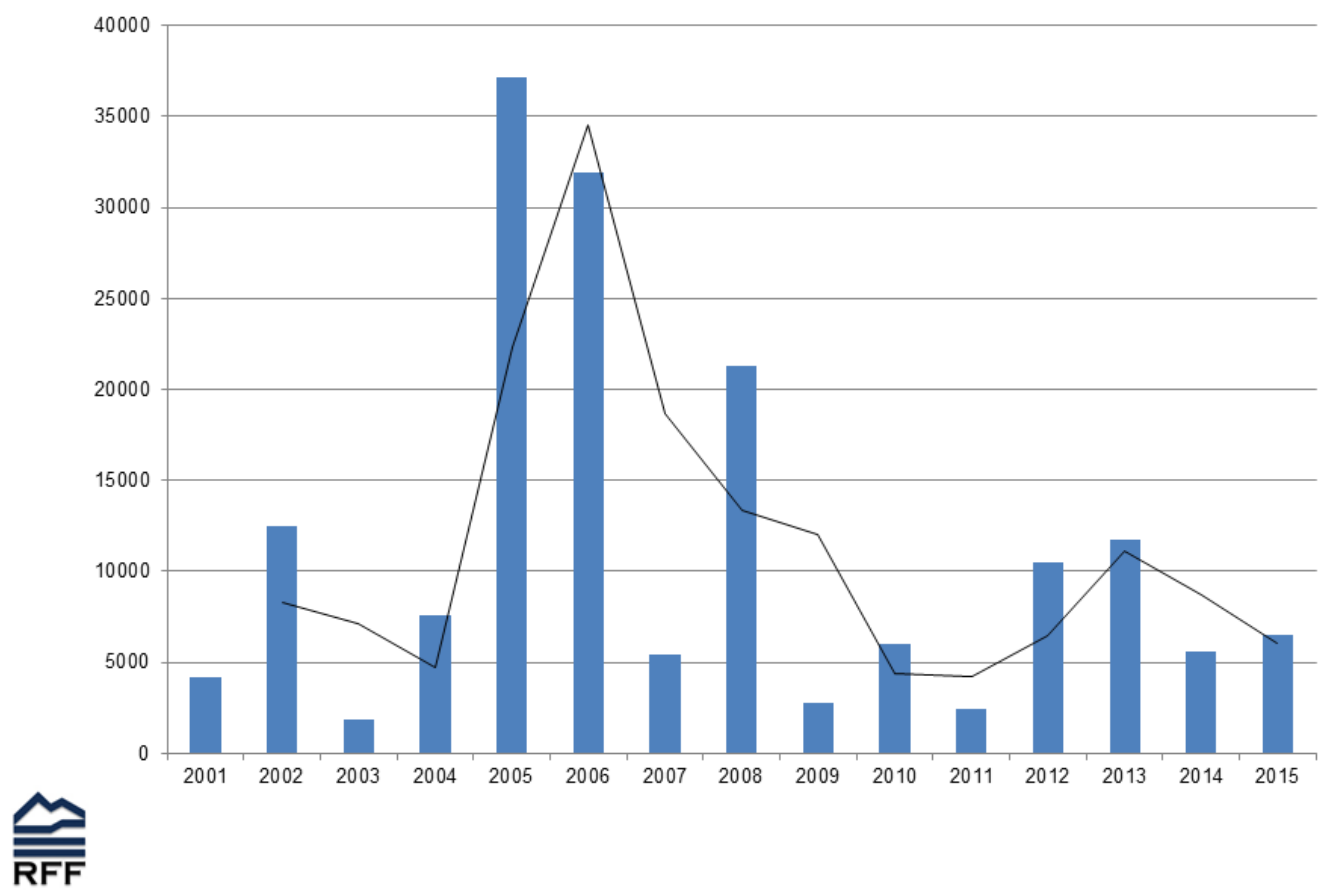

\section{Disaster aid}

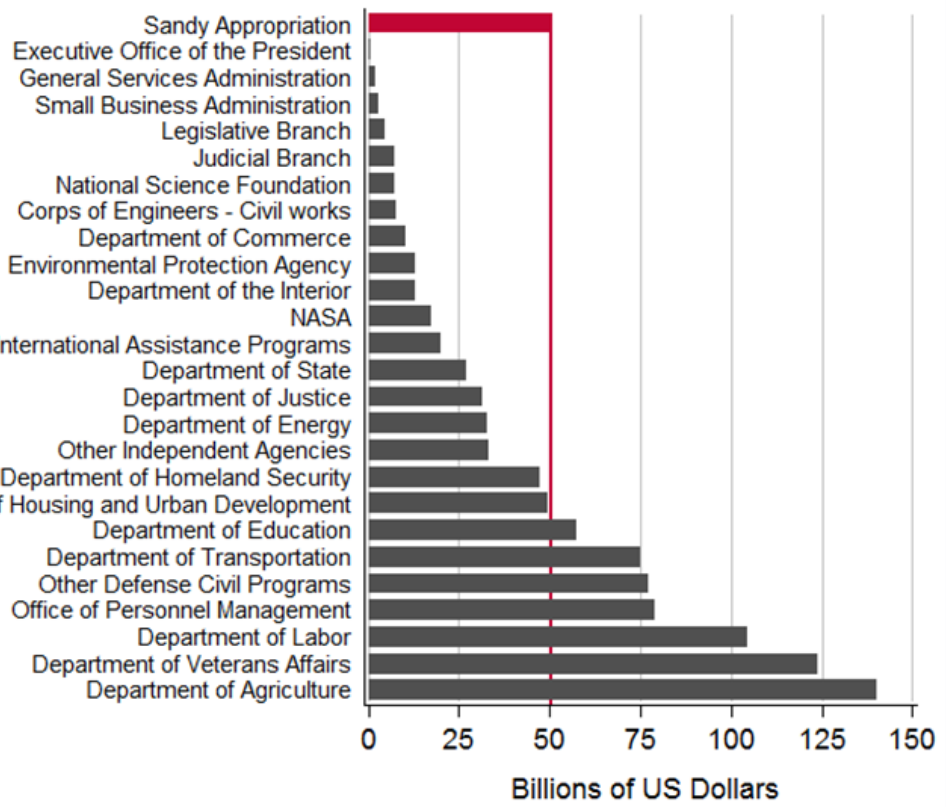




\section{What do households get?}

SBA loans are first line of defense

FEMA Individual Assistance grants

- Many applications are denied

- Amounts capped at $\$ 31,400$ (2012 USD)

- Average grant is only $\$ 3-5 \mathrm{k}$

- Only to make your home safe and habitable again, not bring it back to predisaster condition

\section{Program authorizations}

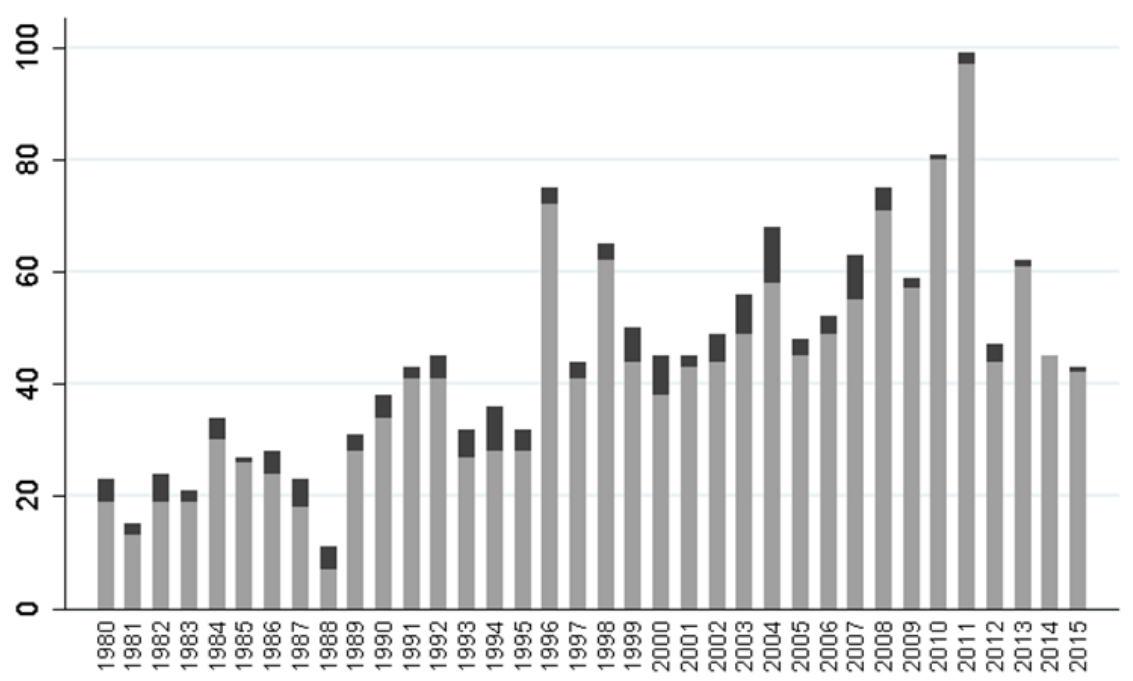

Total declarations with PA 


\section{Sandy appropriations by Department}

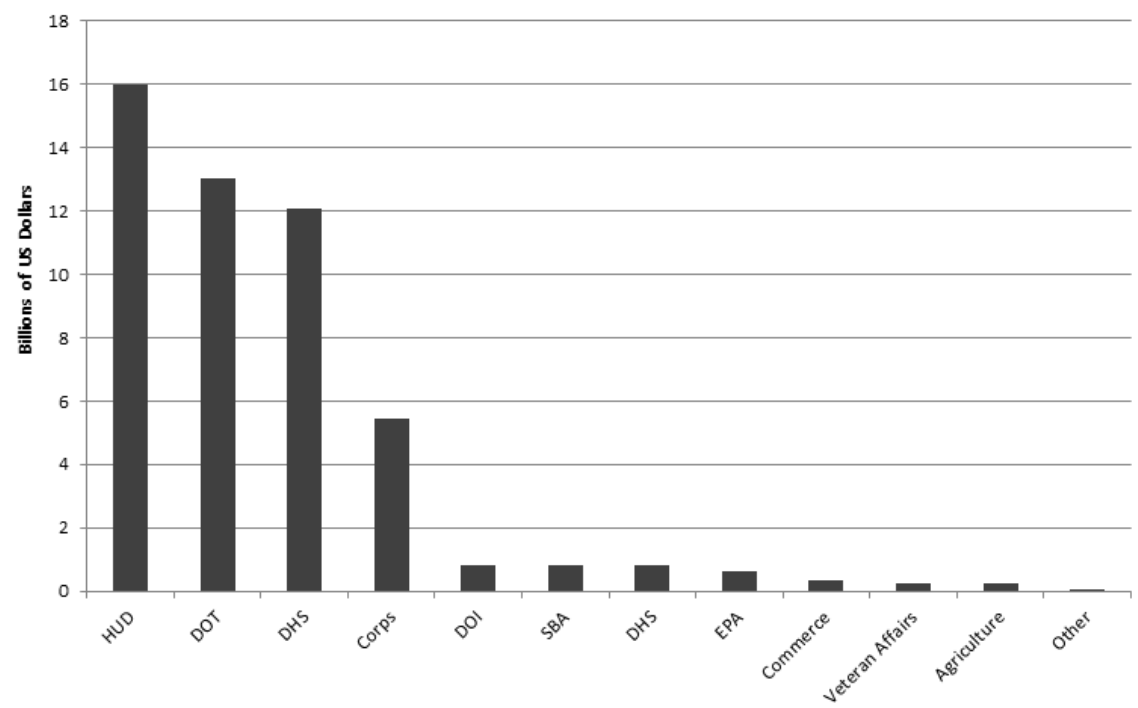

Source: Kousky and Shabman, with data from Public Law 113-2 (2013)

\section{CDBG: A new trend in disaster aid?}

- Local governments have enormous flexibility in how they use the funds

- Hard to track spending

- Capacity issues; long time frames

- CDBG-DR is not just for recovery but also mitigation 


\section{Federal funding for hazard mitigation}

Should we tie mitigation/adaption funding to disasters?

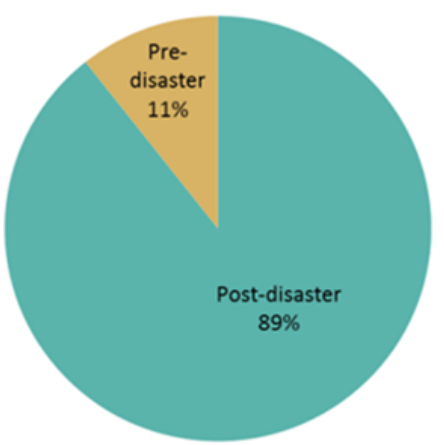

FEMA Spending on Hazard Mitigation;2002-20I4

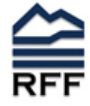

Still...much mitigation is not done

- Upfront costs are large

- Transaction costs high

- No compensation for disruption, loss of use

- Risk perceptions, information again play a role 


\section{Usually piecemeal}
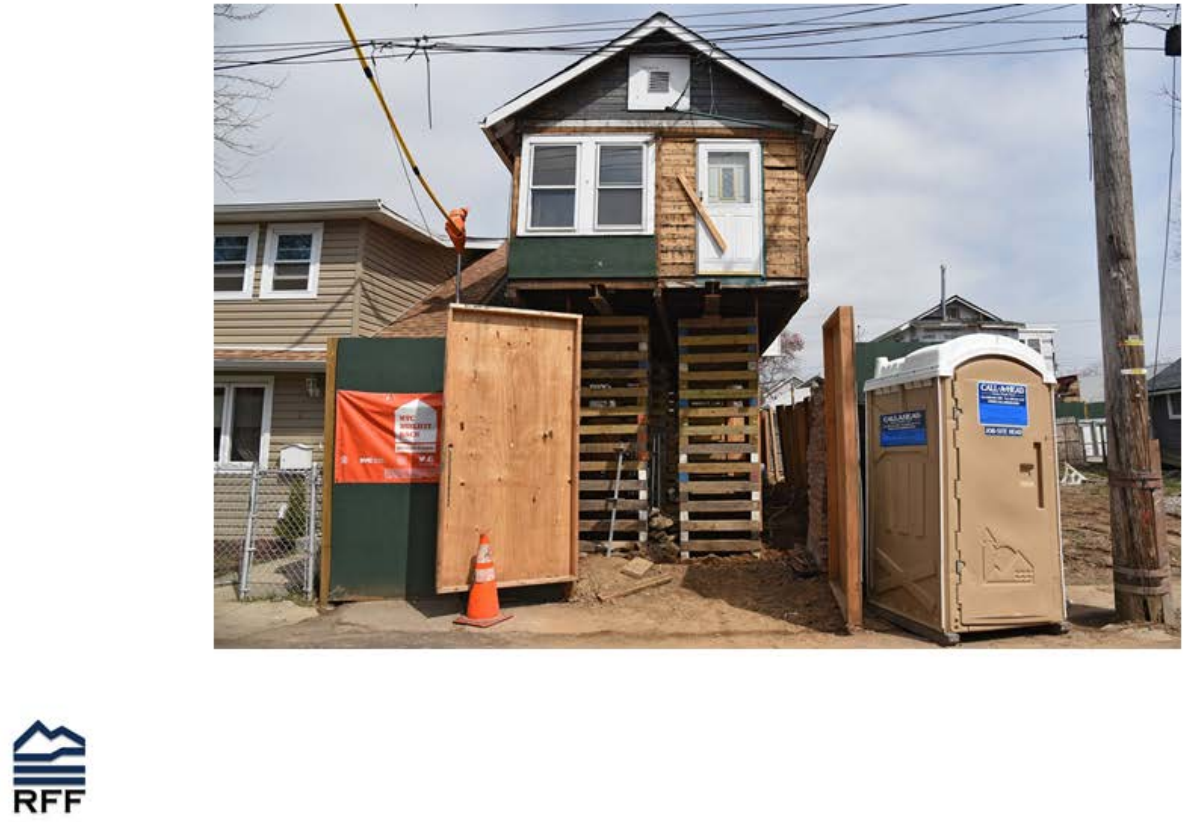

\section{Aggregate measures can be misleading}

- Large heterogeneity in recovery and response

- Some aggregate measures, such as GDP can mask critical distributional impacts of disasters or fail to recognize that GDP does not equate with welfare

- Impacts can be localized

- Distributional consequences can be large, even if aggregate consequences are not 


\section{In sum}

1) Models of recovery need to consider disaster financing since it has implications for reconstruction and recovery

2) Models should pay close attention to what is assumed about homeowner preferences and information since there is empirical evidence these can be drivers of decisions

3) Aggregate models can miss important localized and distributional impacts

\section{䌉}

Thanks!

kousky@rff.org 
Carolyn Kousky is a fellow at Resources for the Future. She has published numerous articles, reports, and book chapters on the economics and policy of natural disasters and disaster insurance markets. She has examined how individuals learn about extreme event risk, the demand for natural disaster insurance, the functioning of the National Flood Insurance Program, and policy responses to potential changes in extreme events with climate change. She is the recipient of the 2013 Tartufari International Prize from the Accademia Nazionale dei Lincei. Kousky was a member of the National Research Council Committee on Analysis of Costs and Benefits of Reforms to the National Flood Insurance Program. She has a BS in Earth Systems from Stanford University and a PhD in Public Policy from Harvard University. 
Title: An integration of Social Vulnerability Data and Mapping Tools for Community Vulnerability Assessments and Recovery Modeling

Researchers: Walt Peacock, Shannon Van Zandt, Nathanael Rosenheim Attendees: Walt Peacock, Nathanael Rosenheim

\section{0-word abstract summarizing our work:}

The last several decades of social science research has established that community resilience is generally characterized as function of hazard exposure (exposure to the forces of disaster agents), physical vulnerability (the vulnerabilities of the built environment) and social vulnerability. Hence, when modeling community resilience our work combines outputs from models that predict structural damage and loss of functionality based on the forces of hazard agents and the physical properties of the built environment, with social vulnerability perspectives. Social vulnerability focuses on the social factors and processes that generate vulnerability in terms of an individual's or group's capacity to anticipate, cope, resist, and recover from the impacts of disasters. This perspective utilizes social characteristics that capture household and individual differences in capacity, access to information, power/influence, and resources. These differentials ultimately have the consequence of disparities in disaster impacts and recovery trajectories. Because population characteristics are not uniformly or randomly distributed within our communities, a critical element of our research depends on spatially locating significant variations that effect disaster impacts and recovery. Our work develops social vulnerability data, maps and mapping tools that can be utilized in community vulnerability assessments, for preplanning of field studies, and most importantly, for the social impact and recovery modeling algorithm development.

\section{Presenter Bio:}

Dr. Nathanael Rosenheim is a Research Scientist for the Hazard Reduction and Recovery Center in the Department of Landscape Architecture and Urban Planning at Texas A\&M University. His areas of interest are spatial modeling, data science, community development, and food system planning. His recent research utilizes public demographic and economic data to improve fact based community planning for hazard mitigation and recovery planning.

\section{Bibliography:}

Highfield, W. E., Peacock, W. G., \& Van Zandt, S. (2014). Mitigation planning why hazard exposure, structural vulnerability, and social vulnerability matter. Journal of Planning Education and Research, 0739456X14531828.

Masterson, J. H., Peacock, W. G., Van Zandt, S. S., Grover, H., Schwarz, L. F., \& Cooper, J. T. (2014). Planning for Community Resilience. Island Press. 
Peacock, W. G., Van Zandt, S., Zhang, Y., \& Highfield, W. E. (2014). Inequities in long-term housing recovery after disasters. Journal of the American Planning Association, 80(4), 356-371.

Van Zandt, S., Peacock, W. G., Henry, D. W., Grover, H., Highfield, W. E., \& Brody, S. D. (2012). Mapping social vulnerability to enhance housing and neighborhood resilience. Housing Policy Debate, 22(1), 29-55. 


\section{An integration of social vulnerability data and} mapping tools for community vulnerability assessments and recovery modeling

National Institute for Standards and Technology Conference

Walter Gillis Peacock, Shannon Van Zandt, Nathanael Rosenheim

International Workshop on Modeling of Physical, Economic, and Social Systems for Resilience Assessment

19-21 October 2016

Washington Dulles Airport Marriott, Reston, VA

\section{Social Science Recovery Modeling Biggest Challenges:}

I. Integrating qualitative and quasi-experimental research with engineering fragility models

2. Model resolutions that show spatial variance in impacts across demographic subgroups

3. Modeling short-, medium- and long term recovery (housing and businesses) when available data is annual or quarterly 4. Modeling variations in infrastructure at neighborhood level to capture investment, disinvestment, and ultimately equity issues 


\section{Fact Basis for Recovery Modeling}

The pre-existing community characteristics can shape and determine the specific impacts of hazard agents:

* hazard exposure (exposure to the forces of disaster agents)

* physical vulnerability (the vulnerabilities of the built environment)

* social vulnerability.

\section{Interacting Components of Disaster Impact Model}

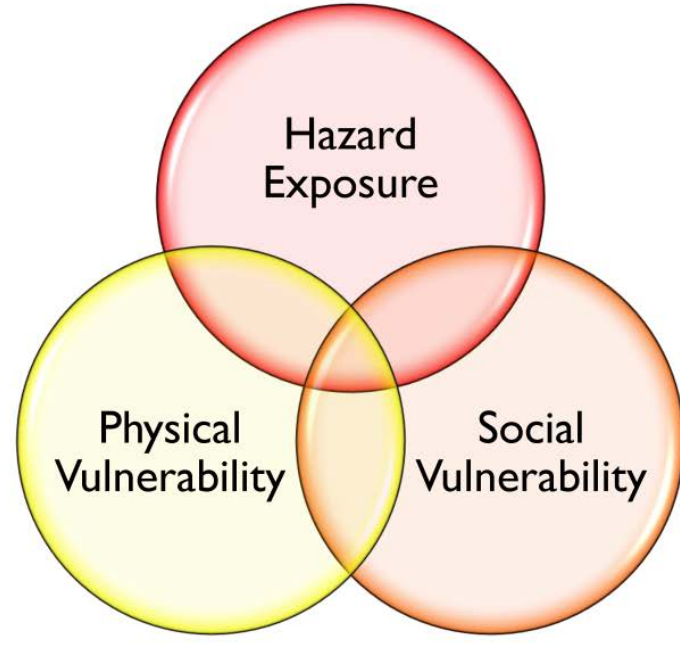

More Vulnerable... Slower Recovery

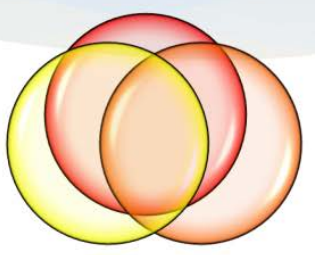

Less Vulnerable... Faster Recovery

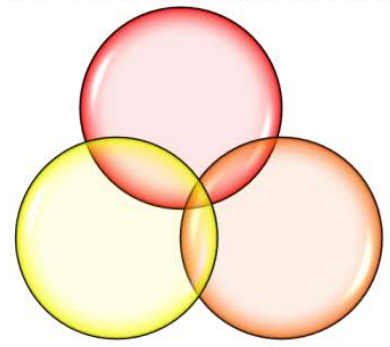

$\widehat{A] \mathbb{M}} \mid$ HAZARD REDUCTION \& RECOVERY CENTER 


\section{Social Science Integration}

* Identify Community Characteristics

* Hazard Exposure

* Physical Vulnerability

* Social Vulnerability

* Identify spatial clusters where vulnerabilities overlap

* Hotspots experience

* Disproportionate losses

* Housing losses

* Greater displacement
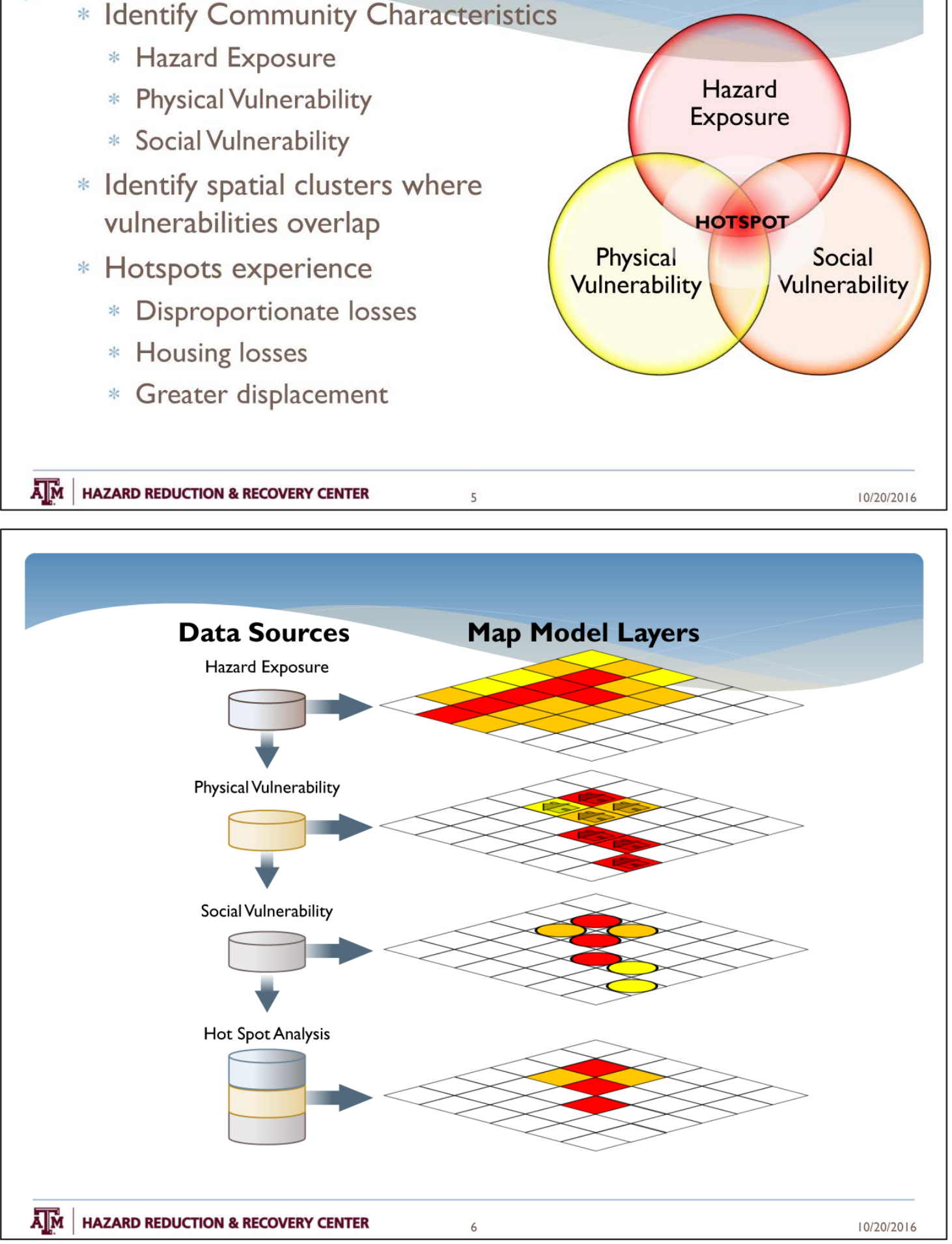


\section{Social Science Research - Consequences}

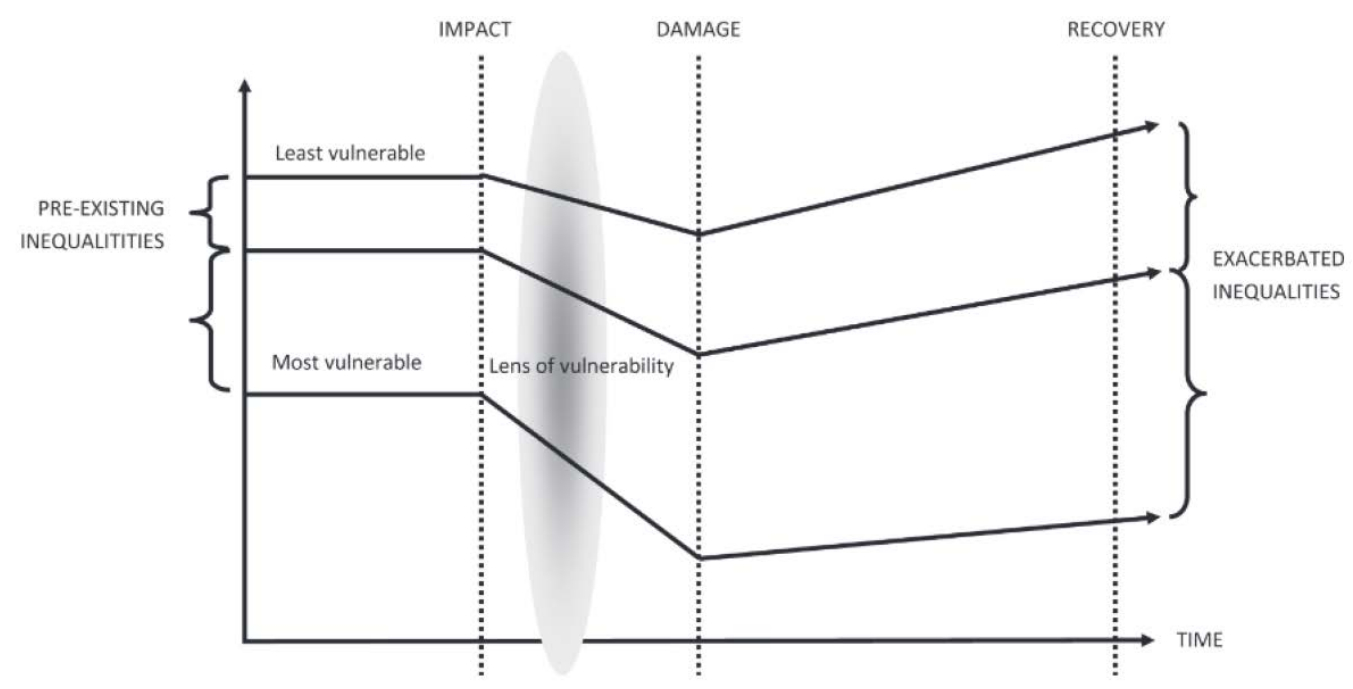

Figure 1. Conceptual model of how housing incqualitics may be exacerbated through the recovery process.

A]

7

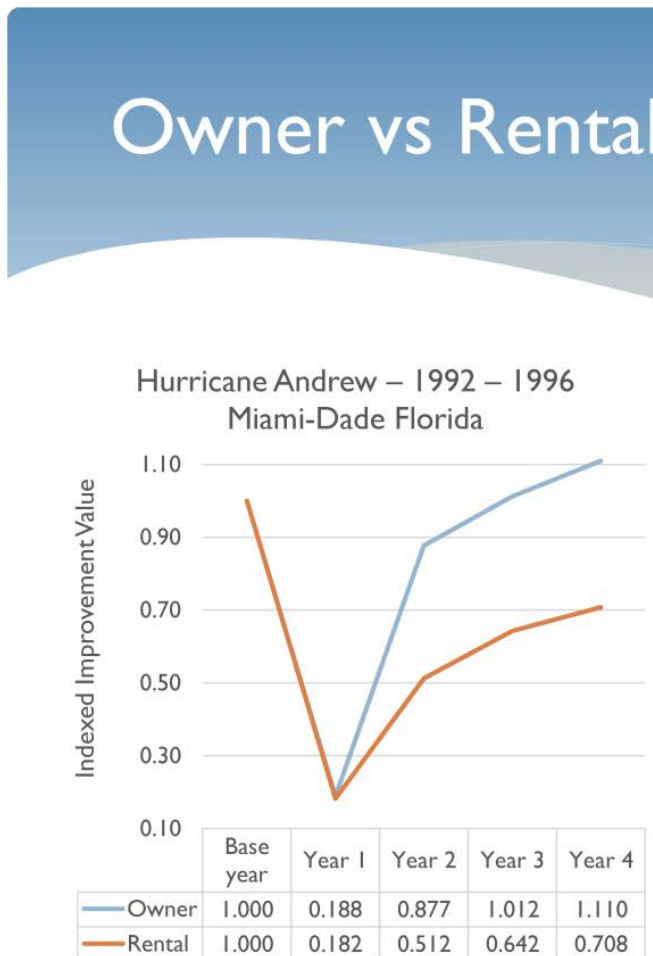

Hurricane lke - 2008- 2012

Galveston, Texas

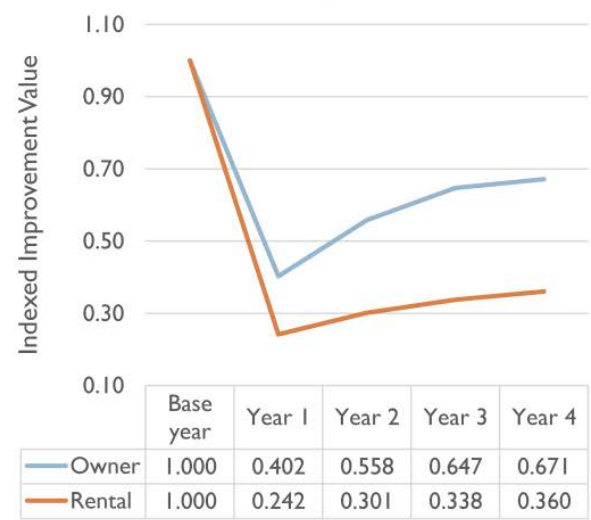

$\widehat{A}[\mathbb{M} \mid$ HAZARD REDUCTION \& RECOVERY CENTER 


\section{Social Science Integration: Example}

* Cameron and Willacy Counties, Texas

* Hazard Exposure

* Hurricane Surge Zones

* Hurricane Evacuation Zones

* Physical Vulnerability

* Location of employee residences
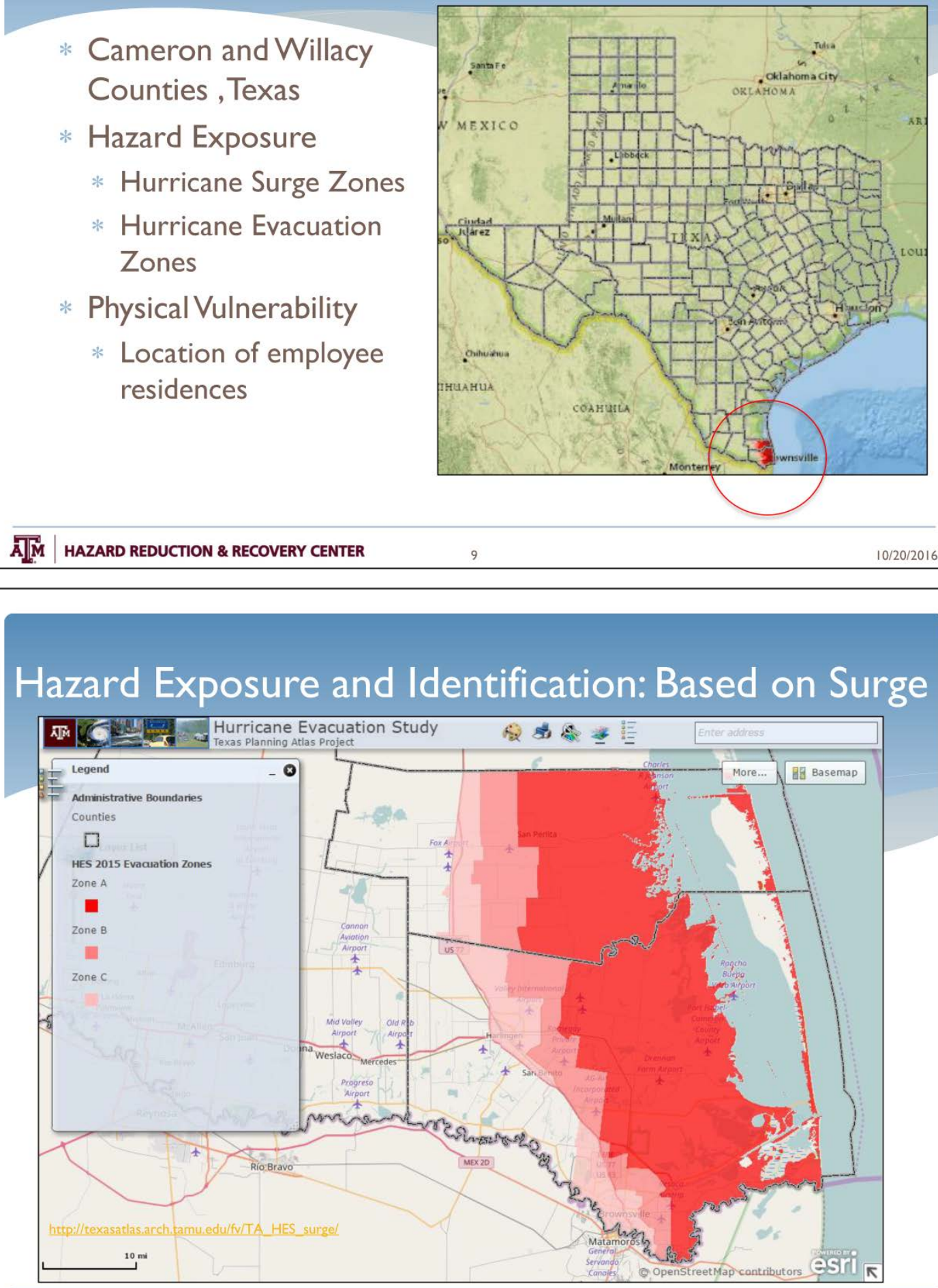

A]

10 
Susceptibility to physical damage and loss based on exposure and:

* Geographic Location

* Social function

* Housing, Economic, Healthcare, Education, etc.

* Critical facilities

* Interdependencies

* Age

* Forms of measurement:

* Vulnerability assessment

* Risk Analysis

\section{Job locations and employee residences} with Evacuation Zones

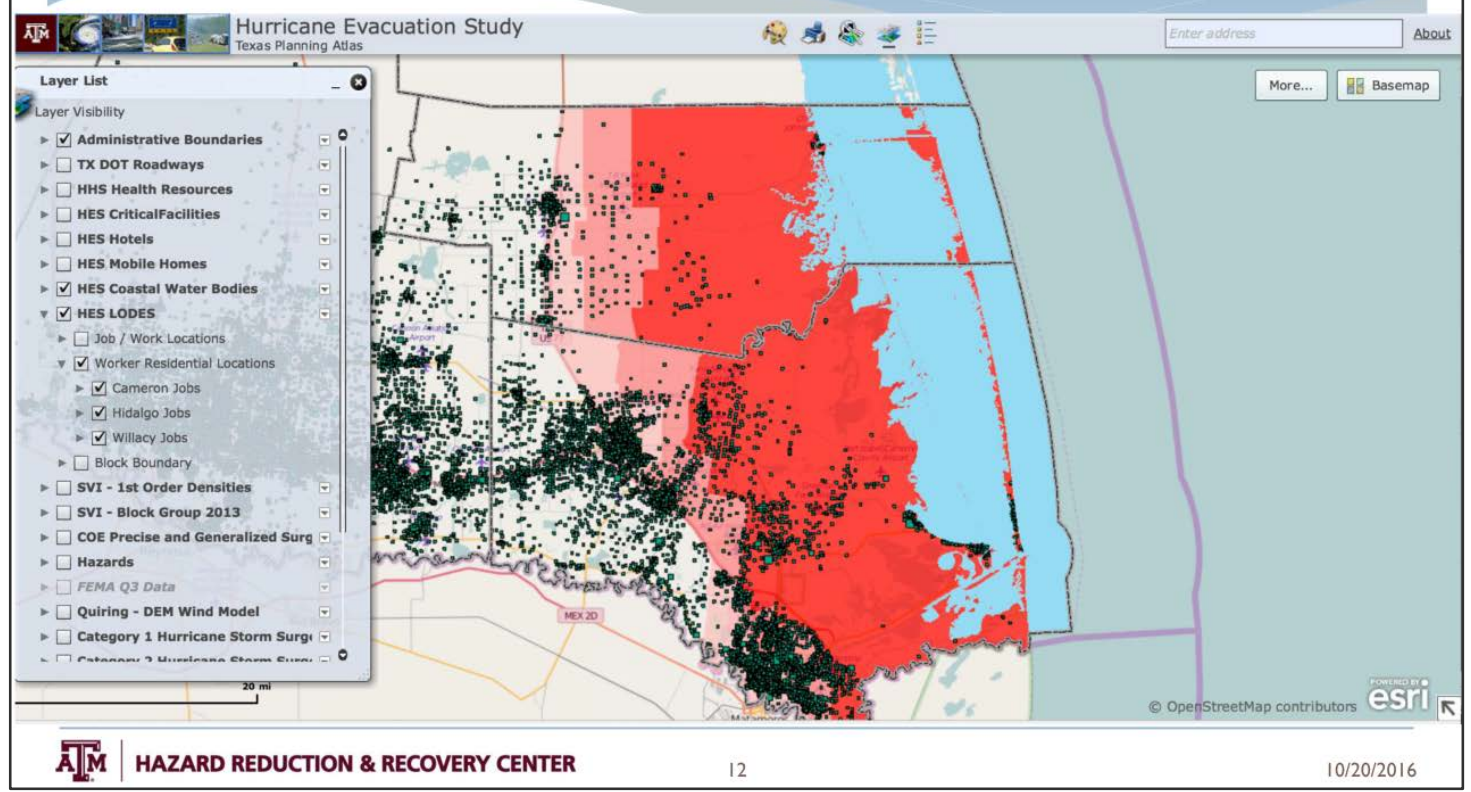




\section{Job locations and employee residences}

\begin{tabular}{|c|c|r|r|r|r|}
\hline & & \multicolumn{4}{|c|}{$\begin{array}{c}\text { Hazard Exposure } \\
\text { Evacuation Zones }\end{array}$} \\
\hline \multirow{2}{*}{$\begin{array}{c}\text { Both } \\
\text { Counties }\end{array}$} & Physical Vulnerability & \multicolumn{1}{c|}{ A } & \multicolumn{1}{c|}{ B } & \multicolumn{1}{c|}{ C } & A+B+C \\
\cline { 2 - 7 } & Residential locations & 20,875 & 34,149 & 67,090 & 122,114 \\
\hline \multirow{3}{*}{ Willacy total residences } & $13.80 \%$ & $22.60 \%$ & $44.40 \%$ & $80.70 \%$ \\
\cline { 2 - 7 } & Residential locations & 1,607 & 343 & 8,475 & 10,425 \\
\cline { 2 - 7 } & $\%$ of total residences & $12.20 \%$ & $2.60 \%$ & $64.30 \%$ & $79.10 \%$ \\
\hline \multirow{2}{*}{ Cameron } & Residential locations & 19,268 & 33,806 & 58,615 & 111,689 \\
\cline { 2 - 7 } & $\%$ of total residences & $14.00 \%$ & $24.50 \%$ & $42.40 \%$ & $80.90 \%$ \\
\hline
\end{tabular}

A] M

13

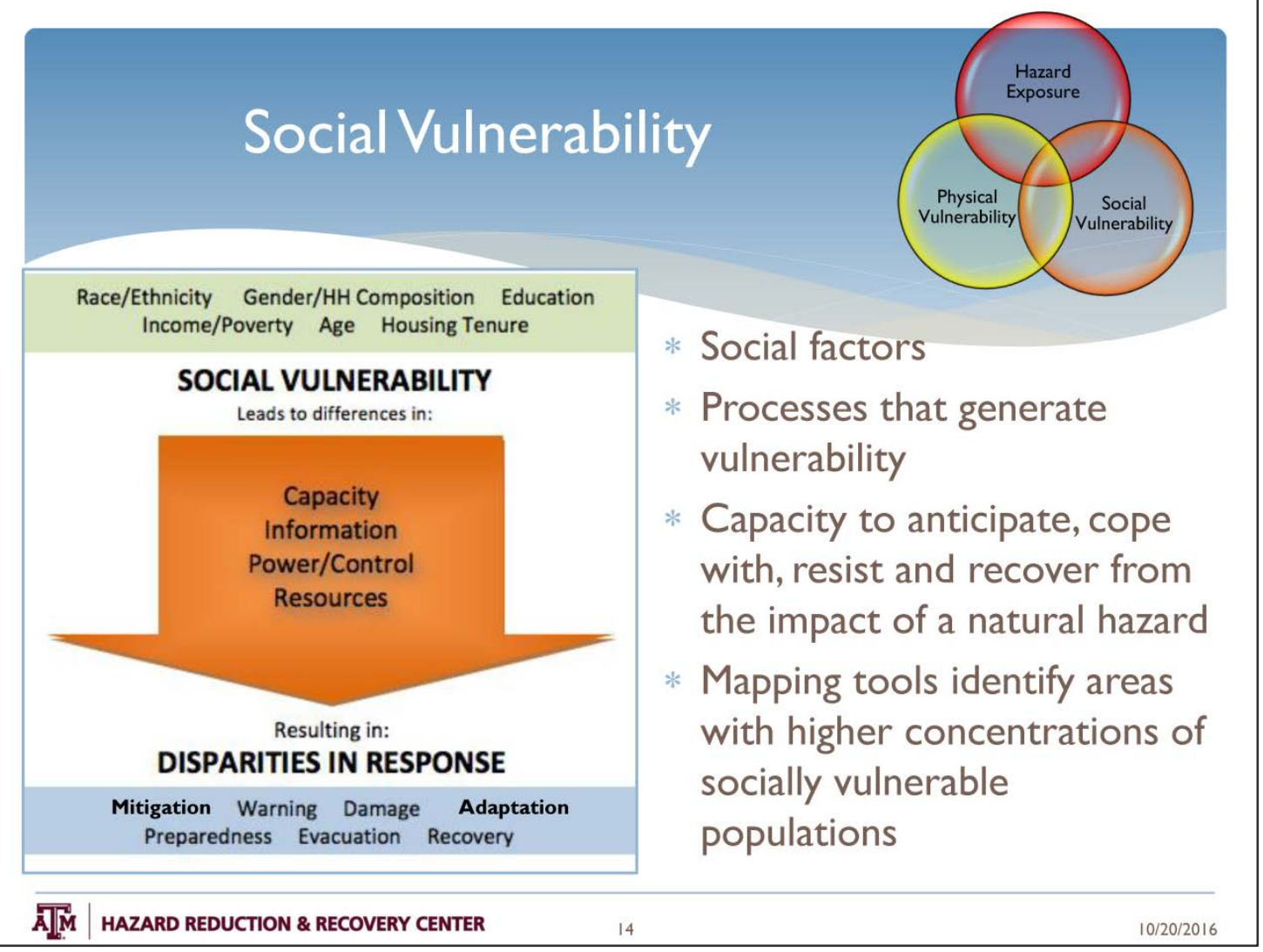




\section{A few examples of social vulnerability measures}

\begin{tabular}{|c|l|}
\hline Household structure & Single parent, particularly female headed households \\
\hline Socioeconomic Status & $\begin{array}{l}\text { Higher levels of wealth, income, prestige, and political power insures enhanced ability } \\
\text { to prepare for, mitigate against and cope with physical impacts. Often have much higher } \\
\text { resources base to draw from to overcome }\end{array}$ \\
\hline Gender & $\begin{array}{l}\text { Women have more difficult time in recovery because of constrained employment } \\
\text { opportunities and lower wages; often must take primary responsibility for child care and } \\
\text { households activities. }\end{array}$ \\
\hline Race/ethnicity & Language, culture, and discrimination issues \\
\hline Age & $\begin{array}{l}\text { Both young and old are at higher risk due to reduce mobility, economic constraints, legal } \\
\text { constraints. Households with these higher risk groups can be limited due to time and } \\
\text { resource constraints. }\end{array}$ \\
\hline Tenure & $\begin{array}{l}\text { Renters more transient, fewer resources, less-control, more dependent on owner for } \\
\text { improvements, repair, mitigation, etc. }\end{array}$ \\
\hline Urban/Rural & $\begin{array}{l}\text { Rural residents are more vulnerable due to isolation, tend to have fewer employment } \\
\text { opportunities, are poorer, etc. }\end{array}$ \\
\hline Special Needs populations & Sick, infirmed, etc. \\
\hline Employment status & Those who are un- or under-employed have reduced resources from which to draw from. \\
\hline HAZARD REDUCTION\&RECOVER CENTER
\end{tabular}

\begin{tabular}{|c|c|c|c|}
\hline \multicolumn{4}{|c|}{$\begin{array}{r}\text { Levels of Social Vulnerability Analysis - develo } \\
\text { Block Groups }\end{array}$} \\
\hline Field Name & 1st Order Descriptions & 2nd Order & \multirow{2}{*}{ 3rd Order } \\
\hline SPHWC & Single Parent Household with Children & \multirow{2}{*}{$\begin{array}{c}\text { Child Care } \\
\text { Needs }\end{array}$} & \\
\hline CHILD & Child Population under 5 Years & & \multirow{16}{*}{$\begin{array}{l}\text { Social } \\
\text { Vulnerability } \\
\text { Hotspots }\end{array}$} \\
\hline ELDER & Elder Population $65+$ Years & \multirow{3}{*}{$\begin{array}{c}\text { Elder Care } \\
\text { Needs }\end{array}$} & \\
\hline ELDERH & Elder Households & & \\
\hline ELDERPV & Elder Households in Poverty & & \\
\hline PTD & Public Transportation Dependent Households & \multirow{2}{*}{$\begin{array}{l}\text { Transportation } \\
\text { Needs }\end{array}$} & \\
\hline HUNOVEL & Households with no Vehicle Available & & \\
\hline VACHU & Vacant Housing Units & \multirow{5}{*}{$\begin{array}{l}\text { Temporary } \\
\text { Shelter Needs } \\
\text { and Housing } \\
\text { Recovery } \\
\text { Needs }\end{array}$} & \\
\hline RENTER & Renter Households & & \\
\hline NONWHITE & Non-White Population & & \\
\hline YEAR1990 & Housing Units Built before 1990 & & \\
\hline MOBILE & Mobile Home Households & & \\
\hline HUPV & Households in Poverty & \multirow{5}{*}{$\begin{array}{c}\text { Civic Capacity } \\
\text { Needs }\end{array}$} & \\
\hline HUNOTEL & Housing Units with no Telephone Service Available & & \\
\hline NOHS & $\begin{array}{l}\text { Less than High School Graduate Population 25+ } \\
\text { Years }\end{array}$ & & \\
\hline UNEMP & Unemployed Population in Labor Force & & \\
\hline SPENW & Population Speaking English Not Well or Not at All & & \\
\hline \multicolumn{4}{|c|}{ The entire set can be combined to capture hyper-vulnerability, or identify hotspots. } \\
\hline HAZARD REDUCTI & DN \& RECOVERY CENTER $\quad 16$ & & \\
\hline
\end{tabular}




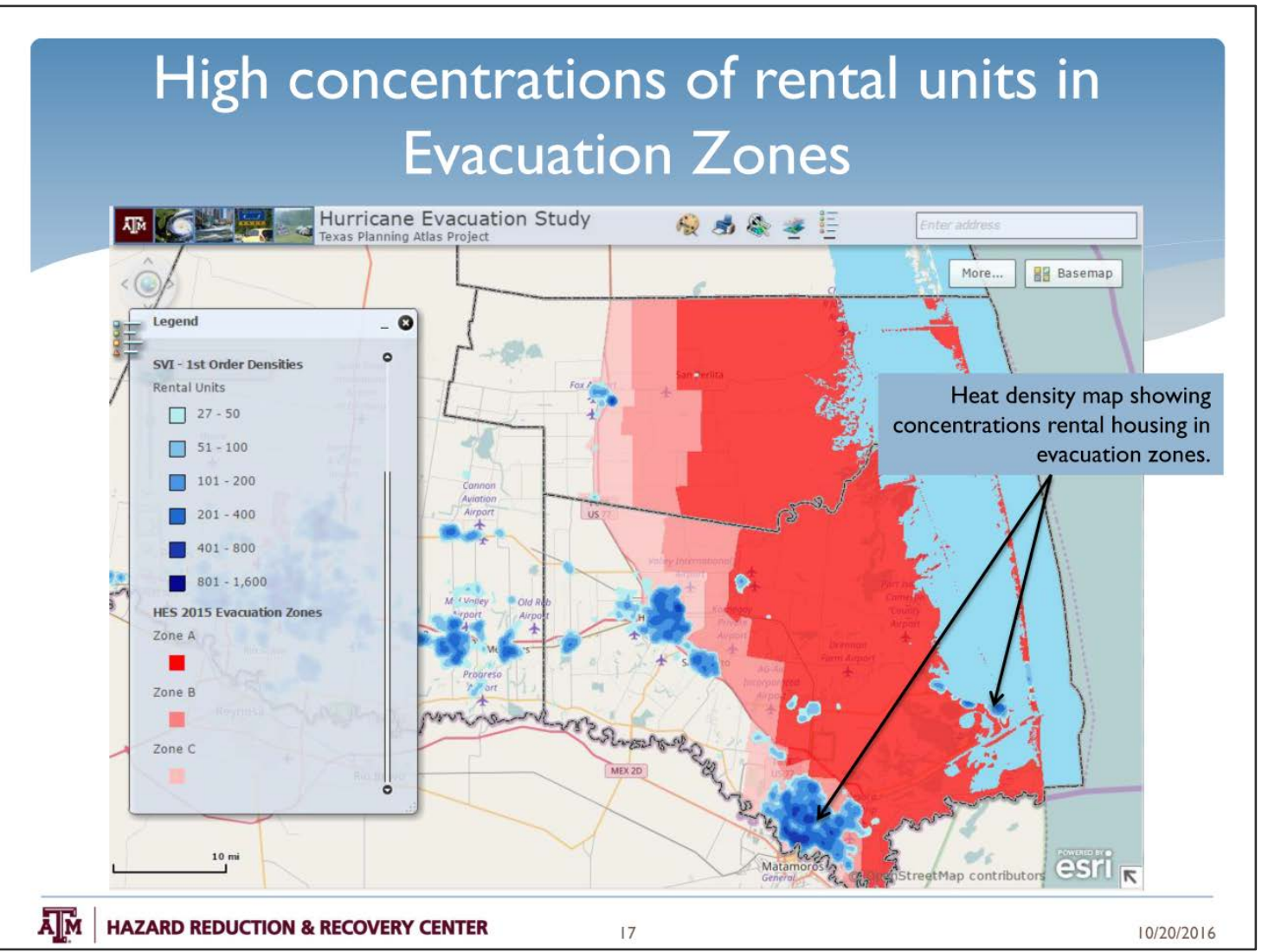

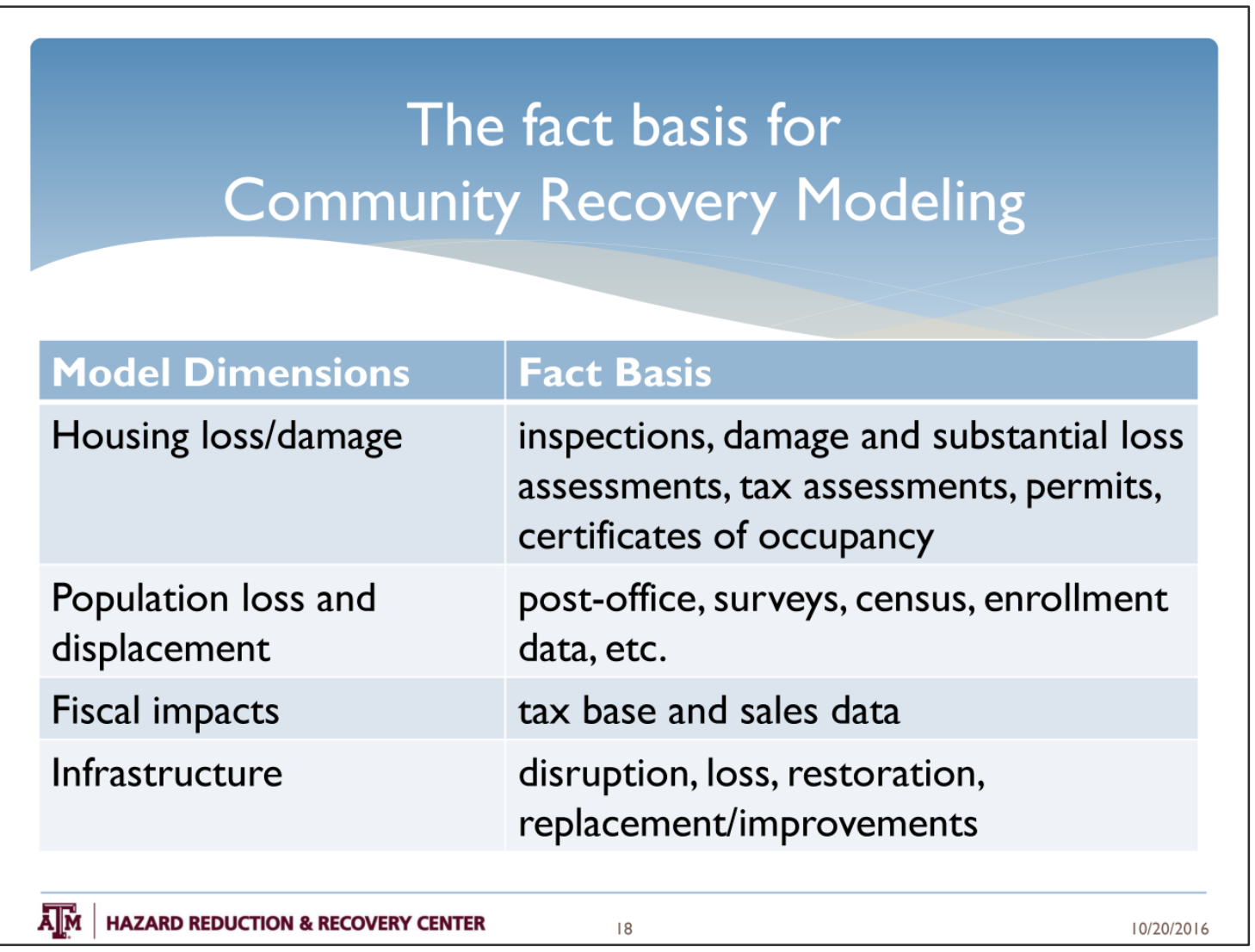




\section{The fact basis for}

\section{Community Recovery Modeling: Issues}

Time scale

* Spatial Scale/resolution

* Uneven and inconsistent data across dimensions (housing, business, infrastructure)

* Incompatible within areas (windshield, inspections, permits, etc.)

* Expensive and time consuming, particularly at the local level.

* Public versus private-proprietary data (insurance, utility, etc.) -

* Difficult to combine into a total picture...

* Should be combined with SV and other factors in analysis.

A $]$ [M $\mid$ HAZARD REDUCTION \& RECOVERY CENTER 19

\section{Social Vulnerability Map Uses}

* Minimize or optimize uncertainty in hazard exposure models

* Confirm appropriate archetypes for structures

Community vulnerability assessments

* Preplanning of field studies

* Recovery modeling algorithm development 


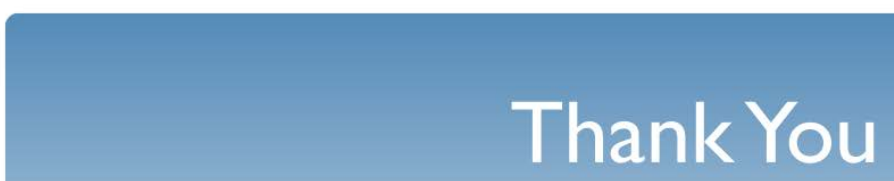

Nathanael Rosenheim

Research Scientist

Hazard Reduction and Recovery Center

College of Architecture

Texas A\&M University

College Station, Texas 77843-31 37

979-845-78। 3

nrosenheim@arch.tamu.edu 


\section{Appendix E: Case Studies and Validation (Session 5)}

GRITT $^{\circledR}$ - a Sustainable Tool for Reducing Weather and Climate Impacts [Cindy

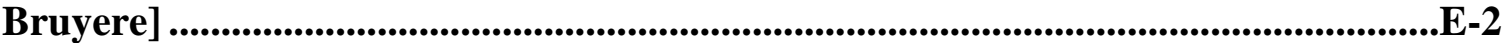

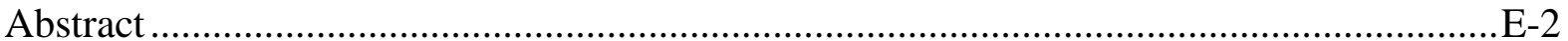

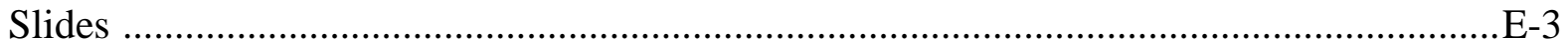

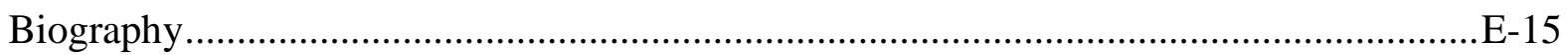

Resilience Modeling Innovations of the HayWired Planning Scenario [Keith Porter].....E-16

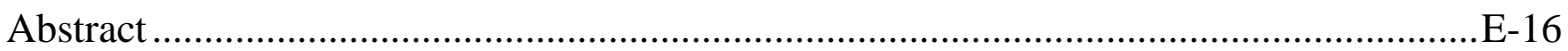

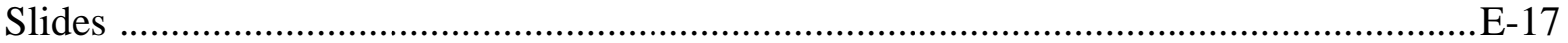

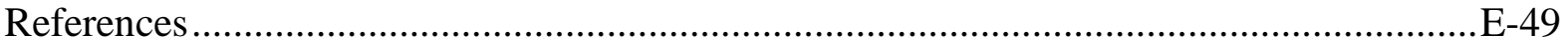

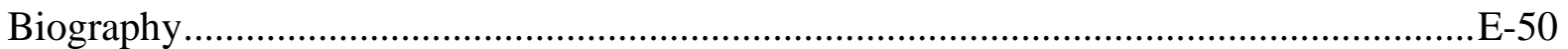

Model Validation: The Joplin Tornado Hindcast [John van de Lindt] ................................E-51

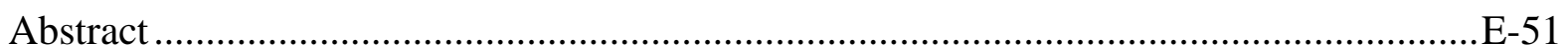

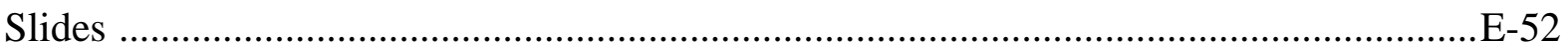

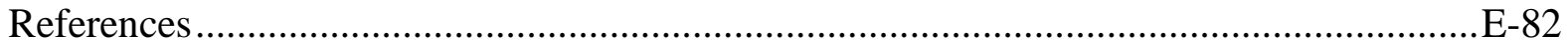

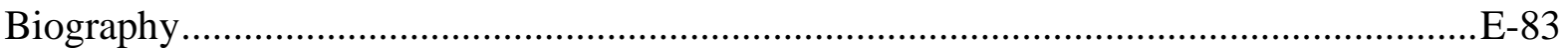




\section{GRRIT $^{\circledR}$ - a Sustainable Tool for Reducing Weather and Climate Impacts}

Cindy Bruyère, C3WE/MMM/NCAR

The economic impacts of weather and climate extremes are rising, as population grows and moves into urban and more hazard-prone areas. Impacts in the form of societal disruption and recovery time, and in the lingering effects of weather and climate events are amongst the less publicized - but nevertheless important - outcomes.

In response to this growing need for actionable information, researchers within NCAR's Capacity Center for Climate and Weather Extremes (C3WE) are partnering with a wide range of research and planning groups to develop GRRIT - the Global Risk Resilience and Impacts Toolbox. GRRIT places the tools and information to advance understanding of extreme events and their effects within reach of decision-makers and planners making society's tough choices. GRRIT uses a sophisticated Framework that provides users with access to hazard, vulnerability, and exposure information and data from a broad variety of public and private sources via tools available within a web interface.

Government agencies, industry, universities and others already have started to develop information and tools that could be used for informing decision makers responsible for making choices that make society less vulnerable to extreme events. However, these data and tools may not: be readily available, exist in formats accessible to the average user, or be adaptable to related regions and requirements.

GRRIT's sustainable, fully supported toolbox is designed to provide a common foundation for these and future developments, ones that aid society in reducing weather and climate impacts, building economic resilience, and improving disaster recovery. In keeping with NCAR practice for community facilities, GRRIT will be freely available and will be maintained and supported by NCAR. 


\section{GRRIT $^{\oplus}$ - a Sustainable Tool for Reducing Weather and Climate Impacts}

Cindy Bruyère (bruyerec@ucar.edu)

Deputy Director

Capacity Center for Climate and Weather Extremes

National Center for Atmospheric Research

Boulder, Colorado, USA

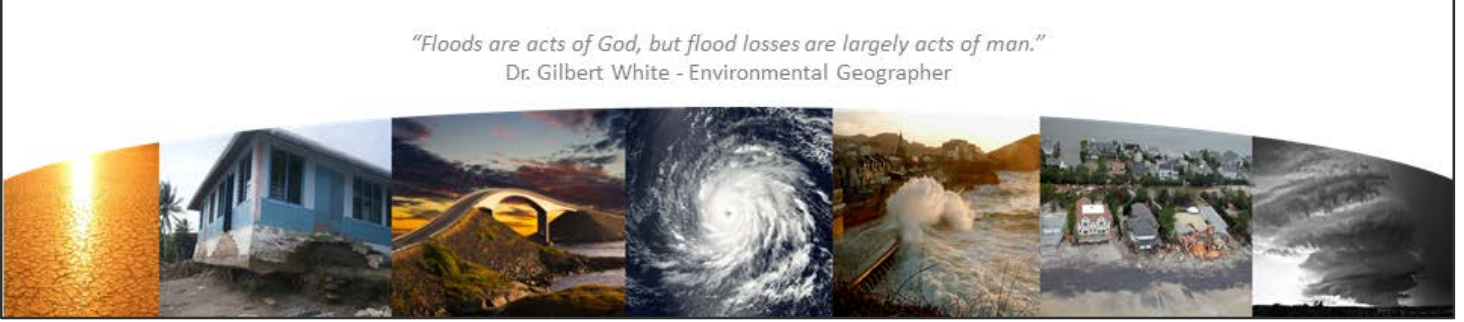

\section{The National Center for Atmospheric Research}

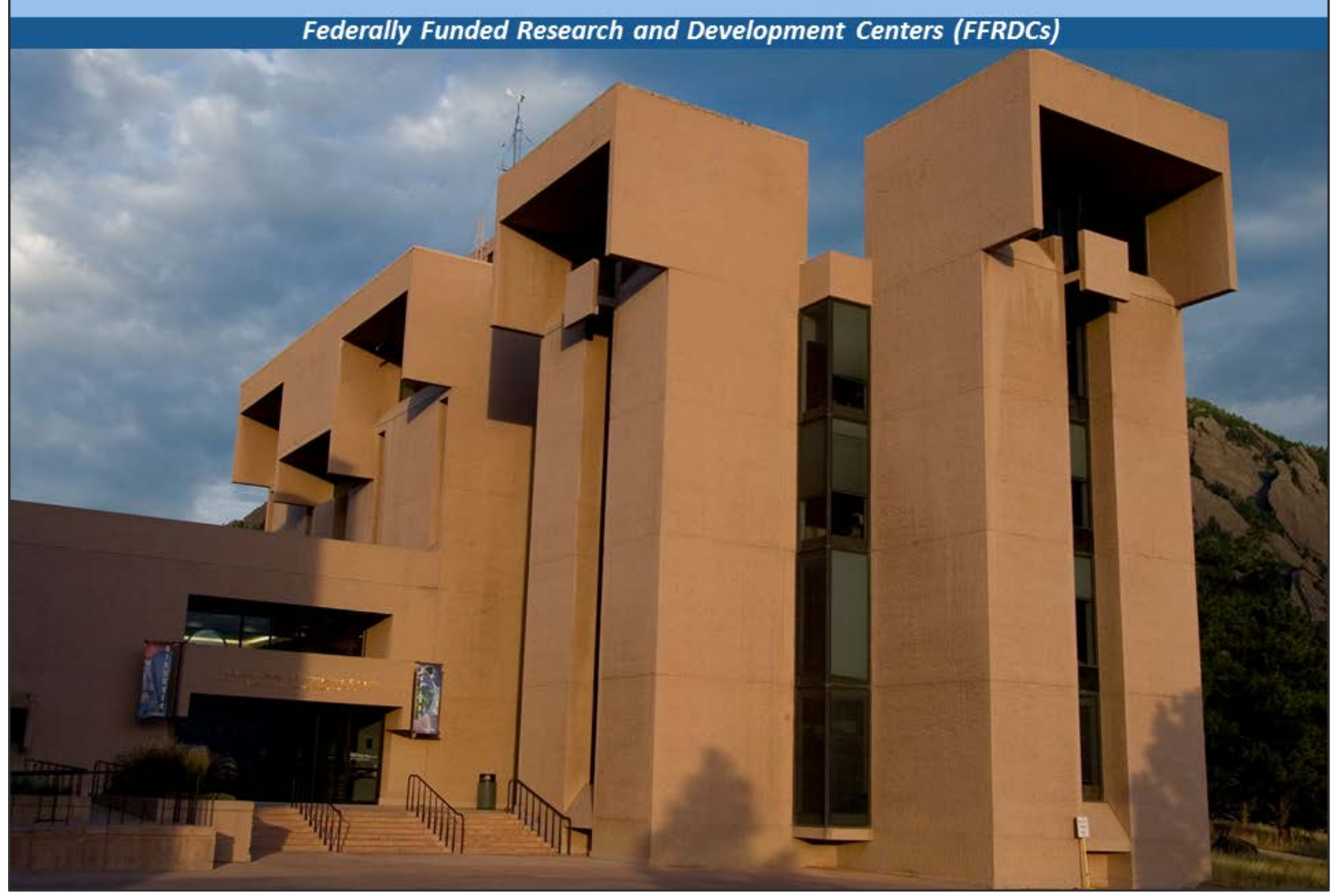




\section{NCAR Imperatives}

1. Conduct innovative research to advance the atmospheric sciences

2. Develop, maintain and deploy advanced observational facilities

3. Develop, deliver and support a suite of advanced community models

4. Develop and sustain advanced computing and data system services

5. Develop and transfer science to meet societal needs

6. Educate and entrain a talented and diverse group of students and early career professionals
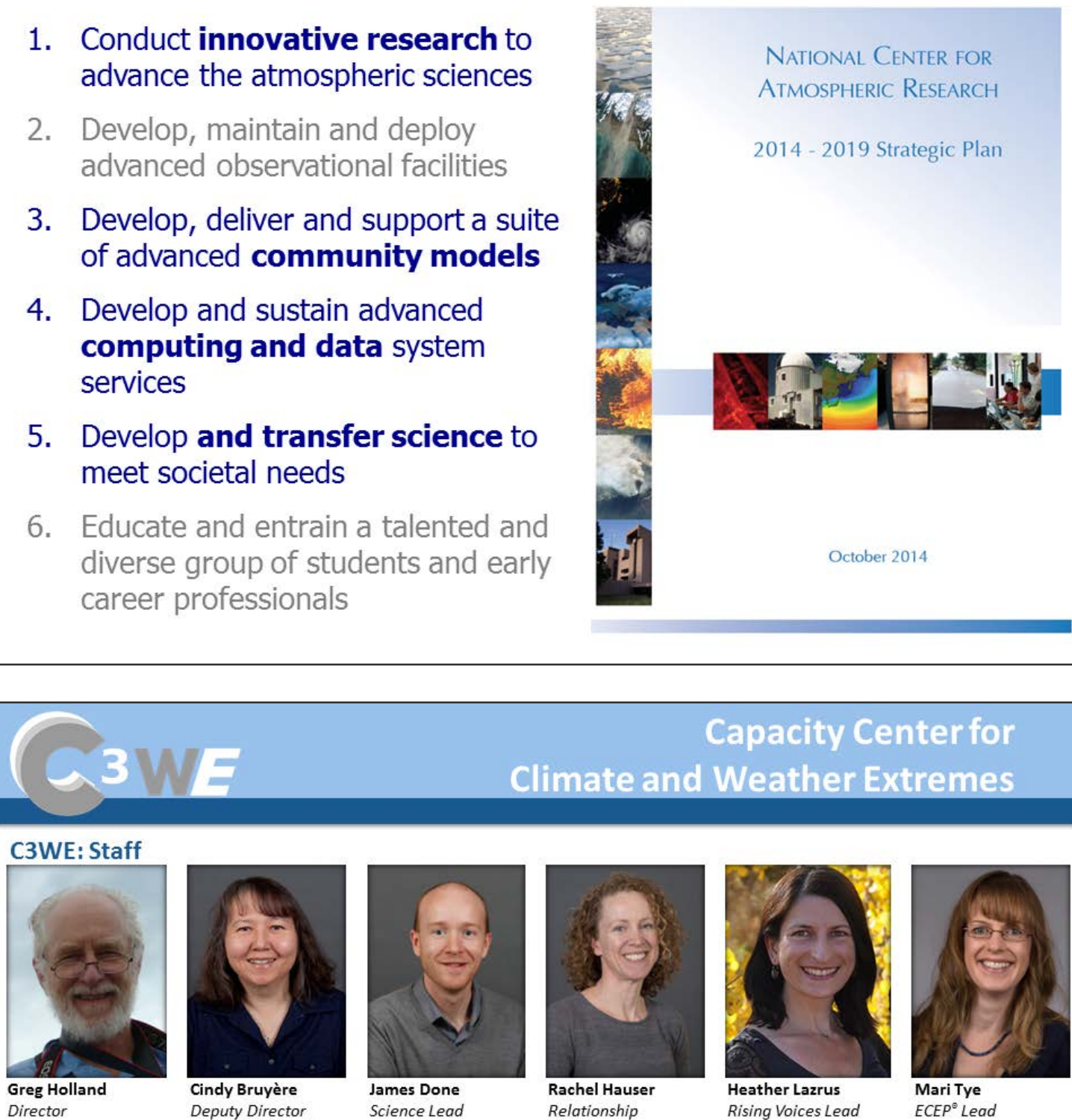

\section{Capacity Center for Climate and Weather Extremes}

Director
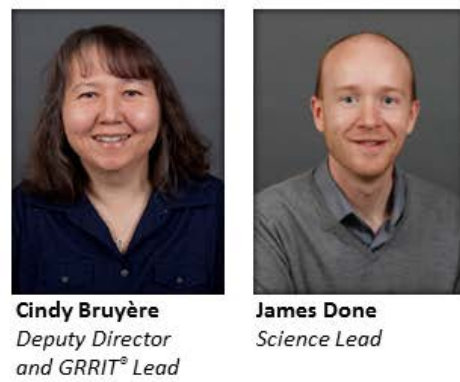

James Done

Science Lead and GRRIT ${ }^{\circ}$ Lead

C3WE: Advisory Panel

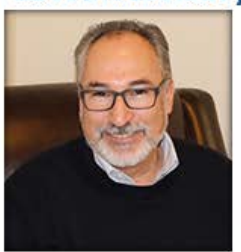

Guillermo (Willy)Accame Joyce Coffee

(Chair)

Environmental Director

Panattoni Develooment

Company, inc

NCAR Industry Advisor

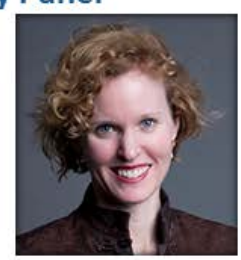

Founder and President, Climate Resilience Consulting
CEO, Capital, Scien Policy Practice Chairman Willis Research Network

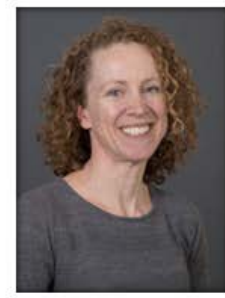

Rachel Hauser

Relationship

Development

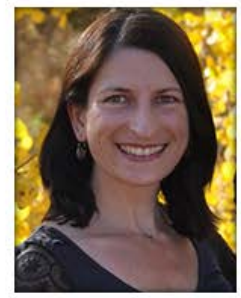

Heather Lazrus

Rising Voices Lead

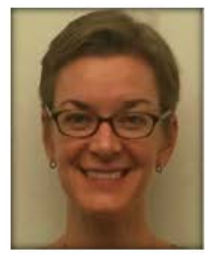

Glynis Lough

Chief of Staff, National

Climate Assessment

U.S. Global Change

Research Program

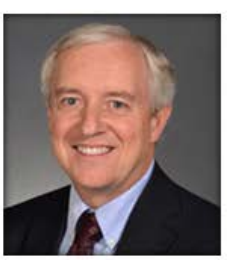

David Titley

Rear Admiral USN (ret) Professor of Practice, Dep. of Meteorology, Director, Center for Solutions for Weather and Climate Risk, PSU

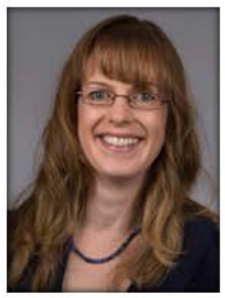

Mari Tye ECEP ${ }^{\circ}$ Lead

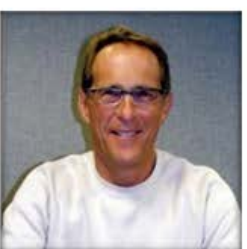

Steve Worley Manager, Data Support Section Computational and Information Systems Laboratory, NCAR 

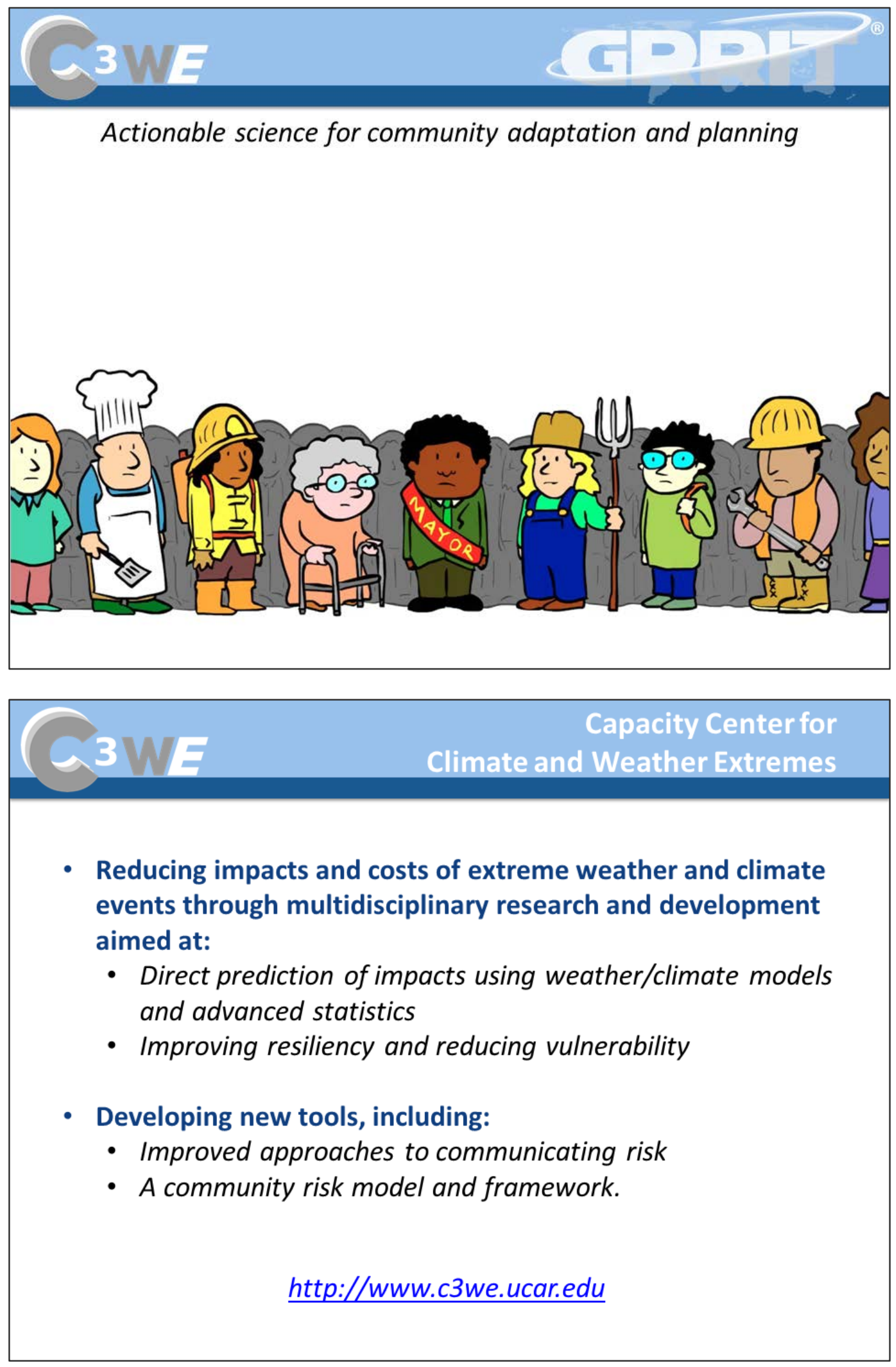


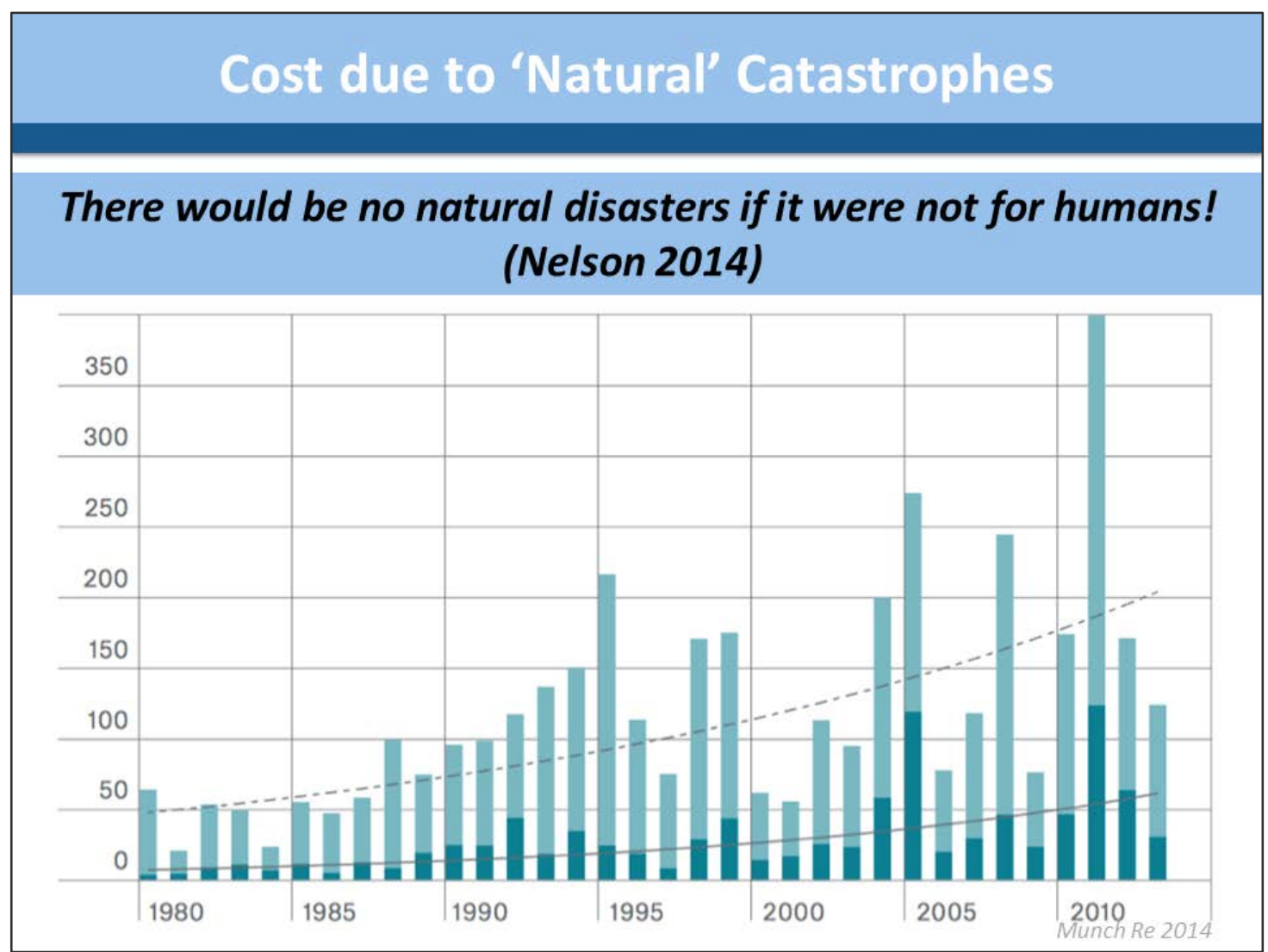

\section{What are the Causes of Increasing Impacts?}

- Climate variability $a^{r}$

- Increasingly vulne \& of ulations and development

- Loss of socie' $\gamma^{d} \Sigma^{t}$ ccy: urbanization, business, - 5 , government

- Lack of $\quad$ slanning to our modern enviroı. 


\section{Primary Causes of Engineering Failures}

- Insufficient knowledge $36 \%$

- Underestimation of influence ......................... 16\%

- Ignorence, carelessness, negligence ................ 14\%

- Forgetfulness, error ............................... 13\%

- Objectively unknown situation ........................ 7\%

- Dolvina unan atharc usithnut cufficinnt rantral 00/

Over $60 \%$ is due to lack of knowledge and communication of information

- Other .............................................. 3\%

\section{Science Products ...}
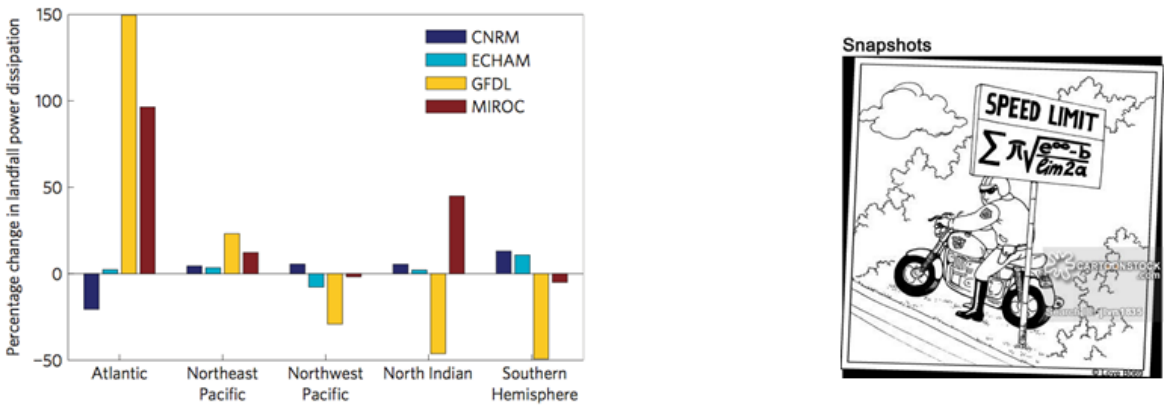

Temporal Range: $\quad$ 1995-01-01 00:00 +0000 to 2055-12-31 21:00 +0000 (Entire dataset) Period details by dataset prodr

Variables:

\begin{tabular}{|l|l|l|l|}
\hline Air Temperature & Geopotential Height & Humidity & Longwave Radiation \\
\hline Outgoing Longwave Radiation & Precipitable Water & Precipitation Amount & Runoff \\
\hline Sea Level Pressure & Shortwave Radiation & Skin Temperature & Snow \\
\hline Snow Depth & Snow Water Equivalent & Soil Moisture/Water Content & Surface Pressure \\
\hline Surface Winds & Upper Level Winds & & \\
\hline
\end{tabular}




\section{... vs Decision Making Tools}
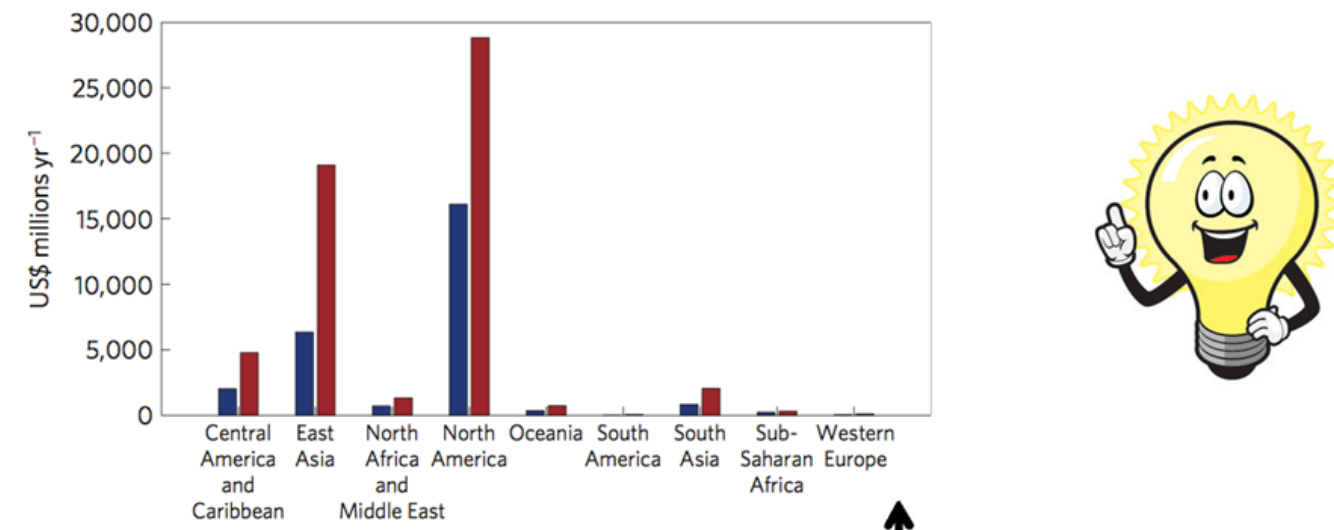

Mendelsohn et al. 2012
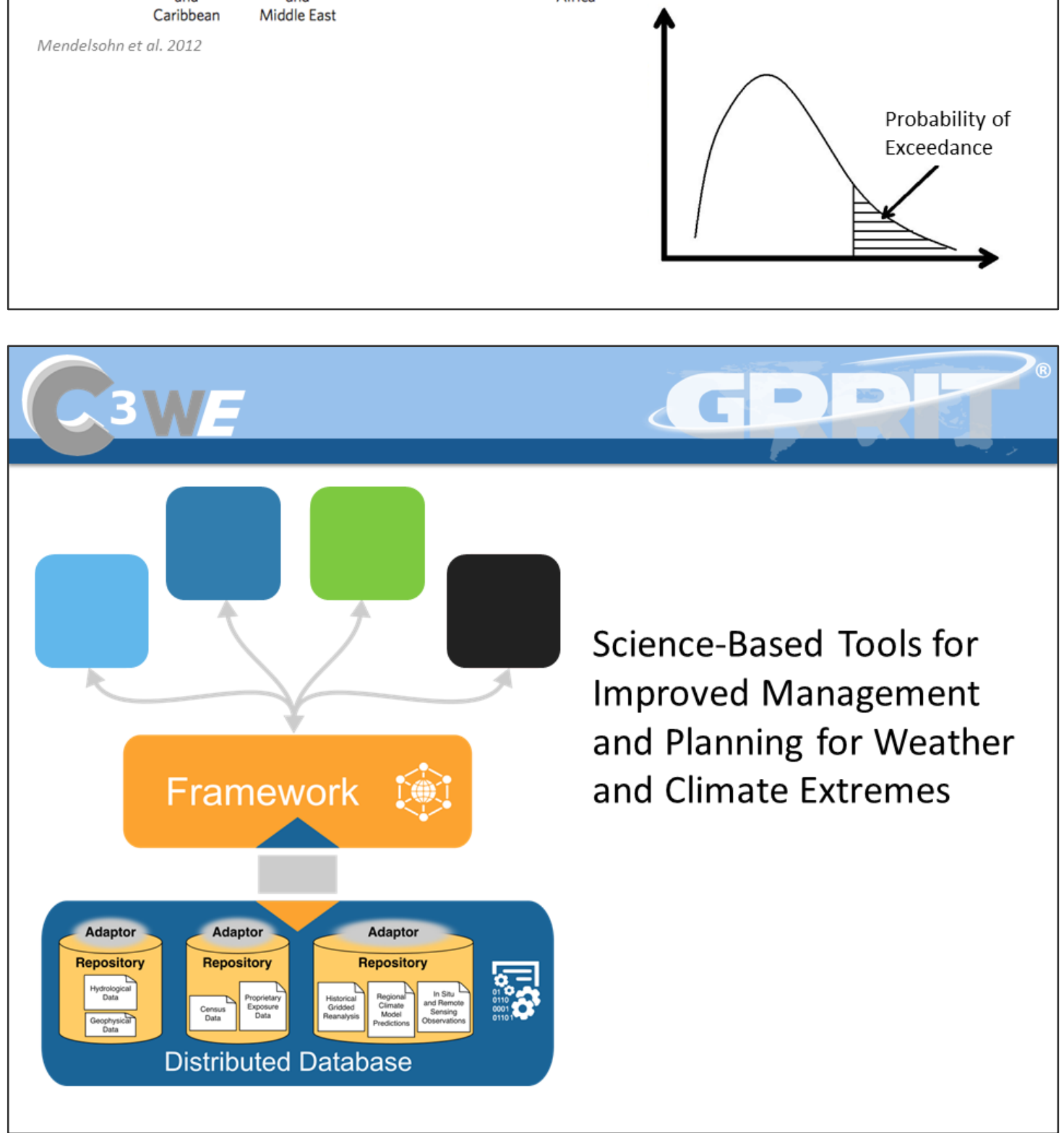

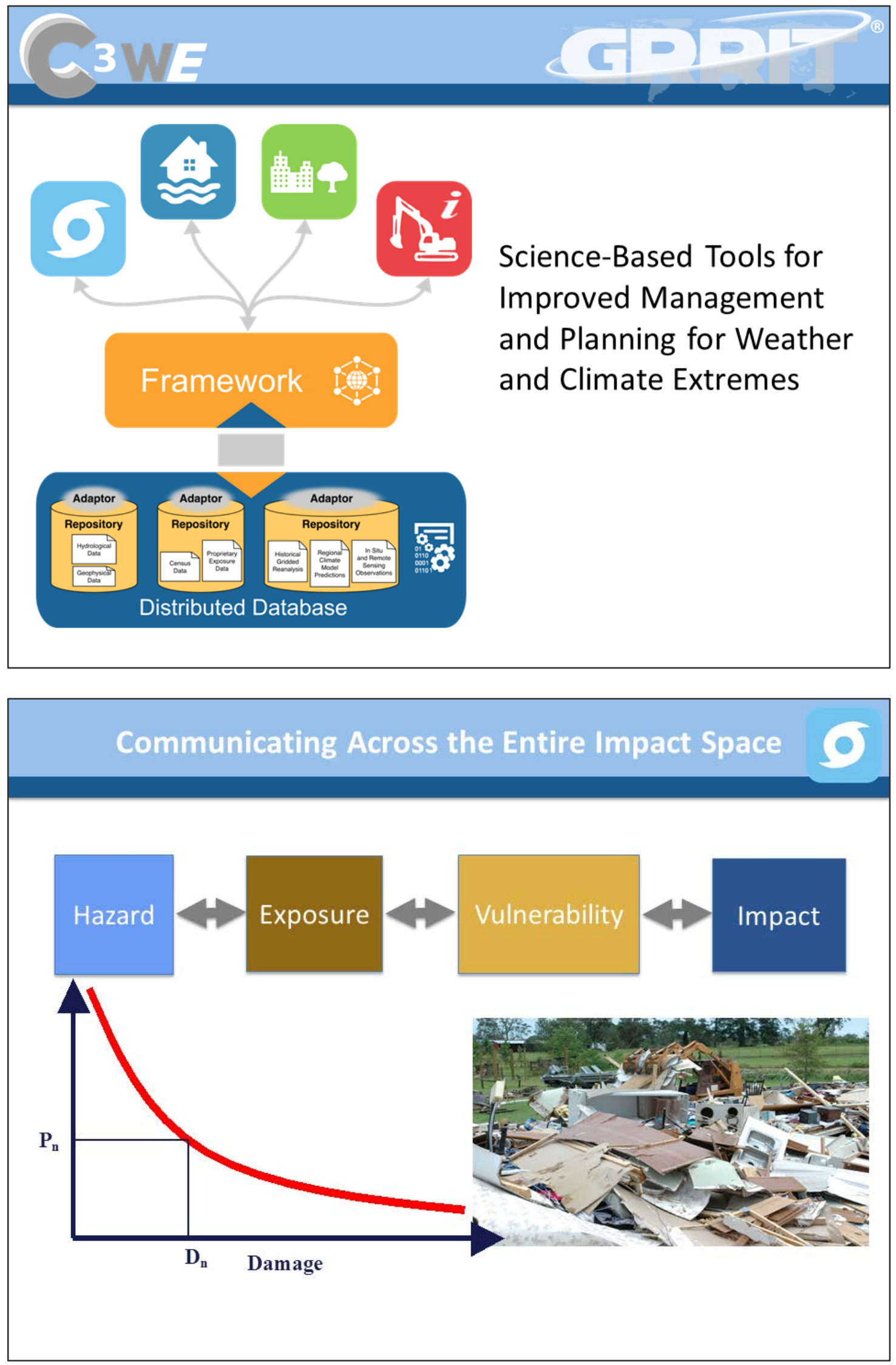


\section{Communicating Across the Entire Impact Space}

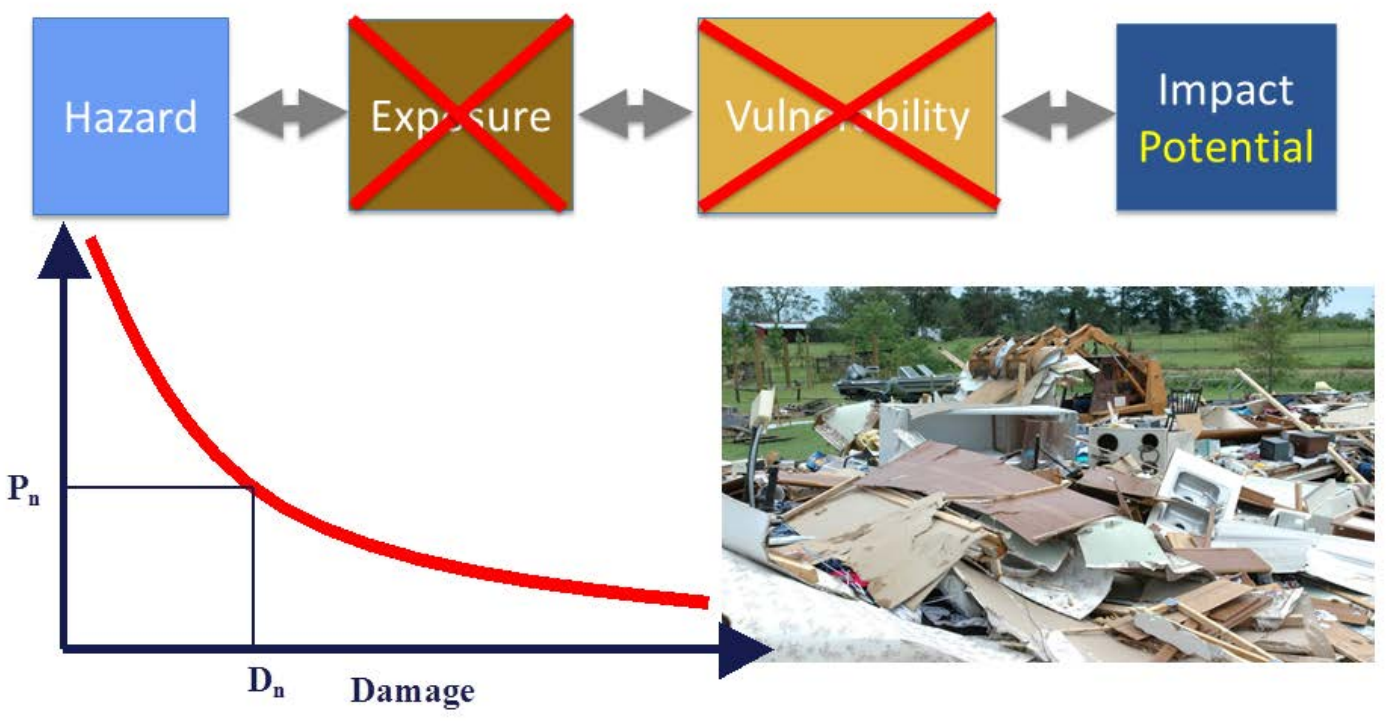

\section{Cyclone Damage Potential Index}

$$
C D P=4 \frac{\left[\left(\frac{v_{m}}{65}\right)^{3}+5\left(\frac{R_{h}}{50}\right)\right]}{v_{t}}
$$

$V_{m}$, the maximum winds (intensity);

$R_{h}$, the radial extent of the hurricane force surface winds (size);

$V_{t}$, the translational speed of the hurricane (speed).
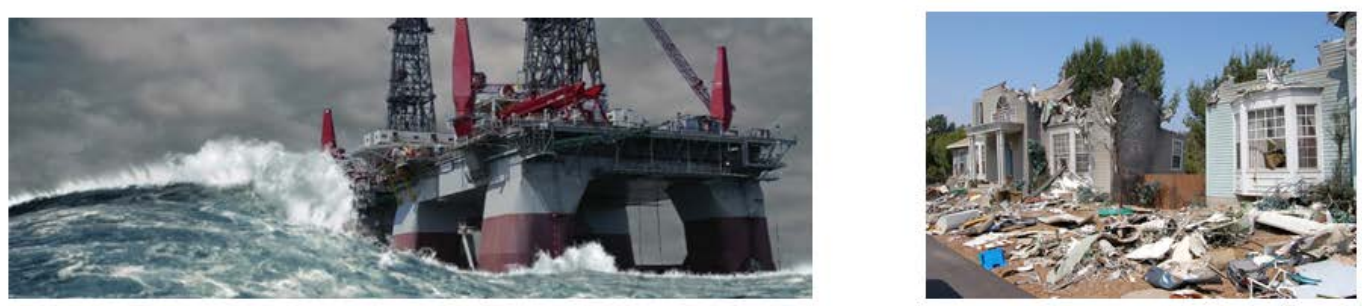


\section{Cyclone Damage Potential Tool}

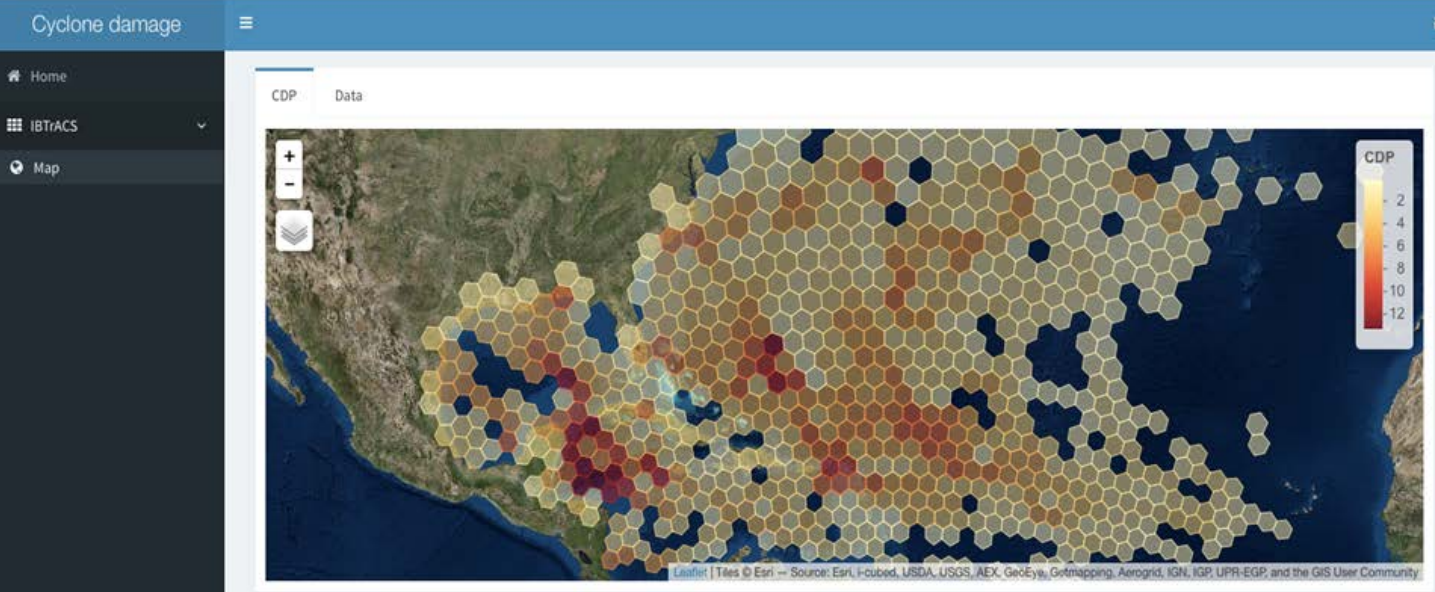

http://grrit.mmm.ucar.edu/CDP/

\section{Dynamical Modeling Approach - CDP}

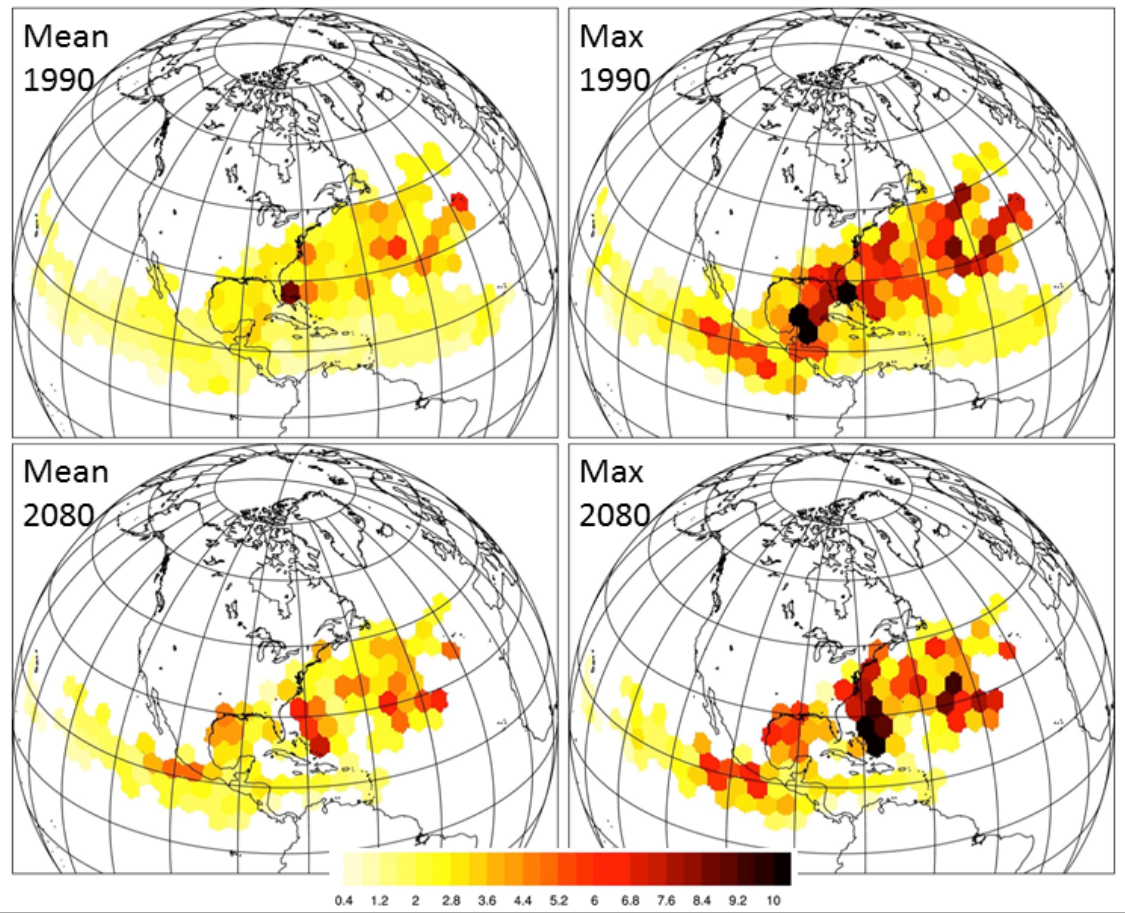




\section{Seasonal Cyclone Damage Potential Forecast}

An alternative view of upcoming North Atlantic basin activity.

Historical average $=3.7$

2005 average $=6.3$

2016 Forecast average $\mathbf{=} \mathbf{5 . 7}$

Does not necessarily correspond to landfalling damage potential.

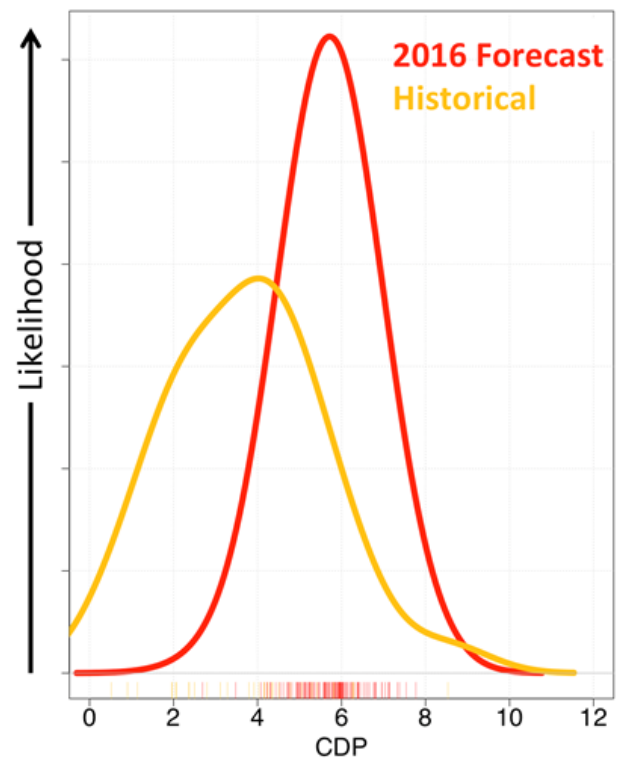

http://www.ecep.ucar.edu/seasonal-hurricane-damage

\section{Climate- $i \quad$ Construction}

A decision-support tool for construction projects to manage weather risk from contract to completion.

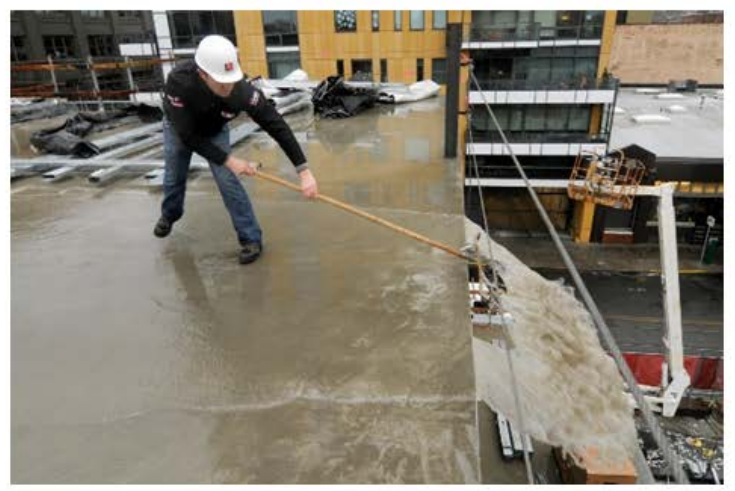




\section{Climate- $i$}

Current practice: Use recent memory or historical average.

Desired: A weather management tool from contract to completion to reduce losses.

\section{Sensitivities:}

Temperature: hot/cold days, hot/cold spells, effective temperature, large temperature fluctuations.

Rainfall: wet days, intense rainfall

Wind: strong wind gusts, strong sustained winds.

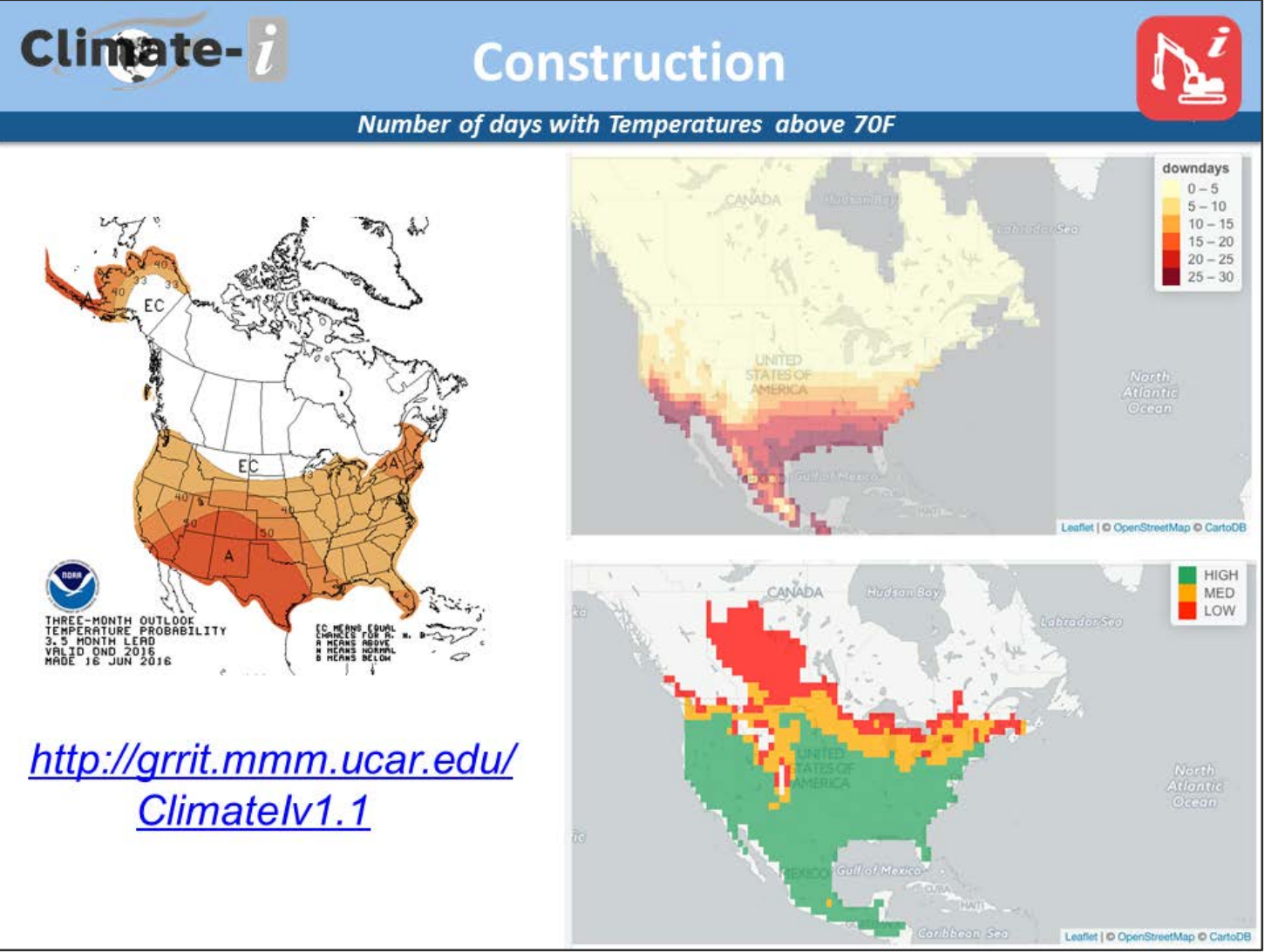



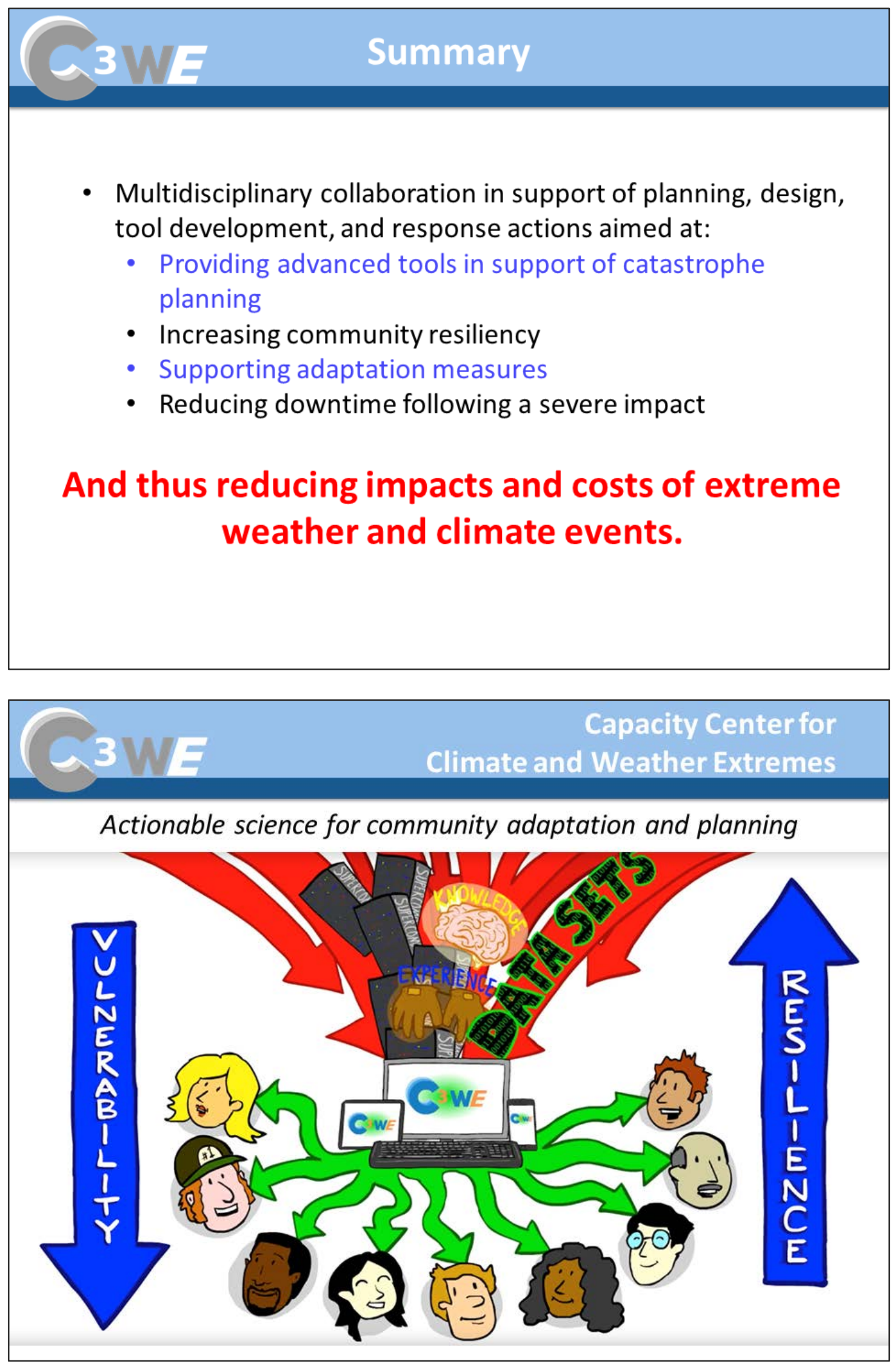


\section{Cindy Bruyere}

PROJ SCIENTIST II

Mesoscale and Microscale Meteorology Laboratory

Regional Climate Research

bruyerec@ucar.edu

$\underline{303-497-8914}$

Cindy Bruyère leads the NCAR-based systems development effort for the Engineering for Climate Extremes. She plays a key role in the development of the Global Risk, Resilience, and Impacts Toolbox (GRRIT), which pulls information from disparate locations, including local drives, the cloud, or servers located in different places. Once captured, relevant data are fed to ECEPdeveloped decision-making tools to provide insights and answers

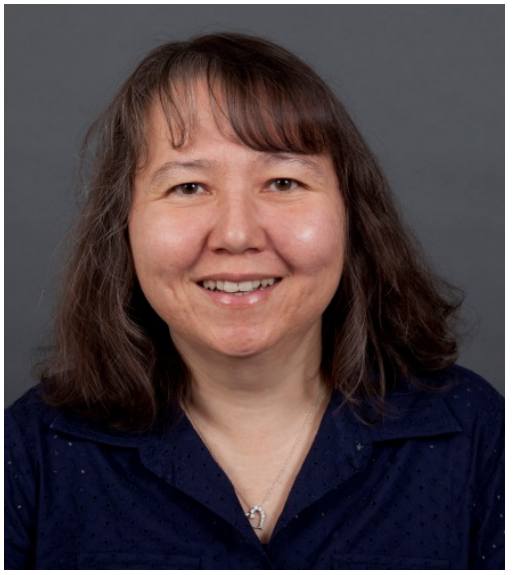
to critical questions related to extreme weather and climate risk and resilience. In her role, Cindy directs efforts to incorporate tools as they are developed into GRRIT, spearheads related database construction, maintenance, and management, and is responsible for climate downscaling efforts conduct by the group.

Cindy has an MSc in Dynamical Modeling and a PhD in Environmental Management. She started her career at the South African Weather Service, where she rose to Assistant Director of research programs, and Project Manager for operational systems. For a number of years she was also associated with the University of Pretoria (South Africa) and involved in meteorological training. Current research activities include understanding and predicting the impact of climate variability and change; dynamical model development; creating useful climate decision-making tools; and the development of statistical downscaling techniques. She trains those within the atmospheric science community in climate modeling techniques. Cindy is also a visiting research fellow at the North-West University of South Africa.

\section{Field of Expertise:}

- $\quad$ Climate Science

- $\quad$ Engineering for Science

- Interdisciplinary Research

- Meteorology

- Research Applications

\section{Specialty:}

- $\quad$ Climate Change

- Decision Support

- $\quad$ Extreme Weather/Severe Storms

- Impacts

- Modeling: Regional 
Resilience Modeling Innovations of the HayWired Planning Scenario

Keith Porter $^{(\mathrm{a})}$, Dale Cox ${ }^{(\mathrm{b})}$, Ken Hudnut ${ }^{(\mathrm{c})}$, Jamie Jones ${ }^{(\mathrm{d})}$, and Anne Wein ${ }^{(\mathrm{d})}$

(a) University of Colorado Boulder

(b) U.S. Geological Survey, Sacramento CA

(c) U.S. Geological Survey, Pasadena CA

(d) U.S. Geological Survey, Menlo Park CA

The USGS's HayWired earthquake planning scenario offers several advances in resilience modeling, only some of which have been published. Its new advances include:

1. Societal impacts of code performance objectives. Life-safety design (ASCE 7's objective) can lead to unacceptable building impairment in a large urban earthquake (the Big One). A simple option for resilient design greatly reduces the potential for dark cities [1].

2. Public preferences for seismic resilience of new buildings. A first-ever large $(\mathrm{N}>800)$ survey shows that the public expects and would pay for resilient design [2].

3. New water supply model with lifeline restoration, interaction, and resilience measures. It measures resilience in lost service-days and quantifies the economic benefit of resilience through two examples [3].

4. A new empirical model of urban search and rescue needs for collapsed buildings and elevators without power. We used NISEE's photo database to quantify, for the first time, the extent of building collapse in earthquakes, i.e., the fraction of a building that collapses when it experiences any collapse [4].

5. Safety benefit of earthquake early warning combined with drop, cover, and hold on (DCHO). HayWired includes the first-ever effort quantifying the time required to complete DCHO via a large $(\mathrm{N}>400)$ survey [5]. 


\section{Resilience Modeling}

Innovations of a Hayward Fault Planning Scenario

International Workshop on Modeling of Physical, Economic and Social Systems for Resilience Assessment

Sterling VA, 19-21 Oct 2016

Keith Porter

University of Colorado Boulder

\section{$\mathrm{M}_{\mathrm{w}}$ 7.0 Hayward Fault earthquake}

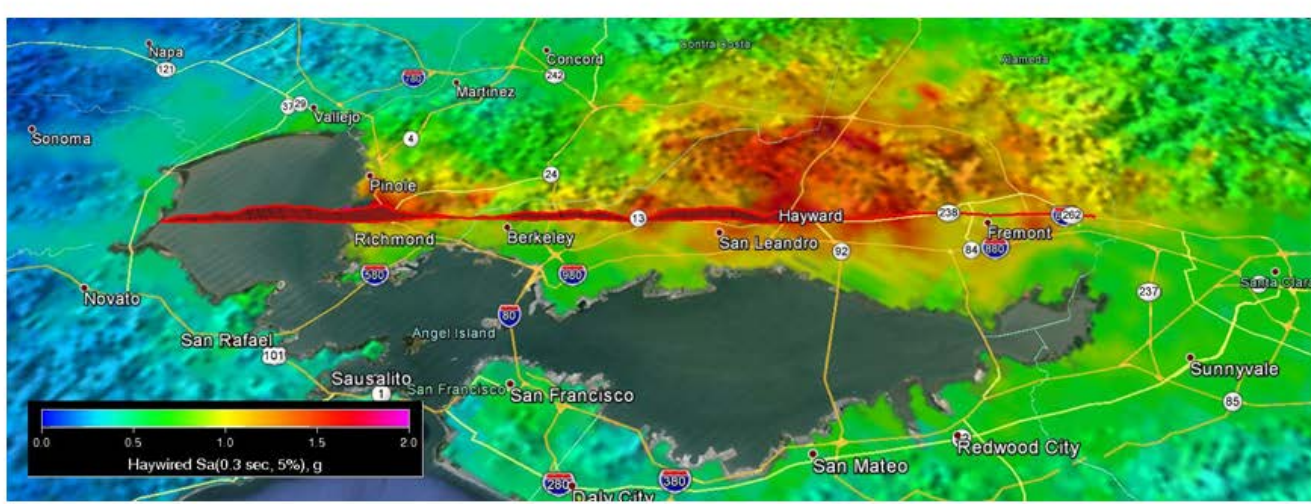




\section{Societal impacts of code life-safety performance objectives}

\section{Life-safety code protects life well}

\section{Peril}

Deaths $/ 100,000$

Where, when pop/yr

Heart disease

194

US, 2010

All accidents

39

US, 2010

Occupational fatality, roofers

32

US, 2011

Auto accidents

11

US, 2009

Firearms

10

US, 2010

New building (earthquake)

0.1

24/7 occupancy

CA earthquakes last $\sim 50 \mathrm{yr}$

0.007

CA, 1964-2014 


\section{A thought experiment}

\section{Life-safety (LS) Beyond life safety (BLS)}
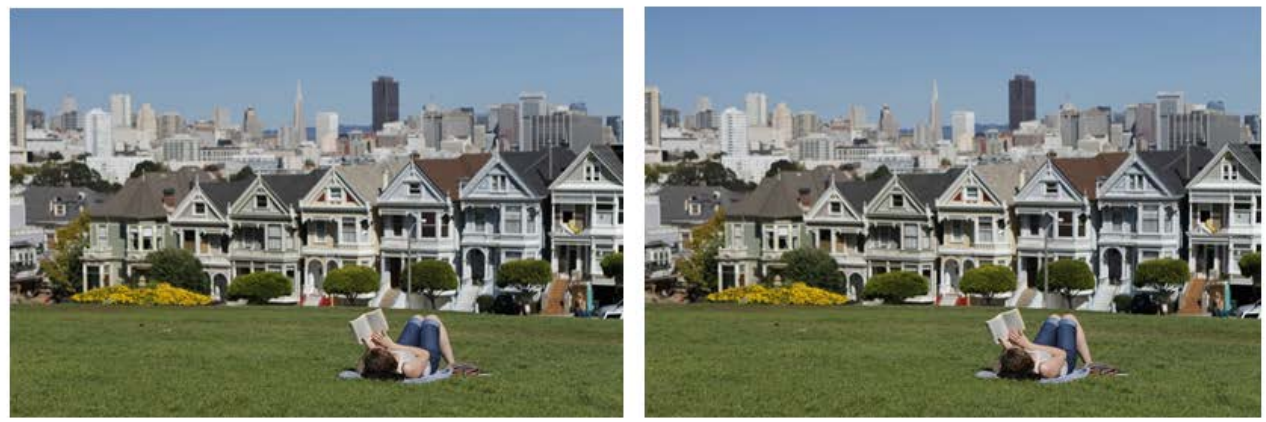

\section{One option for a beyond-life-safety} (BLS) code

$$
\begin{aligned}
& S_{D S}=\frac{H}{B} S_{M S} \\
& S_{D 1}=H_{M 1}
\end{aligned}
$$

Or, in city's code adoption ordinance:

"In recognition of the fact that a more resilient city can be built at a small marginal cost, and that the public prefers resilient buildings, the 2012 edition of the International Building Code is adopted with the exception that all occupied buildings, where it refers to ASCE 7-10, all values of $I_{e}$ in ASCE 7-10 Table 1.5-2 shall be taken as 1.5." 


\section{Let's consider impairment}

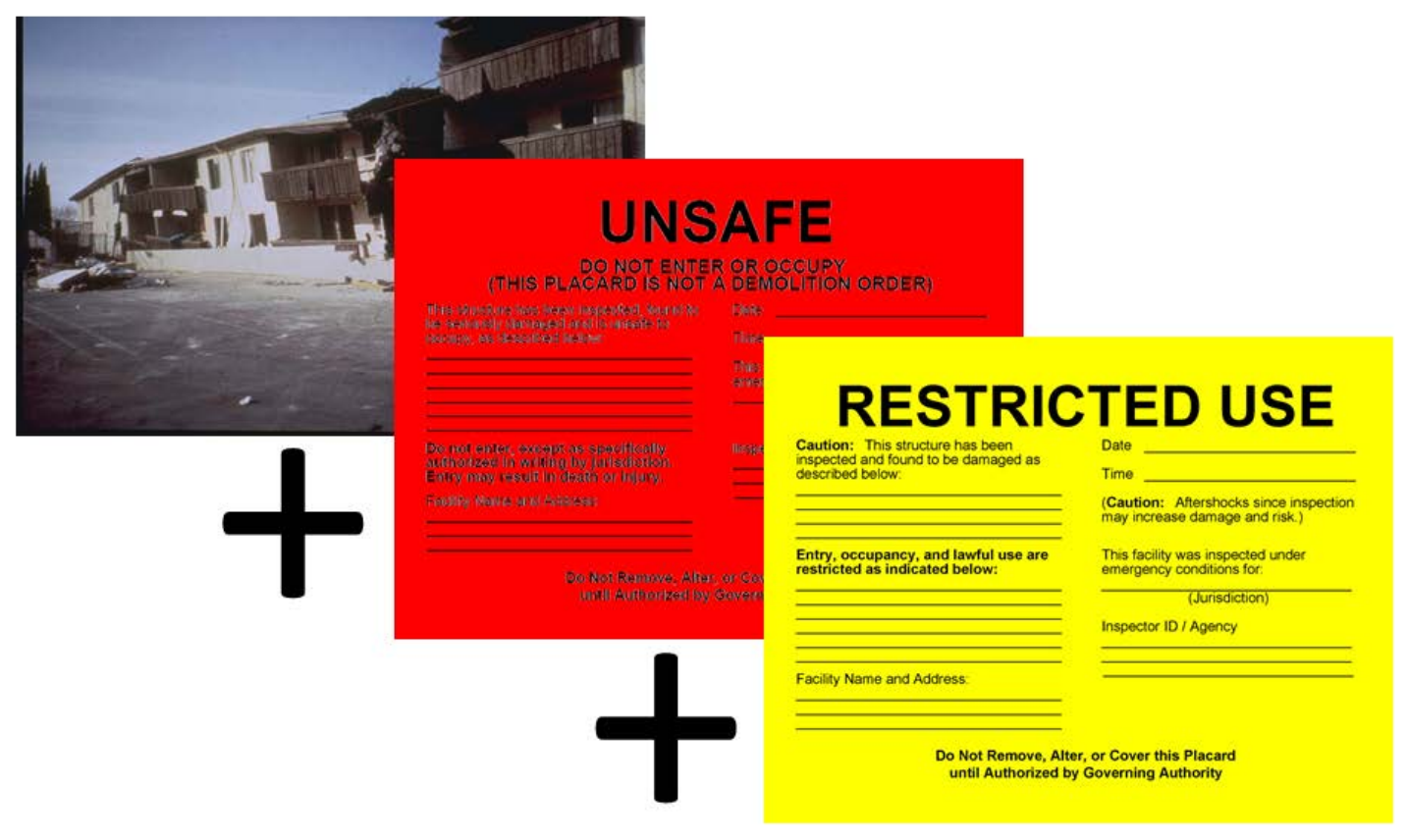

\section{Impairment $\rightarrow$ months of downtime}

Comerio (2006) on yellow and red tags in 1994

"[M]oderate damage to single-family homes was under-counted.... About 40 percent of homeowners began repairs within one year.... For the remainder, it took two to three years to resolve the insurance claim... often longer." 


\section{Collapse fragility}

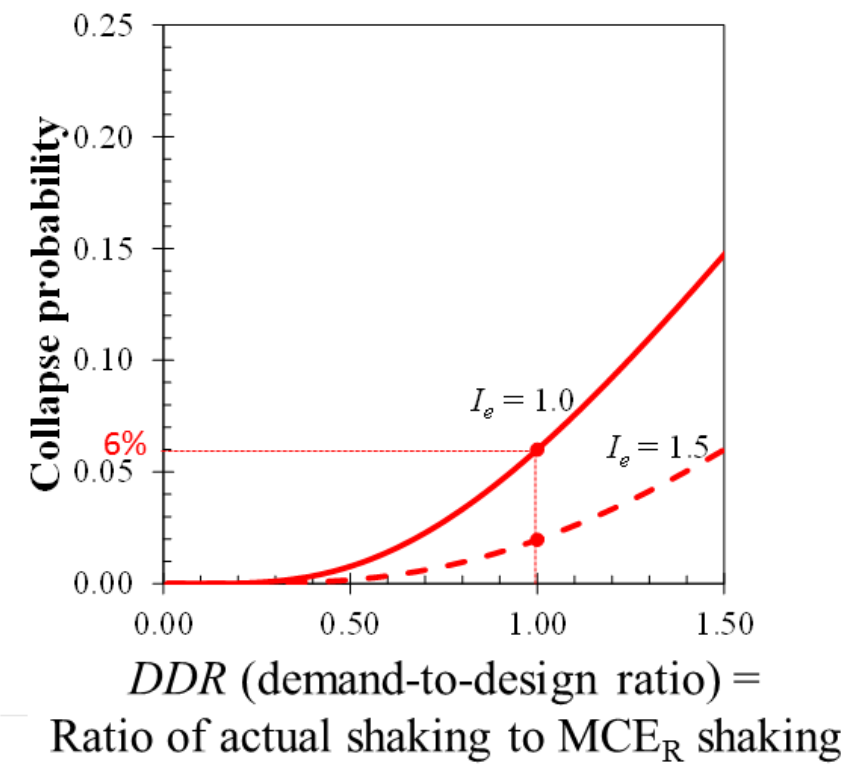

Add red tags

\section{Earthquake $\quad$ Red Collapse Ref}

1989, SF Marina Dist $110 \quad 7 \quad$ NIST (1990), Harris et al. (1990)

1989, Santa Cruz City 10040 SEAONC (1990), Fradkin (1999)

$1994 \quad 2,157 \quad 133$ EQE and OES (1995)

Total

\section{2,367 180 \\ 13 red:1 collapse}




\title{
Add yellow tags
}

\section{\begin{tabular}{l|l|l|l} 
Earthquake & Yellow & Red Ref
\end{tabular}}

1989 Loma Prieta $\quad 3,330 \quad 1,114 \quad$ SEAONC (1990)

Bay Area

1994 Northridge $\quad 9,445 \quad 2,290$ EQE and OES (1995)

Total

$12,775 \quad 3,404$

3.8 yellow:1 red

\section{Total impaired per collapse}

\author{
1 collapse \\ +13 red \\ $+13 \cdot 3.8$ yellow \\ = 63 impaired per collapse
}

So Napa 2014: 57 impaired per collapse 


\section{BLS might cost $1 \%$ more, because}

Memphis TN

Like I = 1.6 relative to 1999 SBC

IBC/Local code Cost

Building N-S E-W Premium

$\begin{array}{llll}\text { Apartment } & 1.36 & 1.46 & 0.9 \%\end{array}$

Office

1.14

1.14

$0.7 \%$

Retail

1.92

$1.94 \quad 0.2 \%$

Warehouse

1.88

1.88

$1.0 \%$

Hospital

0.96

0.96

$0.0 \%$

School

$1.49 \quad 1.49 \quad 0.4 \%$

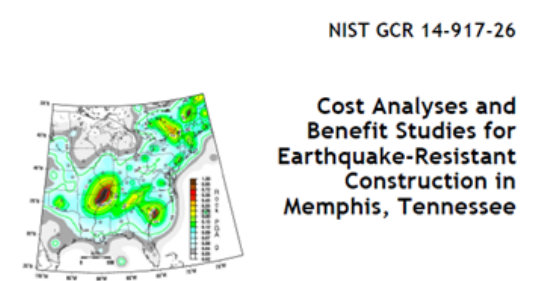

NEHRP Consultants Joint Venaure
Applitied Technology Cotonctl and the
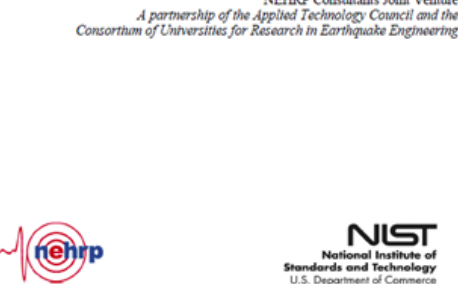

NIST

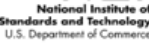

BLS might cost $1 \%$ more, because

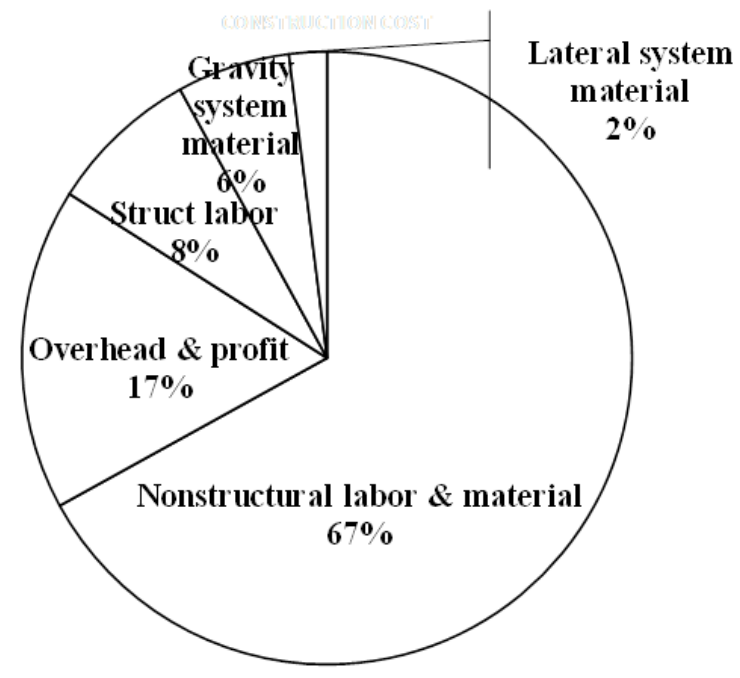




\section{BLS might cost $1 \%$ more, because}

CUREE-Caltech Woodframe Project

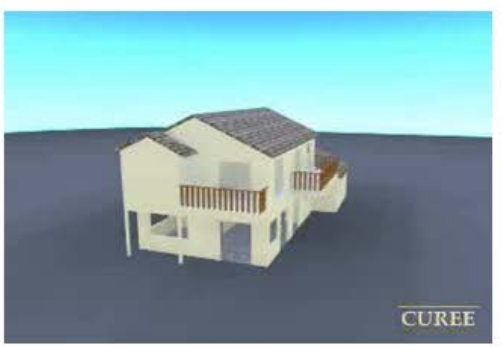

Several experts say so
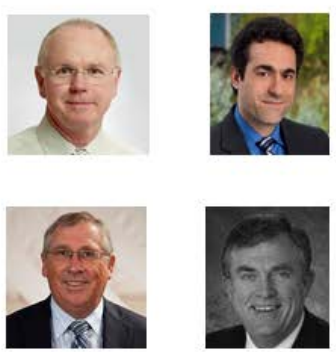

we do it all the time without noticing

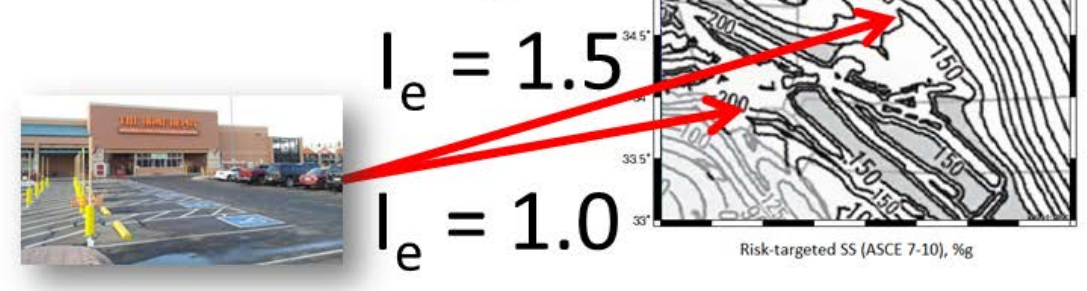

\section{BLS outcomes in Hayward Fault scenario}

$$
\mathrm{I}_{\mathrm{e}}=1.0
$$$$
\mathrm{I}_{\mathrm{e}}=1.5
$$

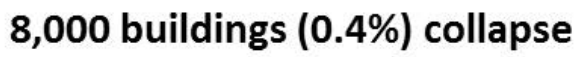

\section{2,000 buildings $(0.1 \%)$ collapse}
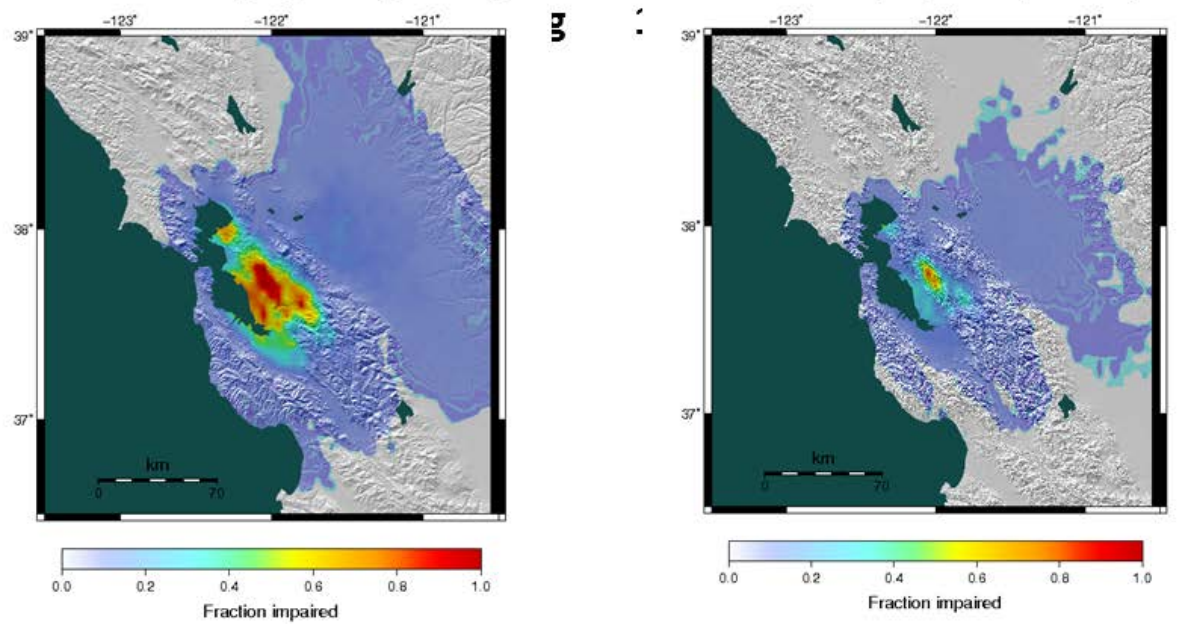


\section{BLS outcomes in Hayward Fault scenario}

\begin{tabular}{|l|c|c|}
\hline \multirow{2}{*}{$\begin{array}{l}\text { Building impairment in } \mathbf{M}_{\mathbf{w}} \\
\text { 7.0 Hayward Fault scenario }\end{array}$} & ...if all $\mathbf{2}$ million Bay Area buildings were new \\
\cline { 2 - 3 } Collapsed & All with $I_{e}=1.0$ & All with $I_{e}=1.5$ \\
\hline Red tagged & 8,000 & 2,000 \\
\hline Yellow tagged & 102,000 & 27,000 \\
\hline Total impaired buildings & 390,000 & 100,000 \\
\hline People in impaired buildings & 500,000 & 130,000 \\
\hline Businesses in imp. buildings & $1,500,000$ & 390,000 \\
\hline Impaired buildings & 150,000 & 39,000 \\
\hline Marginal cost & $24 \%$ & $6 \%$ \\
\hline
\end{tabular}

\section{Similar outcome for SoCal}

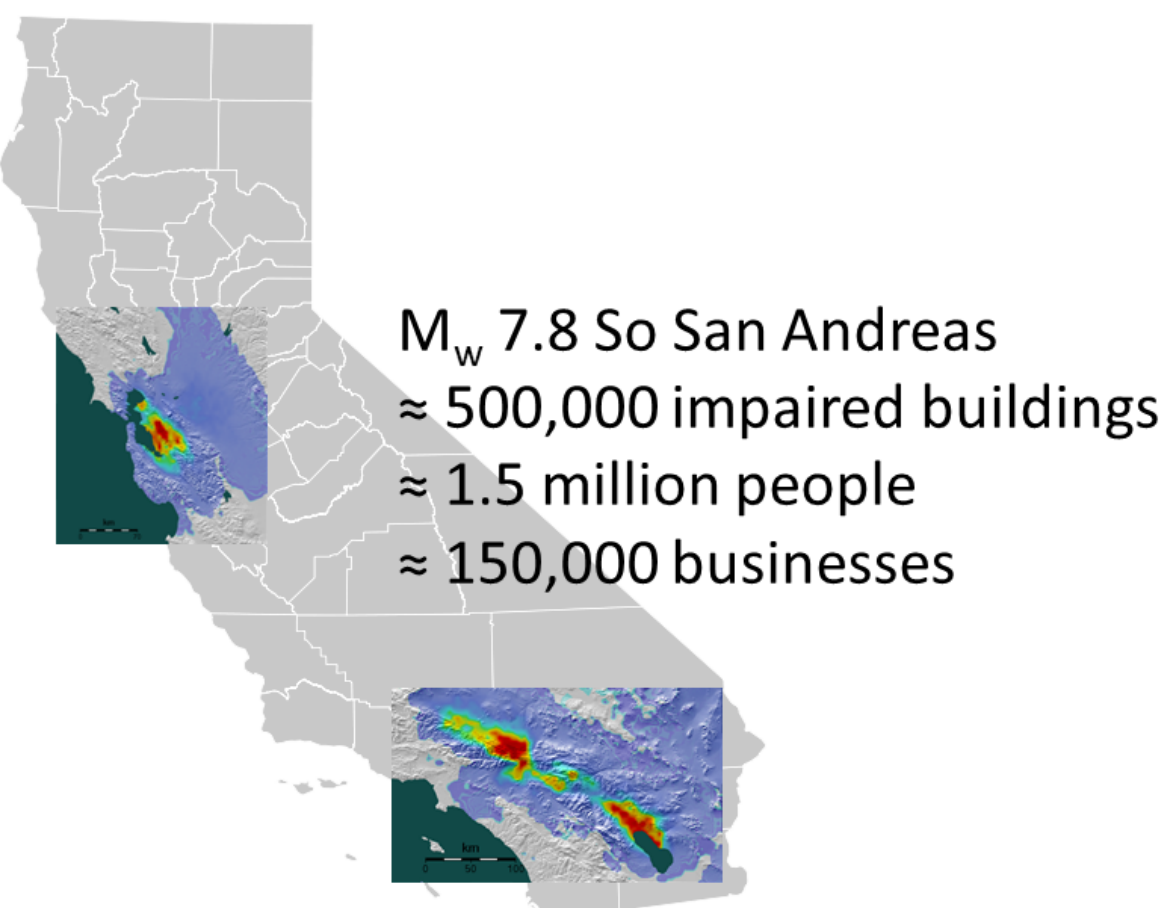




\section{Conclusions}

1. Life-safety design seems to lead to mass outmigration in a large urban earthquake

2. $50 \%$ stronger buildings can meet a $\approx 95 \%$ shelter-inplace objective for $\approx 1 \%$ more cost

What performance does the public prefer? 


\section{How much does the public know?}

Lucy Jones, pers. comm., 19 Nov 2013:

City councils and mayors "absolutely do not know" about the life-safety objective \& how damaged a code-compliant building stock will be in the aggregate, and are unsatisfied when they do learn of it.

\section{What does the public prefer?}

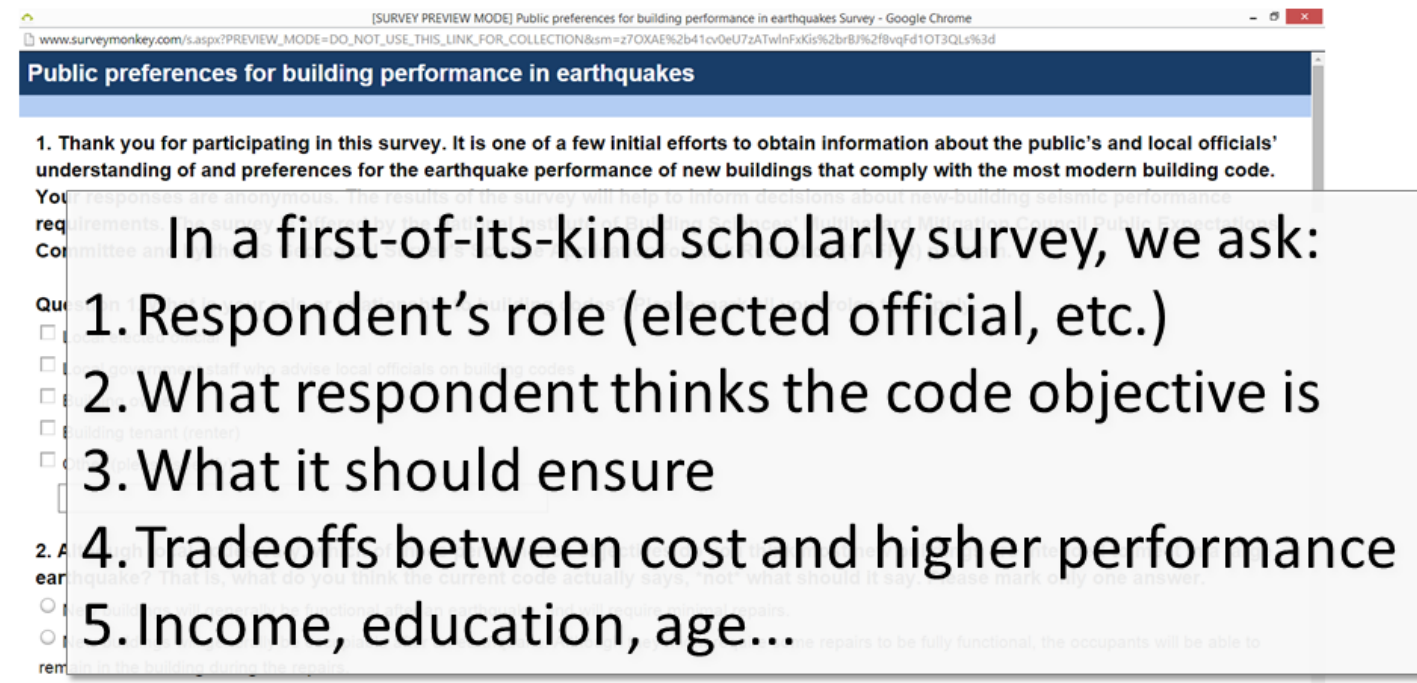

New buildings are safe enough that occupants won't be killed, but are not generally intended to be occupiable after the earthquake. That is, a person will be 
People in California and Central US prefer \& are willing to pay for better performance

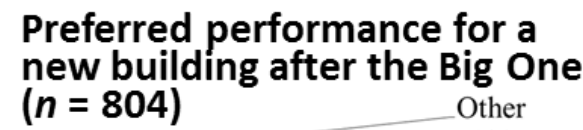

$(n=804)$ $2 \%$

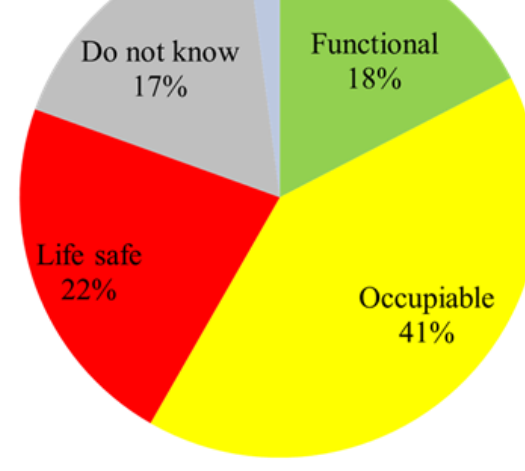

What would you be willing to pay for occupiable or functional?

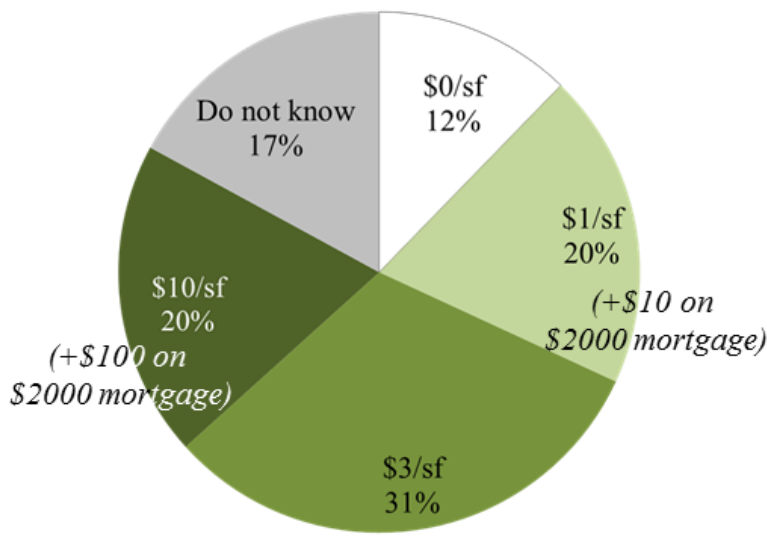

$(+\$ 30$ on

$\$ 2000$ mortgage)

Owners would accept mandatory resilient design

L. Jones 2015 (written commun):

"Most members of BOMA know the code is life safety but they told me they wished it was higher. They don't want to own a building that will be a total loss, but they can't afford to do it alone and be more expensive than their competitors." 
Higher strength does not drive away development

250 Howard St, San

Francisco $\mathrm{CA}, \mathrm{S}_{\mathrm{M} 1}=0.9 \mathrm{~g}$
3555 Caesar Chavez St, San

Francisco $\mathrm{CA}, \mathrm{S}_{\mathrm{M} 1}=1.05 \mathrm{~g}$
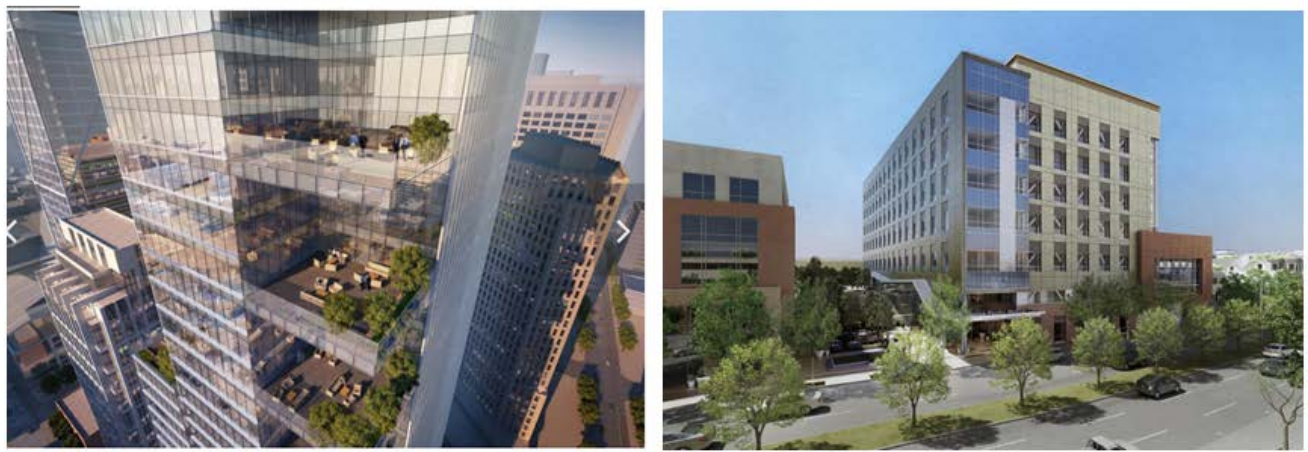

Higher strength does not drive away development

521 Parnassus, San Francisco $\mathrm{CA}, \mathrm{S}_{\mathrm{M} 1}=1.175 \mathrm{~g}$

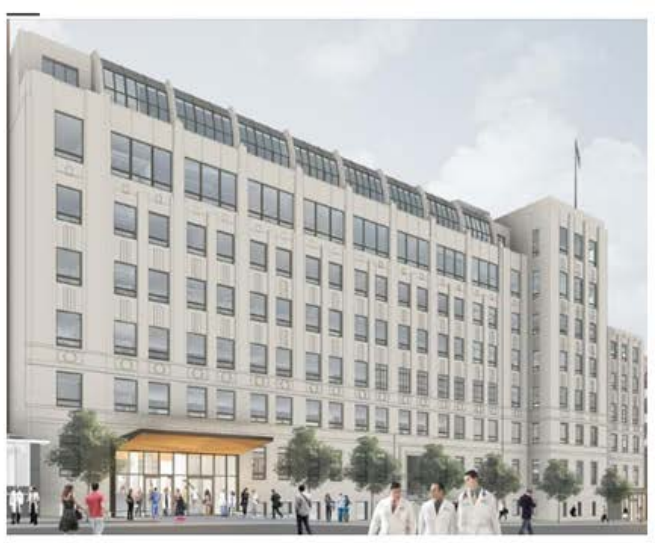

2800 Sloat St, San Francisco

$\mathrm{CA}, \mathrm{S}_{\mathrm{M} 1}=1.70 \mathrm{~g}$

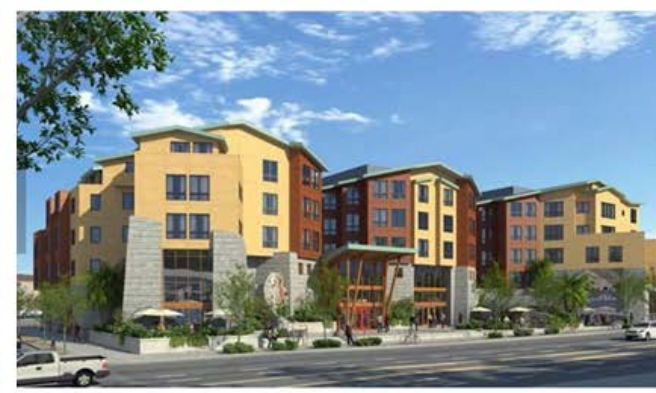




\section{Higher strength does not drive away development}

San Francisco developers said a local code that drove up cost by $1 \%$ but made new buildings survive the Big One, they would not relocate projects to nearby cities without that code:

"It wouldn't matter (wouldn't move location). Profit \%'s so high right now if you can find a good project that $1 \%$ on costs does not change the attractiveness of the deal very much."

"That most likely would not affect a decision on whether or not to build in SF. Usually our development decisions are based on rate of return. If for whatever reason it greatly changed our rate of return it would make a difference."

\section{Conclusions}

3. First-of-its-kind population survey of CA \& central US adults shows that people expect $\&$ are willing to pay for mandatory resilient design

4. Commercial building owners also want mandatory, though not voluntary, BLS design 


\section{New water supply model with lifeline restoration, interaction, \& resilience measures}

\section{Water is the biggest loss in ShakeOut}

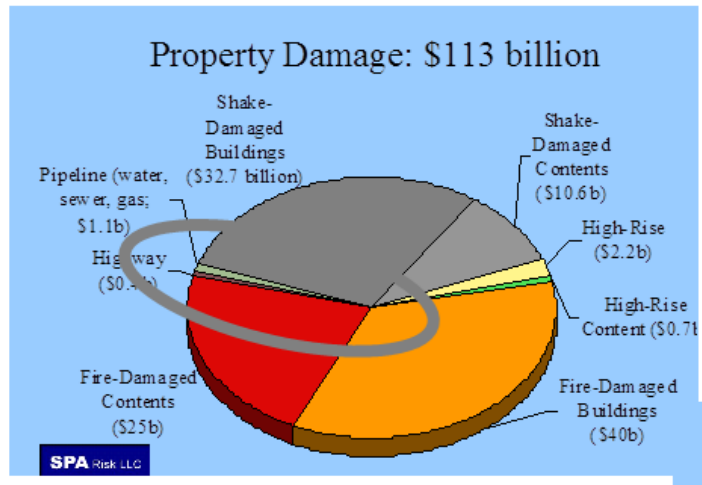

Property damage: $\$ 113 \mathrm{~B}$, Pipeline repairs: $\$ 1 \mathrm{~B}$

Business Interruption: \$96 billion

Business interruption: \$96 B Water supply: \$53 B 
- Asset definition, e.g., pipeline network

- Excitation e.g., shaking at location q, time t

- Damage, e.g., pipe breaks at q \& t

- Repairs, e.g., repaired breaks at q by time $t$

$\mathrm{R}$

- Service, e.g., people with water at q and t

- Loss, e.g., total economic impact

\section{Damage analysis}

Number of pipe breaks \& leaks

\section{Damage from shaking, liquefaction, landslides, coseismic slip, \& afterslip}




\section{Initial service given damage, when $H \& H$ (e.g., EPANET) is too costly}

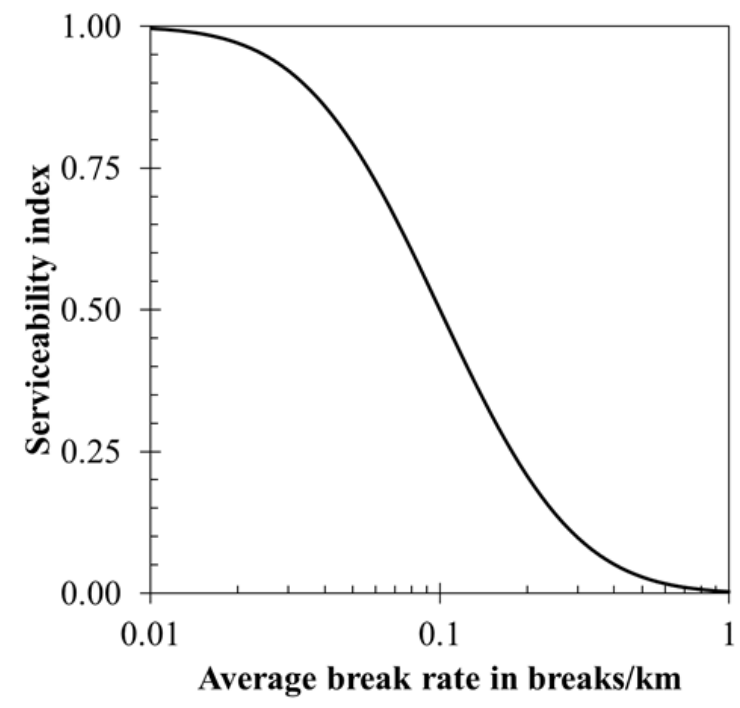

Lifeline interaction, when you can do system-ofsystems analysis

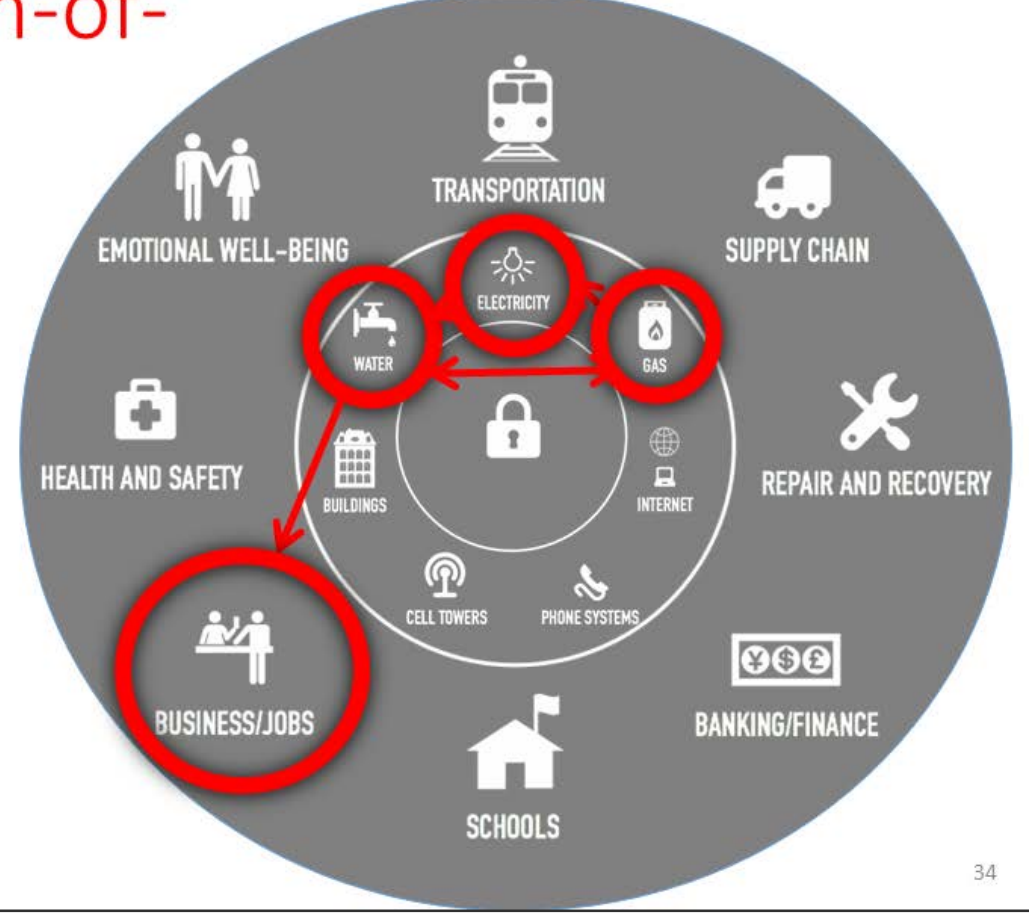


Lifeline interaction, when you can't do system-ofsystems analysis

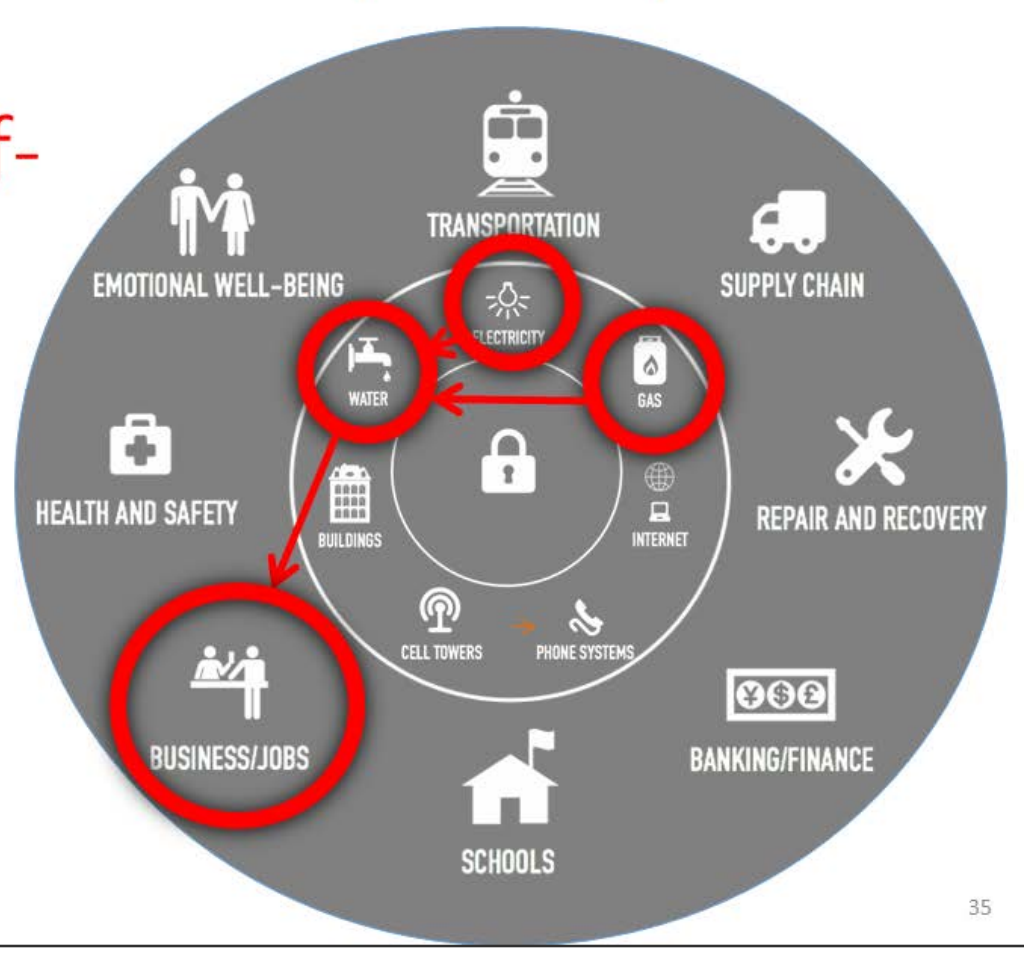

\section{Lifeline interaction as supply chain}

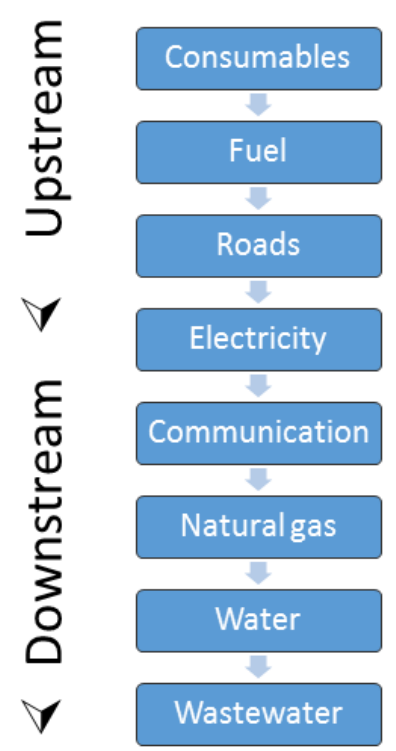




\section{Repairs per unit time}

$$
f(t)=f_{0} \cdot w(t) \cdot c(t) \cdot\left(\prod_{i}\left(1-u_{i} \cdot\left(1-g_{i}(t)\right)\right)\right)
$$

$f_{0}=$ normal productivity (repairs/crew.hour)

$w(t)=$ fraction of day that crews work

$c(t)=$ number of repair crews operating at time $t$

$u_{i}=$ importance coefficient (how lifeline or resource $i$ can slow a repair, e.g., extra time to coordinate repairs without cellphone service)

$g(t)=$ flow of resource $i$ at time $t$

\section{Service restoration}

$$
V(n)=V_{0}+\left(M-V_{0}\right) \cdot\left(\frac{n}{r}\right)^{a}
$$

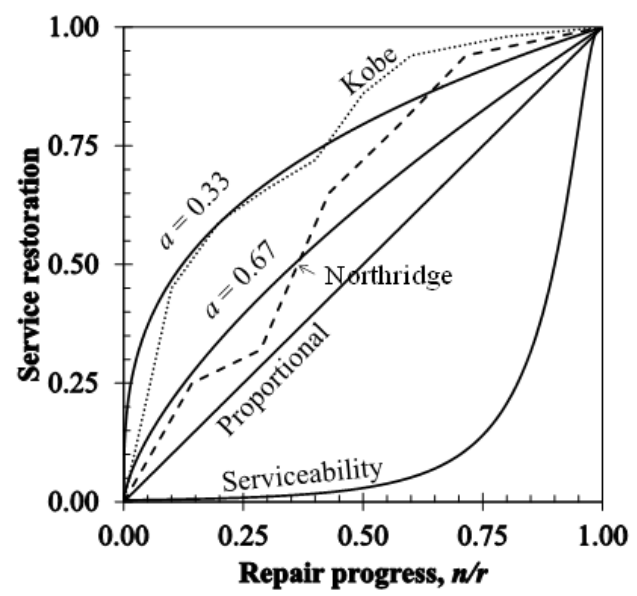




\section{Loss}

Sophisticated \& hard: computable general equilibrium

Simpler \& easier: Input-output

Dumb \& easy: Loss $=\$ /$ service $/$ day $\cdot$ service-days lost

\section{Asset definition: EBMUD pipes}

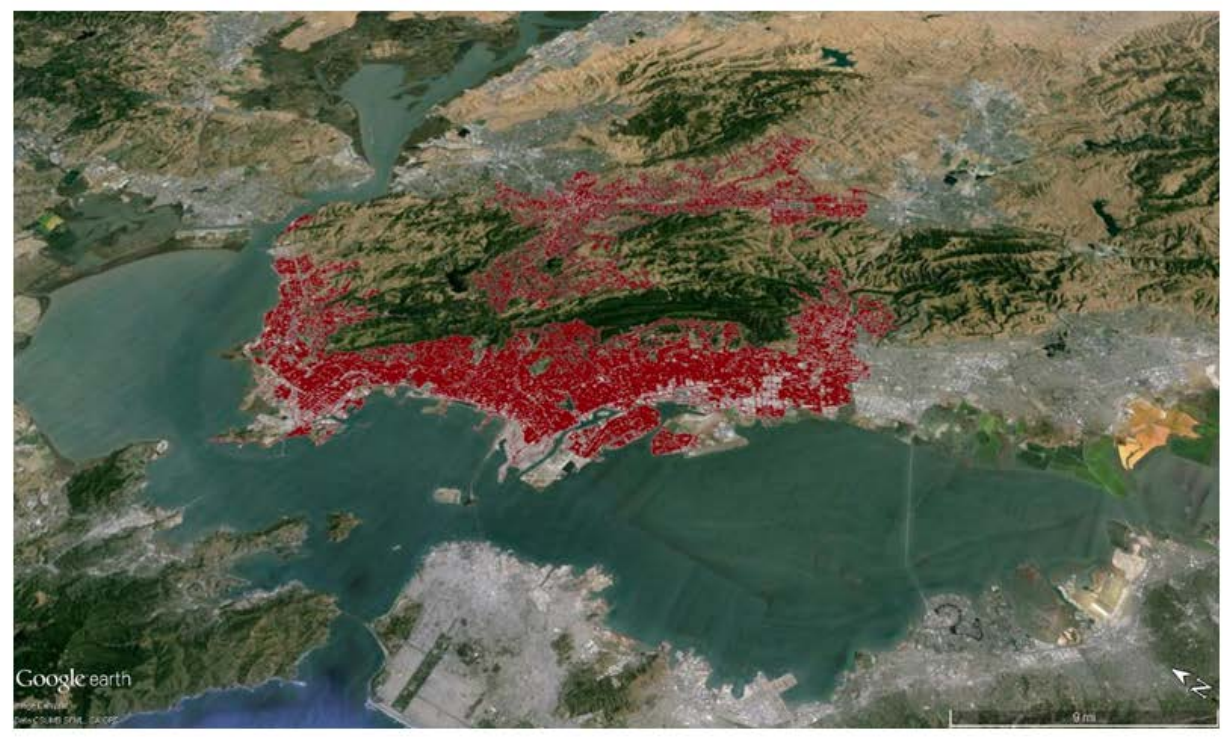




\section{Aagaard et al. (2010) $M_{w} 7.0$ Hayward Fault, Oakland epicenter}

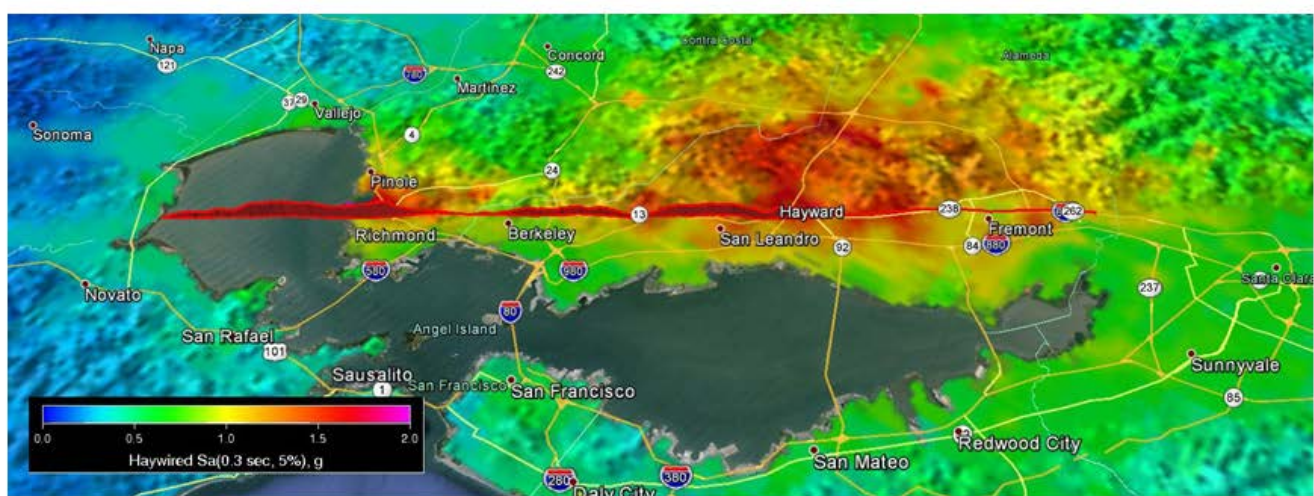

\section{Mainshock damage}

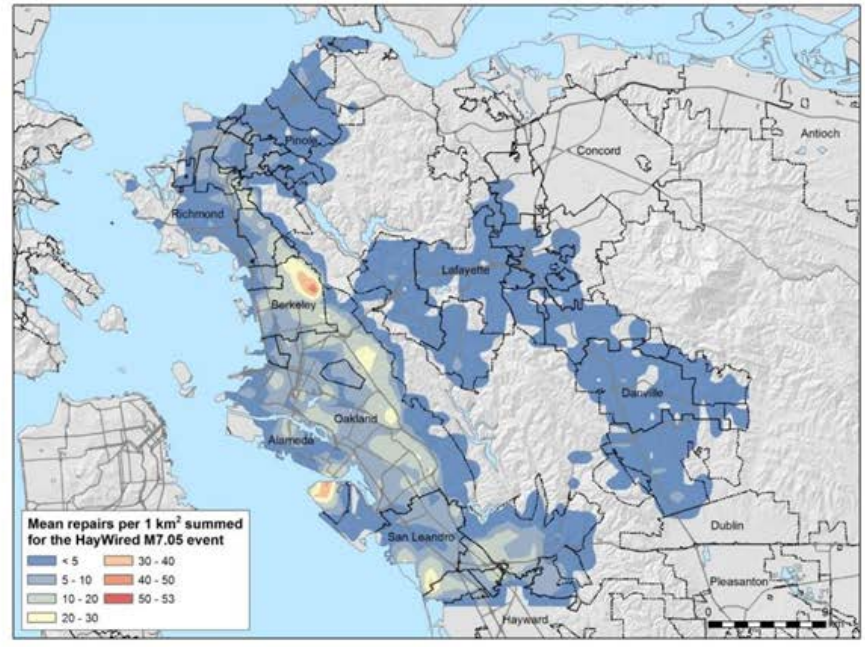




\section{Oakland aftershock}

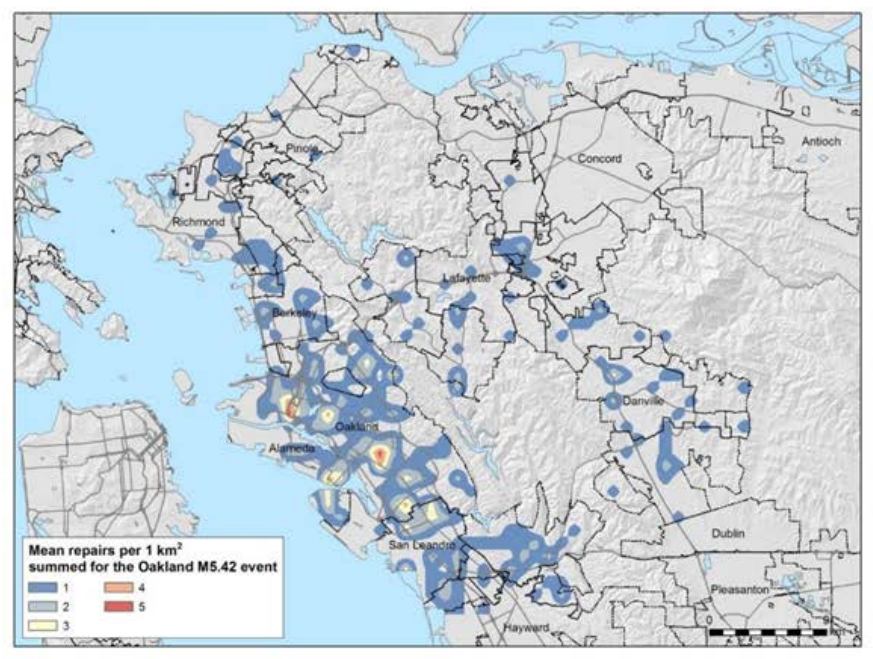

\section{Initial level of service}

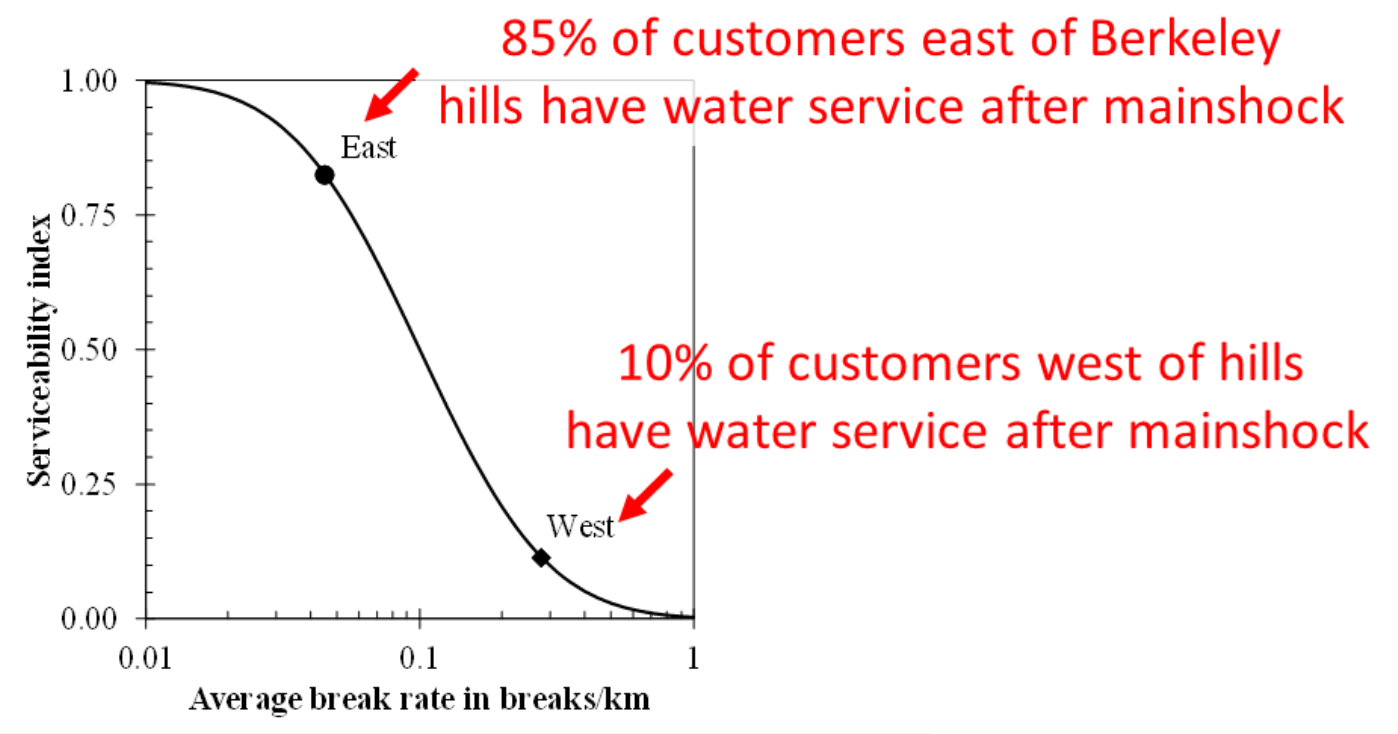




\section{Resources}
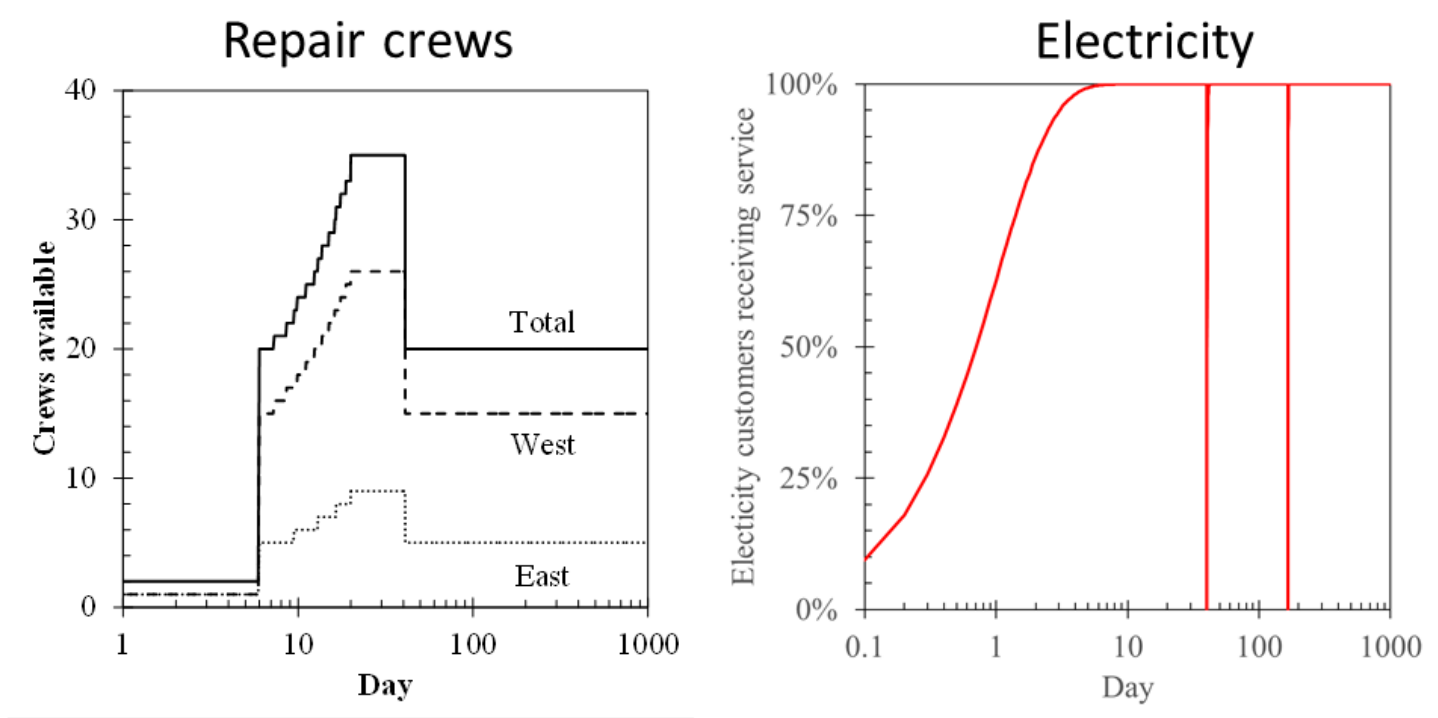

Repair progress

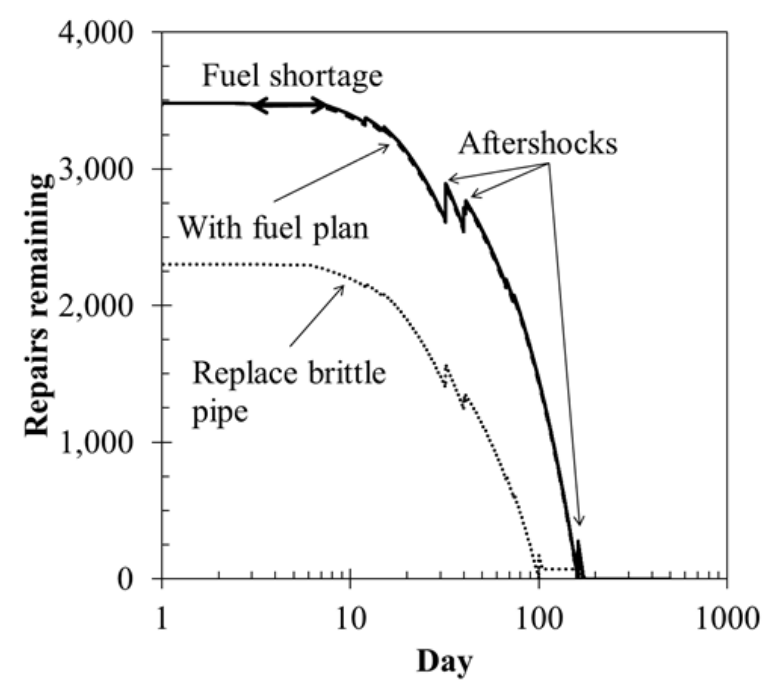


A new model of water restoration with lifeline interaction, fuel, crews, repair resources....

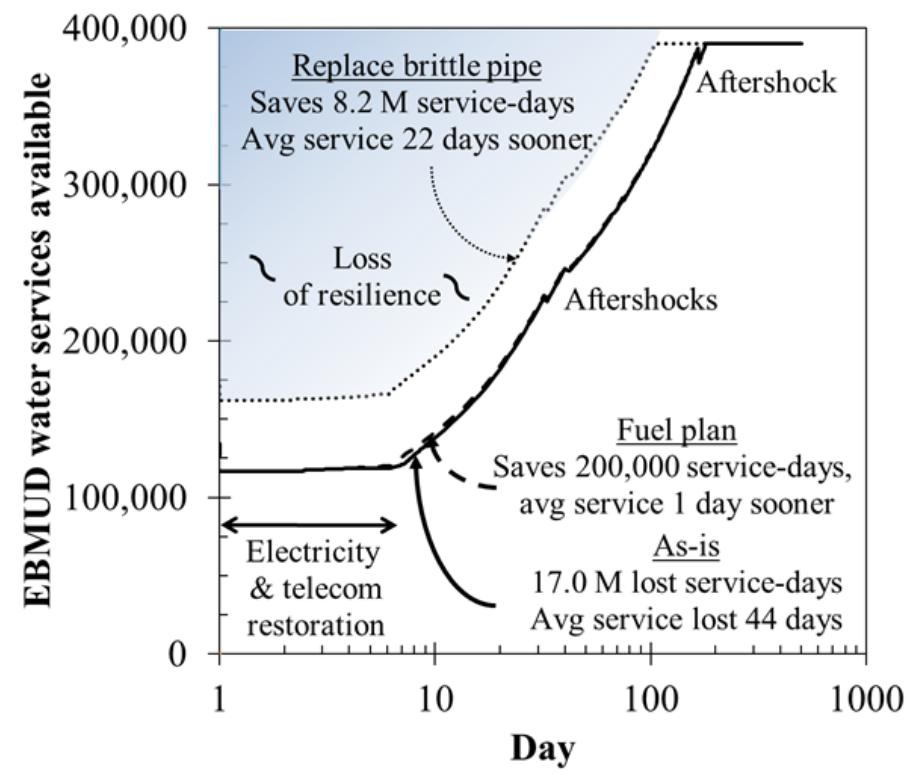

\section{Resilience (EBMUD water)}

\begin{tabular}{|c|c|c|c|c|}
\hline Condition & $\begin{array}{c}\text { Lost service } \\
\text { days }\end{array}$ & $\begin{array}{c}\text { Resilience } \\
\text { benefit } \\
\text { (service days) }\end{array}$ & $\begin{array}{c}\text { Avg } \\
\text { restoration } \\
\text { (days) }\end{array}$ & $\begin{array}{c}\text { Resilience } \\
\text { benefit }\end{array}$ \\
\hline
\end{tabular}
As-is
$17,000,000$
0
44
0

Fuel plan

$16,800,000 \quad 200,000$

43

$\$ 150$ million

Replace all fragile pipe

$8,800,000$

$8,200,000$

22

\$6 billion 


\section{Conclusions}

5. New model to estimate water restoration in terms of service-days lost considering

- Lifeline interaction w/o system-of-systems

- Human agency

- Time-varying human resources and repair supplies

- Resilience options

- Societal economic losses

- Stochastic

- Limited validation by two water agencies

\section{Open questions}

- Human agency

- How long will people tolerate dry taps?

- What motivates \& restrains utility resilience investments?

- More validation, e.g., versus 2014 South Napa

- Better $t=0$ service model short of H\&H model 


\section{A new empirical model of urban search and rescue needs for collapsed buildings and elevators without power}

\section{A model of USAR demands, building collapse only}
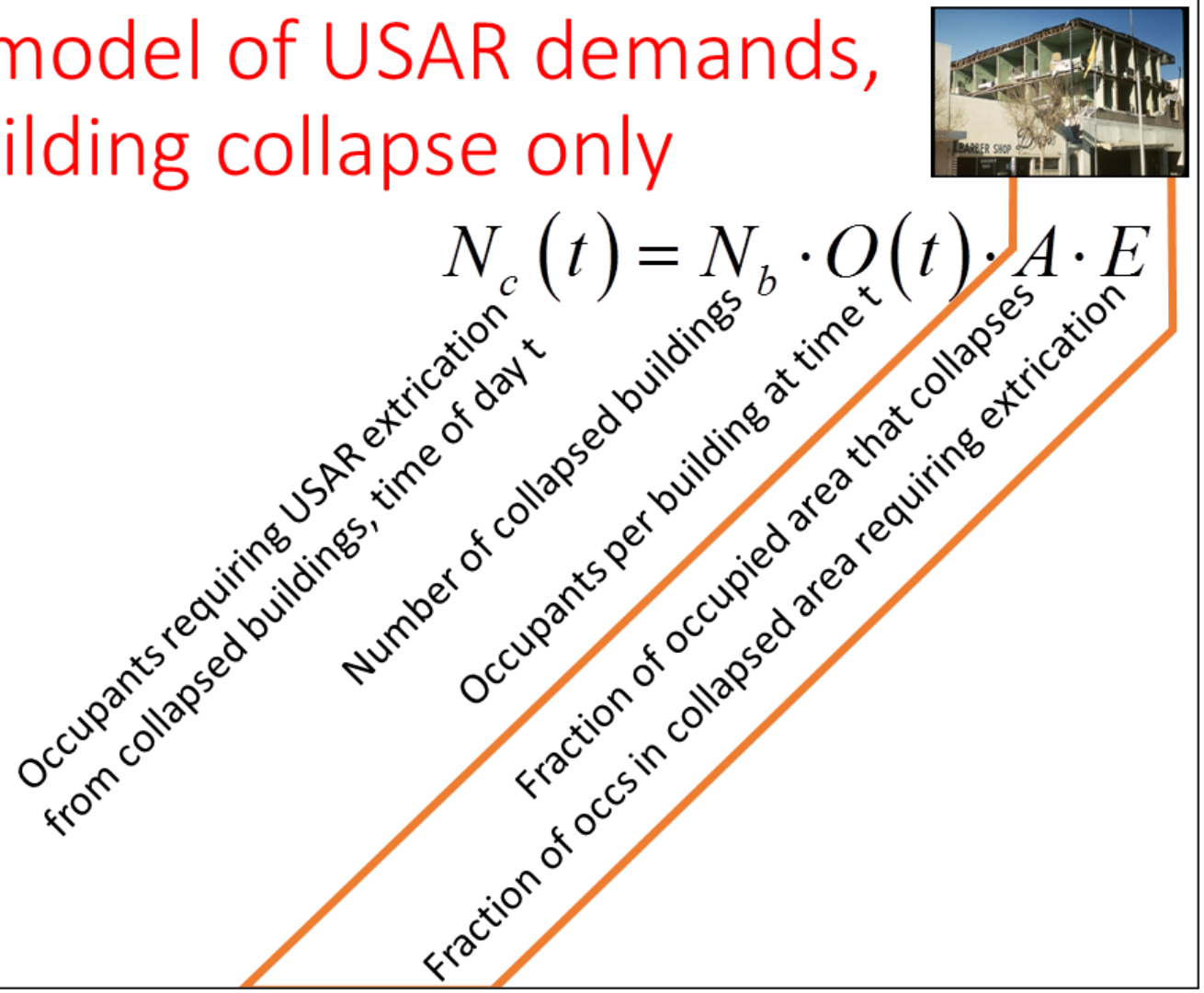

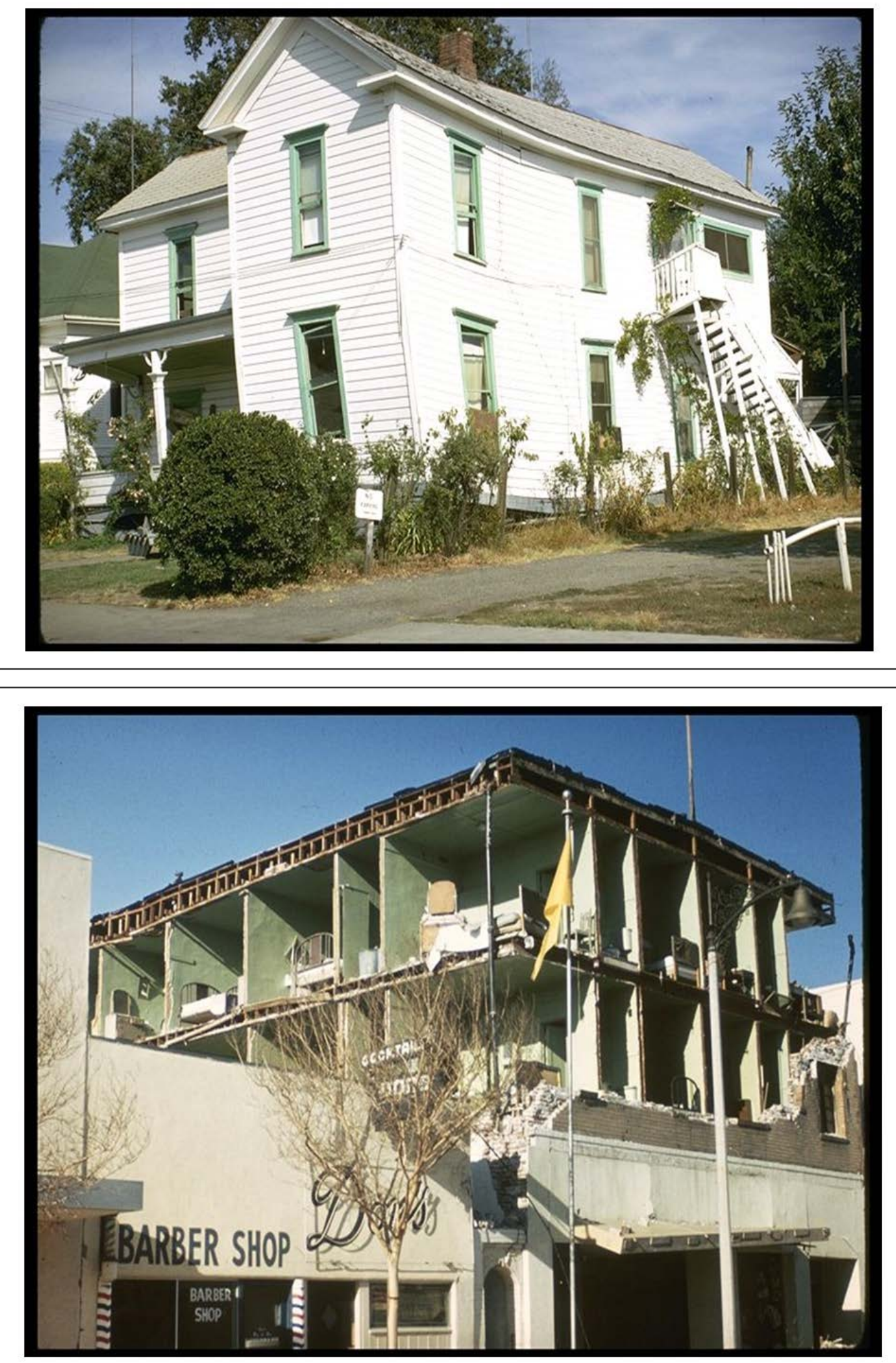


\section{Survey of NISEE e-Library images CA collapses since 1965-2014}

Material

All

Tilt-up concrete

Other concrete

Unreinforced masonry

Wood

All except URM

All except chimneys
Count Collapsed area

73

$23 \%$

14

9

18

32

54

66
$17 \%$

$50 \%$

$28 \%$

$17 \%$

$22 \%$

$25 \%$
Fraction with heavy debris

0.66

0.10

0.94

0.98

0.66

0.56

0.65

\section{Conclusions}

6. New empirical model of USAR demands based on photo evidence from past 50 years of collapses in California earthquakes

- Many more people may be trapped in stalled elevators than in collapsed buildings 
Safety benefit of earthquake early warning (EEW) combined with drop, cover, and hold on (DCHO)

How many more people complete DCHO with EEW?

Number of nonfatal injuries $\quad x$ fraction who can complete with various warning times DCHO within warning time
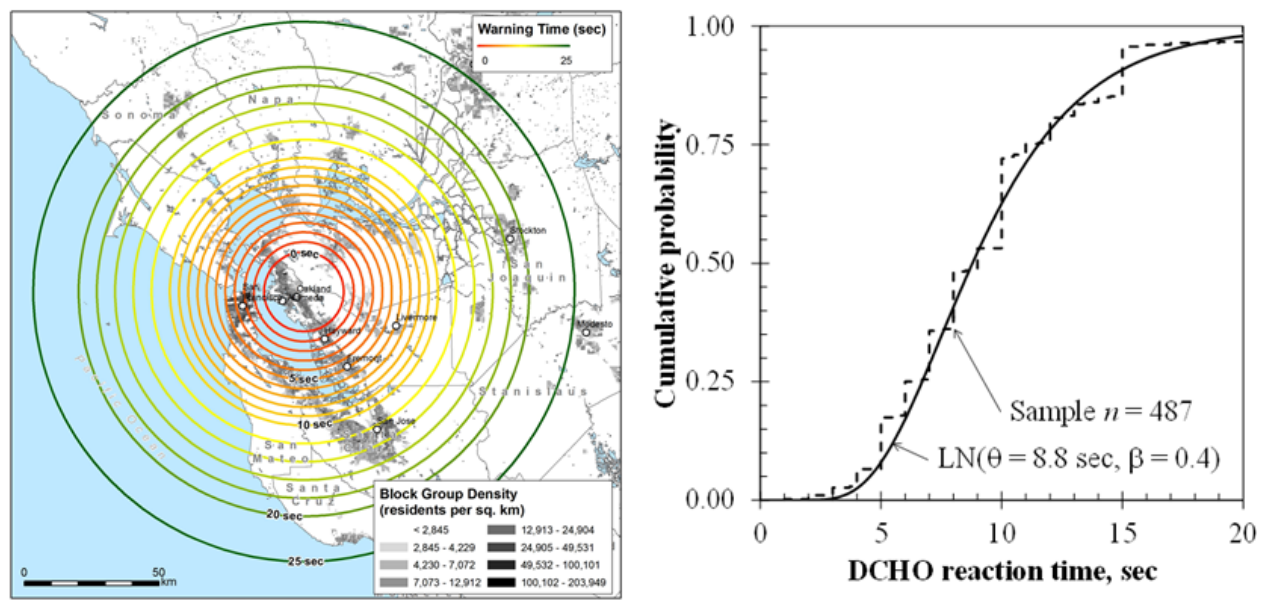


\section{Benefit of EEW + DCHO}

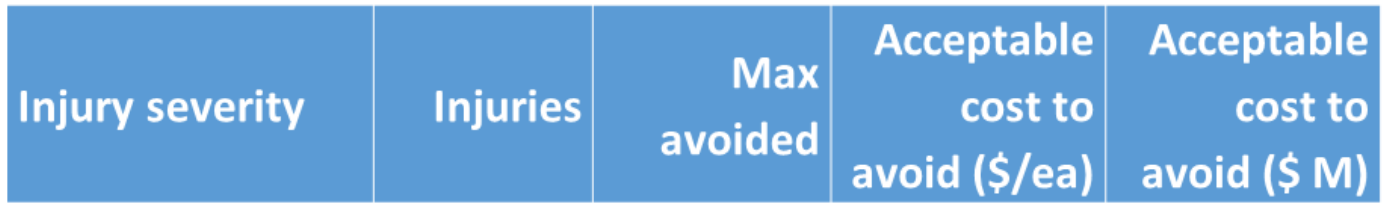

\begin{tabular}{|c|c|c|c|c|}
\hline Basic medical aid & 14,081 & 1,216 & $\$ 28,000$ & $\$ 34$ \\
\hline Moderate, not & 3,491 & 218 & $\$ 660,000$ & $\$ 144$ \\
\hline
\end{tabular}

life threatening

Serious, not fatal

558

$34 \$ 3,700,000$

$\$ 127$

Fatal

971

$0 \$ 9,400,000$

0

Total

19,101

1,468

$\$ 305$

\section{Conclusions}

7. New survey of adults' ability to complete DCHO in the absence of strong motion

- EEW + DCHO could prevent 1,500 additional injuries

- Acceptable cost to avoid those injuries $=\$ 300 \mathrm{M}$ 


\section{Questions?}

Keith.porter@Colorado.edu

\section{References}

- Porter, K., 2016. Not safe enough: the case for resilient seismic design. 2016 SEAOC Convention Maui HI, October 12 - 15, 2016. http://www.sparisk.com/Porter-2016-SEAOC-Resilience.pdf

- Porter, K., 2016. Damage and Restoration of Water Supply Systems in an Earthquake Sequence. Structural Engineering and Structural Mechanics Report Series 16-02, University of Colorado Boulder, 116 p., http://www.colorado.edu/ceae/node/1092/attachment

- Porter, K., 2016. An Earthquake Urban Search and Rescue Model Illustrated with a Hypothetical Mw 7.0 Earthquake on the Hayward Fault. Structural Engineering and Structural Mechanics Report Series 1603, University of Colorado Boulder, 155

p., http://www.colorado.edu/ceae/node/1094/attachment

- Porter, K., 2016. How Many Injuries can be Avoided Through Earthquake Early Warning and Drop, Cover, and Hold On? Structural Engineering and Structural Mechanics Report Series 16-04, University of Colorado Boulder, 32 p., http://www.colorado.edu/ceae/node/1096/attachment 


\section{Other resources}

USGS SAFRR (ShakeOut, ARkStorm, ...)

https://goo.gl/8aay3E

Resilient building code:

www.sparisk.com/pubs/Porter-2016-SEAOC-Resilience.pdf http://www.sparisk.com/pubs/Porter-2015-EQS-SafeEnough.pdf http://www.sparisk.com/pubs/Davis-Porter-2016-EQSEthics.pdf 
Resilience Modeling Innovations of the HayWired Planning Scenario

\section{Bibliography}

[1] Porter, K.A., 2016. Not safe enough: the case for resilient seismic design. 2016 SEAOC Convention,October 12-15, 2016, Maui HI, http://www.sparisk.com/pubs/Porter-2016-SEAOC$\underline{\text { Resilience.pdf }}$

[2] Porter, K.A., 2016. Not Safe Enough-A Survey of Public Preferences for the Seismic Performance of New Buildings in California and the New Madrid Seismic Zone. Structural Engineering and Structural Mechanics Report SESM 16-01, University of Colorado Boulder, http://www.colorado.edu/ceae/node/1090/attachment

[3] Porter, K.A., 2016. Damage and Restoration of Water Supply Systems in an Earthquake Sequence. Structural Engineering and Structural Mechanics Report SESM 16-02, Universityof Colorado Boulder, http://www.colorado.edu/ceae/node/1092/attachment

[4] Porter, K.A., 2016. An Earthquake Urban Search and Rescue Model Illustrated with a Hypothetical Mw7.0 Earthquake on the Hayward Fault. Structural Engineering and Structural Mechanics Report SESM 16- 03, University of Colorado Boulder, http://www.colorado.edu/ceae/node/1094/attachment

Porter, K.A., 2016. How Many Injuries Can Be Avoided Through Earthquake Early Warning and Drop, Cover, and Hold On? Structural Engineering and Structural Mechanics Report SESM 16-04, University of Colorado Boulder, 32 pp. http://www.colorado.edu/ceae/node/1096/attachment 


\section{Keith A Porter, PE PhD Bio Sketch}

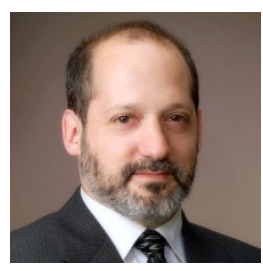

Dr. Porter is a Research Professor in Structural Engineering and Structural Mechanics at the University of Colorado Boulder and Principal of SPA Risk LLC. He specializes in risk from natural disasters and $2^{\text {nd }}$ generation performance-based earthquake engineering. Notable works include the $4: 1$ natural-hazard benefit-cost study for the US Congress (for which he is now leading an update for FEMA and others), the San Francisco Community Action Plan for Seismic Safety's soft-story study, and the engineering risk analyses of the USGS' ShakeOut, ARkStorm, Tsunami Scenario, and HayWired Scenario. He is a licensed Professional Engineer and author of 170 scholarly and professional works. 


\title{
Model Validation: The Joplin Tornado Hindcast
}

J.W. van de Lindt, N. Attary, H. Mahmoud, L. Peek, W. G. Peacock, S. van Zandt, J. TobinGurley, N. Rosenheim, M. Koliou, A. Graettinger, S. Smith, and S. Chen

\begin{abstract}
Many modeling techniques are being developed and used to assess the resilienc of communities to natural and other hazards around the world. These models require systematic validation using the results of real events to ensure that influencing factors not modeled are accounted for when needed. The NIST Center of Excellence: The Center for Risk-Based Community Resilience Planning is developing an Interdependent Networked Community Resilience Modeling Environment (IN-CORE) that will be a robust computational environment known as that allows users to optimize community disaster resilience planning and post-disaster recovery strategies intelligently using physics-based models of inter-dependent physical systems combined with socio-economic systems. The first such validation of IN-CORE v1.0 is the 2011 Joplin tornado. An EF5 tornado struck Joplin MO in May 2011, and became the costliest and deadliest single tornado in U.S. history. In this presentation, different sectors (such as buildings, electrical power network) of Joplin MO community and their dependencies, combined with social and economic aspects are all considered in the hindcast of the 2011 tornado. Building on the NIST investigation of the event in 2011, data on recovery was collected from the community in a recent field study by Center of Excellence (CoE) and were fed into the models developed by the CoE to estimate damage, loss, functionality and recovery of the community. This presentation focuses on the first phase of the modeling effort and explains the process of hindcasting for validation of a model that integrates physical, social, and economic systems. The simulation results were compared with post-disaster available data to check the accuracy of the models.
\end{abstract}




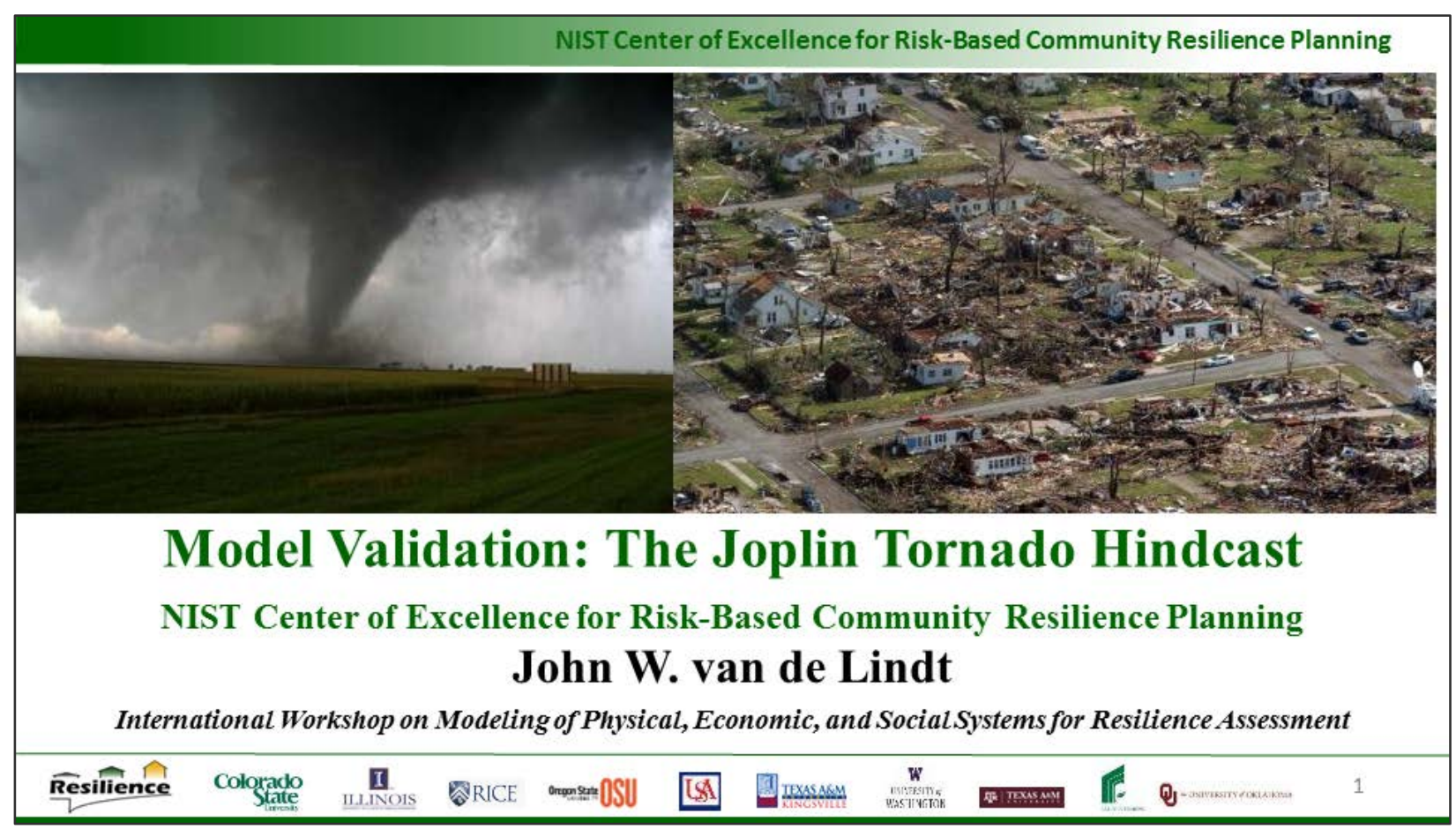

NIST Center of Excellence for Risk-Based Community Resilience Planning

\section{Alternative Titles:}

- Where the Rubber Meets the Road

- Data, what data ? ... OK, messy data.

- Imagine you know what stock is going up 1000\% tomorrow; do you invest? Not if you're doing a hindcast! 


\begin{tabular}{|lc|}
\hline \multicolumn{2}{|c|}{ Prest Center of Excellence for Risk-Based Community Resilience Planning } \\
Colorado State University: & Texas A\&M University: \\
- John van de Lindt & - Walt Peacock \\
- Navid Attary & - Shannon Van Zandt \\
- Lori Peek & - Nathanael Rosenheim \\
- Hussam Mahmoud & - Doug Wunneburger \\
- Jennifer Tobin-Gurley & \\
University of Alabama: & Missouri Southern State \\
- Andrew Graettinger & University: \\
- Shane Crawford & - Steve Smith \\
\hline Resiience & \\
\hline
\end{tabular}

\section{Outline:}

- 2011 Joplin MO Tornado (NIST Report)

- Field Study Data Collection \& Mapping

- Single Sector Validation: Electric Power Network

- Single Sector Validation: Buildings

- Multisector Validation: Buildings-EPN

- Socially Vulnerable Populations

- Next Steps for coupling infrastructure and recovery modeling 
NIST Center of Excellence for Risk-Based Community Resilience Planning

\section{Hindcasting}

Hindcast is a way of testing mathematical models; known or closely estimated inputs for past events, are entered into the model to see how well the output matches the known results.

- Single sector Damage Validation (Each of the Buildings and EPN Individually)

- Multisector Damage Validation (Buildings - EPN combined, including one-way dependencies)

- Social-Economic Modeling (in progress)

- Recovery Modeling (in progress)

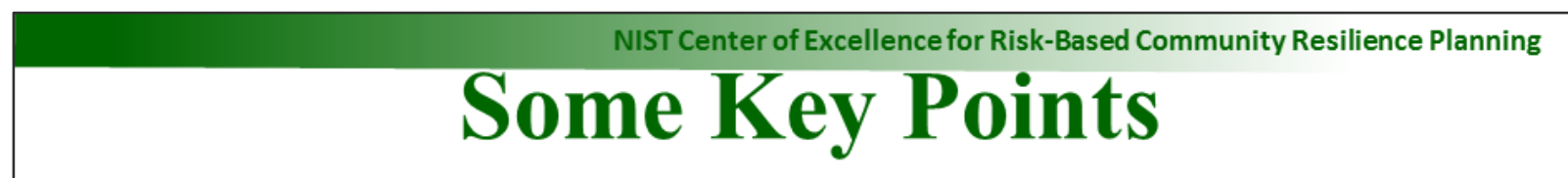

- Step back in time to 2010 - all maps, demographics, costs, information

- Initially conducting a damage comparison for single and coupled infrastructure

- Generic tornado wind model and known path

- Analysis to incorporate population dislocation models as a function of census data

- CGE model to compare economic recovery in one year intervals to present day 


\section{NIST Center of Excellence for Risk-Based Community Resilience Planning}

\section{Joplin, Missouri}

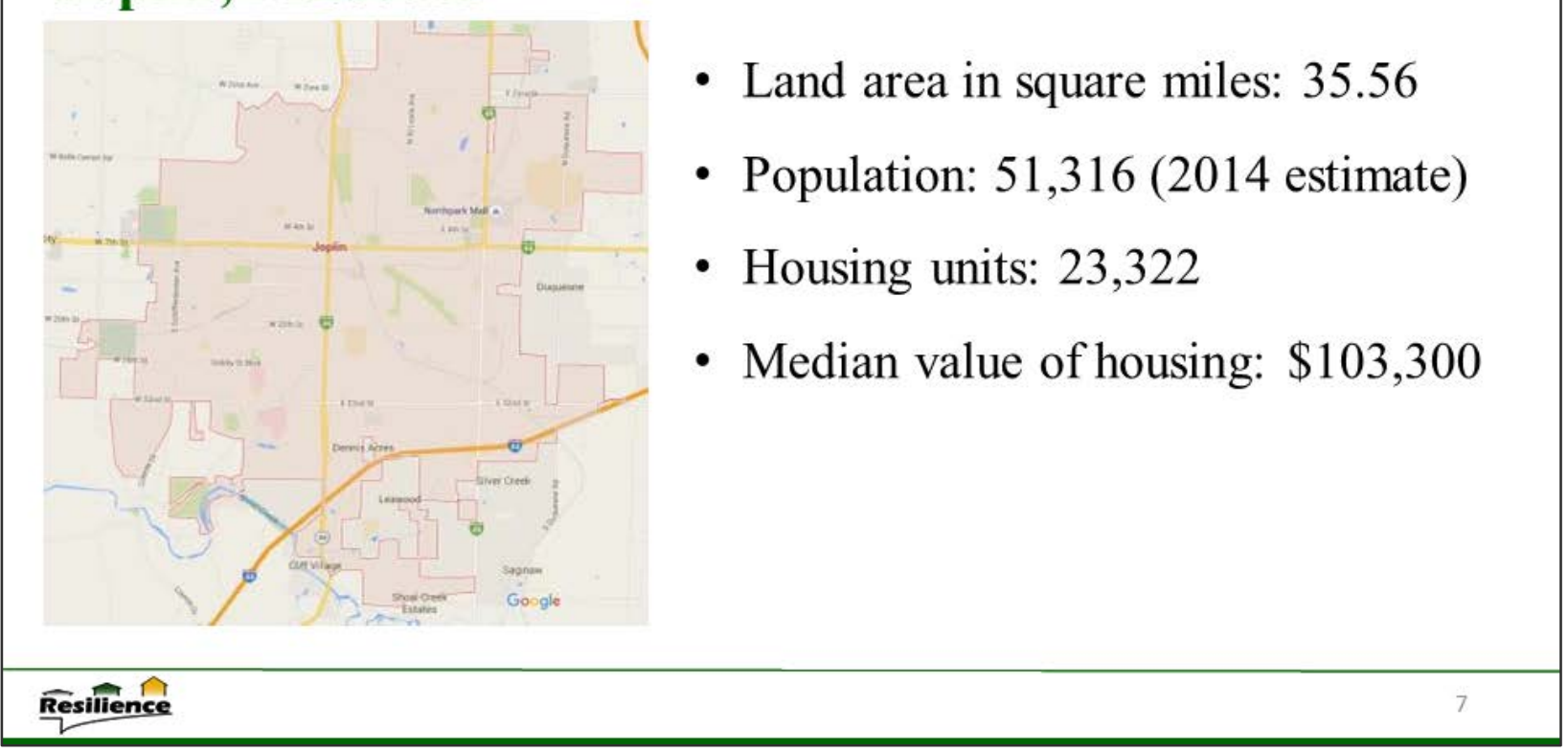

\begin{tabular}{|c|c|c|c|}
\hline \multicolumn{4}{|c|}{ NIST Center of Excellence for Risk-Based Community Resilience Planning } \\
\hline \multirow{2}{*}{\multicolumn{2}{|c|}{ Joplin, Missouri }} & Population & \\
\hline & & Day & 250,000 \\
\hline \multicolumn{2}{|l|}{ Economy } & Gender & \\
\hline \multirow{5}{*}{$\begin{array}{l}\text { In Civilian labor force, total, percent of population age 16+, 2010-2014 } \\
\text { In Civilian labor force, total, percent of population age 16+, 2010-2014 } \\
\text { Total accommodation and food services sales, } 2012(\$ 1,000)\end{array}$} & \multirow[b]{2}{*}{$66.0 \%$} & Female & $52 \%$ \\
\hline & & Male & $48 \%$ \\
\hline & $61.7 \%$ & \multicolumn{2}{|l|}{ Race } \\
\hline & & White alone & $84.7 \%$ \\
\hline & 199,817 & Hispanic & $4.7 \%$ \\
\hline \multirow{2}{*}{ Total health care and social assistance receipts/revenue, $2012(\$ 1,000)$} & $1,049,228$ & African-American & $3.6 \%$ \\
\hline & & Asian & $1.4 \%$ \\
\hline Total manufacturers shipments, $2012(\$ 1,000)$ & 691602 & Two or more races & $3.9 \%$ \\
\hline Total retail sales, $2012(\$ 1,000)$ & $1,819,702$ & \multicolumn{2}{|l|}{ Poverty Rate } \\
\hline \multirow{2}{*}{\multicolumn{2}{|c|}{ Total retail sales per capita, 2012}} & White Non-Hispanic residents & $15.8 \%$ \\
\hline & & Black residents & $61.2 \%$ \\
\hline \multirow{2}{*}{\multicolumn{2}{|c|}{ Income and Poverty }} & Hispanic or Latino residents & $22.5 \%$ \\
\hline & & American Indian residents & $18.9 \%$ \\
\hline Median household income (in 2014) & $\$ 37,889$ & Native Hawaiian and other Pacific Islander residents & $100 \%$ \\
\hline \multirow{2}{*}{ Per capita income (in 2014) } & \multirow{2}{*}{$\$ 23,043$} & Other race residents & $39.7 \%$ \\
\hline & & Two or more races residents & $31.9 \%$ \\
\hline Persons in poverty, percent & $19.0 \%$ & Children & $26.5 \%$ \\
\hline \multicolumn{2}{|l|}{ Transportation } & \multicolumn{2}{|l|}{ Causalities } \\
\hline Mean travel time to work (minutes), workers age $16+$ & 15.6 & $\begin{array}{l}\text { Male } \\
\text { Female }\end{array}$ & $\begin{array}{l}46 \% \\
54 \%\end{array}$ \\
\hline \multicolumn{2}{|l|}{ Source: US Census Bureau } & \multicolumn{2}{|c|}{ Source: http://www.citv-data.com/citv/Joplin-Missouri.html } \\
\hline Resilience & & & 8 \\
\hline
\end{tabular}


NIST Center of Excellence for Risk-Based Community Resilience Planning

\section{Joplin Tornado - May 22, 2011}

- EF5 multiple-vortex

- Fatalities:161

- Injured: 1150

- Total monetary loss: $\$ 2.8$ billion

- Costliest single tornado in US history

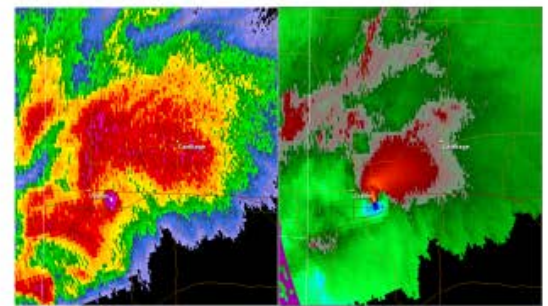

Radar image of the supercell that produced the tornado

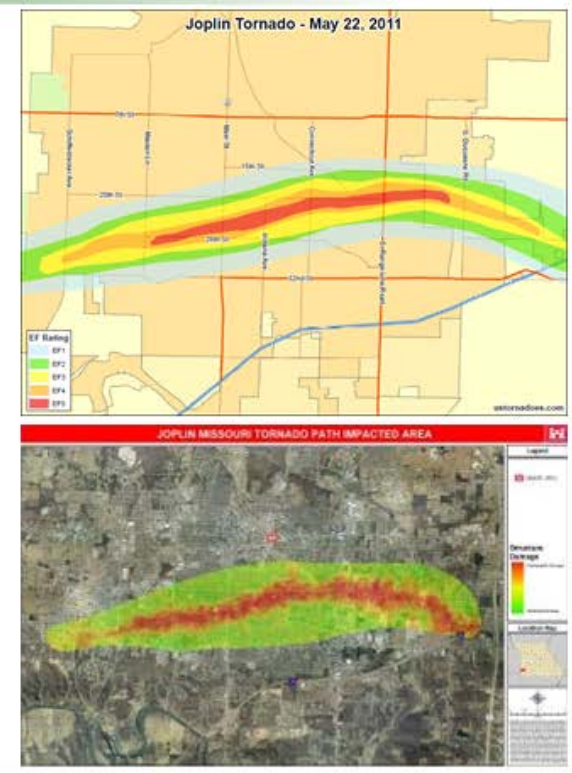

Resilience

NIST Center of Excellence for Risk-Based Community Resilience Planning

\section{Lifeline damage - Water network}

- 4,000 leaks in residential service lines \& 25 torn fire-service lines.

\section{Lifeline damage - Communication}

- Total of 21 cell towers were nonfunctional.

- Wireline network experienced damage to aerial fiber-optic and copper cables

\section{Lifeline damage - EPN}

- Transmission System - 135 transmission towers affected.

- Six power substations with step-down transformers initially impacted.

- Approximately 110 miles of aerial distribution line downed.

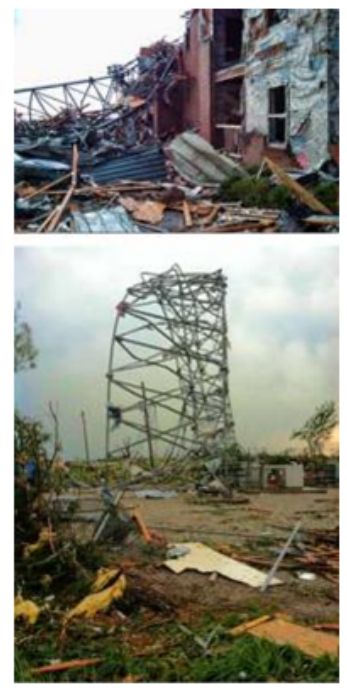




\section{Field Study Data Collection \& Mapping}

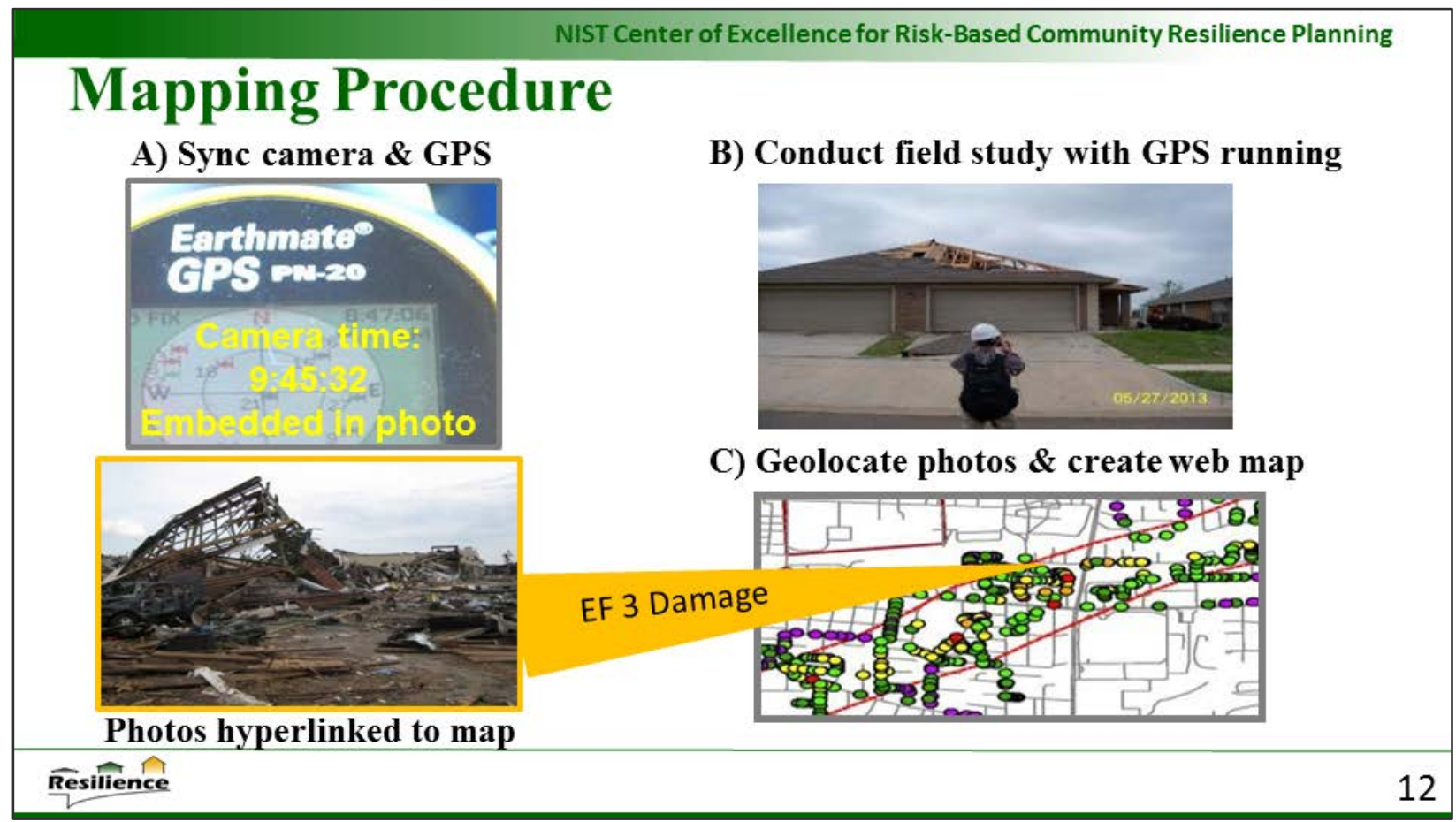


NIST Center of Excellence for Risk-Based Community Resilience Planning

\section{Data Collected in Joplin}

- Damage Assessment Data - May 2011

- 1300 EF-rated, 2600 total photographs

- Damage Contour: GIS-based interpolation of EF-rated photographs showing EF estimate at each location in the damaged area

- Case studies describing construction, failure mechanisms, etc.

- Joplin Hindcast Data - July 2016

- 2000 Recovery photographs

- Interviews with residents \& builders

$-360^{\circ}$ videos along streets in affected area

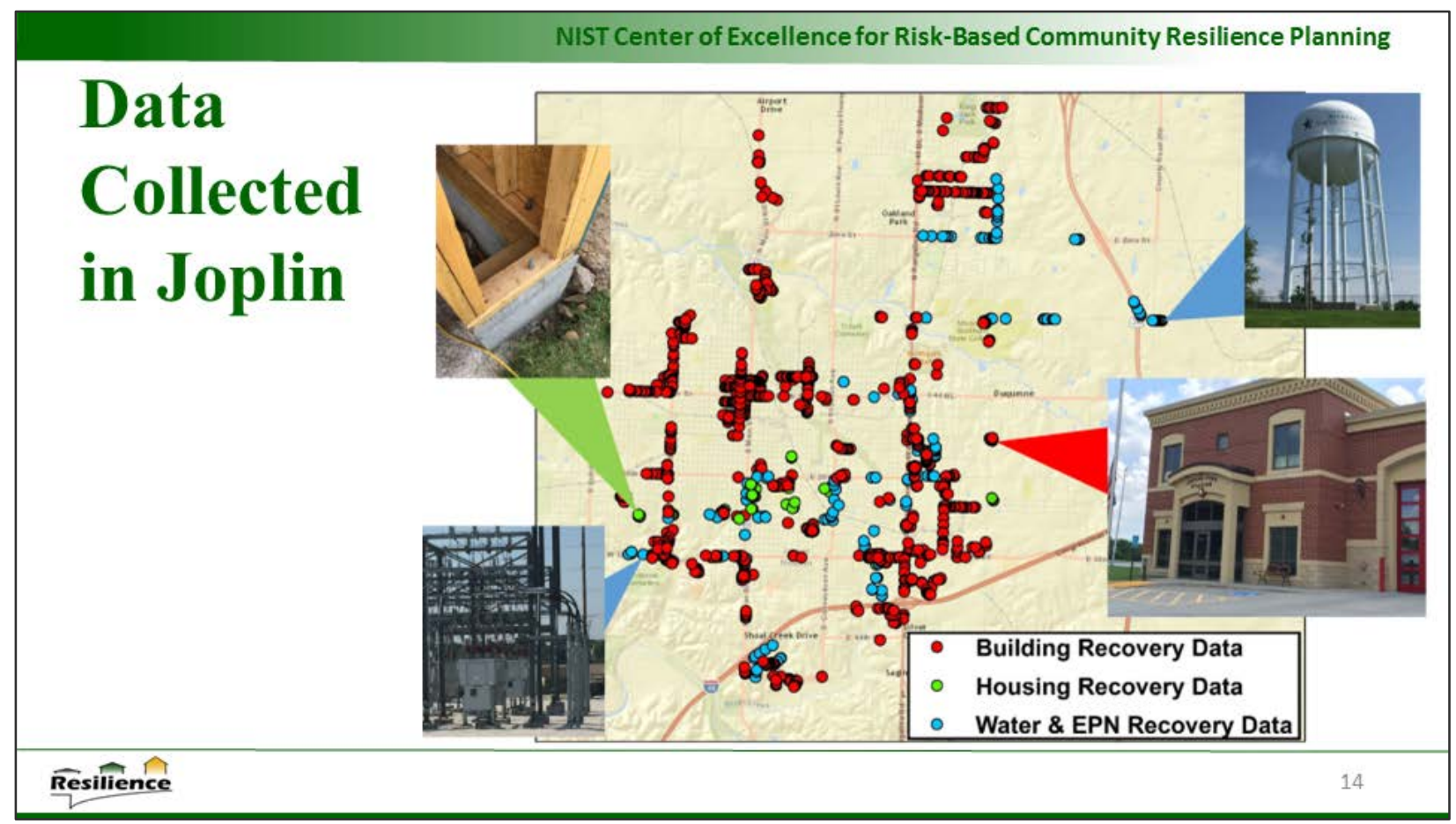




\section{Joplin Field Data- Four Team Types}

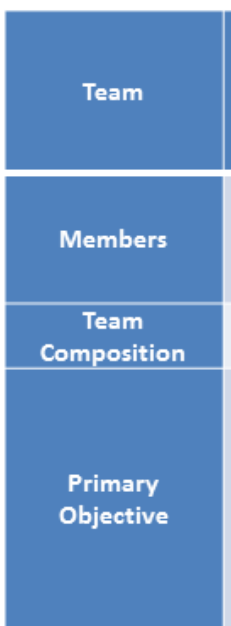

$$
\begin{aligned}
& \text { A. Housing } 1 \\
& \text { Sara Hamideh } \\
& \text { and Shane } \\
& \text { Crawford } \\
& 1 \text { Engineer and } \\
& 1 \text { Sociologist } \\
& \text { To collect data } \\
& \text { that aids in } \\
& \text { developing } \\
& \text { fragilities for } \\
& \text { residential } \\
& \text { buildings. }
\end{aligned}
$$

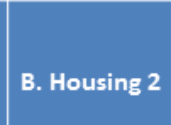

C. Connectivity 1

Social Science (Social Science

and Physical

Infrastructure)

and Physical

Infrastructure)

Sara Hamideh Maria Koliou

and Sam

Lori Peek and

Hassan Masoomi

Jennifer Tobin-

Gurley and Todd

Clapp

2 Engineers

1 Engineer and 1 Sociologist

1 Engineer and 1 Sociologist

To collect

data that

aids in

developing

fragilities for

residential

buildings.

To collect data

To collect data

that aids in that aids in

modeling modeling

connectivities

connectivities

social, and

economic

domains.

social, and
soconomic

economic

domains.

\section{Joplin Field Study: Connectivity Team}

Interviewed Key Community Leaders, July 2016

- Facilities Director, Joplin Schools

- Director of Transportation, Joplin Schools

- Assistant Superintendent of Operations, Joplin Schools

- Vice President Clinical Services, Ozark Center (Mental Health)

- Coordinator of Crisis Services and Accreditation, Ozark Center (Mental Health)

- Associate Professor and Chair, Department of Social Work, MSSU and Chairperson of the Long-Term Recovery Committee

- Chairperson, Joplin Citizens Advisory Recovery Team

- Director of Facilities, Mercy Hospital

- Director of Safety, Mercy Hospital 


\section{Joplin Field Study - School Bus Decisions}

- Sent buses to help St. John's Regional Medical Center (SJRMC) with the evacuation of patients (School Bus Rescue 2012).

- Left the pre-tornado bus routes intact after the tornado and continued to make stops at locations where housing was badly damaged.

- People who had been displaced within thirty-five minutes of Joplin (living with family, friends, or rental) were picked up and taken to the same school that they had attended pre-tornado.

- Bused some high schoolers from school to the location of their after school activities.

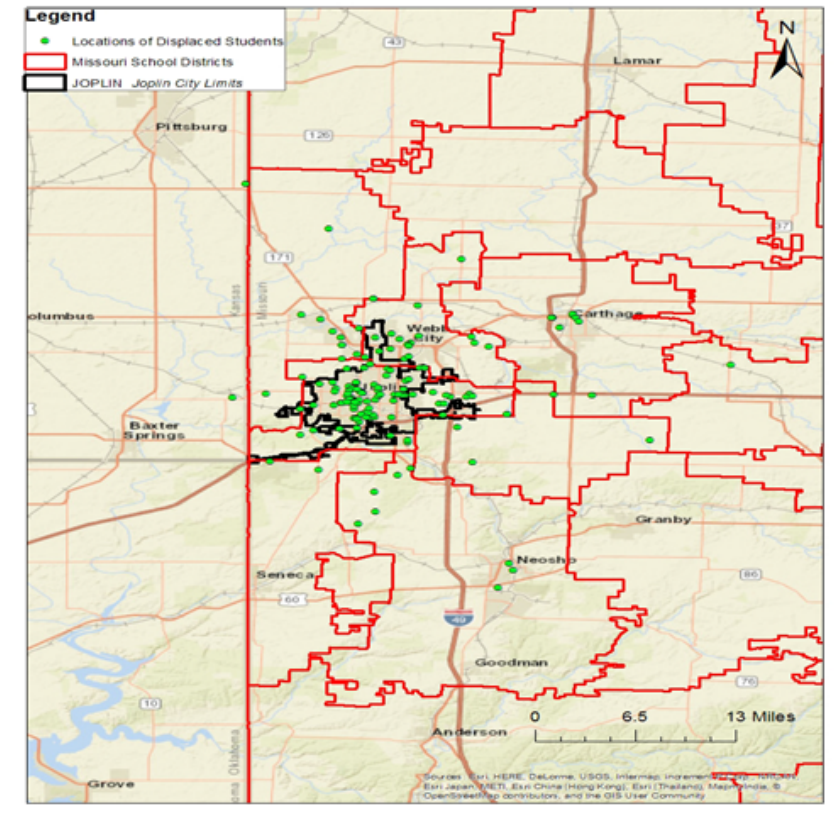

\section{Data Processing Derivation}

The following derivation yields the probability of exceedance of the desired metric as a function of the hazard intensity measure, i.e. a resilience metric fragility function (illustrated on future slide).
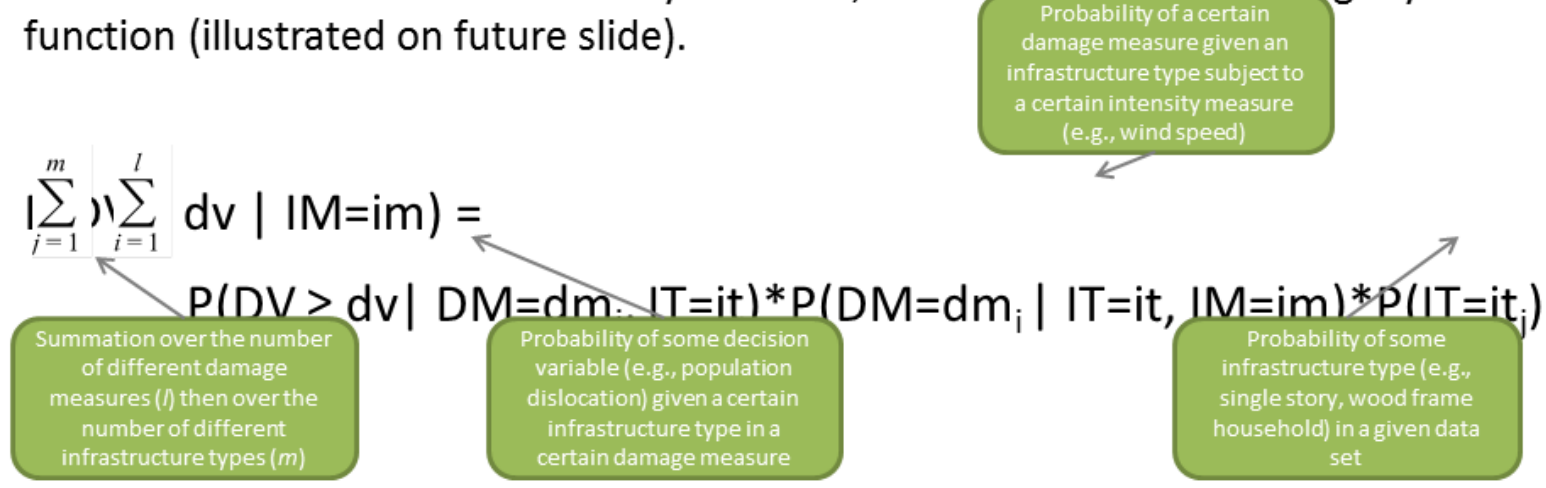

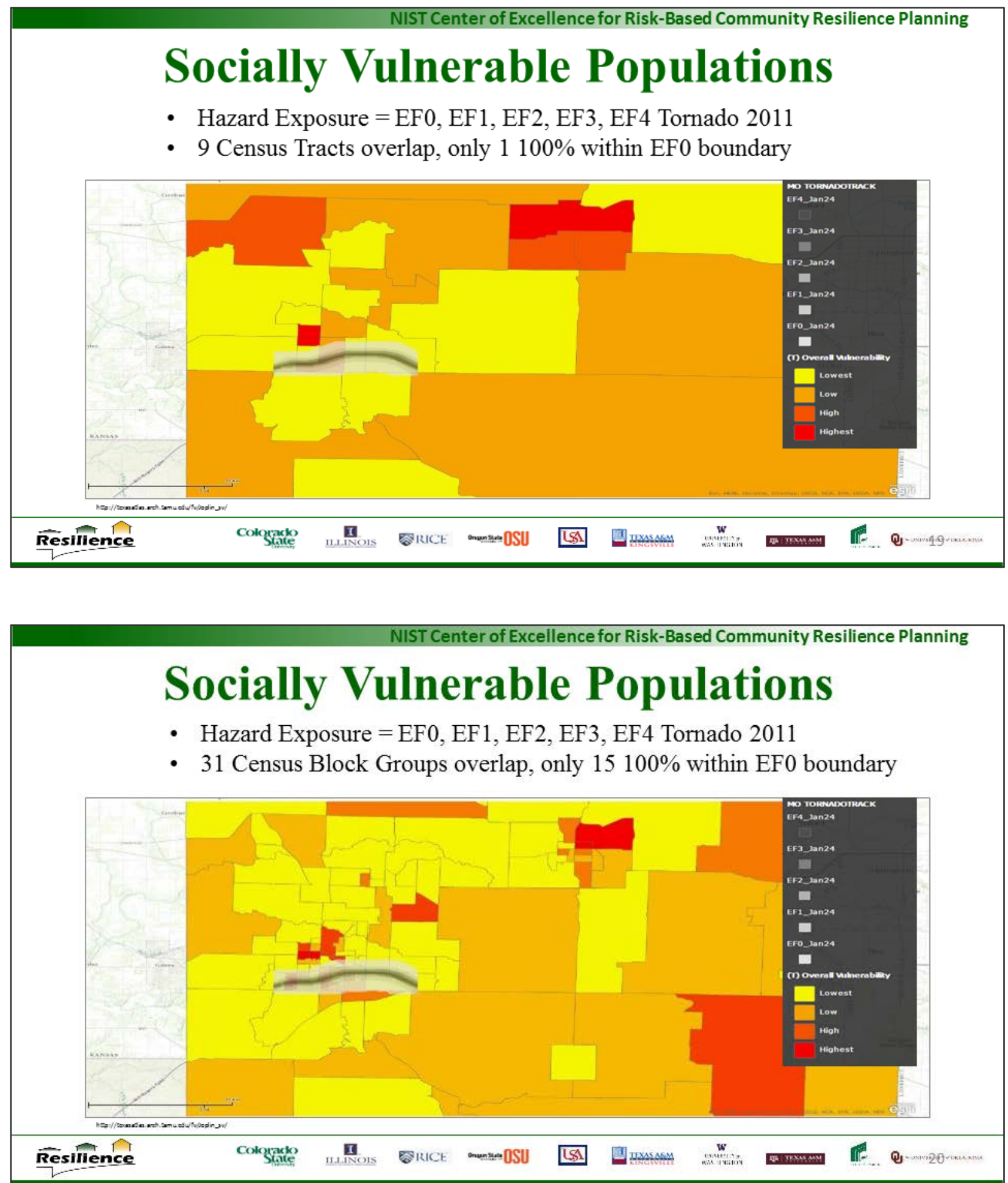


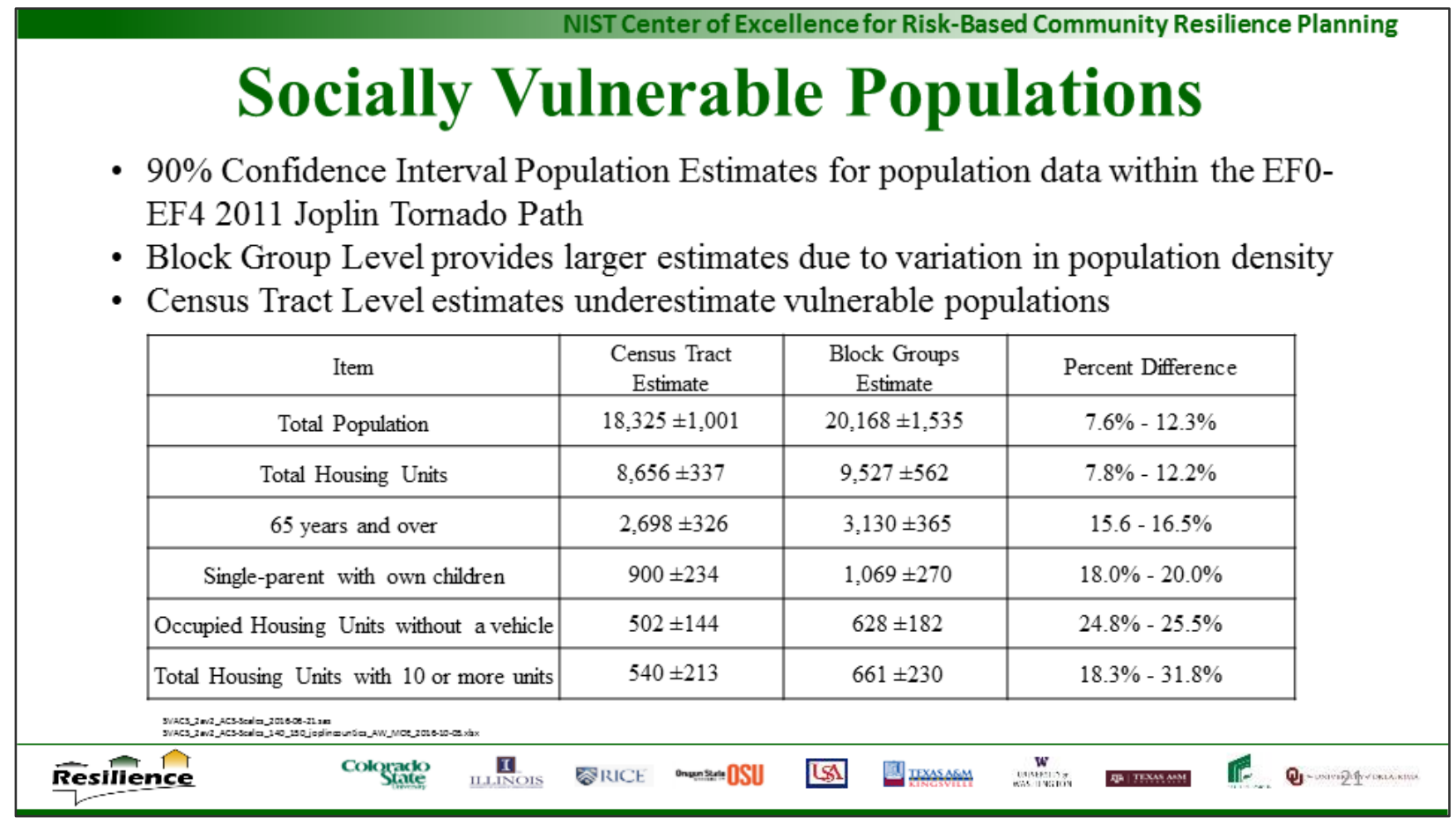

\section{Population Dislocation (Hurricane Andrew Data)}

- Received population dislocation data from Hurricane Andrew.

- Residential damage measure

- Months dislocated

- Race of residents

- Housing type

- Fragilities can be shifted to account for any secondary factors that are collected in the

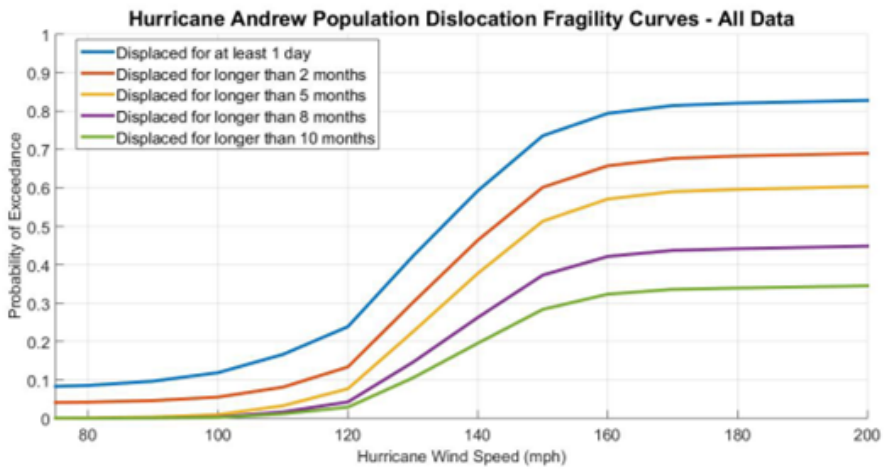

Data from Texas A\&M University field. 


\section{NIST Center of Excellence for Risk-Based Community Resilience Planning}

\section{Population Dislocation}

- 7,500 residential dwellings in Joplin were damaged or destroyed by storm

- Approximately 4,000 homes were completely destroyed

- More than 17,000 people affected by tornado

- 9,200 people were displaced

- FEMA provided temporary housing units to 586 households, across 15 sites throughout the metro Joplin area (last person out of FEMA temporary housing on June 9, 2013)

- Joplin population has grown to 51,300 (July 2015) from 50,175 (2010 Census)

- As of May 2016, a total of 1,657 houses have been rebuilt
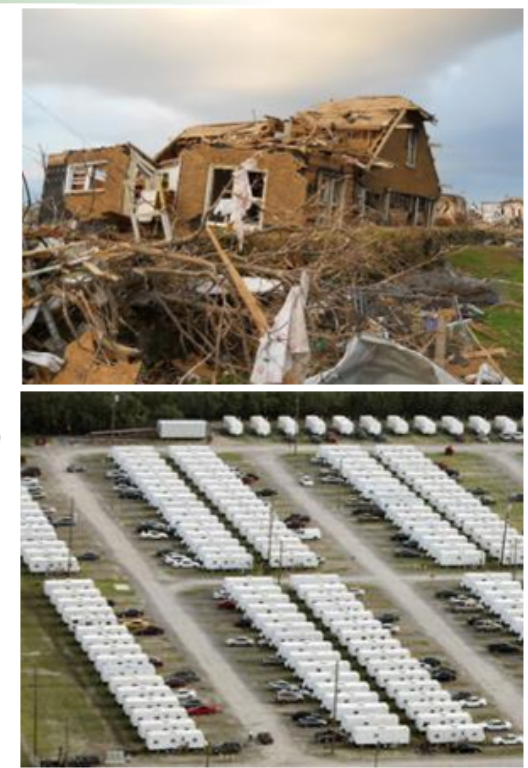

Resiifience

23

NIST Center of Excellence for Risk-Based Community Resilience Planning

\section{Schools}

- 5 of 19 Schools Totally Destroyed: Joplin High School, East Middle School, Franklin Technology Center, Irving Elementary School, Emerson Elementary School
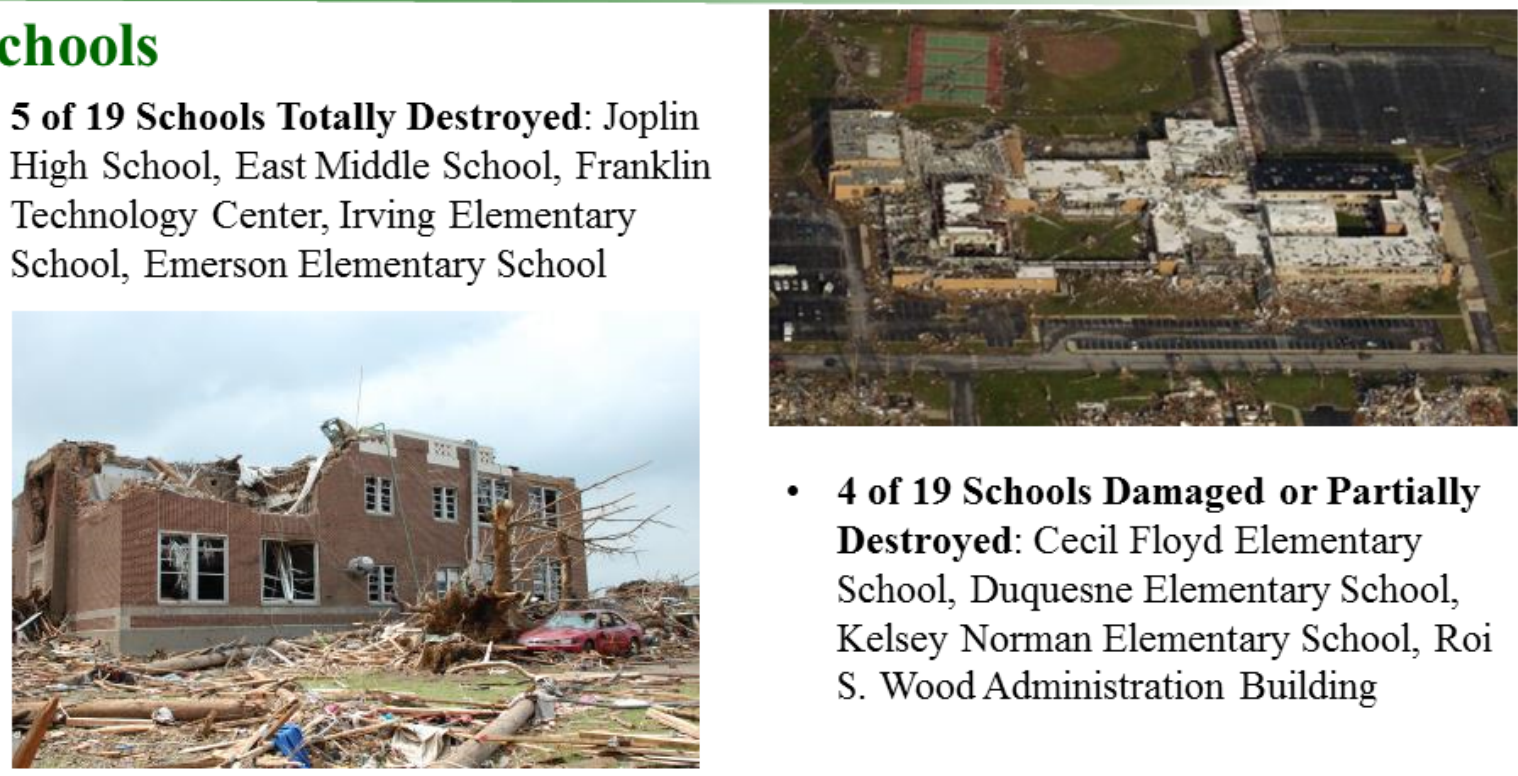

- 4 of 19 Schools Damaged or Partially Destroyed: Cecil Floyd Elementary School, Duquesne Elementary School, Kelsey Norman Elementary School, Roi S. Wood Administration Building 


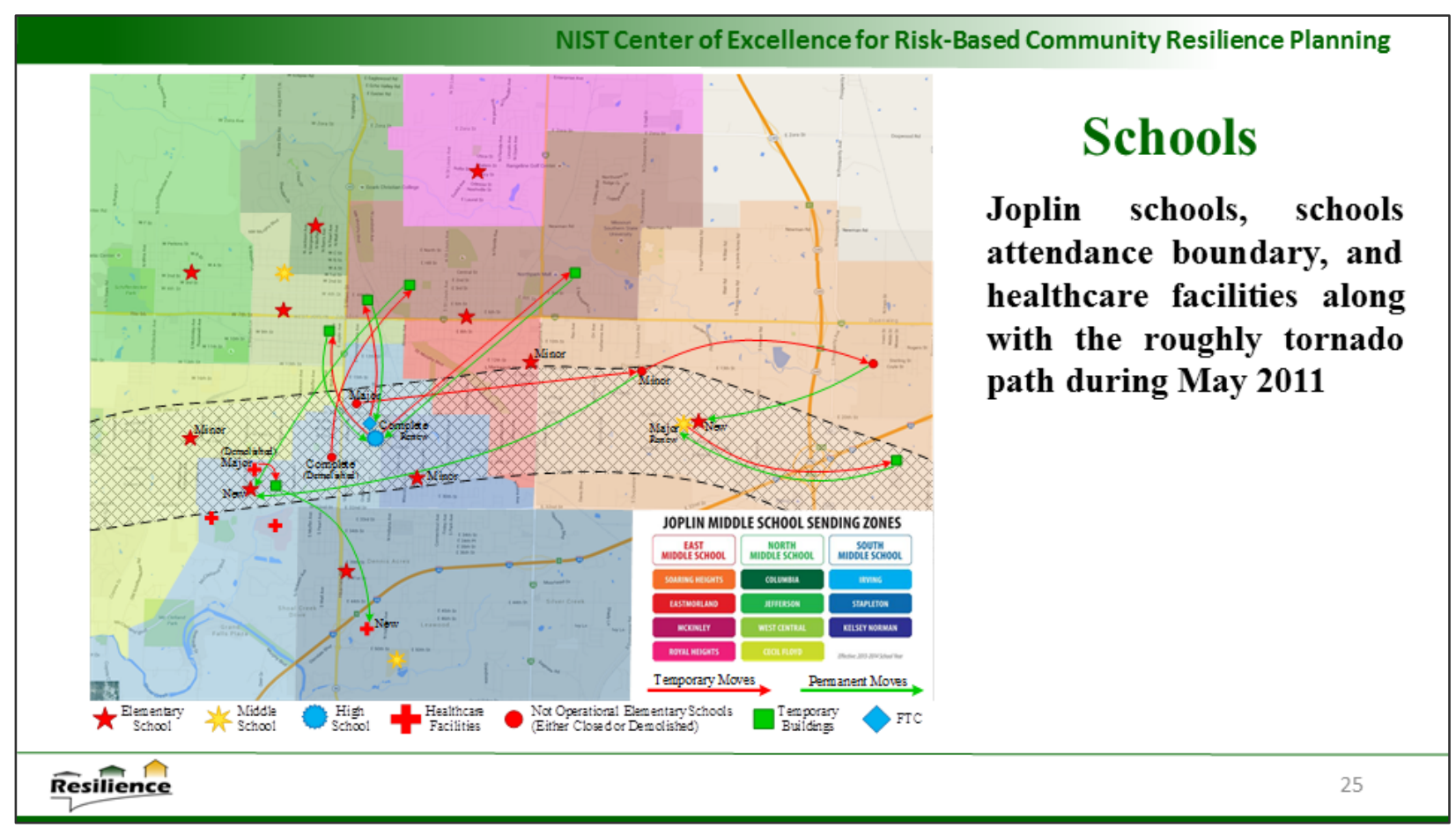

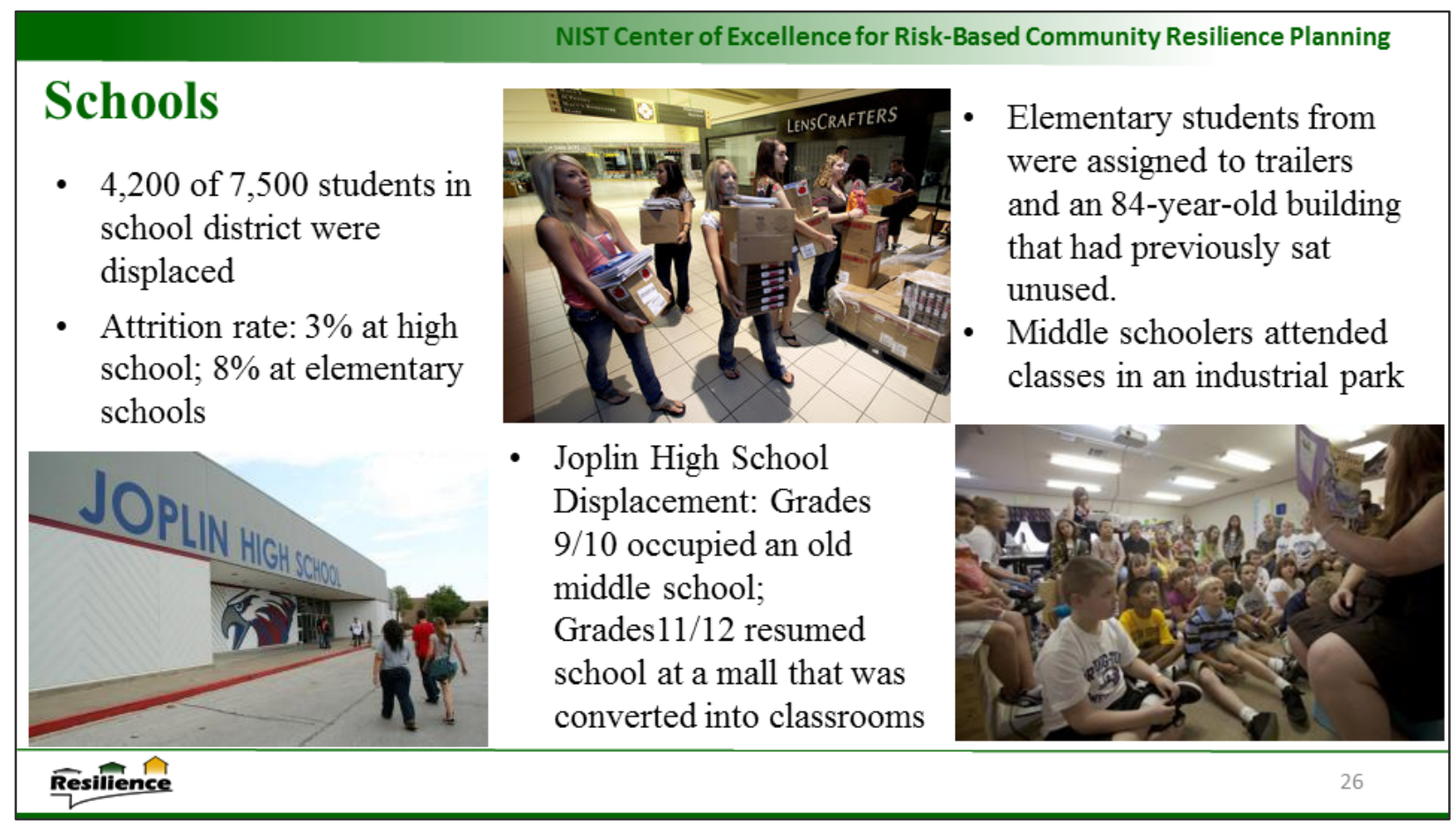


NIST Center of Excellence for Risk-Based Community Resilience Planning

Schools • By 2014, 5 schools were rebuilt

- Every school now has one or more tornado safe rooms

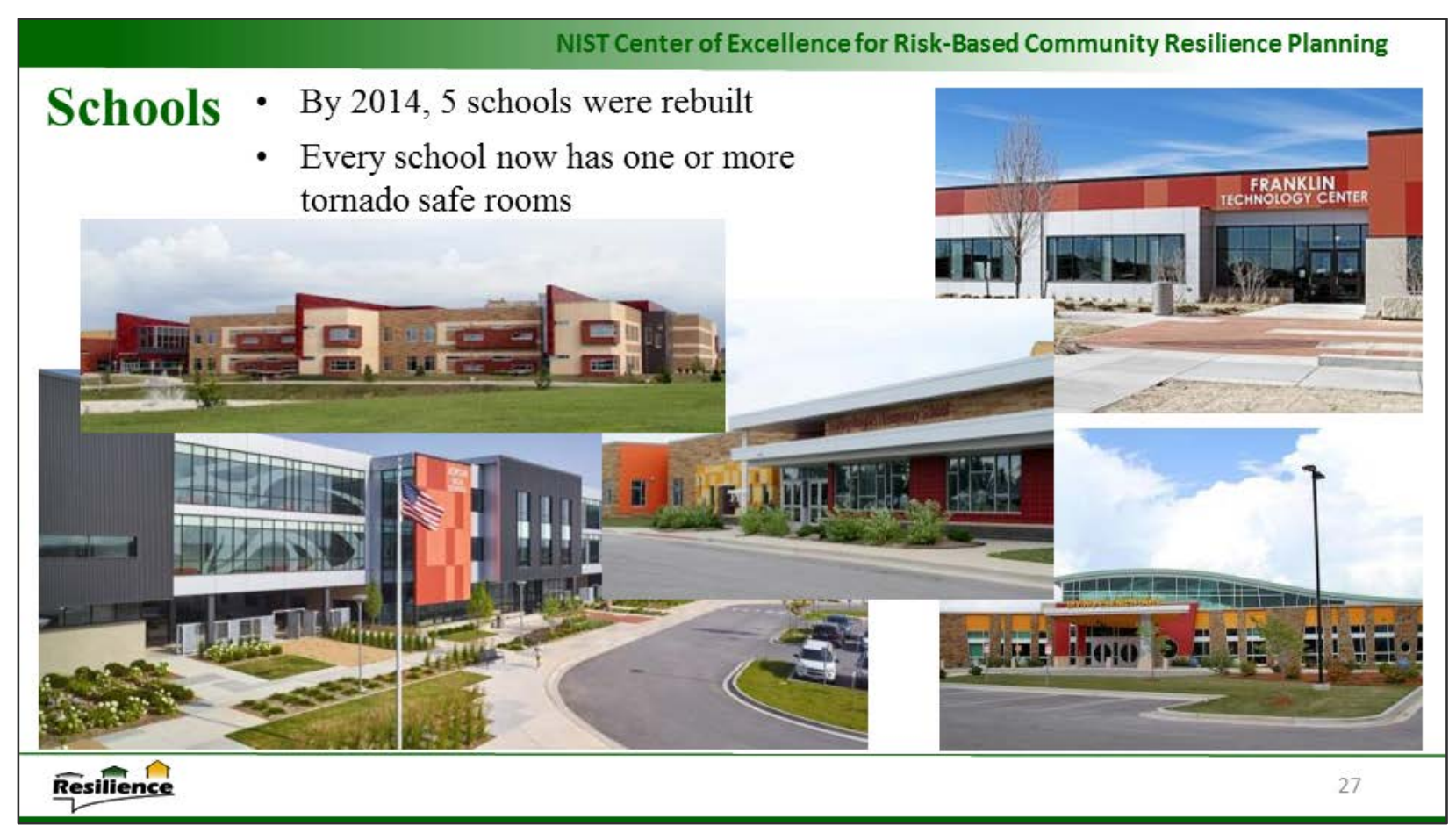

NIST Center of Excellence for Risk-Based Community Resilience Planning

\section{Schools}

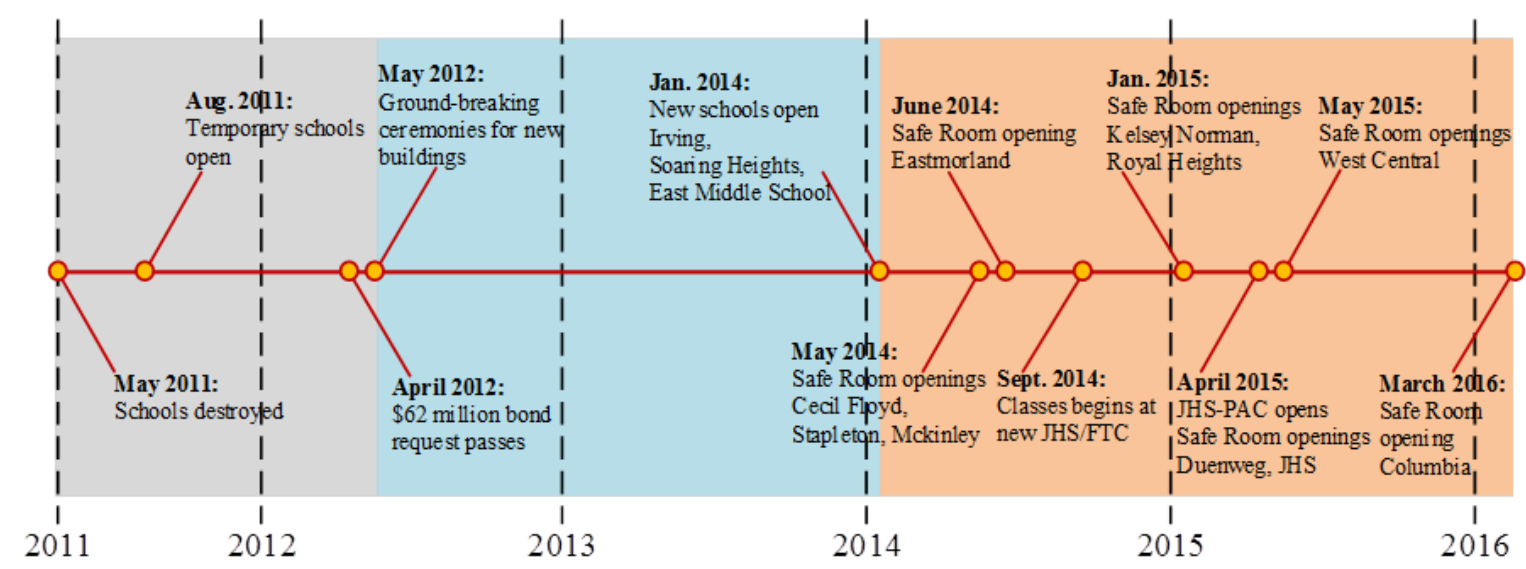

Timeline of schools recovery after the May 2011 outbreak (http://www.joplinschools.org/) 
NIST Center of Excellence for Risk-Based Community Resilience Planning

\section{Mental Health}

- $40 \%$ increase in rates of domestic violence

- $80 \%$ increase in substance abuse

- $10 \%$ increase in suicide, especially among youth

- Ozark Center lost 8 buildings

- 425 employees pre-tornado. In 2016, over 600 employees

- Mental health population has increased from 13,000 to 14,500

- Therapists for children increased from 2 to 14

- $60 \%$ of children seen after the tornado had trauma histories prior to the tornado

- Cumulative losses (losing home and school and job, etc.) resulted more severe symptomology

- Less than .5\% of residents unable to return to work due to mental health issues following the tornado 

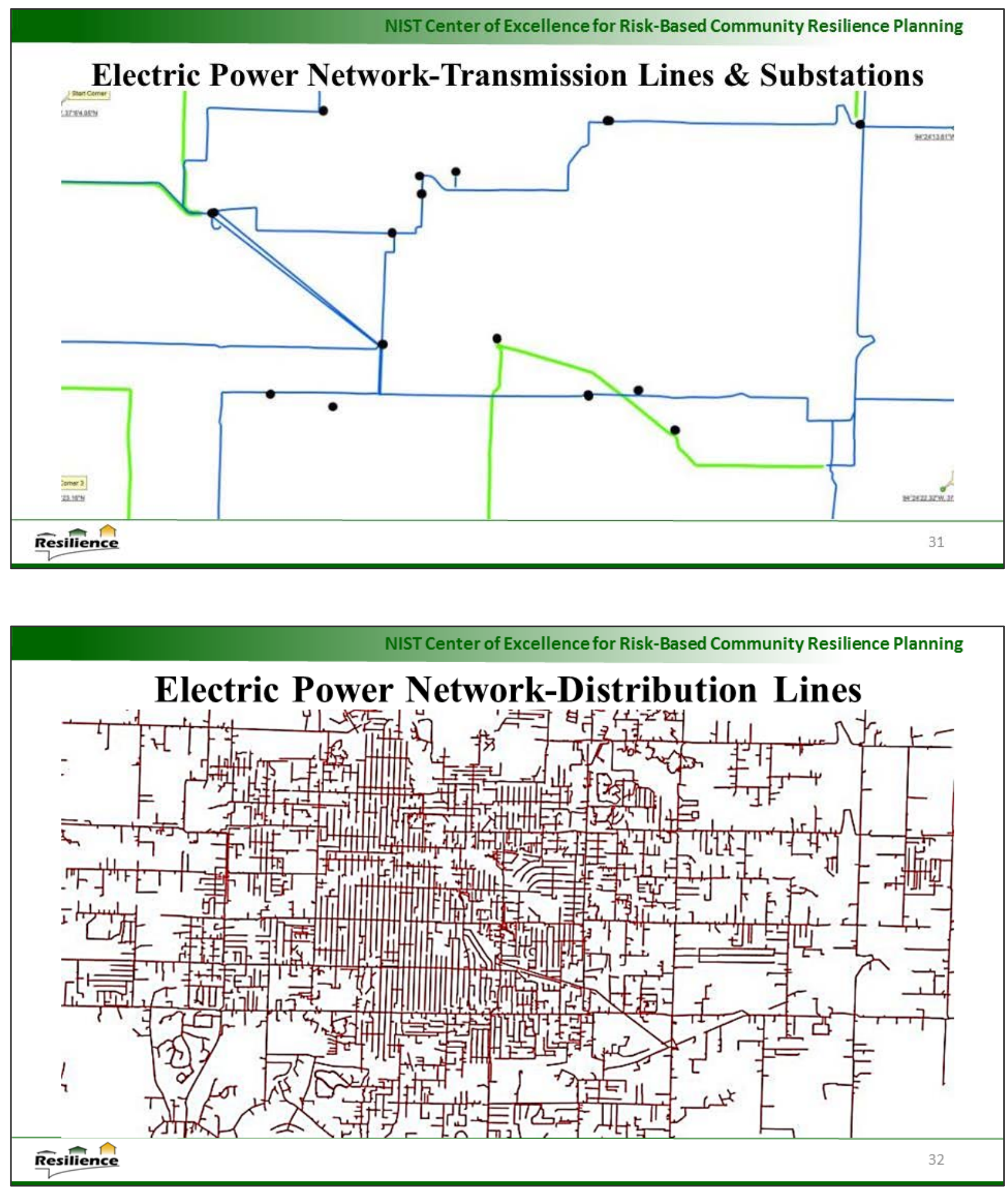
NIST Center of Excellence for Risk-Based Community Resilience Planning

\section{Electric Power Network-Distribution Lines-Poles}

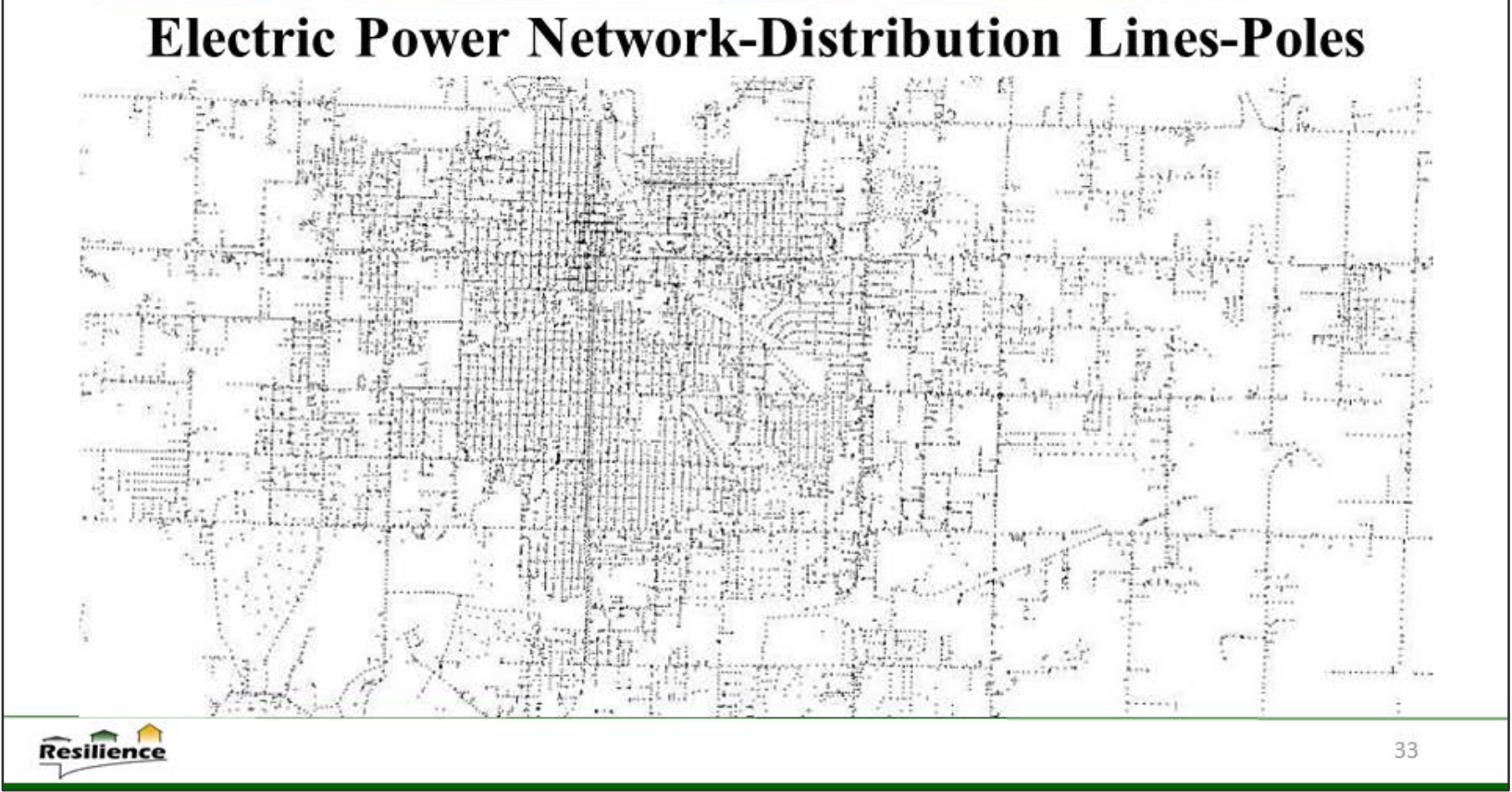

NIST Center of Excellence for Risk-Based Community Resilience Planning

Estimating Service Area of Each Substation Based on

Weighted Cellular Automata Combined with Distribution Lines

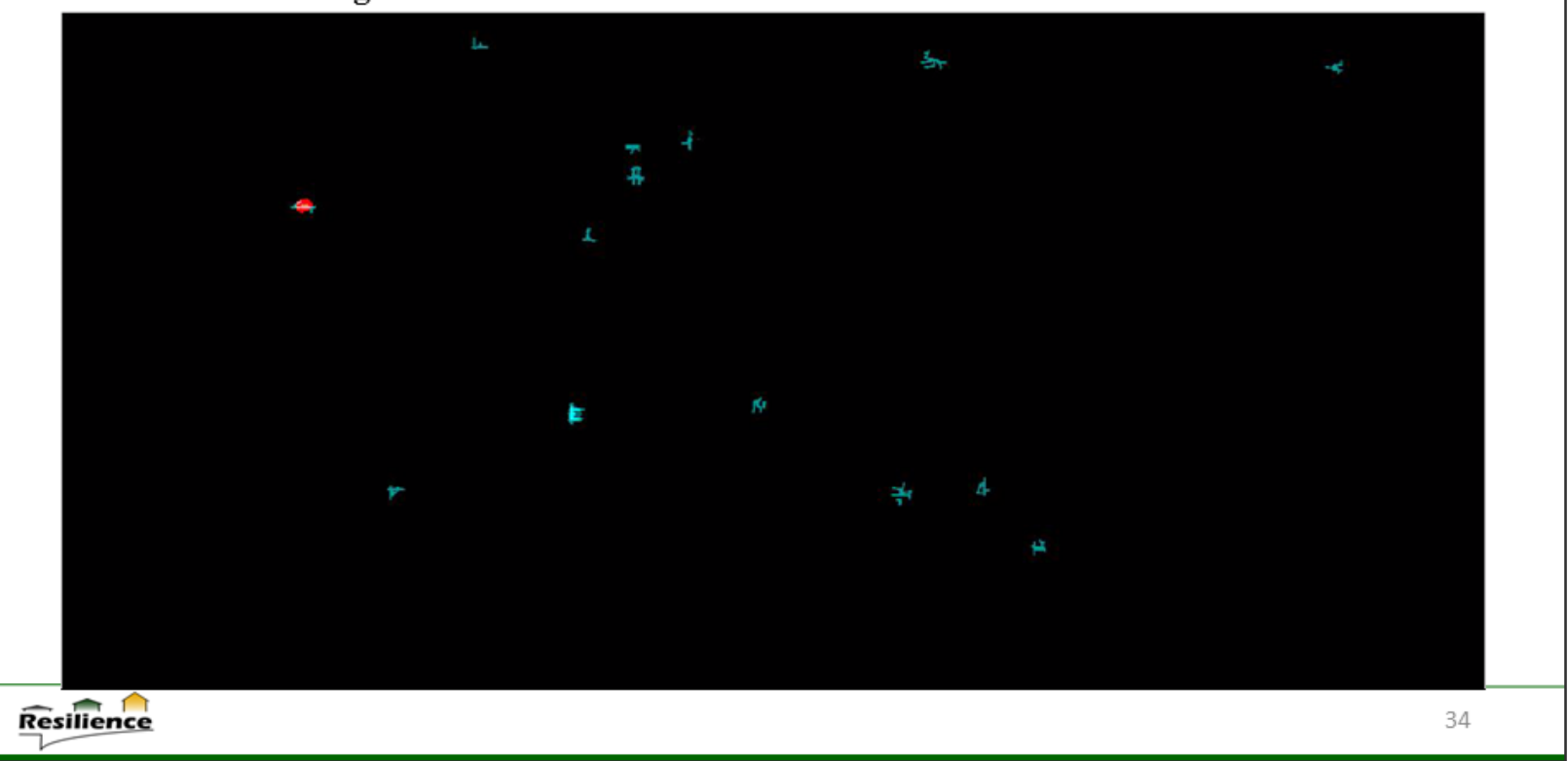


NIST Center of Excellence for Risk-Based Community Resilience Planning

\section{Estimating Service Area of Each Substation Based on}

Weighted Cellular Automata Combined with Distribution Lines

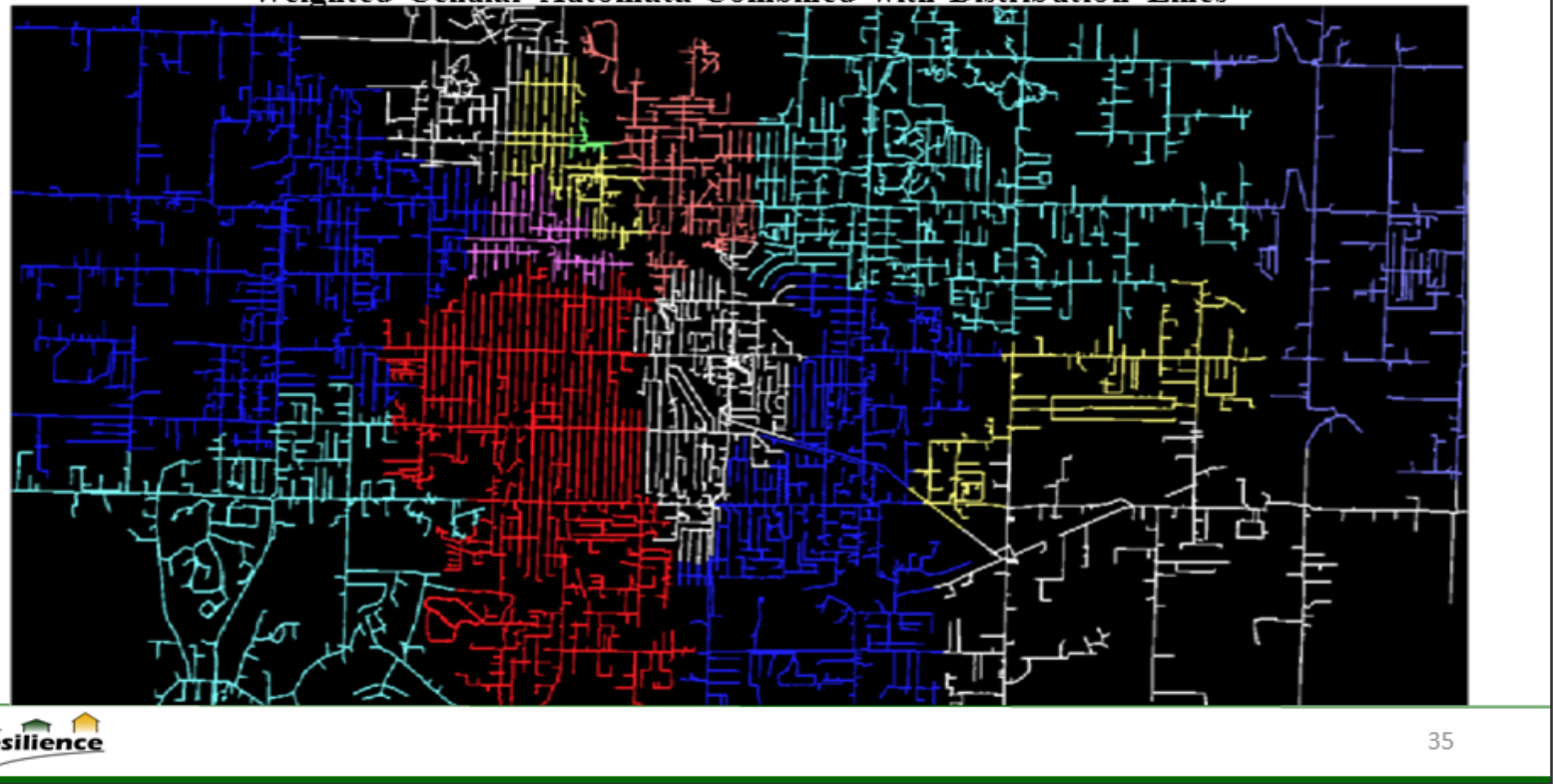

NIST Center of Excellence for Risk-Based Community Resilience Planning Estimating Service Area of Each Substation Comparison of the two Methods

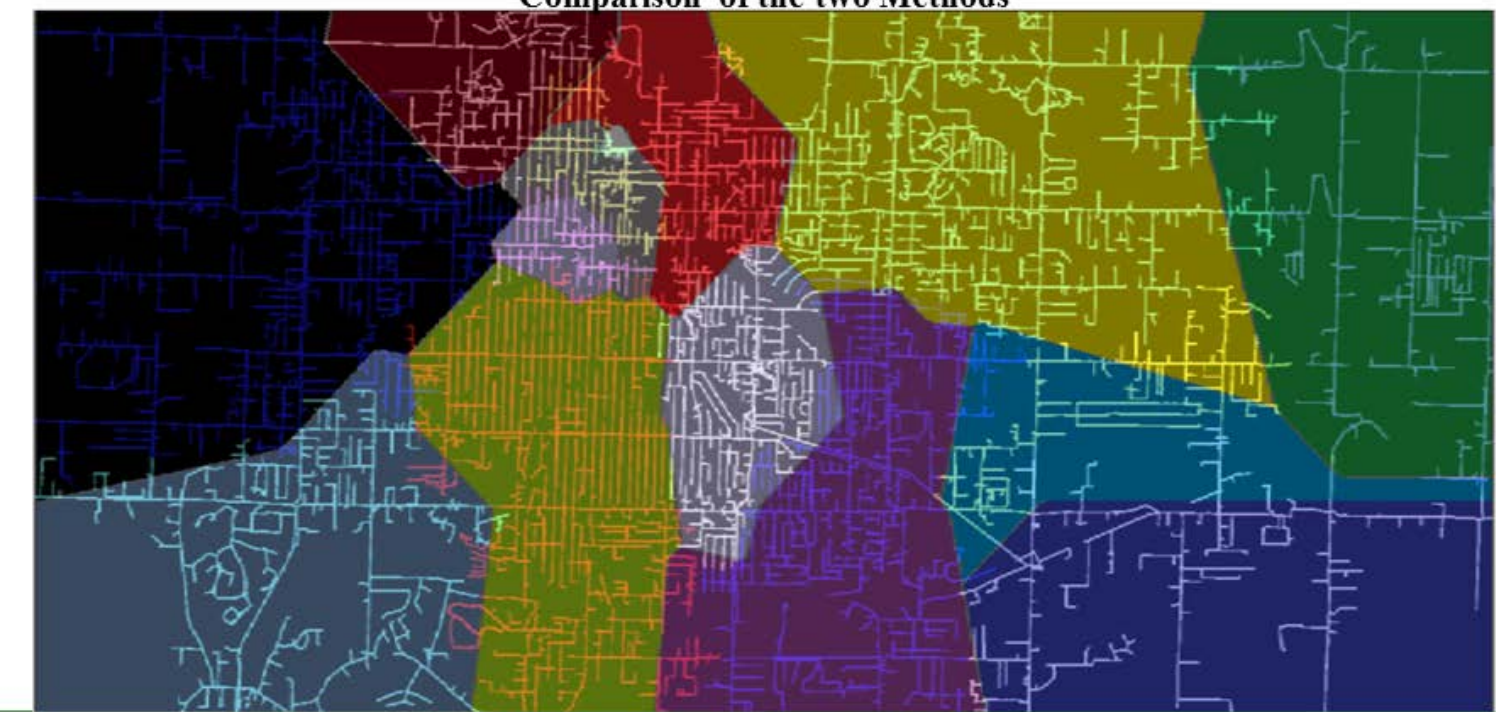


International Workshop on Modeling of Physical, Economic, and Social Systems for Resilience Assessment

NIST Center of Excellence for Risk-Based Community Resilience Planning

\section{Electric Power Network Damage Analysis}

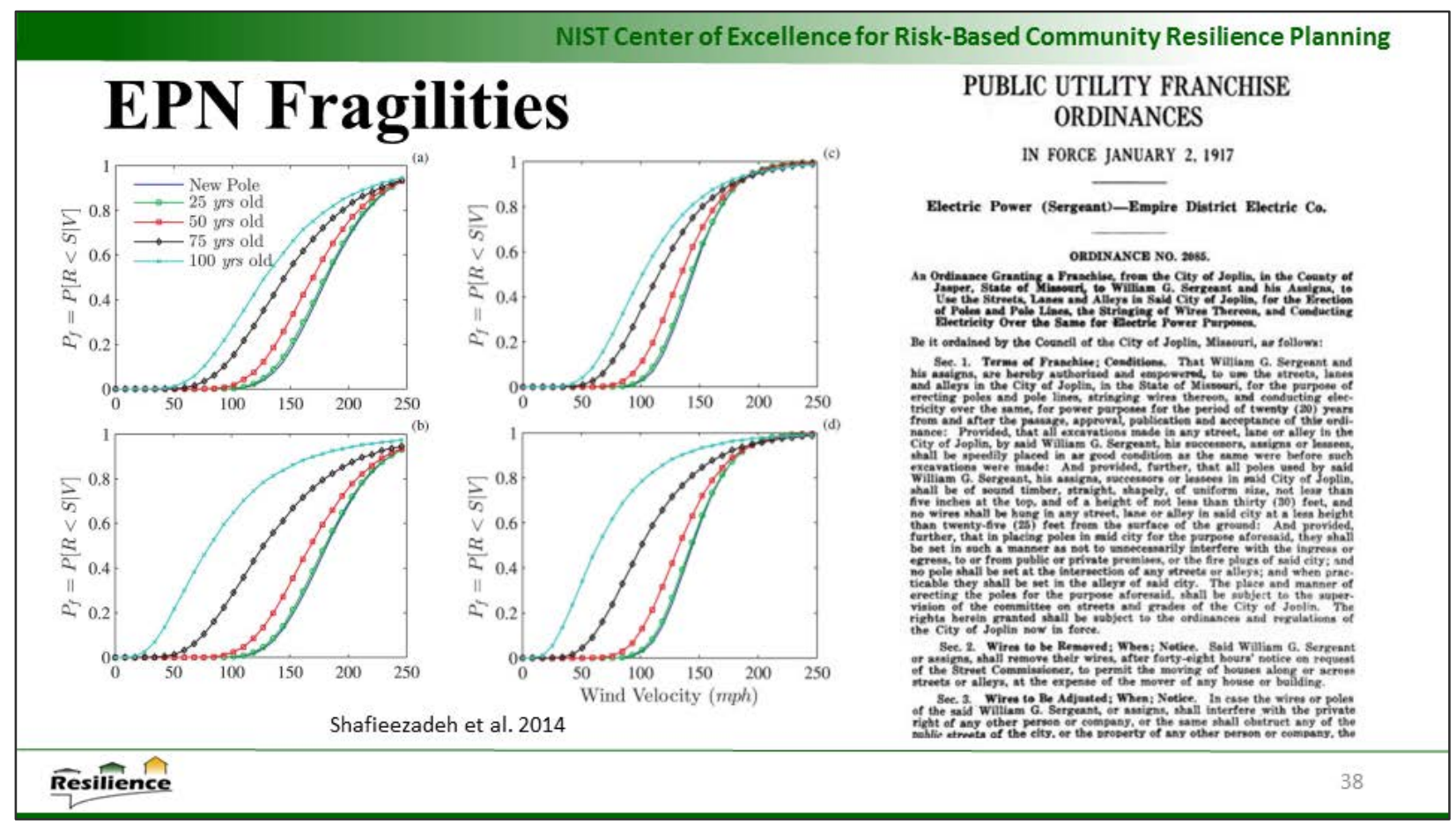




\section{Simplified Tornado Modeling Approach}

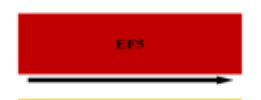

EF3
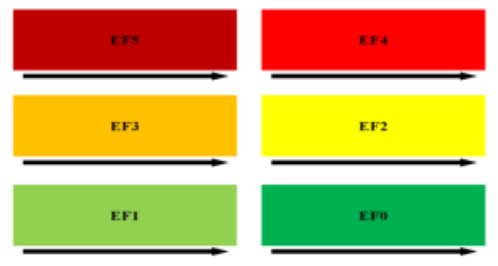
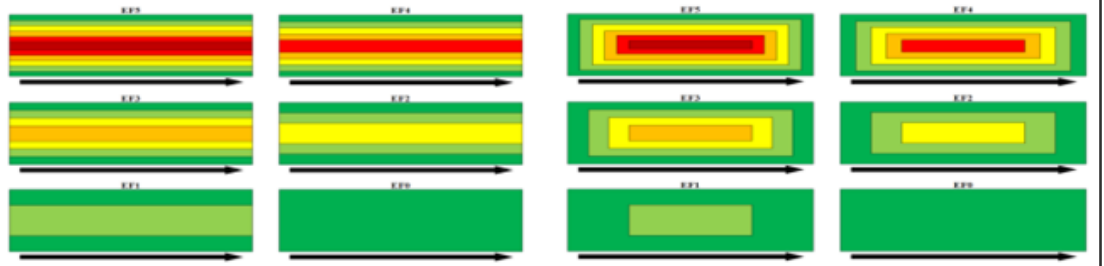

\begin{tabular}{|c|c|c|c|c|c|c|c|c|}
\hline \multicolumn{9}{|c|}{ 1973-2011 } \\
\hline Intensity (EF/F) & Total Number & With Data & \begin{tabular}{|l|}
$\begin{array}{l}\text { Average Length } \\
\text { (miles) }\end{array}$ \\
\end{tabular} & \begin{tabular}{|l|} 
St. Dev. Length \\
(miles)
\end{tabular} & \begin{tabular}{|l|}
$\begin{array}{l}\text { Average Width } \\
\text { (yards) }\end{array}$ \\
\end{tabular} & $\begin{array}{l}\text { St. Dev. Width } \\
\text { (yards) }\end{array}$ & $\begin{array}{l}\text { Average Areal Coverage } \\
\text { (sq. miles) }\end{array}$ & $\begin{array}{l}\text { St. Dev. Areal Coverage } \\
\text { (sq. miles) }\end{array}$ \\
\hline All Tornadoes & 43047 & 42312 & 3.2389 & 7.421 & 100.2788 & 1996614 & 0.5619 & 3.7588 \\
\hline 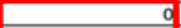 & 22177 & 21677 & 1.0251 & 2.548 & 42.5301 & 67.2193 & 0.0456 & 0.3013 \\
\hline 1 & 13572 & 13434 & 3.3088 & 5.2715 & 99.3349 & 143.561 & 0.2884 & 0.888 \\
\hline 2 & 4906 & 4849 & 7.3543 & 10.0668 & 207.7158 & 273.0966 & 1.246 & 3.4217 \\
\hline 3 & 1468 & 1442 & 15.4504 & 15.9966 & 446.8663 & 457.1184 & 4.6286 & 8.5728 \\
\hline 4 & 386 & 374 & 26.0482 & 26.4238 & 724.8583 & 626.8049 & 13.6711 & 26.1328 \\
\hline & 46 & 44 & 35.7666 & 28.1811 & 892.3864 & 657.8205 & 22.3144 & 33.8861 \\
\hline Unknown & 492 & 492 & 1.7585 & 5.3281 & 36.6301 & 80.6377 & 0.1125 & 0.9154 \\
\hline
\end{tabular}

Resilience

39

NIST Center of Excellence for Risk-Based Community Resilience Planning

\section{Estimating the Damage : Simplified Tornado Path with Mean Values}

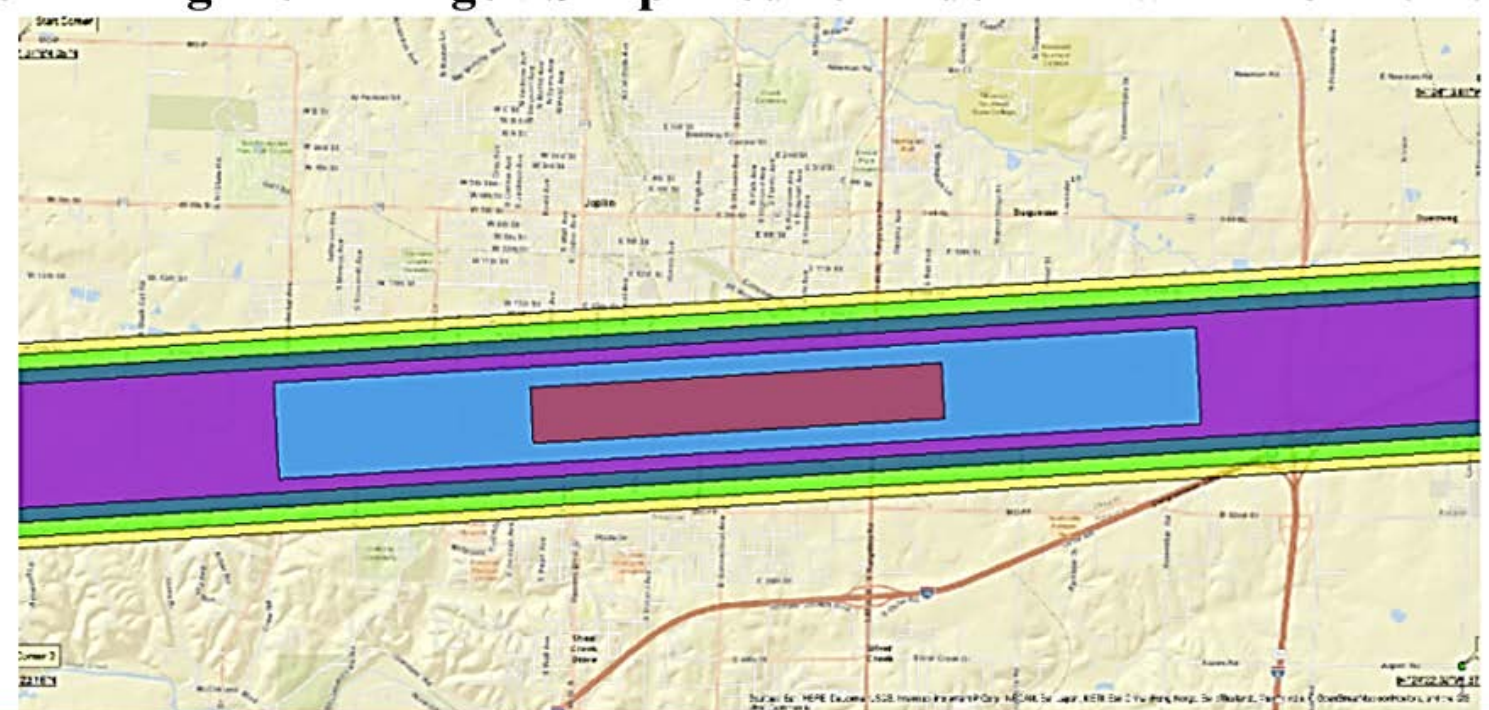




\section{Damage Analysis Results}

\section{In Total, on Average 70 Transmission Towers and 3446 Distribution Poles Damaged}
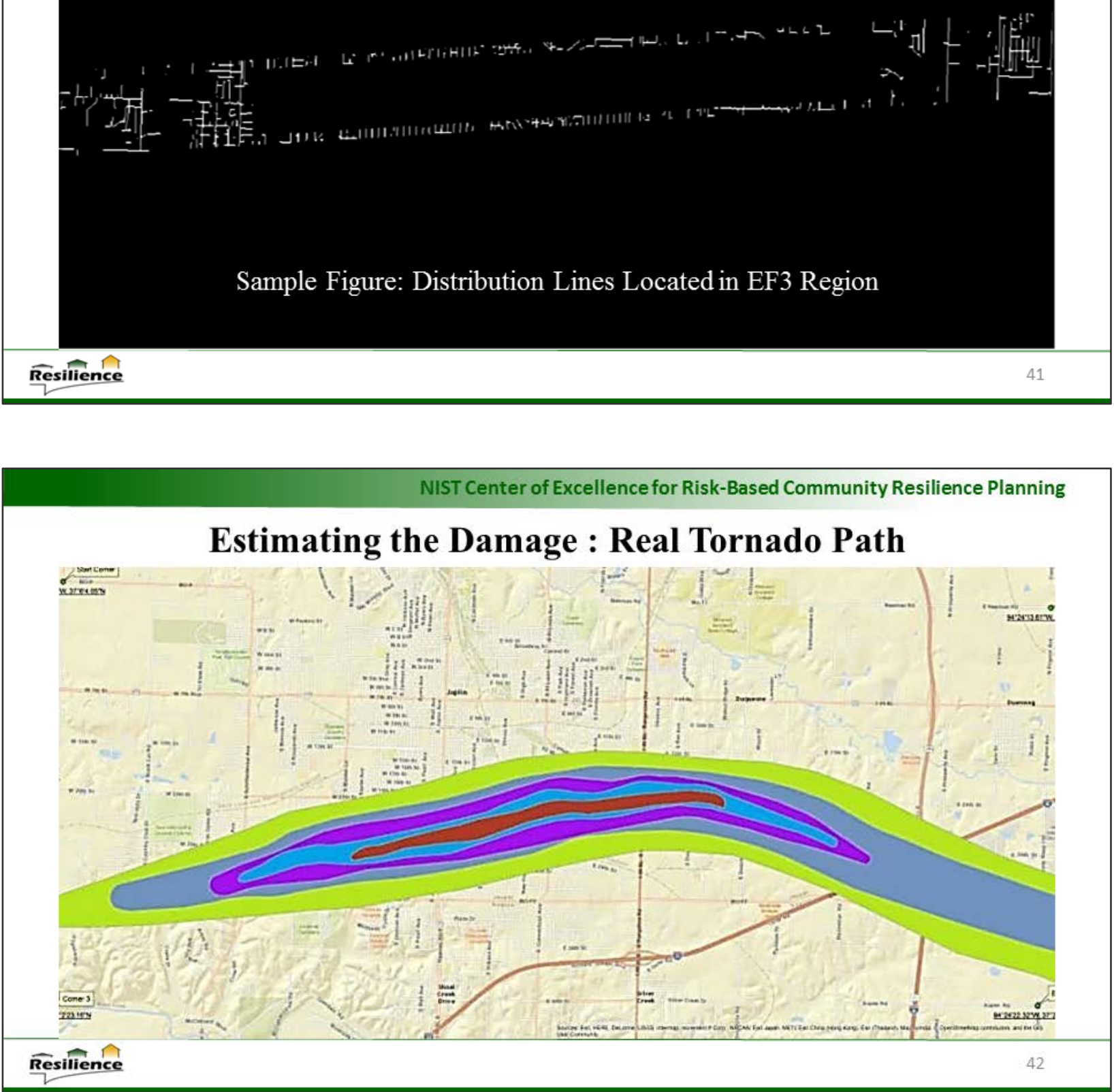


\section{Damage Analysis Results}

\section{In Total, on Average 113 Transmission Towers and 3744 Distribution Poles would be Damaged}

Reality: 135 Transmission Towers and 3850 Distribution Poles

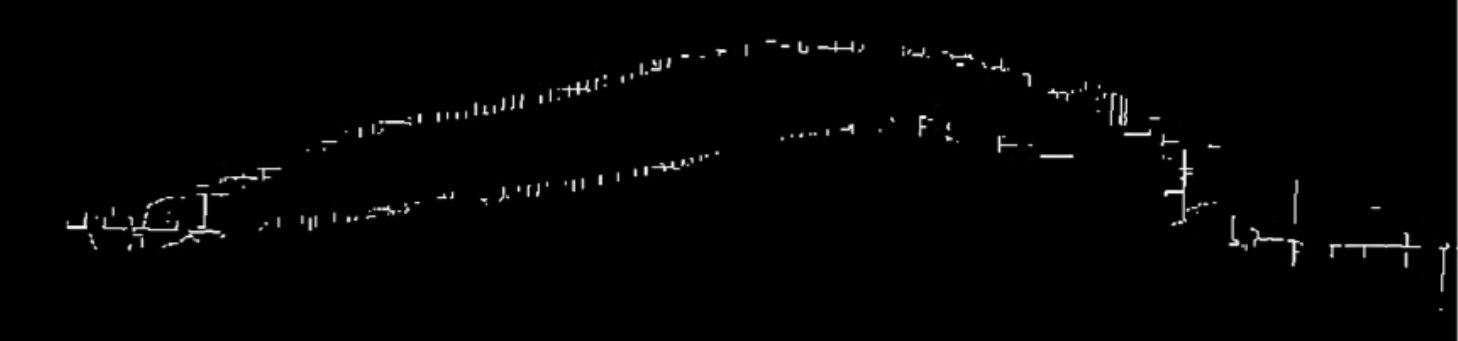

Sample Figure: Distribution Lines Located in EF2 Region

\section{Single Sector Validation: Buildings}




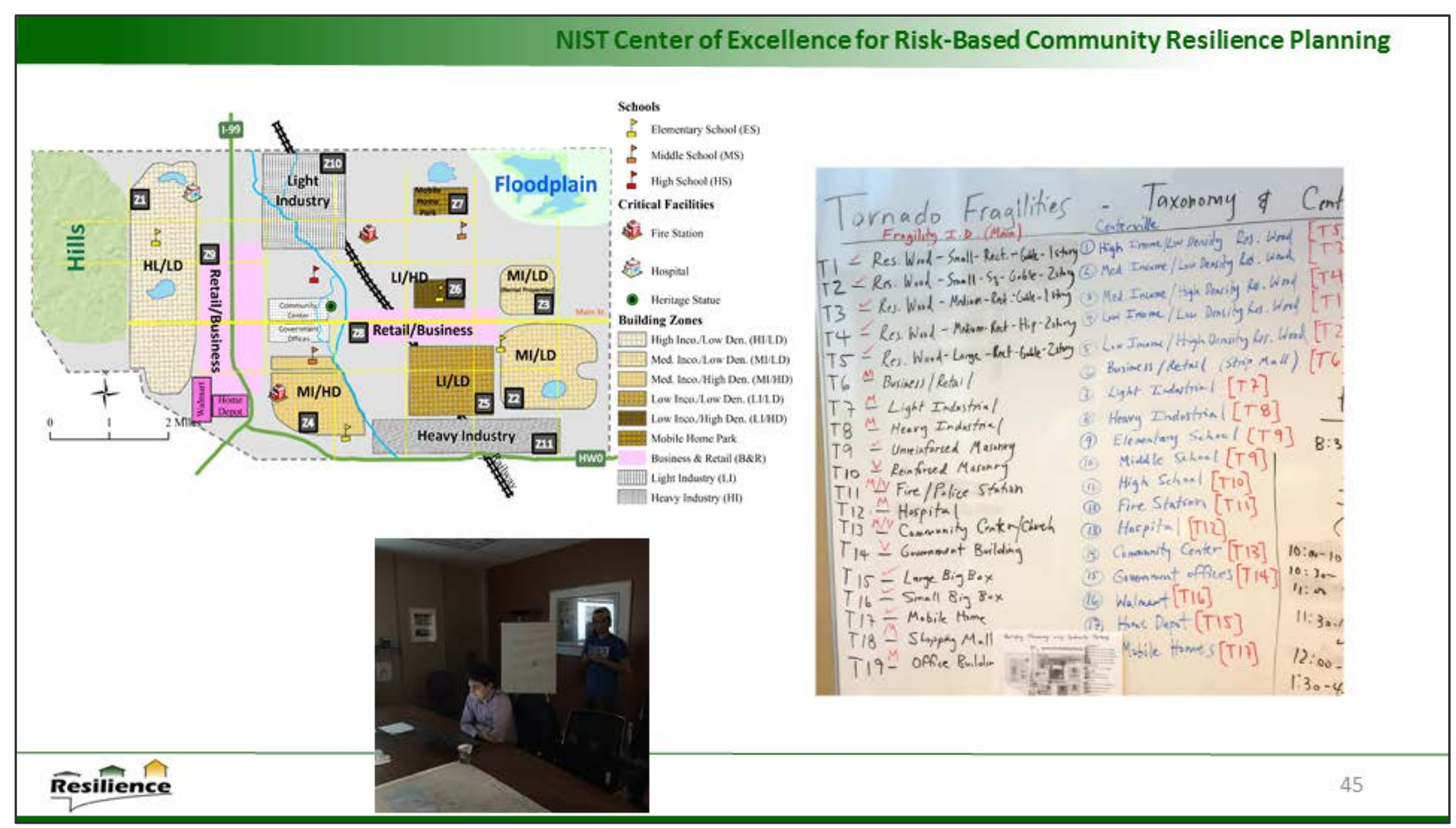

\section{NIST Center of Excellence for Risk-Based Community Resilience Planning}

\section{Fragilities}

\begin{tabular}{|c|c|c|c|}
\hline \multirow{2}{*}{ Bldg. ID } & \multicolumn{2}{|c|}{ Building Description } & \\
\hline & Hurricane Fragilities & Centerville & \\
\hline $\mathrm{T} 1$ & Res Wood Bldg. - Small Rectangular Plan - Gable Roof - 1 Story & Low Income/Low Density Res Wood Bldg. & \\
\hline $\mathrm{T} 2$ & Res Wood Bldg. - Small Square Plan - Gable Roof - 2 Stories & Low Income/High Density Res Wood Bldg. & \\
\hline Тз & Res Wood Bldg. - Medium Rectangular Plan - Gable Roof - 1 Story & Medium Income/Low Density Res Wood BIdg. & \\
\hline T4 & Res Wood Bldg. - Medium Rectangular Plan - Hip Roof - 2 Stories & Medium Income/High Density Res Wood Bldg. & \\
\hline T5 & Res Wood Bldg. - Large Rectangular Plan - Gable Roof - 2 Stories & High Income/Low Density Res Wood Bldg. & \\
\hline T6 & Business and Retail Buildings & Strip Mall & \\
\hline $\mathrm{T} 7$ & Light Industrial Buildings & Light Industrial Buildings & \\
\hline T8 & Heavy Industrial Buildings & Heavy Industrial Buildings & \\
\hline T9 & Unreinforced Masonry & Elementary/Middle School & \\
\hline T10 & Reinforced Masonry & High School & \\
\hline T11 & Fire/Police Station & Fire Station & \\
\hline $\mathrm{T} 12$ & Hospital & Hospital & \\
\hline $\mathrm{T} 13$ & Community Center/Church & Community Center & \\
\hline T14 & Government Buildings & Government Buildings & \\
\hline T15 & Large Big Box & Home Depot & \\
\hline T16 & Small Big Box & Walmart & \\
\hline T17 & Mobile Homes & Mobile Homes & \\
\hline T18 & Shopping Mall & Shopping Mall & \\
\hline lience & & & 46 \\
\hline
\end{tabular}



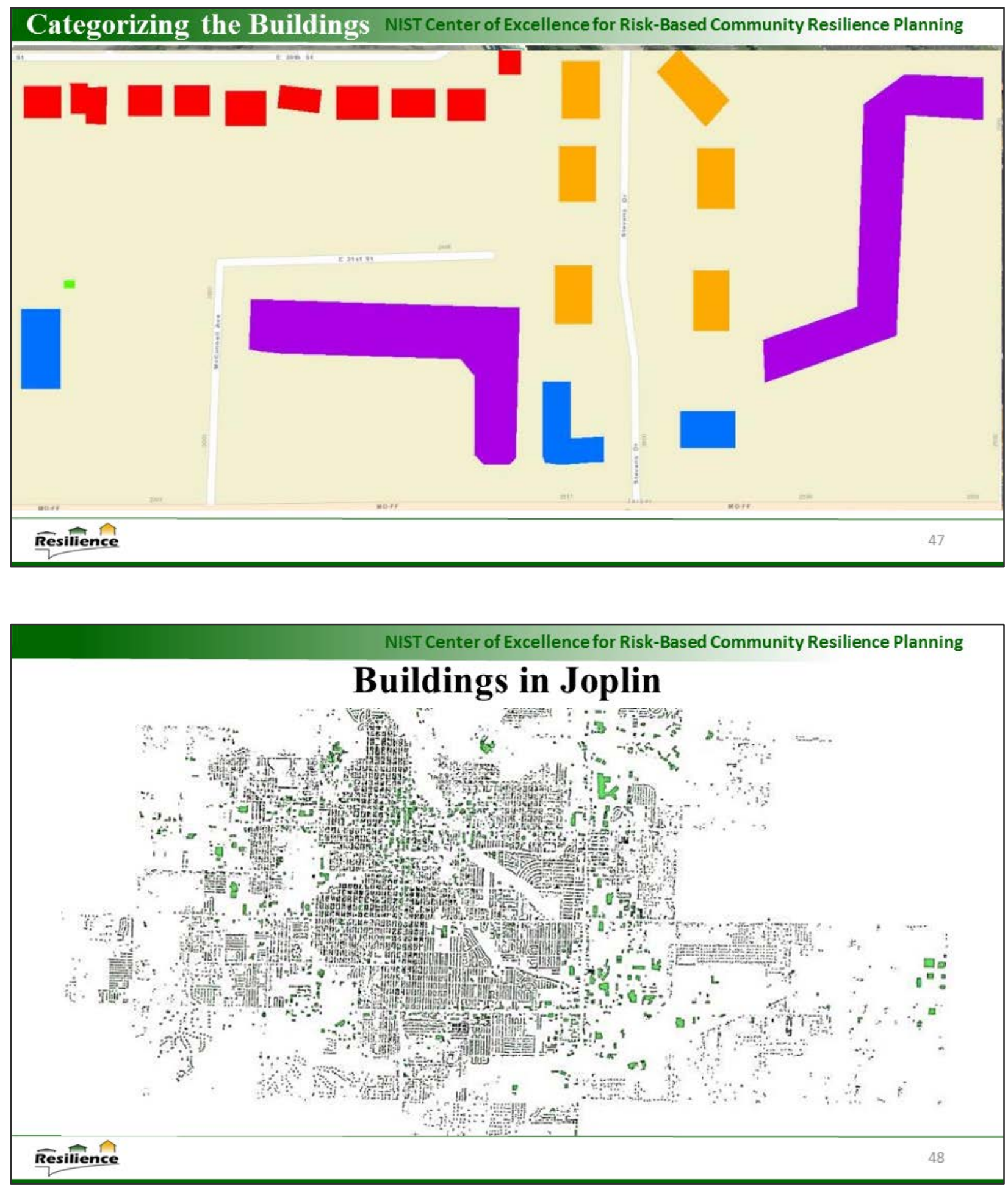
NIST Center of Excellence for Risk-Based Community Resilience Planning

\section{Estimating the Damage}
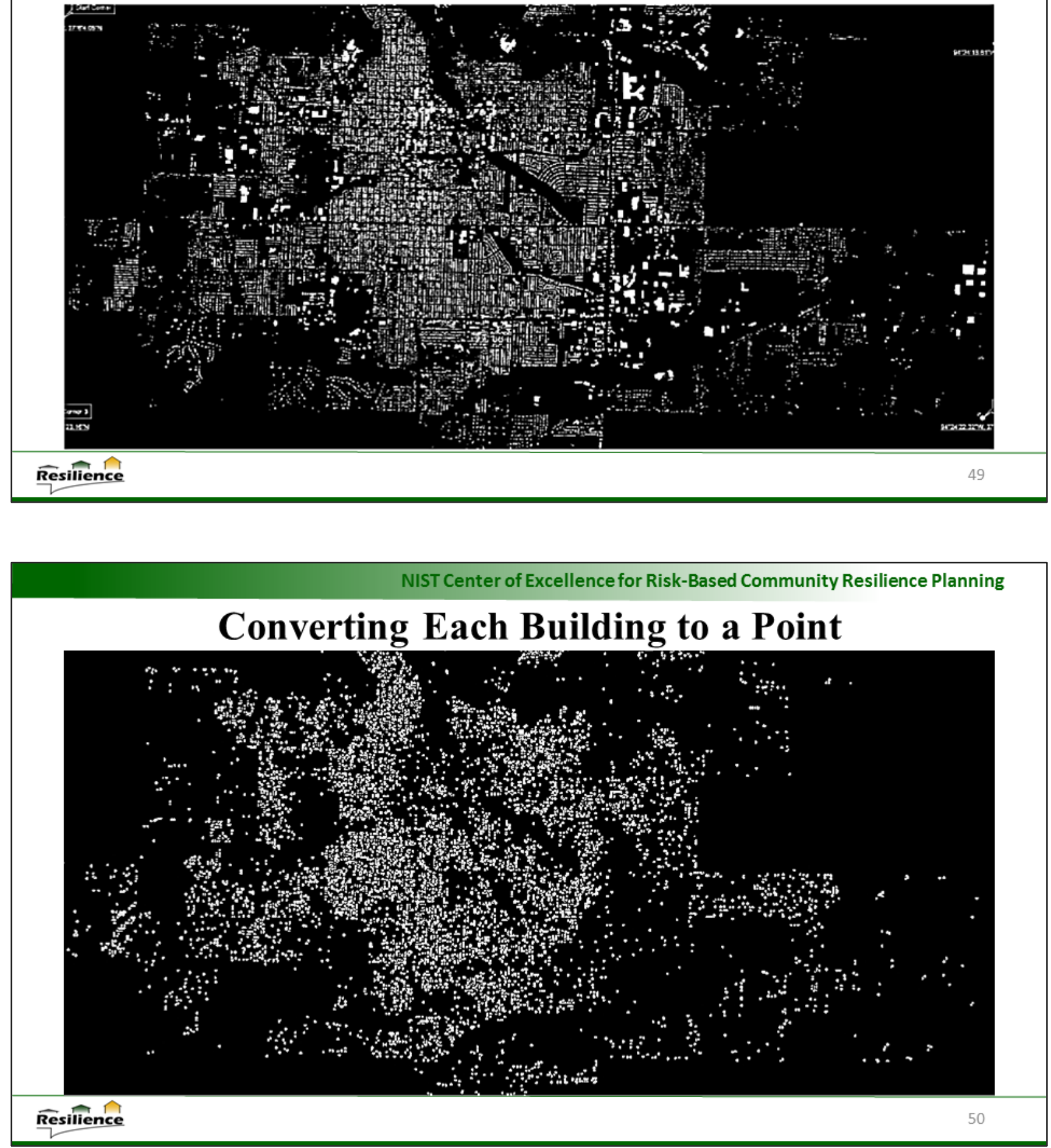

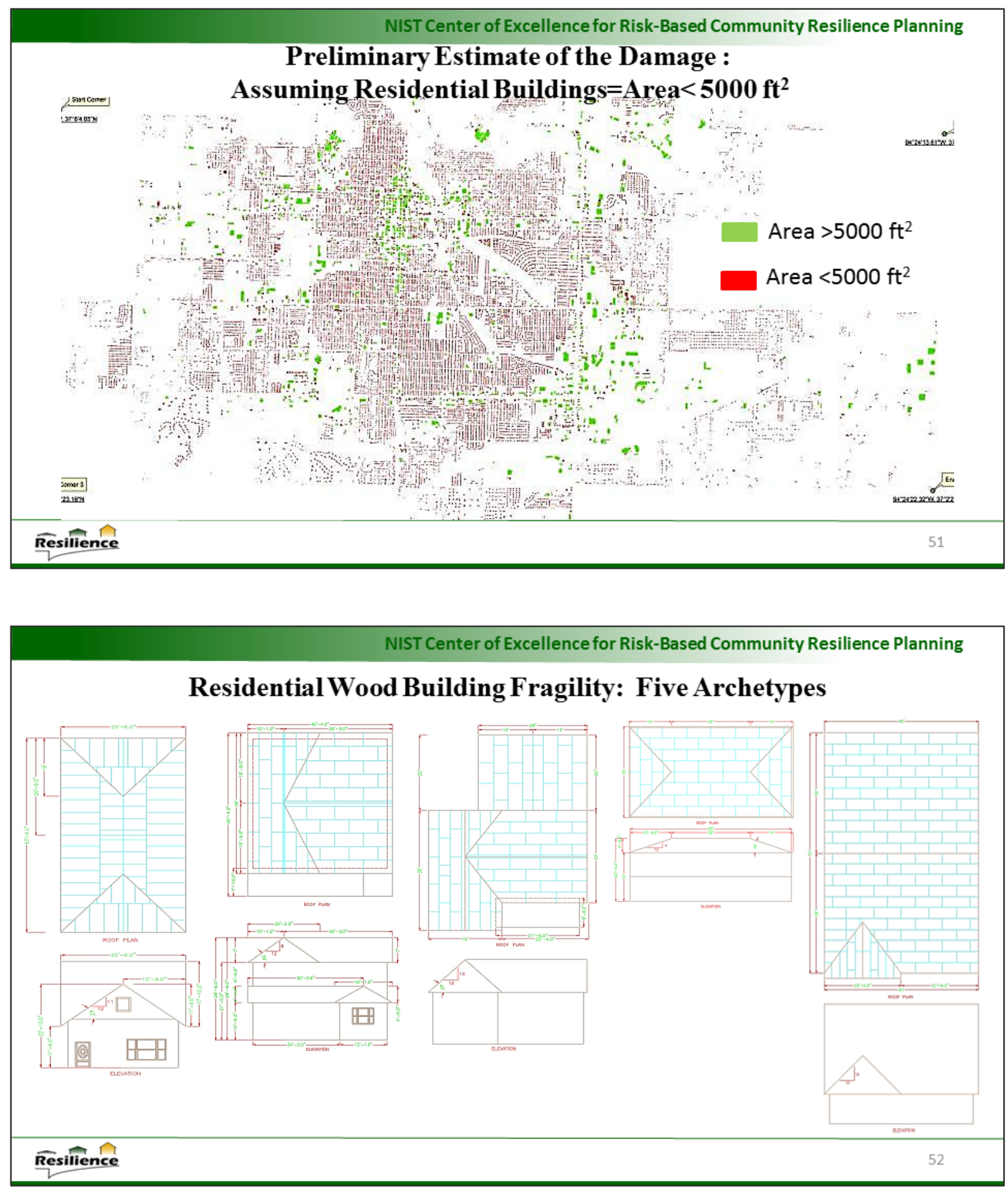
NIST Center of Excellence for Risk-Based Community Resilience Planning

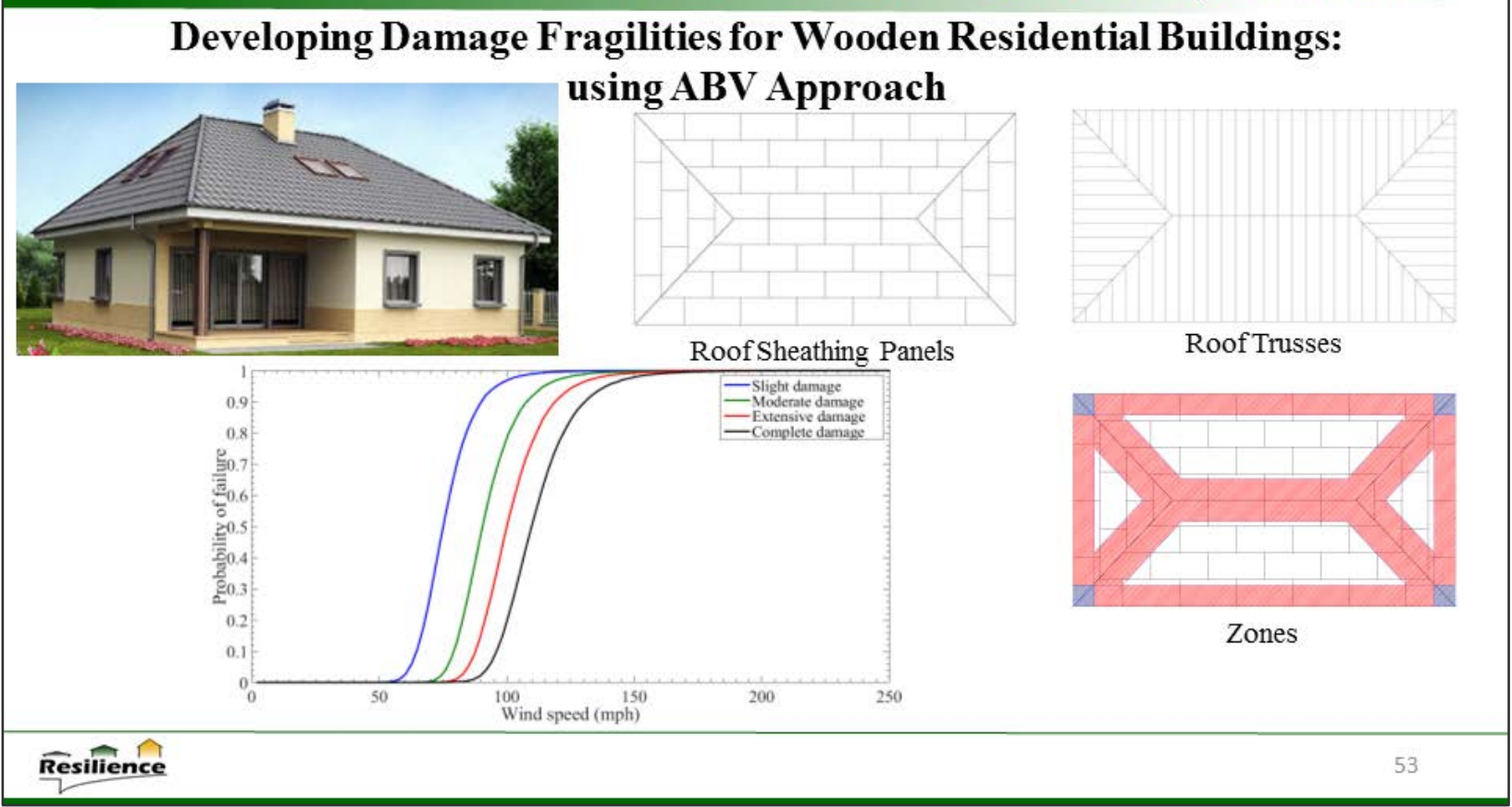

NIST Center of Excellence for Risk-Based Community Resilience Planning

Damage Analysis Results: Assigned to Joplin Buildings

In Total, on Average Total of 2780 Buildings would be Heavily

Damaged or Demolished

Reality: 3181 Buildings

Sample Figure: Houses Located in EF3 Region 


\section{Multisector Validation: Buildings-EPN}

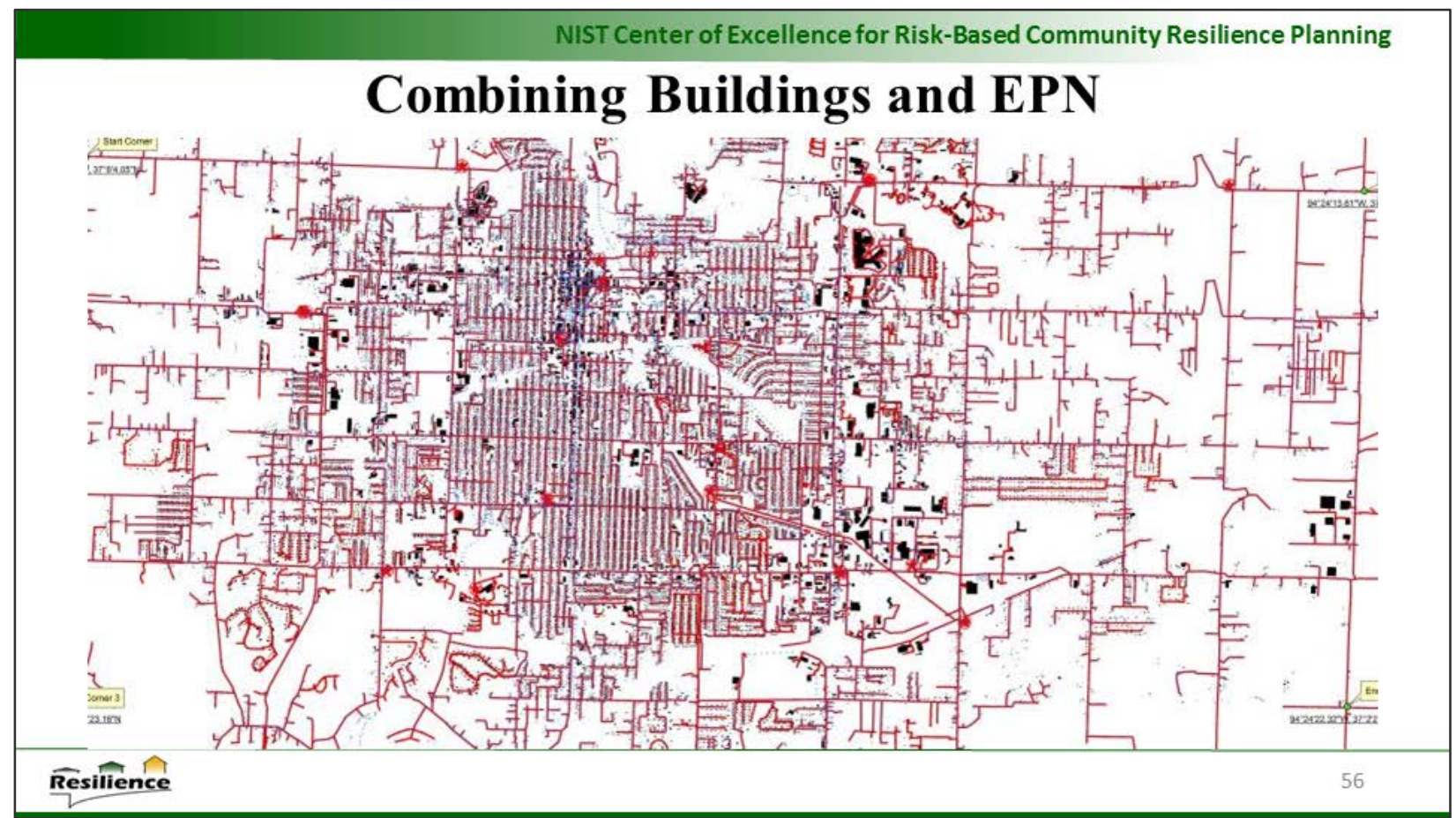


NIST Center of Excellence for Risk-Based Community Resilience Planning

\section{Combining Building and Power Distribution}

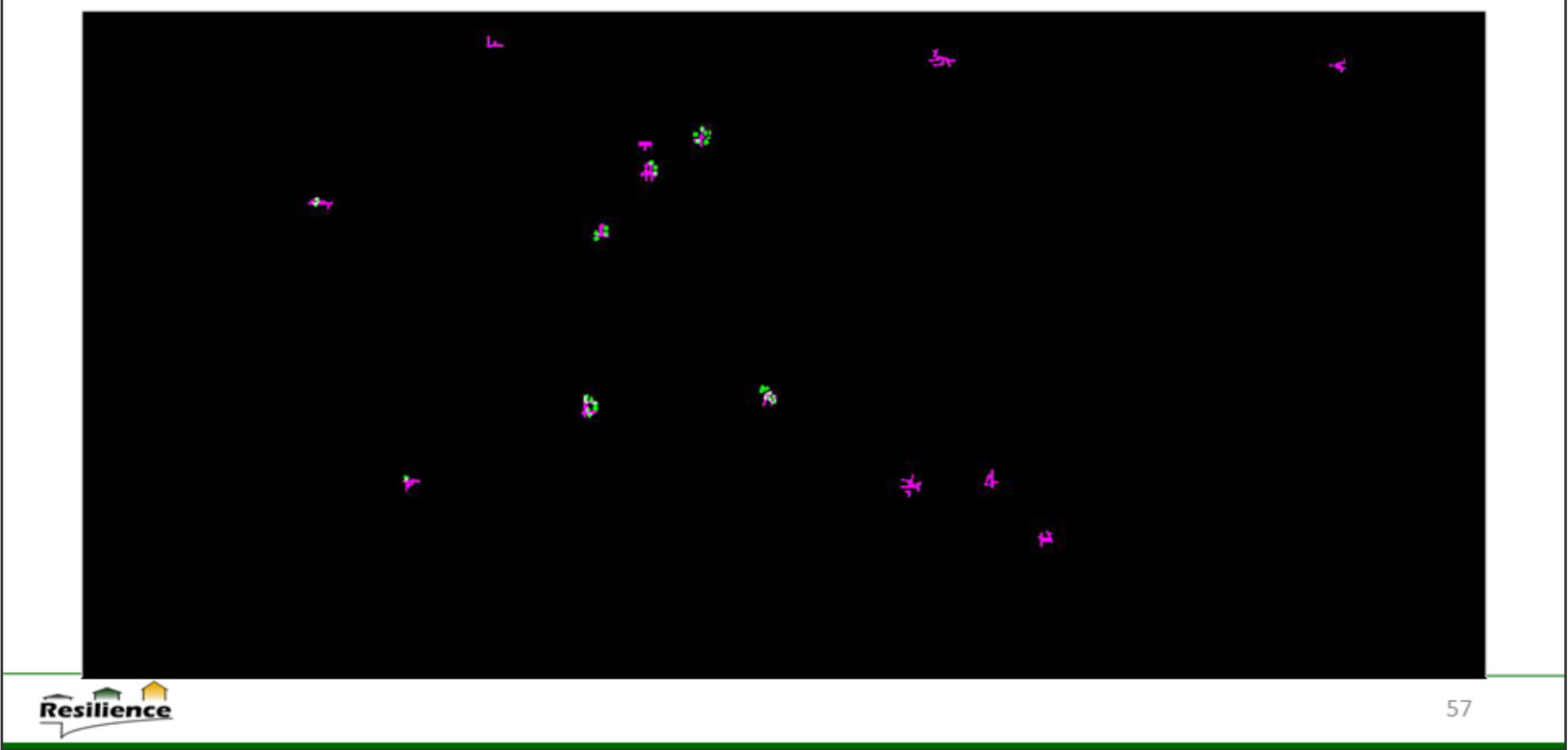

NIST Center of Excellence for Risk-Based Community Resilience Planning

\section{CGE Model Under Development}

- A social accounting matrix (SAM) summarizes the data that reflects the interaction between households, firms and the government.

- SAMS will be constructed for 2010, 2011 and 2012 for Joplin

- CGE model for 2010 and then use it to forecast future SAM values and compare these forecasts to the values in the 2011 and 2012 SAMs.

- The forecast errors (Actual - Forecast) will determine initial ability to forecast the impacts of a tornado. 


\section{Next Steps}

- Accurate fragility assignments to all buildings - aggregation

- Coupling of buildings and EPN; in progress

- Coupling of buildings and socio-econ; in progress

- Application of CGE model; under development

- Other physical infrastructure sectors, debris removal, etc.

- One-way dependencies beyond EPN-buildings

- Recovery functions for buildings; EPN applied

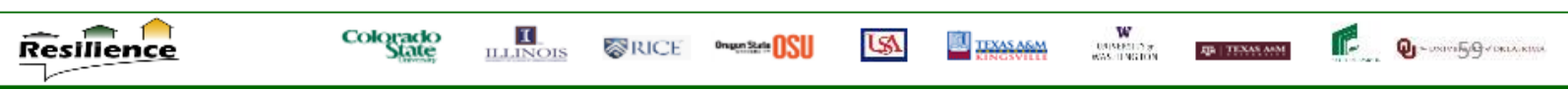

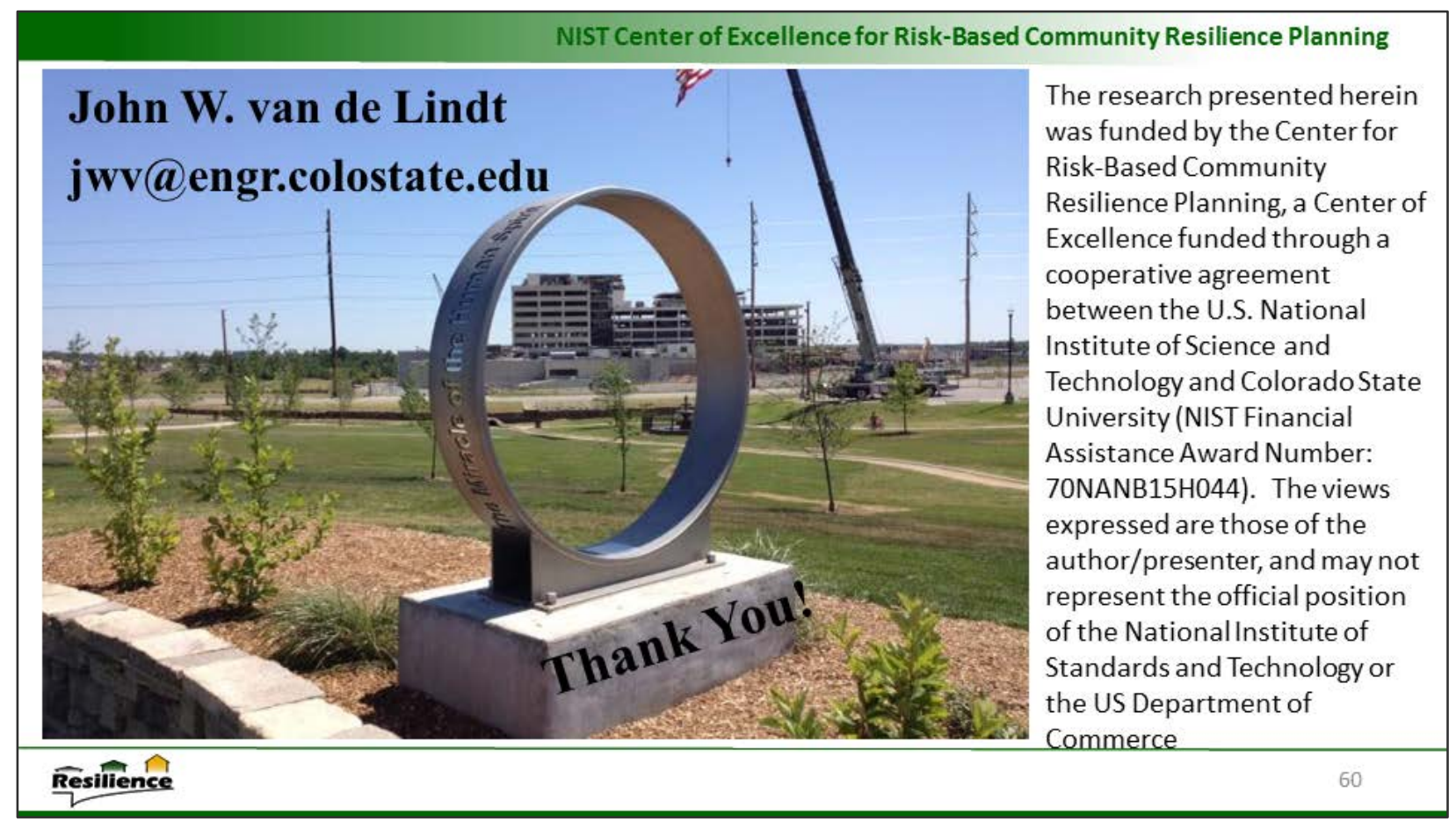




\section{Model Validation: The Joplin Tornado Hindcast}

\section{References}

- Amini, M. O. (2012). Determination of rational tornado design wind speed for residential woodframe structures (Doctoral dissertation, University of Alabama TUSCALOOSA).

- Carreno, E. M., Rocha, R. M., \& Padilha-Feltrin, A. (2011). A cellular automaton approach to spatial electric load forecasting. IEEE Transactions on Power Systems, 26(2), 532-540.

- City-Data. 2016. Joplin, Missouri. Accessed on October 5, 2016 at: http://www.citydata.com/city/Joplin-Missouri.html

- City of Joplin. 2016. Fact Sheet. Recovery update from city of Joplin, May 2016.

- Crawford, P. S. (2014). Methodology for a GIS-based damage assessment for researchers following large scale disasters (Masters thesis, The University of Alabama TUSCALOOSA)

- Fenwick, J. W., \& Dowell, L. J. (1999). Electrical substation service-area estimation using cellular automata: an initial report. In Proceedings of the 1999 ACM symposium on Applied computing (pp. 560-565). ACM.

- Kuligowski, E., Lombardo, F., Phan, L., Levitan, M., \& Jorgensen, D. (2013). Draft Final Report, National Institute of Standards and Technology (NIST) technical investigation of the May 22, 2011, tornado in Joplin, Missouri. NIST NCSTAR 3. Accessed on Dec, 20, 2013.

- Marshall, T. P., Davis, W., \& Runnels, S. (2012). Damage survey of the Joplin tornado. In Preprints, 26th Conf. on Severe Local Storms, Nashville, TN, Amer. Meteor. Soc (Vol. 6).

- Pala, O., Wilson, D., Bent, R., Linger, S., \& Arnold, J. (2014). Accuracy of Service Area Estimation Methods Used for Critical Infrastructure Recovery. In International Conference on Critical Infrastructure Protection (pp. 173-191). Springer Berlin Heidelberg.

- Peek, Lori and Jennifer Tobin-Gurley. 2013. Qualitative interviews with key stakeholders and community members.

- Prevatt, D. O., Coulbourne, B., Graettinger, A., Pei, S., Gupta, R., \& Grau, D. (2011). The Joplin tornado of 2011—Damage survey and case for tornado resilient codes. American Society of Civil Engineers, ed.

- Prevatt, D. O., Roueche, D. B., Van De Lindt, J. W., Pei, S., Dao, T., Coulbourne, W., ... \& Torrent, D. G. (2012). Building damage observations and EF classifications from the Tuscaloosa, AL and Joplin, MO tornadoes. In Structures Congress 2012.

- Van de Lindt, John, Lori Peek, Jennifer Tobin-Gurley, Hassan Masoomi, and Todd Clapp. 2016. Qualitative interviews with key stakeholders and community members.

- Van Zandt, S., Peacock, W. G., Henry, D. W., Grover, H., Highfield, W. E., \& Brody, S. D. (2012). Mapping social vulnerability to enhance housing and neighborhood resilience. Housing Policy Debate, 22(1), 29-55. 


\section{John W. van de Lindt, Ph. D., F. ASCE Curriculum Vitae}

Dr. John W. van de Lindt is the George T. Abell Distinguished Professor in Infrastructure in the Department of Civil and Environmental Engineering at Colorado State University. He formerly was a Professor at the University of Alabama where he held the Garry Neil Drummond Endowed Chair in Civil Engineering. Over the last two decades Dr. van de Lindt's research program has sought to improve the built environment by making structures and systems perform to the level expected by their occupants, government, and the public. This has been primarily through the development of performance-based engineering and test bed applications of building systems for earthquakes, hurricanes, tsunamis, tornadoes and floods. To accomplish this has necessitated coupling nonlinear dynamics, including stochastic approaches in both time and space with structural reliability during extreme loading events. His work includes both the development of new nonlinear numerical models and a large number of experimental investigations to calibrate those models and support hypotheses. Over his career he has successfully led federal, state, and industry sponsored projects totaling approximately $\$ 35 \mathrm{M}$. As a result of these projects he has published approximately 300 technical publications including more than 135 peer reviewed journal papers in scholarly journals and presents work frequently at both national and international conferences. He has recently given a number of keynotes and invited talks around the world including Japan, China, India, Italy, Canada, and New Zealand. Professor van de Lindt current serves as the Co-director for the National Institute of Standards and Technology-funded Center of Excellence for Risk-Based Community Resilience Planning headquartered at Colorado State University. Professor van de Lindt also serves as PI on the three-university NSF-funded project entitled A Risk-Informed Decision Framework to Achieve Resilient and Sustainable Buildings that Meet Community Objectives. That project focuses on optimizing a wood-concrete hybrid building such that it maximizes community resiliency while maintaining a minimum specified level of sustainability.

He also served as the project director and PI for a three-year five-university NSF-sponsored project, which had the objective of reducing seismic risk of soft-story woodframe buildings in North America. Tests at five laboratories around the U.S. (NEES Facilities and other university laboratories) were conducted and the project involved numerous industry and government partners. Professor van de Lindt was also the project director and PI of the four-year five-university NEESWood Project. The final shake table test program took place in June/July 2009 in Miki, Hyogo Prefecture, Japan. The steel moment frame plus light-frame wood building represented the largest building ever tested on an earthquake shake table-designed at almost 17,000 sq ft (1,400 sq meters) and 850,000 lbs. The building was designed using Performance-Based Seismic Design (PBSD) techniques developed during the first three years of the NEESWood Project. Construction took exactly four months in Miki, Hyogo, Japan where Dr van de Lindt lived with his students while supervising construction. All US and Canadian wood products shipped out of Seattle, WA or Vancouver B.C. and steel shipped out of Los Angeles, CA. Shipping consisted of twenty 44,000 lb containers full of numerous products that were donated or discounted by both industry and government, with technical collaboration from the U.S. Forest Products Lab, Simpson Strong Tie, Maui Homes, and FPInnovations-Forintek Division. The building performed very well thereby validating the new design method. Professor van de Lindt solicited and procured approximately $\$ 1 \mathrm{M}$ in additional funds to complete the test program in Japan from both U.S. and foreign governments and industry. 


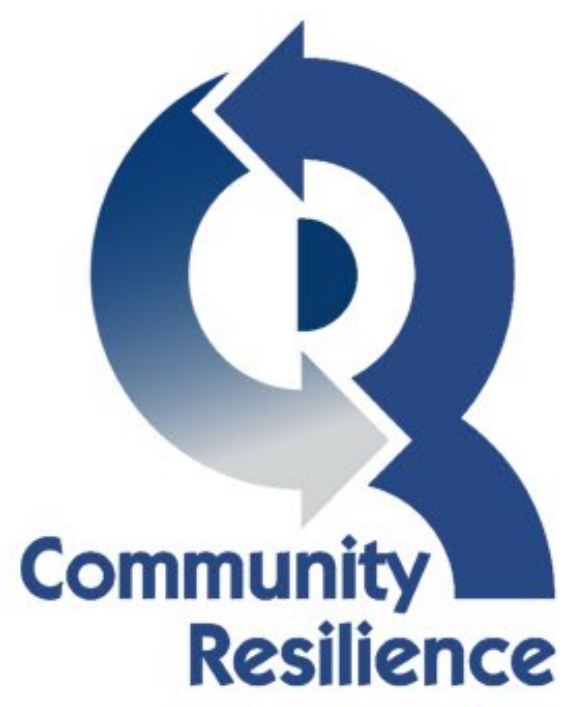

NLT

National Institute of Standards and Technology

U.S. Deparment of Commerce 